\title{
Physicians Poster Abstracts
}

EBMT 2010

\section{Acute leukaemia}

P390

Haematopoietic cell transplantation - Comorbidity Index in AML elderly patients submitted to autologous transplant

D. Capelli (1), M. Chiarucci (1), G. Mancini (1), M. Montanari (1), I. Scortechini (1), A. Poloni (1), S Trappolini (1), F. Saraceni (1), A. Olivieri (2), P. Leoni (1)

(1)Ospedali Riuniti di Ancona (Torrette di Ancona, IT); (2)Ospedale San Carlo (Potenza, IT)

Hematopoietic Cell Transplantation-Comorbitdity Index (HCT-Cl), is a score validated by Sorror et al. to evaluate transplant Transplant-Related Mortality (TRM) incidence, in patients undergoing Allogeneic Transplant. The aim of our study was to assess TRM and the reliability of this score in AML elderly patients undergoing Autologous Transplant. We have treated 101 elderly AML patients aged over 60 years, diagnosed between june 1999 and September 2009. All patients received an induction and consolidation treatment including HD-ARA-C and Idarubicin, with a $30 \%$ reduction dose in over 70 years patients; those obtaining a successful mobilization $\left(\mathrm{CD} 34+\right.$ cell $\left.>3 \times 10^{6} / \mathrm{kg}\right)$ underwent Autologous Transplant, 12 after Busulfan plus Melfalan (BUMEL) and 9 after BEAM conditioning. The remaining patients received alternative consolidation treatment. We obtained a $71 \%$ CR, 49 patients were in CCR and eligible to a second consolidation; 21 of these (43\%) obtained a successful CD34 mobilization and underwent Autologous Transplant. Six patients had a HCT-CI 0 score, 11 had a score between 1 and 2, 4 patients had $a>3$ score. One of the latter patients had a previous cancer, 2 had an heart valve disease and 1 had diabetes, peptic ulcer requiring treatments and previous severe infectious complications. Five out 21 patients died of transplant, with a $25 \%$ cumulative 1-year TRM incidence. Surprisingly no patients with a $>3 \mathrm{HCT}-\mathrm{Cl}$ score died, vs. one patient in the 1-2 score group $(9 \%)$ and 4 patients in the 0 score group $(67 \%)(P=0.02)$. Age $<$ and $>70$ years did not affect the TRM rate, but all the five deaths occurred after the administration of Busulfan plus Melfalan (BUMEL) with an higher TRM rate in this group $(42 \%, P=0.04)$ in comparison with the patients receiving BEAM. In our small series all 6 patients with a $0 \mathrm{HCT}-\mathrm{Cl}$ score received BUMEL as conditioning $(P=0.01)$, suggesting that the higher TRM rate in this group might be related to the administration of a more toxic conditioning regimen.

Elderly patients generally have several comorbidities and HCT$\mathrm{Cl}$ score might be an insufficient tool in assessing their frailty. The high TRM rate in elderly AML patients suggests that some of these patients should receive less toxic conditioning or alternative consolidation regimen. Larger retrospective and multicentric studies are therefore needed in order to better validate this score in this setting.

\section{P391}

Prospective validation of novel myeloablative conditioning regimen:Fludarabine-IV Busulfan in 38 patients with acute myeloid leukaemia

A. Talbi, R.M. Hamladji, M. Benakli, R. Ahmed-Nacer, R. Belhadj, F. Mehdid, N. Rahmoune, F. Harieche, F. Zerhouni Pierre and Marie Curie Center (Alger, DZ)

Introduction: Allogeneic hematopoietic stem cell transplant (HSCT) has the goal of inducing a graft versus tumor immune effect, however the choice of the conditioning may have an impact on the overall out come, in fact more intensive regimens can cause greater morbidity and mortality, on the other hand the reduced intensity conditioning (RIC) may have less anti tumor effect. In this study we used a novel conditioning regimen with Fludarabine (FLU) and IV Busulfan (BU) at myeloablative dose.

Material and methods: Between February 08 and June 09, 38 Adults pts with AML received a geno identical peripheral blood stem cell transplant with FLU $\left(200 \mathrm{mg} / \mathrm{m}^{2}\right)$ and one daily iv BU $(12,8 \mathrm{mg} / \mathrm{Kg})$ total dose conditioning; the median age is 35 years $(22-55)$, the sex ratio is $1,9(25 / 13)$; the cytologic types of AML ( $F A B: M 1=12, M 2=16, M 3=1, M 4=2, M 5=5, M 6=2$ ); $31 \mathrm{HSCT}$ were performed in first remission $(81,5 \%), 5$ in second remission, and 2 in relapse. The median time from the diagnosis to transplant is 4,4 months (2-10).

GVHD prophylaxis associated ciclosporin and methotrexate (Seattle). At September 2009 maximal follow up is 17 months and minimal 3 months.

Results: The overall median time to achieve a neutrophil count $>0,5 \times 10^{9} / \mathrm{L}$ was the day $14(9-18)$, and a platelet count $>20000$ was the day $13(8-16)$; the median duration of the neutropenia is 9 days $(1-16)$. Thirty two pts $(84,2 \%)$ received blood transfusions (1,8 unit/pt); 35 pts $(92,1 \%)$ received platelets transfusions $(2,28$ unit/pt). Six pts $(16,2 \%)$ have a CMV reactivation at day $98(40-210)$ Acute GVHD was observed in 10 pts $(27 \%)$, (Grade $I I=4$, grade $I I I=2$, grade IV $=4$ ). Chronic GVHD was observed in $11 \mathrm{pts}(34,4 \%)$, severe cGVHD: $10 \mathrm{pts}$. Four pts $(10,8 \%)$ relapsed, at a median time of 192 days (102-330), 2 pts were in 1st CR, and 2 pts in 2 nd CR, none of these pts have presented GVHD. Eight pts $(21 \%)$ died; 4 pts after aGVHD; 2 after relapse, 1 after unknown extra hematological cause, and the last one was graft failure. At one year, TRM was about $10,8 \%$. Thirty pts $(84,2 \%)$ are still alive; 28 pts $(73,6 \%)$ are in complete remission; 2 pts are alive in relapse. The median following is $7(3-17)$. Overall survival and DFS are respectively 74,5 and $64,4 \%$.

Conclusion: This study show that FLU-BU myeloablative regimen causes limited toxicity; it can be successfully used in older pts. The rate of relapse seams not higher than what we observed with other myeloablative conditioning regimen $(12,8 \%$ in CPMC study EBMT 08, 37\% GOELAM Blood 97.

\section{P392}

Phase I/II study of haploidentical stem cell transplantation with early transfer of highly purified donor CD56 + CD3NK cells as treatment option for refractory AML

B. Friedrichs (1), N. Basara (2), A. Nogai (1), M. Notter (1), C. Gentilini (1), C. Pfrepper (2), D. Niederwieser (2), E. Thiel

(1), L. Uharek (1)

(1)Charite Berlin-Campus Benjamin Franklin (Berlin, DE);

(2)University of Leipzig (Leipzig, DE)

Objective: Patients with advanced hematological malignancies, especially refratory acute myeloid leukemia (AML) lacking a HLA-compatible donor for allogeneic stem cell transplantation have very poor outcome. For this patient group haploidentical hematopoietic stem cell transplantation (haploSCT) offers an alternative treatment approach. In a prospective phase I-II trial, we evaluated a haploidentical transplantation protocol 
combined with early transfer of natural killer (NK) cells, a cell population known to promote lymphohematopoietic reconstitution and to exert cytotoxicity against leukemic blast.

Methods: Twenty-five patients with hematological malignancies $(A M L=16, A L L=5, C M L=2$, Hodgkin=1, MDS=1), lacking an HLA identical donor received a haploSCT followed by transfer of purified CD56 + CD3-NK cells at day +2 . Conditioning consisted of total body irradiation, thiotepa, fludarabine, and OKT3. NK cells were isolated from the CD34- fraction using an automated two-step procedure of CD3 + depletion and subsequent CD56 + selection

Results: Patients received a mean of $13.3 \times 10^{6}$ CD $34+$ cells/ $\mathrm{kg}$ with $2.9 \times 10^{4} / \mathrm{kg}$ total CD3+ cells. A mean of $9.9 \times 10^{6}$ CD56 + CD3- NK cells $/ \mathrm{kg}$ was adoptively transferred. All of the patients developed early graft-versus host disease (GVHD) of the skin (median day of onset $d+9$ ), which promptly resolved after short term treatment with steroids and CSA. After a median follow-up of 4.1 years (range 0.1 to 7.1 ) 10 of 25 patients are alive and in complete remission resulting in a 3-year overall survival (OS) estimate of $29.3 \%$. Out of 16 patients with AML, 10 had refractory disease prior transplantation. The 3-year OS estimate for this high-risk group of patients is $44 \%(8 / 16)$ with 5 patients now surviving more than 3 years. Causes of death consisted of infections $(n=12)$, chronic GvHD $(n=1)$, relapse $(n=4)$. Treatment related mortality at $d+100$ was $24 \%(6 / 25)$. No correlation was found between KIR-ligand incompatibility and survival.

Conclusions: Our data demonstrate that the early adoptive transfer of high numbers of HLA-mismatched NK shows promising survival rates for a group of patients lacking other treatment options. Best results have been achieved in patients with AML not only in remission but also with refractory disease - a patient group with otherwise extremely poor outcome.

\section{P393}

Cytomegalovirus increases incidence of acute GvHD and reduces the relapse risk for acute leukaemia after transplant: there is a virus-versus-leukaemia effect A.H. Elmaagacli (1), M. Lindemann (1), B. Opalka (1), M. Koldehoff (1), T. Gromke (1), H. Ottinger (1), S. Ross (1), S. Bauer (1), I. Hilgendorf (2), D.W. Beelen (1) (1)University Hospital of Essen (Essen, DE); (2)University Hospital of Rostock (Rostock, DE)

CMV reactivation occurs frequently after HSCT and is associated with an increased TRM.

First, we show that HCMV infects acute leukemia cell line Kasumi-1 (AML) and SD-1 (ALL), inhibits their proliferation and induces apoptosis almost in all cells after 14 days. HCMV further inhibits the proliferation of bone marrow progenitor cells derived from healthy volunteers as demonstrated here by the reduction of the number and size of colony forming units in methylcellulose assays. The induction of apoptosis is unusual because HCMV possesses various strategies to prevent apoptosis in infected cells in order to delay their cell death and maintain its own virus replication. Apoptosis via a caspase-dependent pathway occurred although HCMV induced a significant up-regulation of the anti-apoptotic gene cFLIP and anti-stress gene Gadd45a and simultaneously down-regulation of pro-apoptotic genes p53, Bcl-2, Gadd45a in Kasumi-1 and SD-1 cells, showing that the anti-apoptotic mechanisms of HCMV to prevent cell death failed. Concordantly, the coapplication of the caspasespecific inhibitor zVAD.fmk to HCMV- infected Kasumi-1 cells inhibited the induction of apoptosis to a great extent.

Thereafter we evaluated the clinical outcome of patients with AML after transplant in whom a HCMV reactivation occurred. We evaluated a homogenous group of 140 patients with $A M L$ after myeloablative conditioning, non- $T$ cell depleted allogeneic HSCT from a HLA-identical sibling donor, and compared the clinical results to 60 patients with CML after transplant.CMV reactivation was documented by CMV-related matrix protein pp65 antigenemia test and routinely accompanied by a CMV preemptive therapy with ganciclovir.

CMV reactivation in AML patients was associated with reduced risk for relapse $(11.6 \%$ vs. $50.5 \% ; P<0.0001)$ and improved overall survival estimates $(73.6 \%$ vs. $42.5 \%, P=0.039)$, but increased risk for acute graft-versus-host-disease (GVHD) grade $2-4(75.1 \%$ vs. $37.5 \%, P=0.004)$ and TRM in our analyses, whereas CML-patients had no advantageous effects. Multivariate analyses for risk of relapse confirmed CMV reactivation as an independent factor (RR 0.15, 0.03-0.67; [95\% $\mathrm{Cl}], P<0.013)$ besides chronic GVHD, disease stage, and cytogenetics.

We show here for the first time a strong anti-leukemic effect of HCMV in AML, which improves the outcome of patients after transplant and might be defined as "virus versus leukemia effect".

\section{P394}

Allogeneic haematopoietic cell transplantation with clofarabine/busulfan $x 4$ conditioning exhibits significant anti-leukaemic activity in non-remission AML

J. Magenau, A. Pawarode, T. Buck, D. Jones, K. Kato, A. Harris, D. Frame, L. Kujawski, H. Erba, E. Peres, P. Reddy, C. Kitko, S. Choi, G. Yanik, T. Braun, J. Ferrara, J. Levine, S. Mineishi University of Michigan (Ann Arbor, US)

In an effort to improve the anti-tumor activity of allogeneic hematopoietic cell transplantation (HCT) conditioning, we combined clofarabine as a single daily dose of 20,30 or $40 \mathrm{mg} / \mathrm{m}^{2}$ IV $\times 5 d$ (days -6 to -2 ) with myeloablative doses of busulfan administered as a single daily dose of $3.2 \mathrm{mg} / \mathrm{kg} \mathrm{IV} \times 4 \mathrm{~d}$ (days -5 to -2) (CloBu4) in a phase I/II study in non-remission hematologic malignancies. Pts with acute leukemias had either $\geq 5 \%$ bone marrow blasts or peripheral blasts, while all other hematologic malignancies had active disease at transplant. Pts received dexamethasone $12 \mathrm{mg}$ IV on the days of clofarabine to prevent capillary leak syndrome. No patients received T-depleting antibodies with conditioning.

Characteristics of 44 pts are shown in the Table. Pts received an average of 3 regimens prior to $\mathrm{HCT}$ and $57 \%$ received unrelated and/or HLA mismatched grafts. Pts with AML $(\mathrm{N}=29)$ had either primary induction failure $(41 \%)$ or refractory relapse $(59 \%)$ with an average of $28 \%(2 \%-91 \%)$ bone marrow blasts. CloBu4 was well tolerated. Non-hematologic toxicities included: transaminitis $(52 \%)$, hand-foot syndrome $(12 \%)$, transient hypoxia (11\%), VOD $(5 \%)$, hyperbilirubinemia $(5 \%)$, and seizure $(2 \%)$. All patients engrafted (11 days for neutrophils; 12 days for platelets). Full donor chimerism $\geq 95 \%$ ) at day +100 was seen in $28 / 29(97 \%)$ of pts for CD33+ cells and $23 / 29(79 \%)$ for CD3 + cells. Acute GVHD ( $\geq$ grade 2) developed in $39 \%$ of pts. $80 \%$ of pts achieved CR by day +30 (93\% for AML). With a median follow up of 11 months, overall survival for the entire cohort is $44 \%$ ( $54 \%$ for AML) at 18 months. We compared outcomes for the 29 pts with nonremission $A M L$ to 50 historical non-remission $A M L$ pts who received myeloablative conditioning with busulfan $(N=43)$ or TBI $(\mathrm{N}=7)$ based regimens. The cumulative incidence $(\mathrm{Cl})$ of relapse at 18 months in study pts was significantly lower compared to historical controls ( $26 \%$ vs. $60 \% ; P=0.045)$. The $\mathrm{Cl}$ of non-relapse mortality at 18 months was not significantly different between study and control pts $(25 \%$ vs. $19 \%, P=0.13)$. Disease-free survival at 18 months for study pts was better than control pts, but this did not meet the criteria for statistical significance ( $45 \%$ vs. $21 \%, P=0.11$; Figure). In conclusion, these data suggest that clofarabine combined with myeloablative doses of busulfan is well tolerated, results in reliable engraftment, and has significant anti-leukemic activity in patients with non-remission AML at HCT. 


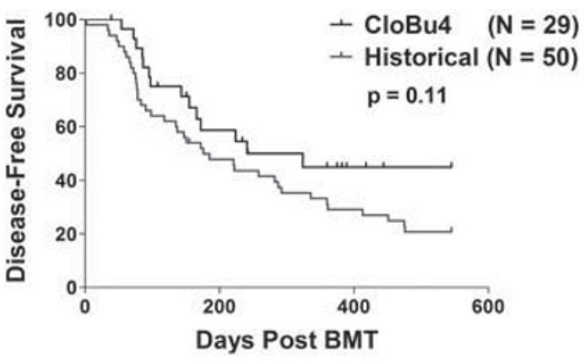

Patient Characteristics ( $\mathrm{N}=\mathbf{4 4}$ )

\begin{tabular}{l|c}
\hline Age, median yrs (range) & $53(1-68)$ \\
Average prior regimens & 3 \\
Average bone marrow blasts, range* & $28 \%(2-91)$ \\
Pts with peripheral blasts, \% (N)* & $69 \%(20)$ \\
Previous allogeneic transplant, \% (N)I & $18 \%(8)$ \\
Unrelated donor, \% (N) & $55 \%(24)$ \\
HLA mismatched donor, \% (N) & $14 \%(6)$ \\
Disease, \% (N) & $66 \%(29)$ \\
AML & $5 \%(2)$ \\
$\mathrm{CML}-\mathrm{BP}$ & $9 \%(4)$ \\
$\mathrm{AlL}$ & $9 \%(4)$ \\
$\mathrm{CLL}$ & $7 \%(3)$ \\
$\mathrm{NHL}$ & $2 \%(1)$ \\
$\mathrm{MDS}$ & $2 \%(1)$ \\
$\mathrm{MM}$ & \\
$\mathrm{Clofarabine}$ dose, $\%(\mathrm{~N})$ & $14 \%(6)$ \\
$20 \mathrm{mg} / \mathrm{m}^{2} /$ day & $48 \%(21)$ \\
$30 \mathrm{mg} / \mathrm{m}^{2} /$ day & $38 \%(17)$ \\
$40 \mathrm{mg} / \mathrm{m}^{2} /$ day &
\end{tabular}

*AML patients $(\mathrm{N}=29)$

\section{P395}

A prospective observational study on the feasibility of donor search and haematopoietic cell transplantation for adults with acute leukaemia

V. Rocha, M. Arat, V. Koza, N.C. Gorin, A. Ferrant, K. Schäfer Eckart, F. Ebeling, G. Milone, P. De Fabritiis, L. Verdonck, A. Bosi, J. Esteve, B. Allione, J. Maertens, A. Olivieri, C. Pilatrino, A.L. Herr, F. Pinto, C. Arrais, S. Nabhan, A. Ruggeri, G. Socié, G. Polge, M. Labopin on behalf of the Acute Leukemia Working Party of EBMT

We have addressed the question of the feasibility of searching HSC donors grafts [HLA-matched sibling donor (MSD), and alternative donors] and performing such transplants or other treatments (such as autologous HSC transplant or chemotherapy) for adults with $\mathrm{AL}$ for whom an indication of allo-HSCT could be planned. The second objective was to compare in an "intent-to-treat" analysis, LFS according to planned strategy treatment. Patients were included at HLAtyping test. From 2003 to 2006, 700 adults were enrolled by 31 EBMT centres, 488 had AML, 212 ALL. HLA-typing was performed for 440 patients at diagnosis, 173 after first CR, 10 after CR2, and 76 in more advanced phase. Definitive analysis was planned to be performed in December 2009. Median follow-up was 38 months, median age was 42-years (18-75); 207 patients were aged more than 50-y. A MSD was found for 311 patients $(45 \%)$. At the end, 269 patients were planned to be transplanted with a family donor. For the remaining 421 patients, the indication of an auto-HCT was made in 84 patients $(20 \%)$, use of chemotherapy as post-remission therapy in 131 (33\%) and indication of an allo-HSCT with an alternative donor in $199(47 \%)$. Of those 199 patients, the transplant centres were keen to search for a cord blood donor in 72 cases and for a haplo donor in 11 cases. At last follow-up, 450 patients of 700 patients included received an HSCT (64\%). Cumulative incidence $(\mathrm{Cl}$, using death as competing event) of allotransplantation for all patients at 1 -year it was $49 \pm 2 \%$. At $1 \mathrm{y}, \mathrm{Cl}$ of allotransplantation was $88 \pm 2 \%$ for patients with a MSD, $57 \pm 3 \%$ for those of whom a donor search was performed and it was $-6 \pm \%$ for those patients for whom another treatment was planned. For those patients with a MDS, $6 \%$ died before the transplantation and $16 \%$ relapsed before receiving a transplant and for those with a donor search, $8 \%$ died and $27 \%$ relapsed. Probability of survival at $2-y$ for 700 patients was $56 \%$. According to treatment planned at HLA-typing, poor risk cytogenetic was present in $46 \%$ of patients searching for an alternative donor, $32 \%$ for those with a MSD, $10 \%$ for autologous treatment and $21 \%$ for chemotherapy. In an intent-to-treat analysis, $2 y$-LFS after HLA-typing was $43 \%$ for patients searching for an alternative donor, $49 \%$ for those with a MSD, $47 \%$ for those with a planned autologous transplantation and $46 \%$ for those with a chemotherapy alone treatment. After transplantation, 2 $y$-DFS was $50 \%$ for autologous, $43 \%$ for unrelated donors and $54 \%$ for MDS transplantation. In conclusion, the majority of patients $(80 \%)$ with AL in European centres are HLA typed at diagnosis or CR1. The majority of patients received a MSD as planned treatment in the first year after HLA typing. Finally, treatment strategies in those 31 centres seem to be linked to patients and disease characteristics and therefore final LFS was not statistically different between all strategies.

\section{P396}

Impact of matched or mismatched unrelated versus matched related donors on outcome of allogeneic haematopoietic stem cell transplantation in AML

B. Federmann, C. Faul, W. Vogel, L. Kanz, W.A. Bethge Hematology \& Oncology (Tubingen, DE)

Current treatment algorithms in AML employ allogeneic HCT from a matched related donor (MRD) in first complete remission (CR). The role of matched/mismatched unrelated donor (MUD/MMUD) HCT remains to be evaluated.

We retrospectively analyzed a cohort of 230 consecutive adult patients (median age 50 (range 18-76)) with AML receiving HCT at our institution 2000-2009 after myeloablative $(M A C=142)$ or reduced-intensitiy conditioning $(\mathrm{RIC}=88) .79$ patients were transplanted from MRD, 85 from MUD and 65 from MMUD. In all but seven patients receiving MMUD grafts, ATG was included in the conditioning. The three cohorts of patients were evenly distributed for age, risk profile and pretreatment. At time of HCT 23 (MRD), 18 (MUD) and 30 (MMUD) patients were not in CR. Current overall survival (OS) is $41 / 79(52 \%)$ in patients transplanted from MRD, 52 of $88(61 \%)$ from MUD and 35 of $65(54 \%)$ from MMUD with a median follow-up of 1331 (range, 136-3530), 904 (range, 100-3267) and 662 (range, 128-1971) days of patients alive. Kaplan-Meier-estimated 3-year OS was similar with $55 \%$ after MRD-, 58\% after MUD- and 49\% after MMUD-HCT $(P=0.4707)$. In patients transplanted in CR, 3-year estimated OS was also comparable ( $60 \%$ MRD vs. $57 \%$ MUD vs. $50 \%$ MMUD, $P=0.90)$. However, in patients transplanted in partial remission (PR) we observed a better OS with a MUD graft ( $31 \%$ MRD vs. $47 \%$ MUD vs. $43 \%$ MMUD, $P=0.18$ ). In patients receiving MAC a better OS compared to RIC was observed (estimated 3 -year OS of $59 \%$ vs. $48 \%(P=0.25))$ mainly due to a lower incidence of relapse. This was also apparent in the subgroup receiving MRD-HCT (61\% vs. $26 \%, P=0.06)$, MUD- $(61 \%$ vs. $49 \%, P=0.80)$ or MMUD-HCT ( $51 \%$ vs. $50 \%, P=0.88$ ) respectively. There were no significant differences in the incidence of aGVHD > II with 24\% (MRD), $33 \%$ (MUD) and $34 \%$ (MMUD) or cGVHD with $38 \%$ (MRD), $44 \%$ (MUD) and 32\% (MMUD), respectively. We observed a significant better OS in patients with limited vs. extensive or without cGVHD (estimated 3-year OS $75 \%$ vs. $35 \%$ vs. $50 \%, P=0.0002$ ). This advantage was present in the subgroup with MRD $(86 \%$ vs. $40 \%$ vs. $43 \% P=0.003)$, but not after MUD ( $68 \%$ vs. $30 \%$ vs. $61 \% P=0.08)$ and MMUD ( $63 \%$ vs. $30 \%$ vs. $47 \%, P=0.32$ ). 
In conclusion, HCT from MUD or MMUD in AML may result in a similar outcome compared to MRD. A graft from MUD and limited cGVHD may improve survival due to an enhanced graft-versus-leukemia-effect particularly in patients in PR.

\section{P397}

The HCT specific comorbidity index fails to predict survival in a large cohort of predominantly high-risk AML patients - single-centre experience

N. Birninger, M. Bornhäuser, S. Parmentier, M. Wermke, M. Schaich, U. Platzbecker, C. Röllig, G. Ehninger, J. Schetelig University Hospital Carl Gustav Carus (Dresden, DE)

Introduction: The hematopoietic cell transplantation specific comorbidity index (HCT-Cl) has been proposed to predict nonrelapse mortality (NRM) and overall survival (OS) in allogeneic HCT recipients. We performed a retrospective study at our center to investigate its capacity in predicting the outcome in comparison with well defined disease-specific risk factors.

Methods: We reviewed the records of 340 patients with AML who underwent allogeneic HCT at our institution between January 2000 and December 2008. Pretransplant comorbidities were assessed by review of medical charts and computer database system. For each patient we assessed the Karnofsky performance score and calculated the HCT-Cl as defined by Sorror et al. (2005). Cox regression modeling was applied for the endpoint overall survival (OS). Non-relapse mortality (NRM) was analysed using univariate and multivariate competing events statistics.

Results: The median age at HCT was 53 years (range, 11 to 76). 133 patients had received myeloablative and 207 nonmyeloablative conditioning regimens. Donors were matched siblings in 116 and alternative donors in 224 patients. Twenty-five percent patients were in first complete remission of standard risk $\mathrm{AML}$. $\mathrm{HCT}-\mathrm{Cl}$ risk was low in $6 \%$, intermediate in $20 \%$, and high in $74 \%$ of the patients. Overall survival at 3 years was $29 \%, 40 \%$, and $44 \%(P=0.7)$ and NRM was $34 \%, 32 \%$, and $26 \%(P=0.6)$, in the low, intermediate and high-risk HCT-Cl groups, respectively. In multivariate analysis, the $\mathrm{HCT}-\mathrm{Cl}$ failed to predict OS and NRM. A reason for this could be, that patients with low comorbidity scores were very rare in this cohort. In part this was due to the high incidence of "heart valve disease" in our cohort, defined in the HCT-Cl as any kind of valvular disease except for mitral valve prolapse. Heart valve disease qualifies on its own for a high HCT-Cl score. In the original publication by Sorror the incidence was $2 \%$. Prior to HCT $45 \%$ of our patients had mostly trace to mild valvular regurgitation, and thus had a high HCT-Cl score. This compares to an incidence of $35 \%$ valvular regurgitation in the healthy population (Framingham Heart Study) and may therefore not be overestimated. Ambiguous definitions could therefore limit the applicability of the HCT-Cl.

Conclusion: In conclusion, in high risk AML patients the HCT-Cl was not associated with NRM or OS in a cohort of predominantly high risk AML patients. Refined definitions might improve the performance of the index.

\section{P398}

Prophylactic donor lymphocyte infusions after allogeneic stem cell transplantation in high-risk myeloid malignancies

Z. Jedlickova (1), R. Conradi (2), H. Baurmann (1), R. Schwerdtfeger (1), M. Schleuning (1)

(1)Deutsche Klinik für Diagnostik (Wiesbaden, DE); (2)Johannes Gutenberg-University (Mainz, DE)

We analysed the toxicity and efficacy of prophylactic donor lymphocyte infusions (pDLIs) in 35 patients (median age 56 years, range $20-70$ ) with high risk myeloid malignancies
(AML, $n=32$; MDS, $n=2$; CML in blastcrisis, $n=1$ ) who underwent allogeneic transplantation between May 2003 and 2008 and were eligible for adjuvant immunotherapy according to the FLAMSA-RIC protocol. All patients received peripheral blood stem cell transplantation. Twenty patients $(57 \%)$ were transplanted from unrelated donors and 15 patients had an HLAidentical sibling donor.

pDLIs were given to patients meeting following criteria: no detectable hematological disease, being off immunosuppression for at least 30 days without active graft-versus-host disease (GVHD), no history of acute GVHD $\geq$ grade $\mathrm{II}^{\circ}$, no active infections.

Two patients received one, 8 patients received two and 25 patients received three pDLIs in escalating doses. Median time from transplantation to first pDLI was 190 days (range 91-439). Minimal interval between two consecutive pDLIs was 4 weeks. Median cell doses were $2 \times 10^{5}, 5 \times 10^{6}$ and $1 \times 10^{7} \mathrm{CD} 3+$ cells/kg at $\mathrm{pDLI} 1,2,3$, respectively. Reasons for administering fewer than three pDLIs were GVHD, relapse, infections or patient's refusal.

At the time of analysis $24 / 35$ patients $(69 \%)$ were alive in complete remission (CR). Median follow-up of survivors was 888 days after transplantation (range 292-2281).

Six patients $(17 \%)$ developed disease relapse (medullary $n=5$; extramedullary, $n=1$ ). Median time to relapse after transplantation was 322 days (range 222-963). Whereas 5 patients died as a result of relapse, one patient survived, being in CR after second transplantation. The 3 -year OS and LFS were 72 and $69 \%$, respectively.

Extensive chronic GVHD developed in 7 patients $(20 \%)$. While in 4 patients chronic GVHD could be controlled, 3 of them died because of refractory GVHD (cutaneous scleroderma, $n=1$; pulmonary fibrosis, $n=1$; polyserositis, $n=1$ ). Two patients developed limited disease not requiring systemic immunosuppressive therapy.

Although further studies assessing the impact of chronic GVHD on the quality of life are warranted, our results indicate that pDLIs are feasible and associated with a low relapse rate and an excellent chance for long term survival in spite of high risk myeloid malignancy in selected patients.

\section{P399}

The EBMT risk-score predicts the outcome of patients with acute myeloid leukaemia receiving allogeneic haematopoietic stem cell transplants

P. Hemmati (1), T. Terwey (1), G. Massenkeil (2), L. Voung (1), C. Jakob (1), A. Vega (1), P. le Coutre (1), B. Dörken (1), R. Arnold (1)

(1)Charite Universitätsmedizin Berlin (Berlin, DE); (2)Städtisches Klinikum Gütersloh (Gutersloh, DE)

Purpose: In chronic myeloid leukemia the EBMT risk-score proofed to be highly valuable in predicting the outcome of patients (pts.) following allogeneic stem cell transplantation (alloSCT). Here, we analyzed whether the EBMT risk-score predicts the outcome of pts. with acute myeloid leukemia (AML) undergoing alloSCT.

Patients and methods: We retrospectively evaluated 225 pts. with AML (median age: 45 years, range: 17-68 years) who underwent alloSCT at our institution between 1994 and 2007. 174 pts. $(77 \%)$ had de novo AML and 51 pts. $(23 \%)$ had secondary or therapy-related AML. A favorable karyotype was present in 11 pts. (5\%), $122(54 \%)$ or 76 pts. (34\%) had an intermediate-risk or a poor-risk karyotype (not evaluable: 16 pts. $(7 \%)) .125$ pts. (56\%) had a myeloablative conditioning (MAC) whereas 100 pts. (44\%) received reduced intensity conditioning (RIC). Transplants were from a matched-related donor (MRD) in 102 pts. (45\%), a matched-unrelated or a mismatched-unrelated donor was chosen in $97(43 \%)$ or in 25 pts. $(12 \%)$. One patient had a mismatched related donor. 120 pts. $(53 \%)$ were in CR1, 40 pts. (18\%) were >CR1, and 65 pts. $(29 \%)$ had active (relapsed/refractory) disease at the time of alloSCT. The EBMT 
risk-score was calculated as described by Gratwohl et al. (Lancet 352, 1998).

Results: Of the 225 pts., 30 (13\%) had an EBMT risk-score of $0-1,48(21 \%)$ had a score of $2,49(22 \%)$ had a score of $3,38(17 \%)$ had a score of $4,47(21 \%)$ had a score of 5 , and $14(6 \%)$ had a score of $6-7$. At ten years after alloSCT, OS in the different groups were $68 \%$ (score of $0-1$ ), $52 \%$ (score of 2 ), $48 \%$ (score of 3 ), $36 \%$ (score of 4 ), $26 \%$ (score of 5 ), or $28 \%$ (score of $6-7$ ) and differed significantly $(P=0.002)$. Likewise, there was a statistically significant difference in relapse between the score groups ranging from $23 \%$ (score of $0-1$ ) to $51 \%$ (score of 4 ) $(P=0.028)$. In contrast, the EBMT risk score did not predict the NRM in the different subgroups $(P=0.084)$. However, a significant trend towards a lower NRM in pts. with a low score was noticed (logrank test for trend: $P=0.004$ ). Of note, the EBMT risk-score failed to predict OS, relapse, or NRM in pts. conditioned with RIC $(n=100)$ whereas it did so in the MAC group.

Conclusions: These data indicate that the EBMT risk-score may be used to predict the outcome of pts. with AML following MACalloSCT. In pts. conditioned with RIC, the EBMT risk-score did not predict the outcome in our patient cohort.

\section{P400}

Allogeneic stem cell transplantation for isolated granulocytic sarcoma (GS): a comparison of outcomes with leukaemic GS on behalf of the ALWP of EBMT

P. Chevallier, M. Labopin, J.J. Cornelissen, G. Socie, P. Ljungman, B. Lioure, H. Tilly, A. Gratwohl, N. Milpied, I. Yakoub-Agha, L. Volin, J.P. Vernant, J.O. Bay, M. Michallet, A. Buzyn, B. Samey, M. Mohty, V. Rocha on behalf of the Acute Leukemia Working Party of the EBMT

Isolated GS is a rare malignant disorder usually presenting as an extramedullary myeloid cell tumor proliferation. GS can be associated with chromosome 8 abnormalities and can be also observed in 3 to $7 \%$ of AML cases (called "leukemic"GS, LGS hereinafter). We have previously shown, that allo-SCT following an AML-like chemotherapy is likely the best available therapeutic option for patients with LGS (J Clin Oncol 2008). However, it is still unclear whether alloSCT is a valid therapeutic option for patients with isolated GS (IGS). The aim of this analysis was to assess the role of allo-SCT in patients with isolated GS as compared to patients with LGS.

We retrospectively analysed the outcome of 68 GS patients including 14 cases of IGS (median age, $39 \mathrm{y}$.) and 54 cases of LGS (median age, $38 \mathrm{y}$.) reported to the EBMT registry between January 1990 and December 2007. In this series, patients received either a standard (IGS, $n=13$ vs. LGS, $n=44, P=N S$ ) or a reduced intensity conditioning (IGS, $n=1$ vs. LGS, $n=10$, $P=$ NS) allo-SCT from a related (IGS $n=10$ vs. LGS, $n=36$, $P=\mathrm{NS}$ ) or a matched unrelated donor (IGS, $\mathrm{n}=4$ vs. LGS, $\mathrm{n}=18, P=\mathrm{NS}$ ).

There were no significant statistical differences between the two groups in terms of year of transplantation, cytogenetics features, disease status, source of stem cell, donor type, conditioning regimen, and recipient CMV serostatus.

Engraftment was observed in all patients. The incidences of grade 2 and grade $3-4$ acute GVHD were $20 \%(n=15)$ and $8 \%(n=6)$, respectively. The incidence of chronic GVHD at 2 years was $37 \pm 7 \%$. With a median follow-up of 56 (range, 7-213) months, the probability of 5-year LFS, relapse incidence (RI), non relapse mortality (NRM) were $44 \pm 7 \%, 37 \pm 7 \%$ and $19 \pm 5 \%$, respectively.

When comparing the two sub-groups (IGS vs. LGS), there were no significant differences in term of major outcomes: neutrophils recovery (IGS:18 days vs. LGS: 19 days), occurrence of grade 2-4 acute GVHD (IGS: $21 \%$ vs. LGS: 33\%), 2-year chronic GVHD (IGS: $54 \%$ vs. LGS: $32 \%$ ), 5 -year LFS (IGS: $54 \pm 14 \%$ vs. LGS: $43 \pm 7 \%$ ) and 5-year RI ( $46 \pm 15$ vs. $34 \pm 7 \%$ ).
Of note, there was a trend for higher NRM in the LGS group $(23 \pm 7 \%$ vs. $0, P=0.07)$.

In all, we conclude that allo-SCT is an effective treatment for patients with GS. Patients with isolated or leukemic GS have similar outcomes after allo-SCT. Therefore, allo-SCT should be presently considered as the optimal therapy for both isolated and leukemic GS.

\section{P401}

Relatively favourable outcome of acute myeloid leukaemia with translocation $\mathrm{t}(6 ; 9)(\mathrm{p} 23 ; \mathrm{q} 34) / \mathrm{DEK}-$ NP214(CAN) rearrangement after allogeneic haematopoietic stem cell transplantation in early phase J. Esteve, M. Labopin, G. Socié, P. Ljungman, A. Bacigalupo, A. Schattenberg, C. Cordonnier, E. Polge, B. Samey, V. Rocha on behalf of the Acute Leukemia Working Party of EBMT

Acute myeloid leukaemia (AML) with translocation $t(6 ; 9)$ (p23;q34)/DEK-NUP214(CAN) rearrangement is a rare AML variety, associated to a poor prognosis, with a long-term survival of less than $20 \%$ in adult patients (Slovak, Leukemia 2006). Nonetheless, a more favourable outcome has been suggested after allogeneic stem-cell transplantation (alloHSCT), although only anecdotal studies with small series of patients have specifically addressed this issue. To investigate the possible role of alloHSCT for the management of AML with $t(6 ; 9)$, we analyzed the outcome of patients with this entity reported to the ALWP. Overall, we identified 68 patients with this AML subtype allografted during the period 19882009 (median year of transplant, 2004). Median age was 39 (range: 16-65) and 51\% were male. Most transplants were performed in complete response (CR1 $=49,72 \%$; CR2 $=8$, $12 \%)$, whereas only a minority were performed in advanced phase (primary refractory, $n=5$; relapse, $n=2$ ). Donor was an HLA-identical sibling in 37 transplants $(54 \%)$, and a matched unrelated donor in $24(35 \%)$. Conditioning regimen consisted of a myeloablative regimen in most patients $(n=56,82 \%)$. After a median follow-up of 52 months (1-179), 5-year overall leukemia-free survival (LFS), relapse incidence (RI), and nonrelapse mortality (NRM) was $42 \pm 6 \%, 28 \pm 3 \%$, and $30 \pm 5 \%$, respectively. In those patients who received an alloHSCT in CR1, 5-year LFS, RI, and NRM was $50 \pm 8 \%, 19 \pm 6 \%$, and $31 \pm 7 \%$, respectively, without significant differences between transplants from HLA-identical sibling and unrelated donors (see figure). In conclusion, alloHSCT in early phase resulted in a favourable outcome in patients with $A M L$ and $t(6 ; 9)$, suggesting this procedure might overcome the adverse prognosis associated to this entity. The analysis of transplant outcome of specific, molecularly defined entities might provide a better knowledge of the role of alloHSCT for the management of AML.

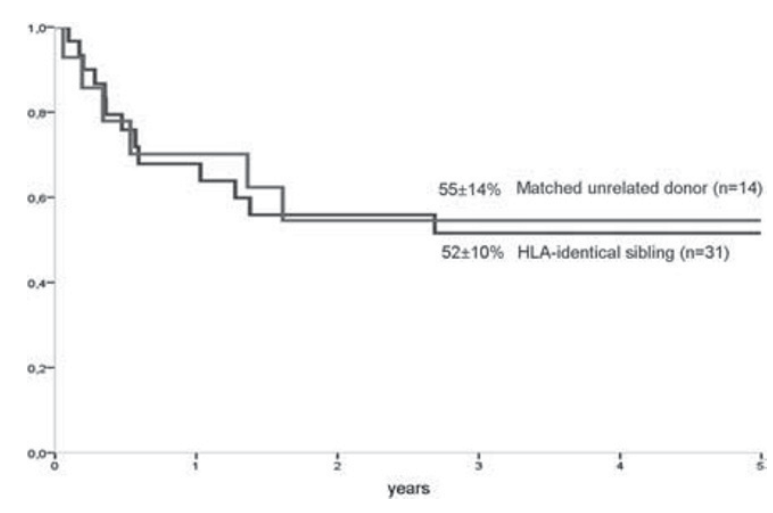


P402

Repeated vaccination with PR1 and WT1 peptides admixed in Montanide adjuvant fails to induce sustained anti-leukaemia responses in patients with myeloid malignancies

K. Rezvani (1), A. Yong (2), S. Mielke (3), B. Jafarpour (2), B. Savani (4), Q. Le (2), R. Eniafe (2), C. Boss (2), R. Kurlander (2), J. Barrett (2)

(1)Hammersmith Hospital (London, UK); (2)NIH (Bethesda, US); (3)Wurzburg (Wurzburg, DE); (4)Vanderbilt (Nashville, US)

In a safety study of one dose of a combination of PR1 and WT1 vaccination we showed that vaccination with PR1 and WT1 peptides can confer transient anti-leukemia immunity in patients with myeloid leukemia. We therefore initiated a phase 2 study of repeated vaccination with PR1 and WT1 peptides in patients with myeloid malignancies. Six patients with acute myeloid leukemia (AML) and 2 patients with myelodysplastic syndrome (MDS) received 6 injections at 2 weekly intervals of PR1 and WT1 peptides in Montanide adjuvant, with GM-CSF as previously described. Six of 8 patients completed 6 courses of vaccination and follow-up as per protocol to monitor toxicity and immunological responses. Responses to PR1 or WT1 vaccines were detected in all patients after only 1 dose of vaccine. However, additional boosting did not further increase the frequency of PR1 or WT1-specific CD8 + T-cell response. In 4/6 evaluable patients the vaccine-induced T-cell response was lost after the fourth dose and in all patients before the sixth dose of vaccine. We found a significant but transient reduction of circulating CD4 + Foxp $3+T$ cells associated with an increase in vaccine-induced PR1 and WT1 specific CD8 + T-cells in patients following vaccination. Vaccination led to preferential expansion of low avidity PR1 and WT1 specific CD8 + T-cell responses that were PD1 negative and was not associated with a significant reduction in WT1 expression, employed as a measure of minimal residual disease. We analyzed bone marrow samples collected from all vaccinated patients 4 weeks following the 6th dose of vaccine and failed to detect PR1 or WT1 specific CD8 + T-cells, thereby excluding preferential localization of PR1 and WT1 vaccine-induced CD8+ T-cells to the $B M$ as an explanation for the failure to detect vaccine-specific CD8 $+T$ cells in the peripheral blood. Three months following the 6th dose of PR1 and WT1 peptides we could not detect the presence of PR1 and WT1 specific CD8 + T-cells by direct ex-vivo tetramer and IC-IFN-g assay or with 1-week cultured IFN-g ELISPOT assay, suggesting that vaccination with PR1 and WT1 peptides in Montanide adjuvant does not induce memory CD8+ T-cell responses. These data support the immunogenicity of PR1 and WT1 peptide vaccines. However new approaches will be needed to induce long-term memory responses against leukemia antigens such as novel adjuvants or induction of CD4+ help.

This study is registered at http://clinicaltrials.gov as NCT00499772.

\section{P403}

Influence of busulfan exposure on allogeneic transplant outcome using a fludarabine/busulfan/thymoglobulin/total body irradiation preparative regimen for adult acute myelogenous leukaemia

V. Lewis (1), F. Naveed (2), S. Kangarloo (2), L. Savoie (2), A. Balogh (2), A. Daly (2), A. Chaudhry (2), N. Zacaria (2), M. Geddes (2), C. Brown (2), N. Bahlis (2), P. Duggan (2), J. Storek (2), L. Larratt (3), R. Turner (3), D. Quinlan (2), M. Yang (2), D. Stewart (2), J. Russell (2)

(1)Alberta Children's Hospital (Calgary, CA); (2)Tom Baker Cancer Center (Calgary, CA); (3)Cross Cancer Institute (Edmonton, CA)

Conditioning-intensity significantly influences outcomes of stem cell transplant for acute myelogenous leukemia (AML). Low-dose total body irradiation (TBI) in a once daily intravenous-busulfan (iBu), fludarabine (Flu) and Thymoglobulin (rATG) regimen improves outcomes. Even without TBI, a daily iBu AUC above $6000 \mu$ M.min, significantly increases non-relapse mortality (NRM) in this regimen. We report the results of a study to determine the effect of iBu exposures below $6000 \mu \mathrm{M}$. min in patients (pts) with AML. 109 adult pts were treated between 2001 and 2008 with Flu $50 \mathrm{mg} / \mathrm{m}^{2}$ daily $\times 5$, iBu $3.2 \mathrm{mg} / \mathrm{kg}$ daily $\times 4$ and TBI $200 \mathrm{cGy} \times 2$ on day -1 or 0 . Acute graft-versus-host disease (aGvHD) prophylaxis was cyclosporine A, methotrexate and rATG (Genzyme) 4.5 $\mathrm{mg} / \mathrm{kg}$ total dose. Patients received a test dose of iBu two days before the first treatment dose of iBu. One quarter of the test dose AUC was added to the AUC of the first or third iBu treatment dose to give an average daily exposure for the complete 4-day treatment course. Five pts with AUC $>6000 \mu$ M.min were excluded. The remainder $(n=104)$ were divided equally into low exposure (LE) (AUC 2547-4321 $\mu \mathrm{M} . \mathrm{min}$ ) and high exposure (HE) (AUC 4322-6000 $\mu \mathrm{M}$.min) groups. Median age was 48 (range 21-63) years in LE pts and 48 (18-66) years for the HE pts. There was no difference in the percentage of recipients with CR1, CR2 and advanced disease $(64,17,19$ vs. $69,19,12)$, alternative (unrelated/mismatched related) donors (58 vs. 54 ), $\mathrm{CMV}+\mathrm{ve}$ donor and/or recipient (67 vs. 60$)$, female donors to male recipients (19 vs. 19), high-risk recipient cytogenetics (25 vs. 18 ) and bone marrow as cell source (4 vs. 6 ) between the LE and HE pts respectively. Follow-up of survivors was 12-104 months (median 34) for LE pts and 13-76 months (median 36) for HE pts.

The incidence of aGvHD grade II-IV at $27 \%$ vs. $20 \%$, aGvHD grade III-IV at $8 \%$ vs. $10 \%$ and chronic GvHD at $48 \%$ vs. $55 \%$ was not different for either group. Neither was there a difference in overall-survival ( $76 \%$ vs. $57 \%, P=0.094)$, disease-free survival $(74 \%$ vs. $56 \%, P=\mathrm{ns})$ or relapse $(22 \%$ vs. $29 \%, P=\mathrm{ns})$ for LE or HE pts respectively. NRM ( $4 \%$ vs. $22 \%, P=0.039$ ) however, was worse for HE pts.

We conclude, when this regimen is used for AML: 1. Higher iBu exposures within the range studied do not reduce relapse. Including TBI in the regimen may abrogate this effect. 2 . Higher $\mathrm{iBu}$ exposures increase NRM. 3. Targeting iBu exposure to between 2500 and $4300 \mu \mathrm{M}$.min may improve outcomes.

\section{P404}

KIR2DL2/3-HLA-C-group 1 and KIR2DL1-HLA-C-group 2 mismatches improve the clinical outcome in patients with acute myelogenous leukaemia after allogeneic stem cell transplantation

R. Richter, G. Bug, S. Mousset, E. Seifried, H. Serve, C. Seidl, H. Martin

University of Frankfurt (Frankfurt/Main, DE)

Background: In patients with acute myelogenous leukaemia $(\mathrm{AML})$, alloreactive donor natural killer (NK) cells are believed to play a significant role in survival after stem cell transplantation (SCT). In this study, we tested the missing-self hypothesis, which indicates that alloreactivity is regulated by inhibitory KIRreceptors on natural killer NK cells and their corresponding HLA ligands on recipient cells.

Patients and methods: Out of a consecutive cohort of 147 patients allografted between 1996 and 2007, blinded samples from 54 donor/patients AML-patients, pairs were evaluated for retrospective KIR-ligand matching. The median patients age was 47 (25-66) years. Patients were transplanted with G-CSF-stimulated PBSC $(n=51)$ or bone marrow $(n=3)$ from HLA matched unrelated $(n=31)$ donors or family related donors $(n=23)$. KIR typing was performed as previously described. Patients were categorized according to their HLA inhibitory KIR ligand group C1, C2, Bw4, A3/A11 and presence or absence of KIR.

Results: A total of $27 / 54$ patients died due to relapse $(n=17)$ or NRM $(n=10)$. The median follow up of surviving patients was 4,2 years (range 1,9-7.2) years. The overall survival (OAS) of all 54 evaluable patients at 5 years was $50 \%$.

In 31 patients HLA ligands for donor inhibitory KIR2DL receptors in the recipient were lacking (mismatch). KIR-ligand mismatch was associated with a significantly superior overall survival 
(0.63 vs. $0.31, P=0.01$ ), superior eventfree survival ( 0.55 vs. $0.25, P=0.009)$, a reduced non-relapse mortality ( 0.12 vs. 0.52 , $P=0.015)$ and a reduced relapse rate (0.36 vs. $0.46, P=0.15)$. Conclusions: The mismatch for KIR2DL2/3-HLA-C-Group-1 or KIR2DL1-HLA-C-Group-2 ligands is associated with a significantly better outcome for AML patients after allogeneic stem cell transplantation.

\section{P405}

Is autologous HSCT a curative option for $\mathrm{Ph}$-positive ALL with negative MRD status? A survey of the European Working Group for Adult ALL (EWALL)

S. Giebel (1), H. Dombret (2), P. Rousselot (3), J.M. Ribera (4), P.P. Piccaluga (5), M. Doubek (6), B. Piatkowska-Jakubas (7), J. Holowiecki (1), D. Hoelzer (8)

(1)M. Sklodowska-Curie Memorial Institute (Gliwice, $P L$ ); (2)Hôpital Saint-Louis (Paris, FR); (3)Hôpital Mignot (Versailles, FR); (4)Hospital Germans Trias i Pujol (Badalona, ES); (5)S. Orsola-Malpighi Hospital (Bologna, IT); (6)University of Brno (Brno, CZ); (7)Collegium Medicum, Jagiellonian University (Cracow, PL); (8)University of Frankfurt (Frankfurt, DE)

Approximately $20 \%$ of adults with ALL presents with $t(9 ; 22)$. The introduction of tyrosine kinase inhibitors in this group of patients increased $\mathrm{CR}$ rate and appeared to prolong leukemia-free survival (LFS). However, the risk of relapse remains high and allogeneic HSCT is still considered the treatment of choice. Patients ineligible for alloHSCT may be offered autologous HSCT although its value in Ph-positive ALL has not been documented so far.

The European Working Group for Adult ALL (EWALL) performed a retrospective analysis of results of autoHSCT in a setting of $\mathrm{Ph}$-positive ALL. Only patients with known status of minimal residual disease (MRD) at the time of autoHSCT were included. 22 patients, aged 50 (21-62) years, treated with autoHSCT in first CR between 1996-2006 in 5 European countries entered the analysis. Median CR duration prior to autoHSCT was 6 (2-13) months. 10 patients were pre-treated with imatinib. Peripheral blood was used as a source of stem cells in all but 2 cases. Conditioning was either TBI+Cy $(n=16)$ or BuCy $(n=6)$. The status of MRD evaluated based on molecular analysis of BCR-ABL fusion gene was assessed as negative in 11 cases and positive in 11 patients. 9 patients received post-transplant maintenance consisting of imatinib $(n=4)$ or chemotherapy $(n=5)$.

With the median follow-up of 2 years, the probability of LFS at 3 -years for the whole group was $53 \%( \pm 15 \%)$. The probabilities of relapse and non-relapse mortality were $38 \%$ and $9 \%$, respectively. The LFS rate was higher for patients with negative MRD status at autoHSCT $(82 \% \pm 12 \%)$ compared to those MRD-positive $(25 \% \pm 20 \%, P=0.08)$. No significant association of the type of induction-consolidation therapy, type of conditioning, the use of post-transplant maintenance and age with outcome was found.

We conclude that autoHSCT apppears a valuable option for adults with Ph-positive ALL achieving negative MRD status. Its role as a curative treatment should be assessed in prospective trial.

\section{P406}

Total body irradiation and total marrow irradiation for patients with advanced haematologic malignanices undergoing an allogeneic stem cell transplant: a pilot study

R. Corvo' (1), S. Agostinelli (1), M. Zeverino (1), S. Barra (1), M.T. van Lint (2), F. Frassoni (2), T. Lamparelli (2), F. Gualandi (2), C. Di Grazia (2), A. Dominietto (2), A.M. Raiola (2), R. Varaldo (2), A. Bacigalupo (2)

(1)Istituto Nazionale per la Ricerca sul Cancro (Genoa, IT); (2)S.Martino's Hospital (Genoa, IT)

Background: Leukemia relapse remains a significant cause of failure after allogeneic hemopoietic stem cell transplantation
(HSCT) (Bone Marrow Transplant. 2007; 39: 341). Increasing the total dose of total body irradfiation (TBI) has the potential of increasing leukemia cell kill, but is associated with increased toxicity (Blood. $1998 ; 92(4): 1455)$.

Total marrow irradiation (TMI) delivered with Helical Tomotherapy $(\mathrm{HT})$ may overcome this problem, by concentrating the radiation dose to the bone marrow, and reducing the dose delivered to vital organs

Aim of the study: This is a feasibility study of TBI/TMI in patients with advanced malignancies, undergoing an allogeneic HSCT. TBI/TMI. Patients received conventional TBI (1200 cGy, as $200 \mathrm{cGy} \times 2 \times 3)$ followed the next day by TMI $(2 \mathrm{~Gy}$ in a single fraction), followed by cyclophosphamide $60 \mathrm{mg} / \mathrm{kg} \times 2$. GvHD prophylaxis was cyclosporine methotrexate. While TBI was delivered with linear accelerator, TMI was HT. Patients were treated in supine position with hands laying on their groins. A couple of thermoplastic masks were used to fix the head/ neck and the thorax and the arms, respectively. Whole body CT imaging was performed by Tomotherapy unit prior to TMI to provide 3-D alignment of patient anatomy to the intended target regions.

Patients: We treated 6 patients beyond CR1 ( 3 sibs, 3 unrelated), median age 35 (19-55). Two were ALL relapsed within 5 months from an allogeneic HSCT, 2 were AML in relapse, 1 was an AML in CR2 relapsing after an autograft, 1 a myelofibrosis with advanced disease.

Results: Median organ doses reduction ranged from $15 \%$ to $80 \%$ with the largest dose reduction $-50 \%$ to $-80 \%$ ) achieved for testes, brain, larynx, liver, lungs and kidney. Small organs as parotids and oral mucosa are less spared $(-35 \%)$ than others. Radiation treatment time for TMI 2 Gy, ranged from $18 \mathrm{~min}$ to 22 min. Target areas (bone marrow sites) were irradiated with an optimal conformity and an excellent dose homogeneity.

Outcome: Follow up is still short ranging from 40 to 140 days. However: tolerance was not different from a conventional CYTBI. All 6 patients treated with TBI/TMI are currently alive in remission. Of the two relapsed ALL, one is VDJ negative and one is VDJ positive.

Conclusions: This study confirms the feasibility of using HT to deliver TMI. Organ sparing by TMI with HT, warrants a prospective dose finding study, with TMI doses up to 6 Gy (and total dose to the marrow of $18 \mathrm{~Gy}$ ).

P407

In AML a high CD34 + peak during consolidation and PBSC mobilization is associated with a poor prognosis in patients treated with autologous HSC transplantation but not in patients submitted to allogeneic HSC transplantation

S. Leotta, G. Avola, M.G. Camuglia, A. Spadaro, E. Marturano, M. Poidomani, C. Consoli, G. Palumbo, E. Mauro, S. Mercurio, A. Di Mercurio, D. Tibullo, G. Milone

Ospedale Ferrarotto (Catania, IT)

In AML patients with a CD34 + high apheretic yield during mobilization, have a poor prognosis independently from cytogenetic risk (Feller 2004, Keating 2004).

We have studied prospectively the prognostic value of CD34 peak reached in P.B. during PBSC mobilization in a group of 50 AML adult patients. PBSC mobilization was obtained, after first consolidation course, by use of G-CSF. Cytogenetic assessment at diagnosis was available in $95 \%$ of patients and FLT-3 status in 30 patients. $49 \%$ of patients received an Allogeneic transplant, $41 \%$ an autologous transplant while $10 \%$ no further chemotherapy. Overall Survival (OS) in the entire cohort of patients was $60 \%$ and Disease Free Survival (DFS) was 50\%. CD34 + peak during mobilization, evaluated as a continuous variable, resulted important for OS (hazard ratio: 1.002; $P=0.002$ ) as well as for DFS (hazard ratio:1.001; $P=0.01$ ). Importance of $C D 34+$ peak was present also in the stratum of patients with a normal cytogenetic (OS: $P=0.003$; DFS: $P=0.002$ ), in stratum of patients having FLT3 wild type (OS: $P=0.02$; DFS: $P=0.04$ ) and in stratum 
of patients that did not receive allogeneic HSC transplant (OS: $P=0.010$; DFS: $P=0.007$ ). Importance of CD34 + peak was however not present after allogeneic HSC transplant (OS: $P=0.36$; DFS: $P=0.95)$. Prognostic significance of CD34 + cells was then evaluated in groups of patients identified in respect of median value of CD34 + peak. Those having a CD34 + cell peak > 200/ $\mathrm{mmc}$ had a OS of $20 \%$, lower than that observed in patients having a CD34 peak $<200 / \mathrm{mmc}$, who had an OS of $69 \%,(P=0.001$ (Figure 1). Group of patients having higher CD34 + peak in P.B. had also a lower DFS $(20 \%)$ in respect to patients having a CD34 + peak $<200 / \mathrm{mmc}(60 \%)$, (log rank: $P=0.008)$. Again these differences were present in stratum of patients with intermediate cytogenetic risk, in stratum of FLT-3 wild type and in stratum of patients treated with autologous transplantation or not transplanted but were not evident in stratum of patients treated with allogeneic transplantation (Table 1). In conclusion a CD34 + peak $>200 / \mathrm{mmc}$ in P.B. during PBSC mobilization has a poor prognostic value independent from cytogenetic and FLT-3 ITD. This adverse prognostic effect may be, however, substantially reduced by allogeneic transplantation.

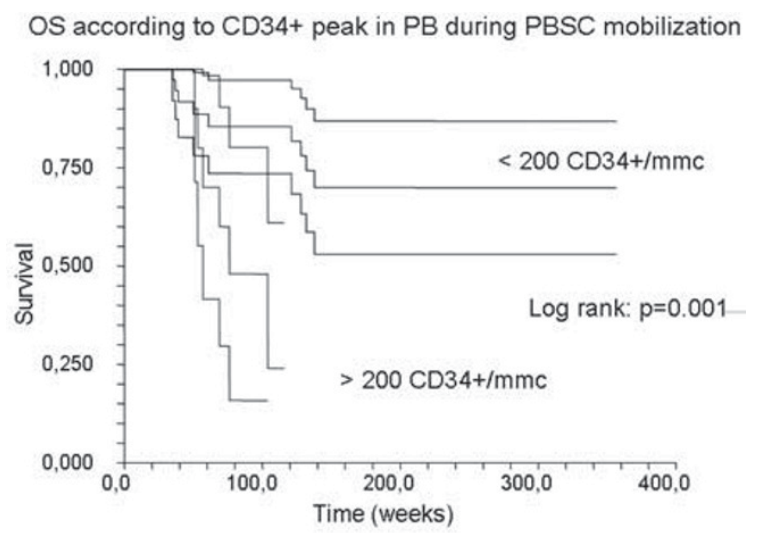

\begin{tabular}{|l|l|l|l|l|l|l|}
\hline & \multicolumn{2}{|c|}{$\begin{array}{l}\text { All post remissional } \\
\text { treatment (n.50) }\end{array}$} & \multicolumn{2}{|l|}{$\begin{array}{l}\text { Group Autologous } \\
\text { Tx (n.21) or no Tx } \\
\text { (n.5) }\end{array}$} & \multicolumn{2}{l|}{$\begin{array}{l}\text { Group Allogeneic } \\
\text { TX (n.24) }\end{array}$} \\
\hline & DFS & RI & DFS & RI & DFS & RI \\
\hline $\begin{array}{l}\text { CD34+Peak } \\
\text { <200/mme }\end{array}$ & 6096 & 4096 & 5096 & $45 \%$ & $65 \%$ & $30 \%$ \\
\hline $\begin{array}{l}\text { CD34+ Peak } \\
>200 / \text { mme }\end{array}$ & 2096 & 7096 & 096 & 10096 & $40 \%$ & $609 \%$ \\
\hline Log rank & 0.008 & 0.005 & 0.002 & 0.0002 & 0.29 & 0.21 \\
\hline
\end{tabular}

\section{P408}

Role of iron overload for the outcome of allogeneic stem cell transplantation in high-risk acute myeloid leukaemia and myelodysplastic syndrome

C. Schmid (1), C. Koenecke (2), G. Wittmann (3), J. Tischer (3), W. Behr (1), M. Eder (2)

(1)Klinikum Augsburg (Augsburg, DE); (2)Medical School (Hannover, DE); (3)Medical Center Ludwig-MaximiliansUniversity (Munich, DE)

Iron overload has recently been identified as an independent risk factor in myelodsyplastic syndrome (MDS), either with conservative treatment or allogeneic stem cell transplantation (SCT). Little data is available on its role in SCT for acute myeloid leukaemia, in particular in advanced disease. Therefore, we performed a retrospective analysis of 113 patients (57 female, 56 male) who had received an allogeneic SCT for highrisk AML or progressive MDS. Serum ferritin levels measured before start of conditioning were evaluated with respect to their influence on post transplant complications and overall survival. Patients were transplanted for de novo AML $(n=50)$, progressive MDS/sAML $(n=51)$, tAML $(n=10)$ and AML secondary to
MPS $(n=2)$, either in untreated disease $(20 \%)$, primary induction failure $(30 \%)$, CR1 $(16 \%)$, or beyond CR1(33\%). Cytogenetics were favourable, intermediate, poor and unknown in 3, 61, 46 and 3 cases. Donors were HLA id siblings (24\%), matched $(50 \%)$ and mismatched $(26 \%)$ unrelated donors. Median ferritin level before start of conditioning was 1900 (76-11008) mg/l.

Median follow up in 59 survivors was 19 months, median overall survival (OS) of the entire cohort was 21.7 months. Relapse or progressive leukaemia was the cause of death in 28 patients, 22 died without evidence of leukaemia and were regarded as treatment-related deaths (TRD).

With respect to ferritin levels, no association could be detected with cardiac or liver toxicity after SCT, or with TRD in general. Nevertheless, patients with a ferritin level $>2700 \mathrm{mg} / \mathrm{l}$, corresponding to the highest quartile, had a significantly inferior $O S$ (median OS: 6 mo vs. 24 mo, $P=.006$ ). This was also confirmed in a multivariate analysis, with stage at transplantation and donor type as competing variables (HR: 2.2, 95\% Cl: 1.2-4.1). In conclusion, extremely high ferritin levels seem to be an unfavourable factor for survival after allogeneic SCT in advanced MDS and high-risk AML. Since a clear association with toxicitiy or TRD after SCT could not be shown, it remains to be elucidated by more detailed analysis, whether high ferritin levels are a surrogate marker for otherwise unfavourable disease, or if the iron overload itself was responsible for the inferior outcome by a so far not yet recognized mechanism.

\section{P409}

Nature of HLA-associated factors of predisposition and resistance in acute lymphoblastic leukaemia

L. Lebedeva (1), T. Potapova (1), T. Puhlikova (1), D. Stavtsev (1), E. Skorobogatova (2), T. Astrelina (1), M. Yakovleva (1) (1)Stem Cell Bank (Moscow, RU); (2)Research Centre of Pediatric Hematology (Moscow, RU)

Objectives: ALL blasts routinely contain somatically acquired genetic abnormalities that provide insight into pathogenesis and strongly influence prognosis. Genetic and environmental factors play an interactive role in the development of childhood acute lymphoblastic leukemia (ALL). The HLA association has been considered as a possible genetic risk factor.

Aims: It is unknown whether there exists a restriction to certain MHC genotypes in leukemia like ALL.

Methods: The material of the study - leukocytes peripheral blood from 162 children with initial diagnosed AML (B-ALL-83, T-ALL-30, ALL-49) and 2060 controls (leukocytes umbilical cord blood from healthy donors). Revealing HLA antigens at low level performed by SSO method on DynalRELI 48 processor. The results received with ambiguous interpretation was using PCR-SSP method (Ivitrogen).

Results: The results showed that in comparison with the control group, the gene frequence of: HLA-A*32 (ALL-10,0\%, B-ALL-11,7\% vs. $-4,7 \%$ ); HLA-A*03 (B-ALL-48,0\% vs. $21 \%$ ); HLA-A ${ }^{*} 23$ (ALL-8,1\%, T-ALL $-8,0 \%$ vs. $4,3 \%$ ), HLA-A*26 (T-ALL- $17,8 \%$ vs. $7,8 \%$ ), HLA-A ${ }^{*} 51$ (T-ALL- $18,5 \%$ vs. $6,2 \%$ ); HLA ${ }^{*} \mathrm{Cw} 04$ (B-ALL-35,2\% vs. 20,0\%), HLA-Cw*07 (B-ALL$68,5 \%$ vs. $40,5 \%$ ), HLA-Cw*01 (T-ALL- $20,0 \%$ vs. $6,6 \%$ ), HLA$\mathrm{Cw}^{*} 05$ (T-ALL-15,8\% vs. 6,0\%), HLA-DRB1*09 (B-ALL-7,9\% vs. $2,3 \%$ ), HLA-DRB1*08 (B-ALL- $11,35 \%$ vs. $5,4 \%$ ), HLADRQ1*05 (T-ALL-51,7\% vs. $30,0 \%$ ) increased significantly, but the gene frequence of: HLA-A*68 (B-ALL- $1,3 \%$ vs. $-6,0 \%)$ and $\mathrm{HLA}-\mathrm{CW}^{*} 01$ (ALL $-3,7 \%, \mathrm{~B}-\mathrm{ALL}-3,8 \%$ vs. $-6,7 \%$ ) allele frequency decreased significantly for patients with ALL. These alleles it is possible to consider as factors of resistency to the predisposition disease at children.

Conclusion: These results suggest that susceptibility to ALL is linked to the histocompatibility HLA molecule itself, although the development of the disease also needs inherited or probably acquired factors not linked to the major histocompatibility complex. 
P410

Allogeneic stem cell transplantation for advanced myeloid leukaemia and MDS following a synergistic combination of clofarabine \pm fudarabine and IV busulfan

B.S. Andersson, B. Valdez, P.F. Thall, L.L. Worth, U. Popat, R.B. Jones, E.J. Shpall, C. Hosing, T. Madden, P. McAdams, A. Alousi, P. Kebriaei, R. Champlin, M. de Lima

M.D. Anderson Cancer Center (Houston, US)

Clofarabine(Clo) is a nucleoside analog (NA) with much improved antileukemic efficacy. In vitro, in a human busulfan(Bu)-resistant AML-cell line, KBM-3/Bu, we found synergy of [Clo + fludarabine] (Flu) compared with either drug alone. Further, $[\mathrm{Clo} / \mathrm{Flu}+\mathrm{Bu}]$ was superior to $[\mathrm{Clo}+\mathrm{Bu}]$ or $[\mathrm{Flu}+\mathrm{Bu}]$. We now investigated [Clo \pm Flu] with IV Bu as pretransplant conditioning in patients with advanced myeloid leukemia and MDS. Methods: Patients were adaptively randomized based on day +30 disease- and chimerism status: Arm I: Clo:Flu 10:30 mg/m², Arm II 20:20 mg/m², Arm III 30:10 mg/m², and Arm IV: Clo only at $40 \mathrm{mg} / \mathrm{m}^{2}$. The Clo and Flu were infused over $1 \mathrm{hr}$ each once daily for 4 days, on each day Bu was given over $3 \mathrm{hr}$ to AUC $6,000 \mu \mathrm{Mol}$ min. GVHD-prophylaxis was tacrolimus + mini MTX. Recipients of unrelated or one Ag-mism. related donor cells received rabbit-ATG.

Results: 24 patients were male, 17 female, median age $45 \mathrm{yrs}$ (range 6-59). 32 had AML (ind. failure: 12, chemo-refr. relapse: 9, untr. relapse: 3, CR2+CR2p: 6, high-risk CR1+CR1p: 2 [cytog. -7 and $t(3 ; 12)$ ]). 17 of the 32 had intermediate, 15 poor prognosis cytogenetics. 9 had CML (BC: 2 , second AP: 3 , and late CP 4). 19/41 (46\%) had related donors (BM:6, PBPC: 13), and $22(54 \%)$ unrelated (BM:11, PBPC:11). All patients engrafted. Day + 30 T-cell chimerism showed Arms I+ II (lower Clo doses) with $85 \%$ (range 17-100) donor-derived DNA, while Arms III + IV (higher Clo doses) had 100\% (64 100) donor-DNA; by day +100 all groups had a median of $100 \%$ donor-DNA The adaptive randomization allocated most patients to Arm III, (Clo $30 \mathrm{mg} / \mathrm{m}^{2}$, Flu $10 \mathrm{mg} / \mathrm{m}^{2}$ ), in keeping with in vitro synergy of [Clo +Flu]. $83 \%(20 / 24)$ of patients with active AML and 7 of 9 with CML achieved CR post transplant. Median follow-up is 18 mos (range 6-34), and as of Dec. 11, 2009, one-year OS and EFS are $52 \%$ and $41 \%$. There were only 2 treatment-related deaths by day $+100(5 \%)$, one pneumonia, one GVHD. Grade 2-3 mucositis was seen in half the patients, but there was no serious hepatic, renal or neuro-toxicity.

We conclude: 1) Clofarabine is immunosuppressive enough for pretransplant therapy, 2) [Clo \pm Flu] + IV Bu is safe and efficacious conditioning in myeloid leukemia, 3 ) In vitro synergy of [Clo + Flu] with IV Bu correlated clinically with the adaptive randomization, favoring Arm III (Clo $30 \mathrm{mg} / \mathrm{m}^{2}$ and Flu $10 \mathrm{mg} / \mathrm{m}^{2}$ ). Additional patients and longer follow-up are needed to evaluate combined nucleoside analogs [Clo+Flu] in pretransplant conditioning

\section{P411}

Improved results of allogeneic stem cell transplantation for adult Ph+ ALL patients in the TKI era - the Swedish experience

H. Hagglund (1), K. Karlsson (2), E. Hulegardh (3), H. Karbach (4), A. Markuszewska (5), M. Åström (6), H. Hallböök (7) (1)Hematology Center (Stockholm, SE); (2)Hematology (Lund, SE); (3)Hematology (Gothenburg, SE); (4)Hematology (Linköping, SE); (5)Hematology (Umeå, SE); (6)Hematology (Örebro, SE); (7)Hematology (Uppsala, SE)

The Swedish ALL group retrospectively evaluated the outcome of 49 adult $\mathrm{Ph}+\mathrm{ALL}$ patients who underwent allogeneic stem cell transplantation at six different centers 2000-2009. Treatment consisted for the majority of patients $(n=33)$ of $3-4$ monthly cycles of polychemotherapy and steroids according to national guidelines or hyperCVAD $(n=9)$ or other $(n=7)$. Time to transplantation from diagnosis was median 5 (range 2-17) months and the majority of the patients were transplanted in first CR. The median age was 41 (range 20-66) years. There were 23 females and 26 males. Forty-three received myeloablative and 6 reduced conditioning. Before transplantation imatinib was included in the treatment protocols in 32 patients (65\%). Twenty-four patients received stem cells from related donors, 24 from unrelated donors and one from cord blood. Twenty-seven patients (55\%) and 22 (48\%) developed any grades of acute or chronic graft versus host disease (GVHD) respectively. The 5-year overall survival (OS) for all patients was $49 \%$, higher in patients $<40$ years $(n=21) 71 \%$ than in patients $\geq 40$ years $(n=28) 35 \%(P=0.02)$. Chronic GVHD was associated with improved 5 -year OS of $74 \%$ compared to no chronic GVHD with OS of $32 \%(P=0.05)$. Four of 17 patients with relapse are still alive $8,12,14$ and 27 months after transplantation on tyrosine kinase inhibitor (TKI) treatment started at relapse. The results have improved compared to our national 1986-2000 study where the 5-year OS was 33\% in Ph+ ALL patients $(n=27$, median age 37 (range 19-57)) grafted in first remission. However, in the present study we have not found superior survival when imatinib was used before transplantation. It can be argued that some patients in the imatinib treated group might not have reached transplantation if imatinib had not been given. According to our limited experience salvage TKI treatment for $\mathrm{Ph}+\mathrm{ALL}$ given at relapse after transplantation is effective in some patients and may prolong overall survival.

\section{P412}

Cryptic genomic alterations in AML patients after allogeneic haematopoietic cell transplantation by SNP DNA profiling

M. Waterhouse, D. Pfeifer, M. Pantic, A. Zerweck, H. Bertz, J. Finke

Freiburg University Medical Center (Freiburg, DE)

Allogeneic hematopoietic cell transplantation (HCT) is the most effective curative therapy in many patients with hematologic malignancies such as acute myeloid leukemia (AML). Cytogenetics studies stratify patients into three groups of prognosis according to the overall survival. These classifications are not fully predictive of response. Genomic technologies can identify molecular subgroups with distinct genomic signatures. We investigated the genomic changes in patients with AML undergoing HCT using DNA microarrays. We analyzed 11 patients before HCT and at relapse after HCT, 6 patients had a normal karyotype at diagnosis and 5 had abnormal karyotype with different types of aberrations. After high resolution of whole genome DNA profiling analysis 5 of the 6 patients with normal karyotype showed genomic aberrations (GA) in the form of gains and losses (5/5 gains/losses) in several regions of different chromosomes with a median of 2 GA per case. The abnormal karyotype group, in addition to the cytogenetically detected aberrations GA were present in 2 out of 5 patients (5/4 gains/ losses). Overall $64 \%$ of the patients had GA, not detected by conventional cytogenetics. All FAB subtypes of AML showed GA, being $M 5$ and secondary AML both with more numerous GA (average: 12 and 17 GA respectively). At relapse 6 patients increased the number of GA, (median of 7 GA per case), while 3 patients presented a reduced number of GA (median 2 GA per case). The remaining 2 patients showed no additional changes. Before transplantation 16 chromosomes showed GA with a median of $3 \mathrm{GA}$ per chromosome, average size of gains and losses were 1568 and $8046 \mathrm{~kb}$ respectively. At relapse 19 chromosomes had GA with a median of $4 \mathrm{GA}$ per chromosome, average size of gains and losses were 697 and $8011 \mathrm{~kb}$ respectively. Chromosomes 11, 14 and 21 showed no GA at any time point. Our preliminary data demonstrate that AML is a genomically heterogeneous disease with a high incidence of small non recurrent DNA GA that can be detected even in cases with normal karyotype. Cryptic genomic changes are likely to play a role in relapse after allogeneic transplantation. 
P413

Comparative outcome of allogeneic peripheral blood stem cell transplantation from related and unrelated donor in acute myeloid leukaemia

S.K. Sohn (1), B.W. Kang (1), J.H. Moon (1), Y.S. Chae (1), J.G. Kim (1), J.S. Jung (2), G.J. Cho (2), D.Y. Jo (3), Y.K. Kim (4), H.J. Kim (4), H.M. Ryoo (5), S.H. Bae (5), H.S. Eom (6), S.M. Lee (7), Y.D. Joo (7), J.H. Won (8), M.R. Park (9), M.K. Kim (10), M.S. Hyun (10)

(1)Kyungpook National University Hospital (Daegu, KR); (2)Pusan National University Hospital (Pusan, KR); (3)Chungnam National University Hospital (Daejeon, KR); (4)Chonnam National University Hwasun Hospital (Hwasun, KR); (5)Daegu Catholic University Medical Center (Daegu, KR); (6)National Cancer Center (Goyang, KR); (7)Inje University Paik Hospital (Pusan, KR); (8)Soon Chun Hyang University Hospital (Seoul, KR); (9)Wonkwang University Hospital (Iksan, KR); (10)Yeungnam University Medical Center (Daegu, KR)

This study compared the results of allogeneic peripheral blood stem cell transplantation (PBSCT) from unrelated and related donors in acute myeloid leukemia (AML), and involved 136 consecutive patients from 10 centers between April 2003 and Dec 2008. Among these patients, 98 received transplantation from matched-related donors (MRD) and 38 matched-unrelated donors (MUD). Twentyeight $(28.6 \%)$ in the MRD and $14(36.8 \%)$ patients in the MUD were high risk diseases $(P=0.349)$. In vivo-T cell depletion (TCD) $(52.7 \%$ vs. $6.1 \%)$ and tacrolimus for graft-versus-host disease (GVHD) $(44.7 \%$ vs. $4.1 \%)$ were more commonly used in the MUD than MRD $(P<0.001)$. The 3 -year overall survival $(\mathrm{OS})$ rate $(66.0 \%$ vs. $62.1 \% ; P=0.323)$, the 3 -year cumulative incidence of relapse $(27.4 \%$ vs. $13.6 \% ; P=0.111)$ and non-relapse mortality $(16.6 \%$ vs. $21.6 \%, P=0.992$ ) were not different between MRD and MUD group. The incidence of cytomegalovirus reactivation was higher in the MUD (49.8\%) than MRD $(22.8 \% ; P=0.005)$. The 3-year cumulative incidence of grade II-IV acute GVHD and extensive chronic GVHD was $21.2 \%$ vs. $27.8 \%(P=0.718)$ and $13.5 \%$ vs. $25.6 \%$ $(P=0.040)$ in MRD and MUD group, respectively. In the multivariate analysis, MUD [hazard ratio (HR) $5.297,95 \%$ confidence interval (Cl) 1.156-24.277; $P=0.032$ ], reduced intensity conditioning (HR 7.318, 95\% Cl 1.355-39.505; $P=0.021$ ), and infused CD34 + stem cells $>6 \times 10^{6} / \mathrm{kg}$ (HR 3.906, 95\% Cl 1.108-13.711; $\left.P=0.031\right)$ were identified as risk factors for the development of extensive chronic GVHD in the multivariate analysis. To adjust the white blood cell (WBC) counts on the differentiating capacity of the leukemic clone, WBC index (WBC X marrow blasts\%/100) was calculated, and distinguished 3 groups of patients with different outcomes (low, $<1.0$; intermediate, 1.0-6.3; high, 6.3 or more). High index showed poor survival compared to the low index $(P=0.038)$. In conclusion, transplantation outcome was comparable between unrelated and related donors in terms of OS and incidence of relapse in AML.

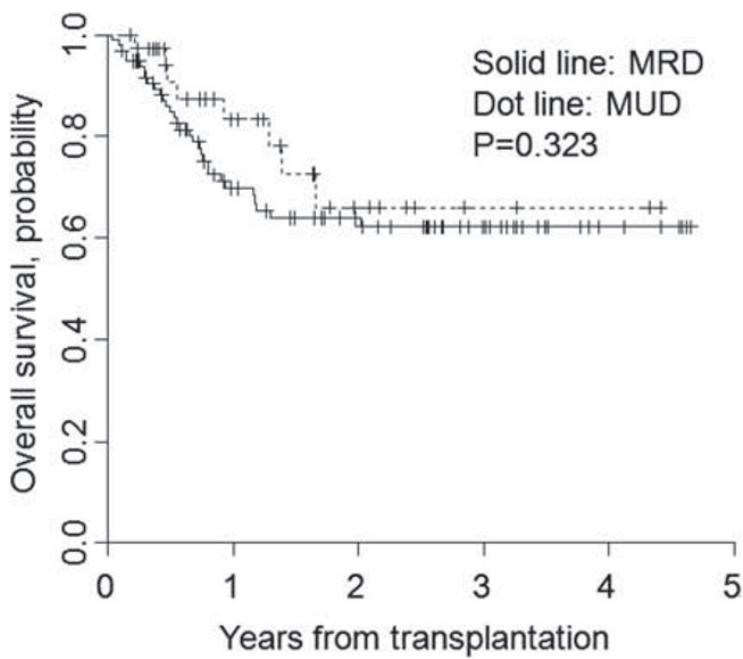

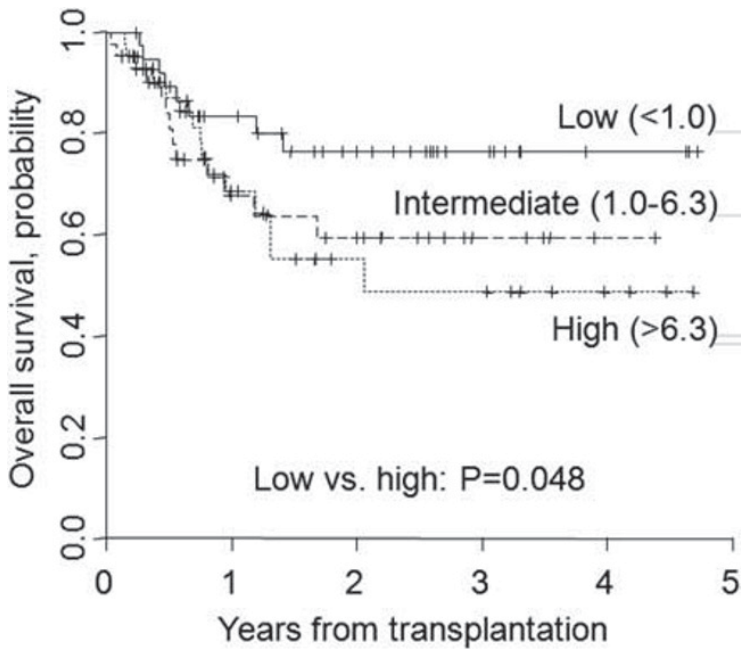

P414

A decision analysis of allogeneic haematopoietic stem cell transplantation for adult patients with Philadelphia chromosome-negative acute lymphoblastic leukaemia in first remission

S. Kako (1), S. Morita (2), H. Sakamaki (3), H. lida (4), M. Kurokawa (5), K. Miyamura (6), H. Ogawa (7), T. Fukuda (8), S. Takahashi (9), H. Kanamori (10), Y. Atsuta (11), Y. Morishima (12), I. Jinnai (13), J. Takeuchi (14), Y. Miyazaki (15), S. Miyawaki (16), K. Onishi (17), T. Naoe (18), Y. Kanda (1) (1)Jichi Medical University, Saitama Medical Center (Saitama, JP); (2)Yokohama City University (Kanagawa, JP); (3)Tokyo Metropolitan Cancer and Infectious Diseases Center, Komagome Hospital (Tokyo, JP); (4)Meitetsu Hospital (Aichi, JP); (5) Graduate School of Medicine, University of Tokyo (Tokyo, JP); (6)Japanese Red Cross Nagoya First Hospital (Aichi, JP); (7)Hyogo College of Medicine (Hyogo, JP); (8)National Cancer Center Hospital (Tokyo, JP); (9)Advanced Clinical Research Center, Institute of Medical Science, University of Tokyo (Tokyo, JP); (10)Kanagawa Cancer Center (Kanagawa, JP); (11)Nagoya University School of Medicine (Aichi, JP); (12)Aichi Cancer Center Hospital (Aichi, JP); (13)Japanese Red Cross Ogawa Hospital (Saitama, JP); (14)Nihon University School of Medicine (Tokyo, JP); (15)Atomic Bomb Disease Institute, Nagasaki University Graduate School of Biomedical Sciences (Nagasaki, JP); (16)Tokyo Metropolitan Ohtsuka Hospital (Tokyo, JP); (17)Cancer Center, Hamamatsu University School of Medicine (Shizuoka, JP); (18)Nagoya University Graduate School of Medicine (Aichi, JP)

Background: The efficacy of allogeneic hematopoietic stem cell transplantation (HSCT) for adult patients with acute lymphoblastic leukemia (ALL) in first remission has been demonstrated through clinical studies using genetic randomization. These genetic randomization studies, however, cannot accurately answer the question whether patients with a human leukocyte antigen (HLA)-matched sibling should undergo allogeneic HSCT or chemotherapy in first remission, since patients without a sibling donor had to choose alternative donor transplantation or chemotherapy alone once they had a relapse in these studies.

Methods: To find out the optimal treatment strategies for adult patients with Philadelphia chromosome-negative ALL in first remission who have an HLA-matched sibling, decision analysis was performed based on the decision tree (Figure 1). Transition probabilities and utilities were estimated from the Japan Adult Leukemia Study Group (JALSG) studies (ALL93 and ALL97), the database of the Japan Society for Hematopoietic Cell Transplantation (JSHCT), and the literatures. Utilities were calculated based on 10-year survival probability with or without 
an adjustment for quality of life (QOL). Subgroup analyses were also performed according to the risk stratification based on the white blood cell (WBC) count and cytogenetics, and according to the age stratification with a cutoff of 35 years.

Results: In analyses without QOL adjustment, the superiority of allogeneic HSCT in first remission was demonstrated in the whole population and also in all subgroups. Sensitivity analyses supported these results in all groups except for the higher-aged group. On the other hand, the superiority of allogeneic HSCT was demonstrated only in the high-risk group if adjusted for QOL. The superiority of chemotherapy appeared to be robust in the higher-aged group by sensitivity analyses.

Conclusions: To improve the long-term survival probability, we should consider allogeneic HSCT in first remission for adult patients with Philadelphia chromosome-negative ALL who have an HLA-matched sibling. Considering QOL, however, chemotherapy might be preferred in higher-aged patients.

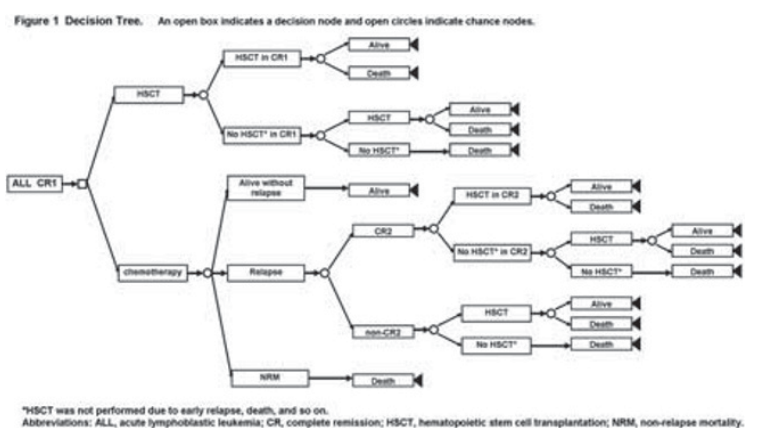

P415

Tandem auto-allo in acute myeloid leukaemia patients in first complete remission

L. Castagna, S. Fürst, T. Prébet, J. El Cheikh, A. Charbonnier, C. Faucher, M. Mohty, N. Vey, D. Blaise

Institut Paoli Calmettes (Marseille, FR)

High and intermediate risk acute myeloid leukaemia (AML) can benefit of reduced intensity allogeneic stem cell transplantation (RIC-ALLO) in first complete remission (CR). To contrast the high relapse rate in RIC-ALLO we hypothyzed that if a better quality of remission could be achieved, the relapse incidence could be lowered.

Patients and methods: From 2001 to 2008, 31 AML patients in first CR received a tandem auto-allo program. The median number of white blood cell was $3 \times 10 \%$ (range 0.9-235). After one or two induction chemotherapies (CT), all but two patients received a consolidation course with high-dose cytarabine (HD-ARAC) CT, followed by autologous stem cell harvest. HD melphalan (HD-PAM $140 \mathrm{mg} / \mathrm{m}^{2}$ ) followed by autologous stem cells reinfusion was administered, followed by ALLO-RIC. RIC consisted of fludarabine plus (2 Gy) TBI ( 3 pts) or fludarabine, oral or intravenous busulfan $(8 \mathrm{mg} / \mathrm{kg})$ in two days, and anti thymocyte globulin (2.5 or $5 \mathrm{mg} / \mathrm{kg}$ ). GVHD prophylaxis was cyclosporine (CyA) plus mycofenolate mofetil ( 3 pts) or CyA alone (28 pts). Donors were all but one HLA identical sibling. The median number of allogeneic CD34 + and CD3 + cells was $6.1 \times 10 e^{6} / \mathrm{Kg}$ (range 1.9-11) and 315 (range 166-609). Prognostic scores (HCT-Cl, PAM, EBMT) were retrospectively calculated for each patient.

Results: The median follow-up was from diagnosis and ALLORIC 40 and 34 months, respectively. The median time between last CT and HD-PAM was 51 days (range 30-77) and HD-PAM and ALLO-RIC was 69 days (55-176). Treatment related mortality after HD-PAM was null. Prognostic scores were: HCT-Cl score $0-2=53 \%$ (16 pts), $\geq 3=47 \%$ (14 pts), 1 pt not evaluable; PAM score $9-16=30 \%$ (10 pts), $17-23=67 \%$ (19 pts), $24-30=3 \%$ (1 pt); EBMT score 1 = 9\% (3 pts), $2=78 \%$ (24 pts), $3=13 \%$ (4 pts). At last follow-up, $42 \%$ of pts $(n=13)$ died: 5 due to disease relapse and 8 because of toxicity. Grade II-IV acute GVHD and chronic GVHD incidence were respectively $26 \%$ and $65 \%$ (extensive $84 \%$ ). GVHD was the cause of death in seven pts. The 5 -year overall survival (OS), relapse free survival (RFS), and 1-year TRM were $60 \%, 60 \%$, and $15 \%$, respectively. In multivariate analysis, prognostic scores did not influence TRM and OS.

Conclusions: This report showed that

i) tandem auto-allo is feasible in AML pts;

ii) acute GVHD incidence is not increased;

iii) prognostic score did not impact on TRM and survival;

iv) the TRM is quite low with GVHD as main cause of death.

A retrospective comparison with a cohort of pts not receiving HD-PAM is on going.

\section{P416}

Reduced-intensity transplantation as a part of standard treatment strategy in patients older than 60 years with acute myeloid leukaemia

M. Karas, K. Steinerova, P. Jindra, D. Lysak, T. Svoboda, S. Vokurka, V. Koza

Charles University Hospital Pilsen (Pilsen, CZ)

Background: Outcome of patients (pts) over 60 years of age with acute myeloid leukemia (AML) treated with intensive chemotherapy is poor. With the aim of improvement of treatment results reduced-intensity transplantation (RIT) was establish as a part of standard treatment strategy for pts older than 60 years with AML in our centre from 2003. To evaluate transplant feasibility and role of RIT in treatment of pts older than 60 years with AML we analysed such treated pts in our centre. As a part of analysis we also tried to evaluate the role of RIT using unrelated donor.

Patients and methods: In period 1/2003-9/2009 from 69 intensively treated pts with AML 30 pts with median of age 62 years (range, $60-68$ years) with AML in 1.CR (19 pts) or AML beyond 1.CR (11 pts) underwent RIT (40\% HLA identical related, 30\% HLA matched unrelated, 30\% HLA mismatched unrelated). Source of stem cells was peripheral blood and the median of infused CD $34+$ cells was $5,79 \times 10^{6} / \mathrm{kg}$ (range, $\left.1,89-14,9 \times 10^{6} / \mathrm{kg}\right)$. The conditioning regimen consisted of fludarabine $\left(30 \mathrm{mg} / \mathrm{m}^{2}\right.$ for 4 days $)$ and melphalan $\left(140 \mathrm{mg} / \mathrm{m}^{2}\right.$ for 1 day). Cyclosporine and methotrexate were used as GVHD prophylaxis. Pts transplanted from related or unrelated donors did not differ for any significant variables except for younger age of donors and higher amount of infused CD34 + cells in unrelated RIT.

Results: The main reasons of impossibility to implement RIT in treatment of older pts with AML were death during remission induction treatment, non-availability of donor, severe comorbidities and refusal of RIT but $43 \%$ of intensively treated pts with AML underwent RIT. All transplanted pts engrafted and achieved complete remission (CR) with full donor chimerism (FDC). 13 pts (43\%) developed acute GVHD (3 pts grade III-IV) and among 25 evaluable pts $10(40 \%)$ of them developed chronic GVHD (1 limited, 9 extensive). With median follow-up 19 months (range, 2-73 months) 17 pts $(57 \%)$ are alive (16 pts in CR). 5 pts $(17 \%)$ relapsed and 4 of them died. 9 pts $(30 \%)$ died due to NRM and $3(10 \%)$ of them till day +100 after RIT. The estimated probabilities of 4 -years EFS and OS are $46 \%$ and $48 \%$ and the type of donor did not show any significant influence of RIT outcome.

Conclusion: Our data show that nearly half of intensively treated pts older than 60 years with AML were able to undergo RIT and that RIT even in case of unrelated or HLA mismatched donor is associated with acceptable NRM and encouraging disease control of unfavourable AML. 


\section{P417}

Improved reponse to gemtuzumab ozogamicin in AML patients with HLA-DR15 antigen positivity

C. Dobbelstein, E. Dammann, A. Wortmann, S. Ehrlich, S. Buchholz, M. Eder, E. Mischak-Weissinger, M. Stadler, J. Krauter, A. Ganser

Hannover Medical School (Hannover, DE)

Objectives: The prognosis of refractory or relapsed acute myeloid leukemia (AML) remains dismal. Salvage therapy including gemtuzumab ozogamicin (GO) can induce remission, but allogeneic stem cell transplantation (allo-SCT) is the only curative option for this cohort of patients. However, factors predicting the response to GO treatment, especially for patients with complex karyotype, have not yet been defined. Here, we present the correlation between response to GO therapy and the presence or absence of HLA-DR15 antigen in a cohort of refractory or relapsed AML patients treated at Hannover Medical School prior to allo-SCT. Methods: We retrospectively evaluated patients with refractory or relapsed AML treated with GO prior to allo-SCT at our center from 2002 until 2009. Patients were divided into two groups: patients who responded to GO (R group; CR, PR, aplasia) and patients who remained refractory (NR group).

Results: 26 patients were evaluable for GO response, and 24 patients have proceeded to allo-SCT so far. Both groups were comparable for sex, age, indication for $\mathrm{GO}$ (induction failure vs. relapse), patients with de novo AML vs. sAML/ t-AML and high-risk cytogenetics. Indications for GO included induction failure $(n=14,54 \%)$ and relapse $(n=12,46 \%)$. Thirteen patients $(50 \%)$ responded to $G O$ treatment. Nine $(69 \%)$ of these were HLA-DR15 positive compared to $1(8 \%)$ in the NR group $(P<0.01)$. Remarkably, the cumulative dose of $\mathrm{GO}$ was higher in the NR group $\left(8 \mathrm{mg} / \mathrm{m}^{2}\right)$ compared to the $\mathrm{R}$ group $\left(5 \mathrm{mg} / \mathrm{m}^{2}\right)$. More patients in the $\mathrm{R}$ group received a combination of chemotherapy with GO than in the NR group ( $54 \%$ vs. $15 \%, P=0.1)$. Also, patients in the NR group had a median of 2 prior salvage therapies vs. one median salvage course in the $\mathrm{R}$ group.

Sinusoidal obstruction syndrome (SOS) is a major complication in patients receiving GO: 6 of the 24 patients $(25 \%)$ of the transplant cohort developed SOS, 3 in each group. Three out of these 6 patients had a myeloablative conditioning regimen with busulfan. The remaining SCT patients received different reduced intensity conditioning regimens (67\% FLAMSA/TBI) without busulfan.

Conclusion: To the best of our knowledge, this is the first report of an association of GO treatment and HLA-DR 15 antigen positivity. If these results were confirmed in larger studies, HLA-DR 15 could become a marker for GO response. Such information is of special interest for developing salvage therapies for highrisk patients.

\section{P418}

Characterization of leukaemia specific T-lymphocyte clones stimulated with AML-derived dendritic cells: value of spectratyping in combination with functional flow cytometry and fluorolysis analyses

S. Reuther (1), F. Schuster (1), C. Grabrucker (2), A. Liepert (2), R. Reibke (2), T. Yang (3), T. Kroell (2), H.J. Kolb (2), A. Borkhardt (1), R. Buhmann (3), H. Schmetzer (2)

(1)Heinrich-Heine University Dusseldorf (Dusseldorf, DE); (2)University of Munich (Munich, DE); (3)Helmholtz Center (Munich, DE)

Introduction: Myeloid leukemic cells can be induced to differentiate into leukemia-derived dendritic cells (DCleu) regaining the stimulatory capacity of professional DCs while presenting the leukemic antigen repertoire. The induced antileukemic T-cell responses are variable both in their specificity and efficacy.

Methods: HLA matched donor T-cells (allogeneic) or autologous T-cells were repeatedly stimulated, either with leukemic blasts or the corresponding DCleu from three different patients with AML. To identify T-cell receptor (TCR) Vbeta-chain-rearrangements in unstimulated and stimulated T-cells, immunoscope spectratyping was performed in CD4 + and CD8 + cells respectively. For functional characterization, flow cytometry and non-radioactive fluorolysis assays were performed. Results: After stimulation with DCleu derived from patient $1 \mathrm{~T}$ cells (allogeneic) showed a lytic activity. In sample 2 (autologous) and 3 (allogeneic), neither blast- nor DCleu-stimulated T-cells were able to lyse blasts. In sample 1 a CD4:CD8 ratio $>1$ was observed in contrary to sample 2 and 3 . Spectratyping revealed a restriction of the TCR repertoire in all three cases indicating a specialization of the T cells against the leukemic blasts: After stimulation with $\mathrm{DCleu}$ distinct restriction was observed in all CD8 + fractions, while in CD4 + T-cells only sample 1 and 2 showed a restriction after DCleu stimulation. In sample 1 a restriction was also detected after stimulation with blasts in both CD4+ and CD8 + fractions. These variabilities may be explained by the different leukemic antigens or the intraindividual immune response of stimulated T-cells. Furthermore, in the patient 2 an identical TCR-Vbeta sequence was identified in the patients CD8 + T-cell fraction after SCT and the DCleu stimulated T-cell culture.

Conclusion: A combined strategy using spectratyping with functional tests may provide useful information about the specificity and efficacy of the intraindividual variable induced T-cell response. It seems that $\mathrm{CD} 4+$ and $\mathrm{CD} 8+$ cells mediate the antileukemic function in combination, however the antileukemic activity was mostly observed at a ratio of CD4:CD8 >1.

Furthermore it may contribute to detect, amplify or monitor T-cell clones (with defined Vbeta-profiles) responsible for antileukemic function, in vitro as well as in vivo. The identification of these Vbeta clones may lead to selection procedures generating GvL-(but not GvH) mediating T-cells for individual adoptive immunotherapy.

\section{P419}

Second allogeneic stem cell transplantation for relapsed leukaemias: a single-centre outcome study H. Schade (1), A. Humpe (1), A. Guenther (1), A. Schrauder (2), T. Valerius (1), M. Gramatzki (1)

(1)Div. of Stem Cell Transpl. \& Immunother. (Kiel, DE); (2)Department of Pediatrics (Kiel, DE)

Objectives: For patients with relapse of leukemia or lymphoma after first allogeneic stem cell transplantation (SCT) therapeutic options are limited. Here, we report our experience at our unit with second SCT and address particularly the role of a graft from an alternative donor.

Methods: Twenty-four patients with a median age of 42 years (range: 6-69) received a second SCT in the relapse situation. Initial diagnoses were AML (14), MDS (4), ALL (4), OMF (1), or CLL (1). In 17 patients the second transplant was performed from a HLA identical (10/10) donor, whereas 7 received grafts with a minimum of one mismatch. The median interval between the first and second SCT was 398 days (range: 47-1856) and in 13 of the 24 patients (54\%) a graft from a different donor was used for the second SCT.

Results: The median overall-survival (OS) after second SCT was 220 days. Treatment-related mortality (TRM) was $22 \%$, acute Graft-versus-Host-Disease (aGvHD) was seen in $50 \%$ of the patients but only $17 \%$ suffered from $\geq$ grade 3 , and extensive chronic GvHD (cGvHD) occurred in 8\%. Relapse was observed in 12 patients. Median time-to-relapse was 227 days (range: 27-717) compared with 274 days (range: 93-1777) after first SCT. However $7(29 \%)$ of the 24 patients after second SCT are still in remission with a median follow up of 526 days. Until now, there is no difference in remission duration but in $4(57 \%)$ of the 7 patients the remission after second SCT is already lasting longer than after the first SCT. The majority of the patients achieving remission received for the second transplantation a reduced intensity conditioning regimen (FLAMSA-RIC) and in 5 of these an alternative donor had donated. In these patients with sustained remission the rate of extended cGvHD was low 
(1 of 7). Conclusions: The rate of TRM after second SCT was acceptable. Complete remissions (CR) after second SCT can be achieved with progression-free-survival even exceeding that of the first SCT. Choosing a different donor might enhance the probability to induce a graft-versus-leukemia effect, although some patients remained in CR with a graft from the original donor. In addition, reduced intensity conditioning is useful for second SCT and long term CR after second SCT did not correlate with extensive cGvHD.

\section{P420}

Allogeneic stem cell transplantation in primary refractory acute myeloid leukaemia: single-centre experience on 55 consecutive patients

A. Algarotti, C. Micò, A. Grassi, A. Rambaldi

Ospedali Riuniti (Bergamo, IT)

Background: The clinical benefit of allogeneic hematopoietic stem cell transplantation (ASCT) in patients with refractory Acute Myeloid Leukaemia (AML) at time of conditioning is still matter of debate.

Patients and methods: From September 1994 to August 2009, 55 patients (34 males and 21 females) with active AML at conditioning underwent ASCT at our institution after a median of 189 days (range 28-2517) from diagnosis. The median age was 45 years (range 18-65). Twenty-three patients were transplanted from matched-unrelated donors, 25 from HLA-identical siblings, 2 from HLA aploidentical and 6 from HLA mismatched (1 related, 1 unrelated and 4 from cord blood). The source of stem cells was peripheral blood in 40, bone marrow in 11 and cord blood in the remaining 4 patients. The conditioning regimen was myeloablative in $29(53 \%)$ and reduced intensity in $26(47 \%)$. Twentyseven patients received ATG in the conditioning program.

Results: The median dose of CD34 + cells infused was 4,7 ×10\% $\mathrm{kg}$ (range $0,01-12,32)$, the median time to leukocyte and platelet engraftment was 16 days (range 10-28) and 17 days (range 10-91), respectively. Thirty-nine patients (83\%) achieved complete remission (CR), 8 patients were refractory and 8 had an early death. Disease relapse $(n=23)$ or progression $(n=7)$ were the primary reasons of treatment failure. With a median followup of 142 days (range 12-3571), 8 patients remain alive $(22 \%$ at 1 year) and in CR. Seven of these latter patients received a conditioning regimen based on Busulfan $(12,8 \mathrm{mg} / \mathrm{kg}, \mathrm{n}=4)$ or TBI (12 Gy, $n=3$ ). By competing risk analysis, the cumulative incidence of relapse/progression and transplant related mortality, at 1 year, were $50 \%$ an $35 \%$, respectively. Acute GVHD was diagnosed in 29 patients (grade I-I, $n=21$; grade III-IV, $\mathrm{n}=8$ ). A limited chronic GVHD occurred in 5 of 36 evaluable patients while it was extensive in 9 patients. Acute (grade I-II) and chronic GVHD were both predictors of survival.

Conclusion: Allogeneic transplantation of refractory AML patients may achieve a durable remission in a minor but definitely sizeable proportion of otherwise incurable patients. New approaches including innovative cellular therapies are needed to reduce the rate of relapse after transplantation.

\section{P421}

The outcome of allogeneic stem cell transplantation conditioned with busulfan and fludarabine in malignant lymphoid diseases

J.H. Moon, S.N. Kim, B.W. Kang, J.G. Kim, Y.S. Chae, J.S. Suh, K.S. Lee, S.K. Sohn

Kyungpook National University Hospital (Daegu, KR)

There is some debate for a conditioning regimen with busulfan and fludarabine (BF) in malignant lymphoid diseases. This study evaluated the outcomes of allogeneic stem cell transplantation (SCT) conditioned with BF compared with BuCy2 in lymphoid malignancies.

The results of allogeneic SCT for 47 patients diagnosed with lymphoid malignancies were retrospectively reviewed. Thirty-three patients were diagnosed with acute lymphoblastic leukemia, 4 lymphoblastic lymphoma, and 10 acute mixed leukemia. Conditioning regimen was BuCy2 for 18 patients and BF for 29. Eighteen $(62.1 \%)$ and $13(72.2 \%)$ patients were high risk diseases including $8(27.6 \%)$ and $2(11.1 \%) \mathrm{Ph}+\mathrm{ALL}$ cases in BF and BuCy2 group, respectively $(P=0.475)$. The 3 -year overall survival (OS) rate was $50.5 \%$ and $31.8 \%$ in standard and high risk group, respectively $(P=0.156)$. The 3 -year OS rate was higher for the patients with chronic graft-versus-host disease (GVHD) than those without chronic GVHD (50.3\% vs. $29.6 \% ; P=0.004)$. The cumulative incidence of grade II-IV acute GVHD $(47.1 \%$ vs. $70.8 \%)$ and extensive chronic GVHD (23.4\% vs. $49.7 \%)$ were significantly lower in BF group than BuCy2 group $(P=0.040$ and $P=0.037$, respectively). The 3-year cumulative incidence of relapse was $28.0 \%$ in BF and $49.0 \%$ in BuCy2 group ( $P=0.277$ ). The 3-year OS (41.3\% vs. $32.4 \%)$ and event-free survival (EFS; $40.0 \%$ vs. $25.9 \%$ ) were not different between BF group and BuCy2 group. However, the treatment related mortality (TRM) was significantly lower in BF group (16.5\%) than BuCy2 group $(50.1 \% ; P=0.035)$. After adjusting age, conditioning intensity, donor type (sibling vs. unrelated), HLA disparity (full match vs. mismatch), and acute GVHD, the BF regimen was an independent favorable risk factor for the TRM (hazard ratio $0.036 .95 \%$ confidence interval 0.002-0.557; $P=0.017$ ).

In conclusion, the BF regimen seemed to be acceptable for conditioning in lymphoid malignancies including high risk diseases in terms of OS and EFS. It could be safely administered with lower TRM rate compared to BuCy2.
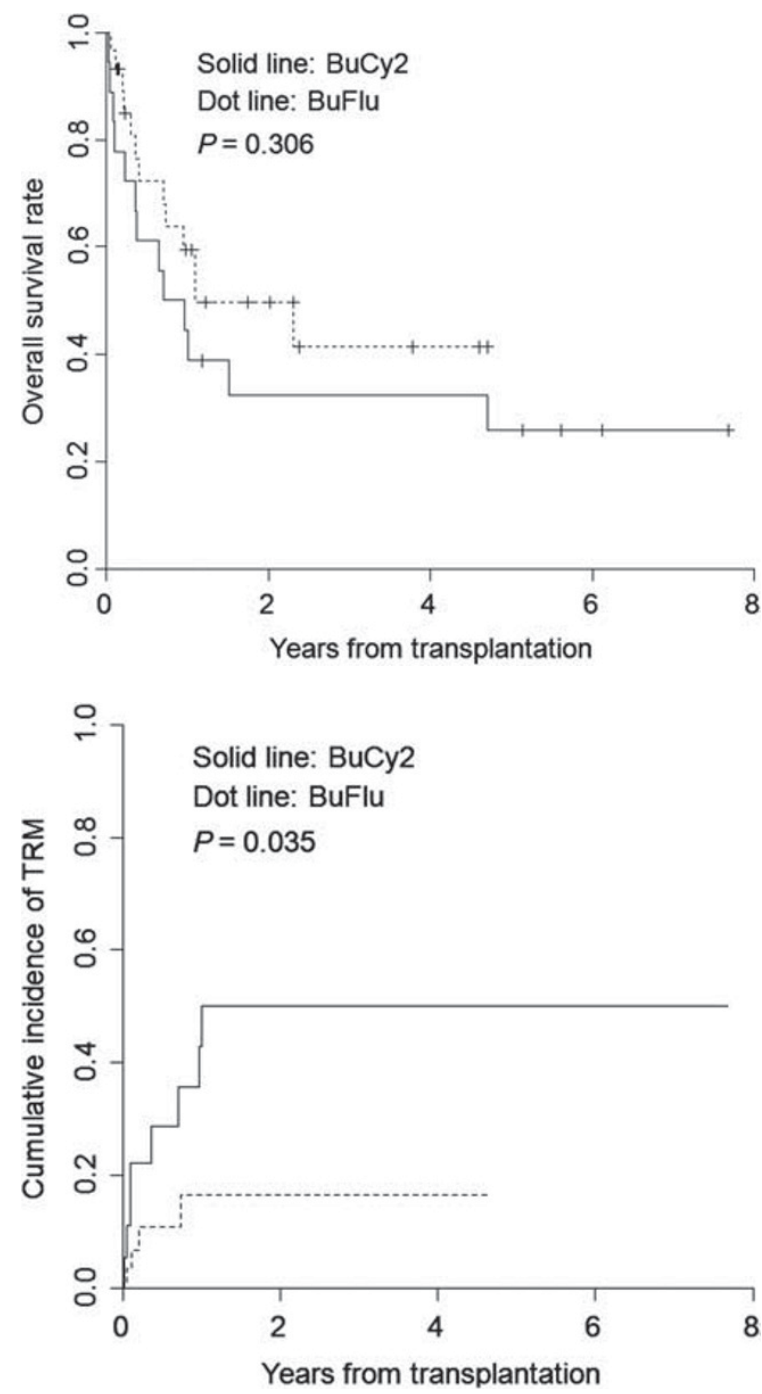
P422

Feasibility and outcome of allogeneic haematopoietic stem cell transplantation in $\mathbf{3 0}$ poor-risk acute myeloid leukaemia patients older than 60 years

A. Candoni, E. Simeone, M. Chiozzotto, R. Fanin

University Hospital (Udine, IT)

Scarce data are available regarding allogeneic SCT (alloSCT) in elderly Acute Myeloid Leukemia (AML) patients. We report on the feasibility and outcome of allo-SCT in 30 poor risk AML patients (pts) older than 60 years. Median age at transplant was 63 years (range 60-70 years) and $18 / 30(60 \%)$ cases were in complete remission. Donor were MUD in $12(40 \%)$ and sibling in $18(60 \%)$ of 30 cases. Twenty-six of 30 pts $(87 \%)$ received a reduced intensity conditioning regimen (RIC). The hematopoietic cell transplantation specific comorbidity index (HCT-Cl) was two or less in $12 / 30$ cases (40\%) and three or more in $18 / 30$ cases $(60 \%)$. All patients engrafted. One year Nonrelapse mortality (NRM) rate was $20 \%$ (6/30 cases). After a median follow-up of 16 months (range 1-64), 17/30 patients $(57 \%)$ were alive and in complete remission while 13/30 (43\%) have died (leukemia refractory or relapse $7 / 13$ and NRM 6/13). Median Overall Survival (OS) for the whole patient population was 28 months. The OS did not differ between unrelated and related donors. The patients transplanted in complete remission have a significantly lower relapse rate compared to those transplanted with refractory or relapsed AML $(P=0,008)$. The cases with a low HCT-Cl (2 or less) have a significantly lower NRM $(P=0,05)$ compared to those with HCT-Cl 3 or more.

Taking into account that this is a retrospective analysis with a small number of cases, these results confirm the feasibility of allo-SCT for high risk AML patients older than 60 years. Outcome was significantly influenced by status of disease at transplant and by HCT-Cl. We also confirm that for older patients lacking a suitable family donor MUD can provide a suitable alternative option.

\section{P423}

Long-term follow-up of autologous stem cell transplantation for elderly acute myeloid leukaemia patients: experience of the Coordinamento del trapianto di cellule staminali emopoietiche della Regione Lazio P. Chiusolo (1), S. Capria (2), R. La Tagliata (2), A. Proia (3), A. Severino (3), E. Zappone (4), S. Sica (1), G. Meloni (2)

(1) Università Cattolica S. Cuore (Rome, IT); (2)"La Sapienza "University (Rome, IT); (3)Ospedale SanCamilloForlanini (Rome, IT); (4)Ospedale Santa Maria Goretti (Latina, IT)

Several trials have suggested the potential utility of autologous stem cell transplantation (ASCT) in acute myeloid leukemia (AML) of the elderly and the introduction of peripheral blood stem cells (PBSC) has reduced morbidity and mortality of the procedure. However the role of ASCT in AML is still a concern expecially in patients $>60$ years, and prospective randomized trials are lacking and difficult to perform.

We report the results of a retrospective multicenter study on 32 patients $(15 \mathrm{M} / 17 \mathrm{~F})$ aged $>60$ years with $A M L$, who underwent an ASCT in 1st CR between May 1995 and May 2009. Patients' characteristics were as follows: the median age of the patients was 62 years (range 60-68). The median WBC at diagnosis was $4.2 \times 10^{9} / \mathrm{L}$ (range 0.9-162). Cytogenetic alterations were found in 6 out 32 pts (2 complex karyotype, $1 \mathrm{t}(8 ; 21), 1$ tris 13,1 tris8, $\mathrm{t}(11 ; 19)$, while 16 pts showed a normal karyotype; in the remaining patients it was not evaluable. Foure out of 19 patients evaluable for molecular analysis showed low risk features (2 AML-ETO, 1 MYH11-CBFB, 1 NPM mutation). The conditioning regimens most used were: BAVC (10pts) and BuCy (14pts). Stem cells sources were PBSC in 29 pts, BM in 2 pts and PBSC+BM in 1 pt. The median dose of CD $34+$ cells reinfused $/ \mathrm{kg}$ was $3.70 \times 10^{6}$ (range 1.85-17).

The median time to granulocyte recovery $>0.5 \times 10^{9} / \mathrm{L}$ was 13 days (range 9-43) and for platelets $>20$ and $>50 \times 10^{9} / \mathrm{L}$ was 14 (range 8-60) and 21 days (range 12-210) respectively. All but one patient achieved independence from RBC and platelets transfusions (96.8\%). Mucosae toxicity grade $3-4$ was observed in $5 / 32$ pts (15\%). The transplant related mortality was $3.1 \%$ and the cause of death was sepsi.

After a median follow-up of 2.7 years from transplantation, the median DFS and OS were $48 \%$ and $50 \%$ years respectively, and the 10 -year rates were $42 \%$ and $50 \%$ respectively (see Figures 1-2). Twelve autografted patients are still in complete remission, 16 have relapsed and 3 had died in remission (1 a second neoplasia, 1 cerebral hemorrhage and 1 sepsi from resistant Pseudomonas aeruginosa early after transplantation).

The long follow up of our data confirms that intensification of remission including ASCT is a feasible and encouraging therapeutic approach, characterized by low transplant related mortality and providing the possibility of long survivorship characterized by low transplant related mortality and the possibility of long survivorship in selected elderly AML patients.
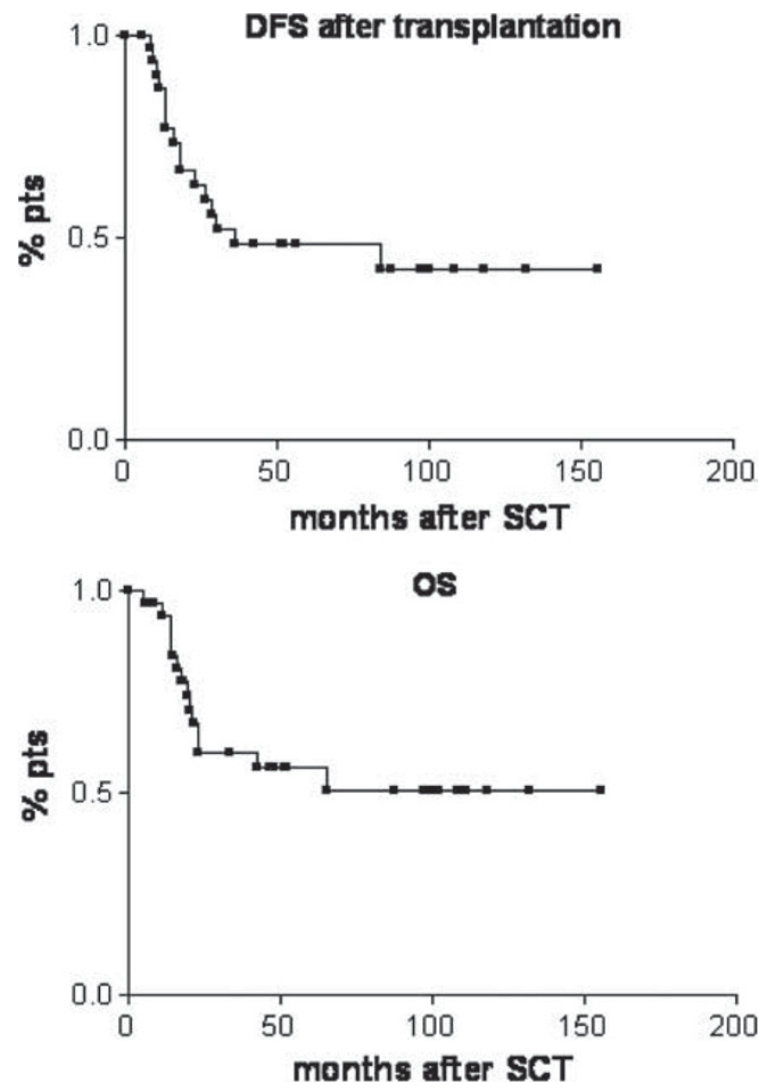
P424

Retrospective analysis of second haematopoietic stem cell transplant. Single-centre experience

E. Carrillo, J. Falantes, F. Marquez, M. Carmona, A. UrbanoIspizua, A. Espigado

Hospital Virgen del Rocio (Sevilla, ES)

Background: Prognosis of patients (pts) with relapse after hematopoietic stem cell transplantation (HSCT) is poor. A second HSCT might be an option in selected pts although treatment related mortality (TRM) is a major concern.

Objectives: Retrospective analysis of overall survival (OS) and TRM of second HSCT in hematologic malignancies. To identify prognostic factors that influence the outcome.

Patients and methods: From September 1989 to June 2009, 44 pts received a second HSCT. Median age was 38y (16-57), 25 were male and 19 female. Diagnoses were: 18 acute myeloid leukaemia (AML), 7 myelodysplastic syndrome (MDS), 5 acute lymphoid leukaemia (ALL), 4 hodgkin lymphoma (HL), 5 non-hodgkin lymphoma (NHL), 3 chronic myeloid leukaemia (CML), 2 solid neoplasms. Autologous SCT was carried out in 10 patients and allogeneic SCT in 34 pts, of which 19 received myeloablative conditioning and 25 reduced intensity conditioning (RIC). Graft source was bone marrow in 4 pts, peripheral blood in 39 pts and cord blood unit in 1 pt. From the initial cohort, we selected a group of 30 patients with AML, MDS and ALL. Median age in this group was $37 y(16-57)$. Disease status previous second HSCT: $11 / 30$ pts were in complete remission $\left(C R_{j} Y 2\right)$ and $19 / 30$ had either partial response (PR) or progressive disease (PD). 25/30 pts received alloSCT and 5/30 pts autoSCT. Conditioning regimen was myeloablative in 12 pts and RIC in 18 pts. The source graft was bone marrow in 3 pts and peripheral blood in $27 \mathrm{pts}$.

Results: The OS rate was $24.5 \%$ at 41 months follow-up in the global group and $19 \%$ in the 30 -patients cohort. A higher survival rate was observed in pts whose time interval between the first and the second transplant was more than 1 year (OS $43 \%$ at 18 months versus $9 \%$ OS for pts with time interval less than a year, $P<0,01)$. 1 -year OS in AML pts in $\mathrm{RC}=$ or $>2$ was $40 \%$ vs. $0 \%$ for AML pts in PR or PD $(P<0,02)$. No difference in OS was observed comparing both types of conditioning regimen. In the 30 -patient cohort the TRM was $36 \%$ and $33 \%$ in AML patients. Conclusion: Despite low long-term OS after the second HSCT, this may be an option in these high risk pts. The remission status at the time of a second transplant and a time interval between both transplants of more than a year, seem to be determinant of the outcome.

\section{P425}

Low relapse without excessive transplant-related mortality following allogeneic stem cell transplantation in patients with high-risk secondary acute myeloblastic leukaemia and myelodysplastic syndromes: long-term outcomes in a single-centre experience

L. Castagna, S. Furst, C. Faucher, A. Charbonnier, N. Vey, D. Blaise

Institut Paoli Calmettes (Marseille, FR)

Allogenic stem cell transplantation (Allo-SCT) as a therapy for secondary acute myeloid leukaemia (sAML) and myelodysplastic syndromes (MDS) is the most powerful treatment option. However, (Allo-SCT) is also complicated by a high risk for treatment-related morbidity and mortality. We analysed retrospectively the data of 70 patients transplanted at our institution from June 1995 to December 2008, 44 patients (63\%) with sAML and 26 patients (37\%) with MDS was treated with (AlloSCT); median age at diagnosis was 41 years, (15-70), and the median age of 42,5 years $(16-70)$ at transplantation; The conditioning regimen was myeloablative in 16 patients $(23 \%)$ and reduced intensity (RIC) in 54 patients $(77 \%) ; 11$ patients $(16 \%)$ were infused with bone marrow $(\mathrm{BM}), 55$ patients $(79 \%)$ peripherical blood stem cells (PBSC), and 4 patients $(5 \%)$ cord blood cells; in 49 cases $(70 \%)$ donor was a HLA identical sibling and in $21(30 \%)$ was a matched unrelated donor;41 patients $(59 \%)$ carried high risk cytogenetic features, like (7q-, 5q-, >3
[P424]

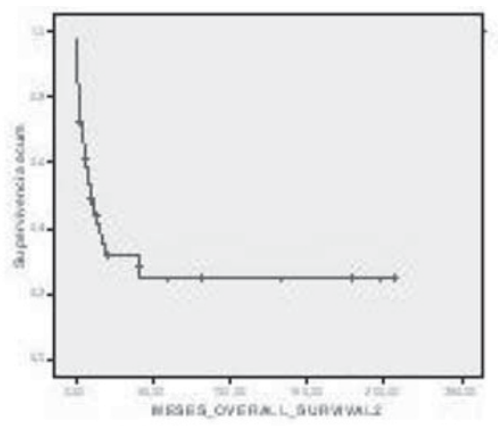

Table 1. 44 pt-cohort os

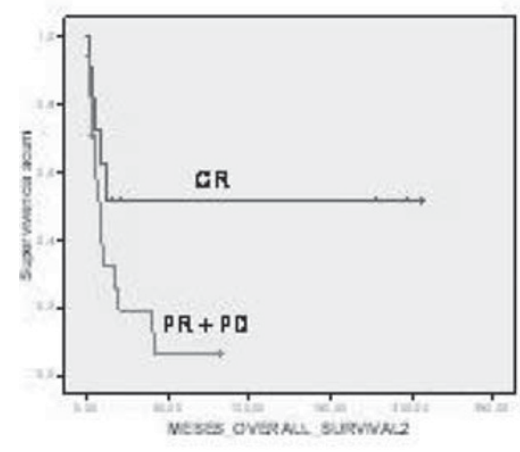

T able 3.30-pt cohort os for type of response

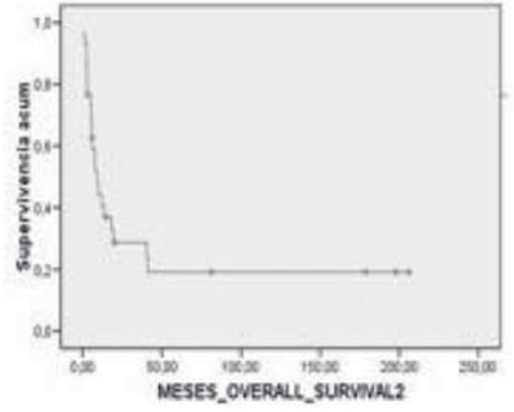

Table 2.30-pt cohort os

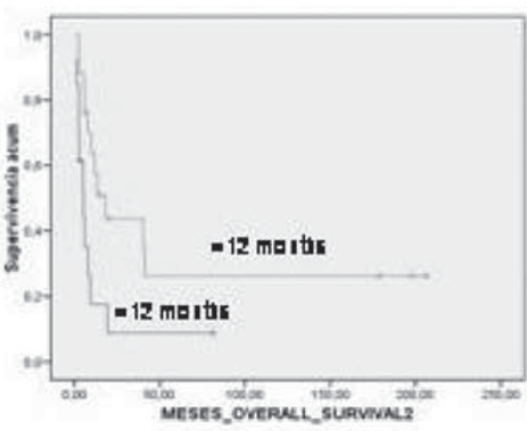

Table 4. 30-pt cohort os for time interval between transplants 
alterations), while was normal in 24 patients (34\%), and in 5 patients $(7 \%)$ was unknown. Disease status at transplantation was as follow: CR in 24 patients (34\%), 34 patients (49\%) was refractory or in progression after treatment, and 12 patients $(17 \%)$ was with a stable disease.

With a median follow-up of 55 months (3-150), 30 patients $(43 \%)$ are alive, the overall survival OS at 2 years and 5 years was $48 \%$ and $39 \%$ respectively, and after ten years of follow up, OS was $30 \%, 95 \% \mathrm{Cl}$ [17.8-50.8]. We observed also that $26 \%$ of refractory patients and $54 \%$ of patients in CR are alive at five years of transplantation. The probability of progression after transplantation at five and ten years was $31 \%$ with $95 \% \mathrm{Cl}$ [20.-46.5]. 2 years and 5 years treatment related mortality (TRM) was $23 \%$ and $26 \%$ respectively, and no modification at ten year, $95 \% \mathrm{Cl}$ [14.3-37.3]. TRM was occurred in 16 patients $(23 \%)$. Cause of death was; infections in 5 patients $(7 \%)$, GvHD in 3 patients (4\%), GvHD and infection in 3 patients (4\%), multi organ failure (MOF) in 5 patients $(7 \%)$. In multivariate analysis; OS, PFS or TRM, were not influenced by donor type (HLA id sibling vs. others), conditioning regimen (RIC vs. MAC), and stem cell source (bone marrow vs. PBSC). Allogenic stem cell transplantation can be considered as a good option of treatment for patients with high risk SAML and MDS, Compared with the remission rate at five years of the other non allogenic SCT therapies.

\section{P426}

Comparison of patients with acute myeloid leukaemia relapsed after myeloablative and reduced-intensity conditioning transplantation. Single-centre experience K. Steinerova, V. Koza, P. Jindra, M. Karas, D. Lysak, T. Svoboda, S. Vokurka

Charles University Hospital (Pilsen, CZ)

The prognosis of the patients (pts) with AML relapsed after stem cell transplantation (SCT) is dismal. We describe treatment and outcomes for patients who relapse following SCT and compare the outcome of the group of pts transplanted with myeloablative and with reduced intensity conditioning regimen (RIC).

Materials and methods: In a retrospective single center study we analyzed 167 pts (96 pts after myeloablative and 71 pts after RIC SCT) with high risk AML transplanted between the years 1993 and 2009. 29 pts (30\%) relapsed after myeloablative SCT and 15 pts $(21 \%)$ relapsed after RIC SCT $(P=0,2161)$. Median of relaps was 4 months in the group of myeloablative SCT (1-49 months) and 5 months (3-28) in the group of RIC SCT $(P=0,1845)$. Both groups were comparable according to disease status, prognostic factors and the donor type (HLA identical sibling vs. unrelated donor, $P=0,3401$ ) with the only difference of age. In the group myeloablative regimen the median age was 40 (21-55 years) and in the group of RIC SCT 55 (29-66 years), $P=0,0015$. 22 pts $(50 \%)$ received aggressive treatment for relapse $(14 / 29$ and $8 / 15, P=1,0)$ including donor lymphocyte infusion (DLI) $(11 / 29$ and $6 / 15, P=1,0)$, chemotherapy $(2 / 29$ and $2 / 15, P=0.5962)$, second transplantation $(2 / 29$ and $0 / 15, P=0.5402$ ).

Results: 7 pts (16\%) archieved complete response(CR) after treatment for relapse ( 5 pts after DLI with or without chemotherapy and 2 pts after second transplantation), 2 pts after RIC SCT and 5 pts after myeloablative SCT, $P=1,0.2$ pts still alive ( 1 from group of myeloablative SCT and 1 from group of RIC SCT) 15 and 51 months after SCT with median of overall suvival (OS) 5 months after myeloablative SCT and 7 month after RIC SCT, the probability of 1 year OS $31 \%$ and $33 \%(P=0,5216)$. 42 pts died, most of them due to disease progression (40/44, $91 \%$ ), 1 pts died due to GVHD after DLI and 1 pts due to respiratory failure. We did not observe difference in the outcome among pts treated with DLI, chemotherapy and second transplantation.

Conclusion: Salvage chemotherapy or cellular therapy for AML relapse after SCT is feasible with low treatment-related mortality, but optimal management of the therapy for AML relapsing after SCT still remains to be defined. Comparing the group of pts transplanted with myeloablative regimen and pts transplanted with RIC no statistical significant difference (except of age) were found, however our results suggest better OS for patients relapsing after RIC SCT.

\section{P427}

Allogeneic haematopoietic stem cell transplantation for adolescent and adult patients with high-risk acute lymphobalstic leukaemia of T-cell phenotype M. Bakr (1), F. Al-Mohareb (1), S.Y. Mohamed (1), N. Chaudhri (1), F. Al-Sharif (1), H. Al-Zahrani (1), M. Patel (1), A.J. Saleh (1), A. Nassar (1), S. Ahmed (1), A. Elghazaly (1), S. O. Ahmed (2), K. Ibrahim (1), F. Hussain (1), W. Rasheed (1), M. Aljurf (1) (1)King Faisal Specialist Hospital (Riyadh, SA); (2)Royal Free Hospital (London, UK)

Introduction: T-cell acute lymphoblastic leukemia (T-ALL) accounts for approximately $20 \%$ of adult ALL. Historically, T-ALL had a worse outcome compared to other ALL. Recent studies suggest improved outcome with the use of intensive treatment. High risk (HR) patients (pts) are often candidates for allogeneic hematopoietic stem cell transplantation (HSCT).

Patients and methods: We analyzed outcomes of 56 consecutive pts (58 transplants) who underwent allogenic HSCT at King Faisal Specialist Hospital from 01/1995 to 07/2009 for HR TALL. HR features included: WBCs $\geq 100,000$, residual disease at D14 post induction, CNS involvement at diagnosis, poor risk cytogenetic features, primary induction failure (PIF) \& disease relapse.

Results: Median age was 18 yr (range, 13-51). Male: female ratio was $6: 1$. T-cell subtypes were pro-T $(n=1,1.8 \%)$, pre-T $(n=21,37.5 \%)$, cortical-T cell $(n=16,28.6 \%)$, mature-T cell $(n=12,21.4 \%)$ and unknown $(n=6,10.7 \%)$. Disease status at transplant: CR1 with HR features at diagnosis $(n=28,50 \%)$, primary induction failure (PIF) $(n=6,10.7 \%)$, $\geq$ second complete remission (CR2) $(n=18,32 \%)$ and relapsed disease $(n=4,7.1 \%)$. HLA matched sibling donor was the main donor type. Stem cell source was Bone marrow $(n=40,69 \%)$, peripheral blood $(n=17,29 \%)$ or cord blood $(n=1,2 \%)$.

Acute (zgrade) II and chronic GVHD occurred in $38 \%$ and $29 \%$, respectively. Thirty pts died, 18 with relapse and $12 \mathrm{sec}-$ ondary to HSCT complications. Transplant related mortality at D100 was $10.7 \%$. With a median follow up of 13 months (rang, 1-168) the estimated 5-year overall survival (OS) and disease free survival (DFS) were both $39 \%$. Cortical cell subtype had a trend for a better OS (54\%) compared with other cell subtypes but this was not statistically significant. Pts transplanted in CR1 had statistically significant superior OS and DFS compared with those transplanted in >CR1 $(50.8 \%$ vs. $29.3 \% p$ value $=0.03$ and $52.4 \%$ vs. $26 \%$ p value $=0.007$, respectively). Seventeen pts had c GVHD. Patients with cGVHD had a trend for lower risk of relapse compared with those without cGVHD (23\% vs. $44 \%$, respectively), but this was not statistically significant. No significant differences in OS and DFS among different age groups were observed.

Conclusion: Allogeneic HSCT represents an effective treatment for HR T-ALL particularly if performed in CR1. The presence of clinically relevant GVL effect needs further evaluation. 


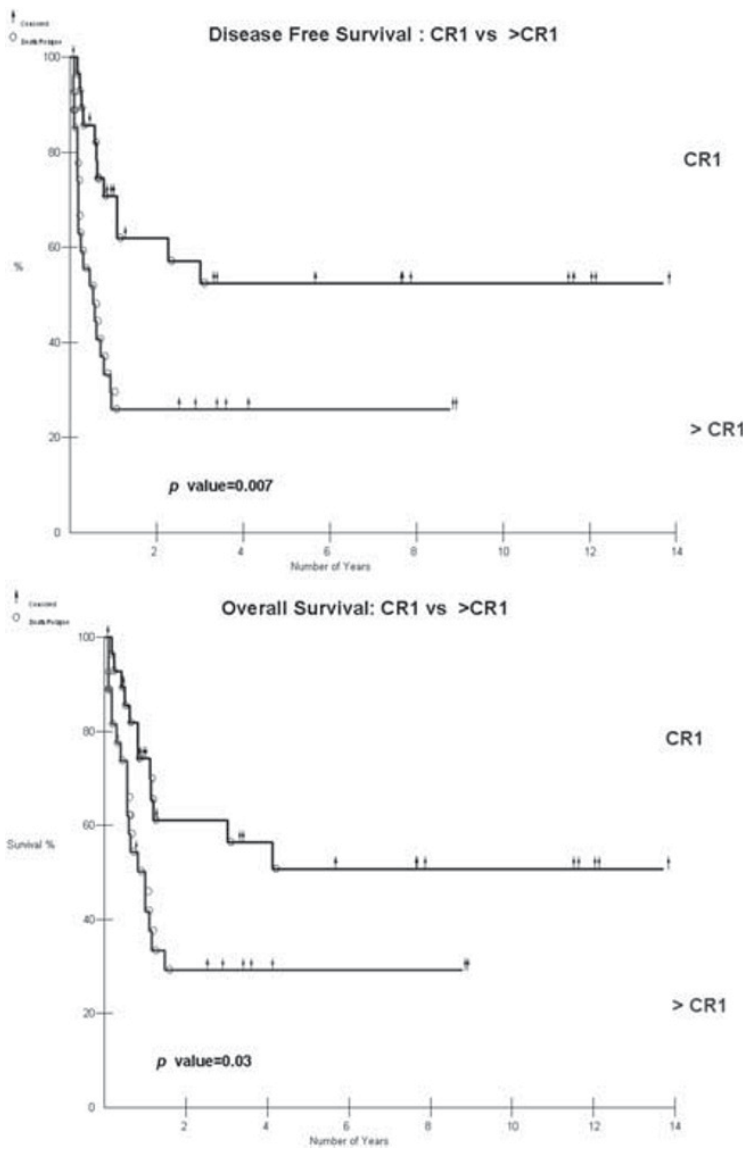

P428

Post-transplant relapses of acute leukemia in children and adolescents

E. Semenova, N. Stancheva, N. Zubarovskaya, E. Babenko, O. Slesarchuk, M. Estrina, L. Zubarovskaya, B. Afanasyev SPb State I. Pavlov Medical University (St. Petersburg, RU)

Relapses of acute leukemias after allogeneic hematopoietic stem cell transplantation (alloHSCT) (AL) are the main unsolved problem because standard therapy for such pts are exhausted. Aim: To analyze pts with AL with relapse after allo-HSCT for the most effective therapy.

Patinets and results: 138 children and adolescences, age from 1 to 21 yo (median 13 y.o.) suffering from AML $-47 \mathrm{pts,}$ ALL-88 pts, biphenotyping $A L-3$ pts.

Relapse were diagnosed in 36 pts $(26 \%)$ in $1-18$ months $(89 \%$ in 3-12 mo) after allo-HSCT (median 7.5 months), 22 pts with ALL (25\%); 13 pts with AML (27\%); 1 pt with biphenotyping AL, bone marrow relapse -24 pts $(67 \%)$, combined relapse -12 pts $(33 \%)$. At the moment of allo-HSCT in I-II CR were 20 pts (55\%); 2 pts (6\%) in III CR; 10 pts (28\%) had relapse, 4 pts $(11 \%)$ - primary resistance. Related allo-HSCT was performed in 12 pts (33\%), MUD-in 14 pts (39\%), haploidentical (haplo-HSCT) - in 10 pts (28\%). Conditioning regimens were MAC-17 pts (47\%), RIC-19 pts (53\%). According to therapy pts were divided into 1 st group - chemotherapy $(\mathrm{ChT})+\mathrm{DLI}$ in 12pts (34\%); 2nd group - ChT in 15pts (43\%), DLI in 3pts (9\%), 2ndallo-HSCT in 5 pts (14\%). DLI: The lymphocytes range was $0,5 \times 10^{5}-1 \times 10^{7}$ on CD3+, infusions number $-1-3$.

1st group: Four pts (33\%) after ChT+DLI are alive in CR; 2 nd group: $4(17 \%)$ pts are alive in CR. 2-years OS of all pts with post-transplant relapses was $14 \%$ but in 1 st group vs. 2 nd group $-31 \%$ vs. $0 \%(x=0.04)$.

In $48 \%(n=10)$ after haplo-HSCT relapse develops; in $32 \%$ $(n=12)$-after related HSCT, and significantly rarer $(x=0.03)$ in $17 \%(n=14)$ after unrelated HSCT.
Conclusions: ChT+DLI-is the most effective therapy of posttransplant relapses of AL but more detailed investigations are needed.

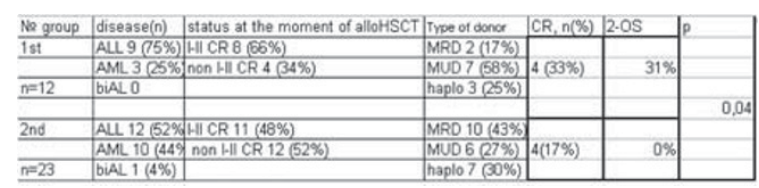

P429

Results of haematopoietic stem cell transplantation in acute myeloid leukaemia over 25 years from a cancer treatment centre, Mumbai

B. Bagal, A. Joshi, M. Shah, R. Singh, S. Kannan, H. Menon, S. Banavali, R. Nair, N. Khattry

Tata Memorial Center (Mumbai, IN)

Background: Allogeneic transplantion is one of the most curative modality of treatment for most patients of acute myeloid leukemia (AML). Autologous transplantation has also shown encouraging long-term survival results in patients who do not have HLA-matched donors. We retrospectively analyzed our results and role of possible prognostic factors.

Material and methods: Fifty-two patients who underwent allogeneic or autologous transplantation either upfront or in relapsed setting were included in this study from March 1983 to September 2009. Patients underwent autologous transplant only if an HLA-matched related donor was not available. Conditioning regimens included busulfan-cyclophosphamide in 27 , cyclophosphamide-total body irradiation (TBI) in 16, highdose melphalan in 4, fludarabine - busulfan in 3, Fludarabine with $2 \mathrm{~Gy} \mathrm{TBI}$ in 1 and busulfan- etoposide-cyclophosphamide in 1 patient. Baseline cytogenetic analysis was not available for most patients. Prognostic factors evaluated for overall survival (OS) in those who underwent allogeneic transplant were disease status at transplant, presence of acute or chronic GVHD, donor-recipient sex match and ABO compatibility.

Results: Fifty-two patients were evaluated, 43 in allogeneic and 9 in autologous setting. At the time of allogeneic transplant, 25 were in first remission (CR-1), 12 in second remission (CR-2) and 6 in refractory state, while in autologous setting, 8 were in CR-1 and 1 in CR-2.Median age at transplant was 27 years. Median time to neutrophil and platelet engraftment was 14 and 15 days respectively. Fifty-three percent and $46 \%$ of allogeneic transplants were sex and ABO mismatched respectively. The incidence of acute and chronic GVHD was $42 \%$ and $19 \%$ respectively. Incidence of veno-occlussive disease was $11.5 \%$. Two patients relapsed after autologous transplant while 13 relapsed after allogeneic transplant. The transplant - related mortality (TRM) after autologous and allogeneic transplantation were $22 \%$ and $28 \%$ respectively. The cumulative probabilities of OS after allogeneic and autologous transplant at 5 years were $38 \%$ and $50 \%$ respectively. None of the above prognostic factors evaluated influenced OS in allogeneic transplant setting Conclusion: This is the first report of long-term survival post transplant for AML from India that shows encouraging OS and patients who do not have an HLA- matched donor may still have long-term benefit from autologous transplant in first remission.

\section{P430}

Allogeneic haematopoietic stem cell transplantation for relapsed/refractory acute leukaemia - a single-centre experience

T.D. Tan, M.C. Wu, J.L.W. Chiou

Koo Foundation Sun Yat-Sen Cancer Center (Taipei, TW)

Purpose: For relapsed or refractory acute leukemia, the curative treatment of choice is allogeneic hematopoietic stem cell transplantation if patients could tolerate transplant therapy feasibly and an HLA mutched donor available. If not, patients could just go into 
clinical trial with novel agents or supportive care. However, the transplant related morbidity and mortality is higher and the eventfree and overall survival are worse than patient in remission. Materials and methods: We evaluated our 20 patients who are relapsed or refractory acute leukemia after the treatment of remission induction and high dose Ara-C and mitoxantrone (HAM) chemotherapy or prior autologous or allogeneic stem cell transplantation between February 2004 and October 2009. All these patients underwent allogeneic hematopoietic stem cell transplantation with matched related or unrelated donor.

Results: Twenty patients included 11 male and 9 female with median age 40.8 years old (range 21 60). Diagnosis included AML $(N=15$, including 4 therapy-related), $A L L(N=3)$, and CML-BP $(n=2)$. There were 16 patients $(80 \%)$ having undergone high dose Ara-C plus mitoxantrone (HAM) chemotherapy. Four patients $(20 \%)$ had had prior transplant treatment. Four patients were graft failure and died in 1 month. For the other 15 evaluable patients, 5 patients were leukemia free but one of them died of pulmonary invasive aspergillosis. Posttransplant relapse occurred in 10 patients $(66.7 \%)$, but in 7 patients underwent salvage chemotherapy alone $(n=1)$ or salvage chemotherapy plus donor leukocyte infusion $(n=6)$ with 5 leukemia-free achieved. In general, 9 patients died of leukemia, 3 patients died of infection, and 1 patients died of unknown cause of hepatic failure. Three year event-free survival is $22 \%$ and overall survival $23 \%$.

Conclusions: As expected, the relapse rate is very high and most patients died of leukemia itself but some patients still could be salvaged by re-chemotherapy plus CD34-riched donor leukocyte infusion or second transplantation. Therefore, the plan of programmed prophylactic donor lymphocyte infusion is mandatory.
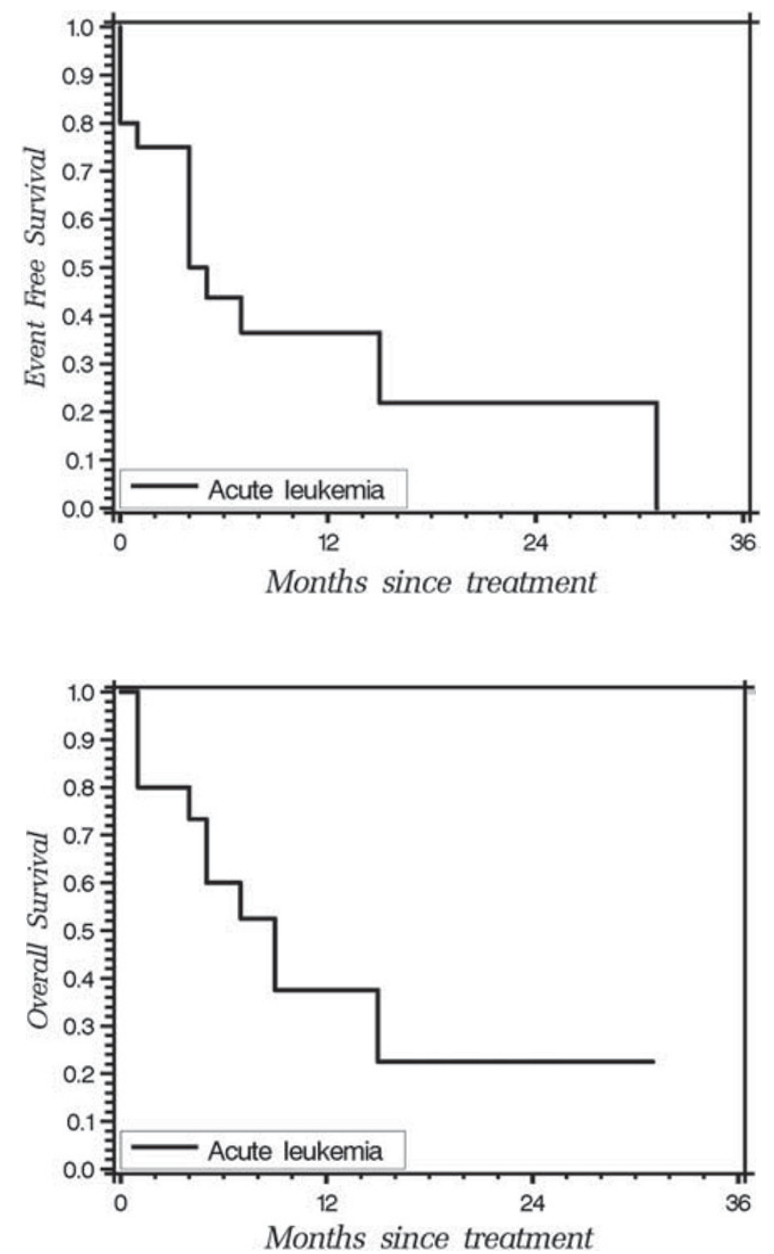

\section{Early side effects / Late effects and quality of life}

\section{P431}

Use of high-throughput genetic polymorphism analysis to predict transplant related mortality after allogeneic haemopoietic stem cell transplantation

F. Bonifazi (1), G. Bandini (1), E. Marasco (2), G. Storci (1), M. Arpinati (1), M. Stanzani (1), M.R. Motta (1), S. Rizzi (1), P. Garagnani (2), V. Mantovani (2), M. Baccarani (1), M. Bonafé (1) (1)University of Bologna (Bologna, IT); (2)S. Orsola-Malpighi Hospital (Bologna, IT)

HSCT is a complex procedure including several life-threatening steps. The success at each step contributes to the global outcome. Some studies have already shown the association of single polymorphisms with GVHD, toxicity, OS, with contrasting results. HSCT outcome may be considered as polygenic quantitative trait, where multiple genes play a role and may also affect different phenotypes (pleiotropy) at different times. Nowadays, high throughput technology allows to simultaneously assay a large amount of genetic variations (single nucleotide polymorphisms, SNPs) at various loci.

DNA obtained from 176 patients who underwent allogeneic HSCT between 2004 to 2008 for hematologic malignancies were assessed for genetic polymorphisms at 92 polymorphisms inside 34 genomic loci (Table 1). Genes were chosen on the basis of their capacity to affect liver drug metabolism and oxidative stress response homeostatic pathway, such as glutathion and folate homeostasis, cytocrome oxidase system, ABC cassettes membrane transporters.

Median age of the study population was 41 years, $43 \%$ were in early phase at transplant, $51 \%$ BM were BM, $47 \%$ were unrelated donor transplants, $65 \%$ received a myeloablative regimen.

Genotypes were assessed by MaldiTOF (Mass analysis light desorption- time of light) SequenomTM technology.

TRM and OS were chosen as endpoints of the study.

Each polymorphism was evaluated in univariate and multivariate analysis, by Cox model incorporating also age, type of donor, intensity of conditioning, phase at transplant as covariates. Multivariate analysis was also performed to assess synergistic effects among multiple polymorphisms. Some of the most representative TRM curves are shown in Figure 1.

Multivariate analysis revealed significant association between TRM and polymorphisms at MTHFR (hazard risk, $\mathrm{HR}=2.3)$, ABCC4 $(\mathrm{HR}=3.6)$, GSTM1 (HR=2.5) loci; a significant increase in the HR for ABCC4 and GSTM1 polymorplysm was found when simultaneuously tested in multivariate analysis. Subgroup analysis according to conditioning regimens revealed drug specific association between polymorphism and TRM and OS (eg CYP3A4 polymorphism was associated with OS in cyclophosphamidetreated patients).

Our results shows that the individual genetic makeup may explain part of the inter-patient variability thus predicting the outcome of HSCT. External validations are requested to extend this approach to clinical use. 
Figure 1.

Transplant related mortality curves according to genetic polymorphisms

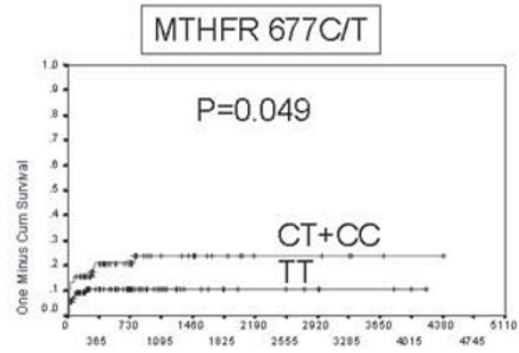

ABCC4 Ser/Arg 304
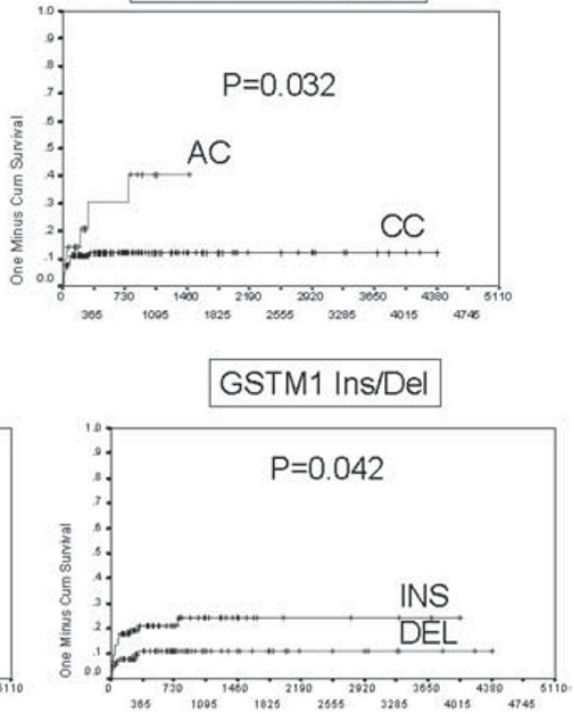

Iable l. List of genes and polymorphisms investigated. rs: reference sequence; wt=wild type

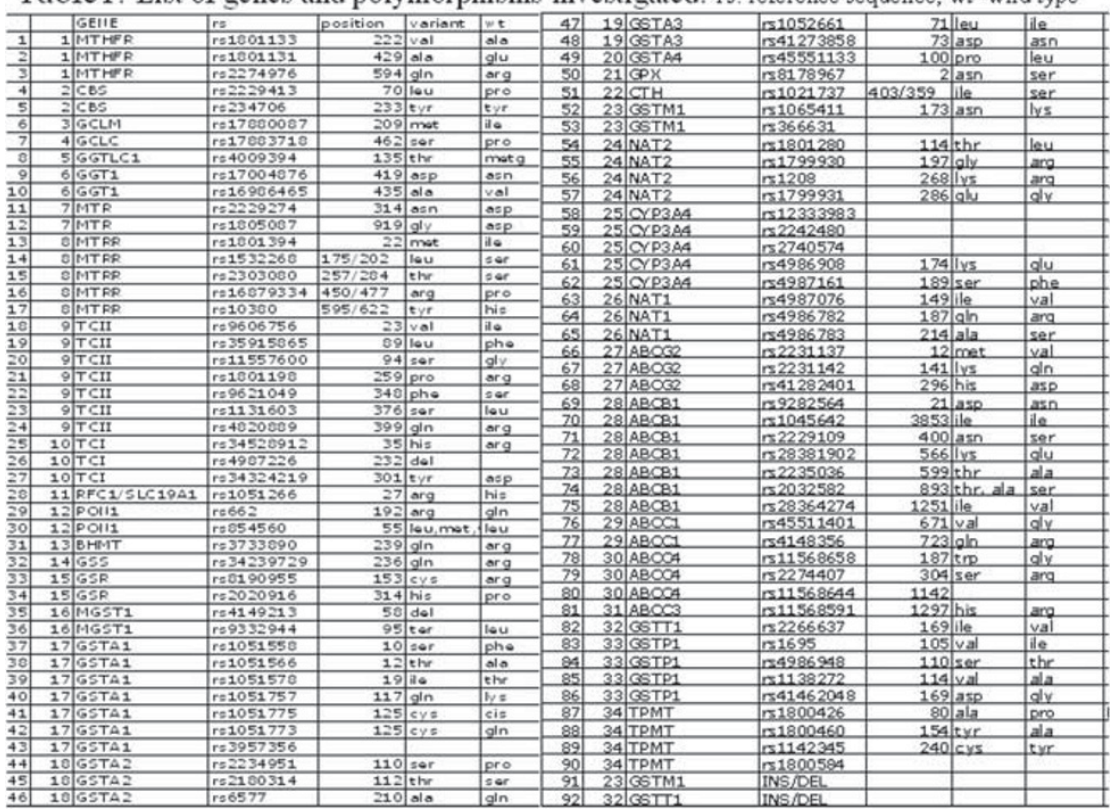

P432

The incidence of secondary malignancy post stem cell transplantation: importance of conditioning regimens without total body irradiation

A. Ghavamzadeh, K. Alimoghaddam, M. Jahani, A.A. Hamidieh, S. Mousavi, M. Iravani, B. Bahar, A. Khodabandeh, F. Khatami, A. Jalali

Hematology-Oncology \& Stem Cell Transplantation Research Center (Tehran, IR)

Introduction: In this study we described the records of 2766 recipients of allogeneic-related and -unrelated or autologous and syngeneic Stem Cell Transplants (SCTs) between 1991 till Dec 2009 to determine the incidence of secondary malignancies after conditioning regimen without Total Body Irradiation (TBI).

Patients and methods: 2766 patients were undergone transplantation for Acute Lymphocytic Leukemia (ALL; $n=388$ ), acute myelogenous leukemia (AML; $n=710)$, chronic myelogenous leukemia (CML; $n=237), 356$ patients for Lymphoma, 164 for aplastic anemia (AA), 442 for Thalassemia and 469 patients for a variety of malignant and nonmalignant disorders. All conditioning regimens included high-dose chemotherapy without total body irradiation (TBI). Patients who were missed to follow-up were excluded from this study.

Result: Eleven patients developed secondary malignancies; One year and five years risk of new malignancy were $0.36 \%$ and $0.47 \%$, respectively. Of all the 11 patients $7(63.6 \%)$ were male, and $4(36.4 \%)$ were female. The median age was 23 years old (range: $13-50$ years). $8(72.7 \%)$ patients received allogeneic stem cell transplantation and $3(27.3 \%)$ received autologous transplantation. 8 patients $(72.7 \%)$ had peripheral blood as graft type. The conditioning regimen for $7(63.6 \%)$ patients was Busulphan and Cyclophosphamide, 2 of them had Ara-C, Etoposide, Melphalan and lomustine, 1 of them had Cyclophosphamide, Etoposide and Ara-C. The 
other 1 patient had ATG, Cyclophosphamaide as conditioning regimen. The median follow-up duration for all our patients was 17.4 months, (1 day to 211.5 months) which was 16.3 months (47 days to 68 months) for these 11 patients who developed new malignancy. At present 4 patients (36\%) are alive and the cause of death in $28.6 \%$ of deaths is new malignancy.

Conclusion: In this study, we described the first Iranian series of malignancies after HSCT. Secondary malignancies are a late complication of HSCT. Others studies have shown that TBI was risk factor for post-HSCT secondary malignancies. In this study, including 2766 patients, the low risks of secondary malignancies post HSCT $(0.4 \%)$ may cause of the absence of TBI receiving in any protocol.

\begin{tabular}{|c|c|c|c|}
\hline $\mathrm{Na}$ & Primary Malignancy & New Malignancy & Months till New Malignancy \\
\hline 1 & CML: $\mathrm{Ph}+; \mathrm{BCR} / \mathrm{ABL}$ Unknown & AML & 9.5 \\
\hline 2 & MDS: unspecified & Lymphoma & 313 \\
\hline 3 & AML: M4, myelomonocytic & Lymphoma & 9.3 \\
\hline 4 & NHL: Burkitt lymphoma/eukemia & Lymphoma & 1.3 \\
\hline 5 & HD. Hodgkin disease & MDS & 48.9 \\
\hline 6 & AML: MS & Lymphangio sarcoma & 14.8 \\
\hline 7 & SAA: idiopathic & Colon & 4.1 \\
\hline 8 & CML: $\mathrm{Ph}+, \mathrm{BCR} / \mathrm{ABL}+$ & AML. & 8.7 \\
\hline 9 & NHL. folhcular, small deaved & AML. & 6.7 \\
\hline 10 & CML: Ph +, BCR/ABL Unknown & ALL(B-cell) & 5.1 \\
\hline 11 & AML: M2, myelocytic & Genitourinary Małignancy & 18.1 \\
\hline
\end{tabular}

\section{P433}

Haploidentical stem cell transplantation by in vivo T-cell depletion: Iranian experience

M.R. Ostadali, M. Vahidfar, K. Alimoghaddam, A.A. Hamidieh, M. Iravani, S.A. Mousavi, B. Bahar, M. Jahani, A. Khodabandeh, A. Shamshiri, M. Jalili, A. Arjmand, B. Chahardouli, A.S. Mousavi, S. Basirpanah, S. Khalilvand, Z. Shahriari, A. Ghavamzadeh Hematology, Oncology, and Stem Cell Transplantation (Tehran, IR)

Introduction: Hematopoietic stem cell transplantation is the only available therapeutic intervention for many congenital and acquired diseases.

For Iranian patients, best available donor sources are full matched relatives (including siblings, parents, and other relatives), one locus mismatched relatives, and unrelated cord blood from Iranian Cord Blood Bank.

Many patients have no suitable donor from above mentioned sources and have no availability to abroad stem cell sources. Besides, unrelated donor search, confirmatory typing, and collection and delivery of cells as a whole is a time consuming process and for many patients, we have no enough time; haploidentical stem cell transplantation could be a life saving option in such cases.

Patients and methods: From 2007 to 2009, we performed 19 haploidentical transplantations by in vivo T cell depletion. Patients were high risk acute leukemia (14), Osteopetrosis, (2), type VI Mucopolysaccharidosis (MPS) (1), Leucocyte Adhesion Deficiency (LAD) (1) and Severe Combined Immune Deficiency (SCID) (1)

Conditioning regimen consisted of standard Busulfan/Cyclophosphamide plus CAMPATH (4 patients) or ATG (14 patients). SCID and LAD patients received Fludarabine, Melphalan, and ATG.

GVHD prophylaxis regimen consisted of Cyclosporin A plus Methotrexate.

Target cell dose was $6-10 \times 10^{8}$ G-CSF primed bone marrow and/or peripheral blood mononuclear cells.

The donors were non inherited maternal antigen (NIMA) mismatched group siblings, mothers, daughter, or fathers.

Results: Six months and one year overall survival was $42 \%$ and $33 \%$ respectively. (Follow up: $20-1101$ days median $=66$ ).
Eight cases of mortalities were transplantation related (TRM) $(42.1 \%)$. Causes of TRM were acute graft versus host disease, (aGVHD) (2 of 8), infection (5 of 8) and veno-occlusive disease (VOD) (1 of 8).

Seven cases experienced grades II-IV acute GVHD (36.84\%). Grades III and IV aGVHD occurred in two cases each.

Conclusion: Hematopoietic stem cell transplantation is a feasible and available therapeutic option for patients that have absolute need for allogeneic stem transplantation and have no available full matched donor.

\section{P434}

Hepcidin synthesis and upregulation in AML patients with iron overload undergoing allogeneic haematopoeitic cell transplantation

H.K. Al-Ali (1), M. Westerman (2), R. Krahl (1), A.K. Eisfeld (1), S. Leiblein (1), U.G. Liebert (1), M. Hehme (3), D. Niederwieser (1) (1)University of Leipzig (Leipzig, DE); (2)LifeSciences LLC (La Jolla, US); (3)Novartis Oncology (Nuremberg, DE)

Hepcidin (hep) is the key regulator of iron homeostasis. As its transcription is normally upregulated by iron and transfusionrelated iron overload is frequent in AML patients (pts) undergoing HCT we analysed the role of chemotherapy, conditioning regimen, liver and renal function and Graft versus Host Disease (GvHD) on hep expression in AML pts undergoing HCT. Also the correlation of hep with blood transfusions (BT), body iron and anemia was studied.

Patients and methods: 42 consecutive pts (23 male/19 female, median age 57 years) with $A M L$ who underwent HCT from February, 2008-February, 2009 at the University of Leipzig were included. Each patient was assessed 10 days prior to and 3 months after HCT. Donors were matched related in $8(19 \%)$ and matched unrelated (MUD) in $34(81 \%)$ pts. Preparative regimen consisted of 12 Gy TBI/cyclophosphamid $120 \mathrm{mg} / \mathrm{kg}$ in $13(31 \%)$ and fludarabin $30 \mathrm{mg} / \mathrm{m}^{2} /$ day for 3 days/2 Gy TBI) in $29(69 \%)$ pts. Acute GvHD > grade II was present in $13(31 \%)$ and chronic GvHD in $17(40 \%)$ pts. Body iron was assessed by serum ferritin (SF) (normal $<400 \mathrm{ng} / \mathrm{mL}$ ). Serum hep was measured by C-ELISA at Intrinsic LifeSciences LLC, La Jolla, CA. (normal: male 29254, female: $17-286 \mathrm{ng} / \mathrm{mL}$ ).

Results: Iron overload was present in all pts prior to HCT with a median SF of 1945 (range: 617-6981)ng/mL after a median of 22 units BT. SF prior to HCT correlated with limited $(P=0.06)$ and extensive chronic GvHD $(P=0.003)$. Median hep was much higher in pts both prior to and after HCT [median 398 (range: $172-941) \mathrm{ng} / \mathrm{ml}$ compared to normal values $(P<0.0001)$. Age and gender had no influence on hep. Also, prior chemotherapies, conditioning regimen, liver and renal function or antifungal-treatment had no impact on hep levels. Hep significantly correlated with BT $(P=0.001)$ and inversely with the degree of anemia $(P=0.002)$ but not with SF values. Hep levels prior to or after HCT did not correlate with acute or chronic GvHD.

Conclusions: The highly elevated hep in iron overloaded AML pts undergoing HCT suggests that despite intensive chemotherapy and HCT hepcidin synthesis and upregulation remain intact. Hep normally leads to a reduction of extracellular serum iron, this may be why serum hep correlates with blood transfusions but not with serum ferritin. Hep might have an important protective role in the setting of HCT as it might prevent an increased iron export from macrophages thereby reducing the parenchymal iron loading and damage. 
P435

Clinical significance of preformed antibodies against HLA and MICA in recipients of unrelated bone marrow transplantation: a case-control study

T. Ichinohe (1), J. Kanda (1), E. Maruya (2), F. Ishimaru (3), Y. Hirata (3), K. Matsuo (4), T. Kawase (4), Y. Morishima (4), H. Saji (2)

(1)Kyoto University (Kyoto, JP); (2)HLA Laboratory (Kyoto, JP); (3)Okayama Red Cross Blood Center (Okayama, JP); (4)Aichi Cancer Center (Nagoya, JP)

Background: Donor-directed HLA antibodies (Abs) in recipients are an emerging barrier that may compromise engraftment after allogeneic hematopoietic cell transplantation (HCT). In solid organ transplants, Abs against MHC class I-related chain A (MICA) have been also reported to adversely affect the risk of graft loss, although their impact on HCT outcomes is elusive. Patients and methods: We performed a nested case-control study to evaluate the role of preformed recipient Abs against HLA and MICA on graft failure (GF) after HCT within cohorts of patients who received unrelated bone marrow (BM) transplantation facilitated by the Japan Marrow Donor Program. Data and stored pretransplant plasma samples were obtained from 79 cases with GF and 229 controls pairs matched for recipient age, diagnosis, HLA compatibility, and infused cell dose. The sample size was defined to detect odds ratio (OR) 5.00 with $88 \%$ statistical power. GF was defined on the basis of survival beyond 21 days after HCT without evidence of sustained engraftment until day 100 . Donor-recipient pairs were retrospectively typed for HLA-A, -B, -C, -DRB1, -DQB1, -DPB1 at the 4 digit level. The presence of Abs against HLA class I, class $\mathrm{II}$, and MICA were evaluated by luminex assays using single antigen-coated microparticles. ORs for GF and 95\% confidence intervals (Cls) were estimated with conditional logistic regression models.

Results: HLA Abs and MICA Abs were detected in $14(18 \%)$ and $18(23 \%)$ cases with GF, respectively, while $45(20 \%)$ and $49(21 \%)$ of controls. Donor-directed HLA Abs were found in 1 patient of the control group but not in the GF group. Among 59 patients with HLA Abs, 55 had Abs directed against HLA class I and 14 had Abs against HLA class II, although anti-DP Abs were not detected. The risk of GF was neither affected by preexisting HLA Abs [OR $(95 \% \mathrm{Cl})$ for class I Abs, 1.03 (0.532.02), $P=0.93$; class II Abs, $0.46(0.10-2.14), P=0.32]$ nor by MICA Abs [OR $(95 \% \mathrm{Cl}), 1.10(0.58-2.08), P=0.78]$. Patients with HLAAbs among the GF group had a lower survival as compared to those without HLA Abs $(P=0.063)$.

Conclusions: In our cohort of unrelated BM recipients, adverse impact of preexisting HLA Abs and MICA Abs on engraftment was not identified. However, since nearly all the detected HLA Abs in our study were not directed against donor antigens, future studies are warranted to clarify the role of preexisting recipient Abs reactive with donor-specific HLA or MICA in various HCT settings.

\section{P436}

Clinical significance of haemophagocytosis in bone marrow clot sections during peri-engraftment period following allogeneic haematopoietic stem cell transplantation

N. Imahashi (1), M. Ito (1), D. Koyama (1), K. Onodera (1), T. Goto (1), A. Seto (1), K. Watanabe (1), S. Tsukamoto (1), T. Yasuda (2), Y. Inamoto (2), Y. Ozawa (1), K. Miyamura (1) (1)Japanese Red Cross Nagoya First Hospital (Nagoya, JP); (2)Nagoya University Graduate School of Medicine (Nagoya, JP)

The effect of macrophage activation on outcomes of allogeneic hematopoietic stem cell transplantation (allo-HSCT) in the clinical setting has yet to be fully examined. We assessed hemophagocytosis in bone marrow clot sections on day $+14 \pm$ 7 and analyzed its impact on subsequent outcomes. We studied 70 adult patients who received their first allo-HSCT at our institution. Twenty patients were diagnosed as having hemophagocytosis (HP group), while 50 were not (non-HP group). There were no significant differences in patient characteristics between the two groups. Neurological symptoms, weight gain, hypoxia, jaundice, and elevated serum creatinine were more frequently observed among the HP group than the non-HP group, with fever and elevated AST tending to be more frequent in the HP group than in the non-HP group. The incidence of graft failure was higher in the HP group than in the non-HP group $(30 \%$ vs. $0 \%, P<0.01)$ and hematopoietic recovery was slower in the HP group than in the non-HP group $(P<0.01)$. The incidence of engraftment syndrome and graft-versus-host disease did not differ between the two groups. The 2-year overall survival (OS) for the HP group and the non-HP group was $30 \%$ and $63 \%(P<0.01)$, respectively, and 2-year nonrelapse mortality (NRM) was $54 \%$ and $31 \%(P<0.01)$, respectively. Multivariate analysis confirmed that the HP group was associated with a lower OS (hazard ratio (HR), 2.3; 95\% confidence interval $(\mathrm{Cl})$, $1.1-4.9 ; P=0.03)$ and higher NRM (HR, 3.5; 95\% Cl, 1.3-9.0; $P=0.01$ ), whereas it was not associated with a relapse rate. The HP group had higher incidence of death due to graft failure $(P<0.01)$ and endothelial complications such as sinusoidal obstruction syndrome and transplant associated microangiopathy $(P<0.01)$. In conclusion, the early macrophage activation is an important complication with a significant impact on outcomes of allo-HSCT.

\section{P437}

Status epilepticus as a main manifestation of posterior reversible encephalopathy syndrome after allogeneic haematopoietic stem cell transplantation in children D.M. Cordelli, R. Masetti, A. Prete, R. Rondelli, V. Gentile, E. Franzoni, A. Pession

Sant'Orsola Malpighi Hospital (Bologna, IT)

Introduction: Posterior reversible encephalopathy syndrome (PRES) is a clinico-neuroradiologic entity represented by symptoms like headache, seizures, visual disturbances and an imaging pattern showing cortical and subcortical edema primarily involving posterior regions of the brain. PRES is a possible complication in allogeneic hematopoietic stem cell transplantation (allo-HSCT) patients (Pts) treated with immunosuppressive agents. Status Epilepticus (SE) is defined as seizure activity lasting 30 minutes or more. Although seizures are a well known manifestation of children with PRES, SE has rarely been described in this setting.

Patients and methods: We reviewed 13 episodes of SE in 10 Pts receiving allo-HSCT aged 1 to 17 years (Table 1) with MRI confirmed diagnosis of PRES. We evaluated data on demographics, etiology of PRES, clinical presentation, seizure semeiology, EEG studies, response to antiepileptic drugs, neuroimaging and outcome.

Results: Pts developed PRES with a median time of onset after allo-HSCT of 58 days (range 5-116). In 8/10 Pts SE was the presenting symptom of PRES. Three Pts had two SE during the course of the disease. Nonconvulsive SE involving posterior regions of the brain (confirmed by EEG monitoring) was observed in 4 cases; convulsive SE was observed in 9 cases. Gaze deviation, oculoclonic movements, nystagmus and altered mental status were the main clinical signs during nonconvulsive SE and preceded convulsive SE in $5 / 9$ cases. SE was responsive to first line drugs (midazolam and/or phenytoin) in $12 / 13$ events but $7 / 10$ Pts needed intensive care admission. Early EEG abnormalities after resolution of SE were prevalent in posterior derivations and consisted in periodic epileptiform discharges (PLEDs) in 8 cases and focal slowing in 5. All Pts showed clinical, EEG and neuroimaging improvement after SE and withdrawal of causative agent.

Conclusions: SE is probably an under-recognized event in alloHSCT children with PRES. In most of our Pts, SE was the first sign of PRES and the onset of the SE was characterized by 


\begin{tabular}{|c|c|c|c|c|c|c|c|c|c|c|}
\hline $\begin{array}{l}\text { Pat. } \\
\text { No. }\end{array}$ & $\begin{array}{l}\text { A } \\
\text { g } \\
\text { e }\end{array}$ & $\begin{array}{l}5 \\
\text { e } \\
\mathrm{x}\end{array}$ & Disease & $\begin{array}{l}\text { Chemo } \\
\text { therapy } \\
\text { before } \\
\text { HSCT }\end{array}$ & $\begin{array}{c}\text { Radiation } \\
\text { therapy } \\
\text { before } \\
\text { HSCT }\end{array}$ & $\begin{array}{c}\text { CNS } \\
\text { involvement }\end{array}$ & $\begin{array}{c}\text { Intrathecal } \\
\text { therapy } \\
\text { before } \\
\text { HSCT }\end{array}$ & $\begin{array}{l}\text { Conditioning } \\
\text { regimen }\end{array}$ & Donor & $\begin{array}{l}\text { GVHD } \\
\text { Profilaxis }\end{array}$ \\
\hline 1 & 1 & $\mathrm{M}$ & $\mathrm{HLH}$ & NO & No & No & No & BU-THIO-FLU & MUD & CSA + ATG \\
\hline 2 & 3 & $\mathrm{M}$ & AML & YES & No & NO & YES & BU-CY-L-PAM & MUD & CSA+ATG $\mathrm{MTX}$ \\
\hline 3 & 5 & $\mathrm{M}$ & BThalassemia & No & No & NO & No & BU-THIO-FLU & MFD & CSA+ATG \\
\hline 4 & 5 & M & $\begin{array}{l}\text { Sickle Cell } \\
\text { Disease }\end{array}$ & No & No & No & No & $\begin{array}{l}\text { TREO-THIO- } \\
\text { FLU }\end{array}$ & MFD & CSA \\
\hline 5 & 7 & $\mathrm{M}$ & AML & YES & No & No & YES & BU-CY-L-PAM & MUD & CSA + ATG + MTX \\
\hline 6 & 7 & $\mathrm{M}$ & AML & YES & No & NO & YES & BU-CY-L-PAM & MUD & $\mathrm{CSA}+\mathrm{ATG}+\mathrm{MTX}$ \\
\hline 7 & 11 & $\mathrm{M}$ & $\begin{array}{c}\text { Blackfan- } \\
\text { Diamond } \\
\text { Anemia }\end{array}$ & No & No & No & No & $\begin{array}{l}\text { TREO-THIO- } \\
\text { FLU }\end{array}$ & MFD & $\mathrm{CSA}+\mathrm{MTX}$ \\
\hline 8 & 12 & $\mathrm{M}$ & AML & YES & No & No & YES & BU-CY-L-PAM & MUD & $\mathrm{CSA}+\mathrm{ATG}+\mathrm{MTX}$ \\
\hline 9 & 16 & F & BThalassemia & No & No & NO & No & $\begin{array}{l}\text { TREO-THIO- } \\
\text { FLU }\end{array}$ & MUD & $\mathrm{CSA}+\mathrm{ATG}+\mathrm{MTX}$ \\
\hline 10 & 17 & $\mathrm{M}$ & ALL & YES & No & NO & YES & BU-CY-L-PAM & MUD & $\mathrm{CSA}+\mathrm{ATG}+\mathrm{MTX}$ \\
\hline \multicolumn{11}{|c|}{$\begin{array}{l}\text { HLH: Hemophagocytic Llymphohistiocytosis, AML: Acute Myeloid Leukemia, ALL: Acute Llymphoblastic Leukemia, } \\
\text { BU: Busulphan,. THIO: Thiotepa, L-PAM: Melphalan, FLU: Ffludarabine, TREO: Treosulphan, } \\
\text { MUD: Matched Unrelated Donor. MFD: Matched Family Donor } \\
\text { CNS: Central Nervous System } \\
\text { GVHD Graft Versus Host Disease } \\
\text { CSA: Cyclosporine, ATG : Antithymocyte globulin, MTX: Methotrexate }\end{array}$} \\
\hline
\end{tabular}

subtle non-convulsive signs involving visual system and mental status. Looking for these suggestive signs may allow prompt recognition and therapy of both PRES and SE. Moreover, our experience confirms that EEG monitoring is a fundamental tool to properly diagnose and treat nonconvulsive SE. An high index of suspicion is required to reduce the high morbidity due to this severe complication in allo-HSCT children.

\section{P438}

Bronchiolitis obliterans after allogeneic haematopoietic cell transplantation: inpredictable and fatal P. Topcuoglu, O.O. Kumbasar, O. Arslan, M. Ozcan, O. Ilhan, H. Akan, M. Beksac, N. Konuk, G. Gurman, M. Arat Ankara University (Ankara, TR)

Bronchiolitis obliterans (BO) is a progressive non-infectious late onset pulmonary complication after lung or allogeneic hematopoetic cell transplantation (allo-HCT). It is associated with chronic graft versus host disease $(\mathrm{GvHH})$ and is frequently resulting in mortality.

We aimed to retrospectively investigate the incidence and outcome of $\mathrm{BO}$ among adult allo-HCT recipients and also determinate the risk factors associated with $\mathrm{BO}$ related survival. We analyzed 366 patients who survived more than 3 months among 439 who received allo-sibling HCT between January 1998 and June 2008. BO was diagnosed in 31 patients (8.4\%) at a median of 9.9 months (range, 1.1-37.5) using NIH criteria. Median age was 34 years and M/F was $17 / 14$. The patients' diagnoses were $15 \mathrm{CML}, 14$ acute leukemia (AML: 11/ALL: 3 ), one severe aplastic anemia and one Hodgkin's Lymphoma. The majority of the patients received peripheral blood stem cells (26/31) and myeloablative conditioning regimen (25/31). Acute GvHD (Gr I-III) was observed in $87.7 \%$. All but one patient had chronic GvHD at the time of BO diagnosis. Pretransplant spirometry and lung diffusion capacity (DLCO) were available for 26/31 and 24/31 BO patients, respectively. Median pretransplant predicted FEV1 was 95.5\% (range, 59-122) and median FEV1/FVC ratio was $85 \%$ (range, 69-105). Median predicted DLCO was 69\% (range, 43-113). Pretransplant smoking history was $45.2 \%$ in BO patients The median time interval between the detection of chronic GvHD and the development of BO was 3.5 months (range, 0-33.7). In our cross sectional analysis 12 patients $(38.7 \%$ ) died of pulmonary insufficiency due to progressive $\mathrm{BO}$ and only one of relapse/progression of primary hematological malignancy. The probability of 5 -year overall survival was $63.3 \% \pm 8.85 \%$ for $\mathrm{BO}$ and $76.8 \% \pm 2.9$ for patients without BO $(P=0.071)$. In univariate analysis, we found that pretransplant smoking history, male patients and female to male donor-recipient direction shortened overall survival in $\mathrm{BO}$ patients (Table). In conclusion, BO is one of the major causes of late onset non-relapse morbidity and mortality after allo-HCT. Pretransplant pulmonary function test and diffusion capacity of lung are not predictive both for the development and outcome of BO. Male gender, female donor and pretransplant smoking history had negative effect on overall survival in our BO cohort.

Table: The statistic al anabys is of pretransplant and transplant-related variables on overall sunvival

\begin{tabular}{|c|c|c|c|}
\hline \multirow[b]{2}{*}{$\begin{array}{l}\text { Pretrunsp smoking history }(+\%), \% \\
\text { Medim }\end{array}$} & \multicolumn{2}{|c|}{$\begin{array}{l}\text { Overall survival }(\% \pm \text { SD } 1) \\
\text { Medin months }\end{array}$} & \multirow{2}{*}{\begin{tabular}{|l} 
P \\
$0.0262^{*}$
\end{tabular}} \\
\hline & $\begin{array}{l}4898 \pm 13.62 \% \\
2880 \text { months }\end{array}$ & $\begin{array}{l}75.63 \pm 10.63 \% \\
\text { Not reached }\end{array}$ & \\
\hline $\begin{array}{l}\text { Pretransplart FEV 1/FVC } \\
\Leftrightarrow \approx 0 \text { vs }>80\end{array}$ & $\begin{array}{l}75.0 \% \pm 153 \% \\
77.13 \text { months }\end{array}$ & $\begin{array}{l}72.7 \% \pm 11.6 \% \\
\text { Not re ached }\end{array}$ & 0.502 \\
\hline $\begin{array}{l}\text { Pretransplart DLC0 } \\
<=70 \mathrm{v}>70\end{array}$ & $\begin{array}{l}71.4 \% \pm 12.1 \% \\
77.13 \text { morths }\end{array}$ & $\begin{array}{l}68.6 \% \pm 15.1 \% \\
\text { Not re ached }\end{array}$ & 0.55 \\
\hline $\begin{array}{l}\text { Age } \\
<=35,>35 y s\end{array}$ & $\begin{array}{l}500 \% \pm 125 \% \\
3894 \text { months }\end{array}$ & $\begin{array}{l}78.97 \% \pm 1081 \\
\text { Not re ached }\end{array}$ & 0.088 \\
\hline Male gender $(\%)$ & $\begin{array}{l}463 \% \pm 123 \% \\
3893 \text { months }\end{array}$ & $\begin{array}{l}85.1 \pm 9.7 \\
\text { Not re ached }\end{array}$ & $0.0054 *$ \\
\hline $\begin{array}{l}F \rightarrow M(n=9) \text { ws other (22) } \\
\text { Median }\end{array}$ & $\begin{array}{l}16.7 \% \pm 10.79 \% \\
27.60 \text { morths }\end{array}$ & $\begin{array}{l}69.0 \pm 14.2 \\
\text { Not re ached }\end{array}$ & $0.018^{*}$ \\
\hline Stem cell source, DB vs BM & $\begin{array}{l}599 \% \pm 99 \% 77.13 \\
77.13 \text { morths }\end{array}$ & $\begin{array}{l}80.0 \% \pm 179 \% \\
\text { Not re ached }\end{array}$ & 0.227 \\
\hline $\begin{array}{l}\text { Onset of BO, early (<1years)vs } \\
\text { late }\end{array}$ & $\begin{array}{l}4922 \% \pm 12.66 \\
3893 \text { morths }\end{array}$ & \begin{tabular}{|l|}
$78.97 \pm 10.81$ \\
Not reached
\end{tabular} & 0.0796 \\
\hline
\end{tabular}

\section{P439}

Imatinib mesylate in T-cell large granular lymphocyte leukaemia associated to chronic graft-versus-host disease

R. Fedele, M. Cuzzola, A. Dattola, E. Massara, T. Moscato, G. Console, C. Garreffa, C. Rigolino, M. Cannatà, G. Irrera, G. Messina, G. Pucci, M. Martino, P. lacopino Azienda Ospedaliera B.M.M. (Reggio Calabria, IT)

Recently, Yang and colleagues1 demonstrated increased expression of PDGF-B gene transcripts and PDGF-BB protein in leukemic cells, suggesting that targeting of downstream components of PDGF signalling may be a potential therapy of T-large granular lymphocyte (T-LGL) leukemia.

We report the first two cases of T-LGL leukemia successfully treated with imatinib mesylate (IM), a compound that inhibits PDGFR kinases. The patients (male, 39 and 57-year old) underwent allo-HSCT from HLA-matched sibling donor for acute myeloid leukemia. After transplant, both the patients suffered from sclerodermatous cGVHD associated with an expanded clonal population of CD3+/CD4-/CD8+/CD57+ 
T-cells of donor origin. Clonality was assessed by analyzing the rearrangement of the T-cell receptor (TCR) gamma gene (region VJ-A). Constitutive phosphorylation of ERK1/2 kinases in peripheral blood mononuclear cell lysate was found. The expression profiles of genes implicated in apoptosis, including BCL2A1, CASP1, CASP6, EGR1, EGR2, FAS, FOS, TNF, TNFRSF1A, were dysregulated.

Because of their cGVHD, the patients received IM according to the trial "Imatinib Mesylate in the treatment of refractory extensive cGvHD with features Scleroderma-like" (Eudract no. 2007-001508-19). During IM treatment, both patients displayed improvement of cutaneous sclerosis, clearance of T-LGL clonotype, ERK inactivation and modulation of genes involving in cell survival. After IM discontinuation, one patient maintained clinical and biological benefit for 4 months (time of last follow-up), while the other one quickly experienced a worsening of cGVHD with T-LGL re-expansion associated with monoclonality of TCR, ERK-autophosphorylation, and deregulation of pro and anti-apoptotic gene transcripts. This second patient restarted IM obtaining clinical and laboratory results similar to those observed after the first course of the drug (Figure 1).

We conclude that, antagonizing PDGF pathway, IM is an effective treatment for T-LGL leukemia that complicates allo-SCT, particularly in patients affected by cGVHD. Since there are no effective therapies for aggressive forms of T-LGL leukemia, we think that further studies need to be performed about the effect of IM in these clinical conditions.

Reference: 1) Yang J, Liu X, Nyland SB, et al. Platelet-derived growth factor mediates survival of leukemic large granular lymphocytes via an autocrine regulatory pathway. Blood. Prepublished online Oct 30, 2009. doi:10.1182/blood-2009-06223719.

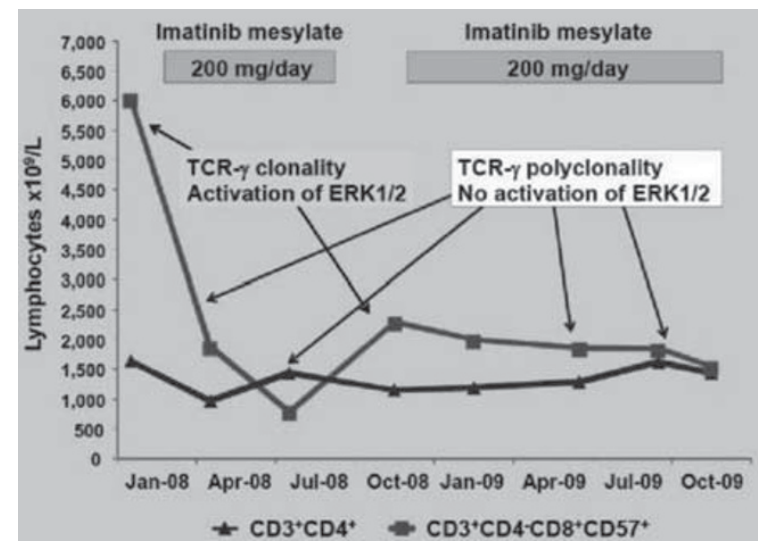

\section{P440}

Early predictor for Bronchiolitis obliterans after allogeneic haematopoietic stem cell transplantation

M. Khalid, M. Aljurf, B. Khan, A. Almobeireek, F. Al-Sharif, M. Zitouni, H. Al-Zahrani, S. Mohamed, W. Rasheed, M. Bakr, S. Saleemi, N. Alezaimi, F. Al-Mohareb, N. Chaudhri

King Faisal Specialist Hospital (Riyadh, SA)

Introduction: Bronchiolitis obliterans (BO) is the most common late non-infectious pulmonary complication after allogeneic haematopoietic stem cell transplantation (HSCT). It is usually part of systemic manifestation of chronic graft-versus-hostdisease (GVHD) but may also be an isolated pulmonary complication. Early detection of airway obstruction on pulmonary function test (PFT) may predict the development of BO and help in early management to prevent development of advanced BO syndrome with respiratory failure.

Objectives: To identify potential early predictors for the development of obstructive airway disease (OAD) in post HSCT patients.
Materials and method: This is a retrospective study covering 10 years (January 1995 to December 2005) involving patients aged 14 years who underwent allogeneic HSCT. Pre and post HSCT PFTs were analyzed. Standard American Thoracic Society (ATS) Guidelines for changes in PFTs parameters were used to indicate significant changes.

Results: Of 580 transplants performed during the study period. 49 patients had serial PFTs were studied to identify potential early predictors for development of OAD post HSCT.

Early in the post HSCT, no significant changes occurred in PFT parameters (FEV1, MEF 25-75, RV, and TLC). However RV/ TLC ratio was noted to be significantly increased in the 1 st post HSCT PFT assessment (mean increase in value was $10.88 \%$ ) Table (1). Subsequent statistical analysis of PFTs suggested that patients with abnormal RV/TLC ratio on their first post HSCT assessment developed a progressive decline in other parameters of OAD like decreased MEF, decreased FEV1, and increased TLC in addition to further deterioration of RV/TLC ratio.

Conclusion: Our study identified an early objective sign of developing $\mathrm{BO}$ in the form of airflow limitation with increase RV/TLC ratio. Conventionally acceptable changes of OAD like decreased FEV1, decreased MEF and increased TLC took much longer time to occur.

Prospective studies are required to evaluate this further and to study whether early therapeutic intervention at this stage will have any impact on the outcome of patients who develop BO post HSCT.

Table (1) SIgnificance between Pre and Post Transplant Measurements:

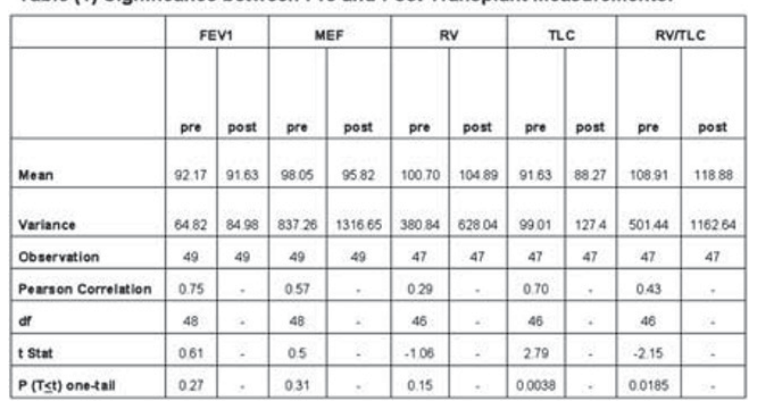

P441

Clinical presentations, treatment and long-term outcome of isolated extramedullary relapses after allogeneic haematopoietic stem cell transplantation for leukaemias: single-centre experience with $\mathbf{5 3 2}$ patients

D. Kata, T. Czerw, G. Helbig, S. Kyrcz-Krzemien

Silesian Medical University (Katowice, PL)

Introduction: Isolated extramedullary relapses (IER) are increasingly reported as late complications after allogeneic hematopoietic stem cell transplantation (alloHSCT) for leukemias. However, the optimal treatment for IER following alloHSCT remains uncertain.

Objectives and methods: We retrospectively analysed incidence, treatment options and long-term outcome of this mode of leukemia recurrence in a cohort of 532 consecutive pts (123 with ALL, 201 with AML, 208 with CML) who underwent alloHSCT in our institution between June 1993 and December 2007. 79 pts ( 34 with ALL, 28 with AML, 17 with CML) relapsed (any site).

Results: $9(11 \%)$ out of all pts who relapsed (4 with B-line ALL, 4 with AML, 1 with CML, F/M 4/5, median age 29 years, range 28-44 years) developed IER after a median time of 17 months (mts) (range, 8-80 $\mathrm{mts}$ ) following alloHSCT. We revealed complete donor chimerism in $7 / 9$ studied pts. 4 pts (3 with ALL, 1 with $A M L$ ) developed skin and/or subcutaneous tissue infiltrates; in one of them ( patient with ALL) leukemic tumor of the peritibial soft tissues was additionally observed. Other 
sites of IER included (No. of cases/diagnosis): leptomeninges of the brain (1/Ph+ALL), paraspinal soft tissues (1/AML), small intestine and the root of mesentery (1/AML), inguinal lymph nodes (1/AML), paranasal sinuses (1/AML). Treatment plans for those IER included (No. of cases/diagnosis): $1 /$ involved-field radiotherapy (IF-RT) followed by chemotherapy (CHT) and interferon-alpha (2/ALL), 2/imatinib + cHT + steroids and methotrexate intrathecally $(1 / \mathrm{Ph}+\mathrm{ALL}), 3 / \mathrm{imatinib}+\mathrm{CHT}$ (1/Ph+ALL), 4/CHT (2/AML), 5/dasatinib (1/CD117+AML,), 6/surgery (1/AML), 7/surgery + IF-RT (1/CML). 7/9 patients died after a median time of $10 \mathrm{mts}$ (range, 1-30) due to resistant systemic relapse and/or infectious complications, 2/9 are currently under $\mathrm{CHT}$.

Conclusions. Our data indicate that isolated extramedullary disease following allo-HSCT occurs predominantly in acute leukemia pts, being rarely observed in pts with CML. Sites of extramedullary infiltrates vary widely among the pts with skin and/or subcutaneous tissue being frequently involved. Local radiation therapy seems to be effective treatment option, but it does not prevent from systemic relapse and should be followed by other therapeutic modalities. According to our experience occurrence of IER following alloHSCT is associated with poor prognosis and the optimal therapy remains a challenge.

\section{P442}

Cardiac toxicity in patients over the age of 60 undergoing autologous stem cell transplantation for lymphoma

L. Lee (1), S.O. Ahmed (1), A. Symeonidis (2), D.C Linch (3), A. Goldstone (3), R. Chakraverty (1), S. Mackinnon (1), P.D. Kottaridis (1)

(1)Royal Free Hospital (London, UK); (2)University of Patra (Patra, GR); (3)University College London (London, UK)

Introduction: Autologous stem cell transplantation (ASCT) has been shown to improve survival in patients with lymphoma. ASCT is generally well tolerated with a treatment related mortality of under $5-10 \%$. Complications are more likely in the elderly patients or in those with comorbidities. We aimed to investigate the incidence of early cardiac complications (ECC) in patients $>60$ undergoing ASCT for Hodgkin's (HL) and Non-Hodgkins lymphoma (NHL).

Methods: We retrospectively analysed records of patients $>60$ who underwent ASCT with a BCNU/ Lamustine + etoposide + ara-c + melphalan/cyclophosphamide (BEAM, LEAM or BEAC) based regimen in 2 centres between 1982 and 2009.

Results: We analysed a total of 76 patients $51(67 \%)$ males, $25(33 \%)$ females aged 60-69 (median 64) transplanted for HL 6(7.9\%), NHL 70(92.1\%), T cell 5(6.6\%), high grade B cell, including transformation from low grade NHL $40(52.7 \%)$, follicular $14(18.4 \%)$, mantle cell $9(11.8 \%)$, lymphoblastic $2(2.6 \%)$. Seventy two $(94.8 \%)$ of patients had BEAM conditioning, $3(3.9 \%)$ LEAM and $1(1.3 \%)$ BEAC. Past medical history (PMH) was available in $37(48.7 \%)$. The median left ventricular ejection fraction (LVEF) was 59\% (38-75\%) and $5(6.5 \%)$ patients had a history of cardiac disease. Patients received a median of 2 (1-4) prior lines of chemotherapy and 35/37 (94.5\%) of patients had had an anthracycline pre-ASCT. Overall $13(17.1 \%)$ had ECC post-ASCT: 5 Atrial fibrillation (AF) and atrial flutter, $1 \mathrm{AF}$ and congestive cardiac failure (CCF), $1 \mathrm{AF}$ and pericardial effusion, 3 supraventricular tachycardia, 1 sinus bradycardia, 1 ventricular arrhythmia (VA), 1 pericardial effusion. Four cases of arrhythmia coincided with sepsis and 1 case of VA was related to $\mathrm{HL}$ insertion. Overall, $15.8 \%$ experienced arrhythmias, $2.6 \%$ pericardial effusion, $1.3 \% \mathrm{CCF}$. One patient required intensive care admission but none of these ECC resulted in death.

$3 / 5(60 \%)$ of patients with a documented cardiac $\mathrm{PMH}$, vs. $5 / 32(15.6 \%)$ without had ECC post transplant $(P=0.056)$. There was no difference in pre ASCT LVEF in those who developed ECC compared to those who didn't $(P=0.80)$.

Conclusion: We report a rate of ECC of $17.1 \%$ in patients aged over 60 post ASCT for lymphoma. The most common ECC was $\mathrm{AF}$; none of the ECC were fatal. A prior history of cardiac dis- ease was associated with an increased risk of ECC but ejection fraction was not. Physicians need to be aware of the risk of ECC in this setting especially in patients with a prior history of cardiac disease.

\section{P443}

Androgen deficency in women after allogeneic stem cell transplantation

Y. Björk (1), C. Ankarberg (2), E. Smith Knutsson (3), A. Broman (3), K. Bergmark (4), E. Wahlström (1), M. Brune (1)

(1)Dpt of Hematology (Gothenburg, SE); (2)Dpt of Pediatrics (Gothenburg, SE); (3)Northern Älvsborg Hospital (Trollhättan, SE); (4)Dpt of Oncology (Gothenburg, SE)

Introduction: Low androgen hormone production in females may contribute to sexual dysfunction and reduced quality of life (QoL) after allo-SCT. This study compares systemic levels of sex steroids and sex hormone-binding globuline (SHBG) in allografted women and healthy controls.

Patients and methods: Twenty-five women in complete remission 52 (3-149) months after allo-SCT for acute and chronic leukemias $(n=22)$ or other. Glucocorticoid treatment of chronic GvHD was ongoing in 9 pts, and 10 pts were on systemic hormone replacement therapy (HRT). Healthy women $(n=26)$, 3 of them being on HRT, served as controls. In both groups, HRT was stopped $24 \mathrm{~h}$ before blood sampling. Serum testosterone concentrations were determined using a modified RIA with a sensitivity of $0.03 \mathrm{nM}$. Free testosterone was calculated from the serum concentrations of testosterone, albumin and SHBG. The analyses were performed in the entire groups of pts (SCT group) and controls, and also in two age cohorts: <50yrs $(n=12+12)$, and $\geq 50 \mathrm{yrs} \quad(n=13+14)$. For comparisons Wilcoxon's rank-sum test was applied.

Results: The SCT group and controls were excellently matched for age (median 51 vs. $52 \mathrm{yrs}$ ), weight (67 vs. $65 \mathrm{~kg}$ ), and salbumin (40 vs. $41 \mathrm{~g} / \mathrm{L})$. Compared to findings in the control group, free testosterone was significantly lower in SCT pts $<50$ yrs (median 3.0 vs. $7.2 \mathrm{pM} ; P=0.03$ ), and also in pts $\geq 50$ yrs ( 3.1 vs. $8.2 ; P=0.008$ ). Dehydroepiandrosterone sulfate (DHEAS) was lower in the entire group of SCT pts (median 0.7 vs. $2.5 \mathrm{uM}$; $P=0.0007$ ), the difference versus controls was significant also in pts $<50$ years $(P=0.006)$, but not in pts $\geq 50$ yrs $(P=0.08)$. Oestradiol was significantly lower in SCT pts $<50$ yrs (median 50 vs. $328 \mathrm{pM} ; P=0.002$ ). Levels of SHBG in the $<50$ yrs cohort were lower (median 55 vs. $85 \mathrm{nM} ; P=0.02$ ) in SCT pts, with no difference in subjects $\geq 50 \mathrm{yrs}$. LH and FSH were significantly higher in younger SCT pts as compared with controls.

Summary: Free serum testosterone was significantly reduced in both younger and elderly allografted women. This new observation indicates that low androgen production after allo-SCT is a common late effect in women. The finding of low DHEAS concentrations suggests that not only ovarian dysfunction contributes to low testosterone levels, but also a reduced adrenal steroid production. The low testosteron production after SCT is likely to be associated with sexual dysfunction and reduced QoL.

\section{P444}

Combination of serum ferritin and disease status at transplantation predicts outcome of patients with acute myelogenous leukaemia or myelodysplastic syndrome undergoing allogeneic stem cell transplantation

H. Kanamori (1), T. Tachibana (1), H. Takasaki (1), M. Tanaka

(1), R. Hyo (2), Y. Ishigatsubo (3), A. Maruta (1)

(1)Kanagawa Cancer Center (Yokohama, JP); (2)Nagoya University (Nagoya, JP); (3)Yokohama City University Graduate School of Medicine (Yokohama, JP)

Background: Recent studies have suggested that iron overload is one of important predictors for outcome in allogeneic stem cell transplantation (SCT). We studied prognostic variables including serum ferritin (SF) in patients receiving SCT and created a 
new scoring system for predicting allogeneic SCT outcome in acute myelogenous leukemia (AML) and myelodysplastic syndrome (MDS).

Patients and methods: We analyzed 119 patients with AML or MDS who underwent allogeneic SCT at Kanagawa Cancer Center between January 2000 and December 2008. The following preand post-SCT variables were collected: age, sex, pre-SCT serum ferritin (SF), disease type, disease status at transplantation, conditioning regimen, graft-versus-host disease (GVHD) prophylaxis regimen, donor type and stem cell source, use of prednisolone within 100 days after SCT, acute GVHD, and chronic GVHD. The log-rank test was used for comparisons of Kaplan-Meier curves. Cox model was used for multivariate analyses.

Results: SF was categorized as low-SF $(<1000 \mathrm{ng} / \mathrm{ml})$ and high $(\geq 1000 \mathrm{ng} / \mathrm{ml})$ in accordance with the median value (973 $\mathrm{ng} / \mathrm{ml})$. In univariate analysis, variables associated with poorer overall survival (OS) included high-SF (vs. low-SF, $P<.0001$ ), active disease at transplantation (vs. remission, $P<.0001$ ), unrelated donor (vs. related donor, $P=.0039$ ), use of prednisolone (vs. no use, $P=.0054$ ), grade II-IV acute GVHD (vs. grade $0-I, P=.0361)$. High-SF and active disease at transplantation were significantly adverse variables for 5-y OS and disease free survival (DFS) by multivariate analysis. Furthermore, patients with high-SF showed a significantly higher cumulative incidence of nonrelapse mortality and relapse compared with those with low-SF. Active disease at transplantation was the significant risk factor associated with increased relapse. Both variables were assigned a score and its sum was used for the prognostic score. This defined 3 risk groups, low (score $=0)$, intermediate (score $=1)$, and high (score $=2)$. The 5 -y OS in low $(n=41)$, intermediate $(n=47)$, and high risk group $(n=31)$ was $78 \%, 50 \%$ and $14 \%$, respectively $(P<.0001) .5$-y DFS in each group was $68 \%, 48 \%$ and $12 \%$, respectively $(P<.0001)$.

Conclusions: This simple scoring system may be useful for predicting the outcome in patients with AML or MDS undergoing allogeneic SCT, although its validation should be performed in other large population.

\section{P445}

Pre-transplant nutrition measures (BMI, albumin, CRP) as prognostic factors in patients undergoing allogeneic haematopoetic cell transplantation

H. Bertz, P. Urbain, R. Claus, M. Kleber, J. Finke, G. Zuercher Albert Ludwigs University Medical Center (Freiburg, DE)

Many risk factors for increased morbidity and mortality in allogeneic transplantation have been recognized actually measured and classified for example by the Sorror-Index. The nutritional status before transplantation may as well be a predictor for survival. We therefore analyzed retrospectively the values for the body mass index (BMI, weight in $\mathrm{kg} /$ height in $\mathrm{m}^{2}$ ), albumin and C-reactive protein (CRP) in all 997 consecutive pts. between 1998 and 2008 at the day of admission for alloHCT. Albumin is a marker for poor nutrition status, which shows conjunction to the protein balance of the patient and CRP as acute phase protein is associated with tumour cachexia. Univariate analysis for the overall survival according the three measures at day +100 , one year and three years post HCT using the log rank test in SPSS $®$ was performed.

Results: The statistical analysis of the BMI (in median $24,7 \mathrm{~kg} / \mathrm{m}^{2}$ ) at the three time points shows no significant difference comparing pts. with a BMI below and above the median. It shows a trend to better survival in pts. with a BMI above the median at one year $(P=0.058)$.

The median albumin is $4,2 \mathrm{~g} / \mathrm{dl}$; pts. with an albumin value at admission $>$ the median had a high significant survival advantage at all three time points $(P<0.001)$.

As well pts. with a CRP $<3 \mathrm{~g} / \mathrm{dl}$ (median value) at admission had a significant better probability of survival at day +100 , one year and three years post $\mathrm{HCT}(P<0.001)$.

Conclusion: Preconditioning albumin and C-reactive protein values significantly predict overall survival after alloHCT. They may be associated with an impaired nutritional status at HCT, but as well albumin and CRP may reflect the severity of illness showing an acute phase reaction. The BMI alone is not a sufficient predictor. Multivariate analysis including disease, remission at HCT, graft source, donor and patient gender as well as conditioning regimens will be presented.

\section{P446}

Assessment of cardiac toxicity associated with haematopoietic cell transplantation with multiple biomarkers of cardiac injury

J.M. Horacek (1), L. Jebavy (2), M. Tichy (1), M. Ulrychova (1), P. Zak (1), M. Lanska (1), J. Maly (3)

(1)University Hospital (Hradec Kralove, CZ); (2)Faculty of Military Health Sciences (Hradec Kralove, CZ); (3)Charles University (Hradec Kralove, CZ)

Aim: Assessment of cardiac toxicity during hematopoietic cell transplantation (HCT) for hematological malignancies with multiple biomarkers of cardiac injury-myoglobin, creatine kinase MB (CK-MB mass), cardiac troponin I (cTnl), heart-type fatty acid binding protein (H-FABP), glycogen phosphorylase BB (GPBB). Experience with new perspective cardiac biomarkers (GPBB, H-FABP) in this context is very limited.

Patients and methods: A total of 53 patients (mean age $49.9 \pm 12.3$ years, median 54 years, 33 males) transplanted for various hematological malignancies were studied. The diagnoses were as follows: AML 27, MM 12, NHL 5, HL 4, ALL 3, CML 1, MDS 1. Thirty transplants were autologous, 23 allogeneic. Cardiac biomarkers were measured on Randox Evidence analyzer the day after completion of preparative regimen (after PR) and the day after infusion of hematopoietic cell graft (after HCT). Values above the reference range recommended by the manufacturer were considered elevated. The cut-off values for cardiac injury were as follows: 76.0 $\mu \mathrm{g} / \mathrm{L}$ for myoglobin, $4.80 \mu \mathrm{g} / \mathrm{L}$ for CK-MB mass, $0.40 \mu \mathrm{g} / \mathrm{L}$ for $\mathrm{CTnl}$, $4.50 \mu \mathrm{g} / \mathrm{L}$ for $\mathrm{H}-\mathrm{FABP}$ and $7.30 \mu \mathrm{g} / \mathrm{L}$ for GPBB.

Results: We found significant elevations in GPBB (above 7.30 $\mu \mathrm{g} / \mathrm{L})$ in $8(15.1 \%)$ patients after PR and in $9(17.0 \%)$ after HCT. $\mathrm{H}-\mathrm{FABP}$ increased slightly above the cut-off after HCT in 1 $(1.9 \%)$ patient. Other cardiac biomarkers (myoglobin, CK-MB mass, $\mathrm{cTnl}$ ) remained within the reference range in all patients. We found a significant correlation between elevation in GPBB and diastolic left ventricular (LV) dysfunction on echocardiography $(r=0.603 ; P<0.0001)$. No patient manifested clinical cardiotoxicity in the peritransplant period.

Conclusions: Our results suggest that administration of PR followed by HCT could be associated with myocardial injury manifested by increased release of GPBB from cardiomyocytes which could correlate with diastolic LV dysfunction on echocardiography. In asymptomatic patients, these findings could be considered a sign of acute subclinical cardiotoxicity. Whether these acute changes will have predictive value for development of treatment-related cardiomyopathy in the future is not clear and should be evaluated during a prospective follow-up. Further studies in a larger number of patients will be needed to confirm our preliminary results and define the potential role of new biomarkers of cardiac injury in this context.

Supported by research projects MO OFVZ0000503 and MZO 00179906.

\section{P447}

Reduced bone mineral density and high bone turnover in long-term survivors of stem cell transplantation

B. Patel (1), E. Terpos (2), J. Todd (1), R. Szydlo (1), J. Apperley

(1), J. Goldman (1), N. Salooja (1)

(1)Hammersmith Hospital (London, UK); (2)University of Athens (Athens, GR)

Reduction in bone mineral density (BMD) is a well recognized complication of SCT for haematological malignancy. There is a lack of data available on BMD and biological bone markers 
in very long term survivors of SCT, however. In this study we have evaluated the incidence of reduced BMD a minimum of 10 years following SCT for CML in consecutive patients attending a dedicated haematology/endocrine long -term follow-up clinic. Patients were evaluated by history, biochemical tests (including vitamin D, PTH, sex steroids and thyroid hormones) and DXA scans. In a subset of patients, markers of bone formation (osteocalcin, bone specific alkaline phosphatase, bALP) and bone resorption (C-terminal cross-linking telopeptide of collagen type I, CTX; tartrate-resistant acid phosphatase isoform $5 b$, TRACP$5 \mathrm{~b}$; RANK-ligand and osteoprotegrin, OPG) were measured in the serum. NTX was also measured in the urine. Forty-two patients $(21=$ female) were evaluated a median of 17 years (range 10-29 years) post-transplant. The median age at evaluation was 50 (range 27-74). A subset of 20 patients had bone markers measured in parallel with 20 age-matched controls. All transplanted patients had received cyclophosphamide/TBI as conditioning. 21/42 $(50 \%)$ had a reduction in BMD. The median $\mathrm{T}$ score at the spine was $-0.64 \mathrm{~g} / \mathrm{cm}^{2}$ (range -2.9-3.8) and at the right hip $-0.4 \mathrm{~g} / \mathrm{cm}^{2}$ (range -2.7-2.7). 16 patients were osteopenic (10 female) and 5 were osteoporotic ( 3 female) according to $\mathrm{WHO}$ criteria. Fourteen patients (33\%) had sub-optimal vitamin $D$ levels but there was no significant difference between those with low compared to normal BMD. We performed a pairwise comparison between patients and controls using Wilcoxon signed ranks test for non-parametric samples for biological markers. Osteocalcin and bALP were significantly higher in patients compared to controls ( $P=0.001$ and 0.001 respectively) indicating an increase in osteoblast activity. Serum TRACP-5b, RANKL and OPG were significantly higher in patients compared to controls ( $P=0.001, P=0.031$ and $P=0.0001$ respectively), and urinary NTX was also significantly higher in transplanted patients $(P=0.014)$. These results indicate an increase in osteoclast function. Our data demonstrate that reduced BMD is common in long term survivors of SCT for CML and that this reduction is mainly due to high bone turnover. These data suggest that such patients may be at increased risk of fracture.

\section{P448}

A comparison of avascular necrosis incidence in patients with acute lymphoblastic leukaemia treated by transplantation versus chemotherapy

S. Ni Loingsigh, N. Chadwick, F. King, L. Brennan, M. Ni Chonghaile, S. McCann, C. Flynn, P. Browne, E. Conneally, A. Fortune, E. Vandenberghe

National Adult Bone Marrow Transplantation Centre, St James's Hospital (Dublin, IE)

Background: Avascular necrosis (AVN) of bone is a serious complication of acute lymphoblastic leukaemia (ALL) treatment and allogeneic stem cell transplant (SCT), with the predominant risk factor being the use of high dose steroids, though other unknown factors may be implicated.

Materials and methods: A retrospective analysis of all patients referred to St James Hospital with ALL between 2002 and 2008 with AVN were included. This population included patients treated with chemotherapy and those treated with both chemotherapy and a myeloablative transplant.

Results: 88 patients were identified: age range 16 to 66 years; 54 male and 34 female. All patients were initially treated with induction chemotherapy, 56 patients subsequently underwent a $\mathrm{SCT}$ in first or subsequent complete remission (CR). Of those who underwent SCT, 50 were in CR1, 5 in CR2, with 1 in CR3. 10 patients (female $n=3$, male $n=7$ ) have confirmed AVN of 24 joints, age range of 16 to 43 years. Of these, 6/10 had chemotherapy alone ( $n=3$ UKALL 2003, $n=2$ UKALL XII, $n=1$ CCSG), and $4 / 10$ were post SCT. Of the patients transplanted $4 / 5$ had GVHD. The median time to AVN development was 17 months, (range 2-44 months). Median follow up was 42 months (range 6-92 months) with an overall survival of $81 \%$.

Conclusions: Avascular necrosis is a significant complication of ALL treatment, leading to considerable morbidity. In our study symptomatic AVN occurred more frequently in patients treated with chemotherapy alone (18\%) versus SCT $(7 \%)$. Concern remains about the morbidity of intensive chemotherapy particularly in young adults. Future studies are required to understand the mechanism underlying AVN, improving early diagnosis and to develop early interventions to prevent this debilitating condition.

\section{P449}

High pre-transplant ferritin is associated with low CD8+ count and infectious complications after allogeneic blood stem cell transplantation

T. Weber, U. Glaeser, M. Christopeit, G. Behre University Hospital Halle (Halle/Saale, DE)

Plasma ferritin levels are surrogate marker for iron overload and easily to access. High pre transplant ferritin levels are associated with increased mortality in allogeneic blood stem cell transplantation (SCT) recipients. This increased mortality is mostly due to infectious complications. The mechanism how iron overload leads to a higher rate of infections in SCT recipients is not fully understood. In patients with thalassemia iron overload is a common problem. In these patients, CD8 + count is significantly decreased and infectious complications are often seen. We addressed the question if secondary iron overload in SCT recipients is also associated with CD8 + depletion.

Data of 68 patients who underwent SCT were analysed retrospectively. Pretransplant ferritin levels (day -100 to +30 ) could be obtained from 31 patients. Ferritin levels were adapted to C-reactive protein (CRP) levels by creation of an index variable ferritin/CRP. Immune reconstitution data were available for 38 patients on day $+30,40$ patients on day +100 and 25 patients on day +180 . Transfusions before and after transplantations and infectious complications were assessed for all patients by chart review.

The median age at transplantation was 58 years. Forty of the 68 patients were male. Reason for transplantation was acute leukaemia in 25, myelodysplastic syndromes in 3 , osteomyelofibrosis in 1 , lymphoma in 26 and multiple myeloma in 13 patients. High ferritin levels were associated with a high number of transfusions (rho=0,384 $P=0,033 n=31$ ). There was no significant correlation between ferritin levels and CD3 + , CD3 + $4+$ +, CD3 +/CD8 +, CD56 + cells on day $+30(n=19)$ and on day $+100(n=22)$. However, high ferritin levels were associated with low CD8 + counts on day +180 (rho $=-0,879 P=0,001$ $n=10$ ). No association was noticed between serum ferritin levels and early bacterial, viral and/or fungal infections. Patients with higher ferritin levels had more bacterial and viral infections after resolved neutropenia than patients with lower ferritin (bacterial infections $P=0,08$, viral infections $P=0,08$ ) and a higher rate of GvHD (MW, SD, $P=0,118$ ).

To our knowledge, we show for the first time that high ferritin plasma levels are associated with a decreased CD8 + count in SCT recipients. As in beta thalassemia, the decreased CD8+ count is associated with an increased risk of infections. The association between low CD8 + count and high serum ferritin should be addressed in larger cohorts.

\section{P450}

Incidence and characterization of taste disturbances following autologous or allogeneic haematopoietic stem cell transplantation

B. Federmann (1), J. Weidmann (1), G. Blumenstock (2), S. Schwarz (3), L. Kanz (1), F. Mayer (1), W.A. Bethge (1) (1)Hematology \& Oncology (Tubingen, DE); (2)Medical Biometry (Tubingen, DE); (3)Biological Chemistry and Nutrition Sciences (Stuttgart/Hohenheim, DE)

After hematopoietic stem cell transplantation (HCT) a high incidence of taste and smell alterations is observed. This could influence physical recovery, general wasting and quality of life as well as impair defense against infections. 
We retrospectively analyzed 181 patients treated in our institution (median age 52 (range 20-79)) using a new standardized questionnaire and the patient chart to evaluate changes in the perception of taste and smell. 148 patients received an allogeneic (allo) graft after MAC $(n=87)$ or RIC $(n=61)$ and 33 patients were treated with an autologous (auto) HCT. Indications for HCT included acute leukemia $=72, \mathrm{MPS}=32$, lymphoma $=29, \mathrm{MDS}=10$ and other $=38$. Moderate to severe changes in taste perception were reported by $71 \%$ during the acute phase of HCT with no significant differences between the three groups (MAC $74 \%$, RIC $66 \%$, auto HCT 73\%). Changes in smell perception were documented in $54 \%$ with a median of 34 days (range, $2-365$ ) to improvement. $30 \%$ of these patients described a reduction in smell perception, $21 \%$ intensification. The symptoms persisted in $62 \%$. In univariate analysis (chi2-tests) we examined the difference between auto and allo HCT as well as the influence of GVHD or immunosuppression. Changes in taste perception were significantly associated with loss of body weight $>5 \mathrm{~kg}(69.2 \%$ vs. $45.6 \%, P=0.002)$. Alterations in body weight and taste perception tended to be more prevalent after allo HCT with $45.9 \%$ and $30.3 \%$ compared to auto HCT with $58.1 \%$ and $45.5 \%$. A complete recovery at the time of survey was reported by $29 \%$, whereas $26 \%$ still suffered from taste alterations. We observed a significant difference in time to recovery of taste alterations between auto HCT vs. allo HCT with a median of 60 days (range, 3-365) vs. 120 (range, 30-600) days $(P=0.03)$. The incidence of cGVHD after allo HCT was $79 / 148(53 \%)$. In patients with cGVHD significantly more changes in taste perception $(64.6 \%$ vs. $49.0 \%, P=0.04)$ and loss of body weight $>5 \mathrm{~kg}(55.7 \%$ vs. $33.3 \%, P=0.004)$ were reported. We observed a significant influence on alterations in taste and a of loss weight $>5 \mathrm{~kg}$ with the use of tacrolimus $(67.2 \%$ vs. $50.0 \%, P=0.04)$ or steroids $(72.7 \%$ vs. $48.4 \%$, $P=0.03$ ) and mycophenolatmofetil ( $53.8 \%$ vs. $34.7 \%, P=0.01$ ). In conclusion taste and smell disturbances are common events after HCT persisting in $25 \%$ of patients receiving allo grafts. In allo HCT a major impact of GVHD and immunosuppression are observed.

\section{P451}

Psychosocial outcomes of long-term survivors after haematopoietic stem cell transplantation in childhood cancer survivors: comparison with survivors without haematopoietic stem cell transplantation and healthy children

H.S. Kim (1), S.C. Won (1), M.A. Rhee (1), K.M. Chung (1), C.J. Lyu (1)

(1) Yonsei University College of Medicine (Seoul, KR); (1)Yonsei University (Seoul, KR)

Background: Long-term survival rate after myeloablative hematopoietic stem cell transplantation (HSCT) in childhood cancer patients has been remarkably increased lately. As considerable number of patients survive these days, quality of life (QOL) as well as psychosocial adjustment of HSCT survivors is getting more attention.

Purpose: The purpose of this study was to investigate psychosocial status and QOL of childhood cancer long-term survivors and to compare differences between those of survivors with/ without HSCT and healthy control group.

Methods: Ninety four children who survived more than two years from off therapy with childhood cancer were included. Forty seven survivors experienced HSCT and the other 47 survivors didn't. Forty seven healthy children were also included. Total 141 children and their mother performed standardized questionnaires (Korean Child Behavior Checklist [K-CBCL], Pediatric quality of life [PedsQL]).

Results: Among six-items (total problems, externalizing problems, internalizing problems, thought problems, attention problems, and social immaturity), survivors with HSCT had significantly higher score of total problems $(P=0.006)$ and externalizing problems $(P=0.003)$ than both survivors without HSCT and healthy children. Both survivors with and without HSCT had significantly higher score of social immaturity $(P=0.001)$ than healthy children, but there was no significant difference between survivors. On PedsQL, the score of physical function was lowest in survivors with HSCT, and survivors without HSCT also had lower score than healthy children. Survivors with HSCT had significantly lower score of social function $(P=0.02)$ and school function $(P<0.001)$ than the others.

Conclusion: Survivors with HSCT had more tendencies of showing delinquency and aggressiveness and experiencing low QOL. Several items showed no significant difference between survivors without HSCT and healthy children. This preliminary study suggests HSCT is thought to be an independent risk factor of poor psychosocial outcomes. These findings propose that childhood cancer survivors who experienced HSCT have more need for attention to their QOL and psychosocial intervention plan to improve their overall outcomes.

\section{P452}

High-dose pulse methylprednisolone treatment of Bronchiolitis obliterans in paediatric stem cell transplantation

H.H. Uhlving (1), C. Heilmann (1), F. Buchvald (1), P. Wehner (2), K.G. Nielsen (1), K. Müller (1)

(1)Rigshospitalet (Copenhagen, DK); (2)H.C. Andersen Childrens Hospital (Odense, DK)

Background: Bronchiolitis obliterans (BO) is a rare complication in paediatric stem cell transplantation (SCT), and is associated with a high risk of mortality. There is no clear evidence that therapeutic interventions have a positive impact on the course of the disease, but a lasting effect on lung function after high-dose pulse methylprednisolone (MP) therapy has been reported. The aim of this study was to evaluate the response of MP treatment in a population based paediatric cohort from the national SCT centre in Denmark.

Material and methods: The study was restricted to patients who had an age sufficient to perform a reliable spirometry. Seven children transplanted between 1999 and 2008 with median age 10 years (4-13) were included. BO was verified by open lung biopsy and/or high resolution computer tomography (HRCT). One patient, who died from acute respiratory failure due to cytomegalovirus infection during the first month, was excluded. Patients were treated with MP $15 \mathrm{mg} / \mathrm{kg}$ for 2-3 consecutive days every 4-6 weeks. In three patients this was combined with infliximab. Basic immunosuppression included cyclosporine $A$ $(n=7)$, mycophenolate $(n=5)$ and prednisolone $(n=5)$. Median time from symptoms to initiation of MP-treatment was 16 days (3-244). Lung function measured by forced expiratory volume in 1 second (FEV1) and forced vital capacity (FVC) was measured before SCT, before start of MP, and then monthly. Data are presented as medians (range), and were analyzed using Wilcoxon rank sum test.

Results: There was a significant reduction in FEV1 $(P<0,01)$ and FVC $(P<0,05)$ from pre-SCT values to the time of diagnosis of BO (Table 1). Three months after initiation of MP therapy, a significant response was seen as FEV1 increased $(P<0,01)$. However, the levels at six months did not differ significantly from pre-treatment levels.

Two patients died from residual cancer within a year from BO diagnosis, whereas five children are alive at median 48 months (9-114) after BO diagnosis. One child is now being referred for lung transplantation. The remaining have a normal to moderately reduced lungfunction after 7,5 (2-43) cycles of MP.

Conclusion: Significant improvement in lung function is demonstrated after initiation of MP treatment in children with BO after SCT, but this effect appears to be only temporary. However, MP may have the potential of stabilizing the level of lung function and prevent further decline. Larger studies are needed to establish long term effect. 
Table 1: Variations in lung function in time from first high-dose pulse methylprednisolone treatment

\begin{tabular}{|l|l|l|l|l|l|l|l|}
\hline & Before SCT & $\begin{array}{l}\text { Before } \\
\text { MP }\end{array}$ & $+1 \mathrm{~m}$ & $+3 \mathrm{~m}$ & $+6 \mathrm{~m}$ & $+1 \mathrm{y}$ & $+3 \mathrm{y}$ \\
\hline No. of patients & $\mathrm{n}=6$ & $\mathrm{n}=7$ & $\mathrm{n}=6$ & $\mathrm{~N}=6$ & $\mathrm{n}=6$ & $\mathrm{n}=2$ & $\mathrm{~N}=3$ \\
\hline $\begin{array}{l}\text { FEV1\% pred } \\
\begin{array}{l}\text { Median } \\
\text { (range) }\end{array}\end{array}$ & $84(75-105)$ & $\begin{array}{l}53(29- \\
62)\end{array}$ & $\begin{array}{l}62(44- \\
83)\end{array}$ & $\begin{array}{l}81(57- \\
89)\end{array}$ & $\begin{array}{l}58(42- \\
96)\end{array}$ & $\begin{array}{l}(61- \\
110)\end{array}$ & $\begin{array}{l}43(41- \\
132)\end{array}$ \\
\hline $\begin{array}{l}\text { FVC\% pred } \\
\begin{array}{l}\text { Median } \\
\text { (range) }\end{array}\end{array}$ & $81(64-100)$ & $\begin{array}{l}61(45- \\
95)\end{array}$ & $\begin{array}{l}64(45- \\
92)\end{array}$ & $\begin{array}{l}84(60- \\
93)\end{array}$ & $\begin{array}{l}72(43- \\
98)\end{array}$ & $\begin{array}{l}(60- \\
105)\end{array}$ & $\begin{array}{l}91(46- \\
129)\end{array}$ \\
\hline $\begin{array}{l}\text { No. of MP- } \\
\text { cycles }\end{array}$ & & & 1 & $3(2-3)$ & $5(2-6)$ & $(11-13)$ & $\begin{array}{l}27(11- \\
33\end{array}$ \\
\hline
\end{tabular}

P453

High prevalence of thyroid dysfunction long-term after autologous and allogeneic stem cell transplantation

I. Sanchez-Ortega, T. Peralta, R. Parody, V. Clapes, M. Arnan, A. Fernandez de Sevilla, R.F. Duarte

Institut Catalá d'Oncologia (Barcelona, ES)

Thyroid dysfunction (TDF) is a recognized late complication of haematopoietic stem cell transplantation (HSCT). For this reason, EBMT/CIBMTR-ASBMT consensus guidelines for longterm HSCT survivors recommend thyroid function testing yearly post transplant in all patients, or if new relevant symptoms develop. The reported incidence of TDF varies between studies, depending not only on patient and HSCT characteristics, but also on the follow-up period of observation. Few studies have analyzed the longer-term prevalence of this complication. In this study, we retrospectively analyse long-term follow-up of thyroid function (TSH and FT4) in 169 long-term HSCT survivors in our center with no pretransplant history of TDF and at least one year follow-up (1993-2008): autologous 115 (68\%), allogeneic 54 (32\%); male $90(53 \%)$; median age at HSCT 43 years (15-68); AML/MDS 29\%, Mieloma 23\%, Non-Hodgkin $17 \%$, Hodgkin $14 \%$, CML $12 \%$, ALL $3 \%$, others $2 \%$. Sixty two of these patients $(37 \%)$ developed TDF during our median period of observation of 75 months (12-186): 54 cases of subclinical hypothyroidism (32\%), six of overt hypothyroidism (4\%) and two of overt hyperthyroidism (1\%). There was no association between TDF and sex, primary disease, conditioning regimen or type of allogeneic donor (related vs. unrelated). A significantly higher prevalence of TDF was observed in younger HSCT recipients $(48 \%$ in $\leq 40$ years vs. $29 \%$ in $>40$ years; $P=0.01)$, and in recipients of autologous vs. allogeneic HSCT ( $43 \%$ vs. $24 \% ; P=0.02)$. Moreover, the median time from transplantation to diagnosis of TDF was longer in autologous HSCT (63 vs. 23 months in allogeneic; $P=0.01$ ), where half of the cases of TDF were observed more than 5 years after HSCT, despite comparable median follow-up periods for survivors in both groups $(P=0.20)$. The prevalence of this complication in autologous HSCT could have been underestimated in previous studies because of a shorter follow-up. These data not only confirm the well-established high prevalence of TDF after allogeneic HSCT, but also do suggest that the prevalence of TDF may be higher than initially described long-term after autologous HSCT, and reinforce a recommendation for sustained long-term monitoring, beyond 5 years post-HSCT to diagnose late-onset TDF, in particular in younger patients.

\section{P454}

High prevalence of dyslipidaemia in recipients of both allogeneic and autologous haematopoietic stem cell transplants - a single-centre experience

J.J. Thomson, R. Barrett, M. Kazmi, L. Bishop, M. Streetly, K. Raj

Guy's \& St Thomas' NHS Foundation Trust (London, UK)

Background: As long-term survival in haematopoietic stem cell transplantation (HCT) improves, managing late complications becomes increasingly important. The leading causes of late death are relapse and chronic graft-versus-host disease (GvHD). Cardiovascular disease (CVD) is now, also emerging as a significant contributor to late morbidity and mortality. Dyslipidaemia is one risk factor for CVD and Joint British Society
(JBS) guidance recommends an optimal total target cholesterol of $<4 \mathrm{mmol} / \mathrm{L}$ in patients at high risk of CVD.

Method: A retrospective review of random lipid levels measured at least one year following HCT is presented. In line with NICE guidance, total cholesterol and HDL are used as markers of dyslipidaemia.

Results: 44 allograft patients were identified from departmental records. $68 \%$ male, median age 29 yrs, $43 \%$ Non-Hodgkin's Lymphoma, 27\% Acute Myeloid Leukaemia, 30\% other. 96\% had a total cholesterol $>4 \mathrm{mmol} / \mathrm{L}(64 \%>5 \mathrm{mmol} / \mathrm{L}$, median $5.5 \mathrm{mmol} / \mathrm{L}) .14(32 \%)$ were classified as high risk (9 raised total:HDL ratio, 2 clinical evidence atherosclerotic disease, 4 diabetes, 2 CVD score $\geq 20 \%$ over 10 years). The median age of the high-risk group was $39 \mathrm{yrs}$, and $93 \%$ were male. $57 \%$ had GvHD, of which $75 \%$ were on steroids, and of these $75 \%$ were also receiving mycophenylate. 3 patients had a total cholesterol of $>7.5 \mathrm{mmol} / \mathrm{L}$ and were being screened for familial dyslipidaemia.

31 autologous HCT patients were also reviewed. $68 \%$ male, median age 47 yrs, 55\% Lymphoma, 45\% Plasma Cell Disorder. $84 \%$ had a total cholesterol $>4 \mathrm{mmol} / \mathrm{L}(52 \%>5 \mathrm{mmol} / \mathrm{L}$, median $5.0 \mathrm{mmol} / \mathrm{L}$ ).

Conclusion: We report a high incidence of dyslipidaemia, which is significantly greater than previously published European data of $16 \%$ (Tichelli et al., Haematologica 2008). Previously suggested mechanisms include endocrine dysfunction and prolonged immunosuppression. However, these do not explain the results in our autologous HCT patients. The median time from transplant to testing was 34 and 28 months in the allogeneic and autologous HCTs, and it may be that with time, some normalization of the lipid profile could be expected. Other possible explanations include poor dietary and lifestyle factors due to the poor socioeconomic status of our population. We propose that all HCT patients should be screened for dyslipidaemia, and statin therapy initiated, with a target total cholesterol of $<4 \mathrm{mmol} / \mathrm{L}$ as per the JBS guidance.

In addition, dietary and lifestyle factors should be addressed where appropriate.

\section{P455}

Measured quality of life does not correlate with the clinical status of patients who receive an haematopoietic stem cell transplantation

S. Giuliani, B. Tedoldi, G. Pantaleo, M. Bernardi, J. Peccatori, C. Corti, A. Assanelli, M.T. Lupo Stanghellini, M. Carrabba, D. Clerici, S. Mastaglio, F. Giglio, C. Messina, R. Greco, S. Malato, L. Sarno, F. Ciceri

San Raffaele (Milan, IT)

Object: Since perceived quality of life promote patients' compliance and satisfaction to medical treatment, supported by many literature's experiences, the main purpose of this study, risen from the collaboration between the Haematology and Bone Marrow Transplant Unit and Health Clinical Psychology Unit at San Raffaele Scientific Institute, was to evaluate factors related to patients' perceived quality of life during the different stages of haematopoietic stem cell transplantation program (HSCT).

Methods: From January 2008 to October 2009, 93 patients undergoing HSCT completed questionnaires measuring level of anxiety and depression (Hospital Anxiety and Depression Scale, HADS), coping strategies (Mental Adjustment to Cancer Scale, MAC), level of distress (Perception of Distress Index, PDI) and quality of life (Medical Outcomes Study Short form-36, SF 36), controlling for sex, age, stage of HSCT (pre-infusion, within 1 week, 1 month, 3 months, over 3 months post HSCT), diagnosis, type of transplant (autologous, allogenic), disease stage. These data were analyzed by the statistical software SPSS.

Results: The results show a significant negative correlation $(P<0.01)$ between perceived quality of life and level of anxiety, depression and level of distress. No correlation between quality of life and type of transplant, stage of HSCT, diagnosis, disease stage was found, so that psychological aspects have 
been related to patients' perceived quality of life more than the clinical measurable variables of the disease and its treatment. Conclusions: Therefore, the psychological status of the patients has to be taken into a proper account in evaluating even the clinical impact of the HSCT on their quality of life and preventive corrections and therapeutical acts, if needed, have to be taken. The HSCT team should include psychologists to work with the clinicians in order to evaluate, during the treatment, the impact of these factors affecting perceived quality of life related to HSCT.

\section{P456}

Validation of the predictive power of the haematopoietic cell transplantation co-morbidity index, performance status for non-relapse mortality and long-term survival after autologous transplantation in patients with haematological malignancies

A. Pivkova, S Genadieva Stavrik, Z. Stojanoski, L. Cevreska, S. Krstevska Balkanov, S. Trajkova, I. Panovska Stavridis, O. Karanfilski, B. Georgievski

University Hematology Hospital (Skopje, MK)

The hematopoietic cell transplantation comobrbidity index (HCT$\mathrm{Cl}$ ) was developed as a sensitive tool to capture pretransplant comorbidities among transplant recipients which will have influence on non relapse mortality (NRM) and overall posttransplant survival (OS). HCT-Cl has not been widely validated among autologous recepients. We retrospectivelly evaluated if $\mathrm{HCT}-\mathrm{Cl}$ and karnofsky performance status (PS) and other readily available pretransplant variables concerning pretransplant mobilization strategies can predict the outcome of autologous recipients in our transplant center.

We stratified outcomes among 120 consecutive adult autologous recipients (47 AML in first remission, $24 \mathrm{HD}, 27 \mathrm{MM}, 16$ $\mathrm{NHL}, 4 \mathrm{ALL})$. HCT-Cl risk was low in $10(12 \%)$, intermediate in 22 $(27 \%)$ high in $45(55 \%)$ and undetermined in $5(6 \%)$. Two year OS was $45 \%(95 \% \mathrm{Cl}: 24-64 \%), 55 \%(95 \% \mathrm{Cl}: 40-68 \%)$ and $42 \%$ (95\% Cl: $24-64 \%)$ in the low, intermediate and high-risk HCT-Cl groups respectively. Two year NRM was 36\% (95\% Cl: $17-36 \%)$, $26 \%$ (95\% Cl: $15-39 \%$ ) and $30 \%$ (95\% Cl: $22-39 \%)$ in the low, intermediate and high-risk HCT-Cl groups respectively. The multivariate analysis revealed that $\mathrm{HCT}-\mathrm{Cl}$ failed in prediction of OS and NRM but KPS $(<90 \%)$ was a strong predictor of NRM as an independent predictor. The variables concerning mobilization of stem cells (chemotherapy with G-CSF versus G-CSF alone and the dose of infused CD34+>4,0 $\times 10^{6} / \mathrm{kg}$ and $<4.0 \times 10^{6} / \mathrm{kg}$ in the three risk HCT-Cl groups revealed that patients with HCT$\mathrm{Cl}$ score $>3$ and intermediate and high risk disease that received $<4.0 \times 10^{6} / \mathrm{kg}$ had 2 year NRM $<30 \%$ and OS $<45 \%$, as well the patients mobilized with chemotherapy + G-CSF showed lower $\mathrm{NRM}$ in the HCT-Cl $>3$ (intermediate and high risk disease). To determine the validity of HCT-Cl, KPS and weather to include the the independent variables concerning the mobilization strategy and stem cell dose that we analyzed, a multi-center collaboration is necessary to produce an adequately powered validation study for risk stratification of autologous recipients.

\section{P457 \\ Genetic polymorphism of NQO1 is associated with an increased treatment-related mortality in patients undergoing allogeneic transplantation \\ M. Koldehoff, D.W. Beelen, A.H. Elmaagacli \\ University of Duisburg-Essen (Essen, DE)}

Background: The widely expressed detoxification enzyme $\mathrm{NAD}(\mathrm{P}) \mathrm{H}$ :quinine oxidoreductase 1 (NQO1) is involved in the cellular response to oxidative stress and irradiation and protects cells against the mutagenicity from free radicals and toxic oxygen metabolities. NQO1 is subject to a genetic polymorphism (C609T) leading to a change in its amino acid sequence. Heterozygous individuals $\mathrm{C} / \mathrm{T}$ have intermediate activity and homozygotes T/T are NQO1 deficient. Never before the influence of genetic polymorphisms of NQO1 on patients who underwent allogeneic transplantation, was evaluated

Methods: Here we genotyped in a retrospective study 198 patients (and their donors) for NQO1 expression who underwent allogeneic transplantation for various diseases and analyzed their outcome. Genotyping of NQO1 was performed by real-time PCR.

Results: 145 patients $(73.2 \%)$ were genotyped as homozygous wild-type gene C/C, 48 patients $(24.2 \%)$ were genotyped as heterozygous genotype $\mathrm{C} / \mathrm{T}$ and five patients $(2.5 \%)$ were genotype as homozygous gene mutation T/T. From the donors 147 donors $(74.2 \%)$ were $\mathrm{C} / \mathrm{C}, 50$ donors $(25.3 \%)$ were $\mathrm{C} / \mathrm{T}$, and one donor $(0.5 \%)$ had a homozygous gene mutation T/T. Calculated genotype frequencies did not differ from that reported earlier by other studies for Caucasians. Five-year estimate for TRM was highest in genotype $\mathrm{C} / \mathrm{T}$ - and T/T-patients with $39 \%+11 \%$ compared to homozygous wild-type gene C/C-patients $(21 \%+$ $3.9 \%[P<0.045])$, whereas the five-year estimate for relapse or overall survival were not statistically different between the groups. No differences for five-year estimates for TRM, relapse rate, or OS were seen in patients with either genotype C/C-, $\mathrm{C} / \mathrm{T}$ - or $\mathrm{T} / \mathrm{T}$-donors. No statistic differences were found in the incidence of acute GVHD grade 2-4 within the study groups.

Conclusions: These results suggest that patients with genetic polymorphism of NQO1 do have an increased TRM after transplantation. Genotyping for NQO1 (C609T) might help to identify patients with higher risk for TRM.

P458

Influence of rKGF on intestinal mucosal damage measured by citrulline in autologous haematopoietic stem cell transplant recipients treated with BEAM: a safety and efficacy study

A. Herbers, J.P. Donnelly, N.M.A. Blijlevens

Radboud University Nijmegen Medical Center (Nijmegen, NL)

Background: Mucositis is a complication of intensive chemotherapy and radiotherapy that is debilitating for patients and plays a distinct role in determining the outcome of haematopoietic stem cell transplantation (HSCT). Despite encouraging data regarding the effectiveness of rKGF (recombinant human keratinocyte growth factor) on oral mucositis there is a dearth of data regarding its activity on intestinal mucosal barrier injury $(\mathrm{MBI})$. Main reason is the inaccessibility of the gut. Citrulline is an aminoacid that can be determined in blood, which is a functional marker of small intestinal enterocytes. Low citrulline concentrations in blood coincide with and are a response to, severe $\mathrm{MBI}$ and associated with bacteraemia. In the present study, we examined the efficacy and safety of rKGF on intestinal MBI induced by cytotoxic therapy using citrulline as parameter.

Patients and methods: Between July 2006 and November 2008 in a single center study 19 patients undergoing autologous HSCT after BEAM were treated with rKGF and retrospectively compared to a matched control group. Outcomes assessed were: safety, daily oral mucositis score (DMS), daily gut score (DGS), course of absolute neutrophil count, C-reactive protein and citrulline, occurrence of neutropenic fever and bacteraemia. Main results: The common side effects of rKGF included rash and erythema, which occurred in all patients and were mild (37\% CTC grade 1 and $63 \%$ CTC grade 2$)$ and transient in appearance. rKGF recipients showed a significant reduction of approximately $30 \%$ in the DMS (between day 10 and 13 ) and DGS (between day 15 and 18). No significant difference in the course of citrulline was seen between the patients. In both treatment groups 9 patients developed bacteraemia (respectively 6 patients with coagulase-negative staphylococci and 3 patients with viridans streptococci in both groups).

Conclusions: The administration of rKGF was generally tolerated and safe. rKGF had a beneficial influence on the signs and symptoms related to oral and intestinal mucositis. However, no impact on the course of citrulline and bacteraemia was seen. 


\section{P459}

The impact of melphalan dose administered per kilogram of body weight on the transplantation results and oral toxicity

S. Vokurka, K. Steinerova, M. Karas, P. Jindra, V. Koza

University Hospital in Pilsen (Pilsen, CZ)

Introduction: Melphalan based conditioning regimens are standard and well-established within hematopoietic stem cells transplantation (HSCT) settings. Several factors may have impact on the treatment results. Objectives: to verify melphalan doses administered per kilogram of body weight in AML patients treated with allogeneic HSCT after fludarabine/melphalan (FLU/ $M E L)$ conditioning regimen and to evaluate impact of the dosing variability on the treatment results and oral toxicity.

Methods and patients: retrospective analysis, $38 \mathrm{AML}$ patients, allogeneic HSCT, FLU/MEL conditioning regimen (fludarabine total dose $120 \mathrm{mg} / \mathrm{m}^{2}$, melphalan $140 \mathrm{mg} / \mathrm{m}^{2}$ ), the period of 10/2001-12/2007.

Results: The median melphalan dose administered per a patient was 3,5 (range $2,5-4,1$ ) $\mathrm{mg} / \mathrm{kg}$ of actual body weight. Two groups of patients were compared in respect to the melphalan dose: $\geq 3,5 \mathrm{mg} / \mathrm{kg}$ ("higher") versus $\leq 3,4 \mathrm{mg} / \mathrm{kg}$ ("lower"). Both groups were relatively well balanced in all important demography, donor-patient transplant characteristics and the follow-up time: $15(1-70)$ vs. $13(2-55)$ months $(P=$ n.s.). No statistically significant differences were observed in relapse rate, transplant related mortality, acute or chronic GVHD incidence and graft rejection. No difference was found in the 3-years OS and DFS probability between the two groups ( $48 \%$ vs. $54 \%$ and $48 \%$ vs. $43 \%, P=0,8)$. In patients with the same body surface area, those with lower body weight had higher melphalan $\mathrm{mg} / \mathrm{kg}$ doses and had higher incidence of severe oral mucositis grade $3-4 \mathrm{WHO}(48 \%$ vs. $24 \%, P=0,05)$.

Conclusions: In patients with the same body surface area, those with lower body weight received higher melphalan $\mathrm{mg} / \mathrm{kg}$ doses. This fact appeared to increase oral toxicity and, probably did not have any significant additional effect on the treatment results within the cohort of the patients. Melphalan dosing based on body weight instead of body surface should be considered and the impact of the median $3,5 \mathrm{mg} / \mathrm{kg}$ melphalan dose within the FLU/MEL conditioning be verified in larger and prospective trial.

\section{P460}

Kidney injury as an early complication after

haematopoietic cell transplantation: incidence,

risk factors and outcome

I. Sakellari, G. Bamichas, A. Barbouti, D. Mallouri, S. Fragidis,

E. Yannaki, C. Apostolou, V. Constantinou, M. Partziali,

K. Sompolos, A. Anagnostopoulos, A. Fassas

G.Papanicolaou (Thessaloniki, GR)

Allogeneic stem cell transplantation (HCT) has been associated with chronic kidney disease (CKD) as a late effect caused by a variety of factors. We retrospectively evaluated the development of CKD in 164 patients (pts), aged 9-65, who underwent HCT for hematological disease from sibling (137) or unrelated donors (27) after myeloablative (144) or reduced intensity conditioning regimens (20) and who survived for at least 3 months post transplantation (median follow up 23.5 months). CDK was defined as a GFR $<60 \mathrm{ml} / \mathrm{min} / 1.73 \mathrm{~m}^{2}$, estimated by the modification of diet in renal disease (MDRD) formula and the Schwartz formula for children. The probability of CKD was $23.8 \%$ at 18 months after a median follow up of $7(3-120)$ months. The mean value of preHCT- GFR was within normal limits $(111.5 \pm 26)$ for pts who did not develop CKD and $97.21 \pm-19$ for those who developed CKD, while the GFR at 12 months post transplant was $108 \pm 28$ and $54.7 \pm 5.4\left(\mathrm{ml} / \mathrm{min} / 1.73 \mathrm{~m}^{2}\right)$, respectively. The course of CKD was asymptomatic until the end-stage disease when 3 pts were on dialysis and 1 patient received a renal transplant from his mother. On univariate analyses, the probability of developing CKD was
$25 \%$ at 18 months for pts with 0 or 1 event of kidney injury vs. $60 \%$ for those with 2-5 preceded events (p: 0.006). On the other hand, the type of conditioning, hyper fractionated TBI, administration of Thiotepa or Fludarabine, acute or chronic GVHD, the toxicity of antiviral or antifungal treatment did not correlate with the CKD. Calcineurin inhibitors were not included as risk factors because of the universal administration as prophylaxis and because of their toxicity appearing in the long term treatment. On multivariate analysis, the only predictive factors were older age $(P=0.01)$, the number of preceded events of acute kidney injury, and the in vivo $T$ cell depletion with antithymocyte globulin or alemtuzumab (0.013). The CKD did not influence the non relapse mortality of the HCT. The profound lymphocytopenia of T-cell depletion might possibly be related to an excess of secretion of the transforming growth factor beta (TGF beta) which may promote early and rapid renal fibrogenesis. Close monitoring of the patients for early events of acute kidney injury due to drug toxicity is necessary for CKD prevention.

\section{P461}

Cause-specific toxicity and second cancer development for relapsed/refractory Hodgkin's lymphoma in complete remission after ifosfamide, gemcitabine, vinorelbine (IGEV) regimen and high dose chemotherapy with autologous haematopoietic stem cell infusion

A. Anastasia, M. Magagnoli, M. Balzarotti, R. Mazza, A. Santoro

Humanitas (Rozzano, IT)

Autologous hematopoietic stem cell transplantation (AHCT) for relapsed/refractory Hodgkin's lymphoma (HL) after one line chemotherapy remains the gold standard, with a long-term disease-free survival in $40-50 \%$ of patients. However the incidence of toxicity and second cancer development in this distinctive subset of patients has not been well established.

The aim of the present study is to assess toxicity and second malignances among 52-months survivors in complete remission after treatment with IGEV plus AHCT.

We retrospectively reviewed a uniform cohort of 121 patients who underwent IGEV induction therapy followed by AHCT from 1997 to 2007.

Among those treated in our Institution, 75 received one ABVD or ABVD-like chemotherapy line before. Radiotherapy was added in $63 \%$ of them with bulky disease.

All the 75 patients were treated with IGEV $\times 4$ cycles. However, only 66 underwent autologous stem cell transplantation. Highdose regimen consisted of carmustine, etoposide, aracytin and melphalan (BEAM) for 18 patients and of a tandem procedure (Melphalan200 plus BEAM) for the other 48 patients.

Treatment related mortality was null. Early haematological and extra-haematological toxicity were similar in the two cohorts of AHCT.

After a median follow-up of 52 months, 50 patients were in complete remission, therefore available for the analysis. Of these patients, $4(8 \%)$ developed a second cancer: 1 acute myelogenous leukemia, 1 pancreatic cancer, 1 papillary thyroid cancer, 1 malignant teratoma.

Nine patients $(18 \%)$ reported late toxicity: 3 thyroid failure, 1 cardiovascular disease, 1 chronic bronchopneumonia, 2 psychiatric and 1 osteopathic disorders. One patient developed Idiopathic thrombocytopenic purpura. Furthermore we observed 2 cases of opportunistic infections (Herpes Zoster visceral disease and Cytomegalovirus pneumonia) during the first year from AHCT. The global related mortality was $4 \%$ (2 tumors).

Salvage therapy using IGEV followed by AHCT was not associated with second cancer development or organ failure in $74 \%$ of $\mathrm{HL}$ patients in complete response.

Late toxicity and second cancers are a significant cause of late morbidity and mortality for relapsed/refractory $\mathrm{HL}$ treated with curative intent by AHCT; while in our experience it does not appear increased in a homogeneous cohort exposed to IGEV regimen followed by AHCT. 


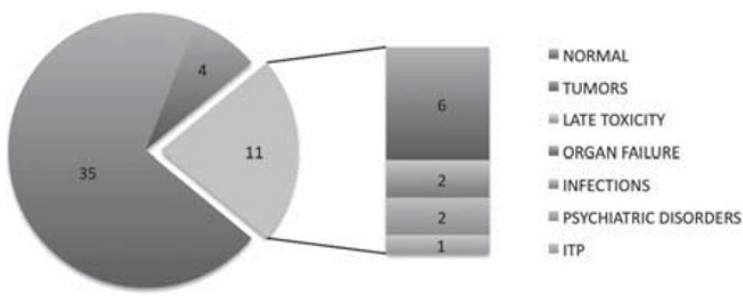

P462

Psychosocial functioning in Hodgkin's lymphoma survivors after ifosfamide, gemcitabine, vinorelbine (IGEV) and high-dose chemotherapy with autologous haematopoietic stem cell infusion

M. Magagnoli, A. Anastasia, M. Mencaglia, M. Balzarotti, R. Mazza, A. Santoro

Humanitas (Rozzano, IT)

Autologous hematopoietic stem cell transplantation (AHCT) for Hodgkin's lymphoma $(\mathrm{HL})$ patients has markedly improved the survival in the last three decades, although, to date, little is known about the psychosocial functioning for long term survivors.

Recently, several investigators have suggested the importance of a psychosocial morbidity assessment in the follow-up of these patients.

Our study concerns the influence of psychosocial dis-functions on the quality of life of patients who underwent AHCT for relapsed/refractory Hodgkin lymphoma $(\mathrm{HL})$ in our Institution from 1997 to 2007.

We retrospectively reviewed the clinical follow-up of 94 patients treated with IGEV plus AHCT, in order to determine the incidence of psychological disorders.

Interviews were performed in between 1 year from AHCT. However, among initial 94 patients, 6 died within 100 days from transplant, 7 were lost during follow-up, so data were available for only 81 of them (43 men and 38 women, with median age of 31years).

Oral reports about mood, household-life, fatigue, depression and anxiety were collected into a specific record called "Performance Status (PS)".

An unexpectedly high number of $\mathrm{HL}$ survivors reported having psychosocial dis-functions $15 / 81(19 \%)$, in spite of young median age and freedom from progression.

Depression occurred in $10 / 15(66 \%)$ of them, anxiety in $3 / 15(20 \%)$ and 2 young women developed alimentary disorders. However, although only 6 patients had second cancer and 12 showed late toxicity, over $70 \%$ of the survivors referred high fears of recurrence or of second tumors.

When we investigated them on "at home drug therapies", our findings showed a high use of benzodiazepine (about $90 \%$ of survivors), whereas 3 patients reported psychotropic treatments. Our data demonstrate a fairly large number of proven psychological disorders in HL.

In contrast only few patients received specific management and psychiatric therapy.

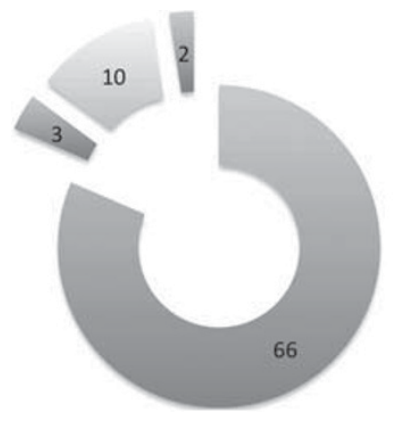

ANXIETY

- DEPRESSION
= ALIMENTARY DISORDER
The analysis also suggests that the most critical host factor for developing psychosocial morbidity was fear of recurrence. Thus we look forward to develop new supporting strategies to improve the quality of life and to identify those patients who need early psychological and psychiatric interventions.

\section{P463}

Rate and outcome of readmission in patients undergoing haematopoietic stem cell transplant

M.P. Gallego, I. Heras, C. Castilla-LLorente, J.M. Torregrosa, B. Muiña, J.B. Nieto, F. de Arriba, V. Vicente Hospital Morales Meseguer (Murcia, ES)

Background: The recent progresses in hematopoietic stem cell transplantations (SCT), especially in the source of the stem cell and the conditioning regimens allow a better tolerance of the transplant proceeding that favors an earlier discharge. Despite this advances, it is still an aggressive therapy not exempt of toxicities that sometimes can lead to the need of readmission to the Hospital.

Aims: To determine the frequency and causes of readmission of patients who underwent stem cell transplant (SCT) in our institution. Methods: A retrospective analysis was performed of 192 consecutive patients who underwent SCT, autologus (autoSCT) or allogenic (alloSCT) between January 2005 and July 2009. We considered readmission as any clinical event that need hospitalization after discharge from transplantation proceeding. Data was collected on the following variables: underlying disease, status at transplantation, presence of comorbodities (mesured by the Charlson index), conditioning regimen, cause and date of readmission. Data on complications during the transplantation procedure were also collected: the occurrence of mucositis, infections, graft-versus-host disease (GVHD), hepatic sinusoidal obstruction syndrome and other relevant complications related with infusion and/or conditioning.

Results: Among 192 patients included in this analysis (120 men, 62'5\%), 127 (66\%) where autoSCT and 65 (34\%) allo$\mathrm{SCT}$, from related (RD: 50 patients) and unrelated donors (URD: 15 patients). The total of readmissions was of 151 among 94 patients: 70 patients were admitted once, 9 patients twice and 15 patients 3 or more occasions. Readmission rate was significantly higher among patients who received alloSCT from URD $(60 \%)$ vs. autoSCT $(46 \%)$ or RD $(54 \%),(P=0.003)$. The median day after transplant of hospitalization was also earlier on the URD group of patients: day +161 (range 13-1095) for autoSCT; +75 (range 20-732) for RD aloTPH and +69 (range 34-575) for NRD aloTPH patients. The principal cause of readmission was infectious disease, followed by GVHD for the alloSCT group. Mortality at day +100 after SCT was 1 ' $5 \%$ among autoSCT, and $4 \%$ and $13 \%$ among RD and URD alloSCT respectively. Those patients who presented complications during the transplant procedure had a higher readmission rate. Discussion: Infections are the principal cause of readmission in our series. Patients who undergo URD alloSCT have a significantly higher and earlier rate of readmission.

P464

A parameter study of bone turnover and prevention of osteoporosis in patients with acute myeloid leukaemia undergoing allogeneic stem cell transplantation

S. Ganguly (1), L. Graves (2), C. Divine (1), O. Aljitawi (1), S. Abhyankar (1), J. McGuirk (1)

(1)University of Kansas Medical Center (Westwood, US); (2)University of Kansas Medical Center (Kansas City, US)

Background: Osteoporosis is one of the well-known consequences of allogeneic stem cell transplantation (Allo-SCT). Several factors might contribute to this- including high-dose chemotherapy, steroids, radiation, prolonged immobilization and malnutrition. Prophylactic measures including provision of sufficient caloric intake, low-dose vitamin D and calcium 
supplementation do not seem to be enough to prevent bone loss following Allo-SCT.

Methods: In an attempt to evaluate safety and efficacy of bisphosphonates in preventing bone loss after Allo-SCT in patients with acute myelogenous leukemia (AML), we enrolled 17 patients from our institution over a period of 3 years. Bone Mineral Density was measured by dual X-ray absorptiometry (DEXA) scan at baseline and at 6 and 12 months after transplantation. Bone metabolism was measured by urinary crosslinked collagen (NTX) and serum Osteocalcin levels at baseline and at 3,6, 9 and 12 months after transplantation. All patients received $4 \mathrm{mg}$ of zoledronic acid intravenously at baseline and at 6 months after transplantation.

Results: Of the 15 evaluable patients, baseline BMD was within normal limits in 10 patients. Four patients had osteopenia and one patient had osteoporosis. DEXA scores did not change significantly in any patient over time including the patient with osteoporosis. Urinary NTX levels dropped over time in the majority of the patients. Median NTX-Telopeptide values in $\mathrm{nmol} / \mathrm{mmol}$ at baseline, and at 3,6, 9 and 12 months were 60, 53, 32, 20 and 11 respectively $(P<0.05)$. Two patients showed increase in urinary NTX values over time and both of them were being treated with high-dose corticosteroids for graft-versus-host disease (GVHD). In comparison, there was no statistical difference in the serum Osteocalcin levels over time when compared to baseline values in individual patients. Four patients expired from transplant related complications. Four patients did not complete follow-up due to disease relapse $(n=2)$ or complications from infection and GVHD $(n=2)$. None of the SAEs were deemed to be related to the study drug. One patient had grade III hypocalcemia that was related to baseline vitamin D deficiency and was treated appropriately without any adverse outcome.

Conclusion: In this pilot study, zoledronic acid ( $4 \mathrm{mg} / 6$ months) was found to be safe in patients with AML undergoing Allo-SCT and is effective in preserving bone mass by decreasing excessive bone loss in the immediate post transplantation period.

\section{P465}

Use of a calcium phosphate mouth rinse for prevention of oral mucositis after haematopoietic stem cell transplantation: single-centre experience

P. Rzepecki, A. Wasko-Grabowska, S. Oborska, B. Mlot Institute of Health Services (Warsaw, PL)

Oral mucositis is a common complication of high-dose chemotherapy and radiotherapy followed by hematopoietic stem-cell support (HSCT). We evaluated the efficacy and safety of calcium phosphate mouth rinse (Caphosol) for prevention and reduction of severity and duration of oral mucositis (OM) in patients treated with HSCT. In 200923 patients were treated. Three received allo-: 2-HD, 1-MM and 20 autologous transplantation: 9-NHL; 8-HD; 3-MM. For ALLO conditioning regimens were composed of: fludarabine $150 \mathrm{mg} / \mathrm{m}^{2}$ and melphalan 140 $\mathrm{mg} / \mathrm{m}^{2}$. AUTO were treated with: BEAM(HD/NHL) and melphalan $200 \mathrm{mg} / \mathrm{m}^{2}$ (MM). Caphosol was administered 4 times daily, starting from the day before the beginning of chemotherapy till the end of hospitalization. Control group was composed of patients, who had been treated with HSCT previously, before "palifermin and caphosol era". The groups were comparable according to number of patients, their age, type of disease, transplant and conditioning regimen. OM was assessed with the use WHO oral-toxicity scale. Treatment with antibiotics was started when neutropenic fever occurred. The number of days with painkillers or antibiotics were estimated. Statistical analysis was performed using Wilcoxon` test for analysis of differences between groups.

Results: In patients treated with calcium phosphate mouth rinse oral mucositis grade 2-4 was not observed. Nobody had to receive opioid analgesics or total parenteral nutrition. $30 \%$ patients developed the first degree of oral mucositis $4-5$ days' duration. In the control group OM was observed in all cases, $50 \%$ patients had III-IV degree. Median duration of OM was
10 and 12 days (range 5-20) for auto- and allogeneic patients, respectively. As compared with control group, treatment with calcium phosphate mouth rinse was associated with significant reduction of the incidence of oral mucositis in II-IV degrees ( 0 percent versus 50 percent, $P<0.001$ ), duration of oral mucositis ( $4 / 5$ days vs. $10 / 12$ days, $P<0.001)$, duration of pain-killers treatment $(0-3$ days vs. $0-22$ days, $P<0.001)$ and number of days with antibiotics' treatment ( $0-7$ days vs. $7-20$ days, $P=0.002$ ). These differences were observed in both types of transplantation. No side effects of calcium/phosphate oral rinse were observed.

Conclusion: Calcium phosphate mouth rinse is a very promising medicine for prevention of oral mucositis for patients treated with high dose chemotherapy supported with hematopoietic stem cell transplantation.

\section{P466}

Frequency and prognosis of patients with graft failure after allogeneic stem cell transplant. Two centres experience

C. Ferra (1), R. Duarte (2), R. Guardia (3), I. Sánchez-Ortega (2), M. Morgades (1), J.M. Sancho (1), M. Batlle (1), M. Arnan (2), A. Fernández de Sevilla (2), D. Gallardo (3), E. Feliu (1), J.M. Ribera (1)

(1)ICO-H. Germans Trias i Pujol (Badalona, ES); (2)ICO-H. Duran i Reynals (Hospitalet Llobregat, ES); (3)ICO-H. Josep Trueta (Girona, ES)

Background and aims: Graft-failure (GF) is an infrequent and poor complication of allogeneic SCT (alloSCT). Strategies for reversing GF will depend on the options available in each situation. We report the frequency and outcome of adult patients with GF in two transplant centers over a 4-yr. period.

Patients and methods: From January 2006 to October 2009 171 alloSCT have been performed in two institutions. Stemcell progenitors were from unrelated cord blood (UCB) in 20, from unrelated peripheral blood (PB) in 37 and from related PB in 113 patients. Conditioning therapy was myeloablative (Myel) and non-myeloablative (N-Myel) in 77 and 94 patients, respectively. Primary GF was defined as ANC $>0.5 \times 10^{\%} / \mathrm{L}$ not reached for three consecutive days by day +28 after SCT from PB progenitors and by day +60 after SCT from UCB progenitors. Secondary GF was defined as a recurrent ANC $<0.5 \times 10^{9} /$ for at least 7 days.

Results: Eleven patients $(6.4 \%$, median age $47 \mathrm{yr}$. [23-62], diagnoses: AML [7], lymphoma [3] and ALL [1]) presented GF. Characteristics of SCT and GF treatment are shown in Table 1. Characteristics of 2nd SCT and patients' outcome are shown in Table 2.

The 2-yr. estimated probability of survival was $51 \%(95 \% \mathrm{Cl}$ : $19 \%-83 \%)$. No differences were found in GF incidence according to the graft source or the type of conditioning.

Conclusions: The incidence of GF in the present series was $6 \%$, without differences according to the graft source or the intensity of the conditioning regimen. The prognosis was poor, although some patients presented long survival if successful recovery of GF was obtained.

Supported by grants P-EF/09 of FIJC and RD06/0020/1056 of RETICS. 


\begin{tabular}{|c|c|c|c|c|c|c|}
\hline & 1st SCT & Hatching & Condtioning & GF & Chimerism at of & Gf Treatment \\
\hline ACE & UCB & 6.6 unrel. & Myel. & Pimary & Mxed & and SCT \\
\hline JPV & PBSC & Rel, matched & N-Myel. & Primary & Pstent & and SCT \\
\hline FBM & UCB & 45 Unrel. & Myel. & Pimary & Patent & Ind SCT \\
\hline APE & UCB & 45 unrel. & Myel. & Primary & Potent & and SCT \\
\hline DLD & PBSC & Rel. matched & N-Myed. & Pimary & Patent & and SCT \\
\hline THC & PBSC & Rel, $\mathrm{Mm}(\mathrm{CAg})$ & N-Myed. & Primary & Pstent & and SCT \\
\hline JPCE & PBSC & Rel. matched & Myel. & Primary & Mxed & Wat-and-see \\
\hline REP & PBSC & Rel. matched & N-Wyel. & Pimary & Mxed & and SCT \\
\hline 158 & PBSC & Peal, matched & Wyel. & Pimary & Potent & 2nd SCT \\
\hline AVR & PBSC & Rat. matched & N-Myel. & Pimary & Maxed & and SCT \\
\hline JCH & PBSC & Unrel. matched & N-Myed. & Secondary & Potsent & and SCT \\
\hline
\end{tabular}

\begin{tabular}{|c|c|c|c|c|c|c|}
\hline & Condtioning & Donor & Chimerism & Status & Last follow-up & Cause of death \\
\hline ACB & FLU-ATG & Ret. Mm ( $(2 \mathrm{Ag})$ (Father) & 2nd donor & Ave & +13 months & . \\
\hline JPV & ATO & seme donor & Atobgous & Nwe & +72 monts & . \\
\hline гвн & None & Aldologous & Abologous & Alve & 15 monhs & . \\
\hline APG & FLU-ATO & Rel. Mm (BAg) (sister) & NA & Dead & 2 monhts & ritection \\
\hline DLO & CY-ATO & Rat. matched & and donor & Ave & +26 months & - \\
\hline тис & $\mathrm{TN} \mid+\mathrm{ATB}$ & Seme denot & NA & Dead & 5 monts & raction \\
\hline JPCE & - & - & 1:tandonat & Ave & +29 monhs & . \\
\hline REP & ATO & Same donor & 1 twend donor & Ave & +24 monns & . \\
\hline iss & ATO & samedencr. & NA & Dead & 3 monns & rection \\
\hline QVRR & 1da-FLAG-Hed & Seme donor & NA & Dead & 8 monhs & CNS henoritinage \\
\hline ЈсH & $T N \mid+A T G$ & Seme dencer & Atoblogous & Dead & 21 months & Relapse \\
\hline
\end{tabular}

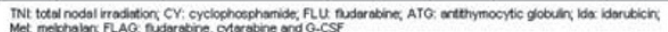

\section{P467}

Cystatin C level as a marker of renal function in allogeneic haematopoietic stem cell transplantation

K. Ohashi, H. Muto, T. Kobayashi, T. Yamashita, H. Akiyama, H. Sakamaki

Tokyo Metropolitan Komagome Hospital (Tokyo, JP)

Hematopoietic stem cell transplantation (HSCT) recipients have an increased risk of acute kidney injury (AKI) or chronic kidney disease (CKD). However, serum creatinine level may underestimate the prevalence of these kidney complications because of decreased lean body mass or concurrent liver disease, which was frequently observed in a HSCT setting. Cystatin C measurement may be more sensitive for detecting impaired kidney function. We retrospectively reviewed medical records of 75 allogeneic HSCT recipients and evaluated cystatin $\mathrm{C}$ as a potential marker of subsequent renal dysfunction. AKI developed in 31 of 75 patients after a median of 46 days (range, 1-502 days), while worsened stage of CKD was observed in 21 patients during the observational period. Cystatin $\mathrm{C}$ level was significantly elevated after allogeneic transplantation $(P<0.001)$. Multivariate analysis revealed use of calcineurin inhibitors as a major cause of cystatin $C$ elevation (odds ratio, $7.26 ; P=0.04$ ). Cumulative incidence of CKD worsening ( $30 \%$ by day 100 ) was significantly higher in the group with cystatin $\mathrm{C}>0.90 \mathrm{mg} / \mathrm{L}$ than in the group with cystatin $\mathrm{C}<0.90 \mathrm{mg} / \mathrm{L}$ (Figure 1). Cystatin $\mathrm{C}$ was elevated in the creatine blind area (GFR 40-70 $\mathrm{ml} / \mathrm{min}$ ) (Figure 2). A strong correlation was also seen between $1 /$ cystatin $C$ and eGFR $(r=0.815$, $P<0.001)$.

Cystatin $\mathrm{C}$ measurement could provide a useful clinical tool to identify hematopoietic stem cell transplantation recipients at increased risk of CKD.

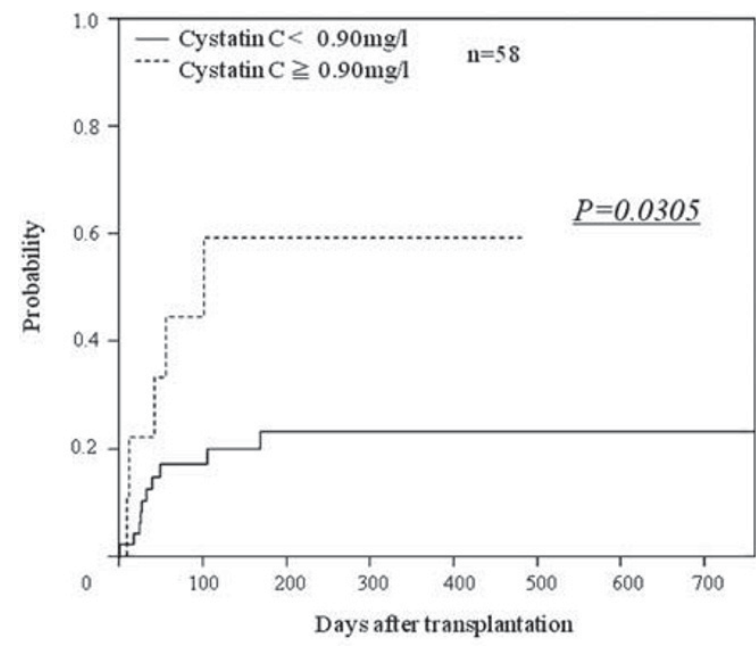

Fig.1 Cumulative incidence of CKD

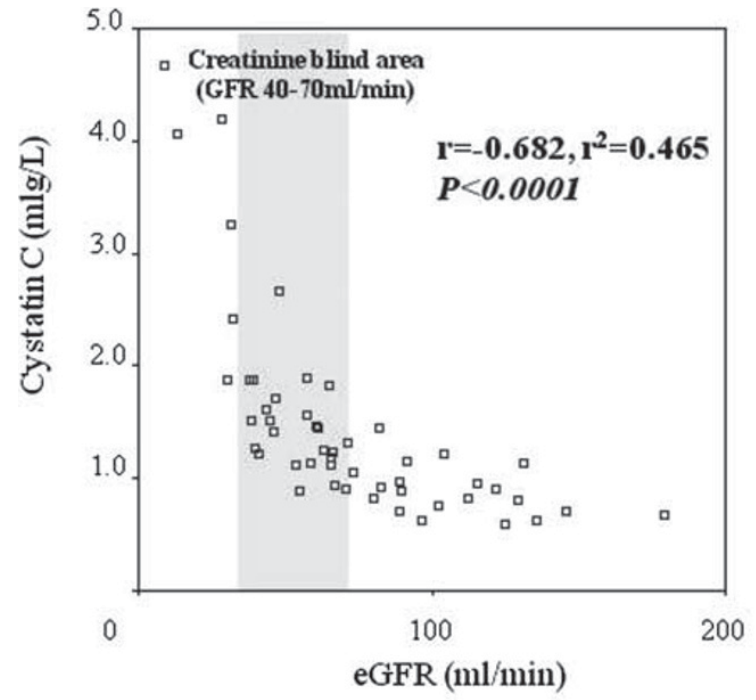

Fig. 2 Correlation between cystatin $\mathrm{C}$ and eGFR

\section{P468}

Severe haemorrhagic cystitis after allogeneic haematopoietic stem cell transplantation: effectiveness of continuous bladder irrigation in prevention, and early cystoscopy, fulguration and instillation of haemostatic agents for treatment

A. Ibrahim, A. Abdou, A. Mugharbil, N. Yassine, G. Nsouli, R. Jalloul, T. Jisr, K. Sayyid

Makassed Hospital (Beirut, LB)

From 9/1997 and 9/2008, 92 pts underwent myeloablative allogeneic haematopoietic stem cell transplantation (HSCT) from HLA identical familial donors. Since $1 / 05$, continuous bladder irrigation (CBI) with normal saline (NS) has been added to hyperhydrationforced diuresis (H-D) and Mesna for the prevention of haemorrhagic cystitis $(\mathrm{HC})$. Nine $(9.8 \%)$ of the 92 pts developed $\mathrm{HC}$. Median age was 18 y (9-34). Five pts were females and 4 males. The diagnosis was CML: 4 pts, AML: 3 pts, beta thalassemia: $1 \mathrm{pt}$ and Fanconi anemia: $1 \mathrm{pt}$. All the pts received $\mathrm{Cy} \pm \mathrm{Bu}$ based conditioning regimens. Median time for the diagnosis of $\mathrm{HC}$ was $18 \mathrm{~d}(2-57)$. HC was of early-onset e (prior to 72 hours following the completion of conditioning) in 4 pts and of late-onset in 5 pts. $\mathrm{HC}$ was severe in all cases. Because all pts were refractory to $\mathrm{H}-\mathrm{D}$ and $\mathrm{CBI}$ with NS, cystoscopy $(\mathrm{C})$ with fulguration $(\mathrm{F})$ were performed in a median interval of $21 \mathrm{~d}(3-29)$ after the onset of 
HC. Instillation of formalin $4 \%$ during $C$ was done in 8 pts. For these 8 pts, median interval between $\mathrm{C}$, F, formalin $4 \%$ instillation and the resolution of $\mathrm{HC}$ was $6.5 \mathrm{~d}$ (3-18). Two pts developed a relapse of $\mathrm{HC} 30 \mathrm{~d}$ and $32 \mathrm{~d}$, respectively after the resolution of the first episode. Both pts were retreated with $\mathrm{F}$ and formalin $4 \%$ instillation. They obtained a complete resolution of their $\mathrm{HC} 3 \mathrm{~d}$ and $18 \mathrm{~d}$, respectively after treatment. The pt who underwent $\mathrm{C}$ and $F$ without installation of formalin $4 \%$ needed $49 \mathrm{~d}$ for the resolution of $\mathrm{HC}$. We failed to confirm an association between GVHD and $\mathrm{HC}$ since $3(33.3 \%)$ out of the 9 pts who developed $\mathrm{HC}$ had GVHD, while $20(24 \%)$ out of 83 pts without HC developed GVHD $(P=0.84)$. There was a significant association between CMV infection and HC since $15(18 \%)$ out of the 83 pts without $\mathrm{HC}$ developed CMV infection while 5 (55.5\%) out the 9 pts with $\mathrm{HC}$ developed $\mathrm{CMV}$ infection $(P=0.03)$. CMV infection preceded the recurrence in the 2 pts who recurred of $\mathrm{HC}$. Polyoma BK virus was investigated in only 2 of the 5 pts who develop late-onset $\mathrm{HC}$, and $\mathrm{BK}$ viruria was negative. Two of the 8 pts treated with instillation of formalin $4 \%$ developed $18 \mathrm{~m}$ after HSCT contracted bladder, hydronephrosis and renal failure. Double $\mathrm{J}$ catheter insertion then augmentation cystoplasty was done in one pt; cystosplasty and renal transplantation were planed for the other pt. Our study confirmed the effective role of CBI (added since 1/05) in HC prevention since $8(18.2 \%)$ out of the 44 pts allografted before 1/05 developed $\mathrm{HC}$ while one $(2.1 \%)$ out the 48 pts allografted after 2005 developed $\mathrm{HC}(P=0.025)$.

\section{P469}

Frequency and risk factors for motor disability in early phase of haemopoietic stem cell transplantation

A. Tendas (1), R. Cinque (1), A. Belleggia (1), A. Spagnoli (2), L. Cupelli (1), A. Siniscalchi (1), T. Caravita (1), A. Bruno (1), F. Cantoni (1), I. De meis (1), P. Niscola (1), T. Dentamaro (1), W. Arcese (2), P. De Fabritiis (1)

(1)S. Eugenio Hospital (Rome, IT); (2)Policlinico Tor Vergata (Rome, IT)

Background: Motor disability (MD) defined as impairment of daily living activities (ADL) related to motor function limitations is frequently observed in hematological malignancies, although rarely explored and reported in patients (pts) undergoing stem cells transplantation (BMT).

Patients and methods: With the aim to assess MD, we studied all pts admitted to BMT during an observation period of 24 months. ADL were tested with Barthel Index (BI) at entry and then weekly; MD was diagnosed in the presence of $\mathrm{BI}$ reduction and classified as: mild $(\mathrm{BI}=67-99 \%)$, moderate $(\mathrm{BI}=33-66 \%)$ and severe $(\mathrm{BI}=0-32 \%)$. Pts with normal $A D L$ (without $M D$ ) were also evaluated for motor function limitation.

Results: Pts examined during the observation period were 46, undergoing 51 BMT (40 autologous and 11 allogeneic). Median age was 50 years (21-74). Diagnosis at BMT was acute leukemia in 10 , multiple myeloma in 22 , lymphoma in 16 , myelodisplasia (MDS) in 2, solid tumor (ST) in 1. Disease phase at BMT was complete remission/very good partial remission in 34, partial remission in 13, untreated in 2 (MDS), progression/relapse in 2. At entry, a mild MD was present in 4/51 BMT (8\%) and a motor function limitation was identified in 7/47 BMT $(15 \%)$ with normal ADL. After BMT, MD was present in $13 / 51(25,5 \%)$, classified as mild in $7 / 13$, moderate in $1 / 13$, severe in $5 / 13$. At discharge, MD was completely, partially or not corrected in 5,4 and 4 pts respectively, so that $8 / 51$ pts $(16 \%)$ were disabled at discharge. Disability was present in $26 \%$ of assistance weeks.

In order to assess risk factors, analysis was then limited to pts with normal ADL at entry (47 BMT). In univariate analysis, age, diagnosis, disease status at transplant and number of lines before transplant were not significantly correlated to risk of MD development. In multivariate analysis, a strong correlation between MD development and both motor function limitation at entry (odd ratio $=13.2 ; \mathrm{Cl} 95 \%=1.6-106.9: P=0.015$ ) and allogeneic versus autologous SCT (odd ratio $=11.2 ; \mathrm{Cl} 95 \%=1.6-$ $75.3 ; P=0.013$ ) was found.
Conclusion: In our experience, MD is a frequent feature in the setting of hemopoietic stem cells transplantation, with more than $1 / 4$ pts experiencing an ADL impairment related to motor function limitation. Identification of risk factors for ADL impairment may help to plan preventive programs. A reduction of MD development, with both pts quality of life improvement and caregiver work-load spearing, is strongly expected.

\section{P470}

Cytopenias after unrelated donor haematopoietic stem sell transplantation: a single-centre retrospective study D. Mallouri (1), C. Apostolou (1), I. Batsis (1), A. Karpouza (1), A. Barbouti (1), A. Tsompanakou (1), E. Parapanisiou (2), I. Sakellari (1), A. Anagnostopoulos (1)

(1)G.Papanicolaou (Thessaloniki, GR); (2)Hippokration Hospital, (Thessaloniki, GR)

Cytopenias consist a well recognized multifactorial complication of allogeneic hematopoietic cell transplantation (HCT). We retrospectively analyzed 64 patients (pts), aged 25 (5-54) years, 44 male, allografted for Acute Leukemia 47, MDS 2, CML 6, HL 1, NHL 4, Aplastic Anemia 3 and X-ALD 1; 27 were in complete remission (CR) (11 in CR1), 32 in refractory and 5 in minimal residual disease. Patients received full (26) or partially (38) matched unrelated donor (MUD) marrow (26) or peripheral blood (38) grafts with a median CD34 + cell dose of $4.55 \times 10^{6} / \mathrm{Kg}$. ABO incompatible were 38 pts (major 15 , mixed $5)$. The conditioning regimen was myeloablative in $63 / 64$ pts, TBI-based in 20/64, with ATG (57) or alemtuzumab (7). Engraftment occurred in $62 / 64$ with neutrophil $>500 / \mathrm{mm}^{3}$ and platelet $>50000 / \mathrm{mm}^{3}$ in 50 pts at a median of 17 days (10-36 and 11-57 respectively). Complete donor chimerism was achieved in a median of 15 days ( +13 day to 4 months) in 39/47 with STR evaluated patients. Thirty three/63 pts developed aGVHD (21 $\geq$ grade II) and 32/55 chronic (20 extensive). Cytopenias were defined as values below normal, i.e. neutrophils $<1000 / \mathrm{mm}^{3}$, platelets $<100000 / \mathrm{mm}^{3}, \mathrm{Hb} \leq 10 \mathrm{~g} / \mathrm{dl}$ post stable engraftment. Their incidence was $66 \%$ (40/61 of evaluable pts): thrombocytopenia (14), pancytopenia (13), anemia (2), thrombocytopenia with anemia (7) or neutropenia (4), presenting at a median of 63 days ( 1 to 8 months). They were associated with refractory disease (8), GVHD (8), GVHD complicated by microangiopathic hemolytic anemia (MAHA), infections or disease (6), MAHA (7), viral infection (3), ABO incompatibility (3), EBV lympoproliferative disease (3), graft failure (1) and hypersplenism (1). Interestingly the incidence of cytopenias was higher in pts who

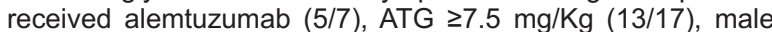
with female donor (8/11) and clinical significant GVHD (15/21 grade $\geq 2$ acute and 14/20 extensive chronic). Patients were treated according to the underlying cause of cytopenias including growth factors, immunotherapy and donor lymphocyte infusion. In the whole cohort of pts $44 \%$ are alive. The outcome was worse in pts with cytopenias (38\% alive) mostly associated with original disease, GVHD and infections complicated by major hemorrhagic events in 2 pts. Cytopenias post HCT remain a complicated, clinically significant entity and its high incidence is possibly related to immune modulations in MUD HCT.

\section{P471}

Kinetics of transplant-related organ injury and recovery after haematopoietic stem cell transplantation using serial assessment of toxicity

A. Chou, M Guo, J. Tay, H. Atkins, I. Bence-Bruckler, M. Sabloff, L. Huebsch, D. Allan

University of Ottawa (Ottawa, CA)

Hematopoietic stem cell transplantation (HSCT) is associated with significant risk of regimen-related toxicity. Grading scales that describe peak toxicity in individual organ systems do not provide temporal information related to onset and recovery. The impact of new interventions aimed to reduce toxicity could be 
better evaluated and compared using a clinical tool that considers the kinetics of organ injury and subsequent recovery. Serial assessment of organ-specific toxicity was performed using the Seattle criteria for patients undergoing HSCT in 57 patients undergoing autologous $(n=39)$ and allogeneic $(n=17)$ transplantation. Oral mucositis (OM) and hepatic toxicity were more severe following allogeneic HSCT than in autologous transplant $(P<0.05)$. OM occurred with a mean toxicity grade of at least 0.5 between days 5 and 15 for autologous transplant recipients and a mean toxicity grade of 1.5 occurred between days 10 and 20 for allogeneic recipients. Resolution of OM was concomitant with hematopoieteic engraftment in the autologous cohort whereas significant OM persisted beyond engraftment in the allogeneic recipients, perhaps owing to added toxicity from methotrexate administered post-transplant to prevent GVHD. The pattern of gastrointestinal toxicity was similar for the allogeneic and autologous groups with gradual resolution following hematopoietic engraftment. A stable but low degree of renal toxicity was observed after allogeneic transplant whereas a second spike of toxicity occurred in autologous recipients following hematopoietic engraftment. Genitourinary, pulmonary, cardiovascular and neurological toxicity was uncommon. The pattern of toxicity is organ-specific and in some cases, recovery was observed concomitant with hematopoietic recovery, suggesting a role for cell-mediated repair. In summary, serial assessment of toxicity provides a means for objective comparison of regimenrelated toxicity in various transplant strategies and may offer potential insight regarding mechanisms of repair.

\section{P473}

Comprehensive symptom profile for symptom assessment in patients undergoing bone marrow transplantation/peripheral stem cell transplantation: applicability and characteristics

A. Novik (1), T. Ionova (2), S. Kalyadina (2), D. Fedorenko (1), N. Mochkin (1), T. Nikitina (2), K. Kurbatova (2), G. Gorodokin (3) (1)Pirogov National Medical Surgical Center (Moscow, RU); (2)Multinational Center of Quality of Life Research (St. Petersburg, RU); (3)New Jersey Center for Quality of Life and Health Outcome Research (New Jersey, US)

Symptom severity is an important treatment outcome for patients undergoing bone marrow transplantation/peripherial stem cell transplantation (BMT/PSCT) Comprehensive symptom assessment and monitoring at early and long-term posttransplantation period is worthwhile. We aimed to develop a new symptom assessment tool - Comprehensive Symptom Profile in Patients Undergoing BMT/PSCT (CSP-BMT) and test its applicability in this patients population.

CSP-BMT is developed to assess the severity of $60 \mathrm{symp}-$ toms specific for patients undergoing BMT/PSCT. It consists of numerical rating scales, scored from "0" (no symptom) to "10" (most expressed symptom). There have been identified 15 clusters of symptoms which were clinically relevant and increased practicability of the tool. Applicability of CSP-BMT with preliminary analysis of psychometric properties was tested in the pilot study which included 58 patients with hematological malignancies and autoimmune diseases who underwent autologous hematopoietic stem cell transplantation (mean age-34.5; male/female-24/34).

The utility of CSP-BMT was shown: all the items were easy to read and understand by the patients; the data of the tool were clear for interpretation by physicians and were used by them in clinical decision making.

Reliability of CSP-BMT was satisfactory (Chronbach's alpha coefficient varied from 0.70 to 0.96 ). The construct validity of CSP-BMT was proved by factor analysis. High sensitivity to changes in symptom status was demonstrated by statistically significant difference $(P<0.05)$ in symptom severity at different time-points post transplant.

Thus, CSP-BMT is an appropriate and practical tool to assess symptom severity in patients before, during and after BMT/
PSCT. The utility of the questionnaire was shown; preliminary psychometric properties appeared to be satisfactory. Further studies are needed before the use of CSP-BMT in clinical practice and clinical trials.

\section{P474}

Plasma endothelin-1, transforming growth factor-beta, fibroblast growth factor and nitric oxide levels as a predictor of liver injury in post-transplant long-term iron overloaded haematopoietic stem cell transplant recipients S.Z. Aký, E. Suyani, H. Pasaoglu, S. Ozenirler, S. Elbeg, G. Sucak

Gazi University Faculty of Medicine (Ankara, TR)

Introduction: Graft versus host disease (GvHD), and iron overload (IO), might contribute to the development of chronic liver injury directly or via the induction of cytokines and formation free radicals. The aim of our study was to analyze the role of plasma fibrogeneic mediators transforming growth factor- beta (TGF- beta), fibroblast growth factor (FGF), endothelin- 1 (ET1) and nitric oxide (NO) levels as a predictor of liver injury in long- term iron overloaded AHSCT recipients.

Patients and methods: Fifthy-four AHSCT recipients (17 women, 37 men), with a median age of 25 years (range 16-53 years) were enrolled in the study. Serum samples for the analysis of endothelin-1, TGF- beta, FGF, NO, ferritin, transferin saturation (TS), non- transferin bound iron (NTBI), and liver function tests were obtained at the 1 year post- transplant follow- up visit. Patients were grouped according to the serum ferritin levels as $<1000 \mathrm{ng} / \mathrm{ml}$ vs. $\geq 1000 \mathrm{ng} / \mathrm{ml}$.

Results: Serum NTBI levels showed a significant positive correlation with serum ferritin $(r=, 471, P=0,000)$ and $T S(r=, 272$, $P=0,047)$. Serum NO levels positively correlated with the serum TS $(r=, 284, P=0,037)$ and creatinin levels $(r=, 283, P=0,038)$. However serum levels of ET- 1 negatively correlated with NO $(r=-, 366, P=0,006)$ and ferritin levels $(r=-, 264, P=0,053)$. Serum alanin amino transpherase (ALT) levels were positively correlated with ferritin and TS $[(r=, 279, P=0,04)$ and $(r=, 308$, $P 0,02)$ respectively]. Serum levels of TGF- beta and FGF did not show any correlation with iron parameters. No correlation was found with serum NO, TGF- beta and FGF levels even with serum ferritin levels $\geq 1000 \mathrm{ng} / \mathrm{ml}(P<0,05)$. Higher ferritin group patients with hepatic GvHD had significantly lower levels of serum ET- 1 when compered to lower ferritin group.

Conclusion: Serum ferritin and TS positively correlated with serum NTBI, and ALT levels which suggests the role of IO in hepatocellular injury in the survivors of AHSCT. Although there was a near significant increase in serum FGF levels in patients with a history of GVHD associated transaminitis, other fibrogenic cytokines did not show any correlation with high ferritin levels. AHSCT recipients should be screened for $\mathrm{IO}$ and removing the excess iron with iron chelators should be considered to prevent the development of hepatocellular injury.

\section{P475}

Evaluation of usefulness of co-morbidity index HCT-Cl and PAM for predicting prognosis of HCT in Japanese patients

K. Tokita

Dokkyo University of Medicine (Mibu City, JP)

Hematopoietic stem cell transplantation comorbidity index (HCT-Cl) and Pretransplantation Assessment of Mortality (PAM) score have been reported useful for predicting mortality risk in patients undergoing $\mathrm{HCT}$. There are several reports from Europe or United States but a few reports from Japan. Here we evaluated the usefulness of both HCT-Cl and PAM scores in Japanese patients who have underwent for SCT.

Totally 68 patients who underwent HCT between January 2000 and December 2006 were enrolled in this analysis. Sixty received HCT under myeloablative preconditioning regimen and 8 under 
non-myeloablative regimen. The median age at transplantation was 39 years. Twenty-four received HLA-matched sibling grafts and 1 received graft from HLA-mismatched sibling donor, and 43 received HLA-matched unrelated grafts. Hemapoietic stem cells were obtained from peripheral blood in 12 , BM in 49 and cord blood in 9 and peripheral blood + BM in 2. Thirty-six\% of patients had scores 1 or more by HCT-Cl (infection $n=7$, liver dysfunction $n=5$, history of solid tumor $n=2$, renal dysfunction $n=1$, diabetes mellitus $n=4$, obesity $n=1$, arrythmia $n=1$, cerebrovascular disease $n=1$ psychiatric disturbance $n=1$, moderate pulmonary disease $n=1$, cardiac disease $n=1)$. HCT-Cl provided discrimination of overall survival (OS) clearly. Whereas PAM score for low intermediate risk and high intermediate risk account for $95 \%$ of the patients and it was difficult to assess the difference of OS among different risk groups.

For conclusion, in our analysis, HCT-Cl enabled to discriminate the risk of HCT patients and was proved to be useful for predicting OS in Japanese patients. However PAM score could not discriminate the risk of HCT patients because $95 \%$ of them were sorted to intermediate risk. Explanation for the difficulty for applying PAM score to our patients might be the stringent selection for HCT patients in our institute. Moreover there is a few patients who have severe obesity and evaluation of renal dysfunction using creatinine level maybe taken severe in Japanese patients who were smaller than patients in Europe or United States and using the creatinin clearance may be appropriate. It might be necessary to improve the index to apply Japanese patients.

\section{P476}

Late effects of haematopoietic stem sell transplantation in children

M.Y. Lu, S. Jou, D.T. Lin, K.H. Lin, H.H Chang, Y.L. Yang National Taiwan University Hospital (Taipei, TW)

Background: As survivors of pediatric hematopoietic stem cell transplantations (HSCTs) increase in number, it is increasingly important to evaluate their well-being. Late effects following HSCT are related to underlying disease process, the transplant process and/or the transplant preparative regimens. Problems related to the disease include relapse and rejection. Problems related to the transplant process include delayed recovery of immune system and chronic graft-versus-host disease (GVHD). Problems related to the transplant preparative regimens include those involving the endocrine system, eyes, lungs, bone and development of secondary malignancies. The incidence of late effects must be assessed. Methods: Among 270 HSCTs in our hospital from August 1986 to December 2007, 161 patients (99 malignant/62 non-malignant, 25 autologous/136 allogeneic) survived over 1 year after engraftment. Here we reported the late effects in these patients before disease relapse or death.

Results: After a median follow-up of 83 months, the 31 patients $(19.3 \%)$ were mortal in the follow-up period. The causes of death were relapse $(24,77.4 \%)$, chronic GVHD with infection $(4,12.9 \%)$, infection $(2,6.5 \%)$, secondary malignancy $(1,3.2 \%)$ and others $(2,6.5 \%)$. Two 2 patients $(1.2 \%)$ had late rejection. Totally 51 patients $(51 / 136,37.5 \%)$ had chronic GVHD (21 mild and 30 extensive). Among 81 patients received thyroid function tests, 2 patients had hypothyroidism with thyroxin replacement therapy, 1 had Hashimoto disease and 5 patients had compensated hypothyroidism. 79 patients received gonadal function study and 27 patients had hypogonadism. One thalassemia major patient had diabetes. In pulmonary dysfunction, there were 4 patients with bronchiolitis obliterans with obstructive ventilatory defect and 5 patients with restrictive ventilatory defect. One patient had avascular necrosis with artificial hip replacement. New neoplasms developed in 8 patients.

Conclusion: HSCTs is a life-saving procedure for an increasing number of children. As an increasing number of patients are long term survivals, late effects are emerging and impacting the mortality and morbidity of these survivors. As more experience is gained, changes in the incidence and approaches to diagnosis and treatment is likely to occur.
P477

Neurologic complication in leukaemia patients after allogeneic stem cell transplantation

M. Ramzi, H. Haghighi Nejad

Shiraz University of Medical Sciences (Shiraz, IR)

Objective: Neurologic complication after allogeneic stem cell transplantation are common and life threatening. The objective of this study was to determine the spectrum and frequency of neurologic complication after allogeneic hematopoietic stem cell transplantation (HSCT) in leukemia patients and to determine a risk profile in these patients during a fourteen years period in our center.

Materials and methods: The records of all patients who received a HSCT for leukemia at Nemazee hospital in Shiraz University of medical sciences between1993-2007 were reviewed. A total of 105 patients $(A M L=55, A L L=26, C M L=24)$ were included in this study. We had 37 female and 68 male patients with mean age of 24.4 year. Conditioning chemotherapy regimen included busulfan14-16 mg/kg and cyclophosphamide $120-200 \mathrm{mg} / \mathrm{kg}$. Graft versus host prophylaxis consisted of cyclosporine A in combination of methotrexate. Only severe neurological complications were included in this study. A severe neurological event was defined as neurological event that contributed to significant worsening of the clinical condition of the patient. Patients with encephalopathy and persistent changes in consciousness, clinical findings suggesting brain edema, cranial nerve involvement, hemiparalysis, visual disturbances (diplopia, ptosis, blurred vision) and those with seizure defined as neuro positive.

Results: The patients with neurological events were $33(32.4 \%)$ of cases. There were 55 patients with AML of whom $15(27.2 \%)$ showed neurologic complication and 26 patients with ALL of whom $11(42.3 \%)$ developed neurologic event. The incidence of neuro + was significantly lower in AML than ALL but no significantly different than that in CML (29.1\%). The most common complications were visual disturbance $(19 \%)$, seizure $(6.7 \%)$ and decrease level of consciousness (4\%). Mean serum cyclosporine level in patient with visual disturbance, seizure disorder and decrease level of consciousness were 218, 208 and 227 respectively which were not significantly different from each other.

Conclusion: We concluded that neurological complication are quite frequent in leukemic patients undergoing HSCT. Major causes are conditioning toxicity, metabolic disturbance, viral infection and cyclosporine toxicity. These patients should be observed carefully due to high risk of neurological complication. By close follow up and effective treatment in spite of high incidence, morbidities and mortalities of these complications can be decreased.

\section{P478}

Reinfusion of autologous peripheral blood stem cells after high-dose chemotherapy via a Port-a-cath is a safe and feasible procedure and does not compromise successful engraftment

A. Alietti, G. Andreola, L. Orlando, M. Negri, C. Rabascio, A. Vanazzi, A. Babic, L. Roveda, B. Lucchetti, S. Sammassimo, L. Nassi, M. Clerici, R. Magri, D. Laszlo, G. Martinelli

European Institute of Oncolgy (Milan, IT)

Introduction: Reinfusion of autologous peripheral blood stem cells (APBSC) via a central venous catheter has been shown to be safe and to lead to successful engraftment. Patients (pts) who undergo APBSC transplants may have already an indwelling Port-a-Cath (PAC) because of previous chemotherapy. Positioning of another temporary central venous catheter may be associated with an increase of infectious and thrombotic complications together with pts' distress. Methods: From January 1st 2008 to November 15th 2009, we performed 80 reinfusions of APBSC in 66 pts after high dose chemotherapy. Pts' median age was 48 years (19-69) with a M/ $F$ ratio of 34/32; 60 pts were affected by hematologic malignancies and 6 by testicular cancer. Fifty-eight pts underwent a single APBSC transplant, while 5 and 3 received a double and a triple procedure, respectively. All pts had already placed a PAC in the subclavian vein for previous treatments. All PBSC reinfusions 
were performed via the transcutanous placement of a Huber point needle into the PAC. Before each reinfusion, the presence of bidirectional flow through the PAC was confirmed and for safety reason a peripheral venous catheter was also put in place.

Results: Overall the 80 reinfusion procedures were not associated with serious adverse events except for a $\mathrm{NCl}$ grade 1-2 hypotension and vomiting episode in 1 patient. In the entire study population, the total duration of PAC use for transplant related procedure, was 1396 days with a median of 18 days (1-28). Median number of CD34 + cells/kg reinfused was $3 \times 10^{6}(1.4-$ 11.9). All pts had a successful engraftment; indeed, an absolute neutrophil count $>1000 / \mathrm{mm}^{3}$ was reached in a median of 11 days (10-51) and a platelet count $>20.000 / \mathrm{mm}^{3}$, in a median of 10.5 days (7-53). We also evaluated infectious complications and short-term occurrence of thrombotic events. A positive blood culture from the PAC developed in 1/66 patient during the marrow aplasia in two consecutive transplants and resolved with medica therapy. PAC removal was necessary, after engraftment, only in 1 patient on a total of 1396 days of use, for mechanical complications. No thrombotic events occurred in the short-term.

Conclusions: Reinfusion of APBSC via the PAC is safe and is not associated with infectious and short-term thrombotic complications. Most of all, it is effective and correlates with successful engraftment, which also suggests that it does not cause any significant damage to the CD34 + cells infused.

\section{P479}

Successful engraftment with second allogeneic transplantation as salvage therapy for young patients after graft failure or progressive disease following a first allogeneic stem cell transplantation

J. Elcheikh, P. Ladaique, J.M. Boher, L. Castagna, S. Furst, C. Faucher, A. Vazquez, N.F. Belmechri, C. Chabannon, D. Blaise Institut Paoli Calmettes (Marseille, FR)

GF or relapse is a common and life-threatening complication of SCT. Here, we report our experience of second transplantation of 22 patients treated at our cancer centre at Marseille from January 1992 at today.

Patients' data were collected from a maintained database and by retrospective clinical chart review.

Gender: M/F (15/7); median age at diagnosis was 35 years (17-42). Median age at the first allo-SCT was 38 years (1849); median age at second allo-SCT was 38 years (20-49). 9 patients $(41 \%)$ had acute myeloid leukemia.

The median interval between the two transplants were 10 months (range 0,5-131). The first transplantation was administered after myeloablative conditioning for hematologic malignancies $(n=10)$, reduced intensity conditioning (RIC) $(n=12)$. The second conditioning consisted of Flu and ATG-based nonmyeloablative regimen in $86 \%(n=19)$.

19 patients $(86 \%)$ received a graft from a family donor, 3 patients had a MUD, and 2 patients received cord blood cells donors. 15 patients $(68 \%)$ had the same donor for the two transplantation. 13 patients $(59 \%)$ received the second allo-SCT because of disease progression and 7 patients $(32 \%)$ because a graft failure. All 22 patients, but one, acquired quick and sustained engraftment after the second transplant. Treatment-related toxicity was minimal. Five patients developed acute GVHD (>grade $\|=1$ ). Five patients developed chronic GVHD (limited=3, extensive $=2$ ). In all, only 3 patients died from transplant related mortality (TRM), 11 patients $(79 \%)$ because of relapse or disease progression. Actually 8 patients $(36 \%)$ are still alive with a median follow up of 25 months IC95\% (13.8-52.7). Overall survival at 2 years and 5 years was $63 \%$ and $41 \%$ respectively. And the TRM was $9,4 \%$ at 2 and 5 years.

These results indicate that a second transplant for young patient after GF or relapse is feasible and can be considered as a good option for salvage therapy.

Target population would need careful selection on individual prognosis factors indicating their poor short term outcome: this represents the ultimate goal for future investigations
P480

Multicentre retrospective analysis of second allogeneic HSCT outcomes for haematologic malignancies in Korea K.H. Kim (1), C.W. Jung (2), J.H. Jang (2), K. Kim (2), H.K. Ahn (2), J.H. Won (1), D.S. Hong (3), J.H. Moon (4), S.K. Sohn (4), Y.K. Kim (5), D.H. Yang (5), J.J. Lee (5), H.J. Kim (5), J.S. Kim (6), Y.H. Min (6), D.Y. Kim (7), J.H. Lee (7), J.H. Lee (7), K.H. Lee (7), I. Kim (8), S.S. Yoon (8), S. Park (8), S.M. Lee (9), Y.D. Joo (9), H.J. Jun (10), D.H. Kim (2)

(1)Soonchunhyang University Hospital (Seoul, KR); (2)Samsung Medical Center (Seoul, KR); (3)Soonchunhyang University Hospital (Bucheon, KR); (4)Kyungpook National University Hospital (Daegu, KR); (5)Chonnam National University Medical School (Gwangju, KR); (6)Yonsei University Severance Hospital (Seoul, KR); (7)Asan Medical Center (Seoul, KR); (8)Seoul National University College of Medicine (Seoul, KR); (9)Busan Paik Hospital (Busan, KR); (10)Konyang University Hospital (Daejon, KR)

Background: Increasing number of autologous or allogeneic allogeneic hematopoietic stem cell transplantation (HSCT) performed in Korea in this decade resulted in substantial requirement for second HSCT as a result of the recurrence of primary disease or graft failure. Since Dec 2008, second HSCT has been approved by Korean government to be covered by national health insurance reimbursement system. The current study analyzed the outcome of second allogeneic HSCT because of no available data on the transplant outcomes following second allogeneic HSCT in Korea.

Methods: Transplant data were collected retrospectively from 8 transplant centers in Korea. Inclusion criteria are as follows. 1) age equal or over 15 years old, 2) hematologic malignancies excluding aplastic anemia, PRCA, PNH or solid tumor, 3) patients who underwent second alloHSCT regardless of source of stem cell except cord blood transplantation (CBT).

Results: Sixty four pts were included with following diagnoses: $\operatorname{AML}(n=29), \operatorname{ALL}(n=5), C M L(n=3)$, lymphoma $(n=19)$, myeloma $(n=6)$, and others $(n=2)$. The median age was 37 (range 16-65). The first transplantation had been performed with autologous $38(59.4 \%)$ or allogeneic $26(40.6 \%)$ donors. The donors for second HSCT were HLA-identical sibling $33(47 \%)$, unrelated $31(49 \%)$, or haploidentical donor $2(3 \%)$. Conditioning regimen included TBI-based myeloablative $6(9 \%)$, non TBI myeloablative $19(30 \%)$, or reduced intensity regimen $38(60 \%)$. With median 16 months of follow up (range, 3 to 93 months), 32 pts died of transplant related toxicity $(n=28 ; 70 \%)$, recurrence of primary disease $(n=10 ; 22.5 \%)$ or other $(n=4 ; 9.5 \%)$. After second HSCT, $56 \%$ were in complete remission, $38 \%$ in partial remission, and $6 \%$ were refractory. The 1 - and 2 - year overall survival rate was $46 \%$ and $35 \%$, respectively. In univariate analysis, factor highly significantly associated with better survival were first HSCT (ASCT vs. allogenic HSCT) and conditioning regimen (non-TBI based regimen vs. TBI based regimen).

Conclusion: Second allogenic HSCT is a reasonable option for selected patients in Korean patients with $35 \%$ of 2 year survival rate.

\section{Graft-versus-host disease: preclinical and animal models}

P481

A xenograft model of human skin to study the role of Langerhans cells in graft-versus-host disease

J. Hemmerling, J. Wegner-Kops, D. Wolff, E. von Stebut, M. Sommer,

E.M. Wagner, M. Theobald, R.E. Schopf, W. Herr, R. G. Meyer

Johannes Gutenberg University (Mainz, DE)

Dendritic cells (DC) of the skin, e.g. epidermal Langerhans cells (LC), are potent antigen-presenting cells (APC). After allogeneic 
hematopoietic stem cell transplantation, persisting host APC activate donor $\mathrm{T}$ cells and thereby play a critical role for initiating acute graft-versus-host disease (GVHD). Manipulation or depletion of APC may be a means for preventing this alloreactivity. However, many potential APC-modifying agents like monoclonal antibodies are species-specific and preclinical models are lacking. We therefore generated a model system using human skin xenografts transplanted between the scapulae of NOD/LtSz-scid IL2Rgamma-null (NSG) mice. First, we analyzed the impact of xeno-transplantation on the distribution of LC as well as dermal HLA-DR positive APC in the grafted skin. During wound healing, LC vanished from the epidermis after the second week and were undetectable until they repopulated the dermis $6-8$ weeks after transplantation. In contrast, HLA-DR positive dermal cells were present at any time after transplantation. The re-establishment of human LC in the xenotransplants supports the hypothesis of a local LC-reconstituting population that is independent of precursors from the peripheral blood.

To induce GVHD, CD8 T cells of a HLA-class I mismatched third-party donor were stimulated with DC generated from peripheral blood of the skin-donors in mixed lymphocyte DC cultures (MLDC) with. We injected the allo-reactive MLDC$\mathrm{T}$ cells into the tail veins of mice 8 to 12 weeks after skin transplantation and detected an erythematous reaction in the xenograft approximately 1 week after T-cell transfer. We also found histological signs of acute skin GVHD as well as infiltrating $T$ cells and a loss of LC. This observation is consistent with studies showing that infiltrating allo-reactive donor $\mathrm{T}$ cells deplete persisting host APC. In contrast, using skin from an area of previous gamma-irradiation lacking LC, we were not able to induce skin rash or histological signs of GHVD by injection of allo-reactive T cells.

In summary, we introduce a model-system for acute skin GVHD using human xenotransplantats and adoptive T cell transfer in NSG mice. We also provide data supporting the hypothesis of a local LC-repopulating precursor population in human skin. This model might improve our understanding of the role of APC in human GVHD and help to determine how APC-modulating strategies could contribute to prophylaxis and treatment of GVHD.

\section{P482}

Inhibition of antigen presentation by statins: a potential treatment approach for GvHD

A. Shimabukuro-Vornhagen, S. Zoghi, T. Liebig, M. von Bergwelt-Baildon

University Hospital of Cologne (Cologne, DE)

Statins are lipid-lowering drugs, which interfere with cholesterol synthesis by competitive inhibition of HMG-CoA reductase, the rate-limiting enzyme of the mevalonate pathway. It has recently been shown that statins, apart from their lipidlowering potential, have pleiotropic effects on the immune system and possess anti-inflammatory and immunomodulatory activity. We performed a gene expression analysis comparing resting $B$ cells with CD40-activated B cells, which are potent antigen-presenting cells (APC), and demonstrated that following CD40-activation expression of the enzymes in the mevalonate pathway was up-regulated. In accordance with these results simvastatin inhibited the proliferation and adhesion of resting B cells which were activated via CD40. In addition, the expression of MHC class II (HLA-DR) and the costimulatory molecules CD80 and CD86 was reduced. This inhibition was dose-dependent and doses as low as $0.1 \mu \mathrm{M}$ simvastatin induced a significant reduction of $\mathrm{B}$ cell activation. This resulted in a decreased antigen-presenting capacity of simvastatin-treated B cells as was shown by a reduction in the proliferation of CD4 and CD8 T cell induced by allogeneic simvastatin-treated B cells. The concentration of simvastatin that was required for the inhibition of $\mathrm{T}$ cell proliferation was at least 10 -times higher than that required for the inhibiton of antigen-presentation by $B$ cells. This effect was mainly dependent on the inhibition of mevalonate production since addition of excess mevalonate almost completely restored B cell activation. Surprisingly, the hydrophilic statin pravastatin did not lead to inhibition of the antigen-presenting function of $B$ cells. In addition to these in vitro results we also observed an inhibitory effect of simvastatin in human subjects in vivo. Our results concerning the effects of statins on human APCs in conjunction with the observation made by others that preemptive treatment with a HMG-CoA reductase inhibitor protected mice from graft-versus-host-disease (GVHD) without impairing the graft-versus-leukemia effect provide the rational for further testing of statins in patients undergoing allogeneic stem cell transplantion. The exquisite sensitivity of APCs to statin-mediated inhibition could result in synergistic effects with conventional immunosuppressants such as cyclosporine A which primarily target $\mathrm{T}$ cells.

\section{P483}

Analyses on migration and homing of human alloreactive T-lymphocytes in a NOD/SCID/IL2Rgc-null transplantation model

A. Brunk (1), M. Nonn (1), V. Lang (2), R. Henschler (2), M. Theobald (1), W. Herr (1), U.F. Hartwig (1)

(1)University Medical Center (Mainz, DE); (2)Institute for Transfusion Medicine \& Immune Hematology (Frankfurt/Main, $D E)$

Donor lymphocyte graft engineering to separate graft-versushost (GVH) and graft-versus-leukemia (GVL) reactivity remains of central interest in allogeneic hematopoietic stem cell transplantation (HSCT). Thus, we established a xenograft-transplantation model using humanized NOD/SCID/IL2Rgc-null (NSG) mice to evaluate human GVH- and GVL-immunity of modified donor T cells in vivo.

In order to study the clinical relevance of human $\mathrm{T}$ cell trafficking to tissues in this model we subcutaneously implanted human skin substitutes composed of collagen embedded primary allogeneic fibroblasts into NSG mice and detected murine vascularisation within 2 weeks. Following transfer of HLA-mismatched or haploidentical T cells, up to $23 \%$ of $T$ cells migrated into substitutes explanted 21 days post injection. As this migration and homing involves both murine and human adhesion molecules we further examined the adhesion mechanisms underlying the egress of human $T$ cells from the murine bloodstream. Using laminar flow chamber and real-time video recording experiments we first demonstrated firm adhesion of human, polyclonally prestimulated $T$ lymphocytes but not naive $T$ cells to the murine endothelial cell line bEND.3 at shear stresses of up to $3.5 \mathrm{dynes} / \mathrm{cm}^{2}$ when compared to human umbilical vein endothelial cells used as positive control. Adhesion and transmigration was significantly enhanced upon preincubation of both human and murine endothelial cells (ECs) with TNF-a $(5 \mathrm{ng} / \mathrm{ml})$ to resemble an activated phenotype. Firm adhesion of activated T cells was suppressed following blocking of anti-integrin-a4 (CD49d) or anti-integrin-aL (CD11a) with antibody (Ab) or when ECs were preincubated with anti-VCAM-1 (CD106). In contrast, no inhibition was observed in the presence of antiEndoglin (CD105) Ab included as specificity control suggesting that CD49d and its ligand VCAM-1 as well as CD11a are required for extravasation of human $T$ cells. In vitro studies using primary murine ECs and in vivo blocking experiments are in progress to verify our results and will be reported. As the same ligand-receptor pairs are described for human T-EC interaction these findings indicate a closely related mechanism of T cell extravasation in human and murine endothelium at least in our model.

In conclusion these results suggest that transferred activated human T cells migrate into allogeneic skin substitutes in NSG mice involving VCAM-1 and CD49d as well as CD11a for firm adhesion followed by transmigration. 
P484

Alloantigen-specific de novo induced FoxP3 + regulatory $\mathrm{T}$-cells revert to effector cells and promote the generation of fatal pro-inflammatory cytokine levels in experimental GvHD

C. Koenecke, A. Ganser, R. Förster, I. Prinz

Hannover Medical School (Hannover, DE)

Background: Recently IFN-g producing exFoxP3 cells, i.e. reverted from FoxP3 + to FoxP3-, have been discussed as particularly harmful effector $\mathrm{T}$ cells. Induced antigen-specific FoxP3 + T cells (iTregs) were discussed as a promising alternative to polyclonal natural FoxP3 + T cells for cell-based therapies. However, we have previously shown that iTregs, in contrast to nTregs, failed to prevent lethal GvHD in a C57BL/6 into BALB/c mouse model of acute GvHD. Here we evaluated the potential of induced and natural Treg cells to produce IFN-g and other cytokines.

Methods: Using FoxP3-reporter mice (C56BL/6), we established an efficient protocol to induce and expand alloantigen-specific iTregs from FoxP3-CD4 + T cells with allogeneic cluster-disrupted dendritic cells (BALB/c). In a C57BL/6 into BALB/c mouse model of acute GvHD we monitored the intracellular cytokine expression of the Treg populations using a congenic marker. For measurement of serum cytokines at different days after BMT we used a cytokine bead array.

Results: Both, nTregs and iTreg populations produced massive amounts of IFN-g in vivo at day 4 and day 8 after allogeneic BMT. Even though IFNg secretion was seen in nTregs, groups of mice receiving nTregs were protected from GvHD. However, we detected large amounts of other proinflammatory cytokines such as IL-6, TNFa and MCP-1 only in the serum of control groups without Tregs and in particular in groups of mice that received iTregs. Interestingly, the production of the pro-inflammatory cytokine IL-17 was exclusively observed in exFoxP3 cells, mainly derived from reverted iTregs.

Conclusions: Our data suggest that IFN-g should not be regarded as an adverse pro-inflammatory cytokine since it is readily produced by protective nTregs under the highly inflammatory environment of acute GvHD. Rather, the capacity to produce IL-17 might highlight the dangerous plasticity of induced regulatory $\mathrm{T}$ cell subtypes in contrast to natural Tregs.

\section{P485}

Indirect presentation HLA class II restricted minor histocompatibility antigens is a characteristic of HLA-DM resistant antigens

A.N. Stumpf, E.D. van der Meijden, R. Willemze, J.H.F. Falkenburg, M. Griffioen

Leiden University Medical Center (Leiden, NL)

HLA-matched allogeneic stem cell transplantation (aSCT) is an effective treatment for hematological malignancies. However, T-cells recognizing minor histocompatibility antigens (MiHA) can mediate detrimental graft versus host disease (GvHD). We recently identified $5 \mathrm{HLA}$ II restricted MiHA which were not directly recognized on non-hematopoietic cells even after HLA class II upregulation by cytokine treatment. However, it was recently shown in mice (Wang, ASH 2009, abstract no. 689) that indirect presentation of host antigens by donor antigen presenting cells (APC) contributed to CD4 + T cell mediated GvHD. Here, we analyzed whether this is a general phenomenon by analyzing processing and inter-cellular transfer of six HLA II restricted antigens.

As a model to investigate recognition by $\mathrm{CD} 4+\mathrm{T}$ cells, we introduced genes of six different MiHA as well as their respective HLA class II alleles into the non-hematopoietic HeLa cell line. Antigen processing was analyzed after retroviral transfer of invariant chain (li) with or without HLA-DM. Inter-cellular transfer of antigens from MiHA positive cells to cells expressing the restriction HLA molecules was analyzed by co-incubation of HLA II +/MiHA- cells with HLA II-/MiHA + cells or their supernatants.

Recognition of all HLA class II restricted MiHA was increased upon transduction of li. Additional transfer of HLA-DM, however, had divergent effects. Following HLA-DM transfer 3 of the MiHA were similarly recognized (DM-resistant), whereas recognition of the other $3 \mathrm{MiHA}$ was completely abolished (DMsensitive).

We observed inter-cellular transfer of DM-resistant, but not DM-sensitive, MiHA to MiHA-/HLA II + cells after co-incubation with $\mathrm{MiHA}+/ \mathrm{HLA}$ II- cells. Also in the absence of direct cell-cell contact the antigen could be transferred by cellderived particles as was shown by incubation with supernatant from MiHA + cells. This transfer was not mediated by free peptides as $30 \mathrm{kDa}$ filtration of the supernatant abolished recognition.

In summary, we show that some antigens can be inter-cellularly transferred leading to $\mathrm{T}$ cell recognition of MiHA-/HLA $\mathrm{II}+$ cells. However, this phenomenon was restricted to a group of antigens that showed HLA-DM-resistant processing and presentation. These data suggest that late after aSCT, when patient-derived APCs are replaced by the donor, only HLA-DM-resistant MiHA may be indirectly presented by donor $\mathrm{APC}$, and therefore not every MiHA mismatch may evoke late onset GvHD.

\section{P486}

Primary alloreactive response to minor histocompatibility antigens with a pro-inflammatory (Th1) differentiation of purified CD4 + naive (CCR7 + cD45RA +) T-cells

M. Cherel (1), S. Thiant (1), J. Trauet (1), P. Cracco (1), F. Dufossé (1), J.P. Dessaint (1), I. Yakoub-Agha (2), M. Labalette (1)

(1)Immunologie (Lille, FR); (2)UAM Allo-CST (Lille, FR)

Background: Allogeneic stem cell transplantation provides donor-derived mature T cells which are involved in engraftment, graft-versus-host disease (GVHD) and graft-versus-leukemia (GVL) activity, and thus play a major role in determining clinical outcome. In a prior clinical study, we showed that receiving a high percentage of CD4 + CCR7 + T cells is correlated with increased incidence and severity of acute GVHD without influencing other clinical events. As recipients and their allogeneic donors were 10/10 HLA-matched, these alloreactive CD4 + T cells recognized minor histocompatibility antigens (mHAs). The aim of the present study was to compare the in vitro response to allogeneic mHAs of the CD4 + CCR7 + CD45RA+ naïve $T$ cells and the CD4+CCR7 + CD45RAneg central memory $T$ cells.

Materials and methods: The alloreactive response to mHAs was investigated by a mixed lymphocyte dendritic cell reaction using five HLA-identical healthy male and female sibling pairs to include at least a $\mathrm{H}-\mathrm{Y}$ mismatch. Stimulators were mature dendritic cells (derived from monocytes of the male sibling) co-cultured with each one of the three highly purified CD4 + T cell subsets (naive, central memory and effector memory) from the female sibling. Alloreactive response was assessed by $3 \mathrm{H}$ thymidine incorporation at day 5 , and functionally at day 4,6 and 10 by IFN-gamma ELISpot and measurement of Th1/Th2/Th17 cytokines in co-culture supernatants.

Results: Four out of five sibling pairs developed an alloreactive response to mHAs. Maximal proliferation was supported by the CCR7 + CD45RA + naive CD4 + T cells with proliferation indices from 1.75 to 3.85 . This proliferation was accompanied by a functional differentiation: only naïve CD4 $+T$ cells were able to produce significant amounts of IL-6, TNF-alpha and IFN-gamma at day 6 and day 10 (120 to 174 IFN gamma spots at day 10).

Conclusion: This study demonstrates the superior capacity of naive CD4 + T cells to mount a primary alloreactive response to 
minor histocompatibility antigens as compared to central memory $\mathrm{T}$ cells. Their proliferative response associated with a proinflammatory differentiation makes naïve CD4 + T cells potential acute GVHD inducers. These in vitro results strengthen the idea that a partial depletion of naïve CD4 $+T$ cells from the graft might reduce the risk of acute GvHD.

\section{P487}

CD107 surface mobilization of peripheral blood T-cells to predict acute graft-versus-host disease

C.A. Bäuerlein (1), C. Brede (1), S.S. Riedel (1), A. Jordan Garrote (1), C. Kiesel (1), M. Ritz (1), S. Schulz (2), G.F. Beilhack (3), T.B. Stuge (4), R.S. Negrin (5), H. Einsele (1), A. Beilhack (1)

(1)University of Wurzburg Medical School (Wurzburg, DE); (2)Technical University (Munich, DE); (3)University Medical School (Vienna, AT); (4)Univesity Hospital of North Norway (Tromso, NO); (5)Stanford University (Stanford, US)

Acute graft-versus-host disease (aGvHD) poses a life threatening complication after allogeneic hematopoietic cell transplantation (allo-HCT). To identify patients at risk for aGvHD we established a functional assay to detect alloreactive $T$ cells in the peripheral blood.

In a murine allo-HCT model of bioluminescence imaging we have identified day +5 as a critical time point for alloreactive $T$ cell detection in the peripheral blood before the onset of aGvHD.

First, we established the CD107 surface mobilization assay with defined activated antigen specific $T$ cells in vitro. We observed more degranulation against antigen expressing targets $\left(1.6 \pm 0.01 \times 10^{6} \mathrm{CD} 8+\mathrm{cD} 107+\mathrm{T}\right.$ cells $\left./ \mathrm{ml}\right)$ than against non-expressing targets $\left(9.2 \pm 0.6 \times 10^{4} \mathrm{cells} / \mathrm{ml}\right.$; t-test $P<0.0001)$. This correlated with intracellular INF-g production $\left(1.4 \pm 0.03 \times 10^{6} \mathrm{~T}\right.$ cells $/ \mathrm{ml}$ against antigen expressing targets versus $4.0 \pm 0.9 \times 10^{3}$ cells $/ \mathrm{ml}$ against non-expressing targets; t-test $P<0.0001$ ).

To assay for alloreactive cytotoxic $T$ cells in vivo, we induced aGvHD in myeloablatively conditioned Balb/c mice $(\mathrm{H}-2 \mathrm{~d})$ that were hematopoietically reconstituted with C57BI/6 wildtype bone marrow and received wildtype $\mathrm{T}$ cells $(\mathrm{H}-2 \mathrm{~b})$. As syngeneic controls we used C57BI/6 $(\mathrm{H}-2 \mathrm{~b})$ recipients. To prove specificity of the functional assay we transplanted ovalbumine specific T cells into conditioned mice that expressed ovalbumine ubiquitously.

In allogeneic recipients (C57BI/6 into Balb/c) we detected $1.7 \pm 0.6 \times 10^{4} \mathrm{CD} 10^{7}+\mathrm{cD} 8+\mathrm{T}$ cells $/ \mathrm{ml}$ in contrast to syngeneic recipients $\left(6.2 \pm 2.1 \times 10^{2}\right.$ cells $/ \mathrm{ml}$; t-test $\left.P<0.001\right)$. We confirmed these findings when we transplanted ovalbumine specific T cells into ovalbumine expressing recipients $\left(2.3 \pm 0.4 \times 10^{4}\right.$ $\mathrm{CD} 107+\mathrm{CD} 8+\mathrm{T}$ cells $/ \mathrm{ml}$ degranulated against ovalbumine expressing versus $1.0 \pm 0.3 \times 10^{4} \mathrm{cell} / \mathrm{sl}$ against non-expressing targets; t-test $P<0.0005$ ).

This CD107 surface mobilization assay allowed us to identify alloreactive $T$ cells before the onset of aGvHD. Subsequent histopathological GvHD-scoring of the same allogeneic recipients correlated with the flowcytometric results. The preclinical data from our in vivo experiments encourage translation of this predictive test to patients undergoing allo-HCT.

\section{P488}

Targeted depletion of cells expressing the IL-2 receptor with IL-2/caspace chimeric protein ameliorates GvHD J. Stein (1), N. Askenasy (1), A. Krauss (1), S. Yarkoni (2), S. Ash (1), I. Yaniv (1)

(1)Schneider Children's Medical Center (Petach Tikva, IL); (2) GSAR Biotechnology (Kfar Sava, IL)

Graft vs. host disease (GVHD), which causes significant morbidity and mortality following stem cell transplantation, can be ameliorated by administration of regulatory T cells (Treg) cells expressing CD25 (a subunit of the Interleukin-2 [IL2]receptor) in conjunction with CD4 and FOXP3. We tested the effects of in-vitro incubation of splenocytes in medium containing a chimeric IL-2/caspase-3 (IL-2/cas) protein on the elaboration of $\mathrm{GVH}$ reactions in mice undergoing stem cell transplantion. IL-2/cas induces apoptosis of CD-25 positive cells that internalize the caspase subunit via cognate IL2 receptors. Although IL-2/cas preferentially deletes CD25+ splenocytes, significant fractions of both CD25low (putative effector cells) and CD25highFoxP3 + cells (putative Treg) remained viable after 48 hours of exposure to the chimeric protein in vitro. The effect of IL-2/cas on GVHD was tested in a parent to $\mathrm{F} 1$ transplant $((\mathrm{H} 2 \mathrm{~K}[\mathrm{~b}]$ to $\mathrm{H} 2 \mathrm{~K}[\mathrm{~b} / \mathrm{d}])$ model. Recipients were infused with parental bone marrow $+2 \times 10^{7}$ viable $\mathrm{H} 2 \mathrm{~K}[\mathrm{~b}]$ splenocytes pre-incubated for 48 hours in either control medium or medium containing IL-2/cas. Lipopolysaccharide was administered on post-transplant day 7 to trigger hyper-acute GVHD. All recipients of control splenocytes died of GVHD within 3 days of LPS injection, whereas $60 \%$ of the recipients of IL-2/cas splenocytes survived for more than 4 weeks. A marked reduction of inflammatory infiltrates in skin, liver, and intestine were seen in mice infused with IL-2/cas splenocytes as compared with tissues harvested from premorbid mice in the control group. Analysis of the splenocytes harvested from surviving mice revealed inverted CD4/CD8 ratios, with preserved lymphocyte proliferative responses to Concanavalin A. Interestingly, lymph nodes harvested from mice who received IL-2/cas incubated splenocytes contained higher numbers of FoxP3 + cells as compared to control mice examined 24 hours post-LPS injection. Administration of IL-2/cas incubated splenocytes to mice with established GVHD had no effect on the course of their disease. Elimination of CD25 + cells by IL-2/cas early in the course of the GVH reaction ameliorated the course of GVHD and dramatically improved survival in this stringent GVHD model, but the effect of this incubation on putative Treg responses requires further evaluation. Future experiments will evaluate the effect of IL-2/cas on graft vs. tumor effects.

\section{P489}

Alloreactivity to HLA class I mismatch alleles mainly resides in CCR7 + naive and central memory CD8 T-cells E. Distler, S. Asdufan, M. Frey, U.F. Hartwig, M. Theobald, W. Herr Johannes Gutenberg-University (Mainz, DE)

Reducing the incidence of severe graft-versus-host disease (GVHD) remains the major challenge in patients who receive stem cell transplants from HLA-mismatch donors. Selective depletion of alloreactive donor T cells can prevent GVHD, but is still a complex technical procedure that requires the in vitro culture of $T$ cells for several days. Here, we studied CD8 $\mathrm{T}$ cell subsets of 5 healthy donors, sorted by flow cytometry according to expression of the differentiation markers CD45RA CD45RO, CCR7 and CD62L for the ability to respond to alloHLA class I alleles in vitro. To detect pure alloreactive T cell responses and minimize the interference by other specificities, we used HLA-deficient K562 cells transfected with single HLAA, -B or -C alleles for weekly stimulations. We observed the strongest anti-HLA mismatch proliferation in sorted CCR7pos and CD62Lpos fractions, comprising naive (TN) and central memory CD8 T cells (TCM). In contrast, the CCR7neg and CD62Lneg counterpart fractions containing effector memory (TEM) and terminal effector CD8 T cells showed much lower allo-HLA induced in vitro expansion. The CD45RApos subset enriched for TN cells and devoid of TCM and TEM cells demonstrated less strong allo-proliferation compared to corresponding CD45RAneg cells. Low allo-proliferation was observed for CD45ROpos and CD45ROneg populations. Interestingly, the growth pattern of CD8 T cell subsets was consistent, regardless of HLA-A, $-B$, or $-C$ mismatch alleles were used for stimulation. Cultures derived from all CD8 $\mathrm{T}$ cell subsets were analyzed 
for allo-HLA class I reactivity by IFN-g ELISPOT assay starting on day 11 . The strongest anti-HLA mismatch reactivity was detected in the CCR7pos subset. Lower level of alloreactivity was observed in CD62Lpos, CD45ROneg, and CD45RApos fractions. All counterpart fractions showed even less strong anti-HLA responses. Again, these findings were consistent for all HLA alleles tested. Our results thus demonstrate that distinct CD8 T cell subsets vary substantially in the ability to respond to HLA class I mismatch alleles. Although all subsets enriched for TN and TCM produced substantial alloreactivity, the CCR7pos fraction exceeded others in terms of proliferation and effector function. Thus, in vitro removal of CCR7pos CD8 T cells may be a straightforward culture-independent allodepletion procedure. Further experiments comparing anti-HLA mismatch and self-HLA-restricted anti-leukemia reactivity of $\mathrm{T}$ cell subsets are ongoing.

\section{P490}

908Arg variant in NOD2/CARD15 gene is associated to gastrointestinal graft-versus-host disease in patients receiving allogeneic haematopoietic stem cell transplantation

A.M. Carella (1), O. Palmieri (1), M. Greco (1), E. Merla (1), A. Latiano (1), L. Savino (1), A. Andriulli (1), G. Palumbo (2), S. Capalbo (2), S. Pinna (3), G. Quarta (3), V. Annese (4), N. Cascavilla (1)

(1)IRCCS "Casa Sollievo Della Sofferenza" (San Giovanni Rotondo, IT); (2)Ospedali Riuniti (Foggia, IT); (3)Ospedale "A. Perrino" (Brindisi, IT); (4)AUO Careggi (Florence, IT)

Acute Graft-versus-Host Disease (aGvHD), an immunological disorder in which the $T$ cells present in the graft react against host tissues, is considered the main complication of allogeneic Hematopoietic Stem Cell Transplantation. Acute GvHD may affects many organ systems, including the gastrointestinal (GI) tract, liver, skin, and lungs. To date, this complication remains a source of significant morbidity and mortality in the setting of allogeneic HSCT. Moreover, while the absence of aGvHD is associated with a higher risk of relapse of the underlying malignant disease, severe aGvHD usually induces the occurrence of life-threatening complications such as severe infections. The NOD2 gene polymorphisms (SNPs)(Arg702Trp, Gly908Arg and L1007fsinsC), which characterizes Crohn's disease, have shown to be associated with the risk to develop GI aGvHD and further transplant-related complications.

Aims: To assess a contribution of Arg702Trp, Gly908Arg and L1007fsinsC NOD2 polymorphisms and to search for likely correlation in clinical GvHD occurrence in patients affected by haematological malignancies receiving allogeneic HSCT in Italian population.

Materials and methods: One hundred and six HLA-matched donors/recipients pairs (R/D) and 824 healthy controls (HC) were genotyped for Arg702Trp, Gly908Arg and L1007fsinsC polymorphisms by using the TaqMan allelic discrimination method. Genotype frequencies were compared in respect to III-IV GvHD, gastrointestinal GvHD, and TRM.

Results: The investigated SNPs were in Hardy-Weinberg equilibrium in both controls, donors and recipients. Significant allele and genotype association with Gly908Arg and recipients group $(P=0.02, \mathrm{OR}=2.4, \mathrm{Cl}=1.1-5.1 ; P=0.008, \mathrm{OR}=2.6 ; \mathrm{Cl}=1.2-$ 5.3 , respectively) was shown, with a subsequently significant difference of allele frequency only in GI GvHD group $(P=0.03$, $\mathrm{OR}=4.74, \mathrm{Cl}=1.4-16.29$ ). After stratifying the R/D pairs on the basis of NOD2 genotypes (NOD2-=no major variants; $\mathrm{NOD} 2+=$ at last one variant), only a trend towards NOD2+ pairs with GI GvHD (33\%), III-IV GvHD (30\%), and TRM (33\%), compared with group without GvHD (23\%), was also demonstrated.

Conclusion: The 908Arg (risk allele) variation has shown to be mainly associated with recipients who developed GI GvHD. In addition, in R+D/NOD2 + pairs, a trend towards in GI GvHD, III-IV GVHD and TRM, was also demonstrated.
P491

CCL8 as a biomarker intimately associated with murine graft-versus-host disease

M. Yamamoto (1), A. Ota (1), T. Hori (2), S.I. Imai (1), H. Sohma (1), N. Suzuki (2), N. Hatakeyama (2), H. Tsutsumi (2), Y. Kokai (1) (1)Department of Biomedical Engineering (Sapporo, JP); (2)Department of Pediatrics (Sapporo, JP)

Objectives: Acute graft-versus-host disease (GVHD) remains a major life-threatening complication and we have recently shown that CC chemokine-ligand motif 8 (CCL8) is a potential biomarker for early diagnosis of GVHD. In this study, we further examined whether CCL8 can serve as a biomarker for GVHD in mice. Moreover, we supposed to analyze how CCL8 protein expresses in vitro.

Methods: Recipient mice (Balb/c) received allogeneic bone marrow (BM) transplantation (BMT) from the donor mice (C57BL/6) after lethal total body irradiation. To make severe GVHD model, allogeneic $2 \times 10^{7} \mathrm{BM}$ cells plus spleen cells of $\mathrm{C} 57 \mathrm{BL} / 6$ ranging from 0.1 to $1.0 \times 10^{7}$ were added. The control mice were transplanted $2 \times 10^{7}$ syngeneic BM cells. Plasma was obtained on days $5,7,14,21$, and 28 after BMT, and CCL8 and IFN-gamma levels were measured using enzymelinked immunosorbent assay (ELISA). Survival rate was monitored daily and GVHD clinical score was assessed weekly. GVHD histopathologic grading was performed on day 14 after BMT. To analyze the expression mechanism of CCL8, Balb/ c derived dendritic cells (DCs) and C57BL/6 derived spleen cells were used for mixed lymphocyte reactions. After 96 hours of culture, CCL8 protein in the culture supernatant could be detected by ELISA.

Results: In vivo analysis revealed that the level of plasma IFNgamma peaked at day 7 and rapidly dropped despite prominent GVHD, whereas CCL8 remained high unless cyclosporine A was administered. Survival rate was found to be parallel to the plasma CCL8 level at day 5. When plasma CCL8 on day5 was more than $2000 \mathrm{ng} / \mathrm{mL}$, the survival rate at day 28 was significantly lower. Furthermore, plasma CCL8 levels at day 5 correlated with both clinical and pathological score at day 14 of GVHD. In vitro study revealed that the host derived DCs and donor derived CD4 + T cells are sufficient to express CCL8. This expression was abrogated by an antibody for MHC class II. Neutralizing IL-2, IFN-gamma and TNF-alpha decreased CCL8 levels in vitro.

Conclusions: These findings suggest that plasma CCL8 might prove diagnostically helpful in evaluating GVHD at least in mice. As a biomarker for GVHD, CCL8 is worth evaluating further with human samples. And further investigation about the role of CCL8 in the pathogenesis of GVHD is required.

\section{P492}

Employing CTLA-4 Ig for the ex-vivo generation of tolerized T-cells to be used for adoptive transfer strategies in murine haematopoietic stem cell transplantation

E. Veith (1), N. Pilat (2), T. Wekerle (2), A. Heitger (1)

(1)Children's Cancer Research Institute (Vienna, AT); (2)Medical University of Vienna (Vienna, AT)

T cells specifically tolerant against donor allo-antigens are critical to provide recipients of HSCT with T cell immunity without promoting graft-versus-host disease (GVHD). Based upon the previously described powerful effect of costimulation blockade by CTLA-4 Ig in vivo for tolerance induction in solid organ-transplantation we began to explore whether CTLA-4 Ig used in an ex-vivo approach is effective to tolerize T cells specifically against allo-antigens without global immunosuppression.

When spleen-derived CD11c+ dendritic cells (DCs) from C57BL/6 mice were co-cultured with allogeneic Balb/c-derived $\mathrm{CD} 3+\mathrm{T}$ cells in a mixed lymphocyte reaction (MLR) we found that the addition of $100 \mu \mathrm{g} / \mathrm{ml}$ CTLA-4 $\mathrm{lg}$ to the co-cultures inhibited the proliferative allogeneic $T$ cell response, (assessed 
by CFSE dilution) by $80 \%( \pm 10 \%)$. Further experiments investigated whether CTLA-4 Ig acts by affecting preferentially the stimulator population (as previously suggested by Grohmann et al., Nat Immunol 2002). By using several isoforms of CTLA$4 \lg (\lg \mathrm{G} 1$ and $\lg \mathrm{G} 3$ isotypes) we found that a pre-exposure of DCs alone to CTLA-4 Ig induced the release of enhanced amounts of interferon-gamma but did not up-regulate the enzymatic activity of the tryptophan-metabolizing enzyme indoleamine 2,3 dioxygenase, and did not down-regulate the subsequent allogeneic $T$ cell proliferative response. Likewise, pre-exposing stimulated T cells alone with CTLA-4 Ig left the capability to subsequently proliferate unaffected. These findings suggested that CTLA-4 Ig neither promoted suppressor activity in DCs nor affected the responsiveness of $T$ cells, but directly affected the interaction of stimulator and responder cells in co-cultures. CTLA-4 Ig, however, when added to cocultures, yielded a $T$ cell population with a CD4 +CD25high, putatively regulatory phenotype. Thus, CTLA-4 Ig, in addition to down-regulating the proliferative response, might induce regulatory activity in CD4 + T cells. This hypothesis is currently tested in an in-vivo approach (supported by the Austrian Science Foundation P\#19865)

\section{P493}

The dynamics of surface marker expression on human peripheral blood T-cells

S. Herz (1), C.A. Bäuerlein (1), A. Jordán Garrote (1), S.S. Riedel (1), C. Brede (1), C. Kiesel (1), M. Ritz (1), M. Chatterjee (1), R.S. Negrin (2), H. Einsele (1), A. Beilhack (1)

(1)University of Wurzburg Medical School (Wurzburg, DE); (2)Stanford University (Stanford, US)

Acute graft-versus-host disease (aGvHD) poses a major risk of allogeneic hematopoietic cell transplantation (allo-HCT). In murine in vivo imaging models we recently demonstrated that aGvHD pathogenesis is tightly spatially and temporally regulated. We showed that alloreactive $T$ cells need to express certain homing receptors in order to reach the aGvHD target organs (intestinal tract, liver and skin). Thus, we reasoned that certain homing receptors on peripheral blood $\mathrm{T}$ cells could serve as predictive markers for aGvHD. This could improve the prevention and treatment of aGvHD.

To develop a clinical test to predict aGvHD we established a multiplex flow cytometry test for cytotoxic and Thelper cell subsets. We investigated the expression of 25 surface markers on human peripheral blood T cells (activation markers, organ specific homing receptors and inflammatory response receptors) that may play a role in the pathogenesis of aGvHD in patients. Here we asked how T cell surface marker expression changes over time in healthy individuals.

For our study we recruited 21 healthy individuals (female and male, mean age 24,9 years). We analyzed peripheral blood mononuclear cells by flow cytometry at 8 time points over 3 weeks. Preliminary mouse experiments had determined these time points as critical for subsequent prospective clinical studies.

We found that the surface marker expression on peripheral blood T cells in healthy individuals can be subdivided into 3 categories: 1. Constant low numbers of cytotoxic and Thelper cells expressed CD25, CD69, chemokine receptor (CCR) 2, CCR10, CXCR1 (on T helper cells only), CXCR2, cutaneous lymphocyte antigen, leukotriene B4 receptor 1 and integrin alpha E. 2. Constant high numbers of $T$ cells expressed CD45RA integrin alpha 4 and L-selectin. 3 . T cell subsets that changed dynamically the expression of CCR1, CCR4, CCR5, CCR6, CCR8, CCR9, CXCR1 (on cytotoxic T cells only), CXCR3 and integrin beta 7 .

These data from healthy individuals established the basis for a recently initiated clinical prospective study. The measured receptor expression profiles were important to determine physiological ranges to compare them to allo-HCT patients. The data from the healthy probands encourage further investigations with patients undergoing allo-HCT to find an efficient and reliable method to predict aGvHD.

\section{Graft-versus-host disease: clinical}

P494

Long-term outcome of patients with severe steroid refractory acute intestinal graft-versus-host disease after treatment with pentostatin

S.A. Klein (1), G. Bug (2), S. Mousset (2), W.-K. Hofmann (1), H. Martin (2)

(1)Medical University (Mannheim, DE); (2)Johann Wolfgang Goethe-University (Frankfurt, DE)

Steroid-refractory intestinal acute Graft versus Host Disease (aGvHD) is a mayor complication after allogeneic stem cell transplantation. In steroid-refractory aGVHD survival rate is low. A promising strategy is the use of pentostatin, an inhibitor of adenosine deaminase. We report the long term results with a median follow up of six years of a single centre study on salvage therapy of aGvHD by pentostatin. 23 patients transplanted between 1999 and 2006 at Frankfurt medical school with steroid refractory severe intestinal aGvHD overall grade III or IV with a follow-up of at least 30 months were included. All patients except one received peripheral blood stem cells. The mean age was 45 (range: $25-61$ ) years. The underlying diseases were ALL (3), AML (17), Multiple myeloma (1), Hodgkin's disease (1) and CML (1). GVHD prophylaxis was ciclosporine based in combination with mycophenolate mofetil or with methotrexate. All patients suffered from severe intestinal aGvHD. 16 patients developed a concomitant skin, five a liver involvement. The overall grade of GvHD was III in 8 patients and IV in 15 patients. The stage of gut GvHD was $2(1 \mathrm{x}), 3(14 \mathrm{x})$ and $4(8 \mathrm{x})$. Pentostatin was applied as salvage therapy $\left(1 \mathrm{mg} / \mathrm{m}^{2} /\right.$ $x 3$ days, 1-4 cycles, q22) after a therapy of at least two days of prednisolone $(\geq 2 \mathrm{mg} / \mathrm{kg} / \mathrm{d})$. Therapy was well tolerated. No severe neutropenia or thrombocytopenia occurred. Except one case of hemolytic uremic syndrome (HUS) renal function was not affected. 16 patients $(82,6 \%)$ achieved a complete, three a partial remission of aGvHD. Clinical improvement occurred typically after $12-15$ days. Four patients died before day 12 without improvement of GvHD symptoms. All patients with an at least 12 day survival responded. Ten patients are still alive (median follow up: 1853 days, range: 953-3049). All one-year survivors stayed alive. 13 patients died (3×relapse of leukemia, 10xtherapy related). The long term overall survival rate is $43,5 \%$. Of the ten long term survivors three experienced no chronic GvHD (cGvHD), three had limited and four extensive cGvHD. Five patients are under ongoing immunosuppressive therapy. In conclusion, pentostatin at a dose of $1 \mathrm{mg} / \mathrm{m}^{2} / \mathrm{d}$ for 3 days is a highly effective and well tolerated drug in severe steroid-refractory intestinal aGvHD with a high rate of long term survivors.

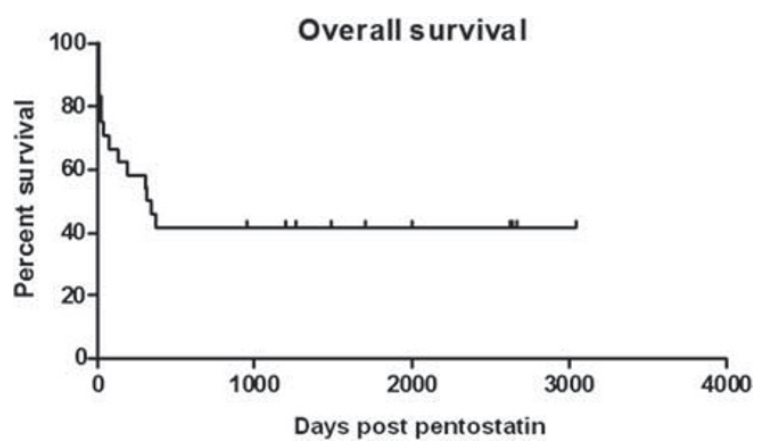




\section{P495}

A biomarker panel associated with acute and chronic graft-versus-host disease

S. Ahmed (1), E. El-Gezawy (1), E. Holler (2), M. Collins (1), G. Jackson (1), A. Dickinson (1)

(1)Newcastle University (Newcastle, UK); (2)Regensburg University Hospital (Regensburg, DE)

Objective: Graft versus host disease (GVHD) is a major complication of haematopoietic stem cell transplantation (HSCT). A number of inflammatory cytokines/chemokines are implicated in GVHD. Several of these have been identified in numerous single centre studies as potential biomarkers for acute and chronic GVHD without validation. We analysed the role of several inflammatory biomarkers including B-cell activation factor (BAFF), IL-33, NALP6, NALP12, CXCL10 and CXCL11, in association with GVHD, in a two centre study.

Method: Each biomarker was evaluated in serums collected pre-transplant and at various time-points post-transplant from patients with and without acute GVHD (aGVHD) and chronic GVHD (cGVHD). The levels of each biomarker were measured using ELISA. The expression of these biomarkers on GVHD target tissue was validated using the skin-explant assay by immuno-histo-chemistry.

Results: Our results showed a significant decrease in soluble BAFF levels following conditioning $(P<0.01)$ indicating BAFF levels are influenced by the conditioning treatment. Although aGVHD patients had increased BAFF levels, we found BAFF was more strongly associated with CGVHD $(P<0.0005)$. Patients with pre-transplant elevated BAFF levels appeared more likely to develop GVHD and a strong association was observed between patients who developed aGVHD and those who developed cGVHD (87\%). IL-33, a interleukin-1 family member was found to be increased following conditioning $(P<0.02)$ and during aGVHD (day 14-100, $P<0.03$ ). IL-33 expression was strongly influenced by the type of conditioning regimen received. Mylobalative conditioning patients had elevated IL-33 levels. IL-33 was not associated with cGVHD. The chemokines CXCL10 and CXCL11 were elevated in grade II-III aGVHD patients compared to controls. However, they were significantly elevated in CGVHD patients 6 months and 12 months post-transplant, respectively $(P<0.001 ; P<0.0001)(P<0.02 ; P<0.04)$. Analysis using the human in-vitro skin explant assay (a model for aGVHD) showed that levels BAFF, IL-33, NALP6, NALP12, CXCL10 and CXCL11 were significantly increased in patients with grade II-III Graft versus Host Reaction (GVHR) $(P<0.04, P<0.004, P<0.002, P<0.049, P<0.007$ and $P<0.01$, respectively).

Conclusion: We show IL-33, NALP6 and NALP12 are associated with aGVHD while BAFF, CXCL10 and CXCL11 are strongly associated with the development of cGVHD. The study identifies potential targets for novel therapies.

\section{P496}

The impact of NOD2/CARD15 SNPs on the severe gastrointestinal GvHD following allogeneic stem cell transplantation in a Sardinian population

M.G. Orofino (1), D. Diana (2), R. Rossino (2), M.C. Addari (1), F. Cossu (1), A. Piroddi (1), F. Rizzo (1), M. Congia (1), A. Vacca (3), G. La Nasa (3), M. Badiali (1)

(1)CTMO Ospedale Microcitemico (Cagliari, IT); (2)Universita Studi Cagliari (Cagliari, IT); (3)CTMO Ospedale Binaghi (Cagliari, IT)

The NOD2/CARD15 gene encodes proteins which are critically involved in innate immunity and Crohn's disease. This proteins activate a nuclear factor NF-kB and are involved in intracellular pathogen recognition. TheNOD2/CARD15 espression is restricted to gut epithelial cells and cells of monocyte and macrophage lineage. In Crohn disease a variety of single nucleotide polymorphisms (SNPs) in the NOD2/CARD15 gene (SNPs: 12,13,8) have been reported to increase the disease risk in European population. The prevalence of SNP13, SPN8 in Sardinian patients (pts) with Crohn disease has been found in $9,1 \%$ and $4,5 \%$ respectively, whilst in Sardinian controls the SNPs13 has been found in $0.1 \%$. Considering the similitude between the pathophysiology of aGVHD with the inflammatory bowel diseases, we hypothesized that genetic variant for NOD2/ CARD15 gene may have an impact on the gut GVHD. In this study we evaluated in 45 patients, receiving hematopoietic stem cell transplantation (HSCT) from 36 related and 9 unrelated donors, the frequency of NOD2/CARD15 SNPs, in severe $(\geq 2)$ gut GVHD. The age of pt at transplant ranged from 1 to 35 years, the underlying diseases were: 41 Beta Thalassemia, 1 Kostman S, 1 LLA, 2 Histiocytosis. All pts received as source of HSCT only bone marrow. Prophilaxis of aGVHD in 36 pts receiving HSCT from unrelated donors. The gut decontamination included polymyxin in all the $45 \mathrm{pt}$. The cumulative severe gut aGVHD was (23/45) $51 \%$. In 23 $R$ and their $D$ with aGVHD and in 9 aGVHD free the SNPs 8 and 13 of NOD2/CARD15 gene have been attributed, using PCR-RFLP technique. The NOD2/CARD 15 SNPs were subdivided in wild "Ctype, in SNP with 1 variant and SNPs with more than 1 variant in both $D / R$ pair with and without gut aGVHD.

Results: In the group with aGVHD both $\mathrm{D}$ and $\mathrm{R}$ resulted negative for NOD2/CARD15 variants whilst in the group without aGVHD 2 pairs of D/R were heterozygous for the SNP13 (9\%). In one D but not in the R the SNP8 was found (4.5\%).

Conclusions: Our preliminary data seem to exclude in our population any involvement of NOD2/CARD15 gene variants in gastrointestinal aGVHD following HSCT. These data differ from data of other European populations and could indicate a lower mutation rate of NOD2/CARD15 gene in Sardinians. However, this different frequency of NOD2/RD15 gene variant must be taken into account when selecting the unrelated donor from BMT registry.

\section{P497}

Reconstitution of B-cell compartment after allogeneic stem cell transplantation and its role in the development of chronic graft-versus-host disease

E. Toffoletti, F. Patriarca, S. Olendo, M. Cavallin, A. Sperotto, M.L. Battista, A. Michelutti, M. Medeot, D. Damiani, R. Fanin Hematological Division of Udine (Udine, IT)

Background: Conflicting results are reported on the status of B-lymphopoiesis after allogeneic stem cell transplantation (SCT) and particularly in patients with chronic Graft versus Host Disease (cGvHD). Several studies documented severe B-cell lymphocytopenia and hypogammaglobulinemia, whereas others data suggested a B-hyperactivity such as the production of pathological autoantibodies and the successful response to Rituximab in certain clinical manifestations of cGvHD.

Objectives of the study: We evaluated the kinetics of B cell recovery and engraftment after allogeneic SCT in comparison with T, NK and myeloid cells. The final objective was to identify if there was a correlation between $B$ lymphocytes recovery and the development of cGvHD.

Patients and methods: We studied prospectively 13 consecutive patients, median age 59 years (range 23-69). The hematopoietic chimerism was studied at day 90,120 and 180 on whole PB, on immunomagnetically sorted CD3+ and CD19+ cells and on granulocyte fraction. Full donor chimerism (FDC) was defined as the presence of at least $95 \%$ donor cells. We evaluated $\mathrm{B}, \mathrm{T}$ and NK cells in PB by immonophenotyping at day $30,90,120$ and 180 .

Results: Thirteen patients developed cGvHD in median at 5 months after SCT, with at least 2 organs involved and a median score of 3 according the Organ Scoring System (Filipovich 
et al., BBMT, 2005). CGvHD progressed from a pre-existent acute GvHD in 7 cases. No patient showed autoantibodies.

We could not analyzed B cell chimerism at day 90 due to the low number of CD19+, but at day 120 we found FDC in CD19+ cells of all patients, as well as in the others fractions.

We observed a similar kinetics of CD $3+$, CD $4+$ and CD $8+$ cells recovery in all patients, with the lowest cells concentrations at day 90 and a subsequent progressive increase at day 120 and 180. Patients with cGVHD showed a lower NK cells concentrations at day 90 in comparison with controls. All patients showed B cells concentrations no higher than 100/microliter from 90 to 180 days. However, the group with subsequent cGvHD showed lower B-cell concentrations at day $120(P=0.03)$ in comparison with controls.

Conclusions: Severe B lymphocytopenia persisted through the first 180 days after SCT. FDC could be observed in B cells of all patients at day 120 after SCT. Patients at risk of cGVHD had a significative reduction of $B$ cells concentrations at day 120 . We are planning to extend the follow-up of the patients and to confirm our results in a larger population.

\section{P498}

Steroid resistance: a second, T-cell independent stage of GvHD with progressive microangiopathy resulting in organ failure

T. Luft (1), M. Andrulis (1), S. Dietrich (1), M. Conzelmann (1), M. Hess (1), F. Neumann (2), C. Falk (3), U. Hegenbart (1), A.D. Ho (1), P. Dreger (1)

(1)University Hospital Heidelberg (Heidelberg, DE); (2)BIOAXXESS (Malvern, UK); (3)National Tumor Centre (Heidelberg, DE)

Steroid-resistant GVHD causes significant morbidity and mortality. The pathomechanism of steroid-resistance is currently not understood. Nevertheless and despite the limited efficacy of the approach, most salvage therapies target the T-cell arm of the disease by intensifying immunosuppression.

Aaim and methods: In order to investigate the individual contribution of T-cells and endothelial cells in steroid-resistant GVHD, we compared kinetics of cytokeratin-18 fragments (CK18F) as quantitative markers of epithelial apoptosis, serum FAS ligand as $T$ cell activation marker, and serum thrombomodulin (sCD141) and angiopoietin-2/VEGF ratio as markers of endothelial damage longitudinally in 21 patients with steroidresistant GVHD and 18 patients with steroid-sensitive GVHD.

Results: Onset of GVHD was associated with a highly significant increase of serum CK18F in both groups $(P=0.007)$. CK18F serum levels decreased following escalation of immunosuppression in patients with therapy-sensitive GVHD $(P=0.03)$, but not in patients with steroid-refractory disease. Late CK18F levels ( +52 after escalation of immunosuppression) were highly predictive for survival $(P<0.001)$ in univariable cox regression analysis. Soluble FAS ligand levels were significantly higher in patients with steroid-sensitive disease $(P=0.007)$, but decreased following immunosuppression in both groups (resistant $P=0.003$, sensitive $P=0.046$ ) indicating efficient suppression of the T-cell response. However, a striking characteristic of steroid-resistant GVHD was progressive endothelial damage as shown by steadily increasing serum thrombomodulin (sCD141) levels coinciding with loss of CD141 expression on the surface of endothelial cells in intestinal GVHD sections. SCD141 was also predictive for survival in cox regression $(P=0.05)$. Finally, the Angiopoietin-2/VEGF ratio in serum which was shown to indicate severe microangiopathic damage could also clearly distinguish between therapy-sensitive and refractory disease and was furthermore highly predictive for survival $(P=0.007)$. Conclusions: "Steroid-resistance" may reflect a second, T-cell independent disease stage of progressive microangiopathy resulting in organ failure, which appears to be associated with defined serum marker signatures. The role of pre-existing or additional causes of endothelial damage contributing to the risk of steroid-refractoriness has to be further investigated.
P499

Predicting chronic graft-versus-host disease on day +100 after allogeneic stem cell transplantation

I. Cavattoni (1), F. Frassoni (2), D. Occhini (2), T. Lamparelli (2), F. Gualandi (2), S. Bregante (2), C. Di Grazia (2), A. Dominietto (2), A.M. Raiola (2), A. Ibatici (2), M.T. van Lint (2), A. Bacigalupo (2)

(1)Bolzano Central Hospital (Bolzano, IT); (2)San Martino's Hospital (Genoa, IT)

Background and aim of the study: We have previously shown that chronic graft versus host disease (cGvHD) and transplant related mortality (TRM) can be predicted by three laboratory values on day +100 : platelets count (PIt), serum cholinesterase (CHE), gamma-glutamyltransferase (g-GT).We have further improved this scoring system by the addition of two new variables, in a larger number of patients.

Patients and methods: The patient population consisted of 1088 consecutive patients with hematologic disorders allografted between 1990 and 2008, alive on day +100 and free of cGvHD. The donor was an HLA identical sibling $(n=647)$ or an alternative donor $(n=361)$. The median follow-up was 1288 days (range 92-7237). The 3 previously described indicators (PIt, CHE, g-GT) remained significant. Additional indicators were serum albumin and total iron binding capacity (TIBC). Median levels at day +100 were used as cut off: Plt $100 \times 10^{9} / \mathrm{L}$, CHE $3500 \mathrm{IU} / \mathrm{ml}, \mathrm{g}-\mathrm{GT} 50 \mathrm{IU} / \mathrm{L}$, TIBC $205 \mathrm{mg} / \mathrm{dl}$, and albumin $3.9 \mathrm{gr} / \mathrm{dl}$. Two prognostic groups were identified: low risk (0-2 negative predictors), and high risk (3-5 negative predictors).

Results: The overall risk of developing moderate/severe cGVHD was $29 \%$ and the risk of TRM beyond day +100 was $19 \%$. The two risk groups identified patients at different risk of cGvHD $(24 \%$ vs. $35 \%, P=0.0002)$ and of TRM $(11 \%$ vs. $28 \%$, $P<0.0001$, see Table 1). Relapse related death was comparable in the 2 groups ( $20 \%$ vs. $23 \%, P=0.2$ ). The actuarial survival of the 2 groups at 10 years was $66 \%$ vs. $43 \%(P<0.0001)$. In multivariate analysis, including donor/recipient data, phase of disease, donor type, and year of transplant, the RR for patients with high day+ 100 score was: RR 1.97 for overall mortality, 2.1 for CGvHD and 3.0 for TRM. In the previous, the RR for overall mortality was 1.5, for chronic GvHD 1.4 and for TRM 1.69. Death due to GvHD with/wo infections was $8 \%$ vs. $21 \%$ respectively in low/high risk patients $(P<0.001)$. Other predictors were year of transplant, donor age, disease phase, recipient gender and donor type.

Conclusions: The use of 5 simple laboratory tests on day +100 (PIt, CHE, g-GT, Albumin and TIBC) predicts cGvHD and mortality, with a greater accuracy as compared with our previous day +100 score. Strategies to prevent cGvHD and delayed mortality may include immune-modulation with/wo intensified infection prophylaxis in high risk patients.

Table 1: comparison between high risk and low risk patients acoording to the score
\begin{tabular}{|l|c|c|c|c|}
\hline & low risk & high risk & $D$ & $R R$ \\
\hline TRM & $11 \%$ & $28 \%$ & $<0.0001$ & 3.0 \\
\hline cGVHD & $24 \%$ & $35 \%$ & 0.0002 & 2.1 \\
\hline Overall survival & $68 \%$ & $43 \%$ & $<0.0001$ & \\
\hline Relapse related death & $20 \%$ & $23 \%$ & $\mathrm{~ns}$ & \\
\hline TRM (cGVHD $+/$ - infections) & $8 \%$ & $21 \%$ & $<0.001$ & \\
\hline Overall mortality & & & & 1.97 \\
\hline
\end{tabular}

\section{P500}

Predicting role of histopathology in graft-versus-host disease. A single-centre experience

I. Cavattoni, E. Morello, S. Deola, N. Pescosta, M. Casini, G. Negri, V. Colombetti, S. Cortelazzo

Bolzano Central Hospital (Bolzano, IT)

Histopathology has a critical role in diagnosis and management of Graft versus host disease (GvHD), a major complication after allogeneic stem cell transplantation (HSCT). The aim of the present retrospective study was to investigate the positive 
predictive value (PPV) of histopathology using a simple predictive score at the time of collecting biopsies for GvHD diagnosis. One hundred and fifty-six consecutive bioptical samples were performed for GvHD diagnosis in 68 adult patients with hematological malignancies, allografted at our institution between 1999 and 2009. The biopsies were collected early at the onset of clinical manifestation suggestive of GvHD: 85 from skin, 67 from the gastrointestinal tract $(\mathrm{GI})$ (stomach $n=43$, colon $\mathrm{n}=24$ ), 4 liver biopsies and 1 from the lung (endotracheal). On the basis of a previous study (Cavattoni, ASH 2009, abstract online), where sensitivity, specificity, positive predictive value (PPV) and negative predictive value (NPV) of the biopsies were calculated, 5 variables improving PPV were identified:peripheral blood (PBSC) as source of stem cells, biopsy collected from upper GI tract, matched unrelated donor (MUD), reduced intensity conditioning (RIC), and biopsy collected from the lower $\mathrm{GI}$ tract. One to 3 points were arbitrarily assigned to the 5 variables which had a PPV $\geq 90 \%$, according to the impact of their predictive value:PBSC with a PPV $=90 \%, 1$ point, biopsy collected from upper $\mathrm{GI}$ tract with a PPV $=90 \%, 1$ point, MUD with a PPV $=92 \%, 2$ points, RIC with a PPV $=93 \%, 2$ points, and biopsy collected from the lower GI tract with a PPV $=100 \%$, 3 points. As previously reported, overall sensitivity and specificity of the histopathological test were $79 \%$ and $80 \%$ respectively. PPV was $86 \%$, NPV was $71 \%$. Applying the previously described score the patients were normally distributed and 3 subgroups were categorized:Group 1 (Score 0 point, 50 biopsies), Group 2 (Score 1-4, 90 biopsies) and Group 3 (Score $>4,17$ biopsies). PPV of the 3 identified groups according to this score was $78 \%$, $90 \%$ and $100 \%$ in groups 1,2 and 3 respectively. The use of 5 simple clinical variables at the time of sampling (RIC, PBSC, MUD, biopsies collected from GI tract, i.e. stomach or colon) correlates with the positive predictive value of the biopsy. These preliminary data must be confirmed by larger prospective case studies in order to optimize the role of histopathology in GvHD, and to reduce the number of false positive and false negative results.

\section{P501}

\section{Prophylactic impact of tyrosine kinase inhibitor} administration after allogeneic stem cell transplantation on the incidence and severity of chronic graft-versushost disease in patients with Philadelphia chromosomepositive leukaemia

H. Nakasone, Y. Kanda, H. Takasaki, C. Nakaseko, T. Sakura, S. Fujisawa, A. Yokota, S. Yano, K. Usuki, A. Maruta, D. Abe, T. Hoshino, S. Takahashi, H. Kanamori, S. Okamoto on behalf of the Kanto Study Group for Cell Therapy

Background: Chronic graft-versus-host disease (cGVHD) is one of the major problems after allogeneic stem cell transplantation (SCT). It has been reported that the fibrotic change in cGVHD is associated with the activation of transforming growth factor (TGF A) and platelet-derived growth factor receptor (PDGFR) pathways, and this is inhibited by tyrosine kinase inhibitors (TKIs), including imatinib. Recent studies showed that imatinib was effective as salvage treatment for refractory cGVHD. It, however, remains unknown whether the administration of TKIs after SCT affects the incidence and severity of cGVHD.

Patients and methods: We retrospectively reviewed the data of transplantation recipients with chronic myeloid leukemia (CML) or Philadelphia chromosome-positive acute lymphoblastic leukemia (PhALL) and compared between those with and without TKIs as prophylaxis against post-transplant relapse of Philadelphia chromosome positive leukemia.

Results: We reviewed 96 patients, including 51 with PhALL and 45 with CML, and classified them into a TKI group $(n=20)$ and a non-TKI group $(n=76)$ according to the administration of TKIs after SCT. The TKI group included significantly more patients with PhALL and those with an alternative donor source. The 3 -year cumulative incidence of cGVHD was $41.1 \%$ and $71.3 \%$ in the TKI and non-TKI groups, respectively $(P=0.0083)$. Cord blood (RR 0.129, $P=0.045$ ) and the use of TKIs as a timedependent covariate $(\mathrm{RR}=0.266, P=0.013$ ) were identified as significant independent predictive factors for a lower incidence of cGVHD. The overall severity of cGVHD was also significantly lower in the TKI group $(P=0.029)$.

Conclusion: Both the incidence and severity of cGVHD appeared to be reduced by a TKI. A large prospective trial is warranted to confirm the prophylactic effect of TKIs on the incidence of cGVHD.

\section{P502}

Common cold viruses leading to life-threatening alloimmune lung syndromes

A.B. Versluys (1), J.W.A. Rossen (2), B. van Ewijk (3), R. Schuurman (1), M. Bierings (1), J.J. Boelens (1) (1)UMCUtrecht (Utrecht, NL); (2)St Elisabeth Hospital (Tilburg, NL); (3)Tergooiziekenhuizen (Blaricum, NL)

In lung transplantation, presence or early infection $(<100 d)$ with a common respiratory virus is associated with either acute or chronic rejection, presenting as Bronchiolitis Obliterans (BO). After hematopoietic stem cell transplantation (HSCT) alloimmune lung syndromes (allo-LS), including Idiopathic Pneumonia Syndrome (IPS) and Bronchiolitis Obliterans (BO), also occur, but the role of respiratory viruses (RV) is unclear. In this prospective study we analysed the influence of common respiratory viruses early during HSCT on the development of allo-LS and survival. We included 110 paediatric patients with a median age of 5 years, who were transplanted for malignant (56) and nonmalignant (54) disease. Conditioning regimen was TBI based in 33 , and chemotherapy based in 77 . Family donors were used in 33 patients, unrelated donor grafts in 77.56 transplantations were HLA matched, 54 had at least one HLA-locus mismatch. Stemcell source was cord blood in 33 , and bone marrow or peripheral blood in 77 . In $50 \%$ of patients RV infection occurred early after HSCT, at a median of day +16 . RV was proven by $\mathrm{qPCR}$ for respiratory viruses on nasopharyngeal aspirate. Rhinovirus was found most frequent, we also found parainfluenzavirus-3, Influenza A virus, Coronavirus, Adenovirus or a combination of multiple viruses. Symptoms were mild, and all patients recovered spontaneously. After a period without symptoms of at least 2 weeks, new respiratory symptoms occurred in about $50 \%$ of the RV positive patients, all of them had allo-LS. In the whole cohort we found allo-LS in $27.3 \%$. Eighteen patients developed IPS (16.4\%) and 12 BO (10.9\%). Multivariable analysis showed that respiratory viral infection early after HSCT is an important predictor for the development of allo-LS $(P<0.0001)$. This was true for all respiratory viruses tested. Acute Graft versus Host Disease (aGVHD) in other organs, had a protective effect on the development of allo-LS $(P=0.004)$, most likely due to the higher and prolonged immunesuppression in this group of patients. Overall survival was $73 \%$, in the allo-LS group survival was only $53 \%$. In multivariable analyses allo-LS was the only predictor for higher mortality $(P=0.04)$. We hypothesize that early infection of the respiratory tract with a common cold virus make the lungs a target for alloimmunity, leading to life threatening lung disease.

\section{P503}

Impact of HLA-DPB1 mismatches on outcome in 10/10 HLA matched unrelated HSCT

J.M. Tiercy (1), F. Bettens (1), A. Gratwohl (2), U. Schanz (3), T. Güngör (4), Y. Chalandon (1), D. Heim (2), G. Stüssi (3), G. Nicoloso de Faveri (5), J. Passweg (1)

(1)Univeristy Hospital Geneva (Geneva, CH); (2)University Hospital Basel (Basel, CH); (3)University Hospital Zurich (Zurich, CH); (4)Kindersspital (Zurich, CH); (5)Swiss Blood Stem Cells Registry (Bern, $\mathrm{CH}$ )

The relevance of HLA-DPB1 incompatibilities on clinical outcome after unrelated HSCT remains controversial, in part 
due to differences in patients studied and to variations in compatibility at the other HLA loci. In order to assess the impact of DPB1 matching we thus retrospectively typed consecutive unrelated HSCT donor/recipient pairs matched by high resolution typing at the HLA-A,B,Cw,DRB1/B3/B4/ B5, and DQB1 loci for DPB1 alleles. The study group comprised 249 patients transplanted in Switzerland between 1990-2008 with acute leukemias (45\%), chronic leukemias (18\%), myeloproliferative/dysplastic syndromes (17\%), lymphomas/multiple myelomas $(10 \%)$, aplastic anemias $(3 \%)$, and other diseases $(7 \%)$. T-cell depletion was performed on $23 \%$ patients. Of these 249 recipient/donor pairs, $30(12 \%)$ were DPB1-compatible, $173(69.5 \%)$ had 1 or 2 DPB1 mismatches, and $46(18.5 \%)$ had a single and unidirectional disparity either in GVH or rejection direction only. Five-year overall survival estimates showed significant differences between donor/recipient pairs with one and two DPB1incompatibilities as compared to matched or uni-directional mismatched pairs $(49 \% \pm 10 \%$ vs. $70 \% \pm 19 \%, P=0.009)$. One or two HLA-DPB1 mismatches and uni-directional mismatches in GVHD but not in rejection direction were significantly associated with grade II-IV GVHD. When DPB1 mismatches were classified as permissive $(P) /$ not permissive (NP) according to previously published algorithms (Crocchiolo et al., Blood 2009; 114: 1437), neither overall survival nor aGVH incidence did correlate with the type of mismatches (Table).

Altogether these data clearly show a deleterious impact of HLA-DPB1 incompatibilities on aGVHD incidence. HLA-DPB1 typing may help in pretransplant risk assessments for GVHD and mortality. In patients with common HLA haplotypes, HLADPB1 matching may contribute to optimal donor selection once several 10/10 matched donors have been identified.

\begin{tabular}{|l|l|l|}
\hline Group & II-IV GVHD (6 mths) & Survival (5 yrs) \\
\hline HLA matched 12/12 & $18 \pm 15 \%$ & $70 \pm 19 \%$ \\
\hline 1 full DPB1 mismatch & $44 \pm 11 \%$ & $48 \pm 14 \%$ \\
\hline 2 full DPB1 mismatches & $49 \pm 12 \%$ & $50 \pm 15 \%$ \\
\hline DPB1 mismatch (GVHD direction) & $50 \pm 28 \%$ & $56 \pm 37 \%$ \\
\hline DPB1 mismatch (rejection direction) & $25 \pm 16 \%$ & $70 \pm 17 \%$ \\
\hline P & 0.021 & 0.133 \\
\hline DPB1 permissive mismatch & $43 \pm 13 \%$ & $55 \pm 19 \%$ \\
\hline DPB1 non-permissive mismetch & $44 \pm 9 \%$ & $51 \pm 11 \%$ \\
\hline
\end{tabular}

\section{P504}

\section{Dissection of GvHD and GvL by FBTA05, a novel} trifunctional anti-CD3 $\mathrm{x}$ anti-CD20 antibody

R. Buhmann (1), M. Stanglmaier (2), B. Simoes (3), H. Menzel (4), C. Peschel (4), P. Ruf (2), H. Lindhofer (2), H.-J. Kolb (3) (1)Klinikum Grosshadern, LMU (Munich, DE); (2)TRION Research (Munich, DE); (3)Helmholtz Zentrum (Munich, DE); (4)Klinikum rechts der Isar, TU (Munich, DE)

Graft-versus-host disease (GvHD), associated in more than half of the patients undergoing allogeneic stem cell transplantation (SCT), is the main contributor to transplant-related mortality. Thus, novel treatment strategies are required augmenting graft-versus-leukemia/lymphoma $(G v L)$ effects while reducing the risk of undesirable reactivity against normal host cells.

In that context we studied whether FBTA05, a novel trifunctional anti-CD20 $x$ anti CD3 antibody, could augment GvL activityafter allo-SCT and donor lymphocyte infusion (DLI), while avoiding GvHD. In a pilot trial, eight patients (four cases with p53-mutated chronic lymphocytic leukemia (CLL) and four patients with high grade non-Hodgkin's lymphoma (HG-NHL) refractory to standard therapy were treated with escalated doses of FBTA05 (range 10-2,000 micro gram) followed by DLI.
Three out of the four CLL patients showed a prompt but transient clinical and hematological response. In one patient with HG-NHL a halt in progression for almost 4 months could be observed. Side effects (fever, chills and bone pain) were tolerable and appeared at antibody dose levels between 40 and 200 micro gram. Human anti-mouse antibodies (HAMA) were not detectable, allowing repeated treatment courses with FBTA05. Moreover, none of the responders evaluable for GvHD (survival $>100$ days following DLI) revealed clinical signs of active GvHD although repeated applications of DLIs up to $1 \times 10^{8}$ $\mathrm{T}$ cells/kg body weight were performed. The cytokine profile was characterized by transiently increased release of IL-6, IL-8 and IL-10. Plasma concentrations of FBTA05 reflected differences in treatment, but strictly correlated with the corresponding dosing schedules. The highest plasma concentrations of FBTA05 with 0.38 micro gram $/ \mathrm{mL}$ were detectable after application of 2,000 micro gram FBTA05 accompanied by the rapid clearance of antibodies within few days. So far no significant correlation with tumor burden or disease activity could be established.

In summary, FBTA05 induced prompt anti-tumor responses in treatment-refractory patients. Moreover, the absence of GvHD after repeated courses of DLI indicates the potency of FBTA05 to redirect the graft-related allogeneic response towards lymphoma cells. Based on these encouraging results, a clinical phase I/II dose-escalation study of FBTA05 and DLI in patients with CD20-positive low and high grade B-cell lymphoma after allo-SCT is planned.

\section{P506}

Significantly elevated $C D 4^{+} C D 25^{\text {high }}$ Foxp $3^{+}$regulatory T-cells in patients with chronic graft-versus-host disease after allogeneic haematopoietic stem cell transplantation R. Weigl, Z. Kuzmina, S. Eder, R. Knobler, N. Worel, C. Zielinski, H. Greinix (Vienna, AT)

Regulatory $\mathrm{CD}^{+} \mathrm{T}$ cells that express high levels of CD25 (Tregs) play a central role in maintaining immune homeostasis, mediate transplantation tolerance and prevent graftversus-host disease (GVHD). However, their role in the pathogenesis of chronic GVHD (cGVHD) is currently unclear. Controversial results with both elevated as well as normal or reduced numbers of Tregs were reported in cGVHD. Here, we investigated Treg cell numbers in patients at day 100 after $\mathrm{HCT}$, at onset of CGVHD and during immunosuppressive therapies (IST).

Patients and methods: Eighty-three consecutive patients (38 males, 45 females) including 27 without cGVHD, 38 with cGVHD and 18 with cGVHD given extracorporeal photopheresis (ECP) were enrolled into our study a median of 156 (range, 69 to 838) days after HCT. Serial investigations of $\mathrm{CD} 4^{+} \mathrm{CD} 25^{\text {high }} \mathrm{Foxp}^{+}$ $\mathrm{T}$ cells and other immune cell populations in the peripheral blood (PB) were performed by flow cytometric analysis and results were compared with clinical assessments of cGVHD activity according to the $\mathrm{NIH}$ consensus criteria.

Results: Around day +100 after HCT significantly higher $\mathrm{CD}^{+}{ }^{+} \mathrm{CD} 25^{\text {high }}$ Foxp3 $^{+} \mathrm{T}$ cells were seen in patients developing cGVHD thereafter compared to patients without cGVHD (mean 14.0 vs. 5.3 cells $/ \mathrm{mm}^{3}, P<0.001$ ). Furthermore, at onset cGVHD patients had significantly higher $\mathrm{CD} 4^{+} \mathrm{CD} 25^{\text {high }}$ Foxp $3^{+}$ T cells compared to HCT patients without CGVHD (mean 15.0 vs. 6.5 cells $\left./ \mathrm{mm}^{3}, P=0.014\right)$. In addition, $C D 4^{+} \mathrm{CD} 25^{\text {high }} \mathrm{Foxp}^{+}$ $T$ cells differed significantly between patients with mild, moderate and severe CGVHD with a mean of $13.4(P=0.02)$ vs. $18.5(P=0.011)$ vs. $12.1 \mathrm{cell} / \mathrm{s} / \mathrm{mm}^{3}$ compared to patients without CGVHD. In serial analyses an increase of $\mathrm{CD} 4^{+} \mathrm{CD} 25^{\text {high }}$ Foxp $^{+} \mathrm{T}$ cells to normal levels was observed in patients without cGVHD until a mean of 270 days after HCT. Patients with CGVHD responding completely to IST remained high with $\mathrm{CD}^{+} \mathrm{CD} 25^{\text {high }}$ Foxp3 $3^{+} \mathrm{T}$ cells with a mean of $16.9 \mathrm{cells} / \mathrm{mm}^{3}$ compared to 26.5 cells $/ \mathrm{mm}^{3}$ and $8.8 \mathrm{cells} / \mathrm{mm}^{3}$ in patients with partial resolution or no response to IST. Also, patients 
responding to ECP remained high with $\mathrm{CD} 4^{+} \mathrm{CD} 25^{\text {high }}$ Foxp $3^{+} \mathrm{T}$ cells (mean 16.0 cells $/ \mathrm{mm}^{3}$ ) during and at the end of therapy. Conclusions: High Treg cells are predictive of cGVHD. Whereas patients with active cGVHD have significantly elevated Treg cells, resolution of cGVHD is not accompanied by normal Treg numbers. Further studies with longer followup will be required to assess Treg reconstitution in cGVHD patients.

\section{P507}

Prospective classification of chronic graft-versus-host disease (cGVHD) according to the new National Institutes of Health (NIH) consensus criteria: no significant difference in overall survival between patients with overlap syndrome and classic cGVHD

Z. Kuzmina, S. Eder, A. Boehm, L. Vormittag, P. Kalhs, V. Petkov, G. Stary, J. Nepp, H. Greinix

Medical University of Vienna (Vienna, AT)

Recently, the NIH Consensus Development Project on Criteria for Clinical Trials in chronic graft-versus-host disease (cGVHD) established new criteria for diagnosis and staging of cGVHD severity based on retrospective data and expert opinion. For both acute as well as CGVHD new definitions were proposed. However, their prognostic relevance with regard to patients' outcome is currently unclear. We performed a prospective study on all consecutive patients undergoing allogeneic hematopoietic stem cell transplantation (HCT) since 2005 to assess the prognostic impact of the new cGVHD staging criteria.

Patients: One hundred seventy-eight patients (85 males, 93 females) with a median age of 40 (range, 20-65) years alive on day +100 after HCT with myeloablative $(n=110)$ or reduced-intensity $(n=68)$ conditioning and a related $(n=37)$ or unrelated $(n=141)$ stem cell donor were enrolled into the study. Fifteen received bone marrow, 162 blood stem cells and 1 unrelated cord blood with a median of 6.6 (range, 1.33-14) $\times 10^{6}$ CD34-positive cells per $\mathrm{kg}$ body weight. Starting on day +100 after HCT all patients were assessed clinically every 3 months in the Outpatient Clinic for cGVHD activity according to the NIH consensus criteria (Filipovich $\mathrm{AH}$ et al., BBMT 2005; 11: 945-956)

Results: One hundred twenty-six $(71 \%)$ patients experienced prior acute GVHD grades I to IV including 11 with recurrent, 15 with persistant, and 11 with late onset acute GVHD. Median time to onset of acute GVHD was 18 (range, 9-120) days after HCT. On day +100 ten patients had acute GVHD whereas 63 (35\%) had cGVHD including 45 with classic cGVHD and 18 with overlap syndrome, respectively. The overall incidence of cGVHD in our cohort was $69 \%$ with cGVHD occurring after a median of 151 (range, 82-510) days after HCT. Ninety-two patients $(75 \%)$ had classic cGVHD and $31(25 \%)$ overlap syndrome. After a median follow-up of 25 (range, 3-60) months 21 patients died transplant-related including 2 with acute, 6 with overlap syndrome, 7 with classic and 6 with no cGVHD. Probability of overall survival $(\mathrm{OS})$ at 4 years was significantly worse for patients without cGVHD with $39 \%(P=0.003)$ compared to $68 \%$ and $67 \%$ for patients with overlap syndrome and classic cGVHD, respectively.

Conclusions: No significant difference in OS between the new categories of cGVHD were observed in our prospective study. Whether other outcome variables differ between these 2 cohorts has to await further analyses.
P508

Pentraxin-3: an easily measurable soluble factor to improve post-transplant graft-versus-host disease monitoring and management

E. Dander (1), S. Bonanomi (1), P. Vinci (1), I. Cuccovillo (2), L. Di Maio (1), G. Lucchini (1), M. Migliavacca (1), P. Perseghin

(3), A. Rovelli (1), A. Balduzzi (1), A. Mantovani (2), C. Garlanda (2), B. Bottazzi (2), A. Biondi (1), G. D’Amico (1)

(1)University Milano-Bicocca (Monza, IT); (2)Istituto Clinico

Humanitas (Milan, IT); (3)San Gerardo Hospital, (Monza, IT)

Graft-vs-host disease (GVHD) is a major obstacle to safe allogeneic hematopoietic stem cell transplantation (HSCT), leading to significant morbidity and mortality. GVHD pathophysiology is still poorly understood and its diagnosis depends on clinical manifestations and invasive biopsies. Specific biomarkers for GVHD would facilitate the early and accurate recognition of this invalidating disease as well as the monitoring of patient response to adopted anti-GVHD pharmacological treatment. With the aim to explore new reliable markers for predicting and monitoring GVHD course, we focused on pentraxin-3 (PTX-3), an acute-phase protein, that has been shown to play a crucial role in orchestrating inflammatory immune responses.

Having obtained an informed consent, we collected plasma samples from 46 patients who received HSCT and from 9 healthy donors (HD) volunteers. After HSCT, 25/46 patients developed skin GVHD (18 acute GVHD and 7 chronic GVHD), while 21/46 never experienced it. PTX-3 plasma levels were monitored by ELISA assays. Concerning GVHD patients, blood samples were collected at the day of GVHD onset/ flare, before the beginning of GVHD-specific drug therapy. Moreover skin biopsies from GVHD patients and HD were collected and stained to evaluate PTX-3 presence by immunohistochemistry. Patients who did not develop GVHD after HSCT showed augmented PTX-3 plasma levels (mean $=3.3 \mathrm{ng} / \mathrm{ml}$, range $=1.1-$ $8.6 \mathrm{ng} / \mathrm{ml}$ ) if compared to $\mathrm{HD}$ (mean $=1.2 \mathrm{ng} / \mathrm{ml}$, range $=0.3-2.5$ $\mathrm{ng} / \mathrm{ml}, P<0.01)$. Interestingly, we observed a strong increase of PTX-3 plasma levels in patients with acute GVHD (mean $=42.2$ $\mathrm{ng} / \mathrm{ml}$, range $=6.7-218.2 \mathrm{ng} / \mathrm{ml}, P<0.01$ ) or with flair-ups of chronic GVHD (mean=15.8 ng/ml, range $=9-44.3 \mathrm{ng} / \mathrm{ml}$, $P<0.05)$. Histological analysis of PTX-3 in skin lesions of patients with active GVHD showed that, similarly to normal skin, PTX-3 was secreted at high levels around the perivascular lymphatics. On the other hands, PTX-3 was specifically produced in the derma of GVHD patients.

These preliminary results show that PTX-3 plasma levels increase very rapidly in patients experiencing active GVHD, candidating PTX-3 as an easily measurable soluble factor useful to corroborate clinical GVHD monitoring. Moreover, the high expression of PTX-3 in GVHD skin lesions suggests a relevant role of this protein in the disease process. Further studies are needed to clarify if PTX-3 could represent a good diagnostic and/or prognostic factor and its role in GVHD pathophysiology.

\section{P509}

An evolving intelligent system for diagnostic gene targets of acute GvHD: an integrated approach

R. Fedele (1), M. Cuzzola (1), M. Fiasché (2), C. Rigolino (1), I. Callea (1), O. lacopino (1), G. Messina (1), M. Martino (1), V. Barillà (1), S. Dattola (1), F. Morabito (2), P. lacopino (1) (1)AO Bianchi Melacrino Morelli (Reggio Calabria, IT); (2)DIMET (Reggio Calabria, IT)

Rationale: Acute graft-versus-host disease (aGVHD) is the major complication after allogeneic hematopoietic stem cell transplantation (AHSCT). Modern approaches are needed to confirm clinical diagnosis of aGVHD to treat patients (pts) correctly as soon as possible.

Aim: To evaluate if it is possible to create and use easily a "computational model" based on an Artificial Intelligence framework 
to analyze biological data in order to formulate the diagnosis of aGVHD.

Patients: Forty-one pts were enrolled in our study from March 2007 to September 2009. All pts underwent HSCT and 12 of them (34\%) developed aGVHD (II-III grade) confirmed by biopsy. Methods: We used peripheral blood (PB) samples from aGVHD (YES) pts taken at the time of diagnosis and PB samples from pts that didn't experienced aGVHD (NO). All together YES/ NO patient groups comprised a validation set. Total RNA was extracted from whole $\mathrm{PB}$ and used to perform a gene expression assay (GEP). GEP were carried out with TaqMan® ${ }^{\circledR}$ Low Density Array Fluidic to test occurrence of aGVHD. The macroarray project included 47 candidate genes involved in immune network and inflammation pathogenesis.

Computational Intelligence Approach. An Evolving Connectionist System has been developed integrating different methods: Multiple Linear Regression, Support Vector Machines, Evolving Spiking Neural Networks and Dynamic Evolving Neuro-Fuzzy Inference Systems.

Results: From first data resulted that not all gene transcripts measured were related to aGVHD classification. Some genes resulted irrelevant and some redundant from the machine learning point of view in order to select gene diagnostic targets and use them for diagnosis of aGVHD. Only a subset of 19 genes carried significant information with different weight. For example, Foxp3, SLP1, CCR4 transcripts weighed $100 \%$, STAT6, BCL2A1, CXCL1, FAS, FOS, IRF1, IL1B, STAT6 weighed 90\%, CCL5, CXCL10, FAS, IL1B and others weighed $80 \%$.

These data confirmed what we observed during GVHD diagnosis and monitoring, so that the profile variations can be associated with both the GVHD presence and the treatment response. Finally, the results told us that it is possible to identify aGVHD using a restricted number of gene transcripts with an accuracy $>92 \%(0,002 \%$ error rate $)$.

Conclusion: Computational Intelligence Approach provides a strong overall accuracy to confirm aGVHD in HSCT pts. Our study outlines the importance and utility of non-invasive tool for aGVHD diagnosis based on GEP.

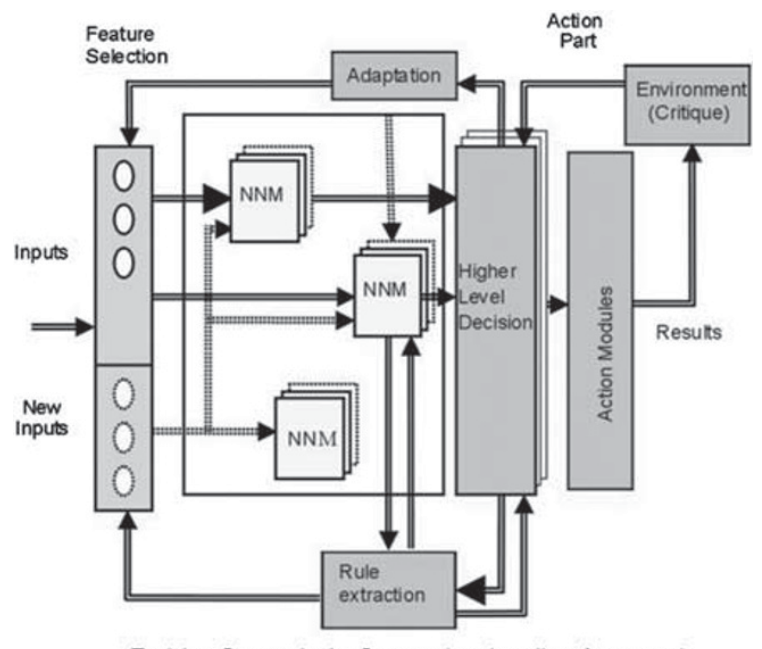

Evolving Connectionist System that describes frame work.
P510

Allogeneic human mesenchymal stem cell therapy (Prochymal ${ }^{\circledR}$ ) as a rescue agent for severe treatment resistant GvHD in paediatric patients

J. Kurtzberg (1), V. Prasad (1), M.S. Grimley (2), B. Horn (3), P.A. Carpenter (4), D. Jacobsohn (5), S. Prockop (6)

(1)Duke University Medical Center (Durham, US); (2)Methodist Healthcare System (SanAntonio, US); (3)University of California, San Francisco (San Francisco, US); (4)Fred Hutchinson Cancer Research Center (Seattle, US); (5)Children's Memorial Hospital (Chicago, US); (6)Memorial Sloan Kettering Cancer Center (New York, US)

Background: Severe acute graft vs. host disease (GVHD) unresponsive to steroid and other immunosuppressive therapy typically leads to poor outcomes and high mortality. Case reports of human mesenchymal stem cells (MSCs) used for the rescue of pediatric patients with severe GVHD resistant to multiple second line agents has generated interest in the therapy. In this study we evaluate the risk/benefit profile of MSCs (Prochymal囚) administered under an FDA Expanded Access Program (Protocol 275) as a rescue agent for treatment resistant GVHD in pediatric patients.

Methods: Children with Grade B-D aGVHD failing steroids and other agents were eligible for enrollment. Patients were given 8 biweekly infusions of $2 \times 10^{6}$ cells $/ \mathrm{kg}$ for 4 weeks, with an additional 4 infusions weekly after day 28 in patients with a partial response, defined as improvement in at least one organ without progression in others.

Results: 59 patients (median age of 8 years, $61 \%$ male, $55 \%$ Caucasian, $83 \%$ recipient of an unrelated graft, and $41 \%$ cord blood) were treated. At baseline, the distribution of aGVHD grades B:C:D was 6 (10\%): $20(33 \%): 33(57 \%)$. The median duration of aGVHD before enrollment was 29 days, and patients failed an average of 3.2 lines of treatment for GVHD. Organ involvement was $60 \%$ skin, $87 \%$ gastrointestinal, and $38 \%$ liver. At day 28 , overall response (OR), defined as organ improvement of at least one stage without worsening in any other, was $64 \% ; 17 \%$ of patients had stable disease or mixed response; and $19 \%$ experienced progression. By grade, 28 day OR was $67 \%$ for $B, 75 \%$ for $C$, and $58 \%$ for $D$. Response by organ was recorded, with $47 \%$ of skin, $23 \%$ of $\mathrm{GI}$, and $39 \%$ of liver GVHD completely responding (stage 0 ) within the first 28 days of treatment. Overall survival through study duration (day 100 ) was $62 \%$. Achieving an OR at day 28 resulted in a significantly higher probability of survival when compared to patients who progressed within the first 28 days $(76 \%$ vs. $9 \%, P<0.05)$. Prochymal was generally well tolerated and there was no evidence of ectopic tissue formation.

Conclusion: Treatment with Prochymal produced a $64 \%$ response rate in patients with otherwise refractory severe GVHD patients by day 28. Response to Prochymal correlated with improved overall survival at 100 days. MSC therapy appears to have an excellent risk/benefit profile and should be considered in pediatric patients with GVHD non-responsive to steroids.

P511

CCR5 expression on circulating blood DC post-allogeneic haematopoietic cell transplant is highly predictive for the development of clinically significant acute graft-versushost disease

M. Sartor, J. Lau, D. Gottlieb, K. Bradstock Westmead Hospital (Sydney, AU)

Dendritic cells (DC) are central to the development of acute graft-versus-host disease (GvHD) following allogeneic hematopoietic cell transplantation (alloHCT). Because DC migration is tightly regulated by the interaction between chemokine receptors on DC and their ligands, we investigated the relationship between the severity of acute GvHD and the expression of the chemokine receptors CCR5 and CCR7 on CD11c+ myeloid and 
CD11c- plasmacytoid from the peripheral blood of 32 patients post alloHCT.

Methods: Peripheral blood was collected twice weekly up to day 100 post transplant from 22 patients who received transplants from matched siblings, 10 from unrelated donors. Nineteen transplants were performed with myeloablative conditioning, 13 with reduced intensity conditioning. The expression of each chemokine receptor on CD11c+ and CD11C- DC was calculated using multiparameter flow cytometry. The percentage of CD11C+DC expressing a given receptor was added to the percentage of CD11c- DC expressing the same receptor to give a maximum score that could vary from 0 to $200 \%$.

Eleven of 32 patients developed moderate to severe acute GvHD (grade II-IV), the remaining 21 patients developed either no GvHD or only grade I GvHD. The percentage of DC expressing either CCR5 or CCR7 was correlated with the development of acute GvHD. CCR7 expression was detected in 13 of 32 patients post-HCT with a median of $2.3 \%$ of DC positive (range 0 to $39 \%$ ). CCR7 expression on DC showed no association with the severity of acute GVHD $(P=1.0)$. In contrast, higher CCR5 expression was detected on DC in patients developing grade II-IV GvHD (median 98.0\%, SEM $9.1 \%$ ) than in those with grade 0-I GvHD (median 5.2\%, SEM $5.1 \%) P<0.0001$. All eleven patients with grade II-IV GvHD expressed CCR5 at a level of $>35 \%$ of myeloid and plasmacytoid DC. Only two of 21 patients with grade $1 \mathrm{GvHD}$ expressed CCR5 at the same level ( $66 \%$ and $94 \%)$. Most importantly, the expression of CCR5 preceded the development of moderate to severe GvHD in all patients by a median of 19 days (range 2 to 47 days, SEM 4.3 days). Using a receiver operator curve analysis, CCR5 expression above $35 \%$ demonstrated a sensitivity of $100 \%$ and a specificity of $90.5 \%$ for predicting grade II-IV GvHD.

Expression of CCR5 on circulating DC post allo-HCT predicts for the development of moderate to severe GvHD, and detection could allow earlier therapeutic intervention prior to the development of clinical GVHD.

\section{P512}

The persistence of recipient antigen presenting cells after stem cell transplantation in humans

M. Haniffa (1), V. Bigley (1), F. Ginhoux (2), S. Pagan (1), X.N. Wang (1), M. Merad (2), M. Collin (1)

(1)Newcastle University (Newcastle upon Tyne, UK); (2)Mount Sinai School of Medicine (New York, US)

Recipient antigen presenting cells (APC) may play a critical role in graft versus host responses, through their activation by conditioning and direct presentation of recipient MHC class restricted minor antigens. Their persistence may influence GVHD tropism and yet also be critical for graft versus leukaemia responses to leukocyte-restricted antigens.

We set out to determine the nature and turnover of APC in the skin, a frequent target of GVHD and accessible peripheral tissue. In addition to epidermal Langerhans cells, we find a complex array of dermal APC including CD14 + dendritic cells (DC), $\mathrm{CD} 1 \mathrm{c}+\mathrm{DC}$ and perivascular macrophages. In parallel with recent murine studies, we find a subset of CD1a + Langerin + DC unrelated to Langerhans cells. Gene expression profiling indicates that blood monocytes, CD14 + DC and macrophages are related cells, which express M-CSF receptors and do not proliferate. In contrast, CD11c + blood DC and dermal CD1c + cells related and show flt-3 dependent proliferation.

After transplantation, APC turnover at strikingly variable rates. CD14+DC and CD1c+DC are rapidly replaced by donor cells, regardless of conditioning intensity or the occurrence of GVHD. Langerhans cells, which may persist indefinitely in murine transplants, are replaced by donor cells within 100 days in almost all human transplants including non-myeloablative and $\mathrm{T}$ depleted transplants. The rate of their turnover is more rapid with full intensity transplantation and is augmented by GVHD. The most long-lived of all human APC are dermal macrophages. Recipient macrophages are found in many patients up to one year post-transplant and are not significantly depleted by prior GVHD. In situ, these cells are located in the perivascular tissue. In vitro they elaborate pro-inflammatory cytokines and stimulate activation and cytokine release by memory CD4 + and CD8 + T cells. In GVHD lesions, recipient and recruited donor macrophages appear to interact with donor T cells and participate in GVHD pathology.

These studies highlight the varied roles of recipient APC in graft versus host responses. Migratory myeloid DC and even LC are present for only short intervals post-transplantation and can only exert transient effects on donor T cell activation. Recipient macrophages survive for much longer periods and may be critical in delayed acute GVHD, chronic GVHD and DLI responses, although their inability to migrate to $L N$ and lower potency as APC are mitigating factors.

\section{P513}

Evaluation of NIH consensus criteria for classification of late acute and chronic GvHD in reduced-intensity conditioning stem cell transplantation

S. Thepot (1), J. Zhou (1), A. Perrot (1), M. Robin (1), R. Peffault de latour (1), L. Ades (2), P. Ribaud (1), R. Porcher (1), G. Socie (1)

(1)Hospital Saint Louis (Paris, FR); (2)Hospital Avicenne (Paris, FR)

Background: The National Institutes of Health (NIH) consensus criteria for the diagnosis of chronic graft-versus-host disease (GVHD) emphasized clinical manifestations of GVHD rather than the classical time of onset. Incidence and impact in term of relapse and no-relapse mortality (NRM) of this new classification is not well known after RIC.

Methods: We retrospectively reviewed 177 consecutive patients (pts) in Saint Louis' Hospital undergoing an SCT for hematologic malignancy and surviving at least day +100 after RIC between Oct 2001 and Dec 2008. The association of occurrence of chronic GVHD with the risk of relapse and non-relapse death was analyzed using time-dependent covariates in causespecific proportional hazards models.

Results: Among 177 pts (M/F: 109/68), with a median age of 52 years old (6-68 years) 106 pts $(60 \%)$ received HLAidentical sibling transplantation. Source of stem cells was mobilized peripheral blood stem cell for 164 pts (93\%), bone marrow for 6 pts (3\%) and 7 cord blood $(4 \%)$. After a median follow-up of 28 months (range 5-92 months), a total of 121 pts $(68 \%)$ developed chronic GVHD according to the Seattle criteria and when using $\mathrm{NIH}$ consensus criteria, 84 pts (47\%) developed chronic GVHD, including 66 pts (55\%) with classic chronic GVHD and 18 pts (15\%) overlap syndrome. Patients reclassified included: 5 pts with late onset acute GvHD, 27 pts had recurrent and 13 had persistent acute GVHD (numbers do not to previous sentence because some of these patients latter developed chronic GvHD). The cumulative incidence of chronic GVHD at 36 months was $74 \%(95 \% \mathrm{Cl} ; 65.9 \%-80.5 \%)$ when using Seattle criteria compared to $53.7 \%(95 \% \mathrm{Cl} ; 45 \%-$ $61.6 \%$ ) with NIH chronic GVHD criteria.Two-year Cumulative incidences of relapse and NRM using both classifications are summarized in Table 1. In Cox model with GvHD as a time dependent covariate, the NRM was significantly higher in patients with late onset, persistent and recurrent acute GVHD compared to no GVHD (hazard ratio (HR) 6.4, 24.5 and 11: $P=0.014, P<0.0001, P<0.0001$, respectively), whereas the NRM was statistically increased in case of chronic GVHD using Seattle criteria (HR: 22.1; $P<0.001$ ).

Conclusion: The cumulative incidence of chronic GVHD "decrease" about $20 \%$ when using NIH consensus criteria compared to Seattle criteria in our cohort of RIC. Most of the NRM occurred beyond 100 days after SCT was due to the increased risk of NRM in patients with late onset, recurrent or persistent acute GVHD. 


\begin{tabular}{|l|r|r|}
\hline & Relapse / progression & NRM \\
\hline Seattle criteria & & \\
\hline Chronic GVHD & $9.70 \%$ & $36.40 \%$ \\
\hline No chronic GVHD & $16.50 \%$ & $1.30 \%$ \\
\hline NIH criteria & & \\
\hline classic chronic / overlap syndrome & $9.80 \%$ & $22.70 \%$ \\
\hline No chronic GVHD & $17.70 \%$ & $15.30 \%$ \\
\hline
\end{tabular}

\section{P514}

Effect of donor regulatory T-cells on CMV-specific CD8 + T lymphocytes reconstitution and acute GvHD after allogeneic haematopoietic stem cell transplantation D. Pastore (1), M. Delia (1), A. Mestice (1), P. Carluccio (1), A. Russo Rossi (1), A. Ricco (1), A.M. Giordano (1), L. Cazzato (2), V. Liso (1), G. Specchia (1)

(1)Hematology (Bari, IT); (2)Transfusion Medicine (Bari, IT)

Introduction: Regulatory T-cells (Treg,CD4+CD25highFoxp $3+)$ are important regulators of allo-reactivity and may therefore represent an important predictor for the risk of acute graft versus-host disease (aGvHD) and immune reconstitution after allogeneic peripheral stem cell transplantation (allo-PBSCT). Patients and methods: To determine the clinical significance of Tregs in peripheral stem cell grafts, we analyzed 33 myeloablative alloPBSCT and correlated the T regs in the donor graft with the aGvHD incidence and immunological recovery (evaluated on recovery of CMV-specific CD8 + T lymphocytes by tetramer analysis). We used fluorochrome-conjugated tetrameric complexes of HLA-A101, HLA-A201, HLA-B702, HLA-B801, HLA B3501 to monitor recovery of CMV-specific CD8 + according to the patient's HLA). Patients were transplanted with unmanipulated peripheral blood stem cells from an HLA identical related donor $(n=28)$ or an HLA identical unrelated donor $(n=5)$. Median age was 32 years (range(r) 18-58); diagnoses were acute myeloid leukaemia $(n=30)$, acute lymphoblastic leukaemia $(n=3)$. The median Tregs dose administered was $5 \times 10^{6} / \mathrm{Kg}(r$ : 1-20). The patients were divided into a high Tregs group (Tregs $>5 \times 10^{6} /$ $\mathrm{Kg}, \mathrm{n}=13$ ) and a low Tregs group (Tregs $<5 \times 10^{6} / \mathrm{Kg}, \mathrm{n}=20$ ) according to the number of Tregs in the grafts.

Results: Median CMV-specific CD8 + T lymphocytes were significantly higher in patients with high than with low Tregs in the graft at 1 (2 cells $/ \mathrm{mmc}$ vs. $0, P<.001), 2$ (6 cells $/ \mathrm{mmc}$ vs. 1 , $P<.001), 3$ (15 cells $/ \mathrm{mmc}$ vs. $3, P<.001)$ months, respectively. During the three months after transplantation, CMV infection/disease was observed in $2 / 13(15 \%)$ patients with high Tregs and in $12 / 20(60 \%)$ patients with low Tregs $(P=.015)$. Moreover, the median recovery of Tregs after transplantation was significantly higher in patients with high Tthan with low Tregs in the grafts at 2 (15 cells $/ \mathrm{mmc}$ vs. $6, P<.001)$ and 3 months $(23$ cells $/ \mathrm{mmc}$ vs. $8, P<.001)$. The incidence of aGvHD (grade II-IV) in high Tregs infused group was lower in the high Tregs group than the low Tregs group ( $1 / 13$ or $7 \%$ vs. $11 / 20$ or $55 \%, P=.009$ ).

Conclusions: We suggest that there is a good correlation between the number of Tregs in the graft and the incidence of GvHD after myeloablative allo-PBSCT. Tregs mediate protective effects toward the aGvHD and the maintenance of an optimal microenviroment for the reconstitution of functional immunity.Our results support further consideration of Tregs immunotherapy for clinical alloSCT.

\section{P515}

Genetic mismatch in GSTT1 is associated with hepatic acute graft-versus-host disease in allogeneic stem cell transplantation

I. Tallon Ruiz, M.J. Martinez Bravo, R. Cardesa, I. Aguilera, A. Nuñez, Á. Urbano Ispizua, I. Espigado Tocino

Hospital Universitario Virgen del Rocío (Sevilla, ES)

Background: Glutathione S-transferase T1 (GSTT1) is a detoxifying hepatic enzyme involved in drug metabolic phase II path- way. It mainly appears in hepatic, renal and blood cells. Twenty percent of Caucasian population and $60 \%$ of Asiatic population present a deletion in the gene encoding the enzyme GSTT1, resulting in the absence of the enzyme, the so called "null genotype". We previously published that a specific antibody-mediated alloimmune response is triggered when a null GSTT1 recipient receives a GSTT1 positive liver or kidney graft. The potential influence of GSTT1 genotype on immunobiologic and toxic reactions in the liver after allo-SCT has not been studied yet.

Aims: Analyzing the association of GSTT1 polymorphic variants with the incidence of hepatic acute GVHD in 73 patients undergoing allo-SCT and its effects on survival.

Methods and results: We retrospectively analyzed a total of 73 HLA-identical siblings Allo-TPH recipients. All patients received myeloablative-conditioning and all donor and recipient pairs were Caucasians. Median follow-up was 75 months (2-171). GSTT1 variants were analyzed by PCR method. The distribution of patients into groups was the following: Group 1: null GSTT1 donor (donor -)/positive GSTT1 recipient (recipient +$)(n=9)$; Group 2: positive GSTT1 donor/null recipient $(n=4)$; Group 3: positive GSTT1 donor/positive recipient $(n=51)$; Group 4: null GSTT1 donor/null recipient $(n=9)$. Hepatic GVHD and toxicity were diagnosed based on accepted international clinical criteria and on ancillary tests, including liver biopsy, when appropriate. Results of the association of GSTT1 genotypes and hepatic alteration is shown in Table 1.

The effect of GSTT1 genotypes disparities on the survival is shown in the graph below (graph 1):

Conclusions:

1. Genetic mismatch in GSTT1 between donors and recipients has a strong association with acute hepatic GVHD. This could help in the differential diagnosis of hepatic alterations after allo-SCT.

2. Null GSTT1 recipients presented a greater incidence of hepatic toxicity after allo-SCT without reaching statistical significance.

3. Genetic mismatch between donors and recipients (donor - / recipient + ) showed a tendency to a decreased survival.

\begin{tabular}{|c|c|c|c|c|c|}
\hline & $\begin{array}{l}\text { Donor - } \\
\text { Recipient } \\
+ \text { (grou 1) }\end{array}$ & $\begin{array}{l}\text { Donor + } \\
\text { Recipient } \\
-\quad \text { (group } \\
\text { 2) }\end{array}$ & $\begin{array}{l}\text { Donor + } \\
\text { Recipient }+ \\
\text { (group 3) }\end{array}$ & $\begin{array}{l}\text { Donor - } \\
\text { Recipien } \\
t \quad- \\
\text { (group 4) }\end{array}$ & $\begin{array}{l}\text { Comparison } \\
\text { between } \\
\text { groups }\end{array}$ \\
\hline $\begin{array}{l}\text { Hepatic acute } \\
\text { GVHD } \\
\text { Day of } \\
\text { appearance } \\
\text { (median) }\end{array}$ & $\begin{array}{c}4 / 9 \\
(44.4 \%) \\
+24.5 \\
(+14 /+34)\end{array}$ & $\begin{array}{c}3 / 4 \\
(75 \%) \\
+180 \\
(+24 /+240 \\
+\end{array}$ & $\begin{array}{c}8 / 51 \\
(15.68 \%) \\
+59 \\
(+18 /+210)\end{array}$ & $\begin{array}{c}1 / 9 \\
(11.11 \%) \\
+13\end{array}$ & $\begin{array}{l}\text { Genetic } \\
\text { mismatch } \\
(1+2 \text { vs } 3+4) \\
\text { p: } 0,001 \\
\text { p: } 0.64\end{array}$ \\
\hline $\begin{array}{l}\text { Hepatic } \\
\text { toxicity } \\
\text { Day of } \\
\text { appearance } \\
\text { (median) }\end{array}$ & $\begin{array}{c}3 / 9 \\
(33.33 \%) \\
+4 \\
(+2 /+7)\end{array}$ & $\begin{array}{c}2 / 4 \\
(50 \%) \\
+5.5 \\
(+4 /+7)\end{array}$ & $\begin{array}{c}12 / 51 \\
(23.50 \%) \\
+5 \\
(+3 /+10)\end{array}$ & $\begin{array}{c}5 / 9 \\
(55.55 \%) \\
+5 \\
(+2 /+6)\end{array}$ & $\begin{array}{l}\text { Null } \\
\text { recipient } \\
\text { genotype } \\
(2+4 \text { vs } 1+3) \\
\text { p: } 0.083 \\
\text { p: } 0.44\end{array}$ \\
\hline
\end{tabular}

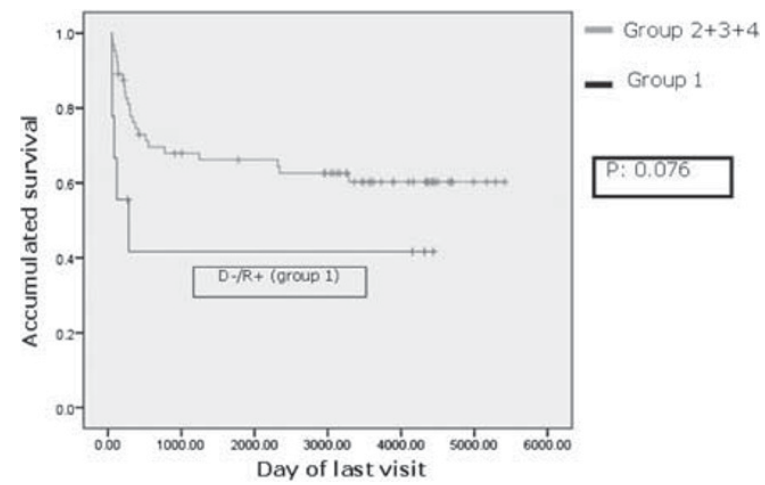




\section{P516}

Citrullinaemia is a helpful marker of gastro-intestinal GvHD in children

E. Merlin, R. Minet-Quinard, E. Doré, F. Isfan, C. Paillard, A. David, J. Kanold, V. Sapin, F. Deméocq

$\mathrm{CHU}$ (Clermont-Ferrand, FR)

The clinical grading of gastro-intestinal GVHD relies on the daily volume of diarrhea. This parameter is often very hard to measure accurately, especially in small children. Plasma citrulline, a nonprotein amino acid produced by enterocytes, has been shown as a marker of remnant enterocyte mass in patients with short bowel. Our objective was to evaluate the utility of citrulline as a marker of severity of acute intestinal GVHD in children.

Patients and methods: 20 children received allogeneic bone marrow transplantation in our center between 2008 and 2009. For all patients, a sample of plasma was collected every week between day-7 and day 28 of BMT; in case of acute GVHD involving or not the gastro-intestinal tract, weekly collections were carried on until resolution of acute GVHD or occurrence of chronic GVHD. Acute GVHD was staged by using the Glücksberg criteria.

Results: All children had normal values of plasma citrulline before the beginning of conditioning regimen $(19 \mu \mathrm{mol} / / \pm 4)$. Citrullinemia decreased at a minimum level on day 0 . On day 28 , all children who did not develop acute GVHD had returned to the initial value $(16.7 \mu \mathrm{mol} / \mathrm{I} \pm 3)$. The drop of citrullinemia was deeper in children who received myeloablative conditioning regimen as compared with non-myeloablative (3.6 \pm 3 vs. $8.1 \pm 5 \mu \mathrm{mol} / /$ respectively, $P=0.02)$. Nine children developed acute GVHD, with gastro-intestinal involvement in 5 of them. Acute GVHD without GI involvement did not impact on citrullinemia. There was a strong correlation between citrullinemia and the clinical stage of GIT involvement (mean values: grade 0: $16.3 \mu \mathrm{mol} / \mathrm{l}$, grade 1: $10 \mu \mathrm{mol} / \mathrm{l}$, grade 2: $6.5 \mu \mathrm{mol} / \mathrm{l}$, grade 3: $4.7 \mu \mathrm{mol} / \mathrm{l}$, grade 4: $0 \mu \mathrm{mol} / \mathrm{l}, P<0.001)$. In patients who responded to GVHD therapy, the increase of citrullinemia occurred in the first days after beginning of treatment, before achievement of clinical improvement.

Conclusion: Citrullinemia is a non-invasive suitable marker of severity of acute intestinal GVHD, which can be useful to assess early and accurately the response to the treatment of GVHD. Its diagnosis value deserves to be assessed in reference to the diagnosis gold-standard (histological examination)

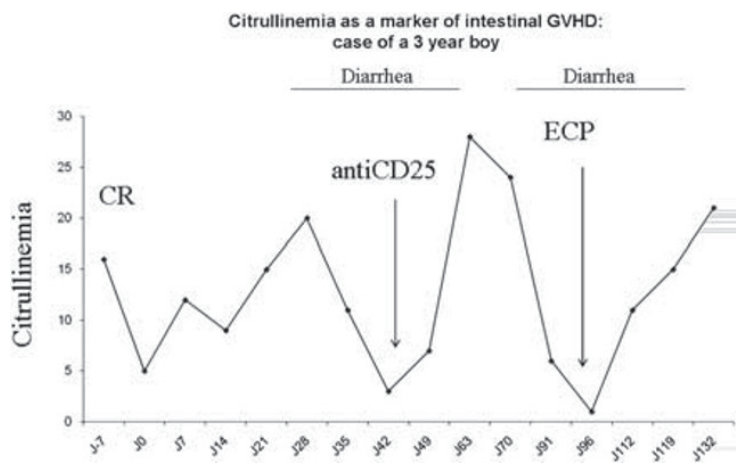

P517

Candidate pathway approach of the single nucleotide polymorphisms involved in cytokine, NFKB, PDGF or transforming growth factor-beta pathway could predict the risk of acute/chronic GvHD following allogeneic HSCT D.H. Kim (1), C.W. Jung (1), S. Park (1), J.H. Park (2), H.J. Jun (3), W. Xu (4), A. Galal (4), V. Gupta (4), J. Kuruvilla (4), H. Messner (4), J. Lipton (4)

(1)Samsung Medical Center (Seoul, KR); (2)Ulsan University Hospital (Ulsan, KR); (3)Geonyang University Hospital (Daejun, $K R$ ); (4)Princess Margaret Hospital (Toronto, CA)

Background: The current study is a hypothesis generating study in order to identify potential predictive surrogate associated with the risk of acute or chronic GVHD which were potentially linked to the Th1/Th2 cytokine activation or transforming growth factor (TGF) mediated pathway. In addition, transplant outcomes after allogeneic hematopoietic stem cell transplantation (HSCT) were also evaluated for SNPs involving multiple candidate pathways.

Methods: The current study was performed to identify genetic surrogates predicting the risk of acute/chronic GVHD, relapse free survival, non-relapse mortality and overall survival in 394 pairs transplanted at the Princess Margaret Hospital, Toronto, ON, Canada. In addition, the predictive markers for organ specific incidence of acute/chronic GVHD were also evaluated (i.e. for skin/liver/gut acute GVHD or skin, eye, oral, lung or liver chronic GVHD)

Total of 261 single nucleotide polymorphisms (SNPs) in 56 genes were determined for donor/recipients' genotypes using MALDI-TOF based platform, involving in the pathways of

1) cytokines (i.e. IL1A, IL1B and its receptor, IL1R1, IL2 \& IL2RA, IL4 \& IL4R, IL6 \& IL6R, IL8, IL10 \& IL10RA, RB, IL12A/B and IL12RB1, IFNG \& IFNGR1/2, TNFTI/II/II),

2) NFKB (NFKB1/2/A, NFKBIA/B, IKB, IKK1, IKBKB, RelB),

3) apoptosis (FAS, TRAIL \& TRAILR1),

4) endothelium nitric oxide regulation (EDN1, NOS1/2A/3),

5) PDGF (PDGFB/C/D \& PDGFRA/B),

6) TGF-beta (TGFB1/2 \& TGFBR1/2/3, TGFRB1),

7) Toll-like receptor (TLR4/5),

8) NOD2/CARD15 and

9) prostaglandin-endoperoxide synthase (PTGS1/2).

Results: The risk of chronic GVHD was significantly associated with SNP of the genes involved in the pathway of NFKB, PDGF, TGF-beta, and some of cytokines (esp. type II, IL6 \& IL4), while that of acute GVHD associates with the genotypes in the pathway of TNF and apoptosis. In addition, survival after allogeneic transplantation was associated with the genotypes in NOS (nitric oxide synthase, endothelial nitric oxide synthesis pathway), IL-2 and TGF pathway.

Conclusion: Because of complex nature of GVHD pathogenesis, multiple candidate pathway SNPs has been explored targeting SNPs in the pathway of cytokines, NFKB, apoptosis, endothelium nitric oxide regulation, NOD2/CARD15, PDGF, PTGS1/2, TGF-beta and TLR. Different involvements were noted of TGF-beta, PDGF or NFKB with chronic GVHD versus TNF and apoptosis-associated SNPs with acute GVHD.

\section{P518}

Six-gene expression model to diagnose chronic graft-versus-host disease

E. Park (1), E. Jeon (2), S.B. Cho (2), N.Y. Kim (2), J. Kim (2), I. Kim (2), S.S. Yoon (2), S. Park (2)

(1)Chung-Ang University Hospital (Seoul, KR); (2)Seoul National University (Seoul, KR)

Introduction: Chronic graft-vs-host disease (GVHD) is one of the most troublesome complications after allogeneic hematopoietic stem cell transplantation (HSCT). The pathogenesis is not clearly understood, and studies to develop standardized diagnostic guidelines are in progress. We have previously reported differentially expressed genes and pathways in chronic GVHD 
using one-dye microarray analysis and also selected diagnostic genes predicting chronic GVHD (Bone Marrow Transplant, $41: 1047,2008)$. In this study, we analyzed microarray data obtained from both one-dye and two-dye microarray experiments, to explore microarray platform-independent results and to improve the prediction accuracy and reproducibility.

Patients and methods: Twenty-four patients who received allogeneic HSCT were included in this study. Eleven patients had chronic GVHD and 13 patients had no evidence of chronic GVHD. The genes of each microarray dataset were ranked by Wilcoxon rank sum statistics between the patients with or without GVHD. The classifier genes highly ranked in both datasets were used as features to diagnose chronic GVHD. The diagnosing performance of the feature genes were evaluated with support vector machine (SVM) and k-nearest neighbor (KNN) classifying algorithm. 10-fold cross validation was applied for the estimation of the prediction accuracy. The microarray results were validated in separate 14 patients (8 patients with chronic GVHD, 6 patients without chronic GVHD) not included in the microarray experiments using quantitative real time polymerase chain reaction (qRT-PCR).

Results: The following six genes were highly ranked (top $2.5 \%$ ) in both datasets: calcium binding and coiled-coil domain 1 , transcriptional regulating factor 1 , isovaleryl coenzyme A dehydrogenase, cadherin 15, type 1, M-cadherin (myotubule), lymphocyte antigen 6 complex, locus $\mathrm{E}$ and proprotein convertase subtilisin/kexin type 9 . The prediction accuracy of the genes was $90 \%$ in the one-dye data and $92 \%$ in the two-dye data with the KNN. The prediction accuracy of the SVM was $87 \%$ and $90 \%$ in one-dye and two-dye data, respectively. The validation study showed that the six-gene expression model had considerable prediction accuracy (SVM: $80 \%$, KNN: $85 \%$ ).

Conclusion: We identified six classifier genes that could be used to diagnose chronic GVHD with favorable prediction accuracy and reproducibility.

\section{P519}

Distinction of acute, chronic inflammatory and chronic sclerotic graft-versus-host diseases of the skin according to cytokine, chemokine and cellular patterns

I. Klein, H. Greinix, W. Bauer, Z. Kuzmina, W. Rabitsch, R. Knobler, G. Stingl, G. Stary

Medical University of Vienna (Vienna, AT)

Graft-versus-host disease (GvHD) is a serious complication of allogeneic hematopoietic stem cell transplantation (HCT) that occurs in an acute, chronic-inflammatory and chronic-sclerotic form. Currently, it is unclear whether the same or different pathomechanisms lead to these different clinical presentations. To address this issue, we analyzed interleukins, chemokines and other relevant molecules in skin biopsies of acute $(n=12)$ and chronic-inflammatory $(n=9)$ GvHD by quantitative RT-PCR and evaluated, by immunohistochemistry, serial biopsies of non-lesional skin at different time points prior and after HCT $(n=14)$.

Results obtained revealed striking differences between acute and chronic GvHD lesions. In acute GvHD skin biopsies we found increased levels of the Th2 cytokines IL-4 $(P<0.01)$, and IL-13 $(P<0.05)$ together with IL-22 $(P<0.001)$ compared with biopsies from chronic-inflammatory GvHD patients. In addition we observed an upregulation of the Th2 chemokines CCL17 $(P<0.001)$ and CCL22 $(P<0.01)$ and a downregulation of the Th1 chemokines CXCL9 $(P<0.001)$ and CXCL10 $(P<0.001)$ in acute GvHD skin lesions as compared to the chronic inflammatory GvHD. Around day +20 after HCT expression of thymic stromal lymphopoietin (TSLP), an inducer of Th2 differentiation of naïve CD4 + T cells, in the epidermis was significantly higher in patients later developing acute GvHD compared to ones without later acute GvHD $(P<0.01)$.

Our data suggest that specific mechanisms are operative in acute and chronic forms of skin GvHD. Identification of acute
GvHD as Th2-mediated disease could provide the basis for the development of novel and more specific dignostic tools by the establishment of an unique marker profile for acute skin GvHD, allowing differentiation from drug or viral rashes.

\section{P520}

Treatment of Bronchiolitis obliterans syndrome following haematopoetic cell transplantation with extracorporal photopheresis and rapamycin

S. Fritsch (1), T. Herold (1), L. Peterson (1), R. Reibke (1), G. Ledderose (1), J. Braess (1), H.J. Kolb (2), J. Tischer (1)

(1)Ludwig-Maximilians-University Munich (Munich, DE); (2)University of Munich (Munich, DE)

Chronic graft versus host disease (GvHD) remains the most common cause of morbidity following allogeneic stem cell transplantation. Bronchiolitis obliterans syndrome (BOS) is an underrecognized manifestation of chronic GVHD and is associated with a very poor outcome. Extracorporal photopheresis (ECP) has shown some activity in the treatment of chronic GvHD as well as in BOS

11 patients (pts) diagnosed with BOS according to the National Institute of Health (NIH) Consensus Criteria for diagnosis of BOS after allogeneic stem cell transplantation were analyzed for improvement of pulmonary function, clinical symptoms and outcome. 2 patients received hematopoetic stem cells from an HLA-identical sibling, 4 patients from an unrelated HLA-identical donor, 3 patients from a mismatched sibling and 2 patients from an unrelated mismatched donor. Progressive chronic GvHD was seen in 2 pts, quiescent in 6 pts and de novo in 3 pts. All patients (median age of 39 years) were refractory to corticosteroids, including inhalative application forms. They received ECP for a minimum of 2 months. The median number of treatments was 35 (range 7-110). The manifestation of BOS was detected in median 281 days after transplantation. Additional treatment of BOS consisted of rapamycin in all pts. Further immunosuppressive agents were used in 3 pts. FEV1 and FEV1/FVC as parameters of pulmonary function were monitored continuously during treatment. Before the start of ECP FEV1/FVC was less than $50 \%$ in 5 pts and less than $60 \%$ in 6 pts. 2 patients showed a FEV1 of less than $35 \%$ predicted, 4 between $36 \%$ and $50 \%$ and 5 between $51 \%$ and $70 \%$. Improvement of lung function was defined as $10 \%$ increase of FEV1. This was observed in 5 of 11 pts $(45,5 \%)$. Response was better in patients with FEV1 more than $50 \%$ at diagnosis. Clinical symptoms such as dyspnea and dry cough improved in 8 of 11 pts $(72,7 \%)$. Overall survival (OS) after a follow up between 216 and 1559 days was $63,6 \%$ and median OS was 1168 days. Cause of death was progressive BOS in 2 patients, relapse of leukemia in one and cardiac failure in one.

ECP in combination with rapamycin proved a valuable therapeutic option for the treatment of steroid refractory BOS. Clinical symptoms and lung function could be improved in more than $70 \%$ and $45 \%$ of the patients respectively.

\section{P521}

Limited chronic graft-versus-host disease is associated with better survival after alternative donor transplants for haematological malignancies: a retrospective GITMO analysis

R. Crocchiolo, F. Ciceri, T. Lamparelli, R. Oneto, B. Bruno, M.P. Sormani, R. Fanin, G. Bandini, A. Bosi, A. Rambaldi, E. Alessandrino, M. Falda, A. Bacigalupo on behalf of the Gruppo Italiano Trapianto di Midollo Osseo

Introduction: Allogeneic hematopoietic stem cell transplantation (HSCT) represents a potential curative option for many hematological malignancies. Its ability to induce a graft-versus-tumor (GVT) effect can lead to eradication of disease; however, graftversus-host disease (GvHD) invariably occurs when a GVT is 
present and its morbidity is responsible for both early and late deaths in complete remission. Chronic GvHD (cGvHD) has been shown to be associated with reduced risk of relapse due to the GVT effect.

Objective: The objective of this study is to evaluate the role of cGvHD on alternative donor HSCT outcome.

Methods: HSCTs performed from January 1999 to March 2009 were retrospectively analyzed from GITMO registry; patients were $>18$ years old and affected by a hematological malignancy. Association between cGvHD and HSCT outcome was evaluated through Cox regression; cGvHD was analyzed as a time-dependent variable. Multivariate models for survival and relapse were obtained after including main patients' and transplants' characteristics in the analysis.

Results: A total of 1536 HSCTs were obtained. Median age at HSCT was 43 (18-69), median follow-up was 393 days (0-3355). 1073 patients were evaluable for cGvHD since they survived more than 100 days after HSCT: of these, 203 and 121 developed a limited and extensive cGvHD respectively so far, and 749 developed no cGvHD. Median time from HSCT to cGvHD was 211 days (100-3004). 302 of 749 (40\%) patients who did not develop any cGvHD died; deaths of patients with limited and extensive cGvHD were $27 \%$ and $39 \%$ respectively $(P=0.02$ for limited vs. extensive, $P=0.01$ for limited vs. no cGvHD, chi-square test). Multivariate analysis confirmed the survival advantage of patients with limited $\mathrm{cGvHD}(\mathrm{HR}=0.48$, $95 \% \mathrm{Cl} 0.33-0.71 ; P<0.0001)$ while no significant differences exist between patients without cGvHD and those with extensive form $(P=0.52)$. Other significant variables were CMV status and disease phase at HSCT (see Table). Multivariate model for relapse is shown in the Table above.

Conclusion: This large analysis confirms that occurrence of cGvHD is associated to reduced risk of relapse after HSCT for a hematological malignancy, compared to absence of cGvHD; limited cGvHD was associated to better survival while extensive form did not result in better survival probably due to severe infections or other fatal complications responsible of deaths in complete remission.

Multivariate Cou regression ana lysis

\begin{tabular}{|c|c|c|c|c|c|c|c|c|}
\hline & & \multicolumn{3}{|c|}{ Survival } & \multicolumn{4}{|c|}{ Relapse } \\
\hline & \multicolumn{4}{|c|}{$95.0 \% \mathrm{Cl}$} & \multirow[b]{2}{*}{$p$} & \multirow[b]{2}{*}{ HR } & \multicolumn{2}{|c|}{$95.0 \% a$} \\
\hline & $p$ & $H R$ & Lower & Upper & & & Lower & Upper \\
\hline NO OGYHO & & 1 & & & & 1 & & \\
\hline Limized & .000 & .485 & .331 & .710 & .178 & .785 & 553 & 1.115 \\
\hline Chronic & 524 & 874 & .577 & 1.323 & .000 & 315 & .168 & 599 \\
\hline CMN status & .027 & 1.462 & 1.045 & 2.045 & & & & \\
\hline Disease phase (adv vs, earty) & .000 & 2018 & 1.481 & 2.788 & .000 & 1.852 & 1371 & 2.500 \\
\hline
\end{tabular}

\section{P522}

A novel therapeutic approach to a severe form of extensive cutaneous chronic graft-versus-host disease: a case report

R. Crocchiolo, S. Ducastelle, L. Lebras, H. Labussiere, G. Cannas, F. Nicolini, X. Thomas, V. Dubois, F. Braye, M. Michallet

Hôpital Edouard Herriot (Lyon, FR)

Objective: We describe the successful treatment of a severe extensive cutaneous form of cGvHD with the transplantation of skin obtained from the same stem cell donor. A 34-year old woman received in May 2008 a myeloablative HSCT for AML in CR2, from HLA-identical sister. In August 2009 an extensive form of cGvHD was characterized by large multiple skin ulcerations with even some visible necrotic areas. Recent pneumocystosis and concomitant multi-resistant Pseudomonas A. cutaneous infection prevented the use of immunosuppressive drugs. A surgical approach was thus decided, consisting in skin transplantation from the same HSC donor.

Methods: After informed consent, a graft of epidermal layer was obtained from patient's HLA-identical sister, with a 8-cm incision in the inguinal area. Epidermal cells were cultured in vitro and expanded in about 3 weeks to obtain large sheets of epidermal layer. Multiple appositions of this in vitro expanded skin were performed under general anaesthesia between September and November 2009; a concomitant immunomodulatory treatment with methotrexate $15 \mathrm{mg} /$ week and extracorporeal photopheresis was administered, together with a continuous enteral nutrition, intensive antimicrobial therapy and pain management.

Results: Lesions disappeared, since the wide skin surface obtained with in vitro expansion enabled to cover all injured sites. A process of cicatrisation is now visible between the patient's and donor's skin in the areas previously characterized by ulcerations. Patient's quality of life dramatically improved, together with nutritional status. Analysis performed on blood and bone marrow showed sustained full donor chimerism.

Conclusion: At our knowledge this is the first described case of successful skin transplantation in a patient with cGvHD, obtained from in vitro expansion of the same HSC donor's skin. This experience showed that this procedure was feasible and well-tolerated. The rationale of using skin from the same HSC donor relies on the lack of expected immunological aggression from the immune system responsible of GvHD to the transplanted skin, both coming from the same donor. So far, the hypothesis was confirmed, in fact skin ulcerations disappeared and we did not need to add any immunosuppressive therapy. However, further follow-up is necessary to evaluate the evolution and long-term tolerability of this multidisciplinary treatment of severe extensive cutaneous cGvHD, still associated to high rate of mortality.

\section{P523}

Human antibodies to thymoglobulin may impair the efficacy of the conditioning in hematopoietic stem cell transplantation

C.M. Jol-van der Zijde, R.G.M. Bredius, A. Jansen-Hoogendijk, R.M. Egeler, A.C. Lankester, M.J.D. van Tol

Leiden University Medical Centre (Leiden, NL)

ATG, a polyclonal rabbit antiserum, is frequently used for in vivo T-cell depletion prior to HSCT to prevent graft rejection and acute Graft-versus-Host disease (aGvHD). In kidney transplant patients, human antibodies directed against ATG are reported to reduce the efficacy of anti-rejection therapy by accelerating the ATG clearance.

Pharmacokinetics of total (rabbit immunoglobulins) and active ATG (capable to bind to a T cell line) in adult and pediatric allogeneic HSCT recipients shows a high inter-patient variability (see Figure). The concentration of ATG has been reported to affect the incidence and severity of aGVHD. Only one study has described the presence of anti-ATG antibodies in HSCT patients, without pointing to the influence on ATG levels or the clinical impact.

In this study, concentrations of total and active ATG and antiATG antibodies were frequently measured in pre- and postHSCT sera obtained from 109 pediatric patients. The children usually received a total ATG (Thymoglobulin) dose of $10 \mathrm{mg} / \mathrm{kg}$ divided over 4 days just prior to HSCT.

In most patients the clearance of total ATG was slow and ATG was detectable until months post-SCT, whereas active ATG was cleared much faster. However, in 11 patients total ATG decreased very fast ( $>\log 2$ decline in 3 days, see Figure). This rapid clearance was associated with a simultaneous appearance of $\lg G$ anti-ATG antibodies.

Five patients with IgG anti-ATG antibodies in the first 4 weeks after HSCT showed serious side-effects, i.e. serum-sickness, aGvHD symptoms or VOD, contrasting patients who mounted an antibody response later post-HSCT. The majority of patients with anti-ATG simultaneously produced IgG, IgM and IgA antiATG antibodies. In all cases, IgG anti-ATG was of low avidity. Donor origin of IgG1 anti-ATG was demonstrated, using IgG1 allotype-specific monoclonal antibodies in all but one patient. 

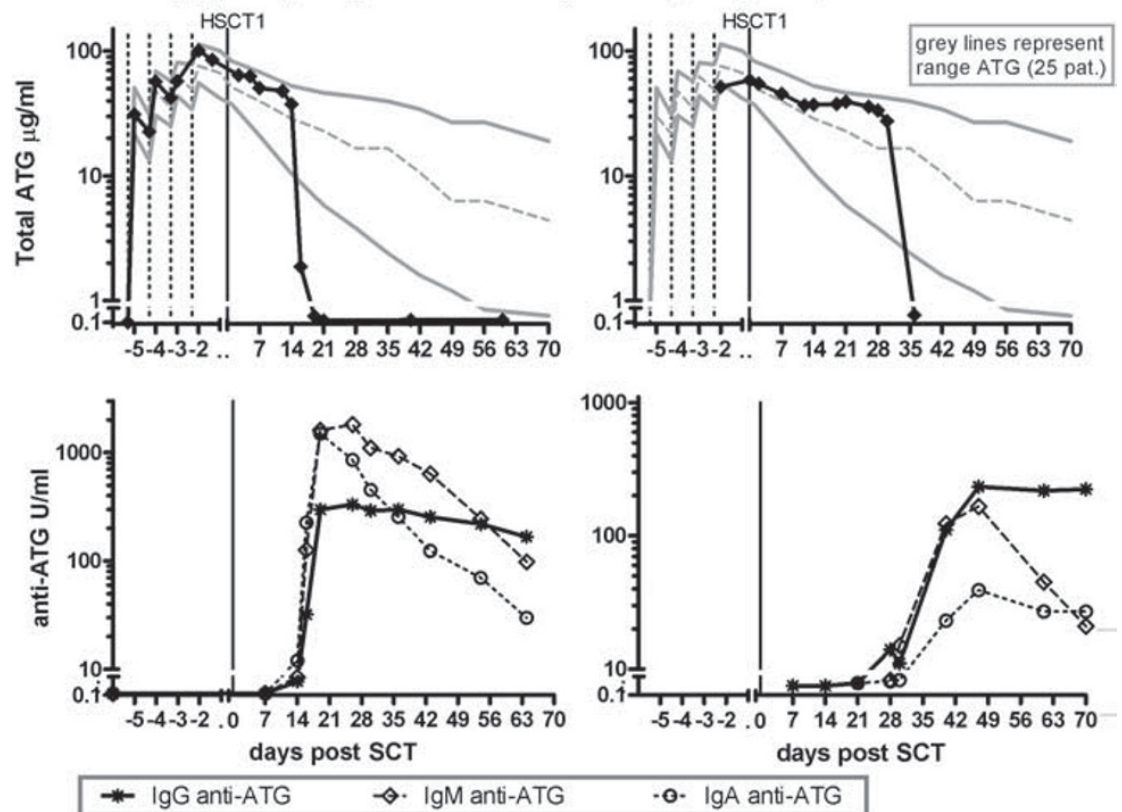

IgG anti-ATG post-HSCT occurred more frequently in older children (11 vs. 5 yrs, $P=0.03$ ).

These data illustrate that monitoring of ATG and anti-ATG levels help to understand the occurrence of transplant related toxicities, i.e. skin rashes and fever, acute GVHD, graft rejection.

\section{P524}

Daily low-dose interleukin-2 administration in steroid-refractory chronic graft-versus-host disease is feasible, safe, and efficacious; with preferential regulatory T-cell expansion

J. Koreth, K. Stevenson, H. Kim, S. McDonough, V. Ho, E. Alyea, C. Cutler, P. Armand, J. Antin, J. Ritz, R. Soiffer Dana-Farber Cancer Institute (Boston, US)

Regulatory T cells (Treg) are low in active chronic graftversus-host-disease (cGVHD). Treg infusion has been proposed as a GVHD therapy. However, ex-vivo Treg expansion is cumbersome and limited by methodologic and regulatory constraints. Daily low-dose interleukin-2 (IL-2) has been demonstrated to expand Treg in cancer patients and early after allogeneic hematopoietic stem cell transplantation (HSCT). We are testing if low-dose IL-2 can expand Treg in-vivo in steroid-refractory cGVHD. We administered daily subcutaneous IL-2 (Novartis) for 8 weeks of a 12-week phase-I doseescalation study: (level A) $0.3 \times 10^{6} \mathrm{IU} / \mathrm{m}^{2}$, (B) $1 \times 10^{6} \mathrm{IU} / \mathrm{m}^{2}$, and $(C) 3 \times 10^{6} \mathrm{IU} / \mathrm{m}^{2}$.

14 patients accrued: 7, 4, 3 at dose-levels A, B, C. Median age was 50.5 (27-65) years. Median time from HSCT and cGVHD onset was 995 (42-2766) and 802.5 (117-2624) days. Active cGVHD sites were skin (14), mouth (7), eyes (5), liver (4), lung (3), esophagus (1). Patients received a median of $3(1-3)$ concurrent agents: steroids (14), sirolimus (8), MMF (7), tacrolimus (6). Prior cGVHD therapies included rituximab (7), ECP (4), MMF (3), sirolimus (2), tacrolimus (2), alemtuzumab (2), cyclosporine (1), denileukin-diftitox (1), thalidomide (1)

At dose-levels $A$ and $B$ there was no cGVHD progression and 2 infectious complications (Hemophilus B bacteremia with HUS; MRSA pneumonia). Dose-level B has been declared MTD, as

dose-level $\mathrm{C}$ induced intolerable constitutional symptoms and 1 case of CGVHD progression.

Of 11 evaluable patients, 6 had a partial response, 1 a mixed response, 3 stable disease and 1 progressive cGVHD. Responses were noted in skin and liver. At study-end, 5 of 6 responders continue on extended duration IL-2 (range, 1-16 months) given their clinical benefit. The 6th, with resolving liver cGVHD, had IL-2 withheld for MRSA pneumonia. He died of progressive liver cGVHD off IL-2. 1 responder on extended duration IL-2 had a nonfatal myocardial infarction, unrelated to IL-2.

Daily IL-2 induced a statistically significant $3-5$ fold rise in peripheral CD4 + CD25 + CD127- Treg, but did not impact circulating NK, NK-T, CD8 or conventional CD4 + T-cells (Tcon). There was a significant increase in Treg:Tcon ratio, which fell towards baseline after IL-2 was discontinued (Table).

Daily low-dose IL-2 is feasible, safe, and has efficacy in refractory CGVHD; with marked preferential Treg expansion in-vivo despite concurrent steroid and tacrolimus use. Further accrual at IL-2 dose-level B is underway.

\begin{tabular}{lccc}
\hline & Week & $\begin{array}{c}\text { Treg/Tcon Ratio } \\
\text { median (range) }\end{array}$ & p-valuet- \\
\hline Pre-IL-2 & Pre & $0.07(0.03,0.30)$ & NA \\
On IL-2 & 1 & $0.17(0.06,0.61)$ & 0.008 \\
On IL-2 & 2 & $0.34(0.10,1.45)$ & 0.004 \\
On IL-2 & 4 & $0.50(0.10,1.22)$ & 0.03 \\
On IL-2 & 6 & $0.31(0.07,2.00)$ & 0.02 \\
End IL-2 & 8 & $0.71(0.14,1.56)$ & 0.02 \\
Off IL-2 & 12 & $0.16(0.04,0.77)$ & 0.03 \\
\hline & & \multicolumn{2}{c}{+ Wilcoxon signed-ranktest }
\end{tabular}

† Wilcoxon signed-rank test 
P525

ABCB1 C1236T single nucleotide polymorphisms are associated with prognostic significance in haematopoietic stem cell transplantation

J.S. Ahn (1), H.N. Kim (2), Y.K. Kim (1), I.K. Lee (2), J.J.Lee (1), H.J. Kim (1), D.H. Yang (1)

(1)Chonnam National University Medical School (Jeollanam-do, KR); (2)Genomic Research Center (Jeollanam-do, KR)

Backgroud: Single nucleotide polymorphisms (SNPs) in the MDR1 gene that are responsible for drug effux can cause toxicities and the other report suggested that calcineurin inhibitors (CNI) such as tacrolimus and cyclosporine bioavailability was related to $A B C B 1$ genotypes. Since the major immunosuppressant used in hepatopoietic stem cell transplantation (HCT) is the $\mathrm{CNI}$, these might influence the graft versus host diseases and clinical outcome in HCT.

Patients and methods: To assess the association between ABCB1 SNP and clinical implication, PCR-RFLP method was used to determine the genotype of the C1236T, G2677T/A and $\mathrm{C} 3435 \mathrm{~T}$ in 78 recipients of allgeneic HCT from a HLA identical sibling $(n=59,75.6 \%)$ and unrelated donor $(n=19,24.4 \%)$. Patients have either AML $(n=55,70.5 \%), \operatorname{CML}(n=12,15.4 \%)$ and lymphoma $(n=11,14.1 \%)$. The genetic variants in ABCB1 on survival were analyzed by Log rank tests and multivariant analysis by Cox regression.

Results: The overall survival the HCT patients was significantly correlated to the C1236T for the CC or CT vs. TT, multivariate analysis showed that $\mathrm{CC}$ and $\mathrm{CT}$ were the independent factor for mortality ( $P=0.038$, HR: $2.51(95 \% \mathrm{Cl}: 1.05-5.98))$. The 5-year survival of C1236T genotype in CC or CT was $35 \%$ and TT was $67.8 \%$. Chronic Graft versus host disease (GVHD) of liver was associated with C3435T in CC (39.4\%), CT $(13.8 \%)$ and TT $(66.7 \%)(P=0.006)$, and in CC or CT vs. variants $(P=0.02)$ but, G2677T/A and C3435T SNPs did not correlated to survival.

Conclusions: Our findings suggest that $A B C B 1$ SNPs affect the survival and chronic GVHD after allogeneic HCT and the correlation between $A B C B 1$ genotype and the overall survival $\mathrm{HCT}$ patients might provide useful information for risk stratification of HCT.

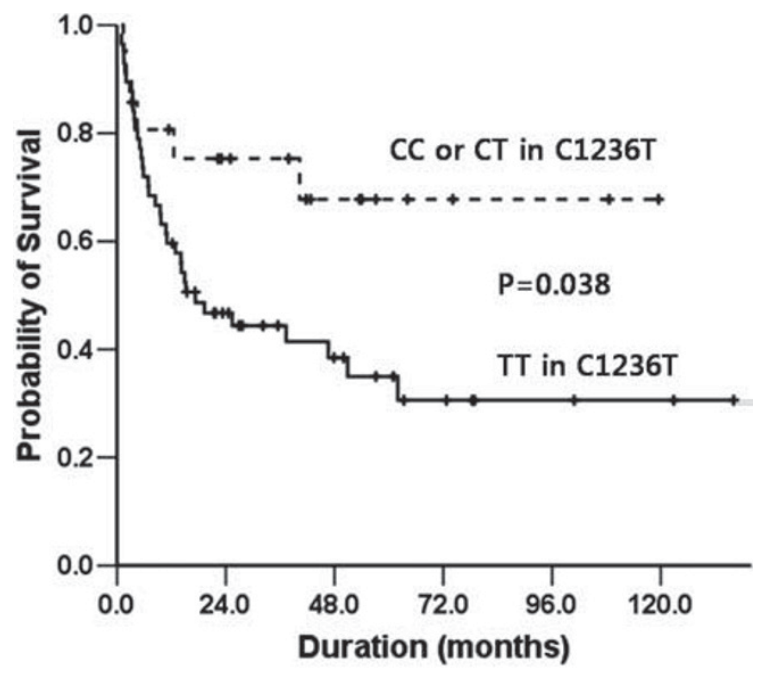

P526

Gastrointestinal steroid refractory GvHD in children successfully treated with mesenchymal stromal cells cannot be solely assessed by clinical response

L.M. Ball (1), J. Schweizer (1), A. Lankester (1), M.E. Bernardo (2), M.J.D. van Tol (1), H. Roelofs (1), J.J. Zwaginga (1), W.E. Fibbe (1), F. Locatelli (2), R.M. Egeler (1)

(1)Leiden University Medical Center (Leiden, NL); (2)University of Pavia (Pavia, IT)

Steroid refractory acute graft versus host disease (GvHD) following allogeneic hematopoietic stem cell transplantation (HSCT) is associated with a high mortality. Mesenchymal stromal cells (MSC) have been used as treatment with reported variable success rates. Most published reports rely on clinical improvement as an evaluation end point to document response.

We report experience in children treated mainly with 3rd party MSC for severe gastrointestinal (GI) steroid refractory GVHD, who have been systematically followed pre and post MSC inclusive of standard clinical grading as well as endoscopic and histological monitoring.

In the LUMC pediatric stem cell transplant unit from 2005 to 2009, 20 children (11 boys; 9 girls) between 11 m-17 y 8 m (median age $6 \mathrm{y}$ ) were treated with MSCs for steroid refractory GVHD, according to an ethically committee approved protocol. Of these, $80 \%$ had $\mathrm{Gl}$ involvement according to above criteria. These patients' details are summarized in Table I.

They received haplo $(n=2)$ or 3rd party derived MSC, with all but 1 receiving 2 infusions, at variable times from GvHD onset.

Seven children had either no response $(n=5)$ or partial resolution $(n=2)$ to MSCs, defined as persistent symptoms and no or minimal endoscopic/histological changes respectively. TRM due to infection and/or progressive GvHD was $85 \%$.

Of the remainder, $8 / 9$ children had persistent diarrhea and increased frequency of stools up to and beyond 30 days of MSCs implying continued GvHD. However, direct visual endoscopy showed widespread mucosal improvement. However, notable villous atrophy (and in some cases patchy fibrosis) was seen, which was considered the cause of clinical malabsorption. In all cases, despite persistent clinical symptoms, histological biopsies at multiple sites of the gastrointestinal tract showed no evident acute GvHD. The procedure was performed safely by experienced pediatric gastroenterologists and subsequently introduced as evaluation post MSC in our unit. TRM in this cohort was $20 \%$ (mainly due to lethal infections).

Such serial endoscopic features directly correlated to histological findings have not been previously reported in children responding to MSC treatment. We demonstrate the lack of correlation with clinical grading namely volume and frequency of diarrhea.

These findings have implications not only for clinical management but also for the design of future clinical trials and evaluation of MSCs therapies in patients with gastrointestinal GvHD. 
TABLE L CHARACTERISTICS OF PATIENTS WITH GIGvHD TREATED WITH MSC'S

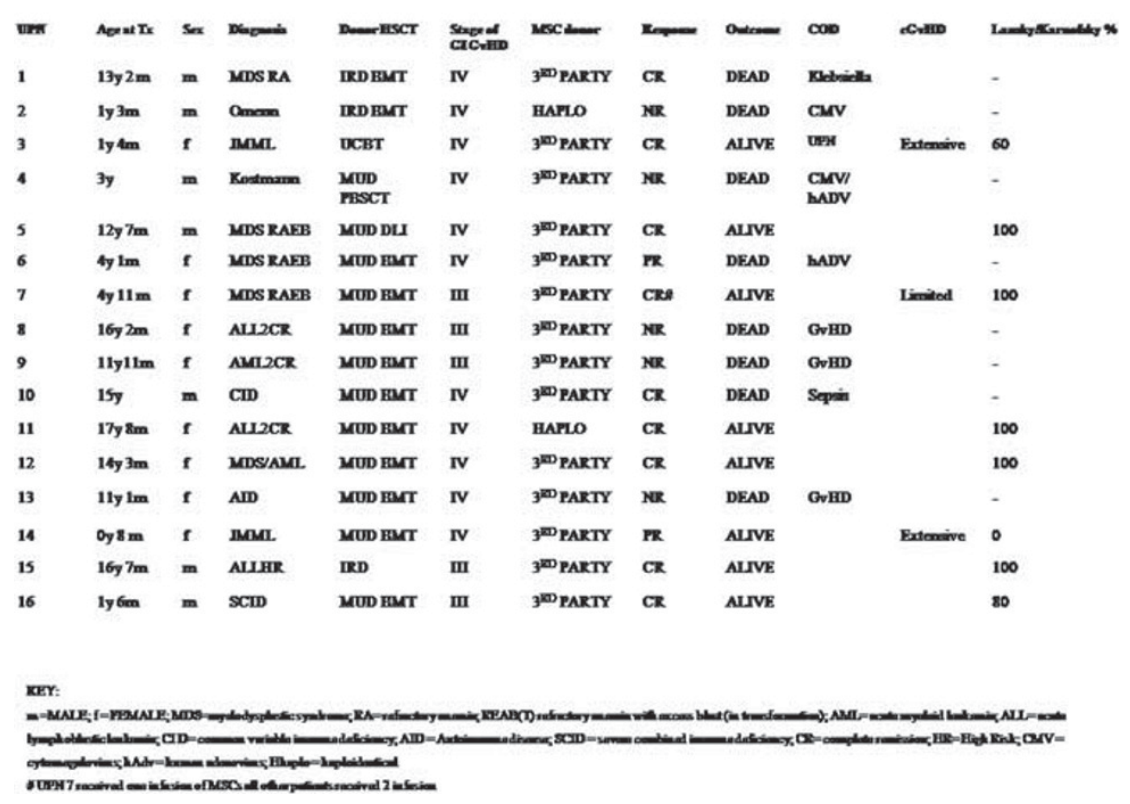

P527

Genetic variations of IL23R(1143A>G) and BPI(A645G) outcompete NOD2 as risk factors for acute GvHD after allogeneic transplantation

M. Wermke (1), S. Maiwald (2), R. Schmelz (1), C. Thiede (1), J. Schetelig (1), G. Ehninger (1), M. Bornhäuser (1), R. Wassmuth (2)

(1)University Hospital (Dresden, DE); (2)Dkms Lifescience Lab (Dresden, DE)

Acute graft-versus-host-disease (aGvHD) remains a potentially lethal complication after allogeneic stem-cell transplantation (allo-SCT). Clearly, new strategies are needed to tailor donor selection and conditioning regimen, according to the individual patient's risk-profile. Recently, single nucleotide polymorphisms (SNP) in genes of the innate and adaptive immune-system were found to be predictive in this regard. Unfortunately, the results are either heterogeneous as in the case of NOD2 or preliminary as with the IL23R(1142A>G)SNP. We therefore sought to determine the effect of these two SNPs and the BPI(A645G)-SNP on posttransplant-outcome in a cohort of 304 allo-SCT patients and their respective related or unrelated donors.

Genotyping was done using real-time PCR assays on a Lightcycler 480. Patients carrying at least one variant allele of NOD2SNP 8,12 or 13 were referred to as NOD2-SNP. Similarly, patients carrying one G-allele at position 1142 of the IL23Rgene were denominated IL23R-SNP. Finally, one G-allele at position 645 of the BPI gene was sufficient for classification as BPI-SNP. The incidence of GvHD was analyzed using competing events statistics and in a multivariate ordinal regression model.

No difference in cumulative incidence $(\mathrm{Cl})$ of aGvHD was found with respect to NOD2 patient- or donor-genotype and recipient $\mathrm{BPI}$ genotype. However, patients receiving a BPI-G graft had a significantly reduced $\mathrm{Cl}$ of aGvHD (day 100: BPI-G $15.4 \%, 95 \% \mathrm{Cl}: 10.8-20.0 \%$ vs. BPI-A $26.1 \%$, $95 \%-\mathrm{Cl}: 15.6-36.6 \%, P=0.047)$. There was also a trend to less aGvHD in those patients transplanted from IL23RSNP-donors (100 day $\mathrm{Cl}$ of aGvHD ${ }^{\circ} 2-4$ : SNP $21.4 \%, 95 \%$ Cl: $7.1-35.8 \%$ vs. wildtype $39.9 \%, 95 \% \mathrm{Cl}: 34.0-45.7 \%$; $P=0.063$, Figure 1). Multivariate analysis identified IL23R$\mathrm{SNP}$ in the donor (HR 0.40, $P=0.044$ ), in vivo T-cell-depletion
(HR $0.35, P=0.001)$ and the use of a multiagent immunosuppressive GvHD prohylaxis (HR 0.53, $P=0.045$ ) as independent factors predicting for less severe aGvHD. A trend towards less aGvHD was observed in patients receiving a BPI-G transplant (HR 0.59) and those with a matched related donor (HR 0.64).

We conclude that at least in our cohort NOD2-SNPs might not be as important as recently suggested. Furthermore, we think that IL23R(1142A>G) and BPI(A645G)-SNP of the donor represent attractive targets for improving donor selection and should therefore be studied in larger prospective multi-center trials.

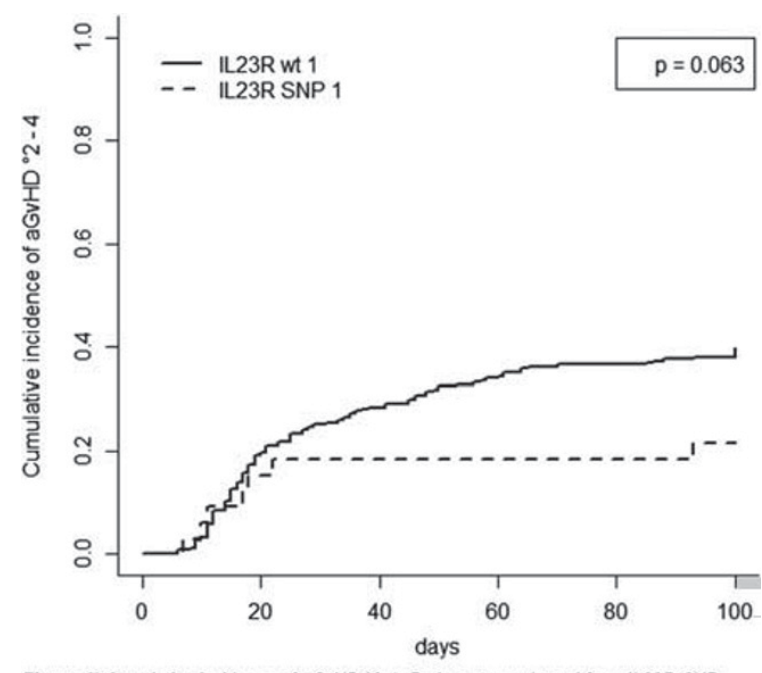

Figure 1) Cumulative Incidence of aGVHD *2-4. Patients transplanted from IL23R-SNP. donors (IL23R SNP 1) experienced less aGVHD than those receiving an IL23R-wildtype graft (IL $23 R$ W 1 ). 


\section{P528}

Regulatory T-cells are more resistant to genotoxic agents compared to effector T-cells

C. Winzler, M. Fantinato, E. Calore, G. Basso, C. Messina University of Padua (Padua, IT)

Background: Both host and graft regulatory T cells (Treg) may provide protection from graft-versus-host disease (GVHD) in the mouse system. Findings from a murine model of syngeneic GVHD suggest that host Treg preferentially survive a lethal dose of irradiation and confer protection from disease. In humans, various combinations of genotoxic agents are actually used for myeloablative conditioning. The sensitivity of human Treg to DNA damage induced apoptosis has so far not been investigated. Objective:To evaluate the resistance of Treg to apoptosis induced by genotoxic agents compared to effector T cells (Teff) in vitro.

Materials and methods: PBMC of healthy donors were exposed to either ionizing irradiation or etoposide. After incubation, samples were labelled with annexin V FITC or anti-activated caspase 3 FITC combined with monoclonal antibody conjugates to CD4, CD25 and CD127 or to surface antigen CD4 and transcription factor FoxP3. Propidium iodide or fixable infrared viability dye were included in the staining protocol as dead cell markers. The frequency of apoptotic cells among Treg and effector CD4 + T lymphocytes was determined by flow cytometric analysis at different time points (up to 72h). CD45RA staining was used to analyse the role of differentiation for resistance to apoptosis. Expression of anti-apoptotic proteins bcl-2 and Bcl-xL by Treg and Teff was compared.

Results: Both activation of caspase 3 and exposure of phosphatidylserine following DNA damage in vitro was observed with significantly higher frequency in Treg compared to effector CD4 lymphocytes. This effect was observed in both etoposide treated cultures and those exposed to ionizing irradiation. Similar results were obtained by identifying Treg as CD25highCD127- or as FoxP3 + events. The resistance to DNA damage by Treg could not be explained by their differentiation to a CD45RA negative memory phenotype. When measured as mean fluorescence intensity, anti-apoptotic proteins $\mathrm{Bcl}-2$ and $\mathrm{bcl}-\mathrm{xL}$ were not more expressed by Treg compared to Teff.

Conclusion: Our observations suggest a higher resistance to DNA damage of regulatory $T$ cells compared to effector $T$ cells in vitro. The higher resistance cannot be explained by differentiation to a memory phenotype or differential expression of anti-apopotic proteins bcl-2 and bcl-xL. The higher resistance to genotoxic agents of Treg could affect the relative size ratio of Treg to effector $\mathrm{T}$ cells following conditioning.

\section{P529}

Immunomodulatory effect of mesenchymal stem cells for the treatment of steroid-resistant GvHD in children

Y. Isaikina, N. Minakovskaya, M. Belevcev, O. Aleinikova

Belarusian Center for Ped OncoHematology (Minsk, BY)

Steroid-refractory graft-versus-host-disease (GVHD) is one of the serious complications after allogeneic hematopoetic stem cell transplantation (HSCT). Application of MSCs due to there immunomodulatory properties may be effective method for therapy of severe GVHD. The aim of our study was the comparison of MSCs infisions, derived from different sources for the treatment of steroid-resistant GVHD.

Patients and methods: 15 children with AML/MDS-6, NHL-1, ALL-5, SAA/AF-3 and steroid-resistant acute and chronic extensive GVHD (grade II-IV) received MSCs on 30-146 days after HSCT. Group I of patient received MSCs derived from bone marrow of HLA-identical donors $(n=7)$ and group II - from third-party HLA-mismatched donors $(n=8)$. The median dose of MSCs was $0.8(0.5-5.4) \times 10^{6} / \mathrm{kg}$ and $1.66(0.62-2.8) \times 10^{6}$ $\mathrm{kg}$ in group I and II, respectively. Immunological parameters $\mathrm{CD} 3+, \mathrm{CD} 3+\mathrm{HLA} \mathrm{DR}+, \mathrm{CD} 4+, \mathrm{CD} 19+, \mathrm{CD} 3+\mathrm{CD} 56+$,
$\mathrm{CD} 4+\mathrm{CD} 25+, \mathrm{CD} 4+\mathrm{CD} 62 \mathrm{~L}+$ were studied in children before and on 30-40 days after MSCs infusion.

Results: After infusion of MSCs reduction of GVHD grade was in $14(93,4 \%)$ patients. Five patients were in complete remission of GVHD, 9 children were in partial remission, 1 child did not response. In all patients we observed the significant reduction of CD3+HLA DR + and increasing the level of CD4+CD25+, $\mathrm{CD} 4+\mathrm{CD} 62+$ after treatment of MSCs $(P<0,05)$. In group I level of CD3 + HLA DR + was significantly lower and CD4 + CD62L + was significantly higher than before MSCs treatment $(P<0,05)$. In group II CD3 + HLADR + and CD3 + CD56 + were reduced and the level of $\mathrm{CD} 4+\mathrm{CD} 25+$ and $\mathrm{CD} 4+\mathrm{CD} 62 \mathrm{~L}+$ were increased after MSCs infusion $(P<0,05)$.

Conclusion: We demonstrated that infusion of MSCs modulate immunity and reduce grade of GVHD severity in patients with steroid-resistant GVHD. MSCs derived from bone marrow of third-party HLA-mismatched donors have more evident immunomodulatory potential then MSCs from HLA-identical donors and may be usefully for treatment GVHD in children in case of the absence of HLA-identical donor for mesenchymal stem cells.

\section{P530}

B-cell depletion as therapy for steroid refractory chronic GvHD is most effective for sclerosis of the skin S. van Dorp, L. te Boome, H. Lokhorst, E. Petersen, M. Minnema, L. Span, S. Ebeling, M. van Dijk, E. Meijer, J. Kuball UMC Utrecht (Utrecht, NL)

Introduction: Chronic graft-versus-host disease (cGVHD) is the major long term complication of allogeneic stem cell transplantation and shares similarities with auto-immune diseases, like systemic sclerosis (SSc). Two observations suggest that B cells contribute to cGVHD. In the first place, autoantibodies can be detected in 15-90\% of cGVHD patients. Secondly, several small-scale clinical studies have demonstrated that in vivo depletion of $B$ cells with the $C D 20$-specific Ab rituximab (Rtx) resulted in amelioration of cGVHD symptoms in steroidrefractory patients. Therefore we prospectively investigated the efficacy of Rtx on different GVHD-related symptoms in patients with steroid-refractory extensive cGVHD.

Methods: In an ongoing prospective phase I/II study 15 patients with steroid-refractory cGvHD were treated with Rtx $\left(375 \mathrm{mg} / \mathrm{m}^{2}\right.$ per week for 4 consecutive weeks). Clinical responses were monitored monthly for 1 year according to the $\mathrm{NIH}$ criteria (EudraCT 2008-004125-42)

Results: A total of 60 Rtx infusions were administered to 15 patients. Toxicity was limited to one infectious event and one allergic reaction. Clinical responses were so far analyzed in 12 patients. Median follow-up was 6.5 months (range 1-13). Eight patients showed a partial response (PR) $(67 \%)$ of which 5 patients $(42 \%)$ had an ongoing response up to 1 year of follow-up and 3 patients (25\%) relapsed. Two relapsed patients were retreated with Rtx 8 months after cessation of the initial Rtx treatment and showed again a PR. In patients with deep sclerosis $(n=10)$, sclerotic features disappeared completely or partially in 8 patients $(80 \%)$. In patients with involvement of eyes $(n=10)$, mucosa $(n=4)$ and gastro-intestinal tract $(n=4)$ response was reached in resp. $50 \%, 25 \%$ and $25 \%$ of patients. Consequently, prednisone dosage could be reduced in 7 patients $(58 \%)$. In 4 patients $(33 \%)$ prednisone treatment was stopped completely.

Conclusion: Rtx treatment is a feasible and effective treatment in patients with steroid refractory CGVHD. In addition, patients with relapsing symptoms of cGVHD can again be treated successfully. Most pronounced responses were seen in sclerotic lesions of the skin. Eye, mucosal and gastro-intestinal tract involvement was less susceptible to treatment. The lack of improvement of eye and mucosal involvement may be explained by an irreversible destruction of lacrimal and salivary glands after long standing cGVHD. This argues for an upfront application of Rtx in patients with cGVHD. 
P531

Low-dose alemtuzumab in vivo confers an efficient GvHD prophylaxis protocol in sibling and matched unrelated allogeneic haematopoietic cell transplantation

A. Spyridonidis, M. Liga, M. Karakantza, E. Triantafyllou, N. Zoumbos

University Hospital Patras (Patras, GR)

Despite standard CyA/MTX prophylaxis, severe acute GvHD occurs after $\sim 35 \%$ of matched sibling HCT. MTX is associated with mucositis, delayed engraftment and pulmonary toxicity. Campath anti-CD52 antibody may reduce GvHD by targeting lymphocytes and dendritic cells. Studies with high doses (100 $\mathrm{mg}$ ) of the humanized Campath1H (alemtuzumab) revealed increased incidence of relapse and severe viral infections. We report our single centre experience of low dose (20-10 mg) alemtuzumab as GvHD prophylaxis in 31 consecutive matched sibling $(n=15)$, related (1) or unrelated transplantations (15) performed during 1/2007-10/2009. Median pt age was 36 y (17-59). Diagnoses were AML(18), ALL(10), MDS-RAEB(2), T-NHL (1), PNH (1). 24 pts were transplanted in CR1 and 7 with advanced disease. Conditioning was $\mathrm{BuCy}=12$, TBI/VP16/ $\mathrm{Cy}=6$, Flu/BCNU/Melphalan (FB/M) or $\mathrm{FB}+$ Thiotepa $=11$, HDARA-C +FB/M=2. All pts received peripheral blood stem cells. The first 11 pts received iv alemtuzumab $20 \mathrm{mg}$ absolute (10 mg on d-2 and d-1), the next 5 pts total $15 \mathrm{mg}$ and the last $15 \mathrm{pts}$ total $10 \mathrm{mg}$ alemtuzumab (5 mg on d-2 and d-1). Campath serum levels were measured and alemtuzumab kinetic up to $d+30$ will be presented in the meeting. All pts engrafted

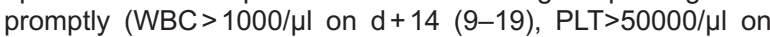
$\mathrm{d}+12$ (7-19)). 2 pts who discontinued Cya on $\mathrm{d}+25$ (incompliance) and $d+30$ (refractory disease) experienced steroid/ATG resistant, fatal GvHD. From the remaining pts, none revealed gut or liver aGvHD, whereas 12 pts (38\%) experienced steroidsensible skin GvHD St I-II at a median d+17 (12-57). 20pts revealed CMV reactivation but no CMV disease occurred. Cya could be discontinued in all evaluable pts at median on $d+120$ (25-210). After discontinuation of Cya, $1 \mathrm{HbsAg}+$ patient experienced fatal acute type Gr IV liver GvHD on d+150 after HCT from her $\mathrm{HbsAg}+$ sibling donor and $1 \mathrm{Gr}$ III gut GvHD. $40 \%$ pts revealed mild skin chronic GvHD, 2 moderate skin cGvHD which resolved with steroids and 1 extensive skin and liver GvHD. After a median follow up of 370 days (24-902), 21/31 pts are alive $(67.7 \%$ ) and in CR and 10 died (relapse $n=6$, TRM $n=4$ ). From the 6 pts who died due to relapse, 4/6 were at advanced stage (>CR-1) at transplant, 5/6 underwent sibling HCT and 5/6 received the higher $(20 \mathrm{mg})$ Campath dose. In conclusion, in vivo low dose Campath confers an efficient GvHD prophylaxis. Campath $10 \mathrm{mg}$ seems to be the optimal schedule for efficient GvHD prophylaxis without compromising relapse in sibling and matched unrelated HCT.

\section{P532}

Pre-engraftment graft-versus-host disease after allogeneic haematopoietic cell transplantation for acute leukaemia

J.H. Lee, J.H. Lee, D.Y. Kim, S.D. Kim, S.N. Lim, Y.S. Lee, Y.A. Kang, M.J. Jeon, M. Seol, K.H. Lee

Asan Medical Center, Ulsan University (Seoul, KR)

Objectives: Acute graft-versus-host disease (GVHD) usually occurs with neutrophil engraftment following allogeneic hematopoietic cell transplantation (HCT), but it can also be observed before engraftment. The pre-engraftment GVHD (pre-GVHD), often called hyperacute GVHD, was associated with a higher non-relapse mortality (NRM) rate in previous studies. In this retrospective study, we investigated the outcomes of pre-GVHD regarding leukemia relapse as well as NRM in patients undergoing allogeneic HCT for acute leukemia in a single institute. Methods: This study included a total of 384 patients who received allogeneic HCT from matched siblings or unrelated volunteers between March 1995 and July 2009 for the treat- ment of acute leukemia. The median age of the patients, 198 males and 186 females, was 36 years (range, 15-63). The phenotype of acute leukemia was myeloid (AML) in 262 and lymphoid (ALL) in 122. The disease status at HCT was the first complete remission (CR1) in 290 (75.5\%). Hematopoietic cell donor was a matched sibling in 243 and an unrelated volunteer in 141. Pre-GVHD was defined as acute GVHD occurring before neutrophil engraftment following HCT.

Results: Acute GVHD occurred in 100 (26.3\%) of 380 patients excluding 4 non-evaluable patients; pre-GVHD in $22(5.8 \%)$ and post-engraftment GVHD (post-GVHD) in 78 (20.5\%). Pre-HCT characteristics were not significantly different between preGVHD and post-GVHD. Severity of acute GVHD was higher in pre-GVHD than in post-GVHD; the proportion of grade I, grade II, and grade III-IV was $9.1 \%, 54.5 \%$, and $36.4 \%$ in pre-GVHD while $48.1 \%, 23.4 \%$, and $28.6 \%$ in post-GVHD $(P=0.002)$. During a median follow-up period of 48.4 months (range, 1.1153.2), 170 patients died and 134 relapsed. The 5-year probabilities of overall survival (OS), relapse-free survival (RFS), and NRM were $52.0 \%, 56.7 \%$, and $19.4 \%$, respectively. When we compared the outcomes following HCT in non-GVHD, preGVHD, and post-GVHD, 5-year OS was $53.2 \%, 37.8 \%$, and $54.6 \%$, respectively $(P=0.056)$; 5-year RFS was $53.6 \%, 46.4 \%$, and $71.7 \%(P=0.040)$; and 5 -year NRM was $12.8 \%, 29.4 \%$, and $33.3 \%(P=0.001)$.

Conclusion: Pre-GVHD comprised $22 \%$ of acute GVHD. NRM was higher both in pre-GVHD and post-GVHD compared to non-GVHD, but RFS was higher only in post-GVHD compared to non-GVHD or pre-GVHD. Pre-GVHD was associated with higher NRM without increased graft-versusleukemia effects.

\section{P533}

Non-classical HLA-E and G gene polymorphisms affect the HSCT outcome

K. Bogunia-Kubik (1), E. Jaskula (1), M. Polak (2), K. Koscinska (2), M. Sedzimirska (2), A. Lange (3)

(1)L. Hirszfeld Institute of Immunology and Experimental Therapy, Polish Academy of Sciences (Wroclaw, PL); (2)Lower Silesian Center for Cellular Transplantation with National Bone Marrow Donor Registry (Wroclaw, PL); (3)L. Hirszfeld Institute of Immunology/Lower Silesian Center for Cellular Transplantation (Wroclaw, $P L)$

The present study aimed to find out whether typing for nonclassical HLA alleles in addition to standard donor-recipient matching may facilitate the outcome of allogeneic hematopoietic stem cell transplantation (HSCT)

One-hundred patients $(F / M=45 / 55$; diagnosed mainly with hematological malignancies $(n=87), 10$ with inborn errors and 3 with anemia; receiving myeloablative $(n=41)$ or reduced intensity conditioning $(n=59)$ regimen, transplanted with PBPC $(n=87)$ or BM $(n=13)$, and grafted from sibling $(n=45)$ or unrelated $(n=55)$ donors) were investigated. There were 39 unrelated donor-recipient pairs matched at 5 classical HLA loci A, B, C, DR, DQ (10/10; high resolution DNA typing), while 1, 4 and 11 pairs were mismatched for 3,2 or 1 HLA specificity, respectively. All patients and donors were typed for the presence of two HLA-E alleles ( $\left.{ }^{*} 0101,{ }^{*} 0103\right)$ coding expressed HLA-E molecules and HLA-G ins/del polymorphism.

The incidence of acute graft-versus-host disease (GvHD) (grade II-IV) was significantly higher in patients transplanted from matched unrelated donors as compared to sibling transplant recipients $(30 / 52$ vs. 9/43, $P<0.005)$ while chronic GvHD was more frequently observed in patients with previous incidence of acute GvHD as compared to those who developed chronic disease de novo (20/34 vs. $12 / 49, P=0.002)$.

The incidence of acute GvHD (grade II-IV) was higher after HLA-E mismatched transplants $(8 / 12$ vs. $19 / 55, P=0.040)$. The latter association was analysed in patients matched for 5 classical HLA loci (HLA-A, B, C, DRB1 and DQB1) at the high resolution level. 
HLA match for 5 classical loci associated with higher incidence of HHV-6 infection before +100 day after transplantation $(>100$ copies/105 blood cells, $16 / 82$ vs. $0 / 18, P=0.069$ ). HHV- 6 infection was also less frequent after HLA-E mismatched transplants (0/17 vs. $17 / 70, P=0.035)$.

Fatal complications were more frequent among acute GvHD cases (24/41 vs. $15 / 54, P=0.003)$. Patients homozygous for the $E^{*} 0103$ allele tended to have better 3 yrs overall survival $(100 \%$ vs. $55 \%, P=0.095)$. This tendency was also seen in patients transplanted with donors carrying the HLA-G del/del genotype (68\% vs. $48 \%$ ). The presence of HLA-E*0103, 0103 in the patients and/or HLA-G del/del in the donors significantly facilitated the survival $(73 \%$ vs. $44 \%, P=0.033$ ).

It appears that the recipient HLA-E*0103 homozygosity and the donor HLA-G del/del may benefit the outcome of allogeneic HSCT.

Supported by the MNiSW grant No. N-N402-193335.

\section{P534}

Clinical impact of cytokine and immune response genes polymorphisms in unrelated haematopoietic stem cell transplantation

I. Mollet (1), K. Gagne (2), M. Malkki (3), J. Bignon (2), T. Gooley (3), E. Petersdorf (3), V. Dubois (1)

(1)HLA laboratory (Lyon, FR); (2)HLA laboratory (Nantes, FR); (3)FHCRC (Seattle, US)

Allogeneic Hematopoietic Stem Cell Transplantation (HSCT) is used as a curative therapy for hematological malignancies such as leukemia and lymphoma, but Graft versus Host Disease (GVHD) remains the most common complication after bone marrow transplantation, and could affect survival when the severity increases. Cytokine polymorphisms can affect occurrence and severity of transplant related complications.

We studied 189 D/R HSCT pairs including patients with malignant hematological diseases transplanted with an unrelated donor, using a myeloablative or non myeloablative-conditioning regimen. All donors and recipients were typed at allelic level for HLA-A, -B, Cw, -DRB1, -DQB1 and -DPB1. We studied polymorphisms of 13 SNPs from 6 different cytokines (IL10, TNFa, IL1, IL2, IL4 and TGFb) using Taqman-based genotyping assays.

We used logistic regression to evaluate the association of cytokine polymorphisms with grades III-IV acute GVHD incidence and Cox regression to compare the hazards of relapse and mortality.

We found a statistically significant association between acute GVHD incidence and IL10 (-1082) polymorphism. Patients whose donors carried a GG genotype increase risk of severe acute GVHD compared with donors carrying AG or AA genotypes ( $P$ value 0.05$)$. There was also a suggestion of association between mortality risk and IL1B (-511 polymorphism): reduced risk of mortality was observed in recipients carried GA at IL1B promoter position -511 than whose carried GG $(P=0.022)$ but this was not confirmed when allele $A$ is present with homozygous status $(P=0.58)$. In other hand, patients whose donors carried a GA polymorphism at position -511 of IL1B receptor have an increased risk of mortality but this was not confirmed with AA genotype $(P=0.05$ and $P=0.35$ respectively). Similarly, we found an increased risk of mortality for patients whose donors had TC genotype for TGFB1 (position -509) not confirmed with TT genotype $(=0.03$ and $P=0.35$ respectively).

We also confirmed previously reported negative associations between acute GVHD and IL1B-511, IL1A-889, IL4-590, IL4R Q576R, IL10-819, TGFB1-509 and TNF-238.

Our results confirmed that IL10 gene polymorphism could be associated with acute GVHD. But as reported results remains variable, we need to increase our study to evaluate more precisely the impact of such cytokines.
P535

The influence of extracorporeal photopheresis on circulating B-cell activating factor in steroid-refractory chronic graft-versus-host disease

R. Whittle, P. Taylor

Rotherham General Hospital (Rotherham, UK)

Introduction: Extracorporeal Photopheresis (ECP) has been successful in the treatment of steroid-refractory chronic Graftversus-host-disease (cGVHD). The role of B-lymphocytes in the pathology of cGVHD has received interest due to observations of allo- and auto-antibody formation, perturbed $B$ cell homeostasis and increased circulating B cell activating factor (BAFF) levels. Serum BAFF levels have been correlated with cGVHD severity and activity. To expand investigations into the role of ECP therapy in influencing $B$ cell behaviour in cGVHD we examined serum BAFF in patients undergoing ECP for steroid-refractory cGVHD.

Methods: 14 patients with steroid-refractory cGVHD were treated by ECP for a minimum of 3 months. Treatment outcome was measured by change in skin score or organ-specific improvement and change in steroid dose over the treatment period. Serum/plasma was collected at ECP start, 4 weeks \& 3 months. BAFF levels were tested by ELISA (R\&D systems) and compared to healthy controls $(n=30)$.

Results: Patients with chronic GVHD had significantly higher levels of serum/plasma BAFF than healthy controls prior to ECP therapy (mean $3.12 \mathrm{vs} .0 .58 \mathrm{ng} / \mathrm{ml})(P \leq 0.0001)$. The interval between HSCT and ECP start had a moderate effect on BAFF level $(>17$ months mean BAFF $=2.0 \mathrm{ng} / \mathrm{ml}$ vs. mean $\mathrm{BAFF}=4.25$ $\mathrm{ng} / \mathrm{ml}$ for patients $<17$ months, $P=0.06)$. Steroid dose had a significant affect on Pre-ECP BAFF level (less than or equal to $20 \mathrm{mg} /$ day, mean $B A F F=5.08 \mathrm{ng} / \mathrm{ml}$ vs. mean $B A F F=1.57$ $\mathrm{ng} / \mathrm{ml}$ for patients receiving over $20 \mathrm{mg} / \mathrm{day}, P=0.02)$. Pre-ECP BAFF level was not significantly correlated with severity of skin disease $(n=10)$. ECP therapy did not generate a consistent affect on BAFF levels with no significant difference in serum/ plasma BAFF levels following either 1 or 3 months of treatment. BAFF levels in $6 / 7$ patients achieving a $\geq 50 \%$ steroid reduction experienced a rise in serum/plasma BAFF $(P \geq 0.05$, ns) whilst patients with a maintained steroid dose displayed a slight fall in circulating BAFF (ns).

Discussion: BAFF may be a driver of alloimmune and autoimmune pathology in CGVHD. The influence of ECP on B cell subset maturity in cGVHD has been reported. Our data does not support a direct application of serum/plasma BAFF measurement to assess cGVHD response to ECP. Pre-ECP factors may have had a confounding influence, masking any correlation between clinical response and BAFF levels. A longer period of ECP therapy with stable steroid doses may reveal more significant associations.

\section{P536}

Treatment of refractory chronic GvHD with rituximab

I. Vicuña, R. Fores, D. Caballero, J. Garcia-Marco, M.J. Pascual, $J$ J De La Serna, R. Duarte, C. Barrenetxea, A. Martinez, M. Diaz, J.M. Ribera, G. Bravo, J. Cartier, A. Sebrango, B. Navarro, J.R. Cabrera on behalf of the GETH (Grupo Español de Trasplante Hematopoyético) Cooperative Group

There is no standard therapy for patients with chronic graftversus-host disease (cGVHD) who do not respond to first-line therapy with steroids. Rituximab has been shown to induce clinical response in a proportion of patients with refractory cGVHD. We report the results of a multicentric retrospective survey of patients treated with rituximab in Spain.

Material and methods: We retrospectively analyzed 31 patients (median age $37 \mathrm{y}$, range 13-65). All had received an allogeneic stem cell transplant, 23 from HLA-identical sibling and 8 from alternative donors. A reduced intensity transplant was performed in 14 cases. Median time from transplantation to diagnosis of cGVHD was 7 months. All cases were refractory 
to several treatment lines, median 2 (range 1-5). Twenty three patients had cutaneous involvement $(\mathrm{NIH}$ grade 1:22\%; grade $2: 35 \%$ and grade $3: 43 \%$ ). Other sites of organ involvement frequently were mouth, eyes and liver. Ten patients had cytopenias of probable immune origin.

Rituximab was given IV at a weekly dose of $375 \mathrm{mg} / \mathrm{m}^{2}$, with $94 \%$ of the patients receiving 4 doses.

Results: Results could be evaluated in 30 patients (1 patient died suddenly after completing 4 doses). Treatment was well tolerated in all cases. The overall response rate was $80 \%, 10 \%$ with complete response (CR) and $70 \%$ with partial response (PR). Organ-specific responses (CR+PR/TOTAL) were skin $70 \%(14 / 20)$, mouth $80 \%(12 / 15)$, eyes $50 \%(5 / 10)$, liver $70 \%$ $(7 / 10)$, joints $62 \%(5 / 8)$, caquexia $25 \%(1 / 4)$, thrombopenia $66 \%(4 / 6)$, gut $66 \%(4 / 6)$, polimyositis $(1 / 3)$, vagina $(0 / 1)$, pure red cell aplasia (1/1), esofagous (1/2), microangiopathic anemia (2/2), immune hemolytic anemia (1/1).

Response was transitory in 16 of the 22 responsive patients with a median of 8 months. Seven patients developed bronchiolitis obliterans $(\mathrm{BO})$ after treatment. Clinical relapse was only observed in two cases of Hodgkin's lymphoma. Disease free survival was $68 \%$ at 5 years. There were 8 deaths due to toxic or infectious complications. Nine patients received additional cycles of rituximab with a low response rate.

Conclusions: Rituximab treatment represents a therapeutic option for patients with refractory cGVHD. Overall response rate is high but transient in many cases. The best response rates were observed in cases with skin, oral or liver involvement and in patients with peripheral cytopenias. We have observed a high percentage of patients who had developed BO after treatment, although the role of rituximab is unknown.

\section{P537}

The handheld dermatoscope as a nail-fold capillaroscopic instrument in sclerodermoid graft-versus-host disease B.N. Akay, H. Sanli, P. Topcuoglu, M. Arat, G. Gurman, A. Akyol

Ankara University (Ankara, TR)

Background: Well-recognized videocapillaroscopic patterns have been described in systemic sclerosis (SS). However no studies have described the capillary abnormalities of sclerodermoid cGvHD (Scl GvHD) developed after allogeneic hematopoietic stem cell transplantation (allo-HSCT).

Objective: The aims of this study were to find the characteristics of nail-fold capillary changes in Scl GvHD after allo-HSCT.

Patients and methods: Eighteen patients affected by Scl GvHD and a control group of 15 lichenoid GvHD patients were evaluated during the period from September 2008 to June 2009. Duration and type of sclerodermoid GVHD, Raynaud's phenomenon (RP), dysphagia, joint contractures, ANA, serum antiSCL70 and anticentromere (ACA) antibodies were investigated parameters. A nail-fold capillary examination using a standard dermatoscope (Dermlite Foto, 3 Gen, LLC, Dana Point, CA, USA) was performed on all fingers.

Results: Twelve patients were male and 6 were female with a mean age of $37 \pm 11.6$ years. Joint retractions and dysphagia developed in $27.8 \%$ and $38.9 \%$ of the patients, respectively. Three $(16.7 \%)$ patients had RP. Autoimmune markers like serum anti-SCL-70 and ACA were negative in all. Capillaroscopy was abnormal in 15 patients with Scl GvHD. A regular disposition of the capillary loops along with avascular whitish linear areas at the level of last row, neovascularization with reticular pattern, capillary disorganization, hemorrhages, enlarged capillaries and avascular areas were the main features. No capillary abnormalities has been observed in patients with lichenoid GvHD. There was no statistically significant correlation between ANA positivity, RP, joint retractions, dysphagia, extension of sclerodermoid GvHD, duration of sclerodermoid lesions and nailfold capillaroscopy analysis.

Conclusions: This study shows the identification of distinct nailfold capillaroscopy patterns in Scl GvHD patients but it does not confer special risk for any other specific clinical symptoms of the disease. Further studies are needed to clarify the diagnostic and prognostic value of capillaroscopy in Scl GvHD.

\section{P538}

Serial intestinal endoscopic evaluation of patients with persistent diarrhoea after allogeneic stem cell transplantation

C. Martínez, M. Rosales, X. Calvo, M. Cuatrecasas, L. Rodríguez, J. Llach, M. Rovira, F. Fernández-Avilés, L. Rosiñol, E. Carreras

Hospital Clínic (Barcelona, ES)

Nausea, watery diarrhea, loos of appetite, bloody or mucoid stool, and abdominal pain are the primary manifestations of gastrointestinal (GI) GVHD; however, there is wide overlap of symptoms and signs with other $\mathrm{Gl}$ diseases such as enteric infections and drug toxicity. GI endoscopic biopsy is the more useful tool to diagnose these patients and to initiate the specific treatment. However, persistence of GI symptoms despite initial treatment is frequent and a second endoscopic evaluation may be helpful to address therapy. We performed a retrospective study to determine the diagnostic value of serial GI endoscopic evaluation of alloSCT patients with persistent diarrhea despite treatment based in the first endoscopic study. We identified 29 patients $(12 \mathrm{~F} / 17 \mathrm{M})$ who had more than one sigmoidoscopy and biopsy (median 2; range 2-5). Simultaneous microbiologic analyses of stools, CMV monitoring by antigenemia and/or $\mathrm{PCR}$, and CMV immunostainig on colonic biopsies were performed. Median time from 1st to 2nd endoscopy was 19 days (range 5-98). Abnormal GI mucosa (edema, erosions and/or ulcers) was observed in 23 and in 24 patients in 1st and 2nd endoscopies, respectively. Of 29 first rectosigmoid biopsies evaluated histologically, $18(62 \%)$ were classified as GVHD, $2(7 \%)$ as GVHD simultaneously with CMV, $4(14 \%)$ as nonCMV infection, and $5(17 \%)$ as normal or unspecific. Second GI biopsies were diagnostic of active GVHD in $6(17 \%)$ cases, GVHD simultaneously with CMV infection in $4(14 \%)$, regenerative changes post-GVHD in $8(28 \%)$, CMV infection in $3(10 \%)$, and normal or unspecific in $8(28 \%)$. Positive predictive value (PPV) and sensibility of endoscopy for GVHD were $70 \%$ and $80 \%$, respectively, with a specificity of $22 \%$. In 21 out of 29 $(72 \%)$ patients the histologic findings of 2 nd endoscopic biopsy varied in comparison to $1 \mathrm{st}$ biopsy, leading to a therapy change in $20(69 \%)$ of them (increase or decrease of immunosupressive treatment and/or treatment of coexistent infection). Most patients with negative CMV antigenemia and/or PCR had no signs of CMV infection in GI biopsies; however, there were 6 endoscopies out of 72 with CMV cytopatic changes and positive immunostaining on biopsies with negative results in blood (PPV of $47 \%$, NPV of $89 \%$, sensibility of $57 \%$, and specificity of $85 \%$ ). In conclusion, our results showed a reliable diagnostic value for serial $\mathrm{GI}$ endoscopic evaluation that could impact on therapeutic decision in patients with persistent diarrhea after allo-SCT.

\section{P539}

Low incidence of pulmonary graft-versus-gost disease

in older patients after reduced toxicity conditioning with fludarabin, carmustine and melphalan prior to haematopoietic stem cell transplantation

J. Duque-Afonso, A. Prasse, R. Waesch, H. Bertz, J. Finke, R. Marks University of Freiburg (Freiburg, DE)

Allogeneic hematopoietic stem cell transplantation (HSCT) of older or comorbid patients has become feasible due to new protocols for reduced toxicity/intensity conditioning. Particularly using fludarabine, carmustine/BCNU and melphalan (FBM) as preparative regimen confers reduced toxicity with substantial anti-leukemic activity. Nevertheless, chronic Graft-versus-Host 
disease (cGvHD) of the lung remains a serious non infectious, late onset pulmonary complication, contributing to treatment related morbidity and mortality of the older patients. Since the clinical entity of pulmonary cGvHD in an older population after reduced toxicity/intensity conditioning has not yet been well characterized, we performed a retrospective analysis of patients, who were transplanted after FBM conditioning at the University Hospital Freiburg between 2003 and 2005 and were alive at least 100 days after HSCT. 92 patients were enrolled in this study (median age of 60 years (range 29-71)). All patients received conditioning with fludarabin $\left(4-5 \times 30 \mathrm{mg} / \mathrm{m}^{2}\right)$, BCNU (or carmustine, patients $>55$ years: $2 \times 150 \mathrm{mg} / \mathrm{m}^{2},<55$ years: $2 \times 200 \mathrm{mg} / \mathrm{m}^{2}$ ) and melphalan (patients $>55$ years $1 \times 110 \mathrm{mg} / \mathrm{m}^{2}$, $<55$ years: $1 \times 140 \mathrm{mg} / \mathrm{m}^{2}$, fo). Peripheral stem cell grafts were used in most of the cases together with cyclosporin based GvHD prophylaxis. Seven patients $(8.2 \%)$ developed a pulmonary cGvHD as defined by $\mathrm{NIH}$ criteria with a median time after HCT of 13.3 months (range 7-19 m). In those patients, pulmonary function tests prior to HCT and on day +100 (prior to pulmonary GvHD) revealed a significant reduction in mean $\%$ of predicted value in FEV1 (88 vs. $71 \%$ ), and of absolute values in MEF50 (3.33 vs. 1.91) and MEF25 (0.96 vs. 0.42 ) as characteristic changes. The patients with pulmonary GvHD showed at the time of diagnosis in comparison to values before HSCT obstructive (\% of predicted FEV1 71 vs. $50 \%$ ), restrictive (mean\% of predicted VCmax 78 vs. $64 \%$ ) and diffusions capacity changes (\% of predicted TLCOc SB 79 vs. $62 \%$ ). In univariate analysis, following risk factors for developing pulmonary cGvHD were found: unrelated donor, chronic GvHD, smoking and lung disease (e.g. infections) after HCT.

In conclusion, we found several risk factors and changes in pulmonary function test associated with developing pulmonary GvHD in HCT after reduced toxicity conditionig. These findings might help to identify a risk population in older patients and therefore result in personalized measures for GvHD prophylaxis.

\section{P540}

Rituximab reduces anti-UL94 and anti-NAG2 antibodies titre and is effective against skin-chronic graft-versus-host disease resembling scleroderma R. Pastano (1), C. Dell'Agnola (2), C. Bason (2), F. Gigli (1), C. Rabascio (1), A. Puccetti (3), G. Cetto (2), M. Negri (1), F.A. Peccatori (1), G. Martinelli (1), C. Lunardi (2)

(1)European Institute of Oncology (Milan, IT); (2)University of Verona (Verona, IT); (3)University of Genoa (Genoa, IT)

We previously described a potential pathogenetic link between human Cytomegalovirus (hCMV) infection and sclerodermalike skin chronic graft versus host disease (cGVHD) in allogeneic stem cell transplant patients (HCT), through a mechanism of molecular mimicry between UL-94 viral protein and NAG-2 molecule, with apoptosis of endothelial cells and fibroblasts proliferation as observed in Systemic sclerosis (SSc) patients. The observation that Rituximab induces a significant clinical response in a proportion of HCT patients with refractory cGVHD, prompted us to evaluate, in our CMV IgG positive HCT patients who developed skin chronic GVHD scleroderma-like, first the efficacy of Rituximab, second weather the potential effect of Rituximab would correlate with the anti-viral antibodies titer. Among 18 patients undergone HCT for hematological malignancies in our Institute, two of them (patients \#2; \#3) both positive for anti-UL94 and anti-NAG-2 antibodies and with clinical evidence of diffuse, SSc-like skin cGVHD following HCT, were treated with Rituximab $375 \mathrm{mg} / \mathrm{msq}$ weekly for 4 weeks and then monthly up to 4 months. Patient \#2 complited the schedule, patient \#3 discontinued the therapy, after the second monthly dose, due to infectious pneumonia. Patients' plasma was monitored for anti-UL94 and anti-NAG-2 antibodies titer, by direct and competitive ELISA assays, before starting Rituximab, after the weekly therapy, at the end of the schedule (patient \#2) or after the last Rituximab infusion, in case of discontinuation (patient \#3). In patient \#2 we observed a dramatic reduction of the anti-NAG-2 antibodies titer immediately after completing the weekly administration of Rituximab, reaching a stable plateau after the monthly administration of the monocolonal therapy in parallel with an excellent clinical response. In the same patient anti-UL94 antibodies titer was already low after HCT and before Rituximab, and it kept stable low values during the entire administration schedule. This patient is still off immunosuppressive treatment. We observed also a reduction of both anti-CMV antibodies titer in patient \#3 following Rituximab, however without reaching a plateau, mainly due to the early discontinuation of the treatment. This patient had also a mild clinical response and is still under immunosuppressive therapy. In conclusion, our data show that Rituximab reduces anti-UL94 and anti-NAG2 antibodies titer and is effective against cGVHD scleroderma-like.

\section{P541}

NIH consensus chronic GvHD following T-replete reduced-intensity conditioning sibling allograft usually develops within a year, causes prolonged morbidity but low mortality and is influenced by prophylactic methotrexate dose

P.G. Medd (1), I. Monk (1), R. Danby (2), R. Malladi (1), R. Clifford (1), A. Ellis (1), J. Birks (3), D. Roberts (4), C. Hatton (1), P. Vyas (1), T.J. Littlewood (1), A.J. Peniket (1)

(1)Oxford Radcliffe Hospitals NHS trust (Oxford, UK); (2)NDCLS, University of Oxford (Oxford, UK); (3)Wolfson College, University of Oxford (Oxford, UK); (4)NHS Blood and Transplant (Oxford, UK)

T-replete reduced intensity conditioned (RIC) allogeneic haematopoietic stem cell transplantation (HSCT) is associated with lower relapse rate but higher acute \& chronic graft-versus-host disease (a/c GvHD) incidence than T-depleted HSCT. We retrospectively analysed the nature of GvHD, treatment required \& transplant variables associated with GvHD development. 75 patients (pts) - 43M 32F, median age 49 yrs (range 26-66) who underwent RIC sibling HSCT between 1998-2009 were analysed. Pts received fludarabine $\left(125 \mathrm{mg} / \mathrm{m}^{2}\right) /$ melphalan $(140 \mathrm{mg} /$ $\mathrm{m}^{2}$ ) conditioning. Ciclosporin A(CsA) was used as GvHD prophylaxis, initial protocol did not use methotrexate (MTX) prophylaxis, subsequent pts were scheduled to receive four doses of MTX (5 mg/m²): 8 pts $(11 \%)$ received no MTX, $28(37 \%) 1-3$ doses \& $37(49 \%)$ the planned 4 doses. aGvHD occurred in $38 / 74$ pts $(51 \%)$, NIH consensus categories: classic acute $24(32 \%)$, delayed $6(8 \%)$, persistent $7(9 \%)$, recurrent $1(1.4 \%)$. Multivariate analysis showed age $>50$ yrs predictive of aGvHD $(P=0.04)$. 69 pts survived > day $100-$ cGvHD was analysed in this group; 49 pts $(71 \%)$ developed cGvHD. NIH categories were classic chronic (CC) 34 pts (49\%), overlap (OC) 15 pts (22\%). Cumulative requirement for systemic immunosuppressive therapy (IST) was $39 \%$ at $6 \mathrm{M} \& 58 \%$ at $12 \mathrm{M}$, only 1 pt $(1.5 \%)$ commenced IST beyond 12M (Figure 1). Median time to IST was significantly less for OC than for $\mathrm{CC}$ (4.5M \& 9M respectively, hazard ratio (HR) for OC 5.4, $P=0.0004$ ), cumulative proportions requiring IST at $12 \mathrm{M}$ were $100 \%$ \& $65 \%$ for OC \& CC respectively. Univariate analysis showed reduced MTX prophylaxis as significantly associated with increased IST requirement (HR for reduced MTX 2.6, $P=0.008$ ). In multivariate analysis reduced MTX showed a trend to significance for predicting IST requirement $(P=0.11)$. Mean CsA level at 21 days \& $3 \mathrm{M}$ did not predict IST requirement. Cumulative proportions of pts still on IST from commencement are shown in Figure 2. Median time to IST cessation was 40M, $26 \%$ of patients $(95 \% \mathrm{Cl} 17-50 \%)$ remain on IST at $5 \mathrm{yrs}$. There was no significant difference in time to IST cessation by $\mathrm{NIH}$ a/ c GvHD category, MTX or CsA level. Cumulative mortality from cGvHD in patients surviving >D100 was $5.4 \%$ at $2 \mathrm{yrs}$.

Conclusions: cGvHD is common after T-replete sibling RIC HSCT; full prophylactic MTX, but not mean CsA level, is important in cGvHD prevention; IST for CGvHD is commonly required for several years; cGvHD is cause of significant morbidity but modest mortality. 

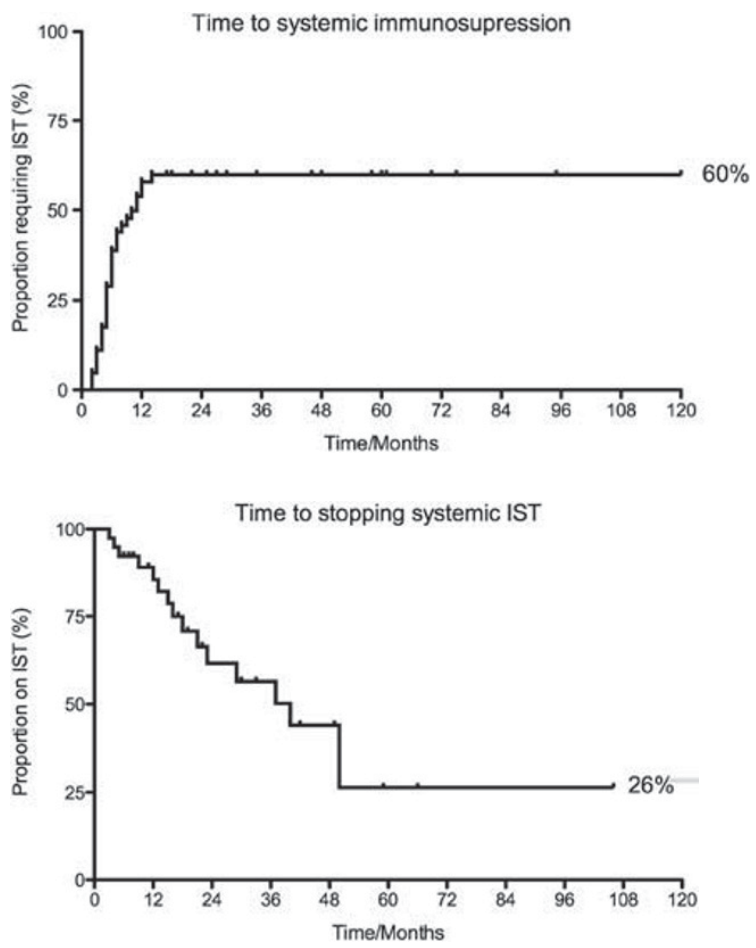

P542

$18 \mathrm{~F}$-fluorodeoxyglucose positron emission tomography in the diagnosis of intestinal graft-versus-host disease:

a new tool?

N. Jacque, P. Maksud, F. Archambaud, R. Chollet, J.F. Finet, M. Uzunov, S. N Guyen, J.P. Vernant, N. Dhédin

Pitie Salpetriere (Paris, FR)

Backgrounds: The diagnosis of gastrointestinal graftversus-host disease (GVHD) after allogeneic stem cell transplantation is usually assessed by clinical symptoms and histological analyses after digestive endoscopy. This approach is frequently not satisfactory because of its lack of specificity. A murin transplantation model has suggested that inflammatory activity associated with intestinal GVHD can be assessed by $18 \mathrm{~F}$-fluorodeoxyglucose positron emission tomography (FDG-PET). Therefore, we evaluated the utility of FDG-PET in the diagnosis of intestinal GVHD in transplanted patients.

Methods: Between February 2008 and March 2009, patients with a suspected diagnosis of gastrointestinal GVHD after haematopoietic stem cell transplantation (HSCT) were enrolled in our study. They were examined by FDG-PET and digestive endoscopy (gastroduodenal fibroscopy, rectosigmoidoscopy and colonoscopy when feasible) with biopsy. Stool samples were screened for bacterial, viral, mycological and parasitic gastrointestinal infections. Patients were systematically screened for adenovirus and cytomegalovirus (CMV) infections by specific PCR in the blood and immunofluorescence stainings in digestive biopsies.

Results: Eleven patients with clinical symptoms of GVHD in a median time of 35 days (4-392) after HSCT were tested. Two patients had a stage 2 digestive GVHD and 9 patients a stage $3-4$. None of them was positive for viral, mycological or parasitic infections in the stool, 6 were colonized with an intestinal bacteria but all were negative for clostridium difficile toxin. One patient presented a CMV colitis based on the biopsy and blood samples. FDG-PET was performed in a median time of 6 days after the beginning of the symptoms (1-159). Seven patients presented a FDG uptake of the gut. All of the 11 patients had sigmoid biopsies, 6 had staged biopsies of all colon and
4 gastroduodenal biopsies. For 6 patients, histological and FDG-PET results were concordant ( 5 were both positive and 1 both negative). In 3 patients, the FDG-PET showed no uptake whereas the histological analysis was positive. For the 2 remaining patients, FDG uptake was localized in the ileum and therefore not accessible to biopsy.

Conclusion: FDG-PET is a non invasive tool that can be useful in some patients with suspected intestinal GVHD. This approach may be interesting to guide biopsy when the diagnosis is difficult with the usual assessment, in particularly in the small intestine localization.

\section{P543}

Extracorporeal photopheresis for steroid refractory acute GvHD in children and adults

E. Calore (1), R. Duce (2), P. Strada (2), F. Gualandi (2), M. Pillon (1), M.T. Van Lint (2), T. Lamparelli (2), S. Cesaro (1), A.M. Raiola (2), A. Dominietto (2), C. Di Grazia (2), S. Bregante (2), B. Buldini (1), C. Winzler (1), S. Varotto (1), S. Zia (2), M. Gazzola (1), R. Destro (1), A. Bacigalupo (2), C. Messina (1), P. Marson (3), T. Tison (3), M.R. Ruzzenenti (2), M.C. De Luigi (2), P. Carlier (2)

(1)Università di Padova (Padua, IT); (2)Az. Osp. San Martino (Genoa, IT); (3)Centro Trasfusionale (Padua, IT)

Acute graft-versus-host disease (aGVHD) is a major cause of morbidity and mortality after allogeneic hematopoietic stem cell transplantion. Extracorporeal photopheresis (ECP) has been shown to be an effective treatment for patients with steroid resistant GVHD. We report our experience on ECP treatment for aGVHD both in adult and pediatric patients.

Methods: Ninety-two patients, 49 children (median age 8 years) and 43 adults (median age 41 years) with steroid-refractory aGVHD, grade II $(n=46)$, III $(n=27)$ or IV $(n=19)$ were treated with ECP in two Italian hospitals. ECP was started after a median interval from aGVHD onset of 48 days in the adult patients and 27 days in the pediatric patients.

The median duration of ECP was 153 days (15-985) in adult patients and 141 days (14-311) in pediatric patients for a median number of $20(1-51)$ and $10(3-45)$ cycles respectively.

Results: Fifty-six patients (61\%) survived and 36 died. Fourteen patients died of leukemia relapse, while 22 of GVHD with or without infections. As regards alive patients, 38 of them $(68 \%)$ had a complete response ( $57 \%$ of the adults and $74 \%$ of the children) and eleven $(20 \%)$ had a partial response $(29 \%$ adults and $17 \%$ children). Complete responses were obtained in $71 \%$, $75 \%, 44 \%$ of patients, respectively, with grade II, III, IV aGVHD. A complete response of aGVHD manifestations of skin, gut and liver was present respectively in $74 \%, 82 \%, 100 \%$ of pediatric patients and in $66 \%, 40 \%, 27 \%$ of adult patients.

Conclusions: Our data confirm the efficacy of ECP in the treatment of resistant aGVHD, both in pediatric and adult patients. Moreover, skin, gut and liver all show good response to ECP.

\section{P544}

Peripheral blood stem cells and granulocyte colony-stimulating factor are associated with acute graft-versus-host disease

O. Ringden, M. Remberger

Karolinska Institutet (Stockholm, SE)

Does granulocyte colony-stimulating factor (G-CSF) increases GVHD or not? A mouse model of GVHD showed that G-CSF induce GVHD after bone marrow (BM) transplantation (Morris et al., Nature Med 15: 436, 2009). Total body irradiation (TBI) increased G-CSF receptors on host dendritic cells, stimulating acute GVHD. Their report prompted us to reassess the role of G-CSF prophylaxis in our HSCT patients. During 19942002, 260 patients were given G-CSF prophylaxis. Patients not given G-CSF $(n=205)$ who underwent HSCT from 1993 to 2003 were controls. We included all patients receiving BM 
$(n=286)$ or peripheral blood stem cell (PBSC) grafts $(n=179)$ from HLA-identical siblings $(n=225)$ or HLA-A, $-B$, or DRß1 genomically identical unrelated donors (MUDs; $n=240$ ). The G-CSF group had more MUD transplants, fewer patients receiving reduced-intensity conditioning (RIC), more patients receiving antithymocyte globulin $(P<0.001)$, and AB0 mismatch was commoner $(P=0.03)$. Patients treated with $\mathrm{G}-\mathrm{CSF}$ had acute GVHD (grades II-IV) of $29 \%$, vs. $19 \%$ in the controls $(P<0.01)$. GVHD occurred in $33 \%$ of patients given PBSC, as compared to $19 \%$ of those given BM $(P<0.001)$. There was no significant difference in incidence of GVHD between HLAidentical sibling transplants and MUD transplants, between those with myeloablative conditioning and those with RIC, or between those treated with TBI and chemotherapy and those treated with chemotherapy only. In patients conditioned with chemotherapy, $34 \%$ in the G-CSF group developed acute GVHD as compared to $21 \%$ in the controls $(P=0.035)$. In patients treated with TBI, those given G-CSF had an incidence of acute GVHD (grades II-IV) of $26 \%$ as opposed to $17 \%$ in the controls $(P=0.10)$. In PBSC recipients, $39 \%$ in the G-CSF group developed acute GVHD as compared to $24 \%$ in the controls $(P=0.025)$. In recipients of $\mathrm{BM}$, the corresponding figures were $22 \%$ and $15 \%$, respectively, in the two groups ( $P=0.19)$. In the present study, a multivariate analysis showed that acute GVHD of grades II-IV was associated with PBSC grafting (hazards ratio $(H R)=2.29, P<0.0001)$, female donors $(H R=1.74$, $P=0.005)$, major $\mathrm{AB} 0$ mismatch $(\mathrm{HR}=1.58, P=0.03)$, and $\mathrm{G}-\mathrm{CSF}(\mathrm{HR}=1.52, P=0.03)$. Differences were adjusted. There were no differences between the two groups regarding nonrelapse mortality, relapse, and survival.

Conclusion: G-CSF induces GVHD in mice, but differs compared to humans regarding the role of source of stem cell and conditioning regimen.

\section{P545}

The impact of CCL5/RANTES SNPs on the outcome of allogeneic haematopoietic stem cell transplantation - a single-centre study

A. Tomaszewska, K. Guz, B. Nasilowska-Adamska, J. Smolarczyk-Wodzynska, M. Lakomy, E. Brojer, B. Marianska Institute of Hematology and Transfusion Medicine (Warsaw, PL)

Introduction: Chemokines are major mediators in the inflammation and immune responses, especially in alloreactivity. RANTES (Regulated upon Activation, Normal T cell Expressed and Secreted) is a member of CC-chemokine family, known as CCL5 (CC-chemokine ligand 5) and is a potent chemoattractant for memory $\mathrm{T}$ lymphocytes, monocytes and eosinophils. This study aimed to determine association between SNPs (single nucleotide polymorphisms) in the CCL5/RANTES promoter gene at positions $-28 \mathrm{C} / \mathrm{T}$ (rs1800825) and $-403 \mathrm{C} / \mathrm{T}$ (rs2107538) with the outcome of allogeneic haematopoietic stem cell transplantation (alloHSCT) performed between August 2003 and April 2009 in our center.

Materials and methods: The allelic variants of these 2 SNPs were determined in 64 patient/donor pairs by real-time polymerase chain reaction (TaqMan-MGB). Patients characteristics were: median age 36 (range 18-65), underlaying diseases-haematologic malignancies in 62 and aplastic anemia - in 2 cases. Donors were HLA-identical sibling-in 46 and unrelated-in 18 transplants, the median age-37 (range 14-65). The studied end points were GvHD, oral mucositis, haemorrhagic cystitis, toxicity and venooclusive disease (VOD) of the liver and survival. Results: The allelic distribution of CCL5/RANTES SNPs was similar to that reported in Caucasoid population. Acute-GvHD was recognized in 31/64 (48,4\%) and chronic-in 34/64 (53\%) of patients. Overall survival in analysed group was $56 \%$. Our study revealed significant correlation between the $-403 \mathrm{C} / \mathrm{T}$ SNP in patients and the occurrence and severity of aGvHD (TT and CT vs. CC genotypes $-41,7 \%$ vs. $31,4 \%$ respectively, $P=0.025)$ and the differences in donors genotypes were indicative of the trend $(P=0.095)$. Besides we found a trend toward correlation between the $-403 \mathrm{C} / \mathrm{T}$ SNP in recipients and development of VOD (TT and CT vs. CC genotypes $-30,8 \%$ vs. $11,8 \%, P=0.070)$ and haemorrhagic cystitis $(30,8 \%$ vs. $17,6 \%, P=0.071$ ). The only statistically significant correlation for $-28 \mathrm{C} / \mathrm{T}$ SNP was increased overall survival obtained by the Kaplan-Meier method for patients carrying CT genotype (100\% vs. $51,7 \%$ for TT-homozygotes, $P=0.048$ ). Besides we couldn't find any correlation between CCL5 SNPs and the rest of analysed end points $(P=\mathrm{ns})$.

Conclusion: Our data revealed that SNPs in the CCL5/RANTES promoter gene at positions -28 and -403 are correlated with aGvHD, toxic complications and overall survival after alloHSCT and should be investigated prospectively and in the larger group of patients.

\section{P546}

Extracorporeal photochemotherapy in small children with acute and chronic GvHD, a single-centre report

V. Witt (1), A. Lawitschka (1), S. Matthes (1), H. Gadner (1), G. Fritsch (1), N. Worel (2), C. Peters (1)

(1)St. Anna Children Hospital (Vienna, AT); (2)MUW Transfusion Medicine (Vienna, AT)

Introduction: ECP is meanwhile a recommended treatment modality in acute and chronic GVHD after BMT. In children data are very rare. Moreover due to the restriction of the available systems to patient weighing more than $40 \mathrm{~kg}$ (Therakos system) the feasability in especially small children is not widely shown. We report here our expirience in the treatment of acute and chronic GVHD in pediatric patients and specially in small children treated with ECP.

Patients and methods: From 1996 to 200932 patients, 9 with a.GVHD and 23 with c.GVHD were treated with ECP. Patients weighing less than $40 \mathrm{~kg}$ were available for the offline system consisting of one leukapheresis on $\mathrm{d} 1$ and 2 reinfusions on $\mathrm{d}$ $1+2$ separately $(n=15)$ or in very small children or critical ill children with a MINI ECP $(n=2)$ consisting of the preparation of MNC from 50 to $100 \mathrm{ml}$ peripheral blood and consecutively the irradiation with UVA after adding 8-MOP, and finally the closed system (UVAR XTS, Therakos Corp., $n=15$ ).

Results: In a.GVHD 8/9 patients responded with improvement overal and $9 / 9$ in skin, $3 / 3$ in gut and $3 / 4$ in liver involvment after 2 or 3 ECP cycles. In median 10 ECP cycles in weekly to 2 weekly interval were necessary before stopping the treatment. In c.GVHD 14 patients improved and 6 patients did not improve. Skin improved in $17 / 22$, gut $2 / 3$ and liver $4 / 4$. In median 27 ECPs were necessary per patient. The interval was 1 month weekly, 2 months 2 weekly and than due to the repsonse 2 weekly or monthly. In chronic GVHD the overal survival was determinated by the response to the ECP treatment $(100 \%$ overal survival in patients who responded to ECP treatment and $40 \%$ overal survival in patients who did not responded to ECP treatment. In chronic GVHD 8/9 with the offline system (1 apheresis and 2 reinfusions) responded and $10 / 15$ with the closed system responded. There was no difference between the methods used for ECP. In total 712 procedures were perfomed (227 (acute GVHD 78) apheresis with 454 reinfusions with the offline method, 16 MINI ECP, and 489 closed ECP with the UVAR XTS) (Neither with the offline nor with the closed system any very severe adverse events were registered.

Conclusion: We used different methods for ECP in pediatric patients and could demonstrate in acute GVHD an overal repsonse of $90 \%$, in chronic GVHD of $74 \%$. This was not depended on the method of ECP used. Therefoe we conclude, that ECP could be safely in pediatric patients with all available methods. 


\section{P547}

A single nucleotide polymorphism of the Fcgamma receptor type IIIA gene in the recipient predicts transplant outcomes after HLA-fully-matched unrelated bone marrow transplantation for myeloid malignancies

A. Takami, J.L. Espinoza, M. Onizuka, Y. Kanda, T. Kawase, Y. Morishima, T. Fukuda, Y. Kodera, S. Nakao for the Japan Marrow Donor Program (JMDP)

Fcgamma receptor type IIIA (FCGR3A), a low affinity receptor capable of interaction with complexed or monomeric lgG, has a functional single nucleotide polymorphism (rs396991), at which a $\mathrm{G}$ to $\mathrm{T}$ point mutation results in an amino acid substitution at position 158 (valine to phenylalanine; V158F) in the second immunoglobulin-like domain. This study examined the impact of the FCGR3A polymorphism in donors and recipients on the clinical outcomes in unrelated HLA-fully-matched myeloablative bone marrow transplantation (BMT) through the Japan Marrow Donor Program (JMDP). The FCGR3A-V158F genotype was retrospective analyzed in a total 99 recipients with myeloid malignancies and their unrelated donors. The presence of the $158 \mathrm{~V}$ genotype in recipients showed a statistically better overall survival (OS; adjusted hazard ratio [HR], 0.49; 95\% confidence interval $[\mathrm{Cl}], 0.26-0.93 ; P=0.03)$ and transplant-related mortality (TRM; HR, $0.30 ; 95 \% \mathrm{Cl}, 0.14-0.67 ; P=0.003$ ) without significant influence on the relapse rate. The recipient $158 \mathrm{~V}$ genotype was also associated with a significantly reduced risk of chronic GVHD (HR, 0.45; 95\% Cl, 0.20-0.99; $P=0.049)$ and a trend toward a reduced risk of II-IV acute GVHD (HR, 0.55; $95 \% \mathrm{Cl}, 0.27-1.10 ; P=0.09$ ), leading to a significantly reduced GVHD-related mortality (HR, $0.22 ; 95 \% \mathrm{Cl}, 0.06-0.77 ; P=0.02)$. The donor FCGR3A polymorphism did not have any impact on the transplant outcomes. These results therefore suggest an association between the recipient FCGR3A genotype and the clinical outcomes after BMT. These findings could therefore be useful for selecting appropriate risk-specific precautions following transplantation.

\section{P548}

Methotrexate protects from severe acute graft-versus host disease without increasing the risk of relapse after unrelated reduced-intensity allogeneic stem cell transplantation

S. Vigouroux (1), R. Tabrizi (1), C. Melot (1), J. Coiffard (1), X. Lafarge (2), G. Marit (1), K. Bouabdallah (1), A. Pigneux (1), T. Leguay (1), M.-S. Dilhuydy (1), A. Schmitt (1), J.-M. Boiron (2), N. Milpied (1)

(1)Centre Hospitalier Universitaire (Bordeaux, FR); (2)Etablissement Français du Sang (Bordeaux, FR)

The combination of a calcineurin inhibitor and short course of methotrexate is a consensual prophylaxis of graft versus host disease (GvHD) after standard myeloablative conditioning regimens (CR). The best prophylaxis is less clearly defined after unrelated reduced-intensity allogeneic transplantation (RIC allo-SCT) following a CR with antithymocyte globulin (ATG). In these circumstances, a calcineurin inhibitor is always used but the role of methotrexate remains a matter of debate.

To explore the effects of methotrexate in this setting, we conducted a retrospective monocentric analysis of unrelated RIC allo-SCT between 2005 and 2009. Sixty-three patients were selected based on a total dose of $5 \mathrm{mg} / \mathrm{kg}$ of ATG in the CR. The median age was 53 years. Diseases were AML $(n=28)$, $\operatorname{ALL}(n=5)$, Iymphoma $(n=13)$, myelodysplasia $(n=7)$, myeloma $(n=7)$, myeloproliferative syndrome $(n=2)$ and $\operatorname{CLL}(n=1)$. Conditioning regimens were Fludarabine-Busulfan-ATG $(n=48)$ or Fludarabine-Treosulfan-ATG $(n=15)$.

Two groups were individualized for the analysis. A group $M+$ $(\mathrm{n}=39)$ received methotrexate at $15 \mathrm{mg} / \mathrm{m}^{2}$ on Day $1,10 \mathrm{mg} / \mathrm{m}^{2}$ on Days 3 and 6. A group $M-(n=24)$ did not. All patients received ciclosporine. There was no significant difference between both groups regarding diseases $(P=0.6)$, status at transplant
$(P=0.5)$, female donor for male patient $(P=0.5)$, HLA mismatches $(P=0.5)$, source of stem cells $(P=0.9)$ or year of tranplant $(P=0.7)$. Patients in the $\mathrm{M}+$ group were older ( 57 vs. 49 years). Patients in the $\mathrm{M}$ - group received more often Treosulfan (14 vs. $1 ; P<0.01$ ). In the $\mathrm{M}$ - and $\mathrm{M}+$ groups, cumulative incidences $(\mathrm{Cl})$ of grade II-IV aGVHD were $52 \%$ and $32 \%$, respectively $(P=0.07)$ while $\mathrm{Cl}$ of grade III-IV aGvHD were $43 \%$ and $10 \%$, respectively $(P=0.002)$. Multivariate analysis indicated that grade III-IV aGvHD was favoured only by the absence of methotrexate. In the $\mathrm{M}+$ and $\mathrm{M}$ - groups, with a median followup of 24 months the 2 -year OS were $69 \%$ and $42 \%$, respectively $(P=0.09)$; the 2-year DFS were $58 \%$ and $46 \%$ respectively $(P=0.2)$, the 2-year TRM were $21 \%$ and $45 \%$, respectively $(P=0.09)$; and the 2-year incidence of relapse were $25 \%$ and $28 \%$, respectively $(P=0.7)$.

These data suggest that methotrexate protects from severe acute GvHD without increasing the risk of relapse after unrelated RIC transplantation with a trend for a better survival. This protective effect was observed despite the older age of patients receiving methotrexate.

\section{P549}

IMPDH1 and UGT1A9 polymorhisms did not affect the outcome after allogeneic stem cell transplantation in patients submitted to GvHD prophylaxis with mycophenolic acid

S. Giammarco, E. Metafuni, P. Chiusolo, S. Bellesi, S. Marietti, F. Autore, L. Laurenti, F. Sorà, G. Leone, S. Sica

Università Cattolica S. Cuore (Rome, IT)

The immunosuppressive (IS) agent mycophenolic acid (MMF) is metabolized by uridine diphosphate glucuronosyltransferase 1 A9 (UGT1A9) and it has as target enzyme inosine 5'-monophosphate dehydrogenase (IMPDH1) which is the rate limiting step of de novo pathway for purine synthesis. Several studies on kidney transplantation demonstrated that pts carrying C440T/T-331C UGT1A9 gene polymorphisms could have different MMF pharmacokinetic and patients with rs2278293 and rs 2278294 IMPDH1 gene polymorphisms could have a different incidence of graft rejection.

The purpose of our study was to evaluate the C-440T/T-331C and rs2278293 and rs 2278294 polymorphisms prevalence in pts submitted to alloSCT submitted to GVHD prophylaxis with MMF and their role in GVHD and other complications after SCT.

We studied 23 consecutive pts submitted to alloSCT between February 2006 and April 2009. Blood samples from pts and their donors were collected after signed informed consent. C440T/T-331C and rs2278293 and rs 2278294 polymorphisms were evaluated by PCR amplification of genomic DNA. Pts' characteristics were: M/F 13/11, median age 55 y (range 1468). They were affected by AML (10), MM (4), ALL (3), CLL (3), $\mathrm{NHL}$ (2), HD (1) and IMF (1). Disease status at transplant was: CR in 4 pts, PR in 12, SD in 2 and PD in 4 pts respectively. All pts were submitted to a RIC followed by CSA and MMF prophylaxis; MMF was administered at $15 \mathrm{mg} / \mathrm{kg}$ until day +28 and +36 after SCT in related and unrelated SCT respectively. Stem cells source was PB in 22 and $C B$ in 2 pts.

The incidence of aGVHD grade III-IV was $25 \%$ while the incidence of cGVHD $33 \%$. The TRM was $16.6 \%$ The DFS was $70 \%$ with a median F-U of 18 months (0-46). We observed the following prevalence of the different genotypes: UGT1A9 -440CC 43 and $30 \%$, CT 39 and $52 \%$, TT $18 \%$ in pt and donors respectively, UGT1A9 -331 TT 43 and $30 \%$, TC 39 and $52 \%$, CC $18 \%$ in pts and donors respectively, IMPDH1 rs2278293 GG 39\%, GA 43\%, AA 18\% in both pts and donors, and rs 2278294 GG 39 and $52 \%$, GA 47 and $34 \%$, AA 14 and $4 \%$ in pts and donor respectively. We did not find any correlation between these polymorphisms and the incidence of GVHD, TRM and relapse incidence.

In conclusion polymorphisms of gene related to MMF pharmacokinetic and activity are not correlated to immunological 
events after alloSCT. One of hypothesis could be the relatively small period of MMF administration in HSCT in comparison to longer period of IS therapy in solid organ transplantation.

\section{P550}

Antiviral agents decrease the risk of acute graft-versushost disease in paediatric patients with human herpes virus 6 reactivation after allogeneic haematopoietic stem cell transplantation

N. Kawashima (1), H. Sakaguchi (1), N. Yoshida (1), K. Matsumoto (1), T. Yoshikawa (2), K. Kato (1)

(1)Japanese Red Cross Nagoya First Hospital (Nagoya, JP); (2)Fujita Health University (Toyoake, JP)

Background: Human herpes virus 6 (HHV-6) is frequently reactivated and detected in plasma of patients in the early phase of hematopoietic stem cell transplantation (HSCT). HHV-6 reactivation has been implicated to be associated with significant morbidity, including encephalitis, bone marrow suppression or acute graft-versus-host disease (aGVHD). However the clinical manifestation and management of HHV-6 reactivation in patients undergoing HSCT are not subject to a clear consensus. We investigated the incidence of HHV-6 reactivation in relation to mortality and morbidity in children.

Patients and methods: Between April 2005 and October 2009 62 consecutive pediatric recipients of allogeneic HSCT were weekly monitored for HHV-6 loads measured by quantitative polymerase chain reaction. Hematological malignancies were diagnosed in 57 patients and non-malignant disorders in five patients. Twenty patients received related bone marrow, 24 patients unrelated bone marrow, 15 patients unrelated cord blood, and three patients related peripheral blood stem cell. Serological HLA disparities were $0(n=44), 1(n=15)$, and 2 $(n=3)$. HHV-6 reactivation was treated in the presence of clinical symptoms.

Results: HHV-6 reactivation was observed in 36 of 62 patients (58\%) by day 100 after allogeneic HSCT. HHV-6 loads more than 500 copies/mcg DNA were associated with higher nonrelapse mortality $(P=.070$ using a generalized Wilcoxon test). Twenty-two patients developed aGVHD and the incidence of HHV-6 reactivation was 14 of $22(64 \%)$ and 22 of $40(55 \%)$ with or without aGVHD. The incidence of aGVHD was 3 out of 10 $(30 \%)$ and 11 out of $26(42 \%)$, with or without administration of gancyclovir or cidofovir, respectively. Administration of antiviral agents was related to the reduced incidence of aGVHD using a multivariate logistic regression analysis $(P=.086)$.

Conclusion: HHV-6 reactivation is common after HSCT in children and is associated with significant morbidity such as aGVHD. Prompt administration of antiviral agents suppresses symptoms caused by the reactivation of HHV-6 and thereby may reduce the development of aGVHD.

\section{P551}

CD4 + lymphocytes of alloHSCT patients are in higher proportions able to produce IL-17 and IFN-gamma under stimulation and it does depend on the manifestation of aGvHD

D. Dlubek (1), J. Lange (2), A. Lange (1)

(1)Institute of Immunology and Exp Therapy (Wroclaw, PL); (2)Lower Silesian Centre for Cellular Transplantation (Wroclaw, $P L)$

In this study we focused on the presence of IL-17 producing cells in patients having and lacking aGvHD post HSCT in the context of FoxP3 + lymphocytes and those with IFNgamma production potential.

The expressions of IL-17 FoxP3 and IFN-gamma were studied in stimulated PBMC (brefeldin A Inomycin and PMA) of alloHSCT pts (48 pts, median age: $45 \mathrm{yrs}$ range; 1.0-64 yrs, with 46 hematological malignancies and 2 SCID, 23 MUD and 25 SIB transplants). The cells were labeled with CD4 IL-17A
IFNg and FoxP3 MoAbs and analyzed in the CD4 + lymph. subpopulation. 21 developed aGvHD among them 8 pts had aGvHD (4 skin and 4 gut) at a later stage after hematological reconstitution and 5 pts suffered from aGvHD.

We found: 1. Percentage of IL-17 producing lymph. was higher in pts at hematological reconstitution as compared to healthy controls $(0.76 \pm 0.13$ vs. $0.19 \% \pm 0.06, P=0.03) 2$. aGvHD cases with an onset of the disease at the time of hematological reconstitution had lower proportions of IL-17A+ producing CD4+ lymph. then those without aGvHD at the similar time post HSCT $(0.29 \pm 0.09$ vs. $0.76 \% \pm 0.13, P=0.02)$. Pts they manifested aGvHD at later time post transplant had an increase in proportions of IL-17 producing cells 3 to 7 days prior the overt maniferstation which significantly declined at the onset of symptoms $(1.07+0.37$ vs. $0.34 \%+0.14, P=0.01) 3$. Gut aGvHD similarly to skin aGvHD characterized with a decrease of IL-17 producing CD4 + cells at the time of aGvHD manifestation. However, lower values of IL-17 producing CD4 + cells were seen in gut aGvHD earlier than in the skin variant of the disease 4 . A high degree of correlation was found between the percentages of $\mathrm{IL}-17 \mathrm{~A}+$ and IFNg + in CD4 + lymph. in blood at the time of hematological recovery $(r=0.586, P=0.002) 5$. Percentages of IFNg-producing CD4 + cells correlated with proportions of FoxP3 + cells in CD4 + lymph. population $(r=0.427, P=0.03)$. Conclusion: HSCT patients had higher proportions of IL-17 producing cells under stimulation as compared to donors. Proportions of these cells further increase prior to the overt manifestation of aGvHD and decrease when the disease is full blown. Proportions of FoxP3 + CD4 + in lymph. increase at the manifestation of aGvHD likely to counter-balance alloreactivity as suggested by a positive correlation with percentages of IFNg producing cells in lymphocytes under the culture conditions.

\section{P552}

Poor oral intake after hematopoietic stem cell transplantation is associated with an increased risk of acute graft-versus-host disease

R. Hanajiri, K. Ohashi, T. Kobayashi, T. Yamashita, H. Akiyama, H. Sakamaki

Tokyo Metropolitan Cancer and Infectious diseases Center Komagome Hospital (Tokyo, JP)

Background: Some studies have shown that early enteral nutrition improves outcome in the intensive care unit setting. However, it is unclear whether early oral intake is of benefit in hematopoietic stem cell transplantation (HSCT). To elucidate the impact of early oral nutrition on clinical outcome, we performed a retrospective cohort study in a single institution.

Methods: 241 patients recieiving allogeneic HSCT between September 2004 and September 2008 were retrospectively reviewed. 13 patients were excluded due to early death $(<35$ days). The remaining 228 patients were analyzed for nutritional history just after day 0 of HSCT.

Results: 87 patients (38\%) were able to eat everyday after day 0 of HSCT; whereas $63(28 \%)$ patiants were not able to eat anything for 1 to 6 days; 78 (34\%) patients for more than 7 days (7-62 days). There were no significant differences in baseline clinical characteristics. Patients with no oral intake more than 7 days ( $\geq 7$ days group), as compared with 0 days ( 0 days group), was associated with an increased risk of grade II-IV or grade III-IV acute graft-versus-host disease (GVHD) (54\% vs. $23 \%, P=0.001 ; 18 \%$ vs. $5 \%, P=0.02$, respectively). The incidence of stage 2-4 skin GVHD and stage 1-4 gut GVHD were also higher in the $\geq 7$ days group compared with 0 days group ( $49 \%$ vs. $32 \%, P=0.03 ; 36 \%$ vs. $14 \%, P=0.001$, respectively). Five-year overall survival and progression-free survival were not different between the three groups.

Conclusions: Our findings demonstrate that no oral intake early after HSCT is significantly correlated with an increased risk of acute GVHD. 


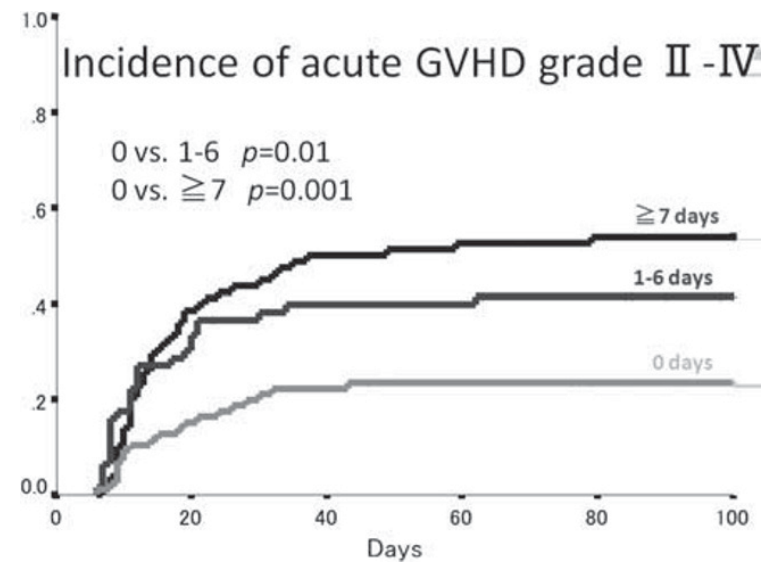

P553

Impact of ABO-incompatibility on acute graft-versus-host disease incidence in allogeneic haemopoietic stem cell transplantation recipients

M. Estrina, S. Alekseev, Yu. Zalyalov, M. Yagmourov, E. Kochina, N. Ivanova, A. Alyanskiy, L. Zoubarovskaya, A. Ganapiev, B. Afanasyev

St-Petersburg Pavlov State Medical Unive (St. Petersburg, RU)

Introduction: Allogeneic hemopoietic stem cell transplantation (allo-HSCT) is currently one of the most effective methods 100 of treatment for various malignant hematological disorders. Recently, the ABO system antigens incompatibility between donor and recipient is given much emphasis as an important risk factor able to influence allo-HSCT recipients post-transplant course.

Materials and methods: 240 allo-HSCT recipients were included in this study, 69 patients were transplanted from matched related donors, 171 patients from matched unrelated donors. 146 of donor-recipient pairs were $A B O$ mismatched, $54(37 \%)$ of the pairs had major, $67(45,9 \%)$ minor and $25(17,1 \%)$ bidirectional mismatch. 94(39,2\%) pairs were ABO-compatible. In 148 cases as stem cell source were used peripheral blood stem cells (PBSC), in 92 cases was used bone marrow (BM). Graft-versus-host disease (GVHD) prophylaxis regimen was identical in all patients groups. Results: We observed no significant differences in engraftment (Leu $>1,0^{*} 10^{9} / \mathrm{l}$, Neu $>0,5^{*} 10^{9} / \mathrm{l}$, Plt $>50^{*} 10^{\%} / \mathrm{l}$ ) in ABO-compatible and $\mathrm{ABO}$-incompatible patient groups $(P=0.6)$, there was also no difference in engraftment between patient groups with different forms of ABO-incompatibility $(P=0,38)$. In 2 patients with major $\mathrm{ABO}$-incopatibility was observed pure red cell aplasia. Incidence $(P<0,01)$ and severity of acute GVHD were significantly higher in ABO-incompatible allo-HSCT recipients $(P=0,005)$. No significant difference in acute GVHD incidence $(P=0,38)$ and severity $(P=0,6)$ was noted between patient groups with different forms of ABO-incompatibility. The risk of acute GVHD development differed in ABO-incompatible HSCT recipients from matched unrelated $(81,3 \%)$ and related $(44 \%)$ donors $(P=0,002)$.

Conclusions: $\mathrm{ABO}$-incompatibility is an important risk factor for acute GVHD development, but doesn't influence the engraftment.

P554

GvHD prophylaxis with everolimus and mycophenolate in allogeneic haematopoietic cell transplantation - a phase I/II study

R. Marks, H. Bertz, R. Waesch, W. Melchinger, A. Baumgarten, J. Finke

University Hospital Freiburg (Freiburg, DE)

Tolerance induction remains to be critical for longterm survivors after allogeneic hematopoietic cell transplantation (HCT). Regulatory T cells (Tregs) are involved in the regulation of graft versus host disease (GvHD) and graft versus leukemia effect
(GvL). Although calcineurin inhibitors are frequently being used for the prevention of GvHD, tolerance induction might be hampered in HCT patients due to deteriorated Treg function. Data from animal models of HCT suggest that inhibition of the mammalian target of rapamycin (mTOR) results not only in suppressed T cell alloreactivity but also in sustained Treg function. Therefore we initiated a phase I/II monocenter trial using everolimus and mycophenolate-sodium (MMF-Na) as GvHD prophylaxis in patients undergoing allogeneic HCT with peripheral stem cell (PBSC) grafts after reduced toxicity conditioning. No additional $T$ cell depleting agents were used for GvHD prophylaxis. So far 13 patients were included (median age: 49,6 years, range: $26-64)$. The diagnoses were: $\operatorname{AML}(n=4)$, sAML $(n=6)$, MDS $(n=1), \operatorname{CML}(n=1), \operatorname{T}-P L L(n=1)$. 8/11 AML/MDS patients were at high risk (not in CR1) for relapse. Grafts were obtained from unrelated $(n=7)$ and related $(n=6)$ HLA-matched donors. With no graft failures, engraftment kinetics for myeloid cells were normal, and reconstitution of the T cell compartment reached median cell counts of $>200 \mathrm{CD} 4+$ cells $/ \mu l$ at day +30 . No grade IV/ $N$ toxicities (according to CTC criteria) were observed due to the study medication. After a median follow-up of 11 months four patients have died. The causes were refractory acute GvHD in a patient with CML, severe pulmonary toxicity/ BOOP, HHV6 associated encephalitis, and one late relapse. Out of 12 patients reaching CR, only two patients relapsed after 8 and 19 months. CMV reactivation could be seen in $3 / 9$ patients at risk. The early recovery of $T$ cell immunity correlated in 9/12 patients with a brief period of acute GvHD grade II-IV. Chronic GvHD could be observed in $8 / 9$ patients, mostly mild to moderate forms. No severe bacterial or fungal infections were observed even in cases with prolonged everolimus treatment. In conclusion, GvHD prophylaxis with everolimus and MMF-Na is feasible but results in an increased frequency of mild to moderate chronic GvHD. Since this sustained mild alloreactivity might reduce the risk of relapse this regimen could be suited for patients undergoing HCT with advanced or uncontrolled malignant disease.

\section{P555}

Addition of omega-3 fatty acids to antithymocyte globulin-containing regimens can decrease occurrence of severe acute graft-versus-host disease after haematopoietic stem cell transplantation: single-centre experience

M. Kucher, Y. Zalyalov, I. Kazancev, S. Andreev, O. Goloschapov, B. Ganapiev, B. Afanasyev

St. Petersburg Medical State University (St. Petersburg, RU)

Introduction: Acute graft-versus-host disease (aGVHD) is a major complication developing in $30-60 \%$ of allogeneic hematopoietic stem cell transplantation (allo-HCST) recipients in spite of the use different prophylactic regimens. Preliminary clinical data showed the influence of omega-3 fatty acids on cytokine profile and inflammatory reactions. We tried to reduce incidence and severity of aGVHD giving allo-HSCT recipients additional nutrition support (NS) with omega-3 fatty acids.

Purpose: The main objective of this study was to evaluate the influence of omega-3 fatty acids NS on incidence and severity of aGVHD in allo-HSCT recipients after antithymocyte globulin (ATG) - containing conditioning regimens.

Materials and methods: We analyzed data from 62 patients receiving allo-HSCT for acute myeloid leukemia $(n=14)$, acute lymphoblastic leukemia $(n=39)$, chronic myeloid leukemia $(n=4)$, Hodgkin disease $(n=4)$, Kostman syndrome $(n=1)$. Median age was 25,4 (17-47) years.

We divided the patients into three groups. All three groups received basic GVHD prophylaxis with CsA and Mtx.

Control group of patients $(n=24)$ received only basic GVHD prophylaxis.

In second group of patients $(n=28)$ we added to basic prophylaxis a course of ATG (ATGAM $20 \mathrm{mg} / \mathrm{kg}$ b.w. for three consecutive days).

The third group of patients $n=10(n=2$ allogeneic related HSCT and $n=8$ allogeneic unrelated HSCT) for GvHD prophylaxis 
received ATG-containing prophylactic regimen with additional NS, starting from D+1 after HSCT-low-bacterial enteral nutrition and parenteral nutrition with addition of omega- 3 fatty acids - Omegaven, Fresenius Kabi $1,5 \mathrm{ml} / \mathrm{kg} / \mathrm{day}$ iv or Lipo Plus, BBraun $1,5 \mathrm{~g} / \mathrm{kg} /$ day iv.

Results: We observed different occurrence of aGVHD in evaluated patients groups. The occurrence of grade III-IV aGVHD was $20,9 \%$ in the first group (only basic prophylaxis), $14,3 \%$ in the second group (addition of ATG) and 10,0\% in the third group (addition of omega-3 fatty acids).

Conclusions: These preliminary results demonstrate lower occurrence of grade III-IV aGVHD in patients receiving NS with omega-3 fatty acids after employment of ATG-containing regimens. This effect requires further investigation.

\begin{tabular}{|l|l|l|l|}
\hline & $\begin{array}{l}\text { CsA+Mix } \\
\mathrm{n}=24\end{array}$ & $\begin{array}{l}\text { CsA+Mtx+ATG } \\
\mathrm{n}=28\end{array}$ & $\begin{array}{l}\text { CsA+Mtx+ATG+NS } \\
\mathrm{n}=10\end{array}$ \\
\hline No engraftment & $12,5 \%$ & $3,6 \%$ & - \\
\hline No GVHD & $33,3 \%$ & $28,6 \%$ & $10,0 \%$ \\
\hline Grade I-II GVHD & $33,3 \%$ & $53,5 \%$ & $80,0 \%$ \\
\hline Grade III-IV GVHD & $20,9 \%$ & $14,3 \%$ & $10,0 \%$ \\
\hline
\end{tabular}

\section{P556}

The comparative characteristic toxicity and immunosuppressive efficacy of both tacrolimus and cyclosporine in patients undergoing allo-HSCT

Y.A. Stankevich, L.S. Zubarovskaya, E. Babenko, B. Afanasyev Pavlov State Medical University (St. Petersburg, RU)

Background: Graft vs. host disease (GVHD) still remains one the major obstacles to successful allogeneic hematopoietic cell transplantation (HSCT). GVHD is a major cause of morbidity and mortality. GVHD has an important impact on survival and the recipient's quality of life. The ability to prevent GVHD is the cornerstone of success. Immunosupression with pharmacological agents such as cyclosporine (CsA) or tacrolimus (Tx) is use for GVHD prophylaxis, because they share a common intracellular target. The toxicity profiles of CsA and Tx are very similar. However, we found that Tx was superior for the prophilaxis of acute GVHD, but more toxic. While hypertension and myelosupression is more commonly seen with CsA, neurological, nephrology and gastrointestinal side effect are more often associated with administration of $\mathrm{Tx}$.

Patients (pts) and methods: From 2000 till 12.2008171 pts underwent 173 allo-HSCT.

Pts and donor characteristics, type of allo-HSCT and preparing regimen are shown at Table 1.

Results: There was no significant difference in survival. The 6 -month overall survival was $53 \%$ for the Tx group and $65 \%$ for the CsA group; 6-month relapse-free survival was $42 \%$ (Tx) vs. $50 \%$ (CsA). There was no difference in rates of relapse between the two groups.

Infections were the most common cause of death (33\%). Other causes of death included acute GVHD (31\%), relapse $(20 \%)$ and multyorgan failure (8\%). The incidence of infections was similar in the two study groups.

Although a significantly greater proportion of pts were treated with CsA, the incidence of grades II-IV acute GVHD was significantly lower in pts who received Tx $(29 \%$ vs. $54 \%$ in related HSCT and $32 \%$ vs. $60 \%$ in unrelated HSCT).

Despite the fact Tx to be superior for the prophylaxis of acute GVHD, it was found to be more toxic. The incidence of hypertension was significantly higher in CsA group (38\% vs. $24 \%$ ), but neurological complications (headache, tremor, paresthesia) were significantly higher in Tx group (44\% vs. $30 \%)$. Gastrointestinal disturbances is more commonly in Tx group (46\% vs. $36 \%$, NS), also as hyperglycemia ( $24 \%$ vs. $16 \%$ ), nephrotoxisity (19\% vs. $15 \%)$ and metabolic disturbances (11\% vs. $7 \%)$.

Conclusion: Use of Tx seems to be preferable in high risk of GVHD group because of more immunosuppressive effect. But
Tx has more toxic complications such as neurological, nephrology, gastrointestinal and metabolic disturbances. On the other hand, hypertension and myelosupression is more commonly seen with CsA.

Table l, Patient's and transplant's characteristics

\begin{tabular}{|c|c|}
\hline & Number of patients \\
\hline Number of allo-HSCT & 173 \\
\hline Related HSCT & 64 \\
\hline Unrelated HSCT & 109 \\
\hline Haploidentical HSCT & 17 \\
\hline Patients age median, y (range) & $31,6(14-66)$ \\
\hline Diagnosis & \\
\hline $\begin{array}{l}\text { AML MDS } \\
\text { remission } \\
\text { relapse }\end{array}$ & $57 / 7$ \\
\hline $\begin{array}{l}\mathrm{ALL} \\
\text { remission } \\
\text { relapse }\end{array}$ & 58 \\
\hline Lymphoma (NHL+HD) & 16 \\
\hline $\begin{array}{l}\text { CML } \\
\text { chronic phase } \\
\text { acceleration phase }\end{array}$ & 25 \\
\hline Aplastic anemia & 6 \\
\hline Other & 4 \\
\hline Source of HSC & \\
\hline $\mathrm{BM}$ & 57 \\
\hline PBSC & 99 \\
\hline $\mathrm{BM}+\mathrm{PBSC}$ & 17 \\
\hline HLA & \\
\hline full match & 118 \\
\hline 1 mismatch & 28 \\
\hline 2 mismatch & 10 \\
\hline haplo & 17 \\
\hline Prophylaxis of GVHD & \\
\hline $\mathrm{C}_{3} \mathrm{~A}+\mathrm{MMF}$ & 14 \\
\hline $\mathrm{C}_{s} \mathrm{~A}+\mathrm{Mtx}$ & 83 \\
\hline $\mathrm{Cs} \mathrm{A}+\mathrm{Mtx}+\mathrm{MMF}$ & 6 \\
\hline$T_{x}+M M F$ & 46 \\
\hline CsA & 8 \\
\hline Other & 12 \\
\hline Mveloablative regimen & 47 \\
\hline Nonmyeloablative regimen & 125 \\
\hline ABO - compatibility & 71 \\
\hline $\mathrm{ABO}$ - incompatibility & 102 \\
\hline Sex - compatibility & 92 \\
\hline Sex - incompatibility & 81 \\
\hline Transplanted donor cells & \\
\hline $\begin{array}{l}\text { NC } \times 108 \% \mathrm{~kg} \\
\text { CD } 34 \times 106 / \mathrm{kg}\end{array}$ & $\begin{array}{c}6,7_{s=19}(2,6-14,4) \\
5,13_{s=21}(0,3-18,3)\end{array}$ \\
\hline
\end{tabular}

\section{P557}

Effect of acute graft-versus-host disease on the overall survival after allogeneic haematopoietic stem cell transplantation

M. Pevna (1), M. Krejci (2), P. Coupek (3), M. Klabusay (1), J. Mayer (2)

(1)Masaryk University (Brno, CZ); (2)University Hospital Brno (Brno, CZ); (3)Czech Geological Survey (Brno, CZ)

Background: Allogeneic hematopoietic stem cell transplantation (HSCT) is an effective treatment of hematological malignancies. Donor lymphocytes can produce graft-versus-leukemia (GvL) effect, but it is often directed against normal tissues as graft-versus-host disease (GvHD), which increases post-transplant morbidity and mortality. The relationship between $\mathrm{GVL}$ and GvHD influences survival. 
Methods: We analyzed data of 88 patients who received allogeneic PBSC (peripheral blood stem cells) graft, mostly from HLA-identical sibling donor. Cyclosporine A with methotrexate or sirolimus and tacrolimus were used for GvHD prophylaxis. Patients included acute myeloblastic and lymphoblastic leukemia $(n=31)$, chronic myeloid leukemia $(C M L, n=31)$, lymphomas $(n=12)$, chronic lymphocytic leukemia $(C L L, n=3)$, multiple myeloma (MM, $n=2)$ and other diagnoses $(n=9)$. The conditioning regimen before HSCT was myeloablative $(52 \%)$ and reduced-intensity (48\%). Acute GvHD was graded according to the Przepiorka's criteria. Kaplan-Meier overall survival (OS) curves were calculated with regard to grade 0-I and II-III acute GvHD, separately for 4 groups according to diagnosis: 1) acute leukemia, 2) CML, 3) lymphomas, CLL and MM and 4) aplastic anemia, myelodysplastic syndrome, and kidney carcinoma.

Results: Average time of patients' observation was 1249 days; the OS time for the whole group was $65 \%$. There were no differences in OS between myeloablative $(67 \%)$ vs. reduced-intensity conditioning (63\%). 43\% patients developed acute GvHD, which was categorized as grades I (18\%), II (19\%) and III $(6 \%$ of patients). No case of grade IV acute GvHD was recorded. Probability of survival, regardless of diagnosis, without GvHD (grade 0 ) and with acute GvHD grades I, II and III were $65 \%$, $59 \%, 76 \%$ and $60 \%$, respectively. In acute leukemias, grades II-III were associated with better survival compared to grades 0 -I of acute GvHD. Conversely, in the group with lymphomas, CLL and MM, grades II-III acute GvHD were associated with worse survival. The log-rank Mantel-Haenszel test in the common survival model for the groups 1$), 2$ ) and 3 ) rejected the homogeneity hypothesis $(P<0.05)$.

Conclusions: GvHD may decrease the risk of relapse and mild GvHD is associated with improved OS in all types of acute leukemias after allogeneic HSCT. Patients with acute leukemia may benefit from grade II of acute GvHD.

Acknowledgements: This work was supported by grants IGA MZ CR NR9671-4 and MSM 021622430.

\section{P558}

Long-term follow-up of minimal chronic graft-versus-host disease after reduced-intensity conditioning with pre-transplant low dose anti-thymocyte globulin in allogeneic stem cell transplantation

S. Langner, P. Staber, W. Linkesch, D. Strunk

Medical University of Graz (Graz, AT)

Objectives: Reduced intensity conditioning (RIC) regimens are increasingly used to lower toxicity in patients not eligible for myeloablative stem cell transplantation (SCT). High rates of morbidity and mortality from acute and chronic graft versus host disease (GVHD) still remain a dilemma. Anti-thymocyte globulin (ATG), in addition to its dominant action as a T cell depleting agent, has been implicated to beneficially influence GVHD by depleting host antigen presenting cells. We analyzed the effect of additional low dose rabbit ATG on the incidence of GVHD and documented infections.

Methods: Forty-seven patients with a hematological malignancy ( $23 \mathrm{AML}, 2 \mathrm{ALL}, 4 \mathrm{CML}, 6 \mathrm{CLL}, 3 \mathrm{NHL}, 9 \mathrm{MM}$ ), not eligible for myeloablative SCT either by age (median 52 years, range 2172) or co-morbidities, received a fludarabine-based RIC-SCT, whereas in the study group $(n=21)$ rabbit ATG $(5 \mathrm{mg} / \mathrm{kg}$ daily for 4 days) was added to standard GVHD prophylaxis consisting of cyclosporine $A$ and mycofenolate mofetil. Six patients had PD, 23 were in PR and 18 in CR.

Results: After a median follow up of 38 months 25 of 47 (54\%) patients were alive. Acute GVHD grade III-IV (24 vs. $27 \%$, $P=\mathrm{ns}$ ) was comparable in both groups. Overall (58 vs. $14 \%$, $P=0.007)$ and extensive chronic GVHD (37 vs. $7 \%, P=0.03$ ) occurred more often in the control group. GVHD related deaths were documented in two ATG treated patients vs. three controls (18 vs. $27 \%, P=n s$ ). Whereas CMV-DNA detected by PCR was present in eight patients in the study group vs. nine in controls, CMV disease was observed in one out of 21 patients after RIC-SCT with ATG and in two out of 26 transplant recipients not treated with ATG, respectively. The three year overall survival, total documented infections and relapse mortality did not differ significantly. Conclusion: RIC including low dose rabbit ATG resulted in a considerably low incidence of chronic GVHD but had no major impact on acute GVHD or infection rates.

\section{P559}

Tacrolimus/methotrexate versus cyclosporine/ methotrexate as graft-versus-host disease prophylaxis in patients who received bone marrow transplantation from unrelated donors

G. Jaimovich (1), V. Milovic (1), G. Drelichman (1), A. Requejo (1), D. Puente (1), G. Saa (1), N. Bonini (1), J. Real (2), J. Trentadue (1), L. Feldman (1)

(1)CEHT Fundacion Favaloro (Buenos Aires, AR); (2)CEHT (Buenos Aires, AR)

Graft versus host disease (GVHD) remains as one of the main causes of mortality and morbidity associated with the use of unrelated donor bone marrow transplantation (UBMT). Factors known to influence the GVHD incidence include the degree of HLA matching, donor sex and pregnancies and the use of an effective prophylactic regimen. We conducted a study in order to compare the efficacy of Tacrlimus/Methotrexate (FK/MTX) and Cyclosporine/Methotrexate (CsA/MTX) in 31 patients given UBMT.

The study population consisted of patients (pts) who had received a UBMT with a full myeloablative regimen and rescued with bone marrow (BM) or peripheral blood stem cells (PBSC). FK was administered through continous i.v. infusion from the day -1 maintaining blood level between 5 and $15 \mathrm{ng} /$ $\mathrm{ml}$. and converted to oral intake when it could be tolerated. CsA was administered from day -2 , in twice short i.v. infusions maintaining blood levels between 200 and $500 \mathrm{ng} / \mathrm{ml}$. Oral way was implemented as in the FK arm. MTX dose for both group was $10 \mathrm{mg} / \mathrm{m}^{2}$ on days $1,3,6$ and 11 . CsA/MTX was used in the first 13 pts: acute leukemia (AL): 6 , chronic myeloid leukemia 4 (CML), others: 3pts. and FK/MTX in the following 18 pts: AL: 14 , CML: 1 , others: 3 pts. Group risk were stratified considering as good risk a 10/10 or 9/10 HLA match and male donor and high risk $8 / 10$ or $7 / 10$ and a female donor for acute GVHD. For chronic GVHD, good risk were pts. without previous aGVHD receiving BM cells. High risk were pts. with previous aGVHD having PBSC. For the entire population mean age was 19 years $(1-38)$.

Results: The probability of aGVHD in the FK group was $50 \%$ and $66 \%$ in the CsA group at 100 days $(P=0.38)$. Thirteen patients $(23 \%)$ in the FK group and $10(30 \%)$ in the CsA group developed cGVHD $(P=0,38)$. There were no significative differences comparing the high or low group risk for both aGVHD or cGVHD. TRM was $45 \%$ for CsA group and $30 \%$ for FK group $(P=0.45)$ and $O S$ was 0.83 and 0.77 years for the FK and CsA respectively $(P=0.86)$.

In conclusion our analysis showed a slight advantage but a non significant difference for the FK/MTX group regarding aGVHD, cGVHD, TRM and OS. However our study was retrospective including a myriad of different diseases. Further studies comparing the same disease population who will receive UBMT may be warranted. 


\section{Multiple myeloma}

P560

The impact of body weight, induction regimen and mobilization technique on stem cell collection in patients with multiple myeloma

A. Nazha, R. Cook, D. Vogl, P. Mangan, K. Hummel, K. Cunningham, S. Luger, D. Porter, S. Schuster, U. O'Doherty, D. Siegel, E. Stadtmauer

University of Pennsylvania (Philadelphia, US)

Introduction: High dose melphalan and autologus stem cell transplant (ASCT) remains an effective treatment for patients with either newly diagnosed or refractory multiple myeloma (MM). Collection of sufficient numbers of stem cells for more than one transplant is optimal. G-CSF with cyclophosphamide (CY/G-CSF) has been a widely used as effective regimen for stem cell mobilization in MM. Recently, Plerixafor with G-CSF has shown in randomized trials to be superior to G-CSF alone. We set out to assess the optimal method for stem cell mobilization at our institution.

Materials and methods: We performed a single institution retrospective analysis of 365 patients with MM who underwent stem cell mobilization and harvest at the University of Pennsylvania from January 2002 to December 2007. 76 patients were excluded from this analysis due to incomplete data on stem cell mobilization regimen, or underwent allogeneic transplants. 289 patients were included in the final analysis; 16 received plerixafor/G-CSF, 198 received CY/G-CSF, and 75 received G-CSF alone.

Results: The median number of collected stem cells was $7.95 \times 10^{6} \mathrm{CD} 34+/ \mathrm{kg}$ in plerixafor/G-CSF group, $7.7 \times 10^{6}$ $\mathrm{CD} 34+/ \mathrm{kg}$ in Cy/G-CSF group and $4.5 \times 10^{6} \mathrm{CD} 34+/ \mathrm{kg}$ in GCSF alone group. The median number of apheresis days was 2 days, 2 days and 4 days respectively. The percentage of the patients who collected $\geq 6 \times 10^{6} \mathrm{CD} 34+/ \mathrm{kg}$ in $<3$ apheresis was $63 \%, 62 \%$ and $19 \%$ respectively. The number of the patients who collected $\geq 6 \times 10^{6} \mathrm{CD} 34+/ \mathrm{kg}$ and weight $>70 \mathrm{~kg}$ was $77 \%$ in the plerixafor/G-CSF group, $77 \%$ in Cy/G-CSF group and $47 \%$ in G-CSF alone group. For patients $<70 \mathrm{~kg}$ collection $>6 \times 10^{6}$ was $100 \%, 50 \%$ and $67 \%$ respectively. The median number of collected stem cells in the patients who received Len/Dex initial therapy and mobilized with plerixafor/G-CSF or $\mathrm{CY} / \mathrm{G}-\mathrm{CSF}$ was high at $8.5 \times 10^{6} \mathrm{CD} 34+/ \mathrm{kg}$.

Conclusion: This analysis suggests that plerixafor/G-CSF and CY/G-CSF mobilization result in similar and adequate stem cell numbers for ASCT in MM patients. Both approaches are superior to G-CSF alone. Body weight may play a role in the selection of the mobilization regimen. Plerixafor and $\mathrm{CY}$ with G-CSF are effective regimens for stem cell mobilization in MM regardless of prior expose to lenalidomide.

\section{P561}

Need of reconsideration of mobilization strategy in autologous stem cell transplantation for multiple myeloma: results of the positive impact of high number of CD34 + infused cells

M. Michallet (1), M. Sobh (1), B. Riche (2), P. Guerre (2), F. Nicolini (1), N. Raus (1), F. Barraco (1), G. Cannas (1), Y. Chelghoum (1), E. Nicolas-Vierlizier (1), G. Clapisson (1), X. Thomas (1), A. Zinai (3), R. Ecochard (1), S. Ducastelle (1) (1)Hôpital Edouard Herriot (Lyon, FR); (2)Biostatistics HCL (LYON, FR); (3)Genzyme France (St Germain en Laye, FR)

This study concerned 130 Multiple Myeloma (MM) patients who underwent ASCT in our center between years 2000 and 2007. There were 79 Males and 51 Females with a median age of 56.8 years (34-72). At diagnosis there were $71 \mathrm{lgG}(49 \mathrm{k}$ 22L), $26 \lg \mathrm{A}(15 \mathrm{~K}, 11 \mathrm{~L}), 2 \mathrm{lgD}(1 \mathrm{~K}, 1 \mathrm{~L}), 27$ light chain $(18 \mathrm{~K}$, 9L), 2 plasma cell leukemia and 2 nonsecretory. There were 11 patients in stage I (10A and 1B), 12 IIA, 96 III (75A and 21B) and 11 not classified. At diagnosis, 24 patients had del (13), and 65 had high levels of Beta2 microglobulin. The median interval between diagnosis and ASCT was 7.8 months (3.5-131). Before transplantation, all patients received G-CSF 5 microg/ $\mathrm{kg} /$ day, PBSC were mobilized in steady state in 135 cases, 62 after G-CSF + cyclo. As conditioning, all pts received Melphalan alone. Sixty six patients received a single ASCT and 64 patients received 2 ASCT in a double ASCT program. After transplantation, there were 2 graft failure, $40 \%$ of patients received red blood cell transfusions, and $64 \%$ received platelet transfusions. The median number of days with neutrophils $<0.5 \mathrm{G} / \mathrm{L}$ was 6 $(0-33)$ and with platelets $<20 \mathrm{G} / \mathrm{L}$ was $17(2-104)$. The median length of hospitalization for auto transplantation was 18 days (14-54). To assess the impact of the infused CD34 + cells number, we have analyzed 2 groups: group $1(n=86)$ for ASCT with a number of CD34 $+\leq 3 \times 10^{6} / \mathrm{kg}$ and group $2(n=107)$ for

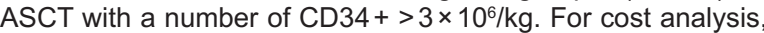
data were collected regarding the mobilisation and harvest of PBSC, and the graft period until hospital discharge.

Results: We found a high significant impact of the high number of infused CD34 + (group 2) on platelets recovery $(P=0.002)$, a trend for the high number of infused CD34 + cells (group 2) on leukocyte recovery O.R. $=0.748[0.5-1.0](P=0.0568)$ and a high significant impact of the same group on neutrophils recovery O.R. $=0.670[0.5-0.9] \quad(P=0.009)$. The multivariate analysis using Cox model showed a significant impact only of poor prognostic factors on overall survival O.R. $=7.94[1.0-$ 59.2] $(P=0.04)$ and also on progression free survival (PFS) O.R. $=2.55[1.1-5.7](P=0.024)$

Conclusion: High level of infused CD34 + appeared to be very optimal for hematological recovery after ATSC in MM, without any significant impact on O.S. and PFS. Our study showed that a high number of CD34 + cells number had an important economical impact including a total cost saving of 1500 euros $(P=0.03)$.

\section{P562}

New strategy for allogeneic peripheral blood stem cell transplantation after reduced-intensity conditioning in multiple myeloma: IFM2005-03 prospective study

M. Michallet (1), M. Sobh (1), M. Mohty (2), A. Sirvent (3), O. Reman (4), J. Cornillon (5), J. El Cheikh (6), A. Praire (1), R. Tabrizi (7), D. Blaise (6), N. Milpied (7), J.L. Harousseau (2), P. Moreau (2)

(1)Hôpital Edouard Herriot (Lyon, FR); (2)CHU de Nantes (Nantes, FR); (3)Hôpital de l'Archet (Nice, FR); (4)CHU de Caen (Caen, FR); (5)ICL (Saint Priest en Jarez, FR); (6)Institut Paoli Calmettes (Marseille, FR); (7)CHU de Bordeaux (Bordeaux, FR)

This is a prospective multicenter study for multiple myeloma (MM) patients (pts) of age $\leq 65$ years receiving RIC followed by allo-PBSCT after achieving at least a PR to auto-HSCT in first line. Pts previously received either VAD or VD as induction treatment followed by Melphalan $200 \mathrm{mg} / \mathrm{m}^{2}$. Pts must have an HLA identical donor either from siblings or unrelated 10/10 HLA donors, and at least one of the following poor prognostic factors (PPF): beta2 microglobulin $>3 \mathrm{mg} / \mathrm{L}$, Del $13, \mathrm{t}(4 ; 14)$ and $\mathrm{Del} 17 \mathrm{p}$. The conditioning regimen combined Fludarabine $30 \mathrm{mg} / \mathrm{m}^{2} / \mathrm{d}(\mathrm{d}-5, \mathrm{~d}-1)$, Busilvex IV $3.2 \mathrm{mg} / \mathrm{kg} / \mathrm{d}(\mathrm{d}-4, \mathrm{~d}-3)$ and ATG $2,5 \mathrm{mg} / \mathrm{kg} / \mathrm{d}(\mathrm{d}-2, \mathrm{~d}-1)$. By day 90 post-allograft, pts not in CR received 4 cycles of Velcade $1.3 \mathrm{mg} / \mathrm{kg}$, and after Velcade, if the CR was not achieved, increasing doses of donor lymphocyte infusions (DLI) were administered. This analysis included 11 pts, 9 males and 2 females of median age 46 years [40-60], there were $8 \mathrm{lgG}$ stageA (7kappa \& 1 Lambda) and 3 IgA (1 kappa stage B \& 2 lambda stage A). There were 3 pts with 1 PPF, 5 pts with 2 PPF and 3 pts with 3 PPF.

Results: Seven pts received 4 cycles of VAD (4 pts $\mathrm{VAD}+\mathrm{DCEP}$ ), 1 patient received 4 cycles of Velcade + dex. and 3 pts received other combinations (1PAD, 2VAD then Velcade). After induction, 7 pts were in PR and 4 in stable 
disease. Pts received auto-HSCT after a median time of 6.6 months [4.5-8.7] from diagnosis. All pts were in PR after auto-HSCT and before allo-PBSCT. The median number of infused CD34 + cells was $6.5 \times 10^{6} / \mathrm{kg}$ [2.6-13.7] from 4 identical siblings and 7 matched unrelated donors. Sex matching was: F..M: 4, F..F:1, M..F: 1 and M..M: 5. At D90, 3 pts were in $C R$ and 8 pts received Velcade after absence of $C R$ (2 VGPR and 6 PR). After Velcade, the 2 VGPR evolved to $\mathrm{CR}$ and pts in PR became $1 \mathrm{CR}, 1 \mathrm{VGPR}$ and 4 remained in $P R)$. One patient in VGPR and 3 in PR received DLI after Velcade, responses were: 1 VGPR and 3 pts progressed. There were 6 acute GVHD (5 grade II and 1 grade III) and 6 chronic GVHD (5 limited and 1 extensive), all GVHD were resolved at the last follow-up. After a median follow-up of 30 months, all pts are alive, 3 pts are in CR, 3 in VGPR, 4 in PR and one in relapse without any active chronic GVHD even after DLI administration.

Conclusion: According to these very promising results, we should reconsider the allo-HSCT as a first line treatment for MM especially for pts with PPF using either RIC or standard conditioning depending on age.

\section{P563}

The graft-versus-myeloma effect using non-myeloablative or reduced-intensity allogeneic haematopoietic stem cell transplantation

O. Ringden, J. Barrett, S. Shrestha, G. Tunes da Silva, M.-J. Zhang, A. Dispenzieri, M. Remberger, R. Kamble, C. Freytes, R.P. Gale, J. Gibson, V. Gupta, L. Holmberg, H. Lazarus, P. McCarthy, K. Meehan, H. Schouten, G.A. Milone, S. Lonial, P.N. Hari on behalf of the Center for International Blood and Marrow Transplantation Research (CIBMTR)

Following myeloablative conditioning due to high treatmentrelated mortality (TRM), some studies have shown an inferior outcome using allogeneic haematopoietic stem cell transplantation (HSCT) compared to autologous transplant. More recently, non-myeloablative (NMA) and reduced intensity conditioning (RIC) for allogeneic HSCT was introduced. A prerequisite for this approach is a significant graft-versus-myeloma effect, which has not clearly been demonstrated.

Between 1997 and 2005, 177 patients were reported to the CIBMTR following NMA $(n=120)$ or RIC $(n=57)$ and an allogeneic HSCT from an HLA-identical sibling donor. Median age was 50 years (range 24-69). Planned tandem autologous transplant followed by allogeneic HSCT was given to 105 of these patients. Most patients were given peripheral blood stem cells $(98 \%)$. Outcomes, with a median follow-up of 55 months (range 3-98) and 25 months (range 3-76) respectively for allogeneic HSCT and autologous transplant followed by allogeneic HSCT, see Table.

The following variables were significant in univariate outcomes analyses and were therefore used in the multivariate modelling: age, sex, performance status, IgG vs. non IgG myeloma, disease status and chemosensitivity, prior lines of chemotherapy, donor-recipient sex match, NMA vs. RIC, year of transplant and GVHD as the time dependent covariate. The only factor on multivariate analysis that increased the risk of TRM was acute GVHD (RR 2.38, $P=0.018$ ). Only chronic GVHD decreased the probability of relapse on multivariate (RR $0.43, P=0.012$ ), but this effect was not seen in patients with IgG myeloma $(n=97$, RR 0.7, $P=0.3$ ) in comparison to all other types of myeloma $(\mathrm{n}=80, \mathrm{RR} 0.11, P=0.004)$. Improved PFS was associated with autologous +allogeneic HSCT (RR 3.6, $P=0.001)$ and absence of acute GVHD $(P=0.001)$, but not chronic GVHD (RR 0.9, $P=0.7$ ).

In conclusion, patients receiving allogeneic HCT for myeloma, chronic GVHD decreased the probability of relapse, but only in patients with non-lgG myeloma. PFS was improved in patients receiving autologous + allogeneic HCT and was decreased in those with acute GVHD.

\begin{tabular}{|l|c|c|}
\hline Outcomes & Allo only & Auto + allo \\
\hline Number of patients & 72 & 105 \\
\hline Acute GVHD at 100 deys, grades (1-4) & $47(37-60) \%$ & $37(28-46) \%$ \\
\hline Chronic GVHD at 3 years & $55(43-67) \%$ & $58(43-72) \%$ \\
\hline Treatment Related Mortality (TRM) at 3 years & $27(17-38) \%$ & $16(10-25) \%$ \\
\hline Relapse at 3 years & $48(36-60) \%$ & $41(29-54) \%$ \\
\hline Progression-free survival (PFS) at 3 years & $25(15-37) \%$ & $42(20-43) \%$ \\
\hline Overall Survival at 3 years & $45(33-58) \%$ & $64(53-75) \%$ \\
\hline
\end{tabular}

\section{P564}

Allogeneic haematopoietic stem cell transplantation with reduced-intensity conditioning in patients with refractory and relapsing multiple myeloma: long-term follow-up

A. Shimoni (1), I. Hardan (1), F. Ayuk (2), G. Schilling (2), D. Atanackovic (2), W. Zeller (2), N. Shem-Tov (1), A. Rand (1), R. Yerushalmi (1), A.R. Zander (2), N. Kröger (2), A. Nagler (1) (1)Chaim Sheba Medical Center (Tel-Hashomer, IL); (2)University Hospital Hamburg (Hamburg, DE)

Allogeneic stem cell transplantation (SCT) with myeloablative conditioning is potentially curative therapy for multiple myeloma (MM) but is associated with excessively high rates of nonrelapse mortality (NRM). Reduced-intensity conditioning (RIC) allows reduction of NRM but relapse rate is increased. The role and timing of allogeneic SCT during the disease course are controversial. There is only limited data on the long-term outcome of RIC in the relapsing/ refractory setting. We retrospective analyzed SCT outcomes in 50 patients (pts) given RIC for relapsing/ refractory MM between the years 2000-2004 from related $(n=27)$ or unrelated $(n=23)$ donors. The median age was 53 years (32-64). This was a relatively heavily pretreated group, a median of 3 years from diagnosis $(0.5-14)$. Fortyseven pts failed one $(n=31)$ or two $(n=16)$ prior autologous SCT. Thirty pts were in PR $(n=26)$ or CR $(n=4)$ at the time of SCT and 20 pts had stable or progressive disease. RIC consisted of fludarabine and melphalan $\left(100-140 \mathrm{mg} / \mathrm{m}^{2}\right)$. Disease response was assessed at day $+100 ; 23$ pts achieved CR; 17 PR, 7 died and 3 have already progressed by day +100 . With median follow-up of 6.4 years (5-7.9 years), 16 pts are alive and 34 have died; 13 had NRM (cumulative incidence $26 \%$ ) and 21 died of relapse. The median survival is 2.3 years and the estimated 7-year overall and progression-free survival (PFS) rates were 34\% (95 C.I. $21-47 \%$ ) and 26\% (95 C.I. 14-38\%), respectively. The PFS curve showed an apparent plateau after 3 years, with no later relapses, suggesting potential cure. In multivariate analysis, adverse prognostic factors for survival included SCT not in remission, long duration of disease and SCT from a female donor to a male recipient. Related and unrelated donor SCT had similar outcome. The 7 year PFS in 19 pts with none of these adverse factors was $47 \%$. Chronic GVHD and achievement of CR after SCT were associated with improved outcome. In conclusion, allogeneic SCT can result in long-term PFS in a subset of MM pts failing prior therapy and should be considered early after failure and preferably after achieving a response with salvage therapy. The treatment goal is to achieve CR as this is associated with better outcome. Relapsing disease is still the major cause of treatment failure. Additional strategies, such as maintenance therapy with novel agents or judicious use of donor lymphocyte infusions merit further investigation for converting PR to CR and reducing relapse risk. 
P565

A phase II study of sequential velcade/thalidomide/ dexamethasone as maintenance therapy post single autologous peripheral stem cell in patients with multiple myeloma

F. Sahebi

City of Hope Medical Center (Duarte, US)

We performed a phase II study investigating the role of sequential velcade/thalidomide/dexamethasone (VTD) as maintenance therapy post single PSCT. We examined the toxicities, CR rate, PFS and overall survival. 4-8 weeks after autologous PSCT using melphalan $200 \mathrm{mg} / \mathrm{m}^{2}$, pts received weekly vel at $1.3 \mathrm{mg} /$ $\mathrm{m}^{2} / \mathrm{wk} \times 3$ and dex at $40 \mathrm{mg} / \mathrm{d} \times 4 \mathrm{~d}$ for 6 months followed by thal at $50-200 \mathrm{mg} / \mathrm{d}$ and dex at $40 \mathrm{mg} / \mathrm{d} \times 4 \mathrm{~d}$ for 6 more months. Thalidomide was continued till progression. Thirty-six pts have been enrolled. Median age is 55 years (29-66). Median time from $\mathrm{dx}$ is $7.5 \mathrm{mo}$. (4.2-145). Disease stage: Salmon-Durie $1 / I I / I I \mid 1 / 6 / 28$ and ISS I/II/III 16/11/7. Induction treatment included thal/dex (14), velcade based (19) and revlimid based regimens (9). Median B2M at enrollment is $1.7 \mathrm{mg} / \mathrm{L}(1.05-1090)$. Disease status at enrollment; CR (9), VGPR (10), PR (14), SD (1). Four pts had ch 13 del.

Results: Thirty-three pts have undergone transplant. Four pts were unable to start maintenance therapy due to grade II neuro toxicities and thrombocytopenia. Twenty-four pts started maintenance vel/dex within 4-8 weeks of PSCT. Eleven pts have completed 6 months of vel/dex. Two pts stopped vel/dex because of low WBC (1) or PN (1). With a median F/U of $5.2 \mathrm{mo}$. (1.2-17.3) nine of 27 evaluable pts (33\%) have achieved CR post PSCT and six out of 11 evaluable pts (55\%) have achieved CR after vel/dex. Two out of $11 \mathrm{pts}(18 \%)$ have upgraded their response with vel/dex. Four pts have completed 6 month of thal/dex and are beyond 1 year post PSCT. Two out of 4 pts remain in $\mathrm{CR}$ at one year post PSCT. One pt is in PR and $1 \mathrm{pt}$ has progressed. Two pts could not complete thal/dex phase of therapy because of relapse (1) and grade III GI toxicity (1). Three pts have relapsed of whom 2 died of relapsed myeloma (leptomeningeal disease $1 \mathrm{pt})$. One pt experienced $\mathrm{Ml}$ while receiving velcade (grade IV). Grade III toxicities have occurred in $7 \mathrm{pts}$; low platelet (1), asthenia (1), mood alteration (1), ileus (1), skin rash (1). Ten pts had PN grade I at enrollment. Only 3 pts have developed PN on the study and all are grade I-II.

Conclusion: Prolonged sequential velcade/thalidomide/dexamethasone maintenance therapy post single autologous PSCT is well tolerated with no severe peripheral neuropathy. Fifty-five percent of pts have achieved CR and $18 \%$ have upgraded their response after six months of velcade/dexamethasone therapy suggesting this is an active and well tolerated maintenance strategy post PSCT.

\section{P566}

Surface molecule CD229 represents a target for the treatment of multiple myeloma

D. Atanackovic, J. Panse, Y. Hildebrandt, Y. Cao, N. Lajmi, A.R. Zander, T. Luetkens, C. Bokemeyer, N. Kröger University Medical Center Hamburg-Eppendorf (Hamburg, DE)

Background: Due to the persistence of marrow-residing myeloma stem cells, most patients with multiple myeloma (MM) will eventually relapse and succumb to their disease even after intensive therapy. Therefore, treatment strategies incorporating new therapeutic targets which are expressed by end-stage myeloma cells and their dormant progenitors are needed.

Methods: We screened myeloma cell lines for the presence of immunoreceptors and verified expression of potential targets on cell lines and patient samples. Protein function was evaluated using gene knockdown and usefulness as target for antibody therapies was investigated using in vitro cytotoxicity assays. Results: Of all immunoreceptors analyzed, SLAM family member CD229 showed the strongest expression and was also expressed on myeloma precursors and on primary plasma cells from myeloma patients while it was absent from most healthy human tissues. Analyse of CD229 expression facilitated flow cytometric detection of myeloma cells within bone marrow. CD229 seemed to promote survival of myeloma cells while CD229 silencing increased their susceptibility towards chemotherapy. Importantly, targeting CD229 with a monoclonal antibody resulted in lysis of the tumor cells through antibody-dependent cellular cytotoxicity as well as complement-dependent cytotoxicity.

Conclusions: Taken together, these results indicate that CD229 might represent an attractive diagnostic and therapeutic target for the treatment of MM.

\section{P567}

The outcome of high-dose chemotherapy and autologous stem cell transplantation in patients with multiple myeloma: a UK and European bench-marking comparative analysis

G. Cook, G. Jackson, K. Kirkland, J. Lee, R. Pearce, D. Marks, A. Pagliuca on behalf of the BSBMT

Introduction: High dose chemotherapy supported with autologous stem cell transplantation (ASCT) in multiple myeloma (MM) is standard first line consolidative therapy. We performed a national outcome analysis in years 1999 and 2005 in order to assess the potential impact of improvements in supportive care and induction therapy.

Patients and methods: Patients with MM who underwent a single ASCT in 1999 and 2005 were identified from the BSBMT $(n=211$ and $n=453)$ and EBMT $(n=1311$ and $n=1978)$ database as eligible for this analysis. All patient and disease-related variables were collected using available MED A data.

Results: In the BSBMT cohort, a trend to more CRs post-ASCT were reported in 1999 compared with 2005 (53\% vs. 46\%, $P=0.14$ ) though an improved conversion to CR post-ASCT (34\% vs. $24 \%$, $P=0.013)$, especially PR CR $(47 \%$ vs. $34 \%, P=0.016)$ was noted. With a median follow-up of 8.7 years (range $0.7,9.9$ years) and 3 (0.1, 4 years) for those transplanted in 1999 and 2005, the 4-year relapse rate (RR) was significantly higher in 2005 compared with $1999(78 \%(\mathrm{Cl} 67,85)$ vs. $53 \%(\mathrm{Cl} 46,60), P=0.0003)$ associated with a significantly shorter time-to-next treatment $(P=0.025)$. The 4-year PFS was significantly lower in 2005 compared with $1999(16 \%(\mathrm{Cl} 9,26)$ vs. $36 \%(\mathrm{Cl} 29,43) ; P=0.0012)$, with year of ASCT $(P=0.001)$ and status at ASCT $(P=0.02)$ being independently significant in a multivariate analysis. The 4-year OS was similar between the year cohorts (1999: $57 \%(\mathrm{Cl} 49,63)$ vs. 2005 : $61 \%(\mathrm{Cl} 53,68), P=0.266)$. In the "benchmarking" analysis, in the EBMT dataset a significant increase in the RR in 2005 compared with $1999(39 \% \mathrm{Cl} 35,40$ vs. $87 \% \mathrm{Cl} 84,90 ; P=0.0001)$ was seen, similar to the BSBMT dataset. When compared collectively, the EBMT 1999 demonstrated the best PFS, though no year of transplant effect could be demonstrated on the 4 year OS rates in the EBMT 1999 and 2005 cohorts $(62 \%$ Cl 58,65 vs. $57 \% \mathrm{Cl} 50$, $63 ; P=0.760)$ or between the BSBMT dataset.

Conclusion: An improvement in supportive care resulting in reduced NRM is evident between the decades, both in the UK and EU. The main cause of treatment failure was disease progression, more significantly in 2005 than 1999. The similarity of OS between the years reflects the introduction of novel agents in salvage therapy. The reduced PFS in 2005 is as yet unexplained and warrants further investigation.

\section{P568}

KIR genotype is associated with progression-free and overall survival following autologous stem cell transplantation for multiple myeloma

I. Gabriel, R. Sargent, H. De Lavallade, R Szydlo, J. Apperley, A. Khoder, A. Alsuliman, D. Marin, L. Foroni, J. Davis, C. Giles, E. Kanfer, A. Rahemtulla, K. Rezvani Imperial College NHS Trust (London, UK)

Myeloma is incurable with median survival of 3-4 yrs. All patients relapse after autologous stem cell transplant (ASCT), 
with a median event-free (EFS) and overall survival (OS) of 2.5-4 and 4-5 yrs respectively. Allogeneic SCT can be curative due to graft-v-myeloma effect but applicability is limited by high transplant related mortality. Identification of independent biological predictors of outcome is required to tailor therapy. Natural killer cells provide defence against tumors and kill allogeneic and autologous myeloma cells in vitro. We investigated the impact of KIR genotype on EFS and OS after ASCT. On univariate analysis of 190 patients post-ASCT factors associated with shorter EFS were haplotype $\mathrm{Bx}(P=0.036)$, $\geq 3$ activating KIRs $(P=0.046)$ and presence of activating KIR genes 2DS1 and 3DS1 $(P=0.045$ and 0.01). Disease status at ASCT was the most predictive factor for EFS. In good risk patients (CR/PR at ASCT) KIR3DS1 + status was associated with significantly shorter EFS, 464d vs. $731 \mathrm{~d}(P=0.003)$ and OS $807 d$ vs. $967(P=0.023)$ but not in poor risk (refractory or relapsed) patients $(P=0.36)$. Median EFS for KIR3DS1+ good risk patients was not significantly different to those with poor risk disease $(P=0.061)$. Patients were stratified by KIR3DS1 and disease status into 3 groups; A: Good Risk KIR3DS1 -ve; B: Good Risk KIRDS1 + ve; and C: Poor risk (KIR3DS1 + or $-\mathrm{ve}), \mathrm{RR}$ of relapse or death was 1.0, $1.9(P=0.002)$ and 3.0 $(P=0.0001)$ respectively. Adjusting for cytogenetics, serum albumin and beta $2 \mathrm{~m}$, this association remained highly significant for poor EFS, RR of 1.0, $2.7(P=0.021)$ and $5.3(P<0.0001)$ respectively. The association between KIR3DS1 and EFS was most significant in patients without HLA-Bw4, the ligand for the corresponding inhibitory KIR3DL1. KIR3DS1 + 3DL1 + Bw4-ve patients had significantly shorter median EFS of 400d vs. 615 $(P=0.048)$. Again this was most striking in good risk patients: 3DS1 + DL1 + Bw4- patients had significantly shorter EFS survival of $372 d$ cf. 3DS1 + 3DL1+Bw4 + patients (509d) and KIR3DS1-ve patients (793d) $(P=0.004)$. Our data suggest KIR3DS1, a gene linked to increased risk of progression to invasive cervical carcinoma, independently predicts for poor EFS and OS post ASCT. Of those patients defined as good risk, $30 \%$ are KIR3DS1 + ve and their EFS is not significantly different from those with refractory/relapsed disease, this is augmented by absence of HLA-Bw4. The mechanisms for our findings are unclear and functional studies are underway.

\section{P569}

Impact of low-dose thalidomide as maintenance therapy in advanced multiple myeloma patients following high-dose therapy

A. Andreini, G. Ruggeri, M. Sorio, C. Tecchio, F. Frattini, S. Ledro, C. Perbellini, D. de Sabata, G. Quaresmini, F. Benedetti Bone Marrow Transplant Unit (Verona, IT)

Introduction: The standard therapy in pts with Myeloma aged $<65$ years was, in the last years, VAD or VAD-like regimens followed by high dose Cyclophosphamide (Cy) and Alkeran (L-PAM) with support of peripheral stem cells (PBSC). Thalidomide (Thali) has showed considerable activity, but Its efficacy as maintenance therapy is still under investigation.

Patients and methods: We considered a conventional CT (3 cycles of VAD or VAD-like regimen), high dose Cy $\left(7 \mathrm{~g} / \mathrm{m}^{2}\right.$ i.v.) and PBSC harvest, followed by single (55 pts) or tandem (71 pts, when disease still present) high dose L-PAM $(200 \mathrm{mg} /$ $\mathrm{m}^{2}$ i.v.) with rescue of PBSC (CD34+>4 $\left.\times 10^{6} / \mathrm{Kg}\right)$. All pts were in stage II/III, according to Durie and Salmon. From October 1999 Thali (100 mg/die) was given to all pts regardless the response and discontinued at the time of relapse or progression or because of toxicities. No anti-thrombotic prophylaxis was administered. Between October 1990 and February 2008, 135 consecutive pts were treated, 129 valuable, median age 55 (33-66), male/female 68/61, M component IgG/lgA/lgD/monoclonal light chain and non-secretory MM were 74/30/6/15 and 3 , respectively. All pts completed induction and high dose $\mathrm{CT}$. One toxic death occurred. Thali (100 mg/day) was started within 3 months from transplant in 79 pts; in 35 cases Thali could not be administered because of refusal (4), progression disease
(9), allergic reactions (3) or neurological toxicities (6); 16/35 pts were enrolled before 1999, when Thali was not available yet. Results: At transplant, 26/129 (20\%) were in very good partial remission or better (VGPR + CR according to EBMT criteria), further 43 pts $(33 \%)$ reached the CR after the first transplant, for a total of $69 / 129 \mathrm{CR}(53 \%)$. We compared the number of responses $(\mathrm{VGPR}+\mathrm{CR})$, the treatment-free interval $(\mathrm{TFI})$ and overall survival (OS) between the Thali group of pts (70 pts) and No-Thali group of pts (35 pts). The VGPR + CR rate was $69 \%(48 / 70)$ in the Thali-group versus $45 \%(16 / 35)$ in the remaining pts. With Thali we obtained further $6 \mathrm{CR}+\mathrm{VGPR}$. Comparing the 2 groups, the TFI was 47 and 22 months $(P=0,002)$, the OS was 70 and 57 months, respectively $(P=\mathrm{ns})$. No thrombotic events occurred. Conclusions: Low dose Thali following single or tandem autotransplant appears to be a safe and feasible treatment not only as maintenance, but also as consolidation therapy, improving the rate of response (VGPR $+\mathrm{CR}$ ), delaying the need of subsequent therapy, without improving the OS, with an acceptable toxicity.

\section{P570}

First-line treatment with bortezomib, doxorubicine and dexamethasone followed by autologous peripheral blood stem cell transplantation in newly diagnosed patients with multiple myeloma

W. Zinke-Cerwenka (1), T. Stojakovic (1), A. Rohn (1), I. Ziegler

(2), W. Emberger (1), S. Sipurzynski (1), W. Linkesch (1), P. Neumeister (1)

(1)Medical University (Graz, AT); (2)TU (Graz, AT)

Background: Induction chemotherapy followed by HDT and autologous peripheral stem cell transplantation is considered standard of care in eligible patients with multiple myeloma. One attempt to improve outcome is to use more effective induction regimens in order to improve overall response rates.

Patients and methods: We analyzed the proteasome inhibitor bortezomib combined with doxorubicine and dexamethasone (PAD) as first line treatment followed by ASCT in 39 newly diagnosed myeloma patients (M/F: 20/19; median age: 59, range 37-74; IgG, IgA, Light chain, others: 24/6/4/5; kappa/lambda: 24/15).

Results: After 4 cycles of PAD the overall response rate was $74.4 \%$ (CR/near CR 35.9\%). All but 2 patients underwent successful stem cell mobilisation with a mean of $8.8 \times 10^{6} \mathrm{CD} 34+\mathrm{cells} / \mathrm{kg}$. In 33 evaluable patients, the overall response rate 3 months after ASCT was $81.8 \%$ (CR/nCR 69.7\%). ASCT after induction therapy with PAD was able to improve the overall response rate from $74 \%$ to $82 \%$ and the nCR rate from $28 \%$ to $58 \%$. After a median followup of 28 months, the OS and PFS for the entire group was $88 \%$ and $69 \%$, respectively. Median OS has not been reached and the median PFS is 32 months. Cytogenetic poor prognosis parameters did not significantly influence survival endpoints. Achievement of $\mathrm{CR} / \mathrm{nCR}$ was associated with a longer PFS.

Conclusion: We conclude that PAD followed by ASCT is an effective first line treatment in newly diagnosed patients with multiple myeloma.

\section{P571}

Autologous haematopoietic stem cell transplantation in multiple myeloma patients with renal insufficiency A single-centre experience

M. Krawczyk Kulis, S. Kyrcz Krzemien, T. Czerw, M. Zielinska Silesian Medical University (Katowice, PL)

Multiple myeloma (MM) might be a cause of the renal insufficiency, often present at diagnosis. Patients (pts) with severe renal failure are generally excluded from high dose therapy even though they display a poor prognosis with conventional chemotherapy. The goal of this study was to evaluate the eligibility and efficacy of autologous hematopoietic cell transplantation (AHCT) in MM pts with concomitant renal insufficiency. In 1998-2009, 236 MM pts were treated in our clinic with high-dose chemotherapy followed by AHCT. $8 \%$ of them were also diagnosed with renal impairment. 
Patients and methods: 20 pts (8f, $12 \mathrm{~m}$, age 40-65 y) were enrolled. Chronic kidney disease (CKD) stage at MM dgn was 2-5 (median $(m)=3$ ). One was on chronic hemodialysis, 2 required plasmapheresis. Before AHCT pts were treated mainly with VAD and CTD.

Mobilization: Hi-dose cyclophosphamide in 8 and IVE (ifosphamide + etoposide + epirubicin) in 12 pts + GCSF. Stem cell collection yield was effective in all pts $\left(m=17.6(1.9-44.7) \times 10^{6}\right.$ CD34 + cells $/ \mathrm{kg}$ ). Disease stage at AHSCT: CR $n=6, V G P R$ $\mathrm{n}=2, \mathrm{PR} \mathrm{n}=12$.

Results: Renal function measured before AHCT significantly improved due to $\mathrm{MM}$ treatment compared to that at dgn, $P=0.008$. CKD stage before AHCT equaled $1-4(m=2)$. Conditioning-melphalan $\left(75-200 \mathrm{mg} / \mathrm{m}^{2}\right)$ was well tolerated. The median of transplanted cells were: NC $2.5(1-7.6) \times 10^{8} / \mathrm{kg}$; CD34+7.9 $(0.8-21.7) \times 10^{6} / \mathrm{kg}$. All patients engrafted. Median regeneration time of granulocytes up to $>0.5 \mathrm{G} / \mathrm{l}$ and of platelets to $>50 \mathrm{G} / \mathrm{l}$ equaled $14(12-19)$ and 14 (12-101) days, respectively. TRM at day 100 was $0 \%$. Regeneration time, hospital stay, days of intravenous antibiotics or antifungal drugs administration and number of transfusion were comparable to pts. transplanted without renal impairment. No renal complications were observed. On the contrary creatinine clearance after transplantation showed trend towards further improvement compared to that before AHCT, $P=0.07$. The probability of OS,PFS for studied group of pts. were $84 \%$ and $63 \%$, respectively. Median observation time 2,5 years $(0,1-12,5)$.

Conclusions: Our observation demonstrates that AHCT is an effective and well tolerated option for MM patients with mild or moderate renal insufficiency. Treatment before transplantation and also high-dose chemotherapy followed by AHCT may even improve renal function. No relationship between CKD and stem cell collection yield, engraftment or severity of post-transplant complications was observed in this group of patients.

\section{P572}

Long-term outcomes of single/tandem autologous

haematopoietic progenitor cell transplantation in

high-risk de novo multiple myeloma patients:

a retrospective analysis

M. Martino (1), R. Fedele (1), G. Console (1), G. Messina (1), G. Irrera (1), V. Callea (2), E. Piro (3), C. Musolino (4), M. Brugiatelli (5), S. Molica (6), A. Abbadessa (7), F. Morabito (8), P. lacopino (1)

(1)U.O. Ematologia con Trapianto (Reggio Calabria, IT); (2)U.O. Ematologia (Reggio Calabria, IT); (3)U.O. Ematologia (Catanzaro, IT); (4)U.O. Ematologia, Policlinico Universitario (Messina, IT); (5)U.O. Ematologia, Azienda Osp "Papardo" (Messina, IT); (6)U.O. Oncologia (Catanzaro, IT); (7)U.O. Ematologia (Caserta, IT); (8)U.O. Ematologia (Cosenza, IT)

High-dose melphalan (HDM) followed by autologous hematopoitic progenitor cell (HPC) support is widely used as intensification treatment in patients with multiple myeloma (MM) responsive to the initial chemotherapy. However, there is growing evidence that only the subset of patients who achieve complete remission (CR) actually benefit from this approach. In our bone marrow unit, in the last 12 years, 152 patients with DurieSalmon stage II-III MM, were intended to undergo two sequential cycles of myeloablative chemotherapy with autologous hemopoietic stem cells (HPC) transplantation after response to primary chemotherapy. Treatment program based on 3 cycles VAD followed by Cyclophosphamide 3-4 gr/mq for HPC collection. The preparative regimen was high-dose melphalan (HDM) $200 \mathrm{mg} / \mathrm{mq}$ for the first and second transplant with a three-four months interval. 63 patients uderwant a single HDM and 89 a tandem HDM. The median follow up is 34 months (range 1-136). The overall survival (OS) and progression-free survival was 51 and 38 months, respectively. PFS and OS at 8 years was $32 \%$ and $45 \%$, respectively. On an intent-to-treat basis, the OS and PFS was significantly higher in patients that underwant a tandem transplant $(P=0.0014$ and $P=0.0001$, respectively) and with a better response (compete remission/near complete remission) after the first transplant $(P=0.002, P=0.02$, respectively). The Cox analysis confirmed a significant association among the best response and clinical results. Our retrospective analysis show that that $\mathrm{CR} / \mathrm{nCR}$ achievement is crucial for longlasting response and prolonged survival after HDM and transplant. The use of the second HDM in the patients in CR/nCR remains of doubtful utility.

\section{P573}

An abnormal non-hyperdiploid karyotype predicts for an adverse outcome after high-dose therapy with autologous stem cell transplanatation in multiple myeloma

D. Tan, Y. Loh, J. Lee, X. Zhang, C. Diong, Y. Lee, G. Kam, A. Ang, R. Yiu, Y. Linn, M. Koh, Y.T. Goh, W. Hwang

Singapore General Hospital (Singapore, SG)

Introduction: Multiple myeloma (MM) is clinically heterogeneous and risk stratification is vital for prognostication. We evaluate the pre-transplant characteristics and the eventual post-transplant response on the survival outcomes of patients (pts) who underwent high dose therapy with autologous stem cell transplantation (HDT/ASCT) at our centre. We sought to identify important prognostic factors that may predict clinical outcomes in the era of novel therapies.

Methods: Seventy-four newly diagnosed MM pts were enrolled to our HDT/ASCT programme from 2000 to 2008. Patient and disease characteristics including age, cytogenetics, international staging system (ISS), induction therapy, bortezomib exposure and the eventual post-transplant response were evaluated. Pts received VAD chemotherapy and thalidomide/ dexamethasone (TD) as induction before and after June 2004 respectively. Bortezomib was available for treatment of relapsed MM and induction treatment of high-risk MM from Oct 2004. After induction, all pts received cyclophosphamide $4 \mathrm{~g} / \mathrm{m}^{2}$ as mobilization followed by peripheral blood stem-cell (PBSC) collection prompted by G-CSF. HDT/ASCT entailed conditioning with melphalan $200 \mathrm{mg} / \mathrm{m}^{2}$ within 1.5 years of diagnosis. Response was defined according to IMWG criteria.

Results: The median age of all pts was 53 years. 30\%, 53\% and $17 \%$ of patients presented with ISS stages I, II and III respectively. Metaphase cytogenetics detected abnormalities in $51 \%$ of pts (hypodiploidy [13\%], hyperdiploidy [25\%], pseudodiploidy [6\%] and near tetradiploidy [3\%]). Interphase FISH results were available for 32 pts, of which $25 \%$ showed highrisk disease. $60 \%$ of pts received VAD induction, while $23 \%$ and $17 \%$ received TD and bortezomib-based induction respectively. Overall, $45 \%$ of pts have been exposed to bortezomib. The ISS and metaphase cytogenetics were discerning of the overall survival (OS) and progression free survival where the medians were 8.7 and 3.5 years respectively. Attainment of at least a very good partial response ( $\geq$ VGPR) was associated with a trend for longer OS and PFS. On Cox regression multivariate analysis, the presence of a non-hyperdiploid karyotype emerged as the single most adverse prognostic indicator after HDT/ASCT (median OS 2.8 years, $P=0.001$ ).

Conclusion: Our study suggests that while HDT/ASCT and achievement of $\geq$ VGPR may prolong the OS of transplant eligible pts, it is still unable to overcome adverse cytogenetics detected on conventional metaphase karyotyping.

\section{P574}

Prognostic value of serum-free light chain monitoring in patients receiving autologous stem cell transplantation Z.N. Özkurt, R. Haznedar, Z. Aki, M. Yagci, G.T. Sucak Gazi University Medical School (Ankara, TR)

Objectives: Autologous stem cell transplantation (ASCT) improves complete response (CR) rates, prolongs overall survival (OS) and progression free survival (PFS) in patients with multiple myeloma (MM). There are several prognostic factors 
used in estimating the clinical course. Free light chain (FLC) level and FLC ratio have been showed as new prognostic markers in newly diagnosed MM patients. However the significance of FLC is unknown yet at pre ASCT setting in MM patients. We hypothesized that pre ASCT FLC levels and FLC ratios may be helpful in predicting transplantation outcome.

Methods: We evaluated $51 \mathrm{ASCT}$ in $47 \mathrm{MM}$ patients [ 31 male and 16 female, median age 54 (28-68) years]. Serum levels of kappa and lambda FLC were measured by the Freelite Binding system at pre-ASCT. Subgroup analysis was performed after excluding patients $(n=13)$ who were in CR both pre-ASCT and at day +90 post-ASCT were excluded, the remaining patients were divided into two groups according to disease status at day +90. Group I $(n=18)$ had improved disease compared to pre-transplantation status and group II $(n=20)$ consisted of patients without any improvement or with progression at day +90 . Patients were followed after ASCT at a median 14.9 (3.0-48.2) months.

Results: OS and PFS were calculated as $\% 76.3$ and $\% 30.4$, respectively. High levels of FLC before ASCT was found to be associated with shorter PFS (\%17.4 vs. $\% 83.3, P<0.05)$ in patients with $\mathrm{CR}$, however without any relation to OS $(P>0.05)$ in all patients. PFS was better in patients in group I than group II (\%54.3 vs. \%8.7, $P<0.05)$. Among patients with normal preASCT FLC ratio, significantly higher number of patients were in group I at day $+90(P<0.05)$.

Conclusion: FLC level and FLC ratio measured prior to ASCT might help to predict PFS and the disease status at day +90 in MM patients providing a more rapid determination of response to ASCT. In the light of these data treatment modalities such as post transplantation maintenance with new generation antimyeloma agents and/or a tandem autologous or allogeneic transplantation might be considered earlier in patients who seem to in the less favourable subgroup with respect to pretransplantation light chain status.

\section{P575}

Impact of thalidomide-based induction regimens on PBSC harvest in multiple myeloma patients

M. Lanska, V. Maisnar, J. Radocha, P. Zak, A. Zavrelova, M. Blaha, J. Horacek, K. Doskocilova, J. Maly

Teaching Hospital (Hradec Králové, CZ)

Thalidomide-containing regimens are currently being used as standard initial therapy for patients (pts) with multiple myeloma. Recent clinical trials have indicated that Thalidomide - based combinations are associated with improvement of response and survival in newly diagnosed pts. The subsequent successful autologous stem cell transplantation (ASCT) is depended on sufficient harvest of peripheral blood stem cells (PBSC). The aim of this report is to compare the PBSC harvest after two different induction regimens: Thalidomide - based regimen - cyclophosphamide, thalidomide and dexamethasone (CTD) versus Adriamycine - based regimens (VAD or AD).

Patients and methods: We retrospectively analysed data of 42 pts with newly diagnosed multiple myeloma eligible for ASCT. 21 pts (median age 59, range 33-68 years) were treated with CTD and 21 pts (median age 58, range 48-65) with VAD or AD. Cyclophosphamide $2,5 \mathrm{~g} / \mathrm{m}^{2}$ plus G-CSF was used as mobilisation regimen in both groups. We evaluated total number of $C D$ $34+$ cells $/ \mathrm{kg}$, number of apheresis days, number of pts who achieved target number of $5 \times 10^{6}$ CD $34+$ cells $/ \mathrm{kg}$.

Results: We analysed 23 mobilisation cycles in CTD group (2 pts were mobilized twice because of insufficient first harvest), 21 cycles in VAD group. Average number of apheresis days was 3,82 (median $2-5$ ) versus 2,1 days ( $1-5$ days). Total number of $C D$ $34+$ cells $/ \mathrm{kg}$ in CTD group was 5,74 × $10^{6}$ CD $34+$ cells $/ \mathrm{kg}(0,99$ $15,9)$ vs. $9,24 \times 106 \mathrm{CD} 34+$ cell/kg in VAD group $(0,26-29,12)$, average number of CD $34+$ cells per one apheresis was $1,5(0,08-$ $9,55)$ vs. $4,21(0,04-17,87)$ CD $34+$ cells $/ \mathrm{kg}$. In CTD group there were more aheresis days needed to reach target number of $C D$ $34+$ cells $/ \mathrm{kg}-82 \%$ pts needed 3 and more apheresis in contrast to second group - only $28 \%$ pts needed 3 and more apheresis days. Target number of CD $34+$ cells $/ \mathrm{kg}\left(5 \times 10^{6}\right)$ was achieved in $61 \%$ of pts in CTD group and 90,4\% of pts in VAD group. 6 pts in CTD group achieved less than $2 \times 10^{6}$ CD $34+$ cells $/ \mathrm{kg}$ vs. only 1 pt in VAD group. 3 pts from CTD group were satisfactory mobilized after subsequent mobilisation regimen with plerixafor.

Conclusion: These results suggest that adding Thalidomide could influence the results of PBSC harvest before ASCT, but we analysed only small group of patiens, so that these observations should be tested in appropriately designed trial with larger patient cohort.

\section{P576}

Relationship between CD34 + cell dose and early immunological recovery in patients with non-Hodgkin's lymphoma and multiple myeloma undergoing autologous peripheral blood stem cell transplantation: a prospective study

T. Moscato, R. Fedele, E. Massara, G. Messina, G. Console, G. Irrera, M. Martino, M. Cuzzola, E. Spiniello, A. Dattola, C. Garreffa, P. lacopino

A. O. Bianchi-Melacrino-Morelli (Reggio Calabria, IT)

There is a growing body of evidence suggesting that early lymphocyte reconstitution following autologous (ASCT) is a good independent prognostic indicator of clinical outcomes for NonHodgkin Lymphoma (NHL) and Multiple Myeloma (MM). In particular, Porrata et al. (Biol Blood Marrow Transpl 2008), confirmed in a prospective cohort study the role of day 15 absolute lymphocyte count (ALC-15) on survival and identified NK cells as the key ALC-15 lymphocyte subset affecting survival after ASCT. Several strategies to enhance early immune reconstitution have been developed in preclinical models but few have made it into clinical trials. In this study, we prospectively evaluated the hypothesis that ALC-15 and absolute NK cell count at day 15 (NK-15) is directly dependent upon the dose of infused CD34 + in the peripheral blood autograft. The study primary end-points were to achieve ALC-15>500 cell/ $/ \mathrm{l}$ and NK-15 > 80 cell/ $\mu \mathrm{l}$ in NHL or MM patients (pts) underwent PBPC autografting with the intent to receive $>6 \times 10^{6} \mathrm{CD} 34+/ \mathrm{Kg}$. The target CD34 + dose was achieved in 13 of $23 \mathrm{NHL}$ and in 12 of $26 \mathrm{MM}$ pts. For data analysis, the pts were divided into four groups, two for each disease, according to the target dose of CD34 + cells (median number of cells infused was: 7.4 and 5.4 in LNH; 7.2 and 5 in MM). Five of $12(42 \%)$ MM pts reinfused with $>6 \times 10^{6} /$ CD34 + cells/Kg achieved the end-point for ALC-15, while 5 pts $(42 \%)$ obtained the end-point for NK-15. Of 14 MM receiving lower than the target dose of CD34 + cells, 6 (43\%) and 6 pts $(43 \%)$ achieved the first and second end-point, respectively. Of $13 \mathrm{LNH}$ pts infused with CD34+ cells $>6 \times 10^{6} / \mathrm{Kg}, 5(38 \%)$ obtained the end-point for ALC-15 and $5(38 \%)$ achieved the NK-15 end-point. Significantly, only one patient achieved the first and only one the second end-point among the pts who received an amount of $\mathrm{CD} 34+$ cells less than the target.

In conclusion, we found that dose of CD34+ cells had no impact on early immunological recovery in MM. However, it is important to note that our $\mathrm{MM}$ pts received in any case more than $4 \times 10^{6} / \mathrm{CD} 34+$ cells $/ \mathrm{Kg}$. On the contrary, our data suggest that immunological recovery in early post-transplant is directly dependent upon the dose of infused CD34 + cells in NHL pts.

\begin{tabular}{|c|c|c|c|c|}
\hline & N. of pts & 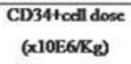 & $\begin{array}{l}\text { End point } \\
\text { ALC15 }\end{array}$ & $\begin{array}{l}\text { End point } \\
\text { NK-15 }\end{array}$ \\
\hline LNH & 23 & $\begin{array}{l}13 p t s>6 \\
10 \text { pes }<6\end{array}$ & $\begin{array}{l}38 \% \text { pts } \\
10 \% \text { pts }\end{array}$ & $\begin{array}{l}38 \% \text { pts } \\
10 \% \text { pts }\end{array}$ \\
\hline MM & 26 & $\begin{array}{l}12 y+s \geq 6 \\
14 y+s<6\end{array}$ & $\begin{array}{l}42 \% \text { pts } \\
43 \% \text { pts }\end{array}$ & $\begin{array}{l}42 \% \text { pts } \\
42 \% \text { pts }\end{array}$ \\
\hline
\end{tabular}


P577

Comparison of 3 different conditioning regimens for a second, salvage high-dose-therapy and autologous peripheral blood progenitor cell transplant in patients with relapsed and refractory multiple myeloma

R. Fenk, V. Liese, I. Bruns, M. Kondakci, S. Balleisen, C. Saure, T. Schroeder, R. Haas, G. Kobbe

Heinrich Heine University Dusseldorf (Dusseldorf, DE)

There is some controversy about the number of transplants to be utilized during first line therapy of MM. For patients who relapse after the 1st HD-T a 2nd transplant may be a valuable treatment option. There is no data whether HD-M still is the best conditioning regimen for the salvage situation. We therefore retrospectively compared toxicity and efficacy of 3 different conditioning regimens in patients with relapsed or refractory MM.

From 1997 to 2007 we treated fifty-five patients with relapsed or refractory MM with a salvage HD-T followed by aPBPCT. All patients had previously received HD-M and aPBPCT as 1 st line therapy. In addition $69 \%$ of patients had received thalidomide and $27 \%$ bortezomib. Time from diagnosis to second HD-T was 45 months, median number of prior treatment lines was 2 (range 1-6) and age was 56 years (median, range 42-69). Forty-three percent of patients were refractory to their last treatment before second HD-T, whereas $57 \%$ relapsed with previous remission duration of 21 months (range 0-71). A re-induction treatment was attempted before 2nd HD-T and included chemotherapy, bortezomib and IMID-based regimens in $56 \%, 40 \%$ and $22 \%$. Conditioning regimens for salvage HD-T were classical HD-M (Mel200, 27\%), HD-BuMel (Bu12Mel140, 38\%) and bortezomib-HD-M (Bortezomib $1,3 \mathrm{mg} / \mathrm{m}^{2}$ with $\left.\mathrm{Mel} 200,35 \%\right)$.

Toxicity of the 3 conditioning regimens was acceptable. TRM was $5 \%$ with all deaths occuring with standard Mel200. Responses were: CR 9\%, vgPR 9\%, PR, 57\%, MR + SD 11\%, PD $4 \%$, not assessable $5 \%$. No significant differences were noted in terms of response, EFS or OS according to the type of conditioning regimen used. Univariate analysis revealed a long remission duration after the 1st HD-T, platelet count $>140 \times 10 \mathrm{E} 9 / \mathrm{I}$ and CR/PR after 2 nd HD-T as favourable prognostic factors for EFS and OS, as well as age >60years for OS. In multivariate analysis remission duration shorter than 12 months after 1st HD-T was the only adverse prognostic factor for EFS (0.002) and OS (0.004). Subgroup analysis identified 3 patient groups with different long term prognosis based on the remission duration after the 1st HD-T (Table 1). Salvage HD-T is an effective treatment for patients who had remission duration $>1$ year after 1st HD-T. BuMel and BorMel are alternative conditioning regimens yielding similar results like HD-Mel in a retrospective single centre analysis. Prospective studies are needed to identify the best regimen for salvage $\mathrm{HD}-\mathrm{T}$ in relapsed and refractory MM.

\begin{tabular}{|l|c|c|c|c|}
\hline $\begin{array}{l}\text { Remission after } \\
1^{\text {st }} \text { HD-T: }\end{array}$ & $\begin{array}{c}1-12 \\
\text { months }\end{array}$ & $\begin{array}{c}13-24 \\
\text { months }\end{array}$ & $\begin{array}{c}>25 \\
\text { months }\end{array}$ & $p$ \\
\hline & & & & \\
\hline EFS & 4 & 15 & 15 & $<0.001$ \\
\hline OS & 7 & 41 & 78 & $<0.001$ \\
\hline & & & & \\
\hline
\end{tabular}

\section{P578}

The different peripheral blood stem cell mobilization methods in patients with multiple myeloma

M. Cioch, M. Wach, D. Jawniak, J. Manko, W. Legiec, A. Walter-Croneck, M. Legiec, A. Dmoszynska

Medical University of Lublin (Lublin, PL)

Introduction: The autologous haematopoietic stem cell transplantation is a standard therapy for patients (pts) with multiple myeloma (MM). The most frequent mobilization regimen in these pts is cyclophosphamide with G-CSF (CY). In 10-20\% pts with initial mobilization failure different remobilization schemes are applied: etoposide + G-CSF (ETO), cytarabine + G-CSF (Ara-C) or plerixafor $+\mathrm{G}-\mathrm{CSF}$.

Patients and methods: In our Department CY $\left(4 \mathrm{~g} / \mathrm{m}^{2}\right)$ is a standard regimen for first-line mobilization in MM pts. ETO $\left(1.6 \mathrm{~g} / \mathrm{m}^{2}\right)$ and Ara-C $\left(2.4 \mathrm{~g} / \mathrm{m}^{2}\right)$ are applied in remobilization and in first-line of mobilization in heavily pretreated pts (> than 2 line of chemotherapy). Pts with refractory, active disease are collected after EDAP regimen. The dose of G-CSF is $5-10 \mathrm{mcg} / \mathrm{kg}$.

Results: We analyzed clinical and laboratory data of 80 pts (48 female and 32 male; mean age 54 years) with MM who underwent 108 haematopoietic stem cell mobilization during last 5 years. Before mobilization pts received median number 2.4 of lines chemotherapy (range: $1-5$ ), $17.5 \%$ of them radiotherapy, $58.3 \%$ thalidomide and $21.2 \%$ bortezomibe therapy. There were $80(74 \%)$ first mobilization (CY 50, ETO 24, EDAP 6) and 28 $(26 \%)$ remobilization (ETO 24, Ara-C 4 ) in pts who not achieved $2 \times 10^{6} / \mathrm{kg} \mathrm{CD} 34+$ cells after CY regimen and in pts with relapse after transplantation. The median CD34 + yield in the whole group was $9.2 \times 10^{6} / \mathrm{kg}$ (range: $0.6-30.0$ ) in a median of 1.4 (range: $1-4)$ aphereses. There was no difference in median CD34 + yield after CY, ETO, Ara-C and EDAP $\left(8.1 ; 10.2 ; 9.9\right.$ and $10.5 \times 10^{6} / \mathrm{kg}$, respectively). The failure rate after $\mathrm{CY}$ was $4 \%$ (2 pts), after ETO in first-line 0\%, in remobilization 4\% (1 pt), after Ara-C 25\% (1 pt) and after EDAP $0 \%$. Finally, only $2 / 80$ pts $(2.5 \%)$ not achieved sufficient number of stem cells from all collections. Haematologic toxicity after $\mathrm{CY}$, ETO and EDAP regimens was moderate; the most toxic scheme was Ara-C. We did not show the significant influence of previous radiotherapy, number of chemotherapy lines, previous treatment with thalidomide and bortezomibe, and remission status on CD34 + cell collection yield. The analysis of post-transplantation engraftment showed no difference in leucocyte recovery and delayed platelet reconstitution after ETO mobilization compared to CY mobilization $(P<0.05)$, but the ETO group received more lines of previous chemotherapy.

Our data showed that qualification to an appropriate mobilization regimen in $\mathrm{MM}$ pts might reduce failure rate to $2.5 \%$.

\section{P579}

Treatment of multiple myeloma with autologous stem cell transplantation: a long-term observation

M. Wach, M. Cioch, D. Jawniak, A. Brzozowski, T. Gromek, M. Kosicka, W. Legiec, M. Legiec, M. Malek, J. Manko, A. Walter-Croneck, E. Wasik-Szczepanek, A. Dmoszynska Medical University of Lublin (Lublin, PL)

Patients with multiple myeloma (MM) treated with conventional chemotherapy have a median survival of approximately 3 years. High dose chemotherapy followed by autologous peripheral stem cell transplantation (ASCT) is now considered as the standard therapy for almost all patients with multiple myeloma.

From November 1997 to October 2006, 122 patients with MM (58 females, 64 males, median age 51.0 (range 30-66) years were transplanted in our Department. 47 patients were in CR or in nCR, 66 patients were in PR, 9 was in SD. Among them there were 95 patients with IgG myeloma, 16 with IgA myeloma, 1 with IgG/lgA, 1 with IgM myeloma, 5 with non secretory type, 2 with solitary tumor and 2 with light-chain myeloma. According to Durie-Salmon 62 patients were in stage III of the disease, 46 in stage II and 4 in stage I. 48 patients were transplanted after one line chemotherapy, whereas 69 patients were transplanted after 2 or more 
lines, 1 patient after surgery and rtg-therapy and 4 patients without prior therapy. In mobilization procedure the patients received a single infusion of cyclophosphamide $\left(4-6 \mathrm{~g} / \mathrm{m}^{2}\right)$ or etoposide 1.6 $\mathrm{g} / \mathrm{m}^{2}$ followed by daily administration of G-CSF until the peripheral stem cells harvest. Median of apheresis were 2.0 (1-5). A median of $7.09 \times 10^{6} \mathrm{CD} 34+$ cells $/ \mathrm{kg}$ were collected $\left(1.8-111.0 \times 10^{6}\right)$. Conditioning regimen consisted of high dose melphalan $60-210 \mathrm{mg} / \mathrm{m}^{2}$. A median of $3.04 \times 106 \mathrm{CD} 34+$ cells $/ \mathrm{kg}$ was transplanted to patient. Fatal complications occurred in 4 patients (treatment related mortality TRM $=3.2 \%$ ). In all patients regeneration of hematopoiesis was observed. The median time to recovery of ANC $>0,5 \mathrm{G} / \mathrm{l}$ was $13(10-38)$ days and PLT $>50$ $\mathrm{G} / \mathrm{l}$ was 25 (12-45) days. Median time of hospitalization was $22(14-50)$ days.

Patients were evaluated on day 100 after ASCT. $74.9 \%$ of them obtained $\mathrm{CR}$ and $\mathrm{nCR}, 14.3 \%$ were in $\mathrm{PR}, 5.4 \%$ of them had SD and $5.4 \%$ had progressed.

Median of OS was 45 months. OS at 3-year was $84 \%$, at 7 -year was $59 \%$. Median of PFS was 25 months. PFS at 3 -year was $68 \%$, at 7 -year was $43 \%$. At present $52(42 \%)$ patients are still alive. High-dose chemotherapy followed by autologous stem cell transplantation is valuable, well tolerated method of treatment for patients with MM who can obtain long-lasting survival.

\section{P580}

Multiple myeloma evolution of survival in patients with autologous bone marrow transplant according to the induction therapy

S. Yantorno, M. V. Prates, J.J. Napal, A. Enrico, J. Milone

Hospital Italiano de La Plata (La Plata, AR)

Objective: To analize retrospectively the evolution of patients (pts) with MM who underwent ABMT, comparing the results of those who received pretransplant treatment with VAD vs. thalidomide - dexamethasone (THAL - Dexa).

Materials and methods: Between January 1991 and August 2009, 180 pts were admitted in our center with MM diagnose. In the 146 evaluated pts, 83 underwent AMBT whereas 63 received treatment without transplant. High doses of melphalan $\left(200 \mathrm{mg} / \mathrm{m}^{2}\right)$ were used as conditioning scheme. The median age of the pts with ABMT was 53 years old (r: 27-68). The first 47 pts transplanted received VAD as induction and the following $15 \mathrm{THAL}-$ Dexa. The features of the pts were similar in both groups median age 51,8 years ( $r$ : 27-66) in the VAD group vs. 52. 9 (r: 31-68) in the THAL - Dexa group; male:female 1,2:1 vs. $1.4: 1$. Stage III A/B $78 \%$ vs. $71 \%$.

Results: After the induction treatment the (GR) global response (CR + VGPR + PR) was $59.9 \%$ with VAD vs. $53 \%$ with THAL - dexa; CR $12.7 \%$ vs. $13.3 \%$. After ABMT the GR and CR was $83 \%$ and $38.3 \%$ with VAD vs. $80 \%$ and $46.6 \%$ with THAL - Dexa respectively.

The overall survival (OS) after 5 years is better in transplant group vs. no transplant group $54 \%$ vs. $42 \%(P=0,05)$.

The transplanted pts who underwent induction with VAD present and OS of 5 years similar to those received THAL - Dexa $(53 \%$ vs. $50 \%, P=0,65)$.

Conclusions: The pts who received high doses melphalan + ABMT showed a greater survival in comparison who didn't received. In our experience THAL - Dexa in induction has not modified the response rates or the evolution post $A B M T$ in comparation with VAD.

\section{P581}

Impact of bortezomib on outcome of autologous stem cell transplant in multiple myeloma: single-centre analysis M. Anguita Arance, P. González Sierra, F. Almagro Torres, M.J. Martinez Quesada, J.A. López López, S. Duran Nieto, A. Alcalá Muñoz

Complejo Hospitalario de Jaén (Jaén, ES)

Introduction: Autologous stem cell transplantation (ASCT) is a procedure usually performed, in patients less than 65 years with multiple myeloma (MM), as consolidation after conventional chemotherapy. This procedure offers good rates of responses and relapse free survival. Objective: Evaluate the results obtained in our center in terms of transplant related mortality (TRM), relapse free survival (RFS) and overall survival (OS) in a group of patients diagnosed of multiple myeloma in which ASCT was performed.

Material and methods: Since the start of our transplant activity nine years ago, ASCT was performed in 23 patients diagnosed of MM,14 men $(60,9 \%)$ and 9 women $(39,1 \%)$, with a median of age of 56 years (rank 41-65). The first line therapy consisted of alternating VBCMP/VBAD chemotherapy in $56.52 \%$ of the patients, and the rest of patients with VAD and Bortezomib. The conditioning regimen was BUMEL in 7 patient $(30,4 \%)$ and the remainder with MEL200 $(69,6 \%)$. The source of stem cells was always of peripheral blood, employing only granulocyte stimulating factor. After the hematopoietic recovery, a therapy of maintenance with interferon and/or prednisone was initiated.

Results: TRM was about $8 \%$ (in 2 of the 23 patients). The causes were septic shock and acute respiratory distress syndrome. We didn't detect statistically significant differences of the TRM due to conditioning regimen $(P=0.52)$. In $60 \%$ of the patients the engraftment was in day +10 of the procedure. Mucositis was the main adverse event in $31 \%$ of the cases. The median of survival was 35 months (rank 13-58). The median of RFS was of 49 months (rank 29-69). At the end of this study $52,2 \%$ of the patients continue alive, with a RFS of the $43.06 \%$. When the patients are stratified by time of the transplant (before or after the appearance of new agents), we found statistically significant differences in the OS $(18 \%$ vs. $83 \% ; P=0,04)$ although this differences couldn't be demonstrated in terms of $\operatorname{RFS}(P=0,1)$. Stimated data related to $1 \mathrm{yr}$ and $3 y$ r of the procedure show us, OS $81 \%$ and $42 \%$; RFS $75 \%$ and $43 \%$, respectively.

Conclusion: ASCT in the course of myeloma has demonstrating a RFS and OS advantage, as we can see in this study.Our results suggest that in the context of ASCT, the use of the new agents, as Bortezomib, in MM is more complementary than exclusive one of another. They would be able to be considered during the induction and/or ASCT to improve the efficacy of this procedure because tend to improve natural history of MM.

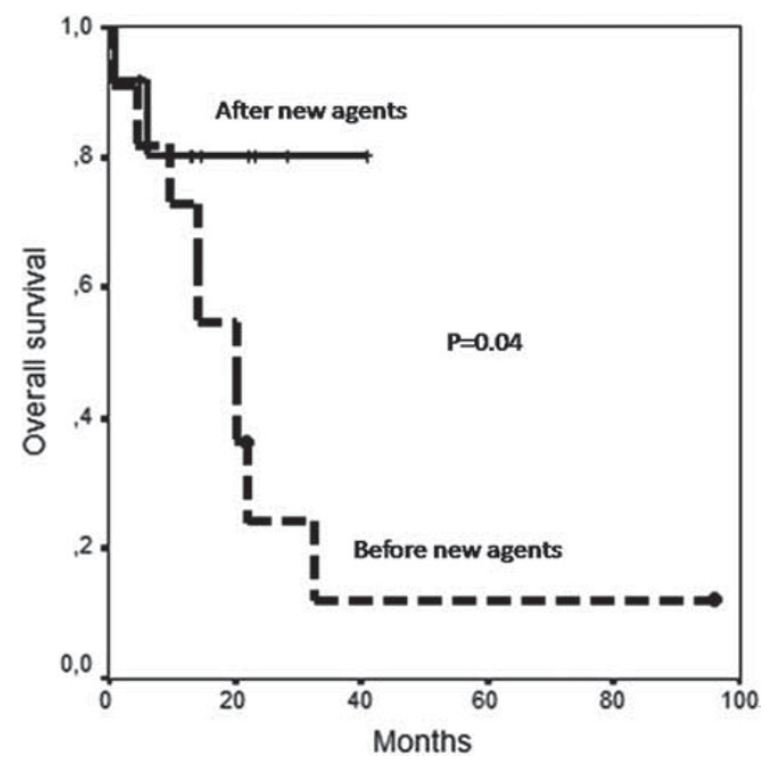




\section{Stem cell source}

P582

CD34 + cell dose is different at different steps of HPC processing and affects engraftment kinetics of autologous HPC transplantation: an analysis of 203 transplantations A. Humpe (1), S. Hengstenberg (1), J. Hedderich (2), U. BuwittBeckmann (1), A. Günther (1), M. Gramatzki (1)

(1)Division of Stem Cell and Immunotherapy (Kiel, DE); (2)Institute of Medical Informatics and Statistics (Kiel, DE)

Objectives: A decisive parameter for durable engraftment after autologous transplantation of hematopoietic progenitor cells (HPC) is the number of CD34 + cells transfused. Based on measurement of CD34 + cells in the freshly harvested graft a minimally recommended threshold dose for autologous transplantation of HPC of $2 \times 10 \mathrm{E}+06$ and an optimal dose of $\geq 5 \times 10 \mathrm{E}+06 \mathrm{CD} 34+$ cells $/ \mathrm{kg}$ of body weight were defined. This does not consider cell losses during cryopreservation (cryo). We analyzed CD34 + cell doses measured at different time points of HPC processing and engraftment kinetics.

Methods: Data were analyzed from autologous patients being transplanted at our centre between January 2004 and December 2007. The CD34 + cell dose was determined according to ISHAGE guidelines at time of harvest (MI), after cryo by analysis of a satellite tube (MII) and by multiplication of the value deduced by MI with the measured viability of CD34 + cells after cryo (MIII). Results: A total of 224 autologous transplantations were performed in the time period and complete data were available for 203 transplantations. Graft failures were not observed. Doses of CD34 + cells derived at MII were significantly lower by a median of $16.8 \%$ when compared to $\mathrm{MI}$ or $13.7 \%$ at MIII, respectively. According to $\mathrm{Ml}$, patients were transplanted with a median number of $6.5(1.2-29.4) \times 10 \mathrm{E}+06$ viable CD34 + cells $/ \mathrm{kg}$ with only 4 patients missing the threshold and 60 the optimal dose. Based on MII data, the median number of transfused viable CD34 + cells was $5.5(0.65-26.6) \times 10 \mathrm{E}+06 / \mathrm{kg}$ with an additional 10 patients missing the threshold and an additional 31 patients missing the optimal dose. There were to little cases missing the threshold in MI or MIII for further analysis. Based on MII dosages, engraftment kinetics were significantly better either for patients transplanted with a cell dose above the threshold compared with those missing this value or for patients being transplanted with the optimal dose compared with those missing this number (leukocytes $>1 / \mathrm{nl}$ : day +11 versus +10 ). Comparable data were obtained regarding platelet engraftment.

Conclusion: Measurement of CD34 + cell numbers after cryo gave significantly lower results compared to those samples evaluated immediately after cell harvest. Apart from other influencing factors cell dose measured after cryo may have an effect on engraftment kinetics and should be considered as additional routine quality control before release of HPC products.

\section{P583}

Moscow Stem Cell Bank: experience of cord blood transplantation

T. Shamanskaya (1), T. Astrelina (1), D. Kachanov (2), O. Paina (3), E. Skorobogatova (2), Z. Dishlevaya (2), E. Podkolzina (1), E. Karpova (1), L. Lebedeva (1), M. Yakovleva (1)

(1)Stem Cell Bank (Moscow, RU); (2)Research Centre of Pediatric Hematology (Moscow, RU); (3)St. Peterburg Pavlov State Medical University (St. Petersburg, RU)

Objective: Umbilical cord blood (UCB) bank was established by the Department of Health Care of Moscow in 2003.

Aims: The aim of this study to assess the results of UCB transplantation (UCBT) of UCB units released for the clinical use by UCB bank.

Materials and methods: Based on stringent selection criteria 3950 UCB units have been collected and processed since
2003 till 2009. 29 UCB units have been released for transplantation that makes $1,09 \%$ from all banked units. UCB units were selected for transplantation based on total nucleated cell (TNC) content and HLA match between donor and recipient.

Results: To date, 16 patients have been transplanted. The male-to-female ratio was $7: 1$. The median age was 2,5 years (range 4 months -20 years). 10(62,5\%) patients had malignant hematological disorders, 6(37,5\%) - non-malignant diseases. $2(12,5 \%)$ patients have received HLA-identical related UCBT. $1(6,25 \%)$ patient has received two UCB units from the related and unrelated donor (8 of 10 alleles matched in both units). $13(81,25 \%)$ patients have received unrelated UCBT. $2(12,5 \%)$ patients were fully compatible (10/10), $11(68,75 \%)$ cases had one or two mismatches. 2 patients have undergone haploidentical HSC transplantation with co-transplantation of UCB unit. $6(37,5 \%)$ patients have received one UCB unit, $3(18,75 \%)-2$ UCB units from one donor, $4(25 \%)-2$ units from different donors, and $3(18,75 \%)-3$ samples (2 UCB units from one donor and the third unit from another donor). The median number of TNC and CD34 + cells infused was $6,0 \times 10^{7} / \mathrm{kg}$ (range 0,2 $21,5)$ and $7,7 \times 10^{5} / \mathrm{kg}$ (range $\left.0,5-40\right)$, accordingly. 9(56,25\%) patients showed engraftment. The median time to neutrophil engraftment was 24 days (range 15-36). Partial engraftment was noted in $2(12,5 \%)$ cases, $5(31,25 \%)$ patients showed no sign of engraftment. Acute GVHD grade I-II was observed in $7(43,75 \%)$ patients, there were no cases of severe GVHD. Chronic GVHD was noted in $1(6,25 \%)$ patient. The transplantrelated mortality at 100 days was $28,5 \%$. The overall survival was $56,2 \%$, with a median time of observation of 116 days.

Conclusions: UCBT has shown encouraging results. The increasing number of banked UCB units will allow using UCB more widely as alternative source of HCS for transplantation.

\section{P584}

Comparison of autologous stem cell grafts viability in patients with multiple myeloma and Hodgkin's lymphoma after cryopreservation

E. Ilatovskaya, O. Sukhova, O. Ivanova, E. Babenko, Y. Zalyalov, B. Afanasyev

Pavlov State Medical University (St. Petersburg, RU)

Objectives: High-dose therapy (HDT) with autologous hematopoietic stem cell transplantation (AHSCT) is intended use for treatment of patients with hematology and oncology malignances. In present peripheral blood stem cells (PBSC) is the main source of hematopoietic stem sells (HSC) using for transplantation. The outcome of the stem sell transplantation depends on several factors: obtaining an adequate CD34 + sell dose after the leukapheresis procedure, appropriate cryopreservation and thawing methods.

The aim of this study is to compare CD34 + sell number in fresh and thawed samples of PBSC from patients with multiple myeloma (MM) and Hodgkin lymphoma (HL).

Material and methods: A total 70 samples of PBSC were characterized by measuring CD34 + cells number, viability (7-AAD +) before and after cryopreservation procedure: 17 samples from patients with $\mathrm{HL}, 18$ samples from patients with MM.

For CD34 + cells, 7-AAD + cells enumeration FACSCalibur flow cytometer with CellQuest Pro Software (Becton Dikinson,USA) was used with triple staining protocol, according to ISHAGE (International Society of Hematotherapy and Graft Engineering) guidelines.

Results: Average number of CD34 + in fresh and thawed sample is summarized in Table.

In fresh PBSC samples from patients with MM the CD34+ number was not differ from patients with $\mathrm{HL}(P=0,19)$. However, in thawed PBSC samples the number of CD34 + cells was reliably higher in patients with $\mathrm{MM}$ than in patients with $\mathrm{HL}(P=0,05)$. According to this study cryopreservation of PBSC resulted more loss of CD34 + cells in patients with $\mathrm{HL}$ than with MM: $31,7 \%$ and $21 \%$ respectively. Difference in CD34 + loss is statistically correlated: $P=0,03$. 
Conclusions: PBSC harvested from patients with MM shows more stability during the freeze/thaw procedure.

\begin{tabular}{|c|c|c|c|c|c|}
\hline & \multicolumn{2}{|c|}{ fresh samples } & \multicolumn{2}{|c|}{ thawed samples } & \multirow{2}{*}{$\begin{array}{c}\text { CD34+ } \\
\text { loss } \\
(\%)\end{array}$} \\
\hline & $\begin{array}{c}\text { CD34+ } \\
\times 10^{4} 6 / \mathrm{kg}\end{array}$ & $\begin{array}{c}\text { viability } \\
(\%)\end{array}$ & $\begin{array}{c}\text { CD34+ } \\
\times 10^{\circ} 6 / \mathrm{kg}\end{array}$ & $\begin{array}{c}\text { viability } \\
(\%)\end{array}$ & \\
\hline $\begin{array}{l}\mathrm{HL} \\
\mathrm{n}=17\end{array}$ & $3,54 \pm 2,37$ & 100 & $2,4 \pm 1,64$ & $68,2 \pm 15,5$ & $31,7 \pm 15,5$ \\
\hline $\begin{array}{l}\mathrm{MM} \\
\mathrm{n}=18\end{array}$ & $4,6 \pm 2,95$ & 100 & $3,64 \pm 2,34$ & $79,0 \pm 12,9$ & $21,0 \pm 12,9$ \\
\hline
\end{tabular}

\section{P585}

Clinical outcome of NHS-CBB units: contribution of ethnic minority donors

F. Regan (1), H. Cockburn (1), J. James (1), A.L. Herr (2), L. McKenna (1), I. Ionescu (2), J. Ellis (3), V. Rocha (2), G. Miqueles (1), C. Brown (1), E. Gluckman (2), C.V. Navarrete (1)

(1)NHS Blood and Transplant (London, UK); (2)Eurocord (Paris, FR); (3)NHS Blood and Transplant (Bristol, UK)

Cord blood transplantation (CBT) provides a unique opportunity for treatment for a variety of patients. One of the aims of the NHS Cord Blood Bank (NHS-CBB) when established in 1997 was to maximize the banking of cord blood units (CBUs) from ethnic minority (EM) donors. We present the results of 12 years of banking experience and the clinical outcomes of the units issued for single transplants, with particular reference to those issued to ethnic minority patients.

As of December 31 2008, 235 CBU out of $>10000$ stored CBUs had been issued for transplantation to 230 patients worldwide. Twenty one percent $(n=2208)$ of the banked units have a total nucleated cell (TNC) content of 40-60 $\times 10^{7}$ TNC, $24 \%(n=2510)$ contain $60-80 \times 10^{7}$ TNC and $54 \%(n=5680)$ contain $>80 \times 10^{7}$ TNCs. Regarding the ethnicity, approximately $30 \%$ of the banked units are from EM and 10\% from mixed ethnic (ME) background. A survival analysis was performed on 139 CBUs issued for single CBT: 104 patients with malignant disease (MD) and 35 with nonmalignant disease with available follow-up. Issued units contained a median of $4.3 \times 10^{7} / \mathrm{kg} \mathrm{TNC}, 20 \%$ were from EM donors and $10 \%$ from ME background where rare HLA types are expressed. HLAmatching (HLA-A and -B antigenic, -DRB1 allelic typing) was $6 / 6$ in $23 \%, 5 / 6$ in $46 \%$ and $4 / 6$ in $37 \%$ of patient-CBU pairs. Ethnicity was matched in $65 \%$ of pairs. Median follow up was 35 months. Median time to neutrophil engraftment was 24 days and the cumulative incidence $(\mathrm{Cl})$ of neutrophil engraftment was $80 \%$. Cl of transplantrelated mortality was $32 \%$ at 2 years. Probability of disease-free survival was $48 \%$ at 1 year and $41 \%$ at 2 years in MD. Probability of overall survival (OS) for the 139 single CBTs was $53 \%$ at 2 years ( $71 \%$ for non malignant disease (NMD) and $39 \%$ for MD). Probability of OS was $81 \%$ in patients with no HLA mismatch CBT, $73 \%$ in patients with $1 \mathrm{HLA}$-mismatched CBT and $33 \%$ in patients with $\geq 2$ HLA mismatches ( $p$ unadjusted 0.05 ).

Over $40 \%$ of the CBUs in the NHS-CBB are from EM donors and $17 \%$ of $\mathrm{CBU}$ used in the 139 single CBTs reported here were from EM or ME background. The clinical outcomes obtained in patients transplanted with our units are comparable to published data.

\section{P586}

Unrelated cord blood transplantation after myeloablative conditioning for adult patients with advanced myelodysplastic syndromes

A Sato (1), J. Ooi (1), S. Takahashi (1), N. Tsukada (1), S. Kato (1), T. Kawakita (1), T. Yagyu (1), F. Nagamura (1), A. Tojo (1), S. Asano (2)

(1) Institute of Medical Science (Tokyo, JP); (2)Waseda University (Tokyo, JP)

The prognosis of advanced myelodysplastic syndromes (MDS) is poor. We analyzed the disease specific outcomes of adult patients with advanced MDS treated with cord blood transplantation (CBT) after myeloablative conditioning and identified pretransplant factors related to the transplant outcomes. Between August 1998 and June 2009, 33 adult patients with advanced MDS were treated with unrelated CBT in our institute. The diagnosis MDS was made for all patients according to the WHO classification. The diagnosis at transplantation included refractory anemia with excess blasts (RAEB1/2) $(n=7)$ and MDSrelated secondary acute myeloid leukemia (sAML) $(n=26)$. All patients received four fractionated $12 \mathrm{~Gy}$ TBI and chemotherapy as myeloablative conditioning. All patients received standard cyclosporine and methotrexate as a graft-versus-host disease (GVHD) prophylaxis. The median age was 42 years (range, 19-52 years), the median weight was $55 \mathrm{~kg}$ (range, 41$75 \mathrm{~kg}$ ), the median number of cryopreserved nucleated cells was $2.51 \times 10^{7} / \mathrm{kg}$ (range, $1.71-4.60 \times 10^{7} / \mathrm{kg}$ ), and the median number of cryopreserved CD34 positive cells was $0.91 \times 10^{5} / \mathrm{kg}$ (range, $0.27-2.14 \times 105 / \mathrm{kg}$ ). 30 patients had myeloid reconstitution and the median time to more than $0.5 \times 10^{9} / \mathrm{L}$ absolute neutrophil count was 22 days (range, 18-35 days). The cumulative incidence $(\mathrm{Cl})$ of neutrophil recovery at day 50 was $90.9 \%$. In multivariate analysis, neutrophil recovery was significantly faster among sAML patients $(P=0.04)$. A self-sustained platelet count more than $50 \times 10^{9} / \mathrm{L}$ was achieved in 29 patients at a median time of 51 days (range, 30-179 days). Cl of platelet recovery at day 200 was $87.9 \%$. In multivariate analysis, platelet recovery was significantly faster among CMV seronegative patients $(P<0.001)$. Cl of grade II-IV acute GVHD and extensive type chronic GVHD was $67.4 \%$ and $34.3 \%$, respectively. In multivariate analysis, degree of HLA mismatch had a significant impact on $\mathrm{Cl}$ of grade II-IV acute GVHD $(P=0.021)$. The 5 -year $\mathrm{Cl}$ of transplant related-mortality (TRM) and relapse was $13.9 \%$ and $15.9 \%$, respectively. No factor was associated with the incidence of TRM and relapse. The probability of event-free survival (EFS) at 5 years was $70.2 \%$. No factor was associated with EFS. These results suggest that adult advanced MDS patients without suitable related or unrelated bone marrow donors should be considered as candidates for CBT.

\section{P587}

Long-term ( $\geq 5$ years) follow-up of a randomized study of allogeneic stem cell transplantation with stem cells from blood or bone marrow

D. Heldal, L. Brinch, Y. Fløisand, I. Dybedal, T. Gedde-Dahl, P.A. Holme, G.E. Tjønnfjord, S.A Evensen

Oslo University Hospital (Oslo, NO)

Objectives/Methods: Between 1994 and 1999, 60 consecutive adult pts with haematological malignancies with a family donor were randomised to myeloablative allogeneic transplantation with blood stem cells (BSC) or bone marrow (BM). Engraftment parameters, incidence of acute and chronic graftversus-host disease (cGvHD), transplant related mortality, 5 yrs leukaemia free survival (LFS), relapse rate and overall survival (OS) have been described in previous publications $[1,2]$. With a landmark of $\geq 5$ years, 40 pts were evaluable, 21 in the BSC group and 19 in the BM group. We recorded the incidence of late relapse, LFS, OS, prevalence of cGvHD, persistent immunosuppressive treatment, secondary malignancies and the ability to work at a median of 13 yrs of observation. Survivors as of 11/2009 were interviewed, 18 in the BSC group, 17 in the BM group.

Results: After 5 yrs, there were 2/21 relapses in the BSC group and $3 / 19$ in the BM group. The total number of relapses was $3 / 30$ and $12 / 30$ respectively $(P<0.001)$. However, there was no statistical difference between the groups with regard to LFS and OS after randomisation. At $\geq 5 \mathrm{yrs}, 12 / 21$ in the BSC group and $9 / 19$ in the BM group had developed cGvHD. At last follow up, there were $4 / 10$ in the BSC group and 6/9 in the BM group who experienced complete resolution of cGvHD symptoms, none of the pts with persistent cGvHD received immunosuppressive treatment. 
Three pts in each group developed secondary malignancy (malignant melanoma (1,7 yrs), ca mammae (4,6 and $8 \mathrm{yrs})$, ca lingua (8,7 yrs), ca labialis (7,7 yrs), basalioma (12,4 yrs)). Ability to work was achieved in $19 / 21$ vs. $13 / 19$ cases in the BSC and BM group, respectively $(P<0.08)$.

Conclusion: Higher relapse rates after 5 yrs in pts transplanted with stem cells from bone marrow was seen, no difference between the groups with regard to late relapse. cGvHD remitted in half of the cases. There was a trend towards increased ability to work in the BSC group. No differences between the groups regarding LFS, OS, persistent GvHD, sustained immunosuppressive treatment or secondary malignancies were seen.

References:

1. Heldal, D., et al., A randomised study of allogeneic transplantation with stem cells from blood or bone marrow. Bone Marrow Transplant, 2000, 25(11): p. 1129-36.

2. Heldal, D., et al., Fewer relapses and increased chronic GVHD in patients transplanted with blood stem cells: a 5 -year follow-up in a single centre study. Bone Marrow Transplant, 2003, 32(3): p. 257-64.

\section{P588}

Unrelated cord blood transplantation in patients with haematologic malignancies in Brazil - a Sociedade

Brasileira De Transplantes De Medula Ossea and Eurocord Collaborative Study

C.A. Rodrigues (1), D.C. Setubal (2), C. Bonfim (2), S.K Nabhan

(2), V.A.R Colturato (3), M.A. Mauad (3), M.P. Souza (3), A.M. Azevedo (4), N. Hamerschlak (5), V.C. Ginani (6), A. Seber (6), H.N. Bittencourt (7), M.V. Gonçalves (8), C.G. Castro Jr. (9), F. Dulley (9), C.A. de Souza (10), W. Chaves (11), J. Zanis Neto (2), R. Pasquini (2), E. Gluckman (11), V. Rocha (11), L.F. Bouzas (4)

(1)Hospital Sirio Libanes (Sao Paulo, BR); (2)Federal University of Parana (Curitiba, BR); (3)Amaral Carvalho Foundation (Jaú, BR); (4)Instituto Nacional de Cancer (Rio de Janeiro, BR); (5)Hospital Israelita Albert Einstein (Sao Paulo, BR); (6)Instituto de Oncologia Pediatrica (Sao Paulo, BR); (7)Federal University of Minas Gerais (Belo Horizonte, BR); (8)Federal University of Sao Paulo (Sao Paulo, BR); (9)Hospital de Clinicas de Porto Alegre (Porto Alegre, BR); (9)University of Sao Paulo (Sao Paulo, BR); (10)University of Campinas (Campinas, BR); (11)Eurocord (Paris, FR)

Umbilical cord blood transplantation (UCBT) has extended the availability of allogeneic transplantation to patients without HLA-identical donors, mainly for countries high frequency of rare haplotypes such as Brazil. We evaluated 173 patients who received a single first UCBT in Brazil between with 1993 and 2009 for hematological malignancies from 13 transplant centers. Median age was 8 years (range 1-60) and $58 \%$ were male. Sixty-nine patients $(40 \%)$ had acute lymphoblastic leukemia, $57(33 \%)$ acute myelogenous leukemia, 23 (13\%) myelodysplastic syndrome, $18(10 \%)$ chronic myelogenous leukemia and $6(4 \%)$ chronic lymphoid diseases. Most patients $(55 \%)$ were transplanted in advanced phases of their diseases. Based on antigen-level HLA-A and -B and allele-level HLA-DRB1 typing, 64 patients $(52 \%)$ received a cord blood unit with 2 mismatches, $54(44 \%)$ with one mismatch and $5(4 \%)$ matched units. Most patients $(94 \%)$ received a myeloablative regimen. Median follow-up time of survivors was 30 months. The cumulative incidence $(\mathrm{Cl})$ of neutrophil engraftment at day 60 was $61 \%$, after a median time of 22 days (range 12-55 days) and the $\mathrm{Cl}$ of platelet engraftment at day 60 was $42 \%$, after a median time of 41 days (range 16-125 days). Experience of centers (more than 30 UCBT performed) was the only factor associated with improved engraftment rate $(P=0.05)$. $\mathrm{Cl}$ of grade II-IV acute graft-versus-host disease (GVHD) at 100 days was 19\% and of chronic GVHD only $5 \%$ at 3 years. $\mathrm{Cl}$ of non-relapse related mortality (NRM) was $41 \%$, being higher in patients older than the median age $(51 \%$ vs. $32 \%$ for younger patients, $P=0.05)$, and receiving a CD34 + cell dose lower than $1 \times 10^{5} / \mathrm{kg}(54 \%$ vs. $25 \% P=0.002$ ). At 3 years, the $\mathrm{Cl}$ of relapse or progression was $20 \%$, and the estimated progression-free survival (PFS) was $36 \%$, being decreased in patients with advanced disease status $(28 \%$ vs. $46 \%$ for patients in remission, $P=0.001)$, and receiving a CD34 + cell dose lower than $1 \times 10^{5} / \mathrm{kg}(21 \%$ vs. $42 \%, P=0.04)$. At 3 years, overall survival was $41 \%$. In conclusion, UCBT in Brazil seems to be associated with lower engraftment rates as compared to the published experience from other countries. Nevertheless, use of HLA-mismatched UCBT is a valuable alternative for patients with hematologic malignancies in Brazil who lack an HLA-matched related or unrelated donor. UCB units with a higher CD34 + cell count and transplants performed for patients in remission may improve outcomes.

\section{P589}

On the role of panel-reactive antibodies in cord blood transplantation-a single-centre study

B. Omazic, M. Remberger, J. Mattson, O. Ringden, D. Hauzenberger

Centre for Allogeneic Stem Cell Transplantation (Stockholm, SE)

Objective: We studied the role of panel-reactive anti-HLA antibodies on outcome in patients undergoing haematopoietic stem cell transplantation (HSCT) with cord-blood.

Methods: Serum samples from 42 patients undergoing HSCT with cord-blood as stem cell source were collected during conditioning, prior to HSCT. Screening for panel reactive antibodies using flow cytometry was performed according to the instructions from the manufacturer (Flow-PRA, One Lambda, USA). Results: 9/42 (21\%) patients undergoing HSCT had panelreactive antibodies (PRA) against HLA class I and/or II prior to HSCT. 5/42 (12\%) had PRA against HLA class I, solely, whereas 2/42 (5\%) had PRA against HLA class II. 2/42 (5\%) patients had PRA against HLA class I and II. 6/42 (14\%) patients had PRA $>20 \%$ against HLA class I and/or II. When comparing the incidence of rejection, defined as $100 \%$ recipient in chimerism post-HSCT, no statistical significant differences between the group with PRA prior to HSCT and the group without PRA prior to HSCT could be observed. However, when comparing time to of engraftment, the group with PRA prior to HSCT had significant delayed engraftment as compared to the group without PRA $(P<0,05)$. Furthermore, a trend to delayed engraftment of platelets could be observed in the group with PRA prior to HSCT as compared to the group without PRA.

Conclusion: We conclude that the presence of PRA prior to HSCT doesn't influence the rate of rejection in HSCT with cordblood, however time to engraftment was significantly delayed in this group. One way of overcoming this problem might be to increase the cell dose in patients with preformed HLA antibodies receiving HSCT with cord-blood.

\section{P590}

Carbohydrate-mediated inhibition of ice recrystallization in cryopreserved human umbilical cord blood

L. Wu, J. Tokarew, J. Chaytor, E. von Moos, Y. Li, C. Palii, R. Ben, D. Allan

University of Ottawa (Ottawa, CA)

Background: Cryopreservation of human umbilical cord blood (UCB) for use in hematopoietic stem cell transplantation is expanding rapidly. Most freezing protocols utilize dimethylsulfoxide (DMSO), however, the risk of infusional toxicity and critical reductions in viable stem and progenitor cells remain a concern. Cell yields in UCB units are limited by the collected volume and damage caused during storage. Ice recrystallization (IR) is an important source of cryopreservation injury that occurs as a result of ice reshaping during thawing. Naturallyoccurring carbohydrates have wide-ranging intrinsic ability to inhibit IR related to structural properties such as hydration index. We investigated the impact of carbohydrate IR inhibition (IRI) on total mononuclear cell (MNC) and CD34 + progenitor 
cell viability, levels of apoptosis and in vitro hematopoietic progenitor function in cryopreserved UCB.

Methods: UCB was collected following informed consent. MNCs were isolated by Ficoll-gradient centrifugation and cryopreserved in liquid nitrogen. Samples were cryopreserved in storage media containing specific carbohydrates $(200 \mathrm{mM})$ and compared to 5\% DMSO. Samples were analyzed under conditions of high IR ("slow" thaw) and low IR ("fast" thaw). Cells were analyzed by flow cytometry for viability (exclusion of 7-AAD) and apoptosis (Annexin-V-positive). Hematopoietic progenitor function was examined in colony-forming unit (CFU) assays. IRI of carbohydrate solutions was determined using the "splat" cooling assay.

Results: Greater IRI capacity of carbohydrates correlated with increased yield of viable MNCs $(r 2=0.92, P=0.004)$ and CD34 $(+)$ cells $(r 2=0.96, P=0.019)$ after thawing under conditions of high IR. The correlations were less apparent under conditions of low IR. Carbohydrates with greater IRI modulate the induction of early apoptosis during thawing, especially in CD34 + cells ( $\mathrm{r} 2=0.96, P=0.0001$ ) as compared to total mononuclear cells $(P=0.006)$, and preserve CFU capacity in vitro $(\mathrm{r} 2=0.92, P \leq 0.0001)$.

Conclusion: Our results suggest that inhibition of IR increases the yield of non-apoptotic and functional hematopoietic progenitors following cryopreservation of human UCB. Developing novel synthetic carbohydrates with enhanced IRI properties is a promising strategy to improve cryopreservation of UCB and facilitate more rapid hematopoietic engraftment following transplantation.

\section{P591}

CD34 (+)-selected stem cell boost without further conditioning for delayed or insufficient engraftment after allogeneic stem cell transplantation. Single-centre experience J. Elcheikh, A. Vazquez, P. Ladaique, J.M. Boher, L. Castagna, S. Furst, C. Faucher, N.F. Belmechri, C. Chabannon, D. Blaise Institut Paoli Calmettes (Marseille, FR)

Poor graft function without rejection may occur after stem cell transplantation (SCT). CD34 (+) stem cell boost (SCB) can restore marrow function but may induce or exacerbate GvHD. We therefore investigated the feasibility and efficacy of CD34 $(+)$-selected SCB in some patients with poor graft function. We present the results for 13 patients transplanted at our cancer centre at Marseille, who received a boost of unmanipulated blood cells from the original donor, without further conditioning, 10/3 (M/F), median age 44 years (range 20-69), transplanted initially for Chronic Myeloide Leukaemia (n.3), NHL (n.3), Myelodisplastic Syndrome (n.2), Medullary Aplasia (n.2), and only one patient with Acute Lymphoblastic Leukaemia, Multiple Myeloma and Myelofibrosis. Nine patients had received HLAmatched sibling donor grafts and four patients a matched unrelated grafts, all patients but one received a peripheral blood stem cells (PBSC); The transplant conditioning was myeloablative in 6 patients, and reduced intensity conditioning (RIC) in 7 patients.

The median interval between transplants and the boost were 5.4 months (range 3.9-9.2). After a median follow up of 25 months post-transplant, none had achieved platelet counts $>50 / \mathrm{nL}, 9$ patients $(69 \%)$ had a platelets transfusion dependence and 8 patients $(62 \%)$ was dependent of red blood cells transfusions at (SCB), even though donor chimerism was $>95 \%$ in all patients.

The patients received a median of $3,94 \times 10(6) / \mathrm{kg}$ CD34 (+) cells and $18,57 \times 10(3) / \mathrm{kg} \mathrm{CD} 3(+)$ T lymphocytes.

After SCB 11 patients (85\%) had a leukocyte recuperation $(>2,5$ $\mathrm{G} / \mathrm{L}), 7$ patients $(54 \%)$ and 6 patients $(46 \%)$ had a platelets and red blood cells transfusion independence respectively. After a median follow-up of 13 months (range10-23), five patients remained alive. No patient had developed acute or chronic GvHD after the boost; four patients died of infections, one patient died of disease progression, one patient of hemorrhagic syndrome, and only one patient died because an resistant preexisting digestive GvHD. At one year and two years, post (SCB) of CD $34(+)$, the OS was $80 \%$ IC95 (39-95) and $60 \%$ IC95 $(16-86)$ respectively.

These preliminary results point to the possibility of safely improving graft function using $\mathrm{CD} 34(+)$ positively selected stem cells without necessarily increasing the incidence of GvHD in patients with poor graft function post-SCT. Experience with more patients and longer follow-up should clarify the optimal role for this procedure.

\section{P592}

Developing a predicting score for poor mobilization in lymphoma patients

E. Morello (1), C. Skert (2), C. Almici (1), L. Arcaini (3), L. Baushi (1), B. Botto (4), A. Castelli (5), A. Nosari (6), G. Pica (7), F. Ripamonti (3), F. Salvi (8), A.M. Carella (7), G. Gaidano (5), A. Levis (8), E. Morra (6), D. Russo (2), U. Vitolo (4), G. Rossi (1)

(1)Spedali Civili (Brescia, IT); (2)University of Brescia (Brescia, IT); (3)University of Pavia (Pavia, IT); (4)AOU S. Giovanni Battista (Turin, IT); (5)Avogadro University of Eastern Piedmont (Novara, IT); (6)Niguarda Hospital (Milan, IT); (7)Azienda Ospedaliera Universitaria S. Martino (Genoa, IT); (8)Ospedale Civile (Alessandria, IT)

Background: Autologous stem cell transplantation (ASCT) is a potentially curative treatment for lymphoma but poor mobilization of stem cells remains a major issue in some patients (pts). New agents were recently developed which may improve the rate of SC mobilization.

Aims: To identify a predictive score for poor mobilization on a large series of lymphoma pts candidates to ASCT according to factors influencing SC mobilization outcome as previously reported (Morello, ASH 2009).

Patients and methods: A total of 415 attempts of PBSC collection, consecutively performed at 7 Italian centres in 388 pts affected by lymphoma, were previously analysed.

A collection of less than $2 \times 10^{6}$ CD34 + cells $/ \mathrm{kg}$ was defined as "poor mobilization". The following parameters were analysed for correlation with poor mobilization: lymphoma diagnosis, disease status at mobilization, type of mobilizing chemotherapy, bone marrow infiltration at collection, $\mathrm{n}^{\circ}$ of previous lines of therapy, prior use of fludarabine, alkylating agents or radiotherapy. The number of CD34+cells/ $\mu \mathrm{L}$ and the ratio between circulating CD34 + cells/ $\mu \mathrm{L}$ and total $\mathrm{WBC} /$ $\mu \mathrm{L}$ on the first day of CD34 + count (SCratio) were also analysed, trying to predict mobilization failure ongoing. Multivariate statistical analyses by logistic regression was performed with NCSS 2007

Results: The identified variables at the multivariate analysis affecting poor mobilization were: CD34/ $\mu \mathrm{l}<30,4 \quad(P=0.003)$, SCratio $<0,004 \quad(P<0.001)$, mobilization with cyclophosphamide (CTX) $(P=0.008)$.

Using the coefficients of the variables selected, a scoring system at the first day of PBSC count was calculated for each patient:

SCORE $=\mid 2,81(C D 34 / \mu \mid<30,4=1)+2,15(C D 34 / \mathrm{WBC}<0,004=1)$ $+1,17($ CTX=1) $-6,2$.

Patients were classified according to their score as high (score $\leq 0,07, \mathrm{~N}=34$; if 3 factors were present), intermediate $(0,07<$ score $\leq 1,5, N=116$, if 2 factors but CTX were present), low $(1,5<$ score $\leq 4,8, N=75$, if CTX in combination with an other factor, or CD34 or SCratio alone) and very low (score $>4,8$, $\mathrm{N}=162$, if $\mathrm{CTX}$ alone or no factors were present)-risk patients. Their risk of poor mobilization was $48 \%, 22 \%, 3 \%$, and $0,2 \%$, respectively.

Conclusions: This prognostic score should be confirmed by a validation set, currently under investigation, and thereafter a prospective trial will be implemented in order to rationally explore the use of new mobilizing agents in patients with lymphoma undergoing SC mobilization with chemotherapy and G-CSF. 
P593

Impact of high-resolution HLA typing on the outcome of dual unit umbilical cord blood transplantation

I. Baltadakis, A. Manaka, F. Panitsas, D. Karakasis, F. Michelis, T. Athanassiades, Z. Poulopoulou, M. Garofalaki, E. Bika, M. Papageorgiou, S. Vasileiou, M.E. Karatza, J. Apostolidis, N. Harhalakis, E. Nikiforakis

Evangelismos Hospital (Athens, GR)

Unrelated umbilical cord blood transplantation (UCBT) is usually performed across major HLA disparities and at a limited cell dose, which necessitates infusion of two partially matched UCB units especially in adult patients. Unit selection for UCBT is currently based on antigen level typing for HLA class I, while the impact of allele match remains unknown. We retrospectively examined HLA compatibility at the allele level in a group of patients who underwent double UCBT at our center From $08 / 2006$ to $10 / 2009,22$ patients lacking an adequately matched volunteer donor received dual unit UCBT for high-risk acute leukemia (AML: 16, ALL: 6), at a median age of 37 years (range, 16-60). The conditioning regimen was myeloablative in 17 and reduced-intensity in 5 patients. The median weight of patients was 74 (range, 48-105) $\mathrm{kg}$. Total nucleated cell (NC) and CD34 + cell doses infused per patient were 2.6-6.5 $\times 10^{7} / \mathrm{kg}$ (median, $4.3 \times 10^{7} / \mathrm{kg}$ ) and $0.6-3.1 \times 10^{5} / \mathrm{kg}$ (median, $1.5 \times 10^{5} /$ $\mathrm{kg}$ ), respectively. Original HLA match of the $44 \mathrm{UCB}$ units was $5 / 6(n=8)$ or $4 / 6(n=36)$. Compatibility was demoted by final match in $57 \%$ of units $(5 / 6$ in 2, 4/6 in 21 and $\leq 3 / 6$ in 21 units. Additional high-resolution typing at HLA-C and -DQB1 demonstrated $8 / 10$ match in $2,7 / 10$ in $6,6 / 10$ in 12 , and $\leq 5 / 10$ in 24 units. Engraftment was achieved in all but one patients, with a median time to absolute neutrophil count (ANC) $>500 / \mathrm{uL}$ of 20 (range, 6-48) days. Time to engraftment did not correlate with total $\mathrm{NC}$ or $\mathrm{CD} 34+$ cell dose and with the original or final HLA match of the engrafting unit either. Notably, the predominant unit could not be predicted by cell dose but was significantly associated with a higher final HLA match $(P=0.008$ and 0.0008 for the degree of matching at 6 and 10 alleles, respectively by Wilcoxon signed-rank test). Final HLA match was not observed to affect the incidence of acute or chronic graft-versus-host disease, transplant-related mortality, relapse rate, and disease-free survival. Nevertheless, there was a trend of superior overall survival with $\geq 6 / 10$ allele match of the engrafting unit compared to $<6 / 10$ match ( $P=0.076$ by log-rank test). In conclusion, a certain degree of allelic match might exert a greater influence on the outcome of double unit UCBT in comparison to a higher cell dose. Further studies are needed to define the role of high-resolution HLA typing in cord blood unit selection.

\section{P594}

Transplantation of unmanipulated haploidentical bone marrow and post-grafting cyclophosphamide in high-risk patients with myeloid leukemia - single-centre experience with an alternative salvage strategy

S. Tuve, M. Wermke, U. Platzbecker, C. Heuchel, N. Shayegi, J. Radke, A. Kiani, K. Hölig, G. Ehninger, C. Thiede, J. Schetelig, M. Bornhäuser

University Dresden (Dresden, DE)

Haploidentical transplantation of allogeneic hematopoietic stem cells remains a high-risk intervention associated with relevant therapy-related mortality. Typically, investigators prefer to use T cell depleted or CD34 + selected grafts after intensive conditioning therapy. Only recently, the use of unmodified bone marrow (BM) grafts from haploidentical donors after reduced intensity conditioning and the use of postgrafting pulsed cyclophosphamide (Cy) have been described.

Patients and methods: Between 10/2008 and 06/2009 5 patients with a median age of 57 years received uniform conditioning therapy as published by Luznik et al (Biol Blood Marrow Transplant 2008) with Cy $14.5 \mathrm{mg} / \mathrm{kg}$ day -6 and -5 , fludarabine
$30 \mathrm{mg} / \mathrm{m}^{2}$ day -6 to -2 and 2 Gy TBI on day -1 , followed by the infusion of BM from HLA-mismatched sibling donors. Donors (son for father, daughter for mother) were matched for $5 / 10$ $(n=1), 6 / 10(n=1)$ and $7 / 10(n=3)$ HLA antigens, respectively. To suppress alloreactive donor T cells $50 \mathrm{mg} / \mathrm{kg}$ Cy was infused on day $3(n=3)$ or day 3 and $4(n=2)$ after transplantation. GvHD prophylaxis with mycophenolate mofetil $(3 \times 15 \mathrm{mg} / \mathrm{kg})$ and tacrolimus started $24 \mathrm{~h}$ after Cy infusion. G-CSF was applied from day 4 until engraftment. Four patients suffered from highrisk acute myeloid leukemia (AML) and had either remained in cytopenia after repetitive salvage chemotherapy $(n=3)$ or had experienced molecular relapse of AML $(n=1)$. One patient with AML had failed two previous transplants. Between $1 \%$ and $48 \%$ marrow blasts had been detectable before conditioning therapy was started.

Results: The BM grafts infused contained a median number of $2.3 \times 10^{6} \mathrm{CD} 34+$ cells $/ \mathrm{kg}$ and $1.8 \times 10^{7} \mathrm{CD} 3+\mathrm{T}$ cells $/ \mathrm{kg}$. Neutrophil and platelet engraftment was achieved in all patients after a median time of 17 and 31 days, respectively. Complete donor chimerism was detectable between day 10 and 17 . There was no grade 3-4 extramedullary toxicity. Acute $\mathrm{GvH}$ grade II occurred in only one patient. With a median follow-up of 7 months, all patients are in CR between 5 and 13 months after transplantation. Interestingly, even patients with residual blasts before conditioning remained in $\mathrm{CR}$ arguing for the effectiveness of GvL reactions with this approach.

Conclusion: We could apply immunosuppressive conditioning followed by haploidentical BMT successfully in heavily pretreated patients without excess morbidity and mortality. The low toxicity allows applying the strategy in high-risk leukemia patients.

\section{P595}

Use of biosimilar GCSF for peripheral blood progenitor cell mobilisation prior to autologous stem cell transplantation: a single-centre experience

A. Publicover, DS. Richardson, K. Hill, C. Hurlock, P. Casey, J. Newman, $\mathrm{KH}$. Orchard

Southampton University Hospitals (Southampton, UK)

Mobilisation of peripheral blood haematopoietic progenitor cells (PBPCs) has become a standard procedure in patients prior to an autologous stem cell transplant. In September 2008 a biosimilar GCSF (Ratiograstim) was granted an EU licence for use in mobilisation of PBPCs. Although licensed for PBPC mobilisation little clinical data has been available regarding the effectiveness of the biosimilar in this context. Ratiograstim was incorporated into clinical use for PBPC mobilisation in the Wessex Blood and Marrow Transplantation Programme from November 2008 replacing the previous form of GCSF. An audit of harvests over the periods Jan-Sept 2008 (standard GCSF) and Jan-Sept 2009 (Ratiograstim) was performed comparing the efficiency of mobilisation in the two groups of patients.

Data were collected regarding the maximum CD34 predictor in peripheral blood, total CD34 per kg body weight collected, time from priming to maximal predictor level and number of harvest procedures required per patient.

Methods: Data were retrospectively collected for 29 patients who underwent PBPC harvest at SUHT from Jan-Sept 2009 using the biosimilar GCSF. The 34 patients who underwent the procedure Jan-Sept 2008 were used as the comparator group mobilised with standard GCSF. Doses of GCSF were essentially the same throughout.

Results: The mean age of the 34 patients from 2008 was 53.6 years (SD 12.8). 24 patients had myeloma, the others lymphoproliferative diseases (LPDs). The mean age of the 29 patients from 2009 was 53.2 years (SD 12.9). 16 of these patients had myeloma and the others LPDs. All patients with myeloma were primed with cyclophosphamide and GCSF. The LPD patients received various GCSF containing regimens, including R-ICE, R-IVE, cyclophosphamide and cytarabine. Results were analysed using the paired t-test. 
The time from priming to day of maximum yield is significantly affected by the priming regimen used but was independent of the type of GCSF. Taking only those primed with cyclophosphamide and GCSF $(2008 n=34,2009 n=23)$, the 2008 mean was 10.5 days (SD 2.0) and the 2009 mean was 10.7 days (SD 1.6), $P=0.80$. The patients were harvested for between one and 3 days. The mean number of sessions required was 1.5 for both 2008 and 2009 . 59\% of patients completed their PBPC harvest in one session in both years.

Conclusions: This study has shown no significant difference in any of the key parameters measured for biosimilar GCSF when compared to standard GCSF.

The $\mathrm{CD} 34$ maximum predictor and $\mathrm{CD} 34$ total collection values are shown below.

\begin{tabular}{|c|c|c|c|c|c|c|}
\hline \multirow{2}{*}{} & \multicolumn{3}{|c|}{ CD 34 Maximum Predictor in blood } & \multicolumn{3}{c|}{ Total CD 34 collection $\times 10^{\wedge} 9 / \mathrm{Kg}$} \\
\cline { 2 - 7 } & Mean & SD & Median & Mean & SD & Median \\
\hline 2008 & 110 & 185 & 54 & 9.1 & 11.4 & 5.5 \\
\hline 2009 & 105 & 126 & 45 & 9.1 & 9.7 & 4.7 \\
\hline P value & \multicolumn{3}{|c|}{0.89} & \multicolumn{4}{|c}{0.9996} \\
\hline
\end{tabular}

\section{P596}

Timing and rate of success in transplant of MUD searches through BMDW: the real case of CIC-813 in 2006-2009

B. Forno, B. Mazzi, E. Zino, M.T. Lupo-Stanghellini, L. Vago, E. Guggiari, M. Coppola, S. Piemontese, S. Claudiani, F. Lunghi, S. Mastaglio, S. Marktel, M. Bernardi, C. Corti, C. Bonini, J. Peccatori, F. Ciceri, K. Fleischhauer, M. Marcatti

San Raffaele Scientific Institute (Milan, IT)

Background: Hematopoietic stem cell transplantation (HSCT) from an allogeneic donor is a curative option for patients (pts) with high-risk hematological diseases. Only $30 \%$ of pts have an HLA identical sibling. The remaining pts need to find a suitable donor within the registries of unrelated volunteers, now enrolling more than 13 million donors in Bone Marrow Donors Worldwide (BMDW) database.

Methods: In our institution, all pts with high risk hematological diseases are HLA typed and if a suitable family donor is missing, a matched unrelated donor (MUD) search is promptly activated. Between 2006 and 2009 we activated 120 searches through Italian Bone Marrow Donor Registry (IBMDR) for the following diagnoses: $\mathrm{AML}(36 \mathrm{pts}), \mathrm{ALL}(17), \mathrm{MDS}(14), \mathrm{NHL}(14)$, $\mathrm{HL}(7), \mathrm{MM}(6), \mathrm{CLL}(6), \mathrm{CML}(6), \mathrm{SAA}(4)$, others(10). The strategy agreed between our transplant(tx) unit and the HLA laboratory was that the confirmatory HLA-A,B,C,DRB,DQB1,DPB1 high resolution typing of the donor should be done in 14 days for standard requests, 7 for urgent ones and 2 for emergencies.

Results: Our HLA laboratory guaranteed typings as scheduled. We identified an at least 8/10 HLA allele matched donor for 85 pts $(70 \%$ of activations) within a median time from activation to donor selection of 63 days, (57 days for pts with acute leukemias). In 38 and 26 cases, the donor was $10 / 10$ or $9 / 10$ HLA allele matched, respectively. We performed 53 tx representing $44 \%$ of all activations and $62 \%$ of the selected donors. Among the group of pts that found a donor, 14(16\%) lost the disease status indication; $7(8 \%)$ were ineligible to tx; for $5(6 \%)$ the donor was not clearable or refused to donate. 8 pts with a selected donor plus 17 others still searching received a tx from an alternative source (haploidentical or cord blood) because of rapidly progressive disease. For pts who underwent MUDHSCT, the median time from donor selection to tx was 47 days; overall, median time from activation to tx was 103 days. In pts with acute leukemia, the median time from selection to $t x$ was of 33 days and median time from activation to tx was 93 days. Conclusions: These data report on a 3 year activity on MUD searches in our Centre: prompt HLA typing and IBMDR coordination allow a donor selection for up to $70 \%$ of candidate pts in a median of 2 months. An activation of donor search at early phases of treatment is warranted for pts with high risk diseases in order to provide the largest chance to receive a transplant from an unrelated donor.
P597

Viability and colony-forming capacity of long-term cryopreserved bone-marrow of CML patients

S. Otten, A. Knauerhase, M. Leeping, S. Sandner, G. Ledderose, J. Tischer

University Hospital Munich-Großhadern (Munich, DE)

Introduction: The higher federal authority for human tissue, the Paul Ehrlich Institute (PEI), requires the declaration of an expiration date for cryopreserved bone marrow (BM). Rare existing data on the durability of long-term (over ten years) cryopreserved BM suggest that the storage in nitrogen is not limited.

We investigated parameters of durability, viability and colonyforming capacity, of bone marrow of CML patients stored in the vapour phase nitrogen as autologous back-up. We compared BM stored between 5 and 10 years (medium-term) with BM stored over 10 years (long-term).

Methods: BM preparations cryopreserved by controlled-rate freezing in $10 \%$ DMSO were thawed in $37^{\circ} \mathrm{C}$ water bath. Immediately after thawing the viability was tested by membrane integrity (trypanblue assay) and the nucleated cell (NC) count was tested by automated cell-counter. As a surrogate marker of proliferation capacity of stem cells we performed colony-forming-unit (CFU) assays.

Results: 6 bags from 3 medium- term stored preparations (median: 6 y) and 7 bags from 3 long-term stored preparations (median: $11 \mathrm{y}$ ) were tested.

The median recovery rate of NCs was $118 \%$ for medium-term and $116 \%$ for long-term stored BM. About $50 \%$ of the preparations showed a viability of $>70 \%$. The median viability of NCs were $60 \%(39 \%-76 \%)$ for medium-term and $76 \%(63 \%-79 \%)$ for long term stored BM. Colony forming units have grown in all 12 preparations (in one no CFU-M and GFU-GMM were found, in 4 no GFU-GEMM were found). The median number of CFUGM per 1x105 mononucleated cells was 20 (1-80) for mediumterm and 15 (10-25) for long-term stored BM.

Conclusion: Our results indicate no significant difference between medium-term and long term stored bone marrow and no limitation in the duration of storage in vapour phase nitrogen. We assume that other not yet analysed factors like previous treatment of the patient and status of the disease have a possible effect on parameters of durability.

\section{P598}

Parameters predicting for successful mobilization and collection of peripheral blood stem cells in patients with haematologic malignacies. A study of 209 patients

M.K. Angelopoulou, T.P. Vassilakopoulos, O. Tsopra, V. Pappi, S. Masouridis, P. Tsirkinidis, M. Moschogiannis, Z. Galani, V. Kalotychou, E. Dimitriadou, S. Sachanas, X. Yiakoumis, C. Kalpadakis, K. Konstantoudakis, M.P. Siakantaris, M.-C Kyrtsonis, Y. Rombos, P. Panayiotidis, G.A. Pangalis, P. Beris University of Athens (Athens, GR)

Objectives: Autologous stem cell transplantation requires successful mobilization and collection of peripheral blood stem cells (PBSC. The aim of this paper was the study of possible predictive factors for successful mobilization and collection of PBSC in multiple myeloma (MM), Hodgkin lymphoma (HL) and non-Hodgkin's lymphoma (NHL) patients.

Methods: 209 patients between 2004 and 2008 were studied. Since more than one mobilization courses and/or apheresis procedures were performed in poor mobilizers, two methods of analysis were applied.

Method A: All mobilization courses were analyzed with the following endpoints: maximum absolute number of circulating CD34 + cells and number of CD34 + cells collected for each mobilization procedure.

Method B: Each patient was considered only once with the following endpoint: mean number of CD34 + cells collected per apheresis. 
Results: Median age was 42 years (13-70), 64\% were men, $64 \%$ had received $0-1,34 \% 2-3$ and $2 \% 4$ or more previous chemotherapy regimens, $43 \%$ had $\mathrm{HL}, 33 \% \mathrm{NHL}, 21 \% \mathrm{MM}$ and $3 \%$ acute leukemia. Eleven\% received G-CSF as mobilizing regimen, $33 \%$ cyclophosphamide +G-CSF, 35\%, $17 \%$ and $4 \%$ regimens containing platinum, ifosfamide and high dose cytarabine + G-CSF, respectively. The maximum absolute number of circulating CD34 + cells and the number of CD34 + cells collected per $\mathrm{kg}$ of body weight were statistically significantly higher for ifosfamide-, platinum-, high dose cytarabine-, cyclophosphamide- and G-CSF- containing mobilizing regimens in this order $(P<0.001)$. The mean number of CD $34+$ collected cells per kg was $8.89 \times 10^{6}, 5.14 \times 10^{6}$ and $1.30 \times 10^{6}$ for patients who had received 1, 2-3 and 4 or more previous chemotherapies $(P=0.001)$. Other significant factors were age $(P<0.001)$, and baseline PLT count $(P<0.001)$. Moreover $\mathrm{HL}$ patients achieved a higher number of CD34 + collected cells compared to $\mathrm{NHL}$ and MM patients $(P=0.002)$. Interestingly, patients with refractory disease had a higher number of CD34 + collected cells compared to patients in relapse and those in remission, $(P=0.003)$. Similar results were obtained with the 2 nd method of analysis.

Conclusions: Factors that affect successful mobilization and collection of PBSC include the mobilizing regimen (superior outcome with ifosfamide-containing regimens), the number of previous chemotherapies (inferior collection with 4 or more), age, baseline PLT count, disease status pre-mobilization (superior collection in refractory patients) and underlying disease (best outcome $\mathrm{n} \mathrm{HL}$ patients).

\section{P599}

Transplantation using BM or PBSC in adult and paediatric recipients of T-cell depleted myeloablative transplants for standard risk leukaemia results in similar outcomes

B.E. Shaw, J. Apperley, N.H. Russell, C. Craddock, E. Liakopoulou, M.N. Potter, R. Wynn, B. Gibson, R. Pearce, J. Lee, K. Kirkland, J.A. Madrigal, G. Cook, J. Byrne on behalf of the BSBMT

Since 2000 the use of GSCF-mobilised Peripheral Blood Stem Cells (PBSC) for unrelated donor (UD) transplantation has increased dramatically. While a number of studies in T-replete transplants have reported significantly different clinical outcomes following PBSC compared to BM, there are few data in T-cell depleted transplants. We present long-term follow-up data on 320 recipients of a myeloablative conditioned, HLA-matched UD allograft using pre-transplant serotherapy (Alemtuzumab $=306, \quad$ ATG $=14$ ). Transplants were January 2000-August 2007: CML in 1CP $(n=102)$ and acute leukaemia in CR1/2 (AML in 105, ALL in 144). 190 patients received $\mathrm{BM}$ and 130 PBSC. The median age of recipients was 28.9 years (10 months -58 years). The groups were similar except for a significantly higher CD34 cell count in the PBSC group (median: 5.8 vs. 2.9 respectively, $P<0.001$ ) and more CML patients in the BM group ( $36 \%$ vs. $25 \%$ in PBSC, $P=0.051)$. The median follow-up was 48 months (BM: 59, PBSC: 38 ). The overall survival at 8 years was $55 \%$. This was not significantly different dependant on the use of BM or PBSC in univariate or multivariable (mva) $(P=0.84)$ analysis. $\mathrm{CML}$ (HR $=0.5, P=0.003$ ) and patient CMV seropositivity (HR 1.4, $P=0.035$ ) were significantly associated with outcome in mva. Graft failure was rare (BM: $4 \%$, PBSC: $2 \%, P=0.306$ ) with a significantly faster time to engraftment using PBSC than BM (14 vs. 20 days; $P<0.001$ ). The incidence of acute GvHD was significantly higher in recipients of PBSC (64\%) than BM (51\%; $P=0.022)$, however there was no increase in grade III/IV $(P=0.420)$ disease in PBSC recipients. No other factor was significantly associated with aGvHD. Chronic GvHD at 6 years was similar (BM: $61 \%$, PBSC: $55 \%$, NS) with no difference in the incidence of extensive disease. The incidence of neither disease relapse nor NRM was significantly different between groups (relapse at 5 years: BM 44\%, PBSC 36\%; $P=0.112$, and NRM at 5 years: BM 22\%, PBSC 24\%; $P=0.751$ ). These findings persisted in mva, where only CML was associated with an increased relapse risk $(P=0.001)$ and CMV seropositive patients with a higher NRM $(P=0.01)$. In conclusion, we have shown that the only significant difference in clinical outcome between PBSC and BM is a higher incidence in the occurrence (but not grade) of acute GvHD. We suggest that either stem cell source can be used with a similar outcome in adult and paediatric recipients of T-cell depleted allografts for standard risk leukaemia.

\section{P600}

Donor stem cell source does not impact outcome of high-risk acute myeloblastic leukaemia patients after reduced-intensity conditioning allogeneic hematopoietic stem cell transplantation

S. Fürst, L. Castagna, T. Prébet, J. El Cheikh, C. Faucher, N. Vey, A. Charbonnier, P. Ladaique, C. Chabannon, D. Blaise Institut Paoli Calmettes (Marseille, FR)

ASCT offers a potential curative treatment approach of highrisk (HR) acute myeloblastic leukaemia (AML) patients (pts). The use of umbilical cord blood (UCB) has featured an attractive alternative stem cell source for those pts lacking a suitable HLA matched related or unrelated donor (UD).

The aim of this analysis was to assess the outcome of 50 pts who received a reduced-intensity conditioning allogeneic hematopoietic stem cell transplantation (RIC-ALLO) in our centre between 2005 and 2008 with a special focus on three different donor sources: identical sibling (IS), UD or UCB.

All pts had HR AML in complete remission (CR) at time of transplant. HR features were defined as at least one of the following criteria: CR1 with unfavourable cytogenetics, secondary AML, more than one induction chemotherapy for obtaining $\mathrm{CR}$ or $\mathrm{CR} 2$ and above. Reasons for RIC regimen were older age, unfit physical condition, comorbidities or prior autologous transplant. All pts received a Fludarabine-based RIC regimen with GVHD prophylaxis consisting in CSA alone or CSA and MMF.

The median age of all pts was 51 (range, 19-70) years. 28 pts had IS, 7 pts have a matched 10/10 (5 pt) or mismatched 9/10 (2 pt) UD and 15 pt received a single or double UCBT. Pts characteristics were comparable between the three groups but for the [IS group: 52 (range, 35-70) years; UD group: 61 (range, 50-66) years; UCB group: 44 (range, 19-61) years].

After a median follow-up of 886 (range, 336-1528) days, 20 pts have died (disease $=6$, treatment related $=14$ ) and 8 pts have experienced relapse. Four year OS and EFS for all pts were $57 \%$ and $54 \%$, respectively with no significant difference between the 3 groups ( 2 year OS: $61 \%, 54 \%$ and $66 \%$; 2 year EFS: $59 \%, 54 \%$ and $45 \%$ respectively). One year TRM was $20 \%$ for the entire group and did not significantly differ between the 3 groups.

Despite the obvious limitations of this small series, results seem to indicate that in this population, donor type does not highly influence outcome. This is quite encouraging as most of the pts lack a compatible sibling donor. This may invite to consider privileging a rapid identification of an alternative donor rather than a given donor type when a match sibling is not available. Thus concomitant search for both graft type should be undertaken in order to perform allo SCT in a larger number of pts otherwise not treated because of early progression as illustrated in the underrepresentation of alternative transplant as compared with familial transplant in this retrospective study (28 familial vs. 22 alternative). 


\section{P601}

Pretreatment with hydroxyurea overcomes limitations of G-CSF mobilization in splenectomized patients with Thalassaemia major

E. Yannaki (1), T. Papayannopoulou (2), E. Jonlin (2), I. Batsis (1), P. Becker (2), F. Zervou (1), A. Xagorari (1), G. Karponi (1), Z. Moustakoudaki (1), P. Kaloyannidis (1), C. Apostolou (1), A. Anagnostopoulos (1), A. Fassas (1), G. Stamatoyannopoulos (2) (1)George Papanicolaou Hospital (Thessaloniki, GR); (2)University of Washington (Seattle, US)

In view of gene therapy of TH, the G-CSF-associated splenic rupture or thrombosis raise safety concerns in $\mathrm{TH}$, because of splenomegaly and hypercoagulability. HU pretreatment (prt) could reduce risks by decreasing splenic size (ss) in nonsplenectomized (nSPL) and high numbers of circulating cells in SPL patients (pts) before G-CSF (G). In a mobilization (mbz) study, we assess the safety/efficacy of $\mathrm{G} \pm \mathrm{HU}$ ptr in 24 adult pts with b-TH. Twenty three pts have been enrolled, $12 \mathrm{SPL}$ and $11 \mathrm{nSPL}$ (9/12 and 5/11 with 1 month-HU prt). Two HU-nSPL pts withdrew during the study. No severe adverse events were observed. In nSPL pts, HU reduced the ss before $\mathrm{G}$, however the max vol during mbz didn't differ between pretreated or not pts. HU negatively affected the CD34 + yield when the 'wash-out' period (W-OP) before $\mathrm{G}$ was 8 days (CD34 +: $1,86 \times 10^{6} / \mathrm{kg} /{ }^{2}$ aph, $\mathrm{n}=2$ ), but it markedly improved when this period increased to 18 days $\left(\mathrm{CD} 34+: 6,5 \times 10^{6} / \mathrm{kg} /{ }^{2}\right.$ aph, $\left.\mathrm{n}=1\right)$. NSPL pts w/o HU-prtm mobilized successfully (CD34+: $6,67 \times 10^{6} / \mathrm{kg} / 2 \mathrm{aph}$, $\mathrm{n}=6)$. SPL pts yielded unexpectedly very low CD34+cells $\left(0.83 \times 10^{6} / \mathrm{kg} / 2 \mathrm{aph}, \mathrm{n}=2\right)$. WBCs were highly elevated during $\mathrm{G}$ (from mean 15.25 to $81 \times 10^{9} / /$ ) despite the reduced and adjusted to the level of WBCs G-doses (mean dose: $3.4 \mathrm{mcg} /$ $\mathrm{kg} / \mathrm{d}$ ). Hyperleukocytosis necessitated early $(\mathrm{d} 3,4)$ therapeutic leukapheresis in all but one pt, who started $\mathrm{G}$ with lower WBCs $\left(12.8 \times 10^{9} / 1\right)$ and aphereses were initiated later (d5) resulting in effective mbz $\left(4,5 \times 10^{6} / \mathrm{kg} / 2 \mathrm{aph}\right)$. HU negatively affected the yield when the $\mathrm{W}$-OP before $\mathrm{G}$ was $<12$ days (mean CD34+0.62 $\times 10^{6} / \mathrm{kg} / 2 \mathrm{aph}, \mathrm{n}=5$ ). However, when this period increased to $\geq 12$ days, to allow bm recovery from HU myelosuppresion, mbz was highly successful (CD34+6.63 $\times 10^{6} /$ $\mathrm{kg} / 2$ aph, $P=0.0007, \mathrm{n}=4)$. Those pts could tolerate 2-fold higher G-doses (mean: $6.75 \mathrm{mcg} / \mathrm{kg} / \mathrm{d}, P=0.02$ ) w/o producing hyperleukocytosis. HU decreased the high PLTs (from 507 to $372 \times 10^{\%} /, P=0,0002$ ) and WBCs (from 17.24 to $9.94 \times 10^{9} / 1$, $P=0,1)$ before $G$, potentially reducing the thrombosis risk and partially preventing hyperleukocytosis during $\mathrm{mbz}$ [maxWBCs ( $\left.\times 10^{9} / l\right)$ SPL-HU: 52,99 vs. SPL-noHU: $\left.76.43, P=0.1\right]$. Overall, $\mathrm{mbz}$ of SPL TH pts is challenging but not inherently inefficient. The poor yields are rather the sequelae of mandatory G-dose modifications to avoid hyperleukocytosis. $\mathrm{HU}$ as prt effectively overcomes this limitation and adds to the safety of the procedure. However, it requires a rather prolonged treatment with a critical W-OP for a sufficient CD34 + cell yield.

\section{P602}

Umbilical cord blood transplantation in adults with haematological malignancies-UK Single Centre Pilot Study M.M. Ceesay, Z.Y. Lim, S. Querol, M. Kenyon, A. Ho, A. Mijovic, S. Devereux, R. Ireland, J. Marsh, G.J. Mufti, A. Pagliuca King's College Hospital (London, UK)

Recent results of adults who received umbilical cord blood (UBC) allografts have been extremely promising, with overall and disease free survival data being comparable to transplantation using adult VUD. However, the UK has been slow in embracing this type of program. We report on preliminary results from a single centre pilot study evaluating the feasibility of UBC allografts for adults in UK.

Between July 2006 and October 2009 a total of 23 adults were transplanted using 11 single UBC and 13 double UCB (one patient had 2 transplants). Use of single or double UCB was based on the recipient weight. Diagnoses at transplanta- tion were $\operatorname{AML}(n=11)$, MDS $(n=5), \operatorname{ALL}(n=3), \operatorname{CML}(n=2)$, aplastic anaemia $(n=2)$ and non-Hodgkin's lymphoma $(n=1)$. Patients received partial HLA-matched UCB grafts using 3 different protocols: Fludarabine, Cylophosphamide, TBI and ATG for the double UCB $(n=10)$, Thiotepa, Fludarabine, Busulphan and ATG for the single UCB $(n=10)$ and FCC (Fludarabine, Cyclosphosphamide and Alemtuxumab $(n=4)$ for marrow failures. Cyclosporine and Mycophenolate were used as the standard GVHD prophylaxis except in FCC where cyclosporine alone was used. The median age was 40 (range 17-72) years. The median total nucleated cell dose (TNC) and cryopreserved CD34+ cell dose were $2.7 \times 10^{7} / \mathrm{kg}$ and $1.0 \times 10^{5} / \mathrm{kg}$ respectively. The median time to sustained neutrophil engraftment was 21.0 (range 6-39) days. Four patients had primary graft failure and 2 others died before engraftment. There were no cases of grade III-IV acute GVHD and only one case of chronic extensive gut GVHD. The day 100 and 1 year transplant related mortality (TRM) was $9 \%$ and $41 \%$ respectively. Causes of TRM included: infection $(n=5)$ (CMV/adenovirus reactivation $=2$, pseudomonas $=1$, aspergillosis $=1$ and CNS toxoplasmosis $=1$, , pulmonary haemorrhage $(n=1)$, intracerebral bleed/EBV PTLD $(n=1)$ and hepatic VOD $(n=1)$. Relapse occurred in only 2 patients and both died at day 63 and 117 post transplant. The overall survival (OS) at one year was $59 \%$. However, the 1-year OS for patients $>55$ yrs was $17 \%$ vs. $72 \%$ for $<55$ yrs $(P=0.03)$. There was no significant difference in outcome between the different protocols or disease types $(P=0.11)$.

Our preliminary UK experience is consistent with existing literature regarding the feasibility of UCB allografts for adults. The use of our protocols has been associated with a low incidence of GVHD and relapse, but infective complications remain a major challenge.

\section{P603}

Adverse events related to autologous and allogeneic peripheral blood stem cell collection: variables related to the collection and safety profile

C. Rabascio, G. Andreola, M. Negri, A. Babic, L. Nassi, A. Alietti, P. Antoniotti, L. Calabrese, F. Bertolini, G. Martinelli, D. Laszlo European Institute of Oncology (Milan, IT)

Background: Thrombocytopenia, hypotension and citraterelated reactions due to hypocalcemia are frequent side effects observed during leukoapheresis (LA), especially during or after large volume LA. We evaluated different biological variables in order to minimize the risk in patients (pts) and healthy donors. Materials and methods: We have retrospectively evaluated 155 LA procedures performed in 81 pts affected by haematological or solid malignancies, mobilized with a combination of G-CSF and chemotherapy and in 17 healthy donors mobilized by GCSF alone. LA were performed by means of a COBE-SPECTRA technology when number of CD34 + before LA were $\geq 5 \mu \mathrm{L}$; patients and donors who showed $\geq 20 \mu \mathrm{L} C D 34$ + were defined as good mobilizers (GM). Target of collection was $\geq 2 \times 10^{6} / \mathrm{Kg}$ CD34+

Results: All subjects underwent at least one LA procedure and reached the target. The median of LA collections was 1 (range $1-4)$. In the entire population the median number of CD34+ at the time of first LA was 36 $\mathrm{L}$ (5-617) with a median percentage of $0.26 \%(0.01-8.39)$. The median of CD34 + collected was $2.98 \times 10^{6} / \mathrm{kg}(0.18-47.6)$. 18/82 (22\%) GM experienced at least one adverse event (EA) during the LA procedure compared to $2 / 16(13 \%)$ of subjects with $<20 \mu \mathrm{L}$ CD34 + (no-GM). The percentage and the absolute count of CD34 + before LA, correlated with $\mathrm{CD} 34+$ cells $/ \mathrm{kg}$ collected $(\mathrm{R} 2=0.7)$. The median of blood volume processed for body weight and the median time of LA was: $9838 \mathrm{ml}(897-15977)$ and $202 \mathrm{~min}$ for GM and $10244 \mathrm{ml}$ (1638-12859) and $207 \mathrm{~min}$ for no-GM. We do not find any correlation between blood volume processed and CD34 + / $\mathrm{kg}$ collected, while we observed that the ratio between number of CD34 +/Kg cells collected and total blood volume processed in GM strongly correlated with the number of CD34 +/Kg cells 
collected $(R 2=0.86)$. In particular, those subjects who reached the target of $\geq 2 \times 10^{6} / \mathrm{Kg} \mathrm{CD} 34+$ cells by one single LA, showed a minimum ratio of 180 .

Conclusions: In our retrospective experience we did not observe any difference in the collection time and blood volume for GM and no-GM to reach the target. The ratio $>180$ suggests that the target of CD34 + cells can be reached by one single LA, with shorter procedures, minimizing the subject's risks. We propose therefore a prospective study in order to adjust the total blood volume reducing the LA time in $\mathrm{GM}$ who showed a ratio $\geq 180$. These data will be confirmed using specific kits with additional bags that allow serial sampling during LA.

\section{$\mathrm{P} 604$}

PBPC collections: procedures, efficiency and risks

Z. Gasova, Z. Bhuiyan-Ludvíková, M. Bohmová, J. Zlabova, E. Kynclova, K. Franzova

Institute of Hematology and Blood Transfusion (Prague, CZ)

Objectives: We tried to evaluate the efficiency, safety and risks of three techniques which were used for autologous PBPC collections: (a) Large-volume Leukapheresis (LVL), (b) Standard Collections, and (c) a new modified technique which was called as "Mix" collections.

Methods: We evaluated the results of 136 PBPC collections which were performed in 98 patients who suffered from multiple myeloma, DLBCL, MCL and Ewing sarcoma. (a) In 93 LVL (more than $3 \mathrm{TBV} /$ total blood volumes/ of the patiens were processed; anticoagulation: ACD - A and Heparin), in (b) 16 Standard procedures (less than 3 TBV were processed; anticoagulation ACD - A), and in (c) 27 "Mix" collections (less than 3 TBV of patiens were processed; anticoagulation: ACD$A+$ Heparin). Collections: Cobe Spectra, Caridian.

Results: In patients (a) with a good effect of mobilization (precollection CD $34+$ cells in blood higher than $20 / u l$ ) we prepared almost the same median dose of CD $34+$ cells from the Standard and "Mix" collections, 3,8 and $4 \times 10^{6} / \mathrm{kg}$. In LVL the median yield of CD $34+$ cells was $8 \times 10^{6} / \mathrm{kg}$. In patients (b) who were mobilized weekly (precollection CD $34+$ cells in blood lower than 20/ul), LVL enabled to prepare $1,5 \times 10^{6}$ of CD $34+$ I $\mathrm{kg}$ from one collection, while the median yield of CD $34+$ cells from Standard and "Mix“ collections was 0,9 and $1,2 \times 10^{6} / \mathrm{kg}$. Three mild adverse reactions in patients were observed - hypotension (1) and hypokalcemia (2) which were corrected in the course of procedure.

Conclusion: We observed the similar efficiency in Standard and Mix collections in well mobilized and weakly mobilized patiens. LVL enabled to get higher yield of CD $34+$ cells than the Standard and MIX collections in well mobilized patients as well as in weakly mobilized patients. We can recommend LVL in all patients who can tolerate it due to a greater chance of collecting high yields of progenitor cells. In the weakly mobilized patients LVL offers a greater chance of collecting at least a minimum amount of CD $34+$ cells needed for transplantation. "Mix" collections may be used as an alternative technique in circumstances in which standard or LVL can not be recommended, e.g. in patients who do not tolerate citrate or the high extent of procedure (cardiac arrhytmia, unstable vital signs etc.)

\section{P605}

Haploidentical stem cell transplantation in children with advanced malignancies

I. Dolgopolov, N. Subbotina, R. Pimenov, V. Boyarshinov, I. Visochin, G. Mentkevich

Institute of Ped. Oncology/Hematology (Moscow, RU)

Haploidentical SCT is the therapeutic modality for patients lacking HLA-identical donor. RIC ensures good tolerability and safety, early stable full donor chimerism with potential GvT effect. Since 2001 we performed 44 haplo-transplantations from relatives in 38 pts with poor-prognostic malignancies: 9-AML,
4-ALL, 4-CML, 5-JMML, 1-MDS, 3-NHL, 7-NBIV, 4-EWS and 1melanoma. RIC regimen included Fludarabine $180 \mathrm{mg} / \mathrm{m}^{2}$, and ATG $40 \mathrm{mg} / \mathrm{kg}$ in combination with Busulfan $8 \mathrm{mg} / \mathrm{kg}(\mathrm{n}=31)$ or Treosulfan $30000 \mathrm{mg} / \mathrm{m}^{2}(\mathrm{n}=7)$. The PBSC with a median number of $6.3(2-12.5) \times 10^{6} \mathrm{CD} 34+$ cells $/ \mathrm{kg}$ were infused after incubation in vitro with vincristine and methylprednisolone. Tdepletion was not performed. GvHD prophylaxis consisted of short methotrexate and cyclosporine A or tacrolimus.

Three pts with leukemia progression at the time of SCT didn't recover and died. Four pts with JMML/MDS rejected and relapsed in first 2 mos after SCT. Thirty three pts $(82,5 \%)$ recovered with full donor chimerism. Incidence of acute GVHD was gr. I-II 22 pts (64\%), gr III- 6 pts (18\%), gr. IV- 0\%. Acute GvHD was successfully treated with steroids and ATG. Incidence of chronic GVHD was $52 \%$ (mainly extensive). Five pts were retransplanted (4-JMML/MDS, 1-CML). Two of them engrafted and one of them is alive and disease free at 3 years. Nine pts are alive and well with a median follow-up 41 (8.1-88.5) mos, 20 pts died from relapse, 2 pts died from acute GVHD, 7 pts from chronic GVHD and infections. Relapse rate at $1 \mathrm{yr}$ was $75 \%$ and $33 \%$ for solid tumor and leukemia/lymphoma pts. DFS and EFS were $54 \%$ and $22 \%$ with a median f-up of 45 and 25 mo for hematological malignancies vs. $20 \%$ and $8 \%$ at 10 and $8 \mathrm{mo}$ in pts with solid tumors, respectively. Haploidentical transplantation following reduced-intensive conditioning is feasible and safe if an appropriate GvHD prophylaxis is given. This strategy is applicable to very high risk and potentially incurable patients if no conventional donor is available. Heavier conditioning regimen does require for kids suffering from JMML/MDS.

\section{P606}

Efficacy, complication rates and cost-effectiveness of chemotherapy + G-CSF and single agent C-CSF as mobilizing regimens for autologous PBSC: analysis of 125 patients with haematological malignancies

A. Pivkova, S. Genadieva Stavrik, Z. Stojanoski, S. Krstevska Balkanov, L. Cevreska, O. Karanfilski, S. Trajkova, B. Georgievski

University Hematology Hospital (Skopje, MK)

Mobilized PBSC have largely replaced conventional, unprimed BM as source during the autologous transplant setting, because of a faster hematopoietic reconstitution, less transfusion requirements, less infective complications and earlier hospital discharge. Choosing the optimal mobilizing regimen is the goal for achieving the sufficient amount of PBSC for autologous transplant. G-CSF is the standard agent commonly administered undergoing PBPC mobilization and collection in a dose of $10 \mathrm{micro} / \mathrm{gr}$ in a 4 days regimen. The additional chemotherapy to G-SCF is still associated with higher rate of hemorrhagic cystitis, prolonged neutropenia, infective complications, secondary malignancies and other toxicities. Therefore in this study we evaluated the efficacy, complication rates and cost-effectiveness of chemotherapy+G-CSF versus single agent C-CSF as mobilizing regimens. We analyzed 125 patients with haematological malignancies (49 AML in first remission, $26 \mathrm{HD}, 30$ $\mathrm{MM}, 16 \mathrm{NHL}, 4 \mathrm{ALL}$ ) who underwent mobilization of PBSC in our centre and the attempt to reach $2 \times 10(6) / \mathrm{kgCD} 34$ cells. In $6 \%$ of patients adequate cell dose was not reachable and overall failure rate of mobilization of $17,5 \%$. Furthermore $15.6 \%$ failed to harvest the optimal $4 \times 10(6) / \mathrm{kgCD} 34+$ cells with $>1$ aphaeresis attempt. The analysis of factors contributing in this effect in the univariante analysis were: $>2$ lines of previous chemotherapy and neutropenic events $(P=0,002$ and $P=0,005)$, those also remained significant in the multivariate analysis (RR:4,4 and 6,2). No differences have been noticed between the diagnostic groups of patients. The mortality rate was $2 \%$ (intracranial bleeding and sepsis). The statistical analysis preformed for analyzed patients transplanted with single G-CSF as mobilizing regimen, compared with the chemotherapy + G-CSF group showed $P<0,0001$ for 
febrile days, microbiological positive isolates, days of hospital stay, transfusion requirements. The median cost of PBSC collection in the Chemotherapy + G-CSF group was E 8550 (E220-10110) compared with the G-CSF group alone E3110 (E2200-4120) that showed $P<0,0001$. Taking these results in consideration for the potential candidates for ASCT, transplant centers should consider the use of less myelosupressive agents or dose reduction strategies for the mobilization and autologous stem cell procurement.

\section{P607}

Peripheral progenitor cell collection is safe in paediatric donors: a single-centre experience from India

R. Raj

Apollo Speciality Hospital (Chennai, IN)

The use of peripheral blood stem cells (PBSC) for stem cell transplantation has greatly increased in adults. This trend is now reflected in paediatrics, where healthy children are donating PBSC or donor lymphocytes via apheresis for their siblings. There have been concerns over the safety of PBSC collection in paediatric donors. We have reviewed 52 PBSC (40 allogeneic and 12 autologous) collections in paediatric donors (aged 18 months to 18 years) at our institution over a period of 13 years from 1996 to 2009. The donors had received a median of 5 days of growth factor $(10 \mu \mathrm{g} / \mathrm{kg}$ body weight). All donors had a femoral apheresis catheter placed on the morning of the procedure under intravenous sedation. Anxiolytic agents like midazolam or lorazepam were used during the process if required. The extracorporeal line was primed with irradiated leucoreduced packed red cells when donors weighed less than $40 \mathrm{~kg}$ (32 procedures were primed). The anticoagulants used were a combination of heparin in bolus and continuous infusion of acid citrate dextrose at a reduced rate to avoid citrate toxicity. All donors were given calcium intravenously in between and at the end of collection.

53 PBSC collections were done with smallest donor weighing $11 \mathrm{~kg}$ and a median of 2.10 times of donor blood volume was processed per procedure. The mean mononuclear cell yield for per $\mathrm{kg}$ of recipient weight was $5.4 \times 10^{8}$ in allogeneic donors and $3.3 \times 10^{8}$ in autologous donors. 8 donors underwent two days of collection and 1 donor for 3 days. Minor side effects like generalized myalgia due to growth factor administration, pain at the femoral catheter site, anxiety, restlessness, perioral tingling due to hypocalcemia and mild hypotension were seen in a majority of the donors. 2 donors had fever after insertion of the femoral catheter requiring intravenous antibiotics. 1 child had atrial fibrillation requiring adenosine. This event occurred in one of our earliest collections and modification of the anticoagulant protocol during harvest has virtually eliminated this potential problem in subsequent procedures.

These results show that PBSC collection in paediatric donors in the allogeneic and autologus setting is possible with minimum adverse events. The yield of MNC by using G-CSF stimulation and the volume processed appear to be adequate and safe for good clinical outcome.

\section{P608}

The duration of the ex vivo transportation of haematopoietic stem cells does not seem to impair the clinical outcome following transplantation - a single-centre study R. Olsson, M. Remberger, O. Ringdén Karolinska University Hospital (Stockholm, SE)

In hematopoietic stem cell transplantation (HSCT), using unrelated donors, stem cells are often harvest at remote hospitals, and may be handled ex vivo for several days before they are infused into the recipient. Thus, the aim of this study was to investigate whether the duration of the ex vivo transportation of hematopoietic stem cells impairs the clinical outcome following transplantation. We retrospectively analyzed 90 consecutive patients who received unrelated donor hematopoietic stem cells at our centre between 2003 and 2008. Both children and adults were included (median age 45, range 0.5-67), whereas retransplantations were excluded. The indications for allogeneic HSCT were: AML (32\%), ALL (16\%), myelodysplastic syndrome $(16 \%)$, CML (10\%), lymphoma $(12 \%)$, solid tumors $(7 \%)$, metabolic disorders (3\%), aplastic anemia (2\%), and multiple myeloma $(2 \%)$. In all recipients, the total ex vivo time of the stem cell transplant (median $28 \mathrm{~h}$, range $5-53 \mathrm{~h}$ ) was calculated and subdivided into the transportation time from the donor hospital to our centre (median $20 \mathrm{~h}$, range $3-38 \mathrm{~h}$ ), as well as the time from arrival at our hospital until the start of the stem cell infusion (median 10 h, 0.5-26h). In univariate analysis, none of the ex vivo times were significant predictors of graft failure, relapse, non-relapse mortality or overall survival. In conclusion, the clinical outcome following HSCT seems to be independent of the time the stem cell transplant is kept ex vivo for transportation purposes.

\section{P609}

Bone-to-bone boost in poor graft function after haploidentical haematopoietic stem cell transplant: safety and feasibility. Two case reports

F. Giglio, S. Malato, D. Clerici, S. Mastaglio, C. Messina, A. Larusso, M. Zambelli, A. Assanelli, V. Matozzo, C. Corti, M. Bernardi, F. Ciceri, J. Peccatori

San Raffaele Scientific Institute (Milan, IT)

Background: Poor graft function (PGF) occurs in $5-27 \%$ of patients (pts) after allogeneic hamatopoietic stem cell transplant (HSCT) and is associated with high morbidity and mortality. Graft function may be poor as result of slow or incomplete recovery of blood counts (primary PGF) or decreasing blood counts after successful engraftment (secondary PGF). Several factors may determine PGF: disease status, conditioning regimen, HSC source, HLA compatibility, T cell content, immunosuppression, GvHD, viral infections, drugs. GCSF and Rhu-EPO are readily available and effective but with no effects on platelet. Second transplantation from the same donor, with or without conditioning therapy, can boost the haematopoietic recovery in these pts. Unfortunately, both a second peripheral CD34 + mononuclear cells (MNC) mobilization and a marrow harvest in the operating room may be contraindicated early after the first donation as not safe for donors. Intrabone SCT can overcome the risk of graft failure even with a low number of CD34 + MNC and we have performed in two adult pts with PGF a bone-to-bone boost (BBB) with a small marrow harvest from respective donors, who were unfit for a second conventional donation.

Aim: To evaluate the feasibility of the BBB technique in 2 pts with PGF.

Methods and results: pts were 2 males $(57,53$ y) with a diagnosis of AML and CMML, respectively. Prolonged pancytopenia and hypoplastic marrow were documented in both cases, with diagnosis of primary PGF and secondary PGF, respectively, donor chimerism ranging from $80-100 \%$ (STR and HLA), without evidence of leukemia. The 2 donors were related, haploidentical. For the BBB procedure small quantities of bone marrow $(<200 \mathrm{ml}$ ) were collected from the posterior iliac crest of the donors, at the bedside, during deep sedation and analgesya. After 30 minutes, necessary for graft treatment according to Institution procedure, the unmanipulated marrow harvested was infused into the posterior iliac crest of the pts at day 30 and 72 days after SCT, respectively. MNC infused doses were 0.9 and $0.4 \times 10^{8} / \mathrm{Kg}$, respectively. No side effects were recorded both for patients and donors.

Conclusion: in this 2 cases the BBB technique proved feasible and safe for both donors and patients. This practice can give the chance of HSC boost to patients with PGF without the need of a GCSF mobilization for donors and with a minimal invasive 
operation. Further studies are necessary to assess the efficacy of this procedure.

\section{P610}

Poor mobilization in patients with acute myeloid leukaemia or myelodisplastic syndrome: risk factors and response of a cytarabine-based rescue mobilization

I. Rodríguez (1), J.M. Sancho (1), R. Guardia (3), D. Gallardo (3), O. Garcia (1), M. Batlle (1), C. Ferrà (1), M. Moreno (1), J. Juncà (1), J.M. Ribera (1)

(1)Hospital Universitari Germans Trias i Pujol (Badalona, ES); (3)Hospital Universitari Doctor Josep Trueta (Girona, ES)

Background and objectives: G-CSF is the most common drug used for peripheral blood progenitor cell (PBPC) mobilization. The objectives of this study were: 1 . To evaluate the prevalence and risk factors of poor mobilization $\left(<1.5 \times 10^{6} \mathrm{CD} 34\right.$ cells $\left./ \mathrm{Kg}\right)$ in AML or MDS patients, and 2. To analyze the results and toxicity of a cytarabine-based rescue mobilization.

Method: First-line mobilization consisted of s.c. G-CSF $(10 \mathrm{mcg} /$ $\mathrm{Kg} / 12 \mathrm{~h}$ for 4 days), whereas rescue mobilization consisted of i.v. cytarabine $\left(800 \mathrm{mg} / \mathrm{m}^{2} / 24 \mathrm{~h}\right.$ for 3 days $)$ plus G-CSF $(5 \mathrm{mcg} /$ $\mathrm{Kg} / 24 \mathrm{~h}$ from the 4 th day), in an inpatient regimen.

Results: From 64 consecutive patients, 46 (71.9\%, 50\% males, mean [SD] age 48.2 [12.2] yr.) mobilized with G-CSF and underwent transplantation, whereas $18(28.1 \%$, 66.6\% males, 50.56 [12.8] yr.) failed. The only factor associated with poor mobilization was the duration of neutropenia and thrombocytopenia in previous induction and consolidation schedules (mean of 19.5 [5.2] days vs. 22.8 [44] days [ $P=0.023$ ] and 15.6 [4.9] vs. 19.5 [4.6] days $[P=0.007]$, respectively). Six out of the 18 non-mobilized patients received rescue mobilization with cytarabine and G-CSF. The mean duration of neutropenia $\left(<0.5 \times 10^{9} / \mathrm{L}\right)$ and thrombocytopenia $\left(<20 \times 10^{9} / \mathrm{L}\right)$ was $9.2(5.1)$ and $10.6(5.2)$ days, respectively. Four patients $(67 \%)$ had neutropenia-related fever. Only one mobilized successfully $\left(2.3 \times 10^{6}\right.$ cells CD34/kg) and underwent transplantation, another patient relapsed at the hematologic recovery and the remaining 4 received a second consolidation chemotherapy.

Conclusions: Poor mobilization is frequent in AML or MDS patients. The duration of pancytopenia following previous chemotherapies was the only factor influencing successful mobilization. Rescue mobilization with cytarabine plus G-CSF was not effective.

Supported by grants P-EF/09 of FIJC and RD06/0020/1056 of RETICS.

\section{P611}

Unrelated cord blood transplant for children: experiences in a SE Asia population; perspective of a single-centre A.M. Tan

KK Women's and Children's Hospital (Singapore, SG)

Haematopoietic stem cell transplants (HSCT) have cured many patients with malignant and nonmalignant conditions since the 70 's. In a population with diverse racial groups and with some ethnic groups not well represented in the international marrow registries, unrelated cord blood transplant gives these children another chance of cure.

Method: We reported 19 cases of unrelated cord blood transplant (CBT) over a period of 6 yrs from Oct 2003 to September 2009 in a Children's Hospital. During the same period we performed 4 cases of matched unrelated donor (MUD) transplant, 27 MSD transplant and 2 related CBT.

Of the unrelated CBT, the majority were $1 / 2 \mathrm{HLAAg}$ mismatched, 12 cases for malignant disorders ( 5 for ALL, 4 for AML, 3 for $\mathrm{CML}$ in accelerated/blastic crisis) and 7 cases for nonmalignant disorders ( 6 for primary immunodeficiency disorders (PID) and 1 for Thalassemia major). The racial groups were mainly Chinese except for 2 Malay, 1 Middle Eastern and 1 Australian descent. The median age was 6 yrs (range 0.25 to $17 \mathrm{yrs}$ ). Most received myeloablative conditioning regimes $\pm R T$, while 4 pts with PID received nonmyeloablative conditioning regimes. Results: Of these, $16 \%$ (3/19) had graft rejection, one pt with ALL remained in remission 4 year post transplant, one with Thalassemia Major had autologous reconstitution and one pt with chronic granulomatous disease (CGD) died from pneumonia 1.5 yrs later. There was no TRM. In the same period for the MUD/MSD HSCT, TRM was $3.2 \%$ (1/31 pts).

The median neutrophil engraftment was 24 day (range D+18 to $D+61$ ) and median platelets engraftment was 30 days (range $D+18$ to $D+50)$. 11 patients had GVHD. Majority had mild to moderate GVHD. OS was $73 \%$ (14/19 pts). The medium follow up was 906 days (range 77 to 2170 days). 5/19 pts died (4 from relapse of leukemia).

Summary: Today cord blood forms an important source of stem cells in unrelated HSCT for SE Asian descents .In the year 2007 out of a total 28 unrelated HSCT for adult/children in Singapore, cord blood formed $45 \%$ the source of stem cells (APBMT 2009). Cord blood formed $80 \%$ (19/23) source of stem cells for unrelated HSCT in this study. With expansion of local Public Cord Bank and International Cord Blood Banks, we expect 50 to $60 \%$ of our children will be able to find a suitable cord unit in future.

\section{P612}

Haploidentical haematopoietic stem cell transplantation as a salvage therapy for patients with high-risk haematologic malignancies. Single-centre experience O. Paina, E. Semenova, E. Babenko, A. Golovacheva, A. Alyanskiy, L. Zubarovskaya, B. Afanasyev Pavlov State Medical University (St. Petersburg, RU)

Background: Haploidentical stem cell transplantation is a treatment option for patients (pts) who don't have an HLA-identical sibling donor, HLA-matched unrelated donor or insufficient amount of cord blood cells. A high rate of TRM limits use of this method of treatment.

Design and Methods: 22 eligible patients with high-risk hematologic malignancies (ALL 9pts; AML 10pts; CML ac. phase 1pts; HL resistant $1 \mathrm{pts}$, Neuroblastoma resistant $1 \mathrm{pts}$ were included in this study. Patient sex M:F 13pts: 9pts (1,4:1). Patient age, median 12,6 years (4-32). We used HSC from three loci mismatched related donors. Unmanipulated bone marrow and PBSCs were infused into the recipient on the day of collection. PBSC CD34 + cells were selected using the CliniMACS device (Miltenyi Biotec). Total quantity of PBSC + BM CD $34+$ cells infused into the recipient were $1,4-13,9 \times 10^{6} / \mathrm{kg}$ for $13 \mathrm{pts}$ and $>14 \times 10^{6} / \mathrm{kg}$ for 9 pts. 3 pts received myeloablative conditioning regimen, which consisted of Ara-C, Bu $(12 \mathrm{mg} / \mathrm{kg}$ p.o. in 12 doses), Cy $\left(1.8 \mathrm{~g} / \mathrm{m}^{2} / \mathrm{d}\right.$, i.v.), Me-CCNU $(250 \mathrm{mg} / \mathrm{kg}$, i.v. $)$. 19pts received RIC regimen, which consisted of Flu $30 \mathrm{mg} / \mathrm{m}^{2}$ + Bu $4 \mathrm{mg} / \mathrm{kg} / \mathrm{d}$ (14 pts) or Flu $40 \mathrm{mg} / \mathrm{kg} / \mathrm{d}$ + Mel $60 \mathrm{mg} / \mathrm{m}^{2}$ + Thio $5 \mathrm{mg} / \mathrm{kg} / \mathrm{d}$ (4 pts) and $1 \mathrm{pts}$ received other conditioning regimen. GVHD prophylaxis: $15 \mathrm{pts}$ received ATG $20 \mathrm{mg} / \mathrm{kg} / \mathrm{d}+\mathrm{CsA} 3 \mathrm{mg} /$ $\mathrm{kg} / \mathrm{d}+\mathrm{MTX} 10 \mathrm{mg} / \mathrm{m}^{2}$ short course. 4pts received: ATG $25 \mathrm{mg} /$ $\mathrm{kg} / \mathrm{d}+$ Tacrolimus $0,03 \mathrm{mg} / \mathrm{kg} / \mathrm{d}+\mathrm{MMF} 30 \mathrm{mg} / \mathrm{kg} / \mathrm{d}$.

Results: 1-year OS was $35 \%$. The cumulative incidence of grade III-IV acute GVHD after "salvage"-haploidentical HSCT without using the CliniMACS device (Miltenyi Biotec) was 58\% $(P=0,01)$. RIC "salvage"-haploidentical HSCT is associated with acceptable rates of TRM (28\%) and aGVHD.

Conclusion: G-PBSC positive selection of CD34 + via selection columns using CliniMACS device combined with BM from haploidentical family donors may be used as a acceptable source of SC for "Salvage"- allo-HSCT. "Salvage"-haploidentical HSCT is the safety and efficacy procedure in heavy pretreated patients with high-risk hematologic malignancies. 
P613

Peripheral blood stem cell collection for autologous transplantation

D. Puente (1), J. Real (2), A. Requejo (2), J. Sosa (2), N. Fernandez (2), V. Milovic (2), G. Jaimovich (3), L. Feldman (4) (1)Fundacion Favaloro (Buenos Aires, AR); (2)Hospital Aleman (Buenos Aires, AR); (3)CEHT Fundacion Favaloro (Buenos Aires, AR); (4)CEHT Fundacion Favaloro Hospital Aleman (Buenos Aires, AR)

Optimal strategy to mobilize hematopoietic progenitors (PHSC) into peripheral blood (PB) remains controversial regarding efficacy, toxicity and costs. Usually a combination of cyclophosphamide (Cy) and granulocyte colony stimulating factor (G-CSF) are used. A number of $2 \times 10(6)$ CD34+cells $/ \mathrm{kg}$ are considered adequate for a prompt hematological recovery after myeloablative conditioning treatment. A substantial failure rate is reported with the current mobilization regimens (MR). We retrospectively analyzed 125 patients (pts), $70(56 \%)$ men with a media age of 45.2 years (Range R: 13-66) who were admitted for an autologous PHSC transplantation. Their diagnoses included $43(34,4 \%)$ non Hodgkin lymphoma (NHL), $43(34,4 \%)$ multiple myeloma (MM), 22 (17.6\%) Hodgkin disease (HD), 12 $(9.6 \%)$ acute leukemias $(A L)$ and $5(4 \%)$ solid tumours.

MR consisted of Cy $1,5 \mathrm{~g} / \mathrm{m}^{2}$ i.v. infusion over 3 hours on day (D) 1 along with MESNA + G-CSF $5 \mathrm{ug} / \mathrm{kg} / \mathrm{D}$ from D 2 to 6 and then G-CSF10 ug/kg/D from D7 until leukapheresis was finished. Collections were performed using a Fresenius AS104 apheresis equipment and a 3 to 4 times total blood volumen (large volume) leukapheresis was performed daily. The procedure was initiated when leukocyte count reached to $5 \times 10(9) / L$.

Results: $107(86 \%)$ pts achieved a target collection of $\geq 2 \times 10(6)$ CD 34 + cells $/ \mathrm{kg} ; 18 \mathrm{p}(14 \%)$ underwent a remobilization attempt. The $M$ yield of CD34+cells $/ \mathrm{kg}$ was $6,91 \times 10(6)$ (R: $0.1-32.25) .75$ pts $(70 \%)$ needed only one leukoapheresis, and $25(23,3 \%)$ were able to achieve the target collection with a two-days procedure. $51(48 \%)$ initiated leukapheresis on D 9 , $36(34 \%)$ on D 8 and $33(31 \%)$ on D10.

Pts who underwent a 2nd procedure included $12(67 \%) \mathrm{NHL}$, $5(28 \%) \mathrm{AL}$ and $1(5 \%)$ MM. 6p (43\%) obtained an adequate harvest with a 2 nd leukoapheresis (mobilized in every case with G-CSF10 ug $/ \mathrm{kg} / \mathrm{D}$ for $5 \mathrm{D}$ previous to procedure), $3(21 \%)$ with a bone marrow (BM) collection, 5 (36\%) combining both procedures. 3 pts were excluded from the autologous BM transplantation programme.

Conclusion: This MR was adequate to achieve PHSC harvest in a high (86\%) number of pts. The optimal approach to remobilization strategy remains unclear.

\section{P614}

Multifactorial dependence of hematopoietic stem cell graft and its impact on the outcome of autologous haematopoietic stem cell transplantation

A. Oprisoni (1), S. Arghirescu (1), C. Jinca (1), L. Balint-Gib (2), A. Isac (2), V. Ordodi (2), M. Baica (2), G. Doros (1), M. Serban (1)

(1)University of Medicine Timisoara (Timisoara, RO); (2)Louis Turcanu Children's Hospital (Timisoara, RO)

Introduction: Hematopoetic stem cell transplantation (HSCT) represents a salvage therapy for many malignant and nonmalignant diseases. The experience of our center is limited due to the many hindrances and disadvantages out which we will only mention those related to the late addressability of patients. Objectives: We aimed at analysing the quantity and quality of the hematopoietic stem cell (HSC) graft in relation to the particularities of patients, disease, therapy and to the procedure of mobilization/harvest of cells as well as the estimation of the impact of the graft on the outcome of HSCT.

Material and methods: The retrospective observational study was conducted on 221 HSC grafts used for 89 procedures of autologous HSCT. Peripheral HSC harvest has been performed in all patients. $53,94 \%$ of the patients were in different stages of advanced disease,in partial remission or progressive disease. Results: Age was an important factor in CD34 + cells collection: the quantity obtained was $5,35 \pm 2,56 \times 10^{6} / \mathrm{kg}$ for the age $<8$ years, $4,15 \pm 5,40 \times 10^{6} / \mathrm{kg}$ for the age $\leq 55$ years and $1,95 \pm 0,40 \times 10^{6} / \mathrm{kg}$ for the age $>55$ years. $(P<0.001)$. The underlying disease influenced the CD34 + cells collection; best molizers have been leukemia patients $\left(7,33 \pm 12 \times 10^{6}\right.$ CD34 + cells $/ \mathrm{kg}$ ) whereas worst mobilizers, patients with solid tumors $\left(2,5 \pm 2,66 \times 10^{6} \mathrm{CD} 34+\right.$ cells $\left./ \mathrm{kg}\right) .(P<0.01)$ The best CD34 + cells collection $\left(5,23 \pm 5,87 \times 10^{6} / \mathrm{kg}\right)$ has been achieved after DHAP regim and the worst with cyclophosphamide $\left(1,92 \pm 5,17 \times 10^{6} / \mathrm{kg}\right) . \quad(P=0.05)$ The quantity of $>20 \mathrm{CD} 34+$ cells $/ \mu$ in peripheral blood has been decisive. $(P=0.012)$ The quantity of CD34 + cells administered in the transplantation procedure correlated with the timelines for granulocytes and thrombocytes engraftment $(r=-0.706, P<0.001 ; r=-0.244, P=0.03)$ without impacting the mortality associated to transplantation $(6,74 \%) .(P=0.4370)$. The administered quantity of CD34 + cells was a determning factor for the quality of engraftment.

Conclusions: Age, underlying disease, mobilization regimens, type of apheresis machine were factors which significantly influenced the prelevation of CD34+ cells. A quantity of $\geq 5 \times 10^{6}$ CD34 + $/ \mathrm{kg}$ was ideal for engraftment, without influencing overall or event free survival rate at 5 years in statistical manner.

\section{Aplastic anaemia}

\section{P615}

Allogeneic haematopoietic stem cell transplantation for 37 patients with Fanconi's anaemia

R. Ahmed-Nacer, F. Mehdid, N. Rahmoune, M. Benakli, R. Belhadj, M. Baazizi, F. Harieche, F. Zerhouni, R.M. Hamladji Pierre and Marie Curie Center (Algiers, DZ)

Introduction: Fanconi anemia ( FA ) is an autosomal recessive disease frequently evolves to bone marrow failure and acute myeloid leukemia. Allogeneic hematopoietic stem cell (HSCT) is the treatment of choice for patients with FA, it leads to normal complete hematopoietic recovery and prevention of acute leukemia.

Materials and methods: From June 1998 to November 2008 (125 months period) 37 patients with FA underwent 38 allogeneic HSCT (from HLA-identical sibling donors: 32 and pheno identical: 4, mismatch cord unrelated donor: 1); median age 11 years (5 to 24$)$; sex ratio $(M / F) 1$; median interval from diagnosis to transplant 24,9 months (5 to 60$) ; 12$ patients $(44,3 \%$ ) had received more than 20 transfusions before allograft. Two conditioning regimens was used: BU-Cy protocol with busulfan $6 \mathrm{mg} / \mathrm{Kg}$ and cyclophosphamide $40 \mathrm{mg} / \mathrm{Kg}$ for 9 patients $(23,7 \%)$ and Flu-Cy-ATG protocol with fludarabin $150 \mathrm{mg} / \mathrm{m}^{2}$, Cy $20 \mathrm{mg} /$ $\mathrm{kg}$ and anti thymocyte globulin (Fresenius) $40 \mathrm{mg} / \mathrm{Kg}$ for 28 patients $(73,7 \%)$. GVHD prophylaxis associated cyclosporin and methotrexate $5 \mathrm{mg} / \mathrm{m} 2(\mathrm{~J} 1-\mathrm{J} 3-\mathrm{J} 6)$. Four patients received bone marrow transplant and 31 patients peripheral blood stem cell and 2 patients died before allograft. At May 2009 maximal follow up is 131 months and minimal 6 months.

Results: The median time to engrafment was 13 days (10 to $21)$. Twenty patients $(54 \%)$ are alive with success engrafment after median follow up 39 months (6 to 126): $2 / 9$ patients (22.2\%) had BU-Cy conditioning and 18/28 (64.3\%) Flu-Cy-ATG conditioning. Late graft failure (J60 to J511) was observed in 5 patients (13,5\%). Acute GVHD occured in 13 patients (43,3\%) with 10 grade II-IV $(33,3 \%)$ and chronic GVHD in 5 patients $(20,8 \%)$ with 3 extensive. Seventeen patients died (45,9\%): 11 patients $(29,7 \%)$ by transplant related mortality (TRM): infectious: 4, grade II-IV acute GVHD: 4, extensive chronique GVHD: 1 , hemorrhage: 2 . Six patients by another causes of death: graft rejection: 4, secondary neoplasia: 1, unknown: 1 . 
Actuarial overall survival (OS) is $49,8 \%$ at 9 years. TRM was higher in Bu-Cy conditioning group (44,4\%) than Flu-Cy-ATG conditioning group $(25 \% ; p=0,0)$ and $O S$ is $63 \%$ at 9 years in Flu-Cy-ATG group.

Conclusion: Flu-Cy-ATG conditioning regimen appears better than BU-Cy. A best outcome can be reached by precoce diagnosis and by reduction of interval from diagnosis to allograft.

\section{P616}

A candidate complement inhibitor for the treatment of paroxysmal nocturnal haemoglobinuria (PNH): preclinical data with tt30, a therapeutic that selectively blocks the complement alternative pathway

C. Pascariello (1), C. Selleri (1), L. Del Vecchio (1), C.J. Hovarth (2), V.M. Holers (2), A.M. Risitano (1)

(1)Federico II University of Naples (Naples, IT); (2)Taligen Therapeutics (Cambridge, US)

Paroxysmal nocturnal haemoglobinuria is characterized by intravascular hemolysis (IVH) due to uncontrolled spontaneous complement alternative pathway (CAP) activation due to the lack of CD55 and CD59 on affected RBCs. By modifying the standard Ham acid hemolysis test, we have developed an in vitro model to investigate both the terminal and the early $\mathrm{C}$ activation, which lead to hemolysis and $\mathrm{C} 3$ fragment (C3frag) opsonization of RBCs, respectively. Both hemolysis and RBC C3frag opsonization were serially measured by double color flow cytometry (Ferreira and Pangburn, Blood 2007; Risitano et al., Blood 2009). TT30 is a fusion protein which links the CAP inhibitory domain of Factor $\mathrm{H}$ (fH) with the $\mathrm{iC} 3 \mathrm{~b} / \mathrm{C} 3 \mathrm{~d}$ recognition domain of $\mathrm{C}$ receptor 2; the resulting molecule is able to supplement $\mathrm{fH}$ activity and control CAP activity specifically at sites of $\mathrm{C} 3$ activation. We have studied RBCs obtained from $8 \mathrm{PNH}$ patients, 5 untreated and 3 on eculi-

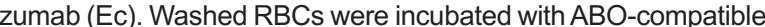
sera from healthy volunteers. Sera obtained $<1 \mathrm{~h}$ after infusion of Ec were used in surrogate experiments for testing Ec in vitro. The Ham test was modified using a pH of 7.0-7.3 to allow slower hemolysis and C3frag RBC opsonization. Using these conditions, survival of PNH RBCs at $24 \mathrm{~h}$ was $15-30 \%$ in untreated patients; survival of PNH RBCs from patients on Ec was lower, likely due to the higher propensity of C3frag + PNH RBCs to lyse even in absence of serum acidification. Peak serum concentration of Ec $(>200 \mathrm{ug} / \mathrm{mL}$ ) resulted in a significantly higher PNH RBC survival (45-70\%); residual C-mediated hemolysis was confirmed by the presence of $\mathrm{C} 3$ frag + RBC membrane fragments. Notably, the majority $(50-80 \%)$ of surviving PNH RBCs became C3frag + after $24 \mathrm{~h}$ incubation. TT30 at $1 \mathrm{uM}(65 \mathrm{ug} / \mathrm{ml})$ or higher concentrations led to $100 \%$ survival of PNH RBCs from untreated patients, which was sustained up to $72 \mathrm{~h}$. In addition, consistent with the mechanism of TT30 activity, no C3frag + PNH RBCs or membrane fragments appeared. In conclusion, our model demonstrates that Ec substantially reduces in vitro lysis of PNH RBCs, but is associated with substantial C3frag deposition on surviving PNH RBCs. In contrast TT30, blocking the CAP and its amplification loop, led to complete inhibition of hemolysis without C3frag deposition on PNH RBCs. These findings suggest that TT30 may inhibit both the MAC-mediated IVH and the C3frag-mediated extravascular hemolysis, providing a strong rationale to test this agent in future clinical trials.

\section{P617}

Comparisons between allogeneic peripheral blood stem cell transplantation and allogeneic bone marrow transplantation in severe aplastic anaemia

A. Ghavamzadeh, S.A. Mousavi, K. Alimoghaddam, A.A. Hamidieh, M. Iravani, B. Bahar, M. Jalili, M. Jahani Hematology-Oncology and SCT Research Center, Tehran University of Medical Sciences (Tehran, IR)

Introduction: Acquired Severe Aplastic Anemia (SAA) is a rare disease and the outcome of these patients has improved con- siderably over the last decades. In young patients bone marrow transplantation (BMT) is the elective treatment who has an HLA-identical sibling donor. We retrospectively analyzed patients who received allogeneic BMT or peripheral blood stem cell transplantation (PBSCT) and compared the differences in hematologic recovery, acute and chronic GVHD, relapse and disease free survival and overall survival in two groups.

Methods: Between March 1991 and October 2009, 163 patients with multi-transfused SAA received allogeneic hematopoietic stem cell transplantation from an HLA-identical sibling donor (158 patients) or HLA full matched other related donor (5 patients). Three patients received both bone marrow and peripheral blood therefore exclude from study. Median age in BMT group was 17 years (range 1 to 32 ) and in PBSCT was 23 years (range 2 to 50 ). All patients received the conditioning regimen containing cyclophosphamide and ATG and graftversus-host disease (GVHD) prophylaxis include cyclosporine and methotrexate at the standard doses.

Result: The median time to neutrophil and platelet recovery in PBSCT group (11 and 17 days, respectively) was significantly lower than BMT group (16 and 19 days, respectively) $(P<0.001)$. There was no difference in the incidence of acute GVHD between patients receiving BMT and those receiving PBSCT $(P=1)$. The cumulative incidence of chronic GVHD for those who survived more than 90 days was $10.2 \%$ in the BMT group, compared to $27 \%$ in the PBSCT group $(P=0.022)$. Eleven $(22.4 \%)$ of BMT recipients and $7(6.3 \%)$ of PBSCT recipients suffered a relapse $(P=0.005)$. Disease-free survival at 1 year was $74 \%$ in the BMT group and $85 \%$ in the PBSCT group $(P=0.318)$ and Overall survival at 1 year was $82 \%$ in the BMT group and $88 \%$ in the PBSCT group $(P=0.1)$. Nine patients in BMT group and 17 patients in PBSCT group were dead.

Discussion: This study results showed that engraftment is rapid in multi-transfused patients who undergoing PBSCT than BMT with significant differences. However there was no difference seen in overall survival and disease free survival in two groups but chronic GVHD which affect long term outcome and quality of life especially in young patients is significantly higher in PBSCT group. Change conditioning and prophylaxis regimen can improve the risk of GVHD in PBSCT.

\section{P618}

Fludarabine, cyclophosphamide and alemtuzumab for stem cell transplantation in acquired aplastic anaemia is associated with a low risk of graft-versus-host disease and sustained engraftment

J. Marsh (1), V. Gupta (2), Z.Y. Lim (1), D. Marks (3), E. GordonSmith (4), G. Mufti (1)

(1)Kings College Hospital (London, UK); (2)Princess Margaret Hospital (Toronto, CA); (3)University Hospitals Bristol NHS Foundation Trust (Bristol, UK); (4)St Georges Hospital (London, UK)

Graft rejection and chronic GVHD remain major obstacles to successful outcome after stem cell transplantation (SCT) for aplastic anemia (AA). We have pioneered the use of Alemtuzumab with CY in AA SCT and shown a reduction in GVHD although graft rejection was high at $24 \%$. More recently, Fludarabine has been added to the conditioning to reduce graft rejection. In this retrospective, multi-center study, we report outcomes for acquired AA in 37 patients using Alemtuzumab, Fludarabine and $\mathrm{CY}(\mathrm{FCC})$ conditioning regimen. Alemtuzumab dose was $0.2 \mathrm{mg} / \mathrm{kg} \times 5$ days $(\mathrm{n}=20), 60 \mathrm{mg} \times 1(\mathrm{n}=12)$, $25 \mathrm{mg} \times 4(\mathrm{n}=4)$ and $40 \mathrm{mg} \times 1+30 \mathrm{mg} \times 2(\mathrm{n}=1)$. All patients received Fludarabine $30 \mathrm{mg} / \mathrm{m} 2 \mathrm{~g} \times 4$ and $\mathrm{CY} 300 \mathrm{mg} / \mathrm{m} 2 \mathrm{~g} \times 4$. Patients were transplanted from 1999 to 2009 . Median follow up of survivors was 641 days. Disease severity was 'very severe' in 10 , 'severe' in 20 and 'non-severe' in 7 patients. SCT was performed using MSD in 15 patients (40\%) and UD in $22(60 \%)$, of whom all but one were matched for $8 / 8$ or $10 / 10$ alleles. Median age was 35 years (range 8-55). Stem cell source was bone marrow $(\mathrm{BM})$ in $21(57 \%)$, peripheral blood stem cells 
(PBSC) in 7(19\%), BM + PBSC in 5(13\%) and G-mobilised BM in $4(11 \%)$. Time from diagnosis to SCT was $<12$ months in $57 \%$ of patients. $8 / 15(53 \%)$ of sibling transplants and $18 / 22(82 \%)$ of UD transplants received IST prior to SCT. There were 5 cases of graft failure. Cumulative incidence of graft failure at 1 year was $15 \% \pm 4 \%$ (14\% for MSD and $15 \%$ for UD SCT). For patients transplanted $>12$ months from diagnosis, graft failure was $25 \%$ compared with $10 \%$ for patients transplanted within 12 months $(P=0.19)$. Acute GVHD occurred in $13.5 \%$ patients, grade I-II in all cases. Extensive chronic GVHD occurred in only one patient. Data for CMV infections was available in 21 patients, and in 20 patients for EBV. CMV reactivation occurred in 2/21 (9.5\%) patients, with one case of CMV disease. EBV infection occurred in $2 / 20$ patients $(10 \%)$ : one responded to Rituximab and one patient died from progressive EBV PTLD. There were no cases of adenovirus infection. 3-yr OS was $89 \%$ (93\% for MSD and $85 \%$ for UD SCT, $P=0.66$ ). For all patients, OS was $95 \%$ when time from diagnosis to SCT was $<12$ months and $80 \%$ for $>12$ months $(P=0.27)$. For patients $>40$ yrs, there was no significant difference in OS compared with patients $<40$ yrs (93\% vs. $82 \%)$. We conclude that Alemtuzumab with Fludarabine and CY(FCC) for MSD and UD SCT for acquired AA is associated with excellent survival, a low incidence of GVHD and viral infections.

\section{P619}

Fludarabine, cyclophosphamide and antithymocyte globulin as conditioning regimen in matched related and unrelated allogeneic stem cell transplantation in severe aplastic anaemia: a cancer centre's ten-year experience P. Anderlini, S. Acholonu, G. Okoroji, R. Bassett, S. Giralt, E. Shpall, M. Qazilbash, M. de Lima, U. Popat, L. Worth, R. Champlin

UTMDACC (Houston, US)

Background: Total body irradiation (TBI) is often employed in matched unrelated donor (MUD) allogeneic stem cell transplantation (allo-SCT) in severe aplastic anaemia (SAA). However, even at low doses, TBI causes serious short- and long-term toxicities. In matched related donors (MRD), outcome in SAA patients over 40 years with high-dose cyclophosphamide (CY) and ATG is often poor due to graft rejection and GVHD.

Methods: Over a 10-year period (1999-2009), twenty SAA patients underwent allo-SCT with a FLU-CY-ATG regimen from a MRD $(n=7$, all age $>40)$ or a MUD $(n=13$, any age). Median age was 34 years (range 1-59). Seven pts (33\%) were $>50$ years old. Bone marrow was used as stem cell source in all but four patients. The regimen employed most consistently (Bacigalupo et al., 2005; 36: 947 Bone Marrow Transpl) included $(\mathrm{n}=13) \mathrm{FLU}(30 \mathrm{mg} / \mathrm{m} \mathrm{sq} \times 4$ days) intravenously (IV), CY $(300 \mathrm{mg} / \mathrm{m}$ sq IV $\times 4$ days), and thymoglobulin (THY, 3.75 $\mathrm{mg} / \mathrm{kg}$ IV $\times 3-4$ days). In the other patients doses were FLU $20-50 \mathrm{mg} / \mathrm{m} \mathrm{sq} \mathrm{IV} \times 4$ days; $C Y$ 40-60 mg/kg IV $\times 2-4$ days; Antithymocyte globulin (ATG) IV 20-30 mg/kg (horse) or $3 \mathrm{mg} /$ $\mathrm{kg}(\mathrm{THY}) \times 2-4$ days. Median time from diagnosis to allo-SCT was 12 months (2-244).

Results: $17 / 19$ evaluable patients engrafted (90\%), with two secondary graft failures $(10 \%)$. Median time to neutrophil recovery was 15 days (range $8-30$ ). Chimerism studies indicated over $90 \%$ donor-derived engraftment in 16/19 evaluable patients $(75 \%)$. Four out of 20 evaluable patients $(20 \%)$ developed acute (grade II-IV) GVHD, and 6/16 evaluable patients (37\%) developed chronic GVHD. Thirteen patients (62\%) are alive (including eight out of the last nine treated) with a median follow-up of 30 months (3-112). Seven patients expired (graft rejection $n=1$, infection $n=2$, GVHD $n=1$, multiorgan failure $n=1$, EBV post-transplant lymphoproliferative disorder/PTLD $n=2$ ). There was one early death (death < day 30 ) and no known late graft failures. Overall 2-year survival (actuarial estimate) for the whole group $(n=20)$ and for the thirteen patients treated according to Bacigalupo et al are $61 \%(95 \% \mathrm{Cl} 42 \%$ $89 \%$ ) and $82 \%(95 \% \mathrm{Cl} 62 \%-100 \%)$, respectively (Figure 1; $A$ and $B$ ).
Conclusion: While the study has limitations (possible selection bias, small sample size, heterogenous patient population, etc), it suggests that fludarabine-based, TBI-free conditioning for MRD and MUD allo-SCT in SAA is feasible, with high engraftment rates and low early mortality. Presumably due to high-dose ATG, GVHD-related incidence and mortality was low, although EBV PTLD was a drawback of this strategy.

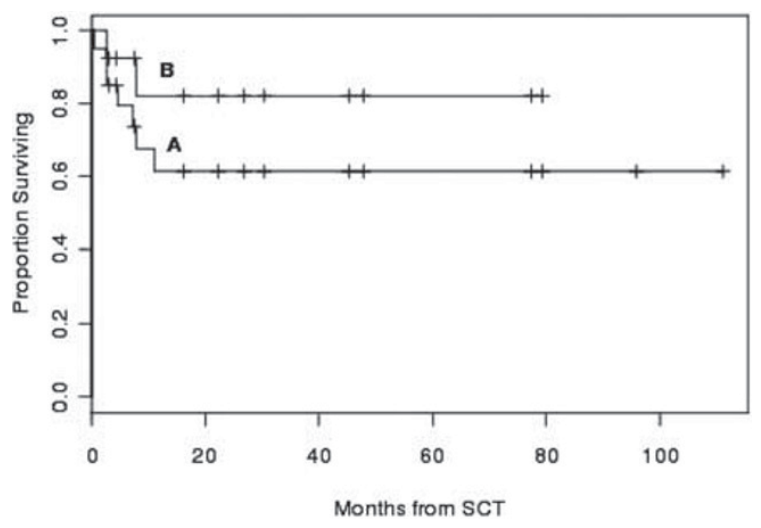

P620

Comparison of three preparative regimens in alternative donor transplant for aplastic anaemia in Japan

H. Yabe, A. Ohara, F. Bessyo, T. Nakahata, R. Kobayashi, M. Tsuchida, S. Ohga, Y. Kosaka, H. Mugishima, A. Morimoto, E. Ito, S. Kojima on behalf of the Japan Childhood Aplastic Anemia Study Group

Objectives: Allogeneic BMT from an alternative donor (AD) is associated with a higher risk of graft failure (GF) in patients with aplastic anemia. To establish the best procedure, we compared three preparative regimens used in AD BMT in Japan.

Methods: Seventy-four patients received AD BMT with following preparative regimens; (1) $3 \mathrm{~Gy}$ of limited field irradiation (thoracoabdominal irradiation; TAI or total lymphoid irradiation; TLI), $100 \mathrm{mg} / \mathrm{m}^{2}$ of fludarabine (Flu), $200 \mathrm{mg} / \mathrm{kg}$ of cyclophosphamide $(\mathrm{CY})$ and $5-10 \mathrm{mg} / \mathrm{kg}$ of rabbit antithymocyte globulin (ATG), (2) $3 \mathrm{~Gy}$ of TAI/TLI, $100 \mathrm{mg} / \mathrm{m}^{2}$ of Flu, $3000 \mathrm{mg} / \mathrm{m}^{2}$ of CY and $5-10 \mathrm{mg} / \mathrm{kg}$ of ATG, (3) 3-10 Gy of TBI, $200 \mathrm{mg} / \mathrm{kg}$ of $\mathrm{CY}$ and $10 \mathrm{mg} / \mathrm{kg}$ of ATG. For the prophylaxis of graft-versus-host disease (GVHD), both short-term methotrexate and tacrolimus were mainly used. Nine patients received AD BMT with LFI/Flu/ CY(200)/ATG regimen, 30 patients with LFI/Flu/CY(3000)/ATG regimen, while 35 patients with TBI/CY/ATG regimen between June 1998 and December 2008. Median age at BMT in each group was 12.6 years (range 2.7-18.1), 13.1 years (2.8-20.8), 9.5 years $3.5-19.5$ ), respectively. Median time from diagnosis to BMT in each group was 18.4 months (range 5.4-107.2), 32.6 months (7.3-186.2), 15.7 months (1.4-211.1), respectively. Mean cumulative units of RBC transfusion and mean time of platelet transfusion in each group was 54.5 (range 8-186) and 26.1 (3-59), 49.2 (3-400) and 34.3 (2-305), $46.1(0-212)$ and 45.3 (0-269), respectively.

Results: Thirty-two patients received marrow from HLA-fully matched unrelated donor, 33 patients from HLA-1 allele/antigen mismatched unrelated donor, 9 patients from related donor other than HLA-identical sibling. Mean dose of nucleated marrow cells in each group was $3.0 \times 10^{8} / \mathrm{kg}(2.3-5.1), 3.5 \times 10^{8} / \mathrm{kg}$ $(1.1-10.3), 3.5 \times 10^{8} / \mathrm{kg}(1.3-8.2)$, respectively. Early or late GF was observed in 0 of 9,3 of 30,3 of 35 patients in each group, respectively. Two of 9 patients in LFI/Flu/CY(200)/ATG group and 3 of 35 patients in TBI/CY/ATG group died of complications, and main cause of death was cardiac toxicity in 3 . Overall survival and event-free survival in each group was 78\% $(95 \% \mathrm{Cl} ; 51-100)$ and $78 \%(51-100), 100 \%$ and $87 \%(74-99)$, $91 \%(82-100)$ and $86 \%(74-97)$, respectively. 
Conclusion: A preparative regimen consisted of low-dose irradiation, reduced-dose $\mathrm{CY}$ and Flu resulted in favorable results with a low rate of graft failure and cardiac toxicity in AD BMT.

\section{P621}

Biological characteristics influencing long-term outcomes in patients with acquired aplastic anaemia after immunosuppressive treatment

A. Kulagin (1), I. Lisukov (1), V. Borisov (2), N. Pronkina (2), I. Kruchkova (2), V. Sergeevicheva (2), A. Gilevich (2), V. Kozhevnikov (2), B. Afanasyev (1)

(1)Pavlov State Medical University (St. Petersburg, RU); (2)Institute of Clinical Immunology SB RAMS (Novosibirsk, $R U)$

Objectives: To evaluate of immunosuppressive treatment (IST) efficacy and biological characteristics influencing long-term outcomes in patients with acquired aplastic anemia (AA).

Methods: We retrospectively analyzed the outcome of 75 patients ( $43 \mathrm{M}$ and $32 \mathrm{~F}$, median age $20,12-61$ ) with moderate (MAA 23), severe (SAA 33) and very severe (VSAA 19) AA treated with ATG and CsA \pm daclizumab (54) or with CsA alone (21) between October 1995 and November 2009. PNH clones, activated T-cells in bone marrow and telomere length in peripheral blood cells were tested by high sensitive flow cytometry and Flow-FISH respectively.

Results: The hematological response was evaluated in 71 patients according to the strict response criteria (B. Camitta, $2000)$. Twenty five $(35 \%)$ and $28(39 \%)$ patients achieved CR and $P R$, respectively. The quality of response was highest in VSAA group $(C R / P R=12 / 1)$ than in SAA $(11 / 12, P=0.011)$ and MAA $(2 / 15, P<0.001)$. There were 3 early and 9 late deaths. Seven patients $(13.2 \%)$ relapsed and 5 responded again after retreatment with ATG and CsA. Late events included MDS/ AML $(n=2)$, trisomy $6(n=1)$, rectal cancer $(n=1)$ and hemolytic PNH $(n=5)$. The cumulative incidence of late events was higher in MAA than in SAA and VSAA $(P=0.043)$. The 5-year overall survival was $79.1 \pm 5.94 \%$ without significant difference between the MAA $(77 \%)$, SAA $(78 \%)$ and VSAA $(84 \%)$. There was a marked trend towards better failure-free survival (FFS) in more severe disease $(33 \pm 13 \%, 43 \pm 11 \%$ and $65 \pm 12 \%$ in MAA, SAA and VSAA respectively). The elevated number of activated CD8 + T-cells and the presence of PNH clones were more frequent in VSAA. The proportion of patients with significant telomere shortening was lower in VSAA (29\%) than in SAA $(50 \%)$ and MAA (58\%). Disease duration before start of IST is longer for MAA as compared with SAA and VSAA $(P=0.0001)$. These biological and treatment related characteristics give the possible explanation for better quality of response and FFS in VSAA.

Conclusions: Modern intensive IST and supportive care allow overcoming early negative prognosis and provide a significantly greater chance to achieve CR and better FFS in VSAA compared with those in SAA and MAA. Our data indicate that the VSAA represents a biological homogeneous entity with predominant immunopathogenesis and relatively intact residual stem cell compartment. Low FFS warrants further investigations of the pathogenic features and optimal treatment strategy in MAA.

\section{P622}

Androgens may boost responses to anti-thymocyte globulin in acquired aplastic anaemia

S. Annunziata, M.T. van Lint, T. Lamparelli, F. Gualandi, C. Di Grazia, A. Dominietto, S. Bregante, A. Bacigalupo

S.Martino's Hospital (Genoa, IT)

Introduction: Androgens have been widely used in the past for the treatment of aplastic anemia, (Blood 1990; 76:2222). A prospective randomized EBMT study has shown that response was improved in females given androgens up front, together with anti-thymocyte globulin (ATG) (BJH, 1993; 83:145). More recently marrow CD34 + cells exposed to androgens, have been shown to have increased telomerase activity and higher TERT mRNA levels (Blood 2009; 114: 2236): these data argue for a role of androgen in the treatment of patients with AA.

Aim of the study: We evaluated the outcome of androgen therapy in 46 patients with Aplastic Anemia (AA) who had received a previous course of ATG and were persistently cytopenic.

Patients and methods: 46 patients AA were selected as having received treatment with testosterone ondecanoato p.o. 40 $\mathrm{mg} /$ day 5-7 days/week, because of persistent cytopenia, in 1-3 cell lines. Median age was 45 years (19-69); there were 16 males and 30 females. The median $\mathrm{Hb}$ levels at start of testosterone, were $(8.6 \mathrm{gr} / \mathrm{dl} 6.2-14)$, the median neutrophil counts $1.6 \times 10^{9} / \mathrm{L}(0.0-5.4)$ and the median platelet count $20 \times 10^{9} / \mathrm{L}$ (1-428). The median interval between ATG treatment and testosterone 173 days $(0-4269)$. The median duration of androgen therapy was 568 days (275181).

Outcome: Of the 46 patients treated, $18(41 \%)$ showed no response; of these 12 were allografted; 26 patients $(59 \%)$ showed increments in blood counts and were considered responders: of these 14 were partial response and 12 were complete responses (platelet count over $100 \times 10 \% / \mathrm{L}$ ). Responses were more frequent in femelaes $(63 \%)$ as compared to amles $(44 \%)(P=0.1)$. The actuarial 5 year failure survival is $\mathrm{XX} \%$; it was $92 \%$ for responders $0 \%$ for non responders. Treatment with testosterone and low doses of cyclosporine, was well tolerated and could be gradually tapered with time, down to a conventional $40 \mathrm{mg}$ of testosterone twice weekly.

Conclusions: This study confirms the potential usefulness of androgen therapy in a proportion of patients with SAA, and may be considered, especially in females, when ATG treatment has not yielded the expected result, while waiting for a suitable donor.

\section{P623}

Sensibilization of severe aplastic anaemia patients to thymoglobulin (ATG) impairs the efficacy of ATG in haematopoietic stem cell transplantation (HSCT) conditioning C.M. Jol-van der Zijde, R.G.M. Bredius, A.M. JansenHoogendijk, R.M. Egeler, A.C. Lankester, M.J.D. van Tol Leiden University Medical Centre (Leiden, NL)

Patients suffering from severe aplastic anaemia (SAA) and lacking a HLA-identical sibling donor are treated with ATG often combined with cyclosporine A. ATG is a polyclonal antiserum raised in horse or rabbit and is used to eliminate autoreactive, disease associated T cells.

Patients will proceed to HSCT if they are unresponsive to firstline treatment with ATG. However, in the conditioning regimen prior to HSCT, ATG is frequently applied to prevent graft rejection and acute Graft-versus-Host disease (aGvHD).

Nineteen patients transplanted for SAA between 1998 and 2008 were included in this study. Ten patients were not previously treated with ATG and nine patients were. Time from ATG treatment to HSCT varied from 6 months to 4 years (median 10 months); two patients received two ATG courses.

Concentrations of total (rabbit immunoglobulins) and active ATG (capable to bind to a T cell line) and anti-ATG antibodies were measured in pre- and post-HSCT serum samples of the SAA patients. Results were compared to non-SAA HSCT patients. In 7 out of 9 SAA patients with a history of previous ATG treatment, IgG anti-ATG and/or IgA anti-ATG, but not IgM anti-ATG antibodies were detectable pre-conditioning. As expected, antiATG antibodies were absent in the patients without previous exposure to ATG

In 3 out of 7 patients with IgG anti-ATG, ATG administration was stopped because of severe and immediate side-effects requiring a switch to Alemtuzumab for further T-cell depletion. In these patients, ATG was instantly depleted from the serum and undetectable at the time of transplantation. 
One patient with detectable IgG anti-ATG pre-conditioning who did receive the complete ATG dosage $(10 \mathrm{mg} / \mathrm{kg})$, showed rash, fever and a high CRP level starting at day +3 post-HSCT. High avidity IgG1 anti-ATG of recipient allotype resulted in rapid depletion of ATG to undetectable levels at day +6 and an autologous lymphocyte recovery and subsequent graft rejection at day +12 post HSCT (see Figure)

We conclude that patients with ATG exposure prior to HSCT may be sensitized to ATG as evidenced by production of IgG anti-ATG. In these patients, a second course of ATG may lead to serious side-effects during ATG infusion and problems like graft rejection and acute GvHD. Replacement of ATG by Alemtuzumab in such patients could be warranted.

Total ATG, active ATG and anti-ATG in SAA patient.

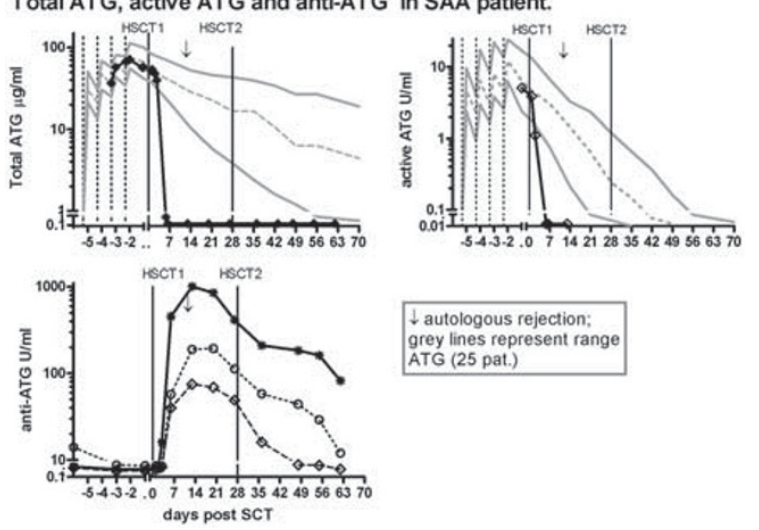

P624

Allogenetic haematopoietic stem cell transplantation for adult patients with acquired aplastic anaemia: multivariate analysis and propensity score matching H. Kim, J.-H. Park, B.-S. Kim, D.-H. Kim, M.-S. Hyun, S.-H. Kim, S.H. Bae, J.-H. Choi, S.-K. Sohn, H.-J. Shin, J.-H. Won, S.-S. Yoon, D.-Y. Jo, Y.-D. Joo, K. Lee on behalf of The Korean Society of Blood and Marrow Transplantation

Backgound: Although allogeneic hematopoietic stem cell transplantation (alloSCT) from matched related donor (MRD) is a standard therapy for severe acquired aplastic anemia (AA), alternative donor $(A D)$ alloSCT is increasing and the survival of alloSCT from $A D$ improves nowadays.

Aims: We planned this study to review the recent result of alloSCT survival and to compare AD with MRD in adult acquired $A A$.

Methods: A retrospective study comparing MRD and AD for alloSCT in patients with adult acquired AA was conducted by Korean Society of Blood and Marrow Transplantation. To adjust the different clinical characteristics between donor types, we calculated propensity scores for choosing donor type. Casecontrol study comparing MRD and AD was prepared by propensity score matching.

Results: Initially a total of 234 patients' data was collected from 15 Korean institutions. One PRCA and $8 \mathrm{PNH}$ patients were included in primary data but these patients were removed from this analysis. Finally 225 adult patients who had AA and had received alloHSCT were analyzed.

Univariate analysis revealed that no prior IST $(P=0.005)$ age at alloHSCT $\leq 31 \mathrm{Y}(P=0.001)$, time from Dx to alloHSCT $\leq 6 \mathrm{M}(P=0.008), \mathrm{RD}(P=0.002), \mathrm{MRD}(P<0.001)$, HLA full match $(P=0.019)$, successful engraftment $(P<0.001)$, no SOS $(P=0.035)$, no aGvHD $(P=0.005)$ and platelet engraftment days $>21 \mathrm{D}(P=0.025)$ were significant favorable factors. Multivariate analysis showed that age at alloHSCT $\leq 31 Y(P<0.001)$, MRD $(P=0.003)$, successful engraftment $(P<0.001)$, no aGvHD $(P<0.001)$ were the independent factors for longer survival. Age at diagnosis $\leq 28 \mathrm{Y}(P=0.090)$,
ABO compatible $(P=0.070)$, no SOS $(P=0.069)$ were marginally significant favorable factors.

Total 50 (each 25 patients from MRD and AD patients) were selected by propensity score matching. Median survivals of MRD and AD were 175.4 months and not reached, respectively $(P=0.514)$. Time to ANC engraftment $(P=0.336)$ and time to platelet engraftment $(P=0.705)$ were not different between MRD and AD. There were not statistically significant difference between MRD and AD in cumulative incidence of aGcHD, cGvHD and TRM.

Summary: In conclusion, our study showed that donor type was a significant factor for survival in multivariate analysis. However, the differences between MRD and AD were not significant in propensity score matching analysis. This might imply that $A D$ could be considered earlier when there is no MRD in patients with AA.

P625

Haematopoietic stem cell transplantation in unclassifiable bone marrow failure syndrome with physical malformations

M. Yabe, H. Yabe, T. Morimoto, T. Shimizu, T. Koike, H. Takakura, S. Kato

Tokai University Hospital (Isehara, JP)

Objective: Aplastic anemia is a rare disease in children that is most commonly acquired /idiopathic and less often a hereditary disorder. The inherited bone marrow failure syndrome (BMFS) is a heterogeneous group of disorders characterized by cytopenias usually in association with one or more physical malformations. Allogeneic hematopoietic stem cell transplantation (HSCT) is a curative treatment for patients (pts) with BMFS, however, very few results are available to make any recommendations for the pts except for classical inherited BMFS. We report the outcome of 16 unclassifiable BMFS pts with physical malformations, who were given allogeneic HSCT.

Methods: Between 1/2002 and 5/2009, 16 pts with physical malformations received HSCT. They are denied to be classical inherited BMFS such as Fanconi anemia and dyskeratosis congenita etc. by clinical features, chromosome instability and/ or genetic subtypes. There were 8 males and 8 females, aged 0.8 to 27 years. Hematological diagnosis included transfusion dependent severe aplastic anemia $(\mathrm{N}=9)$, Refractory cytopenia with multilineage dysplasia $(\mathrm{N}=3)$, Refractory anemia with excess blasts-1 (RAEB-1) $(\mathrm{N}=3)$ or RAEB-2 $(\mathrm{N}=1)$. Three pts received HLA-matched related bone marrow (BM), 1 HLA-mismatched related BM, $5 \mathrm{HLA}$-matched unrelated BM, and $4 \mathrm{HLA}$ mismatched unrelated BM, $1 \mathrm{HLA}$-matched unrelated cord blood (CB), and 2 HLA-mismatched unrelated CB. Conditioning varied: low dose irradiation (3-4.5Gy)+fludarabine (Flu)+cyclophosphamide (CY) based regimen: 10 pts, 7.5-12Gy irradiation + CY: 3 pts, Flu + melphalan based: 3 pts. Graft-versus-host disease (GVHD) prophylaxis included cyclosporine-A (CSA) + methotrexate (MTX) for 3pts received HLA-matched related BM, while tacrolimus + MTX for 13 pts given allograft from alternative donors. Results: All pts engrafted. The rate of acute-GVHD (II-IV) and chronic-GVHD was 6 of $16(38 \%), 7$ of $16(44 \%)$, respectively. Two pts with myelodysplarstic syndrome (MDS) died of bacterial infection with progressive GVHD, and another MDS pt died of hematological relapse. Overall, 13 pts remain alive in first complete remission after HSCT (range 4-90 mo).

Conclusion: Our study indicates that significant advances have been made in the use of HSCT to treat unclassifiable inherited BMFS, but the results are less favorable in MDS patients because of transplant related complication or disease relapse. It is necessary to establish the ideal HSCT conditioning regimens for these disorders. 
P626

Peripheral blood stem cell transplant is safe in heavily pre-transfused aplastic anaemia patients without increased morbidity from chronic graft-versus-host disease

P. Mishra, N. Rathod, M. Mahapatra, T. Seth, S. Rathi, R. Kapoor, N. Gupta, S. Sharma, N. Aggarwal, A.K. Singh, A. Dayama, B. Dhingra

AllMS (New Delhi, IN)

Objective: We studied the outcome of heavily pre transfused aplastic anemia patients receiving peripheral blood stem cell transplant (PBSCT) from matched sibling donor.

Material: The data on 32 consecutive blood/marrow stem cell transplants in 30 aplastic anemia patients over a period of 5 years in non HEPA filtered single rooms was recorded. Fludarabine $30 \mathrm{mg} / \mathrm{m}^{2}$ D-10 to D-5, cyclophosphamide $60 \mathrm{mg} / \mathrm{kg} /$ day D-6 to D-5 and antithymocyte globulin $30 \mathrm{mg} / \mathrm{kg} / \mathrm{day} \mathrm{D}-4$ to D-1 were used as conditioning regimen. Cyclosporine and methotrexate were used for graft versus host disease (GvHD) prophylaxis.

Results: The data is summarised in Table 1. All but 3 had PBSCT. 27 patients received empirical antibiotics and 15 received empirical antifungals for febrile neutropenia in immediate post transplant phase. Positive blood culture for bacteria was recorded in 4 patients and a biopsy proven fungus (2 aspergillus, 2 mucor) in 4 patients. $22 / 30(73 \%)$ patients are alive at median follow up of 351 days ranging from 0-1890 days. Chronic GvHD was seen in the majority and mostly associated with dry skin and changes in pigmentation which generally subsided over time and well tolerated. 1 patient had nephrotic syndrome 3 years post transplant which was controlled with steroids. 1 patient developed pure red cell aplasia which lasted for 3 months and managed with steroids and erythropoietin. The patients who died are summarised in table 2.5 patients developed grade III-IV gut acute GvHD; 4 died. Persistent fever without obvious focus immediately preceding transplant was noted in 3/8 deaths, 2 of whom had grade III-IV gut GvHD (2 had CMV reactivation as well) and the third died of disseminated aspergillus infection. Of the 3 who received bone marrow, 1 patient developed a progressive gut GvHD which lingered on steroids and ultimately died after developing CMV infection 9 months post transplant; 2 had secondary graft failure of whom 1 underwent PBSCT and is a long tem survivor; the third relapsed on stopping cyclosporine and is back on immunosppressants.

Conclusions: PBSCT is safe in aplastic anemia patients whose transplant has been delayed and thereby heavily pretransfused. The outcome and survival appears comparable to those achieved with historical bone marrow transplant data without apparent increase in morbidity on account of chronic GvHD. No pre-treatment variable other than baseline febrile neutropenia could predict adverse outcome.

Table 1: Baseline and follow up data

\begin{tabular}{|l|l|l|}
\hline Median time to transplant & 13.5 months & Range 1-65 months \\
\hline Median transfusion prior to transplant & 22.5 & Range 3-106 \\
\hline Median age of recipients & 30 years & Range 9-37 years \\
\hline Median age of donors & 30 years & Range 4-40 years \\
\hline Gender mismatch & $14 / 32$ & $43 \%$ \\
\hline Major blood group mismatth & $7 / 32$ & $21.8 \%$ \\
\hline Median CD 34 dose & $7.03 \times 1076 / \mathrm{kg}$ & Range 0.7-10.24 X 10 6 6/kg \\
\hline Time to neutrophil engraftment & Day +10 & Range 8-17 days \\
\hline Time to platelet engraftment & Day +20 & Range 10-42 days \\
\hline Median duration of hospital stay & 38 & Range 17-144 \\
\hline Acute GrHD (Consensus/Seattle) & $16 / 32$ & $50 \%$ \\
\hline Grade I & 4 & \\
\hline Grade II & 7 & \\
\hline Grade III-IV & 5 & \\
\hline Clronic GvHD & $18 / 32$ & $56 \%$ \\
\hline Changes in skin colour, dry skin & 18 & \\
\hline Extensive skin & 2 & \\
\hline Dry mouth & 5 & \\
\hline Ocular dryness & 7 & \\
\hline Weight loss > 15\% & 2 & \\
\hline Liver & 4 & \\
\hline & & \\
\hline
\end{tabular}

Table 2: Summary of data from patients who died

\begin{tabular}{|l|l|l|l|l|l|l|l|}
\hline $\begin{array}{l}\text { Serial } \\
\text { Number }\end{array}$ & $\begin{array}{l}\text { Heavy } \\
\text { Transfusion } \\
\text { Pre } \\
\text { transplant }\end{array}$ & $\begin{array}{l}\text { Fever } \\
\text { immediately } \\
\text { prior to } \\
\text { transplant }\end{array}$ & $\begin{array}{l}\text { Intracranial } \\
\text { bleed }\end{array}$ & $\begin{array}{l}\text { Documented } \\
\text { fungal } \\
\text { infection }\end{array}$ & $\begin{array}{l}\text { Graft } \\
\text { failure }\end{array}$ & $\begin{array}{l}\text { CMV } \\
\text { reactivation }\end{array}$ & GvHD \\
\hline 1 & Yes & Yes & No & Yes & No & Yes & Yes \\
\hline 2 & Yes & Yes & No & No & No & Yes & Yes \\
\hline 3 & Yes & Yes & No & Yes & No & No & No \\
\hline 4 & Yes & No & Yes: & No & No & No & No \\
\hline 6 & Yes & No & No & No & Yes & Yes & No \\
\hline 7 & Yes & No & No & No & No & No & Yes \\
\hline 8 & Yes & No & No & No & No & No & Yes \\
\hline
\end{tabular}

"No response to platelet transfusion

\section{P627}

Significance of serial chimerism analysis in allogeneic haematopoietic cell transplantation for bone marrow failure: do stable mixed chimerism matter?

S.M. Bakanay, M. Arat, E. Serbest, P. Topçuoglu, E.A. Soydan, A.U. Bilgin, S.C. Bozdag, K. Dalva, G. Gürman Ankara University Medical School (Ankara, TR)

Introduction: AHCT is core treatment modality for relative young patients with severe aplastic anemia and Fanconi aplastic anemia. In single center cohort, SAA/FAA patients were retrospectively analyzed. Patients and Methods: Fourty (7 PB, 33 BM) infusions have been performed. Two patients necessitated add-back infusions and DLI was administered only in one patient. 40 patients with $\operatorname{SAA}(\mathrm{N}=32)$ and $\operatorname{FAA}(\mathrm{N}=8)$ median age 20 (range, 14-44)] received AHCT from 40 related and 2 unrelated donors. The conditioning regimen consisted of mostly CY-ATG. The patients received CSA and MTX for GHVD prophylaxis. Median donor age 24 years (range, $7-47$ ). Mean pre-transplant ferritin level was $1301 \mathrm{ng} / \mathrm{dl}$. Results: In univariate analysis sex mismatch and peripheral blood as a stem cell source show negative impact on engraftment. Three of the 22 patients showed late graft rejection. Nineteen $(47.5 \%)$ patients are alive with a median post-tx. follow-up of 45.6 mo.s (range, 5.8-221). Since 2002 we established molecular followup of chimerism via determination of STR loci with multiplex PCR. Serial chimerism analysis were available for 19 patients. We observed complete donor chimerism $(n=9)$, stable mixed chimerism (sMC) $(n=5)$; decreasing mixed chimerism $(n=3)$ and increasing mixed chimerism (iMC) $(n=2)$. One of the 2 patients with iMC responded to add-back infusion and we achieved complete chimerism at five years. The second patient failed a second AHCT attempt from a different HLA identical sibling donor. Five patients have been followed up with SMC for a median 42 mo.s (range, 14-134). These patients have normal CBC and none of them are receiving immunosuppressive therapy (IST). The attempt to start tapering IST was done after the 12th month and the dose was increased again whenever the recipient signals increased. Serial lineage specific chimerism analysis of $\mathrm{T}$ cells $50.5 \%$ always showed a higher recipient signal compared to non-T cell lineage $79.8 \%$. None of the patients with sMC experienced acute or chronic GVHD. Patients who experienced acute and chronic GVHD were in complete chimeric status.

Conclusions: Serial monitoring of chimerism is mandatory in AHCT for bone marrow failure. After manipulation with IS agents, interventions like a second transplant or add-back are necessary. Prognosis of SMC were not different than complete chimeric patients. We did not observe any late graft rejection after cessation of IST in this population, who did not show any sign of GVHD. 
P628

Antilymphocyte globulin and cyclosporine in patients with acquired severe aplastic anaemia: a single experience report by the Centre National de Greffe de Moelle Osseuse de Tunis

L. Torjemane, A. Abdelkefi, S. Ladeb, A. Lakhal, S. Krimi, O. Ben Gaied, A. Ben Abdeladhim, T. Ben Othman

Centre de Greffe de Moelle Osseuse (Tunis, TN)

Introduction: Intensive immunosuppressive therapy with antilymphocyte globulin (ALG) and cyclosporine (CsA) is the treatment of choice for patients with severe aplastic anemia who do not have histocompatible sibing donors. We reported the results of this approach in such patients treated in our center.

Patients and methods: Twenty four patients with a minimum follow up of 120 days were treated between July 1998 and January 2009. The median age was 20 years (range, 7 to 45 years) and the median neutrophil count was $0.170 \times 10^{9}$ L. Detection of paroxysmal nocturnal hemoglobinuria clones was positive in 2 patients. Horse ALG $15 \mathrm{mg} / \mathrm{kg}$ was given for 5 days and cyclosporine $(5 \mathrm{mg} / \mathrm{kg}$ ) were given daily for up to 12 months or longer. Patients not responding at 180 days, were either offered a second course with rabbit antithymocyte globulin (rATG) in combination with CsA $(n=4)$, or androgens $(n=3)$.

Results: After a median survival of 47 months (range, 4 to 128 months), nineteen patients responded and were alive $(79 \%)$. The median date of the best response achieved was 15 months (range, 6 to 53 months). Twelve patients (50\%) showed a complete response with normalisation of peripheral blood cell counts. Five others were considered partial responders without transfusion support $(20 \%)$. Two patients were alive with transfusion support after relapse $(n=1)$ or evolution to myelodysplastic syndromes $(n=1)$. All the four patients who received a second course of rATG + CsA were responders and only two patients responded to androgens. Evolution to paroxysmal nocturnal hemoglobinuria was rare $(n=2)$ but sensitive to cyclosporine. Actuarial survival was $78 \%$ at 5 years.

Conclusion: These data support the use of a combination immunosuppressive regimen containing both ALG and CsA as first line therapy for severe aplastic anaemia (SAA) patients who lack an HLA-identical sibling.

\section{P629}

Treatment of acquired aplastic anaemia by bone marrow transplantation and antithymocyte globulin: a 5-year single-centre experience

I. Golubovskaya, Y. Zalyalov, A. Ganapiev, B. Afanasyev Raisa Gorbacheva Memorial Institute of Ch (St. Petersburg, $R U)$

Acquired aplastic anemia (AA) is a serious hematologic disorder characterized by peripheral blood pancytopenia and hypocellular bone marrow. Standard treatment strategies for severe AA are immunosuppressive therapy (IST) including antithymocyte globulin (ATG) with cyclosporine A (CsA) and bone marrow transplantation. The decision of initial treatment depends largely on an availability of a HLA-identical sibling donor and patient age. We report our results in patients with severe aplastic anemia who have received bone marrow transplantation or immunosuppressive therapy as the first line therapy.

Materials and methods: A total of 43 patients (median age 21 , range 3 to 49 ) were included in our study. The allogeneic matched related bone marrow transplantation has been performed to 12 patients who had the HLA-identical sibling donor (related BMT-group). If the number of previous blood transfusions was less than 20 we used conditioning treatment ATG (3 infusions of ATGAM at $30 \mathrm{mg} / \mathrm{kg}$ per day) plus cyclophosphamide (50 mg/kg per day for
4 days). If the number of previous blood transfusions was more than 20 we used fludarabine $(30 \mathrm{mg} / \mathrm{m} 2$ per day for 6 days), cyclophosphamide (60 mg/kg per day for 2 days) and ATG (ATGAM $20 \mathrm{mg} / \mathrm{kg}$ per day for 4 days). GvHD prophylaxis consisted of cyclosporine and methotrexate. Thirty one patients without related donor received IST consisting of antithymocyte globulin and cyclosporine (IST-group). ATGAM was administered at a dose of $20 \mathrm{mg} / \mathrm{kg}$ per day for 5 days and CsA was given orally at a dose of $5 \mathrm{mg} / \mathrm{kg}$ per day. The allogeneic unrelated donor transplants were performed in four patients who did not have response after IST (unrelated BMT-group). The hematologic response was estimated in 6 months. The follow-up period was 5 years.

Results: Overall survival after matched related BMT was $92 \%$, in matched unrelated BMT - group was $50 \%$, in ISTgroup $-84 \%$. Acute GVHD (grade II) in case HLA-matched sibling transplantation was noted only at one patient. In unrelated BMT - group, acute GvHD was seen 3 cases (grade II-IV), in the one case acute GvHD resulted in death. One patient died after matched unrelated BMT due to primary graft failure.

Conclusions: Young patients with HLA-identical sibling donor should received allogeneic BMT as the first line therapy. In the absence of the related donor they should receive IST by ATG and CsA. Patients who failed IST should be considered candidate for unrelated BMT as the salvage therapy.

\section{P630}

The results of immunosuppressive therapy in aplastic anaemia - experience of the Romanian Group for Aplastic Anaemia

A.M. Popp, A.D. Moicean, T. Puscariu on behalf of the Romanian Working Group for Aplastic Anemia

Aplastic anemia (AA) is a rare but heterogeneous disorder, is a potentially life-threatening failure of haematopoiesis characterized by pancytopenia and hypocellular bone marrow. For patients aged more than 30 years, treatment with hematopoietic stem cell transplant and immunosupression give the same good results.

We analyzed 49 patients with idiopathic aplastic anemia diagnosed between 1988 and November 2009 still alive on 30 November 2009. The gender ratio male:female was 1 . There were 17 moderate $A A, 15$ severe $A A$ and 17 very severe AA. Twenty five patients were red blood cell transfusions dependent and 15 were platelets transfusions dependent. Behind blood product supplies 2 patients received corticotherapy only, other 27 patients received cyclosporine A (CsA) alone and 2 patients received combined therapy with thymoglobulin, $\mathrm{CsA}$ and recombinant human granulocyte colony stimulating factor. Other 5 patients received combined therapy with CsA, corticosteroids and ciclophospfamide. We found 15 complete remissions, 16 partial remissions and 6 non-responses, meaning an overall of response rate at $63 \%$. The average follow up and survival was of 39 months. During this period of followup there were no second diseases, except one case. There are a good percentage of persistent remissions after first line immunosuppressive therapy (95\%). In conclusion, the immunotherapy has to be the choice for aplastic anemia patients who are not able (for different reasons) to receive a stem cell allotransplant. 
P631

The outcome of 32 consecutive aplastic anaemia patients reported by the Romanian Working Group for Aplastic Anaemia

A.D. Moicean, A.M. Popp, C.M. Dobrea, C. Calugaroiu, A.C. Catana, M. Brinza, C.M. Ghita, T. Puscariu, I. Constantinescu, E. Benedek on behalf of the Romanian Working Group for Aplastic Anemia

Aplastic anemia is a nonmalignant hematological disease very difficult to manage due to the expansive treatments and the special supportive therapy required. According to World Bank data, released in the 2008 report, Romania has an upper-middle-income economy. In Romania the health budget is low and most of expansive treatment for hematological diseases are not large available.

We analyzed the outcome of 32 consecutive patients with aplastic anemia (AA) taken in charge during 2006-2008. Only one patient had aplastic anemia secondary drug intake. Gender ratio male:female was 1 . There were 14 patients with moderate AA, 7 with severe $A A$ and 11 with very severe $A A$, meaning an overall of $56.2 \%$ severe and very severe forms. Fifteen patients were red blood cell transfusions dependent and 14 were platelets transfusions dependent. Behind blood product supplies, 15 patients received corticotherapy only, other 16 patients received cyclosporine $\mathrm{A}(\mathrm{CsA})$ only and 1 patient received combined therapy with thymoglobulin, CsA and recombinant human granulocyte colony stimulating factor. There were 9 complete remissions, 14 partial remissions and 9 non-responses, meaning an overall of $71.8 \%$ response rate. There were 8 deaths, all patients with non-response to the first immunosuppressive therapy, 4 with sepsis and 4 due to lifethreatring hemorrhages.

In Romania, match unrelated donor transplant is not available and only around of $10 \%$ from hematological patients have a sibling donor. In the same time the thymoglobulin treatment for aplastic anemia is not covered by health insurance system and the irradiated blood products are available only in bone marrow transplantation centers. The immunotherapy without transplant would be an option for aplastic anemia patients therapy in low income countries.

\section{Autoimmune diseases}

\section{P632}

Identification of predictive cellular markers for the development of flares after immunoablation and autologous haematopoietic stem cell transplantation for refractory systemic lupus erythematosus

T. Alexander (1), A. Thiel (1), R. Biesen (1), T. Rose (1), H. Radtke (1), G. Burmester (1), R. Arnold (1), A. Radbruch (2), F. Hiepe (1)

(1)Charite University Medicine (Berlin, DE); (2)German Rheumatism Research Center (Berlin, DE)

Background: We could recently show that clinical remissions in SLE after immunoablation and autologous hematopoietic stem cell transplantation (ASCT) are associated with a depletion of the autoreactive immunologic memory and the de novo generation of a juvenile and tolerant immune system. Nevertheless, relapses of SLE may occur.

Purpose: We further followed the clinical, serological and immunological data of our patients to identify predictive cellular markers for the development of flares after ASCT.

Methods: Since 1998, we longitudinally analyzed the immune reconstitution in seven patients with SLE who underwent CD34-ASCT after immunoablation with CYC and rabbit-ATG as part of a monocentric phase I/II clinical trial. Autoantibody titers were evaluated and peripheral T- and B lymphocyte subsets immunophenotyped using multicolor flow cytometry, including assessment of TCR Vbeta-repertoire.

Findings: Clinical remissions (SLEDAI $\leq 3$ ) could initially be achieved in all patients associated with disappearance of antidsDNA antibodies, recurrence of thymic naïve CD31 + cD4 + T cells of FoxP3 + Tregs with a polyclonal TCR Vbeta-repertoire, and normalization of B-cell disturbances such as expansion of circulating plasma blasts.

While stable long-term clinical remissions could be maintained in patients for up to 11 years after ASCT, three patients suffered a relapse of SLE: p\#3 (+18 mo), p\#4 (+80 mo) and p\#7 $(+36 \mathrm{mo})$. These patients showed recurrence of anti-dsDNA antibodies and ANA, in part with different specificities compared to baseline analysis. Flow cytometric analyses revealed an expansion of memory $B$ cells and circulating plasma blasts and increased coexpression of Siglec-1 on monocytes preceding the flare, and a predominance of CD45RO + memory T cells with a restricted CD4 + TCR-Vbeta-repertoire (distinct from pretransplant) and significantly expansion of FoxP3 + Tregs with reduced coexpression of CD25.

Conclusion: SLE-patients who are at high risk to develop lupusflares after immunoablation and ASCT may be identified by plasma blast expansion, increasing expression-levels of Siglec1 on monocytes as a surrogate marker for interferon-signature and expansion of CD25- FoxP3 + Tregs. The development of antinuclear antibodies with new specificities and a different oligoclonal TCR Vbeta-repertoire compared to pre-transplant may illustrate a de novo development of SLE rather than disease reactivation in these patients.

\section{P633}

Autologous haematopoietic stem cell transplantation induces a prolonged and profound modification of gene expression profiles in patients with multiple sclerosis R. Fedele (1), C. Russo (1), M. Cuzzola (1), B. Mazzanti (2), M. Di Gioia (2), E. Massara (1), C. Rigolino (1), A. Bosi (2), U. Aguglia (1), R. Saccardi (2), P. lacopino (1)

(1)Azienda Ospedaliera B.M.M. (Reggio Calabria, IT); (2)Ospedale Careggi (Florence, IT)

Background: Autologous hematopoietic stem cell transplantation (AHSCT) is being explored in the treatment of severe multiple sclerosis (MS) as a means of high-dose immunosuppressive therapy followed by full immune recovery after the engraftment of the AHSC. AHSCT may be beneficial for the possible 'resetting' of the immune system, thereby becoming tolerant against self-antigens. While there is evidence that long-term suppression of inflammatory activity in MS patients (pts) who receive AHSCT are associated with profound immunological changes (Muraro et al., JEM 2005), very little is known about changes in the gene expression profile (GEP) induced by AHSCT.

Aim of the study. To determine early and late GEP changes, investigating the whole blood from MS pts, before and after receiving AHSCT.

Methods: We analyzed expression changes in 47 genes associated with cell survival functions, transduction signals, intercellular adhesion, immune regulation in 8 patients with secondary progressive or relapsing-remitting MS who underwent AHCT not responding to conventional therapies. Peripheral AHSC were obtained by leukapheresis after mobilization with cyclophosphamide and G-CSF. BEAM + ATG conditioning was used according to EBMT guidelines. Serial samples of peripheral blood were collected before and at 1, 6, 12 and 24 months after AHSCT. The same samples were collected also on the day of AHSC infusion and on the day when complete engraftment was shown. We have used a TaqMan® Low Density Array based on comparative ddCT method to perform a global gene expression.

Results: AHSCT was well tolerated by pts without transplant-related death. At 1 year follow-up (r. 5-39 months) 6 pts improved, one patient showed stabilization and only one patient experienced a deterioration of 0,5 point of EDSS. Base- 
line GEP was similar in all pts. Immediately after transplant, in all pts there were profound modifications of many genes and at 6-24 months after AHSCT a subset of them remained modified. Particularly, CD52 was up-expressed before transplant and stayed markedly down-regulated until 12 and 24 months except in 2 pts, one of them worsening after HSCT.

Conclusions: AHSCT improves clinical outcome in pts affected by severe MS. Our data demonstrates how AHSCT modulates the immune system particularly in responding pts. CD52 may have a central role in pathogenesis of severe MS. Further studies are needed in a larger controlled cohort of patients to better explain these mechanisms.

\section{P634}

Autologous (T-cell depleted) or allogeneic haematopoietic stem cell transplantation for treatment of recalcitrant, severe autoimmune/rheumatic disorders in children?

Z. Nademi (1), M. Slatter (1), A. Gennery (1), T. Flood (1), S. Hambleton (2), J. Clark (1), M. Friswell (3), H. Foster (1), A. Cant (1), M. Abinun (3)

(1)Newcastle upon Tyne Hospitals NHS Foundation Trust (Newcastle upon Tyne, UK); (2)Newcastle University (Newcastle upon Tyne, UK); (3)Newcastle upon Tyne NHS Foundation Trust (Newcastle upon Tyne, UK)

Introduction: Autologous haematopoietic stem cell transplantation (HSCT) following immunosuppressive conditioning and T cell depletion of the graft is "a valuable option for individual" children with severe autoimmune/rheumatic disease recalcitrant to currently used treatments, although recent evidence of only transient benefit suggest this is not a curative procedure. Potentially curative allogeneic HSCT is "generally not recommended" and unacceptable for the paediatric rheumatology community, even for selected patients at the very severe end of the disease spectrum, as graft-versus-host disease (GvHD) and transplant related mortality (TRM) are considered too high a risk [1]

Results: Our data on autologous T cell depleted HSCT in 6 children with juvenile idiopathic arthritis (JIA) have been recently published [2]: 4 are long-term survivors, 3 in complete drugfree remission, 1 relapsed, and 2 died. We compare these with the results of allogeneic HSCT in 8 children with well defined, severe and treatment-resistant autoimmune and autoinflammatory diseases: systemic lupus erythematosus (SLE (1), immunodysregulation, polyendocrinopathy, enteropathy, Xlinked (IPEX) syndrome (3), autoimmune lymphoproliferative syndrome (ALPS) (3), complex periodic fever syndrome due to both mevalonic kinase (MVK) and TNF-receptor (TRAPS) gene mutations (1). Half received HSC from an HLA-matched sibling and half from an unrelated donor; $5 / 8$ received modified myeloablative or reduced intensity conditioning. One child died; 7 achieved remission from their underlying disease: 5 are cured with 2-6 yr follow-up, 1 with slipping donor chimerism requires further treatment and 1 is still $<1 \mathrm{yr}$ post-HSCT. Acute GVHD was frequent but resolved with therapy.

Conclusion: The benefit-risk ratio for allogeneic HSCT using contemporary protocols in specialized centres may have surpassed T-cell-depleted autologous HSCT for severe autoimmune/rheumatic disease.

References:

1. Krauss AC, Kamani NR. Hematopoietic stem cell transplantation for pediatric autoimmune disease: where we stand and where we need to go. Bone Marrow Transplant 2009; 44:137-42

2. Abinun $\mathrm{M}$ et al. Autologous $\mathrm{T}$ cell depleted haematopoietic stem cell transplantation in children with severe juvenile idiopathic arthritis in the UK (2000-2007). Mol Immunol 2009; 47:46-51
P635

Single-centre experience of haematopoeitic stem cell transplantation for patients with complex autoimmune enteropathies

Z. Nademi (1), M. Slatter (1), D. Barge (1), S. Hodges (1), S. Bunn (1), J. Thomas (2), H. Harvey (1), S. Hambleton (3), T. Flood (1), A. Cant (1), M. Abinun (1), A. Gennery (1)

(1)Newcastle upon Tyne Hospitals NHS Foundation Trust (Newcastle upon Tyne, UK); (2)Newcastle upon Tyne NHS Foundation Trust (Newcastle upon Tyne, UK); (3)Newcastle University (Newcastle upon Tyne, UK)

Background: Complex autoimmune enteropathies encompass a wide range of disorders including X-linked immune dysregulation, polyendocrinopathy, enteropathy (IPEX) syndrome with FOXP3 gene mutation, IPEX-like syndrome (similar features as IPEX without gene mutation) and other unclassified autoimmune enteropathies.

Method: A retrospective study of patients with complex autoimmune enteropathies who underwent hematopoeitic stem cell transplantation (HSCT) at Newcastle General Hospital, one of two nationally designated centres for such procedures in the UK, was performed.

Results: 13 patients were identified who fulfilled the inclusion criteria; 2 (15\%) IPEX, 8 (62\%) IPEX-like and 3 (23\%) unclassified autoimmune enteropathy. Median age at the time of HSCT was 2.5 yearss, ranged between 5 months to 19.5 years old. $9 / 13(69 \%)$ patients were male. All underwent HSCT with $10 / 13(77 \%)$ unrelated (URD) including 3 cord transplants and $3 / 13(23 \%)$ matched sibling (MSD). The conditioning regimen used was Fludarabine/Melfalan in $6(46.2 \%)$, Busulfan/Cyclophosphamide in 3 (23\%), Treosulfan/Cyclophosphamide in 2 (15.4\%) and Treosulfan/Fludarabine in 2 (15.4\%). 100\% donor chimerism was achieved in $10(77 \%)$ patients including one after the unconditioned boost infusion 27 days post HSCT. 3 $(23 \%)$ patients have mixed donor chimerism with $60 \%$ donor T cells. $10(85 \%)$ patients are alive with resolution of enteropathy, and have discontinued immunosuppression-1 remains an inpatient after HSCT $<120$ days ago. $5 / 13(38 \%)$ patients had no graft versus host disease and $6 / 13(46 \%)$ had only grade IIIII skin GvHD. 2 (15\%) died from complications related to treatment og grade III skin ang gut GvHD.

Conclusion: HSCT can be curative in patients with severe complex autoimmune enteropathy. GvHD is a common significant complication which can be life threatening.

\section{P636}

Bone marrow mesenchymal stromal cell characteristics in childhood autoimmune diseases

M. Pesmatzoglou, H. Dimitriou, E. Stiakaki, M. Tsirigotaki, L. Sifaki, N. Katzilakis, M. Kalmanti

Pediatric Hematology/Oncology University of Crete (Heraklion, $G R)$

Mesenchymal stromal cells (MSC) compromise a promising source for cellular therapy due to their easy ex vivo expansion. A unique property of these cells is their immunomodulatory and anti-inflammatory function. Based on the successful administration of MSC in children with therapy-resistant acute GVHD, it is important to define the properties of MSC from patients with autoimmune disease (AD) and their possible involvement in the disease pathogenesis with the final goal being their use as therapeutic agents. Although the characteristics and the role of MSC have been studied in adults with $A D$, there are no reports in childhood AD. The aim of this study was the investigation of the phenotypical and functional characteristics of bone marrow (BM)derived MSC from children with AD. Patients and methods BM mononuclear cells (MNC) from 6 children with $A D$, and a control group (CTL) of 8 children with solid tumours without BM involvement, were cultured under conditions that favor MSC growth for five passages (P1-P5). Cell doubling time (DT), surface antigen expression, CFU-F colony development and differentiation 
ability towards adipo-, osteo- and chondrocytes were evaluated. Results AD-MSC and CTL-MSC exhibited similar fibroblastlike morphology. Immunophenotypic analysis showed that both groups express MSC-related markers but not haemopoietic markers. Their proliferation capacity as expressed by DT was similar. Specifically, CTL-MSC in the MNC fraction (D0) required $18 \pm 1$ days to reach confluency and AD-MSC $17.16 \pm 0.87$ days $(P=0.558)$. The DT in all passages was no statistically significant ( $2.50 \pm 0.42$ vs. $2.33 \pm 0.26, P=1.00 \mathrm{P} 2$ ) between the groups. ADMSC developed less medium ( $P=0.026 \mathrm{P} 1, P=0.040 \mathrm{P} 2)$ and large $(P=0.032 \mathrm{P} 3)$ sized colonies in the first 3 passages, indicative of impaired clonogenic potential. CFU-F numbers decreased with the passage progression in both groups. Finally, both CTLMSC and AD-MSC had tri-lineage differentiation potential. Conclusion BM-derived MSC from children with AD exhibit the same phenotypic, proliferative and differentiation potential, compared with the control group. In view of these results, MSC are likely to be a valuable therapeutic option for severe $A D$ refractory to standard treatment. However, further characterization of the properties of MSC and assessment of their immunomodulatory properties are required in order to offer such a therapeutic approach to pediatric autoimmune diseases.

\section{P637}

Development of secondary autoimmune disorders after immunoablation and haematopoietic stem cell transplantation for refractory systemic lupus erythematosus

T. Alexander, S. Schneider, U. Schneider, S. Ziemer, H. Radtke, G. Burmester, R. Arnold, F. Hiepe

Charite University Medicine (Berlin, DE)

Introduction: Stem cell therapy for autoimmune diseases is rapidly developing with promising clinical results. However, it is increasingly recognized that these patients have an added propensity to develop secondary autoimmune disorders, distinct from the underlying autoimmune disease, given their genetic predisposition to autoimmunity.

Methods: As part of a monocentric phase I/II clinical trial, seven patients received immunoablation with $C Y C$ and rabbit-ATG and CD34-ASCT for refractory SLE. Patients were evaluated at 3,6, 9 and 12 months post-transplant and yearly thereafter, including clinical examinations, laboratory and serologic analyses and immunophenotyping of peripheral blood lymphocyte subsets.

Results: With a median follow-up of 90 months, clinical remission could be achieved in all seven patients, defined as SLEDAI $\leq 3$ and in the absence of immunosuppressive drugs. One patient died due to early $(+3 \mathrm{mo})$ infectious complications. From the other 6 patients, three developed secondary autoimmune disorders while in stable clinical remission of SLE: Graves' disease ( $p \# 1$, $+20 \mathrm{mo}$ ), autoimmune thyreoiditis ( $\mathrm{p \# 2}+23 \mathrm{mo}$ ) and acquired factor VIII hemophilia (FVIII AH, p\#7, +9mo) with autoantibodies directed against TSH-receptor, thyroid-peroxidase and factor VIII, respectively. Flow cytometric analyses revealed a significant expansion of peripheral blood CD27++ cD20- plasma blasts during development of the secondary autoimmune diseases in case of the FVIII AH. FVIII AH was refractory to treatment with rituximab and immunoadsorption, cyclophosphamide and intravenous immunoglobulin according to the modified Bonn-Malmö protocol, but disappeared completely during SLE-reactivation with waves of newly generated plasmablasts and high titers of anti-dsDNA antibodies $+36 \mathrm{mo}$ after ASCT.

Conclusion: We propose that the development of these primarily antibody-mediated autoimmune disorders after immunoablation and ASCT in SLE may be promoted on a genetic background through occupation of empty plasma cell niches by differentiated autoantigen-specific plasma cells during the phase of lymphodepletion early after immunoablation. The spontaneous disappearance of the FVIII-inhibitor during the lupus-flare may illustrate a direct competition for plasma cell survival niches in the bone marrow with recurring "lupus-specific" plasma cells outcompeting the FVIII-inhibitor.

\section{Solid tumours}

\section{P638}

Impaired NK cell infiltration into the tumour microenvironment by cancer cell dependent NK cell elimination involves CD16

G. Sconocchia (1), I. Zlobec (2), B. Capuani (3), S. Caratelli (3), G. Donadel (3), R. Arriga (3), B. Iorio (3), L. Terracciano (2), D. Lauro (3), G.C. Spagnoli (2)

(1)CNR, Institute of Neurobiology and Molecular Medicine (Rome, IT); (2)University of Basel (Basel, CH); (3)University of Tor Vergata (Rome, IT)

Allogeneic and autologous NK cells may kill MICA/B + myeloid leukaemia (ML) and colorectal carcinoma (CRC) cells suggesting that NK cell therapy may be utilized for the treatment of MICA/B + epithelial malignancies. To hypothesize NK cell therapy for CRC, there is a need to show NK cell infiltration in the MICA/B + tumor microenvironment (TM). Utilizing a tissue microarray (TMA), we investigated MICA/B expression and NK cell clinico-pathological role in 1414 CRC. More than $90 \%$ of CRC over-expressed MICA/ B molecules. CD16 and CD56 antigens staining showed that only $11.8 \%$ of CRC punches were infiltrated by $<4$ CD $16+$ cells while $61.8 \%$ of CRC punches were infiltrated by $<4$ CD56 + cells $(P=2.2 \times 10-13)$. Similarly, 1070 out of 1259 evaluable CRC contained $\leq 4$ CD $57+$ cells while 189 CRC had $>4$ CD 57 + cells suggesting the myeloid origin of the CD16 + infiltrate. Data validation was obtained by analyzing CD $16+$ cells from 14 freshly removed $\mathrm{CRC}$. The percentage of CD16 + cells identified was higher than that observed for CD56+cells $(1.83 \pm 0.31$ vs. $0.14 \pm 0.07 \%)$ of the CRC $(n=5, P=0.005)$. NKG2D and NKp46 markers were expressed in comparably $(P=0.45)$ low percentages of cells $(\leq 0.1 \%)$ and were accompanied by CD33, CD68 and CD32 markers suggesting that the presence of NK cells in the CRC was negligible. The impact of NK cell infiltration in the CRC TM of CRC patients (pts) overall survival (OS) was evaluated. We found no significant OS differences between CRC with score1 ( $\leq 4$ cells/punch) and score2 ( $>4$ cells/punch) for CD56 + infiltration $(P=0.264)$ and $\mathrm{CD} 57+$ cell infiltration $(P=0.583)$ respectively. To identify mechanisms by which NK cells failed to infiltrate MICA/B over-expressing CRC cells, we assessed the effect of the HCT116, CRC cells, ML2, ML cells, and Caki-1, RCC cells on NK cells upon conjugation. Malignant cells induced NK cell apoptosis, CD16 down-regulation and NK cell elimination. Malignant cell dependent elimination was restricted to CD16+CD56 + NK cell subset without affecting CD16-CD56 + NK and CD16 + NK-T cells while the ligation of CD16 antigen with a mAb prevented NK cells from ML2 induced NK cell elimination, suggesting that CD16 antigen was directly involved. Since NK cells do not easily infiltrate the CRC TM and are rapidly eliminated by malignant cells, the usage of NK cell immunotherapy in CRC is questionable. Novel strategies overcoming the indicated problems could lead to a feasible NK cell immunotherapy of CRC and will improve the GVL effect of NK cell in ML pts.

\section{P639}

Allogeneic stem cell transplantation from HLA identical donor in high-risk sarcoma

A. Prete (1), R. Rondelli (1), F. Locatelli (2), D. Caselli (3), E. Lanino (4), C. Messina (5), R. Masetti (1), P. Pedrazzoli (6), S Ferrari (7), A. Pession (1), F. Fagioli (8)

(1)Università degli Studi (Bologna, IT); (2)Università degli Studi (Pavia, IT); (3)Ospedale dei Bambini "Mayer" (Florence, IT); (4)Ospedale G. Gaslini (Genoa, IT); (5)Università degli Studi (Padua, IT); (6)Istituto Clinico Humanitas IRCCS (Milan, IT); (7)IOR (Bologna, IT); (8)Ospedale S. Anna (Turin, IT)

Objectives: To evaluate the feasibility of an allogeneic stem cell transplantation (ASCT) from HLA identical donor in patients (pts) affected by resistant or relapsed rabdhomyosarcoma 
(RMS) or Ewing sarcoma (ES) in which the 1 year probability of survival is lesser than $5 \%$.

Methods: 20 pts, median age $16(6-22)$ years, affected by resistant (3) or relapsed (8) RMS and resistant (6) or relapsed (3) ES, were enrolled and submitted to an ASCT after a conditioning regimen consisting of Thiotepa + Melphalan + Fludarabine or Cyclophosphamide. The donor was an identical sibling in 14 cases or an unrelated donor (UD) in 6. At time of transplant 18 pts were in PR and 2 (1 RMS and $1 \mathrm{ES}$ ) in progressive disease. Graft versus host disease (GVHD) prophylaxis consisting of Cyclosporin A + Anti-lymphocytic serum and short term methotrexate in UD setting. SC sources were bone marrow in 13 cases and peripheral blood in 7 .

Results: The reconstitution of bone marrow function was obtained in all the 20 pts. Acute GVHD of grade II-IV occurred in 6 pts and a complete marrow donor chimerism was observed after 40 and 60 days in sibling and UD setting respectively. After a median follow-up of $27(1-58)$ months for ES and 4 (1-18) months for RMS, 8 pts (5 RMS and 3 ES) relapsed, 14 dead ( 7 for progressive disease and 7 for TRM) and 6 pts are alive and well. The 1 years probability of OS (SE) and EFS (SE) were $0.43(0.19)$ and $0.29(0.17)$ for ES and $0.37(0.16)$ and 0.14 $(0.12)$ for RMS. The 100 days probability of TRM was 0.00 for ES and 0.29 (0.14) for RMS.

Conclusions: Despite an high TRM in RMS pts, our experience shows the feasibility of an allogeneic ASCT in relapsed or resistant sarcomas even in heavily pre treated pts. On the basis of these observations a prospective national study is now open on behalf of Associazione Italiana di Ematologia ed Oncologia Pediatrica (AIEOP), Gruppo Italiano Trapianto Midollo Osseo (GITMO) and Italian Sarcoma Group (ISG), to evaluate the efficacy of a reduced intensity conditioning regimen followed by ASCT from related or unrelated HLA identical donor for poor prognosis at diagnosis SE and RMS in paediatric and adult.

\section{P640}

Comparison of post-transplant events and outcomes in patients affected by haematological or solid malignancies undergone to a non-myeloablative allogeneic haematopoietic stem cell transplantation in progressive disease D. Caravelli (1), F. Carnevale-Schianca (1), B. Bruno (2), V. Coha (1), R. Sorasio (3), G. Grignani (1), D. Rota-Scalabrini (1), M. Fizzotti (1), M. Gatti (1), L. Giaccone (2), A. Capaldi (1), A. Cignetti (1), D. Sangiolo (1), M. Boccadoro (2), M. Aglietta (4) (1)I.R.C.C. (Candiolo, IT); (2) Università di Torino (Candiolo, IT); (3)Divisione di Ematologia (Cuneo, IT); (4)I.R.C.C. Univeristà di Torino (Candiolo, IT)

The first studies of non-myeloablative allogeneic hematopoietic stem cells transplantation (NMAHCT) in very advanced solid tumors offered evidences of the existence of a graft versus solidtumor effect. Despite these data the low overall response rate and the toxicity reported convinced the oncological community that NMAHCT should be offered only in haematological malignancies. To address these issues, we compared the rate of posttransplant events between patients affected by haematological (Hpts.) or solid malignancies (STpts.) transplanted in a state of refractory/progressive disease. We analysed data on 89 patients (64 Hpts, 25 STpts). Disease status at transplant was: responsive disease (29 pts), refractory but not progressing (22) and progressing (38). All STpts were progressive. Seventy patients $(79 \%)$ were transplanted accordingly to Seattle-regimen and 19 $(21 \%)$ with Fludarabine/Cyclophosphamyde. Patients received

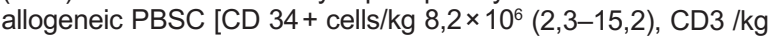
$\left.4,1 \times 10^{8}(1,1-7,3)\right]$ from a HLA identical donor. GVHD prophylaxis was calcineurine inhibitor + Mycophenolate Mofetil in 70(79\%) or + methotrexate in 19(21\%). Myelosuppression and non-hematological toxicity were mild and short-lasting in both cohort of patients. All patients engrafted (median chimerism on CD3 cells at day 90 was $90 \%$ in both cohorts). A total of 60 episodes of bacterial-infections $(67 \%$ in Hpts. and $76 \%$ in STpts. $P=0.42), 47$ of CMV-reactivation (58\% in Hpts and $40 \%$ in STpts $P=0.13$ ) and
19 of fungal-infections ( $20 \%$ in Hpts and $24 \%$ in STpts. $P=0.70)$ occurred. Grades II-IV acute GVHD developed in 47 patients (53\%, 56\% Hpts and 44\% STpts., $P=0.3$ ); extensive chronic GVHD occurred in 22 patients (25\%). Transplant related mortality at day +100 was absent, cumulative TRM in Hpts was $29 \%$ while in STpts. was $20 \%$. No difference was found in immune-reconstitution at day +180 , a faster immune recovery in $\mathrm{CD} 3, \mathrm{CD} 3 / 8$ and CD3/4 was described in STpts at day $+90(P=0.016)$. In a multivariate analysis only status at transplant resulted associated with a better overall survival $(\mathrm{OR}=2.19 \mathrm{Cl} 1.0-4.53 ; P=0.035)$. The analysis suggests that between hematological patients and solid tumor pts. does not exist a difference in post-transplants events; based on these data NMAHCT in STpts should not be prematurely abandoned but investigated in clinical studies admitting only pts. in SD or with low tumor-burden.

\section{P641}

Long-term outcome of salvage high-dose chemotherapy in $\mathbf{1 0 0}$ germ cell tumour patients according to the Indiana University Prognostic Score

U. De Giorgi (1), G. Papiani (2), R. Salvioni (3), M. Marangolo (2), G. Rosti (4)

(1)Istituto Tumori Romagna-IRST (Meldola, IT); (2)Istituto Oncologico Romagnolo (Ravenna, IT); (3)Istituto Nazionale Tumori (Milan, IT); (4)Ospedale Ca' Foncello (Treviso, IT)

Background: We analyzed the long term results of 100 germ cell tumors (GCT) patients with poor prognostic factors who received either a salvage single high-dose chemotherapy (HDCT) cycle or a salvage low dose-intensity multicycle HDCT regimen according to the recent Indiana University (IU) prognostic index for tandem HDCT based on line of therapy, platinum refractoriness and advanced IGCCCG stage (Einhorn, NEJM 2007).

Patients and methods: Between 1986 and 2003, 100 GCT patients received salvage HDCT consisting of high-doses of carboplatin, etoposide \pm cyclophosphamide or ifosfamide. Twenty-four patients underwent a second HDCT cycle and in one case, a third cycle was given with a median interval time of 6 weeks (range, 5-10).

Results: With a median follow-up of 8 years (range, $3-17) ; 6$ of $32(19 \%)$ patients with resistant GCT and 1 of $19(5 \%)$ patients with cisplatin-refractory disease have been continuously disease-free, while none of 16 patients with absolutely cisplatin-refractory GCT have been alive at one year from HDCT treatment. According to the IU prognostic model, a continuously disease-free status was reported in 12 of $17(71 \%)$ low risk (nearly $80 \%$ in the IU cohort), in 13 of $33(39 \%)$ intermediate risk (nearly $60 \%$ in the IU cohort), and 3 of 40 (7.5\%) high risk patients (nearly $40 \%$ in the IU cohort). Our results were substantially inferior to those of IU for refractory tumors (1 of 19 , $5 \%$, patients disease-free vs. 18 of $40,45 \%$, in the IU cohort) and relapsed tumors treated in third-line or later setting (4 of 39, $10 \%$, disease-free vs. 22 of $49,45 \%$, in the IU cohort).

Conclusions: The IU prognostic model for tandem HDCT did categorize our patients treated with a single HDCT cycle or low-dose intensity regimens in a very similar manner, but with inferior overall results. Tandem HDCT regimen only must be considered a major option for refractory GCTs and relapsed tumors in third-line or later therapy.

\section{P642}

Mature results of adjuvant high-dose dense chemotherapy according to baseline features in breast cancer U. De Giorgi (1), L. Frassineti (1), B. Kopf (1), F. Zumaglini (2), G. Rosti (3), D. Amadori (1), M. Marangolo (2)

(1)Istituto Tumori Romagna-IRST (Meldola (FC), IT); (2)Istituto Oncologico Romagnolo (Ravenna, IT); (3) Ospedale Ca' Foncello (Treviso, IT)

Background. The role of adjuvant high-dose chemotherapy and its efficacy according to baseline features in breast cancer has 
not been yet established in breast cancer. We report mature results of adjuvant high-dose dense chemotherapy (HDDC) with epirubicin and paclitaxel and evaluate predictive features in order to identify patients who might benefit from HDDC.

Patients and methods. In a phase II feasibility study, we investigated a HDDC regimen consisting of a mobilizing course with epirubicin and paclitaxel plus filgrastim, followed by three high-dose courses with epirubicin $\left(150 \mathrm{mg} / \mathrm{m}^{2}\right)$, preceded by dexrazoxane and paclitaxel $\left(400 \mathrm{mg} / \mathrm{m}^{2}\right)$ with peripheral blood progenitor cell support every 16-19 days in breast cancer patients with $>4$ involved axillary lymph nodes.

Results. Overall, 73 of 76 enrolled patients were evaluable, median age was 50 years (range 26-62), median number of positive nodes was 15 (range 4-38). Estrogen receptor status was evaluable in all cases, while HER2 status by IHC and/or FISH was evaluable in $64(88 \%)$ cases and results amplified in $21(33 \%)$. At a median follow-up time of 5.5 years (range 3.5-9.8), a continuously disease-free status was reported in 9 of $21(43 \%)$ patients with HER2-amplified tumours and 21 of 43 $(49 \%)$ with HER2-normal tumours. Within the latter subgroup, a disease-free status was reported in 3 of $9(33 \%)$ patients with negative hormone receptors (triple negative tumors) and 18 of $34(53 \%)$ with positive hormone receptors.

Conclusion. Epirubicin and paclitaxel escalated-doses and dose-intensity might not contribute to the benefit of HDC regimens in triple negative breast cancer.

\section{P643}

High-dose chemotherapy supported with autologous haematopoietic stem cell transplantation in patients with advanced germ cell tumours not rersponding to standard treatment. The Warsaw experience

T. Sarosiek, P. Rzepecki, C. Szczylik, E. Bartczak

Institute of Health Services (Warsaw, PL)

To find factors influencing the efficacy and toxicity of the high dose chemotherapy (HDC) and autologous haemopoietic stem cell transplantation (ASCT) in patients with relapsed or cisplatin- resistant advanced germ cell tumors.

Patients and methods: We performed 65 procedures of HDC+ASCT in 51 relapsed or refractory germ cell cancer patients. Our patient population was heavily pretreated with the median of 3 lines of previous systemic therapy and 9 cycles of cisplatin - based chemotherapy. The treatment consisted of one (in 37 patients) or two sequential (14 pts) cycles of highdose carboplatin $\left(1500 \mathrm{mg} / \mathrm{m}^{2}\right)$, etoposide $\left(1800 \mathrm{mg} / \mathrm{m}^{2}\right)$ and alkilating agent (cyclofosfamide $6,4 \mathrm{~g} / \mathrm{m}^{2}$ during 55 procedures, ifosfamide $16 \mathrm{~g} / \mathrm{m}^{2}$ in five patients, or none in four pts) during the four consecutive days. An autologous haemopoietic stem cell infusion was performed at day 6 with the median of $2 \times 10^{2} /$ kg CD34 + cells given.

Results: The most frequent kinds of non-hematological toxicity were: nausea and vomiting, alimentary tract mucositis and renal or hepatic toxicity. In eight of 65 procedures $(12,3 \%)$ treatment toxicity was a direct cause of patient's death. The treatment toxicity increased with Beyer prognostic score, number of chemotherapy cycles administered in previous treatment and ECOG performance status. Responses and survivals: complete durable remission was achieved in $16(31,37 \%)$ patients, partial - in $20(39,215 \%)$, stable disease in two (4\%). 12-months overall survival rate (OS) was equal to $58,5 \%$, and 24 -month OS - 50,6\%. 12- and 24-month progression free survivals (PSF) were $58 \%$ and $54,5 \%$. The only independent prognostic factors for overall survival (OS) were beta-HCG level above 1000 $\mathrm{mlu} / \mathrm{ml}$ and elevated AFP level before transplantation, IGCCCG prognostic group at diagnosis. Beyer prognostic score was an independent prognostic factor for PFS.

Conclusion: High-dose chemotherapy can be curative for some relapsed GCT patients, however it can not be recommended for all patients. Especially those, with upfront poor prognosis (according to Beyer prognostic score), high beta-HCG levels or poor ECOG performance status do not benefit from this kind of treatment.

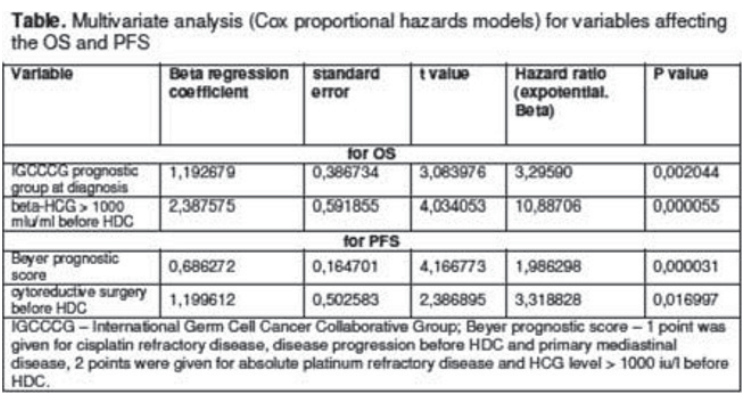

P644

Phase I study of intraperitoneal MHC unrestricted adoptive cell therapy with TALL-104 cells in patients with peritoneal carcinosis

C. Bengala, V. Rasini, R. Sternieri, M. Dominici, A. Andreotti, R. Gelmini, L. Cafarelli, P. Bevini, A. Berti, P. Conte

University Hospital (Modena, IT)

Background: TALL-104 is an irradiated human leukemic T cell line (CD3 +, CD4- CD8 +, CD56 +, CD16-) grown in IL-2-containing medium, that has the ability to kill tumor cells in preclinical models in a MHC unrestricted way. A phase I trial in metastatic breast cancer patients, has shown that multiple i.v. infusions (infs) of TALL-104 cells can be given safely. In order to optimise the tumor:effector cell ratio, we have designed a phase I study of intraperitoneal administrations of g-irradiated TALL-104 cells.

Methods: Patients (pts) with peritoneal carcinosis from solid tumors resistant to standard treatment were eligible for the study. The treatment included 5 i.p. infs (day 1, 3, 5, 15, 30) and the study was designed to test three cell dose levels: $1 \times 10^{8}$, $5 \times 10^{8}, 2.5 \times 10^{9}$. End points were: safety, kinetic of TALL-104 cells on ascites (if present) and peripheral blood (PB), levels of cytokines (TGF-b, GM-CSF, IL-2, IL-4, IL-10, IFN-g, TNF-a and -b, HGF, sIL-2R, sICAM-1) on ascites and serum, and immunological monitoring.

Results: Fifteen pts have been treated: 8 with ovarian and 7 with GI cancer. Five pts have been treated at each dose level. No treatment-related adverse events were observed. TALL-104 cells were detected in ascites (100\% of the pts) and PB (3.3\% of the pts) up to $48 \mathrm{hrs}$ after the infs. Cytotoxicity of autologous MNC showed an increase up to $5 \%$ at day 3 through 7 and it was still evident at day 30. HLA-DR +/CD14 + cells increased up to $21 \%$ and $30 \%$ in the serum and ascites respectively at day 7 through 15 . GM-CSF, IL-2, IL-4, IL-10, IFN-g, TNF were not detectable neither in serum nor in ascitic fluid. sIL-2R showed an increase up to $22 \%$ and $109 \%$ in serum and ascites respectively. IL-10 showed an increase up to $34 \%$ at 1 st dose level and a decrease down to $20 \%$ and $40 \%$ in ascites and serum respectively at 2 nd dose level. TGF-b 1 and -b2 showed an increase both in serum and ascites at 1st and 2nd dose level. sICAM-1 showed an increase up to $22 \%$ at 1 st dose level and a decrease down to $41 \%$ at 2 nd dose level. Six patients had a confirmed stable disease with a median duration of response of 5 months (3-7).

Conclusion: These results show that the i.p. infusion of TALL104 is safe. Moreover, the increased autologous cell-mediated cytotoxicity and the levels of soluble cytokines after i.p. infs indicate that TALL-104 cells may elicit potential antitumor activity and deserve further evaluation in patients with a less severe stage of disease. 


\section{P645}

T-cell therapy with anti-tumour cytotoxic T-cell lines in a patient with metastatic renal cell carcinoma

D. Montagna (1), P. Pedrazzoli (2), I. Turin (1), R. Schiavo (2), E. Montini (1), C. Tullio (2), S. Secondino (2), I. Schiavetto (2), L. Caliogna (1), F. Locatelli (1), R. Maccario (1), S. Siena (2)

(1)Fondazione IRCCS Pol. San Matteo (Pavia, IT); (2)Ospedale Niguarda Cà Granda (Milan, IT)

Despite recent advanced in the medical treatment, the prognosis of metastatic renal cell carcinoma (RCC) remains unfavorable. Although RCC is considered to be one of the most immunogenic tumors $\mathrm{T}$ cell-based immunotherapy in metastatic RCC have shown very limited success. We have recently demonstrated the feasibility of a large scale expansion of anti-tumor cytotoxic T-cell (CTL) lines, derived from peripheral blood of patients affected by different types of solid tumor, by stimulation of CD8-enriched lymphocytes with DC pulsed with whole apoptotic patients tumor cells in the presence of IL-12 and IL-7; anti-tumor CTL are then rapidly expanded in an antigen independent way (Turin et al. Cytotherapy 2007). A 61 year-old patient affected by renal cell carcinoma (RCC) has been treated within a pilot study aimed to evaluate the feasibility and the safety an immunotherapy approach based on the infusion of anti-tumor CTLs. At the time of starting CTL immunotherapy, patient had large tumor masses (kidney, soft tissues) progressing after failing conventional therapies available at that time. Patient received lymphodepleting chemotherapy consisting in fludarabine and cyclophosphamide followed by CTL infusions, on day +14 and +28 and every 2 to 6 months thereafter. He received 17 CTL infusions (median $\mathrm{n}$. of T-cells $1.600 \times 106$, range $830-4.000$ ) and remained in stable disease (RECIST criteria) for 3 years when disease progression required nephrectomy. Following surgery CTL therapy was reintroduced and patient remained in stable disease with a follow up of further two years. CTL infusions have been well tolerated with no acute adverse events and no evidence of clinically significant autoimmunity. Immunological monitoring demonstrated that CTL infusions could induce an increase in specific anti-tumor response measured in the peripheral blood.

In our patient with RCC multiple antitumor CTL infusions proved safe and capable of inducing immunological response and a potential clinical benefit. Because our immunotherapy approach involves the use of autologous whole tumor cells, it requires neither the definition of specific tumor antigens nor the presence of defined HLA specificities. For these reasons this strategy could be potentially applicable for the treatment of patients with a wide variety of solid tumors.

\section{P646}

A proposal for antibody-based immunotherapy combined with haploidentical stem cell transplantation for high-risk neuroblastoma

P. Lang (1), M. Pfeiffer (1), R. Ladenstein (2), H. Lode (3), I. Müller (1), H.-M. Teltschik (1), T. Feuchtinger (1), $R$. Handgretinger (1)

(1)University Children's Hospital (Tubingen, DE); (2)St Anna Children's Hospital (Vienna, AT); (3)University Children's Hospital (Greifswald, DE)

Pediatric patients with relapsed solid tumors have a poor prognosis and additional therapeutic strategies are warranted. We present preliminary results of our institution with haploidentical stem cell transplantation in 25 pediatric patients (soft tissue sarcomas, $n=5$, Ewings sarcomas, $n=5$ and high risk neuroblastomas, $n=10$ ) who relapsed after previous autologous stem cell transplantation as well as a proposal for posttransplant immunotherapy in neuroblastoma patients. 10/25 patients reached complete or very good partial remission after various chemotherapies, $15 / 25$ patients had partial remission or were not in remission prior to transplantation. The conditioning regimen comprised Flud, TT, Mel and OKT3. The CliniMACS $\AA$ device was used for complete $\mathrm{T}$ and $\mathrm{B}$ cell depletion. Median time to reach $>500$ neutrophiles $/ \mu$ was 11 days. Acute GvHD grade $0-1$ occurred in $60 \%, 36 \%$ had GvHD grade II and $4 \%$ had grade III. Chronic GvHD occurred in $28 \%$. No transplant related mortality (TRM) was observed. Over all survival after 2 years was $30 \%$. Thus, transplantation itself seems to be not sufficient for most patients but may be a platform for further immunotherapies. In vitro stimulation with cytokines of donor derived patient NK cells postranplant and/or use of chimeric antiGD2 antibodies resulted in excellent lysis of neuroblastoma cells. Thus, we propose an open, prospective phase I/II study to evaluate the feasibility and safety of $\mathrm{CH} 14.18 / \mathrm{CHO}$ administration in combination with low dose interleukin 2 after haploidentical transplantation. The study design comprises 6 cycles of $\mathrm{mAb} \mathrm{CH} 14.18 / \mathrm{CHO}\left(20 \mathrm{mg} / \mathrm{m}^{2}\right.$ infusion over 8 hours for 5 days; in cycles 4-6, $1 \times 10^{6}$ units $/ \mathrm{m}^{2}$ aldesleukin will be given additionally on days 6,8 and 10). Primary endpoint is "success of treatment" defined as a patient receiving the full protocol treatment, still alive 180 days after end of treatment without progression and without unacceptable toxicity and acute GvHD $\geq$ Grade III or extensive chronic GvHD. Secondary objectives are to evaluate changes in NK cell activation and proliferation.

Conclusions: haploidentical transplantation represents a therapeutic option for patients with relapsed high risk neuroblastoma and other solid tumors and may be a basis for further immunotherapeutic approaches. The proposed study will adresss the question, whether the use of the monoclonal antibody $\mathrm{CH} 14.18$ / $\mathrm{CHO}$ in combination with interleukin 2 will be feasible after this transplantation procedure.

P647

Intensive induction chemotherapy in poor-prognosis adult extra-osseous Ewing sarcomas: a single.centre experience

A. Bertuzzi, L. Castagna, V. Quagliuolo, P. Navarria, P. Colombo, L. Giordano, A. Santoro

Istituto Clinico Humanitas (Rozzano, IT)

Objectives: To evaluate the impact of intensive chemotherapy (CT) as induction treatment before high-dose chemotherapy (HDCT) in adult patients (pts) with poor prognosis extraosseous Ewing's sarcoma (ES).

Methods: We treated consecutively pts with two different induction chemotherapy: conventional and intensive. Conventional-dose induction CT (CDICT) consisted of 4 cycles of ifosfamide/vincristine/epirubicin (IVE) followed by high-dose cyclophosphamide (CY) or etoposide also given for peripheral blood stem cell (PBSC) mobilization. Intensive-dose induction CT (IDICT) consisted of 3 cycles of IVE alternating 2 cycles of carboplatin/CY/ etoposide (CAPEC) supported by PBSC collected following the first cycle of IVE. Only pts in response or stable disease after induction CT proceeded to HDCT. Various conditioning regimens for HDCT were utilized. In case of local advanced disease, surgery and radiotherapy were performed when feasible in course of treatment.

Results: From 1997 to 2006, 31 consecutive pts (16 CDICT, 15 IDICT) with median age of $26 \mathrm{yrs}$, were treated at Our Institution. Disease was advanced in $45 \%$, metastatic in $42 \%$, relapsed in $13 \%$. Five pts had radical surgery at diagnosis and were not evaluable for response. Overall response rate (ORR) after CDICT and IDICT were $66.7 \%$ (CR 8.3\%, PR 58.3\%) and $57.1 \%$ (CR $28.6 \%$ PR $28.6 \%$ ), respectively. HDCT was performed in 23 pts $(74 \%)$. At the end of the whole treatment ORR was $58.3 \%$ (CR 50\%, PR $8.3 \%$ ) and $53.8 \%$ (CR $46.2 \%$, PR 7.7\%) in CDICT vs. IDICT groups, respectively. Completeness of follow-up was of $96 \%$ (follow-up range 2-130 months), median time to progression (TTP) were 10 vs. 11 months ( $p$ value 0.804 ) and median overall survival (OS) were 15 vs. 12 months ( $p$ value 0.894 ) for CDICT vs. IDICT groups, respectively. One transplant-related death occurred.

Conclusions: In our series of adult pts with extraosseous ES the use of intensive CT in the induction phase of treatment did not provide significant benefit over conventional therapy. 

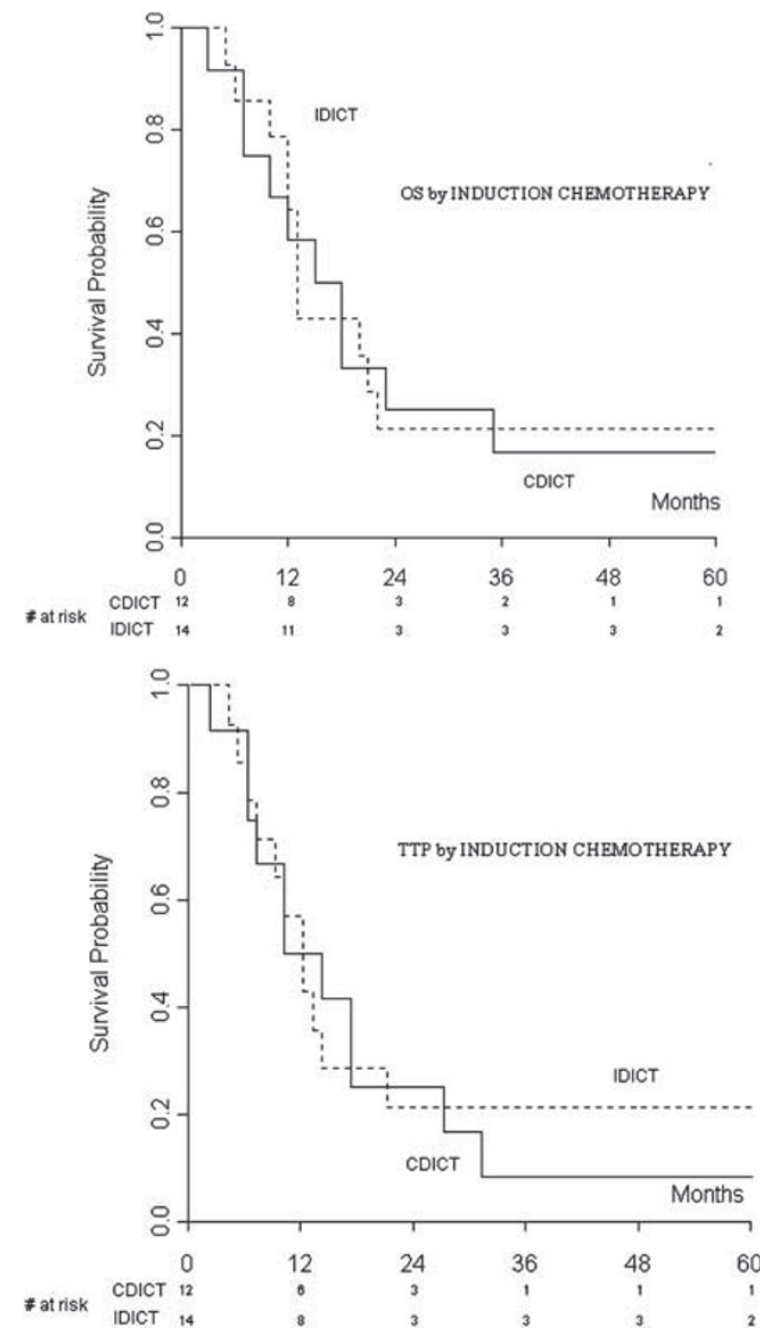

\section{P648}

Effect of the HDAC inhibitor SAHA on the immunmodulatory properties of MSC and tumour stroma cells

A. Kruchen, P.-D. Johann, R. Handgretinger, I. Müller University Children's Hospital (Tubingen, DE)

Histone deacetylase inhibitors (HDACi) affect plasticity of the chromosomes and are extensively investigated as potential new drug targets for treatment of malignancies. Suberoylanilid hydroxamic acid (SAHA) was the first HDACi used in cutaneous T cell lymphoma. HDACi are also known to affect function of physiologic T cells, which may be a new option in treatment of Graft-versus-Disease (GvHD) as has been proposed earlier. Another cellular tool employed in treatment of steroid-refractory GvHD are mesenchymal stromal cells (MSC). Therefore, we analysed if MSC and SAHA may synergise in inhibiting T cell proliferation in vitro. In our hands, SAHA did not add to the inhibitory effect of MSC on peripheral blood mononuclear cell (PBMC) proliferation. By contrast in some settings, SAHA reduced the inhibitory effect of MSC on PBMC. Subsequently, we asked if this observation may be used to improve tumorimmunological approaches as tumor stromal cells (TStrC) share several properties with MSC. Unlike in GvHD, however, inhibition of effector cell functions may be deleterious rather than beneficial and hamper immune therapeutic approaches. Consequently, we analysed the effect of SAHA on neuroblastoma cell lines as well as primary neuroblastoma stroma cells isolated from ten pediatric patients. Neuroblastoma cell lines showed a significantly reduced proliferation when treated with concentrations of SAHA exceeding $5 \mu \mathrm{M}$. Similar results were obtained for MSC and TStrC. TStrC displayed the same morphology as MSC isolated from bone marrow. Moreover, TStrC inhibited proliferation of Interleukin-2 and OKT3 stimulated PBMC and reduced cytotoxicity of NK cells. In these assays, the production of proinflammatory cytokines like Interferon gamma and tumor necrosis factor alpha were markedly reduced. When TStrC were preincubated with SAHA, the inhibitory effect on PBMC proliferation and cytotoxicity was reduced. These effects were also detectable at concentrations below $5 \mu \mathrm{M}$. These findings suggest that SAHA may compromise the TStrC function and improve the function of tumor-infiltrating lymphocytes. Future analyses include animal models to evaluate these effects in vivo.

P649

Cytotoxicity mediated by alloreactive natural killer cells against primary culture cell lines of high-risk paediatric solid tumours: an in vitro model to predict the efficacy of haploidentical stem cell transplantation

A. Bosi (1), L. Ruggeri (2), C. D'Ippolito (1), R.F. Schumacher (1), L.D. Notarangelo (1), L. Berchich (3), S. Lonardi (3), G. Carella (3), C. Gorio (1), S. Pulcini (1), L. Tonegatti (3), A. Velardi (2), F. Porta (1), A. Lanfranchi (1)

(1) Oncohaematology and BMT Units (Brescia, IT); (2)Spedali Civili (Brescia, IT); (3)University of Perugia (Perugia, IT)

Introduction: Natural Killer can have have an antileukemic effect in Haploidentical Stem Cell Transplantation (SCT), triggered by the presence of KIR mismatch between donor and recipient. Graft versus Tumor in high risk pediatric solid tumors remains to be investigated. The aim of our study is to evaluate NK activity against primary tumours in order to predict in vitro the efficacy of an haploidentical SCT.

Methods/materials: We studied 14 patients treated in our centre. We analyzed 2 Rhabdomyosarcoma, 3 Neuroblastoma, 3 Wilms Tumor, 2 Ewing's Sarcoma, 1 Malignant Peripheral Nerve Sheath Tumour, 3 Adult-Type Sarcoma. We HLA I typed parents (potential donors), patients and primary tumour cells evaluating KIR in parents and sons, according to the ligandligand model. In 9 cases we cultured primary tumours with a subsequent histopathology analysis of the cell cultures. In 6 cases we analysed NK activity and cytotoxicity of parental cells against both children PBL and primary tumours. Expression of the KIR ligands (HLAI) and of activating receptor's ligands MIC A-B, CD112 and CD155 on tumor cells with cytofluorimetry were also analyzed.

Results: 8 patients presented all the $3 \mathrm{KIR}$ ligands (HLA C1, HLA C2 and HLA Bw4); 5 parents were potentially alloreactive against their children, whereas in 1 case we found no mismatch because of missing $\mathrm{C} 2$. An alloreactive donor was present in one out of three. The results of the functional analysis of the NK cytotoxicity against children PBL confirmed the ligand-ligand model analysis expected results. The evaluation of the NK activity against tumour cells showed a sensitivity to NK lysis in 2 tumour cell lines. The cytofluorimetric analysis demonstrated poor expression of the activating receptor ligands and downregulation of HLA I in 2 tumour cell lines. The HLA I typing on primary tumours and PBL were overlapping.

Conclusions: These studies in vitro could predict the activity of NK cells against cultured primary tumour in vivo and could be an important element to choose the best parental donor. 
P650

153-Samarium EDTMP administration at escalating doses for relapsed osteogenic sarcoma

M. Berger (1), F. Carnevale-Schianca (2), R.E. Pellerito (3), B. Elia (3), S. Ferrari (4), V. Coha (2), D. Caravelli (2), M. Aglietta (2), F. Fagioli (1)

(1)Ospedale Infantile Regina Margherita (Turin, IT); (2)Istituto per Ricerca e la Cura del Cancro (Candiolo, IT); (3)Ospedale Mauriziano Umberto I (Turin, IT); (4)Istituti Ortopedici Rizzoli (Bologna, IT)

Introduction: Bone metastases of osteogenic sarcoma (OS) have a dismal prognosis even if aggressive chemotherapy is given. 153-Samarium EDTM was proven active on bone metastatic diseases at $1 \mathrm{mCi} / \mathrm{Kg}$ dose. Aiming to overcome the poor radio-sensitivity of OS, we test feasibility and toxicity of escalating doses of 153-Samarium EDTM followed by stem cell infusion on heavily pre-treated patients affected by bone metastatic OS.

Patients and methods: Twenty-one relapsed bone metastatic patients (median age $18 \mathrm{yrs}$ ) underwent escalating doses of 153-Samarium EDTM followed by peripheral blood stem cell infusion (median CD34+5.9 $\times 10^{6} / \mathrm{Kg}$ ). Four patients received 153-Samarium EDTM at $7 \mathrm{mCi} / \mathrm{Kg}$, nine patients received 10 $\mathrm{mCi} / \mathrm{Kg}, 5$ patients received $20 \mathrm{mCi} / \mathrm{Kg}$ and finally three patients received $30 \mathrm{mCi} / \mathrm{Kg}$.

Results: No grade III-IV extra-haematological toxicity was observed. Haematological toxicity was well overcome by stem cell infusion in all but two patients who had no platelet recovery together with OS progression. When we compared patients who had 153-Samarium EDTM $\leq 20 \mathrm{mCi} / \mathrm{Kg}$ with patients who had $>20 \mathrm{mCi} / \mathrm{Kg}$ the $\mathrm{GN}>500 / \mathrm{L}$ and $\mathrm{PLT}>>500 / \mathrm{L}$ recovery were on day 8 and 19 and on day 10 and $19(P=N S)$. When we analyse the day-60 response 12 patients had progressive disease $(60 \%, 1$ patients had first restaging on day 90$), 6$ patients had stable disease $(30 \%)$ and 2 patients had partial response $(10 \%)$. The median time to progression (TTP) was 60 days and finally when we compared patients who received more or less than $20 \mathrm{mCi} / \mathrm{Kg}$ the TTP were 60 and 55 days respectively $(P=\mathrm{NS})$.

Conclusion: Our study shows the feasibility of 153-Samarium EDTM followed by peripheral blood stem cell infusion in metastatic OS patients also as outpatient care. The observed toxicities stand for its clinical use also in the palliative setting. No conclusion can be drawn on anti-tumour effect because of the few patients enrolled, however a phase II clinical trials is warranted.

\section{P651}

Peripheral blood stem cell mobilization in children with brain tumour

S. Frenos, C. Sanvito, I. Sardi, M.P. Mariani, F. Brugnolo, S. Farina, M. Veltroni, V. Tintori, F. Bambi, M. Aricò, D. Caselli AOU Meyer (Florence, IT)

Introduction: Consolidation with high-dose chemotherapy is currently used as part of the treatment program of several childhood cancer types in the attempt to reverse the poor prognosis in high-risk patients. PBSC collection requires a coordinated effort between mobilization and harvesting. The timing of the CD34 + cells count peak in the peripheral blood depends on several issues, including G-CSF stimulation and bone-marrow reactivity. In particular, patients with unaffected marrow may have a more rapid mobilization. We reviewed our experience in the timing of PBSC mobilization and collection in children with solid tumor, and focused the analysis on a comparison between patients with brain tumor versus others solid cancer types.

Patients: 28 patients with solid tumor underwent PBSC mobilization; their age ranged between 1.8 months and 19 years (median, 6.9 years); the diagnosis was of brain tumor in 14 (medulloblastoma $n=7$, astrocytoma $n=3$, glyoma $n=2$, ependimoblastoma or rhabdoid tumor, $n=1$ ), while 14 had a non-CNS solid tumor (Neuroblastoma $n=6$; Ewing Sarcoma $n=5$; nonHodgkin Iymphoma $n=1$; retinoblastoma $n=1$; Wilms Tumor $\mathrm{n}=1$ ). Mobilization was induced by G-CSF 5 microgr $/ \mathrm{kg}$ q12 hrs s.c.; PBSC harvest was usually started in the presence of a CD34 + cell count of $20 /$ microL or more).

Results: Mobilization was achieved in all patients. The time from start of G-CSF to harvesting ranged between 3 and 9 days (median, 6 days). The peak CD34 count on the day of harvest ranged between 12 and 1,840/microL.

Patients with brain tumor had a mean time to harvest of 5.86 days (SD, 1.03; $\mathrm{Cl} 2.14$ to 0.43 ). The peak CD34 count on the day of harvest had a mean value of $369 /$ microL (range, 45 to 1840, median 134.4)

Patients with non-CNS solid tumor had a mean time to harvest of 7.14 days (SD, 1.17 ; Cl 2.14 to 0.43 ). The peak CD34 count on the day of harvest had a mean value of $75 /$ microL (range, 22 to 385$)(P=0.046)$.

Conclusion. Based on our preliminary experience, in children with brain tumor a larger number of PBSC can be harvested even after a significantly shorter time of G-CSF stimulation. We plan to expand this observation on a larger series.

\section{Myelodysplasia}

\section{P652}

\section{Comparison of allogeneic stem cell transplantation and} best supportive care in elderly patients with advanced MDS

N. Kröger, H. Putter, R. Brand, A. Kündgen, T. de Witte, $U$. Germing on behalf of the MDS subcommittee of CLWP (EBMT)

Encouraging results of allogeneic stem cell transplantation (HSCT) in MDS-patients have been reported up to the age of 70 years. However, whether elderly patients with MDS should undergo HSCT is a matter of debate. We used a multistate approach to compare the outcome in elderly advanced MDS patients (aged 55-69 years) with RAEB or RAEB-t who received only best supportive care and were reported to the Düsseldorf registry $(n=137)$, to those who received HSCT and were reported to the EBMT $(n=246)$. We used left-truncation and modelling of transition probabilities to obtain estimates of survival after diagnosis; moreover scenario analyses were performed to quantify sensitivity for untestable assumptions and to estimate survival for various strategies depending on the time between diagnosis and transplant.

In the non-transplant cohort, diagnosis was RAEB in 100 $(73 \%)$ and RAEB-t in 37 patients $(27 \%)$. The median age of the 83 male and 54 female patients was 62 years. Disease status at diagnosis was RAEB (70\%) and RAEB-t (30\%). At time of transplantation, diagnosis was RAEB (54\%), RAEBt $(34 \%)$ and transformed secondary acute leukemia (12\%). Transplantation from HLA-identical sibling $(n=175)$ or unrelated donor $(n=71)$ was performed after standard myeloablative $n=76$ ) or reduced-intensity conditioning $(n=170)$. Median time between date of diagnosis and date of transplant was 7 months. The cumulative incidence of non-relapse mortality of the transplant cohort at 1 year and 3 years was $15 \%$ and $31 \%$, respectively. The $\mathrm{Cl}$ of relapse was $14 \%$ and $29 \%$ at the same time points. Univariately, after accounting for left truncation and modelling of the death rate before transplant, the hazard ratio for survival is estimated as 0.72 in favour of transplant $(P=0.04)$. In a multivariate analysis, adjusting for age at diagnosis, sub-diagnosis (RAEB vs. RAEB-t), cytogenetics (abnormal vs. normal), the hazard ratio for transplantation versus no-transplantation: $0.77(P=0.1)$ while the other hazard ratio's were: 1.4 for "age $>60$ " $(P=0.02) ; 1.20$ for "RAEB-t" $(P=0.2)$ and 1.46 for "abnormal cytogenetics" $(P=0.01)$. 
This study highlighted the difficulties- despite sophisticated statistical methods- to compare non-transplant with transplant cohort, in case death before transplant is possible but unknown and the selection of patients to schedule for transplant is based on assumptions that can not be verified, which all underlines the need for a prospective randomised trial.

\section{P653}

Induction chemotherapy followed immediately by busulfan-based reduced conditioning and allografting in elderly patients with advanced MDS or SAML

N. Kröger, T. Zabelina, C. Wolschke, H. Lellek, T. Stübig, M. Lestin, H. Alchalby, F. Ayuk, U. Bacher, A. Zander University Medical Center Hamburg-Eppend (Hamburg, DE)

Introduction: Allogeneic stem cell transplantation after a dosereduced conditioning has become a reasonable treatment option for elderly patients with MDS/sAML. For patients with high number of blasts prior transplantation, the risk of relapse is considerably. To reduce the risk of relapse after dose-reduced allograft we performed a study using an anthracycline based induction chemotherapy (amsacrine, cytosine-arabinoside, fludarabine) followed immediately by a reduced intensity conditioning therapy consisting of busulfan $(8 \mathrm{mg} / \mathrm{kg})$.

Patients and methods: Between November 2005 and November 2008, 49 patients with MDS $(n=24), \operatorname{CMML}(n=8)$ and sAML $(n=17)$ and a median age of 61 years $(r: 26-73)$ and a median number of $13 \%$ blasts were included. Stem cell source were unrelated $(n=43)$ or related donor $(n=6)$.

Results: No graft failure was observed and the median time to leukocyte engraftment $\left(>1.0 \times 10^{9} / \mathrm{l}\right)$ was only 10 days (r: 7-32). The incidence of acute graft-versus-host disease grade II to IV was $39 \%$ and of grade III / IV was $14 \%$. Chronic GvHD was noted in $57 \%$ of the patients, which was limited in $35 \%$ and extensive in $32 \%$ of the patients. After a median follow-up of 15 months (r: 3-35) the two-years estimated disease-free and overall survival was $49 \%(95 \% \mathrm{Cl} 33-65 \%)$ and $54 \%(95 \% \mathrm{Cl} 39-69 \%)$, respectively. The 1 year cumulative incidence of treatment-related mortality was $29 \%(95 \%$ $\mathrm{Cl} 15-43 \%)$. The 2 year cumulative incidence of relapse was $18 \%(95 \% \mathrm{Cl} 6-30 \%)$. Patients with fully matched related or unrelated donor had a better survival than patients transplanted from mismatched donor ( $69 \%$ vs. $37 \% ; P=0.06)$.

Conclusions: A sequential approach using anthracycline based induction chemotherapy followed immediately by a busulfan based reduced conditioning regimen and allogeneic stem cell transplantation from related and unrelated donors resulted in a fast engraftment and a relative low risk of relapse in elderly patients with advanced MDS or sAML. To lower the therapy related mortality a careful donor selection is mandatory.

\section{P654}

Outcomes of sibling and unrelated donor reduced intensity conditioning allogeneic hsct for patients with MDS RCMD

P. Krishnamurthy, Z.Y. Lim, A. Ho, M. Kenyon, M.M. Ceesay, J. Hayden, R. Ireland, J. Marsh, G. Mufti, A. Pagliuca

Kings College Hospital (London, UK)

While the median survival of patients with MDS RCMD is approximately 49 months; those with transfusion dependency and progressive cytopenias have a poorer prognosis. Treatment options in these patients have been largely limited to supportive care and growth factor therapy. Allogeneic transplantation may be potentially curative but has been limited hitherto by its toxicity. More recent advances in transplantation, particularly the development of reduced intensity conditioning (RIC) regimens, may extend allografting to this group.

We report on the outcomes of 48 patients (median recipient age: 51 years, range 19-64 years) who underwent RIC HSCT between 1999 and 2008 at our institution. Patients had
MDS-RCMD according to the WHO criteria and were staged according to IPSS (low risk $=7$, int- $1=36$ and int-2=5) and cytogenetics (good risk $=29$, intermediate risk $=13$ and poor risk $=5$ ). All patients were either heavily transfusion dependant or thrombocytopenic. 16 patients had chemotherapy prior to transplantation; 14 had received a single course and 2 had received 2 courses. Conditioning comprised Fludarabine $30 \mathrm{mg} / \mathrm{m} 2$ i.v. (Day -9 to Day -5 ); busulphan $4 \mathrm{mg} / \mathrm{kg} /$ day oral or $3.2 \mathrm{mg} / \mathrm{kg} /$ day i.v. (Day -3 to Day -2 ) \& alemtuzumab $20 \mathrm{mg}$ i.v. (Day -8 to Day -4 ) followed by cyclosporine for GvHD prophylaxis. Patients received peripheral blood stem cells $(n=35)$ or bone marrow $(n=13) .10$ patients received stem cells from matched sibling donors, 32 from HLA-matched VUD (6 from mismatched VUD). The median cell dose was $4.57 \times 10^{6}$ CD34/kg (range: $0.44-19.9 \times 10^{6} \mathrm{CD} 34 / \mathrm{kg}$ ).

At a median follow-up of 1102 days (range 52-3659 days), 28 patients remain alive with a median overall survival of 1859 days (range $379-3659$ days) and $5-y r$ OS of $55 \%$. OS was not significantly different by donor type (sibling vs. unrelated), IPSS category or cytogenetic sub-group. The 5-yr non-relapse mortality (NRM) was $26 \%$. 3 patients experienced graft failure; full donor chimerism was achieved (where evaluated) in $7 / 48$ (15\%) at 1 month, $12 / 48(25 \%)$ at 6 months and $13 / 48(27 \%)$ at 1 year. 12 patients $(25 \%)$ relapsed, 9 of whom died. 20 patients received donor lymphocyte infusions, 13 for loss of donor chimerism and the remaining for relapse (5) or graft failure (2). 16 patients (33\%) developed acute GVHD(Grade III-IV: 6 patients) and 15 patients $(32 \%)$ suffered chronic GVHD.

In conclusion, alemtuzumab-based RIC HSCT for selected RCMD patients offers sustained long-term remissions with low incidence of graft failure or GvHD.

\section{P655}

Stem cell transplantation for MDS patients 1987-2008: data from the Austrian transplant registry (ASCTR) O. Krieger (1), S. Machherndl-Spandl (1), H. Hauser (1), M. Binder (1), H. Greinix (2), S. Eder (2), P. Kahls (2), W. Rabitsch (2), D. Nachbaur (3), H. Ludwig (4), W. Linkesch (5), G. Jaeger (5), H. Sill (5), B. Lindner (6)

(1)Elisabethinen Hospital (Linz, AT); (2)Medical University of Vienna (Vienna, AT); (3)University Hospital Innsbruck (Innsbruck, AT); (4)Wilhelminen Hospital (Vienna, AT); (5)Medical University of Graz (Graz, AT); (6)Austrian SCT Registry (Innsbruck, AT)

207 adult patients (94 female, 113 male) with myelodysplastic syndromes (MDS) were treated by allogeneic stemcell-transplantation (SCT) in Austria from 1987 to 2008. The median age was 47 years ( range 18-70, 30pat. $>60$ ys ). 126 patients $(61 \%)$ had primary MDS at time of transplant ( 17 RA/RARS, 44 RAEB, 19 RAEBT, 25 unclass.MDS, 21 MDS/MPS) and 81 patients were treated with SCT after transformation in sec.AML. A peripheral stemcell transplantation (PBPCT) was performed in 165 patients (80\%), 42 patients received bone marrow. Standard conditioning (eg.TBI/CY,BU/CY) was done in 102 pat., 105 pat. were treated by RIC-SCT. SC sources and conditioning changed over the years (from 1987 to 1998 only 3 PBSCT, all RIC-SCT were done after 1999). 105 patients received stemcells from HLA-ID siblings, 78 from HLA-ID unrelated donors; 24 mismatched transplants (related and unrelated) were performed. Time from diagnosis to SCT was 8 months (median) with a range of 1 to 97 months. CSA+MTX (for myeloablative SCT) or CSA+MMF (for RIC-SCT) was used for GVHD-prophylaxis in most cases.

37/126 MDS-patients (group A: without transformation in AML) and 44/81 secondary AML (group B: after MDS-prephase) were transplanted in complete remission. The incidence of acute GvHD (grade II-IV) was 35\%. 73/126 pat. of group A died after a median of 6 months (20 after relapse, 42 due to toxicity/severe GvHD, 11 of other causes), and $53(42 \%)$ are alive 2-185 months (median: 55) after SCT. In the sec.AML group (B) 54 deaths occurred (23 rel/PD, 25 TRM, 6 others) and $27 / 81$ (33\%) 
are alive and leukemia-free after 1-119 months (median:28); $21 / 27$ patients of the long time survivors were transplanted in CR. Patients characteristics (cytogenetic data, comorbidity scales) and transplant toxicities (infectious complications, death due to severe GvHD, incidence of chronic GvHD) will be discussed in detail. Status at transplant was the discriminating factor for outcome; there was no difference in toxicity and survival between sibling and unrelated transplants.

Allotransplantation in MDS-patients (before and after AMLtransformation) is a curable approach, with a transplant-related mortality of $32 \%$, similar to that seen in elderly de novo acute leukemia patients.

\section{P656}

Allogeneic haematopoietic stem cell transplantation in previously untreated myelodysplastic syndrome: better outcome with reduced-toxicity myeloablative conditioning regimens

A. Shimoni, A. Rand, N. Shem-Tov, I. Hardan, Y. Volchek, H. Maayaan, R. Yerushalmi, A. Nagler

Chaim Sheba Medical Center (Tel-Hashomer, IL)

Allogeneic stem-cell transplantation (SCT) is a potentially curative therapy for patients (pts) with myelodysplastic syndrome (MDS). SCT in MDS pts who are often elderly and heavily transfused may be associated with a high incidence of nonrelapse mortality (NRM). Reduced-intensity conditioning (RIC) may reduced NRM, but may be associated with excess relapse rate, such that the optimal conditioning regimen is unknown. Similarly, the need for induction chemotherapy prior to SCT is controversial. We retrospectively analyzed SCT outcome of 66 pts with MDS, all with 5\%-20\% marrow blasts at SCT, and none had prior induction chemotherapy. The median age was 58 years (range, 21-73), 46 male, 20 female. The donor was an HLA-matched sibling $(n=30)$ or matched unrelated $(n=36)$. Thirteen pts had therapy-related MDS. The conditioning regimen was classical myeloablative (BuCy, $n=12$ ), reduced-toxicity myeloablative (RTC) consisting of fludarabine with treosulfan (36 gr/m2, FT, $\mathrm{n}=26$ ) or with high-dose busulfan $(12.8 \mathrm{mg} / \mathrm{kg}$ FB4, $n=13$ ) or RIC consisting of fludarabine and reduced dose busulfan $(6.4 \mathrm{mg} / \mathrm{kg}$, FB2, $\mathrm{n}=15)$. With a median follow-up of 28 months (range, 1-118), 30 pts are alive, 23 died of NRM and 13 of relapse. The estimated 5 -year overall survival (OS) was $34 \%(95 \mathrm{Cl}, 20-48)$. The cumulative incidence of NRM and relapse mortality was $39 \%$ and $27 \%$, respectively. 5-yr OS was $31 \%, 53 \%, 42 \%$ and $17 \%$ after BuCy, FT, FB4 and FB2, respectively ( $P=0.07$ for RTC vs. others). Multivariate analysis (MVA) identified RTC (FT+FB4) as a favorable prognostic factor, HR $0.5(0.3-0.9, P=0.04)$ and unrelated donor as an unfavorable factor with borderline significance, $\operatorname{HR} 1.8(0.9-3.7, P=0.09)$. Conditioning with BuCy and unrelated donor were predicting factors for NRM with HR $2.7(1.1-6.8, P=0.04)$ and $2.7(1.1-7.0$, $P=0.05)$, respectively. NRM was $58 \%$ after BuCy vs. $35 \%$ after the other regimens. Conditioning with BuCy or FT predicted for reduced relapse risk, $\operatorname{HR} 0.3(0.1-1.3, P=0.06)$. Relapse rate was $11 \%$ after BuCy and FT vs. $40 \%$ after the other regimens. In conclusion, RTC is associated with the optimal results in untreated MDS pts. Classical myeloablative conditioning may be associated with excessive NRM in these pts, while RIC may result in excessive relapse rate. Induction chemotherapy may be needed prior to RIC, but can probably be avoided when using more intensive regimens. Treosulfan-based conditioning was associated with favorable toxicity profile and improved anti-MDS activity.
P657

Cord blood transplantation for myelofibrosis: a retrospective analysis from the Japan Cord Blood Bank Network

M. Murata, S. Taniguchi, T. Nagamura-Inoue, K. Kato on behalf of the Japan Cord Blood Bank Network

Idiopathic myelofibrosis (MF) is a clonal myeloproliferative disorder characterized by bone marrow fibrosis, cytopenias, splenomegaly, and constitutional symptoms. There is only one curative therapy for MF: allogeneic hematopoietic stem cell transplantation, which offers a $50-80 \%$ survival rate for patients who received bone marrow or peripheral blood stem cells from related or unrelated donors. Umbilical cord blood, an alternative stem cell source, has been used increasingly for the treatment of patients with various hematological diseases, but its use in patients with MF is extremely limited. Here we summarize registry data in 10 patients with de novo MF who received first cord blood transplantation (CBT) through the Japan Cord Blood Bank Network. The median age was 57 years (range, 43-79), and median body weight was $54 \mathrm{~kg}$ (range, 34-63). The median time from diagnosis to transplantation was 6 months (range, 222). The preconditioning regimen varied according to transplant center and individual patient. Eight patients $(80 \%)$ received regimens including fludarabine with or without reduced total body irradiation ( $\leq 8 \mathrm{~Gy}$ ). As a prophylaxis for graft-versus-host disease (GVHD), seven patients $(70 \%)$ received a tacrolimus with or without short-term methotrexate. All patients received a single cord blood unit including total nuclear cells of $2.6 \times 10^{7} / \mathrm{kg}$ of patient's body weight (range, $1.7-3.6 \times 10^{7} / \mathrm{kg}$ ) with 0 to 2 HLA mismatches. Eight patients (80\%) were successfully engrafted with an absolute neutrophil count $\geq 500 / \mu$ l by a median of day 23 (range, 17-26). Five patients $(50 \%)$ achieved reticulocyte engraftment $(\geq 1 \%)$, and three patients $(30 \%)$ achieved platelet engraftment $(\geq 50,000 / \mu \mathrm{l})$. In eight evaluable patients, five patients (63\%) developed acute GVHD; 2 patients had grade I, 2 had grade II, and 1 had grade IV. Four patients were alive at the last follow-up, with a median survival follow-up of 12 months (range, 4-26). They included three patients in whom sustained engraftment of three lineages was achieved. The most frequent causes of death in six patients were bacterial or fungal infections, and no patient died of progressive disease. The probability of overall survival at 2 years for 10 patients was $36 \pm 16 \%$ (Kaplan-Meier).

In conclusion, CBT can provide a long-term relapse-free survival for some patients with MF. Optimization of preconditioning regimens to obtain sustained engraftment is essential for the improvement of its outcome.

\section{Regulatory issues and Quality management}

\section{P658}

How organizational pre-transplant measures may improve clinical outcomes in haemopoietic stem cell transplantation F.J. Márquez-Malaver, J. Falantes, M.R. López-López, M. Carmona-González, M.I. Montero-Cuadrado, J. GonzálezCampos, F. de la Cruz-Vicente, A. Marín-Niebla, M. MartinoGaliana, R. Parody, V. Osa, A. Urbano-Ispizua, I. Espigado Hospital Virgen Del Rocío (Sevilla, ES)

There is a steady improvement along the years in the clinical results of allo-HSCT. This fact has been mainly attributed to a better medical care, although it might also be due to a better selection of candidates for the procedure and organizational pre-transplant measures.

Objectives: To evaluate the effectiveness of quality improvement interventions for management of HSCT candidates and its consequences on clinical outcomes. 
Methods: 1) Descriptive analysis and comparison of main clinical and demographic features between candidates included in a pre-HSCT program of a tertiary Institution in two consecutive periods (P1: February/05-April/07 and P2: May/07October/09). P2 was started after the evaluation of the clinical results in P1, several measures were implemented in P2 for a more accurate indication, a better interaction with the physician sending the patient (pt), and for shortening the period between indication and the transplant. 2) Survival analysis of the transplanted pts whom the procedure was indicated in each period. Results: 520 pts were consecutively evaluated as candidates for transplant by the HSCT Committee: P1 $(n=195)$ and P2 $(n=325)$. Of them $465(89.4 \%)$ were accepted for transplant, P1 $(35.7 \%)$ and P2 $(64.3 \%)$, while HSCT was considered not indicated in 55 pts $(52.7 \%$ vs. $47.3 \%),(P=0.014)$. Fifty out of 166 pts in P1 and 66 out of 299 accepted candidates in P2 were not finally transplanted $(30.7 \%$ vs. $22.1 \% ; P=0.039)$ due (respectively P1 and P2) to: absence of donor (2 vs. 0), clinical complications and disease progression ( 24 vs. 45$)$, poor autologous stem cell mobilization (11 vs. 6), patient's refusal ( 8 vs. 8 ) and other reasons (6 vs. 7). At the end of P2, 106 pts with the indication established in $\mathrm{P} 1$ and 146 from those considered in P2 (Auto: $58.9 \%$ vs. $52.8 \%$; $P=0.337$ ) were already transplanted, with a median time from program inclusion to HSCT of $3.5 \mathrm{~m}$ vs. $2.8 \mathrm{~m}(P=0.335)$. At last follow-up, the group of transplanted pts whom the procedure was indicated on P1 had lower OS than those with indication established in P2 (59\% vs. $73.6 \% ; P=0.024)$ (Figure 1$)$, lower EFS (47.2\% vs. $64 \% ; P=0.035$ ) (Figure 2), higher cumulative incidence $(\mathrm{Cl})$ of transplant related mortality $(18 \%$ vs. $11 \% ; P=0.095)$ and $\mathrm{Cl}$ of relapse ( $34 \%$ vs. $25 \%$; $P=0.331)$.

Conclusions: Identifying areas of improvement opportunity allows implementation of corrective measures in order to manage a great number of candidates, to reduce the rate of pts not finally transplanted and to obtain better clinical outcomes.

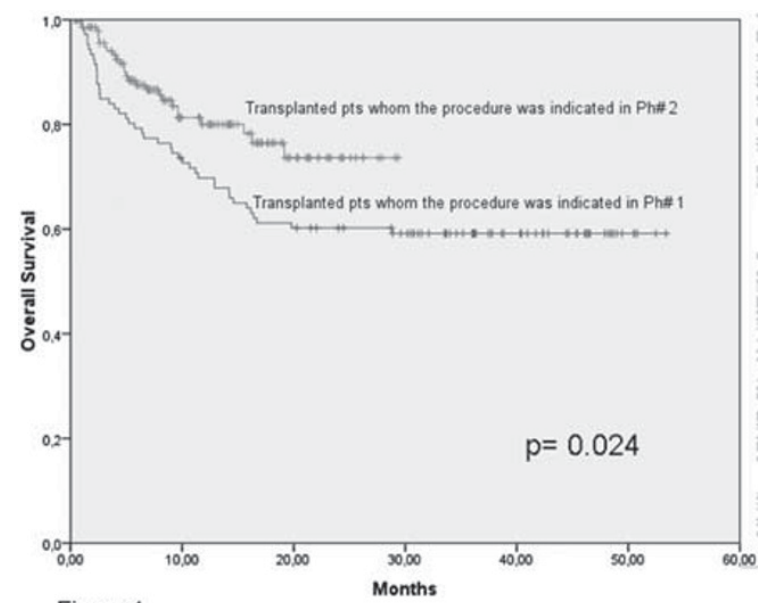

Figure 1

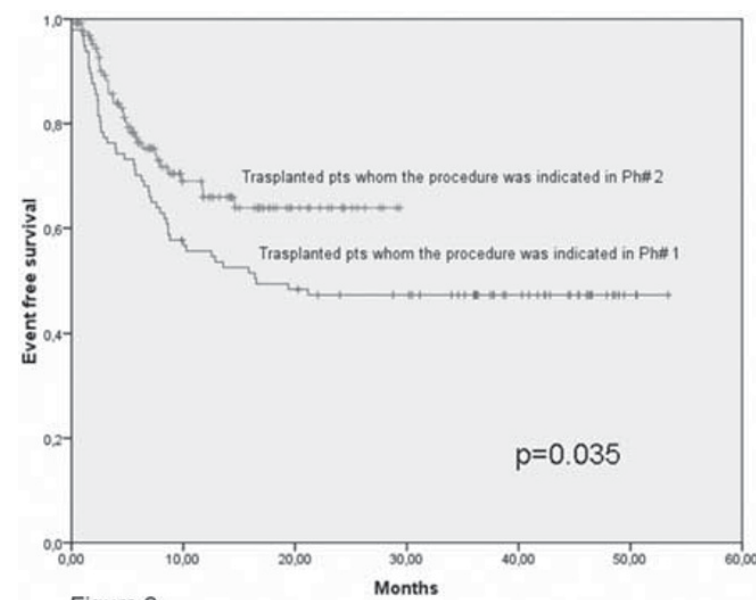

Figure 2

\section{P659}

Incorporation of plerixafor into an algorithm for stem cell mobilization is cost-efficient and successfully allows stem cell collection

J. Schriber, V. Fauble, L. Sproat, A. Briggs

Banner Blood and Marrow Transplant Program (Phoenix, US)

Successful stem cell mobilization typically contributes approximately $10-20 \%$ of the costs associated with autologous stem cell transplantation. Plerixafor is a new agent that has been shown in phase 3 trials to improve mobilization for patients with $\mathrm{NHL}$ and $\mathrm{MM}$ but it has significant upfront costs. It has also been shown to enable collection in patients who have failed prior attempts at mobilization.

Previously at our institution, such patients would have received additional or higher dose growth factors or proceeded to collection following chemotherapy. Both have difficulties associated with them. Chemotherapy carries with it a risk of complications including admissions for febrile neutropenia and prolonged growth factor treatment that may result in long delays between transplant and prior chemotherapy.

To better determine the cost/benefit analysis of earlier use of Plerixafor $(P)$ we reviewed our own costs and based on these have now developed a new standard of therapy for patients who appear to be poor mobilizers.

Costs of Collection

\begin{tabular}{|l|l|}
\hline G 5 days & 12725 \\
\hline SQ Injn & 805 \\
\hline Apheresis (1 day) & 4262 \\
\hline CD34 & 349 \\
\hline CBC & 236 \\
\hline Processing & 1200 \\
\hline Total & 19577 \\
\hline Additional Days & 8492 \\
\hline Plerixafor per day & 13128 \\
\hline
\end{tabular}

Our own experience showed that most patients who had poor peripheral blood CD34 counts would not mobilize easily and even with additional growth factors typically required 4 or more apheresis procedures to reach goal. 
The cost for poor mobilizers including a doubling of G CSF for 4 days and 4 collections was over 60,000 while the addition of Plerixafor for 1 day was approximately 30,000 or 49000 if two days of apheresis were required.

The costs of $P$ use starting on the fifth night in those patients with poor CD34 counts was more than offset by the fewer apheresis days needed.

Based on this data we have now changed our algorithm for those who appear to be poor mobilizers. We now check CD34 counts on day 4 of G CSF therapy. Those with a peripheral CD34 count $<8$, receive Plerixafor that evening and are collected the following day. Despite the high upfront cost of Plerixafor this is more then offset by the fewer days of apheresis and growth factors. Such an approach allows patients to proceed more efficiently to transplant without the need for chemomobilization in patients who have no clinical reason to receive chemotherapy.

P660

Activity survey of haematopoietic stem cell transplantation in the Asian Pacific Blood and Marrow Transplantation Group 2009

M. lida Y. Atsuta, R. Hyo, R. Suzuki, C. Min, T. Wu, D. Ma, A. Ghavamzadeh, A. Lie, L. Chan, S. Jootar, F. Tasneem, W. Hwang, A. Srivastava, T. Binh, T. Chiou, Y. Kodera on behalf of the Asian Pacific Blood and Marrow Transplantation Group

Background: In 2006, Asian Pacific Blood and Marrow Transplantation Group (APBMT) established the Asian BMT Registry which is consisted of Activity Survey and Outcome Registry, and the third survey, APBMT Activity Survey 2009, was performed to collect the data of hematopoietic stem cell transplantation (HSCT) performed up to 2007. We report here the results of the APBMT Activity Survey 2009.
Methods: The APBMT Data Center asked to submit the data about the annual number of HSCT according to disease indications, donor types and stem cell sources until 2007. The data submission was done through the Web from the national or international registries, from the contact persons, or from institutes.

Result: The number of the participating countries in the Asian BMT Registry has increased from 7 to 14 countries/regions. In addition to that, the case numbers from every country/region have also increased, resulting 9,414 transplants performed in 2007 and 84,967 cumulative transplants data have been submitted to the APBMT Data Center (see Table). Hematological malignancies such as acute myelogenous leukemia, acute lymphocytic leukemia, lymphoid malignancy and multiple myeloma were the primary indications for transplantation $(24 \%, 13 \%, 21 \%$ and $16 \%$ respectively), which were similar results of the other international registries. On the other hand, it deemed to be unique finding in our registry that HSCT played an important role for the treatment of hemoglobinopathy in several Asian countries although the absolute numbers was relatively small. As for the donor types and stem cell sources, the proportion largely varied among countries/regions.

Discussion: In this survey, we revealed that HSCT in Asia Pacific area had been continuously advancing and showed the characteristic trends of Asian HSCT in terms of the disease indications, donor types and stem cell sources. We consider these trends depend on the characteristics of this area, that is, vast cultural, social and economical heterogeneity. The APBMT Outcome Registry, whose core search items are shared with the other international registries, is almost ready to start and we expect this would contribute to the further development of HSCT in this area as well as the activity of the Worldwide Network for Blood and Marrow Transplantation (WBMT).

\section{Summary of Collected Data}

\begin{tabular}{|l|r|r|r|}
\hline & $\begin{array}{c}\text { No. of reporting } \\
\text { centers in 2007 }\end{array}$ & $\begin{array}{c}\text { No. of reported HSCTs } \\
\text { in 2007 }\end{array}$ & $\begin{array}{c}\text { No. of reported HSCTs } \\
\text { from 1986 to 2007 }\end{array}$ \\
\hline Australia & 41 & 1175 & 14996 \\
China & 32 & 1170 & 3574 \\
HongKong & 2 & 169 & 1853 \\
India & 15 & 295 & 561 \\
Iran & 2 & 364 & 2057 \\
Japan & 359 & 4065 & 43232 \\
Korea & 37 & 1382 & 10929 \\
Malaysia & 10 & 135 & 1309 \\
New Zealand & 6 & 115 & 1503 \\
Pakistan & 2 & 36 & 190 \\
Singapore & 3 & 121 & 993 \\
Taiwan & 8 & 245 & 2616 \\
Thailand & 5 & 133 & 1092 \\
Vietnam & 2 & 9 & 62 \\
\hline Total & 524 & 9414 & 84967 \\
\hline
\end{tabular}


P661

Family cord blood banking for transplantation among the National Cord Blood Bank Network in Italy

P. Saracco, L. Lombardini, M. Screnci, L. Salvaneschi, A. Tamburini, M. Pagliarino, S. Urbani, S. Pupella, R. Saccardi, L. Bergamaschi, G. Grazzini, A. Nanni Costa on behalf of the Italian Cord Blood Bank Network

Directed family cord blood (DCB) storage provides hemopoietic stem cell source for transplantation (HSCT) for families with an existing or a potentially future recipient with HSCT curable disease (D). To provide best cost-effective practices recommendations it is important to report on DCB procedures and HSCT rate (HSCT-R) among public Banks. The National CB Banks Network in Italy (ITCBN) provides public DCB service for highrisk families, in compliance to GITMO directives for eligibility criteria (HSCT curable D: malignant MD, non MD, inherited ID) By 12.12.2008 almost 1800 DCB units were stored in 18 Italian Banks and $104(9 \%)$ issued for HSCT. The present survey aims at summarizing the over 15 yrs DCB experience among 5 ITCBN Banks active since 1997 (range 1990-1997), and including 670 DCB units.

Results: Preliminary analysis reports a 94\% overall compliance to eligibility criteria directives, and overall HSCT- R for an alive sibling of $12 \%(63 / 522)$; the 63 HSCT were $97 \%$ matched, for curing ID in $84 \%$ and with $72 \%$ overall survival outcome. Parameters of overall stored units were: median volume $105.6 \mathrm{ml}(18-41)$; median total nucleated cell count 9.1 (0.1-27), median CD34 3.1(0.1-7.6). Different policies among Banks were compared (Bank vs. others: 1) eligibility criteria distribution: Bank PV06 DCB for MD <50\% (42\% vs. $55-67 \%$ ), Banks Rm04,R04 significant DCB for a parental recipient with MD (17-24\% vs. $0.5 \%-2 \%)$, Bank T02 with significant (53/261) DCB for future sibling with ID (20\% vs. $2-5 \%)$; 2$)$ Timing of HLA typing: Banks T02,FI03,PV06 with DCB following prenatal HLA (20-27\% vs. 0\%), Banks FI03,Rm04,PV06 with CB HLA typed at birth (61-73\% vs. 23-25\%). Significant transplant rate $(37 / 177=21 \%)$ was reported by PV06 (HLA selection based storage). Lowest HSCT-R by Rm04, R04 (3/101 =3\%-5/98 =5\%) (parental mismatch). At T02 when excluding DCB for future sibling (low birth rate), and autologous (11 ID, in donors) HSCT-R increased from $6 \%(15 / 261)$ to $9 \%(15 / 170)$.

Conclusion: $A$ national public family $C B$ banking program must be ethical, by maintaining equitable access to all needing families and by periodically updating elegibility disease directives (with support of expert consulting third party authority), and it must be cost effective, by providing HLA based selected storage to increase transplant rate and by periodicaly revieweing long-term storage policy(as dismissal of mismatched units).

\section{P662}

1000 autologous haematopoietic stem cell transplantations: single-centre experience

D. Pohlreich, B. Vackova, R. Pytlik, M. Trnkova, M. Hamouzova, M. Trneny

First Faculty of Medicine, Charles University and General University Hospital (Prague, CZ)

Introduction: We present data from our transplant centre, which currently celebrates the number of 1000 autologous hematopoietic stem cell transplantations (ASCT) performed during the last 14 years. The 1st ASCT was on the 10th December 1993. The main indications for ASCT were lymphomas (NHL $41 \%$, $\mathrm{HL} 12 \%$ ) and multiple myelomas (MM, 34\%). Other diagnoses comprise breast carcinoma, CLL, multiple sclerosis, amyloidosis, MDS, AL and solid tumours.

Results: The number of 1000 ASCTs was performed in 817 patients (388 females, 429 males) with median age of 56 years (18-70).

According to diagnoses, ASCT underwent following number of patients: MM (228), DLBCL (198), HL (97), FL (87), breast cancer (46), MCL (41) and other few represented diagnoses. In MM patients we performed 54 tandem and 15 triplet ASCT. Indication criteria for the main disease groups and outcomes are: The disease status for MM before ASCT: 1 st PR (56\%), 1st progression after PR (11\%), 1st CR (10\%), 1st MR or SD $(8 \%)$, other bellow 4\%. Median overall survival (OS) in MM patients is 54 months, 7 -years post -ASCT OS is $28 \%$ and median of progression free survival (PFS) is 21 months. DLBCL was indicated mainly in the 1st CR (37\%), 1st PR (27\%), 2nd CR (9\%), 1 st chemosensitive relapse $(7.6 \%)$, primary refractory $(6 \%), 2$ nd PR $(5 \%)$ and others bellow $5 \%$. Median OS in DLBCL patients unachieved, 7 -years post - ASCT OS is $69.2 \%$. Median PFS is 29 months.

Trends in transplant activities: The significant part of ASCT in $\mathrm{HL}$ and $\mathrm{FL}$ in the beginning of the transplant programe has been moved for the benefit of aggressive lymphomas such as $M C L$ and DLBCL. High number of DLBCL patients transplanted in 1st CR/PR is partially due to R-MegaCHOP-ESAP-BEAM protocol for high-risk patients, where 48 patients were included in 1998-2006. The breast cancer ASCT started 1994 with total number of 46 patients until 2000. The last years there was no ASCT for MDS/AML. From 2001 we have initiated ASCT in multiple sclerosis in BEAM regimen with T-cell depletion (in vivo \pm in vitro), enrolled 26 patients.

Transplant related mortality (TRM) up to 100 days was constantly very low, an average $4.7 \%$ per year with no trend progress.

Conclusion: Although indication criteria for ASCT are developing, ASCT still remains fundamental procedure in the treatment of $\mathrm{NHL}$ and MM patiens in recent years. In the hands of experienced transplant centre, ASCT is safe with acceptable TRM. New indications, such as autoimmune diseases, open new perspectives for ASCT.

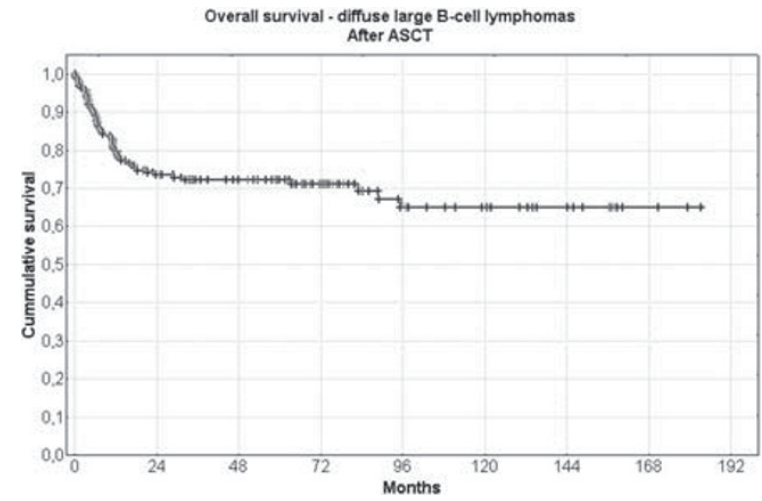

P663

A six-year follow-up report on the workings of the eight networking cell processing laboratories for human haematopoietic progenitor cells in Sydney

V. Antonenas, A. Trickett

BMT Network NSW (Sydney, AU)

In 2002, the Blood \& Marrow Transplant (BMT) Network was established to create a forum for collaboration between the 9 government-funded cell processing laboratories in New South Wales for 14 BMT Hospitals. The Network is centrally managed through an executive committee of elected clinical representatives with a BMT Network Manager and a central and shared basis of 3 Quality Managers with a common document control system linked to all laboratories. The goals of the Laboratory Network Group were set to work collectively as a group to publish data, develop optimum SOPs and produce high quality validation reports, and meet other required criteria to meet national and international regulatory standards. The group meet every three months for meetings and participate an 
on-going education programme. This prepares the 9 laboratories to meet the demanding standards for accreditation for HPC processing and storage from a combined networking group, and not individually as a single facility.

The Network has implemented Q-Pulse for quality management software to aid in meeting the standards applicable to BMT collection, processing and infusion. Standard operating procedures (SOPs) have been developed and are generated as either generic documents to be released to all relevant facilities or, if consensus is not attained, as site-specific documents for release to individual facilities. Laboratories are also using the non-conformance and corrective/preventative action modules of Q-Pulse shared across all labs. The group are about to initiate a common audit programme for labs to be used across the network.

All facilities have developed a professional and sharing relationship in particular for trouble shooting and advice on problems. Enthusiastic input from all laboratories has always been in place for many years, and the scientists have developed a collaborative approach to the collection units and to the clinical units. The BMT Network Laboratory Group in NSW is a driving scientific improvement group and has been successfully existent for 6 years. This networking of laboratories has certainly avoided duplication, competition between sites, and extreme cost savings. The key elements to the success of this setup in Sydney will be outlined, along with discussion about lessons learned along the way.

\section{P664}

Evaluation of CryoMACS $₫$ Freezing Bags for cryopreservation of human peripheral blood stem cells A. Sputtek (1), M. Lioznov (1), N. Kröger (1), A.W. Rowe (2), P. Kühnl (1)

(1)University Medical Center (Hamburg, DE); (2)New York University School of Medicine (New York, US)

The successful cryopreservation of peripheral blood stem cells (PBSC) depends upon many factors, including preservation procedures, cooling rate, and storage in a cryogenic temperature using sterile cryogenically stable plastic bags. The aim of this study was to investigate the bioequivalence of two different plastic freezing bags, namely: i) Cryocyte ${ }^{\circledR}$ Freezing Container from Baxter Healthcare, R4R9955, 500 ml, (="B" bag), and ii) the new CryoMACS ${ }^{\circ}$ Freezing Bag from Miltenyi Biotec, 200074-402, $500 \mathrm{ml}$ (="A" bag), with regard to their performance as containers for cryogenic storage of PBSC.

Methods: The study material consisted of 22 frozen autologous PBSC transplant units (approx. $100 \mathrm{ml}$ containing 10\% dimethyl sulphoxide) from 11 patients and 2 units from an allogeneic PBSC donor which were no longer needed for patients' treatment. As a consequence thereof, 12 pairs (2 identical units from each person) could be used throughout the study. After thawing both units of a pair, one unit was transferred into the "A" bag. This bag was sealed in a corresponding overwrap bag which provides additional safety regarding a potential cross contamination during cryogenic storage. The other unit remained in the original "B" bag. After re-freezing both units at approx. $-1{ }^{\circ} \mathrm{C} / \mathrm{min}$, all units were stored again for 8 to 59 days (on an average for 37 days) under cryogenic conditions using liquid nitrogen. Because of the insulation provided by the additional overwrap bag in the case of the " $\mathrm{A}$ " bag, the thawing time in the shaking water bath was extended from $3 \mathrm{~min}$ to $5 \mathrm{~min}$ to allow for a complete thaw. Results: We found an excellent correlation between the 2 bag types based on the following parameters: cell concentration, percentages of mononuclear cells, lymphocytes, monocytes, membrane integrity, hematocrit, concentration of total hemoglobin, concentration of "free" hemoglobin, and hemolysis rate. With regard to clonogenicity as measured in terms of the total, BFU-E, and CFU-GM number of colony forming units in a semi-solid cell culture assay, there were no significant differences for all 3 parameters depending on the bag type (student's paired t-test).
Conclusion: These data support the conclusion that the CryoMACS ${ }^{\circledR}$ Freezing Bag, 200-074-402, (Miltenyi Biotec) is bioequivalent to the Cryocyte ${ }^{\circledR}$ Freezing Container, R4R9955 (Baxter Healthcare). Based on this comparative study it fulfills the requirements of a cryogenic freezing bag as needed for the cryopreservation of human PBSC for transplantation.

\section{P665}

Short- and long-term outcomes of allogenic haematopietic stem cell transplant performed in non-HEPA filtered single rooms: a single- centre experience from India M. Mahapatra, P. Mishra, T. Seth, N. Rathod, S. Rathi, R. Saxena

AlIMS (New Delhi, IN)

Background: In India, there is an urgent need to enhance the hematopoietic stem cell transplant (HSCT) facilities. It is also important to be certain that these procedures can be carried out within the existing infrastructure of hospitals with requisite expertise. There is a lack dedicated HSCT units equipped with special air-handling facilities and high-efficiency particulate air (HEPA) filter rooms. It is important to ascertain the safety of performing allogenic HSCT in single rooms without HEPA filters.

Aim of study: To evaluate the short and long term outcomes of allogenic HSCT performed in non-HEPA filtered single rooms.

Patients and methods: We analyzed sixty-six consecutive patients, who underwent an allogenic HSCT performed in nonHEPA filtered air-conditioned single rooms with barrier nursing, over a period of 4 years from July 2005 to August 2009 . The preferred source of stem cells G-CSF mobilized peripheral blood stem cells (PBSC). In small donors, where PBSC was technically difficult, bone marrow was harvested under anesthesia. All patients received antibacterial and antifungal and antiviral prophylaxis. At the onset of fever, systemic antibiotics were started. Antifungal agents were added if fever persisted for 3 days.

Results: We present our experience of performing sixty six allogenic transplant. Source of stem cell was peripheral blood in 56 , bone marrow in 9 and combined in 1 . The indications were severe aplastic anemia (SAA)-30, CML-10, AML-8, ALL-5, Biphenotypic AL-1, Thalassemia-9, and Myelodysplastic syndrome -3 . The median age was 24 years (range 2.2-46) with 16 females and 50 males. Median time for neutrophil engraftment was 10 days (range 8-17). Fever occurred in 59 (89\%) for a median of 5 days (range 1-38), Systemic antibiotics were used in $88 \%$ and antifungal in $52 \%$ cases. The 30 -day mortality was $3(4.7 \%)$, and 100 -day mortality was $5(7.8 \%)$. After day 100 , there were seventeen fatalities $(26.5 \%)$ due to chronic GVHD-5, relapse-2, graft rejection-2, infections like disseminated tuberculosis-1 and aspergillosis-3, Veno-occlusive disease (VOD)-2 and platelet refractoriness leading to intracranial bleed-2.

Conclusion: Our experience suggests that availability of dedicated BMT unit with special air handling equipment should not be considered a critical and essential precondition for providing allogeneic HSCT who would otherwise succumb to potentially curable hematological diseases.

\section{P666}

A quality management programme to get JACIE accreditation in a haematopoietic transplant unit: a single centre experience leaded by nurses

C. Muñoz Martinez, P. Balsalobre, J. Gayoso, J. Anguita, A. Arellano Barroso, J.L. Diez-Martín HGU Gregorio Marañon (Madrid, ES)

The FACT-JACIE accreditation program formally recognised those SCT (stem cell transplantation) units implementing the best organisational practices. In October 2007 we started the preparative works to get this accreditation, with the support of an external quality consultant and following the 
designation of a SCT nurse to coordinate the whole process. We proceeded to:

- Define the scope of the accreditation program and inform about the program to all professionals involved.

- Design a flowchart for each type of transplant and other cell therapy procedures.

- Update the Quality Manual Plan, previously designed for the ISO certification of labs.

- Review existing and pending operating procedures, standardizing and encoding them.

- Identify and define job profiles, including those for the clinical program director, attending physicians, SCT coordinator and SCT nurses.

- Define a continuous personnel training and a supervising program for staff without specific training in SCT.

- Implement a software tool to support the quality management plan.

Results: Documentation results are shown in Table 1. To date, a total of 58 abnormal findings, 4 of them relevant for patients, have been reported. The regular analysis of the program performance shows that no deviations concerning predefined ranges have been identified. The internal audit held in November-09 revealed that:

1. The regular practice did not comply with standard B.7.1, regarding the mandatory patient identification by 2 independent nurses prior the conditioning administration.

2. Regarding the donor evaluation information on clinical history was not cleared documented in the donor chart.

3. The nurses' team did not strictly adhere to the written procedure for patient identification.

Conclusions: The implementation of a quality management program (QMP) in our SCT Unit based on JACIE standards, has enabled us to update and complete our written procedures, to check the effectiveness of the SCT program on a regular basis and to standardize a continuous training plan for all professionals involved. We have also implemented a system for detecting deviations in the daily work which contributes to prevent errors. To face the extra workload related to the QMP implementation has been possible thanks to the key role played by a specific clinical professional being nurses a valuable human resource in this setting.

\begin{tabular}{|l|c|c|}
\hline Documentation & $\begin{array}{c}\text { Physicians- } \\
\text { related }\end{array}$ & $\begin{array}{c}\text { Nurses- } \\
\text { related }\end{array}$ \\
\hline $\begin{array}{l}\text { Standard operating } \\
\text { procedures }\end{array}$ & 58 & 13 \\
\hline Technical instructions & 2 & 12 \\
\hline $\begin{array}{l}\text { User's information } \\
\text { sheets }\end{array}$ & 5 & 4 \\
\hline Forms & 30 & 5 \\
\hline Quality indicators & 24 & $\begin{array}{c}\text { In working } \\
\text { progress }\end{array}$ \\
\hline
\end{tabular}

Table 1: Documentation results

\section{Minimal residual disease}

\section{P667}

Pre-transplant minimal residual disease in recipients of allogeneic transplantation for acute myeloid leukaemia is predictive of higher transplant-related mortality F. Dignan (1), C. Anthias (1), M. Ethell (1), R. Morilla (2), A. Morilla (2), F. Davies (1), G. Morgan (1), M. Potter (1), B. Shaw (1) (1)The Royal Marsden Hospital (Sutton, UK); (2)The Institute of Cancer Research (Sutton, UK)

Minimal residual Disease (MRD) monitoring is important in guiding management of acute myeloid leukaemia (AML) treated with chemotherapy. Multiparametric flow cytometry has been evaluated where a molecular marker for MRD is not available. Few studies have investigated the impact of MRD in the setting of allogeneic transplantation for AML. We studied 74 patients who underwent allogeneic transplantation for AML in a single centre from 2004-2008. The median age was 47.1 years (range: $20.8-70.1$ ). Overall survival was $48 \%$ at 2 years (median follow-up:1.96 years). 37 patients had myeloablative conditioning and 37 had reduced intensity. 46 patients had T cell depletion. MRD analysis by MFC (sensitivity $0.04 \%$ ) in the bone marrow was performed pre-transplant and on day 28 and 100 post-transplant. By conventional criteria, 47 patients were in $\mathrm{CR} 1,15$ in $\mathrm{CR} 2,4$ in $\mathrm{CR}>2$ and 8 patients were not in CR pre-transplant. We examined the impact of MRD by MFC in patients who were in CR. Survival at 2 years in MRD negative (MRD-) patients pre-transplant was $70 \%$ compared to $29 \%$ in MRD positive (MRD + ) patients $(P=0.003)$. The MRD analysis at day 28 was not predictive of outcome. In patients alive and without relapse at day 100, MRD analysis was predictive of outcome (2 year: MRD- $70 \%$ vs. $27 \%$ MRD +; $P=0.045)$. In patients who were MRD- at both time-points, survival at 2 years was $81 \%$ compared to $42 \%$ in those who were $\mathrm{MRD}+$ at one or both time-point $(P=0.040)$. The incidence of disease relapse was $32 \%$ at 2 years and non-relapse mortality (NRM) was $8 \%$ at day 100 and $22 \%$ at 1 year. The pre-transplant stage was predictive of relapse (2 years: those in CR $28 \%$ (no difference for CR1 or >CR1) compared to those not in CR 63\%, $P=0.004)$ but being MRD + was not; neither pretransplant $(P=0.47)$ nor at day $100(P=0.29)$. Conversely, the NRM was significantly higher in those who were MRD + pretransplant, but not at day 100 . There was a trend to a higher incidence of acute GvHD in the pre-transplant MRD + patients $(50 \%)$ compared to MRD- patients $(33 \%, P=0.074)$. In multivariate analysis at day 100 there was a RR of NRM of 2.12 $(P=0.014 ; 95 \% \mathrm{Cl} 1.1-3.8)$ to MRD + patients. Pre-transplant MRD appears to be predictive of worse outcome. Interestingly this seems largely due to an increase in NRM rather than disease relapse which may reflect differences in the duration and/or intensity of GVHD prophylaxis. A better understanding of these factors may allow tailored treatment strategies based on MRD status.

\section{P668}

Minimal residual disease post-consolidation therapy affects outcome after stem cell transplantation in acute myeloid leukaemia

J. Falantes, F. Márquez, D. Ramírez, E. Ríos, J. González, A. Marín, M. Carmona, I. Espigado, A. Urbano-Ispizua Hospital Virgen del Rocío (Seville, ES)

Background: Acute myeloid leukemia (AML) is a heterogeneous disease with several clinical and biological prognostic features. Detection of residual leukemic cells after chemotherapy (minimal residual disease; MRD) is related to risk of relapse. The role of stem cell transplant (SCT) in patients with MRDpositive $(M R D+)$ is still unclear.

Objective: Analyse the impact of MRD status in AML pts in complete remission (CR) who underwent SCT.

Patients and methods: 83 AML pts in CR received SCT (auto$\mathrm{SCT}=30$, alloSCT $=53$ ) in 2001-09 period. Baseline characteristics of pts shown in table 1. Quantitation of MRD was performed by flow cytometric detection of abnormal phenotypes after induction and consolidation. MRD after consolidation therapy was used for analysis. $0.01 \%$ level was defined to identify MRD + pts. Statistical analyses were performed by SPSS v.16 for survival and $\mathrm{R}$ package software for relapse risk.

Results: 55 pts are alive (66\%). Relapse-free survival (RFS) and overall survival (OS) were $70 \%$ vs. $44 \%(P=0.022)$ and $75 \%$ vs. $52 \%(P=0.029)$ in MRD- and MRD + pts respectively. Globally (auto and alloSCT), MRD + was associated to shorter RFS $(P=0.053$, HR:2.1; Cl:0.9-4.7), OS $(P=0.045$, HR:2.4; $\mathrm{Cl}: 1.02-5.74)$ and higher relapse risk $(P=0.04, \mathrm{HR}: 2.2)$ in multivariate analysis. Pts who achieved MRD- had better prognosis 
with relapse rate of $22 \%$ after alloSCT (5/23 pts) and $36 \%$ after autoSCT (4/11 pts). Of MRD + pts who received autoSCT $(n=19), 13 / 19$ pts relapsed $(68.5 \%)$. In the allogeneic setting (30 pts MRD + and 20 pts MRD-), only 12/30 MRD + pts (40\%) experienced relapse. MRD + in alloSCT was associated to shorter OS in univariate $(P=0.05)$ and multivariate analysis $(P=0.021$, HR:5.82; Cl:1.3-26.3) and a trend to lower RFS $(P=0.064)$.
Conclusion: MRD status is an independent prognostic factor affecting outcome even after SCT and can be used to design risk based strategies in AML together with other prognostic features. This results seem to confirm the lack of efficacy of autoSCT in MRD + pts. AlloSCT may overcome the negative impact of MRD in this group of patients.

\begin{tabular}{|c|c|c|c|c|c|c|c|}
\hline \multirow[b]{2}{*}{ vartablet } & \multirow[t]{2}{*}{ suto } & \multirow[t]{2}{*}{ Allo } & \multirow[t]{2}{*}{ P } & \multirow[t]{2}{*}{ Total } & \multicolumn{3}{|c|}{ 200 } \\
\hline & & & & & URO- & MRO & $p$ \\
\hline ' & $30(66,15)$ & $53(63,9)$ & & $83(1005)$ & $23(13,65)$ & $x(6,65)$ & \\
\hline Westar age & 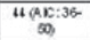 & 30 ax:31-69) & 0.100 & " $\operatorname{ax:32\infty }$ & 30 (ax: $25-12.9$ & $0,5(\mathrm{ax}: 33.00)$ & 0,141 \\
\hline Sex(1). llakt( ) & $12(60 s)$ & $24(45,35)$ & 0.561 & $36(23,65)$ & $11(67,65)$ & $13(43,35)$ & 0,765 \\
\hline 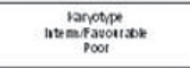 & 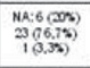 & 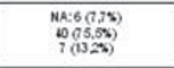 & 02268 & 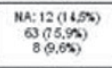 & 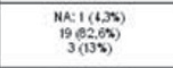 & 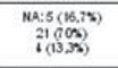 & 0.419 \\
\hline 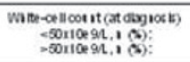 & 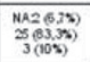 & 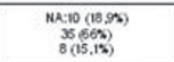 & $022 x$ & 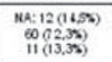 & 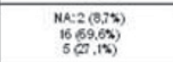 & 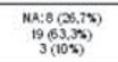 & 0228 \\
\hline Pwiwat u0s,1 (\$) & $5(06 \pi 5)$ & $"(10,85)$ & 0,500 & $15(9 ., 5)$ & $3(105)$ & $8(0, \pi s)$ & 0,384 \\
\hline 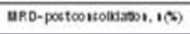 & $1106,75)$ & $23(13.65)$ & 0.56 & $34(615)$ & $23(3925)$ & $30(6,15)$ & 0,550 \\
\hline 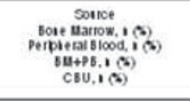 & $\begin{array}{c}3(1005) \\
35(0.75) \\
10505) \\
0\end{array}$ & 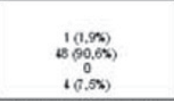 & 0.06 & 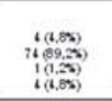 & $\begin{array}{l}1(1,35) \\
20\left(\frac{\beta 15)}{2}\right) \\
2(0,75)\end{array}$ & $\frac{2003.35)}{2(6,75)}$ & 0.508 \\
\hline Coratoothg itghet & $\begin{array}{c}\operatorname{sicy}, 00 \\
(000)\end{array}$ & 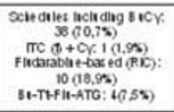 & 0.180 & & 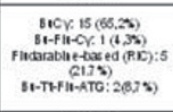 & 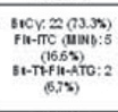 & 0.500 \\
\hline $\cos 4+4 \sec c 00,1$ (\$) & & & & & $7(0,15)$ & $15(500)$ & 0,150 \\
\hline 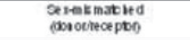 & & & & & $12 \beta 2 . \pi)$ & $12(00)$ & 0,378 \\
\hline
\end{tabular}

Table 1. Baseline charactenstics of pattents. NA: Not avaliable, RIC: Reduced intensity oonditoning
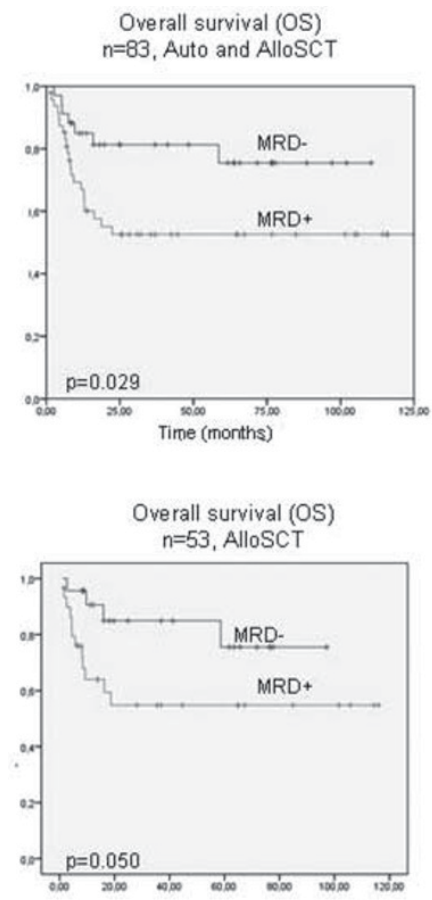

Time (months)
Relapse-Free Survival (RFS) $n=83$, Auto and AlloSCT

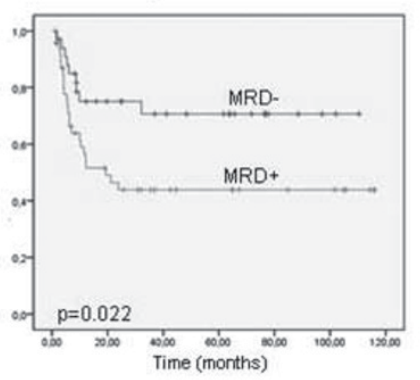

Relapse-Free Survival (RFS) $n=53$, AlloSCT

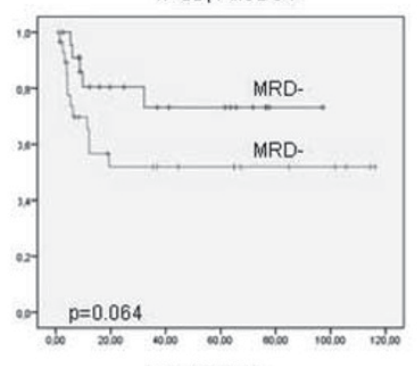

Time (months)
Relapse

$n=83$, Auto and AlloSCT

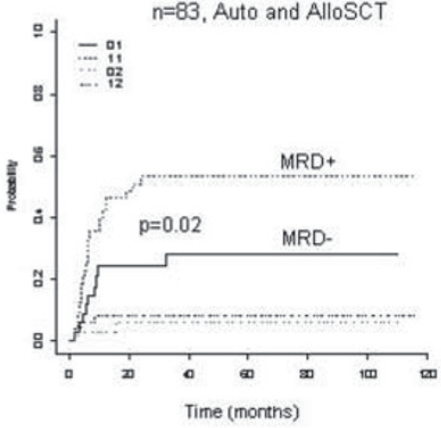

Relapse

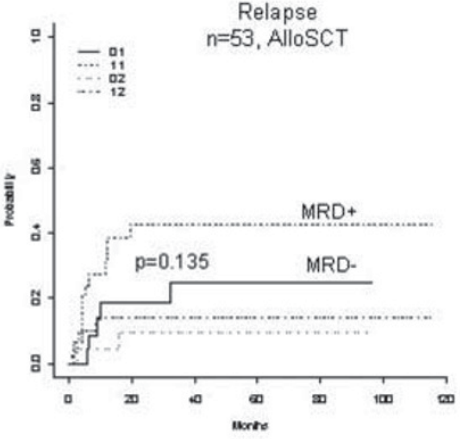


P669

Monitoring of minimal residual disease by quantitative wt1 gene expression following reduced-intensity conditioning allogeneic stem cell transplantation in acute myeloid leukaemia

A. Candoni, E. Toffoletti, R. Gallina, E. Simeone, S. Volpetti, M. Chiozzotto, R. Fanin

University Hospital (Udine, IT)

WT1 is well-known to be a panleukemic marker that is expressed in $90 \%$ of acute myeloid leukemias (AML). Quantification of WT gene expression in bone marrow (BM) samples may be useful as a marker of minimal residual disease (MRD). We evaluated the validity of this AML-MRD marker after Reduce Intensity Conditioning (RIC) allogeneic Stem Cell Transplantation (SCT). The quantitative assessment of WT1 expression by Real-Time Quantitative PCR (RQ-PCR) was measured in 25 AML patients (pts) at diagnosis, at the time of RIC-SCT and after transplant at precise time points. All cases showed high WT1 levels at diagnosis with a mean of 4895 (SD 4462) and a median of 3679 (range 454-16853) copies WT1/104 ABL. At transplant $18 / 25$ pts $(72 \%)$ were in Complete cytologic Remission (CcR) and $7 / 25(28 \%)$ had refractory AML. At the pre-SCT evaluation, bone marrow (BM) samples from pts transplanted in CcR showed significantly lower WT1 expression levels compared to the samples from pts with refractory AML $(P=0.002)$. Median follow up after RIC-SCT was 18 months (range 2-54). On 18 pts transplanted in CcR, those (17/18) who maintained CcR after RIC-SCT displayed WT1 copy numbers persistently low during all the follow-up period. In patients who received RICSCT with active disease obtaining a sustained CcR after transplant (3/25), WT1 levels decreased to normal range in the first two months after RIC-SCT and remained low through the entire study period. All pts who relapsed after RIC-SCT (4/25) had a high WT1 copy number before the cytologic relapse. In $50 \%$ of these cases, an increase in WT1 expression was documented before molecular chimerism decreasing. With this experience, taking into account the limited number of pts, we confirmed a concordance between WT1 expression levels (measured by $\mathrm{RQ}-\mathrm{PCR}$ at precise and sequential time points) and status of AML before and after RIC-SCT and we found a concordance between WT1 expression levels and hematopoietic chimerism status. Our data suggest that, in the RIC-SCT setting, the sequential and quantitative analysis of WT1 may be useful as a leukemia marker for monitoring MRD and as a predictor of overt cytologic relapse.

\section{P670}

Pre-emptive allo-immune intervention in high-risk paediatric acute lymphoblastic leukaemia patients guided by minimal residual disease level before stem cell transplantation

A.C. Lankester (1), M. Bierings (2), E. van Wering (3), A. Wijkhuis (4), R. de Weger (2), J. Wijnen (1), J. Vossen (1), B. Versluys (2), R.M. Egeler (1), M. van Tol (1), T. Revesz (2), J. van Dongen (4), V. van der Velden (4), M. Schilham (1)

(1)Leiden Univ Medical Center (Leiden, NL); (2)UMC Utrecht (Utrecht, NL); (3)SKION (Den Haag, NL); (4)ErasmusMC (Rotterdam, NL)

Relapse of pediatric acute lymphoblastic leukemia (ALL) remains the main cause of treatment failure after allogeneic stem cell transplantation (alloSCT). A high level of minimal residual disease (MRD) prior to alloSCT has previously been shown to predict relapse after alloSCT. Patients at risk might benefit from a pre-emptive, potentially anti-leukemic, allo-immune intervention. In this first prospective, MRD-guided immunotherapeutic intervention study, 48 patients were stratified on the basis of preSCT MRD level. Eighteen children had an MRD level $\geq 1 \times 10-4$ in bone marrow and were, therefore, eligible for an allo-immune intervention, consisting of early cyclosporine A tapering (4-5 weeks postSCT) followed by consecutive, incremental donor lymphocyte infusions (DLI; $n=1-4$ ). The intervention was well tolerated and associated with GvHD $\geq$ grade II in only $23 \%$ of patients who all responded to steroids. Relapse-free survival (RFS) in the intervention group was $22 \%$. However, in contrast with the usual early recurrence of leukemia, relapses were delayed up to 3 years after SCT. In addition, relapses presented at unusual extramedullary sites suggesting that the immune intervention may have altered the pattern of leukemia recurrence. RFS in the conventionally treated MRD negative group $(n=30)$ was similar to that reported in previous studies (RFS: $69 \%$ ). In 8 out of 11 evaluable patients, relapse was preceded by MRD recurrence (median 9 weeks, range $0-30$ ). We conclude that in children with high-risk ALL, immunotherapy based regimens postSCT are feasible and may need to be further intensified to achieve total eradication of residual leukemic cells.

\section{P671}

Monitoring of Willms' tumour gene 1 transcripts in $A M L$ and MDS patients can predict early relapse after allogeneic haematopoietic stem cell transplantation C. Messina, M. Bernardi, C. Tresoldi, M.T. Lupo Stanghellini, A. Crotta, M. Tassara, S. Girlanda, M. Coppola, S. Piemontese, R. Greco, S. Malato, B. Forno, A. Assanelli, D. Clerici, S. Mastaglio, F. Giglio, M. Bruno-Ventre, F. Lunghi, E. Guggiari, M. Carrabba, M. Marcatti, C. Corti, C. Bonini, K. Fleischhauer, J. Peccatori, C. Bordignon, F. Ciceri

San Raffaele (Milan, IT)

Introduction: Various approaches have been used in AML and MDS patients (pts), to detect minimal residual disease (MRD) after haematopoietic stem cell transplantation (HSCT), and predict the risk of relapse, but still the majority of AML cases do not have a useful and sensitive MRD marker. Over-expression of Wilms' tumor gene 1 (WT1) in leukemic blasts has been reported in $>80 \%$ of AML and $>40 \%$ of MDS. Normal hematopoietic stem cells express WT1, but a malignant WT1 expression can be distinguished based on quantitative detection methods such as RQ-PCR; the sensitivity of this assay permit to detect leukemic cells in bone marrow (BM) and peripheral blood (PB) with frequencies as low as 10-3 and 10-5, respectively. We are currently monitoring WT1 in SP and BM after allogeneic HSCT to drive post transplant proper immunotherapy.

Aim: To evaluate if WT1 expression levels can be a useful marker of MRD after allogeneic HSCT.

Materials and methods: $26 \mathrm{AML}$ and $6 \mathrm{MDS}$ pts received an allogeneic HSCT in our Institute between 12/2007 and 11/2009. RQPCR quantification of WT1 was performed in samples of PB and BM at diagnosis, monthly after HSCT, using TaqMan technology on RNA from mononucleated cells. The housekeeping gene ABL was used as the control gene for these quantifications with WT1 level being normalised to 104 copies of ABL per sample.

Results: WT1 at diagnosis: median 6960.02 cp (77.98$31203.57)$. At HSCT 22 pts (68\%) were in CR and $10(31 \%)$ had active disease, with a median WT1 of 82.74 (0.67-5064.91) and 829.94 (44.14-10649.56), respectively. Myeloablative conditioning regimen: Treosulfan $42 \mathrm{~g} / \mathrm{sqm}$, Fludarabine $150 \mathrm{mg} /$ sqm, ATG $30 \mathrm{mg} / \mathrm{kg}$ (HSCT from alternative donors). After HSCT 31 pts were in CR, 9 with active disease before HSCT, with median WT1 $=37.21(11.46-772.92)$. Three pts with low levels of WT1 after HSCT showed a logarimtic increase of WT1 expression only in $\mathrm{BM} \leq 1$ month before hematological relapse; concomitant analysis showed an haematologic and cytogenetic CR with $100 \%$ donor chimerism (STR). Only 1 pt relapsed with a significant, non logarithmic, increase of WT1 levels.

Conclusions: These data show that WT1 could be a useful nonspecific leukemia marker for monitoring of MRD in AML and MDS pts after allogeneic HSCT. WT1 logaritmic increase in PB and $\mathrm{BM}$ can detect molecular relapse, driving post transplant immunotherapy (immunosuppression withdrawal, donor lymphocyte infusion, etc.) to induce an anti-leukemia effect at an early stage of disease. 


\section{P672}

Quantitative real-time PCR detection of Wilms's tumour gene (WT1) transcript in autologous peripheral blood stem cell products predict the risk of acute myeloid leukaemia relapse after autologous transplantation C. Messina, C. Tresoldi, A. Crotta, M. Tassara, S. Malato, J. Peccatori, C. Corti, F. Ciceri, M. Bernardi

San Raffaele (Milan, IT)

Introduction: ASCT is a curative option alternative to allogeneic transplantation for pts with $\mathrm{AML}$; unfortunately, the relapse rate after ASCT is high and can be due to contamination with leukemic blasts of autologous PBSC collected by leukapheresis, although these procedures are usually performed when pts are in proved complete remission (CR). Thus, identification and quantification of a reliable minimal residual disease marker in collected PBSC could be relevant in determining the relapse risk after ASCT. High levels of the WT1 gene transcript in bone marrow and peripheral blood of AML pts after treatment completion predict disease relapse. We retrospectively evaluated the WT1 transcript levels in autologous PBSC of our AML pts who received an ASCT to establish the power of this parameter to predict the risk of AML relapse.

Aim: To correlate the quantitative levels of WT1 in autologous PBSC with the relapse incidence in AML patients who received an ASCT in CR, at our Institute.

Patients and methods: 10 pts, all in morphological and genetic $\mathrm{CR}$ at the time of PBSC collection and before ASCT. PBSC collection by leukapheresis (COBE Spectra cell separator): median CD34+×10\% $/ \mathrm{kg}$ : $9.32(3.79-32)$. RQ-PCR quantification of WT1 was performed in samples of each leukapheresis, using TaqMan technology on RNA from mononucleated cells. The housekeeping gene $A B L$ was used as the control gene for these quantifications with WT1 level being normalised to 104 copies of ABL per sample. Conditioning regimen: treosulfan 10 $\mathrm{gr} / \mathrm{sqm} \times 3$, fludarabine $30 \mathrm{mg} / \mathrm{sqm} \times 5$ cytarabine $2 \mathrm{gr} / \mathrm{sqm} \times 5$. Transplant, median CD34+×106/kg: 5.1 (3.3-8.5).

Results: At last follow-up 4 pts have relapsed after ASCT. Median WT1 copies in leukapheresis of pts who relapsed or did not relapse were 486.65 (89.96-839.63) and 26.66 (4.7982.49 ), respectively. Overall median relapse free survival (RFS) from ASCT was 451 (76-1197) days; median RFS of pts with WT1 copies $>90(n=3)$ or $\leq 90(n=7)$ was $351(93-368)$ and 669 (76-1197) days (log-rank $P=0.003$ ), respectively.

Conclusions: These results suggest the possibility to determine a quantitative cut-off level of WT1 transcripts in autologous PBSC, with higher levels being predictive of contamination with leukemic blasts and indicating an increased relapse risk after ASCT. These data, if confirmed by our ongoing studies, will permit us to discriminate pts who can benefit from ASCT from pts who should be addressed to different therapeutic strategies.

\section{P673}

Durability of minimal residual disease prior to BMT: can we improve on its prognostic value for patients with childhood acute lymphoblastic leukaemia?

P.L. Tan (1), M. Villegas (2), Y.H. Chan (2), A. Yeaoh (2), T.C. Quah (2)

(1)National University Hospital (Singapore, SG); (2)National University of Singapore (Singapore, SG)

We evaluated if the quality of minimal residual disease (MRD) as indicated by the quantitative levels and the duration of sustained MRD level achieved before BMT is predictive of outcome in a retrospective review of consecutive BMTs performed between November 2003 to June 2009 for high risk paediatric acute lymphoblastic leukaemia (ALL) patients. MRD measured by $\mathrm{RQ}-\mathrm{PCR}$ amplification of $\mathrm{IgH}$ and IgK-Kde gene rearrangements was performed at specific time-points before BMT. MRD groups were defined as per Bader P. et. al., JCO 2009: group 1 (undetectable MRD load), group 2 (MRD load less than the quantitative range $(<10-4$ leukemic cells), group 3 (MRD load between $\geq 10-4$ leukaemia cells and less than 10-3 leukaemia cells), group 4 (MRD load $\geq 10-3$ leukaemia cells). Duration of sustained MRD pre-BMT was grouped into $>90$ and $\leq 90$ days. There were 17 BMTs and 15 were evaluable ( 2 had no MRD markers). The median age at BMT was 9.3 (range, 1.3-13.6) years and the median time to BMT was 298 (range, 88-1327) days with 7 patients in CR1, 6 in CR2 and 2 in CR3. Median duration of sustained MRD level pre-BMT was 88 (range, 7-760) days. There were 7 patients in MRD group 2, 6 in MRD group 3, 2 in MRD group 4 and none in MRD group 1. At a median follow-up of 2.4 (range, $0.3-5.6$ ) years, the overall survival (OS) was $86.7 \%$ for the entire cohort. OS and relapse-free survival (RFS) for MRD group 2 was $100 \%$ and $80 \%$, respectively. For combined MRD groups 3 and 4 , OS and RFS were $75 \%$ and $57 \%$, respectively. In the patients who relapsed, the duration of sustained MRD level pre-BMT was shorter (Figure 1). Logistic regression analysis further showed that duration of $M R D \leq 90$ days (regardless of MRD group), predicted relapse, except in patients who developed graft-versus-host disease (GVHD) (Table 1). We verified the findings of recently published data that MRD level pre-BMT predicted outcome. Further, we observed that sustained MRD level with duration >90 days pre-BMT, and more importantly, the presence of GVHD predicted a lower relapse risk even in patients with high MRD levels pre-BMT.

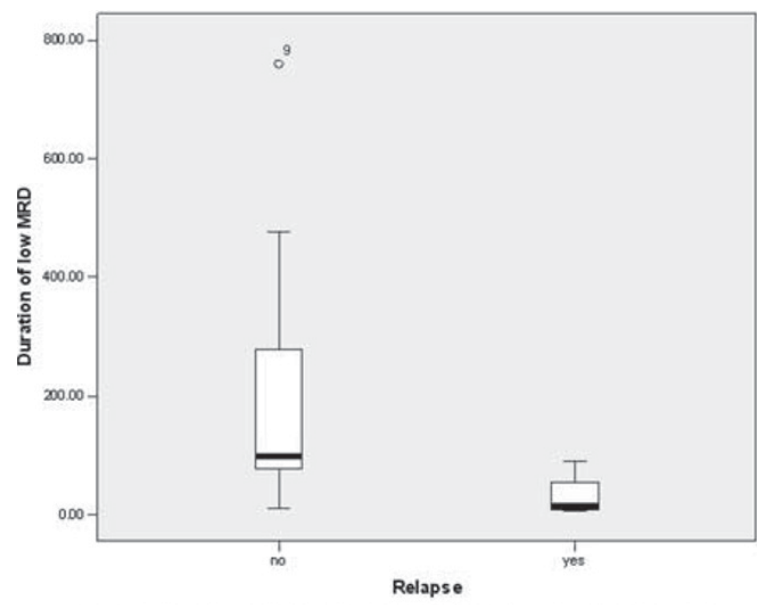

Figure 1: Duration of sustained MRD level and occurrence of relapse

Table1: Prediction of relapse based on GVHD, MRD level and duration of sustained MRD level pre-BMT

\begin{tabular}{|c|c|c|c|}
\hline GVHD & MRD group 3/4 & $\begin{array}{c}\text { Duration of sustained } \\
\text { MRD level } \leq 90\end{array}$ & $\begin{array}{c}\text { Prediction of } \\
\text { relapse }\end{array}$ \\
\hline No & No & No & No \\
\hline No & No & Yes & Yes \\
\hline No & Yes & No & No \\
\hline No & Yes & Yes & Yes \\
\hline Yes & No & No & No \\
\hline Yes & No & Yes & No \\
\hline Yes & Yes & No & No \\
\hline Yes & Yes & Yes & No \\
\hline
\end{tabular}




\section{P674}

Minimal residual disease diagnostics in patients with AML in the post-transplant period: comparison of peripheral blood and bone marrow analysis

T. Stahl (1), A. Badbaran (1), N. Kroeger (1), A. Smirnova (2), S. Asenova (1), T. Zabelina (1), A. Zander (1), B. Fehse (1), U. Bacher (1)

(1)Clinic for Stem Cell Transplantation (Hamburg, DE) (2)Department for Stem Cell Transplantation (St. Petersburg, RU)

Background: Recently, monitoring of the minimal residual disease (MRD) load with quantitative real-time PCR (qPCR) for the nucleophosmin (NPM1) mutations of the subtype $A$ in pts with acute myeloid leukemia (AML) was developed for the posttransplant setting. Also, we perform regular chimerism analyses using our established sensitive qPCR techniques. Due to the rapid kinetics of $A M L$ relapse, short intervals for post-transplant monitoring are desirable, which requires analysis from peripheral blood (PB). We compared qPCR results for the NPM1Amut and chimerism in bone marrow (BM) and PB in the post-transplant period.

Patients: 21 pts with NPM1A mutated AML (10 males, 11 females, 21-70 yrs) were included after allogeneic stem cell transplantation (SCT). 12 pts had a concomitant FLT3-ITD. NPM1Amut levels were quantified with a dilution curve from the OCI/AML1 cell line and normalized for DNA content with HCK. Chimerism was assessed by qPCR for short insertions/ deletions polymorphisms or Y-specific sequences for sexmismatched SCT. Analyses were performed in parallel from unseparated $\mathrm{PB}$ and $\mathrm{BM}$ within different periods post-transplant (days +13 to 1337 )

Results: 8 pts (38\%) were negative for the NPM1Amut in PB and BM. 13 pts $(62 \%)$ tested positive for the NPM1Amut in BM, in 10 cases combined with positive results in PB. In 5/10 with positive PCR results in both materials, the mutation level was higher in BM than in PB (1-3 log scales). Thus, results were discordant in 3 cases (14\%), as the PB was negative for the NPM1Amut while BM was weakly positive. Cases with at least $0.2 \%$ NPM1Amut level in the BM were always positive in PB. Chimerism analysis was possible in 18 pts. $8 / 18(44 \%)$ had complete $(\geq 99.9 \%)$ donor chimerism (DC) in BM and PB. In $4 / 18(22 \%)$, mixed chimerism (MC) was detected in both materials $(55.6 \%-99.7 \% \mathrm{DC}) .1 / 18(6 \%)$ had loss of chimerism in PB and low donor chimerism (39.7\%) in BM. Thus, BM and PB were concordant in 13/18 (78\%). In $3 / 18(17 \%)$, PB showed $\mathrm{DC}$ while the $\mathrm{BM}$ showed $\mathrm{MC}$; in one case, it was the other way around.

Conclusion: qPCR monitoring for the NPM1mut and chimerism from unseparated $\mathrm{PB}$ contributes to post-transplant surveillance in AML. We suggest investigation of PB after SCT in short time intervals (e.g. every other week) within the first 2 years. In case of negative results in the PB, BM analysis might be performed in longer intervals (e.g. every 3 months), but should be done immediately in case of positive PCR results in PB.

\section{P675}

Leukaemia lineage-specific chimerism analysis and molecular monitoring improve outcome of donor lymphocyte infusions

D. Sairafi, M. Remberger, M. Uhlin, O. Ringdén, J. Mattsson Karolinska Institutet (Stockholm, SE)

A retrospective analysis was performed in 118 patients with hematological malignancies who received donor lymphocyte infusions (DLI) after allogeneic stem cell transplantation (ASCT). The overall incidences of acute GVHD grades I-II and grades III-IV acute GVHD were $33 \%$ and $8.5 \%$, respectively. Probability of chronic GVHD was $33 \%$. Pancytopenia developed in three patients. Patients with acute leukaemia and myelodysplastic syndrome showed a three-year survival of $42 \%$ if DLI treatment was given due to molecular relapse, compared to $16 \%$ in haematological relapse $(P<0.006)$. In multivariate analysis, response to DLI correlated with non-hematological relapse (Odds ratio (OR) 11.1, $P=0.0001$ ), chronic GVHD (OR $4.3, P=0.01$ ), and late relapse (OR $4.1, P=0.016$ ). The development of acute or chronic GVHD did not significantly influence the response of DLI in patients with molecular/cytogenetic relapse. However, the development of chronic GVHD was significantly correlated to a better response in patients with hematological relapse since only 4 of 29 patients without chronic GVHD responded compared to 7 of 12 with chronic GVHD $(P=0.007)$. The development of chronic GVHD increased significantly if DLI was given $>12$ months after ASCT (46\% vs. $27 \%, P=0.04$ ). In contrast, time between ASCT and start of DLI treatment did not significantly influence the risk of developing acute GVHD.

To conclude, monitoring of leukemia-lineage specific chimerism is of utmost importance for DLI response after ASCT.

\section{P676}

Constitutive expression of the serine protease inhibitor PI-9 inhibits Granzyme B induced apoptosis in leukaemia cells K. Fritsch (1), J. Finke (1), C. Grüllich (2)

(1)Freiburg University Medical Center (Freiburg, DE); (2)National Center for Tumor Diseases (Heidelberg, DE)

Introduction: Cytotoxic T-lymphocytes and NK-cells (CTL NK) induce apoptosis in malignant cells mainly employing the granzyme B (GrB) pathway. This mechanism constitutes the effector stage of the graft versus leukemia reaction $(\mathrm{GvL})$. We recently reported resistance against $\mathrm{GrB}$ induced apoptosis in leukemia cells. One candidate to mediate $\mathrm{GrB}$ resistance is the endogenously expressed protease inhibitor 9 (PI-9). Aim of this study was to determine the Pl-9 expression in leukemic cells and the influence of expression levels on the activity of GrB directly.

Methods and results: We assessed the proteolytic activity of $\mathrm{GrB}$ with the colorimetric substrate Ac-IEPD-pNA in PI-9 negative and positive cells and consequently the activation of caspase 3 , which is the main downstream target of $\mathrm{GrB}$. We identified $\mathrm{PI}-9$ negative, intermediate and high expressing cell lines. The lymphoblastic cell line Jurkat and the myeloblastic cell line U937 did not express PI-9, while PI-9 expression was high in lymphoblastic Daudi cells, a cell line from a patient with acute myeloblastic leukemia (AML-R) and a U937 subline (U937PI$9+$ ). Myeloblastic K562 cells and two EBV lymphoblastic cell lines (LCL) expressed PI-9 low to intermediatly. When cells were treated with cell permeable $\mathrm{GrB}$, the intracytoplasmatic $\mathrm{GrB}$ activity was inversely correlated to the PI-9 expression level of the respective cells. To verify the influence on apoptosis we measured caspase 3 activation following GrB treatment. Caspase 3 activation was also inversely correlated to the level of $\mathrm{Pl}-9$ expression.

Discussion: In conclusion, we show that PI-9 is a potent inhibitor of $\mathrm{GrB}$. The level of resistance to $\mathrm{GrB}$ in leukemia cells is positively correlated to the level of PI-9 expression. This mechanism may be important for immune evasion of leukemia cells in vivo and could mediate resistance to GvL.

\section{P677}

Nilotinib induced remission of central nervous system relapse of imatinib-resistant $\mathrm{PH}+\mathrm{cML}$ after allogeneic haematopoietic cell transplantation

S. Wang, T. Lange, D. Niederwieser, H.K. Al-Ali

University of Leipzig (Leipzig, DE)

Extramedullary relapse of $\mathrm{Ph}+\mathrm{cML}$ can occur after $\mathrm{HCT}$. Imatinib is an option but offers incomplete protection against CNS leukemia even in patients showing good systemic response. It is known that dasatinib can cross the blood- brain barrier and may be an efficient therapy for CNS leukemia. For the first time, we show that nilotinib can cross the blood-brain barrier and is effective in treating CNS relapse after $\mathrm{HCT}$ in $\mathrm{Ph}+\mathrm{CML}$ resistant to imatinib. 
Patient and methods: A 51 years old female was diagnosed with $\mathrm{Ph}+\mathrm{cML}$ in chronic phase in 1992. Treatment with interferon alpha achieved hematological response. In February 2002, the patient was started on imatinib $400 \mathrm{mg} / \mathrm{d}$. Due to primary resistance with progress to accelerated phase, the patient was offered HCT after RIC [200cGy TBI + fludarabine $30 \mathrm{mg} / \mathrm{m}^{2} /$ day on 3 days] followed by immunosuppression with mycophenolate mofetil and cyclosporine] from a matched related donor in October 2002.Despite extensive chronic graft versus host disease of the skin, the patient suffered a hematological relapse in April 2004. Imatinib was restarted. A complete molecular response (CMR) by RT-PCR of 13 months duration could be achieved. Imatinib was discontinued because of intolerance. The patient lost CMR with low BCR-ABL transcripts detectable in the marrow. July 2007 , the patient suffered a cerebral chloroma and was treated with radiotherapy and dasatinib $40 \mathrm{mg}$ bid. A second CMR could be induced. Due to pleural and pericardial effusions, dasatinib had to be discontinued in February 2009. Four months later CMR was lost with low levels of BCR-ABL transcripts in the marrow. October 2009, the patient developed back pain with numbness in both legs. MRI showed hyperintensities consistent with leptomeningeal disease at the level of the cauda equina. Cerbrospinal fluid (CSF) revealed $\mathrm{Ph}+$ blasts verified by FISH. Simultaneously, marrow examination showed a cytogenetic relapse and $58 \%$ BCR-ABL/ABL transcripts by RT-PCR with no ABL kinase domain mutations.

Results: Nilotinib $400 \mathrm{mg}$ bid was started. Three days later, a dramatic clinical improvement occurred. After four weeks on nilotinib, a CMR could be documented. Both CSF and MRI showed a complete remission of CNS disease. Nilotininb was very well tolerated.

Conclusions: After HCT, minimal residual disease by RT-PCR can herald extramedullary relapse of CML after HCT. For the first time, we show that nilotinib can be a very effective treatment of CNS relapse of $\mathrm{Ph}+\mathrm{cML}$ after HCT.

\section{Tolerance and chimerism}

\section{P678}

Chimerism in children with haematological malignancies given a T-cell depleted haploidentical stem cell transplantation: relationship between lineage-specific mixed chimerism and clinical outcome

D. Lisini (1), R. Cristantielli (1), D. Montagna (2), M. Labirio (1), G. Giorgiani (1), M.E. Bernardo (1), A. Bertaina (1), R. Maccario (1), F. Locatelli (2)

(1)Fondazione IRCCS Policlinico San Matteo (Pavia, IT); (2)Fondazione IRCCS Policlinico San Matteo, Università degli Studi di Pavia (Pavia, IT)

Donor/recipient mixed chimerism (MC) has been reported to be associated with an increased risk of disease recurrence in patients with haematological malignancies given a T-cell depleted haematopoietic stem cell transplantation from an HLA-haploidentical relative (haplo-HSCT). To improve our understanding of the meaning of $\mathrm{MC}$, we investigated the possible persistence or reappearance of host haematopoietic cells in a)peripheral blood (PB) and bone marrow (BM) samples, b)PB T-, B-, and NK-lymphocyte subsets, c) BM cell subsets selected according to the immunophenotype of the leukemia blasts at disease diagnosis, in the relationship to clinical outcome in patients given haploHSCT for haematological malignant diseases.

We analyzed sixty-one consecutive children with acute lymphoblastic leukemia $(n=37)$, acute myeloid leukemia $(n=17)$, myelomonocytic leukemia $(n=1)$ and myelodysplastic syndromes $(n=6)$, who underwent T-cell depleted haplo-HSCT. Nine out of these 61 children received a second transplant from the same donor, due to graft rejection $(n=8)$ or relapse $(n=1)$ and were also analyzed.
Twenty-nine out of the 61 children exhibited MC after transplantation and 15 of them are alive and disease-free; 12/15 showed MC in PB, particularly in T-lymphocyte subsets, at time of engraftment, and finally 8 of them converted to full donor chimerism (FDC) within 32 months after transplantation and 4 are maintaining stable $\mathrm{MC}$ after a median follow-up time of 15 months; $3 / 15$ exhibited $\mathrm{MC}$ in the BM compartment only, but rapidly converted to FDC (in $1 / 3 \mathrm{MC}$ was observed only in the cell subset selected according with the immunophenotype of the leukemia blasts, in $2 / 3$ cell subsets selection was not evaluable). Fourteen out of 29 patients included in the MC group relapsed: 10/14 developed a very quick relapse, while 4/14 showed MC on BM cell subsets 2.5-5 months before disease recurrence.

Thirty-two out of 61 patients exhibited lineage-specific FDC at all time points examined; $21 / 32$ are alive and disease-free, ten died of transplantation-related complications other than relapse and one more child has relapsed and received a second transplant from the same donor.

Early MC, particularly in PB T-lymphocyte subsets, may be maintained months after transplantation and does not predict disease recurrence, by contrast, $\mathrm{MC}$ in BM compartment is strongly associated with subsequent relapse in children with haematological malignancies given haplo-HSCT.

\section{P679}

Outcome of patients with graft rejection after low-dose total body irradiation based preparative regimens for allogeneic haematopoetic stem cell transplantation G.-N. Franke, S. Leiblein, V. Vucinic, W. Pönisch, H.K. Al-Ali, N. Basara, D. Niederwieser, T. Lange

University of Leipzig (Leipzig, DE)

Objectives: SCT after reduced intensity conditioning (RIC) is routinely used as a curative approach for older and medically impaired patients (pts) with hematological malignancies. Graft rejection (GR) occurs in approximately $5 \%$ to $8 \%$ of pts and raises many clinical questions. In order to address these points, the outcome of pts with GR after haematopoetic stem cell transplantation (SCT) with 2 Gy total body irradiation (TBI) in combination with or without fludarabine followed by CsA and MMF was analyzed retrospectively.

Patients and methods: A total of 380 pts underwent allogeneic SCT using a low-dose TBI based preparative regimen at the University of Leipzig between 1998 and 2007. Of these pts, $30(7.9 \%)$ were identified as having either primary $(n=25)$ or secondary GR $(n=5)$, defined as T-cell chimerism $<10 \%$ donor cells. Pts received a transplant from related $(n=3)$ or unrelated $(n=27)$ donors as treatment for $\operatorname{AML}(n=11)$, for $P h+c M L(n=5)$, for MDS/MPS $(n=5)$, for multiple myeloma or $\mathrm{NHL}(n=5)$, for $\operatorname{ALL}(n=2)$, for PNH $(n=1)$ and for solid tumor $(n=1)$.

Results: From the 30 pts with GR, $5(17 \%)$ developed no and $17(56 \%)$ self limiting neutropenia <1000 WBC/ $\mu \mathrm{l}$ with a median duration of 25 (range: 7-69) days. 5 further patients $(17 \%)$ recovered after a 2 nd allogeneic HCT only and 3 pts $(10 \%)$ died in neutropenia. Thrombocytopenia $<50,000 / \mu l$ was observed in 23 pts $(77 \%)$, of which $13(43 \%)$ had spontaneous platelet recovery after a median of 31 (range: 10-69) days. 7 pts (23\%) recovered after the 2 nd HCT and 3 pts (10\%) died before platelet recovery.

The majority of the patients $(n=17 ; 57 \%)$ underwent $2 n d$ HCT after a median of 71 (range 40-1143) days after the 1st SCT using the same $(n=1)$ or another unrelated $(n=16)$ donor. 13 pts $(76 \%)$ showed engraftment, $2(11 \%)$ died of TRM and 5 of relapse $(30 \%)$ after a median of 171 (range 92-1835) days. The 1-, 2-, and 5-year overall survival (OS) for this group was $71 \%, 64 \%$, and $43 \%$, respectively. The $1-, 2-$, and 5 -year OS for pts without a 2 nd transplant $(n=13 ; 43 \%)$ was $45 \%, 18 \%$, and $18 \%$ after a median obsrvation time of 149 (range $40-844$ ) days, respectively.

Conclusion: Patients with GR showed hematological recoveries ranging from no to spontaneous recovery in this minimal 
intensive conditioning regimen. The second transplant was successful in the vast majority of patients, with TRM in the range of the first transplant: The OS of pts with a second transplant was better than those without. Therefore a 2 nd SCT should be urgently considered after GR.

\section{P680}

T-cell chimerism post transplant on day +90 and +180 can predict acute and chronic GvHD but not overall survival and disease-free survival in patients undergoing reduced-intensity conditioning allografts

E. Nikolousis (1), F. Clark (1), C. Brookes (1), S. Nagra (1), S. Jeffries (2), M. Cook (1), P. Mahendra (1), M. Griffiths (2), S. Robinson (3), C. Craddock (1)

(1)University Hospital Birmingham (Birmingham, UK); (2)Womens Hospital Birmingham (Birmingham, UK); (3)Bristol Royal Infirmary (Bristol, UK)

Reduced intensity allografts are used to deliver a GvL effect in older patients with haematological malignancies and a concomitant reduction in the incidence of GvHD. The presence of mixed T cell chimerism posttransplant is indicative of bidirectional haematology outcome and could be predictive for disease relapse. The main curative mechanism is a potent GvL effect and the use of chimerism is the way to determine the percentage of graft cells present.

We have therefore analysed the T cell chimeric status of 133 patients with a median age of 50 years undergoing a reduced intensity Alemtuzumab conditioning regimen for lymphoid (64) and myeloid (69) malignancies and correlated this to the transplant outcomes(overall survival, disease free survival, acute and chronic GvHD). The median follow up was 23 months (range 5-108 months). 77 had a sibling transplant and 56 had an unrelated transplant. 82 patients were in CR at the time of transplant and 51 had PR. Patients with mixed T chimerism at 90 days were almost $80 \%$ less likely to have acute GVHD than patients with full donor $\mathrm{T}$ cell chimerism $(P=0.0004)$. There were no other statistically significant differences between $T$ cell full donor and mixed chimerism patients observed and $T$ cell chimeric status at 90 days was not influential of OS, DFS, relapse free survival or incidence of chronic GVHD in the multivariate setting. Mixed T cell chimerism however, was still associated with a reduction in the incidence of acute GVHD when also accounting for other significant factors, conditioning regimen and disease type.

Multivariate analysis of $T$ cell chimerism at day +180 and outcome found a significant association with chronic GVHD. Patients with mixed chimeric status at 180 days were less likely to have chronic GVHD when also adjusting for CR status at transplant. Despite the inclusion of other prognostic factors chimeric status did not influence either OS or DFS or relapse free survival times. Analysis of chimeric status split by disease type lymphoid and myeloid produced identical results.

Our study showed that T cell chimerism on day +90 and +180 was not predictive of disease relapse and overall survival indicating that probably disease specific factors and pretransplant chemosensitivity could play a more important role in disease relapse. Future studies which could potentially look into $\mathrm{T}$ cell chimerism beyond this time points and higher number of patients might be needed to correlate these results with our study.

\section{P681}

Chimerism quantification in CD4 +/CD25 + regulatory $T$ lymphocytes after stem cell transplantation

V. Noriega, J. Gayoso, P. Balsalobre, D. Serrano, G. RodriguezMacias, J. Anguita, A. Gomez-Pineda, J.L. Díez-Martín, I. Buño Hopital General Universitario Gregorio Marañón (Madrid, ES)

Introduction: Chimerism quantification is useful for the follow up of patients after allogenic stem cell transplantation (Allo-SCT) mostly if performed in purified leukocyte lineages. Regulatory
T lymphocytes (Treg; CD4+/CD25+) have shown to promote donor-recipient tolerance after SCT.

Objective: To analyze the dynamics of chimerism in Treg, compared to that of peripheral blood (PB) and CD3 + T lymphocytes. Patients and Methods: The study included 53 Allo-SCT (18 non-myeloablative and 35 myeloablative, including 14 from MUD). Stem cell source was PB in 44 , umbilical cord blood in 5 , bone marrow in 4 . Chimerism analysis was performed every 2 weeks in PB, Tcells and Treg until complete chimerism (CC) was achieved, and in PB at specific time points $(+30$, $+60,+90,+180,+365$ and every 6 months) thereafter. Follow up was censored at the moment of rejection, relapse, DLI or 2nd Allo-SCT. Isolation of CD3 + Tcells and CD4 +/CD25 + Treg was performed by inmunomagnetic means (Miltenyi Biotec) from mononuclear cells obtained by gradient centrifugation of $1 \times 10^{7}$ PB cells for each procedure. Chimerism was quantified by STR-PCR revealed by capillary electrophoresis (AmpFISTR SG Plus, Applied Biosystems). Considering that $>95 \%$ purity is obtained by immunomagnetic selection of these cell subsets, as determined by flow cytometry, samples with $\geq 5 \%$ of recipient cells were defined as in mixed chimerism (MC).

Results: Figure 1 shows the dynamics of CC achievement within the first year post-SCT in blood, CD3 + Tcells and CD4+ CD25 + Treg in 49 patients (4 patients censored due to early relapse or rejection). Time to CC in Tregs was longer (mean of $42 \mathrm{~d}$ ) in Tregs than in Tcells ( $38 \mathrm{~d}$ ) or PB ( $35 \mathrm{~d}$ ). Therefore, not only patients with $\mathrm{CC}$ in $\mathrm{PB}$ and mixed chimerism in leukocyte lineages, but also patients with complete chimerism in PB and Tcells while the Treg subset maintained mixed chimerism $(n=5)$ were observed. 3 patients maintained MC in PB, Tcells and Tregs beyond day +100 without evidence of graft rejection or disease relapse.

Conclusion: Chimerism dynamics in Tregs correlates with that of Tcells, although time to CC is longer in Tregs. Some patients show MC in Tregs once both PB and Tcells have achieved CC. The analysis of a larger patient cohort would allow to get insight into the possible association between such observations and the development of complications after SCT.

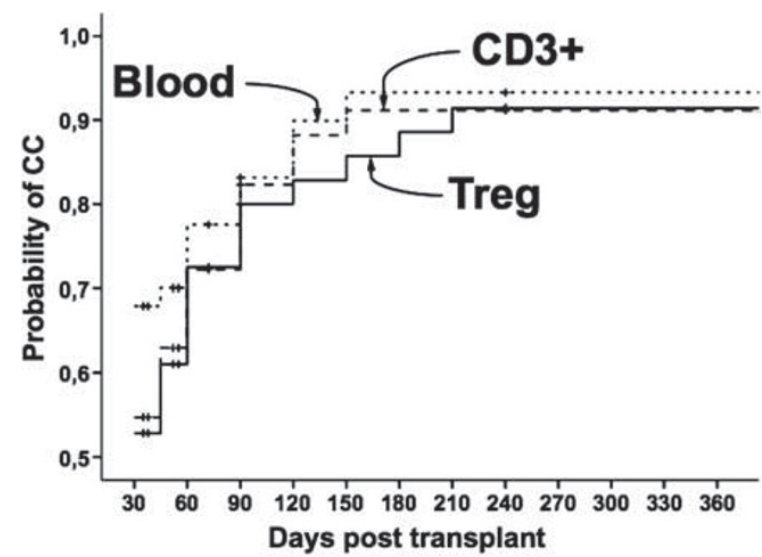

P682

Soluble HLA-G molecules in cord blood: preliminary suggestions for stem cell transplantation

P. Bergamaschi (1), S. Sachetto (2), C. Capittini (1), M. Viola (2), M. Truglio (2), C. Badulli (1), A. Pasi (1), M. Guarene (1), C. Tinelli (1), V. Genovese (1), B. Romano (1), A. Marchesi (1), R. Poma (2), M. Martinetti (1), M. Cuccia (3), L. Salvaneschi (1) (1)Fondazione IRCCS Policlinico San Matteo (Pavia, IT); (2)Fondazione IRCCS Policlinico (Pavia, IT); (3)Pavia University (Pavia, IT)

Soluble HLA-G (sHLA-G) is an immunosuppressive molecule involved in maternal acceptance of the foetus during pregnancy 
and intrauterine growth. In this setting, SHLA-G abrogates maternal NK cell activity against foetal tissue establishing immunotolerance at the maternal-foetal interface. In heart transplantation, high sHLA-G levels have been correlated to low incidence of severe rejection and good prognosis. In haematopoietic stem cell (HSC) transplantation, sHLA-G is advocated in the prevention of graft-versus-host disease (GvHD). As cord blood (CB) is associated to lower incidence of GvHD than other HSC sources, it could be reasonable that this is due (at least in part) to high pregnancy-related SHLA-G levels in $\mathrm{CB}$, which is obtained at time of birth of the infant donor. All HLA-G isoforms are encoded by a HLA class lb gene. As the insertion of $14 \mathrm{bp}$ in the 3'UTR of the HLA-G gene has been associated to low sHLA-G levels, we investigated the correlation between sHLA-G concentration and HLA-G genotype in $\mathrm{CB}$. For this purpose we retrospectively considered $29 \mathrm{CB}$ units of the Pavia CB Bank cryopreserved from 2003 to 2005. sHLA$G$ concentration was determined on thawed aliquots of $C B$ plasma (stored in the same conditions of the cords) by SHLAG ELISA kit (Exbio, Praha, Czech Republic) and 14bp HLA-G genotyping was performed by PCR. 11 samples showed the $+14 \mathrm{bp} /+14 \mathrm{bp}, 9$ the $-14 \mathrm{bp} /+14 \mathrm{bp}$ and 9 the $-14 \mathrm{bp} /-14 \mathrm{bp}$ genotype, respectively. SHLA-G concentration was $46.6,36.9$ and $33.7 \mathrm{ng} / \mathrm{mL}$ on average in the $+14 \mathrm{bp} /+14 \mathrm{bp},-14 \mathrm{bp} /+14 \mathrm{bp}$ and $-14 \mathrm{bp} /-14 \mathrm{bp}$ genotype groups, respectively (see the graphic). No statistically significant variability was observed in CB sHLA$G$ concentration according to the foetal 14bp HLA-G genotype. However, the detection of appreciable levels of sHLA-G supports the hypothesis of a particular microenvironment stimulating sHLA-G secretion in CB. Despite the time (ranging from 4 to 6 years) and conditions of storage $\left(-190^{\circ} \mathrm{C}\right.$, in liquid nitrogen), detectable levels of sHLA-G can be demonstrated in CB also after long-term storage. If confirmed this finding will suggest an inversion to the actual trend of storing $C B$ units after removal of plasma for volume reduction, in order to preserve their beneficial sHLA-G content. Further investigations are needed to increase sample size and identify the mechanisms of sHLA-G expression in $\mathrm{CB}$.

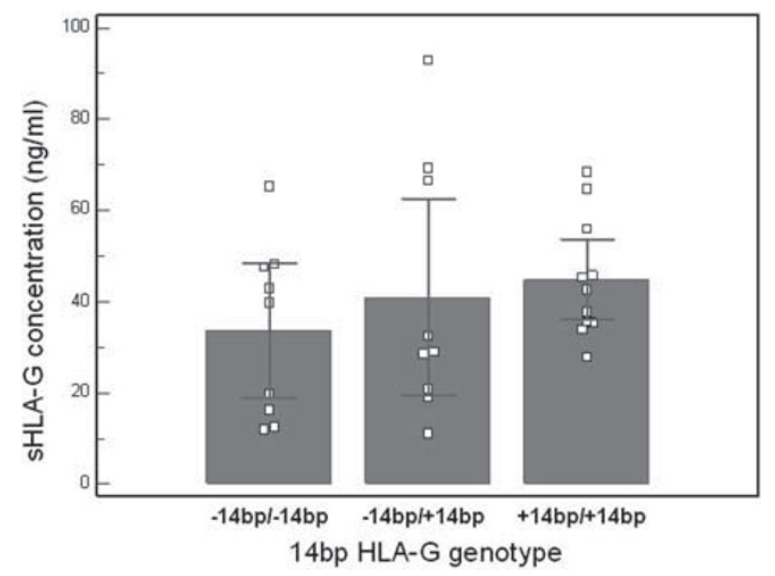

\section{P683}

Indoleamine-2,3-dioxygenase induction and persistence in human monocytes or macrophages versus monocytederived dendritic cells

J. Raberger (1), M. Lanzinger (1), S. Heitzeneder (1), B. Jürgens (1), D. Fuchs (2), A. Heitger (1)

(1)St Anna Children's Hospital (Vienna, AT); (2)Medical University (Innsbruck, AT)

Monocytes (Mo), macrophages (Mph) and dendritic cells (DC) are commonly regarded as discrete cell types with Mo, Mph being components of the innate immune system, and DC acting at the interface with the adaptive immune system. All three have been suggested to be essentially involved in modulating immune responses.

The expression and activity of the tryptophan metabolizing enzyme indoleamine-2,3-dioxygenase (IDO) has been proposed as a critical mechanism in down-regulating $\mathrm{T}$ cell responses and the induction of tolerance. IDO has been described to be active in Mo, Mph and DC with interferongamma (IFN-g) constituting a potent inducer of IDO. Since we aim to create a stable in vitro culture system to generate allospecific tolerant T cells, we investigated potential differences in IDO protein expression and enzymatic activity. Using human blood cells, Mo were identified as CD14+ cells and were differentiated into Mph and DC by culture with M-CSF and with IL-4/GM-CSF, respectively.

The stimulation of Mo, Mph and DC with IFN-g \pm lipopolysaccharide (LPS) resulted in cell surface expression of MHC class I and II, and of co-stimulatory molecules CD80/86 in either cell population with minor differences. IFN-g was critical for inducing IDO expression in either cell type, but IDO enzymatic activity was profoundly different. Upon a 48-hour stimulation period DC completely (>95\%) depleted the cell culture medium of tryptophan, while Mo and Mph showed only about $50 \%$ tryptophan consumption. Furthermore, DC displayed sustained enzymatic IDO activity after removal of LPS and IFN-g, whereas Mo and Mph lost the ability to metabolize tryptophan in re-cultures. This data clearly show that IFN-g can induce potent and sustained IDO activity in DC and, thus, underscore the recently questioned difference in cell biology of DC and Mph (Hume DA, 2008). Furthermore, they identify DC as promising candidates to fully exploit the potential of the tolerogenic potential of an accelerated tryptophan metabolism to specifically eliminate and regulate allo-reactive $T$ cells (supported by Austrian Science Foundation P\#20865).

\section{P684}

Efficacy of a panel constituted by 15 STR markers plus amelogenin locus in evaluation of chimerism status after haematopietic stem cell transplantation

F. Colnaghi, E. Elli, M. Parma, A. Colombo, P. Pioltelli, E. Terruzzi, G. Cazzaniga, A. Biondi, E.M. Pogliani

Ospedale S. Gerardo (Monza, IT)

Introduction: Analysis of Short Tandem Repeats (STR) polymorphism is actually the widely employed assay for evaluation and quantification of Chimerism Status (CS) after haematopoietic stem cell transplantation (HSCT). STRs polymorphism analysis is based on a panel of STR markers largely variable in the population, and it has been mutuated from forensic medicine. Actually, in the transplant setting, many panels of STR markers have been derived from forensic practice.

Aim of the study: In our laboratory we analysed CS on patients submitted to allogeneic HSCT. We employed a STR panel derived from a commercial kit for forensic use (PowerPlex 16 System, Promega, USA). Our panel includes 15 highly polymorphic STR loci (Penta D, Penta E, TH01, D3S1358, D18S51, TPOX, D21S11, D13S317, D8S1179, D16S539, D5S818, D7S820, Vwa, CSF1PO, FGA) and Amelogenin locus. Aim of our study was to verify the efficacy of this panel in CS evaluation in our population.

Methods: We studied STR polymorphism with PCR amplification and semi-quantitative detection of number of repeats of each STR marker. For the CS monitoring we used the "informative markers" between donor and recipient individuals. We analyzed 100 consecutive donor-recipient (D/R) couples in allogeneic HSCT performed in our centre. 95\% of patient population was Italians from all the regions, $5 \%$ was from other countries.

Results: The panel employed permitted to have al least one informative markers for each D/R couple. Particularly $41 \%$ had 2 informative markers, $24 \%$ had 3 informative markers, 18\% had 4 or more informative markers and only $16 \%$ had 1 informative marker. Amelogenin, Penta D, Penta E and TH01 are 
the more variable STRs in our population and at least one of them is informative in $78 \%$ of our population. These four markers summed to D3S1358, D18S51, TPOX, D21S11, D13S317, D8S1179 constitute a panel informative in $99 \%$ of our population. Instead the less informative markers are D16S539, D5S818, D7S820, Vwa, FGA and CSF1PO which are present in $45 \%$ only of our population and are always associated to other more frequent markers.

Conclusion: This panel is useful in CS analysis of the Italian population. We retain that also a smaller panel constituted from 9 STR markers and amelogenin locus could be sufficient to individuate at least one informative marker in our population for CS analysis in transplant setting.

\section{Haemoglobinopathy}

\section{P685}

The impact of genotype on allogeneic stem cell transplantation in beta Thalassaemia major and sickle cell disease

A. Khalil (1), R. Elhasid (2), I. Zaidman (1), D. Filon (3), M. Weyl Ben Arush (1)

(1)Rambam Health Care Campus (Haifa, IL); (2)Sourasky Medical Center (Tel Aviv, IL); (3)Hadassah Hebrew University Medical Center (Jerusalem, IL)

Background: Common hemoglobinopathy (HP) includes betathalassemia major (TM) and sickle-cell disease (SCD). TM is caused by any of approximately 150 point mutations within the beta-globin gene. The most prevalent Arabic mutation in our region is IVS1-110. Clinical severity in TM depends on underlying mutations of the B-globin gene and degree of iron overload. Allogeneic stem cell transplantation (SCT) is the only potentially curative treatment for severe HP.

Objective: To investigate the impact of genotype on the results of SCT and the development of complications in TM and SCD. Subjects and Methods: The study population consisted of 29 (14 male, 15 female) patients of Arabic origin from northern Israel and the West Bank who underwent SCT for severe HP (25 TM, 4 SCD), mean age 8.4 years (10 months-23 years), and followed in Haifa, Israel, from 2000 to 2008. Median follow up was 5 years (1.1-8.9 years). Distribution of patients according to the Pesaro risk classification included 3 in class I, 11 in class II, and 15 in class III. Patients were divided into two groups according to their genotype. Group A (11): TM with the most prevalent Mediterranean mutation: IVS1-110/IVS1110; Group B (18) with the other B-globin mutations: nonsense (NS) 39/39 (2), NS 37/39 (2), IVS1-110/NS 37 (1), NS 37/37 (2), IVS1-6/IVS1-6 (1); IVS2-1/IVS2-1(1), IVS1-110/NS 39 (2), IVS1-1/IVS1-1 (2), frameshift 8/frameshift 8 (1), and 4 SCD. Statistical analysis was performed by Pearson's Chi-Square Test. $P<0.05$ was assumed to denote statistical significance. Results: Patients in Group A were more likely to be distributed in class $\mathrm{I}-1 \mathrm{I}-72.7 \%$ than patients in group $\mathrm{B}-27.3 \%(P=0.039)$ (Table 1), despite their similar mean ferritin levels $(2945 \mathrm{ngr} / \mathrm{ml})$. Endocrine complications (hypothyroidism [HT], diabetes mellitus [DM] and hypogonadism [HG]) were seen only in Group B-33.3\% ( $P=0.032)$ (Table 2). B-globin gene mutations had no impact on SCT outcome, graft versus host disease or hepatic complications.

Conclusion: The underlying genetic defect in HP is a contributing factor for the degree of Pesaro classification and endocrine complications and has no impact on SCT outcome.

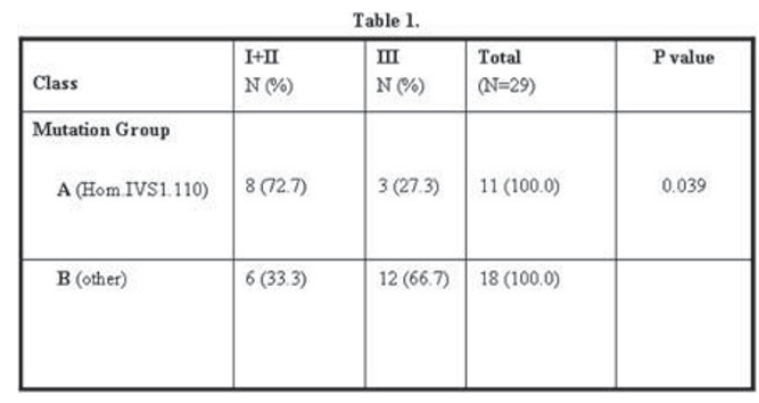

\begin{tabular}{|l|l|l|l|c|}
\hline $\begin{array}{l}\text { Endocrine complications } \\
\text { (DM, HT, HG) }\end{array}$ & $\begin{array}{l}\text { Yes } \\
\text { N }(\%)\end{array}$ & $\begin{array}{l}\text { No } \\
\text { N }(\%)\end{array}$ & $\begin{array}{l}\text { Total } \\
(\mathrm{N}=29)\end{array}$ & Pvalue \\
\hline Mutation Group & $0(0.0)$ & $11(100.0)$ & $11(100.0)$ & 0.032 \\
A (Hom. IVS1.110) & $6(33.3)$ & $12(67.7)$ & $18(100.0)$ & \\
\hline B (other) & & & & \\
\hline
\end{tabular}

P686

Bone marrow transplantation for Thalassemia major using fludarabine-based conditioning

P. Stepensky, M. Shapira, M. Aker, S. Revel-Vilk, S. Samuel, M. Weintraub, R. Or, I. Resnick

Hadassah Medical Center (Israel, IL)

Introduction: Over ten years ago we introduced a fludarabine based conditioning for hematopoietic stem cell transplantation (HSCT) for thalassemia major. We published our early experience for thalassemic patients who received grafts after fludarabine-based conditioning(1).

Aim: In this paper we update previous data on 20 patients and report new data on 25 additional patients.

Methods: Forty five patients (aged 2.4 to 24 years, median 7.2 years) underwent 48 allogeneic HSCT for treatment of thalassemia major. In this work we analyzed the data on first transplantations only. Our experience with second transplantations was published previously(2). The conditioning protocols used were (total doses) Fludarabine (Flu) $180 \mathrm{mg} / \mathrm{m}^{2}+$ Busulfan (Bu) $16 \mathrm{mg} / \mathrm{kg}$ + Antithymocyte Globulin (ATG) Fresenius $40 \mathrm{mg} / \mathrm{kg}$ or Thymoglobulin $10 \mathrm{mg} / \mathrm{kg}$ in 29 patients. Eleven patients were conditioned with the same protocol augmented with Thiotepa $(10 \mathrm{mg} / \mathrm{kg})$; five other patients received TBI (2 patients), Cyclophosfamide (2) or Melphalan (1). Thirty six transplantations were performed from a matched sibling donor, 4 from matched family donors and 5 from matched unrelated donors. Follow up till July 1, 2009 was 4 to 152 months.

Results: In the group of patients who got Flu based protocols disease free survival (DFS) was $80 \%(36 / 45)$ and overall survival (OS) was 93\% (42/45). Fifteen (33\%) patients suffered from acute graft versus host disease (aGvHD), $8(17 \%)$ of them with grade $3-4$. Twelve patients $(26 \%)$ developed chronic GvHD, half of them extensive. Of the 3 mortalities, one died following a matched sibling donor transplantation while two from MUD/MFD group (1/36 vs. 2/9, $P=0.036)$. Two of them were transplanted with Flu-Bu-ATG and one with Flu-Bu-TTATG. Causes of death were aGvHD grade $\geq 2(n=3)$ in combination with sepsis $(n=2)$. Estimated OS using Kaplan-Meier curve was $91 \%$ in Flu-Bu-TT-ATG group vs. $96 \%$ in Flu-Bu-ATG group (Figure 1). Four patients who experienced graft failure were conditioned with Flu-Bu-ATG (14\% of the total group) and three additional patients received Flu-Bu-TT-ATG $(27 \%$, NS). Conclusion: We conclude that combination of fludarabine with busulfex and ATG is safe, demonstrates acceptable rejection rates and is well tolerated by thalassemic patients. 
Our impression that the addition of TT to conditioning regimen does not improve an OS or DFS.

References: 1. Resnick I. et al. Bone Marrow Transplant 2007 Nov; 40(10):957-64

2. Stepensky P. et al. Haematologica 2009 Sep; 94(9):1329-30.

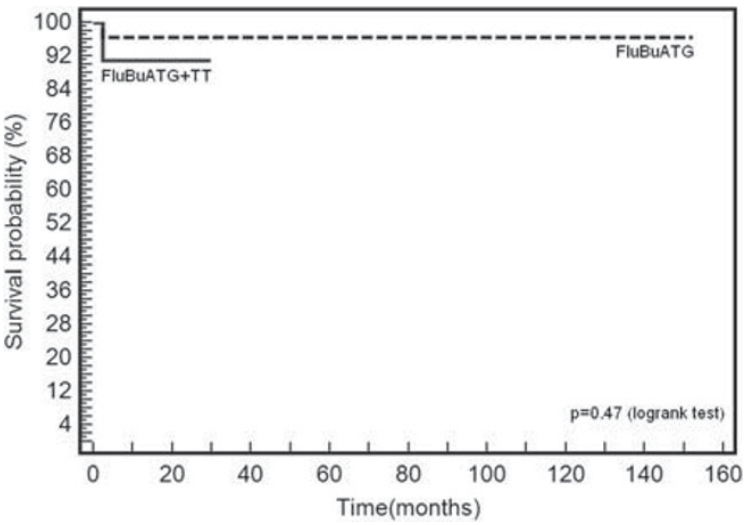

P687

The role of mixed chimerism on transplant outcome in patients with thalassaemia

M. Kazik, G. Karasu, S. Karauzum, V. Uygun, E. Manguoglu, A. Kupesiz, V. Hazar, A. Yesilipek

Akdeniz University School of Medicine (Antalya, TR)

Introduction: The only curative treatment modality for thalassemia major (TM) patients remains allogeneic hematopoietic stem cell transplantation (HSCT). We retrospectively evaluated the transplantation outcomes and chimerism kinetics of the patients transplanted with TM.

Methods: One hundred-three TM patients transplanted in our institution between 1998-2009 with a mimimum 6 months follow-up for alive patients were included in the study. Patients who have chimerism evaluation performed within the first 60 days after transplantation were also analysed in respect to chimerism kinetics. Mixed chimerism (MC) was further classified into three levels according to proportion of residual host cells (RHC) present in the recipient as being MC level 1 (RHC<10\%), MC level 2 (RHC 10\%-25\%) and MC level 3 (RHC>25\%).

Results: Totally 103 TM patients underwent 109 transplantations from matched related donors. Five patients received second and 1 of them received third transplantation because of graft rejection (GR). With a median 38 months (1-131) followup, 80 patients have been alive and thalassemia free. Fifty-five patients out of 80 have full donor engraftment, 22 patients with MC do not require transfusion. The chimerism evaluations of 3 transfusion independent patients could not be done. Twelve patients had GR and have been receiving regular transfusions, 11 patients died. The 5 year disease free survival and overall survival were found as $81,9 \%(95 \mathrm{Cl} 78-85.8 \%)$ and $88.3(95 \mathrm{Cl} 85.1-91.5 \%)$, respectively. The evaluation of chimerism kinetics was available only for 83 transplantations, 44 of which had full donor engraftment, 5 had primary GR with autologous reconstitution and 34 had MC. Among patients with MC, 11 had MC level 1, 11 had MC level 2, 12 had MC level 3. None of the patients with full donor chimerism in the early period after transplantation lost their grafts whereas 10 out of 34 patients with MC (29\%) eventually lost and became transfusion dependent $(P=0.0005)$. Within the patients with MC, GR occurred in 8 out of 12 patients with MC level 3 but only 2 in rest of the total 22 patients with MC level 1 and 2 $(P=0.0019)$

Conclusion: TM patients having more than $25 \%$ of RHC within 2 months after transplantation have higher risk for GR. In patients with $\mathrm{MC}$ but with less than this amount of $\mathrm{RHC}$ at the end of first month, chimerism should be monitored at least monthly until stable chimerism is achieved and in patients with high risk for $\mathrm{GR}$, early intervention should be considered.

\section{P688}

Intensified immunosuppression with ATG reduces graft failure without affecting immune reconstitution or infections in high-risk beta thalassaemia patients after HLA-identical sibling HSCT

E. Biral, B. Cappelli, D. Zugna, R. Chiesa, A. Biffi, L. Zito, K. Fleischhauer, R. Bacchetta, L. Fumagalli, C. Di Serio, F. Ciceri, M.G. Roncarolo, A. Aiuti, S. Marktel

Scientific Institute H S Raffaele (Milan, IT)

Rejection is an unsolved problem following HSCT in high risk (class 3) thalassemia patients (pts). Anti-thymocyte globulin (ATG) could be given in attempt to counteract anti donor immunity. However, it could also delay immune reconstitution, exposing pts to life-threatening infections.

We analyzed immune reconstitution up to 120 days post HSCT in 56 beta-thalassemia pts undergoing HLA-identical sibling HSCT between 2005 and Jan 2009. Median age at HSCT was 8 yrs (range 2-17). 29 pts were classified as low risk (class 1-2) and underwent myeloablative conditioning with weightadjusted iv Bu+Cy200 mg/Kg. 27 pts class 3 high risk pts were treated with weight-adjusted iv Bu and reduced dose Cy160 $\mathrm{mg} / \mathrm{Kg}$ preceded by pre-conditioning (Sodani, Blood 2004). Because of high incidence of rejection in the first 9 pts (class3noATG), we intensified immunosuppression in the following 18 pts with ATG Thymoglobuline $(7.5-10 \mathrm{mg} / \mathrm{Kg}$ ) (class 3ATG). GvHD prophylaxis consisted of CyA and short Mtx. Metylprednisolone (MPD) $0.5 \mathrm{mg} / \mathrm{kg}$ till +30 was associated to class 1-2 and class 3 no ATG.

We compared immune reconstitution in the 3 groups (class 1-2, class3noATG, class3ATG). Immune reconstitution of lymphocytes subpopulation at +30 was best for class $3 A T G$ (Figure 1): CD3 + class $1-2$ vs. 3noATG $P=0.032$, class $1-2$ vs. 3 ATG $P \leq 0.001 ; \mathrm{CD} 3+\mathrm{CD} 4+$ class $1-2$ vs. 3 noATG $P=0.004$, class $1-2$ vs. 3 ATG $P=0.003 ;$ CD $3+$ CD $8+$ class $1-2$ vs. 3 noATG $P \leq 0.001$. No difference was observed for CD19+ Median time to discontinuation of iv Ig supplementation was 81 days for class $1-2,40$ days for class 3 noATG, and 22 days for class3ATG. Frequency of bacterial infections after +60 was higher in class $1-2$ compared to class 3 ATG $(P=0.027)(31 \%$ class $1-2,12,5 \%$ class 3 noATG, $0 \%$ class $3 A T G)$, while no difference was observed in the incidence of viral and fungal infections. GvHD occurred in $28 \%$ of class $1-2$ pts, $12.5 \%$ class 3 noATG and $12.5 \%$ in class3ATG with no significant statistical difference in the 3 classes $(P=0.403)$. None of the pts in class 1-2 rejected the graft, while the cumulative incidence of secondary graft failure was $19.6 \%$ in class 3 ATG and $44.4 \%$ in class3noATG $(P=0.09)$.

In our analysis, high risk pts treated with ATG show a reduced incidence of rejection compared to high risk pts treated without ATG and a satisfying immune reconstitution. Class 1-2 pts have a reduced incidence of rejection but a higher infectious risk, probably due to higher doses of $\mathrm{Cy}$ in the conditioning regimen and the use of MPD in the GvHD prophylaxis. 


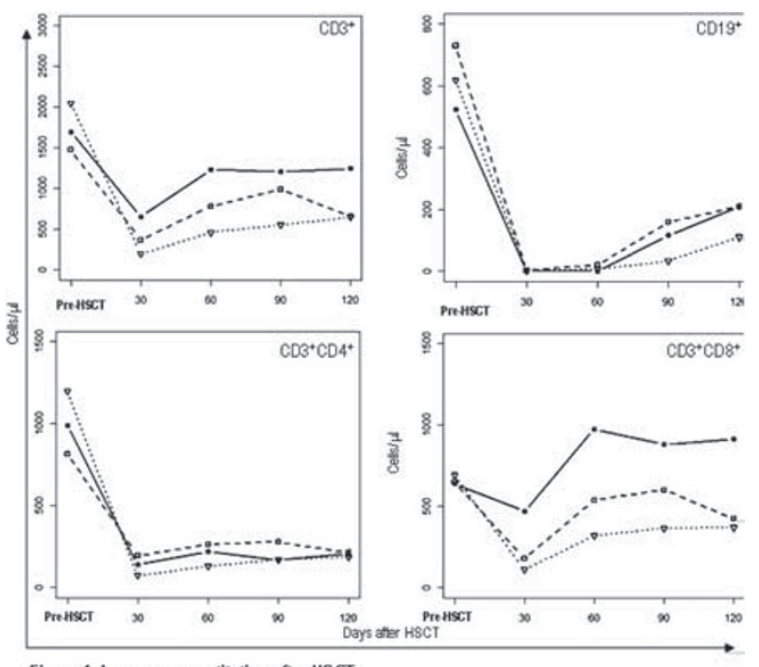

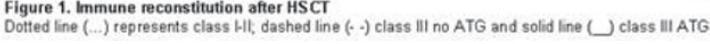

\section{P689}

Late-onset haemorrhagic cystitis in children after haematopoietic stem cell transplantation for thalassaemia and sickle cell anaemia: a prospective evaluation of polyoma (BK) virus infection and treatment with cidofovir (CDV)

J. Gaziev (1), S. Germani (2), R. Miano (2), P. Bove (2), P. Paba (2), C.F. Perno (2), P. Sodani (1), M. Marziali (1), A. Isgro (1), P. Polchi (1), K. Paciaroni (1), C. Gallucci (1), M.D. Simone (1), A. Roveda (1), G. De Angelis (1), C. Alfieri (1), G. Lucarelli (1) (1)Mediterranean Institute of Hematology (Rome, IT); (2)Policlinic of Tor Vergata (Rome, IT)

Little is known about development of late-onset $\mathrm{HC}$ in children following haematopoietic stem cell transplantation (HSCT), its association with BK virus (BKV) and treatment with CDV. We prospectively investigated BKV in pts receiving HSCT from HLA-matched related donors, risk factors for $\mathrm{HC}$ development and treatment efficacy with CDV. 117 pts with thalassemia $(n=107)$ or sickle cell anemia $(n=10)$ with median age of 9 years (range, 1.7-17) were enrolled in this study. All pts received BUCY and/or Thymoglobulin-ATG (in 26 pts) containing regimens. Quantitative BKV-PCR on blood and urine samples were performed before conditioning and weekly thereafter in 64 pts. Risk factors for $\mathrm{HC}$ were evaluated on univariate and multivariate analysis using cumulative incidence curves and competing risk regression analysis. Ten pts received CDV $1.5 \mathrm{mg} / \mathrm{kg} 3$ days/wk and 9 pts $5 \mathrm{mg} / \mathrm{kg} / \mathrm{wk}$. 52 of $64(81 \%)$ pts had 2 or more positive samples for BK viruria and 18 of $64(28 \%)$ pts for BK viremia. Viral load varied from $<556$ to $>55 \times 10^{6}$ copies. Thirty $(26 \%)$ pts had $\mathrm{HC}$ at a median of 38 days (range, 13-114), 24 of them had grade 2, 2 grade 3 and 4 grade $4 \mathrm{HC}$. All pts with $\mathrm{HC}$ had BK viruria. Four pts developed hydronephrosis with ureteral stenosis necessitating ureteral stent placement. The cumulative incidence of grade 2 or $3-4 \mathrm{HC}$ was $21 \%$ and $5 \%$ respectively. Multivariate analysis showed that use of ATG $(H R=10.5 ; P=0.001)$, peak BK viruria $>100000$ copies $(H R=6.2 ; P=0.004)$, and $G V H D(H R=5.3$; $P=0.007$ ) predicted $\mathrm{HC}$. The cumulative incidence of $\mathrm{HC}$ in pts who had 3 risk factors was $88 \%$, compared with $64 \%, 30 \%$ and $0 \%$ who had either 2,1 or none risk factor $(P=0.0001)$. Both dosing schedules of CDV were well tolerated with no cases of dose-limiting nephrotoxicity. The median duration of $\mathrm{HC}$ was 17 days (range, 9-53). The median duration of therapy was 27 days (range, 21-180) with a median of 9 doses given (range, $6-22)$. All pts had a complete clinical response (CCR) but only $6(32 \%)$ had microbiological response at 2 wks after therapy. None of these patients cleared BK viruria when a CCR was achieved. Ten pts with grade $2-3 \mathrm{HC}$ occurred before prospective trial of CDV had spontaneous resolution. The median duration of $\mathrm{HC}$ in these pts was similar to those treated with CDV. In conclusion, high load BK viruria, use of ATG and GVHD are associated with HC. CDV may have some activity against BKV-related $\mathrm{HC}$, although spontaneous resolution of $\mathrm{HC}$ could also occur which rises question regarding the adequacy of CDV therapy for BK virus-related $\mathrm{HC}$.

\section{P690}

Pre-emptive donor lymphocyte infusions for unstable mixed chimerism in thalassaemia

G. Karasu, V. Uygun, S. Karauzum, M. Kazsk, A. Kupesiz, V. Hazar, A. Yesilipek

Akdeniz University School of Medicine (Antalya, TR)

Aim: The assessment of feasibility of pre-emptive donor lymphocyte infusions (DLI) in patients with unstable mixed chimerism (MC) in thalassemia major (TM) patients.

Methods: The indications for DLI were either having more than $25 \%$ residual host cells (RHC) (MC level 3 ) in the early phase after transplantation or increasing proportions of $\mathrm{RHC}$ in serial monitoring with decrease in hemoglobin $(\mathrm{Hb})$ levels. Nineteen patients following 21 transplantations had received DLI out of 109 transplantations performed in 103 patients.

Results: Seven patients received with the indication of having $\mathrm{MC}$ level 3 in the early phase and assigned as early-DLI group. Four patients although had MC level 3 in the early period received DLI because of increasing proportions of $\mathrm{RHC}$ in serial monitoring at post-transplant 4-6 months and assigned as late-DLI group. In the early DLI group, 3 out of 7 patients preserved functional grafts whereas other 4 lost. In the late DLI group, only 1 patient preserved the graft and the difference between these two in respect to preserving functional graft was not found statistically significant. Ten received DLI because of gradual decrease in percentage of donor cells in parallel to $\mathrm{Hb}$ levels. Eight out of 10 patients have been followed as transfusion independent. The increase in $\mathrm{Hb}$ levels was statistically significant $(t=-4,699, P=0.002)$ whereas increase in percentage of donor cells was not found statistically significant.

Discussion: In the early-DLI group, 3 out of 7 patients rescued their grafts, although the probability of graft rejection is around $90 \%$. On the other hand, in the late-DLI group, only 1 patient rescued his graft. Although statistical significance could not be determined between early and late-DLI group, this was thought to be related with differences in the chimerism kinetics of the patients within two groups. If pre-emptive DLI is considered, it seems more beneficial if it is applied earlier for this indication. In patients with gradual decrease in percentage of donor cells in late period, $\mathrm{Hb}$ level should be determinative for $\mathrm{DLI}$ indication but it is difficult to define a critical $\mathrm{Hb}$ level less than which DLI should be proposed depending on our data. However it seems reasonable to delay if $\mathrm{Hb}$ levels are more than $9 \mathrm{gr} / \mathrm{dL}$ even percentage of donor cells decrease more if they have not been recently transplanted.

\section{P691}

First report of haematopoietic cell transplantation for sickle-cell disease from in-vitro fertilization-selected donor G. Del Toro (1), E. Roman (1), A. Hurlet (1), T. Mukherjee (2), G. Jae (1), B. Lubin (3)

(1)Mount Sinai School of Medicine (New York, US); (2)Reproductive Medicine Associates of New York (New York, US); (3)Children's Hospital of Oakland (Oakland, US)

Background: Hematopoietic cell transplantation from human leukocyte antigen (HLA)-matched siblings cures at least $85 \%$ of sickle cell disease (SCD) patients. But most SCD patients lack an HLA-matched sibling. The combination of in-vitro fertiliization (IVF) and pre-implantation genetic diagnosis (PGD) can provide HLA-matched sibling donors for patients with various 
diseases. There are no medical reports of a succesful HCT for a patient with SCD from a sibling donor born via the combination of IVF and PGD.

Report: The parents of an 8-year-old male with SCD requested the opportunity of conceiving a matched donor for their affected child via IVF and PGD. Three embryos who were unaffected by SCD were implanted. Two of the three embryos were HLAmatched to the affected child. Only one embryo survived. At birth, we confirmed that the newborn sibling was HLAmatched to the patient and unaffected by SCD. The total nucleated cell count in the umbilical cord blood unit collected at birth was only 13 million $/ \mathrm{kg}$. Thus, we had to submit the newborn sibling to a bone marrow harvest procedure at the age of 11 months, to complement the cord blood cell count. The combined umbilical cord blood and bone marrow transplant was performed after myeloablative conditioning with the standard regimen combining busulfan, cyclophosphamide, and anti-thymocyte globulin. The patient tolerated conditioning relatively well. Graft-versus-host disease (GVHD) prophylaxis consisted of cyclosporine and low-dose methotrexate. He engrafted rapidly and was discharged home after three weeks. A mixed-donor chimerism test done on Day +30 was $98 \%$ donor. Subsequent tests have shown no $\mathrm{Hgb} \mathrm{S}$. Complications have included Stage 1 GVHD of the skin and marked, unexplained elevation of the liver enzymes with mild non-specific changes in a liver biopsy. The patient is now on Day +156 and is cured from sickle cell disease.

Conclusions: We provide the first medical report of succesful HCT for SCD with a sibling donor born via the combination of IVF and PGD. This technology, readily available in many nations with a high human development index, and frequently reported as a means of achieving succesful transplantation for less common diseases, is grossly underutilized in SCD. We are pursuing the possible reasons for this healthcare gap. Sickle Cell Disease patients without matched siblings in countries such as the United States of America should have easier access to this potential source of the ideal HCT donor.

\section{P692}

Toward $100 \%$ survival following matched related allogeneic haematopoietic stem cell transplantation for thalassaemic patients: from a dream to an achievable goal A. Hussein, A. Al-Zabin, F. Abdel-Rahman, R. Rihani, H. El-Ta'ani, H. Al-Khatib, M. Sarhan

King Hussein Cancer Center (Amman, JO)

Purpose: To evaluate the outcome of children with alpha-Thalassemia major who underwent Hematopoietic Stem Cell Transplantation (HSCT) from matched sibling donors at KHCC.

Patients and methods: Thirty three children less than 18 years of age with Thalassemia major were transplanted from HLA identical related donors between September, 2003 and December, 2008. Seventeen were females and 16 males. The median age was 8 years (1-15 years). Patients were classified into 3 groups according to iron load status, Class I $(n=7)$ and Class II $(n=18)$ patients received myeloablative conditioning regimen with oral Busulphan ( $4 \mathrm{mg} / \mathrm{kg} /$ day for 4 days), Cyclophosphamide (50 mg/ $\mathrm{kg} /$ day for 4 days) and horse Anti-Thymocyte Globulin-ATG$(30 \mathrm{mg} / \mathrm{kg} /$ day for 5 days). Patients with class III $(n=8)$ received non-myeloablative conditioning regimen with Busulphan $(2 \mathrm{mg} /$ $\mathrm{kg}$ twice daily for two days), Fludarabine $\left(35 \mathrm{mg} / \mathrm{m}^{2} /\right.$ day for 5 days), horse ATG (30 mg/kg/day for 5 days), and total lymphoid irradiation (500 cGy on Day zero).

As Graft versus host disease (GvHD) prophylaxis; patients with myeloablative conditioning received Cyclosporine and short course of Methotrexate, while Cyclosporine and Mycophenolate Mofetil (Cellcept) were used for patients who received nonablative conditioning.
Results: After a median follow up of more than 3 years, 32 patients $(97 \%)$ are alive. One low risk patient died with severs sepsis 75 days post transplantation.

All patients showed primary engraftment. Twenty nine $(91 \%)$ out of 32 alive patients had sustained graft. Eighteen patients (56\%) had full donor chimerism, while $11(33 \%)$ having stable mixed donor chimerism. Three patients $(9 \%)$ had unstable mixed chimerism that led to secondary graft failure. Two of them followed non-ablative transplantation (10 and 21 months), while the third one after myelo-ablative transplantation ( 23 months). All of them are doing well now after second (non-ablative) transplantation. The overall survival is $97 \%$ and event free survival $88 \%$.

Nine patients (27\%) developed acute veno-oclusive disorder; all were recipients of myeloablative conditioning HSCT, and all recovered with supportive treatment. Six patients $(18 \%)$ had acute GvHD, 4 of them progressed to chronic GvHD, while 2 others patients had de novo chronic GvHD.

Conclusion: Our results in treating 33 patients with thalassemia major are encouraging. We didn't have any mortality for the last 6.5 years. Using the strategy of careful pre transplantation evaluation and tailoring conditioning regimens accordingly is associated with excellent results.

\section{P693}

Post-transplant chelating therapy with deferasirox in patients with beta thalassaemia

M.A. Yesilipek, G. Karasu, M. Akcan, V. Uygun, Z. Bolat Akdeniz University (Antalya, TR)

Objective: Allogeneic hematopoetic stem cell transplantation (HSCT) is the only radical cure of beta thalassemia. However, iron overload remains a cause of morbidity and mortality in exthalassemic patients.

Method and patients: We present seven patients as a preliminary report who underwent HSCT and received oral chelating therapy (deferasirox) because of poor compliance to phlebotomy and desferrioxamine. Chelating therapy was started 6 to 12 months after HSCT. All patients received deferasirox (Exjade, Novartis) with the dosage of $30 \mathrm{mg} / \mathrm{kg} /$ day orally. Serum blood urea nitrogen and creatinin levels were checked weekly during the first month and then two times in a month. Serum ALT, AST levels and also complete blood counts were also checked periodically. Ferritin levels were measured once a month. Chimerism was evaluated by FISH in patients with sex mismatched HSCT whereas by STRs in others. Median follow up duration was 10 months (range 7-16 months). The patients' parents provided informed consent at the beginning of the therapy.

Results: Three of seven patients were classified as Class II and four were Class III before HSCT. Two of them had Grade II and five of them had Grade III-IV hemosiderosis and median hepatic fibrosis score was II (Ishak system) at time of HSCT. In all patients, deferasirox therapy achieved reduction in serum ferritin levels. No negative effect was seen in AST, ALT, Hb and donor chimerism of the patients while serum ferritin levels significantly reduced $(P=0.022)$. Although serum creatinin significantly increased $(P=0.008)$ it was in normal limits in all patients.

Conclusion: We believe that use of medication to remove iron should only be considered in circumstances in which venous access is prohibitive or anemic patient. In these patients deferasirox may provide safety iron removal in post-transplant period. However, it is necessary to monitor serum creatinin levels of the patients closely. Further studies with more patients and longer follow up are required to define its clinical efficacy and safety. 


\begin{tabular}{|c|c|c|c|c|c|c|c|c|c|}
\hline & $\begin{array}{l}\text { Gender } \\
/ \mathrm{Age}\end{array}$ & $\begin{array}{l}\text { Risk } \\
\text { Class. }\end{array}$ & $\begin{array}{c}\text { Femitin } \\
(\mu / g r / L) \\
\text { Before/After }\end{array}$ & $\begin{array}{l}\text { ALT (Uת) } \\
\text { Before/After }\end{array}$ & $\begin{array}{l}\text { AST (U/L) } \\
\text { Before/After }\end{array}$ & $\begin{array}{c}\text { Creatinin } \\
\text { (mg/di) } \\
\text { Before/After }\end{array}$ & $\begin{array}{c}\text { Hb (grdil) } \\
\text { Before/After }\end{array}$ & $\begin{array}{c}\text { Donor } \\
\text { Chimensm } \\
\text { Before/After }\end{array}$ & $\begin{array}{l}\text { Therapy } \\
\text { period } \\
\text { (months) }\end{array}$ \\
\hline HY & Fin? & III & 741812376 & $53 / 27$ & $41 / 28$ & $0,4310,79$ & $14,3 / 14, ?$ & $100 / 100$ & 16 \\
\hline MO & F/16 & III & $6568 / 2270$ & $180 / 21$ & $5 4 \longdiv { 2 7 }$ & $0,2510,49$ & $11,9 / 10,8$ & $100 \% 100$ & 13 \\
\hline MS & M/6 & II & $2302 / 1729$ & 57162 & 38129 & $0,3 4 \longdiv { 0 , 4 4 }$ & $10,9111,5$ & $100 / 99$ & 12 \\
\hline UIO & M/4 & II & $4137 / 1142$ & $1 5 \longdiv { 2 7 }$ & $29 / 36$ & & & $100 / 100$ & 10 \\
\hline YB & MP & III & 280011127 & $7 4 \longdiv { 3 7 }$ & $5 8 \longdiv { 4 6 }$ & $0,31 / 0,51$ & $12,6 \longdiv { 1 2 , 2 }$ & $100 / 100$ & 10 \\
\hline BK & F/S & II & $1358 / 991$ & $24 / 28$ & $2 8 \longdiv { 2 4 }$ & $0,2610,28$ & $9,2 / 8,8$ & $29 / 30$ & 10 \\
\hline BKa & F/16 & III & $4330 / 3750$ & $91 / 35$ & $57 / 35$ & $0,4010,50$ & $11,4 / 11,8$ & $9 7 \longdiv { 9 7 }$ & 7 \\
\hline
\end{tabular}

P694

Allogeneic haematopoietic stem cell transplantation for children with severe sickle cell disease improved quality of life, resolved severe lung disease and normalized transcranial Doppler measurements

C. Bettoni da Cunha (1), M. Sauer (1), B. Maecker-Kolhoff (1), I. Müller (2), R. Beier (1), M. Tell-Luersen (1), C. Klein (1), K-W. Sykora (1)

(1)Hannover Medical University (Hannover, DE); (2)Tubingen University Hospital (Tubingen, DE)

From 2002 to 2009 we performed 10 bone marrow and blood stem cell transplantations (SCT) in 9 children age 4-18 years with homozygote sickle cell disease. Stem cell sources were matched family donor (MFD) in 7, and matched unrelated donor (MUD) in 3. Indications for SCT were: stroke in 3; severe cerebral vasculopathy without stroke on transcranial Doppler (TCD) in 5; life-threatening hemolysis due to anti- $\mathrm{H}$ and $-\mathrm{HI}$ antibodies in 1 ; sickle cell severe restrictive pulmonary disease and lung fibrosis on chest $\mathrm{CT}$ in 1 patient $(\mathrm{Pt}) .1 \mathrm{Pt}$ suffered 2 episodes of chest syndrome and had the rare blood S-s-Unegative phenotype. For conditioning, 6 Pts received Busulfan (Bu), Fludarabine (Flu) and antithymocyte globulin (ATG); and 2 Flu/Treosulfan/Thiothepa/ATG (1 retransplantation). 2 Pts received reduced-intensity conditioning $(\mathrm{RIC})$ : oral $\mathrm{Bu} 8 \mathrm{mg} / \mathrm{kg}$; Cyclophosphamide (CY) $120 \mathrm{mg} / \mathrm{kg}$; Flu $180 \mathrm{mg} / \mathrm{m}^{2}$ and OKT3 combined with a T-cell depleted PBSC transplant. All others received a full bone marrow transplant with CSA/MTX as GvHD prophylaxis. Toxicity was mainly neurological: $1 \mathrm{Pt}$. seized on CSA and showed posterior leukoencephalopathy on MRI; another developed acute transverse myelitis which resolved after treatment with steroids, immunoglobulins, rituximab, $\mathrm{CY}$ and discontinuation of CSA. Two siblings developed secondary graft failure due to phenytoin hypersensitivity syndrome after MUD-SCT. The boy recovered spontaneously, the girl required a 2 nd SCT from another MUD. She was the only Pt who developed severe chronic skin GvHD, refractory to prednisone, mycophenolate, and rapamicin, with complete resolution after 4 infusions of mesenchymal stem cells.

Results: Sickle cell free survival was $100 \%$. All including 5 heavily pretransfused Pts engrafted. The 2 RIC Pts received donor lymphocyte infusions for mixed chimerism (44-80\% donor). All others had stable chimerism of $90-99 \%$. All Pts had full donor erythropoiesis, and a 100\% Lansky score after SCT. No case of new stroke after SCT occurred, abnormal TCD normalized in $4 / 5 \mathrm{Pts}, 1 \mathrm{Pt}$ is too early for evaluation. The lung function in the Pt with severe restrictive lung disease before SCT normalized. 5/6 Pts had stable pulmonary function after SCT, $1 \mathrm{Pt}$ had normal spirometry but decreased carbon monoxide diffusing capacity. 3 girls developed hypogonadism.

Conclusion: SCT can reverse cerebral vasculopathy, prevent stroke and reverse sickle cell lung disease. Transplantassociated complications were serious but mostly reversible.
P695

Safe and effective reduced-cost bone marrow transplantation for thalassaemia: preliminary experience of the Cure2Children Foundation in Pakistan

S. Ansari (1), T. Shamsi (1), S. Khalid (2), N. Yaqub (3),

K. Rashid (4), T. Farzana (1), Y. Iqbal (4), P. Sodani (5),

C. Gallucci (5), B. Erer (5), L. Faulkner (2)

(1)National Institute of Blood Diseases (Karachi, PK); (2)Cure2Children Foundation (Florence, IT); (3)Pakistan Institute of Medical Sciences (Islamabad, PK); (4)Shifa International Hospital (Islamabad, PK); (5)Mediterranean Institute of Hematology (Rome, IT)

The Cure2Children Foundation has supported both financially and professionally a network of centers in Pakistan performing BMT for transfusion-dependent thalassemia. HLA-matched sibling marrow was administered after conditioning with thiotepa $10 \mathrm{mg} / \mathrm{kg}$, busulfan $14 \mathrm{mg} / \mathrm{kg}$ and cyclophosphamide $200 \mathrm{mg} / \mathrm{kg}$, followed by GvHD/rejection prophylaxis with prednisone, methotrexate, and cyclosporin. Management standards for therapy administration, central venous access, severe pancytopenia, immunosuppression, and hospital infection control have been implemented following on site training assisted by web-based data management and real-time videoconferencing.

Since August 2008, a total of 15 BMTs have been performed in 15 low-risk patients (no hepatomegaly, median age 3.3, range 0.9 to 6.2 ). Liver biopsies were not routinely performed. Nine BMTs were done at an established center (NIBD), and 6 at newly developed services (4 at PIMS and 2 at $\mathrm{SIH}$ ).

Results: At a median follow up of 242 days (range 90-463), actuarial thalassemia-free survival is $80 \%$ and overall survival $100 \%$. Three patients had graft failure followed by autologous reconstitution. One patient developed grade 3 and one grade 2 acute GvHD, 5 had subclinical CMV activation and one developed pulmonary tuberculosis. Other manageable complications included hypertension in 4 patients and hemorrhagic cystitis in 3. No case of veno-occlusive disease of the liver or chronic GvHD has been observed so far.

Costs: The cost of each BMT was in the range of $12,000 €$, including full family support for a minimum of 8 months. Setting up a two-bedded BMT unit (at PIMS) including civil works and basic equipment required approximately $30,000 €$

Conclusions: Early experience with a new international model aimed at enhancing global access to bone marrow transplantation suggests that it can be performed safely and effectively with limited resources. 
Administration of KGF (palifermin) during autologous transplantation for multiple myeloma does not improve thymic function but increases number of effector memory

\section{P696}

Immune reconstitution of naturally occurring Tregs takes place very early after autologous HSCT and is independent from thymic function

L. Perez-Roca (1), A. Perez-Garcia (1), I. Sanchez-Ortega (2), E. Cabezudo (3), J. Lopez-Jimenez (4), I. Marugan (5), T. Peralta (2), M. Arnan (2), P. Ramos-Oliva (4), I. Benet (5), S. Lopez (2), M. Mestre (3), J. Odriozola (4), C. Solano (5), R.F. Duarte (1)

(1)Institut Catalá d'Oncologia-Idibell (Barcelona, ES); (2)Institut Catalá d'Oncologia (Barcelona, ES); (3)Hospital Universitario de Bellvitge (Barcelona, ES); (4)Hospital Ramon y Cajal (Madrid, ES); (5)Hospital Clinico Universitario (Valencia, ES)

Naturally occurring CD4+/CD25high/CD127dim/FoxP3+ Tregs have a regulatory funcion which has been associated not only with GVHD in allogeneic HSCT, but also in animal models with the immunomodulatory effect of autologous HSCT in autoimmune disorders. Despite the critical role of Tregs in auto/allo-immune phenomena after HSCT, their kinetics of immune reconstitution are unkown. We present data in 50 adult recipients of autologous HSCT (34 myeloma = melphalan-200 16 lymphoma =BEAM) in whom prospective peripheral blood samples for analysis of immune reconstitution of Tregs were collected (median age 57, 28-74; 34 men, 16 women). Tregs defined by expression of CD3 +/CD4 +/CD25high/CD127dim, and expressed as a percentage of $\mathrm{CD} 4+\mathrm{T}$ cells recovered surprisingly soon after autologous HSCT, with a 3-fold expansion as early as 2 weeks after transplant $(6,55 \%$ vs. $2,12 \%$; $P<0,001)$. Tregs remain high at day +90 when lymphocyte recovery starts taking place. Tregs early expansion after HSCT correlates with pre-HSCT Tregs levels $(r=0,78 ; P=0,034)$, and mirrors the kinetics of expansion of FoxP 3 mRNA expression at these same timepoints $(10,32$ vs. 3,$0 ; P=0,003)$, in keeping with a true regulatory phenotype, in particular in a clean autologous model, where CD25high and FoxP3 upregulation or CD127 downregulation cannot be caused by alloreactive phenomena. From the perspective of the underlyin mechanism, Tregs expansion early after autologous HSCT in our patients takes place in the absence of thymic output, as meassured by sjTRECs, which remain low up to 6 months after autologous, in keeping with the advanced age of our subjects. Moreover, Tregs expression of CD31, an additional marker of recent thymic output, did not change between timepoints. This is the first study that focuses on early kinetics of immune reconstitution of Tregs after HSCT. Our data show that Tregs recovery occurrs earlier than initially anticipated in only two weeks after the procedure, and that it probably occurs by a peripheral thymic-independent mechanism. This early Tregs expansion in vivo in humans may be a candidate mechanism to explain the immunomodulatory effects of of autologous HSCT in autoimmune disorders, and may identify a novel therapeutic point to modulate such phenomena.

\begin{tabular}{|r|c|c|c|}
\hline $\begin{array}{c}\text { Time } \\
\text { Points }\end{array}$ & $\begin{array}{c}\text { Linfos CD3+ } \\
\left(\times 10^{9} / \mathrm{L}\right)\end{array}$ & $\begin{array}{c}\text { Tregs } \\
(\% \text { of } \text { CD4 }+)\end{array}$ & $\begin{array}{c}\text { CD31 } \\
(\% \text { of Tregs })\end{array}$ \\
\hline Pre & $1.46 \pm 0.67$ & $2.12 \pm 0,96$ & $17.50 \pm 5.58$ \\
\hline D+14 & $1.04 \pm 1.28$ & $6.55 \pm 1,45$ & $19.42 \pm 7.56$ \\
\hline D+90 & $2.07 \pm 1.15$ & $5.87 \pm 2,13$ & $16,92 \pm 4,85$ \\
\hline ANOVA & 0,061 & $<0,001$ & n.s. \\
\hline \multicolumn{4}{|c|}{ Results are mean \pm standard deviation } \\
\hline
\end{tabular}

\section{CD8 + T-cells}

E. Clave (1), C. Douay (1), C. Rabian (1), H. Moins-Teisserenc (1), M. Carmagnat (1), C. Bompoint (2), N. Gorin (2), D. Charron (1), A. Toubert (1), L. Garderet (2)

(1)University Paris Diderot, INSERM UMR 940 (Paris, FR); (2)Hospital St Antoine (Paris, FR)

Human-keratinocyte growth factor (KGF) is currently being evaluated in clinical trials for its safety and efficacy in preventing mucosal damage from irradiation and after Hematopoietic Stem Cell Transplantation (HSCT). It has been suggested that, besides having preventive effects on oral mucositis, it may also have immunomodulating effects through the protection of thymic epithelial cells. Indeed, in experimental mouse models, KGF has a role in post natal thymic recovery in chemotherapy, radiotherapy and allogeneic or autologous HSCT. Taking advantage of the KGF treatment of our Multiple Myeloma patient, we have investigated its potential on immune reconstitution after autologous HSCT in Human.

Thirty myeloma patients from a single institution were treated homogeneously with the induction combination bortezomib (Velcade ${ }^{\circledR}$ ) plus dexamethasone, followed by high dose melphalan $\left(140-200 \mathrm{mg} / \mathrm{m}^{2}\right)$ and an autologous transplantation with peripheral blood stem cells. Patients were randomized for KGF treatment in 2 groups, 14 having received three doses of palifermin (Kepivance $\left.{ }^{\circledR}\right)(60 \mu \mathrm{g} / \mathrm{kg}$ once daily i.v.) pre- and post-conditioning regimen (total six doses). Blood samples were drawn at diagnosis, before HSCT, and at 1, 3, 6, 9, 12 and 18 months post HSCT. To assess thymic function, we analysed signal joint (sj) TREC in peripheral blood lymphocytes using quantitative RT-PCR. Naïve, Central and Effector Memory, and Regulatory T lymphocyte subpopulations percentage and absolute were monitored by multicolour flow cytometry. SjTREC decreased sharply after transplantation and returned to baseline after 1 year without differences between the two groups of patients. Regulatory $\mathrm{T}$ lymphocytes (phenotypically assessed as CD4 + cD25+ cD127-/low) were not modified by KGF treatment either. Notably, the CD3 + cell population was significantly higher during the first 3 months post HSCT in KGF treated patients. This was related to a higher CD8 + cell counts, specifically in the CD8 Effector Memory cells (CD8 + cD45RACCR7- cells) at 1 and 3 month post HSCT. No correlation was found with documented infectious complications, relapse or survival.

These data suggest that, in Human, the main effect of KGF on immunity, in that autologous HSCT setting, is not on thymic function but rather on the peripheral expansion of CD8 + effector memory cells.

\section{P698}

Lymphocyte subset reconstitution after unrelated cord blood or bone marrow transplantation in children

C. Renard (1), V. Barlogis (2), V. Mialou (1), C. Galambrun (2), M.P. Goutagny (1), L. Glasman (2), A.D. Loundou (3), F. Poitevin-Later (4), F. Dignat-George (5), Y. Bertrand (1), G. Michel (2)

(1)Institut d'Hématologie et Oncologie Pédiatrique (Lyon, FR); (2)Hôpital La Timone (Marseille, FR); (3)Faculté de médecine (Marseille, FR); (4)Hospices Civils de Lyon (Lyon, FR); (5)Hôpital de la Conception (Marseille, FR)

We report the lymphocyte subset reconstitution of 226 children who underwent Unrelated Cord Blood transplant (UCBT) $(n=112)$ or Unrelated Bone Marrow Transplant (UBMT) $(n=114)$ for malignant $(n=151)$ or non malignant $(n=75)$ disease in two institutions between February 1997 to June 2008. Conditioning regimens were the same with a Total Body Irradiation in $50.9 \%$ in UCBT and $43 \%$ in UBMT. Graft-versus-host disease (GvHD) 
prevention included cyclosporine $\mathrm{A}$ and antithymocyte globulin in all patients; steroid was added in UCBT.

Immune reconstitution was studied by assaying absolute number of natural killer (NK), B and T cells by flow cytometry at 1, 2, 3, 6 months, 1 and 2 years. Several immunological endpoints were chosen: time to achieve a CD3 + cell count $>500$, 1000 , and $1500 / \mathrm{mm}^{3}, \mathrm{CD} 4+>200$ and $500 / \mathrm{mm}^{3}, \mathrm{CD} 8+>250 /$ $\mathrm{mm}^{3}, \mathrm{CD} 19+>200 / \mathrm{mm}^{3}, \mathrm{NK}>100$ and $250 / \mathrm{mm}^{3}$. These endpoints were analysed through the use of cumulative incidence curves for estimating incidence over time in the context of competing risks and through Fine and Gray models to assess prognostic factors.

The median time to neutrophil and platelet engraftment was respectively 28 and 50 days for UCBT whereas it was 19 and 30 days for UBMT $(P<0.001)$. The cumulative probability of acute grade 2 or greater GvHD was $19.6 \%$ for UCBT and $35 \%$ for UBMT $(P=0.009)$.

The overall survival at 5 year was similar: $57.5 \%$ for UCBT and $62.1 \%$ for UBMT $(P=0.25)$. The Transplantation Related Mortality was $19.6 \%$ for UCBT and $16.7 \%$ for UBMT $(P=0.56)$.

Comparing UCBT and UBMT in a multivariate model, we found that $\mathrm{CD} 8+\mathrm{T}$ cell recovery was delayed after UCBT with a median time to reach CD8 $+\mathrm{T}$ cells $>250 / \mathrm{mm}^{3}$ of 7.7 months whereas it was 2.7 months in UBMT $(P<0.001)$. B cell recovery was better in UCBT with a median time to reach CD19+ cells $>200 / \mathrm{mm}^{3}$ of 3.2 months in UCBT and 6.4 months in UBMT $(P=0.03)$. CD4 + lymphocyte and NK cell recovery was similar in UCBT and UBMT.

Other factors affecting lymphocyte subset recovery in the multivariate analysis were the age for $\mathrm{CD} 4+\mathrm{T}$ cell with a better reconstitution in younger patients $(P=0.002)$, and a positive cytomegalovirus serology which favoured CD8 + T cell reconstitution $(P=0.001)$

This study suggests that naive CD8 + T cells from UCBT have a worst reconstitution than mature CD8 $+T$ cells from UBMT, but $\mathrm{B}$ cell recovery is better in UCBT. Immune reconstitution in other subset of lymphocyte is similar in UCBT and UBMT. Moreover outcome in UCBT is comparable with UBMT, and GvHD is less frequent in UCBT.

\section{P699}

Characterization of T-cell reconstitution in children after CD3/CD19 depleted SCT compared to bone marrow transplant recipients using CDR3 spectratyping and TREC analysis

A. Jakitsch, M. Stais, A. Willasch, J. Soerensen, U. Koehl, S. Kuci, H. Kreyenberg, A. Jarisch, T. Klingebiel, P. Bader

Goethe University (Frankfurt, DE)

In haploidentical stem cell transplantation depletion of CD3 and CD19 positive cells from the graft may facilitate engraftment and immune reconstitution compared to CD34 + stem cell grafts. The quality of the immune reconstitution is predicted by the diversity of the $T$ cell receptor repertoire and the amount of de novo matured $T$ cells through the thymus.

The T-cell repertoire can be assessed by the characterization of rearranged TCR-beta chains (CDR3-Spectratyping). To facilitate the interpretation of the results, comprising the number of TCR families and the grade of polymorphism between each family, a Web-accessible statistical tool (SpA: Spectratype Analysis, Duke University, Durham, USA) was used. It provides a combined graphical reproduction and expresses the degree of diversity by the calculation of the Kullback-Leibler coefficient $(\mathrm{KL})$. In the thymus T-cells undergo somatic recombination of the TCR genes and form thymus excision circles (TRECs) which can be quantified of by RQ-PCR on genomic DNA preparations. The amount of detected TRECs is used as indicator of thymus activity.

We have investigated 52 paediatric patients (pts), who were monitored during the first year after transplantation. 19 pts received reduced intensity conditioning regimen (Flu-TT-MEL) followed by transplantation of CD3/CD19 depleted grafts and
33 pts received a standard myeloablative conditioning and bone marrow (BM) transplantation. For longitudinal investigations peripheral blood samples are collected at day 30,60, 90, 200,300 and 365

During the observation period of $4 / 19$ pts $(=22 \%)$ who received a CD3/CD19 allograft were positive for TRECs, and of 19/57 pts $(=33 \%)$ who received BM showed TRECs $(P=0.112)$. Within both groups (CD3/CD19 and BM) TRECs could be clearly detected starting from day +200 . Vbeta-spectratyping data showed that the $\mathrm{KL}$ values of the BM group were closer to normal values compared to the CD3/CD19 group at day 30 . However, this difference disappears already at day 60 and during further course both groups are not distinguishable.

Conclusion: TCR repertoire reconstitution follows individual courses and is affected by several factors, e.g. the primary disease, infections, relapse, and graft versus host disease (GvHD). Surprisingly, the time to achieve complex TCR repertoires seems to be similar between recipients of BM and CD3/ CD19 depleted peripheral stem cell grafts.

\section{P700}

Patients after allogeneic stem cell transplantation can generate polyomavirus-specific CD8 + T-cell responses D. Schneidawind (1), A. Schmitt (2), D. Bunjes (1), M. Freund (2), M. Schmitt (2)

(1)University Clinic (UIm, DE); (2)University Clinic (Rostock, DE)

Background: In the development of hemorrhagic cystitis in patients after allogeneic stem cell transplantation $(\mathrm{HC})$ polyomavirus BK (BKV) constitutes an important etiologic agent. Potential HLA-A2-binding peptides were synthesized based on computer algorithms to define T cell epitopes of the BKV proteins VP1 and ST towards the development of specific immunotherapies for $\mathrm{HC}$.

Material and methods: CD8 + T cells from peripheral blood (PB) of 25 healthy volunteers and 7 patients suffering from $\mathrm{HC}$ after allogeneic stem cell transplantation (SCT) were coincubated with BKV derived peptides for eight days in a mixed lymphocyte peptide culture (MLPC). Subsequently T cells were screened by enzyme-linked immunospot (ELISPOT) assays and fluorescence-activated cell sorting (FACS) analysis.

Results: We found that CD8 + T cells from 5 of $7(71 \%)$ patients with $\mathrm{HC}$ presensitized with the BKV peptide VP1 p108 (LLMWEAVTV) specifically recognized T2 cells pulsed with VP1 p108. In contrast only 7 of $25(28 \%)$ healthy volunteers had CD8 + T cells reactive with VP1 p108-pulsed T2 cells after presensitization. The presence of VP1 p108-specific T cells in the PB of patients with $\mathrm{HC}$ could be confirmed by staining as HLA-A2/VP1 p108 peptide tetramer + CD8 + T cells.

Conclusion: In summary BKV peptide VP1 p108 seems to play an important role as an immunodominant peptide in the pathogenesis of $\mathrm{HC}$ in patients after allogeneic SCT and might be a promising target for immunotherapies or even strategies to prevent the development of BKV-associated HC. As BKV shares more than $70 \%$ homology with multi-focal leukencephalopathy inducing JC virus on the DNA level, such approaches open also new avenues.

\section{P701}

The role of HLA-C encoded KIR ligands in matched unrelated haematopoietic stem cell transplantation I. Bruns, G. Kobbe, R. Haas, M. Uhrberg, J.C. Fischer University of Dusseldorf (Dusseldorf, DE)

HLA-C-dependent KIR ligand mismatch improves event-free survival (EFS) in AML patients especially after haploidentical HSCT. Recently we could show that in $109 \mathrm{CML}$ patients receiving grafts from matched unrelated donors (MUD), clinical outcome was significantly influenced by specific $\mathrm{C} 1 / \mathrm{C} 2$ donor recipient constellations (Fischer et al. J Immunol, 2007). Based on our previous observation that KIR genes are sequentially 
expressed during NK cell differentiation, we hypothesized that the presence of $\mathrm{C} 1$ but not $\mathrm{C} 2$ ligands in the patient enabled early reconstitution of high numbers of immunocompetent NK cells. To investigate whether this holds true for other patient cohorts, we analyzed the influence of KIR ligand constellations in a second cohort.

89 patients with MDS, $(n=22), A M L / C M L ~(n=34), A L L / N H L$ $(n=33)$ received unmanipulated PBPC after myeloablative $(n=66)$ or non-myeloablative conditioning regimens $(n=23)$ in the Department of Hematology, University of Düsseldorf. All recipients received grafts from MUD (10/10 match) except 16 with an HLA-C KIR ligand mismatch. HLA class I types were determined by direct sequencing and patients classified according to the KIR ligand groups $\mathrm{C} 1 \mathrm{C} 1, \mathrm{C} 1 \mathrm{C} 2$ or $\mathrm{C} 2 \mathrm{C} 2$.

Superior OS rates were found for $\mathrm{C} 1 \mathrm{C} 1$ recipients in $\mathrm{AML} / \mathrm{CML}$ (C1C1 recipients all alive, $P>0,05$ ). This fits to our original hypothesis that the early NK cell reconstitution is dominated by the presence of $\mathrm{C} 1-$ but absence of $\mathrm{C} 2$-specific NK cells. Within the subgroup of patients with $\mathrm{C} 1 \mathrm{C} 2$ or $\mathrm{C} 2 \mathrm{C} 2$ phenotypes 6 patients had an $\mathrm{C} 1 / \mathrm{C} 2$ mismatch, which was associated with significantly increased OS $(P>0.02$, all patients alive, median follow-up of $575 \mathrm{~d}$, range 133-899d) compared to matched HLA-C phenotypes (median survival of 254d). In recipients with C1C1 phenotype the risk for TRM following HLA-C matched transplantation was reduced as reflected by an odds ratio of 0.13 . In turn, patients receiving KIR ligand mismatched grafts had a lower incidence of relapse. These effects were not observed in patients with MDS or ALL/NHL, although there was a trend for a favorable outcome in ALL/NHL patients receiving a KIR ligand mismatched graft $(P>0.07$; mean OS intention to treat $3.5 y$ vs.14y). These findings support our observations that transplant outcome in unrelated matched HSCT is substantially influenced by the distribution of HLA-C encoded KIR ligands (superior clinical outcome in $\mathrm{C} 1 / \mathrm{C} 1$ recipients) and confirms the clinical relevance of NK cell-mediated effects.

\section{P702}

Functionally altered GPI-anchor negative T-cell subsets in patients after alemtuzumab-based T-cell depleted haematopoietic stem cell transplantation

E.M. Wagner, A.N. Lay, D. Wolff, J. Hemmerling, C. Goetze, M. Theobald, W. Herr, R.G. Meyer

Johannes Gutenberg University (Mainz, DE)

The anti-CD52 antibody alemtuzumab is frequently used for in vivo $\mathrm{T}$ cell depletion (TCD) in the context of allogeneic hematopoietic stem cell transplantation (HSCT). We recently demonstrated long-term persistence of CD52-negative T-cell subsets in patients after HSCT following alemtuzumab-mediated TCD. We therefore investigated the functional consequences of this CD52-loss on T cells from our patients.

CD52 is a glycosylphosphatidylinositol (GPI)-anchored protein By staining for CD55 and CD59, we found that CD52-negative $T$ cells also had lost expression of these GPI-anchored molecules. We proved that the loss of CD52 resulted from a reduced density of GPI-anchors on the membrane using a fluorescent aerolysin (FLAER). Subsequently, CD4 and CD8 T cells were sorted from the peripheral blood of our patients according to their CD52-status by flow-cytometry. When we cultured the sorted cells separately, CD52-expression remained unaltered for more than 16 weeks. This observation was in contrast to earlier results with $\mathrm{T}$ cells of healthy donors treated with alemtuzumab in vitro, where CD52-negative populations were only transiently detected. The growth-kinetics of the patients' sorted $\mathrm{T}$ cells did not differ between the CD52-positive and CD52-negative populations when cultured with IL-2 and OKT-3 alone. On the contrary, when we expanded T cells in the presence of overlapping peptides from cytomegalovirus (CMV), only the proliferation of CD52-positive T cells increased. The reduced CMV-specific $T$ cell activity of the CD52-negative T-cell subpopulations was confirmed by IFN-gamma ELISPOT assay with in vitro expanded T cells as well as by IFN-gamma secretion-assay on primary $T$ cells ex vivo. We further analyzed IFN-gamma secretion of $T$ cells following allogeneic stimulation with dendritic cells of non-HLA matched third-party donors and again detected lower levels of IFN-gamma production by CD52-negative compared to CD52-positive T cells.

In summary, we demonstrated that the permanent loss of CD52 in reconstituting $T$ cells after alemtzumab-based TCD is associated with a loss of GPI-anchors. Our data suggest that this loss correlates with altered T-cell effector-functions in response to antigen-specific stimulation. A further comparison of functional changes in CD52-negative T-cell subsets is necessary to understand the impact of alemtuzumab-mediated TCD on immune-reconstitution. This might also lead to an increased insight in the role of GPI-anchors for T cell function.

\section{P703}

The impact of graft composition on early CD4 + T-cell recovery and clinical outcome of patients who underwent allogeneic haematopoietic stem cell transplantation R. Fedele, M. Martino, T. Moscato, G. Messina, G. Console, G. Irrera, M. Cuzzola, E. Spiniello, C. Garreffa, A. Dattola, I. Callea, O. lacopino, A. Pontari, P. lacopino

Azienda Ospedaliera B.M.M. (Reggio Calabria, IT)

Background: Recent studies demonstrated the significant correlation between early immune recovery (IR) and overall survival (OS) of patients (pts) who underwent allogeneic hematopoietic stem cell transplantation (allo-HSCT). There are instead controversial data regarding the influence of graft composition on clinical outcome (CO) after allo-HSCT.

Aim of the study: To evaluate the impact of graft composition on early IR and CO in terms of OS, transplant-related mortality (TRM) and disease-related mortality (DRM), acute graft-versus-host-disease (aGVHD) after allo-HSCT.

Materials and methods: We studied 99 pts underwent allogeneic BM (23) or PBSC (76) transplant. Pts were 51 females and 48 males with a median age of 46 years (r. 11-67). The donors were matched sibling donors $(n=83)$ or alternative donors (family mismached or unrelated donors) $(n=16)$. Conditioning regimens were myeloablative in 48 pts or at reduced intensity in 51 pts. AGVHD grade II-IV was present on 44 pts. Median follow-up of pts was 58 months (r. 24-98). Graft composition was evaluated in terms of CD34 +, CD3 +, CD4 +, CD8 + CD19+, CD56 + cells. Early CD4 + cell count (median 20 days after transplant, r. 12-34) was evaluated in peripheral blood samples.

Results: Multivariate Cox analysis showed that early CD4 + cell count is the main predictive factor of OS $(P=0.012$, HR 2.686, Cl 1.241-5.813). OS also depends on aGVHD $(P=0.025$, HR $0.303, \mathrm{Cl} 0.107-0.860)$ and donor type $(P=0.025, \mathrm{HR} 0.303, \mathrm{Cl}$ $0.183-0.907)$. Roc curve of CD4 + cell count indicated that the cut-off value was 100/microl. At 2 years follow-up, pts achieving this CD4 + cell count had significantly lower cumulative TRM compared to pts who did not have this count $14.5 \% \pm 5 \%$ vs. $39.4 \% \pm 8 \%, P=0.0026$ ). At 5 years follow-up, OS was $77.5 \% \pm 6.5 \%$ and $37.8 \% \pm 7.5 \%(P=0.000)$ in pts with CD4+ cell count more or less than $100 / \mathrm{microl}$, respectively.

The graft composition was comparable in pts with early CD4 + cell count more or less cut-off value and didn't influence significantly the OS, DRM, TRM and aGVHD.

Conclusion: The main predictive factor for $\mathrm{CO}$ of pts who underwent allo-HSCT is early CD4 + T cell count after transplant. Early immune reconstitution is independent of graft composition obtained from actual collect methods. In the future, the use of new mobilizing drugs could modify the graft in order to achieve a product that can improve early IR and consequently OS of pts after allo-HSCT. 
P704

CD4 + CD25highFOXP3 + regulatory T-cells are increased and functionally active after antithymocyte globulin infusion and allogeneic stem cell transplantation in humans - a novel in vivo mechanism of action

S. Theurich (1), A. Reisberg (2), M. von Bergwelt-Baildon (1), D. Riemann (2), G. Behre (2)

(1)University Hospital Cologne (Cologne, DE); (2)University Hospital Halle (Halle/Saale, DE)

CD4 + CD25highFoxP3 + regulatory T cells (Tregs) play a central role in immunologic homeostasis and are able to induce tolerance after allogeneic hematopoietic stem cell transplantation (alloPBSCT). Polyclonal rabbit-anti-human antithymocyte globulins (ATG) are widely used to prevent and treat Graftversus-Host-Disease (GvHD) by a mechanism of action which has been thought to be mainly mediated by $T$ cell depletion. Recent in vitro data have suggested that ATGs might increase the ratio or number of regulatory $T$ cells as a new mechanism of action. So far, it is not known whether such effects exist in humans in vivo. Here, we present the data of a prospective pilot study in which we analyzed the influence of ATGs on the reconstitution of peripheral CD4 +/CD25high/FoxP3 + Tregs in ten patients after alloPBSCT. Ten patients were divided into two groups depending on the requirements of the respective alloPBSCT protocol: those who received ATG during conditioning $(n=6)$ and those without ATG $(n=4)$. ATG treated patients received a total dose of either ATG-Fresenius $30 \mathrm{mg} / \mathrm{kgBW}$ $(n=3)$ or ATG-Genzyme $6 \mathrm{mg} / \mathrm{kgBW}(\mathrm{n}=3)$ respectively. ATG was given day -3 to -1 . All patients received peripheral hematopoietic stem cells (PBSC) from HLA matched donors and the transplanted stem cell grafts contained comparable median numbers of CD3 + T cells and CD34 + PBSC in both groups. All patients recieved prophylactic immunosuppression consisting of mycophenolate mofetil and cyclosporine. Antiviral prophylaxis was with acyclovir. Blood samples were taken before conditioning and after alloPBSCT at distinct time points (day $+30,+60,+90$ and +150$)$ and immune cells were analyzed by flow cytometry (CD3,CD4, CD25,FOXP3). In vitro suppressive activity of Tregs from day +150 samples was measured with CD4 + CD25 + selected Tregs and CD4 + CD25- selected CSFE labelled autologous responder $\mathrm{T}$ cells.

In summary, we found a significant-more than 3-fold-increase of relative Treg numbers in ATG treated patients compared to the control. We could also show that day +150 Tregs were functionally active in an in vitro assay with autologous CSFE labelled responder cells.

Taken together, our results rise first evidence that rATG treatment leads to increased, functional active CD4+/CD25high/ FoxP3 + Tregs in humans after allogeneic stem cell transplantation which supports the thesis that ATG exhibit their immunomodulatory activity by additional mechanisms beyond simple T cell depletion.
Patient characteristics and transplantation parameters

\begin{tabular}{|c|c|c|c|c|c|}
\hline Basefine paraneters & & FATG & \multicolumn{3}{|c|}{ non-AIG } \\
\hline Padients & & 6 & & 4 & \\
\hline Age (median) & \multicolumn{2}{|c|}{$37-68(61)$} & \multicolumn{3}{|c|}{$48-6(6)$} \\
\hline Diagnosis & \multicolumn{2}{|c|}{ MM, Ma, al, NIL } & \multicolumn{2}{|c|}{$\operatorname{AM}(n=3), O M F$} & \\
\hline \multicolumn{6}{|l|}{ Denars } \\
\hline MUD & & 6 & & 2 & \\
\hline \multicolumn{6}{|l|}{ ONV staturs } \\
\hline \multicolumn{6}{|l|}{ Conditioning reginen } \\
\hline $\begin{array}{l}\text { RIC } \\
\text { Standard }\end{array}$ & & $\begin{array}{l}5 \\
1\end{array}$ & & $\begin{array}{l}3 \\
1\end{array}$ & \\
\hline \multicolumn{6}{|c|}{ Iransplanted cell numbers $\times 10^{6} / \mathrm{kg}$ BW recipient, (metian) } \\
\hline $\begin{array}{l}\text { CD34+ PBSC } \\
\text { CO3+T als }\end{array}$ & $\begin{array}{l}185-11.0 \\
150-480\end{array}$ & $\begin{array}{l}\text { (7.6) } \\
\text { (219) }\end{array}$ & $\begin{array}{l}4.91-9.47 \\
120-540\end{array}$ & $\begin{array}{l}\text { (73) } \\
\text { (175) }\end{array}$ & $\begin{array}{l}(p 0.47) \\
(p 0.38)\end{array}$ \\
\hline
\end{tabular}

MRD: matched related donor; MUD: matched unrelated donor; RIC:

Reduced Intensity Conditioning (Treosulfan / Fludarabin or

Cyclophospamide/Fludarabin or 2Gy-Total-Body-Irradiation/

Fludarabin); Standard conditioning (Cyclophosphamide/12Gy-TotalBody-Irradiation). BW: body weight; AML: acute myeloid leukaemia;

OMF: osteomyelofibrosis; CLL: chronic lymphocytic leukaemia; ALL:

acute lymphoblastic leukaemia; MCL: mantle cell lymphoma; MM:

multiple myeloma

PBSC: peripheral blood stem cells. Cellular subpopulations of the

transplanted grafts were calculated to the respective recipient's body

weight. Statistical analysis by SPSS (Mann-Whitney Test).

\section{P705}

Paraproteinaemia after allogeneic HSCT is frequent, may be transient or persistent and is associated with alemtuzumab containing conditioning

P.G. Medd (1), S. Littlewood (1), R. Danby (2), R. Malladi (1), R. Clifford (1), D. Wareham (1), D. Roberts (3), A.J. Peniket (1), T.J. Littlewood (1)

(1)Oxford Radcliffe Hospitals NHS trust (Oxford, UK); (2)NDCLS, University of Oxford (Oxford, UK); (3)NHS Blood and Transplant (Oxford, UK)

Monoclonal gammopathy following allogeneic haematopoietic stem cell transplant (HSCT) has been previously described. We aimed to characterise this finding further and identify transplant variables associated with paraprotein development. We examined a series of 104 consecutive allogeneic HSCT performed at our institution who had a minimum of 6 months post-transplant follow-up. Patients with myeloma (5 patients) or pre-existing paraproteinaemia (2 patients) were excluded. Data were unavailable for 5 patients thus 92 transplants were analysed: HLAidentical sibling donor $61 \%$, matched unrelated donor (MUD) $39 \%$, reduced intensity conditioning (RIC) $77 \%$, myeloablative conditioning $23 \%$. Alemtuzumab was contained in $42 \%$ of conditioning ( 5 sibling, 34 MUD). With a median follow-up of 4.75 years the cumulative incidence of paraproteinaemia was $32 \%(95 \% \mathrm{Cl} 18-44 \%)$ at 2 years; the median time to paraprotein appearance was 5 months. No patient developed paraproteinaemia beyond 22 months post-transplant (Figure 1). In 10 of 29 patients the paraprotein(s) disappeared. Median time to disappearance was 8 months and the cumulative proportion of patients with persistent paraproteinaemia reached a plateau of $88 \%(95 \% \mathrm{Cl} ; 83-98 \%)$ at 32 months. Paraproteins were frequently multiple with a median number of paraproteins per patient of 2 (range 1-6). Forty-one of $49(83 \%)$ assessable paraproteins were of immunoglobulin $\mathrm{G}$ class and were divided equally in light chain restriction (kappa 24 of 49 , $49 \%$ ). Most paraproteins were too small to quantify, the mean concentration of 13 quantifiable paraproteins was $2.85 \mathrm{~g} / \mathrm{L}$. Of paraproteins quantifiable at any time point 5 increased and 9 decreased in concentration from initial detection. Multivariate analysis demonstrated that only patient age (odds ratio (OR) 1.04 per year of increasing age, $P=0.04$ ) and alemtuzumab use 
(OR no alemtuzumab $=0.23, P=0.003$ ) were significantly associated with subsequent paraprotein development (Figure 2). No patient subsequently developed myeloma or a lymphoproliferative disorder. Survival of the paraprotein group was significantly worse than the paraprotein-free group (hazard ration 0.38 for paraprotein-free, $95 \% \mathrm{Cl} 0.16-0.92$ ), which is likely to be a reflection of poorer survival outcomes for T-depleted MUD in comparison with T-replete sibling transplants. We conclude that post-transplant paraproteinaemia is a frequent, probably benign, finding and is influenced by alemtuzumab effects on $B$ and $T$ cell reconstitution.
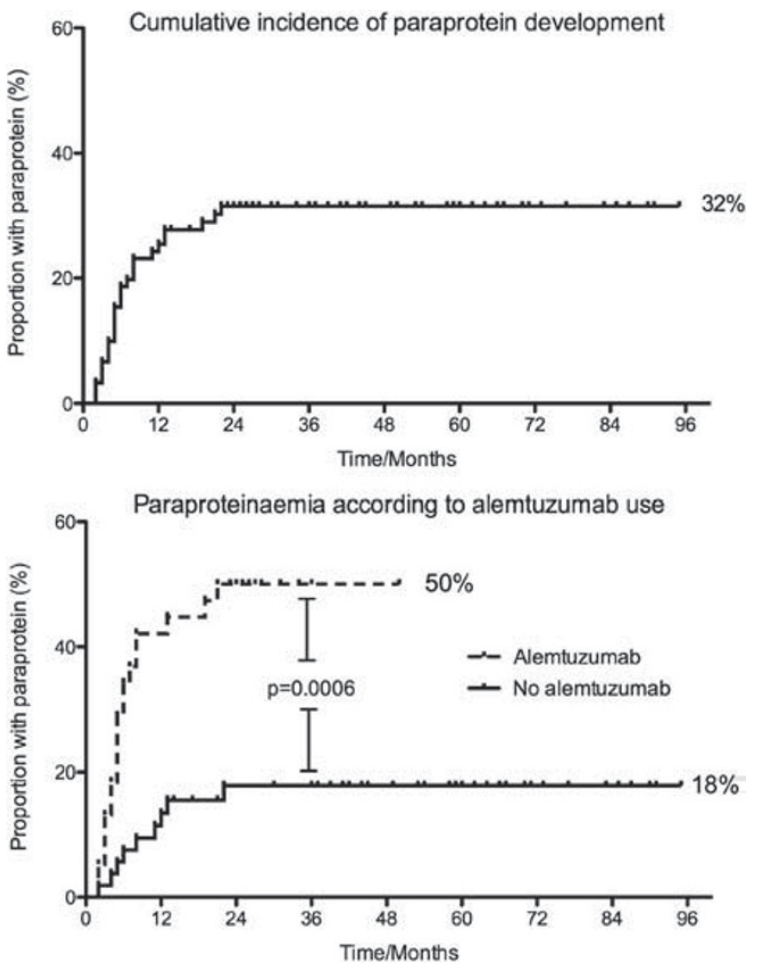

\section{P706}

Rapid recovery of lymphocyte subsets is not associated with protection from relapse of myelodysplastic syndromes and acute myeloid leukaemia after haematopoietic stem cell transplantation using a reduced-intensity conditioning regimen and alemtuzumab

K. Matthews (1), Z. Lim (2), L. Pearce (2), A. Pagliuca (3), A. Madrigal (1), G. Mufti (2), L. Barber (2)

(1)Anthony Nolan Research Institute (London, UK); (2)King's College London (London, UK); (3)King's College Hospital (London, UK)

Graft versus leukaemia (GvL) and graft versus host disease (GvHD) are both caused by alloreactive lymphocytes. We previously reported that GvHD correlates with higher numbers of effector CD4 T cells and NK cells early after allogeneic transplantation using a regimen comprising fludarabine, busulphan and alemtuzumab. Here, we assessed immune cell subset recovery in the same cohort of 25 patients in the context of early myeloid malignant disease relapse. Despite the close relationship between the GvL and GvHD immune responses, rapid recovery of lymphocyte subsets was not associated with protection from disease relapse. Analysis of immune recovery in the context of disease relapse showed no significant differences within the lymphocyte subsets early (day 30 ) in patients that relapsed and those that did not. Indeed by day 60 , twice as many lymphocytes were present in peripheral blood of patients in the relapse group at a median of 770 per microliter compared to 410 per microliter for patients that did not relapse $(P=0.04)$. This was particularly striking for the CD8 T cell population $(P=0.02)$ and was evident in all subsets (naïve, memory and effector). These results indicate GvL may be weak or ineffective in this treatment setting for patients with myelodysplastic syndromes and acute myeloid leukaemia. Consistent with low GVL activity, we previously reported that mixed $T$ cell chimerism has no detrimental effect on relapse in this treatment setting and instead correlates with better outcome because of reduced GvHD incidence. We now report that patients with significantly higher lymphocyte numbers prior to transplantation subsequently maintained the mixed $T$ cell chimeric state (median of 900 per microliter compared to 250 per microliter for patients that progressed rapidly to full donor T cell chimerism; $P=0.04$ ). This pre-transplant profile, together with absence of the early post-transplant signature indicative of GVHD predisposition, could potentially be used to identify patients suitable for early withdrawal of immunosuppression and prophylactic donor leukocyte infusion to boost GvL activity.

\section{P707}

Acute GvHD patients have low proportions of naïve and terminally differentiated memory CD4 + lymphocytes J. Lange (1), D. Drabczak-Skrzypek (2), D. Dlubek (2), D. Wolowiec (3), A. Lange (2)

(1)Lower Silesian Center for Cellular Transplantation (Wroclaw, PL); (2)L. Hirszfeld Institute of Immunology and Experimental Therapy, Polish Academy of Sciences (Wroclaw, PL); (3)Medical School, Departement of Hematology (Wroclaw, PL)

It is known that the pace of immunological recovery influences the outcome of HSCT. Acute GvHD plays a major role in shaping immune reconstruction. Therefore, we studied T-cell populations using three colour flow cytometry post HSCT in association with the presence of aGvHD.

The observation of 41 patients (median age $45 \mathrm{yrs}$, range: 1.064 yrs, with 39 hematological malignancies and 2 SICD, 19 MUD and 22 SIB transplantation) started at the day of hematological recovery ; which coincided in 19 patients with the first symptoms of aGvHD. There was two observation time points, first at the beginning of hematological recovery and second 4 weeks later.

All T cell subsets were defined with the use of two sets of monoclonal; antibodies which had CCR7 or CD27 MoAb, the other two antibodies used always in concert with CD27 and CCR7 MoAb were those against CD4 and CD45RA.

(i) naïve cells were defined as being CD45RA+, CD27+ and $\mathrm{CD} 4+$ in other separate staining procedure instead of CD27 CCR7 positivity described this subset. (ii) central memory cells were CD45RA-, CD27+, CD4 + or defined separately as CD45RA-, CCR7 +, CD4 +, (iii) effector memory cells lacked CCR7 and CD27 in addition they were CD45RA negative and CD4 positive, (iv) terminally differentiated memory cells (TEMRA) were CD4 and CD45RA positive but CCR7 and CD27 negative. Clinical outcome observation included the manifestation of aGvHD.

Results:

1. Naive T cells were in higher proportions in patients lacking than in those having aGvHD at the beginning of hematological recovery (median 30 vs. 11 cells/uL, $P=0.03$ ) and 4 weeks later (median 75 vs. 17 cells/uL $P=0.01$, Mann-Whitney U test) when CCR7 was used for staining. Similar observation was seen when CD27 instead of CCR7 MoAb were used for staining but the difference did not reach statistical significance (not shown).

2. TEMRA cells defined by a lack of CCR7 in CD45RA positive and CD4 positive population were higher in aGvHD patients in two time points at the beginning of hematological recovery (median 22 vs. 6 cells/uL, $P=0.002$ ) and four weeks later (median 31 vs. 9 cells/uL, $P=0.02$, Mann-Whitney $U$ test). 
3. There was no difference when blood central and effector memory CD4 + cells were analyzed.

Therefore, aGvHD patients were handicapped with respect to the number of Naïve and TEMRA CD4 + lymphocytes in blood. It likely reflects an impairment of the thymus function and consequently lymphocytes differentiation into effectors cells in aGvHD patients.

\section{P708}

Serological response to influenza vaccine haematopoetic stem cell transplantation patients

S. Yalcin (1), M. Kondolot (1), N. Albayrak (2), B. Altas (2), Y. Karacan (1), B. Kuskonmaz (1), S. Aksu (1), M. Cetin (1), H. Goker (1), K. Yurdakok (1), D. Uckan (1)

(1)Hacettepe University (Ankara, TR); (2)Refik Saydam Hifzissiha Center (Ankara, TR)

Vaccination is the best strategy to prevent influenza infection that is a potential cause of morbidity and mortality in immunosuppressed patients. Here, we evaluated the factors that may affect serological response to influenza vaccine in patients who have undergone hematopoetic stem cell transplantation (HSCT). Sixty one HSCT recipients were included in the study during the 2007-2008 influenza season. Serum samples prior to vaccination and 6-10 weeks after vaccination were collected. Samples were assayed for antibodies to influenza virus $\mathrm{A} / \mathrm{H} 1 \mathrm{~N} 1, \mathrm{~A} / \mathrm{H} 3 \mathrm{~N} 2$, and B strains by Hemagglutination Inhibition (HAl) Assay. The patients were followed in terms of clinical symptoms up to the next influenza season and for adverse effects within a month after vaccination. Prior to vaccination, $96 \%$ of the patients had protective antibody level against to $\mathrm{H} 3 \mathrm{~N} 2,92 \%$ against to $B$ and $47 \%$ against to $\mathrm{H} 1 \mathrm{~N} 1$. Overall, pre-vaccine seroprotection rate against all vaccine antigens $(\mathrm{A} / \mathrm{H} 1 \mathrm{~N} 1, \mathrm{~A} / \mathrm{H} 3 \mathrm{~N} 2, \mathrm{~B}$ antigens) was $45.1 \%$, post-vaccine seroprotection rate $91 \%$ and seroconversion rate was $28.3 \%$. Seroconversion rates were found to be low against $B$ in patients who were vaccinated in the late influenza season $(P=0.018$; respectively). Five patients $(10.9 \%)$ had no immune response against $\mathrm{H} 1 \mathrm{~N} 1$. Adverse events were reported in $19.6 \%(n=9 / 46)$ of the patients after vaccination. In conclusion, the patients should be vaccinated as early as possible in the influenza season, before they are exposed to the virus.

\section{P709}

Nilotinib administration post-allogeneic stem cell transplantation in advanced chronic myeloid leukaemia and $\mathrm{Ph}+$ acute lymphoblastic leukaemia: Immunological Evaluations

N. Varda-Bloom, R. Somech, Y. Volchek, R. Yerushalmi, N. Shem-Tov, A. Shimoni, A. Nagler

Chaim Sheba Medical Center (Tel Hashomer, IL)

Allogeneic stem cell transplantation (alloSCT) is the treatment of choice for advanced chronic myeloid leukaemia (CML) $(>\mathrm{CP} 1)$ and $\mathrm{Ph}+$ acute lymphoblastic leukaemia (ALL). However, post transplant relapse rates are still high. Nilotinib is a second generation TKI that is active in advanced CML. Administration of Nilotinib post alloSCT is an attractive approach for prevention of relapse. Fast and efficient immune reconstitution is mandatory for the anti leukemic effect. Furthermore, TKIs may mediate an anti GVHD effect. The aim of the current study was to evaluate the post alloSCT immune recovery and function in pts with advanced $\mathrm{CML}$ and $\mathrm{Ph}+\mathrm{ALL}$ that received Nilotinib post-alloSCT and to clinically assess its effect on GVHD and GVL. Twelve pts were recruited so far to the study. Seven with advanced $\mathrm{CML}(\mathrm{BC}-5, \mathrm{AP}-2)$ and 5 with $\mathrm{Ph}+\mathrm{ALL}$, median age 39 years (range, 21-57), males-7 and females-5. Immunological reconstitution was assessed pre- and post Nilotinib administration (200mgx2/d) and includes: FACS analysis of $\mathrm{T}$ and Natural Killer (NK) cells subsets, T cells mitogenic response, $\mathrm{T}$ cells receptor repertoire, $\mathrm{T}$ cells receptor excision circles (TREC) and NK cells cytotoxicity against K562. Results are presented as post vs. pre Nilotinib average values $( \pm S E)$ in 6 pts with $\leq 180 \mathrm{~d}$ follow up.

Results: FACS: Lymphocytes numbers including CD3 (1726.7 \pm 248.2 ; 707.25 \pm 205.5$)$, CD4 (1312 \pm 219.1 ; 581.5 \pm 182.2$)$, CD8 $(554.2 \pm 113 ; 306.5 \pm 76.2)$, CD20 (246.8 $\pm 74.1 ; 15 \pm 7.9)$, and CD56 (99.1 \pm 20.3 ; 83.2 \pm 26.6$)$ increased post Nilotinib administration. T cells mitogenic response increased in $4 / 6$ of the pts (unchanged-1, decrease-1). Increased presentation of normally expressed $\mathrm{Vb}$ was observed in all patients $(57 \% \pm 6.2$ to $67 \% \pm 7.8$ of the tested receptors pre and post Nilotinib, respectively) in conjunction with reduced presentation of under expressed $\mathrm{Vb}$ ( $29 \% \pm 8.5$ to $15.8 \% \pm 4.4$ of the tested receptors, respectively). Average of TRECs was $46.6 \pm 31.8$ copies per 0.5 ug DNA preand $158.3 \pm 67.3$ copies per 0.5 ug DNA post Nilotinib administration. Finally, increased NK cytotoxic activity against K562 was observed following Nilotinib administration in all but one evaluated pts; $27.5 \pm 8 \%$ vs. $10.9 \pm 6.8 \%$, respectively.

Conclusions: Nilotinib administration post alloSCT resulted in increased lymphocyte subsets, T cell mitogenic response, thymic output and repertoire and NK cell cytotoxic activity. In parallel, all pts but one achieved a sustained CMolR indicating a potent GVL effect. GVHD was reduced only in pts that received Nilotinib pre but not post alloSCT (Shimoni A. Leukemia 2009;23:190).

\section{P710}

Early absolute lymphocyte count predicts outcome of patients undergoing autologous haematopoietic stem cell transplantation

E. Romero, D. Hernandez, M. Canales, R. Arrieta, A.R. de la Rúa Hospital La Paz (Madrid, ES)

Background: Absolute lymphocyte count (ALC) $>500 / \mu$ on day 15 post-autologous haematopoietic stem cell transplantation (HSCT) has been associated with good prognosis in patients with hematologic malignancies. ${ }^{*}$

Aims: To asses in our environment, the influence of the early ALC (day 15) in predicting post-HSCT patients with non-Hodgkin lymphoma (NHL), Hodgkin lymphoma (HL), multiple myeloma $(\mathrm{MM})$ and acute myelogenous leukemia (AML) in terms of progression-free survival (PFS) and overall survival (OS) and to analyze other variables that could act as additional prognostic factors.

Patients and methods: Medical records of 158 patients receiving autologous- HSCT between January 2003 and August 2009 were reviewed ( $82 \mathrm{NHL}, 29 \mathrm{LH}, 40 \mathrm{MM}, 7 \mathrm{LAM}$ ). The study was performed using Cox regression model and survival curves of Kaplan-Meier.

Results: With a median follow up of 134 weeks (range: 41-266 weeks) patients with ALC $>500 / \mu \mathrm{l}, \mathrm{n}=94(59.5 \%)$ had a higher PFS and OS compared with those with ALC below the limit, $\mathrm{n}=64$ (40.5\%): PFS 67 months (CI95\%: 60.5-72.5) vs. 23 months (CI95\%: 15.1-30.8), $P<0.001$ and OS of 283.5 weeks (Cl95\%: 262.7-304.4) vs. 211.8 weeks (Cl95\%: 171.3-252.3), $P<0.001$. Multivariate analysis showed that not reaching an ALC $>500$ on day 15 is an adverse factor for PFS with HR 7.72 (CI95\%: 4.05-14.7) $P<0.001$, and for OS with HR 9.7 (CI95\%: 3.1-30.34), $P<0.001$. In this series, we additionally observed that infusing $>$ or $=4.38 \mathrm{CD} 34+\times 10^{6} / \mathrm{Kg}$ was associated with better survival in all groups, $P=0.015$ and $\mathrm{HR}=0.27(\mathrm{Cl} 95 \%$ : 0.094-0.774).

Conclusions: This study confirms:

1) The impact of early ALC post- autologous HSCT in PFS and OS of patients with hematologic malignancies.

2) An absolute lymphocyte count $\leq 500$ is an independent risk factor for decreased PFS and OS.

Reference: *Porrata et al. Mayo Clinic, Rochester 2002. 


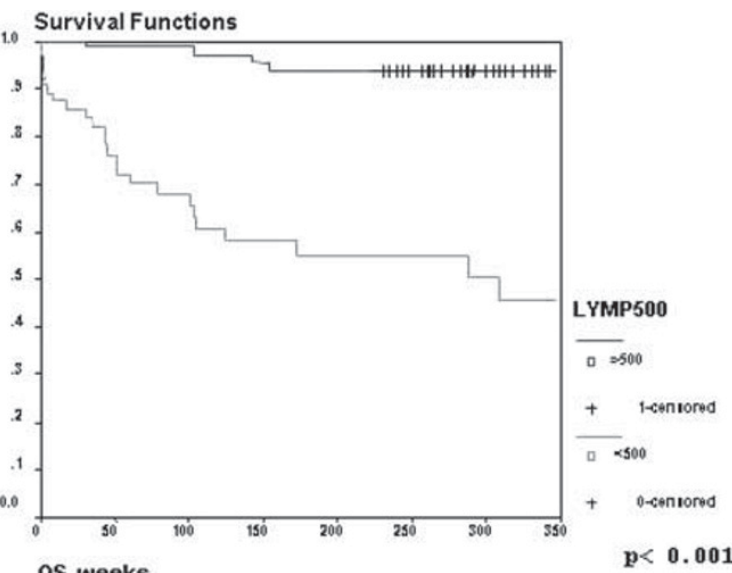

os weeks

Survival Functions

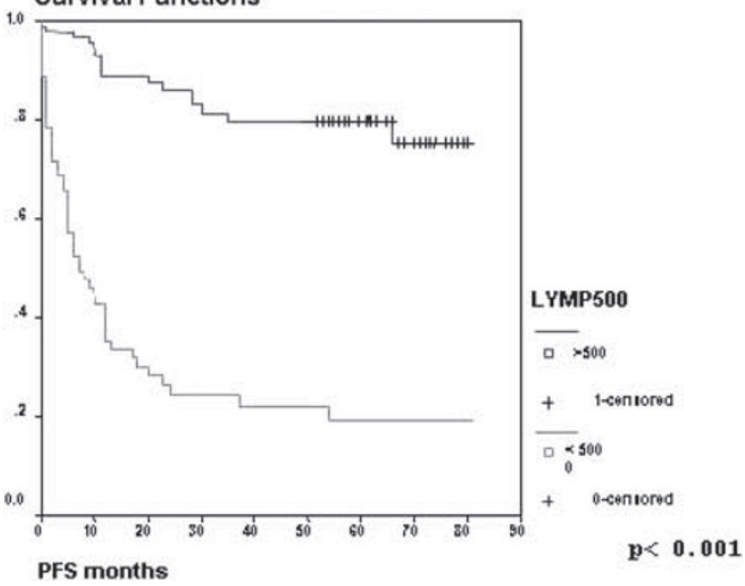

P711

The impact of ATG on gene expression patterns in T-cells after SCT

M. Uzunel, S. Ruhm, O. Ringden

Clinical Immunology (Stockholm, SE)

Antithymocyte globulin (ATG) is often used as part of the conditioning therapy to reduce the risk for GVHD and rejection. ATG treatment not only leads to lymphocyte depletion but also changes in the T-cell subset composition in blood.

In vitro studies show that ATG can increase the expression of FOXP3 protein which has led led to speculation that exposure to ATG might be an effective method to induce Tregs for clinical applications

In this study, we analyzed the gene expression patterns of Foxp3, IL-10, IFN-G, IL18 and beta 7-integrin (ITGB7) in CD4 + and CD8 + T-cells to study how ATG affected the kinetics of these genes after SCT.

Materials and methods: 64 patients were included in the study. Median age was 44 (range 10-67) years. 30/64 patients received a reduced intensity conditioning $(\mathrm{RIC})$ regimen. GVHD prophylaxis was Cy + MTX in most patients $(n=46)$.

ATG (Thymoglobulin, Genzyme) was used in 49 (77\%) patients. ATG was administered for 3 or 4 days with the last dose given the day before SCT.

Blood samples were taken before conditioning, Day +0 (before infusion of graft), D+14, D+21, D+28, Month +3 and $M+6$ post transplant. CD4 + and CD8 + T-cells were separated with immunomagnetic beads and quantitative RNA expression was performed with Real-time PCR and the results were normalized to a reference gene.
Results: Foxp3 expression was significantly decreased after ATG both in CD4 + and CD8 + T-cells for more than 3 months post-SCT. This decrease was seen already at Day +0 and the difference was more than one log of magnitude.

IFN-G and beta 7-integrin gene expressions were also significantly decreased after ATG in CD4 + and CD8 + T-cells and the difference lasted for one month post-SCT.

There was no impact of ATG on IL-10 expression in CD4 + T-cells but higher IL-10 expression after ATG was seen in CD8 + T-cells early post-SCT (Day +0 and Day +14 ).

An increase of IL-18 expression was seen both in CD4 + (Day + 0 only) and CD8 + T-cells (Day +0 and Day +14 ) after ATG.

Conclusion: In contrast to other studies, we could not find an increase of Foxp3 after the use of ATG. Foxp3 RNA levels were decreased considerably after ATG for a long time period after SCT. However, we have analyzed RNA expression which may not be positively correlated to protein levels which others have analyzed.

ATG had a strong negative effect on IFN-G expression while IL-10 levels were increased which may indicate that ATG has a more positive effect on Th2 responses as compared to Th1 responses.

P712

The impact of polyclonal immunoglobulin recovery after autologous stem cell transplant in multiple myeloma R. Bergantim, R. Pinto, F. Trigo, J.E. Guimaraes Hospital S. João (Porto, PT)

Autologous stem cell transplant (ASCT) improves survival in suitable patients with Multiple Myeloma (MM). However, after ASCT there are widely documented impairments in immunological reconstitution such as the delay in recovery of polyclonal immunoglobulin. This abnormality when persistent might be associated with disease relapse or progression. We retrospectively analyzed a series of 65 consecutive patients with MM who underwent ASCT in our centre, from 2000 to 2009, regarding recovery of IgM levels ad day $100( \pm 10)$ post-ASCT. Progression-free survival (PFS) was assessed from the day of the transplant to disease progression or death (using the International Myeloma Working Group). PFS was analysed using Kaplan-Meier curves and univariate and multivariate analysis were done using proportional Cox regression. The median age of patients was 56 years (range- 37 to 67 ), 31 were females. None of those had MM type IgM. Median follow-up was 12 months (range- 1 to 42 months). Twenty-three $(34.8 \%)$ patients recovered normal lgM levels by day +100 and 42 $(63.6 \%)$ did not, 1 patient data missing. PFS was significantly higher in the group who recovered normal $\operatorname{lgM}$ at day $100( \pm 10)$ post-ASCT compared to patients who did not, with median PFS in the group who recovered normal IgM levels of 29 months versus 13 months in the group who did not recover $(P=0.021)$ (Figure 1). Multivariate analysis, using a model including IgM polyclonal recovery, age, B2-mycroglobulin, and lactic dehydrogenase at diagnosis, number of prior chemotherapy schema and MM type, suggests that recovery of polyclonal immunoglobulin is an independent prognostic factor for PFS. It is debatable whether approaches with intravenous polyclonal immunoglobulin administration will translate into improvement of PFS in those patients. 


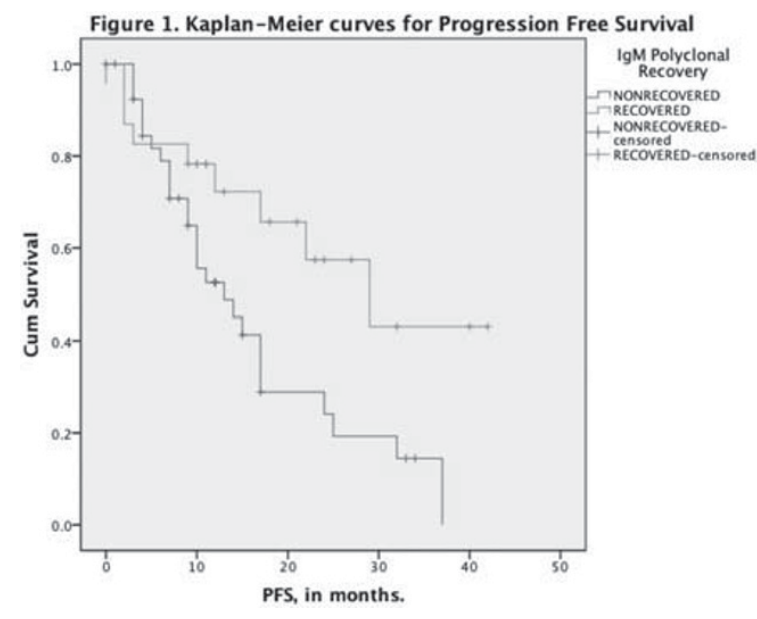

P713

iNKT cell reconstitution in paediatric leukaemia patients following haploidentical stem cell transplantation suggests contribution to leukaemia control and reveals independent CD4 + and CD4- subset maturation programmes

C. De Lalla (1), A. Rinaldi (1), D. Montagna (2), L. Sangalli (3), L. Azzimonti (3), A.M. Paganoni (3), R. Maccario (2), M.E. Bernardo (2), F. Locatelli (2), P. Dellabona (1), G. Casorati (1) (1)H San Raffaele Scientific Institute (Milan, IT); (2)Fondazione IRCCS Pol. San Matteo (Pavia, IT); (3)Politecnico (Milan, IT)

Invariant Natural Killer T cells (iNKT) are a subset of innatelike T lymphocytes that express a semi-invariant TCR and are distributed in two functionally distinct CD4 + and CD4- subsets. iNKT cells recognize both self and bacterial lipids presented by CD1d. Upon recognition of CD1d/lipid complexes, iNKT cells are immediately activated and produce cytokines that contribute to protect against infection and cancer. We investigated the reconstitution of the circulating iNKT cell compartment in 21 pediatric patients undergoing T-cell depleted haplo-identical HSCT (Haplo-HSCT) for hematological malignancies (6 acute myeloid leukemia, 9 acute lymphatic leukemia, 6 myelodisplastic syndrome). Patients' follow-up ranged between 13 and 25 months. In 13 patients in stable remission, CD4 + iNKT cells appear first, followed by the CD4- ones. Both iNKT cell subsets emerge displaying an immature (CD161-) phenotype and undergo independent phenotypic and effector maturation programs, characterized by markedly faster acquisition of the NK-maturation marker CD161 and the ability to produce IFN-g in the CD4- one.

In 8 patients relapsing after haplo-HSCT (median: 5.5; range 3-18 months), we documented a profound depletion of iNKT cells, during all observation time. In order to correlate iNKT reconstitution with relapse, we compared the temporal curves describing the kinetic of iNKT reconstitution in each patient. The statistical analysis indicated a strong difference $(P=0.001)$ between the kinetic of iNKT reconstitution observed in relapsing patients and that of patients in remission (Figure). No correlation was found between iNKT cell reconstitution and infections, or graft-versus-host disease. Analysis performed in the bone marrow confirmed lack of iNKT cells in one patient who experienced leukemia relapse, and their emergence in a patient in remission. These findings define new aspects of human iNKT cell development and suggest a contribution of these cells to leukemia control, possibly via early IFN-g provision. Future strategies of adoptive infusion of donor-derived iNKT cells can be considered in patients with hematological malignancy receiving Haplo-HSCT showing an inability to reconstitute the iNKT cell compartment.
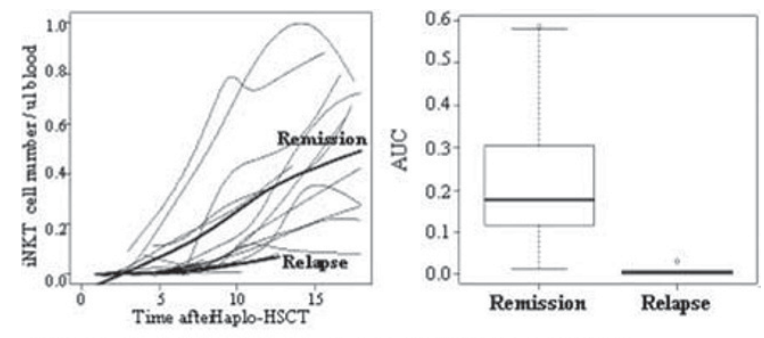

INKT cell emporal reconstitution curves are statistically different behveen remissio and leule mia relapsed patients. A. Temporal curves of INKT coll recorstitution post Heplomears of temporal curves of ANKT cell reconetitution in remission and bukemis relapeed patients, repectively. B. The Area Under esch termporal Curve (AUC) was calculated and the AUC values for patients in remission and patients with relupee are reported The AUC values for pationts in remisgion are statistically different from those for relapsed patients as cakculated

\section{Infectious complications}

\section{P714}

A 12-week course of oseltamivir prophylaxis is well tolerated by haematopoietic stem cell transplant recipients M.Y. Shapira (1), M.G. Ison (2), P. Szakaly (3), G. Krivan (4), A. Nist (5), R. Dutkowski (5)

(1)Hadassah - Hebrew University Medical Center (Jerusalem, IL); (2)Northwestern University (Chicago, US); (3)Medical University of Pécs (Pecs, HU); (4)Szent László Hospital (Budapest, HU); (5)Hoffmann-La Roche Inc. (Nutley, US)

Objectives: Haematopoietic stem cell transplant (HSCT) recipients are at increased risk for severe or complicated influenza and may not develop protective antibody responses after influenza vaccination. Antiviral prophylaxis could be a viable alternative to vaccination in these patients. The safety, tolerability and protective efficacy of a 12-week course of oseltamivir prophylaxis was studied in 477 solid organ transplant (SOT) and HSCT recipients. Here, we present safety and tolerability data from the subset of HSCT patients.

Methods: SOT and HSCT recipients $\geq 1$ year who were influenza negative by rapid test and without signs or symptoms of influenza at baseline were enrolled during periods of local influenza activity. Participants were randomised to oseltamivir $(75 \mathrm{mg}$ for adults and adolescents $\geq 13$ years or weight-based unit doses for children 1-12 years) or matching placebo once daily for 12 consecutive weeks as capsule or suspension. Safety was assessed by physical and laboratory tests and adverse event (AE) reporting.

Results: Eighty-seven (19\%) of the 477 subjects enrolled were HSCT recipients (43 on placebo and 44 on oseltamivir). Most were male $(74 \%)$ and unvaccinated against influenza $(70 \%)$; mean age was 37.5 years. The median time since transplantation was 367 days in the oseltamivir arm and 424 days in the placebo arm. Serious AEs were more common in the placebo arm than in the oseltamivir arm (12\% vs. $2 \%$, respectively), while AEs were reported with a similar overall frequency $(63 \%$ vs. $59 \%$, respectively). The most common on-treatment AEs were infections (30\% vs. $27 \%$, respectively, including one case of influenza in a placebo recipient), gastrointestinal disorders (23\% in both groups) and respiratory, thoracic and mediastinal disorders $(19 \%$ vs. $11 \%$, respectively). The incidence of withdrawal due to AEs was higher in the placebo arm than in the oseltamivir arm ( $9 \%$ vs. $2 \%$, respectively). Graft-versushost disease developed in four patients in each group. Three patients (two on oseltamivir on one on placebo) had grade $3 / 4$ shifts in laboratory parameters from baseline. One patient in the placebo arm died while off treatment due to recurrent acute myeloid leukaemia. Limited patient numbers prevented meaningful evaluation of efficacy. 
Conclusions: A 12-week course of oseltamivir prophylaxis was well tolerated by HSCT patients, with a similar safety and tolerability profile to placebo.

\section{P715}

Clinical features and outcome of influenza A (H1N1) virus infection in onco-haematology patients. Single-unit experience

C. Castilla-LLorente, J. Nieto, I. Heras, F. de Arriba, R. Cesteros, M. Amigo, E. Pérez-Ceballos, V. Vicente

Hospital Morales Meseguer (Murcia, ES)

Introduction: In April 2009, an outbreak of H1N1 influenza A virus infection was detected in Mexico and on June 11, the World Health Organization raised its pandemic alert level to the highest level. Immunocompromised patients have been described as one of the main groups at risk of this infectious disease especially on the most severe forms.

Objective: To describe the clinical characteristics and outcome of patients who develop H1N1 virus infection.

Patients and methods: Prospective observational study on patients followed on our Department from October to December 2009. Respiratory swab from all patient referring respiratory symptoms was obtained and Real Time Reverse Transcriptase Polymerase Chain Reaction (RT-PCR) testing for H1N1 influenza A was performed. Patients were closely monitored for clinical and radiological features.

Results: 44 patients were confirmed positive for the H1N1 Influenza A virus infection. Median age was 43 years and $57 \%$ were male. 20 patients $(45 \%)$ had undergone an allogeneic stem cell transplant (alloSCT) and 5 patients $(11 \%)$ an autoSCT. The median incubation period of the virus was 1 day (range, 1/2-5). The most common symptoms were cough $(86 \%)$ and fever $(84 \%)$ followed by runny nose $(68 \%)$ and sore throat $(31 \%)$. Head and body ache were less common ( 9 and $15 \%$ respectively), only 1 patient presented diarrhea. Dyspnea was reported by 3 patients $(7 \%)$ but only $1(2 \%)$ needed mechanical ventilation (MV). Most patients (93\%) had an X-ray performed at onset of symptoms buy only on 3 patients significant changes were present. CT scan was performed on 9 patients, 1 non SCT, 1 auto and 7 alloSCT patients. On $5(56 \%)$ of this patients viral neumonia was identified even without presenting lower respiratory tract symptoms, all except 1 were alloSCT patients and only the non SCT patient had respiratory failure and needed MV. All patients except 1 received Oseltamivir therapy. 38 at standard dose of $75 \mathrm{mg}$ bid for 5 days and 5 , those who presented neumonia on CT scan at $150 \mathrm{mg}$ bid. $96 \%$ of patients received antimicrobial concomitant therapy. All patients completed therapy and are alive with complete remission of flu symptoms.

Conclusion: The Influenza A in Oncohematology patients can cause a severe and fatal syndrome but in most patients behaves as a mild illness. The early use of oseltamivir may determine the mildness and good outcome of the infection in our series. The use of CT scan may be considered on alloSCT patients to rule out neumonia.

\section{P716}

Pattern recognition of the entire human Cytomegalovirus proteome after HSCT defined by high-content peptide microarray chip analysis

L. Pérez-Bercoff (1), Y. Ngo (2), S. Gaseitsiwe (1), D. Valentini (1), S. Mahdavifar (2), M. Schutkowski (3), P. Ljungman (1), M. Maeurer (1)

(1)Karolinska Institutet (Stockholm, SE); (2)Smittskyddsinstitutet (Stockholm, SE); (3)JPT (Berlin, DE)

Method: We used high-content peptide microarray slides containing 17174,15 mer peptides overlapping by 4 aa covering 214 proteins derived from CMV (193 from AD169, 19 from Toledo and three from the Towne strain). Antibody profiling was carried out on serum samples collected from 20 HSCT pts at 6 ,
12 and 24 months after HSCT. The patients were divided into 4 different groups based on the CMV serological status of donor and recipient: $D-R-, D-R+, D+R-$ and $D+R+$. After quality data extraction and normalization we used three different statistical approaches for each individual group and time point: (i) in- and exclusive analysis to find peptides recognized in individuals in one group but never present in any individual in the control group (D-R-), (ii) PAM (Prediction Analysis for Microarray) to find epitopes differentially recognized between groups and (iii) a 3D-visualization of the breadth and profile of CMV epitope recognition.

Results: 330 peptides, defined by PAM, were differentially detected in the 4 serological groups. Epitope recognition was clustered on individual proteins. The most strongly recognized peptides, differentiating $\mathrm{D}-\mathrm{R}-\mathrm{vs}$. $\mathrm{D}+\mathrm{R}+$ at 6 months were from pp28, UL102, and UL48. In contrast, at month 24, these epitopes were no longer differentially recognized, while epitopes from gB, TRS1, IE1, gO and IE2, pp65 and pp150 among others instead separated the groups.

Conclusions: High-content peptide microarrays allow epitope profiling of entire viral proteomes with minimal amounts of serum and describe common and private CMV specific humoral recognition patterns. This is helpful to describe the immune focus of anti-CMV specific B-cell responses. The humoral immune response against CMV is clearly dependent on pretransplant defined serological groups. Comparisons over time visualize the evolution of CMV epitope focus which, most likely, reflects the B-cell reactivity pattern of the transplanted donor immune system.

\section{P717}

H1N1 following transplantation

J. Schriber, G. Sulski, V. Fauble, L. Sproat, J.A. Tart, R. Chiffelle, C. Boeckman, A. Briggs

Banner Blood and Marrow Transplant Program (Phoenix, US)

Influenza is a common issue that transplant units deal with yearly and routine vaccination is recommended. This year a potentially more virulent strain $\mathrm{H} 1 \mathrm{~N} 1$, not covered by the standard vaccine has been cause for concern. We now report our experience to date with $\mathrm{H} 1 \mathrm{~N} 1$.

The majority of patients who have undergone allogeneic transplantation at Banner Blood and Marrow Transplant Program have active follow up with our center rather than the referring physician. Those who develop flu like symptoms would typically present to our clinic for evaluation and treatment.

We now report 9 patients age range 17-62 years (median 49) who developed $\mathrm{H} 1 \mathrm{~N} 1$ following transplant prior to vaccination. Eight developed disease following allogeneic (5 MUD, 3 Sibling donors) and 1 following autologous transplantation. Time to presentation ranged from 41 (autologous) to 2700 days post transplant with a median of 710 days post transplant. All but two of the allogeneic patients presented beyond 600 days post transplant. Two allogeneic patients and the autologous patient were not on immunosuppressants. Five patients were on steroids, 4 on a calcineurin inhibitor and 2 on additional therapy for GVHD. Patients presented with rhinorrhea, cough, fever and aches. All received Tamiflu at 75 bid for at least five days. In 4 the symptoms were mild and they received treatment as outpatients. Five required hospital admission, however 3 were in hospital for less than 4 days. Two patients had longer hospital stays and required mechanical ventilation. Both were admitted following what appeared to be the initial resolution of symptoms, approximately 2 weeks from the initial diagnosis. One had confirmed reinfection resistant to Tamiflu and died from progressive pulmonary failure. The other had no etiology for their pulmonary failure and is currently improving with aggressive supportive care.

Patients who undergo allogeneic transplantation are at high risk for infection with H1N1. Most appear to have a benign clinical disease but in our small series, $25 \%$ had an aggressive course after what appears to be a reinfection. All patients with URTI 
symptoms should be offered therapy while awaiting the results of testing. Those who are positive require aggressive supportive care and monitoring but it appears that the majority will survive the infection. With the late reinfections seen, patients should be cautioned to notify the transplant center if they develop additional flulike symptoms.

\section{P718}

Norovirus infections in patients after stem cell transplantation: epidemiological and clinical aspects P. Ljungman, J. Mattsson, J. Winiarski, E. Sparrelid, O. Ringdén Karolinska University Hospital (Stockholm, SE)

Background and aim: Norovirus infections have become a major practical clinical problem during the last few years causing outbreaks in hospitals infecting both patients and staff These infections have also been associated with prolonged virus excretion in renal transplant recipients and a risk of mortality in the elderly. Little is known about the clinical impact of norovirus infections on patients who are severely immunosuppressed such as HSCT recipients although a report at the 2009 EBMT meeting suggested prolonged excretion and long-term clinical symptoms in a small series of HSCT patients. The aim of this study was to analyze the impact of norovirus infections in patients after hematopoietic stem cell transplantation (HSCT) Methods: The laboratory records from the Clinical Microbiological Laboratory at Karolinska University Hospital were examined in order to identify HSCT patients with proven norovirus infections diagnosed from 2006 to 2009 . The diagnostic methodology was based on PCR. After identification of cases, the patient charts were reviewed to assess outcome, possible norovirus associated clinical complications, and delay of other therapy. The duration of virus excretion was defined as the time from the first to the last positive sample.

Results: 29 patients were identified; 27 had undergone allogeneic and 2 autologous HSCT. The median age was 34.8 ys $(0.9$ 63). 16 of the detected viruses were typed to genogroup 2, 3 to genogroup 1 , and 10 were not typed. The median duration of viral detection in the entire cohort was 4 days (1-216 days). Four patients have currently ongoing infections. $13 / 29(45 \%)$ patients were PCR positive more than one week, $11(38 \%)$ for more than two weeks, and $6(21 \%)$ for more than four weeks. The majority of patients had minor and quickly resolved gastrointestinal symptoms while two patients had repeated epsiodes with gastrointestinal symtoms. Three patients died while norovirus positive. One patient had repeated hospitalizations due to diarrhoea and other infections, one patient died early after HSCT due to multiple complications including dehydration and electrolyte disturbances, while one patient died from a bacterial infection. One patient had a delay in a planned allogeneic HSCT.

Conclusions: Norovirus infections are important problems in the clinical management of HSCT patients with many patients having prolonged virus excretion although usually not resulting in severe clinical consequences.

\section{P719}

BK virus load in whole blood as a virological marker of late-onset haemorrhagic cystitis in allogeneic haematopoietic stem cell transplant recipients

J. Corneille, N. Dhédin, C. Deback, A. Boudifa, M. Uzunov, J.P. Vernant, H. Agut, D. Boutolleau

Groupe Hospitalier Pitie-Salpetriere (Paris, FR)

Objectives: $\mathrm{BK}$ virus (BKV) infection is associated with late-onset hemorrhagic cystitis $(\mathrm{HC})$ in hematopoietic stem cell transplant (HSCT) recipients. Nevertheless, no virological marker of lateonset $\mathrm{HC}$ has been clearly identified. The aim of this longitudinal study was to determine the incidence and the risk factors of BKV infection in HSCT recipients, to analyse the association of BKV load in whole blood with $\mathrm{HC}$, and to evaluate whether systematic follow-up of BKV infection is warranted.
Methods: BKV load was measured retrospectively using a real-time PCR assay in whole blood specimens from 61 consecutive allogeneic HSCT recipients (38 men, 23 women, median age: 45 years) collected at $0,1,2,3,6,9$, and 12 months post-transplantation.

Results: Fifteen patients (25\%) developed BKV viremia at a median of 2 months after transplantation. The occurrence of BKV infection was associated with acute leukaemia as underlying disease $(P=.03)$ and total body irradiation $(P=.06)$, but not with donor HLA C7 allele. Post-HSCT BKV infection was associated with the occurrence of acute graft-versus-host disease $(P=.003)$ and hematuria $(P<.001)$, but not with renal impairment. The maximum BKV load was higher in patients with $\mathrm{HC}$ than in those without HC (median, 1685 vs. 160 copies $/ \mathrm{mL}$; $P=.05$ ). The development of $\mathrm{HC}$ was statistically associated with a concomitant BKV load $>500$ copies $/ \mathrm{mL}(P=.04)$ and with BKV detection in at least two consecutive blood specimens $(P=.04)$. Lymphocyte recovery in patients with $\mathrm{HC}$ was delayed compared to those without $\mathrm{HC}$. Ten patients experienced both BKV and cytomegalovirus (CMV) infections after transplantation. In all cases, CMV infection appeared either before $(n=5)$ or concomitantly $(n=5)$ with BKV infection. The frequency of BKV detection was statistically higher in CMV-positive than in CMV-negative samples (22\% vs. $5 \%$; $P<.001)$. No adenovirus infection was evidenced in patients with hematuria.

Conclusion: BKV viremia is highly associated with late-onset $\mathrm{HC}$ in HSCT recipients. Prolonged BKV viremia and BKV load over 500 copies $/ \mathrm{mL}$ of whole blood were identified as predictive markers for the development of $\mathrm{HC}$. The analysis of BKV load kinetics and lymphocyte recovery after transplantation suggests the potential role of cellular immunity in the pathogenesis of $\mathrm{HC}$. Our results underline a possible role of CMV in the process of BKV infection. The monitoring of BKV viremia appears useful to identify HSCT recipients at risk for developing late-onset $\mathrm{HC}$.

\section{P720}

Galactomannan-driven diagnostic strategy of invasive aspergillosis in patients with graft-versus-host disease after allogeneic haematopoietic stem cell transplantation - good correlation between serum and bronchoalveolar lavage galactomannan testing L. Gil (1), A. Mol (1), A. Czyz (1), A. Lojko-Dankowska (1), A. Nowicki (1), M. Matuszak (1), J. Styczynski (2), M. Komarnicki (1)

(1)University of Medical Sciences (Poznan, PL); (2)Collegium Medicum, Nicolaus Copernicus University (Bydgoszcz, PL)

Invasive aspergillosis (IA) is a serious complication in pts with graft versus host disease (GVHD) after allogeneic SCT and the outcome of this condition depends on early diagnosis and treatment initiation. Current diagnostic strategy is however tailored mainly for neutropenic patients. Aim of the study was to asses the efficacy of galactomannan (GM)-based diagnostic strategy in SCT recipients after granulocyte recovery and treated because of GVHD.

Material and methods: 81 pts (median age 34; range 15-67) undergoing alloSCT because of hematologic diseases (AML/ MDS 41, CML/MPD 14, NHL/HD 13, ALL 9, SAA 4) were prospectively analyzed. Pts were transplanted with PB (55) or BM (26) stem cells from related (59) or unrelated donor (22). After hematologic recovery all pts were monitored with onceweekly serum GM test up to 100 days or longer in case of GVHD. High resolution chest computed tomography (HRCT) and bronchoscopy with lavage (BAL) were performed in case of positive serum GM test ( $\geq 0.5$ in two consecutive samples), persistent fever or pulmonary infiltrates. GM test was additionally performed in BAL with a result $\geq 0.5$ regarded as positive. The diagnosis of IA was made according to EORTC/MSG criteria. Results: Documented IA was diagnosed in $16(19.8 \%)$ patients (2 proven, 14 probable) and possible in $13(16 \%)$. Median time to diagnosis of documented IA was 76d ( $r$; 23-515). All but one cases of IA were diagnosed in patients with GVHD 
grade $2-4: 15 / 37(40.5 \%)$ vs. $1 / 44(2.3 \%), P=0.00005$, $\mathrm{OR}=29$. $\mathrm{GM}$ serum test was positive in all $16 \mathrm{pts}$ and correlated with GM in BAL detected in 15 pts $(r=0.8, P<0.001)$. HRCT revealed typical changes in 10 pts; endobronchial biopsy confirmed IA (A. fumigatus) in 2 pts. The cumulative risk of development IA was $0.25 \pm 0.05$ at $1 \mathrm{yr}$. All patients with documented IA were treated with voriconazole. In 12 weeks follow-up $8(50 \%)$ patients died because of IA. Probability of $1 \mathrm{yr}$ survival after IA was $0.36 \pm 0.12$, while among non-IA patients $0.69 \pm 0.06(P=0.001)$. Overall risk of death due to IA was $0.124 \pm 0.042$ at $1 \mathrm{yr}$. The mean survival after IA occurrence was $407 \mathrm{~d}(95 \% \mathrm{Cl}=193-621 \mathrm{~d})$.

Conclusions: In alloSCT setting, IA frequently occurs after neutrophil recovery in patients treated because of GVHD. Strategy based on GM screening, HRCT and bronchoscopy allows early diagnosis of IA and early preemptive or targeted therapy in this group of pts, leading to survival in to $50 \%$ of patients with IA. $\mathrm{GM}$ in BAL correlates with GM serum testing.

\section{P721}

Transfer of EBV-specific T-cells: a treatment option for patients with EBV-associated post-transplant lymphoproliferative disease after allogeneic stem cell transplantation J. Tischer (1), A. Moosmann (2), I. Bigalke (3), D. Prevalsek (1), S. Fritsch (1), F. Mumm (1), A. Rank (1), B. Heilmeier (1), M. Bergmann (1), W. Hill (1), A. Hausmann (1), G. Ledderose (1), H.J. Kolb (1)

(1)University of Munich (Munich, DE); (2)Helmholtz Zentrum and Ludwig-Maximilians-University (Munich, DE); (3)Helmholtz Zentrum Munich (Munich, DE)

Epstein-Barr virus (EBV) associated posttransplant lymphoproliferative disease (PTLD) is a rare but severe complication within the first year after allogeneic hematopoetic stem cell transplantation with a high mortality rate. HLA differences, T-cell depletion of the graft, use of antithymocyte globulin and total body irradiation are major risk factors for the development of this rapidly progressive disease. Based on molecular monitoring of the viral load in peripheral blood samples preemptive or prompt treatment including antiviral agents, reduction or cessation of immunosuppression, administration of anti-CD20 monoclonal antibodies and adoptive immunotherapy are important treatment strategies for patients (pts) at risk.

For the treatment of patients with PTLD we developed a rapid overnight procedure for the isolation of EBV specific T-cells by stimulation of donor peripheral blood cells with a pool of EBV derived peptides, interferon gamma based cytokine capture technology and immunomagnetic separation.

6 pts with histologically proven PTLD received one single transfusion of a small number of EBV specific T-cells. In 5 pts (1 pt with SAA, 3 pts with AML and 1 pt with high grade NHL) a haploidentical transplantation including transfer of CD6 depleted stems cells on day +6 and in $1 \mathrm{pt}$ a matched unrelated stem cell transplantation had been performed previously. Clinical symptoms at time of $\mathrm{T}$ cell transfer included fever and lymphadenopathy in all 6 pts plus tonsillitis in 1 pt. 3 pts suffered already from multi organ failure (MOF) including hepatitis, pneumonitis and consecutive respiratory, renal, liver, cardiac and hemodynamic failure. In 2 pts leucaemic spread of lymphoma cells was observed. Immunosuppression was stopped in all pts. All pts had at least one course of treatment with cidofovir and rituximab before and had - despite of this treatment - clinical signs of progressive PTLD. No evaluable response was seen in 3 pts who suffered from late stage PTLD with MOF. In 3 pts with PTLD without MOF we observed a complete and stable remission of PTLD. 2 pts have remained free of EBV disease for more than two years now and show a stable reconstitution of a protective and functional EBV specific T-cell memory. No graftversus-host-disease was seen.

Transfer of directly isolated EBV specific T-cells is a rapid, safe and valuable treatment option for patients with PTLD without MOF after hematopoetic stem cell transplantation.
P722

HHV6-associated limbic encephalitis after reduced-intensity cord blood transplantation

K. Ishiwata, D. Kato, N. Nakano, M. Tsuji, H. Yamamoto, Y. Asano-Mori, N. Uchida, A. Wake, S. Taniguchi

Toranomon Hospital (Tokyo, JP)

Background: HHV6 reactivation has been increasingly recognized particularly in cord blood transplantation (CBT). HHV6 has been associated with limbic encephalitis (HHV6-LE), which causes anterograde amnesia and epilepsy. The CNS disorder confined to the hippocampal regions; the presence of abnormal MRI signals involving the hippocampus, and detection of HHV6 DNA in CSF. However, the incidence and risk factors for HHV6-LE in adult patients receiving CBT with reduced-intensity conditioning have not been established so far.

Methods: In this study we retrospectively reviewed the medical records of $362 \mathrm{CBT}$ recipients with reduced-intensity conditioning between January 2002 and December 2007 at Toranomon Hospital in Japan to determine the incidence of HHV6-LE among these patients. HHV6-LE was defined as: the presence of neurological symptoms; positive PCR results for HHV6 in CSF; and the absence of other identified etiologies of encephalitis. All the patients who manifested suspicious symptoms of HHV6-LE were treated with foscarnet.

Results: HHV6-LE was diagnosed in $25 / 362$ for a cumulative incidence of $7.3 \%$. The median age was 49 years (range 20 68 ). Median symptom onset was on day +19 (range 12-50). Initial symptoms included confusion in 19, retrograde amnesia in 11, and seizures in 5. Median HHV6 DNA levels in the CSF was 20000 (range, 200-500000) copies/ml. Median HHV6 DNA levels in peripheral blood was 1000 (range 0-300000) copies/ $\mathrm{ml}$. Remarkably, 4 patients were negative for HHV6 in peripheral blood even within 2 days prior to the onset of CNS symptoms. MRI showed abnormal high signal intensity on flair and T2 weighted images in the hippocampus and mesial temporal lobes in $70 \%$ of the patients. All were treated with foscarnet at $50-150 \mathrm{mg} / \mathrm{kg}$ of body weight, and clinical symptoms improved in 19 evaluable patients. The virus was cleared in the CSF successfully in 14 patients evaluated. Eighteen died with the median survival of 35 days (range, 5-978) from diagnosis of HHV6-LE. HHV6-LE was not a direct cause of death in any of them. None of the factors analyzed failed to reach statistical significance regarding the impact on the onset of HHV6-LE.

Conclusions: High incidence of HHV6-LE as well as reactivation of the virus was observed in CB recipients. HHV6 DNA monitoring in peripheral blood may not be always sufficient to predict the development of HHV6-LE. Although clinical manifestations were severe in some cases, foscarnet therapy was effective in all evaluable cases.

\section{P723}

Voriconazole or itraconazole for antifungal prophylaxis in patients with grade II-IV acute or extensive chronic graft-versus-host disease

D. Marks, C. Kibbler, G. Cook, A. Pagliuca, A. Reisman, P. Miller, M. Kantecki, H. Schlamm on behalf of the IMPROVIT Study Group

Objective: Antifungal prophylaxis can reduce the incidence of invasive fungal infections (IFI) in patients with acute or chronic graft-vs-host disease (GvHD) after allogeneic hematopoietic cell transplant (alloHCT). We compared outcomes in patients developing grade II-IV acute or extensive chronic GvHD in a study comparing oral voriconazole (VOR) to oral itraconazole suspension (ITR) for primary prophylaxis after alloHCT (the IMPROVIT study).

Methods: We identified study patients who developed Grade II or higher acute GvHD or chronic extensive GvHD at any time during the first 180 days after transplant. We compared baseline patient characteristics, time to first report of GvHD, and time to discontinuation of study drug in patients receiving VOR 
vs. ITR as primary prophylaxis. Distributions by treatment of categorical variables were compared using Fisher's Exact test, distributions of time to event (GvHD or duration on study drug) were compared using Kaplan-Meier estimates. We compared success of prophylaxis at days 100 and 180 using a composite endpoint that incorporated ability to tolerate study drug for at least 86 days, absence of proven or probable invasive fungal infections and survival to day 180 .

Results: 126/489 (25.8\%) patients developed grade II-IV acute GvHD and/or chronic extensive GvHD during the study: 62 received VOR, 64 received ITR. Other than a higher proportion with acute lymphocytic leukemia in the VOR arm (24.2\% vs. $9.4 \% ; P=0.032$ ), there were no significant differences in baseline characteristics between the 2 study arms. Mean time to first report of GvHD was 49.6 for VOR vs. 37.6 days for ITR $(P=0.067)$. Success of prophylaxis was $61.3 \%$ vs. $43.8 \%(P=0.053)$ at day 100 , and $50.0 \%$ vs. $29.7 \%(P=0.029)$ at day 180 after alloHCT for VOR and ITR respectively. Mean duration on study drug was 106.6 vs. 84.4 days (log rank $P=0.009$ ) and proportion completing at least 86 days of study drug was $59.7 \%$ vs. $42.2 \%(P=0.053)$ for VOR and ITR, respectively. Incidence of proven or probable IFI was low $(1.6 \%)$ for both study treatments (1 probable pulmonary aspergillosis in each arm); neither IFI was considered treatment-emergent. Survival at day 180 was $80.6 \%$ vs. $76.6 \%$ (VOR vs. ITR, $P=$ NS).

Conclusion: In patients with grade II-IV acute or chronic extensive GvHD following alloHCT, both VOR and ITR were effective in preventing IFI, but VOR was tolerated for a significantly longer mean duration.

\section{P724}

Immuno-dominance of different CMV-epitopes in patients after HSCT: monitoring antiviral response with tetramers S. Borchers, S. Luther, U. Lips, E. Dammann, M. Stadler, H. Diedrich, A. Ganser, E.M. Weissinger

Hannover Medical School (Hannover, DE)

Reactivation of cytomegalovirus (CMV) in patients after allogeneic hematopoeitic stem cell transplantation (HSCT) remains one of the major complications post-HSCT. The kinetics of CMV-immune-reconstitution are not fully understood, i.e. the time point of immune-reconstitution and the immunodominance of certain epitopes remain unclear. Monitoring the immune reconstitution for CMV using tetramers with epitopes from different immunogeneic viral proteins may help to understand the process more clearly. Here, we present data on 69 patients screened with at least 2 tetramers containing epitopes from either immediate early-1 (IE-1; $B^{*} 0801$ tetramer) or pp50 ( $A^{*} 0101$ tetramer) or pp65 $\left(A^{*} 0201, A^{*} 2402, B^{*} 0702\right.$ and $B^{*} 3501$ tetramers), respectively. Forty-six patients were screened with 2 tetramers $(n=46 ; 67 \%), 22(32 \%)$ had 3 matching tetramers, while 1 patient could be screened with 4 . The combination of IE-1- and pp50-tetramers was used in 23 patients (33\%), pp65 and pp50 in $18(26 \%)$ and pp65 and IE-1 in 11 patients (16\%). The combination of all 3 tetramers was achieved in 17 patients (25\%). Applying screening with these tetramer combinations, we found differences in patient responses within this group: in 10 patients CMV-CTL responded to the pp65-peptide, in another 10 mainly to pp50, while in 8 the IE-1-tetramer seemed to be dominant. $23(33 \%)$ patients had at least one CMV-reactivation. In 9 patients a clear switch of immune-dominance could be shown by tetramer screening. Interestingly, while CMV-reactivation correlated with proliferation of the CMV-CTL recognizing the dominant epitope, the switch of immune-dominance did not correlate with episodes of CMV-reactivations, but followed different time lines. Seven of the 9 patients experienced CMVreactivations, and reactivation and switch occurred at different time points in 6 , suggesting that rather engraftment of the donor immune system influenced immune dominance switching. In 2 patients switch of immune-dominance was associated with the onset of graft versus host disease (GvHD).
Dominance of IE-1, pp65 and pp50 seemed to be equally distributed in this cohort. Using screening with tetramers may give further insight in the mechanisms involved in donor-recipient immune-reconstitution post-HSCT. We shall further analyze the correlation of immuno-dominance switch with CMV-reactivation patterns, the effects of different CMV-strains, onset of acute or chronic GvHD and possible influences of donor leukocyte transfusions.

\section{P725}

Epidemiology of invasive fungal diseases in haematopoietic stem cell transplant recipients: preliminary analysis of a multicentre, prospective, observational study from Italy C. Girmenia, S. Perrone, R. Ricci, A. Algarotti, A. Busca, A. Bosi, P. Chiusolo, G. Irrera, E. Castagnola, L. Cudillo, B. Montante, A. Locasciulli on behalf of the Gruppo Italiano Trapianto di Midollo Osseo (GITMO)

Objectives: The purpose of our study was to describe the current epidemiology, incidence, risk factors and current diagnostic strategies for invasive fungal diseases (IFDs) in allogeneic hematopoietic stem cell transplant (HSCT).

Methods: This prospective, observational, multicenter study involved 30 HSCT Centers from Italy, started in January 2008 and is ongoing. Each Center reported data on consecutive patients who underwent HSCT and data were collected until 1 year from transplant. The data collection is ongoing and preliminary data are now reported.

Results: To date, the baseline characteristics are available for 691 patients. Of them, 71 patients $(10.3 \%)$ had experienced a proven/probable IFD before HSCT. They were 53 (74.6\%) invasive aspergillosis (IA), 12 invasive candidosis (IC) and 6 IFDs by other fungi. Out of 515 patients evaluable at 100 days from HSCT, $53(10.3 \%)$ developed a proven/probable IFD (IA, 46 cases; IC 5 cases; other IFDs, 2 cases). Out of 348 patients evaluable for the follow-up from 100 to 180 days from HSCT, 15 (4.3\%) developed an IFD. Finally, 5 IFDs (2.6\%) were documented among 190 patients evaluable for the follow-up from 180 to 365 days. Overall, according to these preliminary data, the incidence of IFDs at 1 year from HSCT seems higher than $15 \%$. According to the data until now available at least $15 \%$ of patients with an IA diagnosed before HSCT experienced an infection recurrence within 100 days after transplant. Out of 58 cases of proven/probable IA documented after HSCT serum detection of Aspergillus galactomannan antigen contributed to the diagnosis in 45 cases $(77.6 \%)$. At a preliminary analysis, the 3 months mortality rate for HSCT recipients with an IFD was lower than $30 \%$.

Conclusion: This is a prospective study on the "real life" epidemiology of IFDs in allogeneic HSCT population. The available data show that IA remains the most commonly identified IFD and rates of survival appear to have improved, compared with historical literature data.

\section{P726}

IL-7 expression and responsiveness in EBV infection and EBV-associated PTLD in allogeneic HSCT patients

H. Omar, R. Ahmed, L. Rane, A. Björklund, A. GustafssonJernberg, P. Ljungman, M. Maeurer

Karolinska Institutet (Stockholm, SE)

IL-7 is a non-redundant growth- and differentiation factor for $\mathrm{B}-$ and $\mathrm{T}$ - cells. It is essential in early B lymphocyte development and in survival/immune memory formation in T-lymphocytes. IL-7 was found to be expressed in EBV+B cell lines which may impact on B-cell survival and/or B-cell proliferation. Lack of IL-7 responsiveness in T-cells may be associated with reduced cellular immune surveillance. IL-7 may i) contribute to malignant progression of EBV-infected B lymphoblasts in transplanted patients and ii) be used to gauge immune responsiveness in cellular immune responses. This may provide a basis to develop new tools to monitor for early indicators for of the development of PTLD. 
Aim: To study the biological functions of IL-7 and IL-7R in peripheral blood mononuclear cells (PBMCs) and in serum from patients with EBV-PTLD.

Patients and methods: PBMCs from 6 patients with PTLD, harvested at different time points after SCT and in control patients (with EBV + after SCT) were used to test for i) IL-7-induced STAT5 phosphorylation in CD4 + and CD8 + T-cells (functional readout for (L-7), ii) EBV specific $T$ cells by tetramer analysis and iii) expression of IL-7R on B cell subsets. Serum IL-7 and soluble IL-7R concentrations were measured by ELISA.

Results: CD8 + T-cells show decreased IL-7 responsiveness both in PTLD + and PTLD- individuals defined by STAT5 phosphorylation. In contrast, CD4 + T-cells from PTLD patients, but not from $(E B V+)$, PTLD- individuals show a markedly reduced capacity to respond to IL-7. PBMCs from patients with PTLD and control individuals exhibited EBV specific $T$ cells directed against both latent and Iytic EBV targets. IL-7R was not expressed on B-cells obtained from the control patients, but on on B-cells from patients with PTLD.

Conclusion: EBV-specific CD8 + T-cells with a reduced capacity to respond to IL-7 are present in both PTLD + and PTLDindividuals. In contrast, IL-7R expression on B cells as well as reduced responsiveness to IL-7 in CD4 + T-cells could exclusively be detected in PTLD + patients. This immunological profile may aid to monitor patients after SCT for EBV-associated PTLD.

\section{P727}

AmBisome ${ }^{\circledR}$ as antifungal primary prophylaxis in patients undergoing allogeneic stem cell transplantation from alternative donor: preliminary results of phase II "Prophessor" trial

F. Ciceri (1), A. Bacigalupo (2), M. Bregni (1), A. Busca (3), C. Giardini (4), R. Scimè (5), G. Visani (4), R.L. Balzano (6), L.A. Picaro (6), F. Aversa (7)

(1)San Raffaele Scientific Institute (Milan, IT); (2)San Martino Hospital (Genoa, IT); (3)Le Molinette Hospital (Turin, IT); (4)San Salvatore Hospital (Pesaro, IT); (5)Cervello Hospital (Palermo, IT); (6) Gilead Sciences srl (Milan, IT); (7)University of Perugia (Perugia, IT)

Background: Patients (Pts) undergoing allogeneic stem cell transplantation (allo-SCT) are susceptible to invasive fungal infections (IFI), which greatly increase morbidity and mortality. Methods: In this phase II, multicenter, non-randomized study, we evaluated the safety and tolerability of liposomal amphotericin $\mathrm{B}$ as antifungal primary prophylaxis for Pts undergoing alloSCT from alternative donors. The Pts received AmBisome ${ }^{\circledR}$ $3 \mathrm{mg} / \mathrm{kg}$ three times a week from day before transplantation to day +22 (total 8 doses), and $7 \mathrm{mg} / \mathrm{kg}$ weekly from the day 29 to the 8th week (total 4 doses); in case of graft versus host disease (GvHD) requiring immunosuppressive therapy additional $7 \mathrm{mg} / \mathrm{kg}$ weekly doses were administered until week 16 . The efficacy and the incidence of proven or probable IFI during treatment were secondary endpoints.

Results: From August 2007 to December 2008, a total of 34 Pts (mean age 44.9 years, range 20-67; 18 males) were enrolled in the study. $13 \mathrm{Pts}(38.2 \%)$ were affected by acute myeloid leukemia, 9 Pts $(26.5 \%)$ by acute lymphoblastic leukemia and $12 \mathrm{Pts}$ $(35.2 \%)$ by other hematological malignancies; 13 Pts $(38.2 \%)$ were in complete remission, $8 \mathrm{Pts}(23.5 \%)$ in progressive disease; $20 \mathrm{Pts}$ underwent matched unrelated donor (MUD) and 14 haploidentical allo-SCT; 23 Pts $(67.6 \%)$ received a myeloablative conditioning regimen. All Pts received the $3 \mathrm{mg} / \mathrm{kg}$ regimen, while 23/34 Pts continued with $7 \mathrm{mg} / \mathrm{kg}$, with a mean of 7 (range 1-8) and 3 (range 1-4) administration respectively; 1 patient received 7 additional doses for the occurrence of GvHD. No nephrotoxicity was observed in 22 Pts (64.7\%) receiving $3 \mathrm{mg} / \mathrm{kg}$ and in $13(54.2 \%)$ receiving $7 \mathrm{mg} / \mathrm{kg}$; in the 7 $\mathrm{mg} / \mathrm{kg}$ group, 6 Pts (25\%) experienced grade 2 nephrotoxicity, while no grade 3 or 4 toxicity was reported. Hypokalemia was predominantly observed as grade 1 toxicity: 19 Pts (55.9\%) in the $3 \mathrm{mg} / \mathrm{kg}$ group and $11 \mathrm{Pts}(45.8 \%)$ in the $7 \mathrm{mg} / \mathrm{kg}$ group; 3 Pts (12.5\%) in the $7 \mathrm{mg} / \mathrm{kg}$ group experienced grade 4 hypokalemia. IFI were reported in $5 / 34(14.7 \%)$ Pts: 1 proven, 1 probable, 3 possible according to the EORTC criteria 2002. The overall survival rate in the entire population was $50 \%$.

Conclusions: A $3 \mathrm{mg} / \mathrm{kg}$ regimen three times a week followed by $7 \mathrm{mg} / \mathrm{kg}$ weekly administration of AmBisome ${ }^{\circledR}$ is a tolerable and safe antifungal primary prophylaxis in high-risk Pts undergoing allo-SCT from alternative donors. Hypokalemia following the $7 \mathrm{mg} / \mathrm{kg}$ dose should be adequately prevented to further reduce concomitant toxicities.

\section{P728}

Cytomegalovirus reactivation after autologous stem cell transplant for lymphoproliferative diseases: something to deal with. A prospective study on 92 patients

A. Isidori (1), L. Malerba (1), S. Barulli (1), M. Lucesole (1), B. Guiducci (1), F. Loscocco (1), I. Tommasini (2), G. Polenta (2), P. Tontini (2), G. Visani (1)

(1)Hematology and Stem Cell Transplant Center (Pesaro, IT); (2)Pharmacy, Antiblastic Service (Pesaro, IT)

Ninety-two patients consecutively submitted to autologous stem cell transplant (ASCT) for lymphoid malignancies were monitored for Cytomegalovirus (CMV) reactivation. Twentynine patients had multiple myeloma, sixty-three a lymphoproliferative disease (HD, NHL, CLL). Twenty-seven patients received ASCT for a resistant disease, whereas 65 for a newly diagnosed disease. The median number of prior chemotherapy cycles was 2 (range: 1-4). Thirty-six patients received anti-CD-20 antibody as a part of the frontline therapy before ASCT. The conditioning regimen was BEAM for $47 / 92$ patients, high-dose Melphalan for 28/92 patients and BAVC or Busulphan/Endoxan for the remaining 17 patients. Sixty-six patients received peripheral blood stem cells rescue after high dose therapy, whereas 26 got bone marrow stem cells. CMV reactivations were monitored twice weekly with the polymerase chain reaction (PCR) assay in all patients, starting after the engraftment. Twenty-five out of 92 patients $(27 \%)$ presented a CMV reactivation. The vast majority of patients $(17 / 25$, $68 \%$ ) reactivated $\mathrm{CMV}$ after 30 or more days from transplant. Moreover, $15 / 25$ patients $(60 \%)$ presenting a CMV reactivation had received prior Rituximab. Ten out of 25 patients $(40 \%)$ presented symptoms such as fever, vomiting, arthralgia or profound asthenia at reactivation. Moreover, CMV reactivation was associated with moderate to severe neutropenia in $8 / 25$ patients. All patients reactivating CMV were treated with intravenous (iv) ganciclovir given $7.5 \mathrm{mg} / \mathrm{kg}$ once daily. They received antiviral therapy for a median of 20 days (range: 6-40). All patients achieved a negativization of CMV-specific PCR after a median of 12 days of therapy (range: 9-34). Discontinuation of antiviral therapy required at least two consecutively negative PCRs performed at least 3 days apart each other. None of the patient developed a CMV disease. Moreover, no late $\mathrm{CMV}$ reactivations were observed during the longterm follow-up period. In conclusion, our experience clearly demonstrates that a significant proportion of patients submitted to ASCT develop a CMV reactivation after transplant, frequently symptomatic, requiring antiviral therapy. We underline the necessity of a stringent monitoring of CMV reactivation after ASCT, specially for patients receiving therapy with anti-B lymphocytes monoclonal antibodies before transplant. 
P729

Incidence and clinical features of EBV-associated post-transplantation lymphoproliferative disorder after unrelated cord blood transplantation

A. Nishida, H. Yamamoto, Y. Ohta, K. Ishiwata, N. Nakano, M. Tsuji, Y. Asano-Mori, N. Uchida, K. Masuoka, A. Wake, A. Yoneyama, S. Makino, S. Taniguchi

Toranomon Hospital (Tokyo, JP)

Background: Given that unrelated cord blood transplantation ( $\mathrm{UCBT}$ ) is associated with a relatively low number and functionally immature donor T cells, and frequent donor-recipient HLA disparity, it has been speculated that the risk of EBV-associated posttransplantation lymphoproliferative disorders (EBV-PTLD) after uCBT may be increased.

Objective and method: To investigate the incidence and clinical features of EBV-PTLD after UCBT, we retrospectively analyzed 341 patients underwent UCBT from January 2002 to December 2008 at Toranomon Hospital. Diagnosis of EBV-PTLD was made only when both biopsy-proven specimen and EBVviremia were present.

Result: Five cases of EBV-PTLD were identified, with cumulative incidence of $1.8 \%$ at 2 years. The median-age was 52 years (range: $50-66$ ). Three patients underwent transplantation for leukemia and 2 for aplastic anemia. EBV-PTLDs were diagnosed at 120 to 803 days (median, 280 days) after uCBT, with 4 of 5 patients having chronic graft-versus-host disease, and 3 of 5 patients receiving immunosuppressive treatment. Three developed lymphadenopathy, while other symptoms at onset were nonspecific, such as fever in 3 , liver dysfunction in 2 , and diarrhea in 3. Immunosuppressant was decreased in those who were under the therapy, and rituximab was administered to all patients. All had responded to the treatment and, so far, no patient died due to EBV-PTLD.

Conclusion: The incidence of EBV-PTLD after UCBT appears similar to that observed after transplantation using other sources. These findings suggested that UCBT may not be a risk of developing EBV-PTLD. The initial manifestations may not be directly suggestive of EBV-PTLD, and it should be taken into consideration whenever patients have nonspecific symptoms without other reasons.

\section{P730}

Infections with the new influenza H1N1 virus in seven haematopoietic stem cell recipients: a single-centre experience

T. Schroeder, C. Saure, S. Balleisen, M. Kondaki, R. Fenk, I. Bruns, R. Haas, G. Kobbe

University Hospital Dusseldorf (Dusseldorf, DE)

After the first identification of a new Influenza A strain (H1N1) over 450.000 infections resulting in up to 10.000 deaths around the world have been reported. Recipients of autologous or allogeneic stem cell transplantations (SCT) are at substantial risk for infections based on a compromised immune system. However, there is little knowledge about the clinical course of $\mathrm{H} 1 \mathrm{~N} 1$ infections in hematopoietic stem cell transplant (HSCT) recipients.

We report here on the clinical courses of 7 HSCT recipients with H1N1 infections observed at our department.

Four patients presented with fatigue and fever and $\mathrm{H} 1 \mathrm{~N} 1$ virus was detected by PCR. With regard to transplant and disease status two patients had received an auto-SCT after high dose chemotherapy for Hogdkin's Disease (HD) respectively multiple myeloma (MM) 141 and 954 days ago. While the HD patient was in $\mathrm{CR}$ at the time of $\mathrm{H} 1 \mathrm{~N} 1$ infection, the MM patient received dexamethasone/velcade for progressive disease. The 2 other patients had received allo- SCT after myeloablative conditioning. One patient with $A M L$ was in $C R(d+255)$ still requiring tacrolimus for chronic GvHD. The other patient suffered from relapse of CMML $(d+767)$ receiving 5-aza and DLI. Receiving oseltamivir ( $75 \mathrm{mg} 2 \times / \mathrm{d}$ for $10 \mathrm{~d}$ ) all patients got PCR-negative and free of symptoms within 1 week.

Three other patients presented with fever, dry cough and dyspnea. Two of them had received allo-SCT after myeloablative conditioning for $\mathrm{CML}$ and were in $\mathrm{CR}(\mathrm{d}+956 /+1456)$. One patient still required tacrolimus for chronic GVHD. The other had a normal immune status. H1N1 virus was detected by PCR and chest X-ray showed atypical pneumonia in both patients. They were admitted to hospital and received oseltamivir $(75 \mathrm{mg}$ $2 x / d$ for $10 \mathrm{~d}$ ) leading to disappearance of symptoms and virus elimination.

The third patient with progressive light chain MM $(d+904$ after auto-SCT), renal failure and humoral defiency suffered from a H1N1-associated pneumonia with bacterial and viral superinfections. Despite oseltamvir therapy she still requires mechanical ventilation for 37 days being still PCR-positive for H1N1.

Clinical courses of H1N1 infections in HCST recipients seem to range individually and cannot predicted on the basis of disease and immune status. Since symptoms of a H1N1 infection are unspecific and often found in HSCT recipients, but can result in fatal clinical courses, we suggest early testing for $\mathrm{H} 1 \mathrm{~N} 1$ virus in this group of patients especially in this pandemic situation.

\section{P731}

Reconstitution of EBV-specific T-cell memory and control of PTLD after transfer of peptide-selected T-cells

A. Moosmann (1), J. Tischer (1), L. Schirrmann (1), J. Kasten (2), S. Tippmer (2), I. Bigalke (2), H.-J. Kolb (1)

(1)University of Munich (Munich, DE); (2)Helmholtz-Zentrum (Munich, DE)

Post-transplant lymphoproliferative disease (PTLD) associated with Epstein-Barr virus (EBV) is a life-threatening complication after allogeneic hematopoietic stem cell transplantation (HSCT). PTLD can be prevented by adoptive transfer of EBVspecific $T$ cells from the donor. To make EBV-specific T cells available as a therapeutic option in urgent situations, we developed a rapid protocol for their isolation by overnight stimulation of donor blood cells with a mix of EBV peptides, interferongamma surface capture, and immunomagnetic separation. Our generic stimulation cocktail contained 23 EBV peptides derived from 11 EBV antigens and covering 14 HLA class I and II allotypes. Six patients with PTLD received one transfusion of EBV-specific T cells. Progressive disease was seen in three patients who suffered from late-stage disease with multiorgan dysfunction at the time of $\mathrm{T}$ cell transfer. However, in three patients who received $T$ cells at an earlier stage of disease, we observed complete and stable remission of PTLD. Two patients have remained free from EBV-associated disease for more than two years. In these patients, CD8 + T cells specific for EBV immediate-early and early antigens rapidly expanded after T-cell transfer, peaked at over $20 \%$ of peripheral blood lymphocytes, and were maintained at $8 \%$ or more for six months. Later, EBV-specific T cells remained present at lower levels throughout the observation period. For the first months, the overall T cell repertoire was oligoclonal. Later, the repertoire became more polyclonal, coincident with the emergence of naive $T$ cells, an inversion of the CD4 +/CD8 + ratio, and a contraction of numbers of EBV-specific T cells down to levels typical for healthy carriers. Thus, the infusion of small numbers of directly isolated EBV-specific T cells was followed by a rapid, intense and sustained reconstitution of a protective EBV-specific T cell memory. 


\section{P732}

Interactions of human immature dendritic cells with defined recombinant Aspergillus fumigatus antigens M. Ok (1), O. Kurzai (2), F. Ebel (3), C. Baeuerlein (1), M. Mezger (1), M. Topp (1), H. Einsele (1), J. Loeffler (1) (1)Medcal Hospital (Wurzburg, DE); (2)Institut für Hygiene und Mikrobiologie (Wurzburg, DE); (3)Max von Pettenkofer Institut (Munich, DE)

Objectives: One of the major life threatening fungal infections associated with allogeneic stem cell or bone marrow transplantation is invasive aspergillosis (IA). Dendritic cells represent an important element of the innate immune system in the defence against Aspergilli; they acquire antigens in the periphery, migrate into secondary lymphoid tissues, activate T-cells and maturate and secrete cytokines and chemokines. Up until now, the influence of distinct Aspergillus fumigatus molecules on the human innate immune system is largely unexplored.

Methods: We have characterized the interaction between the A. fumigatus antigens Aspf1 and Crf1 and human monocytederived immature dendritic cells (iDC), respectively. Antigens were recombinantly expressed in the Pichia pastoris system and subsequently used for stimulation experiments, which allowed quantifying gene expression (by qRT-PCR) and secretion of cytokines and chemokines (by ELISA assays) in iDC, to localize microscopically Aspf1 and to determine its capacity to induce apoptosis in iDCs. Furthermore, by mixed lymphocyte reactions (MLR), we have analyzed whether iDCs loaded with Aspf1 are able to induce T-cell proliferation.

Results: Compared to Crf1, Aspf1 showed a high ability to specifically stimulate iDCs as demonstrated by an increased expression of genes encoding for pro-inflammatory cytokines (e.g. IL-12p35, TNFa) and chemokines (CXCL10, CCL20, IL8). By using germlings of a deletion mutant for Aspf1 (dAspf1) in confrontation assays with iDCs, increased protein secretion compared to stimulation with wild type morphologies was detectable. Apoptosis of iDCs was markedly induced after cocultivation with Aspf1. MLR revealed that iDCs were not able to present Aspf1 to autologous T-cells.

Conclusion: This study demonstrates that recombinant $\mathrm{A}$. fumigatus antigens are able to efficiently stimulate iDCs in vitro; these observations might potentially lead to the development of a new therapeutic and/or prophylactic option to prevent and treat this devastating infection in high risk patients after allogeneic stem cell transplantation.

\section{P733}

Surveillance cultures for Clostridium difficile in allogeneic haematopoietic stem cell transplantation

A. Ferrant, M.-C. Vekemans, A.-M. Servaty, M. Delmée, X. Poiré, E. Van Den Neste

UCL, Cliniques universitaires Saint-Luc (Brussels, BE)

Clostridium difficile (C. difficile) is a leading cause of nosocomial diarrhea. We performed a study to know whether surveillance cultures and early therapy could avoid toxicities associated with C. difficile in allogeneic hematopoietic stem cell transplantation (allo-HSCT). We also collected data on the prevalence and relapse of $C$. difficile in these patients.

Since 1988, biweekly surveillance stool cultures for $C$. difficile were performed in the patients admitted to the hematology wards. 377 patients have had an allo-HSCT. Data from stool cultures for $C$. difficile and detection of toxin $A$ and/or $B$, were obtained in 368 . The treatment policy was to prescribe metronidazole (500 mg tid for 10 days) as soon as a positive culture result was transmitted.

In 152 patients $(40 \%)$ one or more episodes with a positive culture for $C$. difficile were recorded (before allo-HSCT: 40 patients, at allo-HSCT: 72 , before and at allo-HSCT: 22, after allo-HSCT: 20). In 59 , a positive culture was associated with toxin A or B (tox + ). In 94 patients $(25 \%), C$. difficile (tox,$+ n=41$; tox-, $n=53$ ) was cultured during hospitalization for allo-HSCT. Relapse of $C$. difficile occurred in 22 of the positive patients (35\%). The cumulative incidence of relapse of $C$. difficile did not differ between tox + and toxA positive culture for $C$. difficile (either tox + or tox-) did not affect the toxic mortality in allo- HSCT. C. difficile infection (either tox+ or tox-) was not associated with any increase in graft-versus-host disease. Acute gut GvHD grade $\geq 2$ occurred in 22 patients. In 5 of them C. difficile was cultured during hospitalization (NS).

Conclusions:

1. The prevalence of $C$. difficile infections in allo-HSCT recipients is high.

2. Despite therapy with metronidazole, a third of the patients who have had $C$. difficile relapsed.

3. There is no relationship between acute GvHD of the gut and C. difficile infection.

4. In allo-HSCT, surveillance cultures and early therapy could avoid non-relapse complications caused by $C$. difficile infections.

\section{P734}

In vivo Campath-1h is associated with a high incidence of EBV viraemia but low risk of PTLD following allogeneic stem cell transplantation

B.J.M. Carpenter (1), M. Dimopoulou (1), S. Grace (1), S. Denovan (1), G. Orti (1), C. Atkinson (1), M. Roughton (2), T. Haque (1), P. Griffiths (1), A. Fielding (1), P. Kottaridis (1), S. Mackinnon (1), R. Chakraverty (1)

(1)The Royal Free NHS Trust (London, UK); (2)CR UK \& UCL Cancer Trials Centre (London, UK)

Background: Re-activation of latent EBV infection and posttransplant lymphoproliferative disorder (PTLD) pose a significant risk following T-cell depleted (TCD) allogeneic SCT. Here, we report an analysis of EBV re-activation in 111 consecutive, adult patients receiving allogeneic SCT for haematological malignancy, incorporating either in vivo or in vitro ('in the bag') CAMPATH-1H (CAM) as the method of TCD.

Methods: In vivo CAM was used with a fludarabine-based RIC protocol $(n=75$, median dose $100 \mathrm{mg})$ or in vitro following myeloablative conditioning $(n=36$, dose $20 \mathrm{mg})$. Median follow-up is 2.4 y and median age 43 y (range 16-67 y). Donors were matched sibling $(n=51)$, mismatched $(m m)$ related $(n=3)$, matched VUD $(n=32)$ and mm VUD $(n=25)$. Monitoring for EBV DNA was performed weekly by real time PCR for 100d post transplant and then at follow-up. Patients with a high EBV DNA level (>40,000 copies $/ \mathrm{ml}$ ) underwent clinical exam, CT imaging and bone marrow/CSF evaluations where appropriate, followed by withdrawal of immunosuppression and a single infusion of $375 \mathrm{mg} / \mathrm{m}^{2}$ Rituximab $(R)$.

Results: The cumulative incidence of EBV reactivation at $2 \mathrm{yrs}$ was $40.3 \%$ (46.1\% following in vivo CAM vs. $28.0 \%$ following in vitro CAM, $P=\mathrm{NS}$ ). Re-activation occurred at a median of $118 \mathrm{~d}$ post transplant (range 21-1336d). Patients receiving in vivo CAM had earlier re-activation of EBV than observed for in vitro CAM (at 100 days, $22.7 \%$ vs. $2.8 \%$ respectively, $P<0.05$ ). Median duration of viraemia was 41 days (range 1-461d). Diagnoses of Hodgkin's lymphoma (HR 3.5, 95\% Cl 1.5-8.2) and CLL (HR 3.8, 95\% Cl 1.4-10.3) were associated with a greater risk of EBV re-activation. Day 30 lymphocyte counts, VUD or mm donor and GVHD did not predict for EBV re-activation. $100 \mathrm{~d}$ NRM was $2.2 \%$ in patients with EBV reactivation and $15.6 \%$ of patients without EBV reactivation $(P=N S) .18$ patients had high-level EBV viraemia and in all of these cases, re-activation occurred within $1 \mathrm{yr}$. Treatment with $\mathrm{R}$ resulted in a rapid decline in EBV DNA levels to undetectable levels in all cases. Of these, 1 patient had histological confirmation of PTLD and subsequently attained a CR that persists at $14 \mathrm{~m}$.

Conclusions: CAM-based TCD is associated with a high frequency of EBV viraemia but a very low $(<1 \%)$ risk of PTLD using a strategy of pre-emptive R. Further studies that define the appropriate trigger for pre-emptive therapy (EBV viral load \pm other markers of immune reconstitution) are required to avoid over-treatment with $\mathrm{R}$. 
P735

The effectiveness of liposomal amphotericin in the treatment of invasive fungal infections is not affected by prior azole administration: the Ambi-Prof study

J. de la Serna, I. Jarque, J. López, R. Mar, V. Gómez-García, J. Serrano, A. Báez, A. Sampol, P. Amat, C. Barrenetxea, R. del Campo, J. García, M. Jurado on behalf of the Study Group of Liposomal Amphotericin B

It is a matter of debate whether mold-active azole prophylaxis may reduce the effectiveness of Liposomal Amphotericin (L-AmB).

Objectives: This retrospective study was aimed to determine the non-inferiority of prior azole administration in the treatment of Invasive Fungal Infections (IFI) with L-AmB in hematologic and allogeneic HSCT patients.

Methods: Patients who met the EORTC/MSG criteria for IFI and received treatment with $L-A m B$ were eligible and distributed in two arms according to: (A) mold-active azole exposure prior to $\mathrm{L}-\mathrm{AmB}$, and (B) fluconazole or no prior azole. Patients were stratified according to the type of IFI and evaluated for disease related risk factors and comorbidities. The primary endpoints were favorable response and survival at the end of antifungal therapy, at 4 and 12 weeks.

Results: From Feb/2008 to Sep/2009, 182 consecutive patients were recruited from 26 institutions. The median age was 45 years (range 1-78). Most had acute leukemia (AL) or myelodysplasia (MDS) (129; 70.0\%). Baseline disease was treated for induction, in remission, or refractory/relapse status in $23.6 \%, 45.0 \%$ and $31.4 \%$, respectively. A $40.1 \%$ of patients had allogeneic HSCT. Severe comorbidity and prior IFI were present in $20.3 \%$ and $14.8 \%$, respectively. Arm A included 100 patients with prior itraconazole $39 \%$, voriconazole $35 \%$ and posaconazole $26 \%$. Arm B included 82 patients with fluconazole $49 \%$ or no azole $51 \%$. Patients characteristics were not different in both arms, except for more AL or MDS $(P=0.002)$ and prolonged neutropenia in arm $\mathrm{A}(P=0.021)$, and more use of high dose steroids in arm $\mathrm{B}(P=0.01)$. The rates of possible, probable and proven IFI were $52.7 \%, 28.6 \%$ and $18.7 \%$, respectively (Table 1). Aspergillosis was the proven IFI in 28 of 35 cases. L-AmB was given $3 \mathrm{mg} / \mathrm{kg} / \mathrm{d}$ for a median of $18 \pm 17$ days in $A$ and $15 \pm 13$ in $B$. The favorable response rate to $L$ AmB was $75 \%$ and $74.4 \%$ in both groups, with no differences in the responses at the end of treatment, at 4 weeks or at 12 weeks. The response rates for possible and probable/proven IFI were similar in both groups (Table 2).

Conclusions: Prior exposure to mold-active azoles does not affect the effectiveness of L-AmB for the treatment of IFI in this high risk patient population, indicating that concerns for sequential administration are no longer justified.

\begin{tabular}{|c|c|c|c|c|}
\hline \multirow[t]{2}{*}{$\begin{array}{l}\text { Table } 1 \\
\text { Patent's characteristics }\end{array}$} & $\begin{array}{c}\text { Group A } \\
\text { phor azole } \\
N=100\end{array}$ & $\begin{array}{l}\text { Group B } \\
\text { no azolo } \\
N=82\end{array}$ & \multirow{2}{*}{$\begin{array}{l}\begin{array}{l}\text { Total } \\
N=182\end{array} \\
n(\%)\end{array}$} & \multirow[t]{2}{*}{ pvafue" } \\
\hline & $n \quad($ (क) & $n(\%)$ & & \\
\hline \multicolumn{5}{|l|}{ Diseaso } \\
\hline AM, ALL,MOS & $80(800)$ & 49 (598) & $129(70.0)$ & \multirow{2}{*}{0.002} \\
\hline Other & $20(20.0)$ & $33(402)$ & $53(29.1)$ & \\
\hline \multicolumn{5}{|l|}{ Phase of the disease } \\
\hline Induction & $23(235)$ & $19(23.8)$ & $42(23.6)$ & \multirow{3}{*}{$n s$} \\
\hline Rerrission & $41(41.8)$ & $39(48.8)$ & $80(45.0)$ & \\
\hline RefractoryRelapse & $34(346)$ & $22(227)$ & $56(31.4)$ & \\
\hline Allogeneic HSCT & $44(44.0)$ & $29(354)$ & $73(40.1)$ & $n s$ \\
\hline Severe comorbidity & $22(220)$ & $15(183)$ & $37(203)$ & $n s$ \\
\hline Prior IFI & $18(180)$ & $9(110)$ & $27(148)$ & $n s$ \\
\hline \multicolumn{5}{|l|}{ Type of IFI at L-AmB } \\
\hline Possible & $53(53.0)$ & $43(524)$ & $96(52.7)$ & \multirow{3}{*}{ ns } \\
\hline Proberble & $29(290)$ & $23(28.1)$ & $52(286)$ & \\
\hline Proven & $18(18)$ & $16(195)$ & $34(187)$ & \\
\hline Neutropenia 210 days & $71(845)$ & $41(683)$ & $112(615)$ & 0.021 \\
\hline High dose steroids & $18(18.0)$ & $28(34.1)$ & $46(25,3)$ & 0,013 \\
\hline
\end{tabular}

\begin{tabular}{|c|c|c|c|c|}
\hline \multirow[t]{2}{*}{$\begin{array}{l}\text { Table } 2 \\
\text { LAmB trextment outcome }\end{array}$} & $\begin{array}{c}\text { Group A } \\
\text { phoc asole } \\
\mathrm{N}=100\end{array}$ & $\begin{array}{c}\text { Group B } \\
\text { no azole } \\
N=82\end{array}$ & $\begin{array}{l}\text { Total } \\
N=182\end{array}$ & \multirow[t]{2}{*}{ protion } \\
\hline & $n \quad(\%)$ & $n(x)$ & $n(\infty)$ & \\
\hline Favorable response' & $75(750)$ & $61(744)$ & $196(750)$ & ns \\
\hline Possidie F1 & $44(83.0)$ & $32(744)$ & $76(79.2)$ & ns \\
\hline Proven or Probadie IFI & $31(660)$ & $29(144)$ & $60(698)$ & ns \\
\hline \multicolumn{5}{|l|}{ End of treatment } \\
\hline Favorabio Respome & $73(73.0)$ & $55(87.1)$ & $128(703)$ & ns \\
\hline Surnvall & $83(83.0)$ & $70(854)$ & $153(24.1)$ & ns \\
\hline \multicolumn{5}{|l|}{ At 4 weeks } \\
\hline Favoracio Response & $57(57.0)$ & $50(610)$ & 107 (58 8) & ns \\
\hline Survival & $62(622)$ & $64(780)$ & $126(692)$ & 0.02 \\
\hline \multicolumn{5}{|l|}{ At 12 weoks } \\
\hline Favorable Response & $41(41.0)$ & $41(50.0)$ & $82(45.0)$ & ns \\
\hline Surnval & $42(42.0)$ & $49(598)$ & $91(500)$ & 0.017 \\
\hline
\end{tabular}

\section{P736}

The impact of cytomegalovirus reactivation after cord blood transplantation in adults

S. Takahashi, T. Yamaguchi, J. Ooi, N. Tsukada, S. Kato, T. Kawakita, T. Yagyu, M. Ooiwa-Monna, A. Tojo, S. Asano University of Tokyo (Tokyo, JP)

Study purpose: We have shown cord blood naive T cells could obtain memory and effector function in vivo with antigen-specific manner during early phase of post-CBT without effect of HLA disparity (EBMT, 2009). In this study, we investigated to find graft- and patient-related factors affecting to CMV reactivation after HLA-mismatched CBT and the impact of CMV reactivation to clinical outcome of $\mathrm{CBT}$ in adults.

Patients and methods: We studied the clinical outcomes of 152 consecutive adult patients who received CBT between 1998 and 2009. All patients received myeloablative regimens including 12 Gy of TBI, CsA plus short term MTX for GVHD prophylaxis and almost the same supportive care. In graft-versus-host direction, 7 were antigen-matched, 53 were 1 antigenand 92 were 2 antigens-mismatched in HLA-A, -B and -DR loci by low-resolution method. After engraftment, all patients were monitored using a CMV antigenemia assay with C10/C11 monoclonal antibodies twice a week during hospitalization. Twentyeight $(18 \%)$ were suffered from 10 times or more positive results defended as the higher-frequent positivity $(H)$ and 124 $(82 \%)$ were categorized as lower-frequent positivity or negative (L). If antigenemia assay was positive, $5 \mathrm{mg} / \mathrm{kg}$ ganciclovir was started as preemptive therapy. We evaluated clinical factors correlated with CMV antigenemia positivity by the Chi-square test and the impact of positive antigenemia on clinical results using the Pepe and Mori's test. OS rate was calculated using the Kaplan-Meier method and analyzed by the log-rank test. Multivariate analysis has performed using the Competing risk regression and the Cox regression.

Results: OS and DFS rates were $73 \%$ and $69 \%$ at 3 years respectively. One patient (14\%) in HLA-matched, $7(13 \%)$ in 1 antigen-mismatched and $20(22 \%)$ in 2 antigens-mismatched patients showed $\mathrm{H}(\mathrm{ns})$. CMV antibody positivity $(P=0.032)$ and use of steroid after transplant $(P<0.001)$ significantly affected to $\mathrm{H}$. Patients with $\mathrm{H}$ stayed in hospital significantly longer (median: 161.5 days) than $\mathrm{L}$ (105 days) in multivariate analysis $(P=0.037)$. The incidence of chronic GVHD in patients with $\mathrm{H}$ (65\% at 1 year) tended to be higher than $L(22 \%, P=0.083)$. OS rate in patients with $\mathrm{H}$ was lower $(61 \%$ at 3 years) than with $\mathrm{L}$ (74\%), but the difference was not significant.

Conclusion: HLA disparities in CBT were not affected to CMV antigenemia results. Longer stay in hospital was needed in high-frequent CMV reactivated patients after CBT. 


\section{P737}

Respiratory syncytial virus-induced tracheobronchitis and pneumonia occuring pre-engraftment in allogeneic haematopoietic stem cell transplant recipients can be safely treated with high-dose ribavirin

S. Gueller, U. Duenzinger, T. Wolf, S. Mousset, H. Martin, H. Serve, G. Bug

Klinikum der J.W. Goethe-Universität (Frankfurt, DE)

Objectives: In allogeneic HSCT recipients, respiratory syncytial viruses (RSV) frequently cause severe respiratory disease. Progression of upper respiratory tract infection to life-threatening bronchiolitis and pneumonia is common within the first month post-HSCT leading to a 30-day all cause mortality of $40 \%$ despite aerosolized ribavirin \pm immunoglobulins.

Methods: Concurrent with an outbreak in the community, RSV infection was diagnosed in 10/29 pts (34\%) transplanted in winter 2008. Diagnosis was based on RSV-specific PCR of routinely collected throat swaps. IV ribavirin was dosed acc. to Schleuning et al., 2003 (d1: $33 \mathrm{mg} / \mathrm{kg}, \mathrm{d} 2-5: 4 \times 16 \mathrm{mg} / \mathrm{kg}$, d6-10: $3 \times 8 \mathrm{mg} / \mathrm{kg}$ ) for 8-10 days, followed by oral ribavirin $(1.8 \mathrm{~g} /$ day) until recovery from symptoms. Airflow obstruction (AFO) was defined as a FEV1/FVC ratio $<0.7$ or a drop in FEV1 $>20 \%$ from baseline. All RSV-infected pts (median age $60 \mathrm{yrs}$ ) had RIC-HSCT for high-risk AML (1st/subsequent CR: $n=5$; untreated/refractory relapse: $n=5$ ). GvHD prophylaxis consisted of CSA, MMF with $(n=8)$ or without $(n=2)$ ATG. Donors were HLA-identical siblings $(n=1)$, matched $(n=8)$ or mismatched unrelated donors $(n=1)$.

Results: Median time from HSCT to detection of RSV was 15 (1-40) days. In 7/10 pts, RSV infection was diagnosed preengraftment during neutropenia. Four pts had bronchiolitis or pneumonia and 6 pts tracheobronchitis. High-dose IV ribavirin was administered to all 5 pts with pneumonia and/or diagnosis of RSV infection within 7 days after HSCT. These pts had hypoxia and required oxygen supplementation. Treatment with oral ribavirin was initiated in the other 5 pts with tracheobronchitis on days $12-40$ after HSCT. Nine pts became RSV-negative in throat swabs after a median of 22 days from start of therapy. Severe AFO due to RSV pneumonia in 2 pts improved after treatment. No pt treated for tracheobronchitis progressed to RSV pneumonia. Neutrophil recovery was delayed in the three pts diagnosed with RSV pneumonia pre-engraftment until days 26, $28+$ and 111 after HSCT, due to high-dose IV ribavirin and/ or RSV infection itself. One of these pts died of septic shock without having cleared RSV. No other acute toxicity associated with ribavirin was observed. With a median follow up of 9 months, 9/10 pts are alive and 7 still in CR. Intriguingly, $2 \mathrm{CR}$ pts were diagnosed with a post-transplant lymphoproliferative disorder.

Conclusion: High-dose ribavirin appears to be safe and effective even if administered to neutropenic HSCT recipients.

\section{P738}

Epstein-Barr virus reactivation in allogeneic haematopoietic stem cell transplantation monitored by real-time polymerase chain reaction on different blood compartments

C. Nozzoli (1), M.L. Battista (2), I. Donnini (1), F. Patriarca (2),

S. Guidi (1), M. Di Gioia (1), M. Medeot (2), A. Gozzini (1),

B. Bartolozzi (1), R. Fanin (2), A. Bosi (1)

(1)BMTUnit (Florence, IT); (2)BMTUnit (Udine, IT)

Epstein Barr virus (EBV)-associated post-transplant lymphoproliferative disease (PTLD) is a rare but severe complication arising after haematopoietic stem cell transplantation (HSCT). Known risk factors include unrelated or mismatched donors, Tcell depletion and immunosuppressive treatment. The monitoring of EBV DNA load by quantitative polymerase chain reaction (PCR) is mandatory to reveal early EBV reactivation and start a pre-emptive treatment with anti-CD-20 monoclonal antibody rituximab. Until now no consensus was established on the suitable blood compartment for the quantification of EBV DNAemia between whole blood (WB) and peripheral blood mononuclear cells (PBMC). With this aim, we retrospectively analyzed 146 consecutive pts who underwent allogeneic HSCT at the transplantation centre of Florence and Udine from January 2007 to August 2009. 49 pts received an HSCT from identical sibling, 97 from an unrelated donor. Diagnosis were: AML 64, ALL 19, lymphoproliferative diseases 32 , CMS 11, MDS 6, MM 10, other 3; median age was 43 yrs (range 17-67); 81 were male and 65 female; 91 pts received conventional conditioning and 55 reduced intensity one. 31 pts received a TBI based conditioning; 85 pts received ATG as T-depletion. Stem cell source was peripheral blood in 99 pts, bone marrow in 37 and cord blood in 10 pts (at least 4/6 mismatched). All pts were weekly monitored from the engraftment for EBV DNAemia by real time PCR on PBMC in 76 pts (group 1) and on WB in 70 pts (group 2). EBV reactivation was defined as EBV DNA load $>1.000$ copies/105/ PBMC or $>1000 \mathrm{copies} / \mathrm{ml} / \mathrm{WB}$ and pts received rituximab as preemptive treatment till negativity of EBV DNAemia and resolution of clinical manifestations. 33/146 $(23 \%)$ patients experienced EBV reactivation, with EBV DNA copies $>1000$ (range 1000-1.432.127 viral copies), 9 pts developed PTLD (6\%). The incidence of EBV reactivation was different between the two groups of monitoring: $12 \%$ in group 1 vs. $34.3 \%$ in group 2 (p 0.001). Moreover PTLD incidence resulted higher in group1 ( $7.9 \%$ vs. $4.3 \%$ ). All pts received a median number of 2 cycles of Rituximab: 4 cycles in group 1 vs. one in group 2. In conclusion in our experience real time PCR monitoring allowed early detection of EBV reactivation that resulted significant different on the basis of the blood samples analyzed, suggesting that it could not be applied the same DNAemia cut-off for defining EBV reactivation and starting pre-emptive therapy.

\section{P739}

Guillain-Barré sindrome within Epstein-Barr virus reactivation after allogeneic haematopoietic stem cell transplantation

S. Baldini, C. Nozzoli, A. Gozzini, B. Bartolozzi, S. Guidi, A. Bosi BMTUnit (Florence, IT)

Guillain-Barré syndrome (GBS) is a polineuropathy rarely arising after allogeneic haematopoietic stem cell transplant (HSCT): until now only 23 cases have been reported. We describe the case of a 51-year-old Caucasian woman who underwent allogeneic HSCT from matched unrelated donor for acute myeloid leukemia in second complete remission. The reduced intensity conditioning regimen consisted of tiothepa, cyclophosphamide, and melphalan. Stem cell source was peripheral blood. Graftvs.-host disease (GvHD) prophylaxis was based on anti thymocyte globulin, cyclosporine A (CyA) and methotrexate standard course. Both donor and recipient were pre-transplant Epstein Barr virus (EBV) positive. Antiviral prophylaxis consisted of acyclovir. Engraftment was prompt (day +13$)$ and sustained, with no evidence of GvHD and weekly monitoring of EBV with quantitative polymerase chain reaction (PCR) on whole blood was started. On day +54 EBV reactivation (26510 EBV genome copies $/ \mathrm{mL}$ ) with a mononucleosis-like syndrome was observed CyA was tapered and Rituximab (375 mg/mq i.v.) was started. On day +69 the patient complained of important weakness and paresthesias in toes and fingers with an increase of EBV DNAemia and CyA was discontinued. The neurological evaluation showed weakness on the inferior limbs with impairment in the erect posing, moderate distal sensory disturbance and absence of deep tendon reflexes. The cereberospinal fluid was negative also for neurotropic agents including EBV by PCR. Serum antibodies against anti-GD1b ganglioside IgG (1:2300) and anti-sulfatide IgG (1:3500) were positive. The findings of nerve conduction studies were consistent with a sensorimotor demyelinating polineuropathy mainly to the inferior limbs, and the nuclear magnetic resonance of the encephalon was negative. The patient was treated with high dose intravenous immunoglobulines and then with five plasma-exchange procedures and Rituximab with improvement of both clinical and 
neurological findings and negativity of EBV on PCR assay and of anti-Gd1b ganglioside and anti-sulfatide serum antibodies. After 7 months of follow-up patient is ambulatory on monthly maintenance with rituximab, with full chimerism and no evidence of EBV or chronic GvHD.

Conclusions: in our experience plasma-exchange and rituximab were efficacy in extinguish both B-cells deregulated proliferation and EBV reactivation that could act a trigger for GBS development.

\section{P740}

Impacts of diagnostic and therapeutic advances on a clinical outcome of invasive aspergillosis in haematopoietic stem cell transplantation recipients

Y. Asano-Mori (1), Y. Kanda (2), K. Kandabashi (3), Y. Nanya (4), G. Yamamoto (4), S. Seo (4), M. Ichikawa (4), T. Saito (4), K. Kumano (3), A. Hangaishi (4), Y. Imai (4), T. Takahashi (4), M. Kurokawa (4)

(1)Toranomon Hospital (Tokyo, JP); (2) Saitama Medical Center, Jichi Medical University (Saitama, JP); (3)University of Tokyo Hospital (Tokyo, JP); (4)University of Tokyo (Tokyo, JP)

Objectives: Invasive aspergillosis (IA) remains one of the leading causes of morbidity and mortality after hematopoietic stem cell transplantation (HSCT). Although the diagnostics modalities including CT scan and laboratory adjunct markers, and the therapeutic strategies with newer mold-active agents have been developed, the real impact of these developments on a prognosis of IA in allogeneic HSCT recipients is still unclear Patients and methods: To reveal the alterations in the clinical characteristics and outcome of IA over time, we retrospectively analyzed the records of 221 adult patients who underwent allogeneic HSCT for the first time from February, 2000 to March, 2008 at University of Tokyo Hospital.

Results: Thirty-five patients had proven $(n=9)$ or probable $(n=26)$ IA according to the EORTC 2002 criteria at a median of 178 days (range 21-1526 days) after HSCT, with a cumulative incidence of $17.3 \%$. The development of IA was associated with neutropenia at an early period after HSCT in 4 patients, and the immunosuppressive therapy for graft-versus-host disease in 26 . Thirty-three patients $(94.3 \%)$ had pulmonary disease including 2 with dissemination and 1 with tracheobronchitis, and the remaining one each showed sinusitis and gastrointestinal involvement, respectively. Among 29 patients who underwent chest CT scan, the diameter of nodules or infiltrates was within $1.5 \mathrm{~cm}$ in 17 patients, between 1.5 to $3 \mathrm{~cm}$ in 6 and larger than $3 \mathrm{~cm}$ in 6 , which had no tendency to become smaller across the years. Thirty patients showed positive galactomannan (GM) antigenemia defined as two consecutive results with O.D.I above 0.6 , which occurred at a median of 14 days prior to diagnosis in 17 and served as the initial trigger for further examinations instead of clinical symptoms or CT findings in 11. IA was the principal cause of death in 7 patients. A trend for the declined IA-related mortality was observed after the approval of micafungin in December 2002 (9.4\% vs. 38.5\%, $P=0.073)$. Only one patient died of IA after the approval of voriconazole in June 2005, whose diagnosis was delayed due to the coexisting bronchiolitis obliterans organizing pneumonia. The attributable mortality tended to be lower in patients with CT lesions within $1.5 \mathrm{~cm}$ than with larger lesions $(6.3 \%$ vs. $33.3 \%, P=0.086)$. Conclusion: IA-related mortality tends to decline mainly due to the improved therapeutic approaches. GM screening appears to contribute significantly to the earlier detection of IA.
P741

Polyomavirus JC-targeted T-cell therapy for progressive multiple leukoencephalopathy in an allogeneic haematopoietic stem cell transplantation recipient

A. Balduzzi (1), G. Lucchini (1), H. Hirsch (2), S. Asso (3), M. Cioni (3), A. Rovelli (1), A. Zincone (1), M. Grimaldi (4), P. Corti (1), S. Bonanomi (1), A. Biondi (1), F. Locatelli (3), E. Biagi (1), P. Comoli (3)

(1)Università Milano Bicocca (Monza, IT); (2)University Hospital Basel (Basel, CH); (3)Università di Pavia (Pavia, IT); (4)Ospedale San Gerardo (Monza, IT)

JC polyomavirus (JCV) progressive multifocal leukoencephalopathy $(\mathrm{PML})$ is a rare demyelinating disease of the central nervous system occurring in immunocompromized hosts. The prognosis of PML is almost invariably fatal after hematopoietic cell transplantation (HCT).

We present the case of a 19-year-old recipient diagnosed with PML after prolonged immunosuppression for severe graft-versus-host disease (GVHD) who presented 5 years after HCT with severe slowing, unstable gait, limb tremor, sensitive and motor aphasia, apraxia, agnosia, mental dullness and visual deficiencies. Magnetic resonance imaging (MRI) showed bilateral multiple subcortical lesions and the Mini Mental State (14.6) assessed severe cognitive impairment. Cerebrospinal fluid (CSF) analyses revealed JCV-DNA (8645 copies $/ \mathrm{mL}$ ). No substantial neurological improvement was observed after antiviral treatment with cidofovir and discontinuation of immunosuppression. Donor-derived, JCV antigen-specific, cytotoxic T-lymphocytes (CTL) were generated in vitro after stimulation with a mix of 15-mer viral peptides, spanning the whole JCV VP1 and LT proteins. At 60 and 124 days after PML diagnosis, the patient received two infusions of $0.5 \times 10^{6}$ and $1 \times 10^{6}$ cells/ $\mathrm{kg}$, containing $37 \% \mathrm{CD} 8+, 43 \% \mathrm{CD} 4+, 10 \% \mathrm{CD} 3+/ \mathrm{TCRgd}+$ and $9 \%$ CD56+/CD3-. CTL lines were specific for JCV, proliferating and producing IFNg in response to JCV-VP1 and LT peptide mix, and exerted virus-specific cytotoxicity.

One month after CTL infusion, the emergence of virus-specific CD4 + and CD8 + cell mediated cytotoxicity was assessed and JCV-DNA was cleared in the CSF. Cognitive functions improved markedly (Mini Mental State 27.59); only residual mild impairment in visual-spatial functions, constructional apraxia and deficit in denomination on visual stimulus persisted. MRI showed reduced hyper-intense lesions with diffuse cerebral atrophy.

Twenty-two months after PML diagnosis the patient shows neither further signs of clinical deterioration nor GVHD flare, this finding suggesting that the selection of virus-specific populations, through repeated rounds of antigenic-specific stimulation, efficiently depletes alloreactive T-lymphocytes.

In conclusion we showed that adoptive T-lymphocyte therapy with JCV-specific CTL was feasible and had no side effects in a HSCT recipients presenting with PML. This case suggests that adoptive transfer of JCV-targeted CTL may contribute towards JCV specific immune-reconstitution and PML control in transplanted patients.

\section{P742}

A linked donor recipient study to evaluate B and C hepatitis virus transmission and liver dysfunction after allogeneic bone marrow transplantation

G. Console, P. Scaramozzino, R. Surace, G. Irrera, M. Martino, G. Messina, T. Moscato, R. Fedele, E. Massara, G. Pucci, M. Cuzzola, A. Pontari, I. Callea, A. Dattola, C. Garreffa, E. Spiniello, A. Meliadò, C. Rigolino, O. lacopino, M. Cannatà, D. Marcuccio, P. lacopino

Centro Trapianti Midollo Osseo (Reggio Calabria, IT)

In pairs patient/donor (pt/don) with $\mathrm{HBV}+$ or $\mathrm{HCV}+$, allogeneic transplant is at high risk of developing severe HBV and HCV reactivation. The risk would be even higher if unrelated donor is used. The reduced-intensity is similar, because they are equally immunoablative. No studies have systematically determined the 
impact of HBV + or HCV + of don or pt. Between March 1993 and November 2009, a total of 178 pairs donor/recipient were included in the study, 151 with related donor, 27 with unrelated donor. HCV RNA and HBV DNA PCR and serologic markers, were tested for pt and don. 161 pairs $(90,4 \%)$ were negative for all markers, 17 pairs $(9,6 \%)$ were positive, $2(1,1 \%)$ both don and pt, $7(3,9 \%)$, only don was positive, $8(4,5 \%)$ the pt was positive. About don, 5 were positive for HBV markers, 4 for $\mathrm{HbcAb}, 1$ was inactive carrier, 4 were positive for HCV markers (2 HCV Ab+, 2 HCV RNA+); about pt, 7 were HBV positive (5 $\mathrm{HbcAb}+, 1$ was active carrier, 1 was inactive carrier), $1 \mathrm{HCV}$ $\mathrm{Ab}+, 2$ had coinfection. The outcome of Hepatitis $\mathrm{C}$ was: in two pairs with don positive and pt negative, 1 pt is alive with infection, 1 pt early died for acute GVHD, in two pairs with don negative and pt with coinfection $\mathrm{HBV}+\mathrm{HCV}+$, both pts are alive in treatment with Lamuvidine or Adenofovir. In conclusion, 3 pts of 4 are alive at $+11,+12,+15$ years after allo-SCT, with not evidence of clinical flare of HCV infection. About Hepatitis B, in 1 pairs with HBV DNA +, both pt and don, the pt was in preemptive treatment with Tenofovir, is alive with Hbsag + HBV-, in 2 pairs with pts $\mathrm{Hbsag}+$ and don seronegative, in post transplant the pts are Hbsag + HBVDNA- and alive in treatment with Lamuvidine. In 4 pairs with don $\mathrm{HbcAg}+2 \mathrm{pts}, 1$ in therapy with Entecavir and 1 in therapy with Lamuvidine, are not evaluable because developed aGVHD and Graft failure respectively, 2 pts in therapy with Lamuvidine are alive and the postransplant status is $\mathrm{HbcAb}+$, HbsAb-, HbvDNA-, in 4 pairs with don $\mathrm{HbcAb}+$ $\mathrm{HbsAb}+$ and the pts negative, $1 \mathrm{pt}$ in treatment with Lamuvidine died early for acute liver necrosis, 3 are alive, 1 seronegative, 2 with $\mathrm{Hbcab}+$ and $\mathrm{HbsAb}+$ in preemptive treatment with Lamuvidine (2 pts) or Entevavir (1 pt). In conclusion, HBV infection and HCV infection do not seem increase the rate of aGVHD, cGVHD, VOD. HBV and HCV infection do not prohibit BMT, but hepatitis virus infection can cause liver dysfunction and active prophylaxis of hepatitis virus infection remains necessary in accordance with guidelines.

\section{P743}

Viral reactivation following alemtuzumab-containing conditioning regimens in allogeneic transplantation S. Gomes, I. Ferreira, C. Ornelas, L. Martins, G. Teixeira, N. Miranda, E. Fernandez, F. Leal Costa, M. Abecasis Instituto Português Oncologia (Lisbon, PT)

Introduction: The use of alemtuzumab-based conditioning regimens (ACR) is associated with a high incidence of endogenous viral reactivation and infection with potential life-threatening consequences.

Objectives: to analyse the effects of ACR on cytomegalovirus (CMV), Epstein-Barr virus (EBV), human herpes 6 (HHV-6) and polyoma virus (PMV) reactivation in patients given an allogeneic hematopoietic stem cell transplant (HSCT).

Methods: Retrospective analysis of 39 consecutively transplanted patients given an ACR between June 2002 and September 2009. Viral reactivation was studied by regular quantitative PCR for CMV, EBV and HHV-6 and urine electronic microscopy or quantitative PCR for PMV.

Results: 23 women and 16 male patients aged 3 to $59-y-0$ (median 26$)$ were included in the study. Most $(82 \%)$ had unrelated HSCT. Thirty two $(80 \%)$ received peripheral blood HSCT, 5 (13\%) were given bone marrow and $2(7 \%)$ cord blood cells. The conditioning regimen was myeloablative in 29 patients $(74 \%)$. Thirty four patients (87\%) were CMV positive pre-transplant. Acyclovir was used as antiviral prophylaxis in most patients $(93 \%)$. Ninety percent of patients $(n=35)$ had viral reactivations, with one or more viruses simultaneously. CMV was the most frequently detected (92\% patients), with a median of 3 reactivations per patient, the first at median day +24 (from day +3 to +392$)$. HHV- 6 re-activated in 25 patients $(64 \%)$ at median $d+27(d+11$ to $d+227), 3$ of these patients had HHV-6 pneumonia, with $\mathrm{PCR}$ positive bronchoalveolar lavage (BAL). Eight patients $(21 \%)$ had EBV reactivation at median $d+95(d+13$ to $d+132$ ), one with a post-transplant lymphoproliferative disorder. Ganciclovir and Foscarnet, alone or in combination, were used as antiviral therapy. Polyoma virus infection occurred in 26 patients $(74 \%), 9$ of them with haemorrhagic cystitis $(34 \%)$. Fifteen of the 35 patients (43\%) died with infectious complications and 5 due to disease progression. Two deaths (12\%) were attributed to viral reactivation (pneumonia).

Discussion: Both cases of fatal infection had HHV-6 pneumonia. Despite the high frequency of viral reactivations, even with more than one virus simultaneously, early detection and prompt initiation of therapy was effective in preventing a fatal outcome in most patients.

\section{P744}

Prognosis value of human herpes virus DNA detection in intestinal biopsies of patients undergoing allogeneic stem cell transplantation

A. Bermudez, L. Yañez, S. Cardaba, J. Nuñez, C. Montes, C. Richard, J. Gomez, E. Conde, A. Iriondo

H.U Marques Valdecilla (Santander, ES)

Aims: To study the meaning of Human Herpes Virus infection detected by histopathological and molecular changes in intestinal tissue of patients underwent allo-SCT: to research relation with GvHD, viral disease and long-term follow up.

Patients and methods: Retrospective study of intestinal biopsies of 75 patients underwent allo-SCT in our center from 1999 to 2008. The basic histology study was made with hematoxylin and eosin, DNA viral detection was performed by PCR-ELISA assay for EBV, CMV, HS1, HHV6.In $70 \%$ cases, rectosigmoid biopsies were done.Male/Female: $47 / 28$. The median age was 42 years. Conditioning regimen was myeloablative (47) or RIC (28). Source of stem cells was bone marrow (65), peripheral blood (9) and cord blood (1). Donors were HLA-identical sibling (47). 66 patients were CMVseropositive. Early intestinal biopsies ( $<100$ day) were performed in 46 patients. Previous medical history included: severe mucositis (41 patients), acute GVHD (63), severe GVHD (29), gastrointestinal affectation (52) and cGVHD (26). The median time from symptoms to biopsie was 10 days (5-30). 33 patients had severe digestive symptoms. Previously/coinciding with biopsie, CMV infection was detected by pp65 antigenemia in 33 patients.

Results: Clinical suspected diagnosis was: GvHD 58 (77\%) cases, viral disease $7(9 \%)$ and others $10(13 \%)$. It had correlation between clinical and histological diagnosis in 2/3 cases. 53 biopsies were analyzed by PCR-ELISA and Human Herpes Viruses DNA were detected in $55 \%$ of all cases. If we consider only patients with clinical and histological diagnosis of GvHD, viral DNA was detected in a half of cases (23/43). HHV 6 was virus more frequently isolated $(31 \%)$, alone $(11 / 15)$ or associated with other viruses. CMV was shown in $24 \%$ cases. $15 / 29$ patients with GVHD (clinical and histological) who had PCR positive for viral DNA, had more CMV and HHV6 reinfection (3.2 episodes vs. 1), less complete response of GvHD to steroid therapy ( $40 \%$ vs. $57 \%)$, more duration of steroid treatment (>1.5 month) $(73 \%$ vs. $50 \%)$ and high mortality related with GvHD/infection ( $40 \%$ vs. $28 \%$ ).

Conclusions: Molecular detection of Human Herpes Virus DNA (specially HHV6 and CMV) in intestinal tissue of patients with allo-SCT is frequent. In GvHD cases seem associated with poor prognosis, reduce responses to steroid therapy, more necessity of steroid treatment and viral infection. It is necessary a prospective study to confirm this preliminary conclusion.

Project IFIMAV-API07/13. 


\section{P745}

Adenovirus in urine sample - High predictive value in development of haemorrhagic cystitis

I. Bil-Lula (1), M. Ussowicz (1), B. Rybka (1), D. WendyczDomalewska (2), R. Ryczan (1), E. Gorczynska (1), K. Kalwak (1), M. Wozniak (1)

(1)Silesian Piasts University of Medicine (Wroclaw, PL);

(2)Central University Hospital (Wroclaw, PL)

Objective: Late-onset hemorrhagic cystitis $(\mathrm{HC})$ in patients undergoing hematopoietic stem cell transplantation (HSCT) is a common complication attributed to adenoviral (AdV) infection by serotypes $3,7,11,21,34,35$. An intensified immunosupression or lack of effective antiviral therapy can lead to devastating clinical course and dangerous complications, such as obstructive uropathy and renal failure. The role of adenoviruses in onset of $\mathrm{HC}$ is still controversial because they are also isolated from urine of patients without $\mathrm{HC}$. The main objective of this study was to analyze the role of AdV infection in development of $\mathrm{HC}$ and predisposing factors for late - onset of AdV-HC.

Methods: 84 HSCT recipients from Pediatric Bone Marrow Transplantation Unit were studied. Urine samples were collected on weekly basis. DNA from urine samples was extracted using QIAamp Viral RNA Mini Kit and tested for AdV-DNA with qualitative PCR method. Positive samples ware quantified by real-time PCR method to determine virus load in clinical sample.

Results: In a period of two years, 13 recipients developed HC after HSCT yielding a cumulative incidence of $15,4 \%$. $77 \%$ cases of $\mathrm{HC}$ were caused by adenoviruses (AdV) $(P=0.000)$. The onset of $\mathrm{HC}$ was strongly related to AdV but not BKV infection in urinary tract $(P=0.0004)$. AdV infection preferentially affected younger, male patients $(P=0.013)$ and recipients grafted from matched unrelated donors. AdV-HC occurred medially 41 days after HSCT and was self-limiting, manifesting in most cases $(90 \%)$ as singular or recurrent mild hemorrhagic episodes. Mean viral load in the AdV-HC was similar to patients infected with AdV, who did not developed HC: $2,5 \times 10^{3}$ and $3,4 \times 10^{3}$ copies $/ \mathrm{ml}$, respectively. $\mathrm{HC}$ of etiology other then AdV was characterized by more severe clinical course. Moreover, we observed worsened survival in patients with $\mathrm{HC}$ $(P=0.002)$ (Figure 1), although the etiology of $\mathrm{HC}$ had not a predictive value.

Conclusion: This study confirmed a strong correlation between AdV viruria and symptoms of $\mathrm{HC}$, thus detection of AdV DNA in urine and quantification of viral load can be used as an early predictor for development of HC. Moreover, microscopic hematuria can be an early warning of AdV infection in urinary tract. A detection of AdV-DNA is a useful trigger in tapering of immunosuppression and administration anty-adenoviral treatment. AdV quantitative monitoring is also helpful in determination of infection severity and in therapy evaluation.

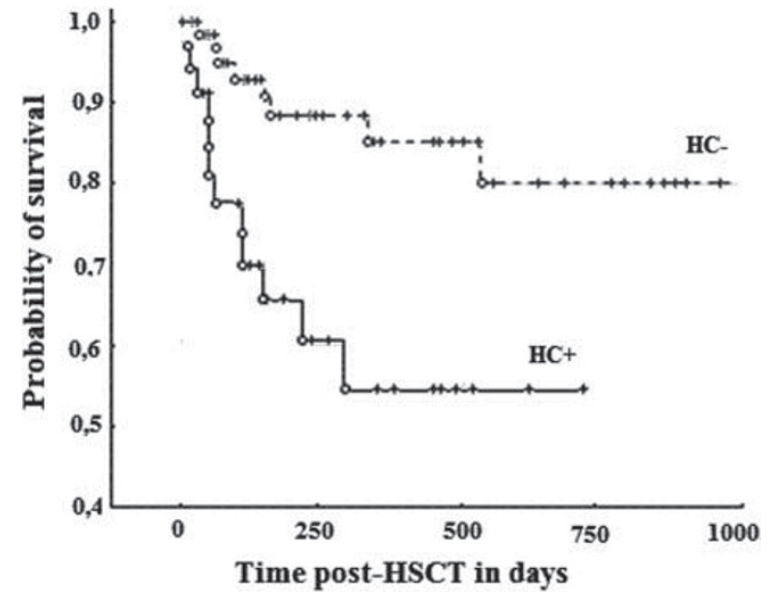

P746

Immunomodulation by IFN-gamma in patients with resistant infections and accompanying Bronchiolitis obliterans following allogeneic stem cell transplantation - first experience in heavily pre-treated patients J. Ammer, K. Landfried, A. Hautmann, B. Holler, D. Wolff, G. Hildebrandt, J. Hahn, R. Andreesen, E. Holler Internal Medicine I (Regensburg, DE)

Objective: Fungal infections such as aspergillosis and severe graft-versus-host disease (GvHD) affecting the lung, especially bronchiolitis obliterans (BO), are the most serious long term complications following allogeneic stem cell transplantation (SCT). Furthermore, patients suffering from BO frequently present with fungal superinfections. Recently, IFN-gamma has been suggested as an additive treatment in haematological patients with infections resistant to conventional antimycotic treatment. In addition, our group has reported heterozygous NOD2/CARD15 single nucleotide polymorphisms (SNPs) indicating a diminished antimicrobial defense as risk factors of BO. As IFN-gamma has been shown to strongly induce NOD2/ CARD15 expression, we speculated that immunomodulation by IFN-gamma might improve antimicrobial defense in patients suffering from $\mathrm{BO}$.

Methods: In a pilot series, 5 patients received a total of 7 courses of subcutaneous injections of IFN-gamma 3 times a week for an average of 182 weeks. Indications were resistant invasive aspergillosis (4 pulmonary, 1 with concomitant $\mathrm{BO}, 1$ sinuses), atypical mycobacterial infection in a patient with $\mathrm{BO}$ and therapy resistant and ventilator dependent $\mathrm{BO}$ with bacterial superinfection in another patient.

Results: Pulmonary aspergillosis and infections improved clinically and by CT scan in all patients. CT signs of BO improved in 2 of 3 patients with $\mathrm{BO}$ and were stable in the 3rd patient. Impressively, the ventilator dependent patient could be wheaned with marked improvement of hypercapnia and another BO patient became oxygen-independent, whereas obstruction did not change in pulmonary function tests. There were no negative effects on extrapulmonary symptoms of acute or chronic GvHD. Hematological side effects were tolerable although moderate anemia might be induced.

Conclusion: Our preliminary results suggest that IFN-gamma can be savely used to treat fungal infections resistant to antimycotic treatment in recipients of allogeneic SCT. In addition, IFNgamma might result in beneficial immunomodulation restoring homeostasis in epithelial GvHD as it is also suggested by recent experimental data on allogeneic SCT in IFN-gamma knockout mice. Further data and a prospective trial addressing this treatment option are needed.

\section{P747}

A phase II dose escalation study of caspofungin for invasive aspergillosis

O. Cornely (1), J.J. Vehreschild (1), M. Rüping (1), D. Arenz (1), S. Schwartz (2), C.P. Heussel (3), G. Silling (4), M. Mahne (1), J. Franklin (1), U. Harnischmacher (1), A. Wilkens (1), F. Farowski (1), M. Karthaus (5), T. Lehrnbecher (6), A. Ullmann (7), M. Hallek (1), A. Groll (8)

(1)University of Cologne (Cologne, DE); (2)Charité Campus Benjamin Franklin (Berlin, DE); (3)University Hospital Heidelberg (Heidelberg, DE); (4)University of Munster (Munster, $D E) ;(5) K l i n i k u m$ Neuperlach (Munich, DE); (6)University of Frankfurt (Frankfurt, DE); (7)Johannes Gutenberg University (Mainz, DE); (8)University Children's Hospital (Munster, DE)

Objectives: Treatment of invasive aspergillosis (IA) fails in up to $50 \%$ and mortality is at least $30 \%$. Antifungal combination treatment has not been proven to be beneficial and dose escalation with liposomal amphotericin B did not improve outcome. New approaches are needed.

Methods: High dose caspofungin was investigated in an escalating Fibonacci approach in IA defined according to modified 
EORTC/MSG criteria. In cohorts at $70 \mathrm{mg}, 100 \mathrm{mg}, 150 \mathrm{mg}$, or $200 \mathrm{mg}$ QD, 8 patients each were to receive caspofungin first-line treatment for proven/probable IA for up to 28 days. Dose limiting toxicity was defined as 2 of 8 patients in the same cohort with the same grade $\geq 4$ non-haematological treatment-related adverse event (TRAE), or 4 of 8 patients with a grade $\geq=3$ non-haematological TRAE. If no dose-limiting toxicity was reached, 12 additional patients were enrolled in the $200 \mathrm{mg}$ cohort. Patients unevaluable for toxicity or pharmacokinetic analysis were replaced.

Results: A total of 46 patients were treated in the 4 cohorts $(9$, $8,9,20$ pts). IA was proven in $2.2 \%$ and probable in $97.8 \%$. Patient characteristics were as follows: Median age 61 years (min 18.3, max 73.7); 21/46 (45.7\%) female. Underlying diseases distribution was: AML 50\%, ALL 8.7\%, lymphoma 19.6\%, chronic lymphocytic leukaemia $10.9 \%$, other $10.9 \%$. Median duration of treatment was 24.5 days. Two (4.3\%) patients with treatment durations $\leq 5$ days were replaced for pharmacokinetic analysis, but evaluated for safety and efficacy. No dose-limiting toxicity was found by investigator or DSMB assessment. At end of treatment (EOT) complete plus partial response, was achieved in the 4 cohorts in $4 / 9,3 / 8,6 / 9,12 / 20$ patients, i.e. $25 / 46(54.3 \%)$ of the total population. Stable disease was achieved in 4 patients $(8.7 \%), 17(37 \%)$ patients failed treatment. Overall survival at 12 weeks was $76.1 \%$. After a 12 week follow-up attributable mortality was $8.7 \%$. Death due to malignancy occurred in $10.9 \%$, to sepsis in $8.7 \%$.

Conclusions: In the first-line treatment of proven or probable invasive aspergillosis no dose-limiting toxicity of caspofungin at doses up to $200 \mathrm{mg}$ QD was found. Complete plus partial response rates at EOT were $54.3 \%$ after dose-escalated caspofungin treatment, and thus in the range of the success rates previously reported with voriconazole and liposomal amphotericin B. Twelve weeks after start of treatment the $23.9 \%$ overall mortality was lower than found in the literature.

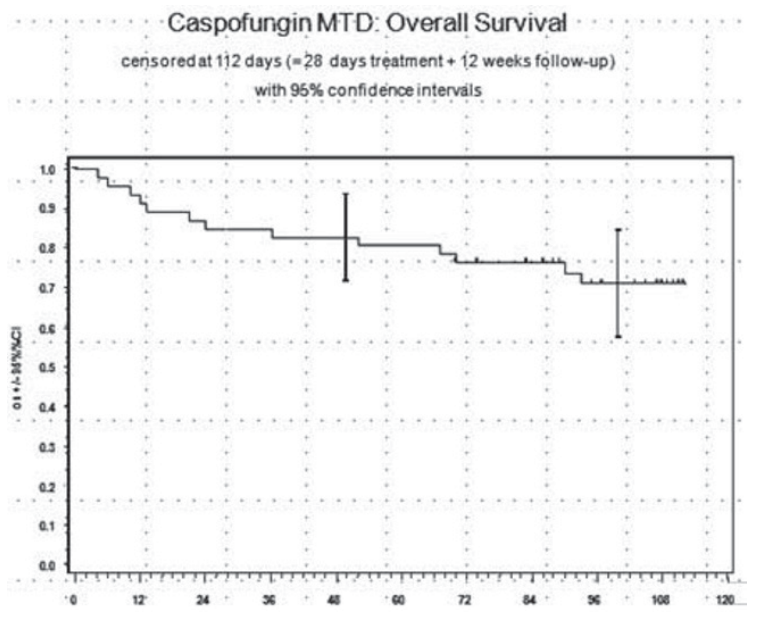

\section{P748}

Voriconazole and posaconazole serum concentrations in allogeneic stem cell recipients - a single-centre observation W.J. Heinz, A.-K. Ulrich, U. Lenker, J. Zirkel, G. Stuhler, A. Kuhn, D. Schirmer, H. Einsele, H. Klinker

University of Wurzburg Medical Centre (Wurzburg, DE)

Objectives: Antifungal therapy and prophylaxis is a common indication for patients after allogeneic stem cell transplantation. Voriconazole (VRC) and posaconazole (PSC) are most often the drug of choice for both indications. Bioavailability can be hampered by gastrointestinal disorders like GVHD or other malabsorptions, as well as by renal or liver impairment or drug interactions. Therefore ECIL guidelines recommend drug monitoring for both compounds.
Methods: Serum samples from patients receiving either VRC or PSC were collected from all hospitalized patients on SCT unit twice weekly. Trough levels of both azoles were determined by a HPLC based method, described previously. Patient characteristics, comedication and treatment duration are documented.

Results: 147 serum concentrations were determined from a total number of $n=47$ patients (20 female, 27 male), including 78 VRC- $(n=27, f=10, m=17)$ and 69 PSC-samples $(n=20$, $\mathrm{f}=8, \mathrm{~m}=12$ ). The patients mean concentrations were between 33 and $6257 \mathrm{ng} / \mathrm{ml}$, median $1107 \mathrm{ng} / \mathrm{ml}$ (confidence interval $1016-2240 \mathrm{ng} / \mathrm{\mu l}$ ) for voriconazole and between 95 and 1634 $\mathrm{ng} / \mathrm{ml}$, median $685 \mathrm{ng} / \mathrm{ml}$ (confidence interval 455-915 ng/ml) for PSC.

Evaluating each serum concentration for VRC a mean of 1660 $\mathrm{ng} / \mathrm{ml}$ (confidence interval 1236 and $2083 \mathrm{ng} / \mathrm{ml}$ ), standard deviation $1897 \mathrm{ng} / \mathrm{ml}$, a median of $1081 \mathrm{ng} / \mathrm{ml}$ and a maximum of $7783 \mathrm{ng} / \mathrm{ml}$ have been determined. For posaconazole the results were $801 \mathrm{ng} / \mathrm{ml}$, (657 and $946 \mathrm{ng} / \mathrm{ml}), 603 \mathrm{ng} / \mathrm{ml}$, $626 \mathrm{ng} / \mathrm{ml}$ and $2799 \mathrm{ng} / \mathrm{ml}$ respectively. $14 \mathrm{VRC}$ and $12 \mathrm{PSC}$ through levels have been very low, defined as below $200 \mathrm{ng} / \mathrm{ml}$, another 14 VRC and 13 PSC concentrations have been only between 200 and $500 \mathrm{ng} / \mathrm{ml}$. In 3 samples more than $6000 \mathrm{ng} / \mathrm{ml}$ VRC could be detected. Correlation between drug levels and toxicity are further evaluated.

Conclusion: For most SCT recipients satisfactory VRC and PSC mean concentrations could be determined. Nevertheless for single trough levels a broad range can be shown and this might result in periods of insufficient therapy or prophylaxis, which supports the recommendation of therapeutic drug monitoring.

\section{P749}

Should pre-emptive antifungal approach be used in febrile neutropenia after autologous peripheral blood stem cell transplantation?

L. Castagna, S. Bramanti, A.I. Gregorini, A. Santoro

Istituto Clinico Humanitas (Rozzano, IT)

Background: Empirical antifungal therapy based on the persistence of fever after 5-7 days of broad spectrum antibiotic treatment is considered the standard treatment in neutropenic patients. This approach can result in overtreatment, increasing toxicity and cost. The aim of this retrospective study was to evaluate the feasibility of pre-emptive strategy.

Materials and methods: We reviewed records of 386 patients who underwent autologous hematopoietic stem cell transplantation (HSCT) for hematological malignancies from January 2002 to December 2008. Patients who met usual criteria to start empirical antifungal therapy such as fever $\left(>38^{\circ} \mathrm{C}\right)$ at least for 5 consecutive days or relapsing after introduction of antibiotic treatment were included. Conditioning regimen and patients characteristics are reported in Table 1. All patients received a yeast prophylaxis with fluconazole $100 \mathrm{mg}$ from the beginning of conditioning regimen to the neutrophil recovery. A diagnostic work up was performed, consisting of chest X-ray, thoracic high resolution computed tomography (HRCT), galactomannan enzyme immunoassay (GM-EIA), urine Legionella antigenemia and clinical oriented investigations. Patients with HRCT positivity underwent to bronchoalveolar lavage or lung biopsy (Figure 1). Primary endpoints were the impact of pre-emptive strategy on antifungal drugs administration and invasive fungal infections (IFI) related mortality.

Results: Twenty one neutropenic febrile episodes were analyzed in this study. A pre-emptive antifungal treatment was given in 5 episodes driven by HRCT positivity. Two episodes met EORTC criteria for IFI possible, the remaining three were classified as pneumonia without microbiologic identification (2) and with positive blood cultures (1). Despite a positive HRCT, three febrile episodes were not given any antifungal therapy since there was a microbiologic identification from blood cultures (2) and a non IFI suggestive imaging (1). No antifungal therapy was started in the remaining 13 episodes, in which diagnostic work-up revealed bacteriemia (4), local catheter 
infection (3), neutropenic typhlitis (2) or fever of unknown origin (FUO) (5). Finally we reported a $76 \%$ reduction in the use of antifungal drugs with an IFI-related mortality of $0 \%$.

Conclusion: The pre-emptive approach is safe and it reduces notably the rate of empirical antifungal use in febrile neutropenic patients after autologous HSCT without increasing IFI-related mortality.
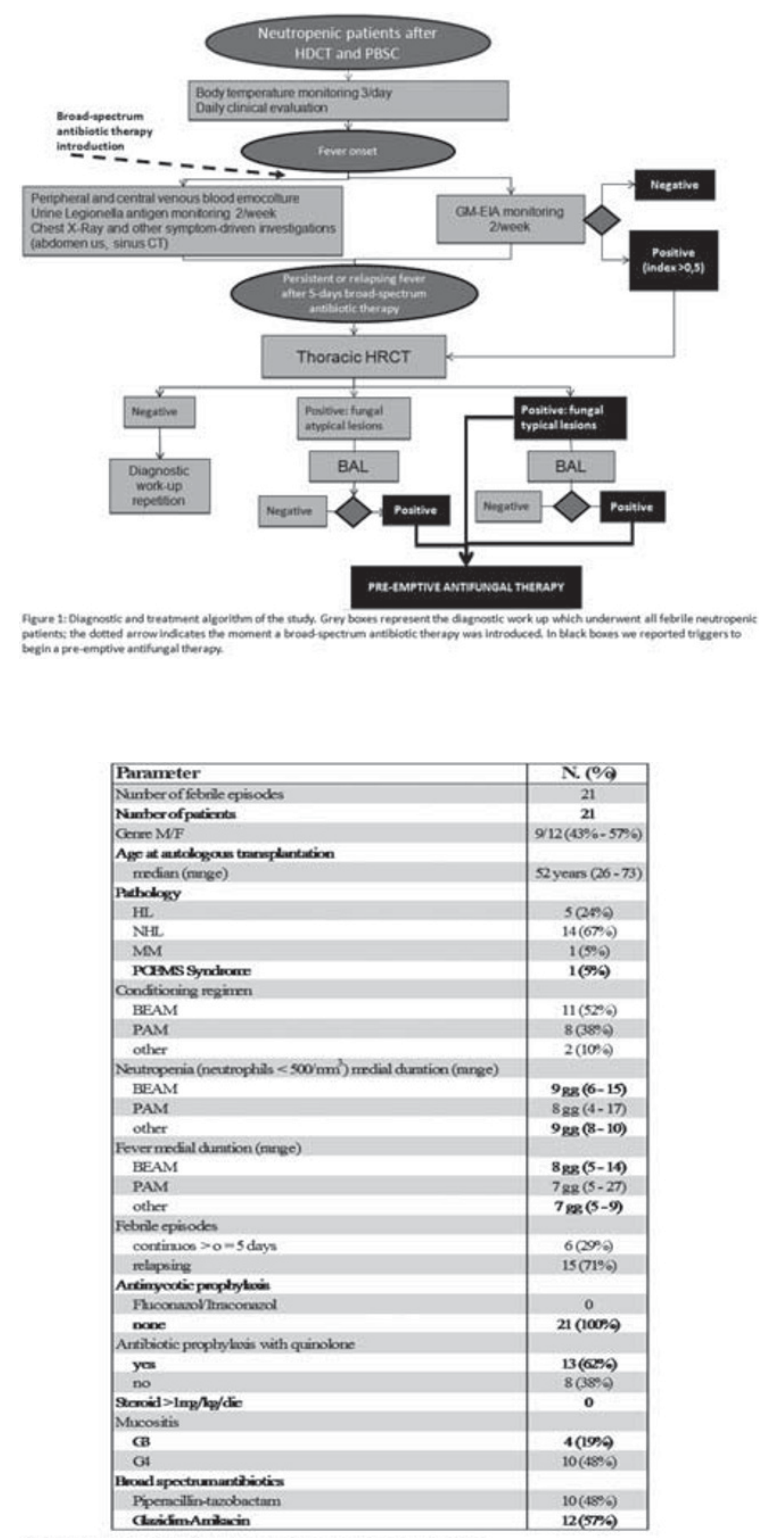

Table 1 Characteristics of included patients and febrile episodes.

HL: Hodplin Lymphoma; NHLL Non Hodgkin Lymphoma; MM: Multiple Myeloma; BEAM: Carmustine, Etoposide, Cytarabine, and Melphalan; PAM: Melphalan $200 \mathrm{mg} / \mathrm{m}^{2}$.
P750

Foscarnet for cytomegalovirus infection after allogeneic haematopoietic stem cell transplantation from related donor

R. Suzuki (1), M. Asakura (1), K. Ikegame (2), S. Yoshihara (2), S. Taniguchi (3), T. Mori (4), T. Etoh (5), A. Takami (6), T. Yoshida (7), T. Fukuda (8), K. Hatanaka (9), H. Kanamori (10), T. Yujiri (11), Y. Atsuta (1), H. Sakamaki (12), H. Ogawa (2) (1)Nagoya University (Nagoya, JP); (2)Hyogo College of Medicine (Nishnomiya, JP); (3)Totranomon Hospital (Tokyo, JP); (4)Keio University (Tokyo, JP); (5)Hamanomachi Hospital (Fukuoka, JP); (6)Kanazawa University (Kanazawa, JP); (7)Toyama Prefectural Hospital (Toyama, JP); (8)National Cancer Center (Tokyo, JP); (9)Rinku General Medical Center (Izumisano, JP); (10)Kanagawa Cancer Center (Yokohama, JP); (11)Yamaguchi University (Ube, JP); (12)Komagome Hospital (Tokyo, JP)

Foscarnet is an active agent against CMV infection after hematopoietic stem cell transplantation (HSCT), as well as ganciclovir. In early studies the dose-limiting toxicities of foscarnet were found to be nephrotoxicity and neurotoxicity, but later studies showed these adverse events were less frequent and did not resulted in the problems for clinical use. Most studies in the literature only involved patients who had received foscarnet as an initial treatment. Therefore, we conducted a survey in Japan of the use of foscarnet against CMV infection after related HSCT and compared its efficacy and toxicity in patients with and without prior ganciclovir treatment. Foscarnet was used in 320 patients ( $F: M=149: 171)$ with a median age of 45 years (range: 15-72). CMV antibody was positive in both the patient and donor in 189 pairs (59\%), in the patient only in 22 cases $(7 \%)$, and in the donor only in 8 cases $(3 \%)$, and it was negative in both patient and donor in 4 pairs $(1 \%)$. The purpose of administration was CMV disease in 65, preemptive use in 248 and prophylaxis in 7 . CMV disease included 48 with gastroenteritis, 12 with pneumonia, 2 with retinitis, and one each for hepatitis, encephalitis, and cystitis. Totally, 194 patients had a history of prior ganciclovir treatment. The reason for foscarnet use was insufficient therapeutic effect of prior ganciclovir in 99, and adverse event including myelosuppression in 95. The initial dose of foscarnet ranged from $7 \mathrm{mg} / \mathrm{kg}$ to $216 \mathrm{mg} / \mathrm{kg}$ (median: $88 \mathrm{mg} / \mathrm{kg}$, Figure 1). The dose was significantly higher in the patients who had received prior treatment with ganciclovir (range: $10-216 \mathrm{mg} / \mathrm{kg}$, median: $91 \mathrm{mg} / \mathrm{kg}$ ) than those who had not (range: $7-180 \mathrm{mg} / \mathrm{kg}$, median: $72 \mathrm{mg} / \mathrm{kg})(P<0.0001)$. The response rate in symptom was $52 \%$ for the CMV disease patients. Antigenemia disappeared in $77 \%$ of the preemptive treatment and improved in $13 \%$ of the patients. No outbreak of CMV disease was recognized. The total effectiveness of therapeutic and preemptive use was significantly higher for patients without prior ganciclovir (91\% vs. $76 \%, P=0.001)$. Adverse events of grade 3 or higher were recognized in $24 \%$, including electrolyte abnormalities in $11 \%$, neutropenia in $8 \%$, and thrombocytopenia in $8 \%$. Renal damage was only observed in $3 \%$ of patients. Foscarnet was concluded to be a safe and effective anti-CMV agent and to be a suitable alternative to ganciclovir. Further prospective evaluations of foscarnet are warranted.

\section{P751}

Efficacy of micafungin in neutropenic patients with invasive candidiasis and candidaemia

B. Sirohi (1), P. Chandrasekar (2), N. Seibel (3), M. Ruhnke (4), R. Powles (5), T. Taylor (6)

(1)Max Cancer Centre (New Delhi, IN); (2)Wayne State University School of Medicine (Detroit, US); (3)National Institutes of Health (Bethesda, US); (4)Campus Charité Mitte, Humboldt University (Berlin, DE); (5)Parkside Oncology Clinic (Wimbledon, UK); (6)Astellas Pharma Europe (Staines, UK)

Patients with neutropenia are at higher risk of contracting invasive fungal infections, e.g. invasive candidiasis. Micafungin 
(MICA) is an echinocandin that has demonstrated non-inferiority to both caspofungin and liposomal amphotericin B for the treatment of invasive candidiasis and candidemia in 2 prospective Phase 3 trials. A pooled post-hoc analysis that examined efficacy in 73 neutropenic patients who received MICA as treatment for confirmed invasive candidiasis/candidemia (IC/C) in these trials, is presented. Patients were defined as neutropenic if the baseline absolute neutrophil count (ANC) value was $<500$ cells/microL. If no ANC value was available, the white blood cell count was used. Patients were further divided into those with neutropenia of duration of $\geq 1, \geq 8, \geq 15$, and $\geq 22$ days. Of the IC/C patients, $66(90 \%)$ had candidemia, and $7(10 \%)$ had invasive candidiasis. C. tropicalis was the most often species isolated (in nearly $40 \%$ of patients) followed by C. albicans and C. krusei in about $20 \%$ of patients. Sixty three percent of the patients had a haematological malignancy (without transplant), and 19 patients $(26 \%)$ were stem cell transplant (SCT) recipients. Overall success (mycological and clinical response) was seen in $66 \%$ of neutropenic patients. Similar results were seen in children $<16$ years of age $(n=13)$ and adults. The number of patients having overall success was comparable in patients with different durations of neutropenia (Table 1). Although the patient numbers were small, a longer duration of neutropenia seemed to show a lower overall success in SCT patients. In conclusion, these results show that micafungin is an effective treatment for confirmed IC/C in neutropenic patients.

Table 1. Efficacy results by duration of neutropenia.
\begin{tabular}{|l|l|l|l|l|}
\hline & $\begin{array}{l}\text { Neutropenia } \\
\text { for } \geq 1 \text { day } \\
(n=73)\end{array}$ & $\begin{array}{l}\text { Neutropenia } \\
\text { for } 28 \text { days } \\
(n=30)\end{array}$ & $\begin{array}{l}\text { Neutropenia } \\
\text { for } \geq 15 \text { days } \\
(n=17)\end{array}$ & $\begin{array}{l}\text { Neutropenia } \\
\text { for } \geq 22 \text { days } \\
(n=10)\end{array}$ \\
\hline & $n(\%)$ & $n(\%)$ & $n(\%)$ & $n(\%)$ \\
\hline Overall success & $48(66)$ & $23(77)$ & $11(65)$ & $5(50)$ \\
\hline $\begin{array}{l}\text { Mycological } \\
\text { eradication/presumed } \\
\text { eradication }\end{array}$ & $56(77)$ & $25(83)$ & $13(76)$ & $6(60)$ \\
\hline $\begin{array}{l}\text { Complete/partial } \\
\text { clinical response }\end{array}$ & $53(73)$ & $24(80)$ & $11(65)$ & $5(50)$ \\
\hline
\end{tabular}

\section{P752}

Use of "high flow" totally implantable central venous access ports for haematopoeitic stem cell transplantation: a prospective study

A. Abdelkefi, O. Ben Gaied, S. Ladeb, L. Torjman, A. Lakhal, A. Ben Hassen, T. Ben Othman

Centre National de Greffe de Moelle Osse (Tunis, TN)

Objectives: A central venous access is always necessary for the management of patients receiving a hematopoietic stem cell transplantation (HSCT). Tunnelled, cuffed silastic catheters are the device of choice for these patients. The aim of this prospective study was to investigate the usefulness of a new "high flow" single lumen port device in these patients.

Methods: Between December 2007 and November 2009, patients with haematological malignancies received a totally implantable central venous access port (TIAP) prior to HSCT. All patients received the same type of port [silicone, 10 French, high flow rate $(3100 \mathrm{ml} / \mathrm{h})$, Reference 40010 , Laboratoires Perouse, Ivry le Temple, France]. All devices were inserted under local anesthesia through direct puncture of the right subclavian vein. All infusions, including the graft itself and all blood drawings, were performed via the port. TIAP was also used for peripheral blood stem cell apheresis in patients receiving autologous stem cell transplantation. For apheresis, the TIAP was punctured with a 19G, 20 mm curved Huber needle. Portrelated bloodstream infection was defined according to Infectious Disease Society of America guidelines.

Results: Eighty five TIAP were placed in 85 patients (50 allogeneic and 35 autologous) [median age: 37 years (20-56 years); 38 female and 47 male], and remained in place for a cumulative duration of 16230 days in situ (range 28-655 days).
Fifty patients received a myeloablative conditioning regimen followed by an allogeneic stem cell transplantation. A total of 53 aphereses were performed in 35 patients receiving an autologous stem cell transplantation. No pneumothorax occurred. Port-related bloodstream infection (candida parapsilosis and staphylococcus epidermidis) occurred in 2 cases (2/85, 2.3\%; 0.12 event per 1000 days). These ports were removed. No port-related thrombosis occurred. No port-related deaths were observed.

Conclusion: To our knowledge, there are no prospective studies investigating the use of TIAP in patients receiving myeloablative conditioning followed by allogeneic SCT. In our study, the use of "high flow" totally implantable ports has resulted in a good option for long-term access to central veins and delivery of myeloablative conditioning followed by allogeneic SCT. Furthermore, the use of TIAP for peripheral blood stem cell apheresis and autologous transplantation was safe and effective.

\section{P753}

Risk pattern of EBV-reactivation following allogeneic stem cell transplantation

A. Bauhardt, E. Dammann, M. Stadler, M. Eder, J. Krauter, A. Ganser, S. Buchholz

Hannover Medical School (Hannover, DE)

Background: EBV-reactivation is a rare complication in patients undergoing allogeneic stem cell transplantation (HSCT) and can lead to post-transplant lymphoproliferative disorder (PTLD) with high morbidity and mortality. Monitoring of EBV DNA levels and preemptive therapy with rituximab may avoid PTLD. We evaluated 197 patients to analyse risk factors of EBV-reactivation and PTLD.

Methods: Patients transplanted in our centre from September 2005 to May 2009 with AML $(n=87), \operatorname{ALL}(n=24)$, secondary $\operatorname{AML}(n=47), \operatorname{MDS}(n=25), \operatorname{MDS} / \operatorname{MPS}(n=4)$ and MPS $(n=10)$ were included in this analysis. Exclusion criteria were as follows: death before day 18, T-Cell-depletion, syngeneic transplantation. HSCT was performed in 1 st CR/1st CP $(n=66),>1$ st CR $(n=16)$ or recurrent/progressive disease $(n=115) .145$ pts. were treated with reduced intensity and 52 with myeloablative conditioning, 176 pts. received Thymoglobuline Genzyme $(n=76)$ or ATG Fresenius $(n=100)$ during conditioning. GvHD prophylaxis was CSA-based. Peripheral blood stem cells and BM were used in 185 and 12 patients respectively, from MRD $(n=57)$, MUD $(n=95)$, and MMUD/MMRD $(n=45)$. Median age of the pts. (107 male, 90 female) was 52 years (18-71). Median follow-up was 13.3 months (0.6-49.1).

Results: EBV-reactivation was detected in 20 pts. (median day $+57(28-166)$ ) by PCR-monitoring of EBV-DNA in peripheral blood; 10 of these pts. developped PTLD and 3 died due to PTLD. 16 pts. with clinical symptoms were treated with rituximab \pm - chemotherapy. Underlying disease, stage of disease at transplantation, conditioning regimen, GvHD-prophylaxis, age, type of donor, sex-match, use of ATG or Thymoglobulin and incidence of aGvHD were evaluated to develop a risk pattern for EBV-reactivation.

In multivariate analysis we confirmed significant associations between donor type (MRD vs. MUD/MMUD/MMRD) and use of Thymoglobulin and increasing risk of EBV-reactivation and PTLD. Patients transplanted from MRD received no $(n=20)$ or reduced doses of anti T-cell antibodies $(n=37)$. In the Pearson Chi-Square-test use of Thymoglobulin $(P=0.002)$ and donor type $(P=0.007)$ were also independent risk factors for EBVreactivation.

Conclusion: In this single-centre analysis the donor type and treatment with Thymoglobulin were independent risk factors to develop EBV-reactivation and PTLD. These subgroups should be monitored for EBV reactivation for at least 6 months after allogeneic stem cell transplantation to initiate early therapy. 
P754

Toxoplasma gondii infection in 35 patients submitted to haematopoietic stem cell transplantation in a single-centre C. Bonfim, R. Vasconcelos, S. Fortier, J. Morando, C.A. Cunha, F. Piazzera, E.N. Coutinho, V. Funke, M. Bittencourt, R. Pasquini, J. Zanis-Neto

Federal University of Parana (Curitiba, BR)

There is a high prevalence of Toxoplama gondii in many countries around the world and many patients (pts) are at risk of reactivation after hematopoietic stem cell transplantation (HSCT). In this setting, toxoplasmosis can be a very serious disease with a high mortality rate.

Objective: Analyze the outcome of 35 pts who developed toxoplasmosis after allogeneic HSCT in a single institution.

Material and methods: Period: 04/1984-05/08; Age: 1-45 ys (M:20 ys). Gender: 17M/18F. 91\% had a positive serology (IgG) before HSCT.

Diagnosis: Bone Marrow failures:14pts; Malignant diseases: 21 pts. Preparatory regimen: Myeloablative: 23 pts; Reduced intensity: 12 pts.Type of donor: related (26 pts); unrelated (9 pts). Toxoplasmosis was classified as definitive, probable, possible or isolated infection according to the diagnostic criteria proposed by EBMTR. In our BMT Unit, PCR tests were performed in blood and/or cerebrospinal fluid from March 2000 in all suspected cases. Toxoplasmosis was identified by PCR, biopsies or necropsy. Statistical analysis was performed using SPSS. Overall survival was estimated using the Kaplan-Meier method. Multivariate analysis was performed by Cox method. Results: 9 pts are alive between 1260-6385 days after HSCT (M: 3956 d) with an OS of 30,9\% (5 ys). Toxoplasmosis was diagnosed between $26-3065$ days (M: 81 ) and $83 \%$ occurred during the first year after HSCT. It was defined as definitive in 23 pts (13 pts-necropsy), probable: 1 pt; possible: 6 pts and infection: 5 pts. Site of infection: central nervous system (CNS): 14 pts; disseminated: 3 pts; lung: 8 pts; liver: 1 pt; ocular: 2 pts; skin: 1 pt; heart: 1 pt. Toxoplasmosis infection (positive PCR only): 5 pts. Primary graft failure occurred in $5 / 35$ pts and all died (4 deaths were related to toxoplasmosis). Acute-GVHD grade II-IV was observed in 22 pts while 11 had Chronic-GVHD (extensive: 9 pts). 26 pts died between 38-4378 days after SCT (M: 90 days) and toxoplasmosis was considered the major cause of death in 14 pts. Only 2 pts with a definitive diagnosis of toxoplasmosis are alive and well. In univariate analysis, survival was significantly influenced by age, type of preparatory regimen and type of disease but no factor reached statistical significance in the multivariate analysis.

Conclusions: Almost half of the patients were diagnosed only after death. We observed a high mortality in this group of patients and a high prevalence of CNS infection.

\section{P755}

Efficacy and safety of caspofungin in paediatric allogeneic haematopoietic stem cell transplantation: a single-centre study

A. Dony (1), M.P. Goutagny (1), Y. Bertrand (1), A. Bienvenu (2), J.M. André (1), N. Bleyzac (1), V. Mialou (1)

(1)IHOP (Lyon, FR); (2)Hôpital de la croix rousse (Lyon, FR)

The aim of this study was to evaluate the efficacy and the tolerance of caspofungin during allogeneic Haematopoietic Stem Cell Transplantation (HSCT).

Methods: We performed a retrospective analysis of 123 consecutive children who received HSCT between January 2004 and December 2008. Sixty one patients were treated by caspofungin at least 3 days, from the beginning of conditioning regimen until the 60th day post-transplant (dose: $50 \mathrm{mg} / \mathrm{m}^{2}$ ). Three groups of caspofungin treatment have been identified: group 1 received empiric therapy, group 2 received secondary prophylaxis and group 3 was treated for fungal infection occurring during HSCT process. In group 3 caspofungin was given in combination with voriconazole (3) or liposomal amphotericin
B (2). Success of the treatment was evaluated according to following criteria: non-occurrence of fungal infection for group 1 , absence of fungal infection recurrence for group 2 and fungal infection resolution in group 3 .

Results: Among the 61 patients treated by Caspofungin, 36 had HSCT for malignancies and 25 for other diseases. Median age at HSCT was 9,2 years. Donors were of unrelated origin for 38 patients, familial HLA identical siblings for 20 and familial haploidentical for 3 . Forty two patients were in the group 1 (empiric), 14 in group 2 (secondary prophylaxis for aspergillosis $(n=4)$ or for disseminated candidiasis $(n=9)$, missing data for 1$)$ and 5 in group 3 (as curative treatment for aspergillosis $(n=4)$ or candidiasis $(n=1)$ ). Results are detailed in the Table 1 .

In group 1 the only treatment failure was the occurrence of a pulmonary aspergillosis.

In group 2, there was no recurrence of the previous fungal infection.

In group 3, all patients received antifungal bitherapy. Among them, 2 patients died of fungal infection (one disseminated candidiasis and one because obstructive endobronchial aspergillosis).

Concerning the tolerance, among the 3 groups of treatment only one therapy has been discontinued, for suspected hepatic toxicity of Caspofungin (not confirmed), in a patient presenting at the same time a grade 4 hepatic acute Graft Versus Host Disease.

Conclusion: Caspofungin displayed favourable safety and may have antifungal efficacy in pediatric allogeneic HSCT.

\begin{tabular}{|c|c|c|c|c|}
\hline \multicolumn{5}{|c|}{ Table 1-Results } \\
\hline $\begin{array}{c}\text { Number } \\
\text { of } \\
\text { patients }\end{array}$ & $\begin{array}{c}\text { Median duration } \\
\text { of treatment }\end{array}$ & Efficacy & $\begin{array}{c}\text { Number of treatment } \\
\text { discontinuation due to toxicity }\end{array}$ \\
\hline $\begin{array}{c}\text { Group } \\
1\end{array}$ & 42 & 10 days (3-77) & $98 \%(n=41)$ & 0 \\
\hline $\begin{array}{c}\text { Group } \\
2\end{array}$ & 14 & 40 days (30-103) & $100 \%(n=14)$ & 1 \\
\hline $\begin{array}{c}\text { Group } \\
3\end{array}$ & 5 & 43 days (8-59) & $\begin{array}{c}50^{*} \%(n=2 / 4) \\
\% 1 \mathrm{NE}\end{array}$ & 0 \\
\hline
\end{tabular}

P756

Randomized PCR-based therapy and risk factors for invasive fungal infection following reduced-intensity conditioning and haematopoietic stem cell transplantation O. Blennow (1), M. Remberger (2), L. Klingspor (3), B. Omazic (4), K. Fransson (4), P. Ljungman (5), J. Mattsson (4), O. Ringdén (4)

(1)Div of Infectious Diseases (Stockholm, SE); (2)Department of Clinical Immunology and Transfusion Medicine (Stockholm, SE); (3)Division of Clinical Bacteriology (Stockholm, SE); (4)Center for Allogeneic Stem Cell Transplantation (Stockholm, SE); (5)Department of Haematology (Stockholm, SE)

Background: Invasive fungal infections (IFIs) are major complications after allogeneic hematopoietic stem cell transplantation (HSCT). Polymerase chain reaction- (PCR-) based assays able to detect DNA from Aspergillus and Candida species have been reported to precede clinical diagnosis of IFI, enabling pre-emptive or early treatment. We performed a prospective study to evaluate a PCR-based pre-emptive approach.

Methods: Ninety-nine patients undergoing reduced-intensity conditioning (RIC) HSCT were followed once a week with fungal PCR during the first 100 days post-transplantation. Patients who tested positive were randomized to treatment with liposomal amphotericin B or to no treatment. After day 100, PCR tests were performed only on clinical suspicion of IFI.

Results: Forty-one patients had at least one positive PCR test (Aspergillus, $n=18$; Candida, $n=29$; both, $n=6$ ). A single positive PCR test was not associated with IFI irrespective of whether or not there had been administration of pre-emptive liposomal amphotericin B. After day 100, PCR tests for Aspergillus did not contribute to diagnosis of proven or probable 
invasive aspergillosis (IA). The cumulative incidence rates of proven or probable IFI, IA, and invasive candidiasis during the first year after transplantation were $12 \%, 9 \%$, and $2 \%$, respectively. Acute graft-versus-host disease (GVHD) of grades II CIV $(P=0.0014)$, CMV-seronegative recipient with CMV-seropositive donor $(P \leq 0.001)$, and conditioning with alemtuzumab $(P=0.014)$ were significant risk factors for developing IA in a multivariate model.

Conclusions: PCR of peripheral blood is a poor predictor of invasive fungal infection after allogeneic HSCT.

Cumulative incidence of Invasive Aspergillosis for patients with 0,1 , or 2 of the risk factors identified in multivariate analysis; aGVHD: acute graft-versus-host disease; conditioning with alemtuzumab; CMV R-D+: $\mathrm{CMV}$-seropositive donor and CMV-seronegative recipient.

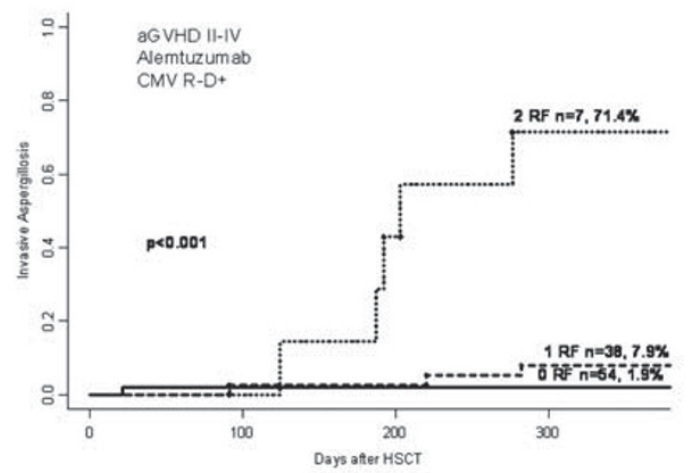

Positive PCR tests and IFI within 100 days of transplant.

\begin{tabular}{|c|c|c|c|c|}
\hline PCR lest & $\begin{array}{l}\text { Number ef pasithe } \\
\text { ists per patient }\end{array}$ & $\begin{array}{l}\text { Number of } \\
\text { patients }\end{array}$ & 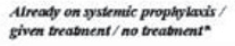 & $\begin{array}{l}\text { Proven or } \\
\text { probable IFI }\end{array}$ \\
\hline \multirow[t]{2}{*}{ Aspergathass } & 1 & 15 & $0 / 4 / 11$ & $0 / 0 / 1^{\circ}$ \\
\hline & $>1$ & 3 & $0 / 2 / 1$ & 0 \\
\hline \multirow[t]{2}{*}{ Cantican } & 1 & 26 & $7 / 6 / 13$ & 0 \\
\hline & $>1$ & $3^{t}$ & $1 / 2 / 0$ & $13 / 0 / 0$ \\
\hline
\end{tabular}

- Within 14 days of positivo PCR test - Probable invasive aspergillosis.

- 1 patient had consecutive positive Aspergillus PCR tests and was treated with liposomal amphotericin B. 2 patients had consecutive positive Candicta PCR tests; both were troated with hposomal amphotericin $B$. Catheter-related candidemia while in ICU for non-transplanted complication. PCR test became positive on day after growth in blood cullure.

Abbreviations: IfFt: invasive fungal infection.

\section{P757}

Bacteraemia with Gram-negative resistant pathogens is frequent in paediatric HSCT patients, but not associated with higher mortality

D. Averbuch, P. Stepensky, R. Or, J. Strahilevitz, M. Shapira, I.B. Resnick, D. Engelhard

Hadassah Medical Center (Jerusalem, IL)

Introduction: Blood stream infections (BSI) are a well-known cause of morbidity and mortality in hematopoietic stem cell transplant (HSCT) patients. In the recent years, a rise in Gram negative rods (GNR) and increase in resistance have been observed. Aim: To analyze if bacteremia with resistant Gram negative pathogens is associated with higher mortality in pediatric HSCT patients.

Methods: A single center study was done on BSI in children who were followed for 1 year. Pseudomonas and Acinetobacter spp that exhibited resistance to three or more antibiotic classes were defined as multi-drug resistant (MDR).

Results: During 2006-2008, 100 children, 58 male, 2 weeks to 17 years old (median 6 years) underwent 110 SCT (101 allogeneic, 9 autologous), for malignancy/premalignancy $(n=62)$ or nonmalignant condition $(n=38) ; 48$ from matched family members, 35 haploidentical, 17 matched unrelated donor and 1 cord blood. 114 pathogens were cultured from blood during the first year of 60 transplantations in 55 patients, including GNR ( $n=63 ; 55 \%)$, Gram positive pathogens (GP, $n=43$; $38 \%)$ and Candida $(n=8 ; 7 \%)$. GNR included $34(30 \%)$ enterobacteriacea, $27(24 \%)$ non-fermenting pathogens.

Resistant GNR included: 19/34 (56\%) enterobacteriaceae resistant to 3rd generation cephalosporins, 9/63 (14\%) carbapenem resistant GNR, including 5 Stenotrophomonas spp., 3 Acinetobacter spp and $1 \mathrm{P}$. aeruginosa. 2/12 P. aeruginosa and 4/7 Acinetobacter spp. were MDR. 12/63 (19\%) GNR were nonsusceptible to ciprofloxacin.

During the first year after HSCT, $10(10 \%)$ children died within 30 days after the BSI episode, in 3 children multiple pathogens were cultured from blood during the 30 days. The 30 -day mortality rate was $3 / 8(38 \%, P=0.013)$ for Candida; $4 / 13(31 \%$, $P=0.02)$ for $\mathrm{E}$. coli; $2 / 12(17 \%, P=0.4)$ for $\mathrm{P}$. aeruginosa, $4 / 43$ $(9 \%, P=0.96)$ for GP pathogens. The 30 -day mortality was associated with $2 / 25(8 \%)$ resistant and $6 / 38(16 \%)$ susceptible GNR episodes $(P=0.36)$

Conclusions: There is a high proportion of GNR bacteremia in transplant children, with a significant high proportion of resistance. Candida and E. coli BSI are associated with 30-day mortality. The mortality associated with BSI after HSCT is significant, but not associated with resistant bacteria.

\section{P758}

Allogeneic haematopoetic cell transplantation in hepatitis $B$ surface antigen positive recipients

P. Topcuoglu, E. Soydan, R. Idilman, C. Ustun, O. Arslan, M. Ozcan, O. Ilhan, M. Beksac, M. Arat, G. Gurman Ankara University (Ankara, TR)

The incidence of hepatotoxic morbidity and mortality after allogeneic hematopoietic cell transplantation (Allo-HCT) in patients positive for hepatitis $\mathrm{B}$ surface antigen $(\mathrm{HBsAg}+)$ may increase, but it has been not well studied. We aimed to analyze the reactivation rate and impact of reactivation on early transplant-related complications in a retrospective single reference center. We detected $23 \mathrm{HBsAg}+$ patients $(3.8 \%)$ who underwent allo-HCT $(n=680)$ in the past decade. Median age was 33 years and M/F: 22/1. In 20 patients pretransplant liver function tests were within normal range and HBV-DNA were negative. The conditioning were based on myeloablative $(n=20)$ or fludarabine based-reduced intensity regimen $(n=3)$. Lamivudin prophylaxis was initiated $100 \mathrm{mg}$ p.o per day in 14 patients with the initiation of conditioning regimen and was continued until 6 to 12 months till the cessation of immunosuppression. Six patients had $\mathrm{HBsAg}+$ donor, 5 were seropositive (antiHBs with antiHBc IgG, 3 antiHBs alone and 6 were HBV naive. The data of HBV serology was not available in 3 donors. HBV reactivation was observed in 7 patients after a median 4.5 months (range, 0.87-23.5) follow-up. Interestingly all the patients with HBV reactivation received myeloablative conditioning. At the time of reactivation there was an increase of serum ALT $(2.5 \times \mathrm{UNL}-54$ $\mathrm{UNL}$ ) and serum HBV-DNA. We observed reactivation only in a single under lamivudine prophylaxis $(7.6 \%$ vs. $60 \% . P=0.012)$ while another patient experienced a reactivation 2 months after cessation of prophylaxis. Two patients with HBV reactivation had HBsAg positive donors. Lamivudin treatment was initiated in 5 patients after reactivation. Lamivudin resistance was observed only in two patients, who were switched to second line antiviral treatment. One of those patients was lost due to fulminant hepatic failure. The incidences of acute or chronic liver graft versus host disease were not affected by the HBV reactivation. The HBV reactivation is one of the most undesired complications of chemo/immunosuppressive treatment in HBsAg positive Allo-HCT recipients. The prophylaxis with lamivudine could significantly decrease the frequency of HBV reactivation. But the development of the lamivudine resistance is another problem reported in patients requiring long-term treatment. In conclusion, our study has shown that $\mathrm{HBsAg}+$ recipients did not have a strong exclusion for allo-HCT under effective lamivudine prophylaxis. 


\section{P759}

A novel haploidentical adoptive CTL therapy as a treatment for EBV-associated lymphoma after stem cell transplantation

M. Okas, J. Gertow, M. Uhlin, J. Mattsson

Karolinska Institutet (Stockholm, SE)

Epstein-Barr virus (EBV)-related malignancies such as posttransplant lymphoproliferative disease (PTLD) are severe complications after allogeneic stem cell transplantation and solid-organ transplantation. In immunosuppressed transplant recipients, the activity of EBV-specific CTLs are often decreased or absent which leads to an increased risk of developing PTLD. If primary treatment modalities of PTLD fail, the most efficient way of treating the malignancy is adopting EBV-specific CTLs from the donor or, more recently, third-party donors. However, both are time consuming and expensive and often it is too late to administer cells to the patient. We have for the first time, using a rapid isolation protocol of EBV-specific T cells, treated and cured a patient suffering from PTLD with multiple-associated tissue lesions, using her haplo-identical mother as a donor. We enriched the EBV-specific CTLs from the blood of the mother by using MHC pentamers containing EBV-derived peptides and beaded out the pentamer-labeled cells using magnetic beads. The administration of cells resulted in dropdown of viral titers and resolvement of clinical symtoms as well as of the organ lesions. Maternal cells could be detected in the patient up to 80 days post-transfer. We had a possibility to perform a second transfer for the same patient at 6 months after the first transfer when the clinical symptoms have developed again and the virus titers began to rise. The second transfer has also resulted in resolution of the symptoms and drop-down of the viral load. This approach demonstrates a new possibility for within-days treatment of a life-threatening EBV-associated malignancy. It also paves the way for new treatment options in other viral infections after stem cell transplantation, such as adenoviral or CMV infections resistant to therapy or of complicated course.

\section{P760}

EBV load after stem cell transplantation: a prospective study E. Metafuni, S. Bellesi, P. Chiusolo, S. Giammarco, L. Laurenti, F. Sora', G. Ausoni, G. Leone, S. Sica

Università Cattolica S. Cuore (Rome, IT)

EBV related post transplant lymphoproliferative disorders (PTLD) are severe complications of allogeneic stem cell transplantation (allo-SCT). In the last years monitoring of EBV-DNA load has been applied to detect patients (pts) at high risk of PTLD. Preemptive therapy in this setting is an emerging option.

We have prospectively monitored EBV reactivation in a cohort of 104 pts receiving SCT between February 2005 and August 2009. Seventy-nine pts received an allo-SCT and twenty-five pts received an autologous SCT (auto-SCT). In the first group, the reactivation rate was $60.7 \%$, while in the second group it was of $17.4 \%(P=0.0002)$. The median time of EBV reactivation was 115 days (range 53-870) in auto-SCT group and 59 days (range 3-840) in allo-SCT group. Hence allo-SCT pts showed a faster EBV reactivation $(P=0.031)$; graft from an unrelated donor was associated with an higher rate of EBV reactivation and with a earlier reactivation. In pts receiving ATG or Campath for GVHD prophylaxis the reactivation rate was $93.3 \%$.

In auto-SCT setting no pts developed PTLD, while in allo-SCT group six pts developed PTLD (7.6\%). In five of these, PTLD followed EBV DNA detection in peripheral blood, while in a pt PTLD was diagnosed by biopsy, in the absence of previous EBV detection. All these pts received an unrelated stem cell graft; five of them received ATG and one received Campath.

Among allo-SCT pts with EBV reactivation, serum protein electrophoresis (PE) shown a gamma-peak in 31 cases (64.6\%), suggesting the presence of a monoclonal gammopathy (MG). In a comparison group of 31 allo-SCT pts without EBV reac- tivation the gamma-peak rate was $35.5 \%$. At the time of EBV reactivation we detected a MG in twenty-two pts (45.8\%). All pts with PLTD presented a gamma-peak at the PE; five of these presented a MG.

Treatment with rituximab $\left(375 \mathrm{mg} / \mathrm{m}^{2} /\right.$ die for $1-4$ doses) was administered to all PTLD cases and to 12 pts with an high viral load, to avoid the progression to PTLD. The $88.9 \%$ of treated pts showed a sharp viral load clearance. In 11 pts (61\%) abnormalities of serum PE normalized after treatment, while in 5 pts $(27.7 \%)$ it persisted. Two pts with PTLD died early after treatment because of multi-organ failure and progression disease. The remaining four pts presented resolution of symptoms and a fall in viral load until its disappearance.

The earlier gamma-peak detection and the MG presence may suggest a prompt identification of pts with EBV viremia at high risk of PTLD.

\section{P761}

Excretion of the novel polyomaviruses $\mathrm{KI}$ and $\mathrm{WU}$ in the stool of haematological patients undergoing stem cell transplantation

M. Ciotti (1), A. Picardi (1), G. De Angelis (2), M. Babakir-Mina (1), M. Ciccozzi (3), C. Alfieri (1), K. Paciaroni (1), C. Alfiere (1), I. Provenzano (1), A. Di Veroli (1), C.F. Perno (1), W. Arcese (1) (1)Tor Vergata University (Rome, IT); (2)Tor Vergata University Hospital (Rome, IT); (3)Istituto Superiore di Sanità (Rome, IT)

Introduction: Infection with human polyomaviruses BKV and $\mathrm{JCV}$ is asymptomatic and lifelong and widespread among the general population. However, in the setting of immuno-compromised patients, due to haematological diseases or viral infections, such as HIV, reactivation can occur and result in severe complications.

Material and methods: In this prospective study, stool specimens were examined from 25 patients who underwent stem cell transplantation (SCT) for haematological disorders to determine whether the novel polyomaviruses KIV and WUV were reactivated and excreted in the gastrointestinal tract. The stool samples were collected in 2 cases of autologous SCT and in 23 cases of allogeneic SCT, of whom 14 HLA matched related, 8 HLA mismatched related and 1 HLA matched unrelated SCT. Viral reactivation was correlated to the appearance of gastrointestinal and respiratory symptoms.

Results: Of the 25 transplanted patients, KIV and WUV were detected in 13 cases $(52 \%)$ as single infection or in combination with BKV, cytomegalovirus and adenovirus. Because of frequent co-infections, a clear correlation between novel polyomaviruses and clinical symptoms could not be established. There was no correlation between demographic variables and detection of KIV and WUV. Phylogenetic analysis of the small t-antigen gene of KIV and WUV isolates showed that the novel polyomaviruses identified in feces clustered with those identified in the respiratory tract suggesting an oral-fecal transmission of these viruses.

Conclusions: The novel polyomaviruses $\mathrm{KI}$ and $\mathrm{WU}$ may reactivate in SCT setting and the oral-fecal contamination is suggested. Further studies on a larger sample size are needed to define their pathogenic role on the clinical outcome of immunocompromised hosts.

\section{P762}

Human herpesvirus 7 (HHV-7) infections in patients with allogeneic haemopoietic stem cellstransplantation in a medical university, Warsaw, 2006-2008

T. Dzieciatkowski, M. Przybylski, T. Torosian, G.W. Basak Medical University of Warsaw (Warsaw, PL)

Objectives: Human herpesvirus 7 is wide-spread in the population and has been recognized as a potential pathogen in haemopoietic stem cell transplant recipients. Different clinical manifestations have been described including fever, skin rash; 
it has been also demonstrated that HHV-7 can induce reactivation of other beta--herpesviruses (CMV and HHV-6).

We want to measure prevalence of the HHV-7 DNA in group of allogeneic haemopoietic stem cells recipients hospitalized in Hematology, Oncology and Internal Medicine Clinics of Medical University in Warsaw from 2006 till 2008.

Methods: A retrospective review of a group of 875 plasma samples taken from 58 adult recipients of allogeneic SCT was made. After transplantation qualitative "in-house" real-time PCR was used to determine viral load in range 0-180 days after transplantation.

Results: Using real-time PCR technique HHV-7 DNA was detected in plasma samples in $26(45 \%)$ of the 58 recipients, especially between day 40 and day 65 of transplantation. All of described patients developed fever of unknown origin and $30 \%$ of them had also GvHD features.

Conclusions: There is a high frequency of detectable HHV-7 viral load in allogeneic SCT recipients in Poland. Limited availability and sensitivity of serological methods along with the necessity of rapid introduction of antiviral treatment has forced the development of molecular diagnostics. Furthermore, establishment of appropriate procedures for monitoring active HHV-7 infection is important to clarify the virus infection in transplant recipients. Investigations of HHV-7 presence in graft recipients may be important to improve the outcome for these patients.

\section{P763}

Pre-emptive treatment with rituximab for Epstein-Barr virus reactivation after allogeneic haematopoietic stem cell transplantation: a safety and efficacy study

S. Ganguly, L. DuBois, C. Divine, O. Aljitawi, S. Abhyankar, J. McGuirk

University of Kansas Medical Center (Westwood, US)

Background: Epstein-Bar virus (EBV) associated post-transplant lympho-proliferative disorder (PTLD) is a serious complication after allogeneic hematopoietic stem cell transplantation (HSCT). Recent attention has focused on early diagnosis and pre-emptive treatment with rituximab, an anti CD20 monoclonal antibody. At present there is no standardized guideline for the management of post-HSCT EBV reactivation.

Methods: In an attempt to establish uniform practice guidelines, we adopted an algorithm based on existing literature for monitoring and pre-emptive treatment for EBV infection in patients considered to be at high risk for the development of EBV PTLD like recipients of haplo-identical or cord blood transplantation, and those who received anti-thymocyte globulin (ATG) or other T-cell depleting therapies. Patients were monitored weekly by quantitative EBV-PCR starting at Day 20 after transplantation. Patients with rising EBV copies of greater than 1000/ml in whole blood (two consecutive samples) were pre-emptively treated with rituximab $375 \mathrm{mg} / \mathrm{m}^{2}$ once a week until viral clearance or up to a maximum of 4 doses. Safety and efficacy were monitored prospectively.

Results: Between 2007-09, 20 out of 59 high-risk patients (34\%), developed EBV reactivation with a median time of occurrence being 63.7 days post-HSCT (range: 26-347 days). Median number of EBV copies $/ \mathrm{ml}$ in blood at the time of diagnosis was 2300 (range: 1000-5000). Eight patients received rituximab secondary to rising titer for a median number of 2 weekly doses (range: 1-4) and all of them showed viral clearance. Two patients required repeat dosing of rituximab secondary to recurrence. CD19 count was undetectable (median 1\%; range $0-1 \%$ ) in all patients for 3-6 months after receiving rituximab and then improved in all surviving patients (6 out of 8 ). None of the 20 patients developed EBV-PTLD. Administration of rituximab was not associated with increased rate of infection, cytopenia or severe infusional reaction.

Conclusion: Up to one-third of high risk patients may develop EBV reactivation after HSCT, but only a minority will require intervention. Pre-emptive treatment with rituximab is relatively safe and effective in viral clearance, but is associated with delayed B-cell immune re-constitution. A prospective randomized trial is necessary to evaluate whether this approach is cost-effective, leads to better survival and improved outcome.

\section{P764}

Determination of human cytomegalovirus and gB 2

polymorphisms in haematopoietic stem cell transplant donors and recipients

M. Ramzi, A.A. Banan, R. Yaghobi

Shiraz University of Medical Sciences (Shiraz, IR)

Background: Human Cytomegalovirus is a member of the herpes virus family. CMV infection in healthy person is usually asymptomatic, however, the virus has the ability to become latent in the host. Primary infection or reactivation of latent CMV in an immunocompromised patient such as HSCT transplant recipients, can lead to morbidity and mortality.

Objectives: Two new nested-PCR protocols were optimized for the accurate detection of HCMV infection in HSCT donors and recipients. The prevalence of HCMV UL55 genotypes was tested pre- and post-transplantation in a cohort of HSCT patients.

Materials and methods: The AD 169 viral genome which is a universal and standard strain used for correct optimization. For this retrospective cohort study of 110 patients during a 7-year period from 2002 to 2009 we obtained pre-transplant blood (plasma and buffy coat) and urine samples and followup samples for 100 days post-transplantation. Blood and urine samples were simultaneously collected from 98 HSCT donors. HCMV UL55-nested-PCR and HCMV UL4-nested-PCR methods were optimized and used to detect HCMV infection in all samples.Genotyping for the HCMV UL55 gene was done for all UL55-nested PCR positive samples. Donor and recipient laboratory and clinical data were collected and analyzed statistically with SPSS v. 15 software.

Results: UL4-nested-PCR positivity was detected in 3045 of 4950 plasma samples $(61.5 \%)$, in $3168 / 4950$ Buffy coat sample $(64 \%)$ and $3020 / 4950$ urine samples $(61 \%)$ from HSCT recipients post-transplantation. UL55-nested-PCR positivity was found in $3540 / 4950(71.5 \%), 3634 / 4950(73.4 \%)$ and $3292 / 4950(66.5 \%)$ samples, respectively. Twenty-five percent of the donors were also infected with HCMV. The relative prevalence of UL55 genotypes was gB2> gB3> gB1> gB4 in plasma, gB2> gB1> gB3> gB4 in buffy coat and gB2> gB3> gB1> gB4 in urine.

Conclusion: we concluded the gB2 genotype is the most prevalent finding in our patients after HSCT, So we suggested that there is need for further research on the HCMV gB2 in HSCT donors and recipients.

\section{P765}

Recombinant plasmid to investigate the structure of the human cytomegalovirus and evaluation of UL-4 nested-PCR protocol for detection of human cytomegalovirus in haematopoietic stem cell transplant patient

A.A. Banan, M. Ramzi, R. Yaghobi

Shiraz University of Medical Sciences (Shiraz, IR)

Background: Human Cytomegalovirus is a member of the herpes virus family. Primary infection or reactivation of latent CMV in an immunocompromised patient such as HSCT transplant recipient can lead to morbidity and mortality, so, monitoring infection plays a critical role in managing clinical complications.

Methods: We obtained the whole genome of Cytomegalovirus strain AD 169 from Dr D. Dargan Medical Research Council, Glasgow, U.K. on the filter-paper, we extracted the whole genome from the filter-paper. (HCMV) DNA was digested with restriction endonucleases and the fragments characterized with respect to molecular weight and relative mole proportions. The HIND III fragments of HCMV DNA were cloned in Escherichia 
coli and recombinant plasmids were characterized by digestion with restriction endonucleases by molecular hybridization with Hind III, Bgl II and Xba I of the virus genome, also, In this research all PCR-mixture components and thermocycling conditioning factors were evaluated for specific amplification of the UL-4 gene that coding a 48 kilo dalton glycoprotein (gp 48) and has role in virus cell penetration. The AD 169 viral genome which is a universal and standard CMV strain and HCMV documented clinical blood samples of HSCT patients as positive controls were used for correct optimization of this nested-PCR method.

Results: Our data helps us to construct physical maps of HCMV DNA for the Hind III, Xba I and Bgl II restriction endonuclease, also in this study, the high sensitivity and specificity of this nested-PCR method for detection of HCMV infections were documented. Positive HCMV-PCR results were detected in 36 out of 56 HSCT patients (64.3\%). These HCMV-PCR results were comparative with presentation of HCMV clinical symptoms and the antigenemia test results in HSCT recipients.

Conclusion: we also,describe the construction of recombinant plasmids which contain the HINDIII Restriction endonuclease fragments of HCMV DNA.

For the widespread HCMV infection in healthy populations and immunocompromised patients especially HSCT recipients, use of sensitive and specific molecular methods like this UL-4 nested-PCR protocol in detection of HCMV infection and /or disease is absolutely needed. This HCMV nested-PCR technique is rapid, sensitive and specific for monitoring of HCMV infections in transplant patients.

\section{P766}

Factors influencing cytomegalovirus seropositivity in haematopoietic stem cell transplant patients and donors A.A. Banan, M. Ramzi, R. Yaghobi

Shiraz University of Medical Sciences (Shiraz, IR)

Background: Cytomegalovirus (CMV) infection continues to have a tremendous impact on recipients of Hematopoietic Stem Cell transplantation (HSCT).

The HCMV status of the donor and the recipient remain one of the key factors influencing outcome after allogeneic HSCT.

Methods: During 2000-2009 at Nemazee hospital in Shiraz University of Medical Sciences 110 patients underwent Allogenic stem cell transplant, To study factors impacting HCMV status in patients we reviewed records of our patients and donors and we evaluate impacting factor on the basis of HCMV status according to serology and Nested-PCR results with Logistic regression models by using the year of HSCT, age, gender, diseases diagnosis as covariates.

Results: Female patients had a higher likelihood to be CMV positive than males $(P<.001)$. The risk of CMV positivity increased by age of patient $(P<.001)$, but decreased yearly since 2006 until $2009(P<.001)$.

The causes of death were determined from archived research files.

CMV death include deaths due to CMV pneumonia, disseminated CMV, as well as gastrointestinal CMV.

Myelosuppression has been observed in association with specific underlying diseases like aplastic anemia or myelodysplasia in HSCT patients.

Conclusions: We conclude that the likelihood of being HCMV positivity is higher in females and increase with age. There are strong differences in the probabilities of being CMV positive with Nested-PCR result between patients transplanted with different diagnosis. We have determined that death due to Mylosuppression in CMV-infected marrow transplant recipients is associated with particular CMV genotypes.
P767

PALIFERMIN after autologous HSC transplantation reduces $\mathrm{FUO}$, severe infections and resources utilization G. Milone, E. Mauro, E. Marturano, A. Di Mercurio, A. Spadaro, G. Lio, M. Musso, M. Poidomani, S. Leotta, V. Pinto Ospedale Ferrarotto (Catania, IT)

The place of palifermin after autologous HSC transplantation remains an unsettled issue and conflicting results have been reported. In our Institution Palifermin has been employed in a cohort of 40 patients treated with autologous HSC transplantation and receiving a conditioning regimens based on chemotherapy. Results have been compared to those obtained in a set of 150 patients not receiving palifermin. The control group were patients transplanted in the 2 years period antecedent and subsequent in respect to the time in which palifermin was administered. The two groups treated with palifermin or not treated were not significantly different for age, sex, diagnosis, stage of disease at transplant, number of previous chemotherapy, number of Neutrophils at admission, conditioning type used, prophylactic use of G-CSF, number of infused CD34 + cells. Treatment with palifermin was well tolerated. 29 pts/40 (72\%) had skin rashes and oedema involving face and extremities but not requiring interruption of treatment. In palifermin treated cohort we observed a significant reduction of NPT need $(11 \%$ versus $88 \%, P=0.0001)$ and of morphine use $(2,9 \%$ versus $25,8 \% ; P=0.01)$, length of hospital stay was significantly reduced (discharge at day +12.0 versus day +16.0 ; $P=0.0001)$. Patients treated with palifermin had a reduced frequency of FUO $(22 \%$ versus $40 \%, P=0.05)$ and a trend for a reduction in severe infections (gram-negative bacteremia or pulmonary infections: $2,5 \%$ versus $11,3 \% ; P=0.09$ ). In patients treated by palifermin as a whole group, we were unable to observed a reduction in mucositis score (maximum score DMS for mucositis: $32 \%$ versus $35 \% ; P=0.75$ ). However, a reduction of mucositis maximum grade was observed when stratum of patients receiving conditioning other than HD-PAM were analysed $(P=0.01)$.

Neutrophils engraftment was not different in the two groups, median day for $\mathrm{N}>0.5 \times 10^{9} / \mathrm{L}$ was 11.5 versus $12.5(P=0.07)$. No treatment related mortality was registered in palifermin group $(0 \%)$ versus $4 / 150$ pts $(2,6 \%)$ in control group $(P=0.2)$.

In conclusion, after chemotherapy-only conditioning, demonstration of reduced oral mucositis is not apparent in palifermin group probably because concomitant epitelial rash and oedema make difficult to assess oral mucositis. Significant beneficial effects of palifermin are, however, evident on many relevant infective and non infective end-points (FUO, severe bacterial infections, use of NPT, use of morphin, length of hospital stay).

\begin{tabular}{|l|l|l|l|}
\hline & $\begin{array}{l}\text { Palifermin } \\
\text { (n.40) }\end{array}$ & $\begin{array}{l}\text { Control } \\
\text { (n.150) }\end{array}$ & p \\
\hline NPT use & $11 \%$ & $88 \%$ & 0.0001 \\
\hline Morphine use & $2.9 \%$ & $25 \%$ & 0.0001 \\
\hline FUO & $22 \%$ & $40 \%$ & 0.05 \\
\hline $\begin{array}{l}\text { Severe } \\
\text { bacterial } \\
\text { infections }\end{array}$ & $2.5 \%$ & $11.3 \%$ & 0.09 \\
\hline
\end{tabular}




\section{P768}

Voriconazole as antifungal prophylaxis in 124 high-risk patients undergoing allogeneic stem cell transplantation D. Clerici, S. Malato, F. Giglio, R. Greco, S. Mastaglio, F. Matteazzi, A. Assanelli, M. Marcatti, M. Bernardi, J. Peccatori, L. Fumagalli, C. Corti, F. Ciceri

San Raffaele Scientific Institute (Milan, IT)

Background: Invasive fungal infections remain a leading complication with high morbidity in haematological patients undergoing allogeneic haematopoietic stem cell transplantation (HSCT). In the last years new drugs were developed with a broad spectum of activity against yeasts and moulds.

Objectives: In our Bone Marrow Transplantation Unit, due to local epidemiology, in the last years we performed antifungal prophylaxis with voriconazole (V.) for patients (pts) undergoing allo-HSCT. We retrospectively analized the incidence of breakthrough fungal infections in the neutropenic period post alloHSCT in this cohort of pts.

Materials and methods: From October 2002 to September 2009 in a cohort of 124 allo-HSCT (44 MUD, 42 haplo without T-cell depletion, 17 haplo with T-cell depletion, 9 cord blood, 12 HLAidentical sibling) for high risk haematological malignancies we performed antifungal prophylaxis (primary or secondary) with V. $200 \mathrm{mg}$ twice daily from the beginning of conditioning until stable engraftment was obtained. We analized the breakthrough fungal infections and the toxicity of $\mathrm{V}$. prophylaxis

Results: In the cohort of MUD with $\mathrm{V}$. as primary prophylaxis (40 pts), 8 pts stopped the drug (4: CT scan suggestive for fungal infection; 1: candidemia; 3: intolerance/reversible toxicity); in the group of $\mathrm{V}$. as secondary prophylaxis (4 pts) 2 pts stopped the drug (CT scan suggestive for fungal infection). In the cohort of haplo without T-cell depletion with V. as primary prophylaxis (35 pts), 6 pts stopped the drug (4: CT scan suggestive for fungal infection; 2: candidemia); in the group of $\mathrm{V}$. as secondary prophylaxis (7 pts) $1 \mathrm{pt}$ stopped the drug (CT scan suggestive for fungal infection). In the cohort of haplo with T-cell depletion with $\mathrm{V}$. as primary prophylaxis (16 pts), 2 pts stopped the drug (intolerance/reversible toxicity); no one pts in the group of $\mathrm{V}$. as secondary prophylaxis. In the cohort of cord blood HSCT with $\mathrm{V}$. as primary prophylaxis (9 pts), 3 pts stopped the drug (CT scan suggestive for fungal infection). In the cohort of HLA-identical sibling donor no one pts stopped V.

Conclusions: Voriconazole is effective and safe as primary or secondary antifungal prophylaxis in high-risk hematological patients undergoing allo-HSCT in the neutropenic period, with a low incidence of breakthrough fungal infections. A prospective study with serum drug monitoring of voriconazole will be run for optimizing the drug serum concentration.

\section{P769}

Invasive fungal infection following allogenic haematopoietic stem cell transplantation adult versus children (single-centre experience)

M. Popova, N. Zubarovskaya, V. Vavilov, A. Volkova, M. Averjanova, N. Klimko, B. Afanasyev

I.P. Pavlov State Medical University (St. Petersburg, RU)

Background: Invasive fungal infection (IFI) is a common cause of morbility and mortality in patients undergoing HSCT. Only $25 \%$ IFI diagnosed while alive.

The aim. To compare incidence, risk factors of IFI in adults and children after allogenic (allo), haploidentical (haplo) HSCT.

Patients: 236 patients (pts) were enrolled between 2000 and 2009. The adult group was 106 pts, age 21-68 (median 33 y.o.), the children group 130 pts, age 1-21 (median 14 y.o.) suffered from acute leukemias - 167, myelo- and lymphoproliferative disorders -31 , lymphomas - 18, inherent disorders -8 , others - 12. At the moment of HSCT in remission were 144 in relapse -92 . The most pts were received allo-HSCT as salvage therapy. Type of the donor: MRD - 130, MURD - 97, haploidentical donor - 9. MAC used in 85, RIC in 151. Sources of HSC: PBSC-154, BM-71, BM+PBSC-11. Median CD34+ $-3-8 \times 10^{6} / \mathrm{kg}$. Acute GvHD prophylaxis was CsA+ short course of MTX or tacrolimus + MMF, plus ALG-60 mg/kg for MUD and haplo-HSCT.

Results: The incidence of IFI following allo-HSCT was $32 \%$. Possible IFI was detected in $30(40 \%)$, probable IFI in $40(53,4 \%)$ and proven IFI in $5(6,6 \%)$ according to the international definition criteria EORTC/MSG 2008. Median date of IFI onset was D+68 (1-940). The main causes of IFI were Aspergillus spp. $94 \%$, Candida spp. $3 \%$, Cryptococcus spp. $4 \%$. The following risk factors were detected: in adults - usage of RIC, ATG, development of mucositis $(P<0,05)$; in children - age older 10 years, mucositis IV, extensive chGvHD $(P<0,05)$.

The multivariate analyses revealed the synergistic effect of the following risk factors: in children - combination of MUD, MAC, and CMV-reactivation; MUD, RIC, relapse and BM as a source of HSCT. In adults - MUD, RIC and CMV-reactivation; MUD, RIC, remission and PBSC $(P<0,05)$. There were no cases of $\mathrm{IFI}$ in pts with inherent disease. Overall survival (OS) in pts undergoing HSCT with IFI versus without IFI was $18 \%$ vs. $55 \%$ (children) and $30 \%$ vs. $30 \%$ (adult). Twelve-weeks OS children vs. adult was $60 \%$ vs. $45 \%$.

Conclusions: We revealed difference of risk factors of IFI in children and adult and in OS in pts with IFI. The main risk factors for children are age older 10 years, mucositis IV, extensive chGvHD, for adults - usage of RIC, ATG, development of mucositis.

\section{P770}

Invasive fungal infections in a pediatric oncology ward - Surveillance study over an 8-year period

G. Kropshofer (1), R. Crazzolara (1), C. Lass-Flörl (2), M. Freund (3), A. Klein-Franke (1), B. Meister (1)

(1)Hematooncology (Innsbruck, AT); (2)Microbiology (Innsbruck, AT); (3)Radiodiagnostic (Innsbruck, AT)

Background: Invasive fungal infections remain an important complication in hematologic malignancies and stem transplantation (SCT). This retrospective single center study analyzed the incidence and the outcome of proven fungal infections in pediatric patients over the past decade.

Methods: Between 2000 and 2008 we evaluated 503 patients at our institution who underwent treatment with either cytoreductive chemotherapy alone or with subsequent hematopoetic stem cell transplantation. Diagnoses were composed as follows: leukemia $n=153$, solid tumors/lymphomas $n=309$, SAA/MDS: 14 , others $n=27$

Results: Among 503 children treated in 13 patients $(2,6 \%)$ a proven (CT guided percutaneous biopsy respectively post mortem diagnosis) systemic fungal infection was documented. Aspergillus spp. $w$ as the predominant fungus $(n=7)$, followed by Zygomycetes $(n=3$, Candida spp. $(n=2)$ and one Irpex lacteus. The lung was the organ predominately affected. Other organs involved were the brain, paranasal sinuses and liver. 3 pts. were clinically cured, 7 pts. died due to relapse of the underlying disease, 1 due to multiorgan failure and two pts. died from fungal disease despite therapy.

Conclusion: The incidence of mould infections has increased in recent years, particulary those due to Aspergillus spp., Fusarium and Zygomycetes. Therefore biopsy of suspected fungal infections in immunocompromised patients is of paramount importance and has direct implication on systemic fungal therapy and hopefully outcome. 
P771

PCR-based molecular diagnostic tool (Septifast $(\AA)$ in septic patients undergoing induction therapy or stem cell transplantation in haematological malignancies

A. Zerweck, D. Raepple, A. Finck, J. Finke, H. Bertz

University of Freiburg Medical Center (Freiburg, DE)

Sepsis in neutropenic patients is a serious complication and a major cause of treatment related mortality (TRM) in hematological patients (pts). Especially pts undergoing stem cell transplantation (SCT) or high dose chemotherapy are prone to develop bacterial or fungal blood stream infections in the induction or peri-transplant (cytopenia until hematological reconstitution, immunosuppression) setting. Early detection and identification of the underlying pathogen play a crucial role in successful treatment. Gold standard diagnostic procedures are blood cultures taken before empirical application or escalation of broad-spectrum antibiotics followed by customized therapy according to the identified species and antibiogram. Usually this requires at least 48-72 hours. PCR based systems detect and identify the presence of the most common microorganisms associated with sepsis within 6 hours. We report our experience of Septifast ${ }^{\circledR}$ system commercially available by Roche on pts undergoing induction therapy and/or SCT for hematological malignancies in our center. Between August 2008 and October 2009. Septifast $₫$ tests were taken additionally to routine blood cultures according to our center's policy. Data were retrospectively analyzed.

53 samples were obtained from 43 pts $(m=24 / f=19)$ with a median age of 60 years (27-77y). 33 samples were acquired in a transplant setting. Median white blood cell count was $0.3 \times 10 \% / \mu$ with 33 neutropenic episodes, median C-reactive protein $193 \mathrm{mg} / \mathrm{l}($ normal $<5)$. A pathogen was identified in 13 (25\%) blood cultures and in 20 (38\%) septifast tests. In a majority of cases $(n=42,79 \%)$ septifast and blood cultures showed identical results. In 7 cases (13\%) both methods differed. In conclusion PCR-based systems like Septifast ${ }^{\circ}$ are additional instruments in improving rapid and reliable diagnosis of blood stream infections in this high risk patient population. If this system allows earlier administration of appropriate antibiotic therapy, while also reducing inappropriate overuse of broad-spectrum antibiotics is subject of further analysis.

\section{P772}

Retrospective study of clinical predictors of positive fungal cultures in recipients of allogeneic and autologous stem cell transplantation

A. Khoder, M. Petrou, C. Thomas, R. Szydlo, E. Kanfer, D. Marin, A. Rahemtulla, A. Luqmani, J. Hung, D. MacDonald, D. Milojkovic, J. Apperley, K. Rezvani

Imperial College NHS trust (London, UK)

Fungal infection is a major cause of mortality in stem cell transplantation (SCT). We retrospectively analyzed all fungal isolates from SCT patients referred to the mycology lab for susceptibility and speciation from 1997 to 2006 . During this period 760 and 455 patients underwent autologous and allogeneic SCT for haematological malignancies respectively. Positive isolates were reported in $78(10 \%)$ of recipients of autologous SCT (30 MM, $27 \mathrm{NHL}$ and 22 others) and in $79(17 \%)$ of alloSCT patients. In the latter group ( $45 \mathrm{CML}, 13$ acute leukaemia and MDS, 11 others), 18 received reduced-intensity-conditioned and 61 myeloblative allografts. The donor was matched sibling (38), matched unrelated (36) and mismatched related or unrelated (5). Two-thirds were T- depleted. Median CD34 + cell dose was $4.3 \times 10^{6} / \mathrm{kg}$ and median time to neutrophil engraftment (TNE) was 21 d. $52 \%$ \& $47 \%$ of patients with positive isolates had grade II-IV aGvHD and moderate-severe cGvHD respectively. Risk factors for positive isolate were aGVHD $(P=0.03)$, unrelated donor (UD) $(P=0.012)$ and T-depletion $(P=0.001)$. No significant association between TNE, CD34+ dose or conditioning and occurrence of positive isolates was seen. There were 494 positive isolates in 157 patients (407 yeasts and 87 filamentous fungi, FF). Non-albicans (NA) candida accounted for $57 \%$ of all yeasts $(74 \%$ C. glabrata and C. krusei). The most frequent species leading to candidaemia were C. glabrata (26\%), C. krusei $(17 \%)$ and C. albicans (17\%). In vitro $64 \%$ of NA candida were resistant to fluconazole (FCZ). Most C. glabrata (88\%) were resistant to itraconazole (ITZ) and $35 \%$ to voriconazole (VCZ). Conversely C. albicans was invariably sensitive to FCZ or other azoles. All yeasts were sensitive to amphotericin $B(A M B)$ and whilst caspofungin was active against Candida its activity against other yeasts was variable. FF were more common in alloSCT $(4.6 \%)$ than ASCT $(1.4 \%)$. The most common FF were Aspergillus species (74\%), Penicillium (6\%) and Fusarium (5\%). In vitro no FF were inhibited by FCZ. Here, we show that T-depletion, an UD and GvHD increase the risk of fungal infection. C. glabrata and C. krusei are the predominant NA candida isolates and have a significant rate of azole resistance. A fumigatus is the predominant FF. This study is limited by its retrospective nature. We are performing prospective studies to define risk factors and analyze the impact of changing anti-fungal prophylaxis and treatment on transplant outcome.

\section{P773}

Differences in cytomegalovirus replication quantified by quantitative PCR and antigenemia pp-65 after allogeneic stem cell transplantation with different prevention strategies F. de la Cruz Vicente, I. Gracia-Ahufinger, P. Perez-Romero, M. Aguilar Guisado, R. Parody Ruiz-Berdejo, J.M. Cisneros Herreros, A. Urbano-Ispizua, I. Espigado

Hospital Universitario Virgen del Rocio (Seville, ES)

Introduction: Cytomegalovirus (CMV) end-organ disease is a serious and frequent complication after allo-stem cell transplantation (Allo-STC). There are two different prevention strategies: universal prophylaxis and pre-emptive therapy. There is no defined the best techniques of detection of infection: antigenemia (the most widespread technique) or real-time PCR (seems to be more sensitive)

Objectives: Systematic quantification of CMV viral replication by RT-PCR and antigenemia pp65 comparing the clinical utility of both in patients (pts) on prophylactic and pre-emptive approach. Patients, material and methods: Allo-STC adult recipients were retrospectively included in the study. One group $(n=34)$ received prophylaxis (ganciclovir $300 \mathrm{mg} / 24 \mathrm{~h}, 3$ days/week/100 days) and the other group $(n=7)$, pre-emptive therapy guide by positive antigenemia (ganciclovir $5 \mathrm{mg} / \mathrm{kg} / 12 \mathrm{~h} / 14$ days). All pts underwent CMV viral load quantification by RT-PCR and antigenemia during 6 months: once a week in the first 100 days and every two weeks thereafter. Antigenemia was carried out by inmunofluorescence in leukocytes with the monoclonal antibody pp65. For RT-PCR the viral DNA was isolated in plasma and was used as a template for amplification.

Results: 41 peripheral blood allo-STC, medium age 40 years (15-58), were analyzed. No differences were seen between both groups in terms of risk factors for CMV infection. Total viral replication quantified by antigenemia was $29.2 \%$. Viral replication quantified by RT-PCR was $65.8 \%$. 2 pts developed an end-organ CMV disease. We compared viral load quantified by RT-PCR in pts with a positive antigenemia against those with negative antigenemia, observing differences on weeks 0,2 , 3 and $6(P<0.05)$, and if the criteria was the median RT-PCR in both groups $(P=0.013)$, before and after day $100(P=0.012$ and $P=0.008)$. We compared the viral load quantified by RT-PCR in prophylaxis vs. pre-emptive therapy groups, observing differences in the median of RT-PCR and median RT-PCR before day 100 . We also saw differences before day 100 and after day 100 in pts in prophylaxis group $(P<0.02)$ (Table 1$)$.

Conclusions: 1) RT-PCR is more sensitive than antigenemia pp-65; 2) CMV positivity and viral load is inferior in the prophylaxis group, but these pts are on viral replication before and after day 100 and received more treatment. 


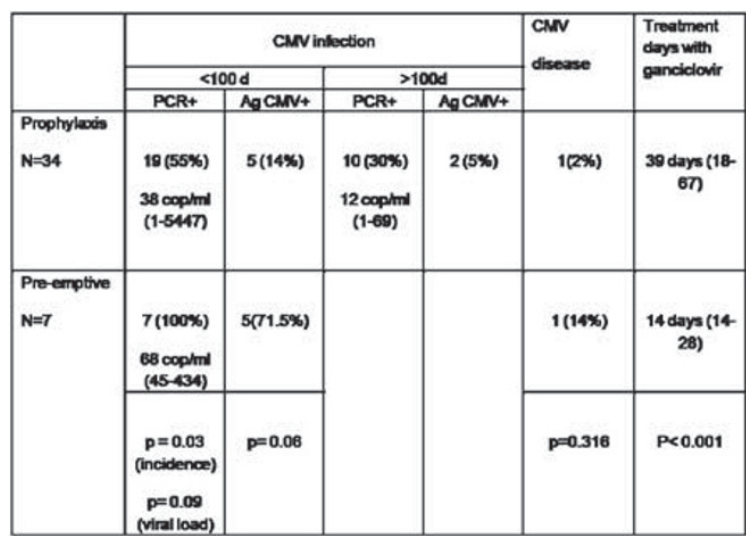

P774

Pre-emptive therapy for cytomegalovirus infection after allogeneic stem cell transplantation guided by quantitative real time-PCR

F. de la Cruz Vicente, I. Gracia-Ahufinger, P. Perez-Romero, M. Aguilar Guisado, R. Parody Ruiz-Berdejo, JM. Cisneros Herreros, A. Urbano-Ispizua, I. Espigado

Hospital Universitario Virgen del Rocio (Seville, ES)

Introduction: Cytomegalovirus (CMV) end-organ disease is a serious and frequent complication after allo-stem cell transplantation (Allo-SCT). Pre-emptive therapy guided by viral replication is a recent prevention therapy, consisting in treatment if viral replication is detected. The best technique for detection of infection is not defined. The PCR real-time seems to be more sensitive but contradictory information exists on its clinical utility. Objectives: To systematically quantify CMV replication by realtime PCR in allo-SCT recipients to determine its efficacy and safety in CMV disease prevention.

Patients, material and methods: Prospective study in consecutive Allo-STC recipients. All patients (pts) underwent CMV viral load quantification by RT-PCR during 6 months: once a week in the first 100 days and every two weeks thereafter. Pts received pre-emptive therapy with ganciclovir $(5 \mathrm{mg} / \mathrm{kg} / 12 \mathrm{~h} / 14$ days, or at least 7 days after a negative quantification) if the viral load increased above $1000 \mathrm{cop} / \mathrm{ml}$ in asymptomatic pts or with any positive viral load if clinical suspicion existed. For RT-PCR the viral DNA was isolated in plasma and was used as a template for the amplification and quantified.

Results: 17 Allo-SCT were performed between June/2008 and May/2009, with a median age 34 years (15-57). $70.6 \%$ were from peripheral blood and $64.7 \%$ from unrelated donors. The conditioning regimen was mieloablative in $64.7 \%$, and $76.5 \%$ of recipients were positive for CMV. The incidence of acute GVHD (II-IV grades) was $66.6 \%$ and of chronic GVHD $58.8 \%$. The incidence of CMV infection was $70 \%(n=12)$, and two pts developed CMV disease (11.7\%). No risk factors for CMV infection were identified among infected pts (conditioning regimen, unrelated donor, GVHD prophylaxis or treatment or previous positive serostatus for $\mathrm{CMV}$ in recipient or donor). The median viral load in infected pts was $7.25 \mathrm{cop} / \mathrm{ml}(0-180)$ and $191 \mathrm{cop} / \mathrm{ml}(35.5-346.5)$ in CMV disease patients $(P=\mathrm{NS})$. The global median days of treatment was 48.5 (10-198), being 10 days (10-150) for infected pts and 186.5 days in CMV disease pts $(175-198)(P=0.036)$. No CMV related mortality was observed.

Conclusions: RT-PCR is a sensitive and specific technique to monitoring CMV viral load after Allo-SCT and clinically useful to guide pre-emptive treatment without increasing incidence of CMV disease nor CMV related mortality.
P775

Indication of empirical antifungal therapy in selected patients with persistent febrile neutropenia according to clinical criteria and risk profile

A. Martín-Peña, M. Aguilar-Guisado, I. Espigado, E. Cordero, J.M. Cisneros Herreros on behalf of the Spanish Network for Research in Infectious Disease (REIPI)

Objectives: Universal empirical antifungal therapy (EAT) in every patient with persistent febrile neutropenia (PFN) is the standard of care, but some recent published data suggest that EAT could be applied only in selected patients. The aim of this study is to investigate a diagnostic and therapeutic protocol based on clinical criteria and risk profile, allowing the indication of EAT exclusively in selected patients with PFN without impact on invasive fungal infection (IFI) incidence and IFI-related mortality.

Methods: Prospective observational study including every persistent febrile neutropenia episodes in patients with hematological malignancies or stem cell transplantation (SCT) recipients admitted in the Hematology Service from October 2007 to November 2009. A previously defined diagnostic and therapeutic approach was applied in every PFN episode and EAT was indicated in patients with: (a) severe sepsis or septic shock; (b) focused infection: lung, central nervous system, sinus, abdominal or skin; (c) individualized clinical decision in patients at high risk of IFI. A comparative analysis of incidence of proven or probable IFI and IFI-related mortality was performed according to whether or not EAT was indicated.

Results: Eighty-five episodes PFN in seventy-two patients were included. The $48.2 \%$ were male and median age in years was 47 (15-75). The most frequent hematological malignancies were acute leukemia (45.8\%) and lymphoma (21.2\%). Thirtytwo patients were SCT recipients, $53.1 \%$ allogeneic, the $24.7 \%$ were IFI-high risk patients. The median of duration of neutropenia and fever were 14 days (range: 6-63) and 10 days (range: 5-37) respectively. EAT was indicated in fifty-two episodes $(61.2 \%)$ during a median of 11 days (range: $2-164)$; in the rest of episodes $(n=33)$ EAT was no indicated. The overall IFI incidence was $14.1 \%(n=12)$. In the group that received EAT, twelve patients developed IFI $(23.1 \%)$, in comparison with noone patient in the group that did not receive EAT. The 30 daysglobal mortality was $16.5 \%, 25 \%$ in the group that received EAT and $3 \%$ in the group that did not received it. The IFI-related mortality was null in the group that did not receive EAT and $3.8 \%$ ( 2 of 52 patients) in the group that received EAT.

Conclusion: These data suggest that, in the management of patients with PFN, the indication of EAT just in patients selected on the basis of clinical criteria and risk profile, may be safe and avoid over-treatment.

\section{P776}

CMV reactivation in recipients of autologous

haematopoietic stem cell transplantation:

single cancer centre experience

H.A. El Taani, F. Abdel Rahman, M. Saad, H. Al Khateeb, M. Al Jamily, A. Badeeb, A. Ahmad, R. Rihani, A. Zabin, N. Hussein, M. Sarhan

King Hussein Cancer Center (Amman, JO)

Objective: To determine the incidence, risk factors and outcome of Cytomegalovirus (CMV) reactivation and disease among recipients of autologous hematopoietic stem cell transplantation (AHSCT) at King Hussein Cancer Centre (KHCC).

Methods: We retrospectively reviewed charts of all AHSCT performed at KHCC for the presence of CMV reactivation and disease. CMV reactivation was assessed by demonstrating the presence of CMV pp65 antigen in circulating leukocytes. We assessed two main outcomes: the development of CMV reactivation/disease and the CMV-related mortality. Risk factors that were assessed for the development of CMV reactivation/disease included age at transplantation, underlying malignancy, 
pre-transplant CMV serology status, lines of chemotherapy used prior to transplantation, conditioning regimen, CMV prophylaxis regimen, use of immunosuppressive drugs and development of sepsis.

Results: Between May 2005 and June, 2009, 122 AHSCTs were performed at KHCC. Fifty one patients $(41.8 \%)$ developed a positive CMV antigenemia at a median of 31 days post AHSCT (range: $6-85$ days); $37 / 51$ patients had positive CMV antigenemia of less than 5 cells/250000 circulating leukocytes and $14 / 51$ had antigenemia $\geq 5$. None of the patients had evidence of CMV disease. Twenty two patients $(43.1 \%)$ received treatment for CMV reactivation (14 who had CMV antigenemia $\geq 5$ and 8 of the patients who had persistent CMV antigenemia $<5)$. The CMV antigenemia subsequently cleared on therapy. All but one of the untreated patients, with CMV antigenemia $<5$, cleared their antigenemia spontaneously. This patient developed sepsis with a multidrug resistant Acinetobacter. Factors that were associated with a significant increase in CMV reactivation included sepsis and the use of immunosuppressive drugs $(P$ value $=0.04$ and 0.02 respectively $)$. Age at transplantation, underlying disease, pre-transplant CMV serology status, CMV prophylaxis regimen, number of chemotherapy lines and conditioning regimen were not associated with a significant increase in CMV reactivation. Finally, CMV reactivation was not associated with a significant increase in mortality.

Conclusion: CMV reactivation occurs frequently in recipients of AHSCT patients. Sepsis and the use of immunosuppressive drugs are risk factors for the development of CMV reactivation.

\section{P777}

Antibacterial prophylaxis reduces the use of broad-spectrum antibiotics in autologous stem cell transplantation H. Hallböök (1), A.K. Lidström (2), K. Pauksens (2)

(1)Section of Haematology (Uppsala, SE); (2)Section of Infectious Diseases (Uppsala, SE)

The use of oral ciprofloxacin as antibacterial prophylaxis in autologous stem cell transplantation (SCT) is debated. Due to an outbreak of Extended Spectrum Beta-Lactamase producing (ESBL) E. Coli and Klebsiella pneumonie, the regimen at the Department of Hematology was changed. Since May 2006, prophylaxis has been given mainly to patients who are treated in the outpatient setting after autologous transplantation. The aim of this study was to analyze the use of broad-spectrum antibiotics and frequency of verified infections between the groups receiving or not receiving antibacterial prophylaxis.

Between October 2006 and May 2009140 patients with lymphoma, myeloma, AL amyloidosis or light chain disease underwent autologous SCT. Four patients were treated with antibiotics at start of conditioning and were excluded from this analysis, leaving 136 patients of which 64 received conditioning regimen with BEAC/BEAM and 72 with melphalan. Prophylaxis with ciprofloxacin $500 \mathrm{mg}$ bid from the day of stem cell infusion until ANC $>0.5 \times 10^{9} / \mathrm{l}$ were given to 60 patients, while 76 did not receive antibacterial prophylaxis. Median age was 58 y (16-68 y) and 45 were female and 91 male with no significant differences between the groups.

Observed frequency of septicemias was significantly lower for the patients receiving prophylaxis; $5 / 60(8 \%)$ cases compared to $28 / 76(37 \%)$ in the group with no prophylaxis $\left(P<0,001\right.$, chi $^{2}-$ test). There was a significant difference between the groups concerning days from stem cell infusion until treatment with antibiotics were started due to neutropenic fever with a median of 9 versus 11 days $(P<0,001$, Log-rank test). The total number of treatment days with i.v. antibiotics was in median $6(0-30)$ for the group with prophylaxis compared to $10(0-25)$ without prophylaxis $(P<0,001$, Mann-Whitney test). A significant difference remained when analyzing the number of treatment days with antibiotics for the patients receiving BEAC/BEAM; 8 (0-30) with prophylaxis versus $11(7-25)$ without $(P=0,002)$ and melphalan; $5(0-25)$ versus $9(0-19)(P<0,001)$.
We conclude that antibacterial prophylaxis with ciprofloxacin might reduce the frequency of septicemias and the use of broad-spectrum antibiotics in autologous SCT.

\section{P778}

Febrile neutropenia and early post-transplant severe bacterial infections after reduced-intensity allogeneic haematopoietic stem cell transplantation

L. Facchini, A. Ferrari, R. Martino, J. Piñana, P. Barba, M. Granell, A. Kerguelen, D. Valcarcel, J. Delgado, J. Briones, A. Sureda, S. Brunet, J. Sierra

Hospital de la santa Creu y sant Pau (Barcelona, ES)

We analyzed the risk factors (RFs) for the development of febrile neutropenia and the occurrence of severe bacterial infections (SBI) in the first 100 days post-transplant in 195 consecutive adult recipients of a reduced-intensity conditioning allogeneic hematopoietic stem cell transplantation (AlloRIC). The RIC regimens consisted in Fludarabine plus Melphalan (62\%) or Busulphan $(38 \%)$. In vivo T-cell depletion with ATG or Campath was used in $13 \%$ of the patients, and GVHD prophylaxis otherwise consisted in Cyclosporine $\mathrm{A}(\mathrm{CsA})$ associated with methotrexate (MTX) $(72 \%)$ or mycophenolate mofetil (MMF) $(28 \%)$. Febrile neutropenia occurred in 141 patients $(72 \%)$, which occurred always in the first 30 days post-AlloRIC. However, a SBI (bacteremia [excluding coagulase-negative staphilococci], pneumonia, urinary tract infection) occurred in 27 patients (14\%) during this early post transplant period (<day +30$)$, while 29 evaluable patients $(15 \%)$ developed a SBI in the intermediate post-transplant period (days +31 to +100 ). In multivariate analysis the RFs for the development of febrile neutropenia included duration of severe neutropenia $>6$ days $(P<.02)$ and grade $3-4$ oral mucositis or grade $2-3$ diarrhea $(P=.03)$. With respect to SBI in the intermediate (day +30 to +100 ) post-transplant period, RFs identified by multivariate analysis included corticosteroid therapy before day $+100(P<.01)$, MMF-based GVHD prophylaxis $(P<.01)$ and having had an early $(<$ day +30$) \mathrm{SBI}(P<.01)$. The rate of SBI from day +30 to +100 increased according to the number of RFs: thus, the rate of SBI was $1 / 74(1,4 \%)$ in patients without any RF, $13 / 77(17 \%)$ in patients with $1 \mathrm{RF}$ and $15 / 40$ $(38 \%)$ in those with 2 or 3 RFs.

In summary, febrile neutropenia and early SBI post-AlloRIC occur mostly in patients with severe mucositis and prolonged neutropenia, while post-engraftment the need for steroid therapy for GVHD was the major risk factor. Knowledge of these RFs may be useful in targeting the antibiotic prophylaxis to high-risk patients in the first 100 days post-AlloRIC.

\section{P779}

\section{Clinical outcomes of febrile neutropenia in the} pre-engraftment phase after haematopoietic stem cell transplantation

T. Yamashita, I. Kamiya, R. Hanajiri, T. Kikuchi, Y. Nagata, S. Wakabayashi, K. Taoka, T. Kobayashi, K. Ohashi, H. Sakamaki, H. Akiyama

Tokyo Metropolitan Komagome Hospital (Tokyo, JP)

Background: Infection is one of the major complications after hematopoietic stem cell transplantation (HCT). As conditioning regimens including high-dose chemotherapy and radiation lead to severe neutropenia and mucosal damage, host defenses are heavily compromised in the pre-engraftment phase after HCT. In this single-institute study, we retrospectively analyze the clinical data of $307 \mathrm{HCT}$ recipients in our hospital to investigate the incidence and duration of febrile neutropenia (FN), the efficacy of antibiotics therapy and factors that affect clinical outcomes. Patients and methods: Data on HCT recipients were obtained from the database and medical charts of Tokyo Metropolitan Komagome Hospital. We included patients with hematological diseases who received HCT between August 2004 and July 2009. The statistical significance of differences was assessed 
with the Student $t$ test and analysis of variance for continuous variables and with the Fisher exact test and chi-square test for categorical variables.

Results: A total of $307 \mathrm{HCT}$ recipients were eligible for this analysis. The median age was 43 years (16-67). Fifty-eight patients received autollogous $\mathrm{HCT}$ and 249 received allogeneic. As a rule, tosufloxacin and fluconazole were used for prophylaxis. Median duration of neutropenia after HCT was 17 days (4-91). In the pre-engraftment phase, 247 patients $(80.5 \%)$ had $\mathrm{FN}$. Variables associated with incidence of FN were age ( $\geq 43 \mathrm{vs}$. $\geq 44)(P=0.021)$, type of transplant (allogeneic vs. autologous) $(P=0.044)$ and stem cell source $(P=0.0016)$. FN after HCT was treated based on IDSA guidelines of 2002. In allogeneic setting, 57 patients received cefepime (CFPM) and 59 received melopenem (MEPM) as initial antibiotic therapy for FN. Success rate of $\mathrm{FN}$ treatment was higher in MEPM group (84.2\%) than CFPM group (32.6\%). But in CFPM group, success rate without vancomycin (VCM) was higher $(21.7 \%$ vs. $8.8 \%)$ and duration of antibiotics use were shorter (7.7 days vs. 11.4 days). Blood stream infections (BSI) were documented in $16 \%$ of FN cases, $60 \%$ of BSI cases were caused by Gram-positive cocci, and $30 \%$ were by Gram-negative rods.

Conclusion: Our study shows that about $80 \%$ of HCT cases had FN in the pre-engraftment phase. Patients' age, type of transplants and stem cell sources affect the incidence of FN. In the treatment of FN after HCT, carbapenem and vancomycin were needed in many FN cases, but in CFPM shows appropriate and rapid response in some cases.

\section{P780}

Impact of long-term low- versus high-dose acyclovir prophylaxis on cytomegalovirus infection and survival after allogeneic stem cell transplantation: a single-centre study A.P. Iori, E. lannella, A. Capobianchi, G.F. Torelli, W. Barberi, F. Natalino, V. Valle, B. Lucarelli, F. Milano, E. Arleo, S. Perrone, R. Foà, G. Gentile University La Sapienza (Rome, IT)

High-dose acyclovir (ACV) has been shown to reduce the risk of CMV infection and improve overall survival in patients undergoing a stem cell transplantion (SCT) (Prentice et al., Lancet 1994). We retrospectively compared two groups of patients who underwent an HLA identical allogeneic SCT, both receiving i.v. ACV $(15 \mathrm{mg} / \mathrm{Kg} /$ day $)$ from day -7 to day +20 after transplant followed by low-dose oral $(200 \mathrm{mg} \times 4$ day) (group A) or high-dose oral ( $800 \mathrm{mg} \times 4 /$ day) (group B) AVC while immunosuppressive therapy was ongoing. Aim of the study was to evaluate whether there was any difference in the rate of CMV infection, CMV disease, adverse events, probability of survival and transplant-related mortality (TRM) between the two groups. We analyzed 113 patients (50 in group A, 63 in group B) at risk of CMV infection (recipient and/or donor seropositive for CMV), managed between January 1999 and December 2008. CMV infection was monitored using the pp65 antigenemia assay. The two groups were comparable for age, sex, hematological disease, disease phase at transplant, CMV serology, conditioning regimens and supportive therapy. No differences were observed between the two groups considering the rate of engraftment and acute and chronic GVHD. Cumulative incidence of CMV infection was $64 \%$ in group $A$ and $50 \%$ in group $B$; this difference was statistically significant $(P=0.029)$. CMV disease was observed in 2 patients in group $A$ and in 3 patient in group $B$. Herpes simplex virus and varicella zoster virus infections were not observed in both groups during the first 6 months after transplant. Observed adverse events that were possibly related to the treatment were nausea and vomiting (55\% group in A vs. $66 \%$ in group B) and creatinine increase $(>1.5 \mathrm{mg} / \mathrm{dl})(45 \%$ group in group A vs. $56 \%$ in group B),the difference was not significant. The 1-year cumulative incidence of TRM was $15 \%$ and $18 \%$ for groups $A$ and $B$, respectively; the difference was not statistically significant. The 1-year probability of survival by the Kaplan-Meier method was $74 \%$ and $76 \%$ for group $A$ and $B$. respectively. In conclusion, high-dose oral acyclovir is effective in reducing the incidence of CMV infection in patients receiving an allogeneic SCT transplantation by an HLA identical sibling without a significant increase of late effects, even though no difference was observed in the incidence of CMV disease, survival or TRM between the two groups. Larger, randomized studies are required to confirm these results.

\section{P781}

Less diagnostic value of serum procalcitonin in febrile patients following allogeneic haematopoietic stem cell transplantation

Y. Nagata, K. Ohashi, I. Kamiya, R. Hanajiri, K. Taoka, S. Wakabayashi, T. Kobayashi, T. Yamashita, H. Akiyama, H. Sakamaki

Komagome Hospital (Tokyo, JP)

Background: The recent reports have suggested the promising predictive value of procalcitonin (PCT) in the diagnosis of bacterial infections following allogeneic hematopoietic stem cell transplantation (HSCT). To validate this hypothesis, we undertook a prospective monitoring of PCT during febrile episodes in 67 consecutive allogeneic HSCT recipients in a single institution.

Material and methods: We have prospectively monitored serum PCT and C-reactive protein (CRP) levels on the occasion of febrile episode $\left(>38^{\circ} \mathrm{C}\right)$ within 100 days after transplantation. In all patients, cultures from sterile sites and measurement of white blood cells (WBC) count were done in parallel. Febrile episodes were classified according to the final diagnosis in: fever of unknown origin, microbiologically or clinically documented infection and non-infectious fever.

Results: During last one year period, a total of 67 patients underwent allogeneic HSCT in our institution and 61 patients developed febrile episodes at median 7 days (range, 1-28 days). The etiology of fever was eventually classified as follows; bacterial sepsis $(n=9)$, clinically documented bacterial infection $(n=29)$, non-infectious fever $(n=20)$, neutropenic fever of unknown origin $(n=3)$. In view of 38 patients with apparent bacterial infection, only 9 patients $(23.7 \%)$ showed high level serum PCT $(>0.5 \mathrm{ng} / \mathrm{ml})$. Thus, sensitivity and specificity of serum PCT elevation in case of bacterial infection following allogeneic HSCT was $15.8 \%$ and $89.7 \%$, respectively. Morbidity prevalence rate was $56.7 \%$. Positive and negative predict value was $66.7 \%$ and $44.8 \%$, respectively. Likelihood ratio of a positive test was $1.53(P=0.39)$. Multivariate analysis disclosed that patients with infection tended to carry higher counts of WBC $(P=0.012)$ and longer febrile duration $(P=0.009)$. Overall survival was significantly shorter when serum PCT level was above $2.0 \mathrm{ng} / \mathrm{ml}(P=0.007)$ or CRP above $4.0 \mathrm{mg} / \mathrm{dl}(P=0.04)$.

Conclusions: Our results with small cohorts could not support the clinical utility of monitoring serum PCT in bacterial infection. Thus, serum PCT may not be a suitable diagnostic marker especially at early duration after allogeneic HSCT.

\section{P782}

Molecular detection of cytomegalovirus in biological

fluids other than plasma. Clinical relevance after allogeneic haematopoietic cell transplantation

A. Papalexandri, T. Touloumenidou, I. Zorbas, M. Gounari, P. Zerva, C. Apostolou, S. Tsimperis, A. Antoniou, C. Smias, I. Batsis, I. Sakellari, A. Anagnostopoulos

G.Papanikolaou Hospital (Thessaloniki, GR)

The precise role of quantitative real-time polymerase chain reaction (RQ-PCR) in the establishment of cytomegalovirus (CMV) infection in biological specimens other than plasma has not been defined conclusively. Several technical aspects remain unresolved, in particular the use of cut-offs for positivity. We retrospectively evaluated the clinical significance of posi- 
tive RQ-PCR tests for CMV DNA in biological fluids other than plasma from 73 patients after allo-HCT, with a special emphasis on samples with a low viral load ( $<500$ copies $/ \mathrm{ml})$. The viral load in plasma and urine was monitored once weekly by RQ-PCR, while other bodily fluid samples were tested only in symptomatic patients. We evaluated $92 \mathrm{CMV}$ positive samples documented in urine $(n=67)$, gastric fluid (GF) $(n=13)$ and bronchoalveolar lavage (BAL), $(n=12)$. RQ-PCR examination of paired plasma samples revealed concordant CMV viremia in only $23 / 92$ cases ( $25 \%$ in urine, $31 \%$ in GF, $17 \%$ in BAL). Overall, forty-six of 92 $(50 \%)$ samples were obtained from cases with clinically symptomatic disease: (i) cystitis, $n=23 / 67(34 \%)$ cases; (ii) gastritis, $n=13 / 13(100 \%$ ) cases; (iii) pneumonia, $n=10 / 12$ (83\%) cases, of which, however, only two were attributed solely to CMV. Viral load was a significant risk factor for CMV disease $(P=0,018)$. To further refine the interpretation of RQ-PCR results and ensure linearity, a cut-off of $500 \mathrm{CMV}$ copies/ml was applied. With this cut-off, samples were assigned to a low- or high-positive group (A or B, respectively).

Among cases with a low-positive urine sample assigned to group $A$, resolution of the infection without treatment was noted in only $14 / 41$ cases (34\%). The remainder (27/41, $66 \%$ ) eventually developed CMV cystitis, as did all group B cases. Concomitant $\mathrm{CMV}$ viremia was more frequent in group B $\{10 / 27(37 \%)\}$ than group $A\{4 / 41(9.7 \%)\}(P=0,013)$. We conclude that the molecular determination of CMV viral load in biological fluids other than blood by RQ-PCR offers diagnostic information of clinical relevance for the detection and prevention of CMV cystitis and, also, CMV gastritis after allo-HCT. A low molecular viral load in urine should alert the clinician to the possibility of impending CMV disease. Larger prospective studies are strongly warranted in order to define universally accepted thresholds that would assist in clinical decisionmaking and thus minimize the toxicity of unnecessary treatment. The clinical utility of this approach in BAL samples remains questionable.

\section{P783}

Posaconazole primary prophylaxis in HSCT recipients

L. Cameron, M. Streetly, M. Kazmi, K. Raj

Guy's Hospital (London, UK)

Background: The data for posaconazole (POS) as primary prophylaxis for invasive fungal infection (IFI) is limited. There are two published studies; Ullmann et al. reported that POS was as effective as fluconazole in preventing IFI in patients with graft versus host disease (GVHD) following allogeneic stem cell transplant (SCT). In neutropenic patients following chemotherapy for AML/MDS, Cornely et al. found POS to be superior to fluconazole/itraconazole in preventing IFI. Based on these large, randomised studies, POS has been adopted by our centre as primary antifungal prophylaxis. We describe our experience with POS which is predominantly in haematopoietic SCT (HSCT) recipients.

Methods: Retrospective analysis of adult patients who received POS prophylaxis between January 2007 and December 2009. Evidence of breakthrough IFI was recorded as per EORTC/ MSG definitions. Liver function tests (LFTs) prior to initiating, during and after treatment with POS were reviewed. Events that could contribute to LFT derangement were identified from the medical notes.

Results: 59 patients received POS prophylaxis $(n=52$ primary, $n=7$ secondary). Of these, 52 were undergoing HSCT $(n=32$ reduced intensity conditioning allograft, $n=10$ full intensity allograft, $\mathrm{n}=4$ autograft including total-body-irradiation or T-cell depletion, $n=2$ umbilical cord transplant, $n=4$ haploidentical transplant) and 7 were undergoing intensive chemotherapy for the treatment of leukaemia.

6 patients developed an IFI, classified as per EORTC/MSG definitions proven $(n=1)$, probable $(n=2)$, possible $(n=3)$. Of these, 3 patients had gut GVHD and 3 were undergoing intensive chemotherapy for the treatment of AML.
The causative organisms identified in the proven and probable cases were Candida glabrata $(n=1)$ and Aspergillus $\operatorname{spp}(n=2)$. LFTs deteriorated in 23 patients on POS ( $n=7$ abnormal LFTS pre POS, $n=16$ normal LFTs pre POS) and the cause was confidently attributed to GVHD $(n=7)$, sepsis $(n=5)$, viral reactivation $(n=5)$, other drugs $(n=5)$ and disease relapse $(n=1)$.

Conclusions: Primary prophylaxis was effective with $10 \%$ breakthrough. This occurred particularly in patients with gut GVHD. Therefore we suggest that an alternative be used. POS can be used safely in patients with deranged LFTs as an alternative to conventional antifungal therapies. This is particularly important following HSCT where the incidence of abnormal LFTs secondary to sepsis, drug toxicity, viral infection (CMV, EBV) and GVHD is high.

\section{P784}

Do infections have impact on early post-transplant mortality? P. Mikulasova

University Hospital Brno (Brno, CZ)

Infections are the most common complications in immunocompromised patients and, not surprisingly, infections are probably the most frequent cause of death in patients after hematopoietic stem cell transplantations (HSCT).

Our aim was to analyze mortality until day +100 after HSCT in all 785 patients (568 autologous, $140 \mathrm{HLA}$ identical sibling, 77 unrelated) transplanted in our center from January 2001 to March 2009.

The post-transplant mortality was $9.7 \%$ (31 autologous, $30 \mathrm{HLA}$ identical sibling, 15 unrelated) and we analyzed clinical, laboratory, and autopsy data from all 76 patients (male 45, female 31 , median age of $50,24-65$ ) who died in the early post-transplant period (until day +100 ). These patients were treated for AML $(n=20), \operatorname{ALL}(n=8), \operatorname{CML}(n=3), \operatorname{CLL}(n=3), \operatorname{MDS} / \operatorname{MPS}(n=8)$, $\mathrm{NHL}(n=9), \operatorname{MM}(n=21)$, others $(n=4)$. Infection was fatal in 14 patients $(18 \%)$. Other causes of death were: disease progression in 27 patients (36\%), bleeding in 9 patients $(12 \%)$, acute graft-versus-host disease in 5 patients $(6 \%)$ and other complications in 21 patients $(28 \%)$. Fungal infections were determined in $16 \%$ of all patients and 5 patients died for fatal fungal infection.

We conclude that infections are the main not-relapse cause of death during the early post-transplant period after HSCT. Moreover, fungal infection seems to be more frequent especially in allogeneic graft recipient and despite using new antimycotics systemic fungal infection is still often fatal. Even if cases, where severe infections are not the primary cause of death, they significantly deteriorate clinical status of patients and worse patient's prognosis.

\section{P785}

Safety and feasibility of posaconazole as oral antifungal prophylaxis in paediatric patients under 12 years of age following allogeneic HSCT

M. Döring, R. Handgretinger, I. Müller

University Children's Hospital (Tubingen, DE)

Pediatric patients undergoing allogeneic hematopoietic stem cell transplantation (HSCT) are at risk to acquire fungal infections. Posttransplant immune suppression, viral infections and acute graft-versus-host disease, are known risk factors for fungal infections. Thus, antifungal prophylaxis early after transplantation is indicated. Data for antimykotic prophylaxis in stem cell transplanted pediatric patients is scarce. Posaconazole is an extended spectrum oral azole which is widely used in adults. It is not metabolized to a significant extent through the cytochrome P450 enzyme system, an increase of the level of immunosuppressive drugs cannot be proven. We opted for oral prophylaxis using posaconazole and assessed safety, feasibility and initial data on efficacy in pediatric patients under the age of 12 following high dose chemotherapy and stem cell transplantation. 
10 patients (age range 1-9 years) were treated with $2 \times 5 \mathrm{mg} /$ $\mathrm{kg}$ b.w. per day after hematopoietic stem cell transplantation. The patient group consisted of pediatric patients with malignant diseases (relapse of acute myeloid leukemia, relapse of acute lymphoblastic leukemia, chronic myeloid leukemia, myelodysplastic syndromes, and neuroblastoma) one child with Morbus Tay-Sachs and one child with Chediak-Higashi Syndrome. We analyzed the effects and side effects of posaconazole during the first hundred days after transplantation. Once a week we analyzed the serum levels of candida antigen and galactomannan antigen. We screened the effects of posaconazole on the number of granulocytes, lymphocytes, and monocytes, the liver enzymes AST, ALT and GGT, bilirubin and inflammation factor C-reactive protein. None of the 10 children suffered from any fungal infection during the observation period. Serum level of candida antigen and galactomannan antigen remained below the critical level. The number of granulocytes, lymphocytes and monocytes did not chance in connection with the treatment with posaconazole. The liver enzymes AST, ALT and GGT as well as the direct and indirect bilirubin showed no changes during the treatment with posaconazole. Side effects were nausea, itching and vomiting.

\section{P786}

Impact of previous fungal infection on the outcome of allogeneic haematopoietic stem cell transplantation: after introducing new antifungal agents

M. Yanagisawa, Y. Inamoto, N. Imahashi, S. Tsukamoto, M. Adachi, M. Sawa, K. Watamoto, A. Kohno, T. Ono, H. lida, M. Murata, K. Miyamura, T. Naoe on behalf of the Nagoya Blood and Marrow Transplantation (NBMTG)

A history of invasive fungal infection (IFI) has been considered a contraindication to allogeneic hematopoietic stem cell transplantation (allo-HSCT). However, use of new antifungal agents, in combination with reduced-intensity conditioning regimen, may give patients with hematological malignancy a chance to receive allo-HSCT. To confirm this hypothesis, we retrospectively reviewed 23 patients with a history of IFI who had undergone allo-HSCT from 1993 to 2008 in the centers of Nagoya Blood and Marrow Transplantation Group.

Patients' median age was 45 years (18-63 years). Twenty-two patients had hematological malignancies. Used graft sources were peripheral blood stem cells $(n=8)$, bone marrow $(n=13)$ and cord blood $(n=2)$. Sixteen patients received fludarabinebased reduced-intensity conditioning. Three patients were diagnosed as proven, 8 as probable and 12 as possible IF before allo-HSCT. Invaded organs were liver $(n=10)$ and lung $(n=11)$. The median onset days of the fungal diseases were 58 days (7-280 days) before allo-HSCT. Four patients received allo-HSCT with fluconazole or amphotericin B syrup, 5 patients with intravenous amphotericin-B, and 14 patients received one of following new antifungal agents: voriconazole, micafungin and liposomal amphotericin B. On the day of alloHSCT, IFI was controlled (defined as afebrile and negative of C-reactive protein ) in 16 patients, and uncontrolled in 7 patients.

After a median follow-up of 238 days (4-738 days) from the day of allo-HSCT, 9 out of 23 patients were alive with an overall survival (OS) rate of $54 \%$ at 1 year after transplantation. Relapse rate at 1 year was $21 \%$ and non-relapse mortality rate at 1 year was $26 \%$. Two deaths were related to fungal infection prior to allo-HSCT; one patient with lung Aspergillosis died of pulmonary bleeding and the other with Candida abscess in liver died of hepatic sinusoidal obstruction. Both of them died within 30 days after allo-HSCT.

$\mathrm{OS}$ at 1 year in patients with reduced-intensity conditioning $(n=16)$ was significantly higher than that in patients with myeloablative conditioning $(n=7)(76 \%$ versus $14 \% ; P=.0045)$. OS at 1 year tended to be higher in patients who received new antifungal agents $(n=14)$ than in those who received other antifungal agents $(n=9)(71 \%$ versus $30 \% ; P=.071)$.
In summary, our data suggest that a history of IFI is not necessarily a contraindication for allo-HSCT if reduced-intensity conditioning and new antifungal agents are used.

\section{P787}

Caspofungin plus posaconazol as salvage therapy of invasive fungal infections in haematopoietic cell transplantation

H. Lellek, F. Dahlke, F.A. Ayuk, C. Wolschke, N. Kröger, A.R. Zander

Clinic for Stem Cell Transplantation (Hamburg, DE)

Background: Substantial improvements of treatment against invasive fungal infections have been achieved by the introduction of new antifungal agents including azoles (e.g. posaconazol) and echinocandins (e.g. caspofungin). However, mortality associated with treatment-refractory aspergillosis remains high. Preliminary data suggest that combination of azoles and echinocandins may increase activity against refractory invasive aspergillosis (IA).

Objective: In the present study we evaluated the efficiency and safety of caspofungin and posaconazol for salvage therapy in hematopoietic cell transplantation.

Patients and methods: In this monocentric, retrospective study, 32 hospitalized hematopoietic cell transplant recipients with IA refractory to primary treatment were treated with a combination therapy of caspofungin $1 \times 50 \mathrm{mg}$ and posaconazol $4 \times 200 \mathrm{mg}$. Efficacy was assessed by clinical symptoms and the degree of pulmonary infiltrate regression.

Results: A favorable response was seen in the majority of patients $(78 \%)$. In two patients $(6 \%)$ clinical improvement, but no decline in pulmonary infiltrates was observed. Five patients $(16 \%)$ did not respond to combination treatment with a fatal outcome in four of them. Combination therapy was well tolerated. No patient discontinued treatment due to toxicity.

Conclusion: This study indicates that the combination of caspofungin and posaconazol may provide an effective and tolerable therapy of IA in immunocompromised patients refractory to primary treatment.

\section{P788}

Meropenem as antibacterial prophylaxis for allogeneic stem cell transplantation recipients

B.S. Muiña, C. Castilla-Llorente, I. Heras, P. Gallego, J. Nieto, F. de Arriba, M. Osma, V. Vicente

Hospital J.M. Morales Meseguer (Murcia, ES)

Background: Bacterial infections are a severe complication of patients undergoing stem cell transplantation despite early aggressive antimicrobial therapy.

Aims: To analyze the efficacy of meropenem as antibacterial prophylaxis during allogeneic stem cell transplantation procedure (alloSCT). To determine the risk factors to develop a febrile episode under this prophylaxis.

Patients and methods: This is a descriptive, observational and retrospective study which included 48 consecutive patients who underwent alloSCT between January 2005 and December 2008 in our institution. All patients were treated with meropenem (1 $\mathrm{g}$ q8h) starting on the first day with a granulocyte count (GC) $<0.5 \times 10 \%$ and it was maintained until myeloid engraftment $\left(G C \geq 0.5 \times 10^{\circ} / \mathrm{L}\right)$. Patients were examined daily for symptoms and signs of infection. A febrile episode was defined as an axilar temperature above $38^{\circ} \mathrm{C}$ for $2 \mathrm{~h}$ or more or a single temperature above $38.5^{\circ} \mathrm{C}$. Statistical analysis: qualitative variables were analyzed using $\mathrm{CH}^{2}$ test and Student's t-test to compare qualitatives vs. quantitative variable. Risk factors for development of fever were identified by univariate and multivariate logistic regression models. Results: The median age of the 48 patients was 42 years (range, $10-67$ ) and $54 \%$ of them were male. A total of $36(75 \%)$ patients received hematopoietic stem cells from sibling donors. Conditioning regimen was myeloablative in about $48 \%$ of the 
cases. $32(67 \%)$ transplantations were performed in patients who had intermediate-advanced disease. During admission $52 \%$ patients developed mucositis and $23 \%$ acute graft-versushost disease (aGvHD). The median days of neutropenia was 14 days (range, $0-57$ ), the median day after transplant to reach a GC $\geq 0.5 \times 10^{9} / \mathrm{L}$ was day +16 after infusion (range, $9-32$ ). The mean day of first febrile episode was day $+10( \pm 8.68)$. $42 \%$ of patients developed fever in the early post transplant period. Multivariate analysis showed that significant predictors of fever were mucositis (OR 22.22 95\% Cl (3.21-153.31); $P=0.002)$ and delayed neutrophil engraftment (OR 1.23 95\% Cl (1.051.44); $P=0.008$ ). Infections were microbiologically documented in 6 patients $(30 \%)$ out of 20 who developed fever.

Conclusions: Prophylaxis with meropenem in patients undergoing allogeneic stem cell transplantation is a good antimicrobial option. Mucositis and delayed neutrophil engraftment were independent risk factors for the development of a febrile episode under this prophylaxis.

\section{P789}

Antifungal prophylaxis with posaconazole in different risk-groups of allograft recipients

J. Sinko, L. Lengyel, A. Barta, E. Torbagyi, L. Gopcsa, A. Batai, Z. Csukly, R. Nikolova, A. Varkonyi, P. Remenyi, T. Masszi

St. Istvan \& St Laszlo Hospital (Budapest, HU)

Background: Posaconazole (POSA) is registered for moldactive antifungal prophylaxis in neutropenic patients with acute myeloid leukemia/myelodysplastic syndrome and for allogeneic hematopoietic stem cell transplant (allo-HSCT) recipients with graft-versus-host disease (GvHD).

Methods: In our single-center observational survey a total of 36 allo-HSCT recipients were given POSA prophylaxis and evaluated for clinical efficacy. In 17 patients a myeloablative, in further 19 reduced intensity conditioning regimen was used. Grafts from HLA identical sibling donors were transplanted in 19 and from matched unrelated donors (MUD) in 17 cases. In subgroup A) POSA was given during the neutropenic phase and stopped at engraftment in cases with no GvHD (11 patients). In subgroup B) prophylaxis was also started with neutropenia but carried on during the subsequent period of acute GvHD and immunosuppression (16 patients). In subgroup C) POSA was started post engraftment in cases of severe GvHD (9 patients). On the basis of fungal history prophylaxis was classified as being primary (31 patients) or secondary (5 patients). The median duration of prophylaxis was $81(11-311)$ days. Patients were followed for median 275 days (21-481). Success was defined as absence of invasive fungal disease (IFD) at the end of the observation in patients on POSA. Success with modification was used for cases receiving empirical antifungal therapy following POSA. All patients developing proven or probable IFD were regarded as failures.

Results: At the end of the follow-up period POSA prophylaxis was successful in 29 patients $(81 \%)$. Success with modification was registered in $3(8 \%)$ and failure in 4 cases $(11 \%)$. Subgroups: A). Success: 10/11, success with modification: 0/11, failure: 1/11. B). Success: $12 / 16$, success with modification: 2/16, failure: $2 / 16$. C).

Success: $7 / 9$, success with modification: $1 / 9$, failure: $1 / 9$.

Failures: 1. Male (36 y), MUD, grade 4 intestinal GvHD, disseminated Aspergillus and Rhisopus infection, died. 2. Female (24 y) sibling donor, grade $3 \mathrm{GvHD}$, probable aspergillosis, survived. 3. Male (20 y), sibling donor, noncompliant, candidemia (C. krusei), died. 4. Male (54 y), sibling donor, grade 4 intestinal GvHD, died, on autopsy disseminated zygomycosis detected. Conclusions: In allo-HSCT recipients POSA prophylaxis seems to be effective for all risk groups in all risk periods. Importance of gastrointestinal absorption and compliance must be emphasized.
P790

Potential benefits of prophylactic intravenous immunoglobulin in allogeneic haematopoietic stem cell transplantation in children

L. Van Bruwaene, I Meyts, V. Labarque, K. De Boeck, A. Uyttebroeck, M. Renard

University Hospital Leuven (Leuven, BE)

Introduction: The routine use of intravenous immunoglobulin (IVIG) after hematopoietic stem cell transplantation (HSCT) is a matter of debate, especially in pediatric HSCT, where data are scarce. Until recently, all pediatric patients undergoing allogenic HSCT in our center, received IVIG $(400 \mathrm{mg} / \mathrm{kg} / \mathrm{w}$ from engraftment until $\mathrm{d}+180 ; 400 \mathrm{mg} / \mathrm{kg} / \mathrm{month}$ from $\mathrm{d}+180$ until $d+365$ ). Adjustments were performed to obtain IgG through levels above $6 \mathrm{~g} / \mathrm{L}$.

Aim: To evaluate the potential benefit of IVIG in pediatric allogeneic HSCT in terms of transplant-related mortality (TRM), incidence of bacteremia, invasive fungal infection (IFI) and CMV infection during the first year post-HSCT.

Methods: We conducted a retrospective study including all allogeneic pediatric HSCTs between January 2001 and December 2007 and compared the results with historic data using PubMed search strategies. For bacteremias and IFI, the incidence of infectious episodes (infection rate (IR)) was calculated as the number of events divided by the patient days at risk and expressed as episodes/100 patient days at risk. Patient days at risk were defined as the days between the day of transplant and day 365 or the date of death, relapse or graft failure. $95 \%$ confidence intervals $(95 \% \mathrm{Cl})$ were calculated by using the Poisson distribution function. Overall survival and cumulative incidence of TRM and relapse were described according to the Kaplan-Meier method. Comparison of the survival curves was made using the Logrank test (MedCalc and JavaStat software). Infection rate (IR) was specified for the pre-engraftment period (days 0-30), the mid-recovery phase (days 31-100) and the late recovery phase (days 101365). Veno-occlusive disease (VOD)-related mortality was also analysed.

Results: 51 HSCTs were performed in 48 patients. Previously published studies were reviewed and data were compared. Only one other study used prophylactic IVIG. A relatively high rate of bacteremias in the pre-engraftment phase was observed as well as of IFI after $\mathrm{d}+100$ (Tables 1-2). CMV infection and disease were comparable. TRM after 100 days and after one year was $8.3 \%$ and $15.3 \%$ respectively. There was no mortality due to VOD despite standard use of IVIG.

Conclusion: Although only minor differences occurred when infection rates ware compared, TRM was slightly less in this study where pediatric allogeneic HSCT recipients received routine IVIG. A prospective trial is needed to investigate the benefit of routine IVIG after HSCT in children. 


\begin{tabular}{|c|c|c|c|c|c|c|c|c|}
\hline \multirow{2}{*}{$\begin{array}{l}\text { Period } \\
\text { (doys) }\end{array}$} & \multirow{2}{*}{$\begin{array}{l}\text { Proce } \\
\text { dures } \\
\text { ot risk }\end{array}$} & \multirow{2}{*}{$\begin{array}{l}\text { Pobent- } \\
\text { doy ot } \\
\text { risk }\end{array}$} & \multicolumn{3}{|c|}{ Bocteremia } & \multicolumn{3}{|c|}{ Invosive fungal infection (IFI) } \\
\hline & & & Orgonigm & $\begin{array}{l}\text { Episodes (\$6 of } \\
\text { procedures) }\end{array}$ & $\begin{array}{c}\text { infectoon Rote } \\
(95 \% 0)\end{array}$ & (if) & $\begin{array}{l}\text { Episcodes(66 of } \\
\text { procedures) }\end{array}$ & $\begin{array}{c}\text { infection Rote } \\
(95 \% \text { C) }\end{array}$ \\
\hline \multirow[t]{4}{*}{0.30} & 50 & 1461 & All & $22(44)$ & $1.51(0.94-2.28)$ & All & $0(0)$ & $0(0-0.25)$ \\
\hline & & & Gram+ & $14(28)$ & $0.96(0.52-1.6)$ & Probable & $0(0)$ & $0(0.0 .25)$ \\
\hline & & & Gram. & $6(12)$ & $0.41(0.15-0.89)$ & Proven & $0(0)$ & $0(0.0 .25)$ \\
\hline & & & Polybacterial & $2(4)$ & $0.14(0.02-0.49)$ & & & \\
\hline \multirow[t]{4}{*}{$30-100$} & 48 & 3123 & All & $6(12.5)$ & $0.19(0.07-0.42)$ & All & $1(2)$ & $0.03(0-0.18)$ \\
\hline & & & Gram + & $4(8)$ & $0.13(0.03-0.33)$ & Probable & $0(0)$ & $0(0-0.12)$ \\
\hline & & & Gram - & $0(0)$ & $0(0-0.12)$ & Proven & $1(2)$ & $0.03(0-0.18)$ \\
\hline & & & Polybacterial & $2(4)$ & $0.06(0.01-0.23)$ & & & \\
\hline \multirow[t]{4}{*}{$100-365$} & 543 & 9652 & All & $11(25)$ & $0.11(0.06-0.2)$ & All & $5(11.6)$ & $0.05(0.02-0.12)$ \\
\hline & & & Gram + & $8(18)$ & $0.08(0.04-0.16)$ & Probable & $3(7)$ & $0.03(0.01-0.09)$ \\
\hline & & & Gram- & $1(2)$ & $0.01(0-0.06)$ & Proven & $2(4.7)$ & $0.02(0-0.07)$ \\
\hline & & & Polybacterial & $2(4)$ & $0.02(0-0.07)$ & & & \\
\hline
\end{tabular}

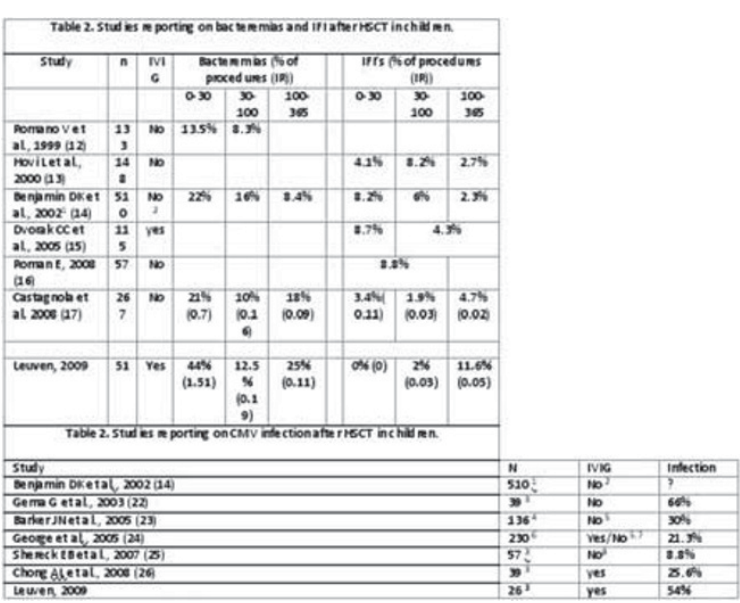

P791

Unusual infections in recipients of cord blood transplantation at a tertiary care centre in India R. Raj

Apollo Speciality Hospital (Chennai, IN)

Infections are the major cause of 100 day mortality in recipients of matched unrelated stem cells. We present data on infections seen in our unrelated cord transplant recipients over a 30 month period from April 2007 to October 2009. A total of 10 transplants have been performed at our unit using NMDP cords. The units were a $5 / 6$ match in 7 cases and $4 / 6$ in 3 cases. All units had a total nucleated cell count of over $3 \times 10^{7} / \mathrm{kg}$ and were thawed at the bedside just prior to infusion. The underlying diagnoses were Fanconi anaemia, relapsed leukaemia and Hurler syndrome. All patients had antithymocyte globulin as part of conditioning.

Klebsiella was the most common gram negative bacteria isolated in the first 48 hours after infusion. This responded to administration of broad spectrum antibiotics. 2 children had Burkholderia cepacia and 2 had Acinetobacter boumanii following prolonged use of broad spectrum antibiotics. One child had vancomycin resistant enterococcus - VRE, which is a rare isolate at our institution. One child had multiple bacterial infections due to a blind loop in the intestine following graft versus host disease of the gut. This included several cultures of Klebsiella, E. coli and a terminal event due to pan resistant Pseudomonas. One child with Hurler syndrome had a mild respiratory illness 5 months post transplantation and succumbed rapidly in 48 hours before any diagnosis could be established. CMV reactivation occurred only in children who needed additional immunosuppression for graft versus host disease and usually settled with pre-emptive therapy. High viral loads of 28,000 copies were only seen in the unrelated transplant setting but responded to ganciclovir.
Infection was the main cause of mortality in 3 of the 10 cases. Sinusoidal obstruction syndrome and graft versus host disease accounted for mortality in two other children. Our experience has shown that multiple blood cultures are essential in the management of fevers in neutropenic children undergoing unrelated transplantation. The duration of neutropenia is prolonged and the median time to engraftment is 23 days. Prolonged use of broad spectrum antibiotics results in resistant bacterial infections which result in mortality.

\section{P792}

Tenofovir for the treatment of hepatitis B virus reactivation in patients with chronic GvHD

I. Hilgendorf, M. Loebermann, K. Borchert, C. Junghanss, M. Freund, M. Schmitt

University of Rostock (Rostock, DE)

Background: Reactivation of resolved hepatitis B virus (HBV) infection is a well known complication in allogeneic haematopoetic stem cell transplantation (alloHSCT) recipients, which is usually treated with lamivudine. In light of the possibility that the prolonged use of lamivudine may foster the emergence of mutant lamivudine-resistant HBV strains, we decided to use the newer anti-HBV agent tenofovir in two patients (pts) with chronic Graft versus host disease (cGVHD) and hepatitis B virus reactivation. Tenofovir disoproxil fumarate (Viread) is a nucleotide analog reverse transcriptase inhibitor with a potent antiviral activity, a favorable safety profile and a higher barrier to the development of resistance. To our knowledge it is the first report on the use of tenofovir in this clinical setting.

Case report: Both female pts had a resolved HBV infection (hepatitis $B$ core antibody-positive, hepatitis $B$ surface antigen-negative, hepatitis $b$ surface antibody-positive) before they received alloHSCT in 2005 for acute/chronic myeloid leukemia. Chronic GVHD occurred 4 and 8 months after HSCT, respectively. First pt: at time of diagnoses of HBV-reactivation the pt received immunosuppressive therapy with everolimus and prednisolon $(10 \mathrm{mg})$ due to severe sclerodermoid cGVHD. The liver enzymes were slightly elevated at time of clinical flare of hepatitis reactivation in this pt. Second pt: the immunosuppression for severe cGVHD of the lung consisted of rapamycin, mycophenolate sodium and prednisolon $(30 \mathrm{mg})$. At time of diagnosis she complained only about fatique and had no signs of liver dysfunction.

Both pts received an oral administration of $245 \mathrm{mg}$ Viread daily and responded with reduction of HBV-DNA replication. The viral load dropped from $4.5 \times 10(9)$ to $1.4 \times 10(4)$ copies $/ \mathrm{ml}$ in pt 1 and from $5.2 \times 10(8)$ to $3.2 \times 10(3)$ copies $/ \mathrm{ml}$ in pt 2 within seven months. The antiviral therapy was well tolerated without any side effects.

Conclusion: Tenofovir is effective as well as feasible in the treatment of hepatitis B virus reactivation after alloHSCT. Due to the lower risk of development of drug-resistance the newer antiHBV agents (tenofovir, entecavir) should be preferred especially in patients with chronic GVHD, who require long term immunosuppressive as well as antiviral therapy. 


\section{P793}

Infection incidence and characteristics in 202 lymphoma patients after autologous haematopoietic cell transplantation at a single-centre

J. Romejko-Jarosinska, L. Poplawska, B. Ostrowska, E. Paszkiewicz-Kozik, K. Domanska-Czyz, H. PolowniakPracka, E. Ochman, M. Pawelec, E.K. Machaj, J.A. Walewski MSC Memorial Cancer Center (Warsaw, PL)

Between January 2005 and December 2008, 202 patients (non-Hodgkin lymphoma - 169, multiple myeloma - 33) underwent high dose chemotherapy (HDT, BEAM - 139, CBV -9 , Mel200 - 33, others - 21) followed by autologous hematopoietic cell transplantation (auto-HCT) at our institution. We performed retrospective analysis of infectious complications after auto-HCT. All patients (pts) were isolated in HEPA-filtered single room, received oral antibacterial and antifungal (including anti-PJP) prophylaxis, and granulocyte colony stimulating factor (G-CSF) support after auto-HCT. All apheresis products were monitored for bacteriologic sterility. Median duration of neutropenia was 9 days. The overall incidence of fever requiring antimicrobial treatment was $75,5 \%$. The origin of fever was unknown in two third of cases. Primary bacteremia occurred in $25,7 \%$, pneumonia - in $2,5 \%$, skin infections - in $1,5 \%$, conjunctivitis - in $1 \%$ of pts. The catheter-related infection was observed in $3 \%$ of pts. In $22 \%$ (45 pts) cases bacterial contamination for apheresis products was found. Sepsis was developed in 12 of these pts. Four pts had invasive fungal infections, and one patient required anti-CMV treatment. Septic shock occurred in $3 \%$ of pts (6 pts) but there were no fatalities. Serum procalcitonin were measured in 20 patients with fever, two of them had increased procalcitonin level. Infectious mucositis was developed in $67 \%$ of pts. Median time to incidence of fever after auto-HCT was 4 days (range 0 to 15 days). The most frequently isolated pathogens were coagulase-negative Staphylococci (62\%). Initial, usually empiric, antibiotic therapy consisted of broad-spectrum beta-lactam agents combined with amikacin. This therapy was successful in $39 \%$ of patients who had fever of unknown origin and in $48 \%$ of patients with documented infections. Median duration of antimicrobial treatment was 9 days. In conclusion, infections were not causing mortality or clinically significant morbidity in patients after HDT followed by auto-HCT under prophylactic measures as used in these patients. The most frequent type of infection was neutropenic fever of unknown origin. More than half of infection episodes were caused by coagulase-negative Staphylococci. Empiric broad-spectrum beta-lactam antibiotics combined with amikacin were not sufficiently efficacious in eradicating all pathogens and ultimate therapy guided by microbiology sensitivity testing was successfull.

\section{P794}

Features of Epstein-Barr virus reactivation after reduced-intensity conditioning unrelated umbilical cord blood transplantation

Z. Peric (1), P. Chevallier (1), X. Cahu (1), T. Guillaume (1), J. Delaunay (1), S. Ayari (1), V. Dubruille (1), S. Le Gouill (1), B. Mahe (1), T. Gastinne (1), N. Blin (1), B. Saulquin (1), N. Milpied (1), R. Vrhovac (2), J.L. Harousseau (1), P. Moreau (1), M. Coste-Burel (1), B.M. Imbert-Marcille (1), M. Mohty (1)

(1)CHU de Nantes (Nantes, FR); (2)University Hospital Merkur (Zagreb, HR)

Because of the slow kinetics of immune reconstitution after UCBT, previous studies showed that Epstein Barr Virus (EBV) reactivation and EBV induced lymphoprolipherative disease (LPD) may be of matter of concern. This single centre study assessed incidence and predictive factors of EBV reactivation and LPD in 33 consecutive patients undergoing reduced-intensity conditioning (RIC) umbilical cord blood transplantation (UCBT)

The median age was 50 (range, $18-66)$ years. $58 \%(n=19)$ of the patients had a myeloid malignancy, $36 \%(n=12)$ had a lymphoid malignancy and $6 \%(n=2)$ had sAA. $91 \%(n=30)$ of the patients received 2 CB units and $9 \%(n=3)$ received a single CB. The patients received a median of $4.0 \times 10^{7} / \mathrm{Kg}$ (range, 2.2-5.8) total nucleated cells and a median of $0.9 \times 10^{5} / \mathrm{Kg}$ (range, $0.2-3.7)$ CD34 + cells. Donors and recipients were mismatched with one mismatch in $43 \%$ of cases and 2 mismatches in $57 \%$. A RIC including fludarabine $\left(200 \mathrm{mg} / \mathrm{m}^{2}\right.$ total dose), cyclophosphamide $(50 \mathrm{mg} / \mathrm{Kg})$ and low dose TBI (2 Gy.) was used in 29 cases ( $88 \%$ ), while 8 patients $(24 \%)$ who were not heavily pretreated before UCBT received ATG. In the first 6 months, patients were screened weekly for EBV reactivation by DNA-PCR. EBV reactivation was defined as viremia of 1000 EBV-DNA copies/105 cells. Patients with EBV reactivation were pre-emptively treated with rituximab.

The median follow-up for surviving patients was 468 (range, 92-1277) days. Engraftment occurred at a median of 12 (range, $8-60$ ) days and $15 \%$ of patients developed grade $2-4$ acute GVHD. EBV reactivation was observed in 5 patients (15\%) at a median of 132 (range, 85-438) days after UCBT. Among the 5 patients experiencing EBV reactivation, 2 patients received ATG as part of their RIC. Four patients were treated with a median of 3 (range, 1-8) rituximab infusions. Two patients responded to Rituximab, but 2 patients developed LPD. One of these 2 patients died before receiving any other anti-EBV therapy. In the other patient, LPD could be controlled after additional chemotherapy and 2 infusions of EBV specific CTLs. Of note, there was no significant difference in overall survival between patients with or without EBV reactivation $(P=0.33)$. Overall, this study shows the rate of EBV reactivation after RIC UCBT to be relatively low $(15 \%)$ and this is comparable to the incidence expected with PBSC or BM mismatched transplants. However, close EBV monitoring and the use of pre-emptive rituximab treatment appears to be mandatory since some cases may progress to LPD requiring additional interventions such as EBV-specific CTLs.

\section{Chronic leukaemia}

\section{P795}

Human leukocyte antigen matching and conditioning Impact on long-term transplant outcome after allogeneic haematopoietic stem cell transplantation for chronic lymphocytic leukaemia: study from the EBMT Registry M. Michallet (1), M. Sobh (1), S. Morisset (1), D. Niederwieser (2), V. Koza (3), T. Ruutu (4), N. Russell (5), L. Verdonck (6), D. Milligan (7), N. Dhedin (8), T. Kozak (9), M. Boogaerts (10), J. Finke (11), V. Dubois (1), A. Van Biezen (12), R. Brand (13), T. De witte (14), P. Dreger (15)

(1)Hôpital Edouard Herriot (Lyon, FR); (2)University of Leipzig (Leipzig, DE); (3)University Hospital Pilsen (Pilsen, CZ); (4)Helsinki University Central Hospital (Helsinki, Fl); (5)Nottingham University Hospital (Nottingham, UK); (6)University Medical Centre (Utrecht, NL); (7)Birmingham Heartlands Hospital (Birmingham, UK); (8)Hôpital Pitié-Salpêtrière (Paris, FR); (9)University Hospital Kralovske Vinohrady (Prague, CZ); (10)U.Z. Gasthuisberg (Leuven, BE); (11)University Medical Center (Freiburg, DE); (12)EBMT (Leiden, NL); (13)Leiden University (Leiden, NL); (14)Radboud University (Nijmegen, NL); (15)University Hospital (Heidelberg, DE)

To evaluate the impact of difference in HLA matching degree among transplants from unrelated donors (UD) and the difference between RIC and standard conditioning on different outcomes in CLL, we have analyzed $370 \mathrm{CLL}$ patients who underwent allogeneic HSCT reported to the EBMT registry between 1995 and 2008. There were 198 HLA identical siblings and among transplantations from UD, there were 31 well matched in high resolution (WMUD), 30 matched in low resolution (LRMUD) and 111 mismatched in high resolution (MMUD). 
Regarding conditioning, 267 (72\%) were RIC and 103 (28\%) standard (patients details in Table).

Results: After transplantation, 359 (97\%) patients engrafted. The cumulative incidence of AGVHD for the total population was $22 \%$ for grade II and $13 \%$ for grade > II [Siblings: $20 \%$ and $13.3 \%$; WMUD: $39.7 \%$ and $6.4 \%$; LRUD \& MMUD: $23.3 \%$ and $17.7 \%$ respectively]. At 1 year after transplant for the total population, the cumulative incidence of limited and extensive cGVHD was $17 \%(15-19)$ and $18.4 \%(13-23)$ respectively [Siblings: $16.2 \%$ and $17.2 \%$; WMUD: $25.8 \%$ and $22.8 \%$; LRUD \& MMUD: $16.3 \%$ and $19.1 \%]$. After a median follow up of 48 months, we found a high significant difference in term of OS between the siblings and WMUD versus LRUD \& MMUD groups $(P=0.002)$ (Figure). We observed also a high significant difference in term of transplant related mortality (TRM) between the same groups $(P=0.0022)$. We did not find any significant difference in term of OS between RIC and standard conditioning groups even after adjusting on the HLA groups, we also found a high significant difference between patients in CR, PR and less than PR at transplantation (5 years OS: $75.5 \%, 55.5 \%$ and $42.8 \%$ respectively). The multivariate analysis using Cox model showed a significant impact of 3 factors on OS: age [HR $=1.04$ (1.01-1.6) $P=0.0004]$, PS $[\mathrm{HR}=2.75(1.5-5.1) P=0.001]$ and HLA MMUD + LRUD group [HR=1.43 $(1.01-2.01) P=0.04]$. The same factors were also highly significant in term of TRM: age $[\mathrm{HR}=1.04(1.001-1.07) \quad P=0.0033]$; PS [HR=2.47 (1.1-5.4) $P=0.009]$ and MMUD group $[H R=1.8(1.07-3.37) P=0.004]$. Conclusion: We pointed out for the first time that there is no difference of OS between HLA identical siblings and WMUD which was significantly higher than observed for LRUD and MMUD related to a significant higher TRM observed within these two later kinds of donors. Moreover we showed no significant difference in terms of OS, relapse and TRM between RIC and standard conditioning.
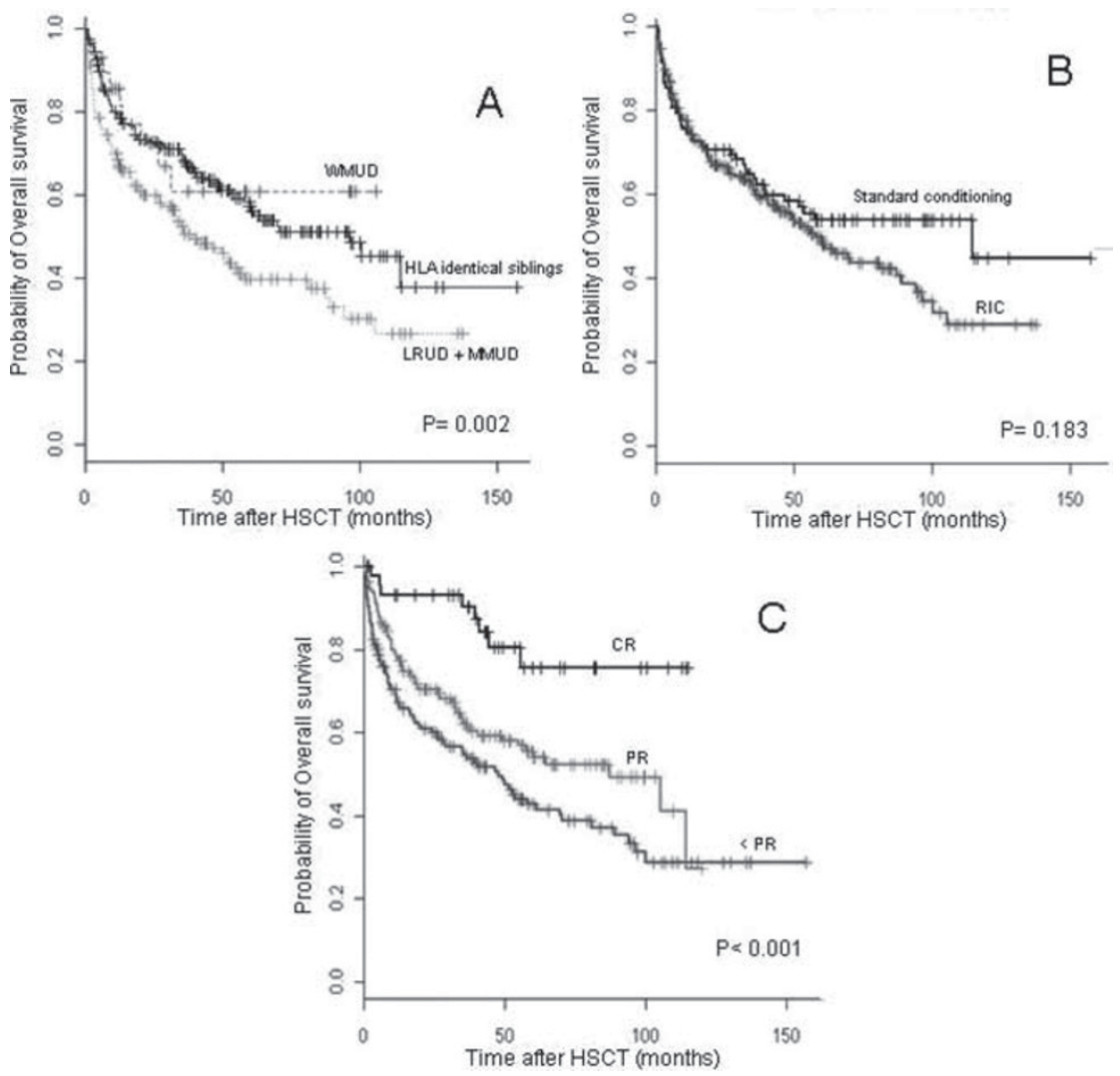


\section{P796}

Allogeneic stem cell transplantation as salvage treatment for relapse after autologous transplantation (autoSCT) in chronic lymphocytic leukaemia: outcome and impact of remission duration after autoSCT

P. Dreger, A. van Biezen, M. Michallet, R. Brand on behalf of the Chronic Leukemia Working Party

We have previously shown that a short duration of remission after autologous transplantation (autoSCT) is associated with poor survival in patients with chronic lymphocytic leukaemia (CLL) (Semin Hematol 44:246, 2007). The purpose of the present analysis was to provide evidence if the poor prognosis of early relapse after autoSCT can be overcome by salvage alloSCT.

Patients and methods: Patients who had received an allogeneic stem cell transplantation (alloSCT) for relapse after a previous autoSCT for CLL were identified from the EBMT database. Survival time data were calculated using the Kaplan-Meier method for OS and RFS while cumulative incidence estimates were calculated in a competing risk framework for REL2 and NRM. Multivariate estimates of hazard ratio's were computed using standard COX modelling.

Results: 111 patients were identified fulfilling the inclusion criteria. The median age was 54 (33-66) years, 91 patients $(82 \%)$ were males, remission status at alloSCT was CR/PR in $48 / 103$ patients $(47 \%)$ and less than PR in the remainder. Reduced intensity conditioning (RIC) was used in 96/108 patients $(87 \%)$, and the graft was obtained from a matched sibling donor in $40 / 108$ cases $(36 \%)$. The median interval from autoSCT to REL1 was 2.8 (0.2-8.9) years. NRM, REL2, RFS, and OS 3 years after alloSCT of all 111 patients was $26 \%, 41 \%, 33 \%$, and $44 \%$, respectively. Patients were classified according to interval from autoSCT to REL1 (TIME) into 3 equally sized categories (TIME0 $<2$ years, TIME1 2-3.7 years, Time $2>3.7$ years) with 37 patients each. Patients from category TIME0 had higher 3y-NRM compared to TIME1 and TIME2 (38\% vs. $23 \%$ vs. $15 \%, P=0.058)$. However, REL2, RFS and OS were not statistically different over all 3 TIME categories (TIME0, TIME1, TIME2; 3y-REL2 30\%, 49\%, 44\%, trendtest: $P=0.6$; 3 -RFS $32 \%, 29 \%, 41 \%$, trendtest: $P=0.36$; $3 y$-OS $41 \%, 45 \%, 48 \%$, trendtest $P=0.20$ ). Even after multivariate adjustment for age, sex, donor, conditioning and remission status at alloSCT, a significant impact of TIME on RFS and OS did not emerge. However, the systematic decrease of NRM was perfectly counterbalanced by a systematic increase of death-after-relapse.

Conclusions: Although limited by small patient numbers, this analysis does not support the hypothesis that a short remission duration ( $<2$ years) after autoSCT adversely impacts on survival after a subsequent salvage alloSCT in CLL, suggesting that the prognostic disadvantage of a short time to relapse after autoSCT might be overcome by alloSCT (first EBMT CLL transplant consensus criterion).
P797

Reduced-intensity allografting with in vivo T-cell depletion is associated with a favourable outcome in chronic lymphocytic leukaemia

S. Richardson (1), E. Vandenberghe (2), R. Benjamin (1), J. Sudak (1), A. Goldstone (1), K. Thomson (1), E. Morris (1), K. Peggs (1), A. Fielding (1), P. Kottaridis (1), S. MacKinnon (1), R. Chakraverty (1)

(1)University College London (London, UK); (2)St James Hospital (Dublin, IE)

Background: The role of T-cell depletion in allografting for chronic lymphocytic leukaemia (CLL) is controversial. We have employed a fludarabine/melphalan-based conditioning protocol that incorporates in vivo CAMPATH-1H (CAM) prior to allogeneic SCT for CLL in order to provide a platform for the directed delivery of donor leukocyte infusions (DLI) to eradicate persistent or minimal residual disease (MRD) or induce full donor chimerism

Methods: Data on 41 consecutive patients treated at 3 centres is presented. In vivo CAM (median dose $100 \mathrm{mg}$ ) was used with a fludarabine and melphalan RIC protocol. GVHD prophylaxis was with cyclosporine alone, withdrawn from 3 months post-transplant. Each centre used uniform criteria for giving DLI post-transplant, which was administered according to an escalated dose regimen (starting at $1 \times 10^{6} \mathrm{CD} 3$ cells $/ \mathrm{kg}$ and additional escalated doses at 3 mo intervals) according to the presence of persistent disease using conventional re-staging or by flow cytometric assessment of MRD from 3 mo. Patients with mixed chimerism (MC) at 6 months (mo) also received DLI.

Results: Median follow-up is 3 years $(y)$ and median age 52 y (range 33-64). Donors were matched sibling $(n=30)$, mismatched $(m m)$ related $(n=2)$ and unrelated donor $(n=9)$. Disease status at the time of transplant was CR $(n=4)$, PR/untested relapse $(n=36)$ and refractory $(n=1)$. The median number of prior therapy lines was 3 (range 1-6). $85 \%$ of patients had received prior purine analogue therapy and $61 \%$ had high-risk cytogenetic $(17 p-n=10)$ or molecular features (non-mutated $\lg \mathrm{H}, \mathrm{n}=15$ ). All patients engrafted. OS at $3 y$ was $83 \%$. Nonrelapse deaths at 3 y occurred in $6 / 41$ patients. DLI was given in 21 patients for MRD alone $(n=2)$, persistent disease or relapse $(n=9)$ and MC $(n=10) .6 / 10$ evaluable patients with MRD or persistent/relapsed disease responded to DLI $(n=5$ MRD negative CR, $n=1$ morphological CR). Overall, of 37 patients not in $\mathrm{CR}$ at the time of transplantation, $27(73 \%)$ subsequently attained MRD negative CR (including 5 of 10 patients with $17 p-)$. Current PFS at $3 y$ is $62 \%$. The overall incidence of chronic extensive GVHD was $41 \%$.

Conclusions: Analysis of this high-risk cohort demonstrates that T-cell depletion with in vivo CAMPATH-1H prior to allogeneic SCT provides a highly effective platform for the delivery of directed immunotherapy for CLL. This approach is associated with a low NRM and the majority of patients are alive and without disease at $3 \mathrm{y}$.
TIME $<2 y$

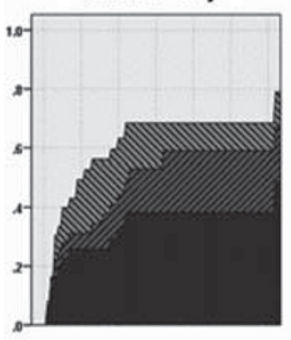

TIME 2-3.7y

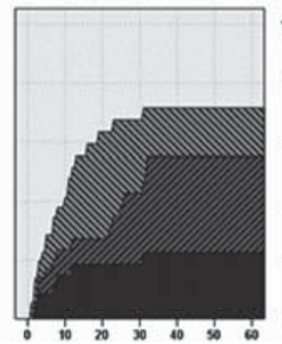

TIME $>3.7 y$

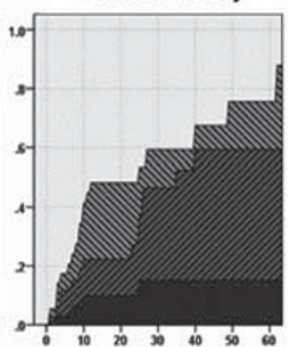

4

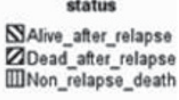


P798

Optimizing patient selection for myeloablative allogeneic haemopoietic cell transplantation in chronic myeloid leukaemia in chronic phase

J. Pavlu (1), A. Kew (2), B. Taylor-Roberts (1), H. Auner (1), E. Kanfer (1), D. MacDonald (1), D. Marin (1), D. Milojkovic (1),

A. Rahemtulla (1), K. Rezvani (1), J. Goldman (1), J. Apperley

(1), R. Szydlo (1)

(1)Hammersmith Hospital (London, UK); (2)Queen Elizabeth II Health Sciences Centre (Halifax, CA)

Chronic Myeloid Leukaemia (CML) in first chronic phase can now be treated very successfully using imatinib. However, approximately $35 \%$ of patients will not obtain long-term benefit from this tyrosine kinase inhibitor (TKI). It is therefore important to identify those with good outcomes after allogeneic haematopoietic cell transplantation (HCT), so it could be considered after imatinib failure.

In 271 patients who underwent myeloablative HCT for CML in first chronic phase we investigated the prognostic value of the HCT comorbidity index (HCT-Cl) together with the level of C-reactive protein (CRP) prior to HCT and the EBMT score. In multivariate analysis the EBMT scores lacked association with TRM, but scores $>3$ predicted for inferior OS. Patients with no comorbidities (HCT-Cl score 0 ) had better TRM as well as OS when compared with patients with comorbidities (HCT-Cl score $>0$ ) in multivariate analysis (relative risk (RR) for TRM 4.29, $95 \% \mathrm{Cl}: 1.8-10.5, P=0.001$; RR for OS $1.58,95 \% \mathrm{Cl}: 1.04-2.4$, $P=0.03$ ). There was no difference in survival or TRM between patients with $\mathrm{HCT}-\mathrm{Cl}$ score $1-2$ and those with $\mathrm{HCT}-\mathrm{Cl}$ score $>2$. Pre-conditioning CRP levels $>9 \mathrm{ml} / \mathrm{L}$ predicted for both inferior TRM (RR 4.22, 95\% Cl: 1.6-11.2, $P=0.004$ ) and OS (RR $2.33,95 \% \mathrm{Cl}: 1.4-4.0, P=0.002)$. The prognostic value of high CRP levels was independent of the HCT-Cl and its individual components and vice versa. Specifically, there was no association between elevated CRP levels and infection, either as a comorbidity or as a cause of death. Figures show probabilities of TRM $(A, B)$ and overall survival $(C, D)$ for patients stratified by $\mathrm{HCT}-\mathrm{Cl}(0$ versus $>0 ; A, C)$ and pre-conditioning CRP levels $(0-9 \mathrm{mg} / \mathrm{L}$ versus $>9 \mathrm{mg} / \mathrm{L} ; \mathrm{B}, \mathrm{D})$.

These data are important for deciding how to treat patients with $\mathrm{CML}$ in first chronic phase who have a suitably matched donor and fail imatinib. Previous cytogenetic response to imatinib, Sokal score and recurrent neutropenia on imatinib are factors which can accurately predict response to second generation TKIs. Therefore, patients with a predicted poor response to these agents, having a low level of CRP, no comorbidities and low EBMT scores may be expected to benefit from HCT as second line therapy.
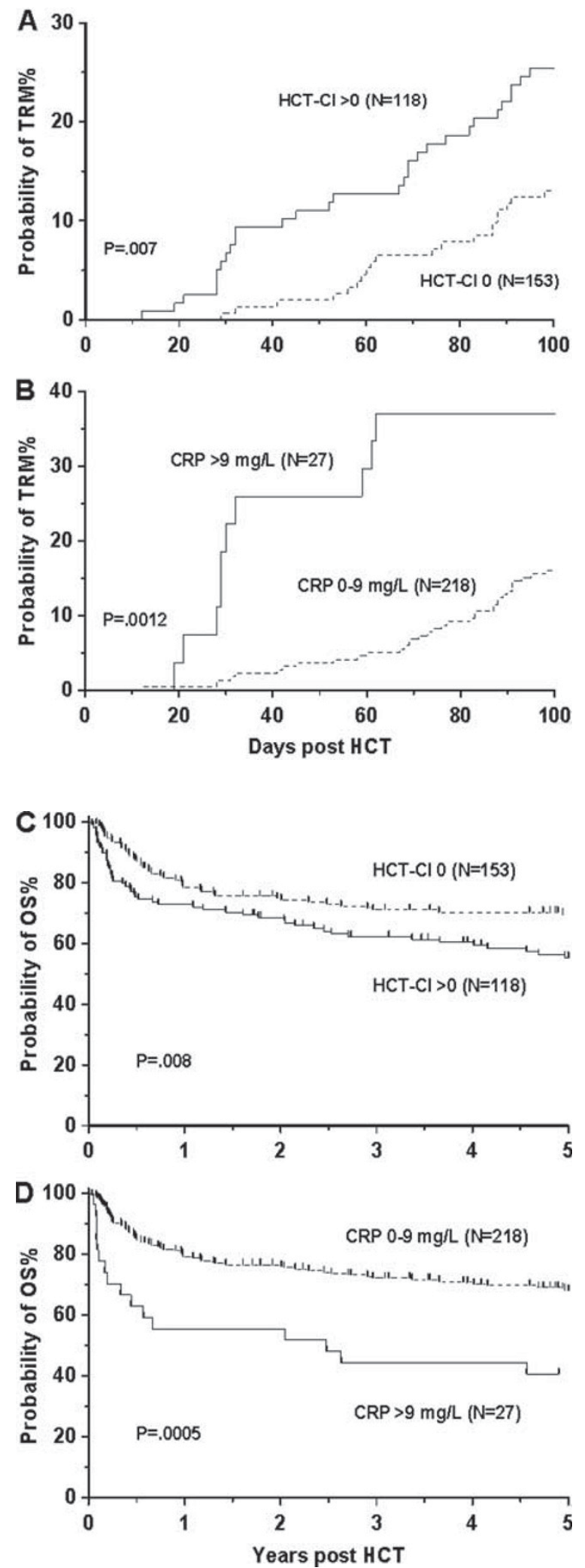
P799

Donor lymphocyte infusions for relapse of chronic myelogenous leukemia after reduced intensity conditioning stem cell transplantation

G.W. Basak, A. van Biezen, R. Brand, R. Szydlo, A. Schattenberg, P.A. Von Dem Borne, N. Kroger, L. Verdonck, J. Finke, D. Bunjes, C. Guglielmi, W. Wiktor-Jedrzejczak, E. Olavarria, T. de Witte on behalf of the Chronic Leukaemia Working Party

Reduced intensity conditioning stem cell transplantation (RIC SCT) in chronic myelogenous leukemia (CML) is associated with higher relapse rate compared to standard conditioning SCT. On the other hand, donor lymphocyte infusions (DLI) constitute a powerful tool in the treatment of CML relapse following SCT but their efficiency after RIC SCT is largely unknown. Therefore, we decided to retrospectively analyze a group of $836 \mathrm{CML}$ patients who relapsed following RIC $(\mathrm{N}=109)$ or standard conditioning $(\mathrm{N}=727)$ SCT and were treated with DLI. The median age of patients was 39 years (range 18-66), at transplantation $84 \%$ were in chronic phase, $11 \%$ in accelerated phase and $5 \%$ in blast crisis. They received cells from HLA-matched related $(64 \%)$, matched unrelated $(30 \%)$ or mismatched $(6 \%)$ donor. SCTs have been performed between 1994 and 2007. Acute GvHD grade II-IV occurred in $20 \%$ and chronic GvHD in $45 \%$ of patients. $88 \%$ of patients with known data achieved complete remission after alloSCT, but all of them relapsed after median of 11.5 months (range $0-132$ ). They were treated with DLI given after a median time of 2.2 months (range $0-84$ ) following SCT. The comparative analysis of groups based on conditioning regimen revealed that the RIC group contained significantly more females (49 vs. $39 \%$ ), older patients (>50) (49 vs. $10 \%)$ and received less frequently $T$ cell depletion (47 vs. $56 \%$ ). They were transplanted more frequently with PBSC (74 vs. $29 \%$ ) and received cells from HLA-identical sibling donors (74 vs. $58 \%$ ). RIC SCT group was characterized by significantly more patients in advanced phase at SCT (22 vs. $15 \%)$, have been performed more recently (mean year 2001 vs. 1999), were associated with lower occurrence of grade II-IV aGvHD (12 vs. $22 \%$ ) and shorter time from transplantation to relapse (mean 9 vs. 19 months). Significantly lower rate of complete remissions after SCT was observed in RIC group (71 vs. $91 \%$ of patients with known data). The actuarial probability of survival at five years from DLI was $64 \%$ in RIC and $69 \%$ in standard SCT group and survival analysis did not show significant differences $(P=0.215)$. The groups did not differ in frequency of grade II-IV aGvHD after DLI ( $18 \%$ for RIC vs. $11 \%$ for standard, $P=0.266$ chi-sq test) while there was a trend towards extensive chronic GvHD in RIC group (60 vs. $38, \%, P=0.059$ chi-sq test). The data show that CML relapse after RIC SCT can be as efficiently treated with DLIs as in case of relapse after standard conditioning SCT.

\section{P800}

Effect of prior therapy with nilotinib or dasatinib on the outcome after allogeneic stem cell transplantation for patients with chronic myeloid leukaemia

M. Schleuning, M. Scholten, A. van Biezen, A. Nagler, J. Apperley, M. Michallet, A. Grigg, R. Silver, D. Niederwieser, T. De Witte, E. Olavarria on behalf of the CML subcommittee of the CLWP of the EBMT

Stem cell transplantation (SCT) will continue to be a treatment option for patients with chronic myeloid leukaemia, despite the introduction of tyrosine kinase inhibitors (TKI). However, many patients will have received prior therapy with TKIs, including Nilotinib or Dasatinib at the time of allogeneic SCT. While the use of Imatinib prior to SCT seems to have no adverse impact on the outcome of allogeneic SCT little is known on the impact of prior use of second generation TKIs. Therefore we conducted a retrospective registry study and identified 56 patients with CML who received an allotransplant after having been treated with Nilotinib and/or Dasatinib. Best responses to second generation TKIs were major molecular response in $11 \%$, complete cytogenetic response in $7 \%$, partial cytogenetic response in $18 \%$, complete haematologic remission in $25 \%$ and no response in $34 \%$, respectively. At SCT, $37 \%$ of the patients were in accelerated or in blast phase, $36 \%$ in CP2 or higher and $27 \%$ in first chronic phase. Graft failure occurred in two patients. The median follow-up for surviving patients is 19 months. At 24 months the estimated non-relapse mortality was $33 \%$ and the relapse incidence $15 \%$. As expected, probability of survival is best in patients transplanted in CP1 with more than $85 \%$ at 2 years. In univariate analysis there was a non significant trend in favor for pretreatment with Nilotinib as compared to the other groups. However, in multivariate analysis only stage of the disease was a predictor for survival. With respect to overall survival no significant differences could be identified for the following variables: patient age, donor type, stem cell source, intensity of the conditioning, time diagnosis to transplant, in or ex vivo T-cell depletion, response to treatment with second generation TKIs. Patients transplanted in blast crisis had a significant higher risk of non relapse mortality. In summary, despite the shortcomings of a retrospective study the numbers reported are comparable to earlier studies on the impact of Imatinib on the outcome of SCT and it should be emphasised that the timing of allogeneic stem cell transplantation remains crucial to avoid unacceptable high treatment related mortality.

\section{P801}

Allogeneic stem cell transplantation for chronic lymphocytic leukaemia: a long term analysis from the Société Française de Greffe de Moelle et de Therapie Cellulaire (SFGM-TC)

M. Mohty, R. Szydlo, J.P. Vernant, I. Yakoub-Agha, D. Blaise, G. Socie, C. Cordonnier, E. Deconinck, J.H. Bourhis, F. Guilhot, A. Buzyn, N. Ifrah, D. Guyotat, J.L. Harousseau, L. Sutton, N. Milpied, M. Michallet on behalf of the SFGM-TC

Chronic lymphocytic leukaemia (CLL) is a common lymphoid malignancy with a heterogeneous natural history. While some patients never require treatment or can be managed effectively with conventional chemotherapy, others experience early disease progression and death. Allogeneic stem cell transplantation (allo-SCT) is increasingly considered as a therapeutic option for patients with poor-risk CLL.

This multicenter retrospective analysis assessed the long term outcome of 153 CLL patients who received allo-SCT between 1987 and 2005, and were reported to the SFGM-TC registry. This series included 120 males $(78 \%)$ and 33 females $(22 \%)$ with a median age at CLL diagnosis of 45.5 (range, 24.4-65.1) y. The median age at time of allo-SCT was 51 (range, 30-68) y. Before allo-SCT, 19 patients (12\%) received a previous SCT in the course of their disease. Patients received either a standard myeloablative conditioning regimen (39\%; Cy-TBI in $90 \%$ of cases) or a so-called reduced-intensity conditioning (61\%). A matched-related donor was used in $90 \%$ of cases and PBSCs were used as source of stem cells in $56 \%$. At time of allo-SCT, only $18 \%$ of patients were in CR, $24 \%$ in first PR and the rest of the series in more advanced phases, including $30 \%$ of patients in progressive disease.

With a median follow-up of 60 (range, 1.6-208) months, 96\% of patients engrafted (ANC>500/ $\mathrm{L}$ ) at a median of 18 (range, 1-41) days after allo-SCT. $7147 \%$ of patients experienced grade 2-4 acute GVHD, including 18\% of grade 3-4 acute GVHD. 33\% of evaluable patients experienced extensive chronic GVHD. At time of last follow-up, the median probability of OS from diagnosis was 124 (range, 109-139) months, and the median probability of OS after allo-SCT was 40 (range, 14-67) months. Of note, there was a statistically significant improved survival in patients developing limited or extensive chronic GVHD. The long term NRM rate was $41 \%$. In multivariate analysis for OS at 5 years, disease status (CR or PR1 vs. more advanced stages), and patient CMV positive serostatus were significantly associated with outcome: $\mathrm{RR}=1.73(95 \% \mathrm{Cl}, 1.05-2.9), P=0.03$ and 
$\mathrm{RR}=1.66(95 \% \mathrm{Cl}, 1.02-2.7), P=0.04$, respectively. The same parameters were also significantly associated with DFS at 5 years: $R R=1.76,(95 \% \mathrm{Cl}, 1.1-2.8), P=0.019$ and $R R=1.63$, $(95 \% \mathrm{Cl}, 1.042-2.6), P=0.03$, respectively.

We conclude that allo-SCT has the potential to induce longterm disease control and overall survival in patients with high risk CLL. Early disease stages (CR or PR1) achieve the best results after allo-SCT.

\section{P802}

Management relapse of CML after allogeneic stem cell transplantation. No advantage of early administration of donor lymphocyte infusions upon molecular relapse Y. Chalandon, J. Passweg, J. Apperley, A. Schattenberg, T. de Witte, N. Kröger, J. Finke, C. Guglielmi, E. Olavarria on behalf of the Chronic Leukemia Working Party of the EBMT

Patients (pts) relapsing with CML after allogeneic hematopoietic stem cell transplantation (HSCT) may be treated by tyrosine kinase inhibitors or by donor lymphocyte infusions (DLI). Best strategies to administer DLI are not known. We analyzed 155 pts relapsing after allogeneic HSCT for CML with disease detectable only by molecular methods who received DLI. Transplants had been done in first chronic phase $(n=125)$ or for more advanced disease $(n=29)$ from identical siblings $(n=84)$ and unrelated donors $(n=71)$ between 1986 and 2003. They received DLI either early with molecular $(n=85)$ disease or upon progression to, cytogenetic $(n=37)$ hematologic in chronic phase $(n=25)$ or more advanced phase $(n=8)$ disease. The median time interval from transplant to molecular relapse was 239 (30-4274) days. The initial DLI dose was $\leq 106$ CD $3 / \mathrm{kg}$ in $44,>106-107 / \mathrm{kg}$ in 58 , and $>107$ in 25. The number of DLI doses given was 1 in 83,2 in 25 and $\geq 3$ in 47 pts. Concurrent imatinib was given in 11 pts. The median interval from relapse to DLI was 210 (1-1673) days. 43 pts developed acute GVHD after DLI and 34 had chronic GvHD. 28 pts did not respond to DLI, response was unknown or unevaluable in 21 and cytogenetic or better responses were seen in 103 pts. Time interval from DLI to best response was 245 (30-1673) days. 9 pts died of DLI related complications. At last follow-up 31 pts had died, of the surviving pts (124) 93 had achieved response (missing information in 15 and no response in 16). Of those who responded, 86 had complete molecular response and 3 of the responders had a subsequent relapse. Median follow-up after DLI was 46 (3-135) mths.

Survival after DLI was $76 \pm 4 \%$ at 5 years. It was $89 \pm 8 \%$ with sibling donors and $63 \pm 13 \%$ with unrelated donors $(P=0.003)$. It was $69 \pm 14 \%$ if DLI were given within 6 mths of molecular relapse and $81 \pm 10 \%(P=0.061)$ if given later; $81 \pm 11 \%$ if given at molecular relapse versus $71 \pm 12 \%(P=0.26)$ if given for cytogenetic, hematologic relapse or advanced phase. In multivariate analysis unrelated donor (RR: $2.79(1.28-6.09)$ ) and DLI later than 6 mths after molecular relapse (RR: $0.53(0.26-1.09)$ were associated with survival. Starting dose, prior T-cell depletion, disease stage at DLI, donor recipient sex combination, and age were not significant.

These data confirm the remarkable efficacy of DLI for this disease. There appears to be no advantage of administering DLI early upon detection of molecular relapse.

\section{P803}

Allogeneic stem cell transplantation in patients with chronic myelomonocytic leukaemia: the impact of WHO classification and of the conditioning regimen on the transplantation outcome

A. Symeonidis, A. van Biezen, G. Mufti, J. Finke, D. Beelen, M. Bornhauser, H. Greinix, J.P. Jouet, L. Volin, R. Schwerdtfeger, $T$. de Witte, N. Kröger on behalf of the Chronic Leukemia Working Party of the EBMT

Chronic myelomonocytic leukemia (CMML) shares hybrid myelodysplastic and myeloproliferative features and is classi- fied in dysplastic (CMML-D) or proliferative (CMML-P) type, on the basis of the initial WBC count. Results of allogeneic stemcell transplantation (SCT) in this disease are usually pooled together with other MDS. We retrospectively analyzed the transplantation outcome in patients (pts) with CMML from the EBMT database and investigated the influence of WHO classification, conditioning regimen, and other factors on the outcome. In this analysis 283 adult pts $(F=92, M=191$, median age 50 years) were included. Among 105 pts with known WHO classification, 45 had CMML-D and 60 CMML-P. The conditioning was conventional in $152(54 \%)$ and reduced-intensity in $87(31 \%)$. Donor was HLA-identical sibling (160), matched unrelated (85) or other (38). Disease status at SCT was CR in $50(18 \%)$, no $\mathrm{CR}$ in $185(65 \%)$ and unknown in $44(16 \%)$. Stem cell source was bone marrow in $108(38 \%)$ and PBSC in 175 pts $(62 \%)$. Engraftment was successful in $245 / 263$ pts (93\%). Grade $0-1$ acute GVHD was reported in 162/258 evaluable pts, grade 3-4 in 85 , and unknown grade in 11. Chronic GVHD was reported in $58 / 102$ pts $(57 \%$, limited in 34 , extensive in 24$)$. At the time of this analysis 120 pts were alive (42\%), of whom $108(38 \%)$ disease-free and $71(25 \%)$ had relapsed. Among 156 deaths, 50 were disease-related (32\%), 95 transplant-related $(61 \%)$ and 11 other cause-related $(7 \%)$. Non-relapse mortality was $37 \%$ and was lower in PBSC recipients $(P=0.023)$ and in pts transplanted after $2002(P=0.015)$. Totally, GVHD was the cause of death in $11 \%$ of the pts. Event-Free Survival (EFS) and overal survival (OS) were higher in pts transplanted $\geq 12$ months from diagnosis $(P=0.012$ and $P=0.036$, respectively). The probability of relapse was lower among pts with grade 2-4 acute GVHD $(P=0.027)$. For pts without chronic GVHD the probability of relapse was higher $(P=0.046)$ whereas EFS and OS were lower $(P=0.019$ and $P=0.012$, respectively). Relapse-free survival was longer in PBSC recipients $(P=0.033)$, in whom EFS $(P=0.033)$ and OS $(P=0.054)$ were higher. Finally, RFS and OS did not differ significantly among the 2 CMML types, or in relation to the conditioning, age or disease status at SCT, cytogenetics, donor-recipient gender match, HLA-type of the donor, stem-cell source, the administration of TBI, T-cell depletion, the manifestation and grading of acute or chronic GVHD and the period SCT was performed.

\section{P804}

Screening and monitoring of MPL W515L mutation with real-time PCR in patients with myelofibrosis undergoing allogeneic stem cell transplantation

H. Alchalby (1), A. Badbaran (1), O. Bock (2), B. Fehse (1), U. Bacher (1), A.R. Zander (1), N. Kröger (1)

(1)Univerity Hospital Hamburg (Hamburg, DE); (2)Hannover Medical School (Hannover, DE)

Monitoring of minimal residual disease (MRD) after allogeneic stem cell transplantation (ASCT) for myelofibrosis allows recognizing the depth of remission and thus guides application of appropriate therapeutic interventions. MPL W515L/K mutations, which are detected in $5-10 \%$ of JAK2V617F-negative patients, may be useful for this purpose. Using a highly sensitive quantitative polymerase chain reaction (PCR) method we tested 90 patients with myelofibrosis who underwent ASCT for the presence of MPL W515L/K mutations. Two patients with primary myelofibrosis were found to harbor MPLW515L while no patient was positive for MPLW515K mutation. Both patients were JAK2V617F negative and cleared the mutation rapidly after ASCT and remained negative for a median follow up of 19 months. The results of molecular monitoring correlated well with other remission parameters such as normalization of peripheral blood counts and morphology and complete donor chimerism. We conclude that MPLW515L can be cleared after ASCT and hence may be used a minimal residual disease marker in a proportion of JAK2V617F-negative myelofibrosis patients. 


\section{P805}

Autologous stem cell transplantation allows long progression-free-survival in high-risk Waldenstrom's macroglobulinemia. Results of a retrospective analysis of Hamburg registry and the Société Française de Greffe de Moelle et de Thérapie Cellulaire

A. Garnier (1), P. Dreger (2), V. Cacheux (3), R. Tabrizi (4), C.E. Bulabois (5), C. Dartigeas (6), S. Le gouill (7), V. Coiteux (8), P. Morel (9), B. Desablens (10), J.O. Bay (3), V. Leblond (1), G. Guillerm (11), A. Delmer (12), O. Tournilhac (3), N. Dhedin (1)

(1)Pitié Salpetriere (Paris, FR); (2)Hambourg (Hambourg, DE); (3)Hotel Dieu (Clermont-Ferrand, FR); (4)Bordeaux (Bordeaux, FR); (5)Grenoble (Grenoble, FR); (6)Tours (Tours, FR); (7)Nantes (Nantes, FR); (8)Lille (Lille, FR); (9)Lens (Lens, FR); (10)Amiens (Amiens, FR); (11)Brest (Brest, FR); (12)Reims (Reims, FR)

Waldenström Macroglobulinemia (WM) usually presents a relative low progression with a median overall survival (OS) of 10 to 15 years. According to the International Prognosis Scoring System for WM, high-risk patients present an OS at 5 years of only $36 \%$. These results could justify innovative approaches and more aggressive treatment modalities such as autologous stem cell transplantation (ASCT).

We examined the long-term outcome of ASCT performed in WM from 1991 to 2007 by studying the records of 46 patients reported in the SFGM-TC database and in Hamburg registry (12 patients). For diagnostic confirmation were applied the second international WM workshop criteria. All confirmed cases were then extensively analyzed directly with the collaboration of the physicians. Response was defined with Kimby et al.'s criteria with few modifications: Patients with disappearance of the monoclonal protein, confirmed by immunofixation, and the complete regression of any adenopathy/organomegaly or signs of WM, even if bone marrow evaluation was not available were considered to have a complete remission (CR). Those with persistent detectable monoclonal IgM $<5 \mathrm{~g} / \mathrm{L}$ or with missing immunofixation data, but who met all the other CR criteria, were considered to have a very good partial response (VGPR).

Median age at transplant was 54 years (34-68) with $37 \%$ of patients who received 3 or more previous lines, including purine analogs in $30 \%$ and Rituximab in $20 \%$. At transplant $20 \%$ of patients had refractory disease. Sixty three percent of patients received a conditioning regimen including TBI. ASCT yielded an overall response rate of $85 \%$ with $9 \%$ of CR and $30 \%$ of VGPR. The median progression free survival (PFS) and OS were 53 and 109 months respectively. With a median follow-up of 110 months (3-145), 5-year OS and PFS rates were respectively, $66 \%(95 \% \mathrm{Cl}: 52-80)$ and $44 \%(95 \% \mathrm{Cl}: 29-59)$, reaching $88.5 \%(95 \% \mathrm{Cl}: 79-98)$ and $65 \%(\mathrm{Cl}: 51-79)$ in patients in CR or VGPR . In multivariate analysis the variables associated with better survival and PFS were the number of previous lines of treatment $(P=0.02$ and $P=0.032$ respectively) and achievement of CR and VGPR. ( $P=0.019$ and 0.004 respectively). Two patients presented at 3 and 42 months post transplantation a therapy related acute myeloid leukemia.

ASCT therefore leads to long PFS in this high risk population and should be evaluated earlier in patients with poor prognostic factors.

\section{P806}

Related and unrelated stem cell transplantation after low-dose total body irradiation based conditioning for the treatment of advanced chronic lymphocytic leukaemia

K. Hebenstreit, C. Pfrepper, T. Lange, R. Krahl, H.-K. Al Ali, C. Becker, W. Pönisch, N. Basara, D. Niederwieser

University of Leipzig (Leipzig, DE)

Introduction: The impact of a minimal intensive conditioning regimen on outcome of patients with advanced CLL was evaluated and the kinetics of the graft-versus-CLL effect analyzed.
Patients: Analysis includes 32 patients with CLL who underwent allogeneic SCT after 200-300 cGy TBI between June 1999 and May 2008 at the University of Leipzig. The median age was 58 (range 47-69) a and 23 patients were male. From 30 patients with cytogenetic analyses, 14 (47\%) had unfavourable cytogenetics and $17(57 \%)$ were resistant to first line therapy. Richter's transformation was found in four patients $(13 \%)$. Unrelated $(n=23)$ as well as related donors $(n=9)$ were matched for HLA-A, $-B$ and C by intermediate resolution and DR-B1 by high resolution typing. Two unrelated recipient/donor pairs had a single HLA-C allele and one DQB1- Ag mismatch. Percentages of donor chimerism were assessed by FISH for $X$ and $Y$ chromosomes and/or by polymerase chain reaction- based amplification of variable number tandem repeat (VNTR) sequences after sorting.

Results: Hematological toxicities after SCT were moderate. Six $(21 \%)$ and seven $(24 \%)$ patients maintained absolute neutrophil counts (ANC) $>500 / \mathrm{il}$ and platelet count $>50000 / \mu \mathrm{l}$, respectively. Complete T-cell donor chimerism (median 100, range 80-100\%) was seen at day +180 , while median $100 \%$ B-cell donor chimerism was observed only 1.5 years after SCT. Absence of B-CLL cells (median CD5+/CD19+: 0 , range $0-73 \%$ ) in bone marrow was already evident by day +180 . Patients who were in CR/PR at SCT had a faster B-cell donor chimerism than those in stable disease/progress [94\% (range: $6-100 \%$ ) versus 9\% (range: 9-100\%) one year post-SCT respectively]. Overall survival (OS) of the whole group was $67 \%$ at 8 years, with 5 of 9 related recipients and 18 of the 23 unrelated recipients being alive. The 8years event-free survival, the incidence of Non relapse Related Mortality (NRM) and the progression/relapse were $41 \%, 25 \%$ and $45 \%$, respectively. The factors significantly associated with increased risk of relapse/progress were intermediate/advanced disease vs. CR/PR1 $(P=0.006)$. The most common causes of NRM were GvHD ( $n=5 ; 16 \%)$ followed by sepsis $(n=3,9 \%)$. Conclusion: Results confirm high OS after low-dose TBI based regimens even in advanced CLL. Full donor T-cell chimerism was reached early after SCT, while B-cell reconstitution was observed only 1,5 years after SCT despite the absence of evident disease by 180 days after SCT.

\section{P807}

Persistent molecular remission in relapsed $\mathrm{CML}$ responding and discontinuing imatinib after allogeneic transplantation A.M. Raiola, A. Garuti, M. Sessarego, M.T. van Lint, A. Dominietto, T. Lamparelli, F. Gualandi, S. Bregante, R. Varaldo, S. Annunziata, F. Frassoni, A. Bacigalupo

S. Martino's Hospital (Genoa, IT)

Background: Imatinib has been reported to be effective in treating post-transplant relapse of CML.

Patients: In this study we analyze $48 \mathrm{CML}$ patients, with a median age of 33 years (range 21-60), who relapsed after transplant, at a median interval of 650 days (range 60-5830), and received Imatinib, at a median interval of 127 days (range 10-1478) from relapse. Results. Group A comprised 4 patients who recovered autologous hematopoiesis after transplant, treatment with Imatinib was ineffective.

Group B comprised 10 patients refractory to Imatinib: 8 had hematologic relapse (2 BC, $2 \mathrm{AP}, 1 \mathrm{CP})$ and 2 had a citogenetic relapse after full donor engraftment.

Group C comprised 31 Imatinib responders: 4 had hematologic relapse (4 CP), 12 citogenetic relapse and 15 had molecular relapse.

Group D: comprised 4 responders to a combination of Imatinib and DLI (2 hematological relapse, 2.molecular relapse).

Responders: We had a total of 35 complete cytogenetic responses, of these, 31 patients $(78 \%)$ had a complete molecular response (CMoIR). Of the $31 \mathrm{CMolR}, 17$ (55\%) discontinued Imatinib, after a median of 500 days (range 90-1272) and have been bcr/abl negative ever since, with median follow up 1825 days (range 730-2190) from discontinuation. Ten patients of $31(32 \%)$ discontinued Imatinib for a median of 120 days, and became positive again: all have been restarted on Imatinib and 
9 continue to be on therapy with MMolR. Four patients never discontinued Imatinib.

Conclusions: This study confirms that response to Imatinib is dependent on disease phase at the time of relapse. The study also shows that (a) molecular responses can be achieved in $64 \%$ of all patients starting Imatinib, (b) imatinib can be discontinued in most patients with molecular responses and (c) molecular responses are maintained after discontinuation in over $50 \%$ of responders.

\section{P808}

Relapse-therapy for myelofibrosis patients after allogeneic stem cell transplantation

E. Klyuchnikov, H. Alchalby, A. Badbaran, C. Wolschke, F. Ayuk, U. Bacher, A. Zander, N. Kröger

University Cancer Center (Hamburg, DE)

Introduction: Up to $30 \%$ of patients (pts) with myelofibrosis (MF) will relapse within the first three years after allo-SCT. Therapeutic options for those include donor lymphocyte infusions (DLIs) and/or a 2nd allo-SCT, but studies on this issue are rare.

Patients and methods: We report our experience with DLIs and 2nd allo-SCT being performed as salvage approach in 10 pts (5 males, 5 females) with relapsed MF (32-63 yrs). Initial transplantations were performed in all pts with reduced conditioning (RIC) (busulfan, cum. $10 \mathrm{mg} / \mathrm{kg}$ p.o. or equivalent i.v.; + fludarabine, cum. $\left.180 \mathrm{mg} / \mathrm{m}^{2}\right)$. Maximal response after initial allo-SCT was complete $(C R, n=3)$ or partial remission $(P R, n=6)$, and stable disease (SD, $n=1)$.

Results: Clinical relapse occurred at a median of 5 months (2-50) following initial allo-SCT. Subsequently, all pts received DLIs at $1-4$ time points (median, $2.5 \times 10^{7} / \mathrm{kg}$ bw; range, $0.5 \times 10^{5}-$ $\left.1.3 \times 10^{8} / \mathrm{kg}\right)$. Thereafter, 2 pts $(20 \%)$ developed grade III acute graft versus host disease (aGvHD) and required adequate immunosuppression. The best responses after DLIs according to IWG-MRT criteria included: CR $(n=3), S D(n=5)$, and clinical improvement $(\mathrm{Cl}, \mathrm{n}=1)$. One pt showed progressive disease (PD). All pts with CR maintain CR 7+, 18+ and 19+ months. 2nd allo-SCT was performed in 7 pts without CR following DLIs. The median interval between both SCTs was 17 months (13-77). RIC regimen being used in this setting consisted of fludarabine (cum. $150 \mathrm{mg} / \mathrm{m}^{2}$ ) with treosulfan (cum. $36 \mathrm{mg} / \mathrm{m}^{2}$ ). All pts received PBSC (median, $7 \times 10^{6} \mathrm{CD} 34$ + cells/kg bw; 4-11) from alternative HLA-matched $(n=3)$ or -mismatched $(n=4)$ unrelated donors. All pts successfully achieved leukocyte engraftment (median, day +18; 10-20). Severe aGvHD (II-III) was observed in 2 of 7 (29\%) pts. There were no TRM cases. Of all 10 pts, 8 were alive at the median follow up of 34 months (range, 16-78) from initial SCT. Of those, according to IWG-MRT criteria, 6 achieved CR, together with JAK2V617F-negativity (3 pts after DLIs and 3 pts after 2nd allo-SCT, including the pt with PD after the 1st allo-SCT). 2 patients attained $\mathrm{PR}$ following the 2nd SCT. Two of 10 pts who were in CR 15 and 11 months after the 2st allo-SCT, respectively, died due to PD 18 and 21 months after the 2nd SCT.

Conclusions: DLIs and 2nd allo-SCT, based on a combination of fludarabine and treosulfan, seem to represent effective and well tolerated salvage approaches for patients with myelofibrosis, who relapsed after initial allo-SCT.

\section{P809}

T-cell and B-cell responses after vaccination against influenza virus and pneumococcus in chronic phase CML patients treated with tyrosine kinase inhibitors

H. de Lavallade, M. Hart, I. Gabriel, P. Kelleher, A. Alsuliman, A. Khoder, K. Stringaris, P. Garland, J. Khorashad, D. Milojkovic, L. Foroni, M. Bua, J. Apperley, J. Goldman, D. Marin, K. Rezvani Imperial College London (London, UK)

Imatinib, nilotinib and dasatinib are remarkably effective as single-agent treatments for chronic myeloid leukemia (CML) in chronic phase (CP). They are also increasingly being used as therapies in the allogeneic stem cell transplantation (alloSCT) setting, both as post allo-SCT maintenance treatment for $\mathrm{Ph}+$ leukemias as well as for their putative immunomodulatory effects. However little is known on their potential impact on the immune system and to date no human in vivo data are available.

To characterize the in vivo immunomodulatory effect of TKIs, $50 \mathrm{CP}-\mathrm{CML}$ patients on standard dose tyrosine kinase inhibitors (TKIs) and 15 healthy controls were vaccinated against Flu and pneumococcus at our institution. Samples were taken pre and at 1 and 3 months post-vaccination. Titers of IgM and IgG anti-pneumococcal were determined using ELISA technology. We analyzed the immunological T-cell response to influenza virus both quantitatively and qualitatively using flow cytometry for intracellular TNF-a, IFN- and IL2 and the cytotoxicity marker CD107a. Preliminary results on 28 patients and 11 healthy controls have been analyzed thus far. Significantly fewer patients on TKls mounted an anti-pneumococcal IgM response (IgM serum titer $>100 \mathrm{U} / \mathrm{ml}$ ) compared to healthy controls (9/28 versus $8 / 11, P=0.033)$. Moreover, patients on TKI had significantly lower levels of anti-pneumococcal IgM at 1 month compared to healthy controls (median, $84.5 \mathrm{U} / \mathrm{ml}$, range 5 to 200 vs. $200 \mathrm{U} / \mathrm{ml}$, range 15 to $200, P=0.006$ ). CD8 and CD4 T cell responses to Flu vaccination have been analyzed in 15 patients on TKI and 5 healthy controls so far. Prior to vaccination, $T$ cell responses against Flu were detected in 4/15 patients on TKI and 1/5 healthy controls, indicating preexisting memory $\mathrm{T}$ cell responses to Flu. A significant T-cell response to Flu was seen in $7 / 15$ patients on TKI and in $3 / 5$ healthy control.

These preliminary results suggest that in patients with $C M L$ on TKIs the IgM B cell response to vaccination with Pneumovax is significantly impaired compared to healthy controls. We have as yet not detected a significant difference in T-cell response following vaccination with Flu in CML patients on TKIs compared to healthy controls. The analysis of the remaining samples is ongoing.

\section{P810}

Factors affecting the curative effect of allogeneic stem cell transplantation in patients with high-risk chronic lymphocytic leukaemia

A.P. Iori, L. Laurenti, A. Piciocchi, A. Severino, P. Chiusolo, F.R. Mauro, G.F. Torelli, I. Della Starza, I. Majolino, A. Locasciulli, G. Meloni, S. Sica, G. Leone, R. Foà on behalf of Coordinamento Trapianti di Cellule Staminali Emopoietiche della Regione Lazio

To date, the only curative approach for chronic lymphocytic leukemia (CLL), in particular in the presence of an aggressive course and/or of adverse biological risk factors, is represented by allogeneic hematopoietic stem cell transplantation (allo-HSCT); the use of reduced-intensity conditioning (RIC) regimens makes this procedure feasible also in elderly patients (pts). We report the results of a retrospective multicenter study on 25 pts with CLL $>45$ years (yrs) who underwent an allo-HSCT from an HLA identical sibling (22 pts) or an unrelated donor (3 pts) between 2001 and 2009; median age was $57 \mathrm{yrs}$ (range 45-70) and the median number of pre-transplant chemotherapy lines was 3 (range 1-6); 6 pts $(24 \%)$ had received a previous auto-HSCT. All pts showed biological high-risk features. At transplant, 2 pts were in CR, 15 were in partial remission and 8 had stable or progressive disease. Ten pts receivedaRICallo-HSCTfollowingfludarabine/cyclophosphamide/ thyotepa, while 15 pts were conditioned with a non-myeloablative conditioning regimen (NMA) with TBI (200) plus fludarabine. The cumulative incidence (Cl) of acute and chronic GVHD was $40 \%$ and $60 \%$, respectively. Transplant-related mortality (TRM) by $\mathrm{Cl}$ was $13 \%$. At 5 yrs, the $\mathrm{Cl}$ of relapse/progression was $47 \%$. The 5 -year actuarial probability of overall survival (OS) and disease-free survival (DFS) was $55 \%$ and $43 \%$, respectively; the most important factor influencing both OS and DFS 
was the hematopoietic cell transplantation-specific comorbidity index $(P<0.0001)$. With a median follow-up of 40 months (5-70), 14 patients (56\%) showed a molecular eradication of their disease. By logistical regression model analysis, the only factor affecting the disappearance of minimal residual disease (MRD) was the presence of chronic GVHD $(P=0.04)$; neither the type of conditioning regimen, nor the pre-transplant chemotherapy, nor the occurrence of acute GVHD had a significant impact; the disease phase pre-transplant showed a trend in affecting MRD $(P=0.08)$. In conclusion, over a prolonged follow-up period, we confirm that allo-HSCT is feasible in CLL patients (including the elderly) and that it is associated with a relatively low TRM, regardless of age. The use of NMA conditioning regimens is a good alternative option to RIC to obtain the eradication of MRD influenced only by the graft-versus leukemia effect in this high-risk population of patients.

\section{P811}

Single-centre experience of allogeneic stem cell transplantation from 2000-2009 in chronic lymphocytic leukaemia

M. Goodyer (1), J. Krawczyk (1), K. Fadalla (1), N. Chadwick (1), J. Kelly (2), M. Ni Chonghaile (1), S. McCann (1), P. Browne (1), E. Conneally (1), C. Flynn (1), E. Vandenberghe (1) (1)St James's University Hospital (Dublin, IE); (2)Our Lady's Children's Hospital, Crumlin (Dublin, IE)

Twenty-three patients (20 males, 3 females) with a median age of 51 (range 33-62) years were transplanted between 2000-2009. Transplant regimens were FluTBI from 2000 to $2005(n=6)$, Fludarabine/Melphalan with T-cell depletion (Campath1H 12, ATG 2) from 2003 to $2009(n=14)$, and CyTBI transplants in 3 patients.

Patients had a median of 3 (range 1-7) lines of treatment pretransplant, and 17 were Fludarabine refractory. The median time from diagnosis to transplant was 51 (range 9-137) months. FISH data was available in 14 patients of whom 4 had del(17p), 3 had del(11q) and 1 had both. Two patients were in complete remission, 20 in partial remission and 1 patient had progressive disease pre-transplant. Eighteen patients had sibling matched and 5 had MUD donors. All patients engrafted successfully, except for 1 patient who died from toxicity pre-engraftment.

Toxicity before 100 days included grade $3 / 4 \mathrm{GvH}(n=11)$ including a death post-Flu/TBI SCT, and 6 patients had NC grade $3 / 4$ infections. Toxicity after 100 days included extensive cGvH in 15 patients including 2 patients post DLI and 1 death, $\mathrm{NCl}$ grade 3/4 infections in 6 patients including 2 deaths, and 5 deaths from relapse. Seven patients had DLI, 5 for mixed chimerism and 2 for relapse. The 2 year overall survival (OS) and progression free survival (PFS) was $72 \%$ and $68 \%$. The 2 year OS and PFS for the 12 patients who received conditioning with Campath $1 \mathrm{H}-\mathrm{Flu}-\mathrm{Mel}$ SCT was $75 \%$ and $75 \%$, with acceptable toxicity.

\section{P812}

\section{Allogeneic haemotopoietic stem cell transplantation} outcomes in imatinib-treated chronic myeloid leukaemia patients

H.K. Ahn (1), C.W. Jung (1), K. Kim (1), J.H. Jang (1), D.H. Kim (1), S.S. Yoon (2), I.H. Kim (2), S.K. Sohn (3), J.H. Moon (3), J.H. Lee (4), K.H. Lee (4), J.H. Won (5), N.S. Lee (5)

(1)Samsung Medical Center (Seoul, KR); (2)Seoul National University Hospital (Seoul, KR); (3)Kyungpook National University Medical School (Seoul, KR); (4)Asan Medical Center (Seoul, KR); (5)Soon Chun Hyang University College of Medicine (Seoul, KR)

Background: The introduction of imatinib mesylate (IM) in the treatment of chronic myeloid leukemia (CML) improved patients' survival and resulted in current trend of avoiding up-front allo- geneic hematopoietic stem cell transplantation (HSCT) in CML patients. Thus, allogeniec HSCT is usually performed in case of failure or intolerance of tyrosine kinase inhibitor. The aim of our study is to understand the outcomes of allogeneic HSCT in Imatinib resistant or intolerant CML patients.

Patients and methods: We retrospectively analyzed $25 \mathrm{CML}$ patients who showed IM resistance or intolerance and underwent HSCT in five tertiary hospitals in Seoul, Korea between 2000 and 2008, before the era of second generation tyrosine kinases. We also compared IM resistant or intolerant patients' survival from HSCT with that of $28 \mathrm{IM}$ naive transplanted CML patients in same hospitals before IM era.

Result: $5 \mathrm{IM}$ resistant and $4 \mathrm{IM}$ intolerant patients underwent HSCT at chronic phase (CP). After HSCT, all patients achieved complete cytogenetic response(CCR), however disease progression appeared in $2 \mathrm{IM}$-resistant patients and $1 \mathrm{IM}$-intolerant patient. Posttransplantation overall survival (OS) and leukemia free survival (LFS) were not significantly different between IM resistant, IM intolerant, and 24 IM-naive patients $(5-y R$ OS $80.0 \%$ vs. $75.0 \%$ vs. $70.8 \%, P=0.954,5$-yR LFS $80.0 \%$ vs. $75.0 \%$ vs. $70.8 \%, P=0.396$ )

HSCT was performed at blastic crisis phase in 13 IM resistant, $1 \mathrm{IM}$ intolerant, and $3 \mathrm{IM}$ naive patients. The best response to glivec and to HSCT were not significantly associated with the relapse rate or survival after HSCT ( $P>0.05$, Chi2 test). Posttransplantation survival was not inferior in IM-resistant group, compared with IM-naive patients $(P=0.752)$.

In two IM resistant patients, molecular progression occurred after HSCT without hematologic relapse. In both cases, complete molecular response (CMR) was achieved again after IM re-administration.

Conclusion: In Korean CML patients, allogeneic HSCT could be an effective salvage therapy for both IM resistant and IM intolerant patients. Long-term outcome was comparable to IM-naive HSCT recipients.

\section{Lymphoma}

\section{P813}

Stem cell transplantation in Hodgkin's lymphoma patients: Iranian experience

A. Ghavamzadeh, K. Alimoghaddam, S. Mousavi, M. Iravani, A.A. Hamidieh, F. Khatami, A. Jalali, M. Jahani

Hematology-Oncology \& Stem Cell Transplantation Research Center (Tehran, IR)

Introduction: Autologous Stem Cell Transplantation (SCT) with High-dose chemotherapy is the responsible therapy for most patients with Hodgkin lymphoma (HL) that is not controlled by conventional chemotherapy. SCT can develop the long-term Disease Free Survival (DPFS) of patients.

Patients and methods: 148 Hodgkin lymphoma Patients, (85 male and 63 female) with a median age of 24.5 years (Range: 12-58 years), have received SCT from January 2003 through December 2009 in this centre. Second complete remission was the most common status of patients before transplantation. The conditioning regimen was cytarabine, etoposide, lomustine and melphalan, followed by infusion of autologous SCT and analyzed the results.

Results: The median time to Absolute Neutrophil Count $\geq 0.5 *$ $10 \% / \mathrm{L}$ was +14 and the median time to platelet count $\geq 20 * 10 \% / \mathrm{L}$ was +23 . The median follow up time was 13 month. At present, $140(94.6 \%)$ out of 148 are still living. Transplant Related Mortality was $2 \%$. The most common cause of death was relapse (6 patients). Two years disease-free survival was $82 \%$ and two years overall survival was $91.4 \%$.

Conclusion: Regarding to our experience and other experiences autologous SCT is the treatment of choice for second remission and good partial remission. 


\section{P814}

Comparison of outcomes after unrelated cord blood transplantation and matched unrelated donor reduced-intensity conditioning regimen transplantation for lymphoid malignancies - a Eurocord-Netcord Group/ LWP and CLWP of the EBMT Study

C.A. Rodrigues (1), C. Canals (2), C. Brunstein (3), J. Finke (4), A. Nagler (5), J. Maertens (6), M. Mohty (7), D. Niederwieser (8), O. Ringden (9), J.J. Cornelissen (10), T. Ruutu (11), N.H. Russell (12), F. Ghilhot (13), B. Rio (14), D. Blaise (15), J.E. Wagner (3), E. Gluckman (16), P. Dreger (17), V. Rocha (16), A. Sureda (2)

(1)Hospital Sirio Libanes (Sao Paulo, BR); (2)Hospital de la Santa Creu i Sant Pau (Barcelona, ES); (3)University of Minnesota (Minneapolis, US); (4)University Medical Center (Freiburg, DE); (5)Chaim Sheba Medical Center (Tel-Hashomer, IL); (6)Uz Gasthuisberg (Leuven, BE); (7)Hôpital Hôtel Dieu (Nantes, FR); (8)University of Leipzig (Leipzig, DE); (9)Karolinska University Hospital (Stockholm, SE); (10)Erasmus MC/Daniel Den Hoed (Rotterdam, NL); (11)Helsinki University Central Hospital (Helsinki, Fl); (12)Nottingham University Hospital (Nottingham, UK); (13)Hopital La Miletrie (Poitiers, FR); (14)Hôtel Dieu (Paris, FR); (15)Institut Paoli Calmettes (Marseille, FR); (16)Eurocord (Paris, FR); (17)University Hospital Heidelberg (Heidelberg, DE)

We have recently reported encouraging results after unrelated cord blood transplantation (UCBT) for patients with lymphoid malignancies. Progression free survival (PFS) was improved in patients with chemosensitive disease, who have received higher cell doses, and low-dose TBI in the preparative regimen. However, whether outcomes after UCBT are comparable to matched unrelated donor (MUD) transplants for lymphoma and CLL remains to be defined. We studied 359 adult patients with non-Hodgkin lymphoma (NHL, $n=168$ ), Hodgkin lymphoma $(H L, n=108)$ or chronic lymphocytic leukemia (CLL, $n=83$ ) who received an UCBT $(n=75)$ or a MUD transplant $(n=284)$ after a reduced-intensity conditioning $(\mathrm{RIC})$ regimen between January 2000 and December 2006. In the MUD group, only patients receiving peripheral blood stem cells and with a 6/6 HLA match (almost $50 \%$ of cases having a $8 / 8$ match) were included. In the UCBT group, 31 patients $(41 \%)$ received a double UCBT and at least one cord blood unit had 2 mismatches with the patient in $75 \%$ of cases. In the UCBT group, patients were slightly younger, and aggressive lymphomas, $\mathrm{HL}$ and chemoresistant diseases were more frequent. Median follow-up time of surviving patients was 28 months. The cumulative incidence $(\mathrm{Cl})$ of engraftment at day 60 was $85 \%$ in the UCBT group and $98 \%$ in the MUD group $(P<0.001)$. At 100 days, $\mathrm{Cl}$ of grade II-IV acute graft-versus-host disease (GVHD) was not statistically different between UCBT and MUD recipients $(32 \%$ vs. $31 \%, P=$ not significant - NS). At 2 years, $\mathrm{Cl}$ of chronic GVHD was higher after MUD as compared to UCBT (48\% vs. $37 \%$, respectively, $P=0.05)$. Non-adjusted 2 years $\mathrm{Cl}$ of NRM was $28 \%$ after UCBT and $31 \%$ after MUD, $(P=N S)$. There was also no difference in 2 -year $\mathrm{Cl}$ of relapse or progression after UCBT or MUD $(38 \%$ vs. $35 \%, P=\mathrm{NS}$ ). Two-year progression-free survival (PFS) was $38 \%$ after UCBT and $41 \%$ after MUD $(P=N S)$ and overall survival was $47 \%$ and $44 \%$, respectively $(P=N S)$. In a multivariate analysis, after statistical adjustments for the differences between the 2 groups, NRM, relapse or progression, PFS and OS were not statistically different between UCBT and MUD. In conclusion, engraftment rate and incidence of chronic GVHD were lower after RIC-UCBT than after RIC-MUD but final outcomes were not different between the two groups. UCBT may be considered as a valuable alternative for patients with advanced lymphoma and CLL lacking an HLA-matched unrelated donor.

\section{P815}

A novel high-dose chemotherapy strategy with bendamustine in adjunct to etoposide, aracytin and melphalan followed by autologous stem cell rescue in resistant/ relapsed Hodgkin and non-Hodgkin lymphoma patients: a phase I-II study

G. Visani (1), L. Malerba (1), P.M. Stefani (2), P. Galieni (3), F. Gherlinzoni (2), C. Giardini (1), S. Falcioni (3), F. Loscocco (1), M. Rocchi (4), A. Isidori (1)

(1)Hematology and Stem Cell Transplant Center (Pesaro, IT); (2)Hematology (Treviso, IT); (3)Hematology (Ascoli Piceno, IT); (4)Insitute of Biomathematics (Urbino, IT)

We designed a phase I-II study to evaluate the safety and the efficacy of increasing doses of Bendamustine for the conditioning regimen to autologous stem cell transplant (ASCT) for resistant/relapsed lymphoma patients. Nineteen patients (median age 54 years, range 18-70) with resistant/relapsed non-Hodgkin (13) or Hodgkin (6) lymphoma were consecutively enrolled in the study. The new conditioning regimen (BeEAM) consisted of increasing doses of Bendamustine coupled with fixed doses of Etoposide ( $200 \mathrm{mg} / \mathrm{m}^{2} /$ day on days -5 to -2 ), Cytarabine $\left(400 \mathrm{mg} / \mathrm{m}^{2}\right.$ on days -5 to -2$)$ and Melphalan (140 $\mathrm{mg} / \mathrm{m}^{2}$ on day -1 ) (BeEAM regimen). Three cohorts of 3 patients each were treated starting with Bendamustine $160 \mathrm{mg} / \mathrm{m}^{2} /$ daily given on days -7 and -6 . The dose of Bendamustine was then escalated according to the Fibonacci's increment rule until the onset of severe adverse events and/or the attainment of the expected MTD, but not higher than $200 \mathrm{mg} / \mathrm{m}^{2}$. Patients were carefully monitored for adverse events. The administration of Bendamustine was safe in all the 3 cohorts of patients, with only 1 patient presenting a grade III oral mucositis. We then fixed the dose of Bendamustine $200 \mathrm{mg} / \mathrm{m}^{2}$ as safe and effective for the Phase II study.

A median number of $7.1 \times 10^{6} \mathrm{CD} 34+/ \mathrm{kg}$ cells (range $2.4-15.5$ ) collected from peripheral blood were reinfused to patients. All patients engrafted, with a median time to ANC $>0.5 \times 10^{9} / \mathrm{A}$ of 10 days. Median times to achieve a platelet count $>20 \times 10 \%$ and $>50 \times 109 / /$ were 13 and 15 days respectively. Ten out of 19 patients presented a fever of unknown origin $(52 \%)$. The median number of days with fever was 2 (range: $0-7$ ), with a median number of 9 days of intravenous antibiotics (range: 3-22). All patients received G-CSF after transplant for a median time of 8 days (range: $8-13$ ).

Fourteen out of 19 patients are evaluable for the response to treatment. All evaluable patients are alive and in complete remission, after a median follow-up of 5 months from transplant. It is of note that $2 / 19$ patients achieved the first complete remission after receiving the high-dose therapy with autologous stem cell rescue.

In conclusion, the new BeEAM regimen is safe and seems to have a high efficacy in heavily pretreated lymphoma patients. All the future studies who want to incorporate Bendamustine on such conditioning regimens for ASCT in lymphoma patients have to use $200 \mathrm{mg} / \mathrm{m}^{2}$ of Bendamustine given overt 2 days.

The study was registered at EMEA with the EUDRACT no 2008-002736-15

\section{P816}

Rituximab and donor lymphocyte infusions are an effective salvage treatment for B-cell lymphoma relapsing after reduced-intensity allogeneic stem cell transplantation

F. Spina (1), F. Patriarca (2), L. Farina (3), V. Montefusco (3), A. Dodero (3), C. Carniti (3), R. Fanin (2), P. Corradini (1)

(1)Fondazione IRCCS Istituto Nazionale dei Tumori-Università degli Studi di Milano (Milan, IT); (2)Azienda OspedalieroUniversitaria Santa Maria della Misericordia (Udine, IT); (3)Fondazione IRCCS Istituto Nazionale dei Tumori (Milan, IT)

Lymphoma patients relapsing after allogeneic stem cell transplantation (alloSCT) have a dismal prognosis and few therapeutic 
options for their salvage. We retrospectively evaluated the role of donor lymphocyte infusions (DLI) alone or after chemotherapy (CT) or after Rituximab as salvage treatment in a cohort of consecutive Hodgkin's (HL) and non-Hodgkin's (NHL) lymphoma patients allografted with reduced intensity conditioning (RIC) including thiotepa, fludarabine and cyclophosphamide. Progression free (PFS) and overall survival (OS) from relapse to event were analyzed by Kaplan-Meier, and relapse incidence (RI) by Cumulative Incidence method with competing risks. Cox multivariate analysis' covariates were disease (HL vs. indolent vs. aggressive NHL), age ( $<55$ y.o. vs. $\geq 55$ ), Sorror comorbidity score ( $\leq 1$ vs. > 1), DLI (DLI vs. no DLI) and Rituximab treatment (Rituximab vs. no Rituximab). Of 146 patients receiving alloSCT, $57(39 \%)$ relapsed, 48 of them (84\%) received a salvage treatment. Median age was 50 years (range 19-65), 12 patients (25\%) had HL, 13 (27\%) indolent and 23 (48\%) aggressive $\mathrm{NHL}$. Median previous CT lines were 3 (range $2-7$ ), $50 \%$ of patients failed a previous autoSCT and $50 \%$ had $>1$ comorbidity score. Donors were siblings $(67 \%)$ or matched unrelated $(33 \%)$. After immunosoppression withdrawal and in the absence of aGVHD, DLI at escalating doses were administered every 45 days. Twenty-four (50\%) B-cell lymphoma patients received DLIs (median 2, range $1-5)$, of whom $10(21 \%)$ after Rituximab \pm CT. One- and 2-years OS, PFS, and RI were 61 and $39 \%, 50$ and $30 \%$, and 45 and $65 \%$, respectively. In univariate analysis DLI treatment improved PFS $(P=0.005)$ and OS $(P=0.06)$ and reduced $\mathrm{RI}(P=0.007)$ compared to CT alone. Rituximab improved OS $(P=0.009)$ without impact on PFS or $\mathrm{RI}$. Compared to CT alone patients treated with Rituximab and DLI had better PFS $(P=0.04)$, OS $(P=0.006)$ and reduced RI $(P=0.05)$. In multivariate analysis, DLI improved PFS (HR=0.3, CI95\% $=0.18-0.85, P=0.02$ ) and Rituximab improved OS ( $\mathrm{HR}=0.2, \mathrm{Cl} 95 \%=0.07-0.66, P=0.006)$, treatment with DLI and Rituximab improved both PFS (HR $=0.3, \mathrm{Cl} 95 \%=0.1-0.8$, $P=0.02)$ and $\mathrm{OS}(\mathrm{HR}=0.1, \mathrm{Cl} 95 \%=0.02-0.5, P=0.005)$, while other covariates did not impact OS or PFS.

In conclusion, DLI treatment may improve survival and reduce $\mathrm{RI}$ as compared to CT alone, and Rituximab alone improves OS. Rituximab treatment and DLI improves both PFS and OS and should be the treatment of choice.

\section{P817}

Characteristics and outcome of relapse after autologous stem cell transplantation for mantle cell lymphoma

S. Dietrich (1), B. Tielesch (1), M. Rieger (1), M. Nickelsen (2), C. Pott (3), M. Witzens-Harig (1), M. Kneba (3), A.D. Ho (1), N. Schmitz (2), P. Dreger (1)

(1)University of Heidelberg (Heidelberg, DE); (2)Asklepios Klinik St. Georg (Hamburg, DE); (3)University Hospital SchleswigHolstein, Campus Kiel (Kiel, DE)

Autologous stem cell transplantation (autoSCT) has been shown to improve the outcome of mantle cell lymphoma (MCL). However, little is known about the patterns and outcome of $\mathrm{MCL}$ recurrence after autoSCT. To address these issues, we have conducted a retrospective study of 118 consecutive patients who underwent autoSCT for MCL from 08/1992 to 08/2008 at three large referral centres.

Patients: 95 patients (80\%) were autografted as part of first-line therapy, and $23(20 \%)$ as part of salvage treatment. Median age at transplant was 57 years (range 30 to 74 ). 76 patients $(64 \%$ ) received rituximab $(R)$ prior autoSCT and 42 patients (36\%) did not. Induction therapy included CHOP-like therapy only in 39 patients $(33 \%)$ and ara-C intensification in 79 patients $(67 \%)$. 74 patients $(63 \%)$ received a TBI-based high-dose regimen.

Results: 49 relapses occurred with a cumulative incidence of $45 \%$ after 5 years. Only 3 patients relapsed later than 5 years (90, 91 and 171 months) post autoSCT. Cox regression analysis of relapse incidence identified no RTX treatment before autoSCT $(P=0.005$ HR 2.68) and salvage autoSCT $(P<0.001$ HR 5.38) as predictive for relapse, whereas ara-C intensification, TBI, age and year of transplant had no influence. More- over, the interaction term $\mathrm{R}^{*}$ salvage autoSCT remained in the final model, implying that the beneficial effect of prior $\mathrm{R}$ was evident only in the subset receiving upfront autoSCT $(P=0.028$ HR 0.15). Median OS from relapse was 22 months. Treatment of relapse comprised a medium of 3 further lines of chemotherapy which contained $\mathrm{R}$ in 34 patients (69\%). 19 patients (38\%) underwent alloSCT for consolidation; 10 of them are still in ongoing complete remission. Notably, there were 4 long-term survivors who lived longer than 5 years after relapse without alloSCT. Cox regression analysis of OS after relapse revealed response duration $<1$ year after autoSCT $(P<0.001 \mathrm{HR} 15.32)$ as an adverse predictor for OS whereas R-containing salvage therapy with alloSCT consolidation tended to result in better OS compared to chemotherapy only $(P=0.079 \mathrm{HR} 0.33)$.

Conclusions: Outcome of patients with relapse after autoSCT for MCL is poor, especially if response duration has been short. However, for a subset of patients alloSCT as part of a rituximab-containing salvage regimen might open perspectives for durable survival, and individual patients may enjoy long-term survival even without alloSCT.

\section{P818}

Unrelated donor reduced-intensity stem cell

transplantation is a promising treatment option for elderly patients with blastic plasmocytoid dendritic cell neoplasia S. Dietrich (1), U. Hegenbart (1), T. Schmitt (1), U. Martens (2), A.D. Ho (1), P. Dreger (1)

(1)University of Heidelberg (Heidelberg, DE); (2)SLK-Kliniken Heilbronn (Heilbronn, DE)

Blastic plasmacytoid dendritic cell neoplasm (BPDC), formerly known as blastic NK cell lymphoma, is a rare hematopoietic malignancy preferentially involving skin, bone marrow and lymph nodes. The overall prognosis of BPDC is dismal. Most patients show an initial response to acute leukemia-like chemotherapy, but relapses with subsequent drug resistance occur in virtually all patients resulting in a median overall survival of only 9-13 months. However, anecdotal long-term remissions have been reported in young patients who received early myeloablative allogeneic stem cell transplantation (alloSCT). As the median age at diagnosis is above 60 years, most patients at risk will not be eligible for myeloablative alloSCT. Here we present our experience with reduced-intensity conditioning (RIC) alloSCT from unrelated donors in elderly patients with BPDC.

Seven consecutive patients with BPDC have been treated at our institution between 2004 and 2008. All patients responded to acute leukemia-like induction therapy. Three patients were ineligible for alloSCT due to comorbidity and experienced rapid relapse. Four patients proceeded to RIC alloSCT as part of first-line $(n=1)$ or salvage treatment $(n=3)$. Median age was 66 (55-69) years. Two patients had active disease at the time of alloSCT. Conditioning was based on submyeloablative doses of busulfan or treosulfan with fludarabine and ATG, followed by transplantation of unmanipulated peripheral blood stem cell grafts obtained from matched or mismatched unrelated donors. Three patients live progression-free 46,9 and 8 months post alloSCT, one patient relapsed 7 months after SCT and died soon thereafter.

We conclude that RIC SCT from unrelated donors is feasible in elderly patients with BPDC and might provide curative potential in this otherwise incurable disease. Prospective analyses of RIC alloSCT in BPDC are warranted.

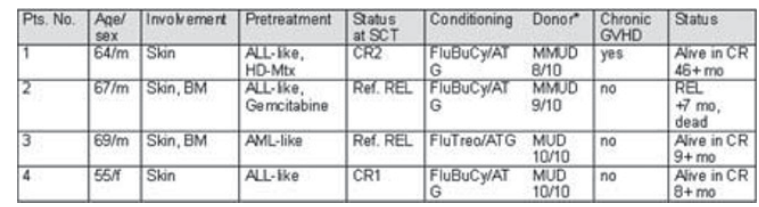


P819

18F-fluorodeoxyglucose (FDG) positron emission tomography (PET) prior to high-dose chemotherapy and autologous stem cell transplant predicts outcome after ASCT in patients with diffuse large cell lymphoma and Hodgkin's lymphoma. Results in $\mathbf{1 1 0}$ patients

S. Akhtar, A. Al-Sugair, M. Abuzaid, Y. Al Kadhi, M. Dingle, M. Abdelsalam, H. Soudy, A. Darwish, A. Eltigani, M. Nabil, I. Maghfoor

King Faisal Specialist Hosp. \& Res. Ctr (Riyadh, SA)

Purpose: There is emerging data indicating poor outcome in diffuse large cell lymphoma (DLCL) and Hodgkin's Lymphoma $(\mathrm{HL})$ patients with positive 18F-fluorodeoxyglucose (FDG) positron emission tomography (PET) before high dose chemotherapy (HDC) and autologous stem cell transplant (ASCT). This study evaluates the impact of PET as a predictor of post $\mathrm{HDC}$ residual / progressive disease and relapse in patients with DLCL and HL undergone HDC ASCT.

Patients and methods: From 2003 to May 2008, 162 consecutive patients with HL and DLCL underwent HDC ASCT. Of these, 110 patients had FDG-PET after salvage chemotherapy/prior to HDC ASCT. 53 reported patients were also enrolled in the prospective trial of FDG-PET. ESHAP was used as salvage chemotherapy, responding DLCL or responding/stable HL patients had ESHAP as mobilization and BEAM as HDC. Patient had CT scan and FDG-PET before starting ESHAP, after 2-3 cycles of ESHAP / before HDC ASCT and 100 days post ASCT. FDGPET, "positive study" was defined as study showing evidence of disease and "negative study" as no evidence of disease. Disease specific (DS) event is defined as presence of persistent disease, progression or relapsed disease after ASCT. DS event free survival (DS-EFS) was calculated from day 0. DS-EFS was calculated using Kaplan-Meier Method and comparison of the 2 groups (FDG-PET-positive or -negative) for event using chisquare method.

Results: There were $66(60 \%)$ male: female: $44(40 \%)$, DLCL: $31(28 \%)$ and HL: $79(72 \%)$. Relapsed: $50(45 \%)$, refractory 60 $(55 \%)$. Median age at ASCT was 28 years (14 to 62 ). Median follow-up from ASCT is 20 months (1.2 to 77 months). Only 13 alive patients have $<12$ months of follow-up ( 2 of them already had an event). As of December 13, 2009, of these 110 patients, $37(34 \%)$ had a disease specific (DS) event, DS event free survival (DS-EFS) is $66 \%$. DS-EFS for PET negative vs. positive is $82 \%$ vs. $53 \%(P=0,002)$. Using Kaplan-Meier method, patients with a positive PET have $51 \%$ probability of DS-EFS vs. $76 \%$ for a patient with a negative PET scan at 77 months $(P=0,0012)$. CT positive patient had DS-EFS probability of $62 \%$ vs. $51 \%$ for CT negative, $(P=0,426)$.

Conclusion: Prior to HDC ASCT, positive PET scan indicates high risk of residual disease/progression or relapse. Many of these patients are likely to suffer from treatment failure. These patients are potential candidate for more aggressive and experimental therapies.

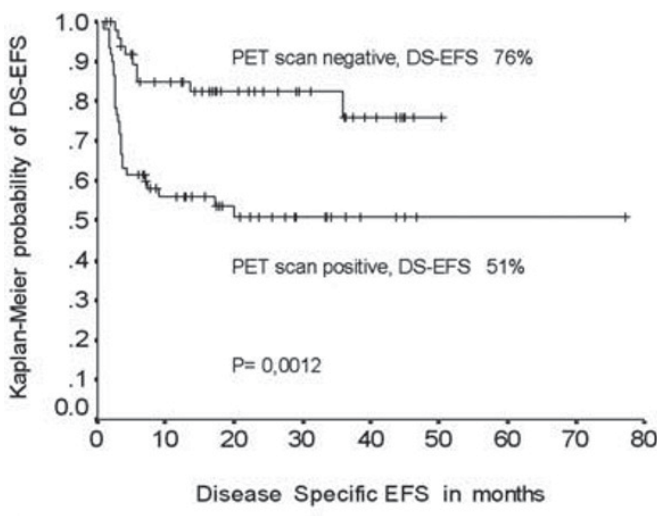

Disease Specific Event Free Survival (DS-EFS) Pre - HDC ASCT PET and CT

\begin{tabular}{|c|c|c|c|c|}
\hline Results & Number & Event & DS-EFS \% & $P$ value \\
\hline $\begin{array}{c}\text { PET } \\
\text { Negative }\end{array}$ & 50 & $9(18 \%)$ & 82 & \multirow{2}{*}{$0.002^{\star}$} \\
\hline $\begin{array}{c}\text { PET } \\
\text { Positive }\end{array}$ & 60 & $28(47 \%)$ & 53 & \\
\hline $\begin{array}{c}\text { CT } \\
\text { Negative }\end{array}$ & 26 & $7(27 \%)$ & 73 & \multirow{2}{*}{$0.407^{\star}$} \\
\hline $\begin{array}{c}\text { CT } \\
\text { Positive }\end{array}$ & 84 & $30(36 \%)$ & 64 & \\
\hline
\end{tabular}

* Pearson chi-square method ( cross tabulation)

\section{P820}

Allogeneic stem cell transplantation for Hodgkin's disease from sibling and unrelated donors: effects of chronic GvHD, prior autografting and disease status on overall and progression-free survival

C. Scheid, P. Dreger, D. Beelen, M. Bornhäuser, M. Stelljes, N. Kröger, M. Topp, G. Stuhler, R. Meyer, W.A. Bethge, J. Schubert, R. Schwerdtfeger, I. Blau, W. Krüger, L. Uharek, M. Stadler, H. Martin, G. Kobbe, R. Naumann, P. Borchmann, M. Hallek, A. Engert on behalf of the German Cooperative Transplant Study Group

High dose chemotherapy with autologous stem cell transplantation is the standard of care for relapsed Hodgkin's disease. However those patients relapsing after autograft have a dismal prognosis with further chemotherapy. Allogeneic transplantation has been offered to these patients, in particular when a matched sibling donor was available. However little information is available on results with unrelated donors and on potential factors influencing outcome.

We performed a retrospective analysis of allogeneic transplants for Hodgkin's disease within the German Cooperative Transplantation Study Group. 18 centres have provided data on patient and donor characteristics, transplant procedure and outcome. Survival data were analysed by Kaplan-Meier and tested for differences by log rank test. Multivariate analyses were done by Cox proportional hazard model.

88 patients with a median age of 30 years (range 14-58) were included. $71(80 \%)$ patients had failed a previous autologous transplantation 582 days (median) before allotransplantation. Disease status at transplantation was CR in $18 \%$, PR in $46 \%$ and stable or progressive disease in $36 \%$. Donors were matched related in $40 \%$ and unrelated in $60 \%$, a mismatch was present in 21 patients. With a median follow up of 23 months the median overall survival (OS) after allotransplant was 34,1 months with a 2 year-probability of survival of $55.1 \%$. The 
cumulative incidence of non-relapse mortality was $21.1 \%$ after 24 months. The median progression-free survival (PFS) was 13.6 months with a 2 year PFS of $41.5 \%$. Better OS was seen with prior autograft and with chronic graft-versus-host disease (cGvHD) both on univariate and multivariate analysis. PFS was higher in patients relapsing after an autologous transplantation, those with cGvHD and better response before transplantation. Only prior autograft and CGvHD remained significant for PFS on multivariate analysis.

Allogenic transplantation is a feasible option for patients with relapsed Hodgkin's disease after failing an autograft. Probabilities of overall- and progression-free survival were similar after transplantation from a related and unrelated donor. The positive effect of cGvHD supports the notion of an alloimmune effect in Hodgkin's disease. Disease status before allotransplantation did not appear to affect outcome when tested in multivariate models.

\section{P821}

A large, single-centre experience with allogeneic stem cell transplantation for T-cell non-Hodgkin lymphoma

E. Jacobsen, J. Aldridge, H. Kim, V. Ho, C. Cutler, J. Koreth, D. Fisher, P. Armand, E. Alyea, A. Freedman, R. Soiffer, J. Antin

Dana-Farber Cancer Institute (Boston, US)

Allogeneic stem cell transplantation is an accepted therapy for B cell lymphoma but the indications for allogeneic HSCT and the outcomes of the procedure are less clear in T cell lymphoma. We conducted a retrospective analysis of patients transplanted with $\mathrm{T}$ cell lymphomas including: peripheral $\mathrm{T}$ cell lymphoma (NOS), angioimmunoblastic, anaplastic, NK/T cell, enteropathy associated, hepatosplenic, panniculitic, mycosis fiungoides/ sezary, cutaneous, and T cell/NK cell other. We identified 55 patients who underwent transplant between April 4, 1997 and February 17, 2009. Eight patients received a prior autologous HSCT. Donors were HLA-matched-related (24), matched unrelated (21), and mismatched unrelated (10). The stem cell sources included peripheral blood (39), bone marrow (8), and umbilical cord blood (8). Thirty-two patients $(58 \%)$ underwent myeloablative conditioning with cyclophosphamide and total body irradiation while $23(42 \%)$ underwent reduced intensity conditioning (RIC). Regimens for acute graft versus host disease (aGVHD) prophylaxis included tacrolimus and sirolimus + MTX in $53 \%$ of patients and tacrolimus and MTX in $22 \%$ of patients. In 51 evaluable patients the incidence of grade II-IV was $26 \%$ by day 100 and the incidence grade III/IV aGVHD was $8 \%$. In 41 evaluable patients the cumulative incidence of extensive chronic GVHD at 2 years was $34 \%$. The median follow-up of survivors is 26.1 months (range 6-102). Transplant related mortality (TRM) at $1 \mathrm{yr}$ was $27.3 \%$. OS at 100 days, $1 \mathrm{yr}$, and 2 yrs was $75 \%, 54 \%$, and $37 \%$ respectively. PFS at day $100,1 \mathrm{yr}$, and 2 yrs was $60 \%, 36 \%$, and $27 \%$ respectively (see figure 1 ). In Cox regression, patient-donor gender mismatch was associated with inferior OS (HR 3.169, $P=0.006)$ and PFS (HR 2.164, $P=0.04)$. More recent year of transplant was associated with superior OS (HR 0.873, $P=0.02$ ). There was no difference is OS or PFS between the patients receiving myeloablative conditioning and those receiving $\mathrm{RIC}$. We conclude that allogeneic HSCT is effective in T NHL with plateaus in both OS and PFS. The incidence of GVHD was acceptable as was TRM. Patientdonor gender mismatch was associated with decreased OS and PFS. There was no difference in outcome between RIC and myeloablative conditioning regimens.

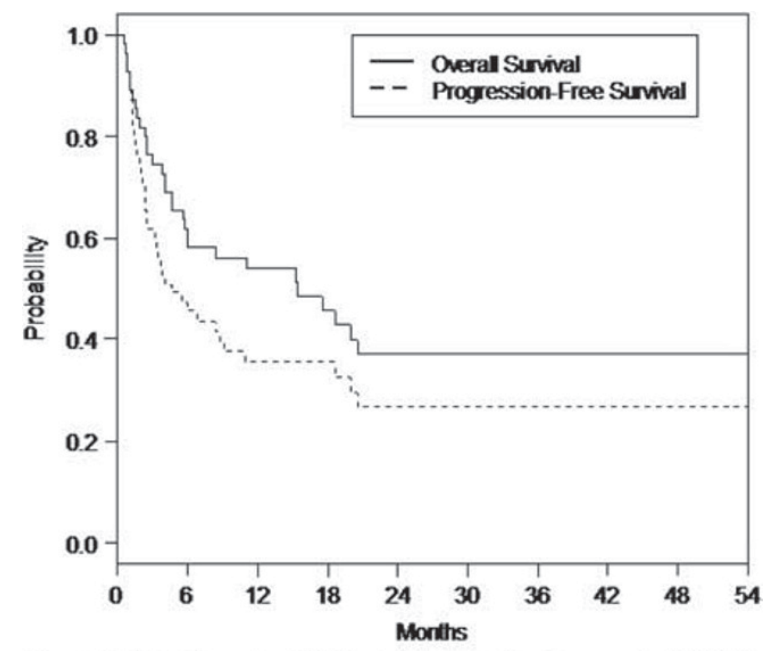

Figure 1. Overall survival (OS) and progression free survival (PFS)

P822

Long-term follow-up of autologous stem cell transplant in AIDS-related Lymphoma patients. Results of Spanish Cooperative Registry GELTAMO/GESIDA

D. Serrano, P. Miralles, R. Carrion, J. Berenguer, P. Balsalobre, J. Anguita, J.M. Ribera, R. Varela, J. Loscertales, E. Conde, R. Arranz, L. Escoda, I. Espigado, G. Rodriguez, J.L. DiezMartin on behalf of cooperative groups GELTAMO/GESIDA, Spain

Introduction: Highly Activity Antiretroviral Therapy (HAART) has improved the outcome of AIDS patients (Pts) allowing the use of intensive treatment for AIDS-related lymphoma (ARL) with similar results to those observed in HIV negative Pts with Lymphoma.

Aim of study: To describe the results, after a long-term followup, of Autologous Stem Cell Transplant (ASCT) in ARL receiving HAART.

Patients and methods: 33 Pts (29 male) with a median age of 42 years old (28-61) received ASCT between years 2000 and 2009 (median year of transplant 2004). Histological Diagnosis: Non Hodgkin Lymphoma (NHLy) in 23 Pts: Burkitt-Ly 4, Burkittlike Ly 2, Diffuse large B cell-Ly 10, Plasmablastic-Ly 3, Peripheral $\mathrm{T}$ cell-Ly 1 , Anaplastic $\mathrm{T}$ cell-Ly 3 . Hodgkin Lymphoma (HLy) in 10 Pts. Clinical Stage: 26 were in III-IV (Ann Arbor), and 14 NHLy presented an IPla $>1$. Pre-ASCT Response: $14 \mathrm{Pts}$ were in first Complete Remission (CR), 10 in second $\mathrm{CR}$, and 9 in Chemo-sensitive Partial Remission. Conditioning Treatment: BEAM en 27, BEAC 3, TBI+Cy+Melf 2, TBI+Cy $1 \mathrm{Pt}$. CD34 + cells infused: median $3.3 \times 10^{6} / \mathrm{Kg}(1.8-21.2)$. HAART was temporary discontinued in $13 \mathrm{Pts}$ due to mucositis (12) or liver toxicity (1).

Results: Engraftment: 32 Pts achieved $>0.5 \times 10^{9} / \mathrm{L}$ neutrophils at a median of 12 days (d) (9-33) and $29 \mathrm{Pts}>20 \times 10^{9} / \mathrm{L}$ platelets at a median of $20 \mathrm{~d}(11-57)$. Toxicity at $+100 \mathrm{~d}$ : Pneumonia was observed in $4 \mathrm{Pts}, 1 \mathrm{Pt}$ presented a multiorgan failure and 2 Pts showed liver veno-occlusive disease. Viral load: Remained undetectable provided HAART was maintained. Lymphoma progression: 7 pts have relapsed at median of 8 months (mo) (2-32), 4 have died and 3 are alive in CR after chemotherapy (+64, +114 mo)and Allogeneic Hematopoietic Transplant (+37 mo). Survival: With a median of follow-up of 58 mo (2-114), 22 pts $(66 \%)$ are alive and 11 Pts have died: 4 pts after Lymphoma progression and 7 due to non relapse mortality (NRM):1 owing to multiorgan failure $(+15 \mathrm{~d}), 4$ as a result of opportunistic infections at $+1,+3,+10,+14$ mo, 2 pts after secondary Acute Myeloid Leukemia at +11 and +61 mo. Cumulative incidence of NRM at $3 \mathrm{mo}$ was $6 \%$, at $12 \mathrm{mo} 16 \%$ and $24 \%$ at $61 \mathrm{mo}$. 
Overall Survival is $61 \%$ at $61 \mathrm{mo}$ and Event Free Survival is $53 \%$ at $61 \mathrm{mo}$.

Conclusions: Autologous Stem Cell Transplantation is a safe and effective option to treat AIDS-related Iymphoma, with a high long-term survival. Nevertheless early diagnosis of infection is critical in order to improve the outcome in this setting.

\section{P823}

Intermediate intensity conditioning and T-cell replete allogeneic transplantation is effective in high-risk relapse and progressive disease of aggressive lymphoma

J. Hasenkamp, G. Wulf, A. Görlitz, P. Dreger, J. Schubert, M. Nickelsen, L. Truemper, N. Schmitz, B. Glass on behalf of the DSHNHL

Patients (pts) failing first-line therapy of aggressive NHL including $\mathrm{mAb}$ and dose-dense chemotherapy have a poor outcome. In addition, there is no standard treatment for relapse after high-dose therapy (HDT) and autologous SCT (ASCT). These pts need vigorous debulking (conditioning) and a GvLymphoma effect (T-Cells with the graft) in order to obtain optimal results. We initiated a trial for pts (18-65 yrs) with aggressive NHL and primary progressive disease, early relapse with $>1 \mathrm{IPI}$ risk factor, or relapse after HDT/ASCT. The intermediate conditioning (Fludarabine $125 \mathrm{mg} / \mathrm{m}^{2}$, Busulfan $12 \mathrm{mg} / \mathrm{kg}$ and Cyclofosfamide $120 \mathrm{mg} / \mathrm{kg}$ ) was followed by GvHD prophylaxis with tacrolimus/ MMF. Pts were randomized to receive rituximab post transplant as GvHD prophylaxis or not. Due to high GvHD incidence we amended the protocol that all pts with unrelated donors received additionally ATG (51 pts). From 6/2004 to 7/2009, 81 pts (median age $49 \mathrm{yrs}$ ) were enrolled and eligible for analysis. 41 pts had DLBCL, 6 pts FCL III, 8 pts blastic MCL, 2 pts aggressive marginal zone lymphoma, 4 pts lymphoblastic B cell lymphoma, and 20 pts suffered from T cell lymphoma. $54 \%$ of pts received HDT/ASCT prior to alloSCT; $75 \%$ had early relapse or primary progressive disease, $59 \%$ chemo-refractory disease and $24 \%$ had progressive disease with high or high intermediate aalPI immediately prior to conditioning. PBSCT were obtained from MRD in 24 pts, from MUD in 40 pts and from one locus mMUD in 17 pts. Median follow-up of surviving pts is $1.9 \mathrm{yrs} .41 \mathrm{pts}$ died, in 26 pts death was treatment related. After one year, estimated OS is $52 \%$, PFS is $46 \%$, RR is $29 \%$, NRM is $37 \%$. The incidence of acute GvHD>I is $66 \%$. The last NHL progress occurred day 288 post alloSCT. Pts with high-intermediate/high IPI at transplant did not differ significantly in PFS, TTP or OS from pts with low/low-intermediate IPI. There are no differences in OS, PFS and GvHD for pts regarding to rituximab. This alternative myeloablative conditioning followed by T-replete allogeneic SCT is an effective treatment in pts with high risk features like active disease at transplantation and high tumor burden. In contrast to the relatively high incidences of GvHD and non-relapse mortality, the relapse rates were low and OS and PFS are promising. Rituximab has no role in the prevention of GvHD in this setting. We do not believe that minimal conditioning and in vivo T-Cell depletion will cure patients with high-risk aggressive lymphoma.

\section{P824}

Tandem high-dose chemotherapy with haematopoietic stem cell transplant yields better outcome in relapsed/ refractory Hodgkin's lymphoma patients in PET complete remission after ifosfamide, gemcitabine, vinorelbine

A. Anastasia (1), M. Magagnoli (1), M. Balzarotti (1), M. Spina (2), R. Mazza (1), L. Giordano (1), U. Tirelli (2), A. Santoro (1) (1)Humanitas (Rozzano, IT); (2)CRO (Aviano, IT)

High dose chemotherapy with autologous hematopoietic stem cell transplant (AHSCT) remains the gold standard in relapsed/ refractory Hodgkin's Lymphoma patients.

Tandem high dose chemotherapy with AHSCT has been already reported in several non randomized studies demonstrating its feasibility and safety. Complete remission (CR) to induction therapy is the mainstay for a better outcome after AHSCT. IGEV is emerging as a powerful induction scheme.

The aim of our study is to compare the outcome of PET-CR patients who underwent tandem high dose chemotherapy with AHSCT versus the gold standard in terms of DFS and OS.

From November 1997 to May 2007121 patients were enrolled in a prospective trial with 4 cycles of IGEV plus high dose chemotherapy with AHSCT.

Conditioning regimens consisted in carmustine, etoposide, aracytin and melphalan (BEAM) for the single procedure and Melphalan200 plus BEAM for tandem transplantation.

Since 2001, we did not perform a specific stratification, as all patients younger than 65 years underwent double transplantation if they reached a satisfactory stem cell collection.

Response assessment was defined by Cheson2007 criteria. For the statistical analysis we used a logistic regression, the Kaplan-Meier method and the Cox proportional hazard model.

After IGEV induction, 56 out of all 121 patients, considered in this study, reached a complete remission (PET negative), with 20 of them receiving a single procedure and 36 a tandem transplant. No differences in terms of prognostic factors could be found in the 2 cohorts. Table 1.

With a median follow-up of 3 years, DFS and OS were $76 \%$ and $90 \%$ in tandem cohort versus $48 \%$ and $80 \%$ in single procedure, respectively. Figure 1.

In our ITT analysis, patients receiving single transplant have a relapse risk 3.3 higher than tandem group (C.I. 95\%. 1.6, 10; $P$ value: 0.025$)$. When we analyzed the program fulfilled, relapse risk was 2.13 higher (C.I. 95\%. 1.05, 4.34; $P$ value: 0.035 ).

In conclusion, our data suggests that patients with relapsed or refractory Hodgkin's Lymphoma which were PET complete responders after IGEV, had a potential benefit by tandem AHSCT treatment.

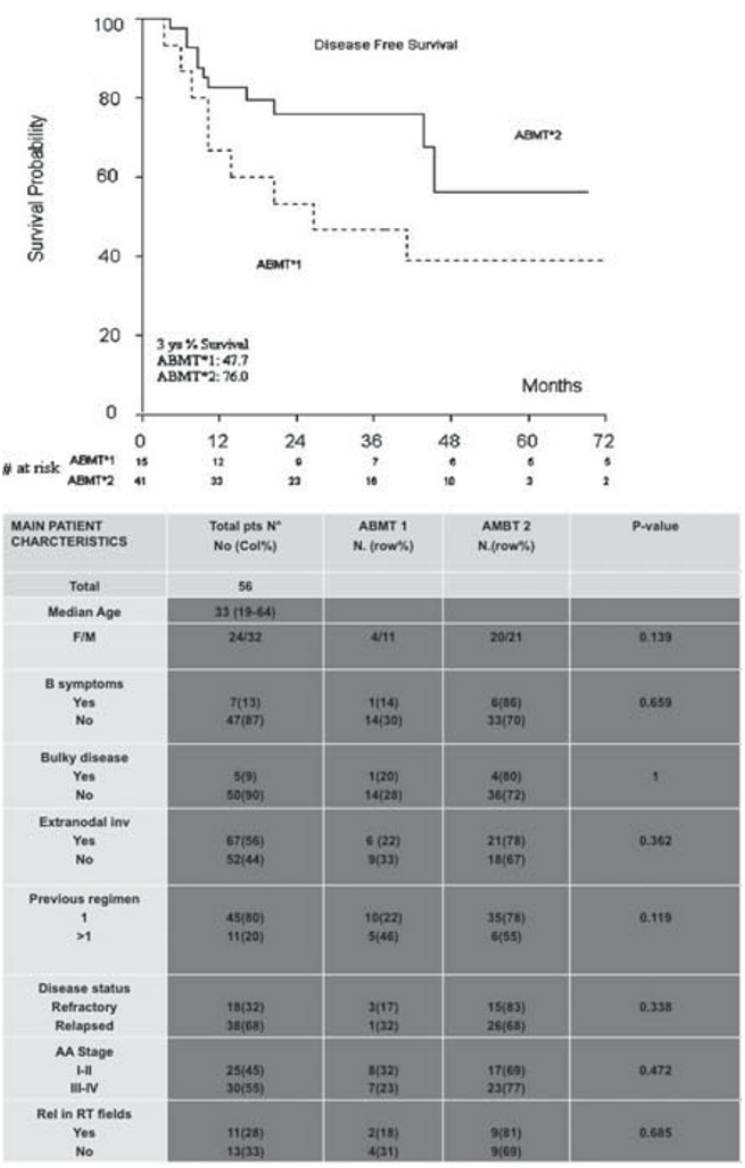




\section{P825}

Retrospective single-centre study of patients with refractory Hodgkin's lymphoma: allogeneic stem cell transplantation compared to conventional treatment using the propensity score matching method H. Labussiere (1), M. Resche Rigon (2), P. Brice (2), P. Ribaud (2), A. Devergie (2), A. Petropoulou (2), R. Peffault De Latour (2), G. Socié (2), M. Robin (2)

(1)Hopital E Herriot (Lyon, FR); (2)Hopital Saint-Louis (Paris, FR)

Although Hodgkin lymphoma is usually characterized by a high chemosensitivity, a minority of patients with primary refractory disease or early relapse presents poor outcome. Allogeneic stem cell transplantation (alloSCT) is one of the treatmentoptions that can be proposed for these progressive patients. We retrospectively studied 31 patients who received an alloSCT in Saint-Louis Hospital and compared their outcome with those of non alloCTpatients with similar characteristics. We only compared patients who relapsed after an autologous SCT (autoSCT) and were alive at least 12 months after autoSCT. To correct the recruitment bias, the propensity score (PS) methodology was used to determine the probability of receiving alloSCT in the group of patients who relapsed after autoSCT, according to a set of baseline characteristics. Parameters used for this PS score were age, primary chemorefractory disease and stage III-IV at first relapse. 31 patients received an alloSCT between 2000 and 2007. Median age at alloSCT was 26 years. Median number of previous lines of chemotherapy was 3.6 and 28 patients were previously autografted. 13 were considered refractory to primary treatment and 11 to secondary treatment. At alloSCT 20 patients were in CR, 9 in PR and 2 in progressive disease. 26 patients received a reduced intensity conditioning regimen. Most of the patients $(n=24)$ received peripheral stem cells and donors were HLA-identical siblings in 15 cases. Median follow-up after autoSCT and alloSCT was 62 and 51 months respectively. All patients engrafted. Grade II-IV acute GVHD occurred in 10 patients and chronic GVHD in 13. 3 months after alloSCT, 19 patients were in remission, 5 presented stable disease and 7 were progressive. 11 patients relapsed despite alloSCT and 11 patients died. The estimated 3 -year overall and event free survival were respectively $69 \%$ $(95 \% \mathrm{Cl}[48 ; 82])$ and $41 \%(95 \% \mathrm{Cl}[23 ; 58])$ and 3-year non relapse mortality was $21 \%(95 \% \mathrm{Cl}[13 ; 29])$. Causes of death after alloSCT were progression in 6 and toxicity in 5 patients. From the 31 alloSCT recipients, 15 matched pairs were successfully constituted according to the PS. Based on these matched pairs, the estimated hazard ratio of death was 0.24 $(95 \% \mathrm{Cl}[0.07 ; 0.80])$ for allografted patients compared with non allografted. AlloSCT in poor-prognosis Hodgkin lymphoma gives promising survival outcomes and provides survival benefit in comparison with non allograft treatment as suggested by PS method.

\section{P826}

Haematopoietic stem cell transplantation in patients with lymphomatoid granulomatosis: a retrospective survey of the Lymphoma Working Party of the EBMT

K. Siegloch, N. Schmitz, B. Friedrichs, E. Hamilton, G.W. van Imhoff, S. Montoto, J. Luan, J.M. Ribera, R. Delage, A. Sureda on behalf of the Lymphoma Working Party of EBMT

Introduction: Lymphomatoid granulomatosis (LG) is a rare, EBV- associated B cell lymphoproliferative disorder. Therapy includes corticosteroids, chemotherapy (COP, CHOP or other), interferon-alpha or Rituximab showing moderate results.

Patients and methods. To describe the results of intensive therapy and stem cell transplantation (SCT) in patients with LG, a retrospective survey was conducted among transplant centers belonging to the European Group for Blood and Marrow Transplantation (EBMT). As LG is not reported as an independent histological entity to the EBMT DataBase, a questionnaire was sent to the centers, thus identifying 8 patients (7 ASCT, 1 alloSCT), who were transplanted from 1998 to 2008. In addition, we were able to collect the follow-up data of a patient with LG, who was subject of a case report (Lemieux et al., Hematology 2002, Vol. 7 (6), pp. 355-358). The patient cohort finally consisted of 4 males and 5 females with a median age of 36 (range 14-52) years. Two patients had localized disease (stage I-1 patient; stage II-1 patient) and 7 patients had stage IV disease, 4 patients presented with $B$ symptoms. The lungs were the most frequently involved extranodal site (7 patients, 3 with liver involvement). EBV serology was positive in 5 of the 7 cases in which the information was known.

Results. Eight patients had received at least 2 lines of therapy before SCT including Rituximab in 5 patients, the median time interval between diagnosis and SCT being 10 (range 6-52) months. All patients had active disease at the time of SCT: 7 patients were in partial remission (PR) and 2 had stable disease (SD). Seven of the 8 autologous recipients were conditioned with the BEAM regimen (including Zevalin in one case), and the remainder was conditioned with fludarabine/busulfan/ cyclophosphamide. A reduced intensity-conditioning regimen (fludarabine plus an alkylating agent) was used prior to alloSCT. One ASCT patient (13\%) died of non-relapse mortality (S. salivarius shock, no evidence of LG at the autopsy) and 1 ASCT patient committed suicide being disease-free 19 months after SCT. The remaining 7 patients (6 ASCT, 1 allo-SCT) are alive and disease-free with a median follow up of 27 (range 8-57) months after SCT.

Conclusions. Hematopoietic SCT is an excellent therapy for patients with relapsed LG. The optimal time for ASCT as well as the optimal transplant modalities need further study.

\section{P827}

Front-line autologous stem cell transplantation as intensive consolidation in patients with newly diagnosed peripheral T-cell lymphomas: multicentre, phase II clinical trial in Korea

D.H. Yang (1), J.S. Ahn (1), S.R. Lee (2), S.K. Sohn (3), J.H. Moon (3), M.K. Song (4), J.S. Jung (4), J.Y. Kwak (5), J.J. Lee (1), H.J. Kim (1)

(1)Chonnam National University (Jeollanam-do, KR); (2)Korea University Medical Center (Seoul, KR); (3)Kyungpook National University Hospital (Daegu, KR); (4)Busan National University Hospital (Busan, KR); (5)Chonbuk National University Medical School (Jeonju, KR)

High-dose chemotherapy (HDT) followed by autologous stem cell transplantation (HDT/ASCT) has represented a rescue option from poor prognosis of peripheral $\mathrm{T}$ cell lymphoma (PTCL). However, the effectiveness of ASCT on distinct PTCL subtypes, the optimal transplant timing during the clinical courses and the available prognostic factors in order to predict the better outcomes are still unclear.

Patients and methods: We investigated the clinical outcomes of HDT/ASCT as frontline intensification in 33 patients with newly diagnosed PTCL from five Korean institutions between Oct. 2003 and Feb. 2009. Eligibility criteria is followings as: 1) age under 65 years, 2) histologically proven PTCL except $\mathrm{ALK}(+)$ anaplastic large cell and primary cutaneous/subcutaneous panniculitis, 3 ) patients who achieved complete (CR) or partial response (PR) after primary chemotherapy, 4) ECOG performance status $\leq 25$ ) no severe concomitant disease. Patients underwent ASCT with a uniform conditioning regimen (busulfex, cyclophosphamide and etoposide).

Results: The median age was 47 years (17-65). The histological subtypes were $48.5 \%$ PTCL-unspecified $(n=16)$, $24.2 \%$ anaplastic large cell $(n=8), 6.1 \%$ angioimmunoblastic T cell $(n=2), 18.2 \%$ extranodal NK/T cell $(n=6)$, and $3.0 \%$ hepatosplenic gamma-delta T cell $(n=1)$. Twenty-one patients (63.6\%) presented in advanced stages (III/IV) and $12(36.4 \%)$ had B symptoms. At diagnosis, 14 patients $(42.4 \%)$ were classified as high-intermediate/high risk by the age-adjusted IPI 
(aalPI) and $6(18.2 \%)$ were classified as high-risk (more than 2 factors) by the prognostic index for PTCL (PIT). Disease status at transplant consisted of 25 patients $(75.8 \%)$ with CR and 8 patients $(24.2 \%)$ with PR. 6 of 8 PR patients improved the response to $C R$ after transplant. There was no significant difference in response rates between patients treated with $\mathrm{CHOP}$ alone or CHOP-like chemotherapy and those treated with another non-anthracycline-based chemotherapeutic regimen. At a median follow-up of 27.5 months, 24 patients $(72.7 \%)$ are alive and 7 patients $(21.2 \%)$ relapsed. The 3 -year probability of progression-free survival (PFS) was $80.9 \pm 7.0 \%$. One patient $(3.0 \%)$ died due to transplant-related mortality (any death within 60 days). The high PIT and high-risk aalPI were poor prognostic factors in PFS.

Conclusion: Our data suggest that frontline HDT/ASCT in patients with PTCL could be performed safely with high response rates and showed a substantial impact on improving outcome for PFS.

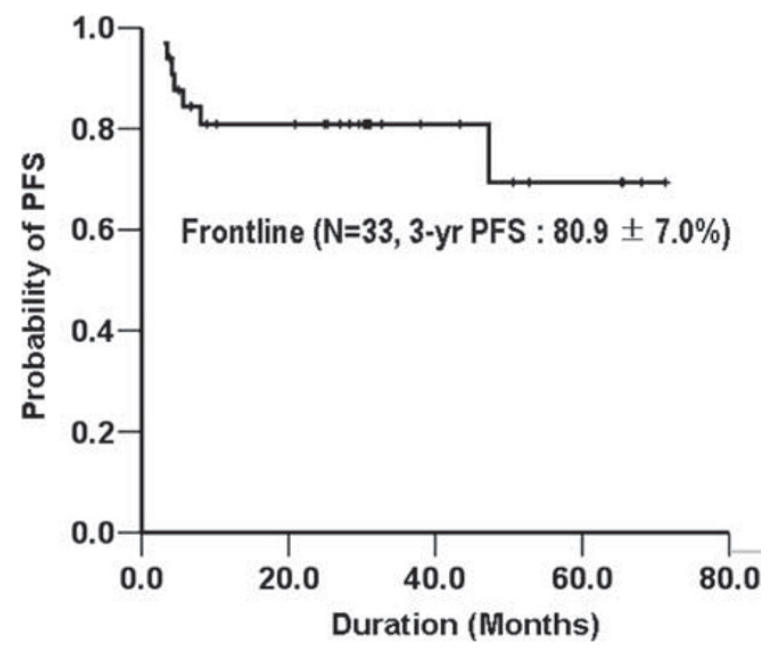

P828

High-dose chemotherapy and in vivo rituximab purged autologous stem cell transplantation as front-line therapy for mantle-cell lymphoma in patients aged up to $\mathbf{7 2}$ years A. Andreini, F. Frattini, M. Sorio, C. Tecchio, G. Quaresmini, A. Bonani, S. Ledro, C. Perbellini, D. de Sabata, F. Randon, F. Benedetti

Bone Marrow Transplant Unit (Verona, IT)

The current treatment of MCL is based either on RituximabCHOP followed by SCT or Rituximab-HyperCVAD regimen. More recently good results were obtained with Rituximab combined with fludara containing regimens. Despite high remission rate, many authors are convinced that new drugs and new approaches are needed.

Some year ago, a long remission was described in a multi-centric Italian study from Istituto Tumori in a group of $28 \mathrm{pts}$, using an aggressive sequential regimen with Rituximab in vivo purging and SCT at the end of therapy (Gianni AM, Blood, 102;749, 2003). The age limit was 60 years.

Methods: After this first study we continued to enroll in the same protocol all the pts with MCL referred to our Institution: 26 consecutive pts (4 females/22 males), median age 60 years (range 38-72), have completed the therapy. All pts had advanced disease and are suitable for evaluation. We obtained a molecular marker $(\mathrm{bcl}-1 / \mathrm{lgH})$, useful for monitoring the response in 21/26 patients; in 3 cases it was negative and in 2 cases not available.

Results: Clinical and molecular complete remission (CR) was reached in $25 / 26$ pts after the transplant procedure. In all cases we tested by PCR analysis the stem cells, and we only used the PCR negative collections. In 4 cases the aphereses collected after Rituximab/Cytoxan were PCR positive, so that we repeated stem cells collection after the following cycle with high dose ARA-C and Rituximab. At the transplant, the stem cells were PCR negative in all the pts. Two bacterial infections, a sepsis and a pneumonia, were the only complications observed. At present, 20/26 are alive (OS at $5 y=90 \%$ ), 17/26 are in continuous CR (PFS 5 y >71\%), with a median follow-up of 43 months (range 7-74). Two pts died because of progression and four because of secondary neoplasia (3 MDS, 1 solid tumor). One patient, relapsed 14 months after treatment was rescued with allogeneic bone marrow transplant, one is in partial remission and one has relapsed. Follow-up has included clinical evaluation 4 times a year, bone marrow biopsy and molecular analysis on marrow and peripheral blood cells twice a year.

Conclusions: This is considered an aggressive treatment, suitable for pts in good Performance Status, aged less than 60 . In this group of pts we have increased the age of a decade, apparently without toxicity. The remission rate, survival and the progression free survival are comparable to those obtained in a younger cohort of patients with the same diagnosis.

\section{P829}

Allogeneic HSCT for Lymphoid Neoplasia, a report by the Swiss Blood and Marrow Stem Cell Transplant

Group (SBST)

J. Passweg, H. Baldomero, M. Stern, C. Bucher, Y. Chalandon, D. Heim, B. Mohty, M. Nagy, G. Stuessi, U. Schanz, A. Gratwohl on behalf of the the Swiss Blood and Marrow Stem Cell Transplant Group (SBST)

Background: Allogeneic HSCT is used with variable frequency in lymphoid neoplasia. Its place in the therapeutic armamentarium is not clear. Allogeneic HSCT is more often used as salvage after failing conventional treatment.

Methods: The comprehensive database of the Swiss Blood Stem Cell Transplant Group (SBST) includes all patients transplanted in Switzerland and we evaluated outcome of allogeneic HSCT for lymphoid neoplasia transplanted 1997-2008.

The study included 205 patients, $136(66 \%)$ male, receiving allogeneic HSCT for non Hodgkin's lymphoma $(n=106$, including 30 with CLL), Hodgkin lymphoma $(n=29)$ or myeloma $(n=70)$. Median age was 48 (9-68) years. Of these 111 had failed prior autologous HSCT, median interval from diagnosis to transplants was 24 months. Stem cell source was bone marrow in 17 and peripheral blood in 188. Donors were identical siblings in 144, twins in 10, unrelated or mismatched related donors in 51. EBMT risk score (incorporating age, disease stage, time interval between diagnosis and transplantation, donor type, and donor recipient gender combination) was $2-3$ in 59, 4-5 in 123 and 6-7 in 23

Results: Survival at 5 years was $61 \pm 12 \%$ for Non Hodkgins lymphoma, $53 \pm 26 \%$ for Hodgkin lymphoma and $47 \pm 15 \%$ for multiple myeloma $(P=n s)$ (Figure 1).

Cumulative incidence of treatment related mortality at 5 years was $11(10-12) \%$ without differences among disease groups. Relapse incidence at 5 years was $50(48-51) \%, 31(22-43) \%$ for $\mathrm{NHL}, 58(40-83) \%$ for Hodgkin lymphoma, and $64(52-78) \%$ for myeloma.

Conclusion: A considerable proportion of patients with lymphoid neoplasia becomes long term survivors after allogeneic HSCT. Weather the higher relapse incidence of Hodgkin lymphoma and myeloma as compared to Non Hodgkins' lymphoma is due to patient selection or differences in graft versus lymphoma effects remains to be elucidated. 


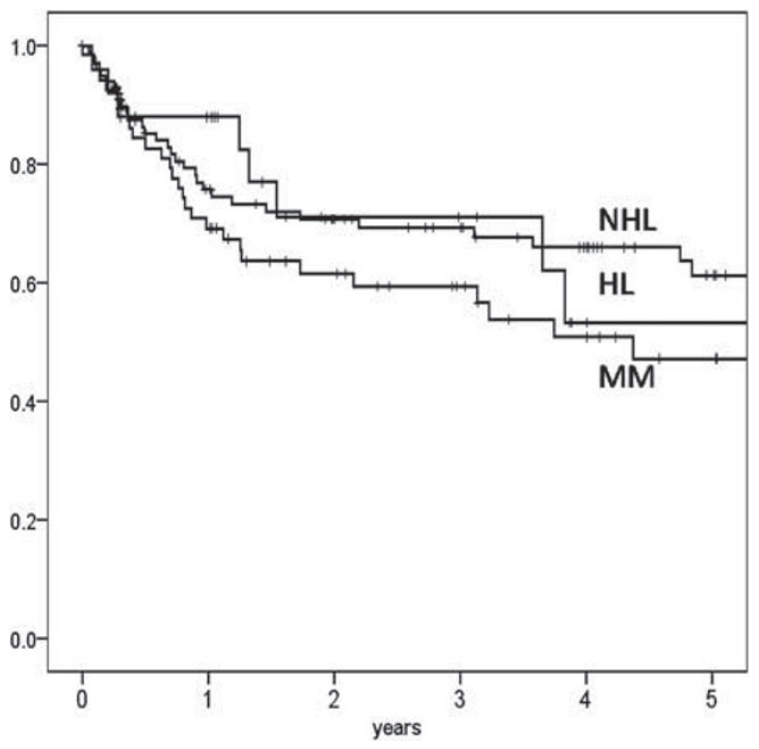

P830

Outcomes of Hodgkin's lymphoma patients with relapse or progression following first autologous stem cell transplantation

P. Kaloyannidis (1), G. Voudiadou (1), A. Balta (2), A. Spyridonidis (3), S. Tsimperis (1), I. Baltadakis (2), A. Symeonidis (3), J. Apostolidis (2), C. Smias (1), D. Karakasis (2), I. Sakellari (1), N. Zoumpos (3), N. Harhalakis (2), A. Anagnostopoulos (1) (1)G.Papanicolaou (Thessaloniki, GR); (2)Evangelismos Hospital (Athens, GR); (3)University Hospital Patras (Patras, GR)

According to recent retrospective analyses, the median survival of patients (pts) with relapsed/progressed Hodgkin's lymphoma $(\mathrm{HL})$ following autologous stem cell transplantation (ASCT) may exceed 2 years regardless of therapeutic approach. We retrospectively evaluated the outcome of 103 (female: 39, male: 64) pts, who underwent 1st ASCT for HL from September 1990 to November 2008 and relapsed or progressed. Sixty-four pts had been transplanted for refractory disease. The median time to relapse/progression was $5(0.7-132)$ months post ASCT, and $<12$ months in $77 / 103$ pts. The median age at relapse was 29 (13-67) years. The stage at relapse was advanced (III or IV) in 58 , and B-symptoms were present in 26 pts. In 41 pts, progression of disease to additional sites was noted. Salvage treatment was chemotherapy \pm irradiation $(R / T)$ in 75 pts, $R / T$ only in 6 , 2nd SCT in 14 (ASCT in 3, and alloSCT with a reduced-intensity regimen in 11), while 2 refused any therapy. Six pts had a positive PET-scan without clinical suggestion of active disease, and did not receive any treatment (watch-and-wait approach). Sixty of the 95 treated pts achieved a complete (38 pts, $40 \%$ ) or partial ( $22 \mathrm{pts}, 24 \%$ ) remission. The interval of $<12$ months from 1 st ASCT to relapse, advanced stage and presence of B-symptoms at relapse, as well as the disease refractoriness before 1st ASCT emerged as significant adverse factors for response rate $(P<0,05$ by $\mathrm{t}$-test). The median survival for all pts was 29.5 months. Multivariate analysis revealed late relapse $(>12$ months), absence of B-symptoms at relapse and disease sensitivity before 1st ASCT as significant factors for prolonged survival $(P<0.05)$. The type of treatment had no impact on survival rates. Three of the 14 re-transplanted pts (2 allo- and 1 autografted) are alive (with 2 in CR for 7 and 5 years, respectively). Eight re-transplanted pts succumbed to progressive disease and 3 to treatment related toxicity. Interestingly, all 6 patients managed with the watch-and-wait approach are alive without disease progression for a median of $12(1.5-45)$ months. Our results confirm that selected pts with relapsed or progressed $\mathrm{HL}$ following ASCT could be rescued with recent treatment modalities. Currently, given the conventional and alternative treatment options, management of relapsed disease post 1st ASCT remains a challenge and prospective studies with large series of pts are needed to establish the appropriate approach.

P831

Outcome of patients with Hodgkin's lymphoma relapsing after autologous stem cell transplantation: comparison between reduced-intensity allogeneic stem cell transplantation and chemo-radiotherapy salvage approaches

C. Martínez, C. Canals, L. Castagna, E. Alessandrino, D. Karakasis, A. Paola, G. Leone, M. Trneny, J. Snowden, E. Kanfer, N. Milpied, A. Bosi, D. Caselli, C.A. de Souza, J. Luan, R. Oneto, R. Willemze, R. Arranz, L. Jebavy, A. Hellmann, C. Gisselbrecht, A. Sureda on behalf of the GITMO and LWP of the EBMT

Patients with HL relapsing or progressing after first ASCT may be offered several therapeutic options. The aim of this retrospective study was to assess if RIT, performed along the first year after relapse, is associated to an improved survival compared to conventional chemotherapy and/or radiotherapy (CHEMO-RT). In order to ensure the comparability of the groups regarding salvage therapy options and follow-up, statistical analysis was restricted to ASCT performed in the period of 2000-2005. Overall survival (OS) from relapse or progression after ASCT was the main endpoint of the study. Cox multivariate regression analysis was performed to adjust for all prognostic factors, and RIT was included in the Cox model as a time-dependent covariate. A total of 195 patients were treated with CHEMO-RT and 49 received a RIT. There were no statistical differences in sex, histological HL subtype, time from ASCT to relapse, stage of HL, presence of B symptoms, bulky disease, extranodal involvement at ASCT, or performance status at relapse between groups. Patients treated with RIT were significantly younger than those treated with CHEMO-RT $(P=.02)$. Donors were HLA-matched related in 26 patients (53\%), unrelated in $13(27 \%)$, and other alternative donors were used in $10(20 \%)$. Fludarabine-based conditioning regimen was used in mostpatients (76\%). Diseasestatus atthetime ofRITwas asfollows: 9 patients (18\%) were in complete remission, $28(57 \%)$ had sensitive disease and $12(25 \%)$ had refractory HL. Seven patients died after RIT of non-relapse causes, giving estimated non-relapse mortality (NRM) of $10 \%$ at 2 years. Disease relapse or progression occurred in 29 patients following RIT (median time 8 months, range $1-19$ ), giving cumulative incidence of relapse of $61 \%$ at 2 years. Median follow-up after relapse for surviving patients was 38 months (range 6-90). Overall survival from relapse after ASCT for RIT and CHEMO-RT patients was $48 \%$ and $32 \%$, respectively, at 4 years. Median survival time after relapse was 45 and 19 months for RIT and CHEMO-RT groups, respectively. In multivariate analysis, RIT was associated to a trend to an improved survival as compared to CHEMO-RT $(P=.08)$. This study suggests that allogeneic RIT may be useful in the management of patients with $\mathrm{HL}$ relapsing after $\mathrm{ASCT}$.

\section{P832}

Tandem autologous/reduced-intensity allograft for relapsed/refractory Hodgkin's lymphoma: early allotransplant after intensive cytoreduction may maximize graft versus lymphoma effect?

E. Todisco (1), L. Castagna (1), A.M. Congiu (2), S. Nati (2), L. Giordano (1), A. Santoro (1), A.M. Carella (2)

(1)Istituto Clinico Humanitas (Milan, IT); (2)Ospedale San Martino (Genoa, IT)

Background: Hodgkin's Lymphoma $(\mathrm{HL})$ patients primary chemorefractory and with disease at the time of autolgous transplantation (ASCT), have high probability of progression after ASCT. Reduced-intensity conditioning allotransplant (RICT) aims to 
exploit graft vs. lymphoma (GvLy) effects while reducing conditioning-related toxicity. RICT is considered as the last therapeutic option and is usually offered to $\mathrm{HL}$ patients failing ASCT and in this contest GvLy responses might be insufficient.

Aims: We pionered that offering RICT as an earlier option after intensive cytoreduction (ASCT) may allow GvLy reaction to be better exploited (Carella et al. JCO 2000; 18:3918).

Patients and methods: thirtyfive refractory $(n=26) /$ relapsed $(n=9) \mathrm{HL}$ patients $(21 \mathrm{M} / 14 \mathrm{~F})$ underwent RICT preceeded by autografting (ASCT). Patients progressing after ASCT were excluded from the analysis. Median $n^{\circ}$ of chemotherapy lines before tandem ASCT-RICT was 2.5 (range 2-4). Median age at diagnosis was 27 (range 15-47). Twenty patients (80\%) had disease before tandem $(C R=7 ; P R=14 ; S D=4 ; P R O=10)$. Highdose therapy consisted of Melphalan $200 \mathrm{mg} / \mathrm{mq}(n=9)$ and $\operatorname{BEAM}(n=26)$. The time interval between ASCT and RIC was 2.5 months (range 1.5-5.5). RIC consisted of fludarabine-cyclophosphamide $(n=20)$ or fludarabine-melphalan $(n=15)$. Thirty patients were allotrasplanted from an HLA identical donor, four from a MUD and one patient from a family-mismatched donor.

Results: The median time to neutrophils and platelets recovery was 10 days and 16 days, respectively. Chimerism studies indicated $100 \%$ donor-derived engraftment. Ten patients $(28 \%)$ developed aGVHD (grade II-IV) and 13 cGVHD (2 limited and 11 extensive). Non relapse mortality (NRM) was $8 \%$. Early response to tandem was as follow: $C R=22 ; P R=2 ; S D=1$; $\mathrm{PRO}=10$. At the last follow up 22 patients $(63 \%)$ were alive, $18(82 \%)$ in RC and $4(18 \%)$ with disease. Thirteen patients expired (37\%), 7 of disease progression, 1 of aGVHD, 1 of cGVHD and 2 of infection and 2 myocardial infarction. With a median follow-up of 48 months (6-127 months), median OS and PFS were 60.5 months and 11.5 months respectively. The three years OS and PFS was 76.5 and $41.6 \%$ respectively. Conclusions: With the limits of a retrospective study, these encouraging results suggest that GvLy may have a role on residual disease after ASCT determinig a disease chonicization. A prospective study based on genetic randomization (ASCT vs. ASCT followed by RICT) would help to answer this important issue.

\section{P833}

Risk-adapted high-dose therapy including autologous and allogeneic stem cell transplantation for relapsed or aggressive non-Hodgkin's lymphoma - a phase II trial by the OSHO (East German Study Group for Haematology and Oncology)

M. Koenigsmann (1), C. Kahl (2), M. Freund (2), N. Basara (3), D. Niederwieser (3), H. Sayer (4), S. Theurich (5), M. Christopeit (5), G. Behre (5), M. Mohren (6), A. Franke (7), A. Reichle (8), A. Ganser (9), B. Metzner (10), L. Uharek (11), L. Balleisen (12), A. Hinke (13), R. Hinke (13), J. Casper (10)

(1)Mediprojekt at Oncology Practice (Hannover, DE); (2)University (Rostock, DE); (3)University (Leipzig, DE); (4)University (Jena, DE); (5)University (Halle, DE); (6)JohanniterHospital (Stendahl, DE); (7)University (Magdeburg, DE); (8)University (Regensburg, DE); (9)Medical School (Hannover, DE); (10)Municipal Clinical Center (Oldenburg, DE); (11)Charité (Berlin, DE); (12)Municipal Hospital (Hamm, DE); (13)WiSP (Langenfeld, DE)

Objectives: Response to salvage therapy in relapsed aggressive non-Hodgkin lymphoma (NHL) depends on primary chemosensitivity and the duration of 1 st remission. While late relapse can be cured with high-dose chemotherapy and autologous stem cell support, pts with early relapse or refractory disease may need additional therapy with allogeneic transplantation. Further, a treosulfan-containing high-dose regimen may be an alternative to the widely used BEAM protocol.

Methods: Pts with aggressive NHL at 1st relapse or with refractory disease received two cycles (R)-DHAP followed by (R)-TEC (high-dose treosulfan, etoposide, and carboplatinum, + rituximab for pts with B-cell NHL). Subsequently, pts with late relapse at study entry (duration of 1 st CR less than 1 year) who achieved
CR after (R)-DHAP or (R)-TEC received no further treatment (A); pts with late relapse who achieved only PR after R-TEC received a 2 nd high-dose treatment with (R)-TEC (B); and pts with early relapse or refractory disease who achieved at least PR after (R)-TEC were scheduled for high-dose fludarabin and treosulfan followed by allogeneic stem cell transplantation (C). Results: Of 61 pts (median age $53 \mathrm{yrs}$ ), $87 \%$ had B-, 13\% T-cell lymphoma. $36 \%$ had refractory disease, $32 \%$ early and $32 \%$ late relapse. So far, 32 pts received (R)-TEC high dose therapy. Of these, 4 had double high-dose (R)-TEC, and 10 allogeneic transplantation. Grade 3 or 4 toxicity with (R)-TEC was infection in $39 \%$, mucositis in $61 \%$, and diarrhea in $39 \%$. Best response in 53 evaluable pts was $\mathrm{CR}$ in $30 \%$, near CR in $8 \%, \mathrm{PR}$ in $17 \%$, NC (no change) in 13\%, and PD in $32 \% .36$ pts (65\%) either progressed at any time or had NC after high dose therapy. Mean follow up time is 13.8 (range $0.5-56.8$ ) months. Median event free and overall survival (OS) was 6.6 and 12 months for all pts. With respect to individual risk, OS in pts with refractory disease, early or late relapse was $9.3,13.1$, and 16.7 months, resp. Among 10 pts who received allogeneic transplantation, OS is 10 months with 4 pts alive to date, 3 of which between 30 and almost 60 months. 28 pts $(52 \%)$ are alive, 5 pts are still under treatment.

Conclusions: 1, high-dose (R)-TEC after initial cytoreduction with (R)-DHAP is effective. 2, double high-dose therapy with (R)-TEC is feasible. 3 , in the setting of this study, a durable remission could be achieved with allogeneic transplantation in pts with early relapse or refractory disease.

\section{P834}

Prognostic value of positron emission tomography/computed tomography (PET/CT) in patients with lymphoma treated with autologous stem cell transplantation for relapsed/refractory or high-risk disease

P.M. Stefani (1), U. Salvadori (1), M. Gottardi (1), S. Bissoli (2), F. Chierichetti (2), F. Gherlinzoni (1)

(1)Haematology Unit (Treviso, IT); (2)Nuclear Medicine Department (Castelfranco Veneto, IT)

PET/CT imaging has increasingly been used for management of lymphoma. This modality combines the advantages of both PET and CT imaging especially improving specificity. Few prospective studies addressed its prognostic value in the setting of high dose therapy with autologous stem cell transplantation (ASCT) so far. We evaluated the predictive role of PET/CT before ASCT (PET/ CT2) and after ASCT (PET/CT3) in lymphoma patients in terms of overall survival (OS) and progression-free-survival (PFS). OS and PFS have been evaluated using Kaplan-Meier estimates; OS and PFS curves have been assessed using Log-rank test.We enrolled 17 patients with Hodgkin Lymphoma (9 in relapse, 8 refractory disease at early PET/CT in induction), 43 patients with non Hodgkin Lymphoma (15 in relapse, 15 in PR after first line treatment, 13 at high-risk while in CR). All patients presented a positive PET/CT (PET/CT1) before starting treatment. 60 patients underwent both $\mathrm{PET} / \mathrm{CT} 2$ and PET/CT3. After ASCT 5 patients never achieved a $\mathrm{CR}$ and all died because of progressive disease; 51 patients after ASCT were in CR (including 24 patients in CR before ASCT); 4 were in $\mathrm{PR}$ and they obtained $\mathrm{CR}$ after radiotherapy. No patients died of treatment-related-mortality. Of note 4 patients presented a PET/CT positivity after ASCT with a decreasing uptake at subsequent controls and without a clear correlation with any lesion detectable by CT: none of them relapsed. Median follow up is 27 months (5-84); Median OS and PFS are not reached. On the basis of PET/CT2 we did not observe statistically significant differences in both OS $(78.1 \%$ negative vs. $60.2 \%$ positive) and PFS (69.2\% negative vs. $60.4 \%$ positive). Conversely, on the basis of PET/CT3, OS was $88 \%$ negative vs. $53 \%$ positive ( $\mathrm{p} 0.003$ ), while PFS was $77.2 \%$ negative vs. $51.9 \%$ positive ( $p$ 0.006 ) (See Figure 1). All relapses have been documented in $\mathrm{PET} / \mathrm{CT} 3$ positive group within 16 months from ASCT (median 6 months). In PET/CT3 negative group 3 patients died, one of TRM after allogeneic stem cell transplantation, one of secondary leukaemia after radioimmunotherapy and the last because of 
progressive disease. 7 PET/CT3 positive patients died: 6 because of progressive disease and one of TRM after allogeneic stem cell transplantation. In this cohort, the role of PET/CT2 in predicting relapse after ASCT is less clear compared to previous published studies, while the role of PET/CT3 seems to be more reliable, especially for early relapses.
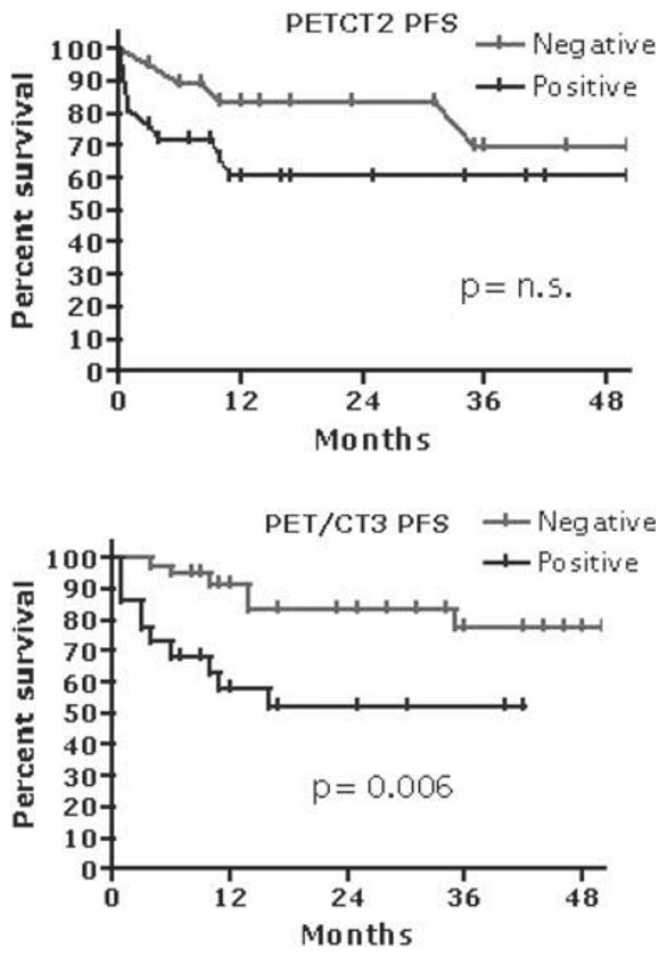

P835

Autologous haematopoietic stem cell transplantation for peripheral T-cell lymphomas - retrospective multicentre analysis of long-term outcomes and prognostic factors A. Czyz (1), L. Poplawska (2), J. Romejko-Jarosinska (2), D. Hawrylecka (3), W. Knopinska-Posluszny (4), P. Boguradzki (5), B. Nasilowska (6), D. Dytfeld (1), L. Gil (1), B. PiatkowskaJakubas (3), P. Mensah (3), J. Walewski (2), A. Skotnicki (3), A. Hellmann (4), M. Komarnicki (1)

(1)Poznan University of Medical Sciences (Poznan, PL); (2)Maria Sklodowska-Curie Memorial Cancer Centre (Warsaw, PL); (3)Faculty of Medicine Jagiellonian University (Krakow, PL); (4)Medical University of Gdansk (Gdansk, PL); (5)Medical University of Warsaw (Warsaw, PL); (6)Institute of Hematology and Transfusion Medicine (Warsaw, $P L$ )

Autologous hematopoietic stem cell transplantation (auto$\mathrm{HCT}$ ) is considered the treatment option for patients (pts) with peripheral T cell lymphomas (PTCL), but its efficacy remains unclear because of the low incidence of the disease and the heterogeneity of the subtypes. We retrospectively evaluated long-term outcome and prognostic factors affecting survival of 63 pts, median age 35 (15-64) years, with PTCL treated with autoHCT following BEAM (44 pts), CBV (8 pts), Cy/TBI (5 pts) and other regimen (6 pts). According to WHO 2001 classification histologic subtypes included PTCL not otherwise specified $(n=25)$, angioimmunoblastic T-cell lymphoma $(n=9)$, ALKnegative anaplastic large cell lymphoma $(A L C L)(n=9)$, ALKpositive ALCL $(n=12)$ and ALK-undetermined ALCL $(n=8)$. The indications for autoHCT were inadequate response to first line therapy (40 pts), consolidation of CR induced by first line therapy (8 pts) and relapse (15 pts). All pts with ALK-positive ALCL were transplanted due to inadequate response to first line therapy or relapse. The patients were treated with the median
2 (range 1-3) chemotherapy (chtx) lines before autoHCT. The disease status at transplant was CR1 (36 pts), CR2 (8 pts), PR1 (12 pts), PR2 (4 pts) and less then PR (3 pts). IPI at autoHCT was 0 in 53 pts, 1 in 6 pts, 2 in 1 pts, and not determined in 3 pts. With a median follow-up of $29(2-163)$ months OS and PFS was $74 \%(95 \% \mathrm{Cl} 61-85)$ and $64 \%(95 \% \mathrm{Cl} 49-79)$ at 5 yrs respectively estimated with the K-M method. The cumulative incidence of relapse and NRM was $34 \%(95 \% \mathrm{Cl} 22-52 \%)$ and $3 \%(95 \% \mathrm{Cl} 0,8-12 \%)$ respectively. In univariate analysis, prognostic factors associated with decreased OS were the disease status at transplant (less then CR vs. CR, P 0.029), IPI at transplant (1-2 vs. $0, P 0.013)$ and the number of prior chtx lines ( $>2$ vs. $1-2$ lines, $P 0.0018)$. PFS was adversely affected by IPI at transplant (1-2 vs. $0, P 0.013)$ and the disease status at transplant (less then $\mathrm{CR}$ vs. CR, $P$ 0.09). In multivariate analysis, IPI at autoHCT (RR 3.8, 95\% CI 1.1-13.2, P 0.03) and the number of prior chtx lines (HR 4.1,95\% Cl 1.2-13.9, P 0.02) remained statistically significant for OS and IPI at autoHCT (HR 4.4, 95\% Cl 1.3-14.6, P 0.01) for PFS.

We conclude that the significant proportion of patients with chemosensitive PTCL treated with autoHCT can achieve long lasting PFS. The outcome of pts is affected by IPI at autoHCT and the number of prior chtx lines.

P836

The prognostic significance of PET scan before and after high-dose therapy and autologous stem cell transplantation in relapsed/refractory Hodgkin's lymphoma patients

M.K. Angelopoulou, T.P. Vassilakopoulos, M. Moschogiannis, V. Pappi, P. Tsirkinidis, O. Tsopra, C. Kalpadakis, M. Dimou, D. Exarchos, K. Datseris, A. Gouliamos, M.-C. Kyrtsonis, P. Panayiotidis, G.A. Pangalis, P. Beris University of Athens (Athens, GR)

Objectives: To study the prognostic significance of PET scan before and after high-dose therapy and autologous stem cell transplantation (HDT/ASCT) in patients with relapsed/refractory Hodgkin's lymphoma (HL).

Methods: Computed tomography and PET scan were performed just prior to ASCT and at 3 months post transplant. Findings were correlated with failure free survival (FFS). PET scan was considered negative, when no uptake was present positive, when any lesion was FDG avid with SUV $\geq 4$ and minimal residual uptake positive (MRUp), when any lesion showed abnormal uptake with SUV $<4$

Results: Among 44 patients included in the study, 55\% were treated for primary refractory disease, $36 \%$ at first relapse and $10 \%$ beyond first relapse. All patients received salvage chemotherapy and $73 \%$ had chemosensitive disease prior to ASCT. A PET scan was available prior to ASCT in 34 patients, post ASCT in 39 and at both time points in 31 . Median follow-up was 14 months (4-64). Eight patients had a negative preASCT scan and one of them relapsed, whereas 13 relapses were observed among 26 patients with a positive or MRUp preASCT PET scan, resulting in a 1 -year FFS of $100 \%$ and $49 \%$ respectively. However this difference was not statistically significant. The figure of $49 \%$ reached a plateau at 8 months posttransplant. Pre-ASCT PET scan was positive or MRUp in 17/25 chemosensitive patients vs. $9 / 9$ chemoresistant ones. On the contrary, post-ASCT PET scan had a strong predictive value for outcome. One-year FFS was $96 \%$ for post ASCT PET negative or MRUp cases vs. $8 \%$ for positive ones $(P<0.0001)$. Among the 31 patients who had a PET scan available at both time points, there were no relapses recorded for those who were pre-ASCT PET scan either positive or MRUp and became post-ASCT PET scan negative (0/12 patients). On the contrary, $12 / 13$ patients with a pre-ASCT PET scan positive or MRUp, who remained or became post-ASCT PET scan positive, relapsed. There was one relapse among 8 patients who were pre-ASCT PET scan negative and remained negative after ASCT. There were no cases who converted from negative to positive. 
Conclusions: Pre-ASCT PET scan positivity does not preclude a favorable outcome for patients with relapsed/refractory HL undergoing HDT/ASCT, since half of them remain relapse free with a plateau at 8 months. Patients who remain or become PET positive after ASCT have an extremely poor prognosis in contrast to those who convert to negativity.

\section{P837}

High-dose therapy and autologous stem cell transplantation (HDT/ ASCT) in relapsed/refractory Hodgkin's lymphoma. Outcome and prognostic factors

M.K. Angelopoulou, T.P. Vassilakopoulos, P. Tsirkinidis, O. Tsopra, Z. Galani, V. Pappi, M. Moschogiannis, E. Dimitriadou, K. Anargyroy, C. Kalpadakis, S. Masouridis, D. Boutsis, S. Sachanas, M. Dimopoulou, E. Variamis, N.-A. Vyniou, S. Kokoris, M.P. Siakantaris, M.-C. Kyrtsonis, P. Panayiotidis, Y. Rombos, G.A. Pamgalis, P. Beris

University of Athens, Medical School (Athens, GR)

Objectives: High-dose therapy and autologous stem cell transplantation (HDT/ASCT) is the most widely accepted therapeutic option with curative intent for Hodgkin's lymphoma (HL) patients who are refractory to or progress after first line treatment. The aim of the present study was the analysis of prognostic factors and outcome for relapsed or refractory HL patients treated with HDT/ASCT in a single Hematology Unit.

Methods: We retrospectively analyzed 87 patients. Failure free survival (FFS) was calculated from time of ASCT to relapse / progression, death of any cause or last follow-up. Overall survival (OS) was calculated from time of ASCT to death of any cause or last follow-up.

Results: Median age at ASCT was 29 years (19-58) and 64\% were males. At diagnosis $13 \%, 55 \%, 15 \%$ and $17 \%$ of the patients had clinical stage I, II, III and IV respectively, $38 \%$ had B symptoms, $37 \%$ bulky disease. $98 \%$ received an anthracycline-based regimen and $2 \%$ MOPP, while $46 \%$ received RT. At relapse $21 \%, 38 \%, 5 \%$ and $36 \%$ of the patients had clinical stage I, II, III and IV respectively, $26 \%$ B symptoms and $10 \%$ bulky disease, while $41 \%$ of the patients were transplanted at first relapse, $16 \%$ after multiple relapses and $43 \%$ were primary refractory. Before ASCT, $54 \%, 33 \%$ and $13 \%$ of patients had received 2,3 and $>3$ prior regimens respectively. Following the last salvage regimen, $35 \%$ of the patients were in complete remission (CR), $44 \%$ in partial remission (PR) and $22 \%$ were chemoresistant. At a median follow-up of 36 months for surviving patients, the 3 - and 5 -year FFS were $44 \pm 6 \%$ and $38 \pm 6 \%$ respectively. The 3 - and 5 -year OS rates were $79 \pm 5 \%$. Prognostic factor analysis revealed chemoresistance prior to ASCT $(P=0.01)$, bulky disease at relapse $(P=0.007)$ and $\mathrm{B}$ symptoms at relapse $(P=0.05)$ as poor prognostic factors for FFS. Pretransplant remission status, after salvage (CR vs. $P R$ vs. no response) proved significant $(P=0.01)$, resulting in a $3 y$-FFS rate of only $11 \%$ for patients transplanted with progressive disease. Moreover, patients transplanted for multiple relapses had the best outcome compared to those in first relapse and primary refractory ones $(P=0.01)$. Age, chemosensitivity, bulky disease at relapse, number of previous regimens and pretransplant remission status were significant for OS.

Conclusion: HDT/ASCT can salvage approximately $45 \%$ of relapsed/refractory $\mathrm{HL}$ patients. Chemoresistant patients, especially those transplanted with progressive disease, as well as those with bulky disease at relapse, have an extremely poor outcome.
P838

LACE-conditioned autologous stem cell transplantation for relapsed or refractory diffuse large B-cell lymphoma: treatment outcome and risk factor analysis from a single centre

J. Pavlu (1), H. Auner (1), S. Ellis (1), R. Szydlo (1), C. Giles (1), D. McLornan (1), A. Contento (2), A. Rahemtulla (1), J. Apperley (1), K. Naresh (1), D. MacDonald (1), E. Kanfer (1)

(1)Hammersmith Hospital (London, UK); (2)Carlos Haya Hospital (Malaga, ES)

High-dose chemotherapy followed by autologous stem cell transplantation (ASCT) is a recognized treatment option for patients with relapsed diffuse large B-cell lymphoma. We have analysed 51 patients who underwent ASCT after LACE [lomustine (CCNU), cytarabine (Ara-C), cyclophosphamide, etoposide] conditioning for relapsed $(n=34,67 \%)$ or primary refractory $(n=17,33 \%)$ diffuse large B-cell lymphoma.

With a median follow-up of 60 months (range 2-216) the probabilities of overall survival and progression-free survival (PFS) at 5 years were $47 \%$ and $42 \%$, respectively. The treatment-related mortality was $10 \%(n=5)$. Probabilities for overall survival and PFS at 5 years for patients with chemosensitive disease were $56 \%$ and $50 \%$ versus $29 \%$ and $27 \%$, respectively, for patients with chemorefractory relapse $(P=0.015$ for survival, $P=0.007$ for PFS). In multivariate analysis abnormal pre-ASCT levels of C-reactive protein (>5 mg/l) were identified as a risk factor for worse oveall survival, whereas abnormal pre-ASCT levels of C-reactive protein and chemoresistance predicted inferior PFS. Probability of survival and PFS stratified by chemosensitivity is shown in Figure 1. Figure 2 shows stratification by pre-ASCT levels of CRP.

LACE followed by ASCT is an effective treatment for approximately half of patients with chemosensitive relapsed diffuse large B-cell lymphoma, and a proportion of chemorefractory patients also benefit.
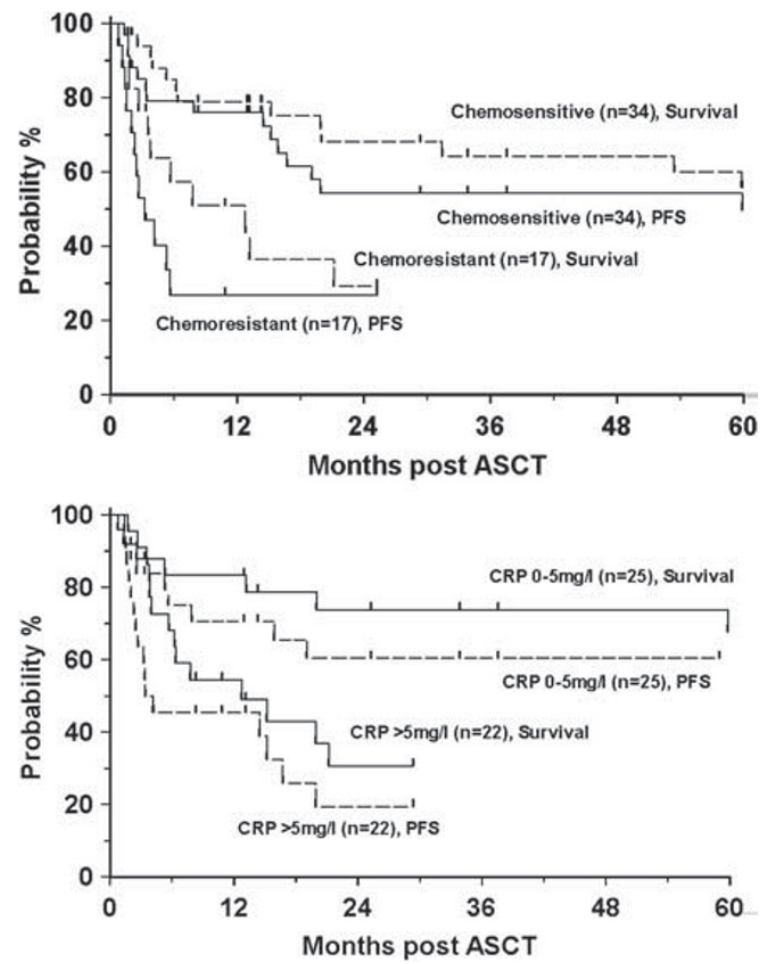


\section{P839}

Allogeneic stem cell transplantation in patients with non-Hodgkin lymphoma who experienced progression after high-dose chemotherapy with autologous stem cell transplantation

J.W. Kim, B.S. Kim, S.M. Bang, I. Kim, D.H. Kim, W.S. Kim, D.H. Yang, J.J. Lee, J.H. Lee, J.Y. Kwak, J.S. Kim, S.K. Sohn, S.S. Yoon, J.S. Lee, K.S. Han, M.H. Park, S. Park, B.K. Kim on belalf of the Korean Society of Blood and Marrow Transplantation

Background: There are few treatment options for patients with non-Hodgkin lymphoma (NHL) who experienced progression after high dose chemotherapy (HDC) with autologous stem cell transplantation (auto-SCT). The role of allogeneic stem cell transplantation (allo-SCT) in these patients has not been clarified yet.

Methods: Patients with NHL who received allo-SCT after disease progression despite prior HDC with auto-SCT from 7 hospitals were eligible for this study. Clinical data of enrolled patients were analyzed retrospectively.

Results: A total of 38 patients (male, 26) were included. Median age was 37 years (range, 16-53). Before allo-SCT, patients had received median 4 prior treatments (range, 2-7) including HDC with auto-SCT. During a median follow-up of 46.7 months (range, 5.3-104.6), 24 patients received allo-SCT from matched related donors $(63.2 \%)$ and 14 patients from matched unrelated donors (36.8\%). Median number of CD34 + cells infused was $5.41 \times 10^{6} / \mathrm{kg}$. Eighteen patients $(47.4 \%)$ underwent a myeloablative conditioning (MAC) and 20 patients $(52.6 \%)$, a reduced intensity conditioning (RIC). Treatment response was evaluable in 30 patients. Overall response rate was $73.3 \%$ : complete remission was achieved in 20 patients $(66.7 \%)$ and partial response in 2 patients $(6.7 \%)$. Median event-free survival (EFS) was 6.3 months (95\% confidence interval $(\mathrm{Cl}), 4.3-8.4$ ). Median overall survival (OS) was 19.0 months $(95 \% \mathrm{Cl}, 4.0$ 34.0 ). Estimated 5 -year survival rate was $33.3 \%$. There were no significant differences in EFS and OS between MAC group and RIC group. Median time to recovery of neutrophil ( $>500 /$ microliter) and platelet (>20,000/microliter) was 13.0 and 19.5 days, respectively. Acute graft-versus-host disease developed in 13 patients $(34.2 \%)$. There were 8 transplant-related deaths $(21.1 \%)$. Incidence of transplant-related mortality (TRM) in MAC group tended to be higher than that in RIC group $(27.8 \%$ vs. $15.0 \% ; P=0.341$ ). Patients with low baseline serum albumin level $(\leq 3.5 \mathrm{~g} / \mathrm{dL})$ had significantly higher TRM $(40.0 \%$ vs. $8.7 \%$; relative risk $7.00(95 \% \mathrm{Cl}, 1.18-41.54) ; P=0.032)$ as well as shorter EFS (2.5 vs. 25.1 months; hazard ratio (HR) 2.35 (95\% $\mathrm{Cl}, 1.04-5.31) ; P=0.039)$ and $\mathrm{OS}(8.9$ vs. 50.0 months; HR $2.73(95 \% \mathrm{Cl}, 1.13-6.58) ; P=0.025)$

Conclusions: Despite high TRM, allo-SCT is a viable option for patients with $\mathrm{NHL}$ who experienced progression after HDC with auto-SCT. Low baseline serum albumin level $(\leq 3.5 \mathrm{~g} / \mathrm{dL})$ was related to higher TRM and shorter EFS and OS.

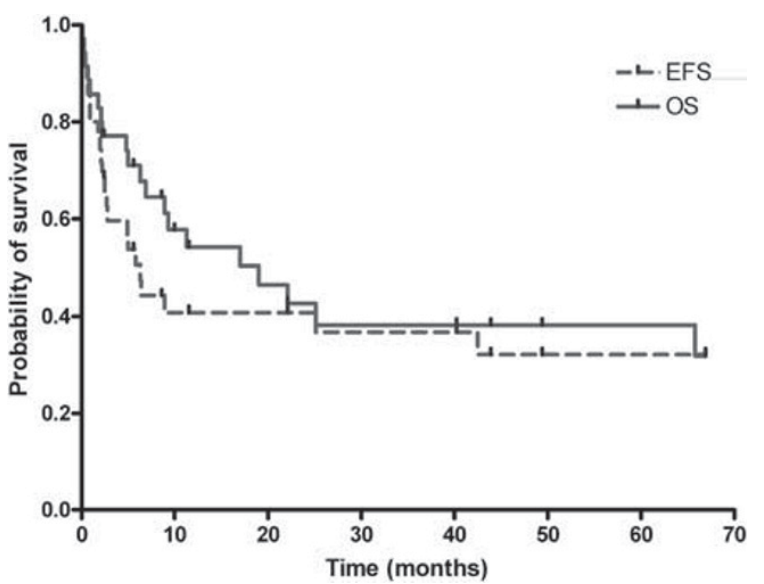

P840

BEAM or rituximab-BEAM for B-cell lymphoma receiving autologous transplantation. Does it matter?

C.T. Lin (1), X.W. Liao (1), C.C. Li (1), S.J. Wu (2), H.A. Hou (2), S.C. Hsu (2), W.C. Chou (2), S.Y. Huang (2), B.S. Ko (2), M. Yao (2), W. Tsay (2), H.F. Tien (2), J.L. Tang (2)

(1)National Taiwan University (Taipei, TW); (2)National Taiwan University Hospital (Taipei, TW)

Background: For relapse or refractory lymphoma, autologous transplantation (auto-SCT) with conventional conditioning regimen, BCNU, etoposide, cytarabine, and melphalan (BEAM), was a good option as salvage therapy. But we are unclear that rituximab is beneficial or not in conditioning regimen for B-cell lymphoma patients receiving autologous transplantation.

Method: Patients with B-NHL received auto-SCT with BEAM or Rituximab-BEAM as conditioning regimens between September 1998 and August 2008 were included in this analysis. Their basic characteristics, relapse free survival, overall survival and complications were retrospectively collected by chart review.

Results: 77 patients (42 male, 35 female) are included, and $57(74 \%)$ patients are diffuse large B-cell lymphoma, and the remaining are low grade B-cell lymphoma. $63(82 \%)$ patients are in advanced stage, and $58(75 \%)$ patients are chemo-sensitive with CR $(n=43,56 \%)$ or PR $(n=15,19 \%)$. The chemotherapy and conditioning regimen are listed in table 1 , and patients are classified as $\mathrm{A}_{i} \mathrm{BB} \mathrm{B}_{i} \mathrm{BC}$ groups.

For group $B$ and $C$, there is no difference in terms of 3-year cumulative incidence rate of relapse ( $32 \%$ versus $29 \%, P=0.592)$ and 3 -year overall survival $(73 \%$ versus $76 \%, P=0.854)$ (Figure 1$)$. For group $A$ versus $B$ plus $C$, there is a trend to have higher 5 -year cumulative relapse rate for group $A$ than group $B$ plus C $(61 \%$ versus $32 \%, P=0.12)$, but they have equivalent 5 -year overall survival $(67 \%$ versus $75 \%, P=0.643)$ and event free survival $(33 \%$ versus $47 \%, P=0.702)$. The major post-transplant complications are not significantly different, except a trend to have more bacteremia, sepsis and shock in R-BEAM group ( $43 \%$ versus $32 \%, P=0.054)$. There is no transplant related mortality in either group.

Conclusions: Our analysis cannot figure out the difference for BEAM and R-BEAM as conditioning regimen in terms of rate of relapse and overall survival. However, rituximab seems to borderline increase the infection events. From the point of view of risk and benefit, rituximab-BEAM as conditioning regimen for B-NHL should be justified.

\begin{tabular}{lll} 
Table 1. Chemotherapy and conditioning regimen & \\
\hline Prior to auto-SCT & BEAM & R-BEAM \\
& $n=40$ & $n=37$ \\
\hline Chemotherapy alone & $n=18$, Group A & $n=4$ \\
Rituximab-chemotherapy & $n=22$, Group $B$ & $n=33$, Group C \\
\hline
\end{tabular}
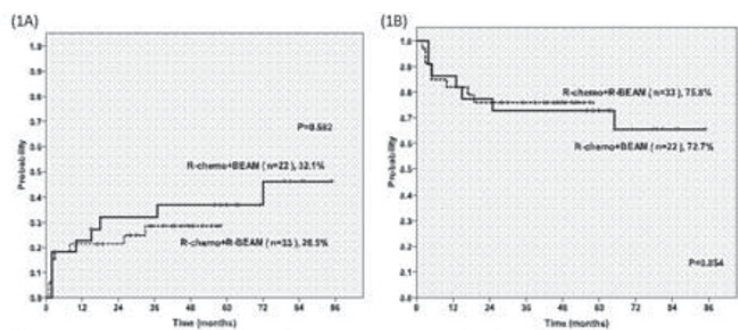

Fig 1. 3-year cumulative incidence rate of relapse (1A) and overall survival rate (1B) 


\section{P841}

Comparison of two different chemotherapy regimens for peripheral blood stem cell mobilization in lymphoma

S. Civriz Bozdag, P. Topcuoglu, S.M. Bakanay, M. Arat, E. Soydan, M. Ozcan, O. Ilhan, T. Demirer, M. Beksac, O. Arslan, N. Konuk, A. Uysal, G. Gurman

Ankara University School of Medicine (Ankara, TR)

Autologous peripheral blood stem cells (PBSC) provide rapid and sustained hematopoietic recovery after the administration of high-dose chemotherapy in patients with lymphoma. Therefore, standard mobilization regimen for PBSC is the combination of chemotherapy and granulocyte-colony stimulating factor (G-CSF). Mostly preferred chemotherapy regimen for mobilization is cyclophosphamide and/or etoposide (CyVP16) followed by G-CSF. In refractory and/or relapse lymphoma patients DHAP (Dexamethasone, high-dose cytarabine, and cisplatin) is usually used for the salvage regimens.

In this retrospective study, we aimed to investigate the effect of two different chemotherapy regimens, DHAP vs. CyVP16 on PBSC mobilization. Between 2000 and 2008, 44 relapse and/ refractory lymphoma patients (27 NHL and $17 \mathrm{HL}$ ) (F/M: 30/14) were evaluated. A target dose of $4 \times 10^{6} / \mathrm{kg}$ CD $34+$ cells $/ \mathrm{kg}$ was determined for the efficacy of PBSC mobilization.

Results: Stem cell mobilization and collection failure was found similar in both groups. Median time period between the first dose of G-CSF and first cycle of apheresis procedure was significantly shorter in DHAP group than CyVP16 $(P=0.002)$ The median number of apheresis for harvesting was $1(1-4)$ and 2 (1-3) respectively. We did not found any significant differences of the median numbers of apheresis cycles, number of CD34 + cells, peripheral blood mononuclear cells (MNCs) collected in both groups (table) Consequently, DHAP regimen reduce the cost with having same efficacy as compared with CyVP16 regimen. These results should be evaluated in a larger and more homogenous disease group by a prospective study design.

\begin{tabular}{|l|c|c|c|}
\hline & $\begin{array}{c}\text { CyVP16 } \\
(\mathrm{n}=22)\end{array}$ & $\begin{array}{c}\text { DHAP } \\
(\mathrm{n}=22)\end{array}$ & $\mathrm{p}$ \\
\hline Median age & $\begin{array}{c}41 \\
(15-55)\end{array}$ & $\begin{array}{c}33 \\
(18-68)\end{array}$ & 0.063 \\
\hline Gender (Male: Female) & 14.8 & 16.6 & 0.517 \\
\hline $\begin{array}{l}\text { Diagnosis } \\
\text { Non-Hodgkin Lymphoma } \\
\text { Hodgkin Disease }\end{array}$ & 10 & 17 & 0.06 \\
\hline SC mobilization failure & 12 & 5 & \\
\hline $\begin{array}{l}\text { Number of doses of G-CSF, days } \\
\text { (median, range) }\end{array}$ & $6 / 22(27 \%)$ & $5 / 22(22 \%)$ & 0.728 \\
\hline Number of apheresis & $9(4-14)$ & $6(5-13)$ & 0.002 \\
\hline $\begin{array}{l}\text { Patients with } 24 \times 10 \mathrm{e} 6 \mathrm{~kg} \text { collected after } \\
\text { one apheresis }\end{array}$ & $8 / 22$ & $11 / 22$ & 0.361 \\
\hline Total mononucleated cellx 10e8/kg & $3.4(0.9-12)$ & $3.2(0.9-21)$ & 0.969 \\
\hline Total CD $34+$ cellsx10e6/kg & $7.1(0.1-23)$ & $6.7(2.4-23)$ & 0.673 \\
\hline
\end{tabular}

\section{P842}

Autologous blood stem cell transplantation for peripheral T-cell lymphoma: a review of $\mathbf{4 4}$ cases

H.G. Yi (1), H.S. Lee (2), D.H. Yang (3), J.S. Kim (4), J.J. Lee (3), S.S. Yoon (5), Y.H. Min (4), S.H. Yang (6), C.S. Kim (1), S. Park (5)

(1)Inha University Hospital (Incheon, KR); (2)Koshin University Hospital (Busan, KR); (3)Chonnam National University Hospital (Kwangju, KR); (4)Yonsei University Severance Hospital (Seoul, KR); (5)Seoul National University Hospital (Seoul, KR); (6)Korea Cancer Center Hospital (Seoul, KR)

For a 10 year period of 1999-2008, a total of 44 patients underwent HDC (high dose chemotherapy) followed by ABSCT (autologous blood stem cell transplantation) for the treatment of PTCL (peripheral T cell lymphoma) in 6 institutions in South Korea. The patients consisted of 28 men and 16 women aged 14-63 (median 36.5), diagnosed as PTCL-u $(n=28)$, ALK negative ATCL $(n=8)$, AITL $(n=4)$, SCPTCL $(n=1)$ and HSTCL $(n=1)$, and initially presented in stage III or greater in the majority $(n=37)$. Remission induction was attempted with CHOP $(n=24)$, CHOEP $(n=5$, $E=$ etoposide), IMEP $(n=5, I=$ ifosphamide, $M=$ methotrexate, $P=$ cisplatin) and others $(n=10)$ resulting in PR in $12(28 \%)$ and CR1 in $32(72 \%)$ among whom $16(50 \%)$ relapsed. Re-induction was attempted for recurrent disease with IMEP $(n=4), \operatorname{ICE}(n=3$, $C=$ carboplatin), and others $(n=9)$ resulting in CR2 in $9(56 \%)$. ABSCT was given aimed at consolidation of CR1 in 16 (group 1 ) as well as salvage for persistent disease in 12 (group 2) or disease in relapse in 16 (group 3). Conditioning for ABSCT consisted in BEAM $(n=15), \operatorname{BuCyE}(n=9), \operatorname{CBV}(n=7), \operatorname{NEAM}(n=7$, $\mathrm{N}=$ mitoxantrone), and others $(\mathrm{n}=6)$. There was no significant difference in age, sex, stage on initial diagnosis, and HDC regimen among 3 groups. Engraftment was documented in 43 patients $(98 \%)$ at a median day of 11 (range 6-80). Although there was no meaningful difference among median days of engraftment, it took longer than 4 weeks for engraftment in $1(8 \%)$ of group 2 , and $3(19 \%)$ of group $3(P<0.05)$. Ten of group $1(63 \%)$ are alive disease free at a median FU (follow-up) of 21.5 months ranging $1-120$, as are 5 of group $2(42 \%)$ at a median FU of 5.5 months ranging $2-56$, and 7 of group $3(43 \%)$ at a median FU of 6.5 months ranging $1-128(P<0.01)$. Taken together, $22(50 \%)$ are alive disease free at a median FU of 16 months. Causes of death included relapse/progression in $15(34 \%, \mathrm{n}=4$ in group 1, 5 in group 2, 6 in group $3, P>0.3)$ and TRM in $7(16 \%, \mathrm{n}=2$ in group 1,2 in group 2, 3 in group 3, $P>0.3$ ). Although it appeared to be better to perform HDC and ABSCT as a consolidation therapy for remission of PTCL rather than a salvage treatment for recurrence, identification of particular factors in CR1 favoring ABSCT was difficult on this limited retrospective analysis.

\section{P843}

\section{Allogeneic stem cell transplantation with reduced-} intensity conditioning in patients with relapsed lymphoma M. Rivas (1), M. Berro (1), S. Belaustegui (1), V. Prates (2), S. Yantorno (2), J. Milone (2), G. Remaggi (3), J. Martinez Rolon (3), G. Milone (3), A. Basquiera (4), J. García (4), G. Sturich (4), G. Kusminsky (1)

(1)Hospital Universitario Austral (Pilar Buenos Aires, AR); (2)Hospital Italiano (La Plata Buenos Aires, AR); (3)Fundaleu (Buenos Aires, AR); (4)Hospital Privado (Cordoba, AR)

Background: The role of reduce-intensity conditioning (RIC) allogeneic stem cell transplantation (allo STC) in patients with relapsed lymphoma remains poorly defined. We performed a retrospective multicenter analysis in 32 patients with relapsed lymphoma receiving a RIC allo SCT.

Design and methods: The median follow up was 2.1 years. Twenty eight $(87 \%)$ patients received SCT from related donor whereas the remaining $4(12 \%)$ were unrelated. The malefemale ratio was $1,6: 1$. Twelve $(37 \%)$ were older than 35 years. Twenty patients (62\%) were Hodgkin lymphoma and $12(37 \%)$ non Hogdkin lymphoma. The majority of patients $(87 \%)$ have recieved a previous autologous transplant. Disease status at time of transplant was complete remission en $11(34 \%)$ patients, partial remission in $17(53 \%)$, stable or progressed disease in $4(12 \%)$. Twenty one $(65 \%)$ patients received fludarabine-melphalan (flu-mel) as conditioning regimen.

Results: The predicted transplant related mortality (TRM) was of $25 \%$. Overall survival (OS) and disease free survival (DFS) were $59 \%$ and $37 \%$ respectively with a relapsed rate (RR) of $37 \%$. Thirty four percent of patients had acute graft vs. host disease (aGvHD)

Patients who received flu-mel regimen had more TRM than other regimens ( $38 \%$ vs. $0 \% P=0.03$ ), not significant after multivariate analysis. There were no differences in RR and OS. Patients with Hodgkin lymphoma had reduced DFS (22\% vs. $66 \%, P=0.01$ ), persisting significant in multivariate analysis (HR $0.66, \mathrm{Cl} 95 \% 0.006-0.711, P=0.02$ ), probably due to a 
higher RR ( $57 \%$ vs. $8 \%, P=0.02)$. We found no significant difference in the OS.

Conclusions: The RIC allo SCT is an adequate therapeutic option for patients with relapsed lymphoma. Flu-mel regimen seems to be more immunosuppressive than other regimens. Patients with Hodgkin lymphoma seem to have less graft vs. lymphoma effect.

\section{P844}

Successful peripheral blood stem cell harvest in mobilization-refractory lymphoma patients

E. Segel, B. Sørensen, F. d'Amore, P. Hokland

Aarhus University Hospital (Aarhus, DK)

Background: Plerixafor inhibits the binding of CXCR-4 to SDF-1 and is approved for mobilization of peripheral blood haematopoietic stem cells (PBHSC) prior to autologous stem cell transplantation (ASCT) for malignant lymphoma.

Aim: The purpose of our analysis was to evaluate the mobilization efficacy of plerixafor in mobilization-refractory lymphoma patients, the majority of which (7 out of 10 ) were also heavily pre-treated.

Methods: A series of 10 lymphoma patients ( 3 Hodgkin, 2 mantle cell, 2 follicular, 1 transformed follicular, 1 'de novo' diffuse large B-cell and 1 peripheral T-cell) were analyzed. All had failed prior mobilization according to standard guidelines (priming chemotherapy regimen + G-CSF in 9 and G-CSF alone in 1) given as part of either 1 st line therapy (3 pts) or relapse treatment (7 pts). The number of CD34 + PBHSC was assessed by flow-cytometry. A biopsy-based bone-marrow status was available for all patients. Plerixafor was administered daily as $0.24 \mathrm{mg} / \mathrm{kg} /$ day s.c. along with G-CSF $12 \mu \mathrm{g} / \mathrm{kg} /$ day s.c.

Results: In 7 out of 10 patients, a sufficient $\left(\geq 2.0 \times 10^{6} \mathrm{CD} 34+1\right.$ $\mathrm{kg}$ ) PBHSC harvest was achieved. In 2 of the remaining 3 patients, harvest after plerixafor yielded 1.8 and $1.6 \times 10^{6} \mathrm{CD} 34+$ stem cells $/ \mathrm{kg}$, respectively. The latter patient had a previously harvested CD34 + stem cell fraction of $0.9 \times 10^{6} / \mathrm{kg}$, which made it possible to perform a successful ASCT. In one patient, persisting low CD34 counts did not allow PBHSC collection. This patient had a relapsed follicular lymphoma, not previously treated with fludarabine, but with ofatumomab $+\mathrm{CHOP}$, radioimmunotherapy (ibritumomab tiuxetan) and bendamustine. The table summarizes clinico-pathological features and stem cell harvest parameters of the 10 analyzed patients.

- Column 1: diagnosis

- Column 2: Neutrophil count

- Column 3: CD34 count in peripheral blood

- Column 4: CD34 + cells/kg

- All values are from days preceeded by plerixafor treatment (at 10 p.m. previous day)

Conclusion: Plerixafor+G-CSF can safely and effectively remobilize patients with stem cell harvest-failing malignant lymphoma, even if heavily pretreated.

\begin{tabular}{|c|c|c|}
\hline NHL (*) & 13,9 & 25 \\
\hline & 19,7 & \begin{tabular}{l|l}
3 & 0,6 \\
\end{tabular} \\
\hline & 22,9 & \begin{tabular}{l|l}
5 & 1,0
\end{tabular} \\
\hline \multirow[t]{3}{*}{$\mathrm{NHL}$} & 0,22 & 과 \\
\hline & 4,19 & 71,1 \\
\hline & 12,1 & 123,1 \\
\hline \multirow[t]{3}{*}{$\mathrm{HL}$} & 0,88 & 1. \\
\hline & 5,70 & \begin{tabular}{l|l}
11 & 1,1 \\
\end{tabular} \\
\hline & 24,5 & 173,3 \\
\hline \multirow[t]{3}{*}{$\mathrm{NHL}$} & 0,06 & 1) \\
\hline & 0,31 & 25- \\
\hline & 6,11 & 174,8 \\
\hline \multirow[t]{3}{*}{$\mathrm{HL}$} & 0,03 & 1 \\
\hline & 1,23 & \begin{tabular}{l|l}
8 & 1,1 \\
\end{tabular} \\
\hline & 7,42 & \begin{tabular}{l|l|}
29 & 2,9 \\
\end{tabular} \\
\hline \multirow[t]{2}{*}{$\mathrm{NHL}$} & 7,27 & 5 \\
\hline & 10,5 & \begin{tabular}{l|l}
22 & 3,3 \\
\end{tabular} \\
\hline \multirow[t]{3}{*}{$\mathrm{NHL}\left({ }^{\circ}\right)$} & 4,76 & 1 \\
\hline & 18,0 & \begin{tabular}{l|l|}
8 & 0,9 \\
\end{tabular} \\
\hline & 43,6 & \begin{tabular}{l|l}
8,6 \\
\end{tabular} \\
\hline \multirow[t]{3}{*}{$\mathrm{NHL}$} & 1,14 & 25- \\
\hline & 10,9 & 8 \\
\hline & 20,9 & 95. \\
\hline \multirow[t]{4}{*}{$\mathrm{HL}$} & 1,07 & 1. \\
\hline & 2,00 & 4 \\
\hline & 4,96 & \begin{tabular}{l|l}
10 & 1,1 \\
\end{tabular} \\
\hline & 9,08 & \begin{tabular}{l|l}
16,3 \\
\end{tabular} \\
\hline \multirow[t]{2}{*}{$\mathrm{NHL}$} & 20,6 & 8 \\
\hline & 24,6 & \begin{tabular}{l|l}
42 & 2,6 \\
\end{tabular} \\
\hline
\end{tabular}

$(*)=$ Additional st em cell product available

P845

The role of high-dose chemotherapy/autologous stem cell transplantation for salvage treatment for relapsed peripheral T-cell lymphoma

Y.J. Cho, M.J. Kang, D.R. Choi, E.K. Kim, S. Kim, D.H. Lee, S.W. Kim, J.R. Huh, J.S. Lee, C.W. Suh

Asan Medical Center (Seoul, KR)

Introduction: Although the role of high dose chemotherapy (HDCT) and autologous stem cell transplantation (ASCT) in the treatment of aggressive lymphoma has been established, optimal second line treatment, including high-dose therapy with autologous stem cell support, is not well established in patients with peripheral T cell lymphoma (PTCL). The purpose of this retrospective study was to compare the outcome of salvage chemotherapy only with that of HDCT and ASCT in patients with relapsed disease after having reaching a complete response (CR).

Methods: We retrospectively investigated the outcome of salvage treatments for patients with relapsed PTCL who had reached a $C R$.

Results: Between January 1999 and February 2009, 39 patients (15 peripheral T cell lymphoma; 14 extranodal natural killer/T cell lymphoma; 5 anaplastic large cell lymphoma; and 6 angioimmunoblastic T-cell lymphoma) were identified to be eligible for this analysis in our institution. Among them, 25 were treated with only salvage chemotherapy and 14 were treated with HDCT/ASCT. The salvage chemotherapy regimens were ESHAP given in $52 \%$ and DHAP given in $12 \%$. Also the conditioning chemotherapy regimens for autologous stem cell transplantation (ASCT) were ESHAP given in $50 \%$ and DHAP given in $14.2 \%$. The median number of chemotherapy regimen was 3 . The overall response rate of salvage chemotherapy was $64 \%$ with a CR rate of $48 \%$ but that of ASCT/ 
HDCT was $92 \%$ with a CR rate of $50 \%$ relatively $(P=0.023)$. With median follow-up time of 20.7 months, there were no statistical differences between the two groups in terms of relapse-free survival (relapse-free survival: ASCT/HDCT $=8.7$ months, Salvage chemotherapy $=8.4$ months, $P=0.794)$ and overall survival $(P=0.390)$. Conclusion: There were no significant differences between the two groups in terms of survival outcome. Therefore, considering the dismal outcome in relapsed PTCL patients even after treated with HDCT/ASCT, incorporation of novel therapeutics into a treatment regimen should be considered.

\section{P846}

The intensification of the conditioning with radio-conjugated antibody improves the outcome in auto-transplant patients with non-Hodgkin lymphoma L. Pezzullo, O. Finizio, U. Sessa, L. Mettivier, L. Bene, G. Nunziata, S. Rocco, V. Mettivier

A. Cardarelli Hospital (Naples, IT)

The Autologous transplantation is an effective therapy in the treatment of non-Hodgkin lymphoma (NHL). Over the last decade the advent of monoclonal antibodies (anti-CD20 conjugated or not) has triggered the possibility of changing the transplantation procedure, or conducting an in purging vivo or intensifying the conditioning with radio-conjugated. From May 2005, at our division, have been auto transplanted 24 patients with CD20+NHL (17 have done a purging in vivo (BEAM-R) and 9 have intensified the conditioning with the addition of radio-conjugated at day -14 to transplantation (Z-BEAM). The aim of our study is to assess whether there are differences in terms of DFS, OS and EFS between these 2 groups of patients. The characteristics of patients in the 2 groups were: $12 \mathrm{M}$ and $5 \mathrm{~F}$ median age of 57 years (range 17-65) in the BEAM-R and $6 \mathrm{M}$ and $3 \mathrm{~F}$ median age of 50 years (range 40-69) in Z-BEAM. In the BEAM-R group 10 patients had high grade lymphomas, 4 mantle cells and 3 follicular, the status at transplant has been: CR: 8; PR: 7 and NR: 2. In Z-BEAM group, 4 patients had high grade lymphomas, 4 follicular, and 1 mantle cells, the status disease at the transplant were: $C R$ in 4 patients; PR: 4 and NR: 1 . The median of CD34 cells infused was $4 \times 10^{6} /$ $\mathrm{Kg}$ in both groups. The Haematological recovery assessed with $\mathrm{N}>1000 / \mathrm{mm}^{3}$ and PLT $>20,000 / \mathrm{mm}^{3}$ were 11 and 12 days respectively in groups BEAM-R and Z-BEAM. The TRM assessed in the 2 groups has been $2 / 17(11 \%)$ in the BEAM-R and $2 / 9(22 \%)$ of Z-BEAM group, this high incidence is due to patients highly treated or in active disease. With a median follow-up of 50 months after autotransplant, $9 / 15(60 \%)$ were in CR in BEAM-R group whereas in Z-BEAM group the median PFS were of 12 months. The EFS median are 26 and 8 months respectively for the BEAM-R and ZBEAM group. This difference is not statistically significant ( $p: 0.07$, Cox F-test). In conclusion, even if the number of the patients is small, the difference in terms of DFS, OS and EFS doesn't seem significant among the two regimes of conditioning. Is necessary a randomized study to confirm these preliminary data.

\section{P847}

Hepatosplenic T-cell and non-hepatosplenic gamma/delta T-cell lymphomas: role of haematopoietic stem cell transplantation: Hungarian experience of 8 cases

L. Gopcsa (1), A. Barta (1), A. Batai (1), Z. Borbenyi (2), J. Csomor (3), Z. Csukly (1), B. Deak (4), Z. Farkas (1), S. Fekete (1), L. Krenacs (5), L. Lengyel (1), A. Matolcsy (3), P. Remenyi (1), A. Rosta (4), J. Sinko (1), A. Sipos (1), A. Szoke (2), E. Torbagyi (1), T. Masszi (1)

(1)St. Istvan and St. Laszlo Hospital (Budapest, HU); (2)University of Szeged (Szeged, HU); (3)Semmelweis University (Budapest, HU); (4)National Institute of Oncology (Budapest, HU); (5)Institute of Biotechnology, Bay Zoltan Fou (Szeged, HU)

Objectives: Hepatosplenic T-cell lymphoma (HTSTCL) is a rare aggressive type of extranodal lymphomas characterized by hepatosplenomegaly, bone marrow involvement, and peripheral blood cytopenias. Non-hepatosplenic gamma/delta (g/d) T-cell lymphomas display marked heterogeneity in clinical and histologic features. Therapeutic strategies efficacious for aggressive B-cell lymphomas usually fail in the caseses of HTSTCL and other $\mathrm{g} / \mathrm{d}$ T-cell lymphomas. The prognosis of these diseases are very poor.

Methods: 8 patients with HTSTCL and other g/d T-cell lymphomas were studied. Clinical histories and possibilities of haematopoietic stem cell transplantation (HSCT) were reviewed.

Results: Our study consisted of 2 men and 6 women with a median age of 39 years (ranging from 29 to 62 years). Histologies consisted of 6 HTSTCL $(\mathrm{g} / \mathrm{d}+5, \mathrm{a} / \mathrm{b}+1), 1$ cutaneous $\mathrm{g} / \mathrm{d}$ Tcell lymphoma and 1 mucosal g/d T-cell lymphoma. All patients presented with splenomegaly and cytopenia. 6/8 patients had initial bone marrow involvement by lymphoma cells. Treatment plan was to performe autologous- and/or allogeneic-HSCT. Despite a broad spectrum of chemotherapeutic approaches including anthracycline based combination chemotherapy (CHOP or CHOP-like), salvage therapy (DHAP, IVE), interferon-a, cladribine, pentostatin and alemtuzumab therapy, only 2 patients responded. 7/8 patients died within 2-24 months, before completing induction or salvage treatment because of disease progression. Finally, only 1 patient with HTSTCL a/ $b+$ phenotype reached the transplant. This $30 y$ old male was received an autograft with BEAM conditioning because of resistant disease, and achieved partial remission. 16 months later, he underwent an allogeneic HSCT with in vitro T-cell depleted graft from an $1 \mathrm{Cw}$ antigen mismatched unrelated donor. Conditioning consisted of Flu-Bu-ATG, GVHD prophylaxis contained tacrolimus + MMF. The patient achieved very-good partial remission. 3 months following the transplant the patient has the best response ever without any sign of GVHD.

Conclusion: Promising results of allogeneic HSCT for HTSTCL patient have recently been reported. Allogeneic HSCT in a short period of remission after chemotherapy or even autologous transplant may be a choice of treatment. Allogeneic HSCT may offer some advantage by providing GVT effect. Allogeneic HSCT, is expected to become potentially a curative treatment option, and GVT effect might play a critical role for sustained remission.

\section{P848}

Zevalin-BEAM conditioning treatment followed by stem cell rescue in follicular lymphoma patient: single-centre experiences

A. Kiss (1), G. Reményi (1), P. Batár (1), R. Szász (1), L. Rejto (1), G. Radványi (2), Z. Fodor (3), L. Galuska (3), M. Udvardy (1) (1)Transplantation Section (Debrecen, HU); (2)Semmelweis Hospital (Miskolc, HU); (3)Nuclear Medicine (Debrecen, HU)

Objectives: The yttrium-90-labelled ibritumomab tiuxetan (Zevalin) is an accepted therapy for relapsed, or therapy-refractaer B-cell non-Hodgkin lymphomas. In the first reports there is a new element: the inclusion of Zevalin in conditioning regimen given prior to autologous peripheral stem cell therapy (APSCT).

Methods: The authors discuss their experiences of 21 follicular lymphoma patients with relapsed or refractory disease treated by Zevalin + BEAM radio-immune-chemotherapy with APSCT.

Results: Six years ago (in September 2003), the fifth Haemopoietic Stem Cell Transplantation Centre of Hungary began its activity. This centre registered as the No648 sub-centre of the European Blood and Marrow Transplantation Committee. Up to November 2009. 214 autologous stem cell transplantations were performed including 94 patients (pts.) with multiple myeloma, 72 with non-Hodgkin lymphoma, 39 with Hodgkin's disease, one pt. with CLL, one pt. with leiomyosarcoma, seven pts. with autoimmune diseases. This centre performed the most use of conditioning protocol Zevalin, bischloroethylnitrosourea, etoposide, cytosine arabinoside, and melphalan (Z-BEAM) in pts. with non-Hodgkin lymphoma in Hungary. Since February 2006 to November 2009, 21 pts. were involved: 10 women, and 
11 men, mean age 49,5 $\pm 11,1$ years, $48,4 \pm 9,7$ months from diagnosis. In cases of 4 pts. the histology was follicular lymphoma grade 3 , grade 2 , in cases of 6 pts., and grade 1 in cases of $11 \mathrm{pts}$. All the $21 \mathrm{pts}$. engrafted the neutrophil granulocytes in a mean $10,9 \pm 9,4$, and the platelet engrafted $13,9 \pm 10,2$ days after the APSCT. $71,4 \%$ of the pts. are in a complete remission $18,6 \pm 14,9$ months after the transplantation. Ten pts. participate in a maintenance Mabthera therapy. One patient from the 21 has an R-CHOP (rituximab, cyclophosphamide, hydroxydaunorubicine, vincristine, prednisolon) and one patient an R-DHAP therapy (rituximab, dexamethasone, high dose cytosine arabinoside, platidium) in the post transplantations relapse. Three pts. died after the APSCT one, two and 13 months.

Conclusions: According to our and others experiences Z-BEAM conditioning is a safe and effective radio-immune-chemotherapy before autologous stem cell transplantation.

\section{P849}

The effect of involved field radiotherapy on survival in patients with recurrent or refractory non-Hodgkin's lymphoma after autologous stem cell transplantation C. Eroglu, A. Ünal, L. Kaynar, O. Yildiz, F. Kurnaz, O. Orhan, C. Sahin, D. Aslan, S. Soyuer, S. Sivgin, B. Eser, M. Çetin Erciyes University (Kayseri, TR)

Purpose: To evaluate the effects of involved field Radiotherapy (RT) on overall survival after autologous stem cell transplantation (ASCT), in patients with recurrent or refractory Non-Hodgkin Lymphoma (NHL).

Materials and methods: Between 1989-2009 forty-four NonHodgkin Lymphoma patients, treated with high dose chemotherapy with ASCT followed by involved field radiotherapy were evaluated. Patients were divided into four groups that earlystage (I-II) and advanced- stage (III-IV), irradiated and unirradiated. The groups were compared in terms of overall survival and survival after ASCT.

Results: The median age was 44 (17-76). Eleven patients were female $(25 \%)$ and 33 were male $(75 \%) .41$ patients $(93.2 \%)$ were B-cell Non- Hodgkins Lymphoma, [31 (83\%) B-cell NHL were diffuse B-cell NHL] Eight of early-stage patients (18.2\%) received RT, 8 did not (18.2\%). Ten advanced-stage patients $(22.7 \%)$ received RT and 18 did not $(40.9 \%)$. Six patients $(20.45 \%)$ underwent ASCT after first-line CT, 22 after secondline CT (50\%) and 13 after third-line CT (29.55\%). 28 patients $(63.6 \%)$ achieved complete response, 16 of them $(36.4 \%)$ were stabilized after CT. 19 patients (43.2\%) achieved complete response, 8 of them (18.2\%) were stabilized, 11 of them $(25 \%)$ had progressive disease and 11 patients (13.6\%) weren't evaluated. Median follow-up was 30 months (2-237). For the unirradiated group of early-stage patients, median overall survival and survival after ASCT were 32 months versus 6 months, respectively. For the irradiated group of early-stage patients, median overall survival and survival after ASCT were 29 months versus 6 months, respectively. There wasn't a significant difference between irradiated and unirradiated patients. For the unirradiated group of early-stage patients, 5 and 10 years survival were $41 \%$ versus $0 \%$, respectively. For the irradiated group of early-stage patients, 5 and 10 years survival were $28 \%$ versus $28 \%$, respectively. For the unirradiated group of advancedstage patients median overall survival and survival after ASCT were 62 months versus 28 months, respectively. For the irradiated group of advanced-stage patients median overall survival and survival after ASCT were 52 months versus 9 months, respectively. There wasn't a significant difference between two groups.

Conclusion: We couldn't find any effect of involved field RT after ASCT on overall survival in patients with recurrent or refractory Non-Hodgkin Lymphoma.
P850

The effect of involved field radiotherapy on survival in patients with Hodgkin's lymphoma after autologous stem cell transplantation

A. Ünal, C. Eroglu, L. Kaynar, O. Yild\&iz, O. Orhan, C. Sahin, F. Kurnaz, D. Aslan, S. Soyuer, S. Sivgin, B. Eser, M. Çetin Erciyes University (Kayseri, TR)

Purpose: To evaluate the effects of involved field radiotherapy (RT) on overall survival after autologous stem cell transplantation (ASCT) in patients with recurrent or refractory Hodgkin Lymphoma (HL).

Materials and methods: Between 1995-2006, forty-five HL patients, treated with High dose chemotherapy followed by ASCT at Erciyes University Medical Faculty were evaluated. Patients were divided into four groups that early-stage (I-II) and advanced-stage (III-IV) irradiated and unirradiated. The groups were compared in terms of overall survival and survival after ASCT.

Results: Median age was 29 (16-60). Seventeen patients were female $(38 \%)$ and 28 were male $(62 \%)$. Sixteen earlystage patients $(35.5 \%)$ received RT, $11(24.5 \%)$ didn't. Four advanced-stage patients $(8.9 \%)$ received RT and $14(31.1 \%)$ didn't. Six patients $(13.3 \%)$ underwent ASCT after first-line chemotherapy (CT), $23(51.2 \%)$ after second-line CT and $16(35.5 \%)$ after third-line CT. Twenty two patients (48\%) achieved complete response, $22(48.9 \%)$ were stable disease and one $(2.2 \%)$ progressed after CT. Median followup was 40 months (12-172). For the unirradiated group of early-stage patients, median overall survival and survival after ASCT was 33 months versus 9 months, respectively. For the irradiated group of early-stage patients, median overall survival and survival after ASCT were 72 months versus 24 months, respectively. For the unirradiated group of early-stage patients, 5 and 10 years survival were $47 \%$ versus $0 \%$, respectively. There was a significant difference between irradiated and unirradiated groups in patients with early stage HL.

For the irradiated group of early-stage patients, 5 and 10 years survival were $69 \%$ versus $20 \%$, respectively. For the unirradiated and irradiated group of advanced-stage patients median survival was 38 months versus 43 months. There wasn't a significant difference between irradiated and unirradiated groups with advanced stage $\mathrm{HL}$.

Conclusion: It was shown the contribution of involved field RT to the overall survival and survival after ASCT in early stage $\mathrm{HL}$ patients.

\section{P851}

Only immunotherapy treatment in auto-transplanted relapsed follicular lymphoma

L. Pezzullo, O. Finizio, U. Sessa, L. Mettivier, V. Mettivier A.Cardarelli Hospital (Naples, IT)

In the last years the follicular lymphoma has benefited of transplantation procedures. The problem is the management of the relapse of the disease in post-transplant, were as patients highly treated. From January 2005 we have auto-transplanted, in our Division, 10 follicular lymphomas. 6/10 patients have relapsed by a median PFS of 18 months (range 9-36).All patients received strict follow-up with CT and PET and were treated early. The 6 patients relapsed were treated with 4 weekly doses of rituximab $375 \mathrm{mg} / \mathrm{mq}$ for 1 month and then re-evaluated, if CR have started maintenance with rituximab $375 \mathrm{mg} / \mathrm{mq}$ every 2 months for 2 years. $5 / 6$ patients (83\%) revalued after 4 weekly doses have documented the $\mathrm{CR}$ and began rituximab maintenance. With a median follow-up of 24 months all patients are in CR and were not documented complications related to treatment. In conclusion, for patients with follicular lymphoma, a strict follow-up may to consent a rapid treatment in relapsed patients after the autologous transplantation and the only immunotherapy may be sufficient to obtain a new CR consolidated by 
maintenance cycles every 2 months. We need a larger cohort and a follow-up longer to confirm these data.

\section{P852}

5 -year experiance in a Sofia BMT Unit (CIC 859) with

haematopoietic stem cell transplantation

G. Mihaylov, P. Ganeva, N. Vasileva, E. Bekrieva, L. Garcheva, I. Tonev, M. Guenova

Specialised Hospital for treatment of Hematological Diseases (Sofia, BG)

Background and objectives: We examined the characteristics and outcome of 75 patients with hematological malignancies (2 of them with solid tumors) treated with high-dose therapy (HDT) and autologous transplantation (ASCT) at our BMT Unit between October 2004 and October 2009.

Design and methods: Analysis was performed examining patients' characteristics, prior chemotherapy regimens, pretransplant disease status, HDT regimen, source of stem cells, time for hematopoietic recovery, complications of transplantation, response rates, overall survival (OS) and relapse-free survival (RFS).

Seventy five (75) patients were analysed, male/female ratio was $43 / 32$, with median age 38.10 years (21-64). Patients undergoing ASCT were with HD (29), NHL (16, MM (20), AML/ ALL ( 7 ), solid tumors (2). All of the patients received peripheral blood stem cell graft.

Patients were treated with salvage chemotherapy (ICE, DHAP, VIHA) and ASCT with conditioning type BEAM (30 patients), LACE (13) and CBV (2) for lymphoproliferative diseases, Melphalan (20) for myeloma patients and BuCy (7) for patients with acute leukemias. For patients with solid tumors we used Mega ICE as conditioning regimen.

Results: Median time to WBC recovery $\left(\geq 0.5 \times 10^{\%} / 1\right)$ was 10 days (7-18), platelets recovered in median of 10.90 days (7-21). The disease status after the HDT with ASCT was as reported from other BMT groups in Europe. Transplant related mortality (TRM ) was $4 \%$ ( 3 patients) - because of infections complications, sepsis or multiorgan faulire.

Conclusions: HDT and ASCT is an effective therapy for treatment of some relapsed and refractory HD patients, relapsed $\mathrm{NHL}$ and standart treatment for MM till 65 years old patient. The toxicity of HDT with ASCT can be considered as acceptable. Disease status and prior treatment before HDT was the most powerful predictive parameter for OS and RFS.

Our results confirmed that HDT with ASCT is associated with a durable RFS in a remarkable proportion of HD/NHL patients, had very good results in MM patients and that the procedure has a very low early toxicity.

\section{Paediatric issues}

\section{P853}

Children responding to treatment with mesenchymal stromal cells for refractory severe graft-versus-host disease have excellent long-term outcome

L.M. Ball (1), M.E. Bernardo (2), M.J.D. van Tol (1), M.A. Avanzini (2), A.C. Lankester (1), H. Roelofs (1), G. Giorgiani (2), J.J. Zwaginga (1), K. Le Blanc (3), W.E. Fibbe (1), R.M. Egeler (1), F. Locatelli (2)

(1)Leiden University Medical Center (Leiden, NL); (2)IRCCS Policlinico S. Matteo, University of Pavia (Pavia, IT); (3)Karolinska Institue, Huddinge University (Stockholm, SE)

Mesenchymal stromal cells (MSC) therapy has been reported to achieve responses of up to $80 \%$ in children with severe steroid refractory acute Graft versus Host Disease (GvHD). No long term comprehensive follow up data is available.
We report our experience of MSC infusions in children with GvHD highlighting the excellent long term outcome in survivors. Between Jan. 2005 and Dec. 2009, 40 children (21 boys; 19 girls) aged between $3 \mathrm{~m}$ and 17 yrs $8 \mathrm{~m}$ (median $11 \mathrm{yrs}$ ) received MSC infusions using a common protocol at variable time points following the onset of steroid refractory grade $3 / 4$ acute GvHD after allogeneic HSCT.

Indications for transplant were $A M L n=8, A L L n=10, \operatorname{MDS} n=5$, JMML $n=5$, immune disorders $n=5$, others $n=7$.

MSC donors were 3rd party $(n=33)$, haploidentical $(n=4)$ or both $(n=6)$. Eighteen children had either no $(N R, n=10)$ or partial resolution $(P R, n=8)$ to $M S C$, i.e. worsening/ persistent symptoms and if available no/ minimal histological changes, respectively. TRM due to infection and/or progressive GvHD was $83 \%$ in this group (see Figure).

Complete resolution (CR) of GvHD was evident in the rest and of these 20/22 children are alive, with a median follow up of 2 yrs $2 \mathrm{~m}$ (range $3 \mathrm{~m}$ to $4 \mathrm{yrs}$ ). Two children died from infection shortly after MSC infusions.

In 28 children transplanted for malignant disease 9 died 7 due to TRM, 2 due to relapse). In the CR group no relapse has been documented (median follow up 1.6 yrs; range $2 \mathrm{~m}-2$ yrs $9 \mathrm{~m}$ ). Chronic (c)GvHD developed in $27 \%$ of evaluable children (limited 5; extensive 5), reducing performance rating to $\leq 80 \%$. Children without cGvHD have ratings $\geq 90 \%$.

Overall survival was $60 \%$. Recent initiation of MSC at the time of steroid failure rather than after weeks of immune suppression may have reduced fatal infections and improved response and outcome. Although infections were evident at the time of immune suppression and mortality was high in NR/PR, response to MSC allows for reduction and eventual discontinuation of pharmacological immune suppression. Long term survival in responders is associated with eventual immune recovery, no late infections and persistent remission status.

Children responding to MSC treatment for severe steroid refractory GvHD have an excellent long term survival. Further studies are required in children to determine the optimal timing of MSC to improve response rates, allowing cessation of other immune suppression and, hopefully, reducing occurrence of fatal infections and $\mathrm{cGvHD}$.

Long term survival related to response from first MSC

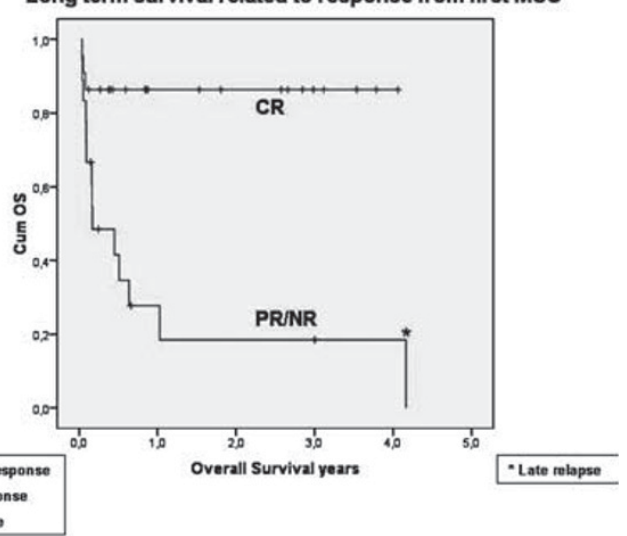

P854

EBMT Survey on adolescents with ALL in second complete remission of given allogeneic HSCT. Analysis on behalf of the PDs and AL WPs

G. Dini, M. Labopin, J. Cornish, S. Lawson, Y. Bertrand, M. Potter, P. Sedlacek, P. Ljungman, F. Locatelli, R. Hough, A. Balduzzi, M. Aljurf, R. Handgretinger, B. Gibson, N. Elarouci, M. Fossati, E. Lanino, V. Rocha, C. Peters on behalf of the EBMT PDs and AL WPS

Adolescents are treated both by paediatric teams (group 1 , defined as centres reporting a majority of pts $<18 \mathrm{yrs}$, or com- 
bined centres, with a dedicated paediatric team) and by adult teams (group 2, defined as centres reporting pts below and above $18 \mathrm{yrs}$, without a dedicated paediatric team). The primary end-point of this study was to evaluate the impact of the type of centre, i.e., paediatric or adult, on outcome after HSCT from a MSD, or from a volunteer UD in adolescents with ALL in 2 nd CR, defined as pts older than 14 yrs and younger than $18 \mathrm{yrs}$ at HSCT, given allogeneic HSCT and reported to the EBMT registry. A total of 334 pts (69\% males and $31 \%$ females in group $1,71 \%$ males and $29 \%$ females in group $2, P=n s$ ) were included in the study; 166 pts were transplanted in paediatric centres, whereas 168 pts were transplanted in adult centres. The 2 groups differed in terms of distribution of patient-and transplant-related variables: the frequency of T-ALL in group 2 pts was about approximately twice as high as in group 1 $(P=0.03)$; the median interval from diagnosis to transplant was higher in group 2; the median age at HSCT was 15,5 and 16 yrs, for groups 1 and 2 , respectively $(P=0.0001)$. A MSD was was used in $45 \%$ of the pts in group 1 and in $63 \%$ of those in group 2; the remaining pts received an UD HSCT $(P<0.001)$; the source of stem cells was bone marrow in $74 \%$ of pts in group 1 and in $62 \%$ of pts in group 2; the remaining pts received PBSCs $(P=0.02)$. The transplant conditioning regimen included TBI in $90 \%$ and $83 \%$ of group 1 and 2, respectively ( $P=n s$ ). As of October 2009, the median follow-up was 50 months. $41 \%$ of the pts from group 1 and $44 \%$ of the pts in group 2 reported acute GVHD grade $2-4$, respectively $(P=n s)$. The cumulative incidence of chronic GvHD was $22 \pm 4 \%$ for group 1 and $34 \pm 4 \%$ for group 2, respectively $(P=.05)$. Five-year Kaplan-Meyer estimates of LFS were $43 \%$ for group 1 , versus $32 \%$ for group 2 , respectively $(P=0.06)$. The 5 year cumulative incidence of TRM and relapse rate were $33 \%$ and $24 \%$ for group $1,26 \%$ and $42 \%$ for group 2 ( $P=\mathrm{NS}$ and $P=0.001)$. Univariate analysis showed that diagnosis to transplant interval $>959 \mathrm{~d}(P<0.000)$, and UD HSCT $(P=0.054)$ were poor-risk factors for LFS, while age $>16$ years, source of stem cells, and conditioning regimen did not impact LFS. Multivariate analysis confirmed for adolescents treated at paediatric centres a significantly lower relapse rate and a trend towards better LFS, while no difference was observed in chronic GvHD.

\section{P855}

Higher CD34 + and CD3 + cell doses in the graft promote long-term survival and have no impact on the incidence of severe acute or chronic GvHD after matched and mismatched unrelated donor haematopoietic progenitor cell transplantation in children

K. Kalwak (1), J. Porwolik (1), M. Mielcarek (1), E. Gorczynska (1), J. Owoc-Lempach (1), M. Ussowicz (1), A. Dyla (1), D. Wójcik (1), J. Musial (1), D. Pazdzior (1), D. Turkiewicz (2), A. Chybicka (1)

(1)Wroclaw Medical University (Wroclaw, PL); (2)Department of Pediatric Oncology (Lund, SE)

The aim of our study was to analyze the impact of infused CD34 + and CD3 + cell doses on survival and incidence of severe graft-versus-host disease (GVHD) in 187 children and adolescents (median age 9.6 years, range 0.6-20.2), who underwent unrelated donor hematopoietic stem cell transplantation (URD HSCT). HLA typing was performed at the high resolution level: there were 159 matched- and 28 mismatched (BFM) donorrecipient pairs, respectively. All patients were given serotherapy with ATG or Campath as GVHD prophylaxis. With a median follow-up of 3.2 years (PB group) and 5.4 years (BM group) there was a statistically significant advantage in disease-free survival (DFS) of pts given PBSC vs. BM $(0.55$ vs. $0.4, P<0.05)$. The most striking statistically significant differences in OS and DFS were observed when comparing groups receiving higher and lower doses of both CD34 + and CD3 + cells in the graft. Patients receiving $>10 \times 10(6) \mathrm{CD} 34+$ cells $/ \mathrm{kg}$ had OS (DFS) probability of $0.64(0.60)$ vs. $0.44(0.44)$ when given lower cell doses. Similarly patients given $>4 \times 10(8)$ CD $3+$ cells $/ \mathrm{kg}$ had OS (and DFS) probability of $0.61(0.60)$ vs. $0.45(0.43)$ when infused lower cell doses. These results were confirmed in a multivariate Cox analysis showing that infused CD34 + cell dose lower than $10 \times 10(6) / \mathrm{kg}$ was a significant, independent risk factor for mortality (RR 1.8, Cl 1.2-2.8, $P=0.009$ ). In terms of DFS, multivariate analysis revealed two independent risk factors for poor outcome: 1. infused CD3 + cell dose lower than $4 \times 10(8) / \mathrm{kg}(\mathrm{RR} 1.8, \mathrm{Cl}$ 1.1-2.7, $P=0.01$ ) and 2. the use of donors mismatched at 2 HLA antigens or $3 \mathrm{HLA}$ allele/antigens (RR 2.4, $\mathrm{Cl} 1.2-5.1, P=0.017$ ). There was a significantly higher incidence of severe aGVHD in the mismatched group $(0.37)$ vs. matched group $(0.20, P=0.04)$, which, however, did not adversely affect OS or DFS. Multivariate analysis showed that: 1 . the use of mismatched donors (mostly high-risk pts) acc. to BFM criteria was significant, independent risk factor for incidence of severe aGVHD (RR 2.2, Cl 1.1-4.5, $P=0.033$ ). Furthermore multivariate analysis disclosed no significant risk factors for development of extensive cGVHD.

The report demonstrates for the first time in a large pediatric cohort that higher doses of transplanted CD34 + and CD3 + cells lead to improved survival without increased risk of severe GVHD. There is no need to limit the number of infused CD34 + and/or CD3 + cells in the graft from URD, at least in pediatric pts.

\section{P856}

Transplantation with CD3/CD19 depleted haploidentical stem cells followed by adoptive therapy with ex vivo IL-2 stimulated NK-cells in children with relapsed neuroblastoma

J. Soerensen, R. Esser, S. Huennecke, A. Jarisch, U. Koehl, T. Klingebiel, D. Schwabe, P. Bader

Clinic of JWG-University (Frankfurt, DE)

Whereas Neuroblastoma in infants often show spontaneous regression without treatment, in older children with advanced disease outcome is still poor despite intensive treatment including autologous stem cell transplantation (SCT). In patients with relapsed tumor, the disease is usually fatal.

In vitro data suggest that Natural Killer (NK) cells are able to kill NB cells. Since NK influence engraftment, graft versus host disease and immune reconstitution, we analyzed haploidentical SCT followed by adoptive therapy with ex vivo IL-2 stimulated NK-Cells in children with relapsed neuroblastoma.

We here present the data of 4 intenisvely pretreated patients (aged 3y, 6y, 8y, 18y) with relapsed neuroblastoma who received in vitro Interleukin 2 (IL-2) stimulated donor NK-cells on day +40 and day +100 after haploidentical SCT.

After conditioning with fludarabine $150 \mathrm{mg} / \mathrm{m}^{2}$, thiotepa 10 $\mathrm{mg} / \mathrm{kgBW}$, melfalan $140 \mathrm{mg} / \mathrm{m}^{2}$ and OKT3 ( day -8 to +10 ) a median of $11.9 \times 10^{6}$ (range 9.7-16.83) CD34 + cells, $39.7 \times 10^{3}$ (24.9-96.8) CD3 + cells, and $13.2 \times 10^{6}(7.15-61.7)$ CD56 + cells per kgBW were administered at day 0 . All patients engrafted until day +16 with complete donor chimerism.

Donor NK Cells were purified and further activated in culture using $1000 \mathrm{U} / \mathrm{mL}$ IL-2 for 2 weeks. At day +40 a median number of $18.26 \times 10^{6} \mathrm{CD} 56+$ cells $/ \mathrm{kgBW}$ ) and of $17.04 \times 10^{6} \mathrm{CD} 56+$ cells $/ \mathrm{kg} \mathrm{BW}$ at day +100 was transfused.

NK-Cell infusion was well tolerated, only fever on the day of administration was seen. Laboratory analyses revealed elevated serum levels of IFN-y, IL-6 and MC-P as well as a decrease of CD14 + cells for a short time period after NK-Cell-infusion.

Graft versus Host Disease (GvHD) (skin, maximum grade II) was observed in 3/4 patients; 2 of these patients developed GvHD before NK cell infusion.

Ten and 23 months after transplantation, 2 of the 4 patients are alive with no sign of active disease; 1 patient relapsed on day +130 and died of progressive disease, 1 patient died due to Epstein-Barr-Virus associated B-Cell-lymphoma 7 months after transplantation.

We conclude that adoptive immunotherapy after haploidentical SCT with ex vivo stimulated NK-cells is feasible with acceptable toxicity and might be a promising therapeutic approach for patients with relapsed or refractory NB. 
P857

Real-time PCR-based post-transplant monitoring of haematopoietic chimerism: an option for earlier detection of impending relapse in paediatric $A M L$

A. Willasch (1), N. Shayegi (1), H. Kreyenberg (1), E. Rettinger (1), R. Handgretinger (2), B. Kremens (3), A. Borkhardt (4), K.W. Sykora (5), D. Reinhardt (5), B. Strahm (6), C. Rossig (7), J.F. Beck (8), T. Klingebiel (1), P. Bader (1)

(1)Goethe University (Frankfurt, DE); (2)Karls University (Tubingen, DE); (3)University Hospitals (Essen, DE); (4)Heine University (Dusseldorf, DE); (5)Hannover Medical School (Hannover, DE); (6)Ludwigs University (Freiburg, DE); (7)Wilhelms University (Munster, DE); (8)Schiller University (Jena, DE)

In pediatric AML impending relapse can be detected by posttransplant monitoring of chimerism. However, the gold-standard method (conventional STR-PCR, quantifiable limit 1E-2) did not highlight all patients with impending relapse in the study by Bader et al. in 2004.(1) Increasing the sensitivity of the method might allow more frequent and earlier detection of significant amounts of autologous cells. Real-time sequence polymorphism PCR (SP-qPCR) monitors chimerism with high sensitivity (quantifiable limit 1E-4 to 1E-3) and reproducibility, principally allowing for more frequent and earlier detection of ongoing relapse.(2)

To study this, a retrospective analysis of real-time PCR based chimerism was conducted including 66 children with $\mathrm{AML}$ after allogeneic SCT. Here we present a re-evaluation of the cohort mentioned above(1) comparing the existing data with real-time PCR (qPCR) based chimerism.

Chimerism by qPCR allowed the definition of two risk groups: High risk (HR) group: Autologous cells in peripheral blood $>0.5 \%$ in two consecutive samples, low risk (LR) group: all others.

Outcome: HR group: 19 patients. CR: $6(32 \%, 3$ patients received immunotherapy), relapse: 10 (53\%), TRM: $1(5 \%)$, rejection: $2(10 \%)$. LR group: 47 patients. CR: $31(66 \%)$, relapse: $3(6 \%)$, TRM: $13(28 \%)$, rejection: $0(0 \%)$. HR patients bared significantly higher risk of relapse. Substantial mixed chimerism (MC) was detected earlier by qPCR (median/mean $7 / 11 d$, range 4-49d) compared to conventional PCR in 11/16 patients $(69 \%)$. Relapsed patients without significant MC ("false negatives") were found less frequently in qPCR $(3 / 47,6 \%)$ compared to conventional PCR $(8 / 62,13 \%)$. Significant MC (qPCR) was found in 15\% (3/19) of patients who remained in CR without intervention.

LR patients showed significantly higher 3-year EFS and RFS estimates compared to HR patients (52\% and $73 \%$ versus $28 \%$ and $33 \%, P=0.013$ and $P<0.0001$ ).

Real-time PCR based chimerism in pediatric AML was reliable and allowed to detect substantial MC earlier (4-49d) in $69 \%$ of cases compared to conventional PCR. However, significant MC was detected in some patients (3/19) who never relapsed.

(1) Bader et al., BMT 33 (8): 815-21, 2004; (2) Willasch et al., Lab. Hematol. 13 (3): 73-84, 2007

Supported by Deutsche José Carreras Leukämie Stiftung e.V. (DJCLS F04/03; AW, PB).

\section{P858}

Reduced-intensity conditioning with fludarabine, cyclophosphamide and ATG for children with severe aplastic anaemia treated with unrelated peripheral blood stem cell transplantation

J.W. Lee (1), P.S. Jang (2), N.G. Chung (1), B. Cho (1), D.C. Jeong (3), H.K. Kim (1)

(1)Catholic University of Korea (Seoul, KR); (2)Catholic University of Korea (Uijeongbu, KR); (3)Catholic University of Korea (Bupyeong, KR)

Purpose: Since January, 2006 we have implemented a reduced intensity conditioning regimen consisting of fludarabine (Flu), cyclophosphamide (Cy) and ATG for severe aplastic anemia (SAA) patients undergoing hematopoietic stem cell transplantation (HSCT). In this study, we reviewed the clinical course of SAA patients who received unrelated, G-CSF mobilized peripheral blood stem cell transplantation (PBSCT), either fully matched or mismatched, to observe the efficacy of our treatment regimen in this significant subset of SAA patients treated with HSCT.

Methods: A total of 9 patients received unrelated PBSCT from January, 2006 to October, 2009 (median age 11 years old, range $1-18,8$ male 1 female). Five patients underwent a conditioning regimen consisting of Cy $50 \mathrm{mg} / \mathrm{kg} /$ day (D -9, -8, -7, -6), Flu $30 \mathrm{mg} / \mathrm{m}^{2} /$ day (D -5, -4, -3, -2), and ATG $2.5 \mathrm{mg} / \mathrm{kg} /$ day (D $-3,-2,-1)$, while 4 patients received a modified regimen in which the Cy dose was reduced to $25 \mathrm{mg} / \mathrm{kg} /$ day (D $-5,-4,-3,-2)$, with the rest of the regimen remaining unchanged. GVHD prophylaxis consisted of cyclosporine and mini-dose methotrexate. Six patients $(67 \%)$ showed either 1 or 2 mismatches on molecular typing for HLA-A, B, C and DR loci. All 4 patients (44\%) diagnosed with very severe aplastic anemia (VSAA) entered the conditioning regimen febrile, 3 with overt sources of infection. Medians of infused cell numbers were as follows: MNC $15.1 \times 10^{8} / \mathrm{kg}(5.9-18.2)$, CD34 + 6.5 × 106 $/ \mathrm{kg}(4.1-14.7), \mathrm{CD} 3+$ $69.4 \times 10^{7} / \mathrm{kg}(25.0-104.3)$.

Results: Median time to engraftment of $1,000 / \mathrm{mm} 3$ neutrophils and $50,000 / \mathrm{mm}^{3}$ platelets was 11 days (10-20) and 20 days (15-35) respectively. All showed complete donor chimerism at $D+30$. The 4 VSAA patients showed defervescence and resolution of infectious signs by the time of neutrophil engraftment. Two patients $(22 \%)$ experienced grade II acute GvHD. Another $2(22 \%)$ were diagnosed with chronic GvHD (1 mild, 1 severe) that has since resolved. Five patients were treated preemptively for CMV DNAemia without major complications. With a median follow-up duration of 325 days (range $86-1,392$ ), all 9 patients are surviving event-free.

Conclusion: A reduced dose conditioning regimen consisting of fludarabine, cyclophosphamide and ATG allowed for rapid engraftment with complete donor chimerism, without major toxicities in our cohort of 9 SAA patients treated with unrelated PBSCT despite significant HLA disparities and comorbid infectious complications.

\section{P859}

HLA-C allele mismatch is the most important factor with regards to chronic graft-versus-host disease incidence in paediatric HSCT: a single-centre study based on the NIH consensus criteria

J.W. Lee (1), P.S. Jang (2), N.G. Chung (1), B. Cho (1), D.C. Jeong (3), H.K. Kim (1)

(1)Catholic University of Korea (Seoul, KR); (2)Catholic University of Korea (Uijeongbu, KR); (3)Catholic University of Korea (Bupyeong, KR)

Purpose and methods: Analyses on risk factors for chronic graft-versus-host disease (GVHD) in the pediatric transplantation population are few, and the importance of stem cell source, either bone marrow (BM) or mobilized peripheral blood stem cells (PBSC), as a significant determinant of later chronic GVHD remains controversial. In order to clarify these issues, a study cohort of 58 patients (13 BMT and 45 PBSCT) who received transplantation from January, 2006 to August, 2008 was analyzed for the incidence of chronic GVHD according to pertinent risk factors, including stem cell source. Chronic GVHD was then classified according to the NIH Consensus Criteria (NCC) and patient response to immunosuppression was followed to determine if NCC global assessment has prognostic implications in the pediatric transplantation group.

Results: Twenty-seven of 58 patients $(46.6 \%)$ were diagnosed with chronic GVHD, 3 of 13 patients in the BMT group $(23.1 \%)$ and 24 of 45 patients in the PBSCT group $(58.8 \%)(P=0.053)$. On univariate analysis, HLA-C mismatch $(P=0.002)$, patient age $(P=0.008)$, preceding acute GVHD $(P=0.008)$, and malignant disease $(P=0.014)$ proved to be significant determinants of chronic GVHD incidence. Other factors such as stem cell source, conditioning regimen, 
infused $C D 34+$ and $C D 3+$ cell dose, proved not to have a significant impact. Multivariate analysis revealed HLA-C mismatch as the most important factor regarding chronic GVHD $(P=0.008)$, followed by patient age $(P=0.012)$ and preceding acute GVHD $(P=0.026)$.

At initial presentation, 11 patients were classified as having mild $(41 \%), 9$ with moderate $(33 \%)$, and 7 with severe chronic GVHD $(26 \%)$ according to NCC. The oral cavity was the most common organ of diagnosis, followed by the lung. Death resulting from GVHD-related complications occurred in only 1 out of 27 patients (3.7\%). Overall, 25 of 27 patients $(92.6 \%)$ had chronic GVHD either resolved or controlled at the end of the follow-up period, resulting in no correlation between global GVHD severity at onset and final response to immunosuppression. Conclusion: In our cohort, donor-recipient HLA-C mismatch was the most important factor with regards to chronic GVHD incidence. The NCC, although providing objective criteria for diagnosing and classifying chronic GVHD, may have limited prognostic ability when applied to children with GVHD.

\section{P860}

NOD2CARD15 polymorphisms have negative influence on the outcome after allogeneic stem cell transplantation in paediatric patients with non-malignant diseases C. Bayer (1), H. Kreyenberg (1), B. Strahm (2), B. Kremens (3), J.F. Beck (4), A. Schrauder (5), S. Burdach (6), H. Kabisch (7), E. Holler (8), T. Klingebiel (1), P. Bader (1)

(1)Goethe University (Frankfurt, DE); (2)University Children's Hospital (Freiburg, DE); (3)University Children's Hospital (Essen, $D E) ;(4)$ University Children's Hospital (Jena, DE); (5)University Children's Hospital (Kiel, DE); (6)University Children's Hospital (Munich, DE); (7)University Children's Hospital (Hamburg, DE); (8)University Medical Center (Regensburg, DE)

Background: In recent years several studies have investigated the role of the polymorphisms (SNP8, SNP12, SNP13) in the NOD2/CARD15 gene for the clinical outcome after allogeneic stem cell transplantation. We recently reported these variants have no influence on outcome in children transplanted for acute leukemias. Here we present a retrospective analysis on the impact of NOD2CARD15 polymorphisms in children who received their transplant for nonmalignant diseases with regard to TRM, GVHD and survival.

Patients: Between February 1997 and March 200874 children (median age $=6.1$ ) could be included who underwent allogeneic SCT for nonmalignant diseases in one of the participating centers. The study was approved by the Ethical Committee of the Goethe University Frankfurt. NOD2CARD15 mutations were assessed using RT-PCR on a BioRad IQ5 System. Diagnosis of aGvHD was based on clinical signs or histo-pathological findings and was graded in line with the Seattle criteria.

Results: Of the 74 transplant pairs polymorphisms were identified in 24 cases $(32.4 \%)$. In 7 pairs polymorphisms were found on the recipient's side, in 11 pairs on the donor's side and in 6 cases both donors and recipients revealed a gene variant. 2 recipients showed coincidence of two polymorphisms. In contrast to the group of patients with malignant diseases we found a significantly higher incidence of aGvHD II-III in patients with nonmalignant disorders carrying the mutations. The cumulative incidence $(\mathrm{Cl})$ was 0.079 in patient/donor pairs with wild type genes compared to 0.33 in pairs with any sort of mutations $(P=0.014)$. The results for cGvHD are likewise: 0.081 in wild type pairs and 0.26 in pairs with NOD2CARD15 variants $(P=0.039)$. The cumulative incidence of TRM was 0.04 in wildtype patient/recipient pairs and 0.38 in pairs showing any mutation in the NOD2CARD15 genes $(P=0.022)$.

Moreover, probability for two years survival (pOS) was significantly reduced if either recipient or donor carries a mutation $(P=0.02)$ from 0.96 in wild type pairs to 0.62 in pairs with mutations, a finding that could be confirmed in multivariate analysis.
Conclusion: The presence of NOD2CARD15 gene variants patient/donor pairs has negative prognostic influence in children who need to undergo allogeneic stem cell transplantation for nonmalignant disorders.

\section{P861}

Graft-versus-host disease-specific survival: are adult prognostic factors correlating with outcome in children? E. Güclü (1), C. Peters (1), T. Fazekas (1), M. Seidel (1), U. Pötschger (2), H. Gadner (1), A. Lawitschka (1)

(1)St. Anna Children's Hospital (Vienna, AT); (2)Children's Cancer Research Institute (Vienna, AT)

Current knowledge of factors predicting chronic Graft-versushost disease (cGVHD)-specific survival (GSS) in pediatric allogeneic hematopoietic stem cell transplantation (HSCT) is based on studies focusing primarily on adults.

We reviewed 84 children with cGVHD who received HSCT (59 bone marrow, 25 peripheral blood stem cells) after myeloablative $(n=49)$ or reduced intensity $(n=35)$ conditioning for malignant $(n=54)$ and non malignant $(n=30)$ diseases between 1982 and 2006 in our center. Donor was an HLA-identical sibling in 36 cases and an alternative donor in 48. A second HSCT was performed in 14, a third HSCT in 3 and donor lymphocyte infusions in 7 patients.

Prior acute GVHD (no, grade I-II, grade III-IV), onset type (progressive, quiescent, de novo), classification (limited vs. extensive), thrombocytopenia $(<150 \mathrm{G} / \mathrm{l})$, kind and extent of skin involvement (sclerodermoid vs. other, $>50 \%$ vs. $<50 \%$ BSA) were analyzed as potential prognostic factors.

$84(40 \%)$ of 209 children surviving more than 100 days post HSCT developed cGVHD. Median follow-up was 8,7 years $(1,1-$ 18,6 ); causes of death in 24/84 patients were: 3 relapses, 1 secondary malignancy, 1 unknown and 19 cGVHD-related causes. 5 -year overall survival was $76 \%$ (10-year $71 \%, 15$-year $68 \%$ ). 5 -year, 10-year and 15-year cumulative incidence of cGVHDrelated deaths was $20 \%, 22 \%$ and $25 \%$, respectively. NonGvHD-related deaths were rare with a 5-year, 10-year and 15 -year cumulative incidence of $3 \%, 7 \%$ and $7 \%$, respectively. Interestingly extent of acute GVHD, onset type, thrombocytopenia and extent of skin involvement did not adversely influence outcome. The only variable predictive of poor GSS was skin manifestations other than sclerodermoid with a 5 year cumulative incidence of GVHD-specific mortality (GSM) for sclerodermoid vs. other or no skin GVHD of $0 \%$ vs. $29 \%(P=0,004)$. Extensive cGVHD (5 year cumulative incidence of GSM $25 \%$ vs. $10 \%$ for limited) showed a trend $(P=0,060)$.

Our findings suggest that adult cGVHD prognostic factors might not be interchangeably used in children and that further analyses are needed, especially considering the new National Institute of Health scoring system.

\section{P862}

Pharmacokinetic study of intravenous busulfan in haematopoietic stem cell transplantation: results of a prospective study with $\mathbf{2 5}$ children

Y. Nagatoshi (1), Y. Okamoto (2), Y. Kosaka (3), A. Kikuchi (4), S. Kato (5), H. Kigasawa (6), Y. Horikoshi (7), K. Chayama (8), M. Kaneda (2), T. Mori (9), H. Mugishima (10), Y. Kawano (2) (1)Kyushu Cancer Center (Fukuoa, JP); (2)Kagoshima University (Kagoshima, JP); (3)Hyougo Prefectural Kobe Children's Hospital (Hyougo, JP); (4)Saitama Childrens Medicla Center (Saitama, JP); (5)Tokai University (Kanagawa, $J P)$; (6)Kanagawa Children's Medical Center (Kanagawa, JP); (7)Shizuoka Children's Hospital (Shizuoka, JP); (8)Okayama University (Okayama, JP); (9)National Center for Child Health \& Development (Tokyo, JP); (10)Nihon University (Tokyo, JP)

This prospective study was performed to investigate the pharmacokinetics of intravenous busulfan (Busulfex®) as a part of 
a conditioning regimen $(0.8-1.2 \mathrm{mg} / \mathrm{kg} /$ dose for total 16 doses $)$ before hematopoietic stem cell transplantation (HSCT) in Japanese pediatric patients. Evaluation of the AUC targeting rate and relationship between the AUC and adverse events were the primary endpoints.

Twenty-five (14 males and 11 females: the median age 6 years; range, $5 \mathrm{~m}-17 \mathrm{y}$ ) of the 26 registered patients received i.v. busulfan after consent was obtained. The patients had acute leukemia $(n=14), C M L(2)$, MDS (5), solid tumors (2), and metabolic disorders (2). Allogeneic (7 related and 16 unrelated) and autologous grafts ( 1 bone marrow and 1 peripheral blood) were infused into 23 and 2 patients, respectively. Five different dose schedules were used according to body weight (BW); $<9 \mathrm{~kg}(1.0 \mathrm{mg} / \mathrm{kg}), 9$ to $<16(1.2 \mathrm{mg} / \mathrm{kg}), 16$ to $23(1.1 \mathrm{mg} / \mathrm{kg}),>23$ to $34(0.95 \mathrm{mg} / \mathrm{kg})$, and $>34 \mathrm{~kg}$ of BW $(0.8 \mathrm{mg} / \mathrm{kg})$, as in a previous European study. Each dose was given over 2 hours and sample blood was drawn at 9 or 11 separate points for analysis by gas chromatography mass-spectrometry. The target AUC after the first dose was set from 900 to 1,500 micro-mol.min/L based on other studies.

We found that the AUC varied from 796 to 1,905 micro-mol. $\mathrm{min} / \mathrm{L}$, and 19 of the 25 patients $(76 \%)$ remained within the target range. The AUC was below and above the target range in 2 and 4 patients, respectively. Three allo-grafts failed to engraft. One patient died in the very early stage of HSCT and two were diagnosed as engraftment failure. These latter patients were JMML (3y, M) and AML (17y, F), and they received grafts of one-locus-mismatched BM from an unrelated donor and mismatched unrelated cord blood. There were no other unknown adverse events. The relationship between the AUC and adverse events is shown below:

The AUC targeting rate was $76 \%$. Since there appeared to be no relationship between the AUC and either graft failure or VOD, busulfan dosing based on BW without adjustment by the AUC appears to be feasible in pediatric patients.

\begin{tabular}{lccc}
\hline AUC $(\boldsymbol{\mu m o l} \cdot \min / \mathbf{L})$ & $<\mathbf{9 0 0}$ & $\mathbf{9 0 0}$ to $\mathbf{1 , 5 0 0}$ & $>\mathbf{1 , 5 0 0}$ \\
\hline No. of patients & 2 & 19 & 4 \\
Early mortality & 0 & 2 & 1 \\
Graft failure & 0 & 2 & 0 \\
Convulsion & 0 & 1 & 1 \\
VOD & 0 & 3, recovered & 1, died \\
\hline
\end{tabular}

\section{P863}

Improved survival after allogeneic HCT in childhood ALLI: a retrospective population-based single-centre study M. Ifversen, K. Müller, H. Baekgaard, C. Heilmann

Rigshospitalet, Copenhagen University Hospital (Copenhagen, $D K)$

Background: Allogeneic hematoipoietic stem cell transplantation (HCT) is indicated in early relapsed or very high risk childhood ALL. Post transplant relapse and early treatment related mortality (TRM) are the major factors influencing overall survival. In recent years pre-transplant chemotherapy, transplant eligibility, donor selection, conditioning regimens, graft versus host disease (GvHD) prophylaxis and quality of supportive therapy has improved. In a retrospective study we analysed the influence of these factors on the improved survival observed over two eight-years time periods from January 1993 to December 2008.

Results: Eighty-nine consecutive transplants performed in Danish children between 1/1-1993 and 31/12-2008 were analyzed. The median age at transplantation was 9.28 years (range 1,1$17,6) ; 67 \%$ were boys. Thirty-five children were transplanted in the first eight-years time period, 54 in the later. Transplant in first complete remission (CR1) was more frequent in the later time period ( $57 \%$ vs. $26 \%$ ).

Survival improved significantly over the two time periods (OS $45 \%$ and $75 \%$ respectively, $P=0.010$ ). The presence of severe acute GvHD (grade 3-4) was significantly lower in the late period $(17 \%$ vs. $4 \%)$. TRM decreased from $18 \%$ to $8 \%(P=0.067)$ and relapse risk was reduced form $50 \%$ to $22 \%(P=0.051)$. Improved survival figures were seen among children who received VP16 and total body irradiation as conditioning. The probability of survival after matched sibling transplant without MTX-prophylaxis was above $90 \%$. Pre-transplant remission status (CR1 vs. later $\mathrm{CR}$ ) and donor selection by high resolution tissue typing did not influence on survival in these data.

Conclusion: Better leukaemia control as well as improved supportive treatment contributed equally to the significantly improved survival after SCT for childhood ALL.

\section{P864}

Needs of paediatric haematopoietic stem cell transplant centres in Eastern Europe - the EBMT Paediatric Diseases Working Party Survey

J. Wachowiak (1), B. Afanasyev (2), O. Aleinikova (3), D. Colita (4), J. Gozdzik (5), A. Kaare (6), N. Kalman (7), J. Kowalczyk (8), G. Krivan (9), A. Lange (10), J. Pretnar (11), J. Rascon (12), S. Sulfiarska (13), D. Vujic (14), M. Wysocki (15), C. Peters (16) (1)Poznan University of Medical Sciences (Poznan, PL); (2)R. Gorbacheva Institute of Children's Haematology and Transplantation (St. Petersburg, RU); (3)Belorussian Centre for Paediatric Haematology/Oncology (Minsk, BY); (4)Fundeni University Institute (Bucharest, RO); (5) Jagiellonian University (Cracow, PL); (6)University Hospital (Tartu, EE); (7)Postgraduate Medical School (Miskolc, HU); (8)Children's University Hospital (Lublin, PL); (9)Pediatric Hematology and HSCT Unit (Budapest, HU); (10)Lower Silesian Centre for Cellular Transplantation (Wroclaw, PL); (11)University Clinical Centre (Ljubljana, SI); (12)University Children's Hospital (Vilnius, LT); (13)University Teaching Hospital (Bratislava, SK); (14)Mother and Child Health Care Institute of Serbia (Belgrade, RS); (15)University Hospital CM UMK (Bydgoszcz, PL); (16)St. Anna Kinderspital (Vienna, AT)

The previous study conducted on behalf of the EBMT Paediatric Diseases WP in 2005 demonstrated the significant overall progress achieved in the domain of paediatric haematopoietic stem cell transplantation (HSCT) in Eastern Europe after 1998, including increasing number of centres performing transplants in children and yearly number of transplants along with important decrease of the 100-day transplant related mortality (Wachowiak et al., 2008). However, simultaneously significant differences between countries of this region were found correlating with national income per capita. The purpose of the current study was to assess needs of centres performing pediatric HSCT in Eastern Europe, nowadays. To accomplish this aim the specific questionnaire has been sent in March 2009 to 46 centres in 14 Eastern European countries. The questionnaire contained list of 12 needs, which could be rated from 1 point for least important need to 15 points for most important one. Fifteen centres from 10 countries responded, including 4 centres from "lower" and 11 centres from "middle" income countries according to the World Bank (www.worldbank.org). The study revealed that as the most important needs centres both from "lower" and "middle" income countries consider 1) clinical training for junior physicians in experienced centres outside the country, 2) close cooperation with experienced, JACIE accredited paediatric transplant centres, 3 ) support in preparation for accreditation procedures, and 4) clinical training for nurses in experienced centres outside the country, whilst as the least important 1) clinical training for senior physicians in experienced centres outside the country, 2) access to medical journals focusing on HSCT, 3) technical training in diagnosis, and 4) basic science training. The only important difference was the significanly higher rating position of the "access to standards obligatory in paediatric HSCT" in the "lower" income countries (5th position), than that one in the "middle" income countries (10th postion). The results of the study could be helpful in the future when planning the support for the paediatric centres in Eastern Europe within the EBMT Pediatric Diseases WP and the EBMT Outreach Program. 


\section{P865}

Outcomes of paediatric patients with acute

lymphoblastic leukaemia receiving two or more allogeneic stem cell transplants

N.B. Heaney (1), P. Veys (2), P. Derbyshire (3), R. Skinner (4), A. Vora (5), J. Layden (1), B. Gibson (1)

(1)Royal Hospital for Sick Children (Glasgow, UK); (2)Great Ormond Street Hospital for Sick Children (London, UK); (3)Birmingham Children's Hospital (Birmingham, UK); (4)Royal Victoria Infirmary (Newcaste-upon-Tyne, UK); (5)Sheffield Children's Hospital (Sheffield, UK)

Little data is published assessing outcomes following multiple allogeneic stem cell transplants (SCT) for paediatric acute lymphoblastic leukaemia (ALL). We report a retrospective study of 17 paediatric patients with ALL treated in 5 centres in the UK receiving 2 or more SCT. The median age at diagnosis of ALL was $7 \mathrm{y}$ ( 1 patient $<1 \mathrm{y}, 121-10 \mathrm{y}$ and $4>10 \mathrm{y}$ ) and mean white cell count $122 \times 10^{9} / \mathrm{L}$ (range 2.7-352). Karyotypic abnormalities were present in 13/17 patients: 9 Philadelphia chromosome $(\mathrm{Ph}+)$; and 4 other abnormalities (complex karyotype, $\mathrm{t}(1 ; 19)$, $t(4 ; 11)$ and hypodiploidy). The patients were initially treated with 1 of the following schedules-ALL97/99 (7/17), UKALL12 (1/17), ALL2003 (6/17), ESPhALL (2/17) and Interfant (1/17). Patients received their first SCT (SCT1) between 1996-2007 with 11/17 transplanted in first complete remission (CR1), 5/17 $\mathrm{CR} 2$ and $1 / 17$ with refractory disease $(\mathrm{Ph}+)$. The median age at SCT1 was 7 y (range $0.8-18$ y). 13/17 received TBI-containing conditioning regimes. Unrelated donors were selected for $10 / 17$ patients with $13 / 15$ receiving bone marrow $(B M)$ and $2 / 15$ peripheral blood (PB) derived stem cells. The median time from SCT1 to relapse was $14 \mathrm{~m}$ (range 3-57 m). Following relapse, patients proceeded to second SCT (SCT2) once CR2 (11/17) or CR3 (5/17) was obtained, with 1 patient transplanted with detectable disease (6\% blasts in BM). For SCT2 4/17 received TBI-containing conditioning. 2 patients received TBI for both SCT1 and SCT2 (180cGy $\times 8$ fractions for SCT1 and then 2Gy $\times 1$ for SCT2). Selected stem cell sources were PB (5/17), BM $(9 / 17)$ and cord blood (3/17). The same donor was used for SCT1 and SCT2 in 6/17 patients. Graft-versus-host disease (GVHD) occurred in 11/17 (6/11 grade I-II and 5/11 III-IV). 3/17 patients received DLI post SCT2. Median follow-up from SCT2 is $24 \mathrm{~m}$ (range $3-57 \mathrm{~m}$ ). 11/17 patient have relapsed at a median of $15 \mathrm{~m}$ from SCT2 (range $1-41 \mathrm{~m}$ ). 8/17 have died at a median of $24 \mathrm{~m}$ from SCT2 (range 3-46 m) with cause of death relapsed disease (4/7), infection (3/7) or GVHD (1/7). Of relapsed patients, $3 / 11$ have received a third SCT-all have died. Kaplan-Meier estimated overall survival and disease-free survival at $24 \mathrm{~m}$ following SCT2 is $72.8 \%$ and $37.6 \%$ respectively. 6/17 patients are alive and in CR following SCT2. Whilst follow-up is short these data support this approach in a select group of high risk patients. We aim to characterise this group more fully expanding the data set to include other UK treatment centres.

\section{P866}

Excellent outcome and engraftment after reducedintensity conditioning regimen in children with high-risk $\mathrm{HLH}$ and $\mathrm{LCH}$

S. Bakhtiar, J. Moskovits, E. Ponstingl, A. Jarisch, J. Soerensen, T. Klingebiel, P. Bader

Goethe University Frankfurt (Frankfurt/Main, DE)

Primary hemophagocytic lymphohistiocytosis $(\mathrm{HLH})$ and Langerhans cell histicytosis $(\mathrm{LCH})$ are rare diseases in children. Patients frequently presented with persistent fever, hepatosplenomegaly and pancytopenia. Some children may achieve remission after courses of chemotherapy. For high risk patients only curative treatment option is allogeneic stem cell transplantation (SCT). These patients have often significant multiorganic manifestations pre transplantation and have gone through diverse courses of chemotherapy regimens. As a consequence these patients are prone to develop organ toxicity and have an increased risk to suffer from graft versus host disease (GvHD). This results in an increased risk of transplant-related mortality (TRM) after conventional intensity conditioning regimens especially in patients with unrelated donors. Recently published data support the thesis of a better outcome in children with HLH and $\mathrm{LCH}$ after SCT utilizing reduced-intensity conditioning (RIC) regimens.

We present five children suffering from $\mathrm{HLH}(n=3)$ and $\mathrm{LCH}$ $(n=2)$, who underwent bone marrow transplantation from a matched sibling donor (MSD, $n=2$ ) or $C D$ 3/19 depleted SCT from a matched unrelated donor (MUD, $n=3$ ). RIC regimen included Fludarabin $\left(5 \times 30 \mathrm{mg} / \mathrm{m}^{2}\right)$, Thiotepa $(2 \times 5 \mathrm{mg} / \mathrm{kg})$ and Melphalan $\left(2 \times 70 \mathrm{mg} / \mathrm{m}^{2}\right)$.

Rapid engraftment and a quickly stabilized hematopoeisis were observed. All patients showed a primary and sustained engraft-

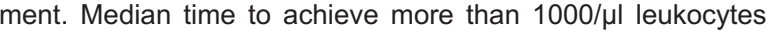
was 14 days (range: $10-29$ ), and 18 days (range: 12-72) to reach more than $>20.000 / \mu$ l thrombocytes.

There was a fast regeneration of T-lymphocytes as well. Median

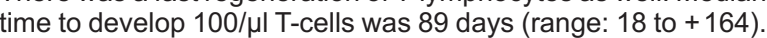
All patients survived and showed complete remission after a follow up of 7 to 20 months (median: 14 months). 1/5 patients developed GvHD grade I.

Conclusion: This reduced intensity regimen allowed rapid and complete engraftment with very low toxicity in patients with $\mathrm{HLH}$ and $\mathrm{LCH}$. This is also true for patients who received a CD3/19 depleted peripheral stem cell graft to avoid GvHD.

P867

Haploidentical stem cell transplantation in paediatric patients with myelodysplastic syndrome

H.-M. Teltschik, T. Feuchtinger, M. Pfeiffer, I. Müller, R. Handgretinger, P. Lang

University Children`s Hospital (Tubingen, DE)

We investigated a cohort of 11 pediatric patients with myelodysplatic syndrome transplanted with T-cell depleted grafts from haploidentical donors. 2 patients had refractory cytopenia, 5 patients had refractory cytopenia with excess blasts in transformation (REAB-T). 4 patients had relapsed MDS (RAEBT) after a previous transplantation from a matched unrelated donor and received a 2 nd allogeneic stem cell transplantation (SCT) from a haploidentical donor. Median age was 7.7 years (1.7-18). 6 patients received Busilvex/Cyclophosphamid/Melphalaned, 5 patients underwent a reduced intensity conditioning with Melphalane/Thiotepa and Fludarabine or Clofarabine. All patients received peripheral stem cells. In 7 patients Graft manipulation was carried out by direct depletion using antiCD3/ antiCD19 coated magnetic microbeads. 4 patients received CD34 positive selected stem cells. Median CD34 + progenitor cell content of the grafts was $13.6 \times 10^{6} / \mathrm{kg} \mathrm{BW}\left(4.6-41 \times 10^{6}\right)$, the median T-cell content of the grafts was $63.9 \times 10^{3} / \mathrm{kg} \mathrm{BW}$ $\left(1.8-162.4 \times 10^{3}\right)$. Primary engraftment occured in 10 of 11 patients. 1 patient failed to engraft but could be rescued with a second stem cell donation from the same donor. 2 patients rejected the graft after primary engraftment. Both patients could be successfully retransplanted from a second parental donor. Thus, Final engraftment could be obtained in all patients. Median time to reach $>500$ neutrophiles/microliter was 11 days (9-15). Independence from platelet substitution was reached after a median time of 10 days $(7-18)$. No severe $\left(>^{\circ} 2\right)$ acute graft versus host disease (GvHD) was observed, 36\% (4 out of 11 ) developed acute GvHD ${ }^{\circ} 1-2$ and $64 \%$ of the patients (7 out of 11) developed no acute GvHD at all. 2 out of 11 patients relapsed (19\%), median time to relapse was 106 days. There was no transplant related mortality (TRM) within the first 100 days after SCT. Over all TRM was 2 out of 11 patients $(20 \%)$. Event free survival (EFS) was $64 \%$. Conclusions: Fast recoveries of neutrophiles and platelets were achieved without occurrence of severe acute GvHD. TRM was low although more than $1 / 3$ of the patients underwent intensive pretreatment 
according to previous allogeneic SCT. Low relapse rate let to a favourable event free survival. Thus, haploidentical SCT with T-cell depleted grafts is a save and effective treatment for MDS if no matched donor is available.

\section{P868}

Time-resolved fluorometric assay for natural killer activity show correlation with RT-PCR minimal residual disease quantification in a neuroblastoma model

C. Paillard (1), P. Halle (1), R. Veyrat-Masson (1), A. Tchirkov (2), F. Quainon (3), C. Confland (1), M. Pfeiffer (4), P. Lang (4), F. Demeocq (1), J. Kanold (1)

(1)CHU Hôtel Dieu (Clermont-Ferrand, FR); (2)Cytogénétique Médicale-Faculté de Médecine (Clermont-Ferrand, FR); (3)EFS Auvergne-Loire (Clermont-Ferrand, FR); (4)University Children's Hospital (Tubingen, DE)

Introduction: Haploidentical stem cell transplantation is increasely used in patients with relapsed neuroblastoma (NB). Although a beneficial impact of alloreactive NK cells has been reported increasing antitumor effect of NK cells may be helpful to improve clinical results.

We developed a haploidentical "parent/child" in vitro model to evaluate the anti-tumor effect of donor derived IL2/IL15 stimulated NK cells against NB cells.

Materials and methods: Six pairs of "parent/child" adult healthy donors were evaluated. NK cells were isolated from "parent's" donors using MACS separators Miltenyi Biotec (France). Enriched NK cells (CD56 + CD3-) were stimulated or not overnight by IL-2 (100 Ul/ml), IL-15 (50 ng/ml) or IL-2 + IL-15 and used as effector cells. PBMCs of "child's" donors irradiated at 30 Grays $\left(\mathrm{PBMC}^{*}\right.$ ) and contaminated with bis(acetoxymethyl) 2,2':6',2"-terpyridine-6,6"-dicarboxylate, (BATDA) labelled IMR-32 NB cell line were used as target cells. Different effector/target ratios were evaluated (10/1, 20/1, 50/1, 100/1). After incubation ( 2 hours at $37^{\circ} \mathrm{C}$ ) of labelled IMR-32 cells with NK cells the TDA released from lysed cells into the supernatant is chelated with Eu3 +. The natural killer cell activity is then quantified by measuring the intense fluorescence of the EuTDA chelates formed: BATDA europium assay (Perkin Elmer, USA). NK cytotoxicity against IMR-32 cell line was also indirectly measured by quantitative RT-PCR for tyrosine hydroxylase (TH) mRNA

HLA C (C1 and C2) and HLA B allotypes (Bw4) were determined for each "parent/child" couple using high resolution PCR sequence based typing.

Results: Cytotoxic activity of NK cells measured by a timeresolved fluorometric assay was correlated with decrease of TH transcripts number measured by RT-PCR (spearman test: ratio 100/1 p: 0.00015, ratio 50/1 p: 0.00012). Cytotoxicity of NK cells against IMR-32 was significantly enhanced by pre-incubation with IL-2 or IL-15 (figure). IL-15 stimulated NK cells show particularly strong cytotoxicity. There was a trend of increased cytotoxicity in 3 cases with KIR mismatch (these results need confirmation).

Conclusion: The presented results provide a framework for constructing an in vitro model for evaluation of anti-neuroblastoma effect of NK cells. In clinical practice we use both techniques to evaluated/compared cytotoxicity against recipient PBMC* contaminated with IMR-32 of parental donor NK cells before transplant, at day +30 and +60 after transplant.

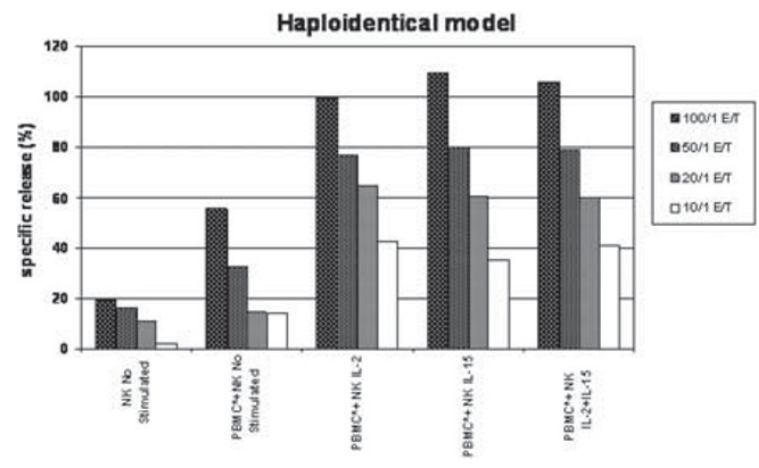

P869

Treatment of patients with high-risk axial and pelvic

Ewing's sarcoma. A single-centre experience

I. Dolgopolov (1), R. Pimenov (1), V. Boyarshinov (1), N. Subbotina (1), I. Visochin (1), S. Siegel (2), G. Mentkevich (1) (1)Institute of Ped. Oncology/Hematology (Moscow, RU); (2)Los Angeles Children Hospital (Los Angeles, US)

This study was initiated in an attempt to improve DFS in pts with high-risk axial and pelvic Ewing's sarcoma (ES) using autoPBSCT. From 1997 to 200735 pts (M/F-15/21) with high-risk ES (localized 25 pts, metastatic 10 pts (only lungs-2, combined or other sites -8 ) received HDCT with autologous transplantation The median primary tumor volume was $739 \mathrm{~cm}^{3}$. CT consisted of 5 courses: 1,3 , and 5 included cyclophosphamide $2100 \mathrm{mg} / \mathrm{m}^{2}$ day on days 1,2 , doxorubicin $37.5 \mathrm{mg} / \mathrm{m}^{2}$ as a 24-hour infusion on days 1,2 , and vincristine $1.5 \mathrm{mg} / \mathrm{m}^{2}$ on days 1,8 , and 15. Cycles 2 and 4 consisted of ifosfamide $2400 \mathrm{mg} / \mathrm{m}^{2} /$ day on days $1-5$ and VP-16 $100 \mathrm{mg} / \mathrm{m}^{2} /$ day on days $1-5$. No G-CSF was given routinely between cycles. RT was given after the fifth cycle of CT at a median dose of $52 \mathrm{~Gy}$ (range, 50-56). Patients with lung involvement received lung irradiation after the second cycle of CT at a dose of $10.8 \mathrm{~Gy}$. All pts received busulfan $16 \mathrm{mg} / \mathrm{kg}$, melphalan $140 \mathrm{mg} / \mathrm{m}^{2}$-based HDCT $(\mathrm{n}=9)$; with the addition of thiotepa (TT) $600-900 \mathrm{mg} / \mathrm{m}^{2}(\mathrm{n}=11)$ or VP16 1400 $\mathrm{mg} / \mathrm{m}^{2}(\mathrm{n}=15)$ followed by ASCT $6.4(1.9-25.3) \times 10^{6} \mathrm{CD} 34+$ cells $/ \mathrm{kg}$. Median number of days to WBC $>1.0 \times 10^{9} / \mathrm{l}$ and PIt $>20$ and $50 \times 10^{\%} / /$ was $10(8-14), 16(0-52)$ and $28(11-66)$ days, respectively. TRM was $6 \%$. Six pts relapsed DFS at $5 \mathrm{yr}$ was $50 \%$ in metastatic pts vs. $88 \%$ in pts with localized tumor $(P<005)$. EFS and DFS were $69 \%$ and $75 \%$ with a median follow-up of 103 and 112 mo, respectively. DFS in TT, VP16 and BuMel groups were $83 \%, 67 \%$ and $79 \%$, respectively $(P>0.05)$.

Pts with high-risk ES may benefit from BuMel-based regimens and that an addition of any drug to BuMel regimen increases toxicity with no influence to DFS.

\section{P870}

Use of preventive extracorporeal photopheresis before myeloablative conditioning for allogeneic stem cell transplantation in paediatric patients

C. Winzler, E. Calore, M. Giordan, M. Pillon, M. Gazzola, R. Destro, S. Cesaro, M. Carli, G. Basso, C. Messina University of Padua (Padua, IT)

Background: Extracorporeal photopheresis (ECP) is currently used to treat acute and chronic graft-versus disease notresponsive to conventional therapy. ECP is believed to have an immunomodulatory effect and may do so by modulating DC function and induction of regulatory T cells (Treg). A significant delay in the time to onset of acute graft-versus-host disease (GVHD) has recently been reported for adult patients receiving preventive ECP, performed prior to myeloablative conditioning regimen. The use of preventive ECP in pediatrics has so far not been addressed. Objective: To evaluate the efficacy and safety 
of preventive of ECP performed before a myeloablative conditioning regimen in pediatrics.

Methods: Eleven pediatric patients receiving an allogeneic hematopoietic stem cell transplant were treated with conventional myeloablative conditioning $(n=8)$ or in addition with prophylactic ECP performed on two successive days before starting myeloablation $(n=3)$ and followed up until d100. The control group $(n=8)$ included five transplants from matched unrelated donors and three from sibling donors, while the treatment group $(n=3)$ included two transplants from matched unrelated donors and one from sibling donor. Regulatory $T$ cell frequency was estimated by FACS analysis before conditioning and, for ECP treated patients, before performing ECP, too.

Results: Preventive ECP significantly reduced the incidence of acute GVHD grade II-IV. While all patients in the control group developed acute GVHD grade II-IV, only two out of three treated patients experienced acute GvHD grade II-IV $(P=0.04338)$. No adverse effects were observed following preventive ECP. The relative size of Treg was increased after the second day of ECP in one sample with low size before treatment, while in two samples with higher basic frequency, Treg did not further increase. Conclusion: Extracorporeal photopheresis incorporated in the myeloablative conditioning regimen was not associated with major adverse effects. A statistically significant reduction of the incidence of acute GVHD was observed. Albeit definitive conclusions cannot be drawn owing to the low size of the cohort, the preliminary results encourage to investigate the usefulness of preventive ECP as a remedy to reduce the incidence and/or severity of acute GvHD.

\section{P871}

A pilot study of levetiracetam (Keppra®) for seizure prophylaxis in children receiving IV busulfan for haematopoietic stem cell transplantation

S. Soni, M. Skeens, V. Pai, R. Bajwa, T. Gross, A. Termuhlen Nationwide Children's Hospital (Columbus, US)

Background: For Busulfan (BU) administration, targeting a narrow therapeutic range is essential to balance toxicities and outcomes. Targeted AUC of 900-1500 microM/L, based on first dose pharmacokinetics (PK) is utilized to assure dosimetry of intravenous (iv) BU. Phenytoin is also used for BU induced seizure prophylaxis in children. Phenytoin is a CYP3A, CYP1A2 and glutathione-S-transferase inducer and increases the clearance of BU. Hence,continued exposure to Phenytoin may actually lead to less BU exposure than targeted. Hence, anti-seizure prophylaxis with a drug that is not a hepatic enzyme inducer is needed for better $\mathrm{BU}$ dose assurance. We provide results of a pilot study of iv Levetiracetam use for seizure prophylaxis in pediatric patients.

Objectives: 1. To monitor the feasibility, toxicity and efficacy of Levetiracetam; and 2. To compare efficacy, toxicity and outcomes with patients that had received Phenytoin.

Methods: All patients received BU at an initial dose of $0.8 \mathrm{mg} /$ $\mathrm{kg}$ to $1 \mathrm{mg} / \mathrm{kg}$, subsequently adjusted to an AUC of $900-1500$ microM/L based on first dose PK. iv Levetiracetam was given at $10 \mathrm{mg} / \mathrm{kg} /$ dose (maximum: $500 \mathrm{mg} / \mathrm{dose}$ ) infused over $15 \mathrm{~min}$ utes every 12 hours. First dose was administered 6 to 12 hours prior to start of $\mathrm{BU}$ and continued for 24 hours after the completion of BU. Data was retrospectively analyzed for seizure events and toxicity. In the control population, iv Fosphenytoin was administered as a loading dose (15 mg PE/kg), 12-24 hours before $\mathrm{BU}$ followed by age appropriate maintenance dose. Both the groups were retrospectively compared for toxicity, seizures, initial busulfan dose adjustments and outcomes.

Results: 27 pediatric patients undergoing HSCT have received iv Levetiracetam. All patients have tolerated it well with no reports of adverse events or toxicity. The case control group that received iv Fosphenytoin $(n=20)$, was matched for age and type of HSCT. Two breakthrough seizures occurred in the Fosphenytoin group vs. none in the Levetiracetam group. There was no difference between the two groups in terms of adjustments required for BU levels; toxicity (similar incidence of mucositis and SOS) and overall outcomes.

Conclusion: This is the first report of administration of iv Levetiracetam as a single agent for seizure prophylaxis during HSCT. iv Levetiracetam is safe and efficacious in preventing seizures. Further prospective trials of Levetiracetam with close monitoring of its effect on $\mathrm{BU}$ dose assurance are required.

\section{P872}

Graft-versus-host disease after bone marrow

transplantation for children with severe aplastic anaemia. Ten-year experience in large transplant centre

I. Zaidman (1), T. Schecter-Finkelstein (2), J. Doyle (2), A. Gassas (2)

(1)Rambam Medical Centre (Haifa, IL); (2)Hospital for Sick Children (Toronto, CA)

Bone Marrow Transplantation (BMT) from HLA matched identical family donor is the treatment of choice for children with Severe Aplastic Anemia (SAA). In 70\% of patients HLA-matched related donor is not available. For SAA patients who failed to respond to immunotherapy the only curative option is BMT from mached unrelated donor (MUD). Historically MUD BMT was associated with high mortality rates and high incidence of GVHD. We illustrate our 10 years pediatric experience of BMT for SAA patients in Hospital for Sick Children (Toronto) in period from 1998-2008. We retrospectively analyzed results of 59 pediatric patients with SAA (median age 10 years) who underwent BMT from related and unrelated donors. 38 children received related donor allograft and 18 had an unrelated donor or unrelated cord. In this study most patients had bone marrow graft. For prophylaxis GVHD, combination of Cyclosporin with Methotrexate or with Methylprednisolone were used. Conditioning regimen for the related donors included cyclophosphamide and antithymocyte globulin (ATG). For the unrelated, either a single dose total body irradiation (TBI) 200 cGy or fludarabine was added. 5-years Overall survival (OS) for whole group was $84 \%$, but it was different in related and unrelated donor and showed $90 \%$ and $71 \%$ respectively.

Incidence of Acute Graft Versus Disease (AGVHD) was 29\% and grade 3-4 AGVHD - 7\%. OS for all patients with AGVHD was $82 \%$, for patients with severe AGVHD - $75 \%$ versus $90 \%$ without AGVHD.

Incidence of Chronic Graft Versus Disease (CGVHD) was $14 \%$ and severe CGVHD - 4\%. Probability of survival among all patients with CGVHD was $80 \%$ and only $50 \%$ for children with severe GVHD. It was no statistically significant difference between incidence of GVHD and conditioning regimen. In group of patients with grade 1-2 AGVHD it was no difference between related or unrelated donor, $54 \%$ and $46 \%$ respectively in comparison with grade $3-4$ AGVHD $-75 \%$ was after unrelated donor and $25 \%$ after related donor. $87 \%$ of children with CGVHD had previously symptoms of AGVHD. $75 \%$ of patients with CGVHD had full donor chimerism. The median duration of immunosuppression among patients with CGVHD was 434 days. Incidence of infection complications was high in patients with GVHD: $76 \%$ of patients with AGVHD and $87 \%$ with CGVHD developed different viral, bacterial or both infections. Incidence of fungal infection among patients with AGVHD and CGVHD was $35 \%$ and $62 \%$ respectively versus $26 \%$ in group without GVHD.

\section{P873}

Double Glutathione S-transferase null mutations may predict hepatic toxicity in patients receiving busulfan based preparative regimens

P.L. Tan (1), S. Blair (1), S.P. Chen (2), L. Wang (2), S. Leow (2), P.L. Koh (1), B.C. Goh (1), A. Yeoh (2)

(1)National University Hospital (Singapore, SG); (2)National University of Singapore (Singapore, SG)

We prospectively studied busulfan (bu) pharmacogenomics in Asian paediatric patients undergoing blood/ marrow trans- 
plantation (BMT) to determine if patients who have glutathione S-transferase (GST) null mutations have different pharmacokinetic and clinical toxicity profiles when treated using an established weight-based bu dosing strategy. From February 2008 to 2009, 10 patients received bu based preparative regimens. GST genotyping was done by multiplex polymerase chain reaction for GSTM1, GSTT1, and GSTA1. Bu drug levels were taken (as per drug insert) to measure area under the curve (AUC) levels and analysed using high performance liquid chromatography. Eight patients ( 5 boys, 3 girls), median age 6 (range, 1-21) years were recruited, 1 patient was ineligible and another declined. Indications for BMT included acute myeloid leukaemia $(\mathrm{N}=4)$, haemophagohistiolymphocytosis $(\mathrm{N}=1), \mathrm{CD} 40$ ligand deficiency $(\mathrm{N}=1)$ and transfusion-dependent thalassaemia major $(\mathrm{N}=2)$. All patients received unrelated donor BMT (5 bone marrow, 1 peripheral blood stem cells, 2 cord blood). Preparative regimens included bu-cyclophosphamide (CPM) $(\mathrm{N}=1)$, bu-CPM-fludarabine $(\mathrm{N}=2)$ and bu-fludarabine-melphalan $(\mathrm{N}=5)$. All patients received oral phenytoin for seizure prophylaxis. Myeloid engraftment occurred in all at median of 20 (range, 13-46) days. While all patients had hyperbilirubinemia, only 2 patients had moderately severe hepatic sinusoidal obstructive syndrome (SOS) with complications. All had oral mucositis; none had seizures. One patient had secondary graft failure, successfully re-transplanted with same donor. All patients are alive and well at a median of 218 days (range, 85-441) days from BMT. Six of 8 patients had bu levels above the target range (900-1500 uM.min). Of the 8 patients, 6 had GSTM1 null and 3 had GSTT1 null mutations. Of those with mutations, 2 patients had double GSTM1 and GSTT1 null mutations. These 2 patients had toxic bu levels and SOS. Using standard weight-based bu dosing regimen designed to improve bu levels targeting without need for therapeutic drug monitoring and dose-adjustment for paediatric Asian patients, only $1 / 4$ of our patients achieved target levels. This is in contrast to the experience in Europe where $>90 \%$ of patients achieves target levels. The observation that clinically significant hepatic toxicity occurred only in patients who carry double GST null mutations is interesting and should be evaluated in a larger series.

\section{P874}

Reduced-intensity conditioning in children with non-malignant inherited diseases: single- centre experience

N. Stancheva, E. Semenova, N. Zubarovskaya, Y. Vasilieva, A. Borovkova, V. Vavilov, L. Zubarovskaya, B. Afanasyev $\mathrm{SPb}$ State I. Pavlov Medical University (St. Petersburg, RU)

Background: Allogenic haematopoietic stem cell transplantation (alloHSCT) is effective approach of treatment for some inherited diseases but efficacy of the usage of different conditioning regimen is controversial. Full intensity myeloablative conditioning is associated with a lot of early and late treatment related toxicity (TRT) that limits its usage especially in patients with advanced stage of disease. This conditioning regimen is connected with high level of treatment related mortality (TRM). Reduced intensity conditioning (RIC) regimens significantly decreases development of different complications and reduces TRM. But this conditioning regimen is associated with increased frequency of graft failure or allograft rejection in inherited diseases.

The aim of this study: To estimate efficacy of RIC in patients (pts) with some inherited diseases.

Patients: From May, 2006 to October, 2009 matched unrelated (MUD) alloHSCT were performed in 6 pts with following diseases: $1 \mathrm{pt}$ - Wiscott-Aldrich syndrome, $1 \mathrm{pt}$ - Krabbe disease, $1 \mathrm{pt}$ - metachromatic leucodystrophy, $1 \mathrm{pt}-\mathrm{x}$-linked adrenoleucodystrophy, $1 \mathrm{pt}$ - malignant osteopetrosis, $1 \mathrm{pt}$ - DiamondBlackfan anemia; 3 boys, 3 girls, median age $-4,8$ year (from 1 to 14 y.o.). Conditioning regimen includes Fludarabine $150 \mathrm{mg} / \mathrm{m}^{2}$ + Melphalan $140 \mathrm{mg} / \mathrm{m}^{2}+$ ATGAM $60 \mathrm{mg} / \mathrm{kg}$ («Pfizer») in $5 \mathrm{pts,}$ $1 \mathrm{pt}$ with Diamond - Blackfan anemia was transplanted by conditioning regimen Fludarabin $150 \mathrm{mg} / \mathrm{m}^{2}+$ Cyclophosphamide 600 $\mathrm{mg} / \mathrm{m}^{2}$. Acute graft versus host disease (aGvHD) prophylaxis was by CsA from D-1 and short course of MTX. Source of HSCT: 5 - PBSC, 1- BMT. Median CD34+-6,8*10\%/kg.

Results: 2 -years overall survival is $75 \%$. Five pts are alive without any signs of disease with follow-up 2-43 months. One pt with metachromatic leucodystrophy died on $D+37$ from CNS complication after alloHSCT. One pt with Diamond - Blackfan anemia was non-engrafted and received 2nd alloHSCT with MAC from the same donors. During RIC alloHSCT none patients developed severe toxicity (III-IV stage according WHO classification). Four pts was engrafted on $D+14$ (range $D+12-$ $D+19$ ) with achievement of full donor chimerism on $D+30$.

Complications: Skin aGvHD II stage - in 3 pts, 1 pt had gut GvHD IV stage, which was successfully treated by steroids and monoclonal antibody therapy.

Conclusion: RIC is effective in children with non-malignant inherited diseases, has low toxicity and is the promising alternative to myeloablative conditioning regimens.

\section{P875}

Mesenchymal stromal cells isolated from children with juvenile myelomonocytic leukaemia show aberrant differentiation compared to healthy paediatric donors F.G.J. Calkoen, C. Vervat, A.G.S. van Halteren, R.M. Egeler, M.J.D. van Tol, L.M. Ball

Leiden University Medical Center (Leiden, NL)

Normal hematopoiesis relies on the functional integrity of mesenchymal stromal cells (MSC) within the bone marrow (BM), which may be dysregulated by malignant cell infiltration. Myelodysplastic syndrome (MDS) is a characterized by hematomorphological dysplasia, cytopenia, cytogenetic abnormalities and leukemic transformation. In children there is an overlap with the myeloproliferative syndromes, many of which are unique to the pediatric age group. The most common is Juvenile Myelomonocytic Leukemia (JMML), a rare form of leukemia which only affects young infants. Marrow stromal defects have been reported in MDS and stromal/hematopoietic stem cell interactions may influence initiation and/or progression of MDS.

We undertook phenotypical and functional analysis of BM derived ex vivo expanded MSC from children with JMML referred for HSCT, in order to determine if MSC differed from those from normal healthy pediatric donors.

BM (10cc) was obtained according to an ethical committee approved protocol. Post-Ficoll separated MNCs were plated in plastic non-coated culture flasks in Dulbecco modified Eagle medium with Glutamax (DMEM; GiboBRL) supplemented with pen/strep (GibcoBRL) and 10\% fetal bovine serum (FBS; Biochrom AG, Berlin, Germany). Cultures were harvested at $80 \%$ confluency, trypsinized (GibcoBRL) and maintained for up to 6 passages at $37^{\circ} \mathrm{C}$ and $5 \% \mathrm{CO} 2$. The adherent cells were analyzed by flow cytometric analysis (positive: CD73, CD90, CD105; negative: CD3, CD31, CD34, CD45, CD86 HLA-DR). Expanded MSC were induced according to published methodology (1) into adipose and osteogenic lineages. Results of 4 children with JMML were compared to those of normal healthy pediatric controls. JMML patient details and results are summarized in Table I.

Two patients with JMML showed aberrant mesodermal differentiation into adipose or osteogenic tissue. Interestingly, both patients showed no PTNP11, RAS or NF1 gene mutations. One harbored mutations in the region of $11 q$ isodisomy ( $C B L$ gene). Both patients with abnormal MSC developed acute VOD and severe toxicity following conditioning which may be related to underlying abnormalities of stromal cells. Whether these abnormalities persist post HSCT requires investigation. To date no other reports of abnormal MSC function has been described in patients with JMML and further research is warranted to determine the significance of these findings in children undergoing HSCT.

(1) In 't Anker PS et al. Haematologica 2003; 88: 845-852. 


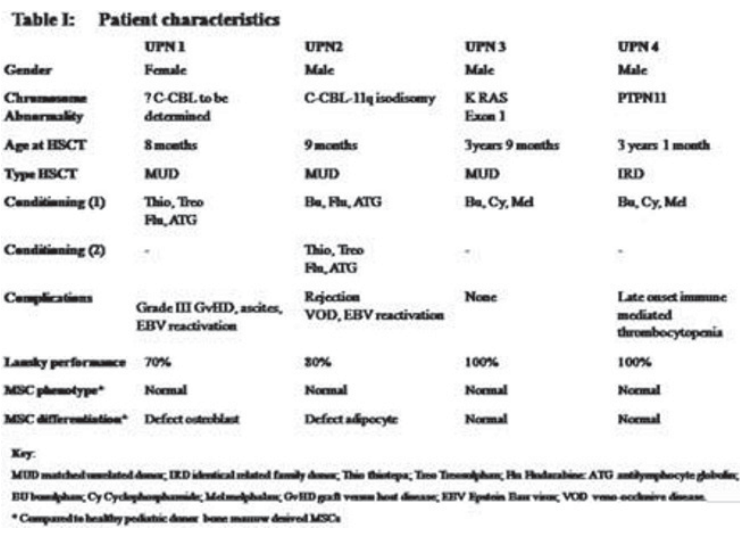

P876

Paediatric haematopoietic stem cell transplantation at King Hussein Cancer Center: a model of success from a developing country

A. Hussein, A. Al-Zabin, R Rihani, F. Abdel-Rahman, H. El-Ta'ani, H. Al-Khatib, M Sarhan

King Hussein Cancer Center (Amman, JO)

Purpose: To analyze our experience in Jordan in performing pediatric Hematopoietic Stem Cell Transplantation (HSCT).

Patients and methods: We reviewed the medical files of all children less than 18 year of age, who received HSCT at King Hussein Cancer Center (KHCC) from establishing the HSCT program in January, 2003 till September, 2009.

Results: A total of 241 children were transplanted, at an average of 35 cases per year. One-hundred-twenty eight patients $(53 \%)$ were males and 113 females (47\%). Thirty-two patients $(13 \%)$ were less than 2 year of age, while 163 patients $(68 \%)$ were between $2-12$ year, and 46 patients $(19 \%)$ between $12-18$ year of age. Thirty seven patients (15\%) received autologus transplantation; mainly for Lymphomas (49\%) and Neuroblastoma (42\%). While 204 patients (85\%) received allogeneic transplantation, that included 174 patients $(72.2 \%)$ from matched related donors, 17 patients $(7.1 \%)$ from cord blood (4 related and 13 unrelated), 12 patients $(5 \%)$ from haploidentical donors, and one patient $(0.4 \%)$ from unrelated donor. Myelo-ablative conditioning was given used in 213 patients (88\%) and non-myeloablative in 28 patients $(12 \%)$. The indication of transplantation was malignant diseases in 138 patients (57\%),and non-malignant disorders in 103 patients $(43 \%)$. The main malignant disease was Acute Myeloid Leukemia $(26 \%)$ followed by Acute Lymphoid Leukemia (25\%) and Chronic Myeloid Leukemia ( $8 \%)$. While the main non-malignant diseases was Thalassemia major (38\%),followed by congenital and acquired bone marrow failure diseases $(30 \%)$ and primary immune-deficiency syndromes $(20 \%)$. The overall survival for all patients at 3 and 5 years was $72.8 \%$. The event-free survival at 3 and 5 years was $64.3 \%$ and $63.2 \%$ respectively. The 100 days transplant-related mortality for all patients (including unrelated transplantation) was $7.1 \%$ (17 patients), 10 patients $(4.1 \%)$ with malignant disease and 7 patients $(2.9 \%)$ with non-malignant diseases. We have good supportive care that includes pediatric intensive care unit, psycho-social service and play therapy. More and detailed results will be presented in the conference.

Conclusion: HSCT for children can be performed safely with an excellent outcome in developing countries. We have a low transplant-related mortality despite most of the cases received allogeneic HSCT. Team work, multidisciplinary action, tailoring management plan according to patient clinical status, and use of available resources effectively can lead to excellent results.
P877

Autologous stem cell transplantation in children with newly diagnosed high-risk or relapsed brain tumours. The Hungarian results

G. Benyó (1), R. Simon (2), P. Hauser (3), K. Kállay (1), V. Goda (1), G. Vértesi (2), G. Márton (2), Á. Tóth (1), Á. Kelemen (2), D. Schuler (3), M. Garami (3), K. Nagy (2), G. Kriván (1)

(1)Unified St István and St László Hospital (Budapest, HU); (2)Borsod-Abaúj-Zemplén County Teaching Hospital (Miskolc, HU); (3)Semmelweis University of Medicine (Budapest, HU)

Introduction: Brain tumors have a high incidence in Hungary comparing to other countries. Conventional treatments for high risk and recurrent medulloblastoma $(\mathrm{MBL})$, supratentorial primitive neuroectodermal tumors (PNET) and atypical teratoid rhabdoid tumors (ATRT) have a very poor prognosis in children. The administration of high dose chemotherapy (HDCT) with autologous hematopoietic stem cell rescue (AHSCT) in the high risk patients has improved survival rates.

Patients and methods: We present 21 patients (males: 10, females: 11) who received HDCT and AHSCT in Hungary between April 1, 2005 and Jun 30, 2009. The median age at transplantation was 5,99 years (range 1,03-16,68 years). High risk (HR) patients were defined as follows: partial removal or anaplastic MBL, PNET or ATRT in patients older than 3 years and all patients younger than 3 years. All relapsed patients were also referred to HR group. Patient's stem cells were mobilized with granulocyte colony-stimulating factor at a dose of 10 microgram $/ \mathrm{kg}$ subcutaneously twice daily. One child was transplanted with BM graft. Mobilization was done in the early phase of the conventional chemotherapy. The conditioning regiment in nineteen patients consisted of busulfan $4 \mathrm{mg} / \mathrm{kg} / \mathrm{day}$, intravenously (iv.) over 4 days in 6-hourly divided doses, thiotepa $250 \mathrm{mg} / \mathrm{m}^{2} /$ day iv. over 2 days and etoposid $750 \mathrm{mg} / \mathrm{m}^{2}$ a single dose iv. Two patients got busulfan (dose above) + melphalan at a dose of $140 \mathrm{mg} / \mathrm{m}^{2} /$ day by iv. on day -1 .

Results: The engraftment was achieved median on day +9 (range 7-21). The median follow up time is 1,3 years (range $0,37-4,65)$. The estimated 3 years overall survival (OS) was: $69,4 \% \pm 11,7 \%$ the relapse free survival (RFS) was $58,6 \% \pm 13 \%$ in this HR patients group. These result were in the newly diagnosed HR MBL group 78,8\% $\pm 13,4 \% / 60 \% \pm 16,9 \%$ respectively. Two of the three lost MBL patients were transplanted in a relapsed disease.

All death occurred due to relapse, there was no treatment related mortality (TRM) during this period of time.

Conclusions: The survival of patients with HR brain tumors highly improved with HDCT plus AHSCT in the risk adapted Hungarian Medulloblastoma protocol (MBL 2004). The improvement was primary due to the better survival of the newly diagnosed HR MBL patients. The good results were achieved without TRM.

\section{P878}

Bone marrow from healthy childhood donors reveal excess of reduced iron reserves related to age at donation S.M. van Walraven, L.M. Ball

Leiden University Medical Centre (Leiden, NL)

Bone marrow examination in healthy children is exceptionally rare. Children acting as healthy sibling donors undergo multiple bone marrow (BM) aspirates in order to donate sufficient volume of stem cells for their sibling. They provide an ideal basis for examining the morphology of BM in normal healthy children. As these donors are screened before donation including routine hematology and biochemistry, and found 'fit', it is assumed that their BM is normal.

We undertook a retrospective analysis of BM aspirated at the time of donation and submitted for routine hemato morphological examination in pediatric donors, in order to screen for abnormalities.

Included aspirates were obtained from pediatric donors underwent BM harvesting between 1981 and 2004 and whom 
representative material was available for review. Inclusion criteria were: younger than 13 yrs at donation. From 145 available samples, $39 \%$ were either clotted, marrow blood or insufficient for iron evaluation and considered not representative and therefore not included in the subsequent analysis.

Review of the original reports was undertaken by a trained pediatric hemato morphologist in order to screen material for further detailed review. Aspirates were routinely stained with Hematoxylin and Eosin as well as for iron deposits, Together with blood smears and routine hematological values these were correlated with donor clinical details.

Representative material was available from 88 donors, aged between 0.6 and 12 years (mean 6.8 yrs). Fifty donor aspirates revealed reduced iron stores defined as either absent $(n=17)$ reduced sideroblasts $(n=33)$. Six children had frank iron deficient anemia, whereas most children had low or iron stores with minimal peripheral blood hypochromia without frank anemia. Infants and pre pubertal children were more likely to have lower iron stores than children aged between 6-8 yrs.

Although children with the most severe iron depletion received either autologous and/or homologous erythrocyte transfusions no routine hematological follow up was undertaken.

Other abnormalities included $6 \%$ white cell abnormalities, mainly undiagnosed hypereosinophilia, lymphocytosis and one case of leucopenia. Two donors had abnormal megakaryopoiesis, but with normal peripheral platelet counts.

We recommend, based upon these findings, that closer attention be paid to hematological parameters in young donors, pertinent to iron stores.

\section{P879}

Early engraftment of G-CSF-primed unmanipulated allogeneic bone marrow transplantation in paediatric patients

H. Kim, J.W. Lee, H.J. Kang, K.D. Park, H.Y. Shin, H.S. Ahn Seoul National University College of Med (Seoul, KR)

Growth factor administration to donors prior to bone marrow (BM) harvest results in enrichment of the graft for myeloid precursors and leads to early engraftment. A total of 41 patients received allogeneic transplantation from HLA-identical siblings, using granulocyte colony-stimulating factor (G-CSF)stimulated BM. All donors received G-CSF $10 \mathrm{mcg} / \mathrm{kg}$ per day subcutaneously for 2 days prior to the harvest. The diseases of the patients were; aplastic anemia $(n=6)$, acute lymphoblastic leukemia $(n=13)$, acute myeloid leukemia $(n=18)$, chronic myeloid leukemia $(n=2)$, myelodysplastic syndrome $(n=1)$, and idiopathic myelofibrosis $(n=1)$. Conditioning regimens varied according to the disease status. The GVHD prophylaxis consisted of cyclosporine and prednisolone was given in all patients. In 18 transplants, the body weight of the donors was lower than the patients with median difference of $8.58 \mathrm{~kg}$ (range 1.1-29.8 kg). The median number of the total nucleated cells infused was $8.4 \times 10^{8} / \mathrm{kg}$ (range $3.93-23.5 \times 10^{8} / \mathrm{kg}$ ), and the median number of CD34 + cells infused was $3.74 \times 10^{6} \%$ $\mathrm{kg}$ (range $0.33-31.9 \times 10^{6} / \mathrm{kg}$ ). The median number of days required for ANC to exceed 500/uL and 1,000/uL was 10 days (range 8-25 days) and 10 days (range 9-26 days), respectively. Spontaneous platelet recovery to more than $20,000 / \mathrm{LL}$ required a median 14 days (range $6-58$ days). The overall survival and event free survival were $69.3 \%$ and $65.1 \%$, respectively. Thirteen events occurred after transplantation resulting in; 9 relapses and 4 non-relapse-mortalities (Pneumocystis jiroveci pneumonia; 1 , adenovirus pneumonia; 1 , chronic GVHD; 2). Reversible acute GVHD occurred in 6 patients (Grade I; 4, II; 1, III; 1) and all transplantation-related toxicities were also reversible.

Our results suggest that the use of cytokine-stimulated BM leads to a successful engraftment even in cases matched for leaner donors and allows reversible toxicities, and tolerable acute GVHD, hence providing a compatible alternative to conservative allo-BM and PBSC.
P880

Cartilage hair hypoplasia: successful reduced-intensity conditioning stem cell transplantation from HLA-identical unrelated donor and invasive cytomegalovirus infection E. Kacinska-Pfaller (1), C. Peters (1), S. Matthes-Martin (1), L. Bonafe (2), L. Mittaz (2), R. Geyeregger (3), T. Lion (3)

(1)St. Anna Children's Hospital (Vienna, AT); (2)University of Lausanne (Lausanne, CH); (3)Children's Cancer Research Institute (Vienna, AT)

Cartilage hair hypoplasia $(\mathrm{CHH})$ is an autosomal recessive inherited disorder that results in short-limb dwarfism associated with T-cell and B-cell immunodeficiency. Severe combined immunodeficiency associated with this rare disease has been recently proven to be curable by allogeneic stem cell transplantation (SCT).

We present a 5-month-old infant with $\mathrm{CHH}$ who suffered from 2-months-long history of liver disease and cytomegalovirus (CMV) infection (gastroenteritis, encephalitis, retinitis). Genetic analysis of the RMRP gene showed in our patient and her brother with the same phenotype, a homozygous mutation ( $g$. $252 \mathrm{~T}>\mathrm{G}$ ) that has not been reported before. Typical phenotype, severe combined immunodeficiency and life threatening infections qualified our patient for a reduced-intensity conditioning regimen SCT from HLA-identical unrelated donor to minimize the risk of severe toxic side effects.

The conditioning regimen included fludarabine $\left(160 \mathrm{mg} / \mathrm{m}^{2}\right)$, thiotepa $(10 \mathrm{mg} / \mathrm{kg})$, melphalan $\left(140 \mathrm{mg} / \mathrm{m}^{2}\right)$. Cyclosporine A, mycophenolate-mofetil and ATG were given for graft-versushost-disease (GvHD) prophylaxis. Hematological engraftment occurred on day +9 . Transplant-related toxicity consisted of orointestinal mucositis grade III, skin toxicity grade II and sepsis in aplasia.

Prior to SCT CMV viraemia has been proven in the host (CMV PCR $10^{3} / \mathrm{ml}$ serum). Despite intensive antiviral treatment with gancyclovir (no resistance found), during immunosuppression CMV load increased in serum until maximum $105 / \mathrm{ml}$. Since day $+10 \mathrm{CMV}$ retinitis has progressed and additional uveitis and vitritis led to total bilateral amaurosis until day +42 (evoked visual potentials not present). The donor serological status was antiCMV IgG positive and as early as on day +16 donor-derived CMV-specific CD8 lymphocytes were detected and increased rapidly in peripheral blood of the host.

One-hundred-twenty days after transplantation the patient is alive, with $100 \%$ donor chimerism, no GvHD signs, spasticity after CMV encephalitis and bilateral amaurosis. The child still receives valgancyclovir and since day $+42 \mathrm{CMV}$ has not been detected in peripheral blood any more.

In conclusion, we demonstrate that SCT with a reduced-intensity conditioning regimen in patients with $\mathrm{CHH}$ may successfully eradicate the severe combined immunodeficiency. CMV specific immunological reconstitution after SCT from a CMV positive donor has been shown to be possible very early after transplantation.

\section{P881}

Clofarabine-based condition regimen in children with acute lymphoblastic and myeloid leukaemia

R. Simon, K. Nagy, G. Vertesi, A. Kelemen

Borsod University Hospital (Miskolc, HU)

The safety and efficacy of clofarabine-based condition regimen were evaluated in children with advanced acute lymphoblastic (ALL) and myeloblastic leukaemia (AML). Clofarabine, a next-generation deoxyadenosine analogue, was developed on the basis of experience with cladribine and fludarabine in order to achieve higher efficacy and avoid extramedullary toxicity. During the past decade this is the only drug granted approval for treatment of pediatric acute leukemia. In our institution over an 8 month period 6 children ( $2 \mathrm{AML}$ and $4 \mathrm{ALL}$ ) with advanced acut leukaemia were transplanted with the use of clofarabinebased condition regimen. The median age was 7.05 years 
(4,5-11.5). The condititon regimen consisted of clofarabine 50 $\mathrm{mg} / \mathrm{m}^{2} / \mathrm{d} 4$ days long, Busilvex $16 \mathrm{mg} / \mathrm{tbwkg}$, Cyclophosphamid $120 \mathrm{mg} / \mathrm{tbw} \mathrm{kg}$ and for unrelated HSCT Anti-Thymocyta Globulin $60 \mathrm{mg} / \mathrm{tbwkg}$. GVHD profilaxis consisted of cyclosporin and methotrexate. This was a first HSCT for 5 pts and a 2nd for one patient. Donors were HLA-matched siblings $(\mathrm{N}=3)$ or HLA matched $(\mathrm{N}=1)$ or mismatched unrelated donors $(\mathrm{N}=2)$. All 6 pts engrafted. Toxicity of the myeloablative chemotherapy included elevation of hepatic transaminases in 5 of 6 patients with a normalization of all patients. No venoocclusiv disease was detected. With a follow-up of 4-11 mo (median $6.5 \mathrm{mo}$ ), 4 of 6 patients are alive, disease-free. 1 patient died of engraftment syndrome at day +40 and 1 patient died of grade 4 gut GVHD caused multi organ failure at day +100 . Four patients developed acut GVHD. No chronic GVHD was detected. Clofarabine was well tolerated as part of a conditioning regimen for allogeneic transplantation. We detected no relapse of original disease. Immunosuppressive effects appeared to be similar to those of fludarabine, Additional studies are warranted to evaluate whether clofarabine can contribute to reduce the risk of relapse in patients with advanced disease.

\section{P882}

Effectiveness and safety of voriconazole in primary antifungal prophylaxis for children undergone allogeneic stem cell transplantation

D. Tallon, J.R. Molina, J. Serrano, P. Gomez, A. RodriguezVilla, C. Cerrato, A. Torres

University Hospital Reina Sofia (Cordoba, ES)

Introduction: Invasive fungal infection (IFI) incur significant morbidity and mortality among children undergone Allogeneic Stem Cell Transplantation (Allo-SCT) due to neutropenic phase and posterior immunosuppression. We have analyzed in this prospective study our experience in pediatric patients who received voriconazole as primary antifungal prophylaxis undergone Allo-SCT.

Methods and patients: A total of 50 children and adolescents $<18$ years were enrolled in this study from Oct-2004 to May2009, median age was 9 years (range: $1-17$ ). All patients received voriconazole at a dose of $5 \mathrm{mg} / \mathrm{kg} / 12$ hours $(\mathrm{n}=23)$ or $7 \mathrm{mg} / \mathrm{kg} / 12$ hours $(\mathrm{n}=28)$ until a highest dose of $200 \mathrm{mg} / 12$ hours, from day -1 to day +75 or later in patients with acute graft versus host disease (aGVHD). Patients were followed up for six months from Allo-SCT. IFI (proven or probable) was diagnosed according to EORTC/MSG criteria.

Results: Only one (2\%) patient developed IFI (Proven Aspergillosis) while prophylaxis with voriconazole and died due to it. In this series $32(64 \%)$ patients completed successfully the treatment without adverse effects, no empirical or preemptive antifungal therapy neither IFI, in 29 of these 32 patients voriconazole was stopped on day +75 , in the rest 3 children, due to aGVHD, it was maintained until a mean day +145 . A total of $9(18 \%)$ receptors needed an empirical $(n=7)$ or preemptive $(n=2)$ antifungal therapy, voriconazole was stopped and replaced with amphotericin $B$ in patients under 14 years $(n=6)$ or caspofungine in patients between 14 and 17 years $(n=3)$ during neutropenia period. In $10(20 \%)$ children was detected adverse effect due to voriconazole prophylaxis and needed a definitive withdrawal. The most frequently adverse effects due to this azole was persistent hepatic damage with cytolysis enzymes elevation $>2.5$ basal levels in $7(14 \%)$ children. Skin rash $(n=1)$, pancreatitis $(n=1)$ and tremor $(n=1)$ were the others secondary effects observed. All adverse effects were solved once stopped voriconazole.

Conclusion: With an overall IFI incidence of $2 \%$, our results suggest that primary antifungal prophylaxis in children undergone Allo-SCT with voriconazole is effective at reducing the incidence of IFI in this type of high risk pediatric patients. Voriconazole is well tolerated despite a high percentage of adverse effects easily solved by drug stopped without life-threatening complications.
P883

Management of severe aplastic anaemia in childhood: a single-centre experience over $\mathbf{3 0}$ years

P. Sovinz, H. Lackner, W. Schwinger, M. Benesch, A. Nebl, C. Urban

Medical University of Graz (Graz, AT)

Background: Treatment of choice for children with severe aplastic anemia (SAA) is a matched sibling donor (MSD) BMT; children without MSD receive combined immunosuppressive therapy (IST) and are eligible for alternative donor SCT as second line therapy in case of insufficient response to IST.

Patients and methods: Between 1978 and 2009, 28 patients (m: $f=13: 15$; median age 11.0 years) were diagnosed with SAA. Causes were hepatitis (6), virus-infections (ParvoB19: 4; EBV: 2), febrile infections (4), drugs (3), pregnancy (1); no cause was identified in 6 patients, one patient presented with constitutional disease.

Primary stem cell transplantation from MSD was performed in 10 patients ("SCT-group"). Stem cell sources were bone marrow (BM: 9) and CD34 + selected peripheral blood stem cells (PBSC: 1). All patients received non-radiotherapy-based conditioning with cyclophosphamide; ATG was added in 4 patients. Graft-versus-host $(\mathrm{GvH})$ prophylaxis consisted of cyclosporine A (6), methotrexate (2) or a combination of both (2).

The remaining 18 patients received IST consisting of ATG, methylprednisolon and cyclosporine A ("IST group").

Results: In the SCT-group, 9/10 patients survive for a median of 12.7 years (4.8-31.5 years); one patient with perinatal asphyxia died 3.5 years after SCT of cardiac failure; 1 patient developed limited chronic GVHD.

In the IST-group, 8/18 (44\%) patients achieved complete remission (CR) after a median of 1.45 years $(0.6-10.6$ years) and survive for a median of 10.3 years $(0.6-22.2$ yrs). Three patients in partial remission are still on IST; one patient died from hepatic failure 19 years after diagnosis. The 6 remaining patients not in CR were transplanted from alternative donors.

(MUD: 4; mother: 1; UCB: 1) after different conditioning regimens; stem cell sources were CD34 + selected PSCS (5) and cord blood (1). Two patients rejected their grafts and required more than one SCT $(2: n=1 ; 3: n=1) .5 / 6$ patients survive for a median of 8 years after SCT (0.3-11.2 years) whereas one patient with chronic GvHD died of sepsis on day +312 .

Conclusions: Over a period of more than 30 years, 25/28 $(89,2 \%)$ patients diagnosed with SAA survive after IST and/or SCT. Primary MSD-SCT remains the best treatment option. Following IST, only $8 / 18$ patients $(44 \%)$ achieved complete remission with a long treatment duration and significant morbidity; $6 / 18(33 \%)$ required alternative-donor stem-cell transplantation as second-line therapy, 5 of whom survive.

\section{P884}

Short-term cryopreserved allogeneic stem cells to optimize graft and recipient conditions in children P. Sovinz, W. Schwinger, H. Lackner, M. Benesch, A. Nebl, A. Raicht, S. Sipurzynski, K. Rosskopf, C. Urban Medical University of Graz (Graz, AT)

Background: Stem cell grafts intended for allogeneic hematopoietic stem cell transplantation are usually collected and transfused "fresh". Data regarding the use of cryopreserved allografts are scarce and limited to adults.

Patients and methods: We report on 4 pediatric patients aged $2.6,1.75,15.9$ and 11.3 years, respectively ( $m: f=1: 3)$ suffering from relapsed acute myelogenous leukemia FAB M5, Mucopolysaccharidosis I Hurler, severe aplastic anemia and metastatic osteosarcoma, respectively, in whom conditioning had to be unexpectedly postponed at short notice for medical reasons (infections $n=3$; non-remission $n=1$ ). Stem cell donors (unrelated $n=3$, HLA match: 10/12, 11/12, 12/12; haploidentical related $n=1$ ) gave their consent to short time stem cell cryopreservation and graft manipulation. Stem cell grafts were manipulated (CD3/19 depletion: $n=4$; additional CD34 + 
selection: $n=2$ ) and cryopreserved for $6,13,11$ and 102 days, respectively. After conditioning with busulfan/ treosulfan-based $(n=2)$ or fludarabine-based $(n=2)$ regimens patients received $10.55,20.3,12.9$ and $15.37 \times 10^{6} \mathrm{CD} 34+$ cells $/ \mathrm{kg}$ together with $3.27 \times 10^{8}, 1.2,0.55$ and $1.0 \times 10^{5} \mathrm{CD} 3+$ cells $/ \mathrm{kg}$, respectively, without relevant transfusion-related side-effects.

Results: All patients showed sustained hematopoietic engraftment. One patient developed acute and chronic intestinal GVHD and died on day +140 of Aspergillus pneumonia in remission of leukemia. The other three patients had an uneventful posttransplant course with a current follow-up of 138, 59 and 21 days without GVHD or other complications.

Conclusions: Short-term cryopreservation of unrelated PBSC allografts in pediatric patients is possible for compelling medical or logistic reasons. The advantages of the use of cryopreserved grafts must be individually outweighed against the concerns raised but not definitely answered by the available data. In view of the increasing use of manipulated grafts short-term cryopreservation might be an option to ensure optimum graft and recipient conditions.

\section{P885}

Haploidentical stem cell transplantation in children and young adults

W. Schwinger, P. Sovinz, H. Lackner, M. Benesch, A. Nebl, S. Sipurzynski, C. Urban

Medical University of Graz (Graz, AT)

Background: In the absence of an HLA-identical donor haploidentical family donors are a useful alternative offering the advantage of being readily available for donation of stem cells and donor lymphocytes.

Patients and methods: Since 199717 patients ( $m: f=5: 12$; median age: 12.4 years; range: 3 months-24 years) underwent haploidentical stem cell transplantation (1 patient was retransplanted). In all patients there was an urgent indication for stem cell transplantation in the absence of a suitable unrelated donor. Diagnoses were hematological diseases in 8 patients (refractory AML: 2; HR-ALL: 2; SCID: 1; SAA: 1; ALPS: 1; relapsed Hodgkin's disease: 1); advanced/relapsed solid tumors in 8 patients (neuroblastoma: $n=3$; osteosarcoma: $n=2$; Ewing's sarcoma: $n=2$; Wilms-tumor: $n=1$ ) and Krabbe's disease:1. All patients were intensely pretreated, including highdose chemotherapy with autologous stem-cell-support $(n=6)$. 15 patients received fludarabine-based conditioning regimens, 1 patient received $\mathrm{Bu} / \mathrm{Cy}$, the SCID-patient was transplanted without conditioning.

Stem cell donors were mother $(n=8)$, father $(n=8)$, brother $(n=1)$, sister $(n=1)$. In 6 donor-recipient-pairs a KIR.mismatch in GvHD-direction was identified. Stem-cell sources were bone-marrow $(n=2)$ and peripheral blood stem cells $(n=16)$. Bone marrow was used unmanipulated; peripheral blood stem cells were CD34 + selected $(n=4), C D 3 / 19$ depleted $(n=1)$ or both $(n=11)$. Median number of CD34 + cells was $11.9 \times 10^{6} / \mathrm{kg}$ (range $2.46-139 \times 10^{6} / \mathrm{kg}$ ).

Results: All patients had complete 3 -lineage engraftment; the patient suffering from $\mathrm{Mb}$ Krabbe rejected on day +33 and was retransplanted from the other parent. Seven patients with malignant disease received donor lymphocyte infusions as adoptive immune therapy ( $n: 4-108)$. Twelve patients survive a median of 26 months (9-78 months; CR: 7, SD: 1; PR: 2, PD: 2). Five patients died of PD:1, GvHD:1, sepsis:1 and MOF:3.

Conclusions: Haploidentical stem cell transplantation with modern stem cell manipulation techniques and immunosuppressive therapy is a feasible alternative for patients without available HLA-identical donor and with urgent need of a stem cell transplant. The graft-versus-tumor/leukemia potential of this technique is currently under investigation.
P886

Efficiency of haematopoietic stem cell transplantation in the treatment of refractory or multiple relapsed malignant lymphoproliferations. A single-centre experience

C. Jinca (1), S. Arghirescu (1), A. Isac (2), A. Oprisoni (1), L. Balint-Gib (2), E. Gai (1), H. Ionita (1), M. Serban (1)

(1)University of Medicine and Pharmacy (Timisoara, RO); (2)Emergency Children's Hospital "L. Turcanu" (Timisoara, RO)

Introduction: Refractory or multiple relapsed malignant lymphoproliferations continue to be a challenge for therapy and remain in many cases subject to palliative treatment.

Objectives: Taking into account the particular status at transplantation of the patients with malignant lymphoproliferations, with multiple relapses or refractory disease, we aimed to evaluate the outcome of haematopoietic stem cell transplantation (HSCT) in this category of patients.

Patients and methods: This prospective study was performed on 77 patients, 43 with Hodgkin lymphoma (HL), 20 with nonHodgkin lymphoma (NHL), and 14 with acute lymphoblastic leukemia (ALL) transplanted in our department between 2001 and 2009. The outcome was assessed by estimation of 5 years projected overall survival ( $\mathrm{pOS}$ ) and disease-free survival (pDFS). From the $\mathrm{HL}$ group, $72.7 \%$ were either in complete remission (CR) 3, 4 or 5 or in partial remission (PR) at the time of transplantation. For the NHL group, $25 \%$ of the patients were $\geq \mathrm{CR} 2$ and $15 \%$ in PR. From the group of patients with ALL, $42.8 \%$ were in $>C R 2$ at the time of transplantation.

Results: A subgroup of patients (63.5\%) had consolidation or maintenance therapy after autologous transplantation: 44\% had only chemo, $17 \%$ chemo- and radiotherapy, $2,5 \%$ had only radiotherapy. Despite the rather unfavorable pre-transplant condition of the patients, pOS was $79 \%$ in patients with $\mathrm{HL}$ and pDFS was $65 \%$. For the group of patients with $\mathrm{NHL}$, survival analysis revealed a pOS of $59 \%$ and a pDFS of $54 \%$. Patients with ALL presented a pOS of $70 \%$ but a lower pDFS of only $47 \%$.

Conclusion: Our data reveals the fact that the indication for HSCT could be extended, being a life-saving procedure for malignant lymphoproliferations even in cases presenting refractory disease or multiple relapses. Consolidation or maintenance therapy may have an influence on the outcome.

P887

Results of allogeneic stem cell transplantation in children with severe aplastic anaemia - A single-centre experience M. Choma, K. Drabko, B. Wojcik, A. Zaucha-Prazmo, J. Kowalczyk

Medical University of Lublin (Lublin, PL)

Aim of the study: We evaluated the clinical results of allogeneic stem cell transplantation (allo-SCT) performed in children with severe aplastic anemia (SAA) in one institution.

Material and methods: Between 2000 and 2009 twenty three children suffering from SAA was referred to our center for transplantation. There were 15 boys and median age of the group was 9 years (range 8 months to 15 years). 14 patients were transplanted from match related donors (MRD) and alloHSCT was primary treatment of those patients, 7 children had match unrelated donors (MUD) and the procedure was introduced as a salvage therapy after failure of immunosuppressive therapy (IST). One patient was transplanted from haploidentical donor and one patient received sibling graft after IST.

Results: $20 / 23$ patients engrafted, $3 / 20$ did not show engraftment ( 2 unrelated donor and one sibling donor after IST) and one boy was rescued by the second graft from the same unrelated donor. $3 / 20$ patients ( 2 MUD and 1 MUD) after primary engraftment rejected their graft 2,3 and 22 months after alloHSCT and 2 of them were successfully re-transplanted from the same donor. Three patients died from transplant related complication: two girls died from multi-organ failure caused by infections (one before engraftment and one after re-trans- 
plantation) one girl died from lymphoproliferative disease which occurred after re-transplantation and IST failure. 20/23 children are alive and with sustained engraftment median 4 years after transplantation (range 0,5-9 years). All 14 children after transplantation performed as primary treatment are alive and well. 9/20 surviving patients have mixed chimerism and 1 boy has autologous recovery.

Conclusion: Our results confirms, that allogeneic stem cell transplantation is the best therapy for children with severe aplastic anemia. In our experience mixed chimerism does not impair the outcome of patients with SAA after transplantation.

\section{P888}

Allogeneic haematopoietic stem cell transplantation in paediatric patients without a matched sibling donor: Iranian experience

A.A. Hamidieh, M.R. Ostadali, M. Jalili, S. Basirpanah, A. Hoseini, O. Bakhti, A. Ghavamzadeh

Hematology-Oncology and SCT Research Center, Tehran University of Medical Sciences (Tehran, IR)

Background: Human leukocyte antigen (HLA)-identical sibling hematopoietic stem cell transplantation (HSCT) is a curative treatment for life-threatening malignant and nonmalignant diseases. However, only $30 \%$ of patients in need of a transplant will have such donors. For other patients, donors can be found in the extended family of the patient or in registries of unrelated donors or cord blood banks.

Methods: To extend the use of allogeneic HSCT to patients without an HLA-matched sibling donor, we investigated HSCT from other related donors. During 2008 and 2009, an early search for a related donor in the extended family was performed for 108 patients who didn't have HLA matched sibling donors who referred to HSCT center. High resolution typing for class I and II alleles was completed for recipient/donor pairs. Twenty one children received a conditioning regimen based on the use of cyclophosphamide in combination with busulfan. The remaining 7 patients were transplanted with a fludarabine based regimen. All patients were prepared for transplantation with antithymocyte globulin. The most common graft-versushost disease (GVHD) prophylaxis regimens used cyclosporine with methotrexate.

Results: The search for an HLA-matched donor in the extended family was successful in 28 of 108 of the pediatric patients $(26 \%)$ lacking a sibling donor. Twenty eight patients (age range: 1-15 years) with thalassemia major $(n=7)$, acute leukemia $(n=4)$, primary immunodeficiency $(n=6)$, hereditary or acquired bone marrow failure $(n=8)$ and metabolic disorders $(n=3)$ were enrolled. Hematopoietic cells were collected from the bone marrow $(n=18)$ or peripheral blood $(n=10)$. The donors were either parents $(n=19)$, grandfather or grandmother $(n=4)$, and uncle or aunt $(n=5)$ of these patients. All the 28 patients engrafted with neutrophils ( $>500 / \mathrm{microL})$ at a median of 14.5 days and achieved complete donor chimerism (>95\%) 15 days after transplantation. Acute GVHD was present in 9 of 28 patients who engrafted (32\%). TRM occurred in 1 patients (3.5\%).

Conclusion: While some countries may have very limited numbers of donor registries and cord blood banks, others will have many choices. On the other hand there is opportunity of finding other relative donors in some countries because of the marriage between relatives. Therefore, any transplant unit especially in countries that marriage between relatives is not forbidden has to evaluate for each single patient, all the possible alternatives to choose the best donor.
P889

The outcome of children with chronic myelogenous leukaemia given allogeneic haematopoietic stem cell transplantation

A.A. Hamidieh, M. Jahani, K. Alimoghaddam, S.A. Mousavi, M. Iravani, B. Bahar, A. Khodabandeh, M. Jalili, B. Darbandi, A. Ghavamzadeh

Hematology-Oncology and SCT Research Center, Tehran University of Medical Sciences (Tehran, IR)

Background: Although hematopoietic stem cell transplantation (HSCT) in children Chronic Myelogenous Leukemia (CML) has several side effects, this method is still being applied as a modality treatment within this age group. We retrospectively evaluated the results of myeloablative HSCT in pediatric CML after standardized pretreatment in the Hematology-Oncology and SCT Research Center, Tehran.

Patients and methods: Fifteen consecutive pediatric patients $(6$ girls, 9 boys) with Philadelphia chromosome-positive $(\mathrm{Ph}+) \mathrm{CML}$ received HLA-matched related marrow grafts between July 1991 and October 2009. Median age at HSCT was 12.5 years (range, $7-16$ years). The graft source was bone marrow in 5 patients and peripheral blood stem cells in 10 patients. Conditioning regimen was: busulfan $4 \mathrm{mg} /$ $\mathrm{m}^{2} /$ day for 4 days, and cyclophosphamide $60 \mathrm{mg} / \mathrm{kg} /$ day for 2 days. Total body irradiation was not used. Cyclosporine and methotrexate were given for graft-versus-host disease (GVHD) prophylaxis. Their response condition to the treatment was examined carefully.

Results: All patients engrafted, (median, 18 days for an absolute neutrophil count $>0.5 \times 10^{9} /$; median, 22 days for platelet count $\geq 20 \times 10^{\%} / \mathrm{l}$ ). At median follow-up of 13 months (range: 3-136), 9 patients $(60 \%)$ are well with complete donor chimerism and stable continuous molecular-genetic remission. One patient developed decrease in his chimerism. Series of chimerism studies over 1 year confirmed that he has stable mixed chimerism. Other 5 patients developed relapse. Acute GVHD of grade II or more developed in $6(67 \%)$ patients and chronic GVHD developed in $5(33 \%)$ patients. Overall survival (OS) and disease-free survival (DFS) at 1 year was $76 \%$ and $60 \%$ respectively. The median time to neutrophil and platelet recovery, intensity of acute and chronic GVHD and OS and DFS did not differ statistically between the peripheral blood and bone marrow transplantation groups.

Conclusion: Findings indicated that the results of the treatment through HSCT in children suffering from CML are similar to those obtained for adults. But it leads to more DFS years in this age group. The results of the treatment did not differ across the two groups of patients divided by stem cell source. This might be due to the small number of patients in each group.

\section{P890}

High-dose chemotherapy and autologous haematopoietic stem cell transplantation for children with relapsed medulloblastoma: the results of the prospective multicentre study KSPNO-S-053

J.E. Park (1), O.K. Noh (1), J. Kang (2), K.W. Sung (3), K.H. Yoo (3), H.H. Koo (3), H.J. Kang (4), K.D. Park (4), H.Y. Shin (4), H.J. Im (5), J.J. Seo (5), H.J. Park (6), B.K. Park (6), H.S. Ahn (4)

(1)Ajou University School of Medicine (Suwon, KR); (2)Northwestern University (Chicago, US); (3)Samsung Medical Center (Seoul, KR); (4)Seoul National University College of Medicine (Seoul, KR); (5)Asan Medical Center (Seoul, KR); (6)National Cancer Institute (Goyang, KR)

Background: High dose chemotherapy (HDCT) and autologous stem cell transplantation (ASCT) have recently been applied in patients with relapsed pediatric brain tumors but have poor outcome. Some researcher tried tandem transplantation to overcome the poor outcome. We evaluate the effect of the HDCT and ASCT including tandem transplantation in relapsed medulloblastoma patients who enrolled at KSPNO-S-053 protocol. Method (Procedure): The first HDCT was administered with CTE regimen (carboplatin $500 \mathrm{mg} / \mathrm{m}^{2} /$ day, day $-8,-7,-6$; 
thiotepa $300 \mathrm{mg} / \mathrm{m}^{2} /$ day, day $-5,-4$, -3; etoposide $250 \mathrm{mg} / \mathrm{m}^{2}$ / day, day $-5,-4,-3)$. The second HDCT was administered with CM (cyclophosphamide 1,500 $\mathrm{mg} / \mathrm{m}^{2} /$ day, day $-8,-7,-6,-5$; mephalan $60 \mathrm{mg} / \mathrm{m}^{2} /$ day, day $-4,-3,-2$ ).

Results: 16 patients, OS rate ( \pm standard error) at 3 years was $16 \%$ and EFS rate ( \pm standard error) at 3 years was $25 \%$. Among 13 patients who received single or tandem transplantations, 2 year OS rate after 1st HDCT in patients with HDCT and ASCT was $39 \%$ and 2 year EFS rate was 31\%. Of the 13 transplantation patients, 7 patients received tandem transplantations and 6 patients received single transplantation. The number of each patient with pre-HDCT tumor status in CR, PR, SD, and PD were 2, 8, 2 and 1 respectively. The patients with SD or PD status before 1st HDCT all died with median survival period of 4 months (4 11). OS and EFS at 2 years after 1 st HDCT were $50 \%$ and $25 \%$ in CR or $\mathrm{PR}$ at first HDCT $(\mathrm{P}<0.001$ and $\mathrm{P}=0.26$, respectively). In 8 patients received transplantation with PR status before 1 st HDCT, 4 patients received tandem transplantation had $75 \%$ at 2 year survival, while the other 4 patients received single transplantation had no survival until 13 months. Total 8 patients who received tandem transplantation; 4 patients did in PR status before 1st HDCT, 3 patients in PD and one patient in SD status before 1st HDCT. 2 year overall survival rate of 4 patients received tandem transplantation in $\mathrm{PR}$ status is $75 \%$, while 4 patients in PD or SD before 1st HDCT had no survival until 12 months. One patient died due to veno-occlusive disease during 13 episode of 1 st HDCT but no TRM following second HDCT in all seven patients who underwent tandem transplantation.

Conclusion: Tandem transplantation was relatively tolerable in the pediatric patients with relapsed brain tumor in children and helpful to the patients with PR status just before HDCT.

\section{P891}

Umbilical cord blood transplantation in paediatric patients: a single-centre experience in Mexico R.M. Navarro Martin del Campo (1), A.L. Orozco Alvarado (1), F. Sanchez Zubieta (1), E. Calderon (2), M.M. Ortiz Sandoval (1), L. Toro-Castro (1), O. Gonzalez Ramella (1)

(1)Hospital Civil de Guadalajara (Guadalajara, MX); (2)Centro Nacional de la Transfusion Sanguinea (Mexico DF, MX)

Introduction: Umbilical cord blood (UCB) as source of hematopoietic stem cell for transplantation offers an opportunity to patients without other suitable donors. In Mexico the availability of public UCB has been difficult until recently. We started our stem cell transplantation program in 2003; the UCB transplant program started in 2008. The purpose of this communication is to describe the experience in UCB transplantation (UCBT) in our center.

Patients: Between January 2008 to September 2009, 13 patients underwent a UCBT. Diagnosis included: Fanconi Anemia (FA) (1), Medular Aplasia (MA) (2), Wiskott Aldritch Syndrome (WA) (2), Acute Lymphoblastic Leukemia (ALL) (4), Acute Myeloblastic Leukemia (AML) (3) and Juvenile Myelomonocytic Leukemia (JMML) (1). Conditioning regimen included non-myoloablative as well as myeloablative regimens.

Results: Mean age at time of transplantation was 5 years (range from 2 to 13). Infused CD34 cells/Kg ranged from 0.1 to 0.666 . Engraftment was achieved in 10 patients, 3 patients had graft failure. Mean Neutrophil engraftment was achieved at day +36 , mean platelet recovery was at day +41 . Acute graft versus host disease was diagnosed in 5 engrafted patients, involving skin (stage 1 to 4), all were primary treated with methyl-prednisolone and resolved without further complications. At the mean time 9 patients are alive; 1 of them with secondary graft loss (ALL) and two with primary graft failure (LLA and MA). Four patients died, 2 of them from transplant related toxicity at days +10 and +27 (AML and FA), the third one died with loss of previous engraftment and active ALL, the last one with primary graft failure and active JMML.

Conclusion: Mexico has a well organized public cord blood bank (accredited by Netcord and FACT certification). Due to ethnical issues, especially that Mexicans are a race that has not been changing in the last 5 centuries; it is easy to find a full or partial matched UCB unit in our bank. Although our serie is small, we found that UCBT is now affordable and promissory in developing countries for pediatric patients who do not have other suitable donor.

\section{P892}

Haematopoietic stem cell transplantation activity and trends of a single paediatric transplantation centre V. Uygun, M. Akcan, G. Karasu, A. Kupesiz, V. Hazar, A. Yesilipek

Akdeniz University Faculty Of Medicine (Antalya, TR)

Aim: To document haematopoetic stem cell transplantation (HSCT) activity and trends of a single pediatric transplantation center in a developing country.

Patients and design: A retrospective analysis of data reported about haematopoetic stem cell transplantations performed in our center over 10 year period (1998-2008). Main outcome measures were types of HSCT performed, transplant-related mortality(TRM), stem cell sources, indications for HSCT, causes of death after HSCT and survival after HSCT.

Results: Over the period 1998-2008 with a minimum one year folow-up, 205 allogeneic procedures (87\%) and 30 autologous (13\%) HSCT procedures, totally 235 HSCT procedures were performed. Stem cells obtained from unrelated donors were used in about $20 \%$ of allogeneic transplants (42/205). Umbilical cord blood as a source of haemopoietic stem cells was used in $11 \%$ $(n=21)$ of all transplants. The most common indications for allogeneic HSCT were hemoglobinopathy (46\%) and acute leukaemias $(20 \%)$, meanwhile for autologous HSCT the most common indication was neuroblastoma (53\%). TRM at 1 year after transplant was $37 \%$ for unrelated donor transplants, $14 \%$ for relative transplants and $30 \%$ for autologous transplants. The most common causes of deaths 1 year after HSCTs were infections (35\%) and relapse or progression of underlying disease $(23 \%)$. In the study period, overall survival of the whole group was $67 \%$ for five years (95\% Cl: 60-74). The causes of deaths are shown in Table 1.

Comment: Haemopoietic stem cell transplantation has an important role in treating children with a range of malignant and non-malignant diseases. TRM for allogeneic transplants was compatible with literature, however in autologous group, it is higher than the literature reported for developed countries, because of the majority of advanced stage neuroblastoma patients. Detailed evaluation of patient selection should be done in this group.

\begin{tabular}{lccc}
\hline Cause of death & Day 0-100 & $\begin{array}{c}\text { Period of Death } \\
\text { Day 101-365 }\end{array}$ & Day >365 \\
\hline Infection & 7 & 13 & 5 \\
Relapse/Progression & 1 & 9 & 7 \\
GVHD & 5 & 4 & 1 \\
SOS & 4 & - & - \\
Other & 5 & 8 & 2 \\
Total & 22 & 34 & 15 \\
\hline
\end{tabular}

\section{Experimental stem cell transplantation}

\section{P893}

Co-transplantation of mesenchymal stromal cells derived from CD271 + bone marrow mononuclear improves the lymphohaematopoietic engraftment of CD133+ haematopoietic stem cells

Z. Kuci (1), H. Kreyenberg (1), E. Deak (2), E. Rettinger (1), R. Henschler (2), U. Koehl (1), T. Tonn (2), M. Grez (3), D. von Laer (3), T. Klingebiel (1), P. Bader (1), S. Kuci (1)

(1)University Children's Hospital III (Frankfurt/Main, DE); (2)Institute for Transfusion Medicine and Immunohematology (Frankfurt/Main, DE); (3)Biopharmaceutical Institute GeorgSpeyer-Haus (Frankfurt/Main, DE)

Several lines of evidence suggest that mesenchymal stromal cells (MSCs) generated through plastic adherence (PA-MSCs) 
support the growth of hematopoietic stem cells (HSCs). However, so far there is no evidence about the potential of MSCs derived from positively selected CD271 + bone marrow mononuclear cells (CD271-MSCs) on the engraftment of hematopoietic stem cells in vivo. In the present study, we co-transplanted both types of GFP- transfected MSCs with CD133 + mobilized peripheral blood HSCs at the ratios 1:1 and 8:1 into knock-out NOD/SCID mice for gamma-chain of IL-2R. Fourteen weeks after co-transplantation the engraftment of CD133+ cells and organ distribution of MSCs was analyzed by flow cytometry, immunohistochemistry and real-time PCR. Cotransplantation of CD271-MSCs with CD133 + hematopoietic stem cells (HSCs) in NOD/SCID mice at a ratio of $8: 1$ promoted a significantly greater lymphoid engraftment than PA-MSCs. They induced a 9-fold increase in the number of T-cells, a 2.5-fold increase in the number of NK-cells, and a 4-fold increase in the number of B-cells, indicating a major qualitative difference between these two MSC populations. Quantitative PCR analysis for the human albumin gene demonstrated that cotransplantation of CD271-MSCs or PA-MSCs with HSCs at a ratio of 1:1 induced their migration mainly to the brain. In contrast, when cotransplanted with HSCs at a ratio of 8:1, CD271-MSCs or PA-MSCs migrated mainly to the lungs and, to a much lesser extent, to the liver, brain, heart, and skeletal muscle. Distribution of MSCs was also analyzed by immunohistochemistry. These results, therefore, suggest that not only can MSCs influence HSC engraftment but also HSCs may affect the migration and homing pattern of MSCs in vivo depending on the cell number of MSCs cotransplanted intravenously. Taken together, our data suggest that CD271-MSCs significantly improve the engraftment of hematopoietic stem cells and their lymphohematopoietic multilineage differentiation.

\section{P894}

Allogeneic mixed chimerism induced by non-myeloablative regimen consisting of co-stimulatory blockade-based minimum intensity conditioning and BMT achieved curative functional reconstitution in X-CGD mouse model Y. Takeuchi (1), M. Otsu (1), E. Takeuchi (2), M. Higashihara (2), M. Abe (3), H. Nakauchi (1)

(1)University of Tokyo (Minato-Ku, JP); (2)Kitasato University School of Medicine (Sagamihara, JP); (3)Kitasato University (Sagamihara, JP)

Background: Allogeneic hematopoietic stem cell transplantation (allo-HSCT) is useful for treatment of life-threatening, non-malignant disease. The procedures, however, inevitably involve substantial risks including, 1) conditioning toxicity, 2) graft rejection, 3) graft-versus-host disease (GvHD). To develop a safe and effective strategy, we have designed non-myeloablative regimen consisting of anti-CD40Ligand monoclonal antibody (aCD40LmAb)-based minimum conditioning with allogeneic bone marrow transplantation (alloBMT). X-linked chronic granulomatous disease (X-CGD) is a primary immunodeficiency characterized by functional defects in phagocytes: affected cells are unable to kill microorganisms due to defective NADPH oxidase. Although allo-HSCT is considered a promising therapy for X-CGD, curative long-term reconstitution of granulocytes has not been established. Thus, we attempted our regimen as a promising treatment option for X-CGD mouse model.

Methods: X-CGD F1 $\left(\mathrm{H}-2^{b+d}\right)$ mice were received $20 \times 10^{6}$ of BCF1 $\left(\mathrm{H}-2^{\mathrm{b}+\mathrm{k}}\right)$ bone marrow cells. 3 Gy TBI on day -1 and aCD40LmAb (2 mg) on day 0 to BMT was given. The degree of donor chimerism and functional reconstitution of granulocytes with production of specific reactive oxygen species (sROS) using APF fluorescence staining in peripheral blood was followed by flowcytometric analysis. Therapeutic effect was investigated using enhanced cutaneous inflammatory reactions to subcutaneous injection of Aspergillus Fumigatus (AF) hyphae. Results: This strategy allowed induction of long-term (>6 months), multi-lineage mixed chimerism (donor B cell; $62.9 \pm 19.1 \%$ at
24 wks) in 18 of 20 X-CGD mice. No clinical signs of GVHD were shown. Granulocytes with production of sROS were reconstituted in chimeric X-CGD mice (APFpositive granulocytes; $65.3 \pm 20.7 \%$ at $24 \mathrm{wks}$ ). Subcutaneous accumulation of granulocytes, appearing granulomatous formation to AF hyphae was apparent in non-chimeric X-CGD mice, while it was improved in chimeric $\mathrm{X}-\mathrm{CGD}$ mice.

Conclusion: aCD40LmAb-based minimum conditioning with allo-BMT is a promising treatment option for X-CGD without GVHD. This strategy may be applicable for other intractable disorders.

\section{P895}

GMP-grade USSC for haematopoietic reconstitution in CB transplantations

T.F. Radke (1), M. Aktas (1), K.S. Jeltsch (2), P. Maier (2), W. J. Zeller (2), G. Kögler (1)

(1)University of Dusseldorf Medical School (Dusseldorf, DE); (2)German Cancer Research Center (Heidelberg, DE)

Because of their high proliferation and differentiation potential, unrestricted somatic stem cells (USSC) from cord blood are promising candidates for future clinical appliance. Accordingly, these cells must be generated and cultivated in compliance with the strict requirements of GMP guidelines to ensure their maximum reliability and safety. In order to meet GMP conformity, fresh $\mathrm{CB}$ were processed by the automated cell processing system SEPAX (Biosafe) to isolate the mononucleated cell fraction (MNC). Following USSC generation, clinical-scale expansion was achieved by usage of multi-layer culture flasks (CellSTACK, Costar/Corning) and products evaluated for infectious disease markers, pyrogen- and endotoxin-levels, immunophenotype, potency, genetic stability and sterility. To date, 19 USSC lines have been generated successfully under GMP conditions, reaching a mean cell count of $8.70 \pm 1.86^{\star} 10^{8}$ within 5 passages.

Furthermore, GMP-grade USSC were tested for hematopoiesissupporting capacities in vitro and in vivo as well as for an eventual tumorgenicity. For in vitro co-culture, freshly isolated CD34+cells from cord blood were seeded on 4 different, mytomycin C-treated USSC-lines as feeder layer, resulting in a 23.29 \pm 8.32 fold mean expansion of total cells, a $3.11 \pm 1.29$-fold expansion of CD34 +-cells and a 1.91 \pm 0.77 -fold expansion of colony-forming units by day 14 . In vivo, hematopoietic support was tested in cooperation with the German Cancer Research Center (DKFZ, Heidelberg) by co-injection of USSC and CD34+-cells versus CD34 +-cells alone in NOD-SCID mice. After 4 and 8 weeks, bone marrow, blood and spleen were tested by flow cytometry for human cells and showed a significantly higher amount of human cells in mice supplemented with USSC versus CD34 +cells alone after 4 weeks ( $30.9 \%$ vs. $5.9 \%, P=0.004)$, but due to wider scatter no significant difference after 8 weeks $(24.2 \%$ vs. $1.3 \%, P=0.112$ ). Finally, USSC were tested in NMRI nu/nu mice for tumorgenicity by subcutaneous injection of $0.5-1 * 10^{7}$ cells. Aside from spontaneous development of murine tumors in 4 out of 30 mice, immunohistochemical staining against human nuclei showed no involvement of human cells. Taken together, these results prove that USSC can be easily produced in amounts and under conditions required for clinical application while showing no potential risk for the recipient.

\section{P896}

Recovery of regulatory T-cells following non-myeloablative DLA-identical littermate allogeneic stem cell transplantation

A. Knueppel, D. Killian, S. Lange, A. Sekora, M. Freund, C. Junghanss

University of Rostock (Rostock, DE)

Background: Regulatory T cells (Treg) are key modulators of immune responses. Treg recovery following allogeneic stem cell transplantation differs between conditioning regimen and is 
not fully understood. Here we report on results of Treg recovery following a 2 Gy total body irradiation (TBI) conditioning regimen in a canine hematopoietic stem cell transplantation model.

Methods: Recipients received a single dose of 2 Gy TBI at day 0 followed by infusion of Dog-leukocyte antigen (DLA) identical littermate bone marrow. Pre- and postgrafting immunosuppression consisted of different regimen: CSA/MMF (group 1, $n=3$ ), CSA/RAD001 (group 2, n=9) and CSA/MMF intra bone marrow transplantation (IBM-BMT, group $3, n=6$ ), respectively. Amounts of Treg within the peripheral mononuclear cells fraction (PBMC) was determined using flow cytometry and CD4/ CD25/Foxp3 staining at different timepoints (d0, d14, d28, d56, d91). In parallel, chimerism of PBMC and granulocytes were determined by PCR-analyses of polymorphic nucleotide repeats.

Results: At day 0 Treg accounted for median $1 \%$ of all lymphocytes in group 1, for median $1.19 \%$ (range $0.51-3.67 \%$ ) in group 2 and for $0.85 \%$ (range $0.61-1.44 \%$ ) in group 3 . At days 28 and 56 Treg accounted for median $0.36 \%, 0.81 \%$, $1.19 \%$ as well as $0.51 \%, 0.87 \% 1.69 \%$ (groups $1,2,3$, respectively). IBM-BMT dogs had at day 28 significant higher Treg values in comparison to dogs receiving CSA/MMF $(P=0.01$, t-test) and CSA/RAD001 ( $P=0.03$, t-test). This difference remained significant at $d+56$ in comparison to group 2 $(P<0.01)$, respectively. Day 56 engraftment in the PBMC compartment was $24.4 \%, 39.9 \%$ and $8.5 \%$ for groups $1-3$, respectively. IBM-BMT dogs had significantly lower donor PBMC and granulocyte chimerisms compared to groups 1 and $2(P<0.01$, both).

Conclusion: Recovery of Treg seems to be faster after intra bone marrow transplantation in combination CSA/MMF immunosuppression compared to intravenous BMT. Whether these higher Treg counts are responsible for the lower donor engraftment in the IBM-BMT group have to be investigated in further studies.

\section{P897}

Intra-bone marrow transplantation of allogeneic

DLA-identical CD34 + cells allows successful engraftment in DLA-identical canine litters following non-myeloablative conditioning

A. Knueppel, D. Killian, S. Lange, A. Sekora, M. Freund, C. Junghanss

University of Rostock (Rostock, DE)

Background: The common injection sites for allogeneic bone marrow transplantation are the veins. Recent data suggest that intra-bone marrow transplantation (IBM-BMT) is feasible and allows faster engraftment compared to intravenous transplantation. This transplantation strategy has not applied in a canine transplantation model, yet. Here, we report on results from a cohort of DLA-identical siblings that received IBM-BMT following nonmyeloablative conditioning. Data is compared to historical controls that received i.v. BMT.

Methods: Recipients received a single dose of 2 Gy total body irradiation at day 0 following IBM-BMT. Bone marrow of identical littermate was aspirated and peripheral mononuclear cells (PBMC) including hematopoietic stem cells were enriched by density gradient centrifugation. The grafts were then suspended in donors 'serum for a final volume of $5 \mathrm{ml}$. Transplantation was performed by IBM-application in the left humerus and femur over an application period of 3-4 minutes. Pre- and postgrafting immunosuppression consisted of a CSA/MMF regimen. Hematotoxicity was determined daily during early posttransplantation period and weekly later on. Analyses of chimerism was performed via polymorphic nucleotide repeat PCR-testing.

Results: All IBM-BMT transplanted animals engrafted. Highest donor chimerisms in the PBMC and granulocytes compartments were median $21.4 \%$ (range 15.5-96\%) and 39\% (range 29$64.7 \%$ ), respectively. Five of six animals had rejected their graft by day 91 . One chimeric dog died at day 57 due to infection. Hema- totoxicity was significantly more intense compared to historic controls with platelets $<50 \mathrm{GPT} / \mathrm{l}$ for median duration of 8 days and with leukocytes $<1.0 \mathrm{Gpt} / \mathrm{l}$ for median duration of 4 days.

Conclusion: Intra bone marrow transplantation of enriched BMcells is feasible in the canine nonmyelobalative hematopoietic stem cell transplantation model. However, long term engraftment could so far not be achieved and hematotoxicity was significant. Whether intra bone marrow transplantation of higher cell dosages improves results has to be investigated.

\section{P898}

Availability of stem cell niches for bone marrow transplantation

K. Forgacova, L. Sefc, J. Michalova, F. Savvulidi, E. Necas Charles University in Prague (Prague, CZ)

Objectives: Bone marrow transplantation (BMT) is a therapeutic procedure utilizing significant regenerative potential of the hematopoietic stem cells (HSC). BMT is usually performed 0-2 days after pretransplantation conditioning. Our aim was to determine "transplantation window", i.e. for how long after conditioning are transplanted HSC able to engraft the microenvironmental niches of the recipient made available by conditioning. We also studied a possibility to "close" niches prematurely by a massive transplant.

Methods: C57BI/6 Ly5.2 and congenic Ly5.1 mice were used. Conditioning by total body irradiation (TBI) was from $60 \mathrm{Co}$ source and doses were 6 Gy (submyeloablative) or 9 Gy (ablative). With a delay of 1 up to 180 days the mice received a large congeneic transplant of $7 \times 10^{6}$ bone marrow (BM) cells. In the experiment aimed at a premature closure of the transplantation window, recipients exposed to 9 Gy were subsequently transplanted with syngeneic BM cells in a dose of about $40 \times 10^{6}$ cells for 5 consecutive days. On the 15th day after TBI they were transplanted with congeneic BM cells of about $20 \times 10^{6}$ cells given in a single dose. Resulting chimerism of the donor derived blood cells was evaluated from 1 to 6 months after the congeneic transplantation by using flow cytometry.

Results: Both ablative and submyeloablative conditioning resulted in a high degree of congeneic donor chimerism. It was possible to transplant recipient mice with a delay exceeding 20 days after TBI. Thereafter the transplantation windows closed rather abruptly. There was a difference between submyeloablative and ablative conditioning regimens regarding engraftment of the short-term and the long-term repopulating cells. The massive repeated syngeneic transplant, delivered during first five days after ablative TBI, closed the transplantation windows effectively by day 15 .

Conclusion: Results correspond to a mechanistic view of haematopoietic niches that need to be "opened" before transplantation by a damage delivered to the BM. Transplantation window depends on the intensity of conditioning and remains "opened" for approximately three weeks. This would allow for fractionated, repeated or delayed BMT modalities.

Support: Projects LC06044 and MSM 0021620806 of the Ministry of Education, Youth and Sports of the Czech Republic.

\section{P899}

Sonic hedgehog transient gene therapy to counteract radiation-induced myelosuppression: development of a non-human primate model

M. Drouet, D. Agay, P. Garrigou, F. Forcheron, B. Ballester, N. Grenier, J.F. Mayol, F. Hérodin

IRBA-CRSSA (La Tronche, FR)

The goal of this study was to set up a model of transient gene therapy in highly irradiated non human primate to determine whether a transient sonic hedgehog (Shh) expression in the stem cell niche could enhance hematopoietic recovery. We chose a gene therapy strategy to combine efficient stimulation and reduction of side effects. Hedgehog signaling role in adult 
hematopoiesis is still debated. Hhh proteins binding to Ptch induces transduction through Smo. Antibodies to Hhh have been demonstrated to inhibit the cytokine-induced proliferation of human hematopoietic repopulating cells (Bhardwaj et al., 2001). In addition transgenic mice (Ptch1 \pm ) exhibiting an increased Shh activity have been shown to recover more rapidly than controls following myelosuppression and prolonged stimulation with Shh deplete the stem cell pool due to cell cycle acceleration. However recent mice studies shows that loss of Hhh signaling through conditional deletion of Smoothened (Smo) has no apparent effect on adult hematopoiesis (Hofmann et al., 2009, Gao et al., 2009). This suggests that hedgehog signaling could be dispensable for adult hematopoieisis. As Shh has also been demonstrated to favor neovascularization in other tissues (skin, myocardiac muscle), it may act on the microenvironment/vascular niche following irradiation, which would favor hematopoietic stem and progenitor cells activity. In the present study autologous or xenogeneic porcine adipocyte derived stem cells were used as vector cells and transduced using a piRES-2-Shh-eGFP plasmid and Nucleofector technology (Amaxa). In this model Shh protein was detected in vitro in the transduced cells during 1 to 5 days and was secreted during 1 to 3 days in accordance with our short term secretion strategy. About $0.5 \times 10^{6}$ autologous Shh-ASC were intrafemorally injected to each monkey $(8.4 \mathrm{~kg}$ and $9 \mathrm{~kg}$ weight) the day after irradiation (whole body, frontal) at a dose inducing a severe and prolonged neutropenia and thrombocytopenia (8Gy 60 Co gamma). In both monkeys, blood cell counts did not significantly differ from irradiated control animals $(n=4)$. In addition the second animal was given a $5 \times 10^{6}$ xenogeneic graft on day 43 which was well tolerated. Further studies are required to validate the therapeutic tools and work is going on to improve the autologous graft size. Moreover, combining transient Shh overexpression at the niche level and systemic cytokine treatment may be required as suggested by in vitro studies.

\section{P900}

Influence of high mobility group Box 1 on allogeneic immune responses

D. Killian (1), A. Knueppel (1), S. Willenbrock (2), H. Murua Escobar (2), A. Heisterkamp (3), H. Lubatschowski (3), I. Nolte (2), A. Sekora (1), M. Freund (1), C. Junghanss (1)

(1)University of Rostock (Rostock, DE); (2)University of Veterinary Medicine Hannover (Hannover, DE); (3)Laser Zentrum Hannover e. V. (Hannover, DE)

Background: High mobility group Box 1 (HMGB1) protein serves intracellular as DNA binding protein and has cytokine function if released. Recent studies suggest that it stimulates immune reactions and might therefore serve as adjuvant in vaccination as well as immunological cell therapy approaches. Here, we investigated the in vitro effects of HMGB1 on autologous and allogeneic immune responses in the canine model. Methods: Peripheral blood mononuclear cells (PBMCs) of dogs were used as responder cells in mixed leukocyte reactions (MLR). Stimulator cells were autologous and allogeneic PBMCs, hematopoietic stem cells $(\mathrm{CD} 34+)$ and dendritic cells (DCs). Bi- as well as monodirectional MLRs were performed. HMGB1 was added at concentrations of 5,10 and $25 \mathrm{ng} / \mathrm{ml}$. Proliferation was determined by thymidin incooperation after 72h. Intracellular cytokine productions (TNFa, IFNg) of T-cells in response to HMGB1 $(5,10,25,50 \mathrm{ng} / \mathrm{ml}) \pm$ leucocyte activation cocktail (LAC) were determined by flow cytometry.

Results: As expected allogeneic compared to autologous stimulation was in general more potent to induce proliferation. Addition of HMGB1 did not significantly enhance proliferation in MLR if PBMCs, CD34+ or DCs were used as stimulators. HMGB1 increased the proportion of TNFa producing T-cells in the MLR from $0.23 \%$ (control) to $0.33 \%(10 \mathrm{ng} / \mathrm{ml}$ ) and $0.31 \%$ (25 ng/ml), respectively. The proportion of IFNg producing T-cells was not altered by HMGB1 addition if measured without LAC. After LAC, it seemed that proportion of IFNg producing T- cells increased with increasing HMGB1 doses $(2.9 \%$ (control) vs. $4.1 \%$ (50 $\mathrm{ng} / \mathrm{ml} \mathrm{HMGB} 1)$ ).

Conclusion: HMGB1-protein at concentrations up to $25 \mathrm{ng} / \mathrm{ml}$ does not increase allogeneic immune responses, but seems to increase the proportion of TNFa and IFNg producing T-cells. If HMGB1 shall be used as adjuvants in vaccination experiments application of higher doses might be neccessary.

\section{P901}

Migration of canine dendritic cells can be detected by radioactive-labelling and varies according to injection sites

D. Killian (1), A. Knueppel (1), S. Lange (1), S. Dunkelmann (1), J. Fitting (2), S. Willenbrock (3), P. Wefstaedt (3), H. Murua Escobar (3), I. Nolte (3), S. Barth (2), A. Sekora (1), M. Freund (1), C. Junghanss (1)

(1)University of Rostock (Rostock, DE); (2)Helmholtz Institute-RWTH Aachen and University Hospital Aachen (Aachen, DE); (3)University of Veterinary Medicine Hannover (Hannover, DE)

Background: Dendritic cells (DCs) as antigen-presenting cells hold a key position between innate and adaptive immune system. With a high stimulatory potential, they are important vaccination tools in research. Here, we aimed at the investigation of the migration of DCs applicated at different sites in the canine model which has been a significant preclinical transplantation model for decades.

Methods: Canine bone marrow CD34 + stem cells were differentiated into DCs (media supplements: GM-CSF, FLT-3, TNFa, harvest day 14). The DCs were labeled with Technetium-99m (20-253 $\mathrm{MBq} /$ injection) and reinjected $\left(1 \times 10^{4}-1 \times 10^{6}\right.$ DCs/injection). There were 3 different ways of application: subcutaneously into the groin ( $n=4$, GROUP 1), intravenously into the vena cephalica $(n=2$, GROUP 2) and intraosseously into the bone marrow of the humerus ( $n=2$, GROUP 3 ). The migration of the Tc-99m-labeled DCs was detected by scintigraphy after $0 \mathrm{~h}, 6 \mathrm{~h}$ and $24 \mathrm{~h}$.

Results: The cell amount of $1 \times 10^{4}$ DCs/injection did not allow a sufficient signal detection. An intense signal throughout all application forms and time points was detected with DC cell numbers $\geq 1 \times 10^{5}$ per injection. In GROUP 1 (sc): DCs migrated into the thyroid lobes and salivary glands as well as the lymphatic tissue of the intestinal tract; the spleen was an invariant destination; a strong DC signal remained at the injection site. In GROUP 2 (iv): DCs migrated into the liver, spleen as well as the lymphatic tissue of the intestinal tract. In GROUP $3(\mathrm{bm})$ : DCs stayed at the injection site (BM), and migrated to the lymphatic tissue of the intestinal tract, the spleen and the liver. In all groups DC migration to distant sites was observed as early as after $6 \mathrm{~h}$.

Conclusion: Technetium-99m labeling allows the observation of DC migration in the canine transplantation model with good sensitivity. For more sensitive migration investigation strategies such as nanoparticle marking are warranted. In dogs, DC migration varies depending on the injection site and might therefore influences specific immune responses.

\section{Stem cell research}

\section{P902}

Human mesenchymal stromal cells activate integrin-mediated adhesion through chemokines

M. Giesen (1), V. Lang (1), E. Deak (1), F. Ciuculescu (1), E. Seifried (1), T. Hartmann (2), R. Henschler (1)

(1)Red Cross Donor Service (Frankfurt, DE); (2)University Hospital Salzburg (Salzburg, AT)

Mesenchymal Stromal Cells (MSCs) have been used in therapeutic approaches including immune modulation, targeting of 
tumors, or in patients with severe sepsis. However, the repertoire of adhesion mechanisms operative in MSCs remains largely elusive. We asked which adhesion receptors are functional in human MSCs. Human MSCs were isolated from normal healthy donors and characterized using differentiation assays, flow cytometry, RT-PCR and in parallel plate flow chambers under shear stress, either preloaded with endothelial cells or recombinant ligands. Of the main selectin-type rolling receptors, MSCs did not detectybly express P-selectin glycoprotein ligand (PSGL)-1 or L-selectin, whereas CD44 which encodes an E-selectin ligand was highly expressed. Beta1 integrins expressed by MSCs comprised Very Late Antigens (VLA)-4 and -5 , whereas beta 2 integrins LFA-1 and Mac1 were hardly found expressed. Chemokine receptors of both, the CCR family (CCR 1, 5, 6, 7) and the CXCR family (CXCR1, 3, 4) were detected intracellularly and, in part, on the cell surface. In shear flow assays, MSCs showed comparable frequencies of arresting cells on activated endothelial cells as human CD34 + cells or blood lymphocytes at shear stresses between 0.1 and 2 dynes $/ \mathrm{cm}^{2}$. Function-blocking antibody experiments and experiments on coated recombinant single ligands showed that E- and, but also P-selectin and VLA-4 can mediate interactions with MSCs. Resistance of MSCs to higher shear stresses of 5-15 dynes $/ \mathrm{cm}^{2}$ was efficiently induced by chemokines co-coated with the beta 1 integrin ligand Vascular Cell Adhesion Molecule (VCAM)-1, but not with beta2 integrin ligands. Chemokines acting on MSCs were CCL 15/MIP5 (normally activating monocytes), CXCL8 (granulocytes), CXCL13/BLR (B-lymphocytes), CCL19/MIP-3beta, CCL20/MIP-3alpha T-lymphocytes) or the more ubiquitously acting CXCL12/SDF-1. Surprisingly, MSCs from different donors showed heterogeneous responses to these chemokines. MSC firm adhesion was blocked after pretreatment with pertussis toxin, an inhibitor of $G$ alpha i and Rac inhibitor NSC23766, confirming involvement of chemokine initiated G protein coupled receptor signalling in this response.

In conclusion, MSCs show a unique profile of adhesion receptor usage when binding endothelial ligands. In part, MSCs adhere with similar mechanisms and dynamics as hematopoietic cells, but display a pleiotropic chemokine receptor usage. These data provide a new basis for MSC graft engineering.

\section{P903}

Hypoxia maintains stemness of somatic endothelial colony forming progenitor cells

N.A. Hofmann, A. Reinisch, K. Schallmoser, E. Rohde, S. Chatterjee, M. Absenger, R. Birner-Gruenberger, D. Strunk Medical University of Graz (Graz, AT)

Endothelial colony-forming progenitor cells (ECFCs) are prototypic vessel wall-derived and circulating somatic progenitor cells which are considered to maintain the vascular integrity. Hypoxia in ischemic tissue seems to be a key factor driving the revascularization machinery. We therefore hypothesize that hypoxic conditions should amplify proliferation and function of ECFCs.

Adult human ECFCs were isolated and propagated directly from whole venous blood using a novel recovery strategy. During cell culture, pooled human platelet lysate (pHPL) entirely replaced fetal bovine serum (FBS). We designated the oxygen level present in vivo in the venous environment at the site of ECFC harvest to be termed EUOXIA $(41,5 \pm 3,4 \mathrm{mmHg})$ throughout this study because the term NORMOXIA has been used variably in the literature describing a wide spectrum of oxygen levels. Oxygen levels above EUOXIA are defined as HYPEROXIA (air oxygen $=139.8 \pm 2.9 \mathrm{mmHg}$ ) and below EUOXIA as HYPOXIA (27.4 $\pm 7.3 \mathrm{mmHg})$. Progenitor cell phenotype, hierarchy, long-term proliferation, wound repair as well as migratory and vasculogenic functions were monitored under EUOXIA as compared to hypoxic conditions or air oxygen levels commonly used in standard laboratory practice. Molecular regulation of cellular responses to different oxygen levels was assessed by flow cytometry and proteomic profiling.
ECFC colony size was decreased under HYPOXIC compared to EUOXIC conditions paralleled by a loss of high proliferative potential (HPP) ECFCs under HYPOXIA. HYPEROXIC conditioning resulted in an increase in proliferation in primary and long-term cultures and significantly augmented HPP colonies $(60 \pm 18 \%$ of total colonies) as compared to HYPOXIC $(0 \%)$ and EUOXIC $(9 \pm 6 \%)$ oxygen levels. Vascular wound repair in scratch assays and in vitro Matrigel ${ }^{\circledR}$ vascular network formation was improved with escalating oxygen supply. Reoxygenation of hypoxic and euoxic ECFCs even lead to an enhanced proliferation and function compared to hyperoxia. Proteomic analysis of ECFCs identified several heat shock and antioxidant proteins involved in the oxygen-dependent regulation of migratory and proliferative responses.

These data indicate that HYPOXIC and EUOXIC oxygen levels maintain the ECFC stem cell characteristic. A new model will be introduced explaining an alternative mechanism for revascularization of hypoxic tissues after ischemic injury by ECFCs.

\section{P904}

Cytogenetic analysis and stability of human bone marrow MSCs during ex vivo expansion

E. Osipova (1), V. Nikitina (2), T. Astrelina (1), E. Skorobogatova (3), Z. Dishlevaya (3), S. Roumiantsev (1), M. Yakovleva (1) (1)Stem Cell Bank (Moscow, RU); (2)Researsch Centre of Medical Geneticts, Russian Academy of Medical Sciences (Moscow, RU); (3)Research Centre of Pediatric Hematology, Oncology and Immunology (Moscow, RU)

Objectives: MSCs are multipotent cells with many potential clinical applications. However, the lack of a precise definition of the cells preparation and the heterogeneity of the obtained product render validation criteria of the final product of the expansion. During the culturing procedures genetic changes can arise which can lead to long term consequences of complications of MSC transplantation.

Aims: to estimate genetic stability (karyotype and aneuploid cell frequency) of MSC from healthy donors during ex vivo expansion.

Methods: MSC $(n=70)$ were prepared by seeding bone marrow MNC in $75 \mathrm{~cm}^{2}$ flasks in low glucose DMEM with $20 \%$ FCS. The adherent were passed every 7 days by trypsinization and replanting at the density of $0.5 \times 10^{6}$ cells $/ 75 \mathrm{~cm}^{2}$ flask until the end of the culture. The immunophenotype of cells from early passages was: CD90 +, CD105+, CD73+ and CD45-, CD31-, CD34-, CD133-, and remained stable up to late passages. Chromosomal preparations of MSC were prepared according to a standard protocol. The karyotype was analyzed by G-banding technique; 15-30 metaphase cells for each culture were analyzed. To analyze the level aneuploidy, fluorescent in situ hybridization (FISH) with chromosome enumeration probes (CEP) studies was performed. For each hybridized probe at least 1000 interphase nucleus were analyzed.

Results: In all cases the karyotype of MSC during expansion were normal-46, $\mathrm{XY}$ or $46, \mathrm{XX}$ and remain stabile. Disomy rate for 6 and 8 chromosomes in the MSC cultures was $98 \%$, tetrasomy rate was $0,9 \%$ and did not change during cultivation. Monosomy rate for sex chrom osomes ( $X$ and $Y$ ) in the MSC cultures was higher then the rate of trisomy $(P=0,05)$. The rate of aneyploidy in MSC cultures did not change during cultivation. FISH-analysis of MSC revealed 3 samples with abnormal clones ( 1 with trisomy 8 , and 2 with monosomy $\mathrm{X}$ ). These clones arose on early passages and remained in cultures during expansion. Conclusions: During the culturing procedures chromosome abnormalities can arise which can lead to long term consequences of cell therapy. Further research is required concerning different passaging techniques and culture conditions to better understand their effects on MSC chromosomal stability. 


\section{P905}

Ex vivo expansion of cord blood and bone marrow haemopoietic stem cells in the presence of mesenchyma stromal cell feeder layer

I. Pelagiadis, H. Dimitriou, E. Stiakaki, C. Perdikogianni, M. Tsirigotaki, M. Kalmanti

University of Crete (Heraklion, GR)

Mesenchymal stem cells (MSC) of bone marrow (BM) origin are part of the supportive microenviroment -the niche- of hemopoietic stem cells (HSC). The exact mechanism implicated in the interaction between HSC and the niche and the regulation of HSC self-renewal/differentiation, quiescence/proliferation are not fully clarified.

The aim of the study was the in vitro evaluation of the ex vivo expansion of cord blood (CB) or bone marrow CD34 + HSC in the presence of MSC used as a feeder layer, with the addition or not of different growth factor (GF) combinations.

CD34 + HSC were obtained from either full term CB $(n=11)$ or BM $(n=5)$ of children with autoimmune idiopathic neutropenia and solid tumors. MSC isolated from the BM of the same pediatric patients $(n=16)$ were co-cultured with either BM-HSC or CB-HSC under different conditions with the addition or not of two GF combinations (GFmix1: SCF $10 \mathrm{ng} / \mathrm{ml}$, IL-3 $5 \mathrm{ng} / \mathrm{ml}$, G-CSF $100 \mathrm{ng} / \mathrm{ml}$, GM-CSF $1 \mathrm{ng} / \mathrm{ml}$, and GFmix2: SCF $20 \mathrm{ng} /$ $\mathrm{ml}$, TPO $25 \mathrm{ng} / \mathrm{ml}$, IL-6 $20 \mathrm{ng} / \mathrm{ml}$, Flt-3 ligand $50 \mathrm{ng} / \mathrm{ml}$ ). After 10 days of liquid culture, cells were immunophenotypically characterized, the CFU-GM and BFU-E development was assessed and total cell as well as CD34 + expansion were evaluated.

The presence of both MSC and GF combinations resulted in the higher expansion of the total cell number and the CD34+ population. CD34 + cells and CD34 + CD33- as well as CD34 + 38- subpopulations, considered more primitive, expanded more efficiently in the presence of MSC only. Higher total cell number and CD34 + cell expansion were observed in the CB-CD34 population compared to the BM-CD34+, in the presence of both MSC and GF or GF only, independently of the GF combination applied. The co-culture of MSC and CB-CD34 resulted in the expansion of cells with greater clonogenic capacity as expressed by the ability CFU-GM and BFU-E colony development. No other significant difference was observed in the various parameters examined.

An effective stem cell harvest in the clinical setting of HSC transplantation, in many cases results unsuccessful and exvivo expansion of the HSC populations seems compulsory. Although growth factors efficiently expand the number of total cells obtained, the presence of MSC may offer a promising, alternative ex-vivo expansion procedure for CB HSC, preserving a population with stem cell characteristics.
P906

Alteration of genomic stability in long-term cultured mesenchymal stromal cells

K. Schallmoser, E. Rohde, A. Reinisch, A.C. Obenauf, C. Bartmann, G. Lanzer, W. Linkesch, D. Strunk

Medical University of Graz (Graz, AT)

Human multipotent mesenchymal stromal cells (MSCs) are currently being tested in clinical trials for immunomodulatory and regenerative therapies. We and others have recently established MSC propagation with pooled human platelet lysate ( $\mathrm{pHPL}$ ) substituting fetal bovine serum (FBS). As serious doubts regarding the use of MSCs cultured with FBS and their possible genomic instability have arisen, we investigated safety aspects of short- and long-term cultures with FBS in comparison to $\mathrm{pHPL}$.

Unmanipulated bone marrow aspirates were seeded in alpha-MEM with pHPL. Clinical-scale expanded MSCs were harvested directly after primary culture (short-term). Representative cultures were continued for long-term expansions where each directly compared $\mathrm{pHPL}$ and FBS stimulation for a maximum of 46 to 51 population doublings until MSC proliferation ceased. Comparative genome hybridization (array-CGH) was carried out with short- as well as long-term expanded MSCs using a whole genome microarray platform and $\mathrm{CGH}$ analysis software. pHPL is highly efficient in stimulating MSC expansion resulting in $780 \pm 150$ million MSCs after one passage. Flow cytometry revealed more than $95 \%$ viability, more than $95 \%$ CD73/90/105 reactivity and less than $2 \%$ hematopoietic contamination. We could show adipo-/osteo-/chondrogenic differentiation potential, endotoxin levels below $0.025 \mathrm{EU} / \mathrm{mL}$ and negative bacterial/fungal/mycoplasma testing. In all shortterm MSC products, array-CGH revealed balanced genomic profiles. We detected several small copy number variations previously found in healthy individuals not associated with phenotype changes. In contrast, after long-term culture with FBS as well as pHPL, MSCs showed de novo copy number amplifications not reported in the database of genomic variants. Despite a high proliferation rate in the short-term pHPLdriven cultures, MSCs showed genomic stability according to array-CGH results. These data support our earlier findings that MSCs expanded under humanized conditions did not form tumors in vivo in animal experiments. It is not clear whether in vitro genomic variations in long-term propagated MSCs under humanized as well as xenogeneic culture conditions may be associated with the risk of malignant transformation rather than representing replicative senescence. Therefore safety concerns have to be vigilantly addressed parallel to the clinical use of MSCs.

\begin{tabular}{|c|c|c|c|c|c|c|c|c|}
\hline & Condition & $\begin{array}{c}\text { Total oels } \\
\text { foldincrease }\end{array}$ & $\begin{array}{l}\text { C130" fod } \\
\text { ncrease }\end{array}$ & $\approx \cos 3$ & OU-GM & Bate & 1034'38 & $\alpha 34 \div 3$ \\
\hline 1 & MSC4CB_CD34 & $8.5+4.3$ & $1.58+0.6$ & $40.8+7.4$ & $166 \div 20.8$ & $54.11+16.7$ & $7.1+3.0$ & $28,1+6,6$ \\
\hline 2 & MSC+OB_СDЗn+GFMX & $164: 4+627$ & 20.995. & $131+0.9$ & $850+20.8$ & $43.3+15.2$ & $135+0.9$ & $4.15+0.9$ \\
\hline 3 & MSC+CB_CD3H+GFTBO & $1082+31.5$ & $9.7 \pm 4.0$ & $7.7+2.2$ & $104.45+8.3$ & $10.75+4.0$ & $0.2 m+0.1$ & $0.88+0.2$ \\
\hline 4 & С__DЗнGPMIXI & 102.94363 & $1373+9.7$ & 10.9155 & $107.0+129$ & $125+4.5$ & $4.2+3.8$ & 1.0+1.1 \\
\hline 5 & С__DЗЗнGPMIXZ & $143.5 \pm 7.2$ & $1389+0.7$ & $9.7 \pm 0.5$ & $101.3+11.2$ & $39+1.0$ & $0.97+0.5$ & $0.6+0.1$ \\
\hline 6 & MSCGEM_CD3 & $10.0+4.58$ & $207+0.7$ & $381+137$ & $72.1+2.5$ & $1.12+0.9$ & $23+4.6$ & $16.3+65$ \\
\hline 7 & MSC.HB__CD3H+GFMD2 & $1546+4.4$ & $1.53+0.4$ & $12.7+34$ & 100.577 .6 & $0.62+04$ & $50+22$ & $1.92+1.4$ \\
\hline 8 & BM_CD3H+GFM1X2 & $11.8+35$ & 0.84+0.2 2 & $7.7+2.0$ & $120.2+10.3$ & o & $2.66+0.9$ & $1.8+0.8$ \\
\hline
\end{tabular}


P907

Co-culture maintenance of functional haematopoietic stem cells and expansion of their progeny

M. Cook, G. Brooke, M. Doran, I. Winkler, J.P. Levesque, K. Atkinson

Mater Medical Research Institute (South Brisbane, AU)

Haematopoietic stem cell (HSC) transplantation is a curative treatment for a number of immunodeficiencies and haematological malignancies. However, adequate supply of HSC from some donor tissues can limit its application. This may be overcome by expanding HSC prior to transfusion which has motivated numerous attempts at expansion processes in this area. We propose that it may be possible to expand HSC using co-culture techniques combined with advances in our understanding of the in vivo bone marrow niche. This study aimed to compare undifferentiated mesenchymal stromal cells (MSC) to osteocytes differentiated from MSC as support monolayers to establish an artificial environment for the controlled expansion of HSC. Using basal medium, supplemented with fetal calf serum, murine HSC demonstrated a proliferative capacity on both these stromal monolayers, with no additional haematopoietic growth factors required. Compared to undifferentiated MSC co-culture, the osteocyte monolayer displayed a significantly superior capacity for expanding both total nucleated haematopoietic cells as well as more primitive HSC, as defined by cell surface phenotyping. However, in both cases, a majority of the expanded cells were found to be committed to mature haematopoietic lineages. The quality and functional capacity of the cultured HSC was then tested in a murine competitive reconstitution assay. Upon transplantation, the cultured haematopoietic cells were capable of engrafting and repopulating lethally irradiated mice in both primary and secondary recipients at 16 weeks post-transplant. HSC co-cultured on MSC showed comparable reconstitution ability to that of freshly isolated HSC, whereas those co-cultured on osteocytes displayed a greater heterogeneity of outcomes. The reason for this is not clear, but we are currently determining if osteocytes injected into mice at the same time as expanded HSC interfere with HSC homing to bone marrow. These results demonstrate that stromal monolayers can be used to maintain functional HSC without the need for additional growth factors. This co-culture assay also presents a tool to determine further niche components that may be used to enhance ex vivo expansion of HSC. As such, the culture conditions for HSC expansion are now being further optimised and implemented into a scalable bioreactor device for controlled regulation of the HSC microenvironment.

\section{P908}

Defibrotide protects human microvascular endothelial cells from fludarabine-mediated pro-inflammatory and pro-apoptotic signals: a microarray (Affimetrix) analysis C. Echart (1), G. Eissner (2), E. Holler (3), M. lacobelli (1) (1)Gentium SpA (Villa Guardia, IT); (2)University of Munich (Munich, DE); (3)Regensburg University Medical Center (Regensburg, DE)

Background: Defibrotide (DF) is a polydisperse mixture of $90 \%$ single-stranded polydeoxyribonucleotides with anti-thrombotic and anti-ischemic functions. DF has been recently showed to be effective in preventing hepatic VOD post SCT. We have previously reported that DF protects endothelial cells (HMEC) from Fludarabine (F-Ara)-induced programmed cell death. In this study we have investigated whether the anti-apoptotic activity of DF occurs at the transcriptional level.

Methods: Microarray analyses of a human microvascular endothelial cell line (CDC.EU/HMEC-1) were carried out on Affymetrix chips and analyzed with Rank products. Cells had been stimulated in biological triplicates with F-Ara $(10 \mu \mathrm{g} / \mathrm{mL})$ and/or DF $(100 \mu \mathrm{g} / \mathrm{mL})$ for $6 \mathrm{~h}$ and $24 \mathrm{~h}$, respectively, prior to
RNA isolation. Functional verification of the gene array results was done with ELISA, RT-PCR, cytometric bead arrays, and intracellular flow cytometry.

Results: DF did not induce or reduce transcriptional activity of control HMEC after $6 \mathrm{~h}$ or $24 \mathrm{~h}$ to a significant extent. However, when cells were co-triggered with F-Ara, DF inhibited a number of F-Ara-induced genes, especially those associated with a pro-apoptotic phenotype, as well as with migration and inflammatory activation. In particular, among 732 differentially expressed genes (comparison DF + F-ara negative to F-Ara as baseline) showing a false discovery rate below $5 \%$, Caspase 3 (apoptosis), IL-8 and Heparanase (migration and metastasis), and ICAM-1 (inflammation) could be found. Cytometric bead arrays and flow cytometry linked the DF-mediated protection against F-Ara-induced cell death to the extrinsic apoptotic pathway.

Conclusion: DF protects endothelial cells against chemotherapy-induced cell death and activation at the transcriptional level by interfering with the extrinsic caspase cascade and by downregulating adhesion molecules and chemokines. DF also inhibits heparanase gene transcription and protein expression.

\section{P909}

Replicative senescence-associated gene expression changes in mesenchymal stromal cells

K. Schallmoser (1), C. Bartmann (1), E. Rohde (1), S. Bork (2), C. Guelly (1), A.C. Obenauf (1), A. Reinisch (1), P. Horn (2), A.D. Ho (2), W. Linkesch (1), G. Lanzer (1), W. Wagner (3), D. Strunk (1) (1)Medical University of Graz (Graz, AT); (2)Medical University of Heidelberg (Heidelberg, DE); (3)RWTH University of Aachen (Aachen, DE)

Based on promising experimental studies with mesenchymal stromal cells (MSCs), a variety of clinical applications have been initiated. Extensive propagation to yield enough MSCs for therapy may result in replicative senescence and thus hamper long term functionality in vivo. Highly variable proliferation rates of MSCs in the course of long-term expansions under varying culture conditions may already indicate different propensity for cellular senescence. We hypothesized that senescence-associated regulated genes differ in MSCs propagated under different culture conditions.

Human bone marrow-derived MSCs were cultured either by a highly efficient two-step protocol or by serial passaging in three different growth conditions. Culture media were either supplemented with fetal bovine serum in varying concentrations or pooled human platelet lysate. All MSC preparations revealed significant gene expression changes upon long-term culture. Especially genes involved in cell differentiation, apoptosis and cell death were upregulated, whereas genes involved in mitosis and proliferation were down-regulated. Furthermore, overlapping senescence-associated gene expression changes were found in all MSC preparations. Long-term cell growth induced similar gene expression changes in MSCs independent of isolation and expansion conditions. A panel of genes will be presented that might offer a practicable approach to assess MSC quality with regard to the state of replicative senescence in advance of therapeutic application.

\section{P910}

Biological and functional characterization of ex vivo expanded mesenchymal stromal cells from bone marrow of patients with primary myelofibrosis

V. Achille, M. Mantelli, M.A. Avanzini, V. Rosti, P. Comite, M.E. Bernardo, F. Locatelli, G. Barosi

Fondazione IRCCS Policlinico San Matteo (Pavia, IT)

Myelofibrosis is classified among myeloproliferative disorders, being characterized by variable degrees of cytopenia and/or cytosis, bone marrow fibrosis and extramedullary hematopoiesis. Mesenchymal stromal cells (MSCs) have been considered for many years a component of marrow stroma with structural support properties only; it 
is now clear that MSCs substantially contribute to the hematopoietic stem cell niche. We characterized the biological and functional properties of MSCs derived from myelofibrotic (MF) patients.

MSCs were isolated and expanded ex vivo from bone marrow (BM; 5 samples) and from trabecular bone fragments (TBF; 5 samples) of 7 MF patients. MSCs were investigated for clonogenic efficiency (CFU-F), proliferative capacity (population doubling, PD), morphology, immunophenotype (flow cytometry) and differentiation potential (histological staining and spectrophotometry). The ability of patient MSCs to suppress in vitro proliferation to mitogens (PHA, OKT3) of both autologous and allogeneic lymphocytes was also assessed at different MSC:PBMC ratio. Results were compared to those obtained in BM-MSCs from 9 healthy donors (HD).

CFU-F frequency was comparable in MF- and HD-MSCs (median \pm SD: $5.07 \pm 3.27$ and $5.29 \pm 0.64$, respectively). Proliferative capacity of BM-MSCs (mean cumulative PD from P1 to P4: 8.42) and TBF-MSCs (mean cumulative PD from P1 to P4: 8.48 ) of patients was comparable to that of HD-MSCs (mean cumulative PD from P1 to P4: 8.24).

MF-MSCs displayed the typical spindle-shaped morphology and were able to differentiate into both adipocytes and osteoblasts. In particular, the osteogenic differentiation capacity of TBF-MSCs resulted higher than that observed in BM-MSC from the same 3 patients as demonstrated by spectrophotometry (mean \pm SD: $251 \pm 69 ; 81 \pm 53 \mu \mathrm{g} / \mathrm{ml}$ ). Surface immunological markers did not differ between MF- and HD-MSCs. MF-MSCs ceased their growth at variable passages (BM-MSCs from P8 to P14 and TBF-MSCs from P10 to P14) and entered senescence, without any change in morphology/proliferation rate.

Both in the autologous (MF-MSCs/MF-PBMCs) and allogeneic (HD-MSCs/MF-PBMCs) setting proliferation of PBMCs was reduced up to $81 \%$ and $93,4 \%$, respectively, and resulted to be comparable to that obtained in the setting HD-MSCs/HDPBMCs (maximum inhibition 95\%).

The biological and functional characterization of MF-MSCs may be relevant for a better understanding of the etiopathological mechanisms responsible for this myeloproliferative disorder.

\section{P911}

Culture of mesenchymal stem cells in presence of human platelet lysate as an alternative for animal-derived serum F.. Aerts-Kaya (1), S. Arslan (2), D. Uçkan (3)

(1)Erasmus MC (Rotterdam, NL); (2)Hemosoft IT (Ankara, TR); (3)Hacettepe University (Ankara, TR)

Objectives: Mesenchymal stem cells (MSCs) are pluripotent cells with the capacity to differentiate into fat, bone and cartilage, and possess immune modulating effects. MSCs can be obtained from bone marrow (BM), but expansion requires presence of animal sera. For clinical applications of MSCs, minimizing the use of animal products is advantageous and may contribute to increased safety. Here, we tested the effects of long-term culture of MSCs in presence of human platelet lysates on immunophenotype and differentiation capacity.

Methods: BM-MSCs from 12 healthy donors (age 1-50 yrs) were cultured in DMEM-LG/MCDB-201 with 1\% Penicillin/Streptomycin, $2 \mathrm{mM} \mathrm{L-Glutamin}$ and $10 \%$ fetal bovine serum (FBS) or $5 \%$ platelet lysates $(\mathrm{PL})$ at $1.0 \times 10^{9}$ platelets $/ \mathrm{mL}$. PL were prepared from $>5$ day old pooled platelet transfusions from 10 donors and obtained by 3 quick freeze-thaw cycles. Differentiation assays were performed as described (Uçkan et al., Cytotherapy 2009). Quantitation of differentiation was calculated by Oil Red O extraction (adipogenesis) and Calcium extraction (osteogenesis). FACS analysis was performed at P2. Results: Passage doublings were compared between MSCs in $10 \%$ FBS and $5 \%$ PL. The relationship between passage doubling $(P D)$ and passage $(P)$ could be described as: $P D=0,28 P+3,6$ for MSC in $10 \%$ FBS and PD $=0,30 P+4,0$ for MSC in $5 \% \mathrm{PL}$. The difference was statistically significant $(P=0.011)$. Differentiation into adipogenic lineage was supported by both FBS and PL up to P8 (Oil Red O: $0,48 \mathrm{mg} / \mathrm{mL}$ each) and into osteogenic lineage up to P6 (Calcium: 10,6 and 10,3 mg/dL, respectively). MSCs cultured in FBS or PL were $>95 \%$ positive for CD29, CD44, CD90 and CD73, dim positive for ALP (FBS: 26,3\%; PL: 35,4\%) and negative for CD106 (<2\%), but displayed differences in CD105 (FBS: 81,4\% pos; PL: 40,4\% pos), CD104b (FBS: $86,3 \%$ pos; PL: $5,9 \%$ pos), CD166 (FBS: 17,3\% pos; PL 0,5\% pos), CD271 (FBS: 19,0; PL: 2,8 ) and SSEA4 (FBS: 0,8\%; PL: 8,8\% pos).

Conclusions: MSCs cultured in PL expand faster than MSCs cultured in FBS, but lose their expansion capacity and differentiation potential after serial passaging, similar to MSCs cultured in FBS. Differences in immunophenotype suggest selection of a slightly different subset of adherent cells. Whether MSCs cultured in presence of $\mathrm{PL}$ are functionally similar to MSCs cultured under standard conditions, ie support hematopoietic stem cells and display immune suppressive effects, should be explored before use in a clinical setting.

\section{P912}

Point-of-care preparation of stem cell concentrates from bone marrow aspirate using the MarrowXpress ${ }^{\mathrm{TM}}$ system A. Conley (1), R. Suzuki (2)

(1)Community Hospital (Oklahoma City, US); (2)Spine Smith (Austin, US)

Background and objectives: The MarrowXpress Platform (MXP) is intended for the preparation of stem cell concentrates from bone marrow aspirate at the point of care or in a laboratory setting. The system consists of a microprocessor-controlled, software-operated device with an optical sensor. The device is utilized with a dedicated disposable set consisting of multiple, sterile bags and a microprocessor-controlled valve to direct fluid movement. The fluid path within the disposable is sterile, non-pyrogenic and functionally closed. The system delivers an operator-selected volume of stem cell concentrate into the product bag in 40 minutes of total processing time. The goal of this study was to evaluate the ThermoGenesis MXP system concentration process of human bone marrow under intended use conditions.

Methods: Bone marrow aspirate was collected in an operating room from patients using heparin and ACD-A as anticoagulants. Bone marrow aspirate $(50 \mathrm{~mL}$ to $120 \mathrm{~mL})$ was concentrated into a buffy coat fraction by the MXP process to a final volume of 10 to $20 \mathrm{ml}$ according to the target volume selected by the operator. Complete blood counts were measured using Sysmex XE-2100 cell counter for pre-MXP processed and postMXP processed (cell product) samples. CD34 + cells and cell viability were measured by flow cytometry using StemKit (Beckman Coulter, Brea, CA). The cell recovery was calculated by dividing the total cell numbers in the final product by the total cell numbers in pre-MXP processed bone marrow aspirate.

Results: A total of twenty-five bone marrow aspirates were processed and evaluated from September 1 to October 15, 2009. The cell counts for pre-MXP and post-MXP samples, cell enrichment factor and cell recovery are reported in the Table. The percent RBC depletion was $91.0 \pm 3.7 \%$ with a total packed $\mathrm{RBC}$ volume of $2.1 \pm 0.4 \mathrm{~mL}$ remaining in the cell product. The percent cell viability was unchanged by the processing $(91 \pm 3 \%$ vs. $93 \pm 5 \%$ )

Conclusion: The MXP system was demonstrated to provide a reproducible method to volume reduce human bone marrow volume for the preparation of stem cell concentrates that are suitable for clinical use at the point of care. The stem cells and platelets were concentrated on average $5.9 x$ and $4.3 x$, respectively above baseline by MXP processing.

\begin{tabular}{|l|c|c|c|c|}
\hline & $\begin{array}{c}\text { WBC } \\
\left(\times 10^{\wedge} 6 / \mathrm{mL}\right)\end{array}$ & $\begin{array}{c}\text { MNC } \\
\left(\times 10^{\wedge} 6 / \mathrm{mL}\right)\end{array}$ & $\begin{array}{c}\text { Platelet } \\
\left(\times 10^{\wedge} 6 / \mathrm{mL}\right)\end{array}$ & $\begin{array}{c}\mathrm{CD} 34^{+} \text {Cell } \\
\left(\times 10^{\wedge} 4 / \mathrm{mL}\right)\end{array}$ \\
\hline Pre-MXP & $21+l-10$ & $4.9+/-2.2$ & $100+l-35$ & $15+/-13$ \\
\hline Post-MXP & $89+/-43$ & $27+l-13$ & $426+l-222$ & $89.3+/-75$ \\
\hline Enrichment Factor & $4.2 x$ & $5.5 x$ & $4.3 x$ & $5.9 x$ \\
\hline Cell Recovery (\%) & $69+/-12$ & $89+/-19$ & $71+/-24$ & $95+/-15$ \\
\hline
\end{tabular}




\section{P914}

Comparison of miRNA expression profile during adipocyte induction of mesenchymal stem cells from bone marrows of malignant infantile osteopetrosis patients and bone marrow donors reveals 15 differentially expressed miRNAs

A. Gunel-Ozcan, E. Kilic, F. Aerts-Kaya, M. Cetin, D. Uckan

Hacettepe University (Ankara, TR)

Mesenchymal stem cells, or multipotent mesenchymal stromal cells (MSC), isolated from various adult tissue sources have the capacities to self-renew and to differentiate into multiple lineages. Both of these processes are tightly regulated by genetic and epigenetic mechanisms. Emerging evidence indicates that the class of single-stranded noncoding RNAs known as microRNAs also play a critical role in this process. Studies with various types of embryonic as well as adult stem cells indicate an intricate network of microRNAs regulating key transcription factors and other genes, which in turn determine cell fate. MicroRNAs are also found to be regulated by extracellular signaling pathways that are important for differentiation into specific tissues, suggesting that they play a role in specifying tissue identity. Objectives: Adipogenesis is the developmental process by which a multipotent mesenchymal stem cell differentiates into a mature adipocyte. This process involves a highly regulated and coordinated cascade of transcription factors that together lead to the establishment of the differentiated state. In our lab, it has been recently observed that mesenchymal stem cells (MSC) expanded from the bone marrows of infantile malignant osteopetrosis patients cannot differentiate to adipocytes (Uckan D et al., Cytotherapy, 2009). In this study, we aimed to investigate the global miRNA profile of bone marrow derived adult mesenchymal stem cells of infantile malignant osteopetrosis patients during their differantiation to adipocytes.

Methods: Bone marrow derived MSCs were immunophenotyped. For adipogenic differantiation cells were transferred to induction media. Adipocytes were identified by Oil red $\mathrm{O}$ staining. Quantitation of differantiation was calculated by Oil Red $\mathrm{O}$ extraction. miRNA was isolated from the cells by SABiosciences kit and CDNA first strand synthesis was performed. Expression profile of miRNAs was detected by using 'human whole genome qPCR miRNA array', which covers 376 of most abundant and well characterized miRNAs (SABiosciences).

Results and conclusion: Our preliminary data from an osteopetrosis patient and a healthy bone marrow donor in the second day of adipogenic differantiation showed that fifteen miRNAs were more than four fold differentially expressed in the patient's cells; two of them were upregulated and thirteen downregulated.

\section{Regenerative medicine}

\section{P915}

MAPK p38-dependent foam cell formation of myeloid cells after pro-angiogenic induction as a risk factor of therapeutic angiogenesis

E. Rohde (1), K. Schallmoser (1), A. Reinisch (1), N.A. Hofmann (1), T. Pfeifer (2), G. Rechberger (3), G. Lanzer (4), W. Linkesch (5), D. Kratky (2), D. Strunk (1)

(1)Stem Cell Research Unit (Graz, AT); (2)Molecular Biology and Biochemistry (Graz, AT); (3)Molecular Biosciences (Graz, AT); (4)Department of Transfusion Medicine (Graz, AT); (5)Department of Hematology and Stem Cell Transplantation (Graz, AT)

Clinical trials for therapeutic angiogenesis use blood- or marrowderived transplants containing endothelial progenitor cells (EPCs), myelomonocytic cells and mesenchymal stromal cells (MSCs) to support vascular regeneration. Safety concerns have emerged since all three cell types can also contribute to athero- sclerosis. We therefore asked whether monocytes, EPCs or MSCs do in fact accumulate lipid droplets (LDs) and form foam cells in vitro as a surrogate marker for potential pro-atherogenic side effects of therapeutic angiogenesis.

LD accumulation was quantified by flow cytometry, confocal laser scanning microscopy and cholesterol measurement in each of the cell types following exposure to low density lipoprotein in vitro. The impact of an initial three-day pro-angiogenic culture on subsequent foam cell formation was studied to mimic a relevant setting already used in clinical trials. The phosphorylation state of intracellular signalling molecules in response to pro-angiogenic stimulation was determined to delineate the operative mechanisms and to establish a basis for interventional strategies.

Foam cells developed from monocytes but not from EPCs or MSCs after pro-angiogenic induction. The mitogen-activated protein kinase (MAPK) p38 phosphorylation related to foam cell development and stress-induced stimuli was enhanced in monocytes after pro-angiogenic stimulation. MAPK p38 inhibition almost abrogated intracellular LD accumulation in vitro.

These data raise serious concerns that cellular therapy with hematopoietic cell preparations containing monocytes may be counterproductive or even aggravate atherogenesis in patients with cardiovascular diseases. We therefore support the argumentation that the role of transplanted cells in the various aspects of vascular homeostasis, regeneration and therapeutic angiogenesis must be re-examined prior to further clinical trials.

\section{P916}

In vitro differentiation of unrestricted somatic stem cells into functional hepatic-like cells

S. Waclawczyk, U. Floegel, A. Buchheiser, G. Kögler

University of Dusseldorf Medical School (Dusseldorf, DE)

Unrestricted somatic stem cells (USSC) are a defined CD34-, CD45- neonatal stem cell population derived from human umbilical cord blood (CB) with high expansion potential and the ability to differentiate into osteoblasts, neural and hepatic cells. USSC share many features with mesenchymal stroma cells (MSC), but are distinguishable by strong expression of DLK1 and, accordingly, by restricted adipogentic differentiation potential. DLK1 is referred to as a fetal tissue marker and is expressed in multiple embryonic tissues, but is undetectable in most adult tissues. Notably, DLK1 seems to be a specific marker for hepatoblastomas and is strongly expressed in hepatoblasts of the embryonic and fetal liver. Arjang Ruhparwar and colleagues have analyzed the impact of transplanted USSC on liver regeneration by using a mature ovine model with acute liver ischemia: USSC were injected into the portal vein resulting in an engraftment into the liver-parenchyma as functional hepatocyte-like cells without fusion (A. Ruhparwar, in preparation). With respect to in vitro differentiation, a three-stage culture protocol resembling embryonic developmental processes of hepatic endoderm was used. Hepatic pre-induction was achieved by ActivinA and FGF4, resulting in enhanced expression of SOX17 and FOXA2. Additional induction with RA, HGF, BMP2 and EGF resulted in simultaneous expression of FOXA2, GATA4, HEX and PROX1. For the subsequent hepatic differentiation HGF, FGF4 and OSM were used sequentially for up to 28 days. Differentiated USSC expressed the hepatic markers HSA, GYS2, G6PC, ARG1 and CYP3A4 as well as hepatic transcription factors such as HNF1a and HNF4a on day 28. Functional studies concerning albumin secretion, urea formation, and cytochrome-p450-3A4 (CYP3A4) enzyme activity confirmed the hepatocyte-like phenotype. In order to analyze the metabolic shift, cells were incubated with $[1-13 C]$ glucose. The fate of the molecule's label was traced by isotopomer analysis using 13C-nuclear magnetic resonance spectroscopy (NMR), allowing detection of gluconeogenesis as well as glycogenesis metabolits. These results clearly show that USSC represent a stem cell source with a high hepatic differentiation capacity in vitro eligible for cell based therapies in future. This work was supported by a grant from the DFG KO2119/ 6-1. 
P917

Clinical use of autologous bone marrow mononuclear cells in patients with severe orthopaedic disorders G. Messina, G. Console, G. Irrera, M. Martino, A. Gervasi, F. Scordino, R. Monteleone, I. Callea, A. Pontari, M. Cuzzola, G. Bresolin, G. Topa, P. Iacopino

A. O. Bianchi-Melacrino-Morelli (Reggio Calabria, IT)

The bone marrow contains hematopoietic cells that can generate the progenitors of blood cells including hematopoietic cells called mesenchymal cells, capable of regenerating tissues of mesenchymal type. This type of cells has a potential self-renewal and osteoblastic or chondrocityc or adipocytic or fibroblasts differentiation. For this reason, in our institution we treated patients with severe bone diseases that had failed conventional treatments. For all cases, we obtained the authorization to process by the local Ethics Committee. We treated 14 patients ( 8 males and 6 females) with median age 38 years (r. 3-67). The orthopedic defects were loss of bone, non union, bone cysts and bone lysis tumor in 6, 5, 2 and 1 case, respectively. The entire procedure takes place within a few hours and provides the collection of autologous platelets, the harvest of autologous bone marrow mononuclear cells (BMNC), its processing, orthopedic surgery to stabilize the bone lesion and, finally, the fixture of BMNC on the lesion. The main characteristics of BMNC are shown below: median volume and median number of BMNC infused was $20 \mathrm{ml}($ r. $8-40)$ and $0.73 \times 10^{9}$ (r. $0.32-1,3$ ), respectively. Briefly, the procedure for laboratory manipulation of bone marrow consists of a centrifugation at 2000 rpmx for 10 minutes. The buffy coat formed is collected and mixed gently with autologous platelets, fragments of autologous bone and calcium chloride to the clot formation. Finally, the preparation is injected into the lesion during orthopedic surgery. No patient experienced postoperative infectious complications. Three patients were lost at orthopedic follow-up, three patients showed no improvement, whereas in 7 patients was documented complete functional recovery of the affected limb. One patient with micromolecular multiple myeloma and lytic lesion in the femoral bone was transplanted with autologous transplantation and then allogeneic transplantation from family HLA compatible donor. At 6 months, with a full donor chimerism she was started to surgical stabilization of pathologic fracture using BMNC. She showed a complete recovery in few months but, after two years, because of the progression of the disease, was observed a severe functional limitation of the limb. In conclusion, these preliminary data, despite the small number of cases, show that it is a safe procedure and effective given the severity of the diseases treated and the failure of conventional therapies.

\section{P918}

Stem cell regenerative therapy for paediatric bone defects A. Lanfranchi (1), F. Bolda (1), C. Paganelli (2), F. Valtancoli (3), P. Brunelli (4), F. Porta (1)

(1)Pediatric Oncohaematology and BMT Units (Brescia, IT); (2)Dental School, University of Brescia (Brescia, IT); (3)Pediatric Orthopedic Unit (Brescia, IT); (4)Pediatric Othopedic Unit (Brescia, IT)

Stem regenerative therapy could be a future option to treat pediatric bone defects such as bone cysts or cleft palate, that at present are treated by dexametazone (bone cysts) with $50 \%$ success or surgery (cleft palate). In our institution fresh BM stem cells have been injected within the lesion of 10 children affected by relapsed bone cysts. In two cases, with a sufficient FU, the lesion resolved, while in 8 the lesions are resolving, but the FU is too short. Promising is the perspective use of decidual teeth pulp expanded mesenchimal stem cells of the affetcted children. Infact dental pulp has a high clonogenic potential in culture (251 fold the initial cell count). Moreover, on week 3-4 of culture, shows elevated expression of immature mesenchimal markers such as CD29, CD90, CD73, CD44. Moreover the expression of CD73 confirms a high replicative potential capability as com- pared with BM cells, also tested in RT PCR. It is also possible to show how these cells differentiate into osteogenic precursors as demostrated by the positivity for Alizarine, opening the option to use these cells for therapeutic purposes. Telomerase activity up to the 21st week remains low. Therefore we studied three techniques of cryopreservation of the decidual teeth and demonstrated that only the directly cryopreserved pulp, as compared with cryopreserving the entire tooth or cryopreserving the predigested pulp, has a comparable dubling capability as the fresh pulp. This technique allows to minimally manipulate the dental pulp stem cells from the decidual teeth of children affected by bone cysts or cleft palate, so that they can be used for therapeutic purposes realizing an autologous regenerative procedure.

\section{Reduced-intensity conditioning}

\section{P919}

The value of PFT prior to reduced-intensity conditioning allogeneic stem cell transplantation in predicting respiratory complications

J.L. Piñana, R. Martino, P. Barba, J. Bellido-Casado, D. Valcarcel, A. Sureda, J. Briones, J. Delgado, M. Granell, S. Brunet, P. Casan, J. Sierra

Hospital De La Santa Creu I Sant Pau (Barcelona, ES)

The pre-transplant pulmonary function test (PFT) utility in predicting the development of post transplant respiratory complications (RespC) and mortality is not well established. We thus performed a retrospective study evaluating the predictive value of PFT in the setting of reduced intensity conditioning AlloHSCT recipients only (Allo-RIC). We retrospectively reviewed RespC in 195 consecutive patients who underwent allo-RIC, mainly based on fludarabine $150 \mathrm{mg} / \mathrm{m}^{2}$ in combination with busulfan $(\mathrm{Bu}){ }^{8}-10 \mathrm{mg} / \mathrm{kg}$ or melphalan $7-140 \mathrm{mg} / \mathrm{m}^{2}$, at our center between 1999 and 2006. Patients were assessed for pulmonary function within 30 days before transplant by PFT, recording the following parameters; forced vital capacity (FVC), forced expiratory volume in the first second (FEV1), the FEV1/FVC ratio. Lung volumes included vital capacity, total lung capacity, residual volume, and the residual volume/total lung capacity ratio. Diffusion capacity was determined by using a carbon monoxide. Pulmonary dysfunction and severity were defined according to the recommendations (Chien JW., BMT 2005, 35, 429-435). The median follow-up for survivors was 6 years $(0.5$ to 10 years)

Results: 130 (66\%) patients were found to have PFT abnormalities before transplant. Fifty-four $(28 \%)$ patients had a reduction on the diffusion capacity, $41(21 \%)$ had an obstructive pattern while $28(14 \%)$ patients showed a restrictive pattern. Seven $(3 \%)$ patients showed a mixed pattern. They were severe in 24 $(12 \%)$ patients, moderate in $65(33 \%)$ and mild in 38 patients (19\%). Ninety-eight (50\%) patients developed RespC (78 and 44 patients developed infectious and non-infectious RespC, respectively). The 6-years cumulative incidence of RespC was $45 \%(95 \%$ confidence interval $(\mathrm{Cl})$ : $38-53)$. Multivariate analysis (MVA) showed that RespC were significantly higher in patients with abnormal pre-transplant $\mathrm{PFT}$ (hazard ratio (HR) $2.1,95 \% \mathrm{Cl}: 1.3-3.2)(P=0.002)$, unrelated donor (HR 2.3, 95\% Cl: 1.3-3.9) $(P=0.003)$ and female-donor to malerecipients (HR 2.6, 95\% Cl: 1.6-4.2) $(P<0.001)$. As well MVA showed abnormal pre-transplant PFT as a risk factor for 6-years NRM (HR 1.8, 95\% CI 1-3) $(P=0.05)$. Six-years overall survival was $43 \%$ vs. $23 \%$, for patients with normal and abnormal PFT respectively, $(P=0.001)$.

Conclusions: This study emphasizes the valuable role of PFT in identifying patients at risk for RespC and NRM. Prospective and larger studies are warranted to establish the predictive value of the PFT in Allo-RIC patients. 
P920

Conditioning with treosulfan and fludarabine followed by allogeneic haematopoietic cell transplantation for high-risk haematologic malignancies. Results of a phase I/II multicentre study in the United States

E.R. Nemecek (1), K. Guthrie (2), M. Sorror (2), R. Hilger (3), K. Doney (2), B. Scott (2), T. Kovacsovics (1), R. Maziarz (1), A. Woolfrey (2), J. Sanders (2), E. Sickle (2), J. Borruel-Rector (1), P. O'Donnell (2), R. Witherspoon (2), F. Appelbaum (2), H.J. Deeg (2)

(1)Oregon Health \& Science University (Portland, US); (2)Fred Hutchinson Cancer Research Center (Seattle, US); (3)University of Essen (Essen, DE)

Objectives: Haematopoietic cell transplantation (HCT) is a potentially curative treatment for patients with haematologic malignancies. The therapeutic success of conventional myeloablative HCT regimens is limited by their associated rates of toxicity and nonrelapse mortality (NRM). Reduced intensity regimens have been used increasingly in patients considered at high risk for NRM. We present the results of the first phase $\mathrm{I} / \mathrm{II}$ trial conducted in the United States, which combined treosulfan and fludarabine for patients with acute myeloid (AML) or lymphoblastic leukaemia (ALL) or myelodysplastic syndrome (MDS) undergoing allogeneic HCT.

Methods: Sixty-two patients with median age of 46 years (range $5-60)$, with AML in first remission ( $n=26), A M L$ in second remission $(n=9), A M L$ in refractory relapse $(n=9), A L L$ in second remission $(n=5)$ or MDS $(n=13)$ were enrolled. Ten patients were younger than 18 years at the time of HCT. The majority of patients were considered at high risk for nonrelapse mortality (NRM). Patients received treosulfan $12 \mathrm{~g} / \mathrm{m}^{2} /$ day (first 5 patients) or $14 \mathrm{~g} / \mathrm{m}^{2} /$ day $(\mathrm{n}=57$ ) on days -6 to -4 , and fludarabine $(30 \mathrm{mg} / \mathrm{kg} /$ day) on days -6 to -2 , followed by matched sibling $(n=30)$ or unrelated donor $(n=32)$, bone marrow $(n=7)$ or peripheral blood stem cell $(n=52)$ transplantation. Graft-versushost disease (GVHD) prophylaxis was with tacrolimus $(n=56)$ or cyclosporine $(n=3)$ and methotrexate; 3 patients received cyclosporine and mycophenolate mofetil.

Results: Neutrophil engraftment was seen in all patients at a median of 18 days (range 11-26). Complete donor T-cell and myeloid cell chimerism (i.e., more than 95\% CD3 and CD33 cells of donor origin) was achieved by day 100 in $85 \%$ and $97 \%$ of patients, respectively. Acute GVHD of grades II-IV and chronic GVHD occurred in $55 \%$ and $50 \%$, respectively. Severe toxicity was noted in 2 patients $(3 \%)$ and consisted of mucositis and acute renal failure, both of which resolved. The estimated NRM at 100 days and 2 years were $5 \%$ and $8 \%$. The 2-year overall and relapse-free survival were $65 \%$ and $58 \%$. The 2 -year estimates of relapse were $15 \%$ for patients with MDS, $34 \%$ for AML in first remission, $50 \%$ for AML or ALL beyond first remission and $63 \%$ for $A M L$ in refractory relapse.

Conclusion: We conclude that a regimen of treosulfan and fludarabine is well tolerated, resulting in low rates of toxicity and NRM and adequate disease control in patients with acute leukaemia and MDS.

\section{P921}

Prognostic role of PET scanning before and after reduced-intensity allogeneic stem cell transplant for lymphoma

S. Mackinnon, J. Lambert, J. Bomanji, K. Peggs, K. Thomson, R. Chakraverty, A. Fielding, P. Kottaridis, M. Roughton, E. Morris, A. Goldstone, D. Linch, P. Ell University College London (London, UK)

The prognostic role of PET scanning pre- and post- reduced intensity allogeneic SCT for lymphoma is unknown. We prospectively investigated: 1 ) whether the pre-transplant PET status was predictive of outcome in patients undergoing reduced intensity allogeneic SCT for lymphoma, and 2) whether posttransplant PET imaging would lead to earlier detection of relapse when compared to standard CT scans, thereby potentially allowing earlier use of DLI. Eighty patients with lymphoma have a median follow-up of 45 months. Paired CT and PET scans which were performed pre-transplant, then at 3,6, 9, 15, 24 and 36 months post SCT. Patients were eligible to receive $\mathrm{DLI} \pm$ chemotherapy if there was evidence of relapse, assessed either clinically, by CT or by PET. All patients were conditioned with an alemtuzumab based conditioning regimen. Pretransplant, 38 patients were PET negative and 42 were PET positive. There were no significant differences in patient characteristics between the PET negative and PET positive groups (Table). There were no significant differences in non-relapse mortality between the two groups. Following transplant, 7 had progressive disease and 21 relapsed during follow-up - 15/28 were PET positive pre-transplant. There was no difference in the relapse rate, overall survival, PFS and current PFS between the 2 groups (Figure). Thirty-four episodes of progression/relapse were observed in 28 different patients. In 17 of these, the diagnosis was made using PET alone, i.e. PET +/CT-, There were 13 episodes where both modalities were positive, and 4 where both were initially negative. Nineteen patients had residual abnormalities on CT post-transplant, with a normal simultaneous PET. None of these patients received anti-lymphomatous therapy and only 4 of 19 patients with PET negative residual masses observed on CT post-transplant subsequently relapsed within these masses. These findings suggest that in contrast to autologous SCT, allogeneic transplantation may result in good outcomes for patients with lymphoma, regardless of pretransplant PET status. Post-transplant surveillance by PET may facilitate earlier administration of DLI in some patients with recurrent lymphoma, and withholding of potentially harmful DLI in those with PET negative masses on CT scans.

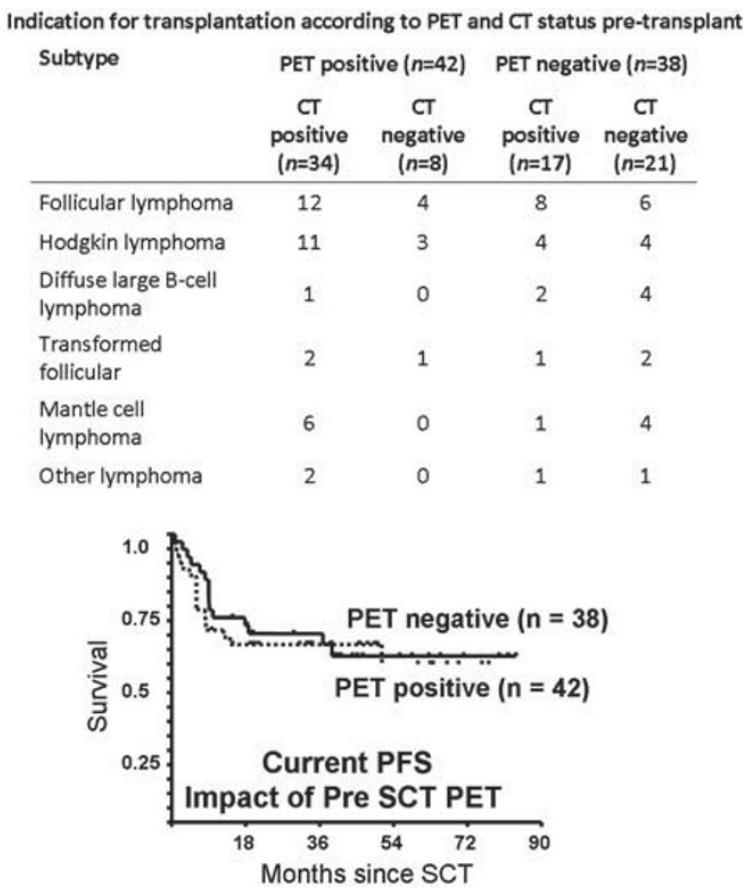


P922

The use of bone marrow graft for haematopoietic stem cell transplantation from matched unrelated donors after reduced-intensity conditioning regimen improves survival: analysis of the Société Francaise de Greffe de Moelle et de Therapie Cellulaire

A. Toma (1), M.L. Balere (2), J.-M. Boiron (3), P. Bordigoni (4), G. Socie (5), J. Vernant (1), M. Renaud (6), N. Milpied (3), M. Michalet (7), E. Deconinck (8), D. Guyotat (9), F. Garban (10), A. Sirvent (11), N. Contentin (12), D. Blaise (13), E. Marry (2), A. Buzyn (14)

(1)Hopital Pitie Salpetriere (Paris, FR); (2)Agence de la Biomedecine (Saint Denis, FR); (3)CHU (Bordeaux, FR); (4)CHU (Nancy, FR); (5)Hopital Saint Louis (Paris, FR); (6)CHU (Tours, FR); (7)Hopital Edouard Herriot (Lyon, FR); (8)CHU (Besancon, FR); (9)Institut de Cancérologie de la Loire (Saint Priest en Jarez, FR); (10)CHU (Grenoble, FR); (11)CHU (Nice, FR); (12)CRLCC Henri Becquerel (Rouen, FR); (13)Institut Paoli Calmettes (Marseille, FR); (14)Hopital Necker (Paris, FR)

The use of peripheral blood stem cells (PBSC) for hematopoietic stem cell transplantation (HSCT) associates with higher risk of chronic graft versus host disease (GvHD) but may favor a greater graft versus leukemia (GvL) effect.

Objective: We proposed to evaluate the role of various parameters including the source of stem cells (SC) on the outcome in the context of HSCT from unrelated donors (UD), because the balance between $\mathrm{GvH}$ and GvL may differ from the context of sibling donors and thus the use of PBSC may be deleterious.

Methods: We analyzed retrospectively 103 patients from the french registry who received a graft from an UD after a reduced intensity conditioning regimen (RIC). $69 \%$ D/R pairs were $10 / 10$ HLA match at the allelic level. Mismatches concerned 5, 6, 15, 2 and 7 D/R pairs for HLA-A, -B, -C, -DRB1 and -DQB1, respectively. The median age was 46 years (18-67). Diagnosis were: AL $(n=35), \operatorname{MM}(n=18), \operatorname{CLL}(n=5), \mathrm{NHL}(n=11), \operatorname{HD}(n=9)$, CML $(n=12)$, MDS $(n=9)$, and MPS $(n=4) .39 \%$ of patients were in an advanced phase of the disease at time of HSCT. RIC was Fluda/TBI 2Gys $(n=26)$, Bu/Fluda/ATG $(n=24)$, Fluda/Melph $(n=16)$ and other for 37 patients. Anti-thymocytes globulins (ATG) were part of the RIC for $77 \%$ of patients. The source of SC was PBSC for 65 patients and bone marrow (BM) for 38 patients. The median follow up of the cohort is 61,3 months $(1,2-113,7)$.

Results: $95 \%$ of patients engrafted. Five patients did not engraft ( 4 in the BM group and 1 in the PBSC group). Acute GvHD grade $2-4$ and grade $3 / 4$ occurred in $47 \%$ and $19 \%$ of patients, respectively. The risk of developing chronic GvHD was $49 \%$ at 2 years. Overall survival (OS) was $36 \%$ at 5 years. The median DFS was 55 months among the 36 patients alive. We performed univariate and multivariate analysis of factors susceptible to impact on GvHD and survival. The multivariate analysis included the impact of HLA mismatch, disease status, diagnosis, source of SC, patient's and donor's ages. The multivariate analysis performed on the global population shows a trend towards an improved OS with the use of BM instead of PBSC. When focusing the multivariate analysis on the 71 patients transplanted with a 10/10 match UD, the most potent factor influencing the outcome is the use of BM which is associated with an improved OS $(P=0.03)$ and DFS $(P=0.02)$.

Conclusion: These results suggest that the use of BM as the source of SC should be reconsidered in the context of matched UD after RIC transplantation.
P923

Clofarabine-melphalan-alemtuzumab conditioning for allogeneic haematopoietic cell transplantation in advanced haematologic malignancies. Immunosuppression and long-term responses compared with fludarabinemelphalan-alemtuzumab

K. van Besien, J. Kline, L. Godley, R.A. Larson, O. Odenike, W. Stock, S. Horowitz, A. Wickrema, S. Allen, P. del Cerro, L. Schroeder, A. Artz

University of Chicago (Chicago, US)

The fludarabine melphalan-alemtuzumab combination is a well tolerated reduced intensity conditioning regimen for HCT. Unfortunately recurrence rates are high, particularly for those with active disease entering $\mathrm{HCT}$. Clofarabine (Clo), a second generation nucleoside analog with excellent activity in acute leukemia, might enhance disease control. We are conducting a phase II study of clofarabine-melphalan and alemtuzumab (CMA) in patients with advanced hematologic malignancies and have accrued 45 patients, median age 54 (21-73). 19 had siblings and 25 had unrelated donors. 25 had acute leukemia/ MDS, $16 \mathrm{NHL}, 2 \mathrm{CLL}, 2 \mathrm{MPD}$. ASBMT risk score was high in 16, intermediate in 15 and low in 14. The phase II dose was initially set at Clo $40 \mathrm{mg} / \mathrm{m}^{2} /$ day $\times 5$ days and melphalan $140 \mathrm{mg} / \mathrm{m}^{2}$ and twenty four pts received this dose. Because of occasional renal toxicity the dose of clofarabine was subsequently reduced to $30 \mathrm{mg} / \mathrm{m}^{2}$. We compared the outcomes of patients receiving CMA with that of a historical control group of 172 pts receiving FMA and identical supportive care. $\mathrm{Pt}$ and disease characteristics of the FMA group were similar to the FMA group, but median follow up was longer. At day 100, full donor chimerism in peripheral blood T-lymphocytes was observed more frequently after CMA than after FMA ( $72 \%$ vs. $41 \%, P=0.02$ ) suggesting more potent immunosuppression by CMA. With a median follow up of 12 months, progression free survival after CMA was equivalent to that after FMA at approximately $60 \%$. Outcomes for myeloid malignancies and for lymphoma are illustrated below.

Conclusions: CMA is overall well tolerated, is more immunosuppressive than FMA and can induce durable remissions after allogeneic stem cell transplantation. Long term outcomes are at least equivalent to those of FMA. The study continues accrual.

Supported by an unrestricted grant from Genzyme Inc. 

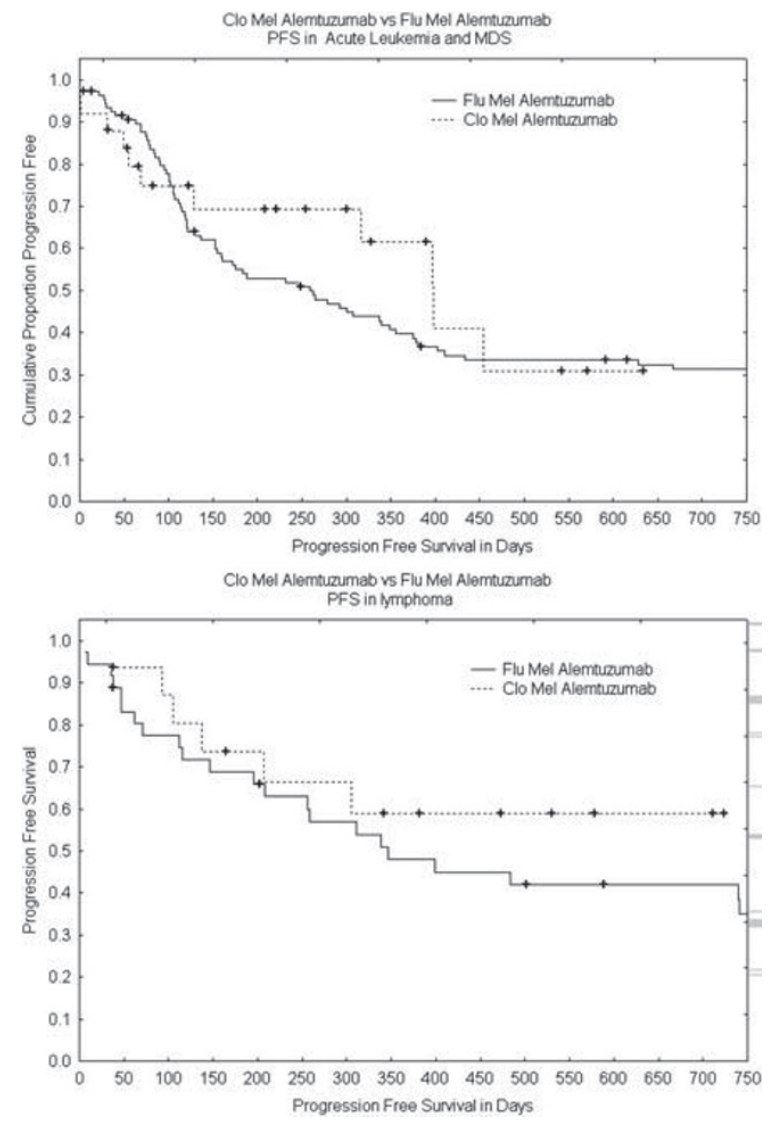

P924

Features of Epstein-Barr virus reactivation after reduced-intensity conditioning allogeneic stem cell transplantation

Z. Peric (1), P. Chevallier (1), T. Guillaume (1), J. Delaunay (1), S. Ayari (1), V. Dubruille (1), S. Le Gouill (1), B. Mahe (1), T. Gastinne (1), N. Blin (1), B. Saulquin (1), N. Milpied (1), R. Vrhovac (2), J.L. Harousseau (1), P. Moreau (1), M. CosteBurel (1), B.M. Imbert-Marcille (1), M. Mohty (1)

(1)CHU de Nantes (Nantes, FR); (2)University Hospital Merkur (Zagreb, HR)

Epstein-Barr Virus (EBV) reactivation is a recognized complication of allogeneic stem cell transplantation (allo-SCT). Since EBV-related lymphoprolipherative disease (LPD) is associated with high mortality, pre-emptive anti EBV treatment with rituximab is now regularly used in this setting.

The aim of this single centre study was to assess incidence, kinetics and predictive factors of EBV reactivation and LPD in 175 consecutive patients undergoing reduced-intensity conditioning (RIC) allo-SCT between January 2005 and June 2009 In this series, the median age was 56 (range, 18-71) years. $49 \%$ of the patients had a myeloid malignancy, while $49 \%$ had a lymphoid malignancy, and $2 \%$ had sAA. $83 \%$ were considered as having high risk disease. PBSC were used in $94 \%$ of cases. Donors were matched related siblings in $48 \%$ of cases, matched-unrelated in $46 \%$ and mismatched-unrelated donors in $6 \% .106$ patients $(61 \%)$ received a fludarabine-busulfan-ATG RIC regimen, while $21 \%$ received fludarabine and low dose TBI. The remaining 32 patients received different chemotherapybased RIC regimens. Patients were screened weekly for EBV reactivation by DNA-PCR until recovery of the circulating CD4 + cells count (usually $>0.3 \times 10^{9}$ ). EBV reactivation was defined as viremia of 1000 EBV-DNA copies/105 cells. Patients with EBV reactivation were pre-emptively treated with rituximab.
With a median follow-up of 655 (range, 92-1542) days for surviving patients, $35 \%$ of patients developed grade $2-4$ acute GVHD at a median of 34 days after allo-SCT. EBV reactivation was observed in 34 patients $(19 \%)$ at a median of 58 (range 0-930) days after allo-SCT. The highest viral load in these patients was found during the 2nd and 12th week after transplantation (Figure 1). Twenty seven (79\%) of these 34 patients were pre-emptively treated with a median of 3 (range, 1-4) rituximab infusions which resulted in complete resolution of EBV viremia in all but one patient (97\%). Of note, none of the patients included in this series developed EBV-induced LPD, and there was no statistically significant difference in overall survival between patients with or without EBV reactivation $(P=0.63)$. In univariate and multivariate analyses, only the use of ATG as part of the RIC regimen was a significant parameter associated with EBV reactivation $(P=0.0027)$.

Overall, this study shows that the rate of EBV reactivation after RIC allo-SCT is relatively low ( $<20 \%)$ without any single case of EBV-related LPD, highlighting the beneficial impact of close EBV monitoring and of the use of pre-emptive rituximab treatment.

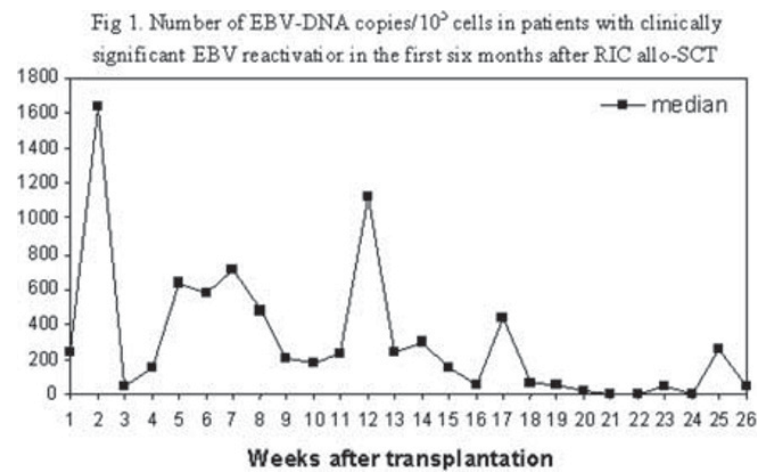

P925

No direct effect of NOD2/CARD15 variants on clinical outcome after non-myeloablative allogeneic stem cell transplantation

H.M. van der Straaten (1), M.M. Paquay (1), M.G.J. Tilanus (2), N. van Geloven (3), L.F. Verdonck (4), C. Huisman (5) (1)UMCU (Utrecht, NL); (2)UMCU (Utrecht, NL), currently azM (Maastricht, NL); (3)AMC (Amsterdam, NL); (4)UMCU (Utrecht, $N L)$, currently Isala Clinics Zwolle (Zwolle, NL); (5)UMCU (Utrecht, NL), currently AMC (Amsterdam, NL)

Background: Single nucleotide polymorphisms (SNPs) in the innate immunity receptor NOD2/CARD15 have been demonstrated to modulate the outcome of allogeneic haematopoietic stem cell transplantation. The effect of the NOD2/CARD15 polymorphism seems to be associated with donor source as well as type of conditioning regimen.

Methods: We reviewed NOD2/CARD15 mutations in all donor/ recipient pairs of 192 consecutive patients who received a nonmyeloablative allogeneic stem cell transplantation (SCT) at our institution between 2002 and 2006. All patients were treated with fludarabine $30 \mathrm{mg} / \mathrm{m}^{2} /$ day for 3 days followed by $200 \mathrm{cGy}$ TBI $(n=154)$ or TBI alone $(n=38)$ and received grafts from HLA-matched related $(n=132)$ or unrelated $(n=61)$ donors.

Results: Mutated NOD2/CARD15 alleles were observed in 36 of $192(19 \%)$ patients and in 35 of $192(18 \%)$ donors. These SNPs, however, did not have a significant impact on clinical outcome $(P>0.05$, Kaplan Meier and Fine \& Gray's test $)$. Acute graft-versus-host disease (GVHD) occurred in 24 of 61 (39\%) patients with and in 65 of $131(50 \%)$ patients without NOD2/ CARD15 SNPs. The cumulative incidence of chronic GVHD was $51 \%$ versus $64 \%$ in patients with variant versus wild type NOD2/CARD15 alleles. Relapse was seen in 23 of $61(38 \%)$ patients with the NOD2/CARD15 variants and in 48 of 131 $(37 \%)$ wild type patients. The incidence of transplant-related 
mortality was $21 \%$ in both groups. Finally, overall survival was $43 \%(26 / 61)$ in patients with and 39\% (51/131) in patients without NOD2/CARD15 SNPs.

Conclusion: These data indicate that mutations in the NOD2/ CARD15 gene do not influence the clinical outcome of nonmyeloablative allogeneic SCT directly. Since NOD2/CARD15 variants are not recognized as a single significant prognostic factor, screening for NOD2/CARD15 when selecting a donor does not seem to have additional value in patients undergoing non-myeloablative SCT.

\section{P926}

Busulfan-based reduced-intensity regimen before allogeneic stem cell transplantation is well-tolerated and effective in patients with haematological diseases L. Castagna, S. Fürst, J. El Cheikh, C. Faucher, N. Vey, R. Bouabdallah, A. Stoppa, M. Mohty, C. Chabannon, B. Esterni, D. Blaise

Institut Paoli Calmettes (Marseille, FR)

Background: Busulfan-based reduced-intensity regimen (RIC) was widely used in the Allogeneic setting, showing good safety profile and efficacy. The intravenous formulation, Busilvex, is considered a forward step compared to oral form because of reproducible biodisponibility and pharmacokinetic. In this study, we evaluated the toxic profile and clinical efficacy of a busilvexbased RIC.

Patients and methods: Since 2005, 78 patients received a uniform RIC consisting of busilvex $3.2 \mathrm{mg} / \mathrm{kg} /$ day for 2 days, Fludarabine $40 \mathrm{mg} / \mathrm{m}^{2} /$ days for 5 days, and thymoglobulin 2.5 $\mathrm{mg} / \mathrm{kg} /$ day for 2 days. Cyclosporine was used as post-graft immunesuppression. The median age was 58 years (24-70), and $41 \%$ of patients suffered from acute myeloid leukemia. $81 \%$ were in complete or partial remission before RIC. Donor was a HLA identical sibling for $64 \%$ of patients. All donors were mobilized with G-CSP and then harvested. $43 \%$ of patients received previous high dose chemotherapy.

Results: The median follow-up was 16 months (2-51). No patients developed venocclusive disease (VOD). The cumulative incidence $(\mathrm{CI})$ for grade II-IV acute graft versus host disease (GVHD) was $31 \%$, and for extensive chronic GVHD was $25 \%$. Overall 9 pts died from TRM for a 2 year $\mathrm{Cl}$ of $16 \%$ (8-28). In addition, 18 pts relapsed or progressed for a 2 year $\mathrm{Cl}$ of relapse progression of $30 \%(17-48)$. Overall the $2-y$ overall survival (OS) and disease free survival (DFS) probabilities were respectively $71 \%(56-82)$ and $58 \%(42-72)$.

Conclusions: This retrospective study shows that busilvexbased RIC is well tolerate with low TRM incidence in a highrisk cohort of patients. Particularly, the extensive chronic GVHD incidence was low probably due to thymoglobulin use for HLA identical donors. VOD was absent even if reversible hepatic toxicity was frequently observed. These promising safety results invited us to asses the impact of higher dose of IV Busulfan in an ongoing trial to improve disease control.

\footnotetext{
P927

Prognostic indexes are not predictive of treatment-related mortality in patients older than 60 years treated with reduced-intensity conditioning and allogeneic stem cell transplantation

L. Castagna, S. Fürst, J. El Cheikh, C. Faucher, M. Mohty, B. Esterni, D. Blaise

Institut Paoli Calmettes (Marseille, FR)

Background: For many diseases, the median age at diagnosis is around the sixth decade of life, precluding myeloablative ALLO. Reduced-intensity conditioning and allogeneic stem cell transplantation (RIC-ALLO) is less toxic and it has been performed in elderly patients (pts), mainly affected by acute leukemia. Prognostic indexes seem to predict treatment related mortality (TRM) and overall survival (OS).
}

Patients and methods: From 2001 and 2008, 63 pts older than 60 years (median age $63 \mathrm{y}$, range 60-70) received RIC-ALLO. Diseases were: acute myeloid leukemia $45 \%$, and chronic lymphoproliferative diseases $54 \%$. Disease status at RIC-ALLO was: complete remission $54 \%$, partial remission $16 \%$, and active disease $30 \%$. RIC consisted of fludarabine-based with thymoglobulin $64 \%$, or low-dose TBI-based $36 \%$. Donors were: HLAid sibling $73 \%$, matched unrelated $21 \%$, and cord blood $6 \%$. Three prognostic scores were evaluated.

Results: The median follow-up was 22 months. The 2-y OS and PFS were 66.8\% (IC95 [55.5-80.4]) and 52.4\% (IC95 [39.569.5]), respectively. Grade II-IV acute graft versus host disease (aGVHD) and chronic GVHD (cGVHD) incidence were $49 \%$ and $43 \%$, respectively. Early infections were fever of unknown origin in $42 \%$ of pts, bacterial infection in 6 cases, pneumonia in 8 , and viral infections in 14 . The early infection-related mortality was null. Late infections were bacterial in 3 cases, pneumonia in 1 , viral infections in 6 , and candidemia in 1 . Seven pts died from late infective complications. Overall, the cause of death was toxicities in $18 \mathrm{pts}$ and disease progression in 6 pts. The $100-d$ and $1-y$ TRM were $6 \%$ and $24 \%$, respectively.

In univariate analysis, $\mathrm{HCT}-\mathrm{Cl}$, EBMT score, and PAM score did not influence TRM or OS. Furthermore, age (60-65 vs. 66-70) was not related to TRM. Only severe aGVHD was predictive. Conclusions: The aim of this retrospective study was to verify if TRM was excessively high in elderly pts, after ALLO-RIC. A secondary objective was to evaluate if several prognostic indexes could predict TRM and OS. TRM was acceptable and not different when compared to younger pts as reported in literature. Furthermore, neither prognostic index nor age help segregate a group of pts with different TRM.

\section{P928}

Reduced-intensity conditioning transplants might be associated with high incidence of fertility preservation in female patients with haematological malignancies

S. O. Ahmed (1), M. Davies (2), D. Marks (3), S. Denovan (1), A. Fielding (1), R. Chakraverty (1), S. Mackinnon (1), P.D. Kottaridis (1)

(1)Royal Free Hospital (London, UK); (2)University College London Hospitals NHS Trust (London, UK); (3)University of Bristol (Bristol, UK)

Introduction: Loss of fertility, is a major side-effect and cause for concern in patients undergoing allogeneic transplantation. Fully myeloablative regimes are more likely to render females nonfertile. Reduced intensity conditioning (RIC) transplants causes less toxicity and may cause a lower incidence of subfertility, as we have previously reported. We have identified 5 female patients who had preserved fertility prior to transplantation who maintained this post transplant.

Methods: We retrospectively collected data on female patients who were being followed up in the late effects clinic and had i) maintained menstruation, ii) normal LH, FSH and oestradiol levels, iii) radiological evidence of ovarian follicles and/or iv) went on to conceive.

Patient characteristics: Our data base includes 46 patients of reproductive age who underwent a RIC for haematological malignancies. Forty one out of 46 were infertile prior to the transplant and remained so and after the transplant. Of the 5 patients who had preserved fertility, the median age was 27 years (range 19-35). Diagnoses were Hodgkin Lymphoma (2), MDS (2), and severe aplastic anaemia (1). One of the HL patients had previously received 4 lines of therapy including an autograft; the other had received previous ABVD and ESHAP. Of the MDS patients, one received ALG and ATG for hypoplastic MDS, and the other received chemotherapy including FLAGIda. The patients received either a sibling allograft $(n=1)$ or matched unrelated donor transplant $(n=4)$ with reduced intensity conditioning (RIC) which consisted of flu/mel/CAMPATH (2 pts), flu/tresulfan/CAMPATH (1 pt), flu/mel (1), flu/cyclo/CAMPATH (1 pt). Of these 5 patients, 2 patients went on to become 
pregnant. Of the remaining 3 patients, all 3 had post transplant menstruation and relative preservation of ovarian function as evidenced by LH, FSH and oestradiol. Two of these patients had an ultrasound showing presence of ovarian follicles.

Conclusions: We report on 5 female patients who underwent RIC transplants for various haematological disorders who had physiological features of maintained fertility prior to and after the transplant. Interestingly, our RIC Flu/Mel/ \pm Campath regime seems to preserve fertility in all 3 females who were fertile prior the transplant and preserved their fertility after the procedure. This is an important consideration in counselling female patients for whom loss of fertility is a major concern.

\section{P929}

Frequencies of severe acute GvHD and relapse after reduced-intensity conditioning allogeneic stem cell transplantation correlate with the kinetics of CD3+ T-cell engraftment

E. Toffoletti, F. Patriarca, A. Sperotto, R. Gallina, A. Chiarvesio, A. Michelutti, M. Cavallin, M.L. Battista, F. Zaja, R. Fanin Hematological Division of Udine (Udine, IT)

Objectives: This study investigated the kinetics of whole peripheral blood (PB) and CD3 + T-cells chimerism in patients receiving reduced-intensity conditioning (RIC) allogeneic stem cell transplantation (SCT). The T-cell chimerism has been correlated with risk of grade II-IV acute graft-versus host disease (GvHD) and relapse.

Materials and methods: Forty-nine patients with a median age of 57 years (range 17-69) affected by lymphoma (27), multiple myeloma (11), acute myeloid leukaemia (9), idiopathic myelofibrosis (1) or paroxysmal nocturnal hemoglobinuria (1) received RIC allogeneic SCT at Hematology Division of Udine between January 2007 and August 2009. Source of stem cell was PB for all patients out one, that received one cord blood unit, and donors were matched unrelated for 35/49 patients. Conditioning regimens were: thiotepa plus cyclophosphamide (27), 2 Gy total body irradiation (TBI) plus fludarabine (3), melphalan plus fludarabine (7), total lymphoid irradiation (1), others (10). In 31 cases anti-thymocyte globulin was used as part of GVHD prophylaxis. Hematopoietic chimerism has been serially assessed at 30,60, 90 and 180 days after SCT in whole PB and sorted CD3 + T-cells. The analysis have been performed by polymerase chain reaction (PCR) based amplification of short tandem repeats (STR) sequences using the AmpflSTR identifler kit (Applied Biosystems).

Results: All patients reached engraftment at day +30 and at day 180 all fractions were $100 \%$ donor.

The mean percentage of recipient cells in CD3 + T-cells was always lower at 30,60 and 90 days in patients with aGvHD in comparison with patients without aGvHD. Patients who subsequently relapsed had a significant lower incidence of CD3+ FDC at day $30(55 \%$ vs. $87 \%, P=0.07)$, at day $60(50 \%$ vs. $88 \%, P=0.03)$ and at day $90(66 \%$ vs. $100 \%, P=0.01)$ in comparison with patients with sustained remission.

Conclusions: We conclude that mixed chimerism at days 30,60 and 90 was associated with an increased risk of relapse and we shown that the development of acute GvHD was correlated with the kinetics of T-cell chimerism. These findings can help the management of immunotherapy after RIC allogeneic stem cell transplantation.

\section{P930}

Increased allogeneic transplant conditioning intensity without greater toxicity: a three-day intravenous busulphan (Busilvex ${ }^{\circledR}$ ) based schedule with promising results in high-risk haematological malignancy

D.S. Richardson, C. Hurlock, K. Hill, J. Newman, K.H. Orchard Southampton University Hospitals (Southampton, UK)

Reduced intensity conditioning schedules containing Fludarabine and 2 days of oral Busulphan are reported as well tolerated.
Published results and our experience indicates that relapse rates, particularly for patients with high risk disease, remain unacceptably high. We have investigated a transplant conditioning schedule containing 3 days Busulphan in combination with Fludarabine and Alemtuzumab, in patients with advanced disease. We elected to use intravenous Busulphan (Busilvex®) in view of its excellent pharmacokinetic profile.

Methods: 45 patients with haematological malignancy underwent allogeneic transplantation using a Busilvex-based schedule: 14 received 2 days Busilvex (FB2C) and 31 received 3 days (FB3C). The 31 patients who received $\mathrm{FB} 3 \mathrm{C}$ were judged to have high-risk disease. The median age for patients receiving $\mathrm{FB} 2 \mathrm{C}$ was 50.5 years (range, 21-58) and $\mathrm{FB} 3 \mathrm{C} 54$ years (range, 23-64), with a median follow-up (survivors) of 21.5 months (range 4.4-68.6). Matched sibling/related donors were used for 26 (FB2C-10, FB3C-16) and unrelated donors for 19 patients (FB2C-4, FB3C-15). All patients received in vivo T-cell depletion with Alemtuzumab.

Results: The non-relapse mortality at day 100 and 6 months was $0 \%$ and $0 \%$ respectively for all patients. Of the patients treated with FB2C, 9 of $14(64.3 \%)$ remain alive, all in CR. Of these 3 have relapsed and have responded to further treatment including a second allograft in 2 cases. 5 FB2C patients have died: 2 relapse, 1 graft versus host disease (GvHD) and 2 from multiorgan failure. 22 of $31(71 \%)$ patients who received FB3C are currently alive. Of the 22 survivors evaluable for response, 20 are in CR and 2 are currently being treated for relapse. 9 patients who received FB3C have died, 6 from relapse; 4 of these had disease present at the time of transplant. Both FB2C and $\mathrm{FB} 3 \mathrm{C}$ schedules were well tolerated with no unexpected toxicities. Two of 33 patients (FB2C-1, BF3C-1) experienced grade 3-4 acute GvHD.

Conclusion: A 3 day schedule of intravenous Busulphan in combination with Fludarabine and Alemtuzumab can be delivered with excellent tolerability, even to heavily pre-treated and older patients and shows promising results in the treatment of high risk haematological malignancy.

\section{P931}

Preliminary results of a phase II trial of clofarabine with busulfan followed by allogeneic related or unrelated donor transplantation for the treatment of haematologic malignancies

E. Agura, E. Vance, R. Berryman, L. Pineiro, G. Saracino, R. Woelfel, M. Tadic-Ovcina, J. Fay

Baylor University Medical Center (Dallas, US)

Background: We tested a novel reduced-intensity transplant (RIT) regimen: clofarabine (CLO) with busulfan (BU) followed by donor allografting, (CLO/BU/Allo).

Methods: Allograft candidates likely to benefit from RIT were selected. Disease criteria included: MDS (IPSS $\geq 2.5$ ), acute leukemia (AML or ALL) in high-risk CR1 or beyond, CLL (fludarabine failures). Donors were matched at HLA A, B, C, DR and DQ using SBT (UD) or mid-level DNA (RD). HLA match grade: $1 \mathrm{Ag} \mathrm{mm}$ or better. Regimen: CLO $40 \mathrm{mg} / \mathrm{m}^{2}$ iv d-8 to $\mathrm{d}-4$, BU $3.2 \mathrm{mg} / \mathrm{kg} / \mathrm{d}$ iv d-3 \& d-2, PBSC infusion d0. AGVHD prophylaxis was tacrolimus + MTX d1,3,6. Endpoints were disease response, chimerism, toxicity and AGVHD.

Results: Treated: $13 \mathrm{M}$ and $7 \mathrm{~F}$ of median $60(28-71) \mathrm{yrs}$. Diseases: AML $(n=16), \operatorname{ALL}(n=1)$, MDS $(n=2), \operatorname{CLL}(n=1)$. Cytogenetic risk groups: fav., $n=2$, int., $n=4$, unfav., $n=14$. Prior therapies: chemo, $n=17$, supportive care, $n=2$, or chemo + allotransplant, $n=1$. Patients with $A L$ were in relapse, $\mathrm{n}=13$ (median BM blasts, $24 \%(7-48 \%)$ or remission, $n=7$. Donor status: related, $n=6$, unrelated, $n=14$. Median KPS, $80(70-90) \%$.

Toxicities: Gr. 4 heme toxicity was seen in $100 \%$. The median time to $A N C>500 \times 3 d$ was $15 \mathrm{~d}$. Marrow engraftment $(>90 \%$ donor @ d 30 ) occurred in 100\% of cases. There were no cases of hepatic, cardiac, or renal RRT. Hepatic VOD requiring treatment did not occur. GVHD: The maximal grade of AGVHD by 
day +100 was $\mathrm{G} 0$ (none)=4, GI=13, GII=0, GIII=3, GIV=0. No deaths due to AGVHD occurred by d100.

Response, survival: All patients achieved a complete remission following the transplant. The CR rate at $d+30$ was $19 / 20$ (95\%). (CCR, $n=6$, new CR, $n=12)$. One case of PR at $d+30$ became $C R$ at $d+90$. Relapse occurred in 4 patients (day +60 , $+62,+120,+335)$. The mean time to relapse is 144 days. With median length of followup of 454 (74-954) days, the overall survival (OS) is $n=11 / 20(55 \%)$. Causes of death include relapse, $\mathrm{n}=4,(+161,+220,+223+415)$, cardiac arrest, $\mathrm{n}=1(+316)$, TTP, $\mathrm{n}=1(+438)$, AGVHD $P$ DLI, $\mathrm{n}=1(+175)$, aspergillosis, $n=1(+158)$, multifactorial, $n=1(+92)$.

Pharmacokinetics: The average concentration of clofarabine declined significantly from day $1(265 \pm 66 \mathrm{ng} / \mathrm{ml})$ to day 5 $(238 \pm 61 \mathrm{ng} / \mathrm{ml})(P=0.03)$. Other clinical correlations with are under study.

Conclusions: The novel RIT regimen CLO/BU/Allo has high activity against myeloid and lymphoid malignancies, facilitates engraftment, and has a low non-hematologic toxicities including AGVHD. These results warrant further study.

\section{P932}

Reduced-intensity conditioning with fludarabine, low-dose busulfan and antithymocyte globulin prior to allogeneic stem cell transplantation

F. Malard, E. Brissot, J. Delaunay, S. Ayari, T. Guillaume, P. Chevallier, V. Dubruille, B. Mahe, N. Blin, T. Gastinne, S. Le Gouill, B. Saulquin, J.L. Harousseau, P. Moreau, N. Milpied, M. Mohty

CHU Nantes (Nantes, FR)

The spectra of patients and diseases for which allogeneic stem cell transplantation (allo-SCT) is now considered have increased considerably, this is mainly due to the introduction of the RIC regimens. There is a wide variability in the degree of myeloablation between the different reduced-intensity conditioning (RIC) protocols and the toxicity profile might vary significantly. The combination of fludarabine and Busulfan (usually $8 \mathrm{mg} / \mathrm{Kg}$ total dose) with or without ATG, is among the most widely used RIC protocols.

In an attempt to decrease the toxicity of the transplant procedure, we hypothesized that reduction $(50 \%)$ of the Busulfan dose can improve transplant outcome. This pilot study tested the combination of Fludarabine $\left(120 \mathrm{mg} / \mathrm{m}^{2}\right)$, Busulfan (4 mg/Kg total dose administered orally over a single day) and Thymoglobuline $(5 \mathrm{mg} / \mathrm{Kg})$ as a RIC regimen prior to allo-SCT in a single centre series of 46 patients. The cohort included 27 males and 19 females with a median age at time of alloSCT of 57y (range, 12-64). Diagnoses included 21 cases of AML, $12 \mathrm{NHL}, 6$ Hodgkin diseases, 3 ALL, 2 myeloproliferative syndromes, $1 \mathrm{MDS}$ and $1 \mathrm{CLL}$. PBSCs were used as stem cell source in 42 patients, 4 patients received classical bone marrow. A matched-related donor was used in 18 cases and an unrelated donor in 28 cases.

With a median follow-up of 30.4 (range, 23.7-50.8) months, the median peripheral blood chimerism of donor origin at day 30 after allo-SCT was 99\%. 20 patients experienced grade 2-4 acute GVHD, including 13 cases of grade 3-4 acute GVHD. 9 patients experienced chronic GVHD. At time of last followup, 26 patients were still alive. Relapse or disease progression occurred in 13 patients at a median of 3.5 (range, 0.6-18) months after allo-SCT. Disease progression accounted for 7 deaths, while transplant-related causes were observed in 13 cases, for a TRM rate of $28.3 \%$. The KM estimates of disease-free survival (DFS) and overall survival (OS) at 3 years after allo-SCT were $46.9 \%$ and $56.5 \%$ respectively. OS was lower in the AML subgroup as compared to the remaining 25 patients with other diagnoses $(42.9 \%$ vs. $68 \%, P=0.09)$.

So low dose Busulfan combined with fludarabine and ATG is a feasible RIC regimen that can allow engraftment after allo-SCT in heavily pre-treated patients with an acceptable toxicity profile. However, in the setting of AML, disease control may be a matter of concern, especially in the early period after allo-SCT, suggesting that this type of RIC should be reserved for patients with lymphoid or indolent malignancies.

\section{P933}

Reduced-intensity conditioning combined with standard GvHD prophylaxis is tolerable and highly effective for patients with primary cutaneous T-cell lymphomas undergoing allogeneic transplantation in advanced disease stage

L. Cudillo (1), R. Cerretti (1), A. Picardi (1), M. Mirabile (1), F. Giannotti (1), G. De Angelis (1), B. Mariotti (1), E. Ceresoli (1), A. Di Veroli (1), M.C. Petti (2), F. Pisani (2), M. Postorino (1), M. Cantonetti (1), W. Arcese (1)

(1)Tor Vergata University (Rome, IT); (2)Regina Elena Hospital (Rome, IT)

Background: Primary cutaneous T-cell lymphomas (CTCL) are an heterogeneous group of non Hodgkin's lymphomas, CTCL in advanced stage have a poor prognosis with conventional therapy. Allogeneic hematopoietic stem cell transplantation (HSCT) provides a potentially curative approach in such patients. A reduced intensity conditioning (RIC), reducing transplant-related toxicities, makes allogeneic HSCT feasible in unfit patients Materials and methods: We are reporting 7 patients (2 F; $5 \mathrm{M})$ (median age 52 years, range 44-64) affected by CTCL. Diagnosis were: panniculitis-like T-cell lymphoma $(n=1)$ large T-cell lymphoma $(n=1)$, NK T-cell lymphoma $(n=1)$, Mycosis fungoides $(n=1)$, Sezary syndrome $(n=3)$. At the time of transplant 5 patients had a relapsed/refractory disease, 1 patient was in partial remission and 1 in complete remission. All patients but 1 were pretreated, having received at least 4 lines of prior therapies and 2 had undergone a previous autologous transplant. Six patients underwent allogeneic transplant from HLA identical sibling and 1 from a mismatched sibling. The RIC regimen consisted of thiotepa $(10 \mathrm{mg} / \mathrm{kg})$, fludarabine $\left(60 \mathrm{mg} / \mathrm{m}^{2}\right)$, and cyclophosphamide $(60 \mathrm{mg} / \mathrm{kg})$. Cyclosporine and methotrexate were used as GVHD prophylaxis in HLA identical HSCT, thymoglobulin (6 $\mathrm{mg} / \mathrm{kg}$ ) was added in mismatched transplant. Sources of stem cells were bone marrow in 5 patients and peripheral blood in 3 . Results: All patients engrafted, a complete chimerism was detected at day 30 in 6 patients and at day 90 after transplant in 1 patient. No early toxicities or infectious complications other than CMV reactivation occurred. A grade I-II acute GVHD occurred in 4 patients; 2 patients developed chronic GVHD: 1 limited and 1 extended with generalized scleroderma. One patient is too early, while 6 patients evaluable for response achieved a complete remission. One patient with Sezary syndrome relapsing at 9 months entered disease remission after donor lymphocyte infusions and she is still in remission at 4 years from transplant. One patient with NK T-cell lymphoma relapsed at 8 months from transplant. To date, with a median follow-up of 28 months (range 2-59), all patients are alive, 5 in complete remission.

Conclusions: The employed RIC regimen combined with standard GVHD prophylaxis is highly tolerable with low transplant related toxicity and suggests a potential curative effect for patients with CTCL also if transplanted in advanced stage of the disease.

\section{P934}

Bone marrow graft as a source of allogeneic CS in patients undergoing intermediate intensity conditioning E. Gomez, V. Coiteux, L. Terriou, L. Magro, E. de Berranger, J.P. Jouet, I. Yakoub-Agha UAM Allo-CST (Lille, FR)

Background: Allogeneic stem cell transplantation (allo-SCT) following reduced intensity conditioning (RIC) and peripheral blood stem cell (PBSC) graft has become the gold standard in patients ineligible for myeloablative conditioning. However, PBSC, usually chosen to overcome graft rejection, could promote chronic 
GVHD. To improve patients' outcome, we performed allo-SCT with marrow as source of SC. Melphalan $100 \mathrm{mg} / \mathrm{m}^{2}$ was added to the classical fludarabine and $2 \mathrm{~Gy}-\mathrm{TBI}$ conditioning (published by Seattle team), in order to prevent graft rejection.

Patients and methods: Between November 1999 and October 2009, 132 patients underwent RIC in our units. Of whom, sixteen received bone marrow graft following RIC-modified conditioning (MIDI) Fludarabin $30 \mathrm{mg} / \mathrm{m}^{2}$ (3d), Melphalan $100 \mathrm{mg} / \mathrm{m}^{2}$ and $2 \mathrm{~Gy}$-TBI. In all those later, GVHD prophylaxis consisted on Cs-A and short-course MTX. The outcomes of patients with MIDI allo-CST (group-A) were compared to those of the other patients (group-B) in terms of patients, donor and transplantation modalities.

Results: There was no difference between the two groups regarding, neutrophil and platelet recovery. However, at day 100 after transplantation, T-cell chimerism was recorded as full donor-type in $100 \%$ and $55 \%$ of patients of the group $A$ and $B$, respectively $(P=0.004)$. With a median follow-up of 607 days, there were non differences between groups in terms of 2-year Disease Free Survival, acute GVHD. In evaluable patients who survived over 120 days, those belonging to group-A developed less often chronic GVH (17\%) than those belonging to group-B $(45 \%), P=0.05$. In univariate analysis, 2 -year overall survival was of $94 \%$ and $51 \%$ in group $\mathrm{A}$ and $\mathrm{B}$, respectively $(P=0.05)$. In multivariate analysis, only donor positive CMV-serology was found to have a protective impact on overall survival $(P=0.04$, $\mathrm{HR}=0.59$ [95 $\mathrm{Cl} \% 0.33-0.98]$ ) while conditionings other than MIDI had a trend of negative impact $(P=0.09, \mathrm{HR}=5.3[95 \mathrm{CI} \%$ 0.8-38.8]).

Conclusion: This study suggests that the use of marrow graft associated with intermediate intensity conditioning is feasible with less chronic GVHD and better outcomes of patients.

\section{P935}

A retrospective study of alemtuzumab level, t-cell chimerism and graft-versus-host disease using intermediate dose alemtuzumab for matched related and matched unrelated reduced-intensity transplantation

T. Morgan (1), W. Cope (2), A. Charlton (2), L. Spence (2), V. Bigley (1), N. Groves (3), G. Hale (3), S. Pagan (1), B. Turner (1), G. Jones (2), G. Jackson (2), A. Dickinson (1), M. Collin (1) (1)Newcastle University (Newcastle upon Tyne, UK); (2)Newcastle upon Tyne Hospitals NHS Foundation Trust (Newcastle upon Tyne, UK); (3)BioAnaLab Limited (Oxford, UK)

Alemtuzumab (CAMPATH $1 \mathrm{H}$ ) at $100 \mathrm{mg}$ effectively prevents GVHD in Fludarabine and Melphalan (FM) reduced intensity transplants but induces profound immunodeficiency. In contrast, FM conditioning alone leads to a high GVHD burden. Recently, many transplant centres have used intermediate alemtuzumab dosing of $50 \mathrm{mg}$ or less. We report our experience of $30 \mathrm{mg}$ alemtuzumab for MRD and $60 \mathrm{mg}$ for MUD transplants, adopted as standard practice at our centre in 2006.

Methods: 24 MRD and 27 MUD transplants were performed. Sera were available from 19 MRD and 15 MUD transplants at day +1 . In addition, day +3 samples were identified from 10 patients transplanted with $100 \mathrm{mg}$ alemtuzumab, 10 receiving $60 \mathrm{mg}$ and 10 receiving $30 \mathrm{mg}$. All patients gave consent according to protocols approved by the local research ethics committee of Northumberland and North Tyneside.

Results: The mean (SEM) alemtuzumab concentration (micrograms $/ \mathrm{ml}$ ) on day +1 was $2.9(0.3)$ after $30 \mathrm{mg}$ and $4.6(0.6)$ after $60 \mathrm{mg}$ (t test $P<0.01)$. On day +3 the levels were 2.4 (0.2); 4.0 (0.6); 8.4 (1.9) after $30 \mathrm{mg}, 60 \mathrm{mg}$ and historical controls of $100 \mathrm{mg}$, respectively $(P<0.05$ between each dosing level). There were significant inverse correlations between patient surface area and alemtuzumab concentration by linear regression for both $30 \mathrm{mg}(\mathrm{r} 20.51 P<0.01)$ and $60 \mathrm{mg}$ dosing (r2 $0.18 P<0.05$ ). All patients achieved $>95 \%$ myeloid engraftment by day 100 . Median (range) T cell engraftment was significantly higher after MUD transplants: $70 \%$ (9-99\%) than
MRD transplants: 21\% (5-85\%; Mann Witney $P<0.05)$. T cell chimerism was inversely correlated with alemtuzumab level in MRD transplants by linear regression ( $r 20.37 ; P<0.05)$ but not in MUD transplants. The incidence of acute GVHD was also greater after MUD transplantation (47\%) compared with $11 \%$ for MRD. There were 2/24 non-relapse deaths after MRD and $3 / 27$ following MUD transplantation; none were due to GVHD. Conclusion: This analysis demonstrates predictable dose and surface-area relationships with alemtuzumab level in patients receiving $\mathrm{FM}$ conditioning. It also reveals that a significantly less than $100 \mathrm{mg}$ alemtuzumab confers reliable GVHD prophylaxis in both MRD and MUD recipients, although at least twice the level of alemtuzumab is required to achieve comparable results in MUD transplants. Finally, it is notable that T cell chimerism at day 100 is directly related to alemtuzumab level at day +1 in MRD transplants.

\section{P936}

Combination of chemotherapy and sequential reduced-intensity conditioning with allogeneic stem cell transplantation in $\mathbf{5 0}$ patients with advanced haematological malignancies

M. Krejci, J. Mayer, Y. Brychtova, M. Doubek, Z. Racil, Z. Koristek, M. Navratil, M. Tomiska, O. Horky, D. Dvorakova, M. Lengerova, J. Vorlicek

University Hospital Brno (Brno, CZ)

Background: Reduced-intensity conditioning (RIC) for allogeneic stem cell transplantation (SCT) with previous course of chemotherapy represents a promising approach for the treatment of high-risk leukemia patients (pts). Here we present our experience with this therapy at cohort of 50 pts with various hematological malignancies, outcomes of pts with lymphoid malignancies have not yet been published.

Methods: Fludarabine $\left(30 \mathrm{mg} / \mathrm{m}^{2}\right)$, cytarabine $\left(2 \mathrm{~g} / \mathrm{m}^{2}\right)$, and amsacrine $\left(100 \mathrm{mg} / \mathrm{m}^{2}\right)$ for 4 days (FLAMSA) were used for cytoreduction. Amsacrine was not administered to pts with lymphoid malignancies (13/50). After 3 days of rest, RIC consisting of 4 Gy TBI, ATG (Fresenius) 10-20 mg/kg/day for 3 days, and cyclophosphamide $40-60 \mathrm{mg} / \mathrm{kg} /$ day for 2 days followed.

We analyzed 50 pts with advanced hematological malignancies (AML, $n=30 ; A L L, n=6 ; N H L, n=5 ; M D S, n=3$; others, $n=6$ ) undergoing chemotherapy and RIC SCT in our centre from March 2006 to May 2009. Disease status before SCT was: CR1, $n=8 ; C R 2, n=10 ; C R 3, n=1$; refractory, relapsing or untreated disease, $n=31$. Types of donors and used grafts were as follows: HLA identical sibling, $n=16$; unrelated donor, $n=34$, PBSCs, $n=47 ; B M, n=3$. Median age of pts was 49 years.

Results: The median time of neutrophil engraftment (above $0.5 \times 10 \mathrm{E} 9 / \mathrm{L}$ ) was 18 days, 43 pts engrafted, 7 died in aplasia. Nonrelapse mortality (NRM) after 100 days and 1 year was $20 \%(10 / 50)$ and $24 \%(12 / 50)$. Causes of death were GVHD $(n=2)$, septic shock $(n=4)$, multiorgan failure $(n=4)$, and brain hemorrhage $(n=2)$. Treatment response was evaluated in 43 pts: remission was achieved in 39 pts (91\%), 4 pts had progression of disease.

Incidence of acute GVHD was evaluated in 43 pts: $63 \%$ (27/43) of pts had GVHD (grade I+ II in 22 pts, grade III in 5 cases). Incidence of chronic GVHD was evaluated in 35 pts, 53\% (19/36) of pts had GVHD (limited in 15 cases, extensive in 4 cases). With median follow-up from SCT 285 days (range 1-1260), $44 \%$ of all pts (22/50) were alive (19 pts in remission, 3 pts with relapse), 28 pts died (12 deaths from NRM, 16 deaths from relapse or progression), 16 relapses (37\%; 16/43) occurred. Median follow-up for 22 survivors was 21 months (range 8-42). Conclusion: FLAMSA-RIC protocol seems to be effective salvage treatment in pts with advanced both myeloid and lymphoid malignancies. Response rate is high $(91 \%)$ and the frequence of serious GVHD is low. However, the NRM is still considerable and relapses occurred after SCT, longer follow-up is needed. 
P937

Reduced-intensity stem cell transplantation with fludarabine and melphalan for children with haematological malignancies

K. Kato (1), N. Maeda (2), H. Kigasawa (3), M. Tsuchida (4), H. Kurosawa (5), T. Taga (6), H. Sano (7), S. Adachi (8), K. Horibe (2)

(1)Japanese Red Cross Nagoya First Hospital (Nagoya, JP); (2)Nagoya Medical Center (Nagoya, JP); (3)Kanagawa Children's Hospital (Yokohama, JP); (4)lbaraki Children Hospital (Mito, JP); (5)Dokkyo Medical University (Mibu, JP) (6)Shiga University of Medical Science (Otsu, JP); (7)Tottori University (Tottori, JP); (8)Kyoto Universit (Kyoto, JP)

Reduced intensity preconditioning before allogeneic stem cell transplantation are recently undergone for children with hematological malignancies to reduce either early or late posttransplant complications. We retrospectively investigated the efficacy and tolerability of reduced intensity stem cell transplantation (RIST) with the most fundamental preconditioning for RIST of fludarabine (FLU) and melphalan (MEL) in 26 children with hematological malignancies from 13 institutions in Japan. Patients and methods: Median patient's age at transplant was 8 years old $(0-16)$, and diagnosis of them were ALL $(n=6), A M L$ $(n=11), C M L(n=3), \operatorname{MDS}(n=5)$, NK leukemia $(n=1)$. In patients with acute leukemia, the disease status at transplant were CR1 $(n=8)$, CR2 $(n=3)$, CR3 $(n=3)$, and relapse $(n=4)$. The stem cell source was bone marrow $(n=22)$, peripheral blood $(n=3)$ and cord blood $(n=1)$. The donor include sibling $(n=6)$, parents $(n=7)$, and unrelated donor $(n=13)$. The serological disparity of HLA were $0(n=17), 1(n=6)$, and $2(n=6)$. Preconditioning was done with FLU of median $125 \mathrm{mg} / \mathrm{m}^{2}(120-150)$, combined with MEL of median $180 \mathrm{mg} / \mathrm{m}^{2}(80-210)$. The reason for choosing RIST were intent to reduce late effects $(n=16)$, concurrent morbidity $(n=4)$, or others $(n=6)$.

Results: Engraftment was obtained in 24 patients with median 19 days (11-35). Grade II-IV acute GVHD and extensive chronic GVHD were observed in 8 and 9 patients, respectively. Relapse and transplant related mortality was observed in 6 patients $(27.2 \%)$ and 5 patients $(28.1 \%)$, respectively. Eighteen patients are alive for median 873 days $(76-2449)$ after transplantation and overall and event free survival was $57.9 \%$ and $39.9 \%$, respectively. In acute leukemia, the event free survival of patients transplanted at CR1 was significantly better than those transplanted at advanced disease status $(68.6 \%$ vs. $17.5 \%$, $P=0.031$ ). Interestingly, no patients transplanted from HLA one or two antigens mismatched donor relapsed, and all patients transplanted from HLA one antigen mismatched donor are alive with significant superiority than those transplanted from two antigens mismatched donor ( $100 \%$ vs. $25 \%, P=0.032$ ).

Conclusion: RIST with FLU + MEL was feasible for children with hematological malignancies in terms of toxicity and anti-leukemic effect. It is mandatory to prove the efficacy of this regimen with large number of patients.

\section{P938}

Second allogeneic haematopoietic stem cell transplantation: a treatment for graft failure

M. Remberger, J. Mattsson, R. Olsson, O. Ringdén

Karolinska Institutet (Stockholm, SE)

A therapeutic option for graft failure (GF) may be a second allohaematopoietic stem cell transplantation (HSCT). We evaluated the results in 20 recent patients treated with a second HSCT after GF at our centre. Ten of the patients were children less than 18 years old. Indications for HSCT were a non-malignant disease in ten patients, leukaemia in seven, MDS/MPS in two and renal cell carcinoma in one patient. In most of the transplantations, fludarabin-based reduced intensity conditioning (RIC) was given. Bone marrow was given to 11 patients, PBSC in seven and cord blood to two patients. For the second transplantation $(n=20)$, a new donor was identified and used in nine cases while the initial donor was used in 11 transplants. Eight patients $(40 \%)$ suffered from a second GF after the second HSCT. Five of these patients were treated with a third HSCT. The probability of survival was $65 \%$ one year and $60 \%$ three years after the second HSCT. No difference in survival was found between patients transplanted with a new donor (56\%) compared to those using the original donor $(64 \%)$. The 3-year survival was $70 \%$ for children compared to $50 \%$ for adults $(P=n s)$. However, patients with a non-malignant disorder showed a 3-year survival of $90 \%$ compared to $20 \%$ in patients with a malignant disease $(P=0.005)$. There was a trend for better survival in patients with $>6$ months between the two transplants, $80 \%$ vs. $40 \%(P=0.08)$.

We concluded that retransplantation using RIC is a valid option for graft failure, especially in patients with non-malignant disorders.

\section{P939}

Allogeneic haematopoietic stem cell transplantation for refractory Hodgkin's lymphoma with modified BEAM-alemtuzumab conditioning is efficient and not associated with severe infections

S. Eder, W. Rabitsch, P. Kalhs, Z. Kuzmina, A. Schulenburg, C. Zielinski, H. Greinix

Medical University Vienna (Vienna, AT)

Patients with refractory Hodgkin's lymphoma $(\mathrm{HL})$ have a dismal prognosis. Allogeneic hematopoietic cell transplantation (HCT) with myeloablative conditioning is associated with high transplant-related mortality (TRM) in this indication. Therefore, reduced-intensity conditioning protocols have been used to decrease TRM. Here, we investigated a BEAM-conditioning protocol with a reduced alemtuzumab dose to reduce infectious complications during immunodeficiency after HCT.

Patients and methods: Eleven patients with a median age of 37 (range, 24 to 44 ) years with refractory $\mathrm{HL}$ were referred for HCT after a median of 4 (range, 4-6) previous chemotherapy regimens including autologous HCT in 7. Conditioning consisted of chemotherapy according to the BEAM regimen, alemtuzumab was given in a dose of $10 \mathrm{mg} /$ day on days -5 to -1 . Graftversus-host disease (GvHD) prophylaxis consisted of cyclosporine $A$ alone. All patients received peripheral blood stem cells with a median of $6 \times 10^{6} \mathrm{CD} 34+/ \mathrm{kg}$ body weight from an HLA-identical related $(n=7)$ or unrelated $(n=4)$ donor.

Results: Ten patients achieved complete hematologic engraftment with absolute neutrophil counts above $0.5 \mathrm{G} / \mathrm{l}$ a median of 15 (range, 12 to 22) days after HCT whereas one rejected the graft. Two patients experienced acute GvHD grade II, four chronic GvHD (one limited and three extensive), and 1 patient died of multi-organ-failure (9\%). Graded according to Cordonnier et al. 8 patients had 34 infectious episodes during the first year after HCT including 4 bacterial, 6 viral, 4 fungal and 20 nonmicrobiologically defined infections. Of note, only one grade 3 infection was observed and thus, most infectious episodes were mild. Data on immune reconstitution will be presented. Best response after HCT was complete remission (CR) in 6/10 evaluable patients (60\%), and partial remission (PR) in $3(30 \%)$. Eight patients achieved complete donor chimerism in myeloid and lymphoid cell lineages including 4 patients after donor lymphocyte infusions (DLI). Cumulative incidence of relapse at 5 years was $39 \%$ and probability of 5 -years-overall survival was $50 \%$. After a median of 37 (range, 13 to 62 ) months of follow-up $6 / 11$ patients $(55 \%)$ are alive including 5 in continuous $C R$. Conclusions: Allogeneic HCT with conditioning according to the modified BEAM-alemtuzumab protocol is an efficient and welltolerated therapeutic option for patients with refractory $\mathrm{HL}$ and was not associated with serious infectious complications. 


\section{P940}

Impact of donor on outcome after allogeneic haematopoïetic stem cell transplantation with reduced-intensity conditioning by Flu-Bu-ATG. A retrospective study of 59 consecutive patients J. Cornillon (1), M. Balsat (1), L. Absi (2), J. Thorin (1), E. Tavernier-Tardy (1), J. Jaubert (1), C. Mounier (1), D. Guyotat (1)

(1)Institut de cancérologie de la Loire (St-Priest en Jarez, FR); (2)Etablissement Français du sang-Auvergne Loire (StEtienne, FR)

Currently, the outcome of hematopoietic stem cell transplantations (HSCT) from unrelated donor (URD) is similar to that of transplantations from HLA-identical sibling (SD). However, immunosuppression used is often more intensive for URD, in particular the dose of anti-thymocyte globulin (ATG) is not equivalent. We retrospectively investigated the role of donor origin in 59 patients (pts) after the same reduced intensity conditioning (RIC) with same immunosuppression.From January 2000 to April 2009, 59 pts) received RIC transplantation, 29 with URD and 30 with SD. The conditioning was identical with busulfan (2 days) and fludarabine. Immunosuppression was by ciclosporine $(5 \mathrm{mg} / \mathrm{Kg}$, starting day-1) and 1 day of ATG (2.5 mg/Kg, day-2), except in 2 URD cases where mycophenolate mofetil was added. Median age was 56 years. Diagnosis was acute leukaemia (29\%), multiple myeloma (15\%), lymphoid neoplasia (32\%) and myeloproliferative syndromes (9\%). Disease status at transplant was complete remission for $42.3 \%$ of cases. A sex mismatch was observed in $56 \%$ of transplants. CMV was positive in $83 \%$ of donors and/or recipients. Median follow-up was 692 days. Median time to engraftment was 15 days. Two patients presented rejection (URD transplant). $32 \%$ patients presented grade $2-4$ and $11 \%$ grade $3-4$ aGVHD. HSCT from URD resulted in more aGVHD than HSCT from SD, respectively $62 \%$ vs. $30 \%(P=.04)$, in particular for grade 3-4 (17.7 vs. $3.3 \%, P=.033)$. The majority of aGVHD was cutaneous and treatment was corticotherapy alone for $66 \%$ of patients. 19 of 48 evaluable pts $(40 \%)$ presented chronic GVHD (cGVHD), 50\% considered as severe. cGVHD are more frequent with URD $(52 \%$ vs. $26 \%, P=.029$. GVHD was also different according to the source of stem cells (bone marrow or peripheral blood stem cells: aGVHD, $25 \%$ vs. $48 \%$ respectively (NS), cGVHD 25 vs. $44 \%$ (NS. 12 pts $(20 \%)$ relapsed and $31(53 \%)$ died. The mean causes of death were relapse $(35 \%)$ and GVHD (22\%). Non-relapse Mortality (NRM) was $26.4 \%$ at 1 year. Overall survival (OS) was $45 \%$ at 5 years. OS is statistically shorter in case of severe aGVHD. NRM was also influenced by aGVH and cGVHD.In conclusion, we show that with the same conditioning and immunosuppressive therapy, outcome is different according to donor type with an increase of GVHD for URD. This difference has no direct impact on OS or NRM but probably an indirect impact through GVHD increase. Increased immunosuppression is probably necessary for URDHSCT with low risk of relapse.

\section{P941}

Reduced-intensity conditioning allogeneic transplant allogeneic transplant in patients with mature lymphoid malignancies: a single-centre experience

M. Cabrero, P. González, L. Lopez, L. Vazquez, F. SánchezGuijo, M. González, J.A. Pérez-Simón, A. Martín GarcíaSancho, J. San-Miguel, D. Caballero

Hospital Universitario de Salamanca (Salamanca, ES)

A Graft versus lymphoma effect have been suggested in patients with mature lymphoid malignancies supporting the role of RIC allogeneic transplant in these malignancies in order to decrease the high Non relapse Mortality (NRM) previously reported after myeloablative conditioning. In 1999 our centre opened the first phase II prospective Trial on RIC allogeneic transplant in haematological malignancies; since then, 75 patients with lymphoproliferative diseases have received an allogeneic transplant with RIC. From them, $12,2 \%$ had a DBLCL, $21,6 \%$ an indolent lymphoma, $5,6 \%$ a MCL, $10.8 \%$ a PTCL, $29,7 \%$ a CLL and $20.3 \%$ a Hodgkin Lymphoma. Median age at transplant was 50 years (range $20-67) ; 49(66 \%)$ were male patients. Median number of therapy lines before the transplant was 3.2 (1-9) $40 \%$ had a previous autologous transplant. At transplant had sensitive disease $79 \%$ (38\% were on CR and $41 \%$ on PR) and $(21 \%)$ were transplanted with refractory disease. Donor was matched related in $57(77 \%)$ and Unrelated in $17(23 \%)$. In most of them, $62(84 \%)$, conditioning regimen consisted of fludarabine $30 \mathrm{mg} / \mathrm{m}^{2}$ intravenously (IV) 5 days and melphalan $140 \mathrm{mg} / \mathrm{m}^{2}$ IV. Median number of CD34 + infused cells was $4.81 \times 10^{6} / \mathrm{Kg}(1.20-11.03)$. GVH prophylaxis consisted on CSA+MTX in most of patients.

Results: all the patients engrafted and median of days to reach more than $500 \times 10^{9}$ granulocytes and more than $20000 \times 10^{9}$ platelets were +17 (range 10-29) and +11 (range 0-25) respectively. With a median follow up of 27 months (range: $1-127$ months), 41 patients $(55,4 \%)$ are alive, 36 disease free and $33(44,6 \%)$ have died, $19(25,7 \%)$ due to NRM and $13(17,6 \%)$ due to progressive disease. NRM at 100 days, one year and 3 years are $5,5 \%, 13 \%$ and $17 \%$ respectively. OS and PFS at 3 years are $58 \%$ and $56 \%$ respectively for the whole group, $62 \%$ and $60 \%$ for related and $43 \%$ and $30 \%$ for unrelated donor. Considering different histologies. OS at 3 years is $82 \%$ for $\mathrm{MCL}, 52 \%$ for $\mathrm{BDLCL}, 80 \%$ for indolent lymphoma, $75 \%$ for PTCL, $80 \%$ for CLL, and $64 \%$ for $\mathrm{HL}$. On univariate analysis the presence of CGVHD is associated with a better OS ( $90 \%$ for cGVHD and $23 \%$ for aGVHD) Chronic GVHD influences in OS and in PFS in the multivariant analysis $(P<0.01)$

Comments: These single center positive results confirm the possible role of RIC allo transplant in sensitive lymphoid malignancies. Full final analysis will be updated at the moment od presentation.

\section{P942}

Reduced-intensity conditioning for allogeneic haematopoietic stem cell transplantation with fludarabine and melphalan is associated with durable disease control in elderly patients with Philadelphia chromosome-positive acute lymphoblastic leukaemia

Y. Ozawa, S. Nishiwaki, D. Koyama, K. Onodera, T. Goto, A. Seto, K. Watanabe, N. Imahashi, S. Tsukamoto, K. Miyamura Japanese Red Cross Nagoya First Hospital (Nagoya, JP)

Purpose: To evaluate the efficacy of reduced-intensity conditioning allogeneic hematopoietic stem cell transplantation (allo-SCT) for Philadelphia chromosome-positive acute lymphoblastic leukemia (Ph (+) ALL) in the imatinib era, we compared the outcome and risk factors between TBI-containing conditioning and fludarabine + melphalan-containing reducedintensity conditioning, and also evaluated several variables such as ages, minimal residual diseases (MRD) and HLA disparities.

Patients and Methods: We retrospectively analyzed 17 adult $\mathrm{Ph}(+)$ ALL patients who received imatinib mesylate containing induction therapy, followed by allo-SCT from April 2002 to November 2009 in our institution.

Results: The median age of the patients was 40 years. Ten patients received $\mathrm{TBI}$ (12Gy)-containing conditioning and 7 patients received fludarabine + melphalan-containing reduced-intensity conditioning (FM). At SCT 11 patients had no MRD and 6 patients had MRD. At median follow-up of 24.1 months, 12 patients were alive, and 3 patients were relapsed, and 4 died of transplantation-related mortality. The actuarial 2-year overall survival (OS) rate, progression-free survival (PFS) rate were $66.9 \%$ and $60.8 \%$, respectively. PFS was significantly superior among patients without MRD than patients with MRD at SCT $(79.5 \%$ vs. $33.3 \% ; P=0.021)$. Multivariate analysis 
demonstrated that MRD positive at SCT and HLA-mismatch were associated with PFS. Seven of 9 patients over 40 years received FM conditioning. No significant difference of PFS was observed between TBI-containing conditioning and FM conditioning (TBI vs. FM: $38.9 \%$ vs. $85.7 \%$ at 2 years, respectively; $\mathrm{P}=.118$ ). Interestingly, patients older than 40 years showed better OS (Age $>40,88.9 \%$ vs. Age $\leq 40,41.7 \%$ ), although no significant difference was observed $(P=0.074)$.

Conclusion: FM conditioning is feasible for elderly patients with Philadelphia chromosome-positive acute lymphoblastic leukemia. It is important that MRD should be disappeared before allo-SCT by imatinib or other tyrosine kinase inhibitors.

\section{P943}

Reduced-intensity conditioning with busulfan, fludarabine and alemtuzumab in children and adolescents: a pilot study

J. Styczynski (1), B. Tallamy (2), N. Fearon (2), C. Van De Ven (2), L. Harrison (2), E. Morris (2), P. Satwani (2), M. Bhatia (2), D. George (2), B. Bradley (2), J. Garvin (2), J. Schwartz (2), L. Baxter-Lowe (3), M. Cairo (2)

(1)Nicolaus Copernicus University (Bydgoszcz, PL); (2)Columbia University (New York, US); (3)University of California (San Francisco, US)

Background: AlloSCT following conditioning with fludarabine, busulfan, and alemtuzumab (BFA) in adult pts with AML/MDS was safe and permitted durable donor engraftment. There is a paucity of data of RIC with fludarabine, busulfan and rATG and none with alemtuzumab (BFA) in pediatric AlloSCT recipients

Objective: To determine the safety and percentage of mixed donor chimerism following a BFA RIC AlloSCT in children and adolescents (C\&A)

Methods: We report results of a pilot study of BFA RIC AlloSCT in 12 recipients $(<21 \mathrm{yrs})$ with malignant $(n=7 ; 5$ average-risk, 2 poor-risk: CML 4, APML 1, NHL 1, HTLV-LL 1), and non-malignant diseases $(n=5$ : SAA 3 , Scleroderma 1 , Wolman's Disease 1). Median age 16 (2-20) yrs, 6F:6M. Stem cell source: 2 unrelated cord blood (UCB) (2 5/6), 1 unrelated BM (10/10), 2 related $(16 / 6,15 / 6)$ and 7 unrelated PBSC (3 8/10, 3 9/10, 1 10/10). Positive CD34 selection was done in 5 unrelated PBSCT. RIC: fludarabine $\left(180 \mathrm{mg} / \mathrm{m}^{2}, 6\right.$ days), iv busulfan $(16 \mathrm{mg} / \mathrm{kg}<4 \mathrm{yrs}$ and $12.8 \mathrm{mg} / \mathrm{kg}$ for $>4 \mathrm{yrs}, 4$ days, total 8 doses)+alemtuzumab (54 mg/m², 5 escalating doses). GVHD prophylaxis: FK506/MMF. Donor chimerism was determined by specific STR loci.

Results: Median time to myeloid engraftment was $16 \mathrm{~d}$ (16d vs. $16 \mathrm{~d}$ for unselected PBSC/BM/CB vs. selected PBSC recipients); median time to platelet engraftment: $31 \mathrm{~d}$ (27d vs. $35 \mathrm{~d}$ for unselected PBSC/BM/CB vs. selected PBSC recipients, ns). The probability of developing > grade I, > grade II AGVHD and CGVHD was $41.6 \%, 25 \%$, and $9 \%$ respectively. There was 1 primary graft failure (GF), and no secondary GF. Median mixed donor chimerism day 100 and 1 yr: $99 \%$ and $96 \%$, respectively; $>90 \%$ of recipients achieved $>80 \%$ donor chimerism. The $3 \mathrm{yr}$ OS was $91.7 \%$ (100\% for malignant vs. $80 \%$ for non-malignant diseases, ns). The probability of $3 \mathrm{yr}$ OS for patients receiving CD34-selected PBSC was $100 \%$ and receiving non-CD34selected PBSC/BM/UCB was $85.7 \%$ (ns). 11 pts remain alive at median $2.8(0.3-6.8)$ yrs. Only $1 / 12$ developed > grade II toxicity (liver grade IV). Incidence of transplant-related mortality was 1/12 (9\%). 1 patient died of IFI at day + 40. Infectious complications included: CVL bacteremia (6/12), 2 IFI and 3 other fungal infections, 3 hematuria of viral etiology (BKVIADV), and 1 each of ADV, CMV, RSV, Parainfluenza, HSV, or Mycobacterium infections.

Conclusions: RI AlloSCT based on BFA conditioning is feasible and tolerable in C\&A, and results in prompt achievement of durable mixed donor chimerism and excellent OS.
P944

Long-term results of allogeneic stem cell transplantation after reduced-intensity conditioning regimen fludarabin, busulfan and ATG

Y. Brychtova (1), M. Krejci (1), J. Muzik (2), M. Doubek (1), J. Mayer (1)

(1)University Hospital Brno (Brno, CZ); (2)Masaryk University (Brno, CZ)

Introduction: In this retrospective analysis we summarized results of allogeneic stem cell transplantation (SCT)with reduced intensity conditioning (RIC):Fludarabin $\left(30 \mathrm{mg} / \mathrm{m}^{2}\right.$, 5 days), Busulfan (8-12 mg/kg p.o.) and ATG Fresenius (10mg/ $\mathrm{kg} / \mathrm{d}, 4$ days) in our transplant center (March 1998-September 2008).

Methods: 71 patients (pts) , median age 50 years, had the diagnosis: 19 AML[17 in 1st.CR, 1 in 2nd CR, 1 in relaps (R) ], 3 MDS $24 \mathrm{CML}$ [21 in chronic phase, 3 in acceleration), 2 myelofibrosis, $3 \mathrm{HD}$ (1R,1 CR,1 PR), 6 agressive B-NHL [3 DLBCL(2 in 2nd $P R, 1$ in 2nd CR), $3 \mathrm{MCL}(2$ in 2nd CR, 1 in 2nd R)], and 9 indolent B-NHL[8 B-CLL/SCLL(5 in relaps, 3 in 2nd PR ), 1 FL(in2nd PR)], 2 T-NHL(1 PR, 1 CR), 3 MM(1R, 2 PR). Peripherial blood stem cells were used in 68 pts, bone marrow in 3 pts. Donors were 65 related and 6 unrelated respectively.

Results: Median time of follow up was 55 months. After SCT a toxicity was low [grade III $5.6 \%$ (4) pts, grade IV $1.4 \%(1)$ patient]. Complete chimerism (CC) was reached in 31 pts $(43.6 \%)$ on day $+100,57$ pts reached CC in median time 82 days, $14(19.7 \%)$ pts didn't reach CC because of rejection of graft (1), giving his autologous back up of stem cells for severe GVHD (1), progression or relaps (9 pts $=12.8 \%$ ), death from other reason (2 infections, 1 bleeding). Twenty-four (33.8\%) pts developed an acute GVHD:11, 8, 3, 2 pts maximal grade I,II,III,IV respectively. A chronic GVHD was presented in $33(46.5 \%)$ pts (25 limited, 8 extensive). Secondary rejection of graft occured in 1 patient with unrelated donor. Seventeen pts had preemptive therapy of CMV. Any infection since day +100 had $34(47.8 \%)$ pts, after day +100 $42(59.1 \%)$ pts. Twenty seven pts $(38 \%)$ died, the causes of death: $17(23.9 \%)$ progresion of disease, $4(5.6 \%)$ infection, $1(1.4 \%)$ infection after treatment of relaps, $1(1.4 \%)$ GVHD and infections, 1 (1.4\%) GVHD and bleeding, $1(1.4 \%)$ only GVHD, $2(2.8 \%)$ from other reason. Early transplant related mortality (TRM) was $11.2 \%$ (8 pts: 5 progressions, $1 \mathrm{GVHD}, 1$ bleeding, 1 infection), late TRM 5.6\%. Median time of overall survival (OS) for all pts wasn't reached, event free survival (EFS) for all pts was 23 months (KaplanMeier). Median time OS on day +100 , 1year, 2years, 4years were $88.7 \%, 74,7 \%, 73.2 \%, 62,6 \%$ respectively. Median time EFS on day +100 , 1year, 2years, 4years were $84.5 \%, 63.4 \%$, $49 \%, 40.3 \%$, respectively.

Conclusions: RIC is associated with statistically significant favorable outcome in patients with aGVHD or CGVHD regarding OS, EFS.

\section{P945}

Allogeneic haematopoietic cell transplantation using reduced-intensity conditioning with intravenous busulfan plus fludarabine in acute myeloid leukaemia. Results in $\mathbf{5 0}$ patients not eligible for myeloablative conditioning D. Serrano (1), J. Sanz (2), J. Sanchez (1), R. Duarte (3), C. Barrenetxea (4), A. Sampoll (5), M. Gonzalez (6), A. Figuera (7), R. Varela (8), J.L. Diez-Martin (1)

(1)Hospital Gregorio Marañón (Madrid, ES); (2)Hospital La fe (Valencia, ES); (3)Hospital Duran i Reynals (Barcelona, ES); (4)Hospital Valle Hebron (Barcelona, ES); (5)Hospital Son Dureta (Plama de Mallorca, ES); (6)Hospital Clinico Universitario (Santiago de Compostela, ES); (7)Hospital La Princesa (Madrid, ES); (8)Hospital Juan Canalejo (La Coruña, ES)

Allogeneic hematopoietic cell transplantation (HCT) with reduced-intensity conditioning (RIC) are used in patients (pts) not eligible for conventional conditioning regimen due to higher 
toxicity. Recently oral Busulfan(Bu) has been changed by intravenous $\mathrm{Bu}(\mathrm{IVBu})$ since it has more predictable pharmacokinetic and less toxicity. We present the results of a multicentre, retrospective study, using Fludarabine(Flu) plus IVBu as RIC conditioning in a cohort of pts diagnosed of Acute Myeloid Leukemia(AML).

Patients and methods: 50 pts, median age of 58 years (22-68), received HCT with RIC, Flu ( $150 \mathrm{mg} / \mathrm{m}^{2}$ in 5 days) and IVBu ( $\leq 8$ $\mathrm{mg} / \mathrm{kg}$ in 2-3 days). The median year of transplant was 2008 (03-09).39 pts were over 50 years (6 relapsed to a previous HCT), 11 pts were below 50 years ( 9 had relapsed post-HCT and 2 presented lung and liver co-morbidity). 41 pts ( $82 \%)$ received an HCT from a relative HLA-identical donor (HLA-id), 8 from non-relative HLA-id and 1 from familiar donor with one HLAmismatch. Ciclosporine (CspA) plus methotrexate was used as Graft versus Host Disease (GVHD) prophylaxis in 37 pts (74\%), 11 received CspA plus Mycophenolate-Mofetil, 2 CspA plus Alemtuzumab. At transplant 41 pts (82\%) were in complete remission (CR): 24 CR1, 14 CR2, $3>$ CR2. 5 pts (10\%) were in partial remission and $4(8 \%)$ had refractory leukemia.

Results: 47 pts $(94 \%)$ engrafted $\left(>0.5\right.$ neutrophils $\left.\times 10^{9} / \mathrm{L}\right)$ at a median of 15 days(d). 3 pts died before engraftment, 2 due to leukemia progression (days $+22,+48$ ) and the third on +11 d after hepatic veno-occlusive disease(VOD). One pt showed secondary Graft Failure and died after a 2nd HCT due to multiorgan failure (MOF) at +15 month (mo). 14 pts (30\% of pts at risk) showed acute GVHD grade II-IV. Survival: With a median of follow-up of $12 \mathrm{mo}(1-74) 29$ pts (58\%) are alive in CR. 21 pts died, 11 due to leukemia progression at a median of $5 \mathrm{mo}$ (1-11), and 10 due to non relapse mortality (NRM): 5 because of GVHD median 3mo (3-15), 3 after toxicity (VOD, MOF, and Neurologic disorder +13 mo), 2 from infection (sepsis, $+7 \mathrm{mo}$, and pneumonia, $+14 \mathrm{mo}$ ). Cumulative Incidence of NRM $15 \%$ at 12 mo. Leukemia Free Survival (LFS) was $45 \%$ at 15 mo posttransplantation for all pts, and $50 \%$ for pts transplanted in CR.

Conclusions: Despite the short follow-up in our patients, HCT in LMA pts using IVBu in RIC present a low toxicity, with a LFS of $50 \%$ for pts in CR before transplant. Relapse and GVHD are the main causes of dead in the first year post-transplantation.

\section{P946}

Haploidentical stem cell transplantation with reduced-intensity conditioning in refractory diseases: current results

P. Lang, H.M. Teltschik, I. Mueller, T. Feuchtinger, M. Pfeiffer, $M$. Ebinger, M. Schumm, R. Handgretinger

University Children's Hospital (Tubingen, DE)

Transplantation of haploidentical, positive selected stem cells in combination with $\mathrm{TBI}$ or Bu based conditioning regimens can result in significant toxicity. New graft manipulation methods and a melphalan based intensity reduced regimen may help to reduce these complications. We present our results with a direct depletion procedure for $\mathrm{T}$ and $\mathrm{B}$ cells using antiCD3/ antiCD19 coated magnetic microbeads. Melphalan $(2 \times 70 \mathrm{mg} /$ $\left.\mathrm{m}^{2}\right)$, fludarabine $\left(4 \times 40 \mathrm{mg} / \mathrm{m}^{2}\right)$ or clofarabine $\left(4 \times 50 \mathrm{mg} / \mathrm{m}^{2}\right)$, thiotepa $(10 \mathrm{mg} / \mathrm{kg})$ and OKT3 $(0.1 \mathrm{mg} / \mathrm{kg})$ was used as preparative regimen. 36 pediatric patients (median age: 11 years) were included. All patients underwent intensive pretreatment according to current study protocols; $18 / 36$ already received previous allogeneic transplantations. The diagnoses were: ALL $(n=19)$, AML $(n=14)$, advanced MDS $(n=3)$; remission status was: $C R 1=2, C R 2=7, C R 3=9, N R=18$. All donors were full haplotype mismatched parents. Primary engraftment occurred in $89 \%$ of patients. After TLI based reconditioning and second haploidentical stem cell donation, final engraftment was achieved in $100 \%$. Median time to reach $>500$ neutrophiles/ $\mu \mathrm{l}$ and independence from platelet substitution was $10(8-15)$ and 9 (5-59) days respectively. GvHD grade 0 occurred in $36 \%$. $54 \%$ and $8 \%$ had GvHD grade I-II and III, respectively. Chronic limited and extensive GvHD was observed in 8 and $11 \%$. Transplant related mortality was $0 \%$ at day 100 and $8 \%$ at one year.
Event free survival at 2 years was $66 \%$ for patients with any $C R$ (1st SCT) $(n=8)$ and $10 \%$ for patients with active disease (1st SCT) $(n=12) .1$ year EFS in patients after $2 n d$ SCT was $30 \%$ $(n=18)$. Causes of death were myocardiopathy $(n=2)$, infection $(n=1), \operatorname{GvHD}(n=1)$ and relapse $(n=22)$. Relapse rates at 2 years were $20 \%$ (CR patients) and $73 \%$ (active disease patients or 2nd trp).

Conclusions: Transplantation of CD3/CD19 depleted haploidentical stem cells with a reduced intensity regimen resulted in a fast recovery of neutrophiles and platelets. Engraftment rates similar to that of patients with myeloablative standard conditioning and positive selected stem cells could be achieved, possibly due to a graft facilitating effect of cotransfused NK cells. The regimen helped to minimize TRM, despite intensive pretreatment, and is suited for 2 nd or 3rd transplantation. Patients in any CR had a favorable outcome, whereas patients with active disease showed high relapse rates. Especially in these patients, further therapeutic elements have to be evaluated.

P947

Safety and efficacy of FLaMSA regimen followed by allogeneic HSCT from related and unrelated donors in high-risk acute myeloid leukaemia: a retrospective study from the Société Française de Greffe de Moelle et de Thérapie Cellulaire (SFGM-TC) registry

A. Huynh (1), G. Cannas (2), S. Morisset (2), M. Roussel (1), M. Sobh (2), N. Raus (3), F. Garban (4), C. Cordonnier (5), S. Maury (5), J.O. Bay (6), J. Cornillon (7), A. Buzyn (8), I. Yakoub-Agha (9), C. Faucher (10), S. Francois (11), P. Bordigoni (12), N. Fegueux (13), L. Fouillard (14), M. Robin (15), E. Deconinck (16), O. Reman (17), N. Dhédin (18), T. De Revel (19), B. Lioure (20), N. Milpied (21), M. Mohty (22), M. Michallet (2)

(1)CHU Purpan (Toulouse, FR); (2)Hôpital Edouard Herriot (Lyon, FR); (3)SFGM-TC (Lyon, FR); (4)Hôpital Albert Michallon (Grenoble, FR); (5)Hôpital Henri Mondor (Créteil, FR); (6)CHU Hotel-Dieu (Clermont-Ferrand, FR); (7)Institut de cancérologie de la Loire (Saint-Priest en Jarez, FR); (8)Hôpital Necker (Paris, FR); (9)CHRU de Lille (Lille, FR); (10)Institut Paoli Calmettes (Marseille, FR); (11)CHU d'Angers (Angers, FR); (12)CHU de Nancy (Nanvy, FR); (13)CHU de Montpellier (Montpellier, FR); (14)Hôpital SaintAntoine (Paris, FR); (15)Hôpital Saint-Louis (Paris, FR); (16)CHU de Besançon (Besançon, FR); (17)CHU de caen (Caen, FR); (18)Hôpital Pitié Salpêtrière (Paris, FR); (19)Hôpital Percy (Clamart, FR); (20)Hôpital de Hautepierre (Strasbourg, FR); (21)CHU de Bordeaux (Pessac, FR); (22)CHU de Nantes (Nantes, FR)

Allogeneic HSCT could improve overall survival (OS) for acute myeloid leukemia (AML) patients (pts) with primary induction failure (PIF) or relapse but new strategies are needed. This study investigates safety and efficacy of FLAMSA sequential conditioning regimen in high risk (HR) AML pts.

We analyzed 74 pts, median age 55 years, 52 were de novo AML, 22 secondary AML. All had allogeneic HSCT after FLAMSA [fludarabine $\left(30 \mathrm{mg} / \mathrm{m}^{2} / \mathrm{d}\right)$, aracytine $\left(2 \mathrm{~g} / \mathrm{m}^{2} / \mathrm{d}\right)$, amsacrine $\left(100 \mathrm{mg} / \mathrm{m}^{2} / \mathrm{d}\right)$ from day -12 to -9 followed by cyclophosphamide $(40 \mathrm{mg} / \mathrm{kg} / \mathrm{d}$ if related donor, $60 \mathrm{mg} / \mathrm{kg} / \mathrm{d}$ if unrelated) on days $-4,-3$, ATG $2.5 \mathrm{mg} / \mathrm{kg} / \mathrm{d}$ on days $-4,-3$, and -2 and either TBI 4 Gy on day $-5(n=45)$ either busilvex $3.2 \mathrm{mg} / \mathrm{kg} / \mathrm{d}$ on days $-4,-5(n=29)]$. At HSCT 6 pts were in PIF, 14 in first early relapse, 11 in late relapse, 30 refractory, 13 had more than 2 treated relapses. None presented complete response (CR). Twenty seven pts had HLA identical siblings SCT, 47 unrelated donors. Source was PBSC (62), BM (4), CB (8). Median time from diagnosis to HSCT was 10.7 months (2.9-313.2). Sixty eight pts engrafted after a median time of 16 days. There were 29 acute GvHD ( 9 grade I, 20 grade II-IV) and 14 chronic GvHD (11 limited). The multivariate analysis showed that, secondary AML, sex matching (Mismatch with Female receiver), CMV positive receiver, minor $A B O$ incompatibility and mismatched unrelated donor, had significant impact on different outcomes (Table). Thirty five pts relapsed, 3 had progressive disease. The 
cumulative incidence $(\mathrm{Cl})$ of relapse at 3, 6, 12, 24 months was $27 \%, 41.9 \%, 45.9 \%, 47.3 \%$ respectively. Nineteen pts without relapse died, $16 \mathrm{pts}$ from transplant procedure. The $\mathrm{Cl}$ of TRM at 6, 9, 24 months were $9.5 \%, 20.3 \%, 25.7 \%$ respectively. At last follow-up, 51 pts died, 23 patients are alive. After a median follow-up of 4.6 months, the probability of OS and PFS at 2 years was $21.9 \%$ and $23 \%$ in very HR AML pts without CR at transplant. This short follow up can be explained by the early high death incidence which can be due to patients with HR factors. Although $\mathrm{Cl}$ of relapse and PFS present a plateau 12 months after HSCT. Results need to be confirmed prospectively, but anti-leukemic activity seems effective, it would be interesting to use cytogenetic and molecular markers in order to precise which AML pts could benefit of such a procedure.
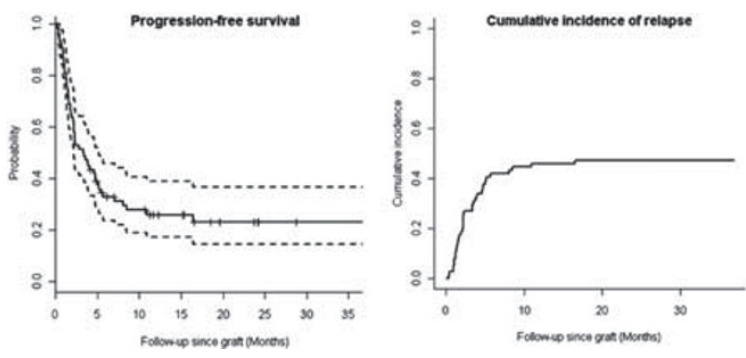

\begin{tabular}{|c|c|c|}
\hline Vuriable & Hanard ratio & Pvalue \\
\hline \multicolumn{3}{|l|}{ Inpact en Overall Survival } \\
\hline Secondury AMIL. & $0.319[0.127-0.804]$ & 0.0153 \\
\hline $\begin{array}{l}\text { Sex matching } \\
\text { Mismatch with } F \text { receiver }\end{array}$ & $0.423[0.183-0.985]$ & 0.0460 \\
\hline CMV positive receiver & $2.530[1.199 .5 .343]$ & 0.0150 \\
\hline \multicolumn{3}{|c|}{ Inpart en Progressien Free Survival } \\
\hline Secondury AMIL. & $0.173[0.065-0.461]$ & 0.0005 \\
\hline $\begin{array}{l}\text { Sexmutching } \\
\text { Mimutch with } F \text { receiver }\end{array}$ & $0.414[0.183-0.938]$ & 0.0345 \\
\hline Minor ABO incompatibility & $2.515[1.038-6.094]$ & 0.0412 \\
\hline \multicolumn{3}{|l|}{ Inpact en Relupse } \\
\hline Secondary AMIL & $0.193[0.061-0.612]$ & 0.0052 \\
\hline Miomatched urrelated donor & $0.942[0.101-8.807]$ & 0.0320 \\
\hline CMV positive receiver & $0.894[0.360-2.222]$ & 0.0260 \\
\hline
\end{tabular}

Multivariate analysis: significant factors on different outcomes

\section{Cellular and gene therapies}

\section{P948}

Development of gene-immunotherapy against human leukaemia with engineered T-cells using WT-1-specific T-cell receptor gene transfer

H. Fujiwara (1), T. Ochi (1), J. An (1), T. Shirakata (1), S. Okamoto (2), J. Mineno (2), K. Kuzushima (3), H. Shiku (4), M. Yasukawa (1)

(1)Ehime University (Toon, JP); (2)TakaraBio Inc. (Otsu, JP); (3)Aichi Cancer Center (Nagoya, JP); (4)Mie University (Tsu, JP)

Purpose: To overcome the clinical insufficiency of anti-leukemia WT1 peptide vaccination, we have been investigating the feasibility of a novel immunotherapy with engineered T cells using WT1-specific TCR gene transfer.

Methods: We have developed the GMP-grade bicistronic retroviral vector carrying full-length of WT1-specific TCR-alpha/beta genes obtained from HLA-A*2402-restricted WT1 235-243specific CTL clone (conventional vector). Additionally we have recently developed a novel TCR vector with built-in silencers for the constant regions of endogenous TCR-alpha/beta genes to reduce the mispairing between transduced and endogenous TCR-alpha/beta chains (si-TCR vector). Using either conventional or si-TCR vector, we transduced GaLV-pseudotyped bicistronic retroviral vector WT1 235-243-specific TCR-alpha/ beta gene in retronectin system. At first, we examined whether the vectors could display the intended TCR with target-specificity on cell surface using Jurkat/MA cells, which lacks endogenous TCR and genetically modified to express CD8 alpha and NFAT-luciferase construct to detect the transduced TCR mediated signaling (kindly gifted from Prof. Erik Hooijberg, Netherlands). The expression level of luciferase by WT1-specific TCR transduced Jurkat/MA was detected after coculture with WT1 235-243 peptide loaded C1R-A24 cells. Next we evaluated the target-specific and leukemia-specific responses of TCR genetransferred CD8 + T cells and CD4 + T cells by flow cytometer, cytokine production assay, and standard $57 \mathrm{Cr}$ releasing assay. Results: Transfection efficacy was around $20 \%$ to $30 \%$. TCRtransduced Jurkat/MA successfully produced luciferase in response to WT1 235-243 in a dose dependent manner. TCR transduced CD8 + cells successfully lysed the only peptide loaded C1R-A24 cells, and leukemia cells positive both for HLA-A*2402 and WT1, but not HLA-A*2402 positive normal cells. TCR transduced CD4 + cells successfully produced type 1 cytokines in response to the peptide-loaded C1R-A24 cells, and HLA-A ${ }^{*} 2402$ positive primary leukemia cells in the HLA-class II restricted fashion. The novel vector, si-TCR vector markedly increased the surface expression of introduced WT1specific TCR on CD8 + T cells in accordance with the successful reduction of endogenous TCR.

Conclusion: We have demonstrated the feasibility of WT1targeting gene-immunotherapy using WT1-specific TCR gene transferred $T$ cells against leukemia. We are about to conduct the clinical trial against human hematological malignancy.

\section{P949}

Platelet-lysate-expanded mesenchymal stromal cells as a salvage therapy for severe resistant graft-versus-host disease in a paediatric population

G. Lucchini (1), M. Introna (2), E. Dander (1), A. Rovelli (1), A. Balduzzi (1), S. Bonanomi (1), A. Salvadè (1), D. Belotti (1), G. Gaipa (1), C. Capelli (2), P. Perseghin (3), E. Lanino (4), P. Chiusolo (5), M.G. Orofino (6), A. Rambaldi (2), J. Golay (2), G. D’Amico (1), A. Biondi (1), E. Biagi (1)

(1)Università Milano Bicocca (Monza, IT); (2)Ospedali Riuniti di Bergamo (Bergamo, IT); (3)Ospedale San Gerardo (Monza, IT); (4)IRCCS Giannina Gaslini (Genoa, IT); (5)Università Cattolica del Sacro Cuore (Rome, IT); (6)Università di Cagliari (Cagliari, IT)

Despite advances in graft-versus-host-disease (GvHD) treatment, it is estimated that overall survival at 2 years for haematopoietic cell transplantation (HCT) recipients, who experience steroid-resistant GvHD, is $10 \%$. Among recent therapeutic approaches for GvHD treatment, mesenchymal stromal cells (MSC) hold a key position. These are multipotent progenitor cells with immunomodulatory properties, capable to escape immune rejection allowing their use in an HLA-mismatched setting. We describe a multi-centre experience of 10 pediatric patients (pts) diagnosed with acute or chronic GvHD treated for compassionate use with GMP-grade unrelated HLA-disparate donors' bone marrow-derived MSCs, expanded in PlateletLysate (PL)-containing medium. Between May 2008 and June 2009, 10 pts (aged 4 to 15 years) transplanted for malignant $(\mathrm{N}=8)$ or non-malignant $(\mathrm{N}=2)$ diseases, received iv MSCs for acute or chronic grade I to IV GvHD, which was resistant to multiple lines of immunosuppression. Twenty MSC infusions were given to 10 pts ( 3 pts had a single infusion, 7 had multiple infusions). The median dose was $1.2 \times 10^{6} / \mathrm{kg}$ (range $0.7-$ $3.7 \times 10^{6} / \mathrm{kg}$ ). No acute side-effects were observed, and no late side-effects were reported at a median follow-up of 8 months (range 4-18 months). 
Overall response was $75 \%$, with complete response in $25 \%$ of the cases. All responder pts could eventually taper ongoing immunosuppression after MSC infusion. None of our pts presented GvHD progression upon MSC administration, but 4 pts presented GvHD recurrence 2 to 5 months after infusion, as already described in the literature. Nine pts are alive in complete remission with a median follow up of 10 months after HCT (range 6 to 24 months). One patient died on day +126 from HCT because of sepsis. Another patient is alive, but with progressive severe GvHD. Two pts developed chronic limited GvHD. Six pts are alive in the absence of GvHD and immunosuppression. To corroborate clinical observations, TNFa and IFNg plasma levels of 4 pts were monitored by ELISA before and after MSC treatment. Interestingly, TNFa and IFNg levels, which were elevated before MSC infusion, transiently decreased in nonresponder pts, while significantly and persistently decreased in responder ones.

The present study underlines the safety of PL-expanded MSC use in children. MSC efficacy seems to be greater in acute than in chronic GvHD, even after failure of multiple lines of immunosuppression.

\section{P950}

Efficient GMP graded method for in vitro induction and expansion of low-frequency antigen specific CD8 positive T-cells

A. Wieczorek (1), K.H. Lee (2), E. Thiel (1), C. Scheibenbogen (1), L. Uharek (1)

(1)Charité Berlin (Berlin, DE); (2)Boehringer Ingelheim Pharma GmbH \& Co. KG (Biberach an der Riss, DE)

Objectives: Efficient and reliable methods for the priming and in vitro expansion of antigen specific cytotoxic T cells (CTLs) are necessary for conducting the adoptive immunotherapy with low-frequency or naïve precursors. Although several methods for the cloning of in vivo induced CTLs have been reported, an efficient protocol to prime and expand low-frequency and naïve CD8 positive $T$ cells in vitro remains a challenge.

Methods: In order to achieve a high avidity specific T cells for adoptive cell transfer we compare induction and expansion possibilities of four different cytokine cocktails including IL-6, IL-7, IL-10, IL-12, IL-15. Peripheral blood mononuclear cells (PBMCs) were obtained from several healthy volunteers (negative for hepatitis B and C, with no evidence of liver disease in the past). Cells were cultivated in the presence of several viral and tumour HLAA2 binding peptides of known immunogenicity. They were supplemented with IL-2 from day 3 of the culture and restimulated weekly with irradiated, peptide pulsed autologous PBMCs. The functional tests included IFN-gamma secretion assay, tetramer staining, cytotoxicity test and immunophenotyping.

Results: In most of our experiments with both viral and tumour associated antigens, we were able to generate and expand the antigen specific CD8 positive $T$ cells. Furthermore, naïve and memory subsets were isolated from PBMCs and cultivated separately according to the protocol. After four weeks cultivation, viral and tumour antigen specific responses were observed only in T cell cultures with naïve precursors. Moreover, by using a cytokine cocktail including IL-6 and IL-12 we successfully adapted the method to the GMP conditions. PBMCs obtained by leukapheresis from a healthy stem cell donor, a sibling sister of our AML patient, were cultivated according to the protocol for a few weeks. As a result we obtained culture with $17 \%$ IFN-gamma positive cells in response to stimulation with three different WT-1 antigens. Generated cells were successfully applied to our patient as a part of conditioning regiment by relapse of AML.

Conclusions: We established a simple and effective approach for the in vitro induction and expansion of CTLs of desired antigen specificity for an adoptive therapy in both viral and malignant diseases.
P951

Detection and rapid ex vivo expansion of adenovirusspecific T-cells for adoptive immunotherapy

R. Geyeregger (1), S. Matthes-Martin (2), G. Fritsch (1)

(1)Childrens Cancer Research Institute (Vienna, AT); (2)St. Anna Kinderspital (Vienna, AT)

Objectives: Adenoviral (ADV) infections after pediatric allogeneic haematopoietic stem cell transplantation (aHSCT) are a significant cause of morbidity and mortality. Effective drugrelated therapies are not available to date. Reconstitution of antiviral immunity by transferring donor-derived virus-specific $T$ cells, was shown to be a promising treatment option. However, the presence of ADV-specific T cells in healthy donors is a prerequisite for such therapies. Since the frequency of AdVspecific T cells is very low in blood of healthy ADV-seropositive individuals, highly sensitive detection methods are necessary. In this study we investigated the sensitivity of ADV peptide-MHC multimers and the IFNg capture assay to detect ADV-specific $T$ cells in freshly isolated PBMCs, developed a method for rapid ex vivo expansion of $A D V$-specific $T$ cells and investigated the effect of ADV-specific T cells in the clearance of ADV infection after aHSCT.

Methods: Depending on the HLA-type, freshly isolated PBMCs were either directly stained with multimers or stimulated for $16 \mathrm{~h}$ with an ADV hexon protein to detect IFNg-producing ADVspecific T cells. For ex vivo expansion of ADV-specific T cells, PBMCs $(5 \mathrm{mio} / \mathrm{ml})$ were stimulated with hexon protein. On day 3 and 9 cultured cells were stimulated with IL-15. On day 6 cells were restimulated with hexon protein-pulsed autologous PBMCs On day 12, expansion of ADV-specific T cells was analysed by multimers or IFNg.

Results: $55 \%$ out of 20 healthy donors have been tested positive for ADV-specific T cells by analyzing freshly isolated PBMCs. In contrast, $90 \%$ have been tested positive after ex vivo expansion. In accordance with these results, only one donor $(20 \%)$ out of 5 of freshly isolated PBMCs and 4 donors (80\%) of ex-vivo expanded T cells have been tested positive by the IFNg capture assay. These results clearly indicate that ex-vivo expansion of PBMCs is necessary to ensure the presence of ADV-specific T cells in healthy donors. In addition, the absolute cell number of ADV-specific $T$ cells was increased 2 to $3 \mathrm{log}$ after ex-vivo expansion compared to freshly isolated PBMCs. Expanded T cells showed functional and cytotoxic activity. Strikingly, clearance of ADV infection was associated with increased numbers of multimer-positive T cells in a patient after aHSCT. In conclusion, this study defines rapid and optimal conditions for detection and ex vivo expansion of ADV-specific T cells with high potential for clinical application.

\section{P952}

Vaccination of multiple myeloma patients with mature dendritic cells expressing multiple tumour antigens following RNA electroporation

H. Dolstra, F. Maas, H. Fredrix, A. Greupink, B. Esendam, S. Bak, T. De Witte, L. Strobbe, B. van Rees, M. Schaap, R. Raymakers

UMC St Radboud (Nijmegen, NL)

In an ongoing trial we vaccinate multiple myeloma (MM) patients using mature monocyte-derived dendritic cells (DCs) electroporated with in vitro-transcribed mRNA encoding for Mage-3, Survivin and $B$ cell maturation protein (BCMA). Preclinical studies showed that RNA electroporation leads to high protein expression and long-lasting peptide presentation in the context of MHC class I, without loss of migratory capacity. To date, six patients with a (near) complete resmission after high-dose Melphalan (HDM) have been vaccinated. Mature DCs were generated from apheresis products, loaded with keyhole limpet hemocyanin (KLH) as an adjuvant and control antigen, and electroporated with clinical grade tumor antigen mRNA. Patients were 
vaccinated 3 times, both intraveneously (8-20 $\times 10^{6}$ per dosis) and intradermally (4-10 $\times 10^{6}$ per dosis), at 2 week intervals. Vaccinated patients were monitored for a) $\mathrm{T}$ cell and antibody responses against $\mathrm{KLH}$ in peripheral blood, b) presence of vaccine-specific T cells in DTH biopsies and bone marrow aspirates using tetramers and ELISPOT, c) Th1/2 cytokine profiles and cytolytic activity of vaccine-induced $T$ cells. Disease activity was monitored by measuring $\mathrm{M}$-component/free light chain and by using a highly sensitive IgH-PCR assay. All patients developed strong anti-KLH T cell responses, but antibodies to KLH could not be detected. Two weeks after the last vaccination DTH-testing was performed and skin biopsies were taken. In 2 patients, vaccine-specific T cells could be detected in the DTHinfiltrating lymphocytes. In one patient, we found CD8 + and CD4 + T cells reactive against Mage- 3 electroporated DCs as well as CD3 $+T$ cells reactive against DCs electroporated with BCMA and Survivin using both ELISPOT and CBA analysis. In the other patient, we detected low numbers of Mage-3 and BCMA-reactive CD8 + T cells using ELISPOT. Vaccination was well tolerated with limited toxicity such as mild fever, redness and swelling at the injection sites. These findings illustrate that TAA mRNA-electroporated mature DCs are capable to induce TAA-specific CD8 + T cell responses in MM patients after highdose chemotherapy. Currently, the specificity of the vaccinespecific $\mathrm{T}$ cell responses are being investigated as well as the effect of vaccination on MRD. The trial will be continued until 12 patients are included.

\section{P953}

Efficient activation of LRH-1-specific CD8 + T-cel responses from transplanted leukaemia patients by stimulation with P2X5 mRNA-electroporated dendritic cells

H. Dolstra (1), I. Overes (1), H. Fredrix (1), M. Kester (2), J.H.F. Falkenburg (2), R. Van der Voort (1), T. De Witte (1) (1)UMC St Radboud (Nijmegen, NL); (2)LUMC Leiden (Leiden, $N L$ )

Alloreactive CD8 $+\mathrm{T}$ cells targeting minor histocompatibility antigens (MiHA) on malignant cells of the recipient play a pivotal role in graft-versus-tumor (GVT) responses observed after allogeneic stem cell transplantation (SCT) and donor lymphocyte infusion (DLI). However, these MiHA-specific CD8 + T cell responses do not result in complete eradication of tumor cells in all patients. Furthermore, CD8 + memory T cells persisting after DLI do not always efficiently expand with recurrence of the disease. Adjuvant immunotherapy using dendritic cells (DC) loaded with hematopoietic-restricted MiHA may boost anti-tumor CD8 + T cell immunity without inducing graft-versushost disease. Here, we explored the use of mRNA-electroporated DC to stimulate MiHA-specific CD8 $+\mathrm{T}$ cell responses. We demonstrate that electroporation of mature DC with P2X5 mRNA encoding for hematopoietic-restricted MiHA LRH-1 results in high expression of both mRNA and protein, and has no negative effect on the mature phenotype and migratory capacity of the DC. Furthermore, these DC can efficiently stimulate LRH-1-specific CD8 + effector T cells to proliferate and produce IFN-ã. In addition, LRH-1-specific CD8+ memory $T$ cells that are present in patient-derived PBMC at long periods post-DLI can be effectively activated by stimulation with P2X5 mRNA-electroporated DC to proliferate and degranulate upon target cell recognition. These results indicate that adjuvant immunotherapy using DC electroporated with mRNA encoding hematopoietic-restricted MiHA mismatched between patients and donors may enhance the GVT response induced by SCT and DLI.
P954

Editing central memory T-lymphocyte specificity for safe and effective adoptive immunotherapy of leukaemia E. Provasi (1), P. Genovese (2), Z. Magnani (1), A. Lombardo (2), O. Muniz Pello (2), J. Kuball (3), A. Bondanza (1), P.D. Gregory (4), C. Bordignon (5), M.C. Holmes (4), P.D. Greenberg (3), L. Naldini (2), C. Bonini (1)

(1)San Raffaele Scientific Institute (Milan, IT); (2)TIGET, San Raffaele Scientific Institute (Milan, IT); (3)Fred Hutchinson CancerResearch Center(Seattle, US); (4)SangamoBiosciences (Richmond, US); (5)San Raffaele University (Milan, IT)

The transfer of high-avidity T cell receptors (TCR) isolated from tumor-specific lymphocytes into polyclonal T cells is an attractive strategy for the adoptive immunotherapy of tumors. However, three major hurdles hinder the effectiveness of this strategy: i) inefficient gene transfer and unstable transgene expression, ii) exhaustion of gene-modified cells, and iii) mispairing of endogenous and exogenous TCR chains. To solve these issues, we used a bi-directional lentiviral vector (LV) expressing a codon-optimized, cysteine-modified TCR for the Wilms tumor antigen 1 (WT1) to genetically modified human central memory T lymphocytes (TCM). Transduced cells showed high $(\sim 16 \%)$ and stable (70 days in culture) levels of WT1-pentamer binding and specifically responded against WT1+ primary AML leukemic blasts. To further enhance the safety profile and potency of modified TCM, we developed a platform to genome edit TCM based on Zinc Finger Nucleases (ZFN). In order to ensure stable and consistent transgene expression, we demonstrated the targeted insertion of the transgene into a predefined genomic locus (CCR5) by homologous recombination in up to $6 \%$ of treated TCM, with concurrent inactivation of $18 \%$ of the targeted CCR5 gene by non-homologous end joining (NHEJ). These findings prompted us to develop ZFN specific for the endogenous TCR beta chain gene to prevent TCR mispairing. We obtained functional inactivation of this gene by NHEJ in 7\% TCM. Targeted lymphocytes failed in expressing the CD3/TCR complex on cell surface, and could be sorted as CD3neg. CD3neg proved stable in culture, maintained a TCM phenotype and were resistant to further TCR-mediated triggering. For a complete editing of T cell specificity, the WT1-specific TCR was transferred with high efficiency $(>20 \%)$ by LV on CD3neg cells. WT1-TCR rescued CD3 surface expression and was expressed in edited lymphocytes at higher levels to that observed upon conventional TCR gene transfer. Accordingly, TCR-edited cells proved superior to cells undergoing conventional TCR gene transfer in promoting killing of relevant targets, and in recognizing low $(10 \mathrm{nM})$ peptide concentrations. Overall, these data show the feasibility of rapid and efficient editing of TCM to generate safe and functional tumor-specific T cells and may open new perspectives to cancer adoptive immunotherapy. (EP and PG: equal contribution).

\section{P955}

Interleukin-15 supports long-term cultures for generation of highly potent clinical grade NK effectors for targeting haematological malignancies

G. Suck (1), V.Y.S. Oei (2), Y.C. Linn (3), A. Choong (1), S. Chu (1), M. Niam (1), M.B.C. Koh (4)

(1)Health Sciences Authority (Singapore, SG); (2)Health Sciences Authority and National University of Singapore (Singapore, SG); (3)Singapore General Hospital (Singapore, SG); (4)Health Sciences Authority and St. George's Hospital and Medical School (Singapore and London, UK)

Objective: Natural killer (NK) cells are promising candidates for cell therapy due to their powerful innate cytotoxic potential. Traditional clinical NK protocols have relied on IL-2, which in clinical applications can cause systemic toxicities. A novel, more tolerable cytokine is IL-15, which increases NK survival, promotes graft versus leukemia effect and immune reconstitution in mice, and enhances NK activation potential. We have 
studied the effects of IL-15 on NK cells expanded in long-term cultures for clinical translation.

Methods: NK were expanded within LAK in CellGro-IL-2 (500 $\mathrm{U} / \mathrm{ml}$ ) or -IL-15 (100-500 U/ml), Okt-3 (Day (D) 0-D4/5), 5\% AB-HS; LAK-NK $=$ CD56 +CD3-LAK population; viability was assessed with trypan blue or 7AAD and cytotoxicity by Flow cytometry.

Results: IL-15 was similarly potent compared to IL-2 in supporting NK cell expansion. After 28 days LAK-IL-2 expanded 40-2500-fold, with LAK-NK 700-6000-fold, vs. 150-500-fold for LAK-IL15, with 300-2000-fold for LAK-NK (4 donors). Both cytokines rendered LAK highly cytotoxic within 2 weeks of culture, with $86 \% \pm 12 \%$ SEM for IL-15 vs. $84 \% \pm 14 \%$ SEM for IL-2 of K562 lysis (E:T 1:1, 2 donors). However, continuation of cultures revealed that potency of LAK-IL-2 decreased more rapidly vs. IL- 15 , with $67 \% \pm 10 \%$ SEM vs. $82 \% \pm 7 \%$ SEM, D20-24, and $16 \% \pm 5 \%$ vs. $44 \% \pm 21 \%$ SEM, D $25-28$ (4 donors). Results for 5 additional donors showed that LAKIL-15 were cytotoxic against other targets, including Molt-4 (T-ALL) $31 \% \pm 11 \%$ SEM and KU812 (CML) $31 \% \pm 10 \%$ SEM (E:T 1:1), and non-toxic against autologous PBMCs (D14-21). Expression of important NK activating and inhibitory receptors, including NKG2D, NKp30, NKp44, NKG2C, NKG2A and KIRs, but not NKp46, CD16 and CD27, was significantly increased at D14 vs. D0. Importantly, IL-15 efficiently expanded effectors from frozen AML (M1, M4) samples, with 24-505-fold LAK and 77-1420-fold LAK-NK, compared to 8-148-fold LAK and 63-770-fold for IL-2 (D23-26, 3 donors). IL-15 could also generate potent LAK from 2 frozen B-ALL samples with 40-44-fold expansion and LAK-NK 1031-1870-fold (D24). Clinical-culture scale up to $400 \mathrm{ml}$ was feasible for CellGro-IL-15, with 109147-fold for LAK and 654-1083-fold for LAK-NK expansion (D20, 2 donors).

Conclusion: IL-15 is a promising novel cytokine to support generation of highly cytotoxic clinical grade LAK, with the potential for longer term sustained potency and preservation of activation receptors in therapy of haematological malignancies.

\section{P956}

Application of purificated CD4 + T-cells for adoptive immunotherapy of haematological malignancies following allogeneic stem cell transplantation

E. Klyuchnikov (1), A. Sputtek (1), O. Slesarchuk (2), M. Lioznov (1), U. Bacher (1), B. Fehse (1), F. Bentzien (1), A. Zander (1), N. Kröger (1)

(1) University Cancer Center (Hamburg, DE); (2)Pavlov State Medical University (St. Petersburg, RU)

Introduction: Donor lymphocyte infusions (DLIs) represent an immunoadoptive approach to induce "graft versus leukemia effect" (GvL) aiming to eradicate the leukemic cells. However, CD3 + DLIs are associated with a high graft versus host disease $(\mathrm{GvHD})$ rate that might be fatal. As some studies proposed a central role of CD8 + cells in the development of GvHD, the use of separated CD4+ donor cells seems to be an option to reduce the incidence of this event. Here we reported on clinical use of selected CD4 + cells by the CliniMACS $₫$ device aiming to evaluate its efficacy and safety after allo-SCT.

Patients and methods: 24 patients (pts) (16 male; 8 female) with hematological malignancies (multiple myeloma, $n=11$; myelofibrosis, $n=6$; CML: blast phase, $n=2$; chronic phase, $n=1$; MDS, $\mathrm{n}=3$; CLL, $\mathrm{n}=1$ ) were enrolled at median of 14 months (range, 2-76) from allo-SCT. The median age was 49 years (range, 21-65). 19/24 (79\%) pts received dose-reduced, whereas 5/24 $(21 \%)$ myeloablative conditioning. 11 pts received grafts from related, 13 from unrelated donors (matched, $n=8$; mismatched, $n=5)$. The reasons to perform CD4 + selected DLI were relapse $(n=10)$, minimal residual disease $(n=8)$, prophylaxis due to high relapse risk $(n=4)$, and severe herpetic stomatitis $(n=2)$. 24 leukapheresis products were enriched for CD4 + cells using magnetic cell sorting (MACS) with an automated device (CliniMACS $®)$.
Results: MACS resulted in a mean of $16 \times 10^{6} \mathrm{CD} 4+$ cells/ $\mathrm{kg}$ body weight (bw) corresponding to a 3.4 -fold enrichment. Mean yield and purity were $63 \%$ (range, 25-99.8) and $81 \%$ (range, 53.7-95). Median dose of received CD4 + cells was $1.1 \times 10^{6} / \mathrm{kg} \mathrm{CD} 4+$ cells $/ \mathrm{kg}$ bw (range, $0.1-15$ ). Acute GvHD grade II-III was observed in one (4\%) pt, chronic limited GvHD developed in two $(8 \%)$ pts; there were no cases of chronic extensive GvHD. 8 of $18(44 \%)$ patients with measurable disease and two patients with severe herpetic stomatitis showed a response after a median of 1.8 months (range, $0.1-5$ ) following the infusion. After a median follow up of 45 months (range, 4-100), 23 of 24 pts were alive; one pt died due to disease progression (PD). All 18 pts, who experienced signs of disease before infusions were alive $(C R, n=14$; VGPR, $n=2$; PD, $n=2$ ).

Conclusions: We conclude that the use of CD4 + enriched DLI provides an efficient and tolerable method of immunoadoptive therapy for patients with signs of disease or infections following allo-SCT, associated with low incidence of acute and chronic GvHD.

\section{P957}

Umbilical cord derived mesenchymal stromal cells modulate monocyte function to suppress T-cell proliferation

A. Cutler, V. Limbani, J. Girdlestone, C. Navarrete

NHS Blood and Transplant (London, UK)

Objectives: Originally derived from Bone Marrow (BM), mesenchymal stromal cells (MSC) may be derived from alternative sources with umbilical cord (UC) providing an abundant and non-invasive source. MSC possess interesting immunological properties, they can suppress $\mathrm{T}$ cell activation, modulate the function of several leucocyte subsets and do not induce strong allogeneic responses. MSC are currently being used in clinical trials as a novel anti-inflammatory agent. Multiple mechanisms have been proposed to explain the suppressive potential of MSCs, and we are dissecting the cellular interactions required for suppression of allo-specific $T$ cell proliferation.

Methods: MSC generated from UC were maintained in DMEM, $10 \%$ FCS. Peripheral blood mononuclear cells (PBMC) were purified from buffy coats by Ficoll centrifugation. Monocytes, CD4 + T cells and monocyte or B cell depleted PBMC were purified using magnetic microbeads. PBMC, monocyte or B cell depleted PBMC were stimulated using $T$ cell activation beads \pm MSC. Monocytes purified from MLR or PBMC \pm MSC co-culture using percoll centrifugation and bead separation were cultured with HLA-mismatched CFSE labeled CD4 + T cells at a ratio of $1: 10$.

Results: UC-MSC share the surface phenotype as well as the immunosuppressive abilities of MSC from BM and cord blood. We found that suppression of mitogen induced T cell proliferation by MSC from all 3 sources required monocytes. Removal of monocytes but not B cells from adult PBMC preparations reduced the immunosuppressive effects of MSC on T cell proliferation. Conversely, addition of purified monocytes to UC-MSC and CD4 + T cell co-cultures restored the suppressive effects of UC-MSC. There was rapid modulation of a number of cell surface molecules on monocytes when PBMC, MLR or purified monocytes were cultured with UC-MSC. Monocytes purified from UC-MSC co-culture had significantly reduced accessory cell and allo-stimulatory function when tested in subsequent $T$ cell proliferation assays, an effect mediated in part by UC-MSC prostaglandin production. Finally, UC-MSC and MLR co-culture induced prostaglandin-E2 independent IL-10 production by monocytes.

Summary: Monocytes were found to be essential for the immunosuppressive function of MSC on alloantigen induced T cell proliferation. UC-MSC rapidly altered the phenotype, induced IL-10 production and reduced allo-stimulatory function of the monocyte population following co-culture, an effect mediated in part by prostaglandin-E2. 
P958

Enhancing cytotoxicity of cytokine-induced killer cells against leukaemia

E. Rettinger (1), S. Selim Kuçi (1), Z. Kuçi (1), M. Anzaghe (1), A. Willasch (1), H. Kreyenberg (1), U. Koehl (1), S. Fulda (2), T. Klingebiel (1), P. Bader (1)

(1)Goethe University (Frankfurt, DE); (2)University UIm (UIm, DE)

Haploidentical hematopoietic stem cell transplantation (HSCT) has become an important treatment modality for high-risk leukemia patients. Nevertheless, the success of HSCT is mainly affected by relapse. Pre-emptive immunotherapy may prevent impending relapse in some cases. Cytokine-induced killer (CIK) cells with reduced propensity for GvHD may offer an alternative approach to conventional donor lymphocyte infusions (DLI). In this study, we investigated the feasibility of enhancing CIK cell mediated cytotoxicity against leukemias without influencing the alloreactive potential of CIK cells.

CIK cells are non-MHC restricted effector cells consisting of T, NKT and NK cells. CIK cells can easily be generated from peripheral blood mononuclear cells (PBMC) in vitro. Expanding CIK cells showed an increase of NKT cells and a decrease of regulatory $T$ cells (Treg). The number of NKT cells expressing CD8 + cytotoxic phenotype, activating NK receptors and effector molecules increased during cell culture.

Cytotoxicity of CIK cells was assessed by europium release assay. At an effector to target ratio of $50: 1$ CIK cells showed potent antileukemic activity against standard target K562 $(51.73 \pm 17.69 \%)$, T- ALL cell lines Jurkat $(57.50 \pm 10.61 \%)$, H9 $(44.29 \pm 25.58 \%)$, MOLT-4 $(49.38 \pm 17.70 \%)$ and subtype M4 AML cell line THP-1 $(60.17 \pm 31.18 \%)$. Furthermore, we could show that CIK cell mediated cytolytic activity is NKG2D receptor and granzyme/perforin dependent and that extrinsic death signals are transferred via TRAIL. These findings and the fact that CIK cells can easily be expanded even according to GMP terms may spark clinical interest in these cells.

To even enhance CIK cell mediated cytotoxicity CIK cell generation was modified by adding IL-2 plus IL-15 or IL-15. In fact, addition of IL-15 significantly augmented cytotoxicity of CIK cells.

Irrespective of the mode of ClK cell expansion, the alloreactive potential against allogeneic PBMCs remained low indicating a low risk to induce GVHD.

In conclusion, in vitro expanded CIK cells may be a useful therapeutic option in case of impending relapse following haploidentical transplantation.

This work was kindly supported by grants of the Else KrönerFresenius-Stiftung.

\section{P959}

Clinical relevance of in vitro generated dendritic cells of leukaemic origin (DCleu) in patients with AML

M. Freudenreich (1), C. Schmid (2), J. Dreyßig (1), A. Kremser (1), T. Kroell (1), J. Tischer (1), H. Kolb (1), H. Schmetzer (1) (1)University Hospital (Munich, DE); (2)Municipal Hospital (Augsburg, DE)

The GvL effect is based on the antileukemic activity of transplanted donor T-cells and can be reinforced by donor-lymphocyte-infusions (DLI). Ex vivo myeloid blasts can regularly be induced to differentiate to DCleu (Kremser 2009; Schmetzer 2007), presenting unknown/known leukemic antigens to T-cells. GM-CSF is a main component in most of these methods. Our hypothesis is, that myeloid leukemia respond better to SCT/DLI than lymphoid malignancies because of the in vivo generation of DCleu from blasts.

The aim of our study was to enlighten the role of a successful ex vivo DC-generation with respect to its 'predictability of an in vivo behaviour of blasts', and the prognostic significance for the clinical course of AML-pts after SCT ( $n=21)$ or (GM-CSF-containing) DLI-relapse therapy $(n=17)$.
Results: 1) Pts who had responded successfully to SCT $(n=18)$ presented with more mature DC $(57 \%$ vs. $44 \%)$, more DCleu (16\% vs. $8 \%)$ and a higher blast-coversion rate to DC $(56 \%$ vs. $46 \%)$ than nonresponders $(n=3) .2)$ Pts responding to DLI $(n=10)$ presented with more mature DC $(73 \%$ vs. $52 \%, P=0.04$ ) and a higher blast conversion rate (44 vs. $21 \%$ ) than non-responders $(n=7)$. 3) Pts receiving $G M-C S F$ in vivo had a probability of $69 \%$ to respond to DLI and a Øsurvival time of $602 \mathrm{~d}$ compared to $25 \%$ responders in pts without GM-CSF and a Øsurvival of 152 d. 4) Transplanted pts with a blast conversion rate of $>20 \%,>15 \%$ DCleu or $>50 \%$ mature DC had a probability of $92-100 \%$ vs. $71-82 \%$ to respond to SCT compared to nonresponders. Moreover cases with $>20 \%$ blasts converted to DCleu were characterized by a Øsurvival of 460 vs. $106 \mathrm{~d}(P=0.02)$, cases with $>10 \%$ DCleu/MNC by a surivival of 491 vs. 246 d. 6)Relapsed pts after SCT with a blast conversion rate of $>20 \%(P=0.06),>10 \%$ DCleu/MNC or $>60 \%$ mature DC $(P=0.03)$ had a higher probability to respond to DLI.

In sum a better prognosis is associated with 1) relapsed pts receiving GM-CSF in combination with $D L I$ in vivo 2 ) Pts with a higher ex vivo blast conversion rate to DCleu, more mature and more DCleu have a higher chance to respond to SCT or DLI-relapse therapy and have a better survival. That means, that proportions and composition of DC generated ex vivo are predictive for the pts' response to SCT or DLI, what could be explained by an improved DC-generability and T-cell-priming in vivo. Our data could contribute to estimate prognosis of pts after SCT/DLI and to improve GM-CSF/DCbased immunotherapies in AML.

\section{P960}

Leukaemia-derived DC for T-cell therapy against AML

H. Schmetzer (1), A. Liepert (1), C. Grabrucker (1), D. Fischbacher (1), M. Merle (1), R. Reibke (1), J. Tischer (1), T. Kroell (1), C. Schmid (2), H.J. Kolb (1)

(1)University Hospital of Munich (Munich, DE); (2)Municipal Hospital (Augsburg, DE)

Adoptive immunotherapy is an important therapy option to reduce relapse rates in AML-patients. Myeloid blasts can regularly be induced to differentiate into leukemia-derived-dendriticcells (DCleu), regaining the stimulatory capacity of professional DCs while presenting the known/unknown leukemic antigenrepertoire. So far induced antileukemic T-cell-responses are variable or even mediate opposite effects. To further elicit DC/ DCleu induced T-cell-response-patterns we generated DC from 17 AML- and 2 high grade MDS-cases and performed flowcytometry and (functional) non-radioactive fluorolysis-assays before/after mixed-lymphocyte-cultures (MLC) of matched (allogeneic) donor-T-cells $(n=6), T$-cells prepared at relapse after stem-cell-transplantation $(n=4)$ or (autologous) patients'T-cells $(n=7)$ with blast-containing mononuclear-cells ('MNC') or DCleu ('DC'). Compared to 'MNC' 'DC' were better mediators of anti-leukaemic-T-cell-activity, although not in every case effective. We could define cut-off proportions of mature $\mathrm{DC} / \mathrm{DCleu}$,/proliferating/CD4+/CD8 + and non-naive T-cells after 'MNC'- or 'DC'-stimulation, that were predictive for an antileukemic activity of stimulated T-cells as well as a response to immunotherapy. Interestingly especially ratios $>1$ of CD4:CD8 or CD45RO:CD45RA T-cells were predictive for antileukemic function after DC-stimulation.

In summary the composition and quality of DC and T-cells after a MLC-stimulating-phase is predictive for a successful ex-vivo and in-vivo anti-leukemic response, especially with respect to proportions of mature and leukemia-derived DC or proliferating/CD4 +/CD45RO + T-cells. Successful cytotoxicity and the development of a T-cell-memory after 'DC'- stimulation could be predictive for the clinical course of the disease and may pave the way to develop and refine DC-vaccination or adoptive immunotherapy strategies, especially for patients at relapse after SCT. 
P961

Isolation and characterization of naturally occurring HLA-G expressing cells with immunoregulatory properties in peripheral blood of healthy individuals

I. Lazana, M. Themeli, A. Zoudiari, D. Kokkinou, N. Zoumbos, A. Spyridonidis

University Hospital Patras (Patras, GR)

Introduction: HLA-G is a nonclassic MHC class I with unique features that functions as an immunomodulatory molecule. HLA-G was found to be expressed in extravillous trophoblasts during pregnancy and protects fetal tissues from the maternal immune system. HLA-G has been shown to regulate the rejection process after solid organ transplantation but its role in allogeneic hematopoietic cell transplantation (HCT) is unknown.

Objectives: The aim of this study was to detect and characterize naturally occurring HLA-G + cells and to investigate their possible immunoregulatory properties.

Methods: Peripheral blood mononuclear cells (PBMC) were obtained from 22 volunteer donors and were analyzed by FACS for the presence of HLAG + (MEM-G/9 MoAb) cells, both in whole PBMC and in specific cell compartments. Moreover, we isolated HLA-G + cells by FACS sorting (Vantage, BD) and analyzed their tolerogenic properties by using them as third party cells in mixed lymphocyte cultures (MLC) and in PHA stimulated lymphocyte cultures. The lymphoproliferative response in the cultures was measured using the CFSE cell proliferation assay. The immunmodulatory effect of HLA-G was calculated in comparison to lymphocyte proliferation in control cultures without addition of HLA-G + cells.

Results: The mean percentage of HLA-G + cells in whole PBMC was $4.4 \%$ with a Specific Fluorescent Index (SFI) of 1.2. The mean percentage of HLA-G + cells was significantly higher in CD14 + $(23.2 \%)$ than in CD $3+$ cells $(1.4 \%), P=0.0002$. Inversely, we isolated by FACS sorting HLA-G + cells from PBMC and found that $93 \%$ of them were CD14+. CD14+/HLAG + cells showed significant lower expression of HLA-DR (SFI 81.3) as compared to CD14+/HLAG- cells (SFI 186), $P<0.001$. CD3+/ HLAG- cells were found to express membrane HLA-G upon their ex vivo exposure to azacytidine (HLA-G induced T-cells). When FACS sorted CD14 +/HLAG + cells were added as third party cells in MLC or in PHA-stimulated lymphocyte cultures we observed a dose dependent suppression of lymphoproliferation. Concentrations $>1 / 1$ of responder/HLA-G + cells showed significant suppression of the allo-response by $>42 \%$ over the control, $P=0.0012$.

Conclusions: Our results indicate the existence and the capacity to isolate HLA-G + cells with immunosuppressive function from PB of healthy individuals. Naturally occurring HLAG + monocytic cells (and maybe also HLA-G + induced T-cells) suggest a promising strategy for adoptive cell immunosuppressive therapy after HCT.

\section{P962}

Superior performance of the novel suicide gene TK.007 in adoptive immunotherapy models both in vitro and in vivo E. Preuß (1), A. Treschow (2), S. Newrzela (3), D. Brücher (3), K. Weber (1), J. Otte (3), U. Felldin (2), E. Alici (2), D. von Laer (4), S. Dilber (5), B. Fehse (1)

(1) Univ. Medical Centre Hamburg-Eppendorf (Hamburg, DE); (2)Karolinska Institute (Huddinge, SE); (3)Frankfurter Stiftung für krebskranke Kinder (Frankfurt, DE); (4)Georg Speyer Haus (Frankfurt, DE); (5)Karolinska Institute (Huddinge, SE)

Adoptive immunotherapy (adIT) with suicide gene-modified allogeneic T lymphocytes has been shown to be a potent strategy to facilitate immune reconstitution and to treat leukaemia relapses or infectious complications after allogeneic HSCT. Despite several imperfections, including slow and incomplete killing as well as unspecific toxicity of high ganciclovir (GCV) doses, the Herpex simplex virus thymidine kinase (HSVtk) gene together with its prodrug GCV has been the suicide gene- of-choice in most clinical gene therapy studies so far. In order to address these shortcomings, we developed a codon-optimised HSVtk [coHSVtk] and modified this by introducing the A168H mutation (initially described by Karlsson and colleagues) to generate the novel suicide gene TK.007. Performance of coHSVtk and TK.007 as compared to the frequently used splice-corrected wild-type scHSVtk was assessed in different preclinical adIT models. Gene transfer was accomplished using retroviral MP71 vectors encoding fusion constructs of the different suicide genes with one of the selection marker tCD34 or OuaSelect. Using these tools we observed (i) better expression characteristics and (ii) strongly improved killing kinetics for TK.007 as compared to both other HSVtk's in haematopoietic cell lines and primary T cells in vitro. We next tested TK.007 in vivo in a murine graft-vs.-host disease (GvHD) model. We found that infusion of TK.007-transduced allogeneic T cells induces severe, lethal GVHD which could readily be abrogated by GCV treatment. In a further experimental setting we intended a direct comparison of TK.007 vs. the commonly used scHSVtk in vivo. To this aim, we established a modified adIT model which facilitates the quantitative assessment of HSVtk activity in vivo. This allowed us to demonstrate that TK.007 mediates both significantly faster and higher absolute killing rates at low GCV concentrations (10 and $25 \mathrm{mg} / \mathrm{kg}$ ) than scHSVtk. In summary, we have shown that TK.007 combines better killing performance with reduced unspecific toxicity (as compared to the frequently used splice-corrected wild-type scHSVtk gene) thus representing a promising alternative suicide gene in the context of adoptive immunotherapy.

\section{P963}

A preliminary investigation into the kinetics of MSC induced immuno-modulation during the allo-reactive phase of an MLR

M. Rae, X.N. Wang, M. Collin, A. Dickinson

Newcastle University (Newcastle, UK)

A novel cellular therapy for Graft versus Host Disease (GvHD) is the co transplantation of mesenchymal stromal cells (MSCs). This investigation intended to further elucidate the immunosuppressive properties of mesenchymal stromal cells (MSCs), particularly in the context of GvHD. Three of the key mechanisms of MSC immunosuppression (indoleamine-2,3, deoxygenase (IDO), inducible nitric oxide synthetase (iNOS) and HLA-G) were studied in the context of a primary mixed lymphocyte reaction (MLR). FACS analysis and blocking experiments were used. In order to investigate whether the mechanisms described could be demonstrated to act in a synchronised manner levels of activity for each mechanism for days 0,1 and 3 were measured and given a score. The score was expressed as the percentage of the maximum mean activity for each mechanism and grouped into four categories; very low or zero expression (0-30\%); low (30-50\%); high (50-70\%) and maximal(70-100\%)expression. Furthermore MSCs significantly reduced the Graft versus Host Reaction (GvHR) in the in vitro human skin explant model. The Graft versus Host alloreactivity (GvHR) seen in this model is analogous to graft versus host disease (GvHD) in a clinical transplant setting. Briefly, MSCs and responder cells were taken from a MLR then co cultured with a skin biopsy from the stimulator for 3 days. Various conditions were tested. MSCs showed immunomodulatory effects even when trytophan levels were restored $(P=0.018)$. Blockade of iNOS also altered the inhibitory effects of MSCs in this context.

HLA-G was strongly expressed on the MSC cell surface $(n=4)$ and it was shown using ELISA and FACS that HLA-G is shed from the MSC cell surface and taken up and expressed by responder cells via a process known as trogocytosis during an $\operatorname{MLR}(P=0.053)$.

Results demonstrated that when overall expression was analysed IDO and iNOS showed a reciprocal activity during the lag phase of the alloresponse. There was no obvious link to HLA-G activity. Priming MSCs with IFNg and TNFa altered the 
kinetics of IDO and iNOS expression by prolonging the activity of IDO and iNOS during the lag phase of an allo-reaction but had no effect upon HLA-G. This may explain the augmentary effects of these cytokines on MSC function. MSC immunomodulatory activity is multi-phasic -perhaps indicative of an important homeostatic in vivo role which can be manipulated using cytokines. This may have relevance in their future use in transplantation.

\section{P964}

An allo-restricted peptide-specific CTL clone directed against the universal tumour antigen survivin

P. Circosta (1), A.R. Elia (1), M. Todorovic (2), F. Orso (1),

C. Tarella (1), A. Cignetti (1)

(1)Molecular Biotechnology Center (Turin, IT); (2)IRCC (Candiolo, IT)

High-avidity T cells specific for self-peptide/self-MHC ligands are eliminated in the thymus by negative selection. An attractive strategy to circumvent tolerance to self-protein is the generation of peptide-specific, allo-restricted cytotoxic T cells (CTL). We have isolated an allo-restricted CTL clone with high avidity against a modified, HLA-A2 restricted survivin epitope. Survivin is the prototype of "universal" tumor associated antigens, because it is expressed at high levels in nearly all tumor types and at low levels in normal tissues. A survivin specific CTL clone was generated by priming CD8 + cells from an HLAA2 negative donor with peptide-pulsed T2 cells. Primed CD8+ cells were restimulated with peptide-pulsed PHA-blasts from 2 different donors who were mismatched only for the HLA-A2 allele. One day after each restimulation, CD137 expression was evaluated on bulk cultured CTL and after the fourth cycle a significant CD137 upregulation was observed (8\%). The CD137 + fraction was purified by magnetic beads and cloned by limiting dilution. 135 clones were screened by IFN-g elispot using peptide-loaded T2 cells pulsed with the modified survivin peptide or with an irrelevant peptide. 109 clones were not reactive, 25 clones were reactive against both the relevant and irrelevant peptide and one clone (4C12) was highly reactive only against the survivin peptide. The activity of clone $4 \mathrm{C} 12$ was tested against T2 cells pulsed with escalating doses of the modified (LMLGEFLKL) and of the naïve (ELTGEFLKL) survivin peptide. The functional avidity against the modified peptide was higher than against the naïve peptide, and 4C12 activity against $T 2$ cells pulsed with the naïve peptide was similar to that against HLA-A2 + leukemic cells expressing endogenous survivin (THP-1). We have also tested clone 4C12 against HLA-A2 + normal fibroblasts and EBV-transformed Bcells and found that its functional activity is strictly correlated with the amount of survivin transcript expressed by each cell type. We are currently extending this observation to a broader panel of normal and malignant HLA-A2 cells, including stem cells. To evaluate the degree of non-specific alloreactivity, we will test the activity of clone 4C12 against a set of HLA-A2 negative normal and malignant cells. If clone $4 \mathrm{C} 12$ will confirm its high degree of specificity and activity against tumor cells overexpressing survivin, it will be suitable for T-cell receptor gene transfer.

\section{P965}

Cytokine-induced killer cells for haematological malignancy: feasibility and efficacy in autologous and allogeneic settings

Y.C. Linn (1), M. Niam (2), S. Chu (2), A. Choong (2), G. Suck (2), Y.T. Goh (1), W. Hwang (1), Y. Loh (1), T.J. Lim (2), L.H. Ng (1), H.X. Yong (1), M. Koh (2)

(1)Singapore General Hospital (Singapore, SG); (2)Health Sciences Authority (Singapore, SG)

Objective: CIK cells are polyclonal T cells generated from peripheral blood or bone marrow (BM). Since Aug 2006 we started on a clinical trial with infusion of ClK cells for 3 groups of patients: (1) adjuvant infusion post autologous haemopoietic stem cell transplant (HSCT) for acute myeloid leukemia (AML), (2) untreated AML and (3) relapse post allogeneic HSCT.

Method: CIK cell was cultured from frozen or fresh leukapheresis cells under GMP condition. At D26-28 cells were washed and frozen in aliquots.

Results: Expansion was a median of 7.7(0.7-171.1) fold for CD3 + subset and 28.3(1.7-350.9) fold for CD3 + CD56 + subset, which comprised $26.6 \%(3.8-76.9 \%)$ in the final product. CIK could be cultured even from untreated AML patients with $20-87 \%$ circulating blasts and post allo-HSCT patients where donor cells are unavailable.

At E:T ratio of $40: 1$, cytotoxicity was a median of $29 \%$ vs. auto AML targets $(n=10)$ and $37 \%$ vs. a common allogeneic AML target $(n=43)$. CIK products post-thaw showed comparable cytotoxicity as pre-freeze sample.

A total of 85 infusions were given to 28 patients. For group $1(n=11)$, RFS was $32 \%$ at 34 months. For group $3(n=14)$, 2 patients with Hodgkin's disease that progressed on mild oral chemotherapy and donor lymphocyte infusion (DLI) had shrinkage of lymph node after CIK infusion. 8 acute leukemia patients received donor CIK at cytopenia post salvage chemotherapy. 1 patient with refractory relapse of T-ALL had a 5th salvage followed by $\mathrm{CIK}$ and achieved BM remission for $10 \mathrm{mo}$ despite progression of extramedullary (EM) leukemia. Another with relapsed pre-B ALL post cord HSCT achieved remission after salvage and underwent leukapheresis for CIK culture. BM remission lasted 5 mo while leptomeningeal relapse occurred at 1 mo post infusion. Both cases suggest a GVL effect known to be active for BM but not EM leukemia. CIK was ineffective in 4 patients $(A M L=3, H D=1)$ with untreated relapse post allo-HSCT and 2 non-HSCT patients (group 2) with untreated AML.

Fever was the only acute side effect and was self-limiting. Acute GVHD of skin $(n=2)$ and liver $(n=2)$ occurred in 4 of the 14 patients and all responded to short course corticosteroids. CIK was tolerated up to a dose of $200 \times 10^{6} \mathrm{CD} 3 / \mathrm{kg}$ and the dose did not correlate with GVHD.

Conclusion: The efficacy of autologous CIK as adjuvant therapy to prevent AML relapse needs a larger sample size to assess. CIK has superiority over DLI in some patients post allo-HSCT, but efficacy is limited to BM and not EM leukemia.

\section{P966}

Preclinical validation of an NK cell preparation: phenotype and function of NK cell products isolated via CliniMacs and subsequent ex vivo activation with IL-2 or IL-15

M. van Ostaijen-ten Dam, D. Verhoeven, K. Kraal, R. Bongaerts, J. van Bergen, L.M. Ball, A. Lankester, M. van Tol, J. Zwaginga

LUMC (Leiden, NL)

Background: Recent advances in understanding of the biology of Natural Killer (NK) cells indicate that cell-based immunotherapy for malignancies utilizing haplo-identical NK-cell infusions in the setting of haplo-identical Stem Cell Transplantation (SCT) may be an attractive approach.

Methods: GMP-NK cell preparations were isolated from apheresis material $(n=5)$. In three donors, two types of CliniMACS NK-cell enrichment procedures were tested: CD3-CD56 + or CD3-CD19- CliniMACS. Preparations were analysed regarding purity and $\mathrm{B} / \mathrm{T}$ cell contamination. Products were cultured in RPMI human serum alone or supplemented with IL-2 or IL-15. After 17, 38 and $112 \mathrm{hrs}$ cells were analysed for their viability, phenotype and lytic activity. In two donors, the effect of cryopreservation of (cytokine activated) CD3-CD56 + cliniMACS NK-cell preparations on phenotype and function was analysed.

Results: Average NK-cell recovery/purity was $72 / 88 \%$ for CD3CD56 + cliniMACS and 80/26\% for CD3-CD19- CliniMACS 
procedures. Monocytes, approximately $25 \%$, were only present in the cytokine stimulated CD3-CD19- CliniMACS product displayed enhanced expression of the activation marker CD64. With increased duration of culture with cytokine, expression of activation markers and lytic activity of NK-cells increased significantly. Cryopreservation of activated NK-cells had dramatic effect on their lytic activity, which could be restored partially by overnight incubation with cytokine. No major differences were observed between IL-2 or IL-15 stimulated cells.

Discussion: The CD3-CD56 + cliniMACS procedure results in a product of higher purity, viability and absence of activated monocytes with only slightly lower yield compared to CD3CD19- CliniMACS. The activated phenotype and lytic capacity of IL-2 or IL-15 stimulated cultures is comparable. Cytokine induced activation of cryopreserved CD3-CD56 + cliniMACS NK-cells is feasible.

\section{P967}

Targeting Naïve CD8 + T-cells allows the generation of gene-modified lymphocytes with high proliferative potential and early differentiation phenotype

N. Cieri, E. Provasi, Z. Magnani, B. Camisa, A. Bondanza, C. Bonini

San Raffaele Scientific Institute (Milan, IT)

Adoptive cellular therapy, potentiated by gene transfer technologies, holds great promise for the treatment of cancer. However, current gene transfer protocols result in terminally differentiated, often exhausted, cells mediating only transient anti-tumour responses. Our lab previously showed that activation of donor lymphocytes with cell-size beads conjugated with antibodies to CD3 and CD28 and culture with low doses g-chain cytokines generates genetically modified $T$ cells with a functional central memory (TCM) phenotype. In contrast to effector and effector memory (TEM) cells, TCM are the ideal source of long-term anti-tumor immunity, having a longer lifespan and stem/progenitor cell features, such as self-renewal and the ability to differentiate into a progeny of effectors. In the present work we therefore asked whether gene-modified TCM cells represented a homogenous population or whether initial targeting of Naïve cells could further improve the potency of transduced cells. Thus we activated with our protocol and efficiently transduced FACS-sorted Naïve, TCM and TEM CD8 + cells by retro and lentiviral vectors. We observed that CD8 $+\mathrm{T}$ cells from FACS-sorted Naïve cells have a greater expansion potential than cells from FACS-sorted TCM and TEM. IL7-Ra was the most informative marker in defining cell function, origin and potential as its expression was maintained only by cultured Naïve T Cells and not by TCM and TEM-sorted lymphocytes. In FACSsorted cultured Naïve cells we observed a predominant population of CD45RA+CD62L + CCR7 + lymphocytes. Although this phenotype traditionally defines Naïve T cells, CFSE dilution revealed that these cells are post-mitotic and re-acquire the phenotype upon cell division. These cells display features common to early differentiated cells, such as expression of $\mathrm{CD} 27+\mathrm{CD} 28+\mathrm{CD} 127+\mathrm{Bcl} 2+$, and markers proper of memory lymphocytes, such as CD122+CXCR3+CCR5 \pm , and produce low levels of IFNg and Granzyme A. Further phenotypic analysis showed that cultured Naïve express higher levels of c-Kit, CXCR4 and IL18R, while they express lower levels of activation markers such as HLA-DR, CD40L and CD69 compared to TCM and TEM. Althogether our protocol, when applied to sorted Naïve cells, allows the generation of lymphocytes that, based on their phenotypic and functional features, are a promising target for gene and adoptive cellular therapy. We are currently investigating in vivo alloreactivity and anti-leukaemic function in humanized mice.
P968

Erythroid and granulomonocyte colony-forming cells predict haematopoietic recovery after autologous peripheral blood progenitor cell transplantation G. Martin Henao (1), P. Barba (2), D. Moreno (3), M. Moreno (4), J.M. Sanchez-Villegas (5), A. Lopez-Iglesias (2), M. Panades (1), A. Pinacho (5), J. Muñoz (6), A. Ester (7), M. Pujol (8), C. Azqueta (1), C. Barrenetxea (2), J.M. Sancho (4), A. Fernandez de Sevilla (3), R. Duarte (3), J.M. Ribera (4), J.R. Grifols (7)

(1)Banc de Sang i Teixit (L'Hospitalet de Llobregat, ES); (2)Hospital Vall d'Hebron (Barcelona, ES); (3)Institut Català d'Oncologia (L'Hospitalet de Llobregat, ES); (4)Institut Català d'Oncologia (Badalona, ES); (5)Banc de Sang i Teixit (Lleida, ES); (6)Banc de Sang i Teixit (Bellvitge, ES); (7)Banc de Sang $i$ Teixit (Badalona, ES); (8)Banc de Sang i Teixit (Barcelona, ES)

Aim: To analyze variables that might influence the hematopoietic recovery after autologous peripheral blood progenitor cell transplantation (auto-PBPCT)

Patients and methods: A total of 398 patients, 252 men and 146 women, median age 54 years (range 4-76), with multiple myeloma (42\%), non-Hodgkin or Hodgkin lymphoma (40.5\%), acute leukemia/myelodisplastic syndrome (13.3\%), solid tumors $(2 \%)$ and other $(2.3 \%)$ underwent auto-PBPCT between January 2003 and August 2009. Patients were mobilized with G-CSF $(92 \%)$ or with chemotherapy and G-CSF $(8 \%)$. Factors potentially influencing the engraftment kinetics were examined using a Cox regression model. To compare continues variables the Mann-Whitney test was used. Results are expressed in median and range. Cellularity was measured before cryopreservation and colony forming cells after thawing.

Results: Total nuclear cells (NC) infused was $10 \times 10^{8} \mathrm{NC} / \mathrm{Kg}$ (1.4-74), mononuclear cells $7.5 \times 10^{8} / \mathrm{Kg}(0.8-32)$, CD34 + $3 \times 10^{6} / \mathrm{Kg}(1.1-56)$, granulo-monocye colony forming cells (GM) $2.2 \times 10^{5} / \mathrm{Kg}(0.0-14)$ and erythroid burst-forming and mix units (BFU-E-Mix) $8 \times 10^{5} / \mathrm{Kg}(0.0-57)$. Cloning efficiency (CE) per CD34+ seeded cell was $32 \%(0-97)$. Time to neutrophil recovery more than $0.5 \times 10^{\%} / \mathrm{L}$ and platelet count more than $20 \times 10^{9} / \mathrm{L}$ was $11(6-50)$ and 13 days (0-190), respectively. The most powerful predictors of neutrophil engraftment were the total $\mathrm{GM} / \mathrm{Kg}$ infused $(P=0.003)$ and $\mathrm{G}-\mathrm{CSF}$ administration post-infusion $(P=0.002)$ and of platelet engraftment, the total BFU-E-Mix/Kg infused $(P=0.01)$ and the infusion of more than $3 \times 10^{6} / \mathrm{Kg} \mathrm{CD} 34+$ cells $/ \mathrm{Kg}(P=0.02)$. In addition, bone marrow involvement at the time of mobilization and the diagnosis of acute leukemia had a negative impact for neutrophil $(P=0.01)$ and platelet recovery $(P=0.004)$, respectively. Additionally, when CE was inferior to $10 \%(n=31)$, median days to neutrophil and platelet recovery was 16 and 19 days, and when CE was equal or superior to $10 \%(n=365) 11$ and 13 , respectively, $(P<0.005)$. Variables not influencing hematological recovery in the multivariate analysis were age, sex, stage of disease, mobilization regimen, number of cycles of chemotherapy and type of conditioning regime.

Conclusions: Our data suggest that total GM and BFU-E-Mix infused, in addition to CD34 + cells, may be helpful in predicting the engraftment potential after auto-PBPCT and that cloning efficiency may be a good in vitro test for quality assessment of hematopoietic progenitor cells from cryopreserved apheresis.

\section{P969}

Contruction, expression and function of a CMV-specific chimeric immunoreceptor

F. Full (1), M. Lehner (1), V. Thonn (1), G. Goetz (1), B. Scholz (1), K.B. Kaufmann (1), M. Mach (1), H. Abken (2), W. Holter (1), A. Ensser (1)

(1)University Hospital (Erlangen, DE); (2)University Hospital (Cologne, DE)

Cytomegalovirus (CMV) infections in haematopoietic stem cell transplant (HSCT) patients are associated with substantial 
morbidity and mortality despite specific pharmacologic antiviral prophylaxis and therapy. Adoptive immunotherapies with virusspecific cytotoxic effector T cells have been successfully used to treat or prevent viral reactivations, this approach, however, is not feasible in the high risk constellation of a CMV-positive HSCT patient and a CMV-negative stem cell donor. We have engineered human effector $T$ cells directed to a viral protein that is expressed on the surface of lytically infected cells. A CMV glycoprotein gB specific chimeric immunoreceptor (cIR) was constructed and expressed in primary human T cells using lentiviral vectors or by electroporation of in vitro transcribed RNA. Such cIR-engineered T cells produced IFN-g and TNF in response to CMV infected fibroblasts, showed antigen specific degranulation and lysed gB expressing target cells. Redirected CMV-specific $T$ cells may open a new perspective for an HLA independent adoptive immunotherapy of CMV infections in high-risk transplant recipients.

\section{P970}

Peptide extension skews the HA-1 presentation towards activated dendritic cells but reduces the presentation efficiency

L. Hambach (1), Z. Aghai (1), N. Kröger (2), E. Goulmy (1)

(1)Leiden University Medical Center (Leiden, NL); (2)University Medical Center Hamburg-Eppendorf (Hamburg, DE)

Minor histocompatibility antigens (mHags) are the molecular targets of the graft-versus-tumor (GvT) effect and of graft versus-host disease (GvHD) after human leukocyte antigen (HLA)matched allogeneic stem cell transplantation (SCT). MHags are HLA-restricted polymorphic peptides expressed on normal and leukemia cells. The ubiquitously expressed mHags, e.g. $\mathrm{H}-\mathrm{Y}$, are the prime in situ targets of GvHD. Some mHags, e.g. mHag $\mathrm{HA}-1$, are only expressed by human normal and malignant hematopoeitic cells and by solid tumors. Therefore, vaccination with peptides derived from hematopoiesis-restricted mHags like HA-1 may boost the GvT effect with low risk of GvHD. However, the optimal approach of mHag peptide vaccination after allogeneic SCT is still unclear. Peptides exactly reflecting immunogeneic T-cell epitopes in HLA class I molecules ("short peptides", SP) can induce tolerance in some animal models potentially due to systemic antigen spreading. Peptides can spread in unbound form or directly bound to HLA molecules of circulating peripheral blood cells. Extension of the peptide length ("long peptides", LP) may optimize immune responses by focusing and prolonging antigen presentation on dendritic cells (DCs). Here, we compared the in vitro characteristics and T-cell stimulatory capacities of a 30-mer HA-1 LP with the SP. Activated DCs presented both the HA-1 LP and SP and expanded cytotoxic HA-1 specific T-cell lines. As hypothesized, HA-1 LP presentation-in contrast to SP presentation - was largely restricted to activated DCs and almost absent on other hematopoeitic cells. However, activated DCs presented the HA-1 LP 2-3 log levels less efficiently than the SP. Finally, the decays of HA-1 LP and SP presentation on DCs were comparable. We conclude that HA-1 LP and SP show different in vitro characteristics that may translate into different effects on HA-1 specific immune responses in vivo. Therefore, only comparative clinical studies after allogeneic SCT may reveal the optimal HA-1 vaccine.

\section{P971}

Therapeutic potential of gamma delta T-cells in controlling CMV and leukaemia after allogeneic stem cell transplantation

S. van Dorp, S. Kersting, F. Pietersma, S. Hol, S. Heijhuurs, V. Marcu-Malina, D. van Baarle, J. Kuball

UMC Utrecht (Utrecht, NL)

Introduction: Allogeneic stem cell transplantation (allo-SCT) is substantially hampered by GvHD, infections like CMV and relapse of disease. Gamma delta T cells (gdT cells) seem to be important in virus control but also in malignancy control by MHC independent recognition of antigens up regulated on stressed cells, however they do not mediate GvHD. In particular CMV infections are associated with an increased expansion of gdT cells, expressing the Vd1 chain. Therefore, we investigated frequency and function of gdT cells after allo-SCT in order to assess their therapeutic potential.

Methods: PBMCs at time points within 3 months after allo-SCT of 17 patients were sampled. CMV viral load was monitored by real-time PCR. Phenotype and frequency of gdT cells and alpha beta $T$ cells (abT cells) were analyzed by flow cytometry. Two patients with CMV reactivation were selected and gdT cells were isolated and expanded from time points before, during and after CMV reactivation. Two patients without CMV reactivation were used as controls. Frequency and clonality of $\mathrm{Vd} 1+, \mathrm{Vd} 2+$ and $\mathrm{Vd} 3+\mathrm{gdT}$ cells were measured with spectratyping. The reactivity against CMV AD169 infected fibroblasts, uninfected fibroblasts and primary AML blasts of $\mathrm{Vd} 2-$ and $\mathrm{Vd} 2+\mathrm{gdT}$ cells was tested using an IFN-g ELISPOT.

Results: We observed an increased polyclonal expansion of specifically Vd1 + gdT-cells after allo-SCT in patients during CMV reactivation and contraction after resolution as assessed by flow cytometry and spectratyping. Furthermore $\mathrm{Vd} 1+$ but not Vd2 + T-cells from these patients reacted against CMV AD169 infected fibroblasts, but not against uninfected fibroblasts. This suggests that $\mathrm{Vd} 1+\mathrm{gdT}$-cells have the potential to dampen CMV infection after allo-SCT. Moreover, following pp65-reactive abT cells in HLA-A2 + patients indicated that $\mathrm{Vd} 1+\mathrm{gdT}$ cells precede an abT-cell response. Finally, selectively $\mathrm{Vd} 2+$ gdT cells had the potential to recognize primary leukemic blasts, but only in the presence of pamidronate, a phosphoantigen stimulating agent.

Conclusion: GdT cells are present in patients after allo-SCT, have the potential to eradicate CMV infected fibroblasts and maybe the potential to spread an immune response to abT cells. Furthermore $\mathrm{Vd} 2+\mathrm{gdT}$ cells have the potential to recognize primary AML blasts. This strongly supports the idea to explore gdT cells as cell population for immune interventions after allo-SCT and the application of pamidronate post allo-SCT in patients suffering from AML.

\section{P972}

Umbilical cord blood transplantation: the co-infusion of cd34 + cells from a third party donor offers better overall survival compared to single cord blood transplantation M. Kwon, P. Balsalobre, D. Serrano, J. Gayoso, I. Buño, A. Perez Corral, J. Anguita, J.L. Diez-Martin

Gregorio Marañon G. U. Hospital (Madrid, ES)

Umbilical cord blood transplantation (CBT) with the co-infusion of CD34 + cells from a non-HLA-identical donor (dual SCT) has shown to reduce the period of posttransplant neutropenia and related early morbidity and mortality of single CBT. We describe the main clinical results of this strategy in our institution, and compared them with those of single CBT. Methods: Fourteen patients (pts) underwent dual SCT from March-04 to June-09, and 8 pts received a single CBT between September-06 and December-07. All patients had high-risk hematologic malignancies (Table 1).

Results: There were no significant differences between age, sex, HLA disparities, median of transplanted cells, and pre-SCT status between both groups. However, a higher proportion of patients in the single CBT group received more than 1 line of therapy before SCT. Engraftment was reached sooner in the dual SCT group ( $P=0.03$, Table 2). One dual SCT pt showed primary graft failure and received successfully a second dual SCT, and another showed CB failure but engraftment of the third party donor. One pt of the single CBT group showed graft failure rescued by a haploindentical donor. No GvHD $\geq$ grade III were present in any group. Relevant infectious complications were more frecuent in the single CB group, including mortality 
associated in 3 cases. OS and DFS for the dual group were $66 \%$ and $50 \%$ respectively (Figures)with a median follow-up of 39 months (5-60). The cumulative relapse incidence was $50 \%$, and all the relapses occured within the first year posttransplantation (one of the relapsed pts underwent successfully an unrelated SCT). OS for the single CBT group was $12 \%$, with a median time to death of 7 months (1-23 months). Remarkably, NRM was $7 \%$ for the dual group and $75 \%$ for the single CB group.

Conclusions: In our experience, CBT with co-infusion of CD34 + cells from a third party donor showed to be a safe strategy for patients with no available donor. Remarkably, NRM mortality rate was very low in this group, especially compared to the single CB group. More cases and a longer follow-up are needed to assess the causes of these differences, including worse preSCT status of the single cord group and/or shorter period of neutropenia in the dual group.
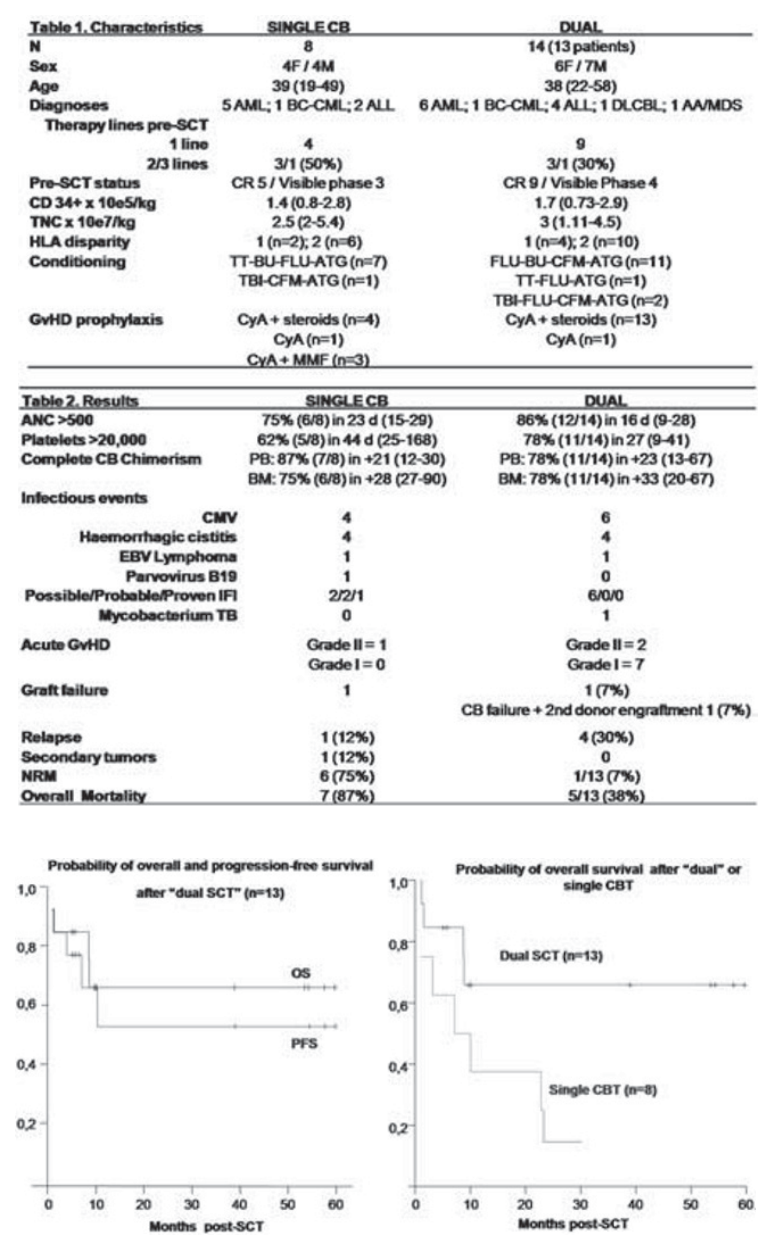

P973

High-dose chemotherapy followed by autologous stem cell transplantation in treatment-refractory severe acquired paraproteinemic neuropathy - a single-centre experience

H.D. Hummel (1), J.C. Rath (1), H. Wiendl (1), W. Hetzel (2), R.C. Bargou (1), K.V. Toyka (1), C. Sommer (1), H. Einsele (1), M.S. Topp (1)

(1)Universtity Hospital (Wurzburg, DE); (2)Arzt für Neurologie und Psychatrie (Wurzburg, DE)

Background: Paraproteinemic neuropathies (PN) represent a heterogenous group of peripheral nerve disorders. Current treatment options include immune-modulating approaches with intravenous immunoglobulin (IVIg), plasmapheresis, immunosuppression, e.g. with corticosteroids or cyclophosphamide. A subset of patients remains refractory to these treatment modalities. We describe four patients with progessive PN despite various treatment attempts who underwent an intensified immunosuppressive regimen with high-dose melphalan (HD-Mel) therapy followed by autologous stem cell transplantation (ASCT).

Methods: As induction therapy one cycle of cyclophosphamid $\left(1-3 \mathrm{~g} / \mathrm{m}^{2} \pm\right.$ etoposid $200 \mathrm{mg} / \mathrm{m}^{2}$ ) was given and stem cells were collected after several days of GCSF stimulation. High-dose melphalan (HD-Mel) (between $150 \mathrm{mg}$ and $200 \mathrm{mg}$ abs.) was administered on day 1 and 2 with consecutive ASCT on day 4 . One patient received a second course of HD-Mel and ASCT. Responses were assessed including clinical and electrophysiological examination.

Results: All patients suffered from severe disabling neuropathies associated with monoclonal gammopathy of undetermined significance (MGUS) and failed to respond to various immunomodulatory or immunosuppressive treatments. Remarkably, pretreatment in two of the four patients even included immunoselective B-cell depleting therapy with anti-CD20 monoclonal antibodies (Rituximab). All patients with a follow-up of up to 46 months showed sustained clinical and electrophysiological recovery after ASCT. 3 patients with previous tetraparesis and wheel-chair dependency and 1 patient with severely limiting distal prevailing hypesthesia are now able to work again, in part in their former jobs. All patients had uncomplicated peri-transplant periods.

Conclusion: We conclude that high-dose therapy with melphalan and ASCT, under strict patient selection and monitoring, may be an experimental treatment option in very severe forms of treatment-refractory progressive PN. A prospective multicenter trial is warranted to substantiate these observations.

P974

Induction of prostate-specific T-cells and graft-versus-tumour effect in patients with advanced prostate cancer after allogeneic stem cell transplantation J. Mattsson (1), J. Gertow (1), M. Okas (1), S. Berglund (1), L. Henningsohn (2), O. Gustavsson (2), M. Uhlin (1)

(1)Centre for allogeneic stem cell transplantation (Stockholm, SE); (2)Dept of Urology (Stockholm, SE)

Therapies for localized prostate cancer include curative surgery and radiotherapy while treatment for metastatic disease is palliative and based on depriving the tumor cells of their androgen stimulation that reduces tumor growth rate. Therefore, we started to investigate the potential of allogeneic SCT as a treatment for non-curable prostate cancer.

Two patients underwent allogeneic SCT with PBSC from HLAidentical sisters after a non-myeloablative conditioning regimen as treatment for metastatic prostate adenocarcinoma. Frequencies of prostate-specific T cells in the peripheral blood of the patients, sibling donors and a group of control individuals were determined by flow cytometry using tetrameric and pentameric HLA A2 complexes containing peptides derived from the prostate specific antigen (PSA). Cytotoxic activity of PSApeptide-specific T-cells against peptide-pulsed target cells was analyzed ex vivo by $51 \mathrm{Cr}$-release assays.

Stable clinical and laboratory remission lasting for more than 4 years was observed after SCT for the first transplanted patient. The patient is now 6.5 years after SCT but now slowly increasing in PSA indiacting tumor progression. The PSA level is today $12 \mathrm{ng} / \mathrm{mL}$. The second patient has decreased in PSA from $257 \mathrm{ng} / \mathrm{mL}$ before SCT to $70 \mathrm{ng} / \mathrm{mL}$ and without clinical signs of progression one year after SCT. Both patients are doing clinically excellent and have a good quality of life. In the second patient a dramatic increase was detected in PSA levels during development of GVHD. After treatment of GVHD the PSA levels decreased continuously. We believe that the sudden increase of PSA was due to an antitumor attack of prostatespecific $T$ cells indicating a graft-versus-tumor effect. Using 
HLA containing pentamers with PSA-derived peptides we could detect prostate-specific CD8 $+\mathrm{T}$ cells in both patients in high frequencies over several months. Furthermore, higher frequencies of PSA-specific T-cells were revealed in the peripheral blood of the patients and female controls as compared with male healthy controls.

Our data suggest that allogeneic SCT led to the generation of a T cell mediated prostate-specific immunity in both patients. The in vitro and ex vivo immunological monitoring performed indicate an adjuvant anti-tumor effect of PSA-specific T cells. This report presents a novel treatment approach involving allogeneic SCT in prostate cancer patients who do not respond to chemotherapy and/or cannot undergo prostectomy.

\section{Stem cell donor}

\section{P975}

Haematopoietic stem cell transplantation: HLA analysis of non-transplanted versus transplanted patients

P.A Gourraud, M.L. Balere, E. Marry, F. Garnier

Registre France Greffe de Moelle, Agence de la Biomédecine (Paris, FR)

Allogeneic haematopoietic stem cell transplantation (HSCT) can cure patients with malignant and non malignant haematological diseases. Only about $30 \%$ of them have a suitable family donor. Therefore, a full HLA-matched unrelated donor (UD) should be found out through national and international registries for those patients who lack a suitable donor. Various levels of HLA polymorphism could argue for further difficulty for those recipients to find a compatible donor. A comparative retrospective study of HLA characteristics of the patients who did and did not find a full- HLA matched donor or CBU was performed.

Method: Between 2000 and 2007, 772 recipients, were "not transplanted because of the absence of an HLA-compatible donor worldwide" (status double-checked with transplantation centres). An extensive analysis of their HLA phenotypes was performed, by comparing them to 3,123 patients transplanted with full-HLA matched UD over the same time frame. Estimated haplotype frequencies for HLA-A -B -C -DRB1 -DQB1 from both the French UD (Low Res.) Registry, and the 4 various ethnic backgrounds haplotype frequencies (High Res., (data available at http://bioinformatics.nmdp.org website) were used.

A "qualitative" analysis consisted of checking that each HLA recipient phenotype was found among all possible pairwise combination of 2 haplotypes of the different sets of haplotypes. Various "quantitative" analyses calculated the likelihood associated to each recipient phenotype for a given set of HLA genes, in a given population, at low versus high resolution typing.

Results: 1- HLA-C was shown to be the major contributor for HLA rareness in non transplanted patients. The most significant difference found between transplanted and non transplanted patients correlates with the introduction of HLA-C in the likelihood model (median: 8.6e-6 vs. 1.1e-6 $P<0.0001$ ).

2-Ethnic background/ancestry based analysis, also provide a framework for assessment of patient's chance to find a donor. Frequent HLA phenotypes of transplanted patients are consistently found among these different backgrounds $(P<0.001)$. Discussion: The HLA rareness of non-transplanted patients can be categorised in several groups: Type of allele resolution, extended loci typing such as HLA-C and non causasians haplotypes are the main sources of rareness. The use of haplotype frequencies to identify rare HLA phenotypes, underlines the interest of haplotype-based computations for a prospective analysis as implemented by Haplologic and Optimatch.
P976

Immediate and long-term physical side effects of donating bone marrow in early childhood

S.M. van Walraven (1), L. Straathof (1), A. Brand (2), G.E. Switzer (3), E.T. Korthof (1), L.M. Ball (1)

(1)Leiden University Medical Centre (Leiden, NL); (2)Sanquin Blookbank, Leiden University Medical Centre (Leiden, NL); (3)University of Pittsburgh (Pittsburgh, US)

Bone marrow (BM) donation in early childhood is uncommon. Late effects of childhood donation have not been investigated. We aimed to study the immediate and long-term side effects (SE) in pediatric donors. Participants donated BM between 1968 and 2002 in the pediatric transplant unit in Leiden. Inclusion criteria were: younger than 13 yrs at donation, minimum follow up of 5 yrs and at least 12 yrs at study entry. Self reporting questionnaires were used. Surviving recipients acted as controls $(n=43)$. Of 204 eligible donors, 145 were invited of whom $54 \%(n=79)$ responded (Figure I). Additionally, 196 case files were used to record immediate post donation SE.

At donation 13 children had previous medical conditions (congenital heart disease $n=3$; prematurity $n=6$; hypothyroidism $n=1$; neurological $n=2$; hepatomegaly $n=1$ ). These were not considered contraindications to donation.

An average $18 \mathrm{mls} B M / \mathrm{kg} / \mathrm{donor}$ weight was harvested (range 6-47 ml) with significant hemaglobin loss (mean 1,58 mmols/ $\mathrm{L} ; \mathrm{SD}=0.46 ; P=0.000$ ). Forty-five percent of the children were exposed to donor blood transfusion.

From self reports, $91 \%$ considered themselves healthy. Twenty one reported one or more health issues since donation (cardiovascular $n=3$; respiratory $n=14$; auto immune $n=2$; epilepsy $n=2$; anemia $n=2$; psychological $n=2$; fatigue $n=5$; persistent back pain $n=2$ ). The mean total score of the General Health Questionnaire did not differ significantly from the healthy adult Dutch population. With the Short Form 36 Health Survey, all donors showed significantly higher raw scores in the subdomains than the healthy Dutch population (Figure 2).

Body Mass Index (BMI) was classified by age and sex. In donors where the BMI was available pre-donation the underweight index has doubled. Only $3 \%$ of donors were morbidly obese in contrast to the general Dutch population.

Participants $<18$ with a living recipient scored high on the health related quality of life "physical health" domain $(P=0.025)$. There was no correlation between donor and recipient scores.

Our results show that young children both in the immediate and long term physically tolerate BM donation. In parallel to this study psychosocial evaluation is ongoing. In contrast to adults, nearly half receive blood products following donation, directly related to the amount of marrow harvested. Presently no specific guidelines in relation to donation in young children have been established. Our results are important in the development of such recommendations.

Figure 1: Flow diagram of donor inclusion

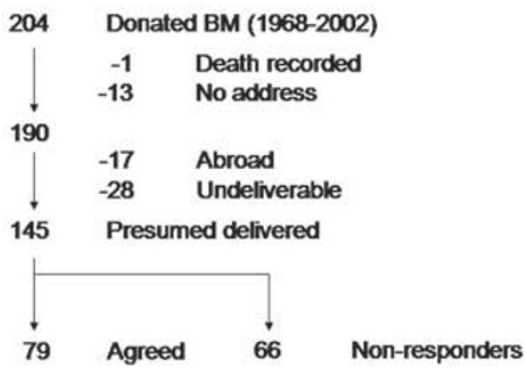




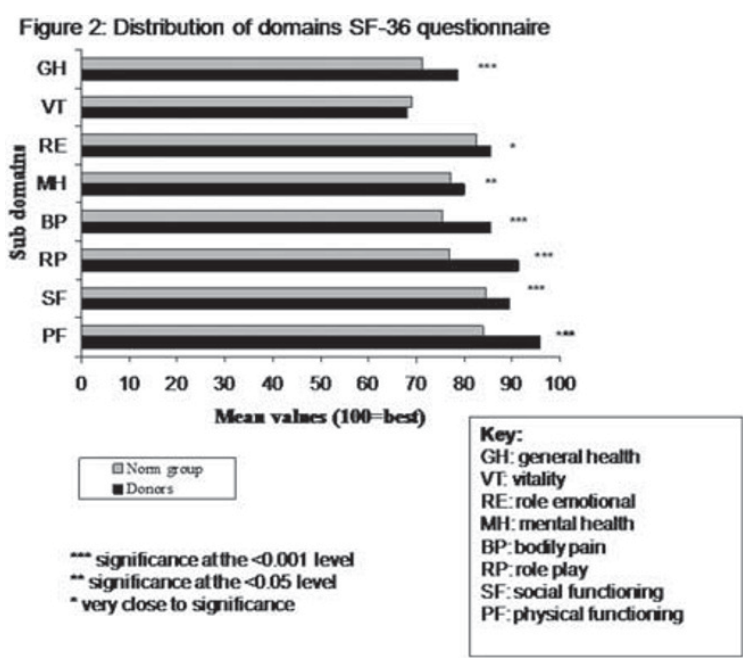

P977

Educational achievement and employment choices in adults who have donated marrow in early childhood S.M. van Walraven (1), L. Straathof (1), C.M.J. VrijmoetWiersma (1), A. Brand (2), L.M. Ball (1)

(1)Leiden University Medical Centre (Leiden, NL); (2)Sanquin Blookbank, Leiden University Medical Centre (Leiden, NL)

The psychosocial impact of donating bone marrow (BM) in early childhood to save a sibling's life could influence career choice and further education. We studied the schooling and employment of adults who had donated BM as a child. Inclusion criteria: less than 13 yrs at donation, minimum follow up of $5 \mathrm{yrs}$ and at least $12 \mathrm{yrs}$ at study entry. All were admitted from 1968 to 2002 in our pediatric department. Self reported Course of Life questionnaires were used. Surviving recipients acted as controls $(n=44)$.

Of 204 potential donors, 145 available donors were invited, of whom $54 \%$ responded. Donor and recipient gender was $44 \%$ M: 56\% F. Donors mean age was 23 yrs (range 12-47) and recipients mean 22 yrs (range 10-38). Age at donation/BMT was the same for donors as recipients (mean $6.9 \mathrm{yrs}$ range $0.6-12$ and $0.6-15.8$ resp.). Results of donor and recipient education were compared to Dutch population norms (Statistics Netherlands). Data was available in 77 donors who were/had attended secondary school. These were: 1st stage secondary (higher academic); 2nd stage secondary (academic) and vocational training. Over $50 \%$ of donors were/had attended higher level secondary education $(n=52)$, which exceeds the Dutch norm. Of 44 recipients $38 \%$ achieved this level, more consistent with the Dutch norm.

Most donors (95\%) were employed full time with $30 \%$ $(n=19 / 59)$ involved in medicine, teaching, child care or science, compared to $17 \%$ of recipients. More donors (17\%) were involved in physically demanding work, compared to only $2 \%$ of the recipients.

Educational achievement was high in our donor group. The results may be biased by the number of non-responders, and not reflect the group as a whole. However the differences from Dutch norms are so great that we can at least conclude children acting as donors were not disadvantaged in the Dutch system. Many child donors later follow a "caring" profession compared to recipient siblings. This may reflect their involvement in the donation process. Recipients are less likely to be involved in physical work, which may be health related. These results need to be confirmed in prospective studies but are encouraging as to the long term social effects of childhood BM donation.
P978

HLA-identical sibling donors search and identification:

3 years of experience of an ISO 9001-certified centre

B. Calmels (1), B. Puddu (1), B. Mansueto (1), C. Picard (2),

C. Lemarié (1), L. Castagna (1), S. Fürst (1), C. Faucher (1),

D. Blaise (1), P. Ladaique (1), C. Chabannon (1)

(1)Institut Paoli-Calmettes (Marseille, FR); (2)EFS AM (Marseille, FR)

Identification of an HLA-identical sibling represents the first step towards allogeneic hematopoietic stem cell transplantation (HSCT) process. A few years ago, we split responsibilities for donor search and patient care, as it preserves donor independence. The search is thus carried out by a transplant coordinator center (TCC) at the cell therapy facility, and starts with construction of a family tree: unless obvious exclusion criterions exist, siblings contact the TCC in order to organize blood drawn for HLA typing. Disposables and instructions are sent to the laboratory of their choice and samples are then shipped to the same histocompatibility laboratory that performs HLA typing for our transplantation program. Conclusion of the family study is communicated to the patient as a basic information (you have or not a sibling donor). The whole process is monitored by the TCC using a database accessible by the transplant team.

Between 2006 and 2008, 552 family trees were received, and 511 family studies were initiated. 1460 siblings did contact the TCC, and $79 \%$ were considered suitable for HLA typing. $12 \%$ of siblings were not typed because of the patient disease evolution, $7 \%$ because of a medical contraindication to donation and $2 \%$ declined donation. An HLA-identical sibling was identified for 216 of the 552 patients (39\%). 112 of the 216 identified siblings underwent consecutive stem cell collection, and 104 were not collected, mainly for reasons associated with the patient (85\%): unfavourable evolution of the disease $(37 \%)$, loss of HSCT indication (28\%), patient's refusal of HSCT (13\%), or contraindication for HSCT (7\%); only 16/216 HLA-identical siblings were not collected because of reasons associated with the donor, either contraindication (13) or late refusal of donation (3). Ultimately, from the 552 patient's families explored, $20 \%$ (112) continued to HSCT from a sibling. The median time necessary to identify a donor was 40 days, and identification occurred 120 days (median) before stem cell collection.

We conclude that the process that leads to the identification of an HLA-compatible sibling can be conducted within a delay that is compatible with the organization of an HSCT, provided that the process is initiated early enough. Anticipation is especially important for those patients for whom no HLA-compatible sibling will be identified, as a second search may then be initiated in order to identify an unrelated donor or cord blood unit(s).

\section{P979}

Role of HLA-DPB1 mismatch on the outcome of unrelated donor stem cell transplantation

A. Manaka, F. Panitsas, I. Baltadakis, D. Karakasis, I. Kakkas, T. Athanassiades, F. Michelis, J. Apostolidis, N. Harhalakis, C. Papasteriades, E. Nikiforakis

Evangelismos Hospital (Athens, GR)

The main determinants of the outcome of unrelated donor stem cell transplantation (URD-SCT) are disease phase and HLA compatibility. Donor selection is currently based on the degree of matching at the HLA-A, -B, -C, -DRB1, and -DQB1 loci. Few studies have addressed the role of HLA-DPB1 implicating allelic or alloreactive T cell epitope (TCE) mismatch in acute graft-versus-host disease (GvHD), relapse, or survival. We retrospectively analyzed the influence of DPB1 mismatch on the outcome of URD-SCT performed in our center. From 01/1994 to $04 / 2009,108$ patients underwent URD-SCT for hematologic malignancies. In 74 cases, HLA-DPB1 alleles were retrospectively typed in donor and recipient. The median age of patients was 35 years (range, 15-62). The disease phase at transplant was early in 23 , intermediate in 16 , and advanced 
in 35 patients. Forty-six patients received myeloablative and 28 reduced-intensity conditioning. The graft source was mainly peripheral blood $(n=65)$. In vivo T-cell depletion (ATG or CAMPATH-1H) was undertaken in only 16 cases. Donor selection was based on allele compatibility at HLA-A, -B, -C, -DRB1, and -DQB1, without considering -DPB1. The outcomes were analyzed in relation to transplant-related mortality (TRM), acute and chronic GvHD, relapse, disease-free survival (DFS), and overall survival (OS). Risk factors included in the multivariate analysis were age and CMV-status of donor and recipient, disease phase, type of conditioning regimen, degree of compatibility at HLA-A, -B, -C, -DRB1 and/or -DQB1 (i.e. out of 8 or 10 alleles), as well as mismatch at DPB1. Donors were matched at $10 / 10$ and $8 / 8$ alleles in 27 and 34 cases, respectively. DPB1 compatibility was noted only in 7 transplants. In 58 cases, DPB1 mismatch in the GvH direction was found. OS was associated with disease phase and compatibility (HR: 2.37 and 0.47 , $P=0.047$ and 0.06 , respectively). Allelic DPB1 mismatch in the $\mathrm{GvH}$ direction emerged as an independent factor for relapse (HR: 0.23, $P=0.006$ ) and DFS (HR: 0.31, $P=0.01$ ). A favorable effect was also observed with non-permissive TCE mismatch on relapse and DFS (HR: 0.28 and $0.47, P=0.02$ and 0.049 , respectively). The incidence of acute or chronic GvHD, TRM, and OS were not affected by DPB1 mismatch. HLA-DPB1 incompatibility is common in URD-SCT and seems to enhance the graft-versus-tumor (GvT) effect. DPB1 mismatch in the GvH direction may be relevant in donor selection for patients with high-risk hematologic malignancies.

\section{P980}

Adverse events during haematopoietic progenitor cells collection with aphaeresis in healthy donors: a retrospective analysis

M. Martino, G. Console, R. Fedele, G. Irrera, E. Massara, T. Moscato, G. Messina, P. lacopino

U.O. Ematologia con Trapianto (Reggio Calabria, IT)

Apheresis is a special collection procedure in which peripheral hematopoietic progenitor cells (HPC) are separated from the other parts of the blood and returned to the patient/donor. Collection of HPC by apheresis machines takes approximately three-four hours and it' s generally regarded as a safe procedure. However, data about adverse events in HPC harvesting are scarce. The data of HPC collections in a period of 14 years in healthy donors (HDs), mobilized using G-CSF, were reviewed. A total of $211 \mathrm{HDs}$ (92 female, 119 male, median age 43.8 years, range 15-70) underwent 422 HPC harvests. We used the Fresenius, Bad Homburg, Germany, AS 204 and COM.TEC cell separators for continuous flow centrifugation. HDs-related side effects was observed in the $23.7 \%$ of procedures. The most frequents side effects were mild $(17.1 \%)$, especially paresthesias due to anticoagulant-citrate toxicity $(15.2 \%)$. Paresthesias were treated by oral or intravenous calcium supplementation. Problems with venous access have been rare $(1.2 \%)$, resulting in blood flow alarms and blockades in the return line and 10 procedures had to be discontinued for this reason. $14 \mathrm{HDs}$ experienced gastrointestinal troubles (vomiting/nausea). Mild bleeding at the site of venipuncture was observed in $2 \mathrm{HDs}$. Severe side-effects were described in 15 procedures $(3.3 \%)$. Severe hypotension with faintness has been reported in 8 donors; a state of clinical hypocalcemia was observed in $6 \mathrm{HDs}$; 1 apheresis had to be discontinued because the HD presented chest pain and mild dyspnea and the computerised tomography showed a pulmonary microembolism. Following the development of side effects, 22 HDs required hospitalization (10.4\%).

In conclusion, although HPC collection is associated with a low incidence of severe side effects, requires a considerable commitment of donors' time and energy and ability to comply and adhere to the collection. Apheresis team caring for HDs receiving HPC collection offer teaching and provide ongoing survey of potential risks and complications.
P981

A comparison of once-daily versus twice-daily recombinant human granulocyte colony-stimulating factor (lenograstim) for haemopoietic progenitor cell mobilization in healthy donors for allogeneic transplantation

M. Martino, R. Fedele, G. Console, G. Messina, A. Dattola, C. Garreffa, E. Massara, T. Moscato, G. Irrera, P. lacopino U.O. Ematologia con Trapianto (Reggio Calabria, IT)

The optimal schedule of administration for rhG-CSF in hemopoietic progenitor cell (HPC) donors are still being defined. Much of the attention has been devoted to the rhG-CSF dose. Although most centres use once-daily rhG-CSF administration, some investigators have recommended a twice-daily schedule. To evaluate the schedule of granulocyte colony-stimulating factor (G-CSF) (Lenograstim) for HPC collection, we conducted a retrospective comparison in healthy donors mobilized with one subcutaneous daily injection of Lenograstim (Group A, $10 \mathrm{microng} / \mathrm{kg}$; n. 28) compared with twice injections daily of Lenograstim (Group B, 5 microng/kg every 12 h; n. 85). The two groups were well balanced for age, body weight and sex. G-CSF application was performed on an out-patient basis and leukapheresis was started in all donors on day 5 . The side effects of G-CSF were significantly higher in the group with one daily injection (mild to moderate bone pain, $P=0.004$; mild fatigue, $P=0.000$; mild splenomegaly, $P=0.015$ ). The peak number of CD34 + cells $/ \mathrm{ml}$ blood observed on day 4 and 5 was not significantly different in the two groups (mean values: Group A 55 and 89 vs. Group B 52 and 84, respectively, $P=$ ns). Leukocyte kinetics were substantially equivalent. In particular, leukocyte peak values were $41 \times 10^{9} / \mathrm{L}$ and $45 \times 10^{9} / \mathrm{L}$ in the Group $A$ and in the Group B, respectively ( $P=\mathrm{ns})$. There was no statistically significant difference in the apheresis yield of CD34 + HPC $\left(\times 10^{6}\right)$ collected on day 5 (464 vs. $434, P=0.6$ ) and per liters of blood processed. Our data suggest that both Lenograstim administration schedules are similarly effective for the mobilization and collection of CD34 + HPC in normal donors, at least when daily leukapheresis begins on day 5 of Lenograstim mobilization. In conclusion, we were unable to show any clinical significant advantage of twice-daily lenograstim administration in terms of HPC mobilization. Once-daily schedule is associated with higher short-terms side effects, but remains presumably simpler and more convenient for the donors.

\section{P982}

A prospective study to identify clinically factors that could influence the effectiveness of cd34+cell mobilization in healthy donors after administratration of lenograstim M. Martino, A. Dattola, C. Garreffa, G. Console, G. Irrera, G. Messina, R. Fedele, P. lacopino

U.O. Ematologia con Trapianto (Reggio Calabria, IT)

We conduct a prospective study to identify clinically significant factors that could influence the effectiveness of CD34 + cell mobilization and collection in healthy donors after administration of lenograstim. A total of 35 consecutive first-time normal donors received lenograstim before leukapheresis for collection of HPCA for allogeneic transplantation. Donors received G-CSF subcutaneously at a median (range) dose of $10(8-13) \mu \mathrm{g} / \mathrm{kg}$. HPC collections were started on day 5 , i.e., after four days of lenograstim in every donor. Five different determinations of CD34+ cells were done in each donor: First, baseline CD34 + cell count (before G-CSF administration); second, third and fourth, enumeration of CD34 + cells in peripheral blood on day 3, 4 and on the morning of collection (after G-CSF); finally the number of CD34 + cells in the apheresis bag (CD34 + cells collected). The total number of $\mathrm{CD} 34+$ cells $/ \mu \mathrm{L}$ in peripheral blood on the first day of apheresis (after G-CSF administration) was used to evaluate the effectiveness of mobilization. This variable was examined separately by linear regression analysis against independent variables (sex, age, weight, dose of G-CSF, white cell count, hemoglobin, platelet 
count, and CD34 + cell count). In a second step, logistic regression analysis was done to determine the variables influencing the number of CD34 + cells collected $/ \mathrm{kg}$ of donor body weight $(<5 \mathrm{vs}$. $>5 \times 10^{6} \mathrm{CD} 34+$ cells $/ \mathrm{kg}$ ). The independent variables analyzed in this step were the same as those in the previous one with the addition of the volume of blood processed (normal vs. Large Volume Leukapheresis). As concerns mobilization of CD34+ cells, the median number of CD34 + cells in peripheral blood on the day of the apheresis was $74 \mu \mathrm{L}$ (range, 9-240). By univariate analysis, no variables correlated with the number of CD34+ cells in the peripheral blood the day of apheresis. The median number of CD34+ cells collected in the whole series was $5.6 \times 10^{6} / \mathrm{kg}$ (range, $0.8-14$ ) and a CD34 + cell count $>5 \times 10^{6} / \mathrm{kg}$ was obtained in 23 cases. The donor's weight $(P=0.025)$, volemia $(P=0.023)$ and number of $\mathrm{CD} 34+$ on day $4(P=0.016)$ were the only variable that significantly correlated with the number of CD34 + cells/ $\mathrm{kg}$ collected $\left(>5 \times 10^{6} / \mathrm{kg}\right)$. Multivariate analysis didn't not show any correlations. Our study confirms that some donors are poor responders to G-CSF, but we didn't find significant predictive clinical factors for the apheresis yield of CD34 + cells.

\section{P983}

The potential application of the allogeneic transplant according to a widespread donor search policy: an intention-to-treat analysis from the Rome Transplant Network

A. Picardi (1), P. De Fabritiis (2), T. Dentamaro (2), G. De Rossi (3), M. Caniglia (3), M.C. Petti (4), A. Mengarelli (4), L. Annino (5), A. Chierichini (5), B. Monarca (6), A. Ferrari (6), G. Avvisati (7), M.C. Tirindelli (7), I. Mangione (1), R. Pinto (3), E. Montefusco (6), O. Annibali (7), L. Cupelli (2), L. Cudillo (1), R. Cerretti (1), G. De Angelis (1), F. Giannotti (1), A. Di Veroli (1), I. Provenzano (1), M. Mirabile (1), W. Arcese (1)

(1)Tor Vergata University (Rome, IT); (2)S. Eugenio Hospital (Rome, IT); (3)Bambin Gesù Pediatric Hospital (Rome, IT); (4)Regina Elena Hospital (Rome, IT); (5)S. Giovanni Hospital (Rome, IT); (6)S. Andrea University Hospital (Rome, IT); (7)Campus Biomedico University (Rome, IT)

Background: To date, all retrospective studies report similar results in terms of survival for patients lacking an HLA identical sibling and undergoing to an allogeneic transplant from alternative HSC sources: Matched Unrelated Donor (MUD), Cord Blood (CB) or Haploidentical Related Donor (HRD). The policy of the Rome Transplant Network (RTN), a metropolitan network of transplant Centers, is the identification of a suitable donor in order to perform transplant within $<3$ months.

Material and methods: The criteria for MUD selection are: a 8/8 HLA loci matching tested at low resolution for class I and at high resolution (HR) for class II HLA; one difference of C Ag is accepted in case of both class I and II identity defined at HR. Criteria for $\mathrm{CB}$ selection are based on cell dose $\left(\mathrm{TNC}>2.5 \times 10^{7} /\right.$ $\mathrm{kg}$ and CD34+>1 $\times 10^{5} / \mathrm{kg}$ ) and on a compatibility $\geq 4 / 6 \mathrm{HLA}$ loci. From April 2006, the haplodentical option was also simultaneously considered for all candidates, so all closer family members have been tested for HLA. Here, we report the results of a transplant policy based on the intention to treat (ITT) for patients registered in the RTN database.

Results: From April 2006 to September 2009, 236 patients have been candidate to receive an allogeneic HSCT for hematological disease. Eighty-five out of $236(36 \%)$ underwent HSCT from HLA identical sibling, while a search process for an alternative donor was activated for 140 patients. Of these 140 patients, 40 were not transplanted: 23 died in most cases because of early disease progression, 5 withdrew the consent and in 5 cases, despite an alternative donor could be identified within 3 months, the search process was temporary discontinued due to clinical causes. A suitable alternative donor was not found in only 7 (5\%) cases. To date, $100 / 140(71 \%)$ evaluable patients lacking an HLA identical sibling have been transplanted ( $n=85: 27$ MUD; 34 CBU; 24 HRD) or are willing to proceed towards the transplant $(n=15)$. In summary, for all 236 candidates to an allogeneic transplant the eligibility was confirmed for $225(95 \%)$, a suitable donor was identified for $218(92 \%)$ of all patients and an allogeneic transplant could be performed for $185 / 225(82 \%)$ of those eligible.

Conclusions: From this ITT analysis, we can conclude that, by adopting the RTN policy of widespread donor search and multiple transplant options, the allogeneic transplant can be offered as potential therapeutic procedure to a large majority of patients.

\section{P984}

Pegfilgrastim for the mobilization of allogeneic peripheral blood stem cells in related donors

V. Vucinic, R. Stiegler, K. Bartsch, C. Kliem, G.-N. Franke, S. Leiblein, D. Niederwieser, N. Basara

University of Leipzig (Leipzig, DE)

Introduction: The standard procedure for obtaining peripheral blood stem cells (PBSC) is mobilization with G-CSF given s.c. BID for $4-5$ days. Pegfilgrastim is a covalently bound conjugate of G-CSF and mono-methoxypolyethylene glycol, making it a larger molecule of approximately $39 \mathrm{kDa}$ with a longer half-life due to decreased plasma clearance. In the current study we investigated the administration of a single-dose Pegfilgrastim for mobilization of CD34 + cells for stem cell transplantation from healthy donors.

Material and methods: From July 2006 to August 2009, 28 related healthy donors ( $50 \%$ male) were treated with a single dose of 12 $\mathrm{mg}$ Pegfilgrastim for the mobilization of PBSC. The harvests were performed as large-volume, continuous-flow collections using a Cobe Spectra blood cell separator on day 4 and if necessary on day 5 after pegfilgrastim. In case of inadequate CD34 + counts (less than $4 \times 10^{6} / \mathrm{kg}$ recipient body weight on day 5 ), stimulation was continued with filgrastim. In addition, the serum level of filgrastim was determined twice daily in 15 of the 28 donors.

Results: Sufficient PBSC were harvested from all 28 donors. In $22(79 \%)$ of them, a single apheresis was required to reach the target of $4 \times 10^{6} / \mathrm{kg}$ recipient body weight and two of the donors required additional treatment with filgrastim. The maximal concentration of circulating CD34 + cells was achieved on day +4 with a median of 67,5 (range 24.6-136.6) CD34+/ $\mu$ l followed by a decrease on day +5 to 52,1 (range 24.6-136.6) CD34 +/ $/$ l. The median yield was 5,9 (range $3-14.5) \times 10^{6} \mathrm{CD} 34+/ \mathrm{kg}$ recipient body weight and 3.25 (range 1.4-6.2) $\times 10^{8} \mathrm{CD} 3+/ \mathrm{kg}$ recipient body weight. Serum pegfilgrastim levels peaked on day 2 after administration with a median of 226 (range 35 to 1123 ) $\mathrm{ng} / \mathrm{ml}$ preceding the increase of CD34 + cells in the peripheral blood. The main adverse events were WHO grade 1 including headaches, bone pain and transient elevations of alkaline phosphatase and lactate dehydrogenase.

Conclusion: PBSC mobilization with a single dose of pegfilgrastim is feasible in healthy donors and represents an interesting alternative to G-CSF. The graft composition was comparable to that resulting from a conventional regimen using short term GCSF stimulation. Further studies will include a long term follow up of healthy donors treated with pegfilgrastim.

\section{P985}

Outcomes from medical assessment of asymptomatic sibling donors

K. Hill, C.A. Hurlock, J. Newman, N. McKeag, D.S. Richardson, K.H. Orchard

Southampton University Hospital (Southampton, UK)

Introduction: The medical review of sibling donors is an important component of an allogeneic transplant. We report the outcomes for the medical reviews of 106 sibling donors seen in the Wessex Blood and Marrow Transplantation unit in the period from 2002-2009.

Results:

106 donors underwent a detailed assessment by a transplant physician for their suitability for stem cell donation. Age range 13-64 years (median 46 years; mean 42 years). 
$3 / 106(3 \%)$ had a heart murmur on examination; on echocardiography 2 were normal and 1 was confirmed as having moderate mixed aortic valve disease with a bicuspid aortic valve.

$5 / 106(5 \%)$ had an elevated blood pressure; 3 requiring initiation of therapy.

4/106 (4\%) were found to have microscopic haematuria; 3 had a positive urine culture and 1 with a negative urine culture had no abnormality on urological review.

$37 / 106(35 \%)$ had abnormal blood results:

- 9/106 (9\%) showed mild elevations in gamma-glutamyl transferase; improved with alcohol abstention

-9/106 (9\%) pre-menopausal women were iron deficient; 4 were mildly anaemic

$-4 / 106(4 \%)$ showed isolated elevated bilirubin levels consistent with Gilbert's syndrome

$-3 / 106(3 \%)$ showed a raised haematocrit secondary to lifestyle factors

- 3/106 (3\%) were diagnosed with a new malignancy; 2 myelodysplasia, 1 monoclonal gammopathy

$-2 / 106(2 \%)$ were hypothyroid; 1 requiring therapy

- 7/106 (8\%) showed either a raised APTR (no evidence of lupus anticoagulant/clotting factor deficiency), a raised prostate specific antigen (normal prostatic biopsy), a raised ferritin (haemochromatosis carrier on testing), low B12, alpha-1antitrypsin deficiency, absolute Immunoglobulin A deficiency or both raised triglycerides and a fatty liver

$42 / 106(40 \%)$ had a chest x-ray performed; all were normal

$56 / 106(53 \%)$ had an electrocardiograph performed of which 7 were abnormal (5 left ventricular hypertrophy, 1 each of either left or right bundle branch block).

Conclusions: A variety of asymptomatic medical conditions were diagnosed during assessment for stem cell donation. $17 / 106(16 \%)$ had further investigation after the initial assessment and 11/106 (10\%) required treatment and/or on-going follow-up for their newly identified condition. Apart from the recognised benefits of being a sibling stem cell donor, in a small but significant number of donors, a major medical condition may be diagnosed and treated with a subsequent potential improvement in the donor's lifespan.

\section{P986}

Factors predicting peripheral blood progenitor cell collectionc from paediatric healthy donors

G. Karasu, V. Uygun, M. Kazik, P. Kurt, Z. Ozturk, A. Kupesiz, V. Hazar, A. Yesilipek

Akdeniz University School of Medicine (Antalya, TR)

Introduction: The use of allogeneic peripheral blood stem cells (PBSC) as a source of stem cells has greatly increased and the studies adressing normal pediatric donor collection efficacy is very limited. The main objective of this paper is to document our own experience as a single center experience and to yield predictive factors for PBSC collection in pediatric donors.

Methods: Datas of all allogeneic PBSC pediatric donor apheresis performed to be used in transplantation for children in our institution were retrospectively collected.The factors affecting CD34 + cell collection being more than $2 \times 10^{6} \mathrm{~kg}$ recipient body weight were evaluated by univariate and multivariate analysis. Results: A total of 151 apheresis performed in 101 pediatric (49 males, 52 females) donors were included in this study. The mean values for ages and weights were 10 years (range: 2-18 years) and $35,4 \mathrm{~kg}$ (range 11-84 kg) respectively. Sixty -three donors in pediatric age group $(62 \%)$ underwent only one apheresis. $63 \%$ of the pediatric donor apheresis were done via a dual lumen catheter and $54 \%$ of the apheresis were done as large volume leukapheresis. Eighty-five out of 151 pediatric donor apheresis provided CD $34+$ cell count more than $2 \times 10^{6} / \mathrm{kg}$ recipient body weight. The median number of CD34+ cells $\times 10^{6} /$ $\mathrm{kg}$ recipient body weight in the first apheresis was 3.99 (range: $0.03-33$ ) whereas it was 1.95 (range:0.3-17.25) in the second one $(P<0.05)$. On univariate analysis, we found that the factors affecting to reach this amount of target cell were: donor weight
( $P=0.041)$, donor to recipient weight ratio $(P<0.001)$, first day of apheresis $(P=0.015)$, baseline WBC count $(P=0.012)$, peripheral blood CD $34+$ cell count $(P<0.001)$ and decrease in hemoglobin level of the donor after procedure $(P=0.008)$ in that donors with a more decrease in hemoglobin levels were those who yielded higher numbers of CD34 + cells. In multivariate analysis, donor to recipient weight ratio $(P=0.01)$, first day of apheresis $(P=0.04)$, and decrease in hemoglobin level of the donor after procedure $(P=0.01)$ were found to be significant predictors of yield.

Conclusion: In this small preliminary study, we found that the variables that influenced the yield were the donor to recipient weight ratio, the first day of apheresis and decrease in hemoglobin levels of donors. Further validation of these findings in larger series will help to optimize stem cell yields.

\section{P987}

Outcome of unrelated donor searches in a large multicultural transplant centre

S. Lloyd, L. Casey, R. Sergeant, J. Apperley, R. Szydlo Imperial College / Hammersmith Hospital (London, UK)

In July 2003 a comprehensive database was initiated to register and follow the progress of unrelated donor (UD) searches in a large transplant centre based in London. From 01/01/2004 to $31 / 12 / 2008$, searches were commenced for 193 patients (125 M/ $68 \mathrm{~F})$. The underlying diseases were CML $(n=99,51 \%)$ Acute Leukaemia $(n=39,20 \%)$ and other $(n=56,29 \%) .139$ patients $(72 \%)$ were Caucasian, 15(8\%) African/Caribbean, 24(12\%) Asian and 15(8\%) other. A total of 149 donors were identified, 107 fully matched at high resolution HLA-A, B, Cw and DR (8/8), 31 with $7 / 8$ matches and 11 at $6 / 8$. The median time taken to find a donor was $3.4 \mathrm{~m}$ (range 1-55), with a median number of 7 donor samples tested (tissue-typed) (range 1-20). Of the 149 patients with a donor identified, 61 received an SCT from an UD, 72 are alive and on treatment (median time from donor identification $25 \mathrm{~m}$ (range 1-68)), and 16 have died. 44 patients had no UD identified - of these, 17 patients received a non-UD SCT, 10 searches are on hold, 5 unsuccessful searches have closed, 3 searches are still ongoing, and 9 patients have died. Donors were identified for $120(86 \%)$ Caucasian patients but only $29(54 \%)$ non-Caucasian patients $(P=0.0001)$. There was no statistical difference in the degree of matching $(P=0.70)$, the number of searches carried out $(P=0.97)$, nor the duration of the search period $(P=0.38)$ between donors found for Caucasian and non-Caucasian patients. Donors were identified from registries in the UK $n=66(44 \%)$, Germany $n=25(17 \%)$, USA $n=45(30 \%)$ and from 9 other countries $n=13$ $(9 \%)$. The value of Bone Marrow Donors Worldwide (BMDW) is thus highlighted, as is the discrepancy in donor availability between Caucasian and non-Caucasian patients.

\section{P988}

The effect of different bone marrow harvest strategies on the yield of bone marrow stem cell collection

T.F. Wang (1), S.C. Chu (1), S.H. Chen (1), Y.C. Su (2), D.K. Li (2), M.H. Shyr (1), K.P. Huang (1), C.Y. Chang (3), S.S. Tsai (1), R.H. Kao (1)

(1)Tzu-Chi General Hospital (Hualien, TW); (2)Tzu-Chi General Hospital (Dalin, TW); (3)Tzu-Chi Stem Cell Center (Hualien, TW)

An adequate bone marrow (BM) cell dose is one of the most important donor factors for successful transplantation. In order to improve BM harvest of the volunteer donors in our institute, we changed the single-hole needle to the multi side-hole needle in Mar. 2002 and started to check the midway total nucleated cell (TNC) count during collection after Sep. 2004. The aim of this retrospective study was to evaluate the yields of the BM harvests by different strategies. The correlation of TNC and harvested BM volume was also analyzed. The distribution of BM harvests by different strategies was 235 donors with single-hole needle (group A), 389 donors with 5 side-hole needle (group B) and 365 donors with 5 side-hole needle and checking the midway TNC (group C). The 
final harvested $\mathrm{BM}$ volume and the BM volume per $\mathrm{kg}$ of recipient were higher in the group C (944 vs. 927 vs. $995 \mathrm{~mL}, P<0.001$ and 18.6 vs. 20.2 vs. $20.7 \mathrm{~mL} / \mathrm{kg}, P<0.001$, respectively). The recipient weight of the group $A$ was significantly heavier than those of group B but not group C (56.1 vs. 52.1 vs. $53.9 \mathrm{~kg}, P=0.045$ ). The baseline WBC count was slightly higher in group $C\left(5.97 \times 10^{3} / \mathrm{uL}\right.$ vs. $5.95 \times 10^{3} / \mathrm{uL}$ vs. $6.25 \times 10^{3} / \mathrm{uL}, p=0.02$ ). The final TNC count, marrow cell density and collected cell dose were all significantly improved by modification of the harvest strategy $\left(189 \times 10^{8}\right.$ vs. $201 \times 10^{8}$ vs. $245 \times 10^{8}, P<0.001 ; 0.020 \times 10^{8} / \mathrm{mL}$ vs. $0.219 \times 10^{8} /$ $\mathrm{mL}$ vs. $0.250 \times 10^{8} / \mathrm{mL}, P<0.001 ; 3.79 \times 10^{8} / \mathrm{kg}$ vs. $4.46 \times 10^{8} / \mathrm{kg}$ vs. $5.19 \times 10^{8} / \mathrm{kg}, P<0.001$, respectively). The percentage of unacceptable cell dose $\left(\mathrm{TNC}<2 \times 10^{8} / \mathrm{kg}\right)$ was also decreased $(5.9 \%$ vs. $3.6 \%$ vs. $0 \%, P<0.001)$. The multiple regression analysis revealed that the donor weight, WBC count and strategy groups were positively correlated with the cell density of the BM harvest $(P<0.001)$, while the total volume of the harvested BM was negatively correlated with the cell density $(P<0.001)$. The mean cell density of final harvested BM was significantly lower than the midway harvested product $\left(0.250 \times 10^{8} / \mathrm{mL}\right.$ vs. $0.292 \times 10^{8} / \mathrm{mL}$, $P<0.001)$. Base on a linear regression, a highly significant correlation $(n=730, r=0.7937, P<0.001)$ was noted between the harvested $\mathrm{BM}$ volume and the TNC count. The regression equations were $\operatorname{TNC}(y)=40.08+0.2057 x B M$ volume $(x)$. By changing the harvest needle and checking the midway TNC count, the harvest outcome was improved. This may help to have a sufficient volume of BM to ensure a better recipient outcome and minimize the side effects of the harvest at the same time.

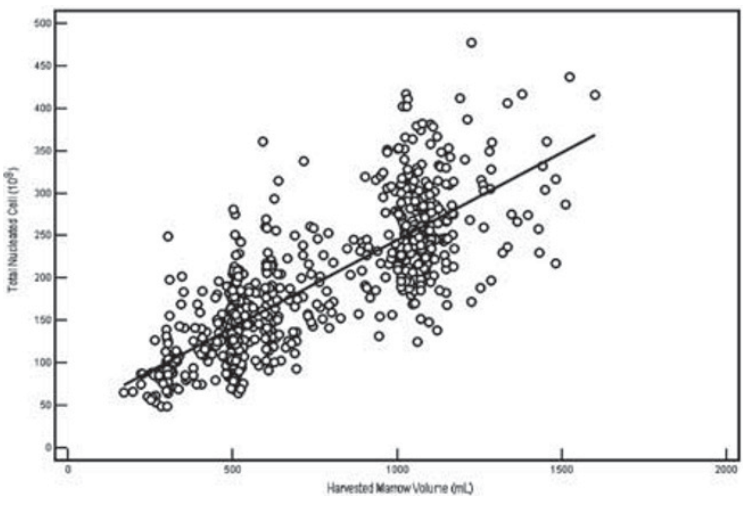

\section{P989}

Second donation from volunteer haematopoietic stem cell donors in Taiwan

T.F. Wang (1), S.H. Chen (1), S.H. Yang (1), C.Y. Chang (2), Y.W. Chiu (2), T.L. Lin (1), M.H. Shyr (1), K.L. Yang (2)

(1)Tzu-Chi General Hospital (Hualien, TW); (2)Tzu-Chi Stem Cell Center (Hualien, TW)

Occasionally, unrelated hematopoietic stem cell (HSC) donors may be asked to provide subsequent donations of stem cells or lymphocytes. In this study, we evaluated the frequency of and reasons for a second donation and whether donors prefer peripheral blood stem cell (PBSC) collection or bone marrow (BM) harvest. In addition, we compared the yields of and side effects associated with twice BM harvests.

Donor characteristics, reasons for the second donation, type of second donation, and yield of BM stem cell product were abstracted from medical records. The psychosocial and physical experiences of donors who underwent two different HSC collection procedures (BM and PBSC) and their preference for one procedure over the other were collected via a self-reported questionnaire. Among donors who underwent two BM harvests, we compared the yields of and the side effects associated with the first and second harvest. Among the 1738 donors analyzed, 72 had made more than one donation. The procedures included lymphocyte donations in 55 donors, BM harvest in 9 donors, and PBSC collection in 8 donors. A total of 13 donors under- went both BM and PBSC donations and 4 donors underwent two BM harvests. The median recovery time associated with PBSC (1 day) was significantly shorter than that associated with BM harvest ( 7 days) $(P=0.0083$ ). Although most of the donors who underwent both procedures felt that BM harvest was more physically demanding, caused more pre-procedural anxiety, was more time consuming, and was more inconvenient than PBSC collection, $63.6 \%$ of them preferred BM donation. There was no significant difference in yield of stem cells between the first and second BM harvests $(P=0.25)$. In addition, there were no significant differences in the incidence of side effects or recovery time between the first and second BM donation. Approximately $30 \%(5 / 15)$ of the donors had difficulty before agreeing to donate HSC a second time. Among the 17 donors, 10 encountered disagreement from their relatives or friends.

Although the case number in our study is relatively small, more donors' preference for BM harvest over PBSC collection is different from that reported in previous studies despite similar side effects. Discomfort of PBSC apheresis procedure can explain this result partially. Further understand of the donors ' preference and donation side effects might help the registry improving their work.

\section{P990}

Mobilization and collection of peripheral blood stem cells in small children with low blood volume

C. Landefeld, K. Kentouche, A. Müller, B. Gruhn, F. Zintl, J.F. Beck

University of Jena (Jena, DE)

Background: High-dose chemotherapy with autologous stem cell transplantation is an established procedure in the treatment of oncological diseases in childhood. Because of the extracorporeal blood volume the collection of the peripheral blood stem cells (PBSC) with apheresis procedures is difficult in small children.

Patients and Methods: We analyzed the apheresis results from 36 children whose weight less than $20 \mathrm{~kg}$ (range 7-20 kg) and had an average blood volume of $1244 \mathrm{ml}$ (range 591-2000 $\mathrm{ml}$ ). The collection of the PBSC was performed with the Cobe Spectra PBSC System. The extracorporeal blood volume in the system is about $285 \mathrm{ml}$, and so priming the system with an erythrocyte/plasma concentrate was necessary in most cases to prevent hypotensive dysregulations.

Results: Overall we performed 47 stem cell mobilizations with 89 apheresis (average 1,89). In the middle we started the collection after 8,2 days of cytokine (G-CSF) stimulation. On the first day of collection the mean blood stem cell (CD34) count was $43,7 / \mu \mathrm{l}$. The average yield of collected CD34 was $8,9 \times 10^{6} /$ kg bodyweight. In 27 of 36 patients (75\%) we reached a CD34 yield about $2 \times 10^{6} / \mathrm{kg}$ with the first mobilization. When second and third mobilization was performed all patients reached the expected stem cell count. The apheresis procedure was well tolerated and no critical side effects occurred.

Conclusion: Also for small children with low blood volume we find good overall results for the collection of stem cell in childhood malignancies. The priming of the extracorporeal system prevents hypotensive dysregulations. When the first collection was insufficient the second and third stem cell mobilization/collection can be more effective. Mobilization and collection of PBSC from small children are save, well tolerated and best performed in children specialized apheresis centres.

\section{P991}

Comparison of outcomes between transplantation from unrelated donor umbilical cord blood and from unrelated donor bone marrow or peripheral blood in children with acute leukaemia

S.H. Lee, H. Chueh, K.H. Yoo, K.W. Sung, H.H. Koo

Samsung Medical Center (Seoul, KR)

Introduction: Although umbilical cord blood (UCB) is an accepted alternative to bone marrow or peripheral blood (BM/ 
PB) for transplantation, allele-matched marrow or peripheral blood is generally regarded as the preferred graft source. Our aim is to compare leukemia-free survival after transplantation of unrelated donor UCB and allele-matched BM/PB, and to provide guidelines for selection of an appropriate donor and graft source in children with acute leukemia.

Patients and methods: Outcomes of 22 children with acute leukemia and transplanted with single unit UCB were compared with outcomes of $34 \mathrm{BM} / \mathrm{PB}$ recipients. All transplantations were performed in Samsung Medical Center between May 2003 and June 2009. Recipients of UCB were transplanted with grafts that were HLA-matched $(n=3)$ or HLA-mismatched for one $(n=15)$ or two antigens $(n=4)$. BM/PB recipients were transplanted with grafts that were HLA-matched $(n=20)$ or mismatched $(n=14)$. While allele-level typing for HLA-A, HLA-B, HLA-DRB1 and antigen-level typing for HLA-C was used for $B M / P B$ transplantation, antigen level typing for HLA-A, HLA-B and HLA-DRB1 was used for UCB transplantation.

Results: The median times to neutrophil and platelet recovery were 15 (range 10-21) and 22 (12-29) days after BM/PB transplantation, and 17 (13-44) and 34 (24-148) days after UCB transplantation, respectively. The 4-year probabilities of leukemia-free survival were $58.0 \%$ after HLA-matched BM/PB transplants, $65.9 \%$ after HLA-mismatched BM/PB transplants, $66.7 \%$ after HLA-matched UCB transplants, $67.5 \%$ after onemismatched UCB transplants with high cell dose $\left(\mathrm{NC}>3.0 \times 10^{7} /\right.$ $\mathrm{kg}), 40.0 \%$ after one-mismatched UCB transplants with low cell dose $\left(\mathrm{NC}<3.0 \times 10^{7} / \mathrm{kg}\right)$, and $33.3 \%$ after two-mismatched UCB transplants. The incidence of grade 3-4 acute graft-versus-host disease (GVHD) were $12.1 \%$ after BM/PB transplantation and $5.3 \%$ after UCB transplantation, respectively $(P=0.64)$. Rates of extensive chronic GVHD were $53.8 \%$ after BM/PB transplantation and $23.1 \%$ after $U C B$ transplantation, respectively $(P=0.09)$. Transplant-related mortality rates were higher after UCB transplantation $(21.7 \%)$ than $\mathrm{BM} / \mathrm{PB}$ transplantation $(2.9 \%)(\mathrm{P}=0.03)$. Relapse rates were similar after BM/PB transplantation (29.4\%) and UCB transplantation $(21.7 \%)(P=0.56)$.

Conclusion: These results support the use of HLA-matched or one-antigen HLA mismatched UCB in children with acute leukemia who need transplantation.

\section{P993}

Successful enhancement of stem cell mobilization in G-CSF mobilized allogeneic donors by application of plerixafor (AMD 3100)

K. Hölig, M. Blechschmidt, M. von Bonin, A. Rosner, F. Stölzel, K. Zimmer, M. Bornhäuser, G. Ehninger

University Hospital Dresden (Dresden, DE)

Background: Engraftment and outcome after allogeneic peripheral blood stem cell (PBSC) transplantation are closely related to the dosage of CD 34 + cells in the graft. Until now, "poor mobilizers" among allogeneic donors remain difficult to identify 'a priori' and alternative salvage mobilizing agents would be highly desirable. Methods: We report on 4 allogeneic female donors (age 25-70 a) who did not respond sufficiently to our standard mobilization protocol (G-CSF, lenograstim 7.5-10 $\mu \mathrm{g} / \mathrm{kg} 5$ days). Plerixafor was administered within a compassionate use programme during a 2nd course of stem cell mobilization on day 4 (10 p.m.) at a dosage of $240 \mu \mathrm{g} / \mathrm{kg}$ in 2 donors. In the other 2 donors plerixafor was administrated during the 1st course of stem cell mobilization at 10 p.m. on day 5 because of a very low stem cell yield after the 1st apheresis. Written informed consent was obtained from all participating individuals. 2 grafts underwent T-cell depletion because of HLA-mismatch. Recipients ( 3 males, 1 female) suffered from acute myeloid leukemia ( $A M L, n=2)$, high-grade non Hodgkin lymphoma (NHL) and chronic myeloid leukemia (CML). Results: Apheresis yields with G-CSF alone and after administration of G-CSF and plerixafor are shown in table 1 . The amount of CD 34 + cells collected could be enhanced by $167 \%$ to $300 \%$ by the addition of plerixafor. All donors reported only mild or no side effects after receiving plerixafor. All 4 recipients had been transplanted with these grafts and showed timely and sustained trilineage engraftment.

Conclusion: Our preliminary data suggest that the administration of G-CSF and plerixafor may be a successful and well-tolerated regimen for PBSC mobilization in allogeneic donors who do not achieve a given CD34 + PBSC target with G-CSF alone. The stem and progenitor cells obtained by this strategy seem to have a high engraftment potential. This novel approach could possibly avoid graft failure in the recipients and the need for bone marrow collection in poor mobilizing allogeneic donors and should therefore be further evaluated in future studies.

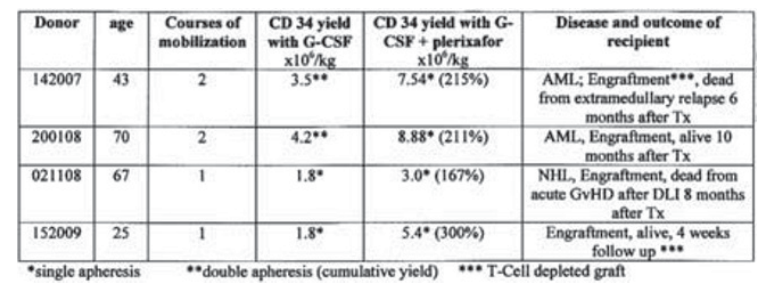

\section{P994}

Influence of filgrastim mobilization of HLA identical familiar healthy stem cell donor in allogneic transplant setting

S. Genadieva-Stavrik (1), A. Pivkova (2), Z. Stojanoski (2), V. Milenkov (2), S. Krstevaka-Balkanov (2), L. Cevreska (2), B. Georgievski (2)

(1)University Hematology Clinic (Skopje, MK); (2)Clinical Center (Skopje, MK)

Mobilized peripheral blood stem cells (PBSC) have become an increasingly used alternative to bone marrow for allogeneic transplantation. Granulocyte colony-stimulating factor (G-CSF) -primed peripheral stem cells harvesting may result in a graft with increased mononuclear cells collected, increased progenitor cell dose and potential for more rapid engraftment resulting in improved survival. Filgrastrim is not only known to mobilize $\mathrm{CD} 34$ + progenitor cells but acts as a pleiotropic immune modulator. So, systematic donor follow-up in healthy donors is needed. The aim of this study is to evaluate safety and feasibility of G-CSF primed hematopoietic peripheral stem cells in familiar HLA-identical donors. The follow-up focused on laboratory testing including reports of adverse event.

Granulocyte colony-stimulating factor (G-CSF) is administrated in 49 healthy donors to reach sufficient mobilization in the period 2000-2009. The donors were characterized as follows: 43 years median; female $60 \%$ of the donors. G-CSF was administrated in the dose $10 \mu \mathrm{g} / \mathrm{kg}$ of donor weight in five day and PBSC collections started on the fifth day using COBE Spectra cell separator. The aim was to collect mononuclear cells $2 \times 10^{8} / \mathrm{kg}$ of recipient weight. Three donors were mobilized twice (for second transplant). Aphaeresis needed to reach target number of CD34+ cells were: 1 apherese in 50\%, more than two apherese need in only 1 patient. The most frequent adverse event that was noted by patients was bone pain associated with increasing number of white blood cells. Better mobilization and higher PBSC yield correlated significantly with younger age. Four years after GCSF -primed peripheral stem cells harvesting, a young female 48 years old was diagnosed with acute myeloblastic leukemia. Four years ago when she was 44 years old, she donated for her HLA identical sister with acute myeloblastic leukemia.

G-CSF is safe and very effective for PBSC mobilization in our healthy donors. This method allows certain collections of sufficient numbers of progenitors in virtually all donors. We demonstrated that filgrastim mobilization for peripheral blood stem collection is effective and result with successful engraftment in all the recipients. Daily injection of $10 \mu \mathrm{g} / \mathrm{kg}$ of G-CSF and first aphaeresis preformed at day 5 seems to be the best strategy to obtain the CD34 + cell count required for an allogeneic hematopoietic stem cell graft. 
P995

Effect of the search of an unrelated stem cell donor in patients with high-risk acute myeloid leukaemia over a long follow-up: a prospective, single-centre study

A..P. Iori (1), V. Valle (1), A. Piciocchi (2), G.F. Torelli (1), G. Meloni (1), S. Trisolini (1), M. Breccia (1), C. Minotti (1), S. Capria (1), W. Barberi (1), A.M. Testi (1), M.L. Moleti (1), P. Perrone (1), L. Laurenti (1), M. Screnci (1), E. lannella (1), F. Natalino (1), E. Arleo (1), R. Ricci (1), W. Arcese (1), R. Foà (1) (1)University La Sapienza (Rome, IT); (2) GIMEMA Data center (Rome, IT)

To assess the impact of the donor search period on the outcome of patients (pts) with high risk (HR) acute myeloid leukemia (AML), we prospectively analyzed 80 pts-median age 34 years (1-59), median body weight $60 \mathrm{Kg} \mathrm{(10-120)-who} \mathrm{underwent} \mathrm{a} \mathrm{search} \mathrm{of}$ an unrelated donor through the BMDWW Registry and cord blood (CB) banks, between April 1995 and September 2009. At the start of the search 34 pts were in I complete remission (CR), 28 in > II CR and 18 in relapse. The probability of finding a donor, $\mathrm{CB}$ or unrelated volunteer donor (UD) by cumulative incidence (CI) was $52 \%$ at 4 months $(95 \% \mathrm{Cl}: 51-52.3), 66 \%$ at 8 months $(95 \% \mathrm{Cl}: 65.3$ $66.4), 67 \%$ at 12 months (95\% Cl: 66.7-67.9), respectively; after this time, no increase in the $\mathrm{Cl}$ of finding a donor was registered. The median time from the start of the search to finding a donor was 1.8 months (range: $0.5-6$ ) for a CB and 3 months (range: 1.4-12) for a UD, the difference being statistically significant $(P=0.02)$. Out of 54 pts with a donor, $36(66 \%)$ underwent a transplant, while the remaining $18(33 \%)$ could not undergo a transplant because of lost eligibility mainly due to a relapse occurring during the search, observed at a median of 4 months after the start of the search. The overall survival (OS) probability at 10 years was $21 \%$ for the whole population. In univariate analysis, to address the search at the time of relapse $(P=0.008)$, a body weight less than the median $(P=0.03)$ and the absence of relapse during the search $(P<.0001)$, analyzed as covariate time dependent, showed a positive effect on survival. In multivariate analysis, relapse during the search remains the most important factor affecting OS $(P<.0001)$. In conclusion, relapse during the search represents the major cause of failure of the search of an UD for pts with HR AML, in terms of OS, compromising the eligibility to transplant. Since an increase in the research time beyond 4 months, the $\mathrm{Cl}$ of finding a donor increases slightly compared to the increased risk of relapse, the time of the research should not exceed this time point. Addressing the search, at the same time, in CB banks and UD registries increases the chance to carry out a transplant in helpful time. Over 4 months, the indication for an alternative transplant should be given.

\section{Inborn errors of metabolism}

\section{P996}

Allogeneic haematopoietic stem cell transplantation in patients with alpha-mannosidosis: analysis of $\mathbf{1 3}$ patients M. Mynarek (1), J. Tolar (2), M. Albert (3), J.J. Boelens (4), M.J. Cowan (5), M.L. Escolar (6), A. Glomstein (7), J.S. Kühl (8), D. Malm (9), P.J. Orchard (2), T. Lücke (1), J. Kurtzberg (10), K.W. Sykora (1)

(1)Hannover Medical School (Hannover, DE); (2)University of Minnesota (Minneapolis, US); (3)Ludwig Maximilians University (Munich, DE); (4)University Medical Center (Utrecht, NL); (5)University of California San Francisco (San Francisco, US); (6)University of North Carolina at Chapel Hill (Chapel Hill, US); (7)University Hospital Oslo (Oslo, NO); (8)Charité University Medicine Berlin (Berlin, DE); (9)University Hospital of North Norway (Tromsoe, NO); (10)Duke University Medical Center (Durham, US)

Alpha mannosidosis is a rare lysosomal storage disease with variable developmental delay and skeletal dysplasia. Hematopoietic stem cell transplantation (HSCT) is considered stand- ard therapy for severe variations of the disease. The first successful HSCT for mannosidosis was described in 1998. Here we present a retrospective analysis of 13 patients with alpha-mannosidosis that were diagnosed at a median of 2.9 (1.6 to 23) years and underwent HSCT at a median of 3.8 (2 to 23.2) years. Three patients received bone marrow, three cord blood, four CD34 selected peripheral blood stem cells (PBSC), and one a cord-haploidentical PBSC cotransplantation. In two patients, stem cell source was not documented.

Transplant course: Twelve of these patients are alive (92\%) after a median follow up of 6.9 (1.1 to 10.5) years. Three patients $(23 \%)$ developed respiratory insufficiency that required mechanical ventilation, one patient $(8 \%)$ died from multi organ failure after HSCT. Of the surviving patients, two (17\%) developed severe acute GvHD ( $\geq$ grade II) and four (33\%) chronic GvHD (two limited, one extensive, one not documented). Two patients $(17 \%)$ required re-transplantation because of graft failure. All twelve showed stable engraftment at last follow up. Disease-specific follow-up: Developmental data before HSCT were available in nine patients. The extent of these patients' developmental delay varied over a wide range. Post-HSCT neurologic follow up was available for six patients and all of them showed developmental improvement after HSCT. Before HSCT, six of eleven patients $(55 \%)$ required hearing aids, after HSCT five of twelve patients $(42 \%)$. In three patients hearing aids could be discontinued, while two required new hearing aids after HSCT.

Conclusion: Children seemed to tolerate conditioning and HSCT without unusual or unexpected complications. The final survival rate with engraftment was $92 \%$ making HSCT a feasible therapeutic option which may promote mental development. However, the natural history of alpha-mannosidosis without transplantation is so far not well characterized, and it is difficult to predict before HSCT how severely affected a patient would become without HSCT. Therefore, quantification of the developmental benefit of HSCT for patients with mannosidosis remains difficult and careful evaluation of neurodevelopmental outcome of transplanted and non-transplanted patients is an important task for the future.

\section{P997}

Transplantation of CD3/CD19 depleted PBSC leading to viral clearance and no graft-versus-host disease in SCID M. Slatter (1), Z. Nademi (1), S. Patel (1), D. Barge (1), M. Valappil (2), K. Brigham (3), S. Hambleton (4), T. Flood (1), A. Cant (1), M. Abinun (1), A. Gennery (1)

(1)Newcastle upon Tyne Hospitals NHS Foundation Trust (Newcastle upon Tyne, UK); (2)Health Protection Agency (Newcastle upon Tyne, UK); (3)Newcastle Haematopoietic Stem Cell Laboratory (Newcastle upon Tyne, UK); (4)Newcastle University (Newcastle upon Tyne, UK)

A haplo-identical parental T cell depleted HSC can be used in severe combined immunodeficiency (SCID). Until recently, CD34 + cells positively selected using organic iron beads and a magnetic column were the product of choice, but there are concerns regarding speed and quality of immunoreconstitution1. Using CD3/CD19 deleted product, retains mature precursors and stem cells, and may lead to faster immunoreconstitution. Previous studies have reported significant GvHD. We report 2 SCID patients with viral infection, successfully treated with CD3/ CD19 depleted PBSC. Both were conditioned (table) but had no serotherapy. CSA and MMF were used as GvHD prophylaxis.

Case 1 presented with recurrent respiratory infections and failure to thrive. She had pneumocystis jeroveci (PJ) and respiratory syncytial virus isolated at bronchoalveolar lavage at 8 months of age and was found to have IL7R alpha SCID. She received CD3/CD19 depleted haplo-id maternal PBSC after conditioning. MMF was weaned from $D+28$, stopped at $D+43$. CSA was run at $<50 \mathrm{ng} /$ $\mathrm{ml}$ and stopped at $\mathrm{D}+147$. She received weekly Palivizumab and IVIG through the transplant period. RSV cleared at D+60. She has $100 \%$ donor chimerism in all cell lines at 19 months post transplant, with normal $\mathrm{T}$ and $\mathrm{B}$ cell number and function. 
Case 2 presented with recurrent respiratory infections and failure to thrive. Age 6 months he was ventilated for PJP. RAG1 SCID was confirmed. He received CD3/CD19 depleted haploid maternal PBSC after conditioning. MMF was weaned from $\mathrm{D}+28$ and stopped at $\mathrm{D}+50$. CSA was run at $<50 \mathrm{ng} / \mathrm{ml}$ and stopped at $D+124$. On $D+4$ routine viral surveillance PCR revealed adenovirus in stool, blood and NPS (maximum adenoviremia, $6.1 \times 10^{5}$ copies $/ \mathrm{ml}$ ). He was treated with cidofovir but the viral load continued to rise. Fever and adenoviraemia were controlled by naïve T cells on $\mathrm{D}+80$. He was discharged home 4 months post transplant and is now 10 months post transplant, off IVIg and receiving primary vaccinations.

CD3/CD19 depleted HSCT leads to more rapid immune reconstitution and viral clearance than CD34 + selected HSCT. GvHD can be avoided by use of low dose CSA and MMF.

1. Slatter MA, et al. J Allergy Clin Immunol 2008;121:361-7

\begin{tabular}{|c|c|c|c|c|c|c|c|}
\hline Case & Conditioning & $\mathrm{NC} / \mathrm{kg}$ & $\underset{\mathrm{Ng}}{\mathrm{CD} 34+}$ & $\underset{\mathrm{hg}}{\mathrm{CD} 3+}$ & $\underset{\mathrm{kg}}{\mathrm{CD} 19+1}$ & $\begin{array}{c}\text { Neut } \\
>0.5 \\
x \\
10^{\circ} \pi\end{array}$ & $\begin{array}{l}\text { chimerism/ } \\
\text { FU }\end{array}$ \\
\hline$T$ & $\begin{array}{c}\text { treo } 42 \mathrm{~g} / \mathrm{m}^{2} \\
\text { cyclophos } 200 \mathrm{mg} / \mathrm{kg}\end{array}$ & $3.7 \times 10^{4}$ & $\begin{array}{l}8.1 x \\
10^{\circ}\end{array}$ & $\begin{array}{l}1.0 x \\
10^{4}\end{array}$ & $\begin{array}{l}2.2 x \\
10^{4} / \mathrm{kg}\end{array}$ & +11 & $\begin{array}{l}100 \% \\
19 / 12\end{array}$ \\
\hline 2 & $\begin{array}{c}\text { treo } 36 \mathrm{~g} / \mathrm{m}^{2} \\
\text { cyclophos } 200 \mathrm{mg} / \mathrm{kg}\end{array}$ & $4.2 \times 10^{2}$ & $\begin{array}{l}8.8 \mathrm{x} \\
10^{\circ}\end{array}$ & $\begin{array}{l}6.0 x \\
10^{4}\end{array}$ & $\begin{array}{l}6.7 x \\
10^{\prime} / \mathrm{kg}\end{array}$ & +10 & $\begin{array}{l}100 \% \text { Ly } \\
92 \% \mathrm{My} \\
10 / 12\end{array}$ \\
\hline
\end{tabular}

\section{P998}

Haploidentical stem cell transplantation in 2 siblings with mucopolysaccharidosis type $\mathrm{VI}$ as a rescue for cord blood graft failure

J. Toporski (1), D. Turkiewicz (1), D. Papadopoulou (1), J. Dykes (1), J.E. Mansson (2), A.N. Békássy (1)

(1)Lund University Hospital (Lund, SE); (2)University of Gothenburg (Gothenburg, SE)

Two pre-symptomatic siblings with mucopolysaccharidosis type VI (MPS-VI) were accidentally diagnosed when hyper granulated neutrophils were seen in the PB smear of the 11 months old boy. His sister was screened 3 months later at birth with the same disease. Homozygous p.C192R ARBS mutation was identified, considered usually "mild" but still leading to considerable organ damages and "a point of no return".

Decision was taken to transplant both sibilings with unrelated cord blood (UCBT) after myeloablative preparation. The boy experienced multiple viral reactivations post transplant, leading to secondary hemophagocytic lymphohistiocytosis and eventually loss of the graft. While the 2nd transplantation was performed with PBSC from his haploidentical father, he was kept on mechanical ventilation due to persistent HSV-1 infection with respiratory tract involvement. His sister received the same conditioning prior to UCBT but had an autologous recovery and became subsequently retransplanted with the cryopreserved cells from her father. The haplo-graft was T-cell depleted with the use of immunomagnetic selection of CD34+ cells (CliniMACS). The children received reduced intensity conditioning before the second graft.

The post-transplant follow up was not complicated; both children achieved fast and sustained hematological recovery, eventually with $100 \%$ donor chimerism with no aGvHD. Both children are passing developmental milestone on time, being well 16,5 and 6 months after the transplant with no cGvHD nor symptoms of the disease. See tables for details. Arylsulfatase activity in leukocytes is normal and urinary elimination of glycosaminoglycan (GAG) is decreasing.

MPS VI is a progressive disorder with significant morbidity and early mortality. Enzyme replacement therapy (ERT) is a lifelong therapy administered intravenously and has been shown to improve physical capabilities; however, it is costly and burdensome to the family and society.

In our hands haplodentical stem cell transplantation was safe and rescued the children after unsuccessful UCBT. We observe biochemical correction of enzyme activity in the blood as well as decreasing secretion of glycosaminoglycan (GAG) in urine. Long term follow up is necessary in order to judge the haplo procedures' impact on the course of the disease.

Table 1. Characteristics of the UBCT.
\begin{tabular}{|l|c|c|c|c|c|c|l|}
\hline $\begin{array}{l}\text { Pat } \\
\text { No }\end{array}$ & Sex & $\begin{array}{l}\text { Age at } \\
\text { diagnosis } \\
\text { (months) }\end{array}$ & $\begin{array}{l}\text { Age } \\
\text { at TX }\end{array}$ & Conditioning & $\begin{array}{l}\text { HLA } \\
\text { match }\end{array}$ & $\begin{array}{l}\text { NC dose } \\
\left(\times 10^{\wedge} 7 / \mathrm{kg}\right)\end{array}$ & $\begin{array}{l}\text { Outcome } \\
\text { after 1* TX }\end{array}$ \\
\hline 1 & M & 11 & 20,5 & $\begin{array}{l}\text { IV BU + CY, } \\
\text { ATG }\end{array}$ & $6 / 6$ & 7,5 & $\begin{array}{l}\text { HLH } \\
\text { Day }+30\end{array}$ \\
\hline 2 & F & 0,0 & 15 & $\begin{array}{l}\text { IV BU + CY, } \\
\text { ATG }\end{array}$ & $4 / 6$ & 4,4 & $\begin{array}{l}\text { Autologous } \\
\text { recovery } \\
\text { Day }+52\end{array}$ \\
\hline
\end{tabular}

\begin{tabular}{|c|c|c|c|c|c|c|}
\hline $\begin{array}{l}\text { Pat } \\
\text { No }\end{array}$ & $\begin{array}{l}\text { Time from } \\
1^{\text {ts }} \text { to } 2^{\text {ad }} \\
\text { TX (days) }\end{array}$ & Conditioning & $\begin{array}{l}\text { CD34+ dose } \\
\left(x 10^{\circ} 6 / \mathrm{kg}\right)\end{array}$ & $\begin{array}{l}\text { T-cell dose } \\
\left(\mathrm{x} 10^{-4 / \mathrm{kg})}\right.\end{array}$ & $\begin{array}{l}\text { Take } \\
\text { (day) }\end{array}$ & $\begin{array}{l}\text { Follow up } \\
\text { (months) }\end{array}$ \\
\hline 1 & 61 & $\begin{array}{l}\text { Fludarabine, } \\
\text { Etoposide, OKT3 }\end{array}$ & 27 & 0,47 & +10 & $\begin{array}{l}16,5 \text { alive } \\
\& \text { well }\end{array}$ \\
\hline 2 & 59 & $\begin{array}{l}\text { Fludarabine, } \\
\text { Thiotepa, } \\
\text { Melfalan, OKT3 }\end{array}$ & 14.7 & 0.24 & +16 & $\begin{array}{l}6,0 \\
\text { alive \& } \\
\text { well }\end{array}$ \\
\hline
\end{tabular}

\section{P999}

Early functional improvement following allogeneic unrelated stem cell transplantation for an adult patient with late-onset Krabbe disease

H. Baurmann (1), G. Ay (1), M. Alicke (1), J. Müller (1), E. Rudnay (1), J. Bayerl (2), M. Schleuning (1), R. Schwerdtfeger (1) (1)Deutsche Klinik fuer Diagnostik (Wiesbaden, DE); (2)Diakoniekrankenhaus (Mannheim, DE)

Objective: Krabbe disease or globoid-cell leukodystrophy is a rare neurodegenerative disorder caused by hereditary deficiency of the lysozymal enzyme galactocerebrosidase (GALC). Central and peripheral demyelinisation usually develop in early infancy - however some adults with late-onset disease are described. Allogeneic stem cell transplantation (alloSCT) is able to restore normal leukocyte GALC levels and probably establish donor derived GALC competent microglia and astroglia in the central nervous system. We here report on an adult patient with late-onset Krabbe disease who received unrelated alloSCT for severe and progressive neurological impairment.

Patient and methods: A 37 year old female was diagnosed with Krabbe disease in February 2009 after a 17 year history of progressive lower limb paraplegia. Her GALC level was 0 to $<0.5 \%$ of lower normal. MRI of the brain showed an obvious increase in signal intensity of the pyramidal tract and the pericentral region. Since 2007 she was permanently confined to a wheel chair. On admission she had no motor and little sensory function of the lower limbs, no bladder control, progressive paresis (force 2/5) of the right arm, severe headache, vertigo, diplopia, mild dysarthria, neuropathic pain and an ECOG performance status of 4 . No HLA identical donor could be found. After fully informed consent the patient therefore received peripheral blood stem cells of a 9/10 matched, unrelated donor following reduced intensity conditioning with iv Busulfan 6.4 $\mathrm{mg} / \mathrm{kg}$, Fludarabin $150 \mathrm{mg} / \mathrm{m}^{2}$ and Thymoglobulin $® 7.5 \mathrm{mg} / \mathrm{kg}$ body weight. Immunosuppression consisted of Cyclosporin A and short course Methotrexate.

Results: Except for fever of unknown origin day (d) +14 and CMV reactivation $d+20$ the patient had an uneventful course. Take and full donor chimerism was documented $d+16$ and $d+19$, respectively. Pain was relieved $d+2$, a substantial gain in right hand function occurred $d+8$. Sitting on the edge of the bed and use of the right arm was possible $d+17$. A first step was documented $d+27$ and at discharge $d+42$ the patient was continuously improving and able to walk $30 \mathrm{~m}$ with a walking frame.

Conclusion: To our knowledge this is the first adult patient with Krabbe disease grafted from an unrelated donor, and the second adult patient receiving alloSCT for this condition. An impressive gain in neurological function was seen within days after transplant despite the presence of longstanding structural changes of the nervous system. 


\section{P1000}

Systemically administered MSCs following BMT home to inflamed tissues including CNS

J. Böhringer, M. Vaegler, R. Handgretinger, I. Müller

University Children's Hospital (Tubingen, DE)

Human multipotent mesenchymal stromal cells (MSCs) are increasingly used for clinical applications mainly in regenerative medicine and immune modulation. Immunomodulatory properties of MSC are exploited in treatment of steroid refractory GvHD following allogeneic SCT. In addition, autoinflammatory diseases such as systemic sclerosis, Crohn's disease and multiple sclerosis have shown responses to MSC in animal models and clinical pilot trials. Application of MSC in regenerative medicine has been largely focused on the osteogenic differentiation potential of MSC, e.g. in osteogenesis imperfecta. However, in a growing number of reports MSC mediate their effects in tissue regeneration by secretion of signalling molecules and other proteins. This feature may also be used in the treatment of patients with neurometabolic diseases, e.g. metachromatic leukodystrophy (MLD). We show that MSCs produce arylsulfatase A (ASA), which is the deficient enzyme in MLD patients. Moreover, MSCs release significant amounts of ASA into the media. In a coculture system of healthy MSC and fibroblasts from MLD patients separated by a semipermeable membrane, ASA deficient cells take up substantial amounts of the enzyme. This uptake was blocked by mannose and mannose-6-phosphate indicating that receptor mediated processes involving the mannose-6-phosphate receptor are important. However, in order to exert their role of enzyme replacement in the brain, MSC need to home to the CNS. This has not been shown in humans so far, but could have great impact also on strategies in multiple sclerosis and other autoinflammatory or degenerative diseases of the CNS. Therefore, we analysed the case of a DNA ligase IV-deficient boy, who underwent allogeneic bone marrow transplantation from a matched unrelated donor and obtained MSCs from his haploidentical father for treatment of steroid refractory hepatic and intestinal GvHD ${ }^{\circ} \mathrm{IV}$ four weeks apart. Two weeks after the second application he succumbed to GvHD and generalized aspergillosis. An autopsy was performed and tissue samples were analysed by immunohistochemistry. Parental MSCs were located in inflamed organs, mainly liver and gut as well as the CNS in areas close to infiltrates of aspergillosis. Our results demonstrate that MSC may cross the blood-brain barrier in an inflammatory environment and home to the CNS.

\section{Cytokines}

\section{P1001}

Haematopoietic stem cell mobilization with Pegfilgrastim for multiple myeloma: quality of life, usage and results A. Leon (1), C. Bompoint (2), L. Garderet (2), T. Bokobza (2), J.M. Segier (1), B. Birebent (1), H. Rouard (1), P. Bierling (1) (1)EFS lle de France (Paris, FR); (2)Saint Antoine Hospital (Paris, FR)

Multiple Myeloma (MM) is one of the more frequent indications for autologous stem cell (PBSC) transplantation - as intensification after induction. The optimal method of mobilizing cells is not established. We tested the utilization and ability of Pegfilgrastim to mobilize PBSC and compared it to our group of Filgrastim mobilized MM. We employed either in group 1: $2(12 \mathrm{mg})$ to $3(18 \mathrm{mg})$ Pegfilgrastim injections on a single day (J0) or in group 2: Filgrastim-twice a day until the end of leukaphersis (LK) at $5 \mathrm{microg} / \mathrm{kg}$. Material and method: Group $1: 18 \mathrm{MM}: 9$ with $12 \mathrm{mg}$ and 9 with $18 \mathrm{mg}$. Group $2: 37 \mathrm{MM}$. Number of analyzed LK group 1/ group 2: 29/84. Patients were tested at J3 and before each LK for WBC and CD34 + cells. LK were performed on Spectra (Caridian) and there goals were 4 to $8106 \mathrm{CD} 34 / \mathrm{Kg}$ depending on simple or tandem autograft request. Results are expressed in median.
Results: Mobilization with Pegfilgrastim: problem to organize the injections: none. Adverse effects: bone pain requiring more potent analgesic: $3 / 18$. Evolution in peripheral blood of WBC $10^{3} / \mathrm{mm}^{3}$ and CD34 103/ $\mathrm{mm}^{3}$ : J3-42.6/36. J4: 41.5/34. J5$26.2 / 27$. At J3, $84.2 \%$ of patients were able to be collected. Comparison Group 1/2-CD 34 106/Kg per LK: 2.54/ 2.68-CFUGM 104/Kg per LK: 18.64 / 20.8.

Conclusion: Pegfilgrastim is easy to use and facililtate the life of patients. Adverse effects are comparable to those usually described with filgrastim. The rising of WBC is moderate, even after $18 \mathrm{mg}$ of Pegfilgrastim. In term of CD34 + cells and functionality, the results are comparable to the filgrastim mobilized group. The optimal dose of Pegfilgrastim still needs to be determine in that context.

\section{P1002}

Does adminstration of rh-GCSF to healthy volunteers induce epigenetic alteration in lymphocytes?

G.C. Leitner (1), M. Faschingbauer (1), G. Fischer (1), G. Weigel (2)

(1)Department for Bood Group Serology and Transfusion Medicine (Vienna, AT); (2)Department of Cardiothoracic Surgery (Vienna, AT)

Background: The short-term safety profile of rhG-CSF in the allogeneic stem cell setting seems acceptable, but only few data on long-term safety are available. The role of rhG-CSF in inducing cancer in previously healthy individuals is still uncertain. Nagler et al. (Exp Hematol. 2004 Jan;32(1):122-30) have shown that lymphocytes in rhG-CSF mobilized stem cell donors show epigenetic and genetic alterations which are comparable to those observed in patients suffering from malignomas. We therefore investigated in a prospective study the occurrence of changes in DNA methylation in lymphocytes of healthy unrelated donors after rhG-CSF administration.

Material and methods: In the present study, 20 stem cell donors (14 male/6 female, median age: 40 yrs, range 22-54) were included (active group). Twenty, gender and age-matched routine blood component donors (from our pool) served as untreated controls. Sampling was done before rhG-CSF (Filgrastim, Neupogen $®$ ) administration, at the time of donation and on days $+1,7,30$ 100,180 and 360 in both groups. After Ficoll gradient isolation, the mononuclear cell fraction was analyzed by flow cytometry after intracellular staining with an anti-methylcytidine antibody. Analysis of DNA-methyltransferase (DNMT) activity in nuclear extracts was performed using a modified radionuclide assay.

Results: At no time point, significant differences in overall DNMT activity and DNA methylation could be detected between the groups. The proportion of methylated lymphocytes varied between $95 \%$ and $99 \%$ in the active group and between $93 \%$ and $98 \%$ in the control group. Moreover, flow cytometric analysis of lymphocyte subpopulations revealed no differences in the extent of DNA-methylation between CD3 + T-cells and CD19+ B-cells. Conclusion: In our prospective study no evidence was found for the induction of DNMT activity or enhanced DNA-methylation after rhG-CSF administration in healthy stem cell donors.

\section{P1003}

Gene expression changes of haematopoietic progenitor cells collected from healthy donors

E. Spiniello, M. Cuzzola, M. Martino, G. Console, R. Fedele, E. Massara, T. Moscato, R. Surace, A. Meliadò, O. lacopino, G. Irrera, P. lacopino

AO Bianchi Melacrino Morelli (Reggio Calabria, IT)

Introduction: Hematopoietic progenitor cells (HPC) mobilization requires the administration of cytokines as granulocyte colony-stimulating factor (G-CSF). As more healthy donors are exposed to pharmacological doses of G-CSF, it is essential to know its effects to protect their safety. Accumulating evidence suggests that G-CSF in healthy subjects may exercise more 
complex and heterogeneous effects that originally thought. Therefore, changes in molecular pathways induced by mobilization and homing are not yet clear. The gene expression profile (GEP) of healthy donors was evaluated to better explain the effects of G-CSF. Moreover, in order to assess the duration of these effects, sequential studies were also performed. Method: Peripheral blood (PB) samples were harvested before and after G-CSF administration: at baseline, at +5 days and at +30 days post G-CSF. We used a TaqMan ${ }^{\circledR}$ Low Density Array based on comparative dd CT method to perform relative quantification of mRNA. Expression of each gene was normalized to the reference gene 18S mRNA, 1 unit was assumed as the normal reference value. About the project of macroarray card, we selected 47 candidate genes involved in immune network and inflammation pathogenesis.

Results: After G-CSF administration, we observed an increased expression of genes which specifically acts on endothelial cells: VEGFA, eNOs, MMP9. The transcriptome of Th1, Th2, TH17 response was characterized by no significant modulation. Similar behavior is showed by a cluster of genes coding for chemokines/cyclines, inflammation mediators. Only IL-10 was significantly up-regulated. Genes involved in apoptosis regulation (CASP1, CASP6, BCL2A1) were unchanged, except for transcription factors $(E G R-1,2)$ that were serially increased until +30 days. All others genes returned to baseline values at +30 days. The oncogenes JUN and FOS did not change.

Conclusion: Based on all information currently available, we believe that the administration of G-CSF to healthy donors in order to collect HPC continues to have a favourable risk-benefit profile. The increase of endothelial pattern might be due to an increased endothelial fenestration. In vivo, release of VEGF by progenitor cells may result in a biological mechanism which supports proliferation of endothelium and progenitors cells and may facilitate transendothelial migration during cytokineinduced HPC mobilization.

\section{P1004}

Serum IL-7 and IL-15 kinetics drive lymphopenia-induced proliferation after high-dose chemotherapy and autologous stem cell transplantation

D. Focosi, M. Metelli, M. Petrini

University of Pisa (Pisa, IT)

Introduction : Murine models have shown that interleukin (IL)-7 and IL-15 are required for the 2-step recovery of the absolute lymphocyte count (ALC) after high-dose chemotherapy, a phenomenon termed "Iymphopenia-induced proliferation (LIP)". No human study to date has ever validated preclinical models. We studied kinetics of serum IL-7 and IL-15 levels during high-dose chemotherapy supported by autologous hematopoietic stem cell transplantation (HSCT) in patients with multiple myeloma (MM), Hodgkin's lymphoma (HL) or non-Hodgkin lymphoma (NHL). Methods: Informed consent was collected from 60 MM (Mel100 or Mel200), $15 \mathrm{NHL}$ (BEAM) and $12 \mathrm{HL}$ (BEAM) patients undergoing autologous HSCT. Blood samples were collected at admission, and on days $+3,+6,+10$, and +40 after HSCT. Plasma IL-7 and IL-15 levels were measured by high sensitivity $(>0.1 \mathrm{pg} / \mathrm{ml}$ ) Quantikine ELISA kits (R\&D Systems, Minneapolis, MN) according to manufacturer's instructions.

Results: Serum IL-7 and IL-15 levels inversely correlated with the ALC. Serum IL-7 peaked at day 3 in Mel100 and day 6 in Mel200 and BEAM. Serum IL-15 peaked on day 10 in all subgroups. The degree of the peak directly correlated with the dose of melphalan administered during conditioning $(100 \mathrm{mg} /$ $\mathrm{m}^{2}<200 \mathrm{mg} / \mathrm{m}^{2}<$ BEAM)

Conclusion: The differential day of peak of serum IL-7 and IL15 is concordant with what is known from murine models about LIP. LIP is a dose-dependent phenomenon which in our model correlated linearly with increasing doses of melphalan. These results have implications for adoptive immunotherapy in the setting of high-dose chemotherapy.

\section{P1005}

Plerixafor for stem cell mobilization in poor mobilizers: results from the German Compassionate Use programme K. Hübel (1), M. Fresen (1), F. Lange (1), H. Salwender (2), N. Basara (3), C. Bogner (4), O. Galm (5), R. Hartwig (6), F. Heits (7), P. Petersen (8), W. Rösler (9), M. Albert (10), S. Dressler (11), M. Ebinger (12), N. Frickhofen (13), B. Hertenstein (14), M. Kiehl (15), S. Liebler (16), M. von Lilienfeld-Toal (17), E. Weidmann (18), C. Weigelt (19), N. Kröner (20)

(1)University Hospital of Cologne (Cologne, DE); (2)Asklepios Hospital Altona (Hamburg, DE); (3)University Hospital (Leipzig, DE); (4)Klinikum Rechts der Isar (Munich, DE); (5)University Hospital of the RWTH (Aachen, DE); (6)Katholisches Krankenhaus(Duisburg,DE);(7)DiakoniekrankenhausRotenburg (Rotenburg, DE); (8)Städtisches Klinikum (Braunschweig, DE); (9)University Hospital (Erlangen, DE); (10)Ludwig Maximilian University (Munich, DE); (11)Klinikum Nord (Nuremberg, DE); (12)University Hospital (Tubingen, DE); (13)Dr-Horst-SchmidtKliniken (Wiesbaden, DE); (14)Klinikum Bremen Mitte (Bremen, $D E) ;(15) K l i n i k u m$ Frankfurt an der Oder (Frankfurt/Oder, DE); (16)Philipps-University Hospital (Marburg, DE); (17)University Hospital (Bonn, DE); (18)Krankenhaus Nordwest (Frankfurt/ Main, DE); (19)Heinrich-Heine-University Hospital (Dusseldorf, $D E) ;$ (20)University Hospital of Hamburg (Hamburg, DE)

The CXCR4-inhibitor Plerixafor mobilizes hematopoietic stem cells (HSC), thereby amplifying the effects of granulocyte colony-stimulating factor (G-CSF). Before achieving European approval Plerixafor was applied in a compassionate use program (CUP) including patients who failed a prior mobilization. In the German CUP 48 patients (23 NHL, $15 \mathrm{MM}, 2 \mathrm{HL}, 8$ others; median age 58.5 years (range $4-75$ years); 20 centers) received Plerixafor $240 \mu \mathrm{g} / \mathrm{kg}$ SC 9-11 hours prior apheresis. $77.1 \%(37 / 48)$ of patients received G-CSF for a minimum of 4 days before Plerixafor administration; $70.3 \%(26 / 37)$ of these patients were able to collect at least $2.0 \times 10^{6} \mathrm{CD} 34+\mathrm{cells} / \mathrm{kg}$. The median cell yield was $2.8 \times 10^{6} \mathrm{CD} 34+\mathrm{cells} / \mathrm{kg}$ (range 0-24.79 cells $/ \mathrm{kg}$ ). Seven patients received Plerixafor alone or with G-CSF for less than 4 days mobilizing a median of $3.3 \times 10^{6}$ CD34 + cells $/ \mathrm{kg}$ (range 1.6-5.6 cells $/ \mathrm{kg}$ ). There was no significant difference between G-CSF application for four days compared to a shorter period of time $(P=0.68)$. Forty-two patients received Plerixafor plus G-CSF in combination with chemotherapy harvesting a median of $3.2 \times 10^{6} \mathrm{CD} 34+$ cells $/ \mathrm{kg}$ (range $0-24.79$ cells $/ \mathrm{kg}$ ). Thirty patients $(62.5 \%)$ proceeded to transplantation receiving a timely and stable engraftment. The most common side effects were gastrointestinal disorders $(6.25 \%)$ and exhaustion (4.17\%). In conclusion, HSC mobilization with Plerixafor in poor mobilizers is safe and results in a sufficient stem cell harvest in a majority of patients.

\section{P1006}

A low dose of IL-3 could enhance ex vivo expansion of cord blood committed progenitors in HP01 medium without impairing stem cell activity

Z. Ivanovic (1), P. Duchez (1), J. Chevaleyre (1), M. Vlaski (1), B. Dazey (1), X. Lafarge (1), E. Robert-Richard (2), F. Mazurier (2), J.-M. Boiron (1)

(1)Etablissement Francais Du Sang (Bordeaux, FR); (2)INSERM E0217 (Bordeaux, FR)

We upscaled (Duchez et al., J Hematother Stem Cell Res 12: 587,2003 ) the experimental procedure (Kobari et al, Exp Hematol 28: 1470, 2000) of cord blood CD34 + cell expansion in two-step clinical-scale cultures in presence of SCF, FLT3 ligand, MGDF (100 ng/ml each) and G-CSF (10 ng/ml).

To test our hypothesis (Ivanovic 2000, ECN 15: 6, 2004) that an appropriately low dose of IL-3 could enhance ex-vivo production of committed progenitors (CP) without impairing the stem cells, the expansion of CD34 + cells, selected from previously cryopreserved and thawed cord blood units by Isolex (Duchez et al., J Hematother Stem Cell Res 12: 587, 2003) 
or Miltenyi, (Ivanovic et al., Stem Cells. 22: 716, 2004)) was performed in two-step cultures (diluted 1:4 after 6 days) in presence of SCF, FLT3 ligand, MGDF (100 ng/ml each) and GCSF (10 ng/ml) $\pm \mathrm{IL}-3(0.5 \mathrm{ng})$ (VueLife culture bags) in Macopharma HP01 serum-free medium (Ivanovic et al., Transfusion 46: 126, 2006). The activity of stem cells has been evaluated on the basis of detection of human cells in NOD/Scid mice bone marrow (primary recipients) 6-8 weeks after transplantation (i.v.). The maintenance of very primitive stem cells was studied by a serial engraftment: the secondary recipients were engrafted by an intramedullary injection of primary recipient bone marrow cells. Six weeks later, injected femurs were analyzed (human CD45, CD33, CD19 as well as the number of clonogenic progenitors (CFC) of human-origin, as in primary recipients).

The low IL-3 dose used was $50 \%$ efficient (5 of 10 samples responded by doubling-tripling the expansion of total cells and CP with respect to cultures without IL-3). The activity of primitive stem cells-SRC (SCID Repopulating Cells) at the end of culture, was maintained at Day-0 level as judged either by the phenotypical markers (corresponding to relatively less primitive subpopulation of SRC), or by human CFC content in primary recipient bone marrow (SRCCFC, representing relatively more primitive subpopulation of stem cells). IL-3 did not impair the engraftment of secondary recipients (considered to be the measure of very primitive stem cells).

Thus, IL-3 applied at low dose in our clinical-scale serum-free cultures of CD34 + cord blood cells could enhance the expansion of CP without impairing stem cell activity.

\section{P1007}

Clinical and economic benefits of using pegfilgrastim post autologous stem cell transplant

S. O. Evans (1), F. Dignan (1), B.E. Shaw (2), F. Davies (1), M.A. Smith (1), C. Dearden (1), E. Matutes (3), M. Ethell (1), G.J. Morgan (1), M.N. Potter (1)

(1)The Royal Marsden Hospital (Sutton, UK); (2)Anthony Nolan Trust, London, The Royal Marsden Hospital (Sutton, UK); (3)The Royal Marsden Hospital (London, UK)

Background: Pegfilgrastim has a longer half-life than filgrastim (thus requiring only a single dose) and recent studies have shown equivalent efficacy in enhancing neutrophil recovery post autologous peripheral blood stem cell transplant (PBSCT). There are few data on the cost-effectiveness of pegfilgrastim in this setting.

Methods: To investigate this issue we compared healthcare resource utilisation for 53 consecutive patients receiving a single $6 \mathrm{mg}$ dose of pegfilgrastim on day +1 post transplant, to an historical control group $(n=53)$ who received daily filgrastim or no filgrastim (clinical decision). Resource utilisation was assessed by collating costs of the following interventions during admission: in-patient bed stay, critical care admission and usage of blood products, anti-infectives and parenteral nutrition.

Results: The median age was 57 years (range 19-72). 76 patients had myeloma (72\%), 19 Lymphoma (18\%), 11 other haematological malignancies. Conditioning was with melphaIan (78), BEAM (17), or other protocols (11). 20 patients were receiving a second PBSCT. Median CD34 + dose was $2.25 \times 10^{\%} /$ $\mathrm{kg}(0.94-7.18)$. Day 100 TRM was $1 \%$. There were no significant differences between the groups with regard to any of these factors. Filgrastim was administered to 32 patients in the control group (60\%) and median number of doses was 2 (range $0-7)$. There was a significantly reduced time to engraftment in the pegfilgrastim group versus the control group (median: 10 days (range: $8-28$ ) versus 13 days $(6-26) ; P<0.001)$ ). Likewise median in-patient stay was significantly reduced ( 17 days $(11-34)$ versus 19 days $(16-78) ; P<0.001)$. No adverse effects attributable to pegfilgrastim were observed. Reductions in time to engraftment and in-patient bed stay translated into the following cost savings: $13 \%$ on IV antibiotics, $40 \%$ on IV antifun- gals, $75 \%$ on parenteral nutrition, $23 \%$ and $30 \%$ on platelet and blood transfusions respectively, totalling $24,000 €^{*}$. These savings more than offset the drug acquisition costs of pegfilgrastim over baseline filgrastim $(16,600 €)$. In addition, approximately $150,000 €$ were saved by the reduction in haematology and critical care bed usage.

Conclusion: In our institution the use of pegfilgrastim post autologous PBSCT was cost effective and associated with clinical advantages compared to previous practice. ${ }^{\star} 2008$ conversion.

\section{P1008}

Effect of Th1/Th2 balance on PBPC allogeneic transplantation outcome

S.M. Lopes, I.L. Barbosa, S. Roncon, S. Ferreira, F. Amado, F. Campilho, A. Campos, A. Carvalhais

Instituto Portugues de Oncologia Porto (Porto, PT)

The immune system is a finely tuned tool that allows for the protection of the human body from both foreign and internal threats. One of the major mechanisms for this fine tuning is the balance between an inflammatory (type 1 or Th1) or a humoral (type 2 or Th2) response. Allogeneic PBPC transplantation has evolved to a true form of immunotherapy, relying on the immune system of the host to eliminate residual cancer cells and to prevent disease relapse.

In this work, we aimed evaluate the influence of IFN-g and IL-4 secreting T lymphocytes (Th1 and Th2 lymphocytes, respectively), present in the graft, in the outcome of allotransplanted patients.

We analyzed the PBPC grafts collected to 25 healthy adult donors, and the transplant outcome of the corresponding patients, in IPO Porto. All patients presented a malignant haematological disease, with a median age of 48 years (18-59), and were transplanted with a median CD34 + cell dose of $5.09 \times 10^{6} / \mathrm{kg}$. After the transplant, patients were followed for an average of 297 days (139-481) and evaluated for neutrophil and platelet recovery, lymphocyte recovery, degree of aGVHD and CGVHD, disease free survival (DFS) and overall survival (OS).

The group showed a median of 14 and 10 days to neutrophil and platelet recovery, respectively, while the median lymphocyte recovery was of 16 days. Of the 25 patients, 16 developed no aGVHD, 1 had grade I, 6 had grade II and 2 had grade III disease. In this group, 14 patients had no cGVHD, 3 developed limited and 8 extensive disease. Survival analysis showed that 7 patients experienced disease relapse with 4 eventually dying. An infused Th1 cell dose greater than $0,30 \times 10^{8} / \mathrm{kg}$ was associated with a quicker platelet engraftment (12 vs. 5 days) $(P<0.05)$. Neither the Th1 nor the Th2 subpopulations correlated with the degree of aGVHD. Nevertheless, patients infused with a higher dose of Th2 cells developed more extensive cGVHD $(P<0.05)$. The patient's survival wasn't affected by any of the populations studied.

Due to relative small size of the population studied, careful data interpretation is needed. Despite not affecting the survival, the dose of Th1 and Th2 cells infused affected the patient's engraftment and severity of cGVHD developed, showing that a type 1 response is associated with better engraftment and less severe cGVHD, which is consistent with published works.

We can conclude that the Th1/Th2 balance of the PBPC graft does affect the outcome of an allogeneic transplant.

\section{P1009}

Higher concentration of IL-10 in bone marrow-derived mesenchymal stem cell cultures from healthy donors as compared to their recipients

K. Drabko, D. Winnicka, A. Bojarska-Junak, J. Kowalczyk Medical University of Lublin (Lublin, PL)

Aim of the study: In the study we aimed to compare the levels of IL-2, IL-4,IL-10 and TNF-alpha in bone marrow and in 
supernatant of mesenchymal stem cells cultures (CFU-F) from pediatric hematopoietic stem cell transplantation recipients and their donors.

Material and methods: 15 consecutive donors and 12 recipients of allogeneic stem cell transplantation in our institution were included into the study. Samples of bone marrow were obtained from the recipients at the time of pre-transplant evaluation before conditioning regimen. Samples from the donors were obtained at the time of transplantation. Cytokines levels were measured using high sensitivity human immunoassay (R\&D and Bender) in bone marrow plasma and in supernatant form CFU-F cultures. CFU-F cultures were performed according to research protocol and reagents provided by manufacturer (StemCell Technologies). Three cultures were performed using $0,5 \times 10^{6}, 1 \times 10^{6}$ and $2 \times 10^{6}$ mononuclear cells isolated from each bone marrow sample.

Results: Level of IL-10 was significantly higher in supernatant of CFU-F then in bone marrow plasma derived from healthy donors, irrespective of the concentration of cells in the culture $(P<0,05)$. In treated patients increase of IL-10 concentration in CFU-F as compare to bone marrow plasma was not significantly different. II-2 concentrations were significantly increased in CFU-F supernatant as compared to bone marrow plasma in both: patients and donors, however significant negative correlation was found between number of CFU-F in the culture and II-2 concentration $(r=-0,84)$. Levels of IL-4 and TNF-alpha were not significantly different in CFU-F and bone marrow plasma in our study.

Conclusion: Results of our study confirm, that the immunomodulatory effect of bone marrow derived mesenchymal stem cells may be dependent on IL-10. Further study are planned to explain the difference between MSCs from patients and healthy donors regarding IL-10 secretion.

Supported by grant of MNISW of Poland N407 11735.

\section{P1010}

Plerixafor plus G-CSF can successfully mobilize CD34 + cells from patients who have previously failed chemotherapy and/or cytokine mobilization: the compassionate use experience in Austria N. Worel (1), K. Rosskopf (2), D. Nachbaur (3), H. Kasparu (4), G. Russ (5), K. Namberger (5), G. Leitner (1), P. Kalhs (1), E. Schögl (6), V. Witt (7), H. Greinix (1), F. Keil (8), W. Linkesch (2) (1)Medical University of Vienna (Vienna, AT); (2)Medical University of Graz (Graz, AT); (3)Medical University of Innsbruck (Insbruck, AT); (4)KH Elisabethinen (Linz, AT); (5)St. Johanns Hospital (Salzburg, AT); (6)Hanusch Hospital (Vienna, AT); (7)St.Anna Childrens Hospital (Vienna, AT); (8)KH Leoben (Leoben, AT)

The failure to harvest a CD34+ cell target dose of $2 \times 10^{6} / \mathrm{kg}$ for autologous hematopoietic stem cell transplantation (HCT) remains a significant problem for many transplant centres. Recently, Plerixafor+G-CSF has shown to be safe and more effective than placebo+G-CSF in mobilizing hematopoietic stem cells. On a named-patient basis, Plerixafor has been used in patients failing to mobilize stem cells by conventional means. We present our initial experience with Plerixafor in 33 mobilization attempts in 30 patients with either myeloma $(n=8)$, lymphoma $(n=19)$ or germ cell cancer $(n=3)$ pretreated with a median of 8 (range, 3-27) chemotherapy cycles who failed at least one previous mobilization attempt. In four patients bone marrow harvest had been performed prior to a renewed mobilization attempt resulting in a CD34 + cell dose of $0.16-0.89 \times 10^{6} / \mathrm{kg}$. In the compassionate use program patients received four days of G-CSF at $10 \mu \mathrm{g} / \mathrm{kg}$ followed by Plerixafor at $240 \mu \mathrm{g} / \mathrm{kg}$ subcutaneously, 10-11 hours prior to first apheresis. G-CSF and Plerixafor were repeated daily until a defined cell dose of at least $>2 \times 10^{6} \mathrm{CD} 34+$ cells $/ \mathrm{kg}$ was obtained. Between days 4 and 5 of cytokine stimulation the CD34 + cells in peripheral blood increased a median of 5 fold (range, 1-12), reaching 24 (range, 5-61) CD34 + cells/ $\mu$ l after Plerixafor administration.
Twenty-one of 30 patients $(70 \%)$ achieved the target cell dose of $>2 \times 10^{6} \mathrm{CD} 34+$ cells $/ \mathrm{kg}$ (median 4.15 , range $2.03-8.07 \times 10^{6}$ CD34 + cells $/ \mathrm{kg})$ after one $(n=20)$ or two $(n=1)$ courses of Plerixafor stimulation. In 6 patients $(20 \%)$ an insufficient cell dose of 0.42 to $1.46 \times 10^{6} \mathrm{CD} 34+$ cells $/ \mathrm{kg}$ were collected and 3 patients $(10 \%)$ failed to mobilize CD34 + cells. Patients failing Plerixafor re-mobilization had a median of 10 (range, 5-18) cycles of pretreatment with $\mathrm{NHL}(\mathrm{n}=7)$ and $\mathrm{MM}(\mathrm{n}=2)$. Common side effects to G-CSF + Plerixafor stimulation included abdominal discomfort $(n=3)$, diarrhoea $(n=5)$, and paresthesia $(n=1)$. Thirteen patients underwent HCT and received a median of $3.19 \times 10^{6}$ (range, 1.46-5.6) CD34 + cell dose/kg without side effects. All patients achieved sustained neutrophil and platelet engraftment a median of 12 (range, 9-16) and 15 (range, 9-38) days after stem cell infusion. In our experience, Plerixafor appears highly effective for the mobilisation of hematopoietic stem cells for transplantation in patients failing to mobilize by conventional means, with generally acceptable toxicity and a high rate of success.

\section{P1011}

Rescue from failed growth factor and/or chemotherapy haematopoietic stem cell mobilization with G-CSF and Plerixafor in patients with non-haematologic diseases N. Worel (1), K. Hübel (2), H. Agis (1), W. Rabitsch (1), G. Russ (3), N. Frickhofen (4), P. Kalhs (1), H. Greinix (1) (1)Medical University of Vienna (Vienna, AT); (2)Medical University of Cologne (Cologne, DE); (3)St. Johanns Hospital (Salzburg, AT); (4)HSK (Wiesbaden, DE)

Autologous hematopoietic stem cell transplantation (HCT) can be a curative procedure for a variety of haematological and oncological malignancies. Successful HCT is dependent on transplantation of sufficient CD34 + cells to ensure prompt and durable engraftment. However, a number of patients fail to mobilize the threshold of $>2 \times 10^{6} / \mathrm{kg}$ CD34 + cells. Recently, Plerixafor + G-CSF (G) has shown to be safe and more effective than placebo $+G$ in mobilizing $C D 34+$ cells in patients with $\mathrm{MM}$ and Iymphoma. In addition, Plerixafor has been used in a named-patient basis for PBSC mobilization from patients failing to mobilize by conventional means. The aim of this report is to evaluate the effect of Plerixafor $+G$ among patients with non-hematologic diseases. Patients with previous CD34 mobilization failure were included in this analysis. Mobilization consisted of 4 days of $\mathrm{G}$ at $10 \mu \mathrm{g} / \mathrm{kg} / \mathrm{d}$ followed by Plerixafor at $240 \mu \mathrm{g} / \mathrm{kg}$ on day 4, 10-11 hrs prior to first apheresis. $\mathrm{G}$ and Plerixafor were repeated daily until a defined cell dose of at least $2 \times 10^{6}$ CD $34+$ cells $/ \mathrm{kg}$ was obtained. Nine patients with a median age of 50 years diagnosed with germ cell (7) and ovarian cancer (1) and Ewing sarcoma (1), were enrolled. Pretreatment consisted of a median of 5 (range, 5$20)$ chemotherapy cycles. In 2 patients previous stem cell collection resulted in $0.9 \times 10^{6} / \mathrm{kg}$ CD34 + cells prior to a renewed mobilization attempt. Between day 4 and 5 (10-11 hrs after Plerixafor), the CD34 + cells in the peripheral blood increased a median of 5 fold (range, 1-24), reaching 33 (range, 12-52) CD34 + cells $/ \mu \mathrm{l}$

Eight of $9(89 \%)$ patients achieved the target cell dose of $2 \times 10^{6}$ $\mathrm{CD} 34+$ cells $/ \mathrm{kg}$ together with previous harvested cells (median 3.19 , range $1.8-25.33 \times 10^{6} / \mathrm{kg}$ CD34 + cells) after $1(2), 2(6)$ or $4(1)$ days of collection, 1 patient failed to collect an insufficient cell dose $\left(0.89 \times 10^{6} \mathrm{CD} 34+\right.$ cells $\left./ \mathrm{kg}\right)$. Common side effects to $\mathrm{G}+$ Plerixafor stimulation included abdominal discomfort (1) and diarrhoea (2). Three patients underwent HCT receiving a median of 3.36 (range, 2.6-54.14) $\times 10^{6} / \mathrm{kg}$ CD34 + cells. All patients achieved sustained neutrophil and platelet engraftment between days 10-12 and on day 10 after HCT, respectively. These data demonstrate that Plerixafor $+G$ is effective in patients with non-hematologic diseases after mobilization failure. In patients eligible for HCT who have failed prior mobilization attempts, Plerixafor can provide an opportunity to still pursue a potentially curative procedure. 


\section{P1012}

The kinetics of transforming growth factor beta1 mRNA expression and its serum concentration in graft-versus host disease after allogeneic haemopoietic stem cell transplantation for myeloid leukaemias

P. Zielinska, G. Helbig, A. Wieczorkiewicz, M. Markiewicz, S. Krzemien, U. Mazurek

Silesian Medical University (Katowice, PL)

Introduction: Graft-versus-host disease (GVHD) still remains one of the major complication following allogeneic haematopoietic stem cell transplantation (alloHSCT). Recent data indicates that transforming growth factor beta1 (TGF-beta1) may play a role in the development of GVH reaction.

Patients and methods: The study included 40 adult patients who underwent allogeneic bone marrow transplantation for myeloid leukemias between 2003-2005. A median follow-up was 28 months (2-39). In all cases, the expression of TGFbeta1 mRNA by real-time quantitive polymerase chain reaction (RT-QPCR) using ABI PRISMTM 7700 (Taqman) and TGFbeta1 serum concentration by commercial ELISA were measured fourfold in the peritransplant period (on days: $-10,0,+30$ and +100 ).

Results: Acute GVHD (aGVHD) experienced 20 patients, in 4 patients the symptoms were classified as severe (grades IIIIV). Chronic GVHD (cGVHD) occurred in 11 patients (in 4 in diffuse form). In all patients a prompt and significant decrease in TGF-beta1 mRNA expression and its serum concentration was demonstrated after conditioning regimen $(P<0.005$ and $P<0.00006$, respectively). In patients who developed symptoms of acute GVHD, TGF-beta1 mRNA expression and its serum concentration remained low until day +30 after transplant if compared to the transplant day $(P<0.03$ and $P<0.006$ respectively). TGF-beta1 mRNA expression and its serum concentration significantly increased on day +100 if compared to the transplant day $(P<0.0009$ and $P<0.02$, respectively) in patients who developed chronic GVHD.

Conclusions: We demonstrate the kinetics of TGF-beta1 changes both on molecular and protein level in patients who underwent alloHSCT for acute myeloid leukemias. These results show that TGF-beta1 is likely to be an additional regulator of donor engraftment, probably its low levels contributing to the development of acute GVHD, which may suggest that TGF-beta1 exerts beneficial effects in the early post transplant period. In contrast, chronic GVHD symptoms seem to correlate significantly with high TGF-beta1 levels, suggesting that TGFbeta1 is likely to increase the risk of cGVHD by causing fibrosis of target organs, which remains in coherence with TGF-beta1 profibrotic activity.

\section{P1013}

Influence of combined application recombinant human erythropoietin (rH-EPO) and granulocyte colony stimulating factor (G-CSF) on haemopoetic recovery after high-dose chemotherapy with autologous stem cell transplantation

H. Kuzmich, V. Zmatchinski, N. Novoselova

Research Institute of Haematology, United Institute of Informatics Problems (Minsk, BY)

From 1994 to 2006 years in Belarus haematology and transplant centre 132 patients have been treated by method high dose chemotherapy (HDC) with autologous stem cell transplantation (SCT). All patients have been divided into 2 groups. The first group (control; $n=90$ ) did not receive any haemopoietic growth factors, and the second group $(n=42)$-combination of recombinant human erythropoietin (rH-EPO) (10000 UI per dose three times a week) and granulocyte colony stimulating factor (G-CSF) (300 mkg per day).

Criteria of haemopoesis restoration after autologous SCT were following: $\mathrm{Hb}>90 \mathrm{~g} / \mathrm{l}$, leukocytes $>1,0 \times 10^{9} / \mathrm{l}$, neutrofiles $>0,5 \times 10^{9} / \mathrm{l}$. For comparison of efficiency of haematologic recovery in investigated groups used day of achievement specified above indicators of peripheral blood after autologous SCT. Time of $\mathrm{Hb}$ restoration in group of patients received $\mathrm{rH}-\mathrm{EPO}+\mathrm{G}$ CSF was $8,0+4,6$ vs. $11,2+6,8$ in control group $(P=0,006)$. Time of leukocytes restoration in group of patients received $\mathrm{rH}-\mathrm{EPO}+\mathrm{G}-\mathrm{CSF}$ was $9,3+1,3$ vs. $12,2+3,1$ in control group $P=0,0001)$. Time of neutrofiles restoration in group received rH-EPO + G-CSF was $9,3+1,4$ vs. $13,0+3,8$ in control group $(P=0,0001)$.

For an estimation of age influence on haemopoesis restoration after autologous SCT patients have been divided into 3 subgroups by age sign: till 30 years, from 31 till 40 years, senior than 40 years.

Time of restoration of $\mathrm{Hb}$ in the senior age group (> 40 years), received $\mathrm{rH}-\mathrm{EPO}+\mathrm{G}-\mathrm{CSF}$ was shorter $(6,3+5,1$ vs. $11,6+5,6$; $P=0,03)$ than in control group. Terms of leukocytes and neutrofiles restoration were shorter in all age groups $(P<0,05)$.

Thus, efficiency of rH-EPO application after HDC and autologous SCT was most expressed at patients senior than 40 years.

\section{P1014}

Plerixafor plus G-CSF can mobilize autologous haematopoietic stem cells from heavily pre-treated patients failing previous mobilization attempts: analysis of the French compassionate use programme

M. Mohty (1), F. Lefrere (2), D. Caillot (3), N. Azar (4), J.M. Miclea (5), F. Broussais (6), J.P. Marolleau (7), M. Attal (8), N. Milpied (9), C. Gisselbrecht (5), P. Moreau (1), C. Chabannon (10)

(1)CHU Hôtel-Dieu (Nantes, FR); (2)Hopital Necker (Paris, FR); (3)CHU de Dijon (Dijon, FR); (4)Hopital La Pitie-Salpetriere (Paris, FR); (5)Hopital Saint Louis (Paris, FR); (6)CHU de Lyon (Lyon, FR); (7)CHU d'Amiens (Amiens, FR); (8)CHU de Toulouse (Toulouse, FR); (9)CHU de Bordeaux (Bordeaux, FR); (10)Institut Paoli-Calmettes (Marseille, FR)

Classical haematopoietic stem cells (HSCs) mobilization approaches (G-CSF \pm chemotherapy) result in mobilization failures in a significant number of cases. Plerixafor, a CXCR4 antagonist, has gained recently EMEA approval in combination with G-CSF to enhance mobilisation of HSCs in patients with lymphoma and multiple myeloma whose cells mobilise poorly, adding to the HSC mobilizing regimens currently available. However, the role of Plerixafor in mobilization failures in the most heavily pre-treated patients, which were excluded from registration trials, is still under investigation.

We present here the first analysis of the French Plerixafor compassionate use program (called "ATU") which included 39 consecutive patients with mobilization failure prior to receiving Plerixafor.

Patients characteristics were as follow: median age 58 (range, 4-77) y.; 22 men (56\%); 22 myeloma (56\%), 14 lymphomas $(36 \%)$ and 3 other diagnoses (8\%); median weight 73 (range, $47-100) \mathrm{Kg}$. Prior to Plerixafor administration, patients received a median of 2 lines of chemotherapy (range, 1-6) and a median number of 7 (range, $4-18$ ) therapy cycles. 3 patients (8\%) also received radiation therapy. OF NOTE, per program, patients needed to have failed at least TWO mobilization procedures before being eligible to receive Plerixafor + G-SCF.

The median delay between the last mobilization attempt and Plerixafor administration was 44 (range, 16-664) days. During the last mobilization attempt prior to salvage with Plerixafor, the median number of circulating CD34 + cells achieved was 4 (range, $0-21$ ) cells $/ \mu \mathrm{L}$. During mobilization with Plerixafor, patients received a median of 2 (range, $1-4$ ) injections. No Plerixafor-related severe or serious adverse events were observed. After the first injection of Plerixafor, the median number of circulating CD34 + cells achieved, was 21 (range, $0-126$ ) cells $/ \mu \mathrm{L}$, and the median number of collected CD34 + stem cells after the first aphaeresis session was 2.12 (range, $0.3-10) \times 10^{6} / \mathrm{Kg}$. The median total number of collected CD34 + cells after salvage 
with Plerixafor+G-CSF was 2.5 (range, $0.3-15.2) \times 10^{6} / \mathrm{Kg}$. Overall, 14 patients were not able to collect $\geq 2.0 \times 10^{6} \mathrm{CD} 34+/$ $\mathrm{Kg}$ despite salvage mobilization with Plerixafor, yielding a success rate of $64 \%(95 \% \mathrm{Cl}, 49-79 \%)$.

We conclude that Plerixafor in combination with G-CSF is an efficient salvage mobilization approach to collect sufficient autologous CD34 + cells from heavily pre-treated patients who have failed several classical mobilization methods.

\section{P1015}

The addition of plerixafor allows adequate PBSC collection in multiple myeloma and lymphoma patients poor-mobilizers after chemotherapy and G-CSF

A. D'Addio (1), A. Curti (1), N. Worel (2), M.R. Motta (1), S. Rizzi (1), E. Dan (1), S. Taioli (1), V. Giudice (3), H. Agis (4), G. Kopetzky (5), M. Baccarani (1), R.M. Lemoli (1)

(1)University of Bologna (Bologna, IT); (2)Medical University of Vienna (Vienna, AT); (3)S.Orsola-Malpighi Hospital (Bologna, IT); (4)Medical University (Vienna, AT); (5)General Hospital (St. Pölten, AT)

Peripheral blood stem cell (PBSC) mobilization and subsequent autologous stem cell transplantation (ASCT) is the most effective strategy in many patients with Multiple Myeloma (MM), Hodgkin's Disease (HD) or non-Hodgkin's Lymphoma (NHL). However, a significant proportion of patients are not able to mobilize an adequate number of cells after conventional stem cell mobilization strategies [i.e. chemotherapy and granulocyte colony-stimulating factor (G-CSF) or G-CSF alone], and can not proceed to ASCT.

In the present work, we report 8 heavily pre-treated or treatment refractory MM or lymphoma patients (4 males and 4 females, median age 57 years) who were failing PBSC mobilization, after disease-specific chemotherapy (DHAP, $=2$; Cyclophosphamide, = 4; R-ICE, =2) and G-CSF but PBSCs were successfully harvested after addition of Plerixafor. Study patients showed a peak concentration of PB CD34 + cells lower than 10 cells/ $\mu \mathrm{L}$ during the recovery phase after chemotherapy and G-CSF and/or were predicted to have inadequate PBSC collection to proceed to the planned stem cell transplantation program.

The timing for Plerixafor administration was not planned in advance. The rapid biological activity of Plerixafor (i.e. peak time of CD34 + cell mobilization occurring 10-12 hours after injection) allowed its administration when PBSC mobilization was felt to be inadequate, considering WBCs and CD34 + cell kinetics.

Plerixafor $(0.24 \mathrm{mg} / \mathrm{Kg})$, was administered subcutaneously for up to 3 consecutive days (median injections $=2$ ), while continuing G-CSF, 10-11 hours before the planned leukapheresis. Plerixafor was safe and no significant adverse events were recorded. We observed a remarkable fold-increase (median value $=4$ ) in the number of circulating CD34 + cells following Plerixafor as compared to baseline CD34+ cell concentration. In all patients more than $2 \times 10^{6} \mathrm{CD} 34+$ cells/Kg were collected. Four/8 patients collected the target cell dose: $4 \times 10^{6}$ CD34+ cells/Kg for single ASCT in NHL and $6 \times 10^{6} \mathrm{CD} 34+$ cells $/ \mathrm{Kg}$ for tandem ASCT in MM with a median of 2 apheresis.

Four patients have already undergone ASCT with Plerixaformobilized PBSCs showing a rapid and durable hematological recovery. Our results demonstrate that the addition of Plerixafor to G-CSF, after tumor-specific chemotherapy, is safe and allows the rescue of heavily pre-treated or poor-prognosis $\mathrm{NHL}$ and MM patients who need to perform ASCT as consolidation therapy but are failing stem cell mobilization.

\section{P1016}

The use of plerixafor in Czech transplant centres

Z. Koristek (1), F. Folber (1), D. Lysák (2), D. Pohlreich (3), M. Lánská (4), Z. Rusináková (5), T. Papajík (5), T. Kepák (1), J. Novák (6), J. Mayer (1), J. Erba (1)

(1)University Hospital Brno (Brno, CZ); (2)Charles University Hospital Pilsen (Pilsen, CZ); (3)Charles University Hospital (Prague, CZ); (4)University Hospital Hradec Králové (Hradec Králové, CZ); (5)University Hospital Olomouc (Olomouc, CZ); (6)University Hospital Královské Vinohrady (Prague, CZ)

Approximately $20 \%$ of patients with hematologic malignancies fail to mobilize adequate numbers of peripheral blood progenitor cells (PBPC) and therefore cannot receive high-dose chemotherapy that requires PBPC support. Plerixafor, a novel CXCR4 antagonist could be recently used with G-CSF in heavily pretreated or not mobilizing patients as a new stem cell mobilizing agent inhibiting binding of stromal-derived factor- $1 a$ to its receptor CXCR4 present on CD34 + cells. Thanks to Genzyme Company Named Patient Program we have had an opportunity to use plerixafor in 7 Czech transplant centers (see above).

Our aim was to assess the safety and mobilizing potential of plerixafor in 35 patients of median age of 63 years (7-72) with myeloma $(n=17)$, NHL $(n=15)$, Hodgkin lymphoma $(n=1)$, and pediatric solid tumors $(n=2)$. Plerixafor was used together with G-CSF for the first mobilization in 10 heavily pretreated patients, as a second mobilization attempt in 22 previously poor mobilizers, and in 3 patients as a boost at the end of usual mobilizing regimen (chemotherapy + G-CSF) because of poor mobilization (in 1 patient was plerixafor used twice). Mobilization using plerixafor was successful in 25 patients $(71 \%)$ and median number of collected CD34 + cells was 4,9 $\times 10^{6} / \mathrm{kg}(1,3-$ $37,6)$. All patients who have been already transplanted, recover hemopoiesis quickly and completely. The adverst events were slight and were observed in 10 patients $(28 \%)$, mainly as musculoskeletal pains (14\% of patients) and diarrhea or nauzea (14\% of patients). The concentration of CD $34+$ cells in blood before apheresis correlated closely and significantly with the leukapheresis yield $(r=0,89)$.

Based on our experience, we can conclude that plerixafor is very valuable tool for obtaining PBSC in heavily pretreated or previously poorly mobilizing patients. More then two thirds of poor mobilizers could be, in our hands, successfuly mobilized using plerixafor and having a safe autologous transplant we can offer them high-dose chemotherapy which could be potentialy curative.

\section{P1017}

Stem cell mobilization with plerixafor in poorly mobilizing myeloma and lymphoma patients - A single-centre experience and strategies

G.W. Basak (1), E. Urbanowska (1), M. Witkowska (2), D. Zdunczyk (2), A. Waszczuk-Gajda (2), K. Skwierawska (2), J. Drozd (2), M. Skibinska (2), M. Glazer (2), K. Madry (1), K. Halaburda (1), T. Torosian (2), M. Krol (2), W. WiktorJedrzejczak (1)

(1)Medical University of Warsaw (Warsaw, PL); (2)Central Clinical Hospital (Warsaw, PL)

Plerixafor is a new CXCR4 receptor inhibitor, which is used for stem cell mobilization in myeloma and lymphoma patients. Since April 2009, we performed 18 mobilizations in 16 patients using plerixafor in combination with G-CSF. 10 patients suffered from multiple myeloma, 3 from Non-Hodgkin's lymphoma and 3 of Hodgkin's lymphoma. Median age was 59 years (range, 19-71) and 9 of patients were males. They have been treated with median of 10 courses of chemotherapy (range, 4-27) and radiotherapy (5 patients). 5 of patients already underwent autologous stem cell transplantation. The majority of them failed at least one mobilization attempt, while in three patients we added plerixafor to the first-line chemotherapy-based mobilization. This approach was based on their predicted poor mobilizer 
status confirmed by low peripheral blood CD34 + cell count. At the time of mobilization, only 3 patients were in complete remission while the majority had active disease. The majority of patients were treated according to protocol consisting of daily s.c. injections of G-CSF $(10 \mathrm{ug} / \mathrm{kg})$ and plerixafor $(240 \mathrm{ug} / \mathrm{kg})$ on day 4,5 and 6 . Plerixafor was administered about 11.5 hours before planned leukapheresis. The mobilization regimen resulted in satisfactory peripheral blood CD34 + cell number (median 22 cells/ul; range, 11-62). However due to high leukocytosis (median 43.3G/L; range, 11.4-72.5) their proportion was low (median $0.075 \%$, range $0.030-0.215$ ). We managed to collect the minimum required No. of $>2.0 \times 10^{6} \mathrm{CD} 34+\mathrm{cells} / \mathrm{kg}$ b.w. in $12 / 16$ patients $(75 \%)$. The median CD34 + cell number was $2.6 \times 10^{6} \mathrm{CD} 34+$ cells $/ \mathrm{kg}$ b.w. (range, $0-4.5 \times 10^{6} \mathrm{CD} 34+$ cells $/ \mathrm{kg}$ b.w.) collected in median of 3 leukaphereses (0-3). It was associated with high total nucleated cell (NC) count (median $9.35 \times 10^{8} \mathrm{NC} / \mathrm{kg}$ b.w.; range 0-24.05). This resulted in high volumes of frozen stem cell product (median $1280 \mathrm{~mL}$; range $0-2050$ ). The median No. of BFU-E was $3.84 \times 10^{4}$ (range, 1.03-16.09) and No. of CFU-GM + GEMM was $3.12 \times 104 / \mathrm{kg}$ b.w. (range, 2.08-13.76). The contamination of stem cell product with plasma cells in MM patients was not remarkable in majority of patients $\left(0.28 \times 10^{6} \mathrm{CD} 138+\right.$ cells $/ \mathrm{kg}$; range, $\left.0-.67\right)$. 10 of patients have already been transplanted and median time to neutrophil (>0.5 G/L) engraftment was 13 days (11-14) and platelet engraftment (>20 G/L) 14 days (10-15). In conclusion, stem cell mobilization with plerixafor is efficient and may find application even in poorly mobilizing patients.

\section{P1018}

\section{Effective stem cell mobilization with plerixafor +} G-CSF followed by large volume leukapheresis in poor mobilizers: the experience of the Croatian cooperative group for haematologic diseases (KROHEM)

O. Jaksic (1), S. Basic-Kinda (2), I. Maricic (1), I. Bojanic (2), D. Nemet (2), V. Pejsa (1), B. Labar (2)

(1)Dubrava University Hospital (Zagreb, HR); (2)University Hospital Center Zagreb (Zagreb, HR)

Autologous stem cell transplantation is an important treatment option for a number of hematological malignancies as well as some other malignancies and autoimmune diseases. Main source of hematopoietic stem cells is peripheral blood (PBSC). Our standard approach to mobilize adequate number of PBSC includes chemotherapy+G-CSF and large volume leukapheresis (LVL) to further improve PBSC yield. This approach is quite effective, however in a number of cases mobilization failure still occurs. In this report we have evaluated efficacy of plerixafor + G-CSF followed by LVL in a group of patients that previously failed to mobilize by our standard approach including LVL.

There were 11 patients $(4 \mathrm{M}, 7 \mathrm{~F})$, median age 59yr (range 26-63 $y r)$ with multiple myeloma $(n=2), \mathrm{NHL}(n=7)$ Hodgkin lymphoma $(n=1)$ and testicular cancer $(n=1)$. They were pretreated with median 2 lines of chemotherapy (range 2-5). Standard protocol for mobilization was the one proposed by manufacturer and included G-CSF $(10 \mu \mathrm{g} / \mathrm{kg} \mathrm{SC})$ for 4 days, followed by plerixafor administration $(240 \mu \mathrm{g} / \mathrm{kg}$ ) in the evening on day 4 (11 hours before apheresis) and G-CSF (10 $\mathrm{gg} / \mathrm{kg} \mathrm{SC})$ administered on day 5 , one hour before apheresis. Plerixafor administration, G-CSF administration and apheresis continued until the patient collected sufficient number of CD34+ cells. Total of $9(82 \%)$ patients were successfully mobilized with plerixafor achieving minimum of $\geq 2 \times 10^{6} \mathrm{CD} 34+$ cells $/ \mathrm{kg}$ while $6(55 \%)$ patients achieved $\geq 5 \times 10^{6} \mathrm{CD} 34+$ cells $/ \mathrm{kg}$ with median number of 2 apheresis (range 1-4). Total of 22 apheresis procedures were performed with mean of 4.96 blood volumes (BV) processed (range 4.3-6.0). Mean CD34 + yield per apheresis procedure was $2.65 \times 10^{6} / \mathrm{kg}$ (range $0.15-13.44$ ). Mean CD34 + yield per apheresis per BV was $0.52 \times 10^{6} / \mathrm{kg}$. Subsequently 7 patients were successfully transplanted and there was 1 tandem transplant. Median number of CD34 + cells infused $\left(\times 10^{6} / \mathrm{kg}\right)$ was 5.64 (range 4.74-6.96). Median time to leukocyte recovery
(WBC $>1$ ) was 10 days and median time to platelet recovery $(>20)$ was 14 days.

In conclusion, plerixafor with G-CSF is effective mobilization regimen that increases peripheral blood CD34 + count (median $2.9 \times$ following plerixafor) resulting in collection of adequate cell dose with LVL in patients who previously failed to do so with standard protocols with LVL (9/11). Mobilization with plerixafor is safe and subsequent transplantation and engraftment is consistent with our prior results.

\section{P1019}

Addition of plerixafor to a chemotherapy plus granulocyte-colony stimulating factor mobilization in hard-to-mobilize patients

E. Jantunen, K. Penttilä, M. Pyörälä, E. Mahlamäki, T. Nousiainen

Kuopio University Hospital (Kuopio, FI)

A significant proportion of patients with lymphoid malignancies are hard-to-mobilize with currently used mobilization regimens including granulocyte-colony stimulating factor (G-CSF) with or without chemotherapy (CT). Plerixafor combined with G-CSF has been shown to be more effective in blood stem cell mobilization than G-CSF alone and has been used also in patients who have failed a previous mobilization attempt. Limited data is available on the addition of plerixafor to CT+G-CSF mobilization in hard-to-mobilize patients.

We have used add-on plerixafor in five patients with lymphoid malignancies after CT + G-CSF mobilization. There were three males and two females with a median age of 64 years (55-68). Four patients had non-Hodgkin lymphoma and one patient multiple myeloma. Two patients had failed a previous mobilization course (CT+G-CSF). The mobilization regimen was high-dose cytarabine plus G-CSF in three patients and cyclophosphamide plus G-CSF in two patients. B-CD34 + counts were low $\left(<1-3 \times 10^{6} / l\right) 11-15$ days after start with mobilization in all but one patients who had decreasing B-CD34 + counts $\left(7 \times 10^{6} / \mathrm{l}\right)$. The patients received $1-3$ injections of plerixafor (median 2). B-CD34 + counts rose in four patients to $9,39,39$, and $40 \times 10^{6}$ / I, respectively; one patient did not respond. In four out of five patients the collections were successful after addition of plerixafor. The number of collections was one in two patients (2.1 and $3.1 \times 10^{6} / \mathrm{kg} \mathrm{CD} 34+$ cells, respectively), two in one patient $\left(4.0 \times 10^{6} / \mathrm{kg} \mathrm{CD} 34+\right.$ cells $)$ and four in one patient $\left(2.2 \times 10^{6} / \mathrm{kg}\right.$ CD34 + cells). Two patients have received BEAM with stem cell graft support with successful engraftment.

Addition of plerixafor to a mobilization regimen consisting of chemotherapy plus G-CSF in poor mobilizers is efficient and frequently leads to successful collection to facilitate high-dose therapy with blood stem cell support.

\section{P1020}

Subpopulation cell mobilized with plerixafor and granulocyte colony-stimulating factor J. Vela-Ojeda, F. Tripp-Villanueva, J. Ayala, AG. RamírezAlvarado, E. Gil, L. Montiel

IMSS (Distrito Federal, MX)

Background: Plerixafor (AMD3100) functions as a reversible inhibitor of Stromal cell-Derived Factor-1 (SDF-1) and its chemokine receptor (CXCR4) given with granulocyte colonystimulating factor (G-CSF) has been shown to mobilize CD34 + cells in non-Hodgkin's lymphoma (NHL), multiple myeloma (MM), and Hodgkin's disease (HD) patients who could not collect sufficient cells for autologous transplant following other mobilization regimens

Objective: Measure cell populations mobilized with plerixafor in a patient who previously failed G-CSF alone and chemotherapy mobilization

Methods: This is a case report study, the patient provided written informed consent. We measured cell populations 
mobilized with Plerixafor and G-CSF in a 32 years old patient with a previous diagnosis of Follicular Lymphoma Grade I, Ann Arbor Stage IVB, Intermediate Risk Group of Follicular Lymphoma International Prognostic Index, in second Complete Remission after receive 6 cycle of C Cyclophosphamide, Epirubicin, Vincristine, Prednisona (CEOP)-Rituximab, 4 cycle of Mesna Ifosfamide Mitoxantrone, Etoposide (MINE) with disease progression, and 2 cycle of Fludarabine, Cyclophosphamide, Dexamethasone. The patient previously failed G-CSF alone mobilization and chemotherapy mobilization. In this time the patient received G-CSF as a subcutaneous dose of $10 \mathrm{mg} / \mathrm{kg}$ each morning for 4 days. At approximately 22.00 hrs on the fourth day of treatment they were given a subcutaneous dose of $240 \mathrm{mg} / \mathrm{kg}$ Plerixafor. On the morning of the fifth day G-CSF was administered and apheresis began at approximately $10 \mathrm{~h}$ after the Plerixafor dose. Administration of G-CSF, apheresis and administration of Plerixafor were repeated daily until seventh day. Peripheral blood sample at baseline and before each apheresis was obtained. Using four-color flow cytometry (with antibodies CD 34, CD3, CD4, CD8, NK, NKT) the populations cell mobilized with plerixafor and G-CSF was measured.

Results: We obtained in the baseline and the median of peripheral blood sample of the 3 apheresis CD3 50.1/58.2 CD4 18.3/22.1 CD8 26.3/27.0 NK 2.1/3.2 NKT 1.9/3.2 and CD34 $1.7 / 16.2$ cell/ $\mu \mathrm{L}$ respectively. We collected $6.1 \times 10^{6} \mathrm{CD} 34$ cell/ $\mathrm{kg}$ in three apheresis, obtaining subpopulation cell count CD3+ 113.3, CD4 + 73.3, CD8 + 36.0, NK+ 22.4, NK T $10.1 \times 10^{6}$. Conclusion: We obtained an adequate account of CD34 with low total lymphocyte subpopulations mobilized with the scheme Plerixafor and G-CSF.

\begin{tabular}{|ccc|}
\hline CELLS & BASAL & MEDIAN \\
\hline CD3 & 50.1 & 58.2 \\
\hline CD4 & 18.3 & 22.1 \\
\hline CD8 & 26.3 & 27.0 \\
\hline NK & 2.1 & 3.2 \\
\hline NKT & 1.9 & 3.2 \\
\hline CD34 & 1.7 & 16.2 \\
\hline
\end{tabular}
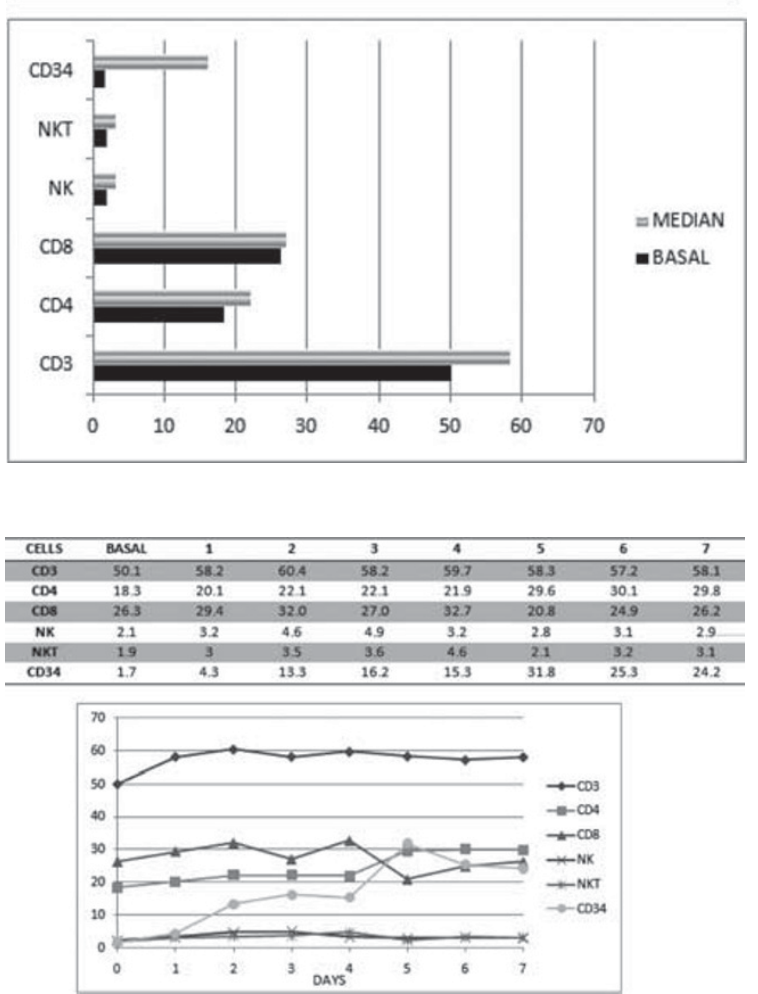

P1021

Bioequivalence of a biosimilar filgrastim and reference filgrastim: impact on the mobilization of CD34+ peripheral blood progenitor cells in a randomized, phase I study C.F. Waller (1), M.H. Bronchud (2), S.J. Mair (3), R. Challand (4) (1)Medical Iniversity Clinic Freiburg (Freiburg, DE); (2)Genral Hospital Granollers (Barcelona, ES); (3)Charles River Clinical Services (Edinburgh, UK); (4)Hospira UK Ltd (Royal Leamington Spa, UK)

Objectives: To demonstrate the bioequivalence of Hospira filgrastim (HF) and Amgen filgrastim (AF) with respect to the mobilisation of CD34 + peripheral blood progenitor cells (PBPCs).

Methods: Healthy, male or female subjects aged $18-50$ years were enrolled in a randomised, double-blind phase I study. Subjects were first randomised to $5 \mathrm{mcg} / \mathrm{kg}$ or $10 \mathrm{mcg} / \mathrm{kg}$ filgrastim dose groups, before further randomisation to order of agent administration. Subcutaneous injections of HF or AF were administered under double-blind conditions on five consecutive days (days 1-5), with crossover to the alternative agent after a washout period of $\geq 13$ days. Blood samples were taken at day 1 (pre dose), day 3 (6 h post dose), day 5 (6 h post dose), day 7 (48 h post dose) and day 10 (120 $\mathrm{h}$ post dose). Mean CD34 + cell counts were evaluated by flow cytometry. Bioequivalence was assessed using a mixed effects analysis of variance model. Bioequivalence was concluded if the $90 \%$ confidence intervals $(\mathrm{Cl})$ for the ratio of 'test' (HF) to 'reference' (AF) mean CD34 + cell counts at day 5 were within the conventional bioequivalence limits of $0.80-1.25$.

Results: Twenty-four subjects were randomised to the $5 \mathrm{mcg} / \mathrm{kg}$ group and 26 to the $10 \mathrm{mcg} / \mathrm{kg}$ group. At both doses, CD34 + cell counts were similar with $\mathrm{HF}$ and $\mathrm{AF}$ across all time points. Regardless of agent or dose, mean CD34 + cell count at day 1 was $2.2-2.8 \mathrm{cells} / \mathrm{microl}$, increasing to a maximum count at day 5 . In the $5 \mathrm{mcg} / \mathrm{kg}$ group, mean CD34+ cell count at day $5(n=24)$ was 47.2 cells $/ \mathrm{microl}(95 \% \mathrm{Cl}: 36.1,61.7)$ with $\mathrm{HF}$ and 46.0 cells/microl $(95 \% \mathrm{Cl}: 33.6,63.0)$ with $\mathrm{AF}$. In the $10 \mathrm{mcg} / \mathrm{kg}$ group, mean CD34 + cell count at day 5 $(n=23)$ was 81.9 cells/microl $(95 \% \mathrm{Cl}: 64.5,104.0)$ with $\mathrm{HF}$ and 77.5 cells/microl $(95 \% \mathrm{Cl}: 59.4,101.3)$ with AF (Figure 1). At both doses, $90 \% \mathrm{Cls}$ for the ratios of test to reference mean CD34 + cell counts at day 5 were within the predefined range required to demonstrate bioequivalence. The incidence of adverse events (AEs) was slightly lower with HF than with $\mathrm{AF}$ at both doses $(5 \mathrm{mcg} / \mathrm{kg}, 79$ vs. $83 \% ; 10 \mathrm{mcg} / \mathrm{kg}, 77 \mathrm{vs}$. $92 \%)$. The most frequently reported AEs were back pain (38$62 \%)$ and headache $(44-58 \%)$, which could be treated with standard analgesics.

Conclusions: Hospira filgrastim and Amgen filgrastim are similar in their ability to stimulate mobilisation of CD34 + PBPCs. The bioequivalence of these agents should enable Hospira filgrastim to be used without the need to adapt PBPC harvesting protocols.

Figure 1. Mean CD34+ cell counts (with $95 \%$ confidence intervals) following administration of Hospira filgrastim $10 \mu \mathrm{g} / \mathrm{kg}$ or Amgen filgrastim $10 \mu \mathrm{g} / \mathrm{kg}$

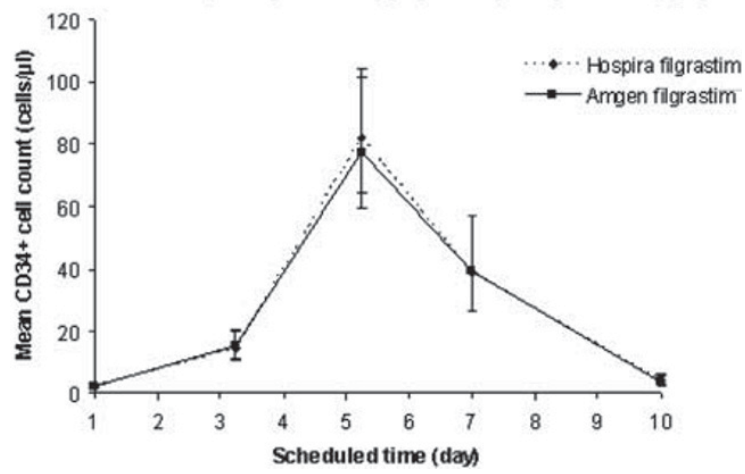




\section{P1022}

Predictability of stem cell mobilization following GCSF plus/minus plerixafor based on day 4 peripheral blood CD34 + cell levels: basis for a pre-emptive model of plerixafor use in patients with lymphoma and myeloma who mobilize poorly

R.F. Duarte (1), G. Martin-Henao (2), A. D'Addio (3), J. Gayoso (4), B.E. Shaw (5), N.H. Russell (6), R. Lemoli (3), N. Worel (7) (1)Institut Catalá d'Oncologia (Barcelona, ES); (2)Banc de Sang $i$ Teixits (Barcelona, ES); (3)Instituto de Ematologia "Lorenzo e Ariosto Seragnoli" (Bologna, IT); (4)Hospital General Universitario Gregorio Maranon (Madrid, ES); (5)Royal Marsden Hospital (Sutton, UK); (6)Nottingham University Hospital (Nottingham, UK); (7)University Hospital of Vienna (Vienna, AT)

Plerixafor has been recently approved in Europe to enhance mobilisation of peripheral blood stem cells (PBSC) in combination with GCSF in patients with lymphoma and multiple myeloma who mobilise poorly. Following approval, clinical algorithms tailored to local institutions are being developed to appropriately manage these patients. One of such possible algorithms is a preemptive model of plerixafor use, which takes advantage of the easy applicability of the drug in the evening before planned apheresis and relies on PB CD34 cell counts on day 4 of GCSF as a trigger. We have identified 38 patients (20 myeloma, 18 lymphoma; 23 men; age 56, 33-67) with suboptimal PB CD34 cell levels on day 4 prior to plerixafor treatment $(C D 34 / u L<5, n=17 ; 5-10, n=15$; $10-20, n=6)$ and compared them with 175 controls from the same period of time (57 myeloma, 118 lymphoma; 92 men; age $59,22-70$ ) with day 4 CD34 level monitoring who did not receive plerixafor treatment $(\mathrm{CD} 34 / \mathrm{uL}<5, \mathrm{n}=71 ; 5-10, \mathrm{n}=39$; $10-20, n=65)$. Plerixafor administration on day 4 increased the PB CD34 level 5.9-fold $( \pm 3.9)$, even for the subgroup with $<5 \mathrm{CD} 34 / \mathrm{uL}$ (6.4-fold \pm 3.7$)$, and overall, enhanced PBSC mobilisation, in keeping with results from registration trials. In particular, patients with $<10$ CD34 + cells/uL on day 4 , who are more likely to mobilize poorly, collected higher numbers of PBSC with plerixafor compared to GCSF alone in the first apheresis (2.3 vs. 0.7 CD $34 \times 10^{6} / \mathrm{kg}$; $P<0.001)$ and overall (3.4 vs. 2.1 CD34 $\left.\times 10^{6} / \mathrm{kg} ; P<0.001\right)$. Even in patients with baseline PB CD34 levels $<5 / \mathrm{uL}$, plerixafor provides a CD34+ yield of $1.5 \pm 0.9 \times 10^{6} / \mathrm{kg}$ in the first apheresis, and $2.8 \pm 1.3 \times 10^{6} / \mathrm{kg}$ overall, successfully achieving $\geq 2 \times 10^{6} / \mathrm{kg}$ CD34 cells $/ \mathrm{kg}$ in $76 \%$ of these pacients. Twenty six patients $(68 \%)$ have undergone autologous HSCT so far, with a median CD34+ cell dose of $2.9(1.5-5.6) \times 10^{6} / \mathrm{kg}$. All patients engrafted neutrophils $>0.5 \times 10^{9} / \mathrm{L}$ at median day +12 $(9-30)$ and platelets $>20 \times 10^{9} / \mathrm{L}$ at median day $+14(9-38)$, with no cases of secondary graft failure. These results set the ground for a preemptive model of plerixafor use based on day four PB CD34 + cell count monitoring. This strategy may allow centers to identify patients, even those with very low PB CD34 counts, for whom the benefit derived from the enhanced mobilization capacity of plerixafor can be maximized, improving the success of first-line mobilisations and avoiding treatment delays and costs derived from secondary attempts.

\section{P1023}

Stem cell mobilization using out-patient ICE and single dose of pegfilgrastim is associated with inferior mobilization outcomes

V. Antonenas (1), F. Garvin (1), K. Yehson (1), B. George (2), M. Hertzberg (2), D. Gottlieb (2)

(1)Sydney Cellular Therapies Laboratory, Westmead (Sydney, AU); (2)Blood and Marrow Transplant Unit, Westmead Hospital (Sydney, AU)

Aim: This pilot study was aimed at assessing the feasibility of mobilization using outpatient fractionated ifosfamide, carboplatin and etoposide (ICE) as salvage therapy along with a sin- gle dose of pegfilgrastim in patients with relapsed or refractory Hodgkin's and non-Hodgkin's lymphoma.

Methods: Between 2005 and 2008, 10 patients (5 Hodgkin's and 5 diffuse large cell lymphoma) underwent salvage therapy with ICE and 9 with evidence of responsive disease received pegfilgrastim (6 mg SC) on Day 4 following the 3rd cycle of ICE towards stem cell mobilization. Stem cells were harvested once the peripheral blood CD34 dose was $>20 /$ ul.

Results: Six patients (66.6\%) successfully mobilised at a median of 31 days (range: 22-36) from administration of pegfilgrastim while 3 had failure of mobilization. The median peripheral blood CD34 on the day of harvest was 31.5/ul (range: 22-38) while a median dose of $3.1 \times 10^{6} \mathrm{CD} 34 / \mathrm{kg}$ (range: 2.6 to 4.1) was harvested. Four of the six patients required 2 harvests to obtain an adequate cell dose. Compared with a retrospective cohort of patients mobilised with ICE chemotherapy and G-CSF (5-10 ug/kg/day), mobilization results were clearly inferior (Table 1). Of the 3 patients with mobilization failure, 2 were mobilised with AMD3100 and 1 with Cyclophosphamide/ G-CSF. All patients underwent autologous stem cell transplant with median neutrophil engraftment of 15 days (range: 11-21) and platelet engraftment of 19 days (range: 13-35). At a median follow up of 12 months (range: $6-37$ ), eight patients $(80 \%)$ are alive.

Conclusion: Stem cell mobilization using ICE chemotherapy and pegfilgrastim has inferior mobilization characteristics compared with ICE chemotherapy and daily filgrastim. In patients who had successful mobilization, neutrophil and platelet engraftment following autologous SCT was prompt and not delayed.

Table1: Mobilization data of patients mobilized using different mobilization regimens

\begin{tabular}{|l|c|c|c|}
\hline & $\begin{array}{c}\text { ICE with } \\
\text { Pegfilgrastim }\end{array}$ & $\begin{array}{c}\text { ICE with G-CSF } \\
(5 \mathrm{ug} / \mathrm{kg})\end{array}$ & $\begin{array}{c}\text { ICE with G-CSF } \\
(10 \mathrm{ug} / \mathrm{kg})\end{array}$ \\
\hline $\begin{array}{l}\text { Mobilization } \\
\text { success (\%) }\end{array}$ & $66.6 \%$ & $100 \%$ & $87.5 \%$ \\
\hline $\begin{array}{l}\text { Median time to } \\
\text { harvest (days) }\end{array}$ & $\begin{array}{c}31 \\
(22-36)\end{array}$ & $\begin{array}{c}14 \\
(10-20)\end{array}$ & $\begin{array}{c}14 \\
(12-18)\end{array}$ \\
\hline $\begin{array}{l}\text { Median peripheral } \\
\text { blood CD34(/ul) }\end{array}$ & 31.5 & 44 & 115 \\
\hline $\begin{array}{l}\text { Median number of } \\
\text { harvests }\end{array}$ & $\begin{array}{c}(22-38) \\
(1-2)\end{array}$ & $\begin{array}{c}11-427) \\
(1-3)\end{array}$ & $\begin{array}{c}25-1159) \\
(1)\end{array}$ \\
\hline $\begin{array}{l}\text { Median cell dose }(\mathrm{x} \\
\left.10^{6} \mathrm{CD} 34 / \mathrm{Kg}\right)\end{array}$ & 3.1 & 5.0 & 5.1 \\
\hline
\end{tabular}

\section{P1024}

Plerixafor for PBSC mobilization in myeloma patients with advanced renal failure: safety and efficacy data in a series of 12 patients from the U.S. or European Compassionate Use Programmes, and from the U.S. post-licensing

K. Douglas (1), P. Hayden (2), A. Rahemtulla (3), A. D'Addio (4), K. Rao (5), M. Maris (6), A. Pagliuca (7), J. Uberti (8), C. Scheid (9), R. Noppeney (10), T. Balasubramaniam (11), G. Cook (11)

(1)Beatson West of Scotland Cancer Centre (Glasgow, UK); (2)University College Hospital (Galway, IE); (3)Hammersmith Hospital (London, UK); (4)University Hospital of Bologna (Bologna, IT); (5)North Carolina Cancer Hospital (Chapel Hill, US); (6)Rocky Mountain Blood \& Marrow Transplant Program (Denver, US); (7)King's College Hospital (London, UK); (8)Barbara Ann Karmanos Cancer Institute (Detroit, US); (9)University of Cologne (Cologne, DE); (10)University Hospital of Essen (Essen, DE); (11)Leeds Teaching Hospitals (Leeds, UK)

We present 12 patients with myeloma from 11 centres, all dialysis-dependent at diagnosis. For 11 patients, plerixafor was used after one or (in one case) two initial attempts at PBSC mobilisation had been unsuccessful $(n=7)$ or only partially successful ( $n=4$; median PBSC dose collected prior to plerixafor $\left.1.54^{*} 10^{6} / \mathrm{kg}\right)$. Initial PBSC mobilisation used G-CSF only $(n=7)$ or cyclophosphamide plus G-CSF $(n=4)$. Median peak peripheral CD34 + count achieved during initial mobilisation (prior to plerixafor) was 9.5 per microlitre. For one patient, plerixafor was used first-line. 
Ten patients received plerixafor along with G-CSF 10 micrograms $(\mathrm{mcg}) / \mathrm{kg} /$ day but without prior chemotherapy; 2 patients received plerixafor following cyclophosphamide $2 \mathrm{~g} / \mathrm{m}^{2}$ with G-CSF. For 6 patients, plerixafor was dose-reduced to $160 \mathrm{mcg} /$ $\mathrm{kg} / \mathrm{day}$, with a subsequent dose increase in one case to $240 \mathrm{mcg} /$ $\mathrm{kg} /$ day due to poor response. The remaining 6 patients received $240 \mathrm{mcg} / \mathrm{kg} /$ day. For patients on haemodialysis, 9 remained on their usual thrice-weekly schedule with plerixafor administered in the evening post-dialysis; 2 patients had dialysis immediately following apheresis over and above their usual thrice-weekly schedule.

Following plerixafor administration, all patients underwent apheresis. Median Day 1 peripheral CD34+ count was 36 per microlitre. A median CD34 + cell dose of $2.95^{*} 10^{6} / \mathrm{kg}$ was achieved after $1(n=2), 2(n=7)$ or $3(n=3)$ aphereses. Only 1 patient failed to achieve a sufficient cell dose for transplant: she subsequently underwent delayed re-mobilisation using G-CSF with plerixafor 240 micrograms $/ \mathrm{kg} /$ day, resulting in a CD34+ dose of $2.12^{*} 10^{6} / \mathrm{kg}$. One patient suffered immediate plerixafor-related toxicity (nausea).

One patient died of sepsis at 75 days post-plerixafor, without undergoing transplant: the sepsis did not appear to be plerixafor-related. 2 patients were not transplanted due to concerns over fitness $(n=1)$ or disease progression ( $n=1$; subsequently responded to bortezomib): both remain alive. The remaining 9 patients have undergone transplant. One patient died of sepsis at Day +33 , though neutrophil engraftment was achieved; the remaining 8 transplanted patients are alive without disease progression at a median of 11 months from plerixafor administration. Two patients recovered endogenous renal function post-transplant; a third achieved CR from myeloma and underwent successful renal transplant. Plerixafor is highly effective in mobilising PBSC in this difficult patient group.

\section{Graft engineering}

\section{P1025}

No impact of long-term storage on engraftment potential of haematopoietic progenitor cells stored at $-80^{\circ} \mathrm{C}$ after uncontrolled rate freezing: a preliminary report from 2 European transplant centres

L. Calvet (1), G. Detry (2), A. Cabrespine-Faugeras (1), N. Straetmans (2), C. Ravoet (2), H. Petre (2), B. Husson (2), N. Boiret-Dupré (1), E. Merlin (3), C. Paillard (3), P. Gembara (3), M. Berger (1), J.O. Bay (1), O. Tournilhac (1), A. Delannoy (2), P. Halle (3)

(1)CHU(Clermont-Ferrand, FR); (2)Centre Hospitalier (Jolimont, BE); (3)INSERM CIC 501, CHU (Clermont-Ferrand, FR)

Controlled rate freezing and storage of HPC in liquid nitrogen are considered standard procedures in the setting of autologous transplantation. Uncontrolled rate freezing and storage of hematopoïetic stem cells in a $-80^{\circ} \mathrm{C}$ freezer are less complex and expensive, but not used as routine technic mostly due to their unknown long-term viability. We therefore examined if the engraftment of stem cells frozen at $-80^{\circ} \mathrm{C}$ in $3.5-5 \%$ DMSO was affected by their storage duration.

Six hundred seventy two autologous transplants from two european transplant units between 1995 and 2008 were performed in 573 patients, aged from 1 to 82 years (median 52.6 years), from our 2 transplant units. Diagnosis were myeloma $(n=231)$, lymphoma $(n=222)$, solid tumor $(n=155)$, acute leukemia $(n=43)$, chronic leukemia $(n=15)$ and "uncommon" disorders $(n=6)$. The median storage time was 56 days (3-4148) with 570 autografts cryopreserved less than 6 months, 41 between 6 to 12 months and 61 more than 12 months.

Age and sex had no impact on hematological recovery to $0.5 \times 10^{9} / /$ neutrophils and to $20 \times 10^{9} / /$ platelets, while diagno- sis, number of CD34 + cells infused, and use of growth factors, correlated with early blood cells recovery. Otherwise, diagnosis impacted on neutrophil recovery $(P=0.002)$, number of CD34 + cells infused correlated both with neutrophil and platelet recovery $(P<0.0001$ and 0.001 , respectively). Moreover, growth factors use was associated both with neutrophil $(P=0.03)$ and platelet recovery $(P=0.0001)$.

Median time to reach $0.5 \times 10^{9} / /$ neutrophils was 11 [0-299] versus 10 [0-18] versus 11 [0-46] days for storage time of less than 6 months, between 6 to12 months and more than 12 months respectively ( $P=0.08$, Kruskal-Wallis test). Similarly, storage time had no impact on platelet recovery $(P=0.12)$ : with median times of 12 [0-318] versus 11 [0-47] versus 12 [4-120] days respectively.

These data suggest that uncontrolled rate freezing and longterm storage of stem cells at $-80^{\circ} \mathrm{C}$ even beyond several years does not alter their engraftment potential. This supports the use of this inexpensive and easy technique, especially in the context of limited financial and technological resources.

\section{P1026}

Ex vivo expansion of regulatory T-cells is enhanced by the inhibition of Fas-induced apoptosis

W. Zhang (1), R. Danby (2), S. Naid (1), A. Peniket (3), D. Roberts (1)

(1)NHS Blood and Transplant Centre (Oxford, UK); (2)Nuffield Dept of Clinical Laboratory Sciences (Oxford, UK); (3)Churchill Hospital (Oxford, UK)

Optimal methods for ex vivo expansion of Tregs may lead to novel therapies for GVHD. We have examined the importance of Fas-FasL interaction in Treg growth. Tregs were enriched from PBMCs using CD25 magnetic beads. The proportion of Tregs in PBMCs was $3.6 \%$ FOXP3 + cells out of CD3 + CD 4 + . Three sequential purification on magnetic columns yielded CD25Pos 1 50.2\%; CD25Pos2 66.5\%; CD25Pos3 78.8\% respectively. High purity was offset by lower Treg recovery: CD25Pos1 $17.7 \%$; CD25Pos2 7\%; CD25Pos3 4.5\% respectively. The CD25Pos2 population was thus identified as optimal for subsequent expansion (Exp). Two rounds of expansion (Exp-1 and Exp-2) were performed using anti-CD3/CD28 beads. Exp-1 gave an 11-fold and Exp-2 a 21-fold expansion in cell number. The highest levels of Treg purity were found in the Exp-1 (81.4\%) vs. Exp-2 $(67.5 \%)$. The cell number required to suppress MLRs by $50 \%$ were lower in Exp-1 $\left(2 \times 10^{*} 3\right)$ compared to Exp-2 $\left(3.3 \times 10^{*} 4\right)$ $(P<0.01)$, indicating a loss of suppressive function in Exp-2. We assessed Fas and Fas-L expression of expanded cells. Fas$L$ was negative in freshly isolated T-cells. During CD3/CD28 expansion of CD25Pos cells, FasL was mainly expressed intracellularly on CD4 + and CD8 + cells, extracellular expression of FasL detected only on a small percentage of T-cells. However, Fas levels were found to be positively correlated to FOXP3 levels. To assess the functional significance of the graded expression of Fas according to FOXP3 levels, we next measured the sensitivity of the CD25 + cells after Exp-1 to Fas-mediated apoptosis induced by the anti-Fas mAb CH11. $\mathrm{CH} 11$ caused a dose-dependent reduction in $\mathrm{CD} 4+\mathrm{Fas}+\mathrm{FOXP} 3+$ cells $(P<0.01)$ and especially CD4 + Fas + FOXP3Hi cells $(P<0.001)$ whilst concomitantly enriching those with no FOXP3. We also tested if activation-induced apoptosis can be prevented by the Fas-blocking antibody ZB4. We found a significant dosedependent increase in viable CD4 +FOXP3High cells after 24 hours $(P<0.01)$, while the CD4 + FOXP3- percentage remained unchanged; there was also significant $(P<0.05)$ protection of the CD4+FOXP3 + cells by the higher doses of ZB4. Staining for FLICA (a marker of caspase 8 activation) was greatest in cells expressing high levels of FOXP3. FLICA staining was reduced in experimental groups by ZB4 at 0.5 or $1.0 \mathrm{mcg} / \mathrm{ml}$ suggesting dose-dependent protection from Fas/Caspase 8-mediated apoptosis. These studies indicate that reducing apoptosis via Fas-FasL pathways may enhance the ex vivo expansion of Tregs. 


\section{P1027}

Effective CD3 T-cell depletion using the CliniMacs ${ }^{\circledR}$ System to produce peripheral blood progenitor cell products for haploidentical transplantation in $\mathbf{2 3}$ children and adults: the updated Lund experience

J.H. Dykes (1), J. Toporski (2), D. Turkiewicz (2), G. Juliusson (3), A.N. Békássy (2), S. Lenhoff (3), A. Lindmark (3), S. Scheding (3)

(1)Clinical Immunology and Transf. Med. (Lund, SE); (2)Dept. of Pediatric Oncology (Lund, SE); (3)Dept. of Hematology (Lund, SE)

Background: Rigorous depletion of T-lymphocytes in the progenitor cell graft is an absolute prerequisite in haploidentical transplantation. In the current study, we investigated the performance of two CliniMACS direct T-cell depletion programs, the Depletion D2.1 program and the large-capacity program D3.1, which are attractive alternatives to standard stem cell enrichment procedures.

Study design and methods: Peripheral blood progenitor cells were harvested from 23 human-leukocyte-antigen (HLA)haploidentical donors. T-cells labelled with anti-CD3 coated beads were depleted with the CliniMACS device using either the Depletion $2.1(n=23)$ or the D3.1 $(n=13)$ program. If indicated, additional positive CD34-selections $(n=16)$ were performed. Twenty-three patients received T-cell depleted grafts after reduced-intensity conditioning, with a target cell dose of $\geq 5 \times 10^{6} \mathrm{CD} 34+$ cells and $\leq 1 \times 10^{5} \mathrm{CD} 3+$ cells per kilogram of recipient body weight.

Results: The median log10 T-cell depletion rate was significantly better with the D2.1 compared to the D3.1 (log 3.6 vs. $\log 2.3, P<0.05)$ and could be further improved by blocking of non-specific binding using $0.15 \%$ Gammagard immune-globulin (IgG) (log 4.5 and $\log 3.4$, respectively) and surprisingly only to a remarkably lesser extend with $0.15 \%$ Kiovig IgG (log 3.8 and $\log 2.9$, respectively). A negative impact of platelet contamination on T-cell depletion rate was observed.

The D3.1 was superior to the D2.1 $(P<0.05)$ in median recovery of CD $34+$ cells $(90 \%$ vs. $80 \%)$ and in median recovery of CD3-negative cells ( $87 \%$ vs. $75 \%)$. The median processing time/1010 total cells was 0.90 hours (D2.1) and 0.35 hours (D3.1) The transplanted grafts (directly T-cell depleted products \pm CD34 + selected cells) contained a median of $12,9 \times 10^{6} /$ $\mathrm{kg} \mathrm{CD} 34+, 0.90 \times 10^{5} / \mathrm{kg} \mathrm{CD} 3+$, and $18,6 \times 10^{6} / \mathrm{kg} \mathrm{CD} 56+$. Rapid engraftment was achieved in 22 patients. The incidence of acute Graft-versus-host disease was $22 \%$ (grade I/II) and $0 \%$ (grade III/IV)

Conclusion: Our results indicate that non-specific binding of cells to anti-CD3 micro beads considerably impair depletion results. This effect can be overcome by blocking with a carefully selected IgG preparation. We, conclude that both, the D2.1 and the novel D3.1 program enable effective, time-saving large-scale T-cell depletion. Combining direct depletion techniques with standard CD34-selection facilitates the composition of grafts optimized to the specific requirements of the patients.

\section{P1028}

Depletion of CD3 and CD19 positive cells from leukapheresis products with the CliniMACS device

M. Schumm (1), P. Lang (1), W.A. Bethge (2), C. Faul (2),

T. Feuchtinger (1), M. Pfeiffer (1), R. Handgretinger (1)

(1)University Childrens Hospital (Tubingen, DE); (2)Dep. Int. Med. (Tubingen, DE)

Depletion of $T$ and $B$ cells of stem cells is mandatory for allogeneic stem cell transplantation from HLA non identical donors. We summarize our results using the CliniMACS device for depletion of apheresis products intended for transplantation from haplo-identical donors.

Procedure was slightly modified from the original protocol. Cells were washed and stained with antibody reagent in centrifuge tubes instead of bags. Separations were performed using the LS tubing set and CliniMACS program 2.1 with up to three devices run in parallel. Cells were analyzed and stored overnight for separation on day of transplantation or cryopreserved.

252 separations were performed. Apheresis products with $4,50 \pm 2.12 \times 10^{10} \mathrm{MNC}$ were used with a mean of $358 \pm 50 \times 10^{6}$ CD34 + cells. T-cell load was $27.7 \pm 10.7$ percent and B-cells were $4.6 \pm 2.8$ percent in the apheresis product. After depletion, a mean of $0.0082 \pm 0.011$ percent of CD3 + cells could be detected and B-cells, as determined as CD20 + cells were reduced to $0.0028 \pm 0.004$ percent. By this T-cells were depleted by $\log 4.08 \pm 0.44$ and B-cells by log $4.41 \pm 0.89$. Recovery of mononuclear cells was $44.9 \pm 10.5$ percent and recovery of CD34 + cells was $67.64 \pm 16.58$ percent. Recovery of CD56+ NK-cells was $68,61 \pm 45,68$ percent. Viability of cells was $96.8 \pm$ 4.0 percent after separation.

Flow cytometry: Viable T-cells were defined by their cell scatter characteristics of lymphocytes, the absence of CD14 and CD33, negativity for propidium iodide staining and positivity for CD3 and CD4 or CD8, respectively. Furthermore, a sample of depleted cells was spiked with about 3 percent of cells from the positive fraction and the gate for $\mathrm{CD} 3+$ cells was set using the dot plot of this sample. A minimum of $1 \times 10^{6}$ events was acquired for analysis and the gate for detection of CD3+ cells was set first on the CD3 + population in the CD3 + CD19+ positive cell sample.

Detection of residual B-cells could not be performed using standard CD19 antibody, possibly due to the strong internalization of CD19 after the incubation of cells with CD19-reagent. Therefore, CD20 was used for detection instead.

Profound depletion of T-cells could be achieved with the CliniMACS system at a acceptable recovery of stem cells. However, the number of cells per run is limited and the procedure takes more time than CD34 enrichment. Detection of residual T and B cells by flow cytometry is difficult and needs special attention.

\section{Non-haematopoietic stem cells}

\section{P1029}

Galectin-1 contributes to the immunomodulatory properties of human multipotent mesenchymal stromal cells

F. Gieseke, J. Böhringer, R. Handgretinger, I. Müller University Children's Hospital (Tubingen, DE)

Human multipotent mesenchymal stromal cells (MSC) are of clinical interest in allogeneic stem cell transplantation for several reasons including support for haematopoietic stem cells and immunomodulatory effects in graft-versus-host disease (GvHD). In vitro studies have shown that MSC suppress lymphocyte proliferation and effector functions of $\mathrm{T}$ cells and NK cells. However, the underlying molecular mechanisms of these immunomodulatory properties are not completely understood, even though several molecules and mechanisms have been identified. We performed a cDNA array analysis of MSC and found a very high expression of galectin-1 mRNA in MSC. As galectin-1 was described to be a potent inhibitor of $T$ cells and to ameliorate GvHD in a mouse model, it is possibly contributing to the immunomodulatory functions of MSC in this setting. At the protein level we found galectin-1 efficiently translated and expressed intracellularly and on the cell surface of MSC. Furthermore, galectin-1 is released into the supernatant by MSC in high amounts as shown by ELISA. In addition to galectin-1, other members of the galectin family have similar immunodulatory functions. Thus we analysed the expression of different galectins in MSC by RT-PCR and detected expression of galectin-3 and -8 mRNA as well as weak expression of galectin-2, -4 , -9 and -12 mRNA.

In order to demonstrate function relevant and involvement of galectin-1 in the antiproliferative effect on peripheral blood 
mononuclear cells (PBMC) exerted by MSC in vitro, we established a galectin-1 knockdown in MSC using a retroviral transfection system. Therefore we constructed a retroviral vector containing an $\mathrm{H} 1-\mathrm{RNA}$-promotor followed by a small hairpin targeting galectin-1 or control sequences, respectively. Retroviral transfection did not alter immunophenotype or plasticity of MSC. A stable and efficient knockdown of galectin-1 in MSC was achieved as confirmed by immunoblot and ELISA. In functional assays, MSC with a galectin-1 knockdown (MSC-G1-knockdown) showed significantly reduced antiproliferative potential compared to MSC infected with control RNAi sequences. In addition, MSC-G1-knockdown were less efficient in suppressing interferon-gamma production by activated PBMC. Taken together, these data indicate that galectin-1 contributes to the immunomodulatory properties of MSC and furthermore other members of the galectin family with redundant functions may further contribute to this inhibitory pathway.

\section{P1030}

CD146 expression of primary bone marrow MSC progenitor/stem cells is regulated by hypoxia and correlates with in vivo location

A. Tormin (1), O. Li (1), J. Brune (1), S. Scheding (2)

(1)University of Lund (Lund, SE); (2)University Hospital Lund (Lund, SE)

MSC progenitor/stem cells (MSC-PC/SC) are important constituents of the hematopoietic stem cell niche, thus playing an essential role in supporting, maintaining and controlling hematopoiesis. The enrichment of primary MSC progenitors, i.e. CFU-F, has been recently reported using surface markers such as CD271, CD146, GD2, SSEA4, etc. Based on the expression of CD271 and CD146 on primary MSC, we have now identified two bone marrow (BM) subpopulations (CD271 +/CD146 +/CD45- and CD271 +/CD146-/low/ CD45-) that were highly enriched for primary MSC-PC/SC. Both populations coexpressed typical MSC markers (CD90, CD105, PDGFR-alpha, STRO-1), but not GD2, SSEA4, and endothelial markers. Both populations contained all assayable CFU-F at comparable frequencies, they both gave rise to typical cultured MSC (surface markers, differentiation capacity), and their gene expression profile was similar (single cell multiplex PCR for Oct4, Sox2, Nanog, CEBPA, LPIN1, ALPL). Interestingly, confocal microscopy analysis of normal human BM sections showed that reticular CD271/CD146 double positive cells were mainly located adjacent to larger vessels and sinusoids but were also found in the marrow space. In contrast, CD271 single positive cells were primarily found in the endosteal space. These cells were furthermore negative for CD45 in contrast to CD45 coexpressing CD271+/low cells in the marrow space. Thus, expression of CD146 in CD271+/ CD45- cells correlated with localization (primarily endosteal versus primarily perivascular). Moreover, CD146 expression of CD271+/CD146-/low/CD45- cells cultured in normoxia was lower up to the 1st passage when compared with cultures initiated with sorted CD271+/CD146+/CD45- cells. Thereafter however, CD146 expression was comparable. Furthermore, when established MSC (CD146+) were cultured in hypoxia, CD146 expression was clearly downregulated over time, and CD146 was re-expressed again when cells were re-transferred to normoxia. No hypoxia-related changes were observed for other surface markers, such as CD90 and CD271.

In summary, CD271 +/CD146 +/CD45- and CD271+/CD146-/ low/CD45- bone marrow cells are putative primary MSC stem/progenitor populations. Differences in CD146 expression correlated to localization and are caused by differences in oxygen levels. We therefore conclude that CD146 expression allows to distinguish the vary rare primary endosteal niche MSC (CD271+/CD146-/low/CD45-) from vascular niche MSC (CD271+/CD146+/CD45-).

\section{P1031}

Characterization of human ESC cell-derived MSC compared to bone marrow-derived MSC indicates that hES-MSC might be a possible alternative and unlimited cell source for clinical off-the-shelf MSC therapies O. Li (1), A. Tormin (1), J. Brune (1), J. Hyllner (2), S. Scheding (3) (1)University of Lund (Lund, SE); (2)Cellartis AB (Gothenburg, SE); (3)University Hospital Lund (Lund, SE)

Mesenchymal stroma cells (MSC) have a high potential for novel cell therapy approaches in clinical transplantation due to their intriguing properties, e.g. high proliferation and differentiation capacity, stromal support and immune-modulation. Commonly, bone marrow-derived MSC (BM-MSC) are used for clinical MSC cell therapies. However, BM-derived MSC have a restricted proliferative capacity and cultured BM-MSC are heterogeneous and are thus difficult to standardize. Human embryonic stem cell-derived mesenchymal stroma cells (hESMSC) have recently been developed and might represent an alternative and unlimited source of MSC. We therefore aimed to characterize human ES-cell-derived MSC, i.e. the hES-MSC line hES-MP002.5 (Cellartis) and compare its properties with normal human bone marrow (BM) derived MSC. We found that hES-MP002.5 cells have lower yet reasonable CFU-F capacity when compared with BM-MSC $(6+3$ vs. $25+1$ CFU-F per 100 cells). hES-MP cells showed similar surface immunophenotypic properties compared with BM-MSC (flow cytometry): Both cell types were positive for CD105, CD73, CD166, HLA Class I, CD44, CD146 and CD90, and cells were negative for surface markers such as CD45, CD34, CD14, CD31, CD19, and HLA-DR. hES-MP, like BM-MSC, could be differentiated into adipocytes, osteoblasts and chondrocytes upon induction in vitro. In order to test whether MSC were capable of homing to the bone marrow after intravenous injection, hES-MP and BMMSC were infected with GFP expressing lentivirus, and sorted GFP-positive cells were injected intravenously into NOD-SCID mice. GFP-positive cells could not be detected in the bone marrow 24 hours after injection, neither when hES-MP cell were injected, nor-and as expected-when cultured BM-MSC were used. Intra-femoral transplantation experiments of hES-MP into NOD-SCID mice to test for stroma reconstituting capacity as well as LTC-IC experiments to test for in-vitro stroma support are ongoing. Furthermore, characterization of the immunemodulatory properties of hES-MP will be performed. In summary, our experiments conducted thus far demonstrated that MSC derived from hESC have similar biological properties and functions compared with conventional BM-MSC. ES-cell derived MSC might be an attractive and reliable alternative and unlimited source for obtaining MSC for clinical use.

\section{P1032}

Suppression of allogeneic reaction by mesenchymal stromal cells generated from human bone marrow CD271 + mononuclear cells

S. Kuci, Z. Kuci, S. Zircher, S. Koller, S. Huenecke, H. Kreyenberg, U. Koehl, T. Klingebiel, P. Bader University Children's Hospital III (Frankfurt/Main, DE)

Bone marrow mesenchymal stromal cells (MSCs) generated by plastic adherence (PA-MSCs) are able to suppress T- lymphocyte proliferation induced either by cellular or non-specific mitogenic stimuli. Quirici et al. demonstrated that MSCs derived from CD271 + bone marrow mononuclear cells (CD271-MSCs) possess a higher clonogenic and proliferative potential than PA-MSCs (Exp. Hematol. 2002,30:783-791). In the present study, we asked therefore whether these cells possess the inhibitory activity on proliferation of T-cells as a result of allogeneic reaction or mitogen stimulation. In order to study molecular mechanisms which mediate the immunosuppressive effect of CD271-MSCs on the allogeneic reaction, we estimated prostaglandin E2 (PGE2), nitric monoxide (NO) and cytokines in the supernatants of mixed lymphocyte reaction (MLR). 
Our results demonstrated that addition of CD271-MSCs to the double-way MLR on day 0 induced a significant inhibition of alloreactivity on day 6 . This effect was associated with the increased levels of PGE2 and was reversed by addition of indomethacin as a specific PGE2-inhibitor. However, attempts to block this inhibitory effect of CD271-MSCs with an NO synthase inhibitor (L-NAME) had no effect. In addition, in the presence of CD271-MSCs the levels of inflammatory cytokines secreted as a result of allogeneic reaction was significantly decreased. Furthermore, CD271-MSCs at the ratio 1:1 were able to significantly suppress mitogenic response of peripheral blood mononuclear cells.

In conclusion, our data suggest that CD271-MSCs are potent inhibitors of T-lymphocyte proliferation induced either by allogeneic or nonspecific mitogenic stimuli and therefore would be a reasonable alternative to the PA-MSCs for the treatment of patients with a steroid- resistant acute GvHD.

\section{P1033}

A potential role for mesenchymal stem cells in allogeneic bone marrow transplantation for familial haemophagocytic lymphohistiocytosis

M. Abecasis, C. Lobato, I. Ferreira, A. Arsenio, G. Teixeira, N. Miranda, E. Fernandez, F. Costa, J. Cabral Instituto Portugues Oncologia (Lisbon, PT)

Familial hemophagocytic lymphohistiocytosis $(\mathrm{FHLH})$ is a rare autosomal recessive disorder affecting mainly young children, being rapidly fatal if untreated. It is caused by genetic defects that impair T cell-mediated and natural cytotoxicity with a highly stimulated but ineffective immune response. The use of immunossupressive agents has resulted in improvements in survival during the first months after diagnosis, however allogeneic hematopoietic stem cell transplantation (HSCT) is considered the definitive therapy. FLHL patients have high levels of circulating inflammatory and hematopoietic-inhibitory cytokines as a result of T lymphocyte and histiocyte activation that may result in a hypercytokinemia syndrome with considerable mortality in conventional intensity transplant conditioning. Mesenchymal stem cells (MSC) have inherent immunomodulatory properties inhibiting activation of $\mathrm{T}$ lymphocytes and histiocytes and great capacity for secretion of anti-inflammatory soluble mediators. MSC could therefore be advantageously used as an adjunct cell therapy to HSCT in FLHL.

Two patients with FLHL were submitted to allogeneic HSCT supplemented with double infusions of third party MSC after conditioning with busulphan and cyclophosphamide. Both had previously received the $\mathrm{HLH}-04$ protocol with a partial remission. Patient characteristics are summarized in the Table.

$50 \mathrm{ml}$ of MSC donor's bone marrow were obtained under sterile conditions. Ficoll separated mononuclear cells were plated in tissue culture flasks in DMEM-low glucose supplemented with $10 \%$ fetal calf serum (nanofiltered, gamma-irradiated, Hyclone) and incubated at $37 \mathrm{C}$ with $5 \% \mathrm{CO} 2$. At $80 \%$ confluence cells were harvested using Accutase (Sigma) and replated at 3-6 cells $/ \mathrm{cm}^{2}$ until the target dose of $1-26 / \mathrm{kg}$ recipient body weight was obtained (Passage 2-3). Quality control testing included bacterial culture, mycoplasma PCR testing, MSC immunophenotype, morphology and multilineage differentiation ability. All procedures were performed under GMP conditions in a nationally accredited laboratory. Approval of the local ethics committee was obtained.
Cryopreserved MSC were infused iv on day $0,4 \mathrm{~h}$ before donor HSC cells and on day +4 with no toxicity. Hematopoietic engraftment was prompt with fast complete chimerism and no evidence of the hypercytokinemia syndrome nor graft-vs-host disease. These results suggest that MSC may help decrease the regimen related toxicity and support engraftment in HSCT for FHLH.

Table: Patients characteristics
\begin{tabular}{|c|c|c|c|c|c|c|}
\hline $\begin{array}{c}\text { Patient } \\
\text { Sex/ag }\end{array}$ & HSC donot & $\begin{array}{c}\text { MSC donor } \\
\text { Dose } 10 \text { 6/kg }\end{array}$ & Trunsplant & N $>500$ & Chimerism & $\begin{array}{c}\text { Disease } \\
\text { assessment }\end{array}$ \\
\hline F/2y & Brother & $\begin{array}{l}\text { Father } \\
1.5 \\
1.8\end{array}$ & $\begin{array}{c}\text { March } \\
2008\end{array}$ & +14 & $\begin{array}{c}\text { Full donor } \\
+14\end{array}$ & $\begin{array}{c}\text { Normal } \\
\text { NK cells at } \\
6 \text { months }\end{array}$ \\
\hline F/1 y & $\begin{array}{c}\text { Unselated } \\
10 / 10 \text { dllele } \\
\text { matched }\end{array}$ & $\begin{array}{c}3 \text { rd party } \\
1.4 \\
2.1\end{array}$ & $\begin{array}{c}\text { September } \\
2009\end{array}$ & +13 & $\begin{array}{c}\text { Full donot } \\
+13\end{array}$ & NA \\
\hline
\end{tabular}

